\title{
A GUIDE TO \\ EXPERIMENTAL ELEMENTARY PARTICLE PHYSICS LITERATURE (1985-1989)
}

\author{
S.I. Alekhin, V.V. Bazeeva, V.V. Ezhela, B.B. Filimonov, S.B. Lugovsky, \\ A.S. Nikolaev, N.L. Petrova, S.R. Slabospitsky, S.I. Striganov, \\ Yu.G. Stroganov, A.N. Shelkovenko and O.P. Yuschenko \\ Institute for High Energy Physics, Serpukhov, Protvino, Moscow Region, USSR \\ G.P. Yost, A. Rittenberg, F.E. Armstrong, R.M. Barnett, \\ K.H. Simpson, T.G. Trippe, G.S. Wagman, and C.G. Wohl \\ Berkeley Particle Data Group," Lawrence Berkeley Laboratory, \\ 1 Cyclotron Rd., Berkeley, California, 9\{720, USA
}

\begin{abstract}
We present an indexed guide to experimental high energy physics literature for the years 1985-89. No actual data are given, but approximately 3500 papers are indexed by Beam/Target/Momentum, Reaction/Momentum (including the final state), Final State Particle, and Accelerator/Experiment/Detector.

- The Berkeley Particle Data Group is supported by the Director, Office of Energy Research, Office of High Energy and Nuclear Physics, Division of High Energy Physics of the U.S. Department of Energy under Contract No. DE-ACOS76SF00098, and by the U.S. National Science Foundation under Agreement No. PHY86-15529. Partial funding to cover the cost of PDG publications is also provided by an implementing arrangement between the governments of Japan (Monbusho) and the United States (DOE) on cooperative research and development.
\end{abstract}




\section{TABLE OF CONTENTS}

\section{Introduction}

1. Overview . . . . . . . . . . . . 1

2. Scope of this Compilation . . . . . . . . . . . 1

3. The Names of Hadrons . . . . . . . . . . . . 1

4. Using this Compilation . . . . . . . . . . . 3

5. Accessing the IHEP DOCUMENTS Database . . 4

6. Other Related Databases . . . . . . . . . . 4

7. Some History . . . . . . . . . . . . . . . . 7

\section{Indices}

ID/Reference/Title . . . . . . . . . . . . . . 19

Beam/Target/Momentum . . . . . . . . . . . 123

Reaction/Momentum/Data-Descriptor . . . . 153

Particle/Decay . . . . . . . . . . . . . . . . 297

Accelerator/Experiment/Detector . . . . . . . 359

\section{Vocabularies}

Particles . . . . . . . . . . . . . . . . 379

Accelerators . . . . . . . . . . . . . 395

Detectors . . . . . . . . . . . . 397

Data Descriptors . . . . . . . . . . . . 401 


\section{Overview}

This report is a guide to experimental particle physics papers issued during the vears 1985 89. It is based on the DOClMENTS database. maintained on the BDMS/4 sistem under VMS at Serpukhov. The database is accessible from many sites around the world.

Papers covered in this report are those containing neu experimental data. Thus a theoretical paper that extracts new information from an experiment would be included. No actual data are presented in this report. We include papers published or preprinted during the years 1985 through 1989 . Papers appearing earlier may be found in the previous edition of this report.

This Introduction describes how to use this book and the DOCLMENTS database. Section 2 discusses the scope of this compilation. and the sources of information. Section 3 discusses the particle naming scheme we use. Section 4 tells how to use this book. Section 5 tells how to access the DOCLMENTS database on which this book is based. Section 6 lists some other publicly accessible databases. Section 7 gives a short graphical sunmary of the contents of our database.

The bodr of this report is organized as follows: Each paper is referenced by an "ID" giving the first auchor's name and the year of first preprinting or publication. e.g. Smith 84. The first Index is a complete list of all these ID's. each with the title and preprint number and/or publication reference.

Following this are four other Indices. When you find the ID of a paper in any of these indices, you can then find the full reference in the Index of ID's. The Beam/Target/Momentum Index lets you locate papers by beam particle, target particle. and beain momentum (or center-of-mass energy). The Reaction/Momentum/ Data-Descriptor Index, lets you locate papers by both the initial and final state of the reaction. The FinalState-Particle/Decay Index directs you to papers by a specific particle and its decay in the final state of a reaction. Finally, the Accelerator/Experiment/Detector Index ' rganizes papers according to the facility at which the experiment was done.

Following the Indices are four Vocabularies. Our "spelling conventions" for particle names are given $\mathrm{i}: 1$ the Particle Vocabulary. We use the same nomenclature for particles as is used in the "Review of Particle Properties." 2 as explained in Section 3 of this Introduction. and some furcher general rules are given in Section 4 .

Names and abbreviations assigned to accelerators and detectors appear in the Accelerator and Detector Vocabularies. The Data Descriptor Vocabulary contains abbreviations used in the Reaction/Momentum/Data Descriptor Index.

Please bring any errors and unissions you may find to sur attention.

\section{Scope of this Compilation}

The starting point for our compilations is bibliegraphic data from a scan of the literature available at Sorpukhor. Ardditional bibliographic data comes from the SLAC-SPIRES HEP database, a joint project of the SLAC and DESY libraries. Then the INIS database is used to check for completeness. From the full list of papers in these databases. we then select those with experimental data. All decisions are made by a physicist: in cases of uncertainty about the "newness" or "originality" of data. we include the paper.

"Data" means not only the obvious experimentally measured quantities. but also some derived quantities, such as partial-wave phase shifts. We exclude instrumentation papers and st udies of properties of the cosmic-ray flux itself (although cross sections and other properties of reactions or particles measured in cosmic-ray experiments are included). We also exclude papers mostly of interest to nuclear physicists, such as nuclear-level or other nuclear-structure measurements. There are of course "gray" areas: many elementary particle physics experiments measure scattering phenomena off nuclei. Heary-ion experiments are also frequently of interest to elementary particle physicists. In these areas. we generally include papers that report more than just nuclear structure parameters and that involve beam energies above about $1 \mathrm{GeV} /$ nucleon, or that report measurements on light nuclear targets (such as the isotopes of hydrogen, helium. or lithium). Other papers are decided on a case-by-case basis by a physicist.

A separate publication. "Current Experiments in Elementary Particle Physics," LBL-91 Revised (1989) ${ }^{3}$ is also available, covering current, approved experiments at the major world accelerators. It includes a spokesperson index and summaries of fixed-target beams arailable at many accelerators.

\section{The Names of Hadrons}

\subsection{Introduction}

In the 1986 edition of the "Review of Particle Properties," 4 we introduced a new naming scheme for hadrons. The virtues sought after were as follows. The symbols were to be as few and as simple as possible. with those already in common use retained where possible; the symbols were to convey unambiguously the important quantum numbers of the particles they name; and the quark model was to guide the whole scheme, without limiting it. Some compromise between sinplicity and long-established usage was unavoidable.

Changes from older terminology affected main] $y$ the heavier mesons made of $u$. $d$. and $s$ quarks. Otherwise. the only important change was that the $F^{ \pm}$became the $D_{s}^{ \pm}$. None of the lightest pseudoscalar or vector meson mames rhanged. nor did those of the $\bar{c} \vec{c}$ or $b \bar{b}$ mesons (we do. however, $n$ w use $\backslash c$ for the $c \bar{c}$ a states), nor did any of the established baryons. 
We follow custom and use spectroscopic names fo.n.. $\Upsilon(1 S)]$ as the primary name for must of those $4 . \Upsilon$. and $x$ states whose spectroscopic identity is known. Wc continue to use the nominal mass form [e.g. $\Upsilon(9-460)]$ as an alternate or as the primary name when the spectroscopic identity is not known.

\section{2 "Neutral-fiacor" mesons ( $S=C=B=T=0$ )}

Table 1 shows the naming scheme for mesons having the strangeness and all heary-flavor quantum numbers equal to zero. The naming scheme is designed for all mesons, whether ordinary or exotic. First, we assigned names to those states with quant um numbers compatible with being $q \bar{q}$ states. The rows of the Table give the possible $q \bar{q}$ content. The columns give the possible parity/charge-conjugation states, $P C=-+,+-,--$, and ++ ; these combinations correspond one-to-one with the angular-momentum state ${ }^{2 S+1} L_{J}$ of the $4 \vec{q}$ sistem being

$$
{ }^{1}(L \text { even })_{J}{ }^{1}(L \text { odd })_{J} \cdot{ }^{3}(L \text { even })_{J} \text {, or }{ }^{3}(L \text { odd })_{J} .
$$

The entries in the Table give the particle symbol. The spin $d$ is added to the symbol as a subscript except for pseudoscalar and vector mesons. Then the mass is added in parentheses for any meson that decays strongly: however, for the lowest-mass meson resonances. we sometimes shorten the names [e.g.. $\rho$ for $\rho(770)]$.

Table 1. Symbols for mesons in which the strangeness and all heary-flavor quant um numbers are equal to zero.

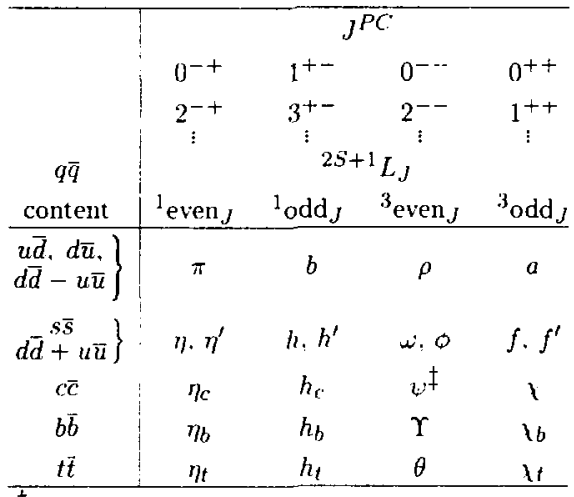

$\mp$ The $J / 2(1 S)$ reme ns the $J / \psi(1 S)$.

Experimental determination of the mass, quark content (where relevant), and quantum numbers $1, J . P$. and $C^{\prime}$ (or $G$ ) of a meson thus fixes its symbol. C'onversely, these properties may be inferred unambignously from the symbol.
If the main simlol camot be assigned becalso the quantum numbers are unknown. $x$ is tosed. Sometimes it is not known whether a meson is mainly the isospin-0 mix of $u \bar{u}$ and $d \bar{d}$ or is mainly s $\bar{s}$ : a prime for symbul of may be used to distinguish two such mixing st ates.

Names have beenl assigned for the anticipated $t \bar{t}$ mesurns.

Gluonium states or other mesons that are not $q \bar{q}$ states are, if the quantum numbers are not exotic. to be named just as the $q \bar{q}$ mesons are named. Such non- $q \bar{q}$ states will probably be difficult to distinguish from $q \bar{q}$ states and will likely mix with them: that is, our scheme makes no at tempt to distinguish the "mostly gluonium" or "mostly $q \vec{q}$ " nature of a particle.

An "exotic" meson with quant um numbers that a $q \bar{q}$ system cannot have, namely $J^{P C}=$ $0^{--} .0^{+-}, 1^{-+} .2^{+-} .3^{-+} . \cdots$, will use the same svmbol as would an ordinary meson that has all the same quantum numbers as the exotic meson except for the $C$ parity: Then a caret or "hat" is added to the symbol. For example. an isospin-1 $0^{--}$meson would be a $\hat{\pi}$, an isospin-0 $1^{-+}$meson would be an $\dot{\omega}$.

The results of this scheme are as follows. None of the lowest-mass pseudoscalar or vector mesons $(\pi, \eta$. and $\eta^{\prime} ; \rho, \omega^{\prime}$. and $\left.\phi\right)$ changed names, nor did any of the $c \bar{c}$ or $b \bar{b}$ mesons (except for $\nmid$ beconing $x_{c}$ ). Established mesons whose names changed slightly are:

\begin{tabular}{|c|c|c|c|}
\hline Old name & New name & Old name & New name \\
\hline$H(1170)$ & $h_{1}(1170)$ & $A_{2}(1320)$ & $a_{2}(1320)$ \\
\hline$B(1235)$ & $b_{1}(1235)$ & $f^{\prime}(1525)$ & $f_{2}^{\prime}(3525)$ \\
\hline$A_{1}(1260)$ & $a_{1}(1260)$ & $\omega(1670)$ & $\omega_{3}(1670)$ \\
\hline$f(1270)$ & $f_{2}(1270)$ & & \\
\hline
\end{tabular}

Established mesons whose names changed completely are:

\begin{tabular}{cc} 
Old name & New name \\
\hline$S(975)$ & $f_{0}(975)$ \\
$\delta(980)$ & $a_{0}(980)$ \\
$D(1285)$ & $f_{1}(1285)$ \\
$\epsilon(1400)$ & $f_{0}(1400)$ \\
$E(1420)$ & $f_{1}(1420)$ \\
$\iota(1440)$ & $\eta(1440)$
\end{tabular}

\begin{tabular}{cc} 
Old name & New name \\
\hline$A_{3}(1670)$ & $\pi_{2}(1670)$ \\
$g(1690)$ & $\rho_{3}(1690)$ \\
$\theta(1720)$ & $f_{2}(1720)$ \\
$X(1850)$ & $o(1850)$ \\
$h(2030)$ & $f_{4}(2050)$
\end{tabular}

Note that the $S(975), D(1285), \epsilon(1300), E(1420)$, $\theta(1690)$, and $h(2030)$ all became $f$ mesons. The new schenic reveals that all have $P C=++$ and are ${ }^{3}(L \text { odd })_{J}$ states.

\subsection{Mesons with nonerwo $S, C . B$, and/or $T$}

A meson with nonzero $S, C, B$, and/or $T$ cannot be an eigenstate of charge conjugation. Alsu. in each such 
meson one of the quarks must be heavier than the other. The naming rules are:

- The main symbol is an upper-case italir 'etter indjcating the heavier quark as follows:

$$
s \rightarrow \bar{K} \quad c \rightarrow D \quad b \rightarrow \bar{B} \quad t \rightarrow T .
$$

- If the lighter quark is not a $u$ or a $d$ quark, its identity is given by a subscript.

- If the spin-parity is in the "normal" series, $J^{P}=$ $0^{+}, 1^{-}, 2^{+}, \ldots$, a superscript "**" is added.

- The spin is added as a subscript unless the meson is a pseudoscalar or a vector.

Thus the pseudoscalar and vector $K, K^{*}, D, D^{*}$, and $B$ mesons did not change names. Established mesons whose names did change were:

\begin{tabular}{ccccc} 
Old name & New name & & Old name & New name \\
\cline { 2 - 4 }$Q_{1}(1270)$ & $K_{1}(1270)$ & & $L(1770)$ & $K_{2}(1770)$ \\
$Q_{2}(1400)$ & $K_{1}(1400)$ & $K^{*}(1780)$ & $K_{3}^{*}(1780)$ \\
$\kappa(1430)$ & $K_{0}^{*}(1430)$ & $K^{*}(2045)$ & $K_{4}^{*}(2045)$ \\
$K^{*}(1440)$ & $K_{2}^{*}(1440)$ & $F$ & $D_{s}$
\end{tabular}

Most notably, the $F$ (the $c \bar{s}$ state) changed to $D_{s}$. However, with the prospect of $B_{s}, B_{C}, T_{s}$, and similar mesons, there was no consistent and simple alternative. The rules can lead to cumbersome symbols, such as $D_{s 2}^{*}$, but such particles are unlikely to be often seen.

\subsection{Baryons}

No change has been made to the symbols $N, \Delta$, $\Lambda, \Sigma, \Xi$, and $\Omega$ that have been used 25 years for the baryons made of light quarks $(u, d$, and $s)$. These symbols indicate the isospin and quark content, as do the symbols used for the baryons containing one or more heavy quarks ( $c, b$, and $t$ quarks). The following system was invented earlier and independently by Hendry and Lichtenberg and by Samios. The rules are:

- Baryons with three $u$ and/or $d$ quarks are $N$ 's (isospin 1/2) or $\Delta$ 's (isospin 3/2).

- Baryons with two $u$ and/or $d$ quarks are $\Lambda$ 's (isospin 0 ) or $\Sigma$ 's (isospin 1). If the third quark is a heavy quark (not an $s$ quark), its identity is given by a subscript. This nomenclature was already used for the $\Lambda_{c}(2285), \Sigma_{c}(2455)$, and $\Lambda_{b}(5500)$.

- Baryons with one $u$ or $d$ quark are $\Xi$ 's (isospin $1 / 2$ ). One or two subscripts are used if one or both of the remaining quarks are heavy: $\Xi_{c}, \Xi_{c c}, \Xi_{b}$, etc.

- Baryons with no $u$ or $d$ quarks are $\Omega$ 's (isospin 0) with subscripts indicating any heavy-quark content.

In short, the total number of $u$ and $d$ quarks together with the isospin determine the main symbol, and subscripts indicate any content of heavy quarks. A $\Sigma$ always has isospin $I$, an $\Omega$ alwavs has isospin 0 , etc.

\section{Using this Compilation}

Each paper is assigned a unique "ID," comprised of the first author's name and the date of the first preprinting or publication. In case of duplicates, we append a letter "B," " $\mathrm{C}$," etc., as in

Jones 84

Jones $84 \mathrm{~B}$

Jones $84 \mathrm{C}$.

The maximum length of the ID is 16 characters, so long author's names are truncated.

All references for the paper corresponding to an ID are given in the ID/Reference/Title Index. When a paper has been preprinted and published, both references are given. In these cases, the year in the ID, which is usually that of the preprint, may not match the year of the published reference. In a very few cases, the first author of the preprint may not be the same as that of the publication, in which case the ID usually reflects the preprint's first author.

To see a paper's full author list or to search for a set of papers by the name of one or more authors, one may query the DOCUMENTS or HEP databases.

The first page of each index explains its use. It is worthwhile to understand a few of our conventions.

- Some "particle" names actuelly represent groups of particles. For example,

* " $\mathrm{X}$ " is used for inclusive measurements or, if used as the only particle in the final state, for total cross-section measurements.

* "(vees)" means zero or more unspecified neutral vees,

* "vee(s)" means one or more unspecified neutral vees, and

* "vees" means two or more of the same.

* "Mult[charged-hadron]" means a collection of reactions for which the multiplicity distribution of charged hadrons has been measured.

* "Inelastic" means a sum over all inelastic final states.

* "Jet" means a jet of particles, treated as a single entity.

* " $0 \gamma$ " means final states in which the occurrence of photons has been excluded.

- In using the computer database, all antiparticles commonly written with a bar over the name are spelled with the letters "BAR" appended to the particle name. Thus, KBAR, LAMBDA/CBAR-, etc.

- Particles tend to be encoded in the same language the experimenters used, leading to some inevitable ambiguity. For example, "charged" in one paper may be called "charged-hadron" in another paper.

- Reactions are listed in the shortest form possible. Identical particles are grouped tngether, so the reaction $\pi^{-} p \rightarrow \pi^{+} \pi^{+} \pi^{-} \pi^{-} \pi^{-} p$ appears as $\pi^{-} p \rightarrow p 2 \pi^{+} 3 \pi^{-}$. 


\section{Accessing the IHEP DOCUMENTS Database}

Anyone who has an account on VXCERN can directly access the IHEP databases, including $D O C U$ MENTS. (See the subsection on 'Databases under VMS (at CERN)' for a list of the other databases.)

Otherwise, remote interactive access can be achieved from other VAXes with DECNET access to VXCERN (where the databases themselves reside). The remote software $(20,000$ blocks) can be obtained from either VXCERN::YGSCD or LBL::PDG.

In the following description, words in Typewriter Font must be typed as given. Only the letters in UPPER CASE are necessary and these must be entered in upper case. Words in italics in brackets $\langle\ldots\rangle$ are "variables" for which the user substitutes an appropriate value, again in upper case.

Access to the IHEP-CERN databases can then be initialized by the system manager (as is done on VXCERN) or by having each user type:

Olisk: [directory. COMPAS. BDMS. COM]BDMSINI

(For example, on the CSA cluster at LBL, substitute DISK\$PHYSICSOO for 'disk' and WAGMAN for 'directory'.) To enter the system and obtain general information, type:

\section{PPDS}

or, in particular (to select DOCUMENTS), type: PPDS DOCuments

- For a short explanation of the database, type: HElpbase

- For a list of database commands, type: ?

- For an explanation of a particular database command, type:

?<command-word>

(e.g., ?FInd, ?HElpbase, ??)

- To see the record structure and names of key data elements for searching, type: FDT

- To browse the index of a key data element, type: INDex, <key data element name> (e.g., INDex, AC)

The following are typical examples of the search command FIND. Notice the use of the '**' to terminate each search statement and the use of the ';' to separate data elements. Previous search results can be combined with a current search by use of 'set numbers':

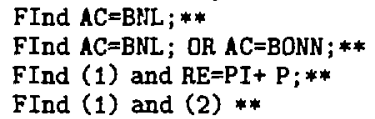

The last example combines the results of two searches labelled SET (1) and SET (2). Notice that ';' is not used when searching for 'SETS'. Each successful search produces a list of all previous SET numbers along with the search command. Enter DIR, to get a list of these SET numbers and commands.

- To do a truncated search:

FInd $\mathrm{DE}=\mathrm{HBC} /$; **

Finds all detectors that begin with HBC.

- To do a string search:

FInd DE/C=BC;**

Finds all detectors that have $B C$ anywhere in the name.

- The following examples are WRONG:

FInd AC BNL;** (Error: no ' $=$ ')

FInd $A C=B N L * *$ (Error: no ';')

FInd $A C=B N L$ OR $A C=B O N N ; * *$

(Error: no ';' after BNL)

FInd $A C=B N L$ OR BONN ; **

(Error: no ';' and no ' $\mathrm{AC}=$ ')

- To see the results of a search with key data element names, type:

LISt

LISt , AC , RE, SC. (for individual data elements)

- Or for an attractive listing, type:

DOcument then Lookfile

- To save the results of a search in a file, type one of the following:

Document

DUmp

PRInt

The results are stored in files DOC.DOC, DOC.DUM, or DOC.PRN respectively. The first file contains a user-friendly listing, the second one contains a highly compressed dump of each record (with data element and value), and the third one is a line-by-line decompressed version of the second file. Another file automatically created, DOC.AUD, contains a history of your commands.

The searchable information includes two groups of key data elements:

BIBLIOGRAPHIC: ID (short code-SC), references, date of document (year), authors and affiliations, and experiment number.

TOPICAL: beam particle, target particle, reaction, particle in the final states of reactions, momentum in initial states, type of data obtained, particle whose property has been measured, accelerator and/or detector, and initial state polarization. It is possible to construct complex queries including any set of key data elements.

\section{Other Related Databases}

\subsection{Databases under VMS (at CERN)}

Large user-friendly databases are now available to anyone with DECNET access to VXCERN by using the commands PPDS DDCUMENTS, PPDS EXPERIMENTS, etc. (See the section on 'Accessing the IHEP DOCUMENTS Database' above.) They are maintained by the Serpukhov COMPAS group and the CERN-HERA group 
with input from the world-wide Particle Data Group collaboration. They are managed by BDMS $/ 4$, a menudriven database managenent system with on-line help information. This system consists of:

- the archival databases DOCUMENTS, EXPERI. MENTS, and REACTIONS,

- the evaluated data compilations PP (Particle Properties) and $C S$ (integrated reaction cross sections). and

- the supplementary database VOCABULARY (the vocabulary used by the other databases).

The DOCUNENTS database contains information extracted from experimental papers (but no actual data). It covers 1974 to the present with earlier papers as far back as 1936 .

The EXPERIMENTS database contains information in the DOCUMENTS format extracted from laboratory proposals. It covers 1961 to the present.

The REACTIONS database contains actual physics data extracted from experimental papers. It covers 1952 to the present.

The $P P$ database contains information from the "Review of Particle Properties" Summary Tables. ${ }^{2}$

The $C S$ database contains data from CERN-HERA, UCRL, and LBL cross-section compilations. All data are double checked. It is regularly updated from the $R E A C T I O N S$ database. It covers 1950 to the present.

These databases (except for $C S$ ) overlap in large part those maintained at SLAC, where they are called $E X$ PERIMENTS, DATAGUIDE, REACTIONS, and PARTICLES, respectively. (See the next subsection.) They are not, however, even when titled the same, identical to the SLAC databases. For example, the $P P$ database contains only the Summary Table information from the "Review of Particle Properties" 2 instead of the Full Listings which are available in the SLAC database PARTICLES. As another example, the DATAGUIDE database at SLAC is out-of-date and will eventually be replaced with data taken from DOCUMENTS.

\subsection{Databases under SLAC-SPIRES}

SLAC and the Berkeley and United Kingdom Particle Data Groups, in collaboration with other groups and institutions, maintain several particle physics databases on SLAC's IBM computer in the SPIRES database management system. For detailed information and examples of their use, see the "User's Guide" 5 available from the Berkeley Particle Data Group, and the "Search Gujde to HEP" available from the Library, SLAC, P.O. Box 4349, Stanford, CA 94309, USA. Or contact Louise Addis at SLAC: ADDISOSLACVM, phone (415) 9262411.

The HEP database contains bibliographic information on particle physics papers (journal articles, preprints, reports, theses, etc.). It covers 1974 to the present, is maintained by the SLAC Library in collaboration with the DESY HEP Index Group and is updated daily. It is searchable by author, institution, title, topic, report number, citation, and other bibli- ographic items. It is used to produce the biweekly "Preprints in Particles and Fields."

The DATAGUIDE database was used to produce the previous edition of this report. ${ }^{1}$ It covers 1976 to 1985 (thus is out-of-date) and will eventually be replaced by a DOCUMENTS database maintained by the Serpukhov COMPAS Group and the Berkeley PDG. It is searchable by reaction, lab momentum, c.m. energy, particle studied. accelerator, detector. and other items. The previous edition ${ }^{1}$ tells how to access and use it.

The PARTICLES database contains the Full Listings from the "Review of Particle Properties," 2 but no Particle Properties Summary Tables or Miscellaneous Tables. Figures. and Formulae. It is maintained by the Berkeley PDG in collaboration with the entire authorship of the "Review." It is updated around April each year. It is searchable by particle and particle property (e.g., mass, lifetime, etc.).

The REACTIONS database contains numerical data on ruactions: differential and total cross sections, structure functions, polarization measurements, and many other items from most current aspects of experimental particle physics. It covers 1978 to the present. It is compiled by the United Kingdom Particle Data Group (University of Durham and Rutherford Appleton Lab) in collaboration with the Serpukhov COMIPAS Group. It is updated approximately annually, and is searchable by first author, reference, reaction, lab moment um, quantity measured, and final-state particle.

The EXPERIMENTS database contains summaries of approved experiments at the major laboratories. It covers approximately 1975 to 1989 , with coverage since 1980 being more complete. It is maintained by the Berkeley PDG in collaboration with correspondents at various labs and is updated periodically. It is searchable by experiment numbur, author, accelerator, detector, reaction, beam momentum, journal paper, and other items. The report "Current Experiments, in Elementary Particle Physics," 3 is produced from it.

The CONF database contains names and dates of past and future conferences of interest to particle physicists.

The HEPNAMES database has electronic-mail addresses of many people working in high-energy physics.

The INST database has addresses (including phone and fax numbers) of high-energy physics institutions.

\subsection{QSPIRES Access to SLAC-SPIRES}

People without a SLAC computing account can use QSPIRES (see 'NOTE' below) to access the databases at SLAC either interactively via BITNET using the 'tell' command ('send', 'bsend', or a similar command on some systems) or using electronic mail.

Here is an interactive search on HEP; the query is refined as QSPIRES sends responses to your screen:

tell QSPIRESGSLACVM FIND TITLE E- E-

(response) 


\section{tell QSPIRES QSLACVM AND Zo}

(response)

tell QSPIRES@SSLACVM AND DATE 1988

(response)

To recejve the search result on your screen $(\leq 10$ records):

tell QSPIRES@OSLACVM OUTPUT (TYPE

To receive the search result instead as electronic mail:

tell QSPIRES@SLACVM OUTPUT PRINT BRIEF

You may combine search criteria in a single command (FIND TITLE HADRON AND PION AND DATE 1988), but the contmand 'OUTPUT PRINT BRIEF' must be separate. Also note that a QSPIRES search defaults to the HEP database. To search another database. like CONF:

tell QSPIRES@SLACVM FIND PLAC: VIENNA (IN CONF

tell QSPIRES@SLACVM OUTPUT PRINT BRIEF

or tell QSPIRES: QSLACVM OUTPUT (TYPE

Or to access the electronic version of the "Review of Particle Properties" (results always being returned as mail):

\section{tell QSPIRESOSLACWM}

EXPLAIN PARTICLES (IN PARTICLES

tell QSPIRESQSLACVM

FIND PP ETA MODES IIN PARTICLES

For the HEPNAMES and INST databases, you may use the special short-cut searches:

tell CSPIRES@SLACVM WHOIS ARMSTRONG.B

tell QSPIRES@SLACVMI WHEREIS FERMILAB

If your system does not support interactive BlTNET communication or is not on the BITNET network, send electronic mail to one of the following:

For BITNET: QSPIRES AT SLACVM

For non-LBL DECNET: LBL::"QSPIRES@SLACVM.BITNET"

For LBL DECNET: ST\%"QSPIRES@̈SLACVM.BITNET"

For Internet: QSPIRES\%SLACVM.BITNET@LBL.GOV

as in the examples above. You must remove the tell QSPIRES@SLACVM' from all messages:

FIND PLACE VIENNA (IN CONF

Each mail message must contain only one line, and the mail 'subject line' must be blank. QSPIRES will send its responses as nuail. For other networks, contact your local system manager.

For more information. you can send electronic mail to HEPNAMIESGSACVMI and request material on the QSPIRES commands. You can get the 'HELP' file by mailing the command 'HELP' to QSPIRES@SLACVM.

- NOTE: Use of QSPIRES is free. Anyone may use the special short-cut searches for the HEPNAMES and INST databases. Other use of QSPIRES requires that your specific conputer node be registered with SLAC: an individual account is not required. Send mail to QSPIGSLACVM for questions about node registration.

\subsection{SPIRES HEP Databases at other Institutions}

SLAC/DESY HEP and several of the other databases mentioned above are available on SPIRES at DESY, KEK. and Kyoto Lniversity, RIFP. Clone copies of HEP are kept current by nightly updates.

Contacts at these institutions are:

DESY Hart mut Preissner (LOOHTP@DHHDESY3);

KEK - Y. Miura (MIURA@JPNKEKVM);

Kitoto L'niversity, RIFP-- K. Aoki (AOKI@JPNRIFP). Kiyoto also operates a 'remote SPIRES' for Japan.

\subsection{The CERN Preprint Database}

CERN maintains a database of high-energy physics preprints, PREP. similar to the SLAC/DESY HEP database. (C'ERN proposes adding journal articles, making their database comparable in scope to $H E P$.) For information on QALICE, a QSPIRES-like facility for accessing this database, contact Maja Gracco (MGR@CERNVM).

The PREP database will also run on an IBN PC (or compatible) using Mlicro C.DS/ISIS, an information storage and retrieval system developed by UNESCO. The system is call MicroPREP and is intended for use in countries without direct access to BITNET or other electronic mail capabilities. For further information, contact Alec Hester, CERN Scientific Information Service, $\mathrm{CH}-1211$ Geneva 23, Switzerland.

\subsection{The Durham-RAL Particle Physics Databases}

These databases contain compilations of experimental particle physics data (e.g., reaction cross sections, polarizations. etc.) and may be searched interactively using VM/CMS on both the Rutherford Appleton Laboratory (RAL) and CERN central computers. The topics include:

- two-body (and quasi-two-body) reactions;

- hadron and photon one- and two-particle inclusive distributions:

- lepton-produced inclusive data (i.e., deep inelastic scattering, structure functions, etc.);

- data from $e^{+} e^{-}$annihilations.

A subset of the SLAC/DESY HEP literature-searching guide (from 1980 onwards) is linked to the reaction data to inform users when new data is available. Also available are the EXPERIMENTS and PARTICLES databases from the SLAC system. (See above.)

The databases run under the Berkeley Database Management System and are menu-driven with full on-line help information for easy use. They can be accessed by anyone having network access to the RAL or CERN computers. For PSS access to RAL, the relevant address is 23422351919169 , then .2) - a guest account, PDG (password HEPDATA), is available at RAL for those without a CMS account. An EXEC file, HEPDATA, resident on the user-disk (UDISK). gives interactive access to the databases. The data are retrieved using simple kevword-based searches, and resulting data records can be listed on the terminal, sent 
to a printer, or transferred to the user's own machine as desired.

To insure that the databases are current. experimentalists are urged to send their data to the compilers as soon as they are available.

For more information or a user guide (1988 edition), contact Mike Whalley at Durham Lniversity. South Rd.. Durham City DH1 3LE. England (MRW Q UKACRL or MRW (CERNVM) or Dick Roierts at Rutherford Appleton Lab. Chilton, Didcot. Oxon. OX11 0QX. England (RGR@LKACRL). At (ERN, user guides may' be obtained from Alec Hester of the CERT library (HES@CERNVM).

\section{Some History}

We present a few figures showing the development of major accelerator centers and a historical perspective of accelerator usage. The presentation is three-fold. Figure 1 presents the total publication rate in worldwide particle physics from the very beginning, as it reflected in the DOCUMENTS and REACTIONS databases. Figures 2 through 5 show cumulative curves of major laboratories' contributions to the literature. Figures 6 through 10 show the evolution of accelerator usage by home experimental groups (i.e., the number of journal papers generated by experiments involving home accelerators divided by the total number of journal papers generated by that laboratory).

\section{REFERENCES}

1. G.P. Yost et al.. "A Guide to Data in Elementary Particle Physics," LBL-90 Revised (1986).

2. Particle Data Group, "Review of Particle Properties." Phy's. Lett. B239 (1990).

3. Particle Data Group. "Current Experiments in Elementary Particle Physics," LBL-91 Revised (1989). See also G. Gidal et al., "Major Detectors in Elementary Particle Physics." LBL-91 Supplement. Revised (1985).

4. Particle Data Group, "Review of Particle Properties," Phys. Lett. 170B (1986).

5. A. Rittenberg et al.. "A User's Guide to Particle Physics Computer-Searchable Databases on the SLAC-SPIRES System." LBL-19173 (1986). 


\section{EXPERIMENTAL PARTICLE PHYSICS}

PUBLICATION RATE

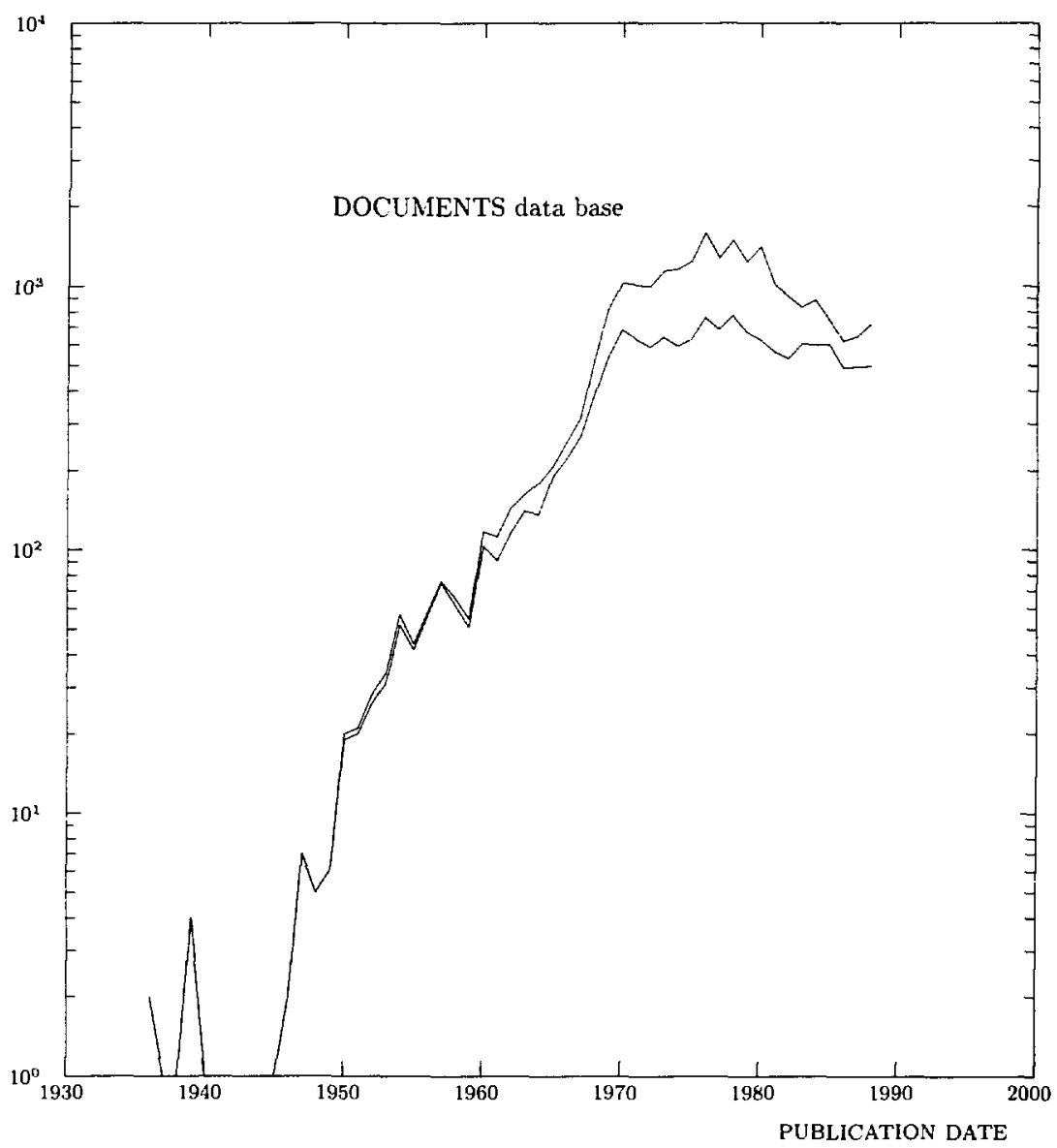

Fig. 1. The number of experimental papers produced each year. The lower curve gives the number of journal papers, and thus doesn't include unpublished preprints. 

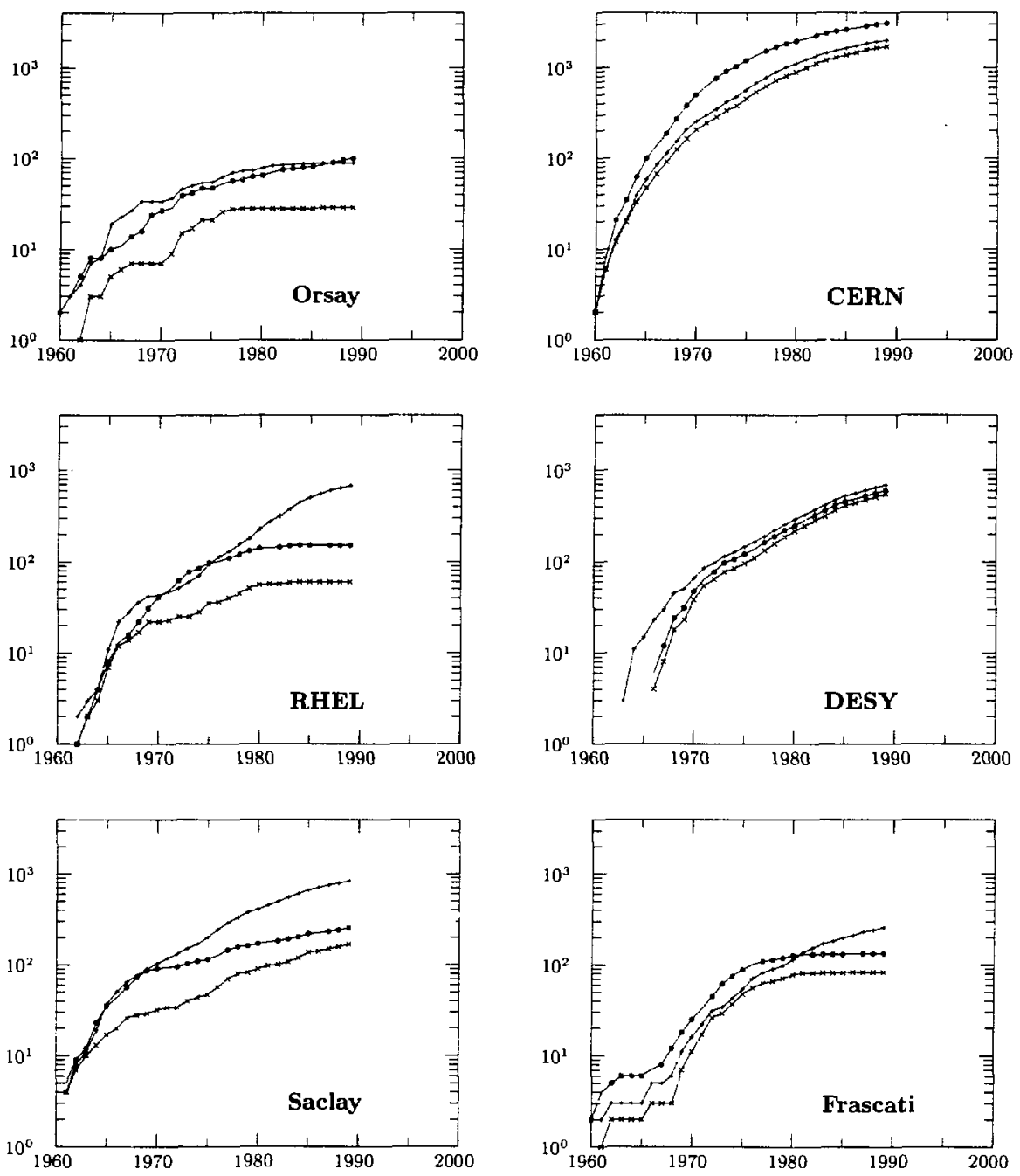

Fig. 2. The cumulative number of papers from major European accelerator laboratories. A + indicates papers with authors from the laboratory, a - indicates papers on data taken at an accelerator at that laboratory, and an $x$ indicates papers both with authors from the laboratory and data from its accelerator(s). 

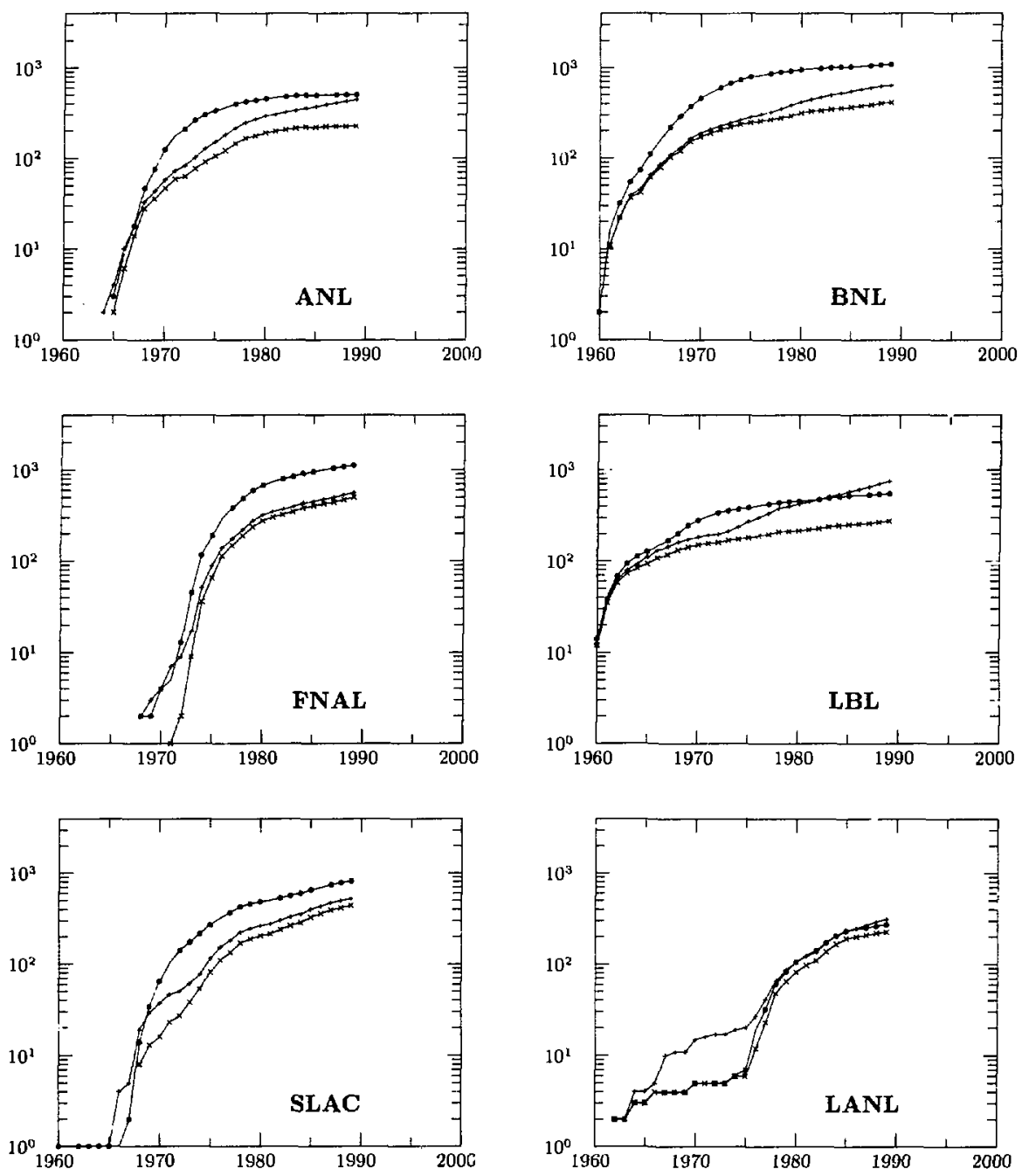

Fig. 3. The cumulative number of papers from major USA accelerator laboratories. A + indicates papers with authors from the laboratory, a * indicates papers on data taken at an accelerator at, that laboratory, and an $x$ indicates papers both with authors from the laboratory and data from its accelerator(s). 

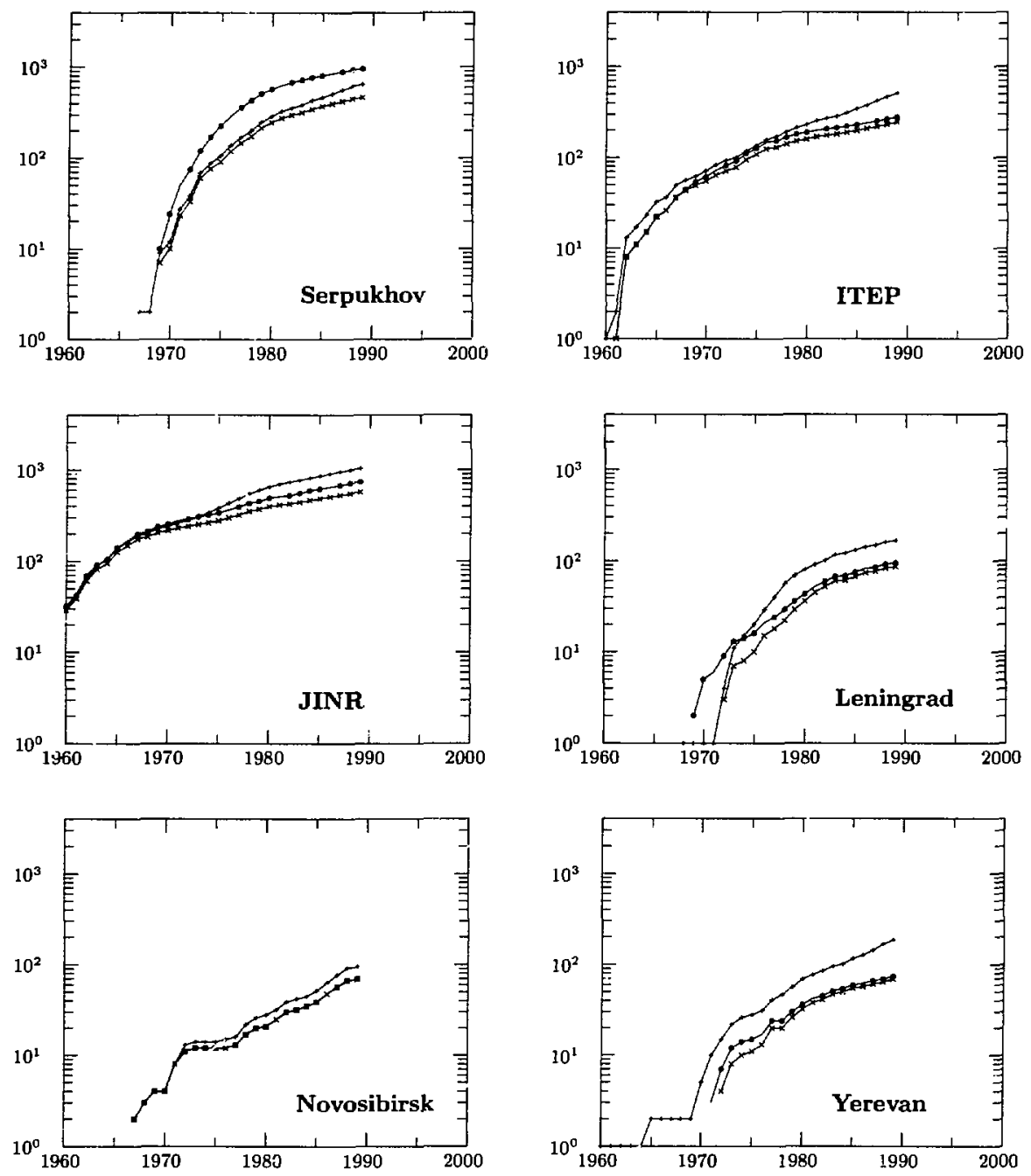

Fig. 4. The cumulative number of papers from major USSR accelerator laboratories. $\mathrm{A}+$ indicates papers with authors from the laboratory, a • indic "es papers on data taken at an accelerator at that laboratory, and an $\times$ indizates papers both with authors from the laboratory and data from its accelerator(s). 

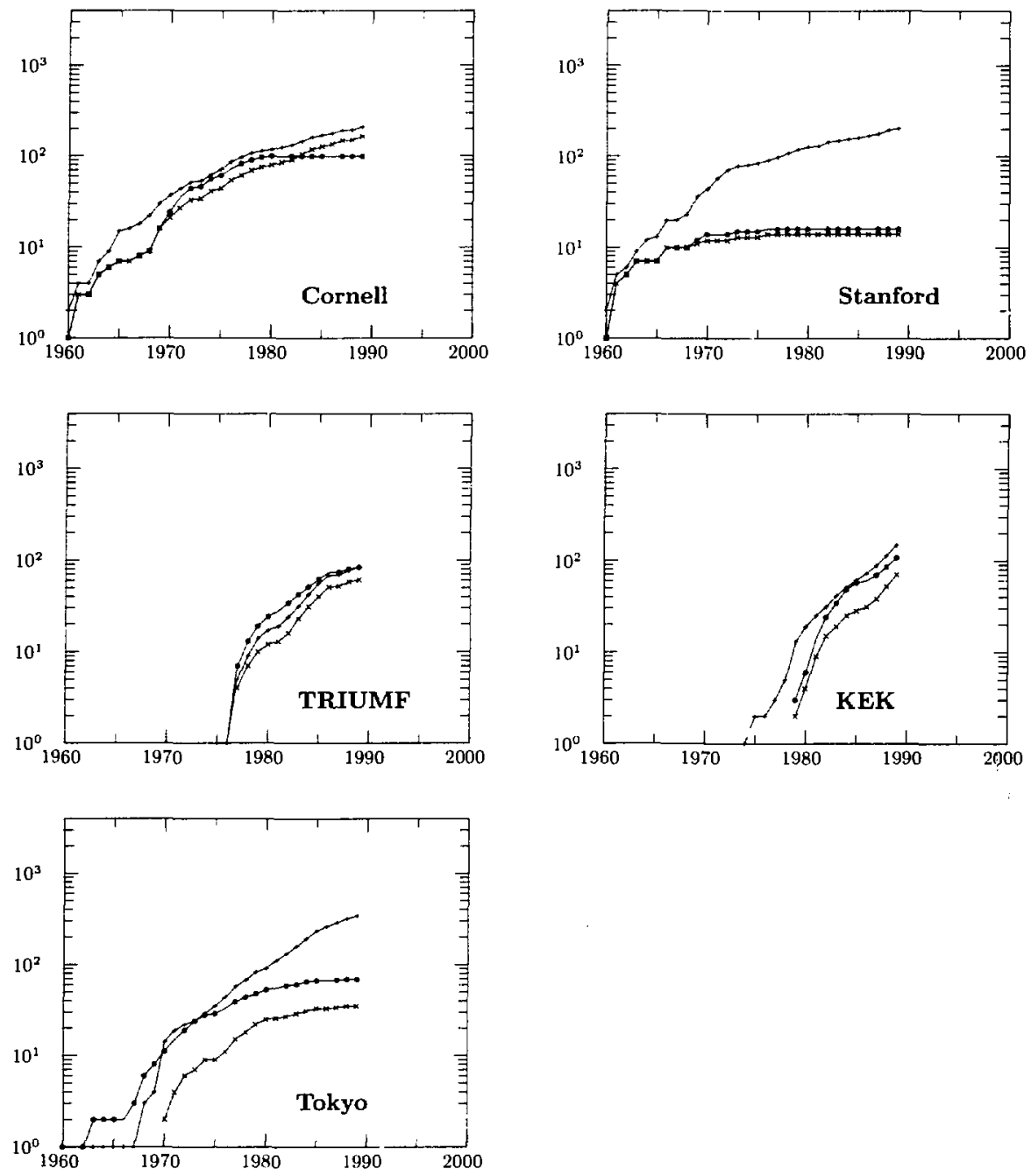

Fig. 5. The cumulative number of papers from some USA, Canadian, and Japanese accelerator laboratories. A + indicates papers with authors from the laboratory, a $\bullet$ indicates papers on data taken at an accelerator at that laboratory, and an $\times$ indicates papers both with authors from the laboratory and data from its accelerator(s). 


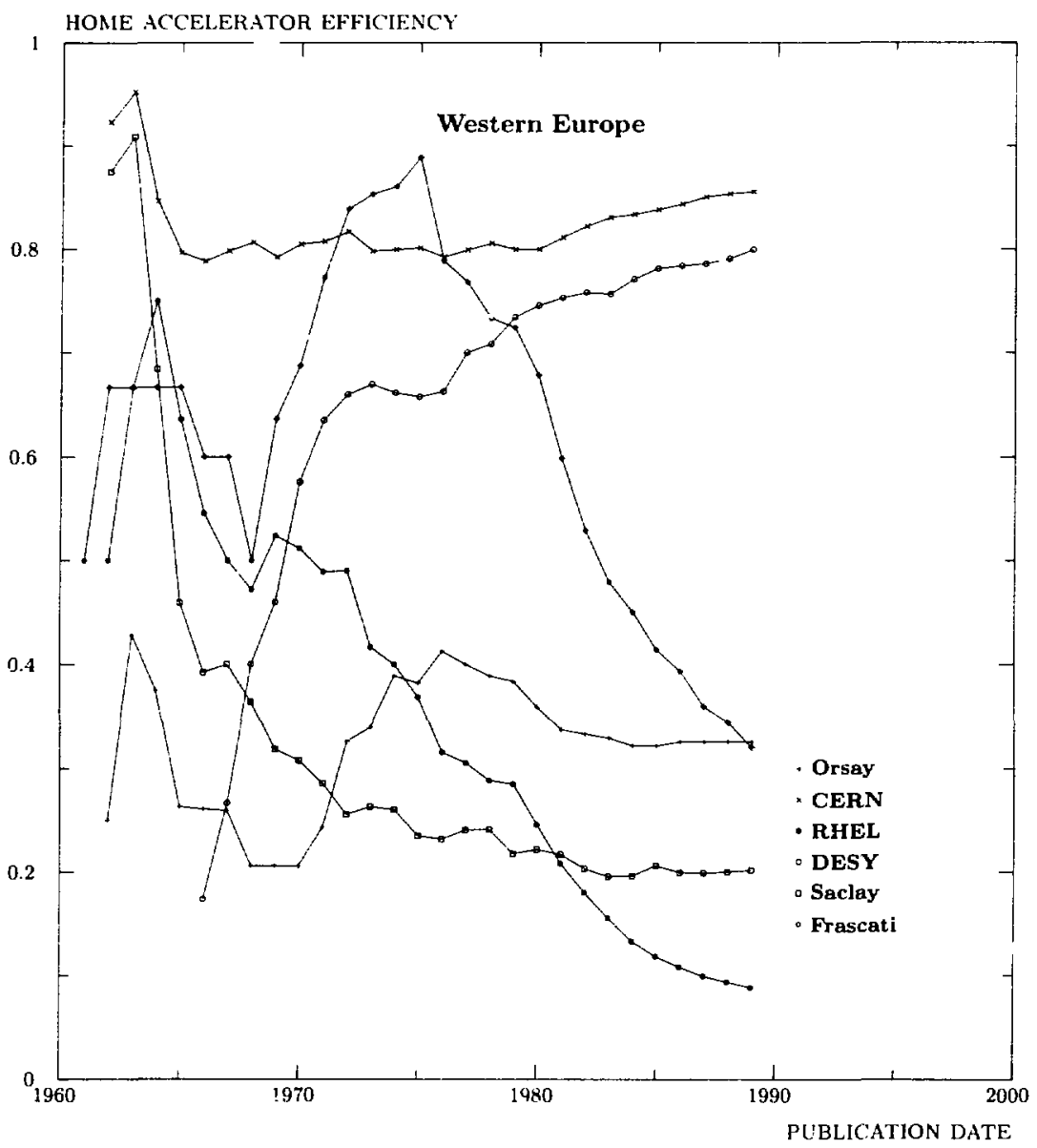

Fig. 6. The fraction of all the experimental papers with authors from a given laboratory that present data taken at an accelerator at that laboratory. 


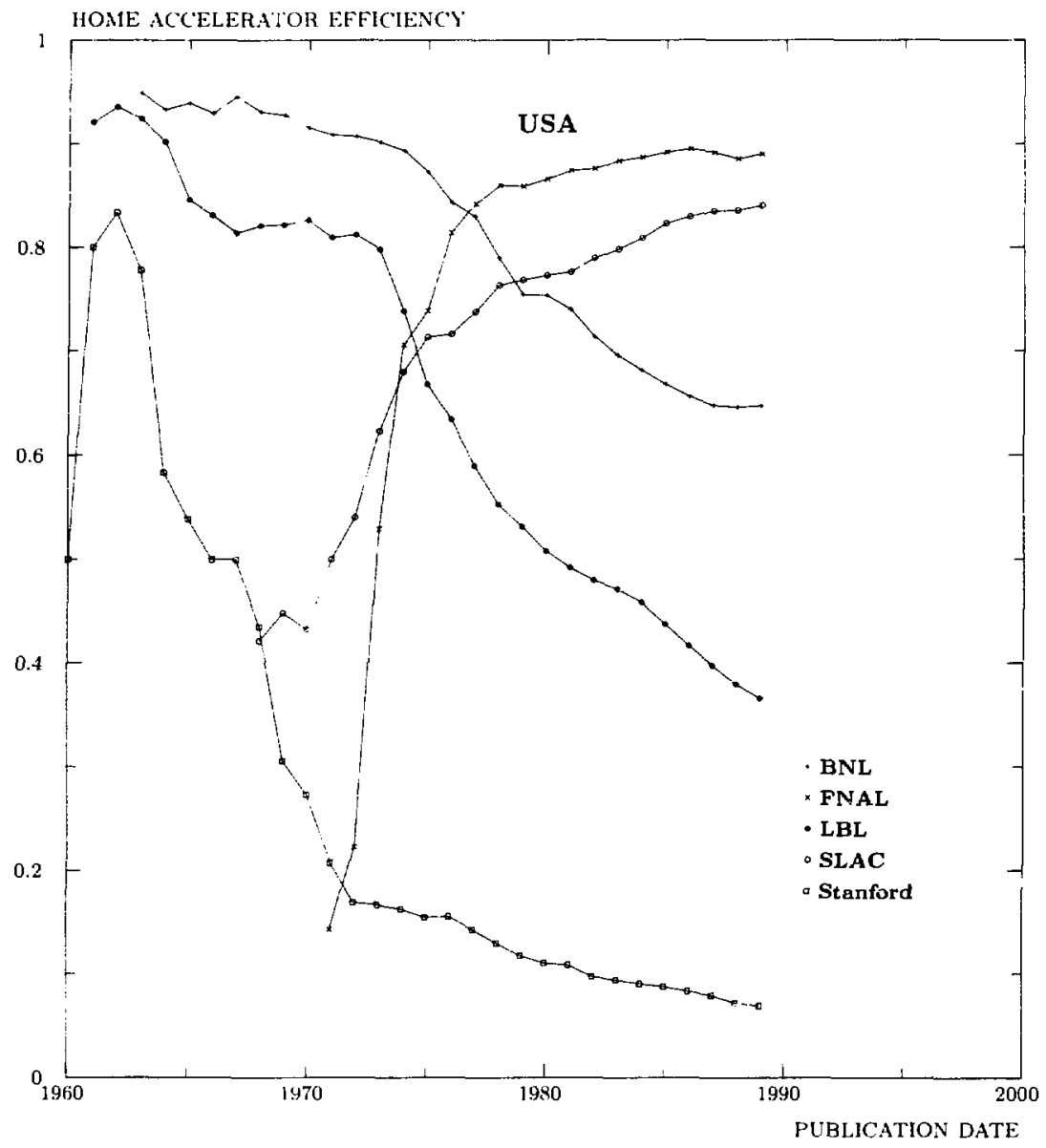

Fig. 7. The fraction of all the experimental papers with authors from a given laboratory that present data taken at an accelerator at that laboratory. 


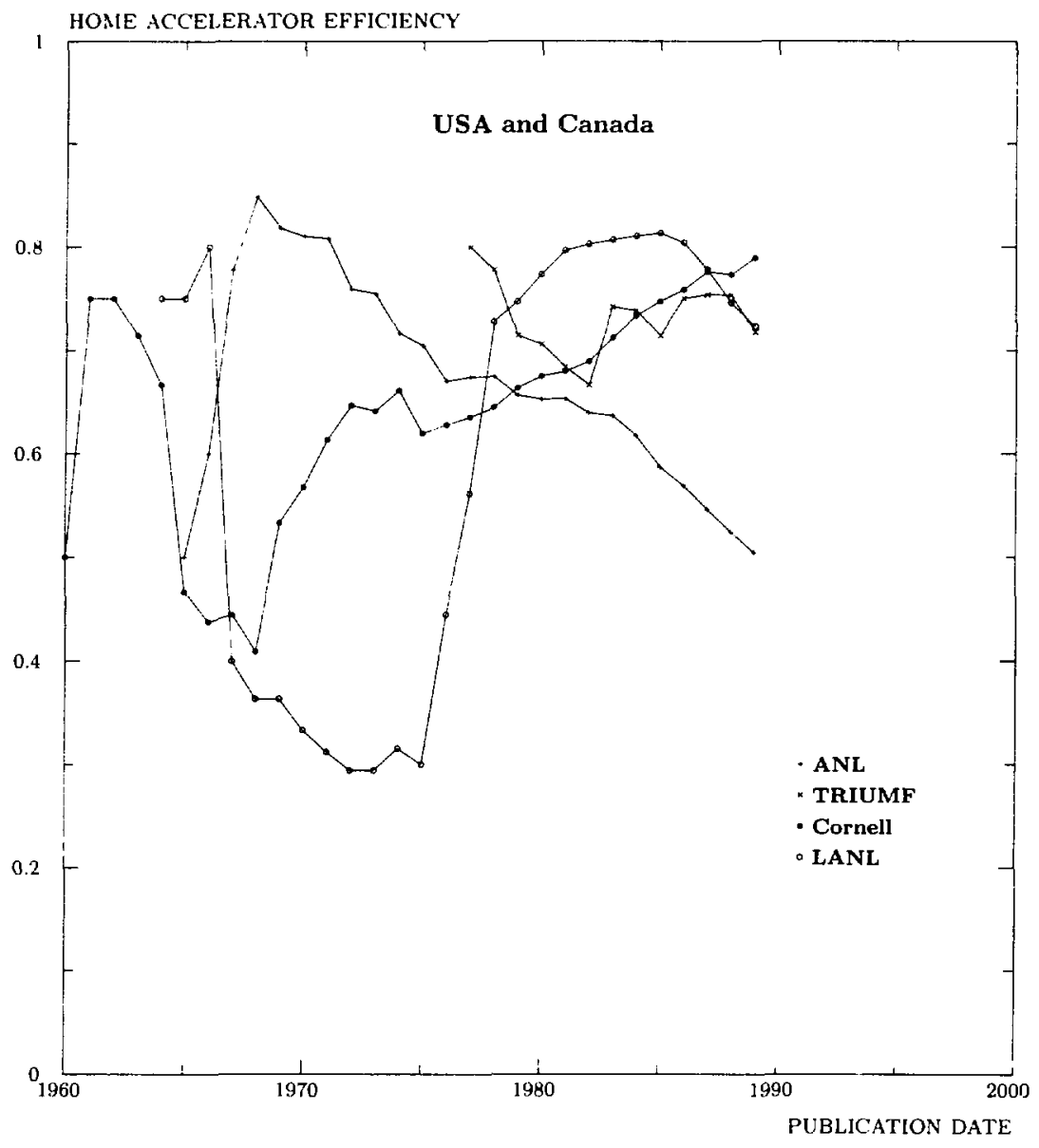

Fig. 8. The fraction of all the experimental papers with authors from a given laboratory that present data taken at an arcelerator at that laboratory. 


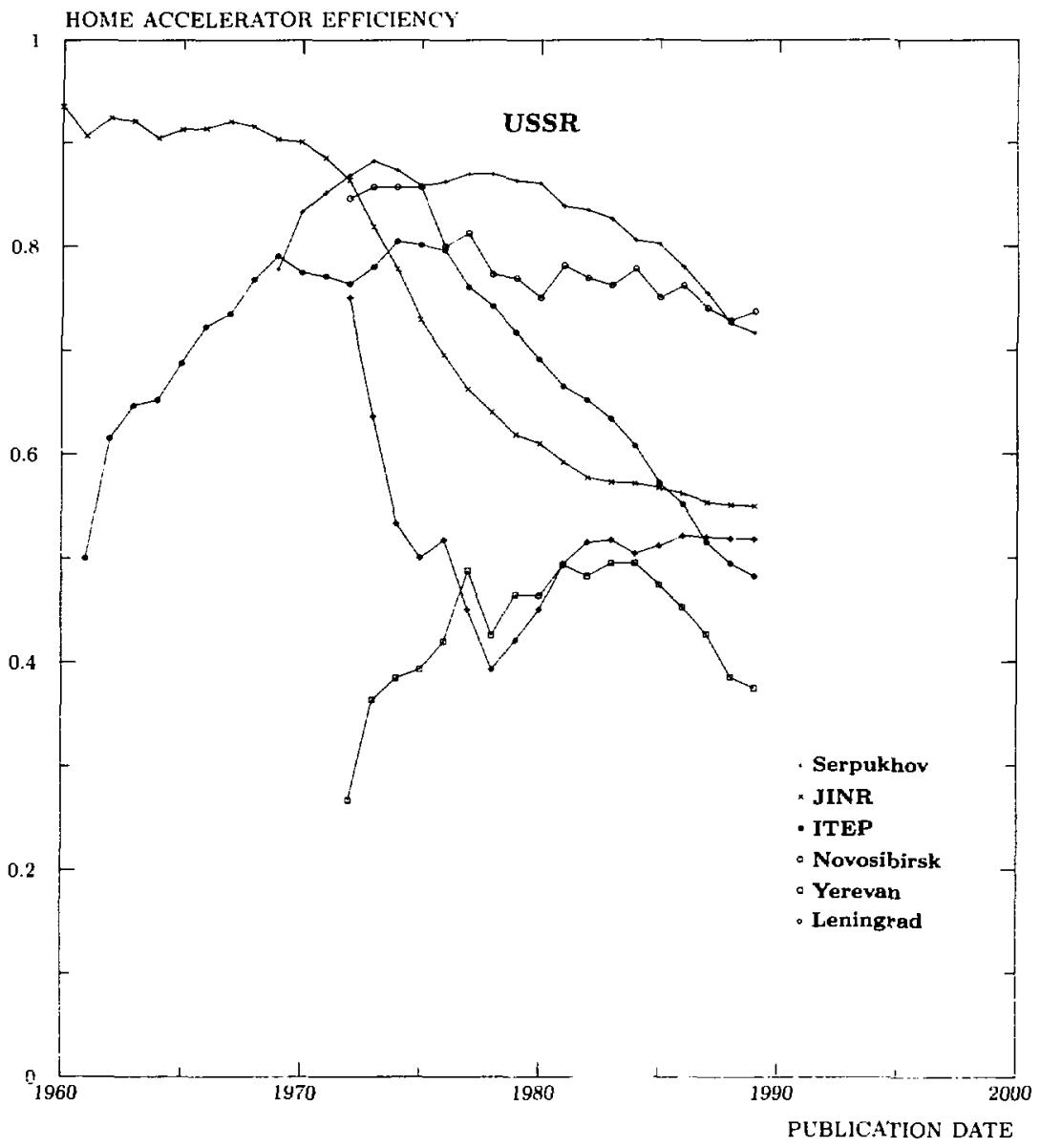

Fig. 9. The fraction of all the experimental papers with authors from a given laboratory that present data taken at an accelerator at that laboratory. 
HOME AC'CEIERATOR EFFICIENC'

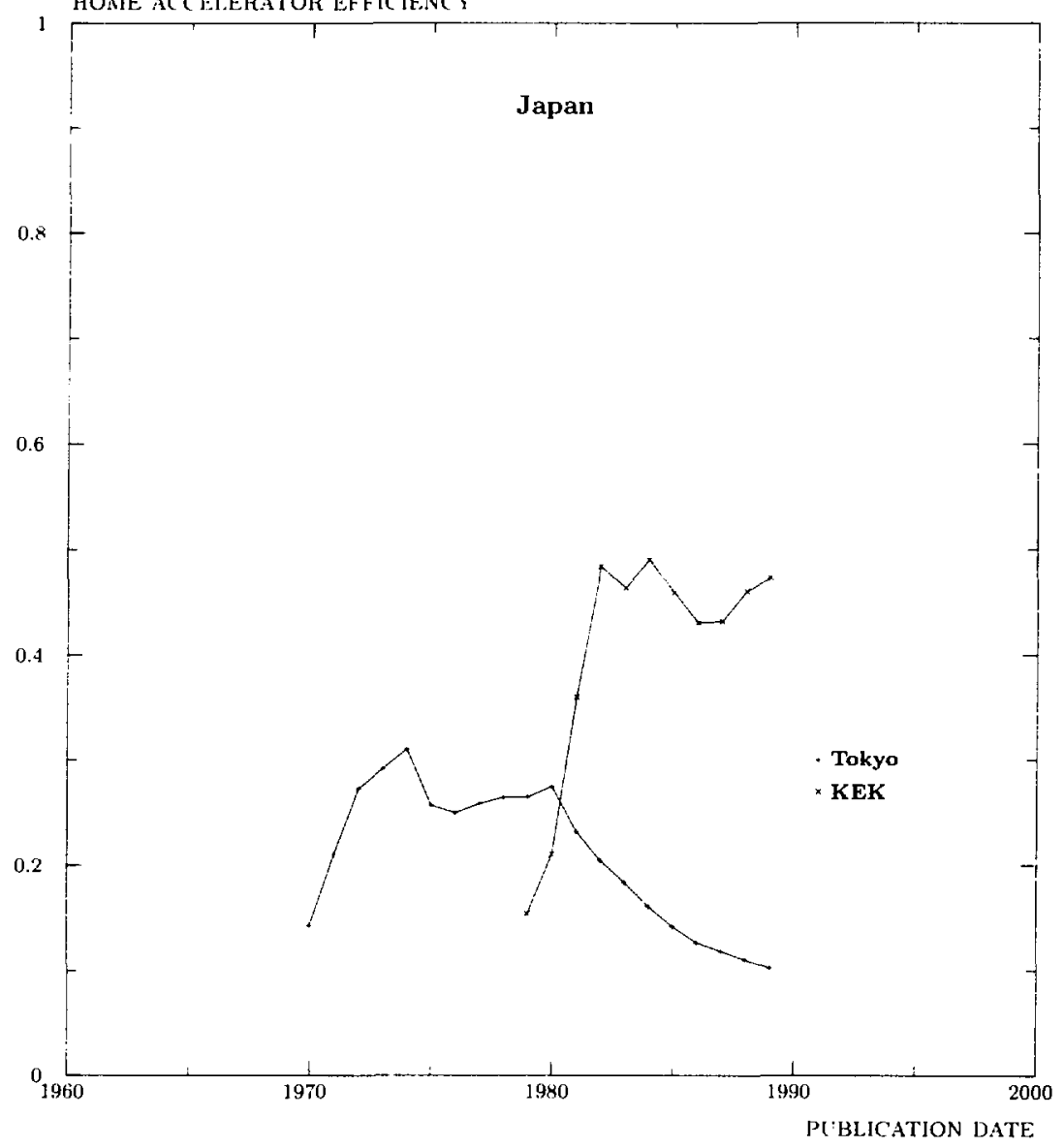

Fig. 10. The fraction of all the experimental papers with aut liors from a given laboratory that present data taken at an accelerator at that laboratory. 


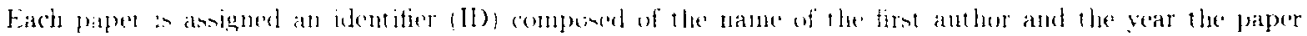

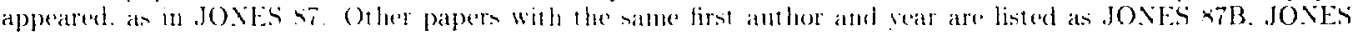

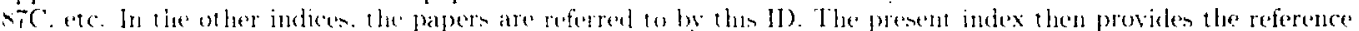
and the title of the paper. Due to text procensing procedures. titles ef papers in this index may differ slightly from the or ginal hitem. enpeciatly concerning particle natuen.

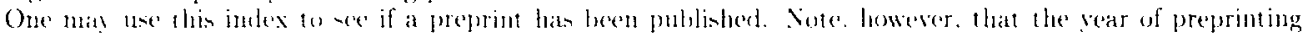
and the reat of fublishimn are oftend different. and our ID in usually that of the vear of preprinting.

\section{Illustrative Key}

Document ID: all other indices in this volume refer to this pit]er be this II).

Primary Reference: 1 the iournal reference for this paper. if the paper was published.

Additional References: the preprint number (orcationally. there is more than one)

Title of Paper
Dougherty is

Dowell is

Drechisel kn

Dropesky $\times 6$

Druzhinin \&8

Dubar 89

Dubinina \&

Duffy 85

Dugan $85 \mathrm{~B}$

Dukhorskoj $x \overline{7}$
L.BL-263013:

An Experimental Investigation of Double Beta Decay of ${ }^{100} \mathrm{Mo}$

('FRN-EP-Xri-I5t:

Recent Results from the UA1 Experiment

Phis. Rev. Lett. 54:30.1985,

Search for Anomalous Fragments of ${ }^{56} \mathrm{Fe}$ Using Plastic Nuclear Track Detectors

Phys. Rey. C'32:1305.1985:

Excitation Functions for the Production of ${ }^{18}$ Fl and ${ }^{24} \mathrm{Na}$ from $\mathrm{Al}$ and Si with Fast Pions

Z. Phrs. ( $37: 1.1988$ NOVO-87-52:

Search for Rare Radiative Decays of the $\phi$ Meson at VEPP $-2 \mathrm{M}$

Yad. Phys. 49:1239.1989:

Parametrization of Total Cross Sections at Intermediate Energies

Pisma Zh. Eksp. Teor. Fiz. 48:233.1988:

Observation of the Slow Pion Production in the Nucleus Nucleus Interactions

Phys. Rev. Lett. 55:1816.1985:

A-dependence of Charm Production

Phys. Rev. Lett. 55:170.1985: HUTP-85/A033:

New Neutrine Constraints on Majorana Mass Matrices

Iad Phvs. 47:1816.1988; ITEP-87-198:

Measurement of the Total Cross Sections of the Proton Interactions with Nuclei ${ }^{6} \mathrm{Li},{ }^{7} \mathrm{Li}$, and ${ }^{9} \mathrm{Be}$ at $2 \mathrm{GeV} / c$ 


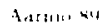

1:211, 111

Andus: andk

Nhat bis

Aliath sti

Alation atils

Airat bat at.

Abitita atil

Abach 4 -

Abasin $x+13$

Abachlo $x \mathrm{C}^{\circ}$

Alach: $\times-5$

Abachi RTE

Abach $\propto$ i $F$

Abachi $x$

Aluath: Axps

Abech

A'ach sy

Aloarte 6th

Alische $x$ ac

Alactu xal)

Alines 4

Aliaes 5

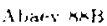

Blimblester 4

Abrobietae as

Abanturtar mols

Alectean toil

Albent 4 ;

Alifut (y)

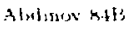

Afidents ot

Antrotex atis

Atelderes bier

Aistinas $x$ th

Aluison

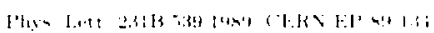

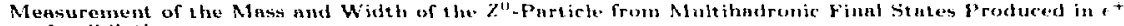
Annibilations

( H.k.

Study of Hadronic Decays of the $Z^{\prime \prime}$ Boson

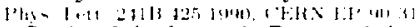

Study of the Leptonic Decays of the $Z^{0}$ Busth

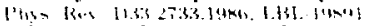

Search for P'roduction of Fractional chargen. New Particles. and Sibthreshold $A$ nt iprotons. in Relativistic Nuclear Collisions

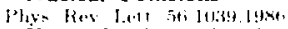

Upper Limit on the Tatu-nentrino Alasm

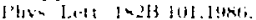

Search for Wrong Sign $D^{\text {th }}$ Decuys

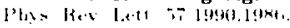

Observation of Tensor and Scalar Mesoms Produced in ${ }^{t}$, Annibilation at 29 GeV

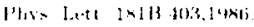

Asymmetry in the Angular Distribution of Inclusive A Baryons from ${ }^{+}$. Annihjlations at Em $=20$ GeV

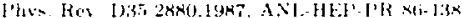
Tau-neutrino Mass Limit

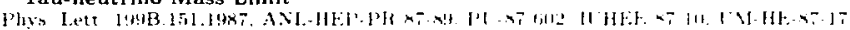

Charged $K^{*}(892)$ Production in ${ }^{+}+$Annihilations at 29 GeV

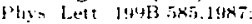

Meesurements of the Spin Density Matrix of $D^{*}(2010)$ Mesons Produced in $c^{+} e^{*}$ Annihilations

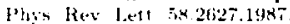

Production of Strange Baryons in + Annihilation at 20 GeV

Whyt Rev l.t.t. 5y:2519.iykt.

Measurement of the Tau-Lepton Lifetime

Phys Lett 197B.241,1987. Inclusive $\eta$ Production in $T^{t}$ Decays

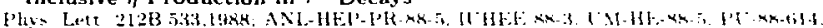

Measurement of Upper Limits for the Decay Width of $D^{*}(2010)^{+}$and $D^{*}(2010)^{0}$

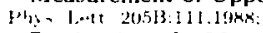

Production of $\eta$ Mesons in $e^{+} e^{-}$Annihilation at $\sqrt{s}=2 \theta$ CeV

I'hy. Let 20is b:411.19x.

Measurement of the $D^{0} \rightarrow K \quad \pi^{+}$Branching Fraction

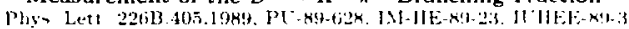

Mensurement of the Branching Ratio for $\tau^{-} \rightarrow * \quad \vec{\nu}$, 's $_{r}$

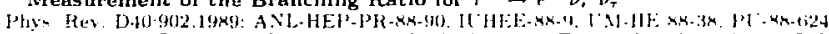

Production Cross Section and Topological Decay Branching Fractions of the Tau-Leptor

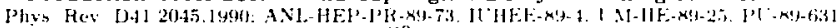

Quark Hadronization Probed by $k^{\circ}$ Mesons

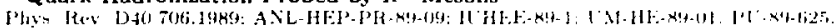
Study of Vector Meson Production in $e^{+} \epsilon^{-}$Annihilation at $\sqrt{s}=28 \mathrm{GeV}$

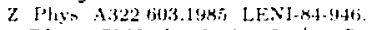

Phase Shift Analysis of $\pi^{+} p$ Scattering in the Region $194 \mathrm{MeV} \quad 600 \mathrm{MeV}$

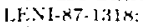

Measurement of Narrow Dibaryon Resonances with the Isuspin $\mathbf{T}=\mathbf{2}$

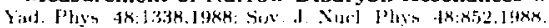

Measurement of the Spin Rotation Parameters $R$ and $A$ in Elastic $\pi$ - $p$ Scattering at 450 and 560 MeV

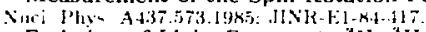

Emission of Light Fragmems ${ }^{3} \mathrm{H},{ }^{3} \mathrm{He}$, and ${ }^{2} \mathrm{He}$ in ${ }^{2} \mathrm{He}$ nucleus Collisions at $3.33 \mathrm{GeV} / \mathrm{Nucleon} \mathrm{Kinetic}$ Energy

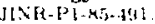

Differential Cross Section for the Formation of Helium and Lithiurn Isotopes in the Interaction of Alpha Particles with Silver and Gold

Yarl Phys $42: 3 \times 3,1 !$ !x:

Factorization of Fragmentation Cross Sections it Relativistic p Nuclei and He Nuclei Interactions

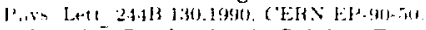

$A$ and $\Lambda$ Production in Sulphur-Tungiten Interactions at 200 GeV/r per Nucleon

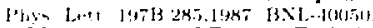

Measurement of Energy Emission from ${ }^{10} \mathrm{O}$ nucleus and $p$ nucleus Collisions at $14.5 \mathrm{GeV} / \mathrm{per} \mathrm{Nucleon}$ with a Lead Glass Array

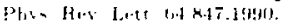

Kaon and Pion Production in Central Si Au Collisions at $14.6 \mathrm{~A} \mathrm{GeV/r}$

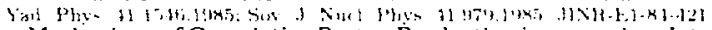

Mechanisms of Cumulative Proton Production in $\pi$ nucleus Interactions at 5 GeV/c: A Phenonenological Analysis

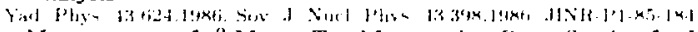

Measurenent of $\rho^{\circ}$ Meson Total Interaction Cross Section for Internuclear Nucleons in $\pi$ C Collisions at 5 GeV/r

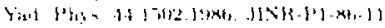

Search for Multibaryon Resonances in the $\pi$ 12C Interactions at 5 GeV/r

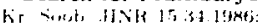

Observation of Narrow Diproton Resonnnces with 1066 and $1989 \mathrm{haV} / \mathrm{r}^{2}$ Masses

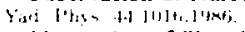

Absorption of Slow Secondary $\pi$ Mesons in Haciron Nuclear Internetions

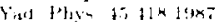

Dependence of Averaged Inclusive Characteristics of Charkerl pione nue Jrotous on the Cumalative Number in $\pi C$ Interactions at 5 GeVir 
Abdullin 87

Abudulin 88

Abdullin $88 \mathrm{~B}$

A todullin $88 \mathrm{C}$

Abdullin 88D

Abdullin 89

Abo :llin 89B

Abdullin 890

Abdullin 89D

Abdullin $89 \mathrm{E}$

Abdullin 89F

Abdullin $89 \mathrm{G}$

Abdullin 89H

Abdullin 90

Abdurakhimov 88

Abdurakhimov 88B

Abdurakhimov 89

Abdurakhimov $89 \mathrm{C}$

Abdurazakova 87

Alsdurazakova 88

Abduzhamilov 87

Abduzhamilov 88

Abduzhamilov $88 \mathrm{~B}$

Abduzhamilov $88 \mathrm{C}$

Abduzhamilav 89

Abe 85

Abe $85 \mathrm{~B}$

Abe 86

Atse $86 \mathrm{~B}$

Abe $86 \mathrm{C}^{\circ}$

Abe $86 \mathrm{D}$

Abe 87

A be $87 \mathrm{~B}$

Abe 870

Abe $8 B$

Alse $88 \mathrm{~B}$
ITEP-87-24:

Analysis of the Reaction ${ }^{3} \mathrm{He} p \rightarrow p$ p $n$ at $5 \mathrm{GeV} / \mathrm{c}^{3} \mathrm{He}$ Nuclei in the Pole-Graph Model

Yad. Phys. 48:917.1948:

Analysis of the ${ }^{3} \mathrm{He} p \rightarrow$ deuteron $p \mathrm{n} \pi^{+}$Reactions at $5 \mathrm{GeV} / \mathrm{c}^{3} \mathrm{He}$ Momentum in the Entire Phase Space ITEP-88-130:

An Investigation of the Reaction ${ }^{3} \mathrm{H} \rightarrow 2 p 2 n\left(\pi^{0}\right)$ at the ${ }^{3} \mathrm{H}$ Momentum of $5 \mathrm{GeV} / \mathrm{c}$

ITEP-88-159:

Analysis of the ${ }^{3} \mathrm{He} \rightarrow 2 p 2 n \pi^{+}$Reaction at the ${ }^{3} \mathrm{He}$ Momentum of $5 \mathrm{GeV} / \mathrm{c}$ in the Total Phase Volume ITEP-88-161:

Analysis of the ${ }^{3} \mathrm{He} \rightarrow$ deuteron $p n \pi+$ Reaction at the ${ }^{3} \mathrm{He}$ Momentum of $5 \mathrm{GeV} / c$ in the Total Phase Volume

Y'ad. Phys. 49:169.1989:

Crass Section of Tritium Interactions with Protons and ${ }^{3} \mathrm{H}$ p Elastic Scattering at $5 \mathrm{GeV} / \mathrm{C}$

ITEP-89-17:

Quesielastic ${ }^{3} \mathrm{H} p$ Scattering at $5 \mathrm{GeV} / \mathrm{c}^{3} \mathrm{H}$ Momentum

Yad. Phys. 50:400.1989: ITEP-89-7:

Reaction ${ }^{3} \mathrm{H} p \rightarrow$ deuteron $p$ at $5 \mathrm{GeV} / c^{3} \mathrm{H}$ Moment um

Pisma Zh. Eksp. Teor Fiz. 49:413.1989: [TEP. H91-51:

Determination of the Nucleon Momentum Distributions in the ${ }^{3}$ He and ${ }^{3} \mathbf{H}$ Nucleus from the Reactions ${ }^{3}$ He $p \rightarrow p$ deuteron and ${ }^{3} \mathrm{H} p \rightarrow p n$ deuteron

Yad. Phys. 49:16+5,1989: Sov. J. Nucl. Phys. 49:1018.11489

Total-Phase-Space Analysis of Reaction ${ }^{3} \mathrm{He} \mathrm{p} \rightarrow 2 \mathrm{p} 2 n \pi^{+}$at $5 \mathrm{GeV} / \mathrm{c}$ Moment um of Nuclei

Yad. Phys. 52:15.1990: ITEP-89-96:

Cumulative Nucleon Production in ${ }^{3} \mathrm{He} p$ and ${ }^{3} \mathrm{H}$ p Interactions at the $5 \mathrm{GeV} / \mathrm{c}$ Nucleus Momentum

ITEP-89-359.

The Total Phase Space Study of the Reaction ${ }^{3} \mathrm{H} p \rightarrow$ deuteron $p p \pi^{-}$at Nucleus Momentum of $5 \mathrm{GeV} / c$ Pisma Zh. Eksp. Teor. Fiz. 50:213.1989: ITEP-89-117:

A Search for Anomalons with Charge $Z=2$ in the $p^{3}$ He Interactions at $5 \mathrm{GeV} / c^{3} \mathrm{He}$ Momentum

Yad Phys. 51:1215.1990:

Search for a $\Delta\left(1232 P_{3 a}\right)++n n$ Configuration in ${ }^{3} \mathrm{He}$

JINR-P1-88-106:

Size of $\pi^{-}$Meson Emission Region in Inelastic and Centra) Nuclear-Nuclear Interactions at $E=3.60$ A GeV

JINR-PI-88-903.

Emission of Rigid Gamma-Quanta in $\pi^{-}$Xe Interactions at $3.5 \mathrm{GeV} / \mathrm{c}$

JINR-PI-89-272:

Longitudinal and Transverse Dimensions of $\pi^{-}$Meson Emission Region in Central ${ }^{12} \mathrm{C}+\mathrm{Cu}$ Collisions at 3.66 A GeV Energy

Nuovo Cin. 102. :645,1989:

Experimental Study of Relativistic Hypernuclei Using the HYBS-Spectrometer

Acta Phys. Polon. B18:249.1987:

Interaction of the $800 \mathrm{GeV}$ Protons from Fermilab with Emulaion Nuclei

Yad. Phys. 47:1299.1988;

The Shape of Relativistic "Fe Transverse Momentum Distribution in High Energy Nucleus-Nucleus Collisions

Phys. Rev. D35:3537,1987

Charged Particle Multiplicity and Angular Distributions in Proton Nucleus Interactions at $800 \mathrm{GeV}$

Yad. Phys. 48:446,1988: Sov. J. Nucl. Phys. 48:280.1988:

Azimuthal Correlation in Six-Prong $p$ p Interactions at 200 and $400 \mathrm{GeV} / \mathrm{c}$

Z. Phys. C40:1,1988;

Angular Distributions in Proton-Nucleus Interactions in Emulsion at $800 \mathrm{GeV}$

2. Phys. C40:223.1988:

Multiplicity in Proton-Nucleus Interactions in Emulsion at $800 \mathrm{GeV}$

Plyss. Rev. D39:86.1989:

Central Collisions of $800 \mathrm{GeV}$ Protons with $\mathrm{Ag} / \mathrm{Br}$ Nuclei in Nuclear Emulsion

Phys. Rev. D32:2288.1985:

Test of a-Channel Helicity Conservation in Inelastic $\rho^{0}$ Diffraction in $20 \mathrm{GeV}$ Photoproduction

Phys. Rev. D:32:2869.1985:

Inclusive Photoproduction of Strange Baryons at $20 \mathrm{GeV}$

Phy's. Rev. D33:1.1986; RAL-85-055: SLACC-PUB-3722; UTHEP-85-(1601:

Lifetimes, Cross Sections and Production Mechanisms of Charmed Particles Produced by 20 GeV Photons

Phys. Rev. Lett. 56:1107.1986:

Precise Determination of $\sin ^{2} \theta_{W}$ from Measurements of the Differential Cross Sections for $\nu_{\mu} p \rightarrow \nu_{\mu} p$ and $\bar{\nu}_{\mu} p \rightarrow \bar{\nu}_{\mu} p$

Phys. Rev. D34:1950,1986

Inclusive $A$ Polarization in Proton Nucleus Collisions at $12 \mathrm{GeV}$

Phys. Rev. Lett, 58:636.1986:

Measurement of the Weak-Neutral Current Coupling Constants of the Electron and Limits on the Electromagnetic Properties of the Muon Neutrino

KEK-87-80: KOHE-HEP-87-02; KUNS-812: OLLNS-87-04: TMUL-HEL-87-21:

Measurement of the Reactions $e^{+} e^{-}-e^{+} e^{-}$and $e^{+} e^{-}-\gamma \gamma$ at $\sqrt{s}=52 \mathrm{GeV}$

Phys. Rev. D.36:1302.1987:

Production of Neutral Strange Particle $K_{S}$ and $\Lambda$ by $12 \mathrm{GeV}$ Protons on Nuclear Targets

KEK-87-79: KOBE-HEP-87-01: KLNS-8111: OLLNS-N7-3: TNLP-HEL-87-20;

Search for the Top Quark in $e^{+} e^{-}$Annihilation at $\sqrt{s}=50 \mathrm{Gev}$ : The First Result from the VENUS Detector at TRISTAN

Phy's. Lett. 200JB:266j, 1984:

Leading Particles Distribution in $200 \mathrm{GeV} / \mathrm{c} p+$ Nucleus Interactions

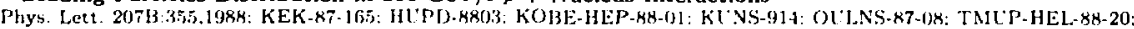
Study of Multihadron Events with Isolated Leptons in $e^{+} e^{-}$Annihilation at $50<\sqrt{s}<55 \mathrm{GeV}$ 
Abe $88 \mathrm{C}$

Abe $88 \mathrm{D}$

Abe $83 \mathrm{E}$

$\mathrm{A}$ be $88 \mathrm{~F}$

A be 89

A be $89 \mathrm{~B}$

Abe $89 \mathrm{C}$

Abe 89D

Abe $89 \mathrm{E}$

Abe $89 \mathrm{~F}$

Abe $89 \mathrm{H}$

Abe 891

Abe 89J

Abe $89 \mathrm{~K}$

A be $89 \mathrm{~L}$

Abe 89M1

Abe $89 \mathrm{~N}$

Abe 890

Abe 89P

Abe $89 Q$

Abe $89 \mathrm{R}$

Abe $89 \mathrm{~S}$

A be $89 \mathrm{~T}$

Abe 90

Abe $90 \mathrm{~B}$

Abe $90 \mathrm{C}$

A begg 85

Abegg 89

Abegg $89 \mathrm{~B}$

Ableev 84B

Ableev 85

Ableav 86

Ableev 87

Ableev 87B

Ableev $87 \mathrm{C}$

Ableev 87D
Phys. Fur. Le1t, 61:1819,1988; ANL-HEP-PR-KN-32.

Transverse Moinentum Distributions of Charged Particles Produced in $\bar{p} p$ Interactions at $\sqrt{8}=630$ and $1800 \mathrm{GeV}$

Phys. Rev. Lett, til 915.1988 :

Search for New Charged Leptons Decaying into Massive Neutrinos and Stable Charged Leptons in $e^{+} e^{-}$ Collisions

Phys. Lett. 213B:400.1988; KEK-88-33: KOBE-HEP-48-02: KLNS-937: HLPD-8808: OLLNS-88-04 I'MILP-HEL-88-22: Search for Excited Electrons in the $e^{+} e^{-}$Feactions up to the $\sqrt{8}=56 \mathrm{GeV}$

Phys. Rev. D39:3524.1989: KEK-88-111: HTPD-8815: KOBE-HEP-88-06: KLNS-954: OLLNS-88-08: TMLP-HEL-88-23: Search for a Fourth Generation Quark with $Q=e / 3$ in $e^{+} e^{-}$Collisions at $\sqrt{s}=56-57 \mathrm{GeV}$

Phys. Huv. Lett. 62:613,1989; FERMILAB-PUB-88-213-E:

Measurement of the Inclusive Jet Cross Section in $p p$ Collisions at $\sqrt{s}=1.8 \mathrm{TeV}$

Phys. Rev. Lett. 62:1005.1989; FERMILAB-PLB-88-207-E:

Measurement of $\boldsymbol{W}^{ \pm}$-Boson Production in $1.8 \mathrm{TeV} \bar{p} p$ Collisions

Phys. Rev. Lett. 62:1825,1989:

Limitz on the Masses of Supersymmetric Particles from 1.8 TeV $p \bar{p}$ Collisions

Phys. Rev, Lett. 62:3020.1989; FERMILAB-PLB-89-62-E: Dijet Angular Distributions from $\bar{p} p$ Collisions at $\sqrt{s}=1.8 \mathrm{TeV}$

Phys. Rev. Lett. 62:1709,1989:

Determination of $\sin ^{2} \theta_{W}$ from Measurements of Differential Cross Sections for Muon-Neutrino and Antineutrino Scattering by Electron

2. Phys. C45:175.1989: KEK-89-66; OLLNS-89-06: KOBE-HEP-89-113: HLPD-8909: KUNS-982: TMUP-HEL-89-20; Measurement of the Differential Cross Section of $e^{+} e^{-} \rightarrow \gamma \gamma$ and $e^{+} e^{-} \rightarrow \gamma \gamma \gamma$ at $\sqrt{8}=55,56,56.5$ and $57 \mathrm{GeV}$ and Search for Unstable Photino Pair Production

Phys. Rev. Lett. 63:1447.1989: FERM1ILAB-PUB-89-161-E; ANL-HEP-['R-89-71: Search for Heavy Stable Charged Particles in $1.8 \mathrm{TeV} \bar{p} p$ Collisions at the Fermilab Collider

Phys. Lett, 232B:431,1989; KEK-89-68; TMUP-HEL-39-21: OLLNS-89-07: KOBE-HEP-89-04; HLPD-8910; KUNS-983; A Study on Single Phaton Production at $\sqrt{8}=54.0-81.4 \mathrm{GeV}$

Phys. Rev. Let t. 63:1776,1989: KEK-89-39: Search for Isolated Photons from Flavor-Changirg Neutral-Current Decay of a New Quark at the KEK $e^{+} e^{-}$Collider TRISTAN

Phys. Lett. 232B:425,1989: KEK-89-89; HUPD-8911: OULNS-89-08: KOBE-HEP-89-05: KUNS-984: TNLP-HEL-89-22; Charge Asymmetry of Hadron Jets and Limits on the Compositeness Scales in $e^{+} e^{-} \rightarrow \bar{q}$ Reaction at $\sqrt{8}=57.6 \mathrm{GeV}$

Phys. Rev. D40:3791,1989:

$K^{0}$ Production in $\bar{p} p$ Interactions at $\sqrt{s}=630$ and $1800 \mathrm{GeV}$

Phys. Rev. D41:2330,1990; FERMILAB-PUB-80-201-E:

Pseudorapidity Distributions of Charged Particles Produced in $\bar{p} p$ Interactions at $\sqrt{s}=630$ and $1800 \mathrm{GeV}$

FERMILAB-PUB-89-206-E:

Two Jet Differential Cross Section in $\bar{p} p$ Collisions at $\sqrt{s}=1.8 \mathrm{TeV}$

Phys. Rev. Lett. 64:348,1990: FERMILAB-PLB-89-171-E:

Measurement of $D^{*}(2010)$ Produced in Jets from $\bar{p} p$ Collisions at $\sqrt{s}=1.8 \mathrm{TeV}$

KEK-89-192;

Measurement of the Reactions $e^{+} e^{-} \rightarrow \mu^{+} \mu^{-}$and $e^{+} e^{-} \rightarrow \tau^{+} \tau^{-}$between $E_{\mathrm{em}}=50$ and $60.8 \mathrm{GeV}$

FERMILAB-PL'B-89-245-E;

Measurement of the Ratio $\Sigma\left(W^{\ddagger} \rightarrow \mathrm{e}^{ \pm} \nu\right) / \Sigma\left(Z^{\circ} \rightarrow e^{-} e^{+}\right)$in $\bar{p} \rho$ Collisions at $\sqrt{s}=1.8 \mathrm{TeV}$

Phys. Rev. D41:1717,1990: FERMIILAB-PLB-89-250-E:

Search for a Light Higgs Boson at the Tevatron Proton-Antiproton Collider

Phys. Rev. D41:1722,1990; FERMILAB-PUB-89-229-E;

The Two Jet Invariant Mass Distribution at $\sqrt{s}=1.8 \mathrm{TeV}$

Phys. Rev. Lett. 63:720,1989; FERMILAB-PUB-89-160-E; ANL-HEP-PR-89-66:

Measurement of the Mass and Width of $Z^{\circ}$ Boson at the Fermilab Tevatron

Phys. Lett. 234B:382.1990; KEK-89-135: HUPD-8916: KOBE-HEP-80-09: KLNS-990; OULNS-89-10: TMUP-HEL-89-31; Measurement of $\mathbb{R}$ and Search for New Quark Flavors Decaying into Multi-Jet Final States in $e^{+} e^{-}$ Collisions between $54 \mathrm{GeV}$ and $61.4 \mathrm{GeV}$ c.m. Energies

FERMILAB-PLB-90-71-E;

Jet Fragmentation Properties of $\bar{p} p$ Collisions at $\sqrt{s}=1.8 \mathrm{TeV}$

KEK-90-4: HUPD-9004; KOBE-HEP-90-01: KLNS-1005: OULNS-89-13: TMIL:P-HEP-90-10:

Determination of the QCD Scale Parameter $\Lambda_{M f S}$ with QCD Cascade on the Basis of the Next-to-Leading Logarithmic Approximation

Phys. Rev. Lett. 56:2571.1986; TRI-PP-85-70:

Test of Charge Symmetry in $n$ Elastic Scat tering at $477 \mathrm{MeV}$

Phys. Rev. D39:2464,1989; TRI-PP-87-60:

Charge Symmetry Breaking in $n p$ Elastic Scattering at $477 \mathrm{MeV}$

Phys. Rev. C40:2406,1990; TR1-PP-89-56;

$n$ p Elastic Scattering Analyzing Power Characteristics at Intermediate Energies

Yad. Phys. 42:205,1985; JINR-P1-84-476:

The Search for Anomalous Deuterons in the ${ }^{3} \mathrm{He} \mathrm{C} \rightarrow$ deuteron X Reaction at $10.8 \mathrm{GeV} / \mathrm{c} \mathrm{Momentum}$

Acta Phys. Pulon. B16:913,1985: JINR-P1-85-924:

Alpha Nuclear Differential Cross Sections at $4.45 \mathrm{GeV} / c$ per Nucleon

Kr. Soob. JINR 13:5,1985;

The Search for Exotic Longlived Diharyons

Yad. Phys. 46:549.1987; JINR-Pl-86-435:

Charge-Exchange $p\left({ }^{3} \mathrm{He},{ }^{3} \mathrm{H}\right)$ at Momenta $4.4-18.3 \mathrm{GeV} / c$ with $\Delta\left(1232 P_{33}\right)$ Isobar Production

Pisma Zh. Eksp. Teor, Fiz. 45:467,1987; JINR-P1-87-93;

Momentum Distribution of Protons and Deuterons from ${ }^{3} \mathrm{He}$ Fragmentation at $10.78 \mathrm{GeV} / \mathrm{c}$ on Carbon at Zero Angles

Yad. Phys. 48:27,1988: JINR-P1-87-374;

Charge-Exchange of ${ }^{3}$ He Relntivistic Nuclei to Tritons on Carbon with $\Delta$-Isohar Excitation in the Target Nucleus

JINR-E1-87-797:

$\Delta$-Isober Excitations of Nuclei in Charge-Exchange Reactions 
Ableev $87 E$

Ableev 88

Ableev 89

Aboy 89

Abraanyan $8 \mathrm{H}$

Abraámyan 89

Abramov 8.

Abramor $84 \mathrm{C}$

Abramov 84D

Abramov 84E

Abramov 85

Abrano: 86

Abramoy 86B

Abramov 87

Abramov 88

Abramov 89B

Abramov 89C

Abramowicz 85

Abramowicz 86

Abrams 89

Abrams $89 \mathrm{~B}$

Abrams $89 \mathrm{C}$

Abrams 89D

Abrans 89E

Abrams $89 \mathrm{~F}$

Abreu 85

Abreu 89

Abreu 90

Abreu $90 \mathrm{~B}$

Abreu $90 \mathrm{C}$

Abreu 90D

Abreu 90E

Abreu 90F

Abrosinov 85

Abrosimov 85B

Absemetova 8:5

Achasov $84 \mathrm{~F}$
JINR-E1-87-24ti:

Observation of the Dominance of the Target $\Delta\left(1232 P_{33}\right)$ Excitation and Their Collective Nature in the $\left({ }^{3} \mathrm{He}^{3} \mathrm{H}\right)$ Charge Exchange at High Energies

Pisma 2h. Eksp. Teor. Fiz. 17:558,1988; JINR-El-k8-250;

Measurement of Tensor Analyzing Power for the ${ }^{12} \mathrm{C}$ (deuteron, p) Reaction at $P=\theta .1 \mathrm{GeV} / \mathrm{c}$ and Zero Angle Proton Emission

JINR-E1-80-3H1:

Proton and Triton Momentum Distributions from ${ }^{4}$ He Fragmentation at Relativistic Energieg

Plyys. Lett. 217B:225.1989: The Investigation of Parity Violation in the Process ${ }^{207} \mathrm{~Pb}(n, \gamma){ }^{208} \mathrm{~Pb}$

JINR-P1-88-334:

Inclusive Production of $\pi^{0}$-Mesons in $p C$-Collisions at $4.5 \mathrm{GeV} / \mathrm{c}$

Yiad. Phy's. 51:150.1989: JINR-P1-89-240:

Inclusive $\pi^{0}$ Production in He C Interactions at $4.5 \mathrm{GeV} / \mathrm{c} / \mathrm{Nucleon}$

Nucl. Plays. B245:1 1984; Yad. Phys. 41:137.1985: Sov. J. Nucl. Phys. 41:87.198i; IF VE-84-12;

Production of Charged Hadron Pairs with Large Symmetric Transverse Momenta in $p$ p Collisions at 70 GeV

Yad. Phys. 41:700.1985; Sow. J. Nucl. Phys. 11:445.1985: IFv'E-8.4-88;

Large Transverse Momentum Inclusive Hadron Production in $p$ p Collisions at $r 0 \mathrm{GeV}$

Z. Phys. C27:491.1985: IFVE-84-143;

Observation of Puzzling A-Dependence of Symmetric Hadron Pairs at High pT

Z. Phys. C24:205.1984: Yad. Plyys, 41:357,1985: IFVE-84-26:

High pr Hadron Production off Nuclei at $70 \mathrm{GeV} / \mathrm{c}$

ITEP-85-160:

Backward $\pi^{-}$deuteron Elastic Scattering from 1.75 to $3.1 \mathrm{GeV} / \mathrm{c}$

Yad. Phys, 45:1362,1987: IFVE-86-56:

High pt Deuteron and Antideu. con Production in $p p$ and $p$ Nuclei Collisions at $70 \mathrm{GeV}$

Yad. Phys. 45:725.1987: 1FVE-86-119:

A Search for A nomalously Interacting Stable Particles in the Mass Range from $1.0 \mathrm{GeV} / \mathrm{c}^{2}$ to $1.8 \mathrm{GeV} / \mathrm{c}^{2}$

Phys. Lett. 189B:295.1987;

Backward $\pi$ - Jeuteron Elastic Scattering from 1.75 to $3.1 \mathrm{GeV} / \mathrm{c}$

Yad. Phys. 48:154,1088; Sov. J. Nucl. Plyss. 48:96.1988:

Measurement of $\pi^{ \pm} n \rightarrow p$ X Inclusive Cross Sections at $1.84,2.22$ and $2.63 \mathrm{GeV} / c$ and Test of Inelastic Intermediate States Model for Pion-Deuteron Backward Scattering

Yad. Phys. 50:1042.1989; ITEP-89-106:

New Results on Backward Pion-Deuteron Scattering from 0.83 to $1.16 \mathrm{GeV} / \mathrm{c}$

ITEP-89-29:

A Search for Exotic Baryon $E^{+++}$in the Baryon Exchange Reaction $\pi^{+} p \rightarrow p \pi^{+} \pi^{+} \pi^{-}$at $4 \mathrm{GeV} / \mathrm{c}$

Z. Phys. C28:51.1985:

Measurement of the Neutral to Charged Current Cross Section Ratios in Neutrino and Antineutrino Nucleon Interactions and Determination of Weinberg Angle

Phys. Rev. Lett. 57:298.1986: CERN-EP-86-38

A Precision Measurement of $\sin ^{3} \theta_{W}$ from Sernileptonic Neutrino Scattering

Phy's. Rev. Lett. 63:724,1989; SLAC-PUB-5037; LBL-27518:

Initial Measurements of $Z^{\circ}$-Boson Resonance Parameters in $e^{+} e^{-}$Annihilation

Phys. Rev, Lett. 63:2173,1989; SLAC-PLB-51]3: LBL-27857:

Measurements of $Z^{\circ}$-Boson Resonance Parameters in $e^{+} e^{-\cdot}$ Annihilation

SLAC-PL'B-5124; LBL-27898;

Measurement of $Z^{\circ}$ Decays into Lepton Pairs

Phys. Rev. Lett. 63:1558,1989; SLAC-PUB-5045: LBL-27557: CALT-68-1605:

First Measurements of Hadronic Decays of tine $Z^{0}$ Boson

Phys. Rev. Lett. 64:1334,1990; SLAC-PUB-5092; LBL-27740;

Measurements of Charged Particle Inclusive Distributions in Hadronic Decays of the $Z^{\mathbf{0}}$ Boson

Phys. Rev. Lett. 63:2447.1989; SLAC-PUB-5176; LBL-27838:

Searches for New Quarks and Leptong Produced in $Z^{\circ}$ Boson Decay

Z. Phys. A324:1,1986; CERN-EP-85-99:

Inclusive Proton and Antiproton Production in $\pi^{I} p$ and $\pi^{ \pm}$nucleus Interactions at $30 \mathrm{GeV} / c$

LAPP-EXP-89-15: C89/09/06:

The Production of $\phi$ in $200 \mathrm{GeV} /$ Nucleon S-U and O-U Interactions

Phys. Lett. 241B:449,1990; CERN-EP-90-33:

Search for Heavy Charged Scalars in $Z^{\circ}$ Decays

Phys. Lett. 242B:536.1990; CERN-EP-90-40;

Search for $t$ and $b^{\prime}$ Quarks in Hadronic Decays of the $Z^{0}$

CERN-EP-90-60:

Search for Pair Production of Neutral Higgs Bogons in $Z^{0}$ Decays

Phys. Lett. 247B:137.1990; CERN-EP-90-78;

A Study for Intermittency in Hadronic $Z^{C}$ Decays

Phys. Lett. 247B:148,1990; ; CERN-EP-90-79:

Search for Scalar Quarks in $Z^{\circ}$ Decays

Nucl. Phys. B342:1.1990; CERN-EP-90-44:

Search for Light Neutral Higgs Particles Produced in $Z^{0}$ Decays

LEN1-85-1073:

Measurement of $\pi^{+}$Meson Lifetime

Pisma Zh. Eksp. Teor. Fiz, 43:214,1986: LENI-85-1146:

$K^{+}$Meson Production in Proton-Nuclear Interactions from $\mathrm{Be}, \mathrm{C}, \mathrm{Cu}, \mathrm{Sn}$, and $\mathrm{Pb}$ in the Energy Range from $800 \mathrm{MeV}$ to $1000 \mathrm{MeV}$

Yad. Phys. 42:1434.1985:

Energy and Azimuthal Characteristics of Slow Particles in Inelastic Interactions of High-Energy Hadrons with Emulsion Nuclei

Phys. Lett. 156B:434,1985; TF-83-1.22.

Mystery of $J / \psi(15) \rightarrow \gamma \rho \rho, J / \psi(1 S) \rightarrow \gamma \omega \omega$ and $J / \psi(1 S) \rightarrow \gamma \gamma \rho^{0}$ Decays 
Ackleh 89

Adachi 87

Adachi 88

Adachi 88B

Adachi $88 \mathrm{C}$

Adachi 88D

Adachi 89

Adachi 89B

Adachi 89C

Adachi 89D

Adachì $89 \mathrm{E}$

Adachi 90

Adachi 90B

Adachi $90 \mathrm{C}$

Adamovich $86 \mathrm{~B}$

Adamovich $86 \mathrm{E}$

Adamovich 88

Adamovich $88 \mathrm{~B}$

Adamovich $88 \mathrm{C}$

Adamorich $88 \mathrm{D}$

Adamovich 89

Adamovich 89B

Adamovich $89 \mathrm{C}$

Adamovich $89 \mathrm{D}$

Adamovich $89 \mathrm{E}$

Adamovich 90

Adams 87

Adams 89

Adamuy 86

Adamus $86 \mathrm{~B}$

Adamus $86 \mathrm{C}$

Adamus 87

Adamus $87 \mathrm{~B}$

Adamus $87 \mathrm{C}$

Adarnus 870
SLAC-PIB-4473; LTHEP-87-1101:

A Search for a Short Lived Axion Decaying to $e^{+} e^{-}$in a 20 G.V Photoproduction Experiment

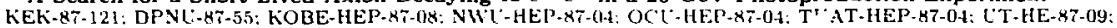

Charge Asymmetry Measurement in $e^{+} e^{-} \rightarrow \mu^{+} \mu^{-}$and $\tau^{+} \tau^{-}$Reactions at $\sqrt{\theta}=52 \mathrm{GeV}$

Phys. Lett 200B:391.1988: KEK-87-103: DPNL-87-53: KOBE-HEP-87-06: NUT'-HEP-87-(12: OCL-HEP-87-02: TCATHEP-87-02: UT-HE-87-07:

Measurement of the Processes $e^{+} e^{-} \rightarrow e^{+} e^{-}$and $e^{+} e^{-} \rightarrow \gamma \gamma$ at $\sqrt{s}=50 \mathrm{GeV}$

Phys. Rev. D37:1339,1988; INS-661: KEK-87-109; UT-HE-87-0S: DPNC-HT-54: KOBE-HEP-87-07: NWL-HEP-87-03: OCL'-HEP-87-03: TUAT-HEP-B7-03:

Search for Sequential Heavy Leptons in $e^{+} e^{- \text {Collisions at } \sqrt{9}=52 \mathrm{GeV}}$

Phys. Rev. Lett. 60:97.1988: CT-HE-ki-116:

Search for Top Quark in $e^{+} e^{-}$Collisions at $\sqrt{8}=52 \mathrm{GeV}$

Phys. Lett. 208B:319.1988:

Charge Asymmetry Measurement in $e^{+} e^{-} \rightarrow \mu^{+} \mu^{-}$and $\tau^{+} \tau^{-}$Reactions at $\sqrt{8}=52 \mathrm{GeV}$ and $55 \mathrm{GeV}$

Phys. Lett. 218B:105.1989: KEK-88-101:

Search for SUSY Particles at TRISTAN

KEK-89-31:

Search for New Particles in $e^{+} e^{-}$Annihilation at TRISTAN

Phys. Lett. 227 B:495.1989; KEK-89-37:

Measurement of $\alpha_{S}$ in $e^{+} e^{-}$Annihilation at $\sqrt{s}=53.3 \mathrm{GeV}$ and $59.5 \mathrm{GeV}$

Phys. Lett. 228B:553,1989; KEK-89-90: UT-HE-89-07: TUAT-HEP-89-02: TL-HEP-89-02: PL-89-63: OCC-HEP-89-03; NWL-HEP-89-03; KOBE-HEP-89-07: (NS-REP-767: DPNL-89-45:

A Search for Excited Leptons in the Energy Region $\sqrt{8}=52-60.8 \mathrm{Gev}$

Phys. Lett 229B:427,1989; KEK-89-75:

A Search for a Fourth-Generation Charge $-1 / 3\left(b^{\prime}\right)$ Quark Using inclusive Muons in $e^{+} e^{-}$Annihilations at $\sqrt{s}=56.5$ to $60.8 \mathrm{GeV}$

Phys. Lett. 234B:185,1990; KEK-89-156:

A Study of Pion Pair Production in the Two-Photon Process

Phys. Lett. 234B:525.1990: KEK-89-136: DPNC'-69-5]: INS-779: KOBE-HEP-89-10; NWW-HEP-89-04; OCC-HEP-89-04; PU-89-636: TU-HEP-89-03: TUAT-HEP-89-03: LTT-HE-89-08;

Measurement of the Total Hadronic Cross Section in $e^{+} e^{-}$Annihilation and Determination of the Standard Model Parameters

Phys. Let t. 240B:513,1990:

A Search for Charged Higgs Bosons at TRISTAN

Phys. Lett. 187B:437,1987; CERN-EP-86-69;

Cross Sections and Some Features of Cherm Photoproduction at 7 Energies of $20 \mathrm{GeV}$ to $70 \mathrm{GeV}$

Eur. Lett. 4:887,1987: CERN-EP-86-77:

Measurement of Charmed Particle Lifetimes and Decay Branching Fatios

Phys. Lett. 201B:397,1988;

Multiplicities and Rapidity Densities in 200 A GeV ${ }^{10} \mathrm{O}$ Interactions with Emulsion Nuclei

Phys. Rev. Lett. 62:2801.1989; LUJP-8813:

Limiting Fragmentation in Oxygen Induced Emulsion Interactions at 14.6, 60 and 200 A GeV

Phys. Lett. 223B:262,1989: LU1P-8814:

Scaling Properties of Charged Particles Multiplicity Distributions in Oxygen Induced Emulsion Interactions at 14.8, 80 and $200 \mathrm{~A} \mathrm{GeV}$

HZPP-88-01:

Charged Particle Spectra in Oxygen Induced Reactions at $14.6 \mathrm{GeV} /$ Nucleon and $60 \mathrm{GeV} / \mathrm{Nucleon}$

Phys. Lett. 227B:285.1989: LUIP-8905: Rapidity Densities and their Fluctuations in Central $200 \mathrm{~A} \mathrm{GeV}{ }^{32}$ S Interactions with Au and $\mathrm{Ag} / \mathrm{Br}$
Nuclei

LUIP-8904:

Substructural Dependence of the Multiparticle Production in Relativistic Heavy-Ion Interactions

Phys. Lett. 234B:180,1989: LU1P-8906;

Target Nucleus Fragmentation in ${ }^{10} \mathrm{O}+(\mathrm{Ag}, \mathrm{Br})$ Interactions at $200 \mathrm{~A} \mathrm{GeV}$

Phys. Lett. 230B:175,1989; LU1P-8907:

A Study of Recoil Protons in Uitra-Relativistic Nucleus-Nucleus Collisions

LUIP-8912;

On the Multiplicity Fluctuations in Relativistic Heavy-Ion Collisions

Mod. Phys. Lett. A5:169.1990:

On the Energy and Mass Dependence of the Multiplicity in Relativistic Heavy Ion Interactions

KEK-8T-38:

Spin Observables in Small-Angle Elastic $p$ deuteron $\rightarrow p$ deuteron Scattering with an $L$-type Deuteron Target at $800 \mathrm{MeV}$

Phys. Lett. 219B:399,1989:

Analyzing Powers for $p$ (polarized) deuteron $\rightarrow{ }^{3} \mathrm{He} \pi^{0}$ at $800 \mathrm{MeV}$

Phys. Lett. 177B:239.1986: IFVE-86-209:

Rapidity Dependence of Negative and All Charged Multiplicities in Nondiffractive $\pi^{+} p$ and $p$ Collisions at $250 \mathrm{GeV} / \mathrm{c}$

2. Phys. C $32: 475.1986$ :

Cross Sections and Charged Multiplicity Distributions for $\pi^{+} p, K^{+} p$ and $p p$ Interactions at $250 \mathrm{GeV} / \mathrm{c}$

Yad. Phys 47:429,1988; Z. Phys. ('35:7.1987: IFVE-86-210;

Inclusive $\pi^{j}$ Production in $\pi^{+} p, K^{+} p$ and $p$ Interactions at $250 \mathrm{GeV} / \mathrm{c}$

Phys. Lett 198B:427.1987: IFVE-87-1i1:

Strangeness Suppression and Inclusive $\phi$ and $K^{\bullet}(892)^{\circ}$ Production in $K^{+} p$ Interactions at $250 \mathrm{GeV} / \mathrm{c}$

Phys. Lett. 183B:42t, 1987: Yad. Phys. 47:136.1988: 1FV'E-86-211: Suppression of Valence Quark Recombination in $\pi^{+}$Fragmentation into $\rho^{+}$

Phys. Lett. 185B:200.1987: IFV'E-87-33;: C'ERN-EP-86.218:

Maximum Particle Densities in Rapidity Space of $\pi^{+} p, K^{+} p$ and $p p$ Collisions at $250 \mathrm{GeV} / \mathrm{c}$

Phys. Lett. 1808:223.1987: IFVE-87-29:

$\pi^{+} p$ and $K^{+} p$ Elastic Scattering at $250 \mathrm{GeV} / c$ 
Adamus 87E

Adamus 88

Adamus $88 B$

idamus $88 \mathrm{C}$

Adamus 88F

Adamyan 84C

Adamyan 86

Adamyan 88

Adamyan 89

Adeishvili 87

Adelberger 87

Aderholz 86

Aderholz 89

Adeva 85

Adeva 85B

Adera $85 \mathrm{C}$

Adeva 86

Adeva 86B

Adeva 86C

Adeva 87

Adeva 88

Adeva 89

f.deva $89 \mathrm{~B}$

Adeva 90

Adeva 90B

Adeva 90C

Adeva 90D

Adiels 86

Adiels 86B

Adiels 88

Adier 87

Adler $87 \mathrm{~B}$

Adler $\mathbf{8 8}$

Adler $89 B$

Adler 88D
Adamus 88G

Adler 88C

Phys. Lett. 198B:292,1987: 1FVE-87-112:

A Comparison of Inclusive $\rho^{\circ}, \rho^{+}$and $\omega$ Production in $K^{+} p$ Interactions at $250 \mathrm{GeV} / \mathrm{c}$

2. Phys. C.37:347,1988;

Bose-Einstein Correlation in $\mathrm{K}^{+} p$ and $\pi^{+} p$ at $250 \mathrm{GeV}$

2. Phys, C37:215.1988:

Phase Space Dependence of the Multiplicity Distribution in $\pi^{+}{ }_{F}$ and $p p$ Colltsions at $250 \mathrm{GeV} / c$

Phys. Lett. 205B:4Gl,1988:

Multiplicit: Distribution in Separated Phase Space Intervals

2. Phys. C39:301.1988.

Single Difraction Dissociation in $\pi^{+} p$ and $K^{+} p$ Interactions at $250 \mathrm{GeV} / \mathrm{c}$

Z. Phys. C39:311,1988; IFVE-88-121;

Charged Particle Production in $K^{+} p, \pi^{+} p$ and $p p$ Interactions at $250 \mathrm{GeV} / \mathrm{c}$

Pisma Zh. Eksj. Teor. Fiz. 42:345.1985; YFRE-722(37)-84:

Cross Section Asymmetry of the $\gamma n-\pi^{-} p$ Reaction by Polarized Photons in the Third and Fourth Resonance Regiong

Czech. J. Plyys. B36:945,1986:

Cross Section Asymmetry for the Reactions $\gamma$ deuteron $\rightarrow p n, \gamma$ deuteron $\rightarrow \pi^{0}$ deuteron by Linearly Polarized Photons in the Energy Range $E(\gamma)=0.4-0.8 \mathrm{GeV}$

YERE-106](24)-88:

Experimental Study of the Photoproduction $\gamma$ deuteron $\rightarrow$ deuteron $\pi^{\circ}$ and Photodisintegration $\gamma$ deuteron $\rightarrow p n, \gamma^{\theta} \mathrm{Li} \rightarrow p n \mathrm{X}$ and $\gamma^{a} \mathrm{He} \rightarrow p \mathrm{n} X$ Processes by Linearly Polarized Photons in Energy Fange $0.3-$ $1 \mathrm{GeV}$

Phys. Lett. 232B:296.1989;

Cruss Section Asymmetry for the Reaction $\gamma$ deuteron $\rightarrow$ deuteron $\pi^{0}$ in the Energy Range $0.5-0.9 \mathrm{GeV}$

Jetp 65:882,1987; Zh. Eksp. Teor. Fiz. 92:1574.1987:

Experimental Study of $780 \mathrm{MeV}$ Electron Scattering by a Silicon Crystal

Pliys. Rev. Lett. 59:849.1987;

New Constraints on Composition Dependent Interactions Weaker than Gravity

Pliys. Lett. 173B:2 11,1986; CERN-EP-86-31:

Measurement of Total Cross Section for Neutrino and Antinetirino Charged Current Interactions in Hudrogen and Neon

Phys. Rev. Lett, 63:2349.1989: 1IHE- on us,

Coherent Production of $\pi^{+} \pi^{-}$Mesons by Charged Current Interactions of Neutrinos and Anti-neutrinos on Neor Nuclei at the Fermilab TEVATRON

Phys. Lett, 152B:439,1985;

New Particles Searches

Phys. Rey. Lett. 55:665,1985: Measurement of $e^{+} e^{-} \rightarrow \mu^{+} \mu^{-}$: A Test of Electroweak Theories

Phys. Rev. Lett. 54:1750,1985:

Measurement of Strong Coupling Constant $\alpha_{S}$ to Second Order for $22 \leq E_{\mathrm{cm}} \leq 46,78 \mathrm{GeV}$

Phys. Rev. D34:681,1986;

Scudy of Hadron and Inclusive Muon Production from $e^{+} e^{-}$Annihilation at $39.79 \leq E_{\mathrm{cm}} \leq 46.78 \mathrm{GeV}$

Phys. Lett. 179B:177,1986;

The Production and Decay of Tau Leptons

Phys. Lett. 180B:181,1986:

Measurement of Strong $C$,upling Constent $\alpha_{S}$ to Complete Second Order

Phys. Leti. 194B:167,1987;

Search for SUSY Particles photinc, $\tilde{e}, \tilde{\nu}$, wino' in Electron Positron Annihilation at $44 \mathrm{GeV}$

Phys. Rev. D38:2665,1988:

Electroweak Studies in $e^{+} e^{-}$Collisions $12<\sqrt{s}<46.78 \mathrm{GeV}$

Phys. Lett. 231B:509.1989; L3-001;

A Determination of the Properties of the Neutral Intermediate Vector Boson $Z^{0}$

Phys. Lett. 233B:530.1989; L3-002;

Mass Limits for Scalar Muons, Scalar Electrons, and Winos from $e^{+} e^{-}$Collisions Near $\sqrt{a}=81 \mathrm{GeV}$

Phys. Lett, 237B:136,1990; L3-004;

Measurement of $Z^{0}$ Decays to hadrons, and a Precise Determination of the Number of Neutrino species

Pliys. Lett. 236B:109,1990; L3-003:

Measurement of Ga and Gv the Neutral Current. Coupling Constant to Leptons

Phys. Lett. 238B:122,1990; L3-005:

$A$ Measurement of the $Z^{0}$ Leptonic Partial Widths and the Vector and Axial Vector Coupling Constants

Phys. Lett. 241 B:416,1990: L3-006;

Measurement of $Z^{0} \rightarrow$ b $\breve{b}$ Decay Properties

Z. Phys. C35:15,1987; Z. Phys. C35:19,1987; CERN-EP-86-154;

Experimental Determination of the Branching Ratios $p \bar{p} \rightarrow 2 \pi^{0}, \pi^{0} \gamma$ an's $2 \gamma$ at Rest

Phys. Lett. 182B:405,1986; CERN-EP-86-155;

Search for Narrow Signals in the ', Spectrur, from $p \bar{p}$ Annihilation at Rest

Z. Phys. C42:49,1989; CERN-EP-8:-142:

Experimental Study of the Inclusive $\eta$-Spectrum from $p \bar{p}$ Annihilations at Rest in Liquid Hydrogen

Phys. Let L. 196B:107,1987; SLAC-PUB-4346;

Resonant Substructure in $K \pi \pi$ Decays of Charmed $D$ Mesons

Phys. Rev. Lett. 59:1527,1987; SLAC-PUB-\$205; SLAC-PUB-4205;

Measurement of the Decay $\tau^{ \pm} \rightarrow \rho \nu$

Phys. Rev. Lett. 60:1375,1988; Phys. Rev, Lett. 63:1658,1989; SLAC-PUB-4343:

Search for the Decay $D^{-4} \rightarrow \mu^{+} \nu_{\mu}$ and Upper Limit on the Pseudoscalar Decay Constant

Phys. Rev. D37:2023,1988; Phys. Rev. D40:3788,1989: SLAC-PUB-44:38:

Search for the Decay $D^{0} \rightarrow e^{+} e^{-}$

Pliys. Lett. 208B:152.1988; Pinys. Lett. 227B:501.1989: SLAC-PUB-4518;

Measurement of the $D^{*}(\mathbf{2 0 1 0})$ Branching Patios

SLAC-PUB-4686;

Measurements of $f_{1}(1285) \rightarrow \pi^{+} \pi^{-} \pi^{+} \pi^{-}, \eta_{c}(1 S) \rightarrow 0^{0} \rho^{0}$ and $\eta_{c}(1 S) \rightarrow f_{2}(1270) f_{2}(1270)$ in Radiative $J / \psi(15)$ Decays

See the legend on page 19 . 
Adler $88 \mathrm{~F}$

Adler 89

Adler $89 \mathrm{~B}$

Adler $89 \mathrm{C}$

Adler $89 \mathrm{D}$

Adler 89E

Adyasevich 85

Adyasevich 85B

Adyasevich 85C

Adyasevich 87

Adyasevich 8\%B

Adyasevich $88 B$

Adyasevich 89

Afanasyev 88

Afanasyev 90

Afanasyev 90B

Afonin 85

Afonin $85 B$

$A$ fonin $85 \mathrm{C}$

Afonin 86

Afonin 87

Afonin $87 \mathrm{~B}$

Afonin $87 \mathrm{C}$

Afonin 88

Afonin 88B

Agababyan $85 B$

Agababyan 85C

Agababyan 89

Agábabyan 89B

Agakishiev 84B

Agakishiev $84 \mathrm{E}$

Agakishiev $\mathbf{8 5}$

Agakishiev 86B

Agakishie: 87

Agakishiev 87B
Phys. Rev. Lett. 60:89.1988: S1,AC.PUB-4291:

A Reanalysis of Charmed D Meson Branching Fractions

Phys. Rev. Lett. 62:1821.1989. SLAC-PUB-4745.

Measurement of the Branching Fractions for $D^{0} \rightarrow \pi^{-} e^{+} \nu_{e}$ and $D^{0} \rightarrow K^{-} e^{+} \nu_{r}$ and Determination of $\left(\mathrm{V}_{\mathrm{CD}} / \mathrm{V}_{\mathrm{CS}}\right)^{2}$

Phys. Rev. Lett, 63:1211,1989: S1.AC-PL'B-4952;

Observation of $D_{S}^{+} \rightarrow \bar{K}^{\circ} K^{+}$and $D_{S}^{+} \rightarrow \bar{K}^{*}(892)^{\circ} K^{+}$and an Upper Limit on $D_{S}^{+} \rightarrow K^{0} \pi^{+}$

Phys. Rev D40:906.1989: SLAC.PLB-4671:

A Search for the Decay $D^{0}-\bar{K}^{\circ} e^{+} e^{-}$

SLAC-PUB-5130; CALT-68-1606:

Resonant Substructure in $\boldsymbol{K}^{-} \boldsymbol{\pi}^{+} \boldsymbol{\pi}^{+} \boldsymbol{\pi}^{-}$Decays of $\boldsymbol{D}^{0}$ Mesons

SLAC-PLB-5052;

Recent Results on Hadronic $D_{S}^{ \pm}$and $D$ Meson Decays from the MARK-III

Phys. Lett. 161B:55.1985:

Universal Proton Rapidity Distributions in High Energy Nucleus-Nucleus Collision

KIA E-85-4152-2;

Invariant Cross Sections of Protons Emitted in the Interactions of ${ }^{3} \mathrm{He}$ Nuclei with C, Cu and P!b Targets at $4.9 \mathrm{GeV} / \mathrm{Nucleon}$

KIAE-85-4148-2:

Invariant Cross Sections of Deuterons Emitted in the Interactions of ${ }^{12} \mathrm{C} \mathrm{Nuclei}$ with $\mathrm{C}, \mathrm{Cu} \mathrm{Sn}$ and $\mathrm{Pb}$ Targets at $3.6 \mathrm{GeV} /$ Nucleon

Yad. Phys. 45: 436.1987 ;

Inclusive Spectra of Protons Emitted in Interactions of $4.9 \mathrm{GeV} / \mathrm{Nucleon}{ }^{3} \mathrm{He}$ Nuclei with $\mathrm{C}, \mathrm{Cu}, \mathrm{and} \mathrm{Pb}$ Nuclei

Yad. Phys. 46:1353.1987:

Emission of Compound Light Fragments in Collisions Between Relativistic Nıclei

Vopr. At. Nauki i Tecin. Ser. Obsch. 2-42:13,1988:

The Measurement of Two-particles Correlations of Fragments in the Interaction of ${ }^{12} \mathrm{C}$ Nuclei at the 3.6 GeV/Nucleon Energy with Pb Nuclei

vopr. At. Nauki i Techn. Ser. Yad. 2:9,1989;

The Measurement of Two Particle Correlation in the Reactions $\mathrm{C}+\mathrm{Pb}(3 . \mathrm{G} \mathrm{GeV} / \mathrm{N})$

Yad. Phys. 4T:1656.1988;

Cross Sections of $K^{ \pm}$Meson Interaction with C, Al, Ti, and Cd Nuclei in $1.5 \mathrm{GeV} / c$ Range

Phys. Lett. 236B:116,1990:

Measurement of the Branching Ratio for $\pi^{0}$-Meson Decay into a Photon and a Positronium Atom

Yad. Phys. 51:1040.1990;

Measurement of the Photon Internal Conversion Coeffleient into Positronium Atom and of the $\pi^{0} \rightarrow \gamma$ positronium Branching Ration

Pisma Zh. Eksp. Teor. Fiz. 11:355,1985; Jetp Lett. 41:435,1988:

Neutrino Experiment at Rovno AES: The Cross Sections of Inverse $\beta$-Decay

Pisma Zh. Eksp. Teor. Fiz. 42:230,1985; Jetp Lett. 42:285.1988:

Search of Neutrino Oscillations at Rovno AES

Yad. Phys. 42:1138.1985: Sov. J. Nucl Phys. 42:719,1985;

Measurement of Inverse $\beta$-Decay Section by Means of Scintillation Detector at Rovno AES

Pisma Zh. Eksp. Teor. Fiz. H:111,1986;

Comparison of $\bar{\nu}_{e}$ Intensities at Two Distances from Rovno AES Reactor

Pisma Zh. Eksp. Teor. Fiz. 45:201,1987;

$\bar{\nu}_{e}$ Spectra at Two Distances from Rovno AES Reactor: Search for Oscillations

Yad. Phys. 46:1304.1987;

Measurement of the Cross Section of Inverse $\beta$-Decay at Reactor of Rovno AES: Results from Scintillaticn Method

Yad. Phys. 46:1590,1987:

Total Cross Section of Inverse $\beta$-Decay (Results of Experiment at Reactor of Rovno AES, Mede by Integral Method)

KIAE-88-4746-2:

Measurements in the Neutrino Flux of the Rovno AES by Spectrometer RONS

Zh. Eksp. Teor. Fiz. 94(2):1,1988:

Investigation of the $\bar{\nu}_{e} p \rightarrow e^{+} n$ Reaction in a Nuclear Reactor

YERE-820(4\%)-85;

Investigation of Incoherent Production of Pion Systems in Reactions $\pi^{-}$Nuclei $\rightarrow \pi^{-} \pi^{-}$Nuclei and $\pi^{-}$ Nuclei $\rightarrow \pi^{-} \pi^{-} \pi^{+}$Nuclei on Nuclei at $5 \mathrm{GeV} / \mathrm{c}$

Yad. Phys. 41:1401,1985:

Study of Deuteron Photodisintegration by Linearly Polarized Photon in the Energy Range $0.4-0.8 \mathrm{GeV}$

Z. Phys. C $41: 539.1989$; IFVE-89-120:

Inclusive Meson Res mance Production in $\mathrm{K}^{+}{ }_{\mathrm{p}}$ Interactions at $250 \mathrm{GeV} / \mathrm{c}$

Yad. Phys. 50:1341.1989:

Measurement of Polarization Parameters $\Sigma, T$, and $P$ in $\pi^{0}$ Photoproduction at $E(\gamma)=0 . \theta-1.35 \mathrm{GeV}$

Yad. Phys. 40:1209.1984; Z. Phys. C27:177,1985; JINR-P1-84-35:

Multiplicities, Moment um and Angular Characteristics of $\pi^{-}$Mesons Produced in Interactions of Protons, Deuterons, Alphas and Carbon Nuclei with Carbon at $4.2 \mathrm{GeV} / \mathrm{c}$ per Nucleon

Yad. Phys. 11:1562.1985; JINR-P1-84-551:

Correlation Phenomena in Multinucleon C C Interaction at $4.2 \mathrm{GeV} / c$ per Nucicon

Yad. Phys. 43:366.1986: Sov. J. Nucl. Phys. 43:234,1986: JINR-P1-85-220:

$\Lambda$ and $K^{0}$ Production in $C p$ and $C$ C Interactions at $4.2 \mathrm{GeV} / c$ per Nucleon

Yad. Phys. 45:1373.1987: JlNR-P1-86-370:

Analysis of Behaviour of $\pi^{-}$Mesons and Protons Produced in nucleus nucleus Interactions at $4.2 \mathrm{GeV} / c$ per Nucleon Depending on the Number of Interacting Protons

Yad. Phys. 47:1292,1988: JINR-P1-87-442;

Two Particle Correlations and Regions of Proton Emittance Regions in $p$ Ta Interactions at $P=10 \mathrm{GeV} / \mathrm{c}$ JINR-P1-87-924;

Sizes of Cumulative Pion and Proton Formation Region According to the Data on $p$, $p$ Ta and $\pi^{-} \mathrm{C}$ 
Agakishiev $87 \mathrm{~B}$ (cont $\mathrm{d}$ ) Interaction at $p(p)=10 \mathrm{GeV} / \mathrm{c}$ and $p\left(\pi^{-}\right)=40 \mathrm{GeV} / \mathrm{c}$

Agakisher $37 \mathrm{C}^{\circ}$ Yad. Phys $45: 423,14 \mathrm{kT}$.

Inclusive Distributions of $\pi^{-}$Mesons Produced in $\pi^{-} \mathrm{C}$ Interactions at $P=40 \mathrm{GeV} / c$ and in $p \mathrm{C}$. nd $p$ Ta Interactions at $P=\theta . \theta \mathrm{GeV} / \mathrm{C}$

Agakishiev $8 \mathrm{H}$

Vat. Phys f9:481 1984. IIVR-P1-K8-15.4:

Dependence of the Chnracteristics of Proton-Nucleus Interactions at 4.2 and $10 \mathrm{GeV} / \mathrm{C}$ on a LeadingParticles Energy

Agakishiev 89 JINR-Pl-8S-5is:

Characteristics of Cumulative $\Lambda$ - Hyperons Produced in Nucleus - Nucleus Collisions

Agakishiev 89B

Yad. Phiv- 51:1591.1990: JINR-Pl-k9-793:

Dependence of $\pi^{-}$Meson Spectra at Fixed Angles on the Atomic Weight of Projectile in Interactions between Light Nuclei (p,deuteron, ${ }^{4} \mathrm{He}, \mathrm{C}$ ) and Carbon Nucleus at $4.2 \mathrm{GeV} / \mathrm{c}$ per Nucleon

Agakishiev $89 \mathrm{C}$

Yad. Phys. 51:759.1990: JINiR-P1-89-488.

Comparative Characteristics of Central and Noncentral C C Interactions at $\$ .2$ GeV per Nucleon

Aggirwal 8.5

Aggarwal 85B

2 Phyit ( $26: 323.19 k 5$.

Experimental Study of Proton Emulsion Nuclei lnteractions at High Energy

Phys. Rev. C31:1233.1985:

Momentum and Boltzmann Distributions of Neon Fragments at Approximately $300 \mathrm{MeV} / \mathrm{Nucleon}$

Aglamazov 85

Agliet ta $\mathrm{R6B}$

Aglietta 89

Aguilarbenit 85

Aguilarbenit $85 \mathrm{C}$

Fiz. 49:1380,1985

Cross Section of Inelastic Interaction of Cosmic Ray Muons

Nuovo Cim. 9C: 588,1980 Monopole Search with the Mont Blanc LSD Experiment

Eur. Let t. 8:611,1989;

Experimertal Study of Atmospheric Neutrino Flux in the NUSEX Experiment

IFIE-85-21:

New Results on Neutral $D$ Meson Properties in $360 \mathrm{GeV} / c \pi^{-} p$ Interactions

Phys. Lett 156B:444.1985; CERN-EP-\$5-02:

A Search for $D_{S}^{ \pm}$Production in $380 \mathrm{GeV} / c \pi^{-} p$ Interactic is

Aguilarbenit $85 \mathrm{D}$

Phys. Lett 16+B:404.1985: CERN-EP-85-123:

$D \bar{D}$ Correlations in $380 \mathrm{GeV} / c \pi^{-} p$ and $p$ Interactions

Aguilarbenit $85 \mathrm{E}$

Aguilarbenit $85 \mathrm{~F}$

Inclusive Properties of $D$ Meson Produced in $380 \mathrm{GeV} \pi^{-} p$ Interactions

Phys. Lett. 160B:217,1985: CERN-EP-85-105: Search for Anomalons in $360 \mathrm{GeV} / c \pi^{-} p$ and $p$ Interactions

Aguilarbenit 86

Phys. Let t. 168B:170.1986: Measurement of $D$ Meson Branching Ratios

Aguilarbenit $86 \mathrm{~B}$

Aguilarbenit $86 \mathrm{C}$

Aguilarbenit $86 \mathrm{D}$

Aguilarbenit 87

Aguilarbenit $87 \mathrm{~B}$

Aguilarbenit $87 \mathrm{C}$

Aguilarbenit $8 \pi \mathrm{D}$

Aguilarbenit 8TE.

Aguilarbenit $87 \mathrm{~F}$

Aguilarbenit $87 \mathrm{H}$

Phy's. Lctt. 169B:106,1986; CERN-EP-86-06; IF VE-k6-16is: Neutral and Charged $D^{*}(2010)$ Production in $360 \mathrm{GeV} / c \pi^{-} p$ Interactions

2. Phys. C34:419.1987; Yad. Phys, 46:1098.1987: IFVE-86-196: CERN-EP-86-165: Inclusive $\pi^{\circ}$ and $\eta$ Meson Production in $\pi^{-} p$ Interactions at $360 \mathrm{GeV} / c$

2. Phys. C31:491.1986: CERN-EP-85-103;

Charm Hadron Properties in $360 \mathrm{GeV} / \mathrm{c} \pi^{-} p$ Interactions

Phys. Lett. 199B:462.1987; CERN-EP-87-126; [FVE-88-30;

$\Lambda_{c}^{+}$Production Characteristics in Proton-Proton Interactions at $400 \mathrm{GeV} / \mathrm{c}$

Phy's. Lett. 193B:140.1987; CERN-EP-87-38: D-Meson Lifetimes

Phys. Lett. 189B:476.1987: Yad Phys 48:757 1988. CERN-EP-87-45: JFVE-47-189

$D$-Meson Production from $400 \mathrm{GeV} / c$ p $p$ Interactions

2. Phys. C34:1+3.1987: CERN-EP-86-16\%:

Determination of $D$-Meson Lifetimes

Z. Phys C36:551.1987: CERN-EP-87-135:

Inclusive D-Meson Branching Ratios

Z. Phys. C36:559,1987; CERN-EP-87-61:

Exclusive Hadron Branching Ratjos of the $D$ Meson

Phys. Lett 189B:254.1987: Yad. Phys. 48:1310.1988: Sov. J. Nucl. Phys. 48:K3:3.1988: 1FVE-N7-114. CEHN-EP-87-17: Lifetime Measurement of $\Lambda_{c}^{+}$

Aguilarbenit 88

Aguilarbenit 888

Phys. Lett 201B:176.1988. CERN-EP-8T-212.

$D$-Meson Produ-tion from $400 \mathrm{GeV} / \mathrm{c} p$ interactions. Evidence for Leading Diquarks?

2. Phys. C: $40: 321, i 98 d:$

Charm Hadron Properties in $400 \mathrm{GeV} / \mathrm{c} p \mathrm{p}$ Interactions

Z. Phys. C41:191.1988.

Comparative Properties of $400 \mathrm{GeV} / \mathrm{c}$ Proton-Proton Interactions with and without Charm Production

Aguilarbenit s8C

Aguilarbenit 89

2. Phys. C44:531,1989: CERN-EP-89-58:

Vector Meson Production in $\pi^{-}$Interaction at $380 \mathrm{GeV} / \mathrm{c}$

Ahlen 87

Phys. Lett. 195B:603,1987;

Limits on Cold Dark Mateer Candidates from an Ultralow Background Germanium Spectrometer

Ahlen 88

Phys. Pev. Lett 61:145,1988;

New Limit on the Low-Energy $\bar{p} / p$ Ratio in the Galactic Cosmic Radiation

Ahmad 84

Ahmad $85 \mathrm{~B}$

Phys. Lett, 152B:135.1985: CERN-EP-84-167; Search for Monochromatic Pion Emission in $\bar{p} p$ Annihilation from Atomic $P$ States

Nucl. Phys. B254:441,1985:

Multiparticle Production in $\pi^{-}$Emulsion Collision at $340 \mathrm{GeV} / \mathrm{c}$

Phys. Lett. 157B 3333.1985: CERN-EP-85,-05;

First Observation of $K X$ rays from $\bar{p} p$ Atoms

TR1-PP-86.45:

Antiproton-Proton Annihilation into Collinear Charged Pions and Kaons

Ahmad 86

Phys. Rev. Lett. 59:970.1987:

Searches for $\mu^{-} e^{-}$and $\mu^{-} e^{+}$Conversion in Titanium

Phys. Rev. D38:2102.1988; TRj-PP-88-51;

Search for Muon-Electron and Muon-Positron Conversion

See the legend on page 19. 
Ahmad 89

Ahrrad 90

Ahn 86

Ahn 87

Ahrar 86

Ahrens 85

Ahrens $85 B$

Ahrens 86

Ahrens 87

Ahrens $87 \mathrm{~B}$

Ahrens $87 \mathrm{C}$

Ahrens 88

Aibergenox 86

Aihara 8.4F

Aihara $84 \mathrm{G}$

Aihara 85

Aihara 85B

Aihara $85 \mathrm{C}$

Aihara $85, \mathrm{D}$

Aihara 85E

Aihara $85 F$

Aihara $85 \mathrm{G}$

Aihara 86

Aihara $86 \mathrm{~B}$

Aihara $86 \mathrm{C}$

Aihara 86D

Aihara $86 \mathrm{E}$

Aithara $86 \mathrm{~F}$

Aihara $86 G$

Aihara $86 \mathrm{H}$

Ailuara 86I

Aibara 86J

A ihara 87

Aihara 87 Is

Aihara 87C

Aihara 87D

Aihara $87 \mathrm{E}$

Aihara $87 \mathrm{~F}$
Acta Plyys. Polon. B20:701.1189:

Multiparticle Production in $340 \mathrm{GeV} \pi^{-}$Nucleus Collisions

Nuovo Cim. 103A:517.1990:

Characteristics of Hadron Nucleus Interactions with and without Emission of Hadrans in the Backward Hemisphere

Phys. Let 1. 177B:233.1986: FERMILAB-PlB-86-74-EXP.

Valence Quark Effects in Beam Remnants in High $E_{f} p$ pollisions at $E_{\mathrm{cm}}=27.4 \mathrm{GeV}$

Phys. Lett. 1835:115.1987:

Scaling and Fragmentation Distributions of Beam Remnants in High $E_{t}$ Proton Proton Colligions at $E_{\mathrm{cm}}=27.4 \mathrm{GeV}$

Phys. Rev. D33:25.1986:

Study of Inelastic Interactions of $340 \mathrm{GeV} / \mathrm{c}$ Pions with Emulsion Nuclei

Phys. Rev. Lett. 54:18.1985;

Measurement of the Ratio of Cross Sections for Neutrino and Antineutrino Scatering from Electrons

Phys. Rev. D31:2732.1985:

New Limit on the Strength of Mixing between $\nu_{\mu}$ and $\nu_{r}$

Phys. Rev. D34:75.1986:

Determination of the Neutrino Fluxes in the Brookhaven Wide Beams

Phys. Rev. D3f.702.1987:

Comparison of Narrow-Band and Wide Band Neutrino Beams in the Search for $\nu_{\mu} \rightarrow \nu_{\mathrm{r}}$ Oscillations

Phys. Lett. 1948:586.1987:

A Search for Heavy Neutrino Decays in a Neutrino Beam

Phys. Rev. D35:785.1987;

Measurement of Neutrino-Proton and Antineutrino-Proton Elastic Scattering

Phys. Lett. 202B:244.1988.

A Study of the Axial-Vector Form Factor and Second-Class Currents in Antineutrino Quasielastic Scattering

Czech. J. Phys. B36:948,1986:

Radiat ive Photoproduction of Pions and Pion Compton Scat tering

Z. Phys. C27:187.1985: LBL-18326:

Inclusive $\gamma$ and $\pi^{\circ}$ Production Cross Sections and Energy Fractions in $e^{+} e^{-}$Annihilation at 28 GeV

Z. Phys. C28:31.1985: LBL-18408:

Tests of Models for Quark and Gluon Fragmentation in $e^{+} e^{-}$Annihilation at $20 \mathrm{GeV}$

Phys Rev. Lett. 53:2378,1985: LBL-18325:

$K^{*}(892)^{\circ}$ and $K_{S}$ Meson Production in $e^{+} e^{-}$Annihilation at $29 \mathrm{GeV}$

Phys. Rev. Lett. 54:274.1985; LBL-18382:

$\Lambda$ Production in $e^{+} e^{-}$Annihilation at $29 \mathrm{GeV}$

2. Phys. C27:495.1985: LBL-18911:

Quark Fragmentation Functions and Long Range Correlations in $e^{+} e^{-}$Annihilation at $2 \theta \mathrm{GeV}$

Phys. Rev. Lett. 54:2564.1985; UClK-TPC-85-08:

Exclusive Production of $\mathrm{K}^{+} \mathrm{K}^{-} \pi^{+} \pi^{-}$in Photon-Photon Collisions

Phys. Rev. D31:2719.1985;

Prompt Muon Production in $e^{+} e^{-}$Annihilation at $29 \mathrm{GeV}$

2. Phys. C27:39,1985: LBL-17545:

Prompt Electron Production in $e^{+} e^{-}$Annihilation at $29 \mathrm{GeV}$

Phys. Rev. Lett. 55:1047.1985: LBL-19566:

Baryon Production in $e^{+} e^{-}$Annihilation at $29 \mathrm{GeV}$ : Clusters or Diquarks?

Phys. Rev. D33:844.1986:

Study of $\eta$ Formation in Photon Photon Collisions

Phys. Rev. Lett. 57:945.1986: LBL-21543:

Comparison of the Particle Flow in $q \bar{q}$ gluon and $q \bar{q} \gamma$ Events in $e^{+} e^{-}$Annihilation

Phys. Rew, Lett. 57:51.1986:

Experimental Limit on $\eta(1440)-\cdots \gamma$ and the Interpretation of the $\eta(1440)$ as a G lueball

Phys. Rev. Lett. 57:-404.1986: LCR-TPC-86-01:

Pion and Kaon Pair Production in Photon Photon Collisions

Phys. Rev. D34:1945,1986: IS-J-1848:

Charged $D^{*}(2010)$ Meson Production in $e^{t} e^{-}$Annihilation at $\sqrt{s}=2 \theta \mathrm{GeV}$

Phys. Rev. Lett. 57:3140.1986: LBL-22037:

Study of Baryon Correlations in $e^{+} e^{-}$Annihilation at $29 \mathrm{GeV}$

Phys. Rev. Lett. 57:1836.1986; LBL-21766:

Measurement of the Inclusive Branching Fraction $\tau^{-} \rightarrow \nu_{\tau} \pi^{-} \pi^{\circ}+$ Neutral Meson(s)

Plyys. Rev. Lett. 57:3245,1986:

Search for High Mass Nerrow Resonances in Virtual Photon Photon Interactions

Phys. Rev. D.35:155.3.1986:

Measurement of $\tau^{ \pm}$Branching Ratios

Phys. Rev. Lett. $57: 2500.1986$ :

Evidence for Spin-1 Particle Produced by Two Photon

Phys. Rev. D35:2650.1987:

Study of $\eta^{\prime}$ Formation in Photon Photon Collisions

Phys, Lett. 184B:114.1987: J.BL-21544:

Comparison of $\pi^{ \pm}, K^{ \pm}$and $p \tilde{p}$ Production in the Central Rapidity Region in Hadron Hadron Collisions and in $e^{+} e^{-}$Annihilation

Phys. Letl. 184B:299.1987;

Pion and Kaon Multiplicities in Heavy Quark Jets from $e^{+} e^{-}$Annihilation at $20 \mathrm{GeV}$

2. Phys C $34: 1.19 k 7$ :

Measurement of the Photon Structure Function $F_{2}\left(x, Q^{2}\right)$ in the Region $0.2<Q^{2}<7 \mathrm{GeV}^{2}$

Phys. Rev. D36i:3506i.1987: Exclusive Production of Proton-Antiproton Pairs in Two-Photon Collisions

Phys. Rev. Let1. $5,8: 97,1987$

Observation of Scaling of the Photon Structure Function $F_{2}$ at Low $Q^{2}$ 
Aihara $87 \mathrm{G}$

Aihara 88

Aihara 88B

Aihara $88 \mathrm{C}$

Aihara $88 \mathrm{D}$

Aihara $88 \mathrm{E}$

Aihara $88 \mathrm{~F}$

Aihara 89

Aihara $89 \mathrm{~B}$

Aihara $89 \mathrm{C}$

Aivazyan 86

Aivazya. $86 \mathrm{~B}$

Aivazyan 88

Aivazyan 89

Ajaltouni $85 \mathrm{~B}$

Ajaltouni 87

Ajaltouni 88

Ajaltouni 88B

Ajinenko 83B

Ajinenko 8.1

Ajinenko 8.4B

Ajinenko $84 \mathrm{C}$

Ajinenko 85

Ajinenko $86 \mathrm{~B}$

Ajinenko $86 \mathrm{C}$

Ajinenko 87

Ajimenko $87 \mathrm{~B}$

Ajinenko 89

Ajinenko $89 \mathrm{\theta}$

Ajinenko $89 \mathrm{C}$

Ajinenko 89D

A jinenko $89 \mathrm{E}$

Ajinenko 90

Ajinenko $90 \mathrm{~B}$

Ajnutdinov 88

Akchurin 89

Akerlof 8.5
Phys. Rev. Lett 59:751,198T, LBL-23176:

Experimental Limit on the Decay $\tau^{-} \rightarrow \nu_{t} K^{-} K^{\circ}$

Phys. Rev, Di37:28,198\%.

Production of Four-Prong Final States in Photon-Photon Collisions

Phys. Rev. Lett. 60:2355.1988:

Charmonium Production in Photon-Photon Collisions

Phys. Rev. Lett 61:1263.198R: LBL-24k96;

Charged Hadron Inclusive Cross Sections and Fractions in $e^{+} e^{-}$Annihilations at $\sqrt{\theta}=29 \mathrm{GeV}$

Phys. Rev, D38:1.1988:

Formation of Spin-1 Mesons by Photon-Photon Fusion

Phys. Lett. 209B:107.1988.

$f_{1}(1285)$ Formation in Photon-Photon Fusion Reaction

LBL-23737:

Charged Hadron Production in $e^{+} e^{-}$Annihilation at $\sqrt{\theta}=28 \mathrm{GeV}$

Phys. Rev, D40:2772.1989: NIKHEF-H-89-19:

Exclusive Production of $p \bar{p} \pi^{+} \pi^{-}$in Photon Photon Collisions

Z. Phys. C44:357,1989; [T-HE-89-02:

Evidence of Soft and Collinear Gluon Emission in $e^{+} e^{-}$Hadronic Events

SLAC-PUB-5]01: LC-IIRPA-89-02:

A Measurement of the Total Hadronic Cross Section in Tagged $\gamma \gamma$ Reactions

YEHE-859(10)-86:

Inclusive Spectra of He, Li, Be, Bor Fragments Produced on Nuclei by Electrons with Energies of 2 and $4.5 \mathrm{GeV}$ at Angles 50 and 130 Degrees

YERE-857(8)-86;

The Dependence of Inclusive Electroproduction Cross Sections of $\mathrm{He}$, Li, Be Fragments on the Charge, the Angle of Emission, the Energy of Electrons, the Mass Number of the Target Nucleus and their A nalysis

Phys. Lett. 209B:193.1988: HEN-286:

Multiplicity Dependence of the Average Transverse Momentum in $\pi^{+} p, K^{+} p$ and $p p$ Collisions at 250 GeV

2. Phys. C42:533,1989:

Forward-Backward Multiplicity Correlations in $\pi^{+} p, K^{+} p$ and $p$ Collisions at $250 \mathrm{GeV} / c$

LAL-85-45:

Results on Photon-Photon Physics with the DM2 Detector

Phys. Lett. 194B:573.1987; LAL-87-15; Pion Pair Production in Photon-Photon Collisions at DCI

LA L-88-30:

Three and Five Photon Decays of the $J / \psi(1 S)$

LAL-88-06:

Study of the Decays $J / \psi(1 S) \rightarrow$ Vector + Pseudoscalar

Z. Phys. C25:103.1985; IFVE-83-153:

Inclusive $K^{*}(892)^{+}, K_{3}^{*}(1430)^{+}$and $K^{*}(892)^{-}$Production in $K^{+} p$ Interactions at $32 \mathrm{GeV} / \mathrm{c}$

Sov. J. Nucl. Pliys. 41:214.1985; Yad. Phys. 41:338.1985: IFVE-84-107:

Reactions with Two Neutral Kaons Inclusive Production in $K^{+} p$ Interactions at 32 GeV/c

Sov. J. Nucl. Phys. 41:972,1985: Yad. Phys. 41:1535,1985: IFVE-84-180:

Upper Limits on Production Cross Sections of Charmed Particles in $K+p$ Interactions at 32 GeV/c

Sov. J. Nucl. Phys. 41:593,1985: Yad. Phys. 41:925,1985: IFVE-84-154:

Inclusive $K_{S} \Lambda, K_{S} \bar{\Lambda}$ and $\Lambda \bar{\Lambda}$ Pair Production in $K^{+} p$ Interactions at $32 \mathrm{GeV} / c$

Yad. Phys. 43:1195.1986: Sov. J. Nucl. Phys. 43:765.1986: IFVE-85-108;

Study of the Reaction $K+p \rightarrow p \Lambda \times$ at $32 \mathrm{GeV} / c$

Yad. Phys. 45:1026.1987: IFVE-86-115:

Inclusive $\pi^{ \pm}$Mesons Production in Association with Two Leading Particles in $K^{-} p R_{e a c t i o n s}$ at 32 GeV/c

Yad. Phys. 44:6-44,1986: Sov, J. Nucl. Phys. 44:417,1986:

Cross Sections of Exclusive $K^{+} p$ Reactions at $32 \mathrm{GeV} / \mathrm{c}$

Phys. Let.. 197B:457,1987: [FVE-87-113;

Indicat on of an Onset of Hard-like Effects in $K^{+} p$ and $\pi^{+} p$ and $p$ Collisions at $250 \mathrm{GeV} / c$

Yad. Phys. 46:818.1987: IFVE-86-177;

Difraction Processeg in 6 body Exclusive $K^{+} p$ Reactions at $32 \mathrm{GeV} / \mathrm{c}$

Z. Phys. C42:377.1989:

Multiplicity Distribution in $K^{+} \mathrm{Al}$ and $K^{+} \mathrm{Au}$ Collisions at $250 \mathrm{GeV} / \mathrm{c}$ and a Test of the Multiple Collisions Model

Z. Phys. C43:15.1989; IFVE-89-70:

A Study of $4^{-}$and $B$ body Reaction in $K^{+} p$ and $\pi^{+} p$ Interactions at $250 \mathrm{GeV} / c$

Phys. Lett. 222B:306.1989: HEN-309:

Intermittency Patterns in $\pi^{+} p$ and $K^{+}{ }_{p}$ Collisions at $250 \mathrm{GeV} / \boldsymbol{c}$

Z. Phys. C43:37.1989:

Charge and Energy Flow in $\pi^{+} p, K^{+} p$ and $p p$ Interactions at $250 \mathrm{GeV} / c$

2. Phys. C44:573.1989: IF V'E-89-172:

Strange and Non-Strange Baryon Production in $\pi^{+} p$ and $K^{+} p$ Interactiong at $250 \mathrm{Gev} / \mathrm{c}$

Phys. Lett, 235B:377.1990:

Intermittency Effects in $\pi^{+} p$ and $K^{+} p$ Collisions at $250 \mathrm{GeV} / c$

2. Phys. C.46:56!1,1990:

Negative Binomial and Multiplicity Distributions in $250 \mathrm{GeV} / \mathrm{c} \mathrm{K}^{+}$and $\pi^{+}$Interactions on $A l$ and Au Nuclei

ITEP-88-118

The Track Experiment for Double $\beta$-Decay of ${ }^{138} \mathrm{Xe}$

Pliys. Lett. 229B:269.1949. ANL-HEJ-PR-69-82:

Analyzing-Power Measurement of Coulomb-Nuclear Interference with the Polarized-Proton and Antipraton Beams at $185 \mathrm{GeV} / \mathrm{c}$

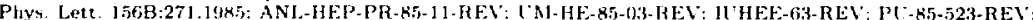

Experimental Limits on Monojet Production in $e^{+} e^{-}$Annihilations at $24 \mathrm{GeV}$ 
Akerlof $85 \mathrm{~B}$

Akerlof 88

Akesson $84 \mathrm{~B}$

Akesson 85

Akesson $85 \mathrm{~B}$

Akesson $85 \mathrm{C}$

Akesson $85 \mathrm{D}$

Akesson $85 \mathrm{E}$

Akesson $85 \mathrm{~F}$

Akesson $85 \mathrm{G}$

Akexion 86

Akesson $86 \mathrm{~B}$

A kesson $86 \mathrm{C}$

Akesson $86 \mathrm{D}$

Akesson $86 \mathrm{E}$

Akesson $86 \mathrm{~F}$

Akesson 87

Akesson $87 \mathrm{~B}$

Akesson $87 \mathrm{C}$

Akesson $87 \mathrm{E}$

A kesson 88

Akesson 88B

Atesson $88 C$

A kesson $88 \mathrm{D}$

Akesson 89

Akesson $89 \mathrm{~B}$

Akesson 89D

Akesson $89 \mathrm{E}$

A kesson 90

A kesson 901

Akesson 90C

Akhababian 8

Akkmerov 8 ?

Akiba 85

Akimenko 85

Akimenko 89

Akimereks, git
Phys. Rev. Lett 55:570.1985:

Measurement of the Topological Branching Fractions of the Tau Lepton

Phys. Rev, D37:57T,196k:

Experimental Limits on Massive Neutrinos from $e^{+} e^{-}$Annihilation at $29 \mathrm{GeV}$

Phys. ' ett. 152B.140.1985; CERN-EP-44-155:

A N-easurement of Alpha-Alpha Elastic Scattering at the CERN ISR

Phys. Rew. D31:976.1985:

Rapidity and Charge Correlations of Centrally Produced Charged Particles in Event with a High Momentum $\pi^{0}$ near 11 Degrees

Phys. Lett. 155B:128.1985; CERN-EP-85-12:

Bose-Einstein Correlations between Kaons

Phys. Lett. [52B:411.1985: CERN-EP-84-169:

Production of Prompt Positrons at Low Transverse Momentum in 63 GeV p $p$ Collisions at the CERN Intersecting Storage Rings

Nucl. Phys. B264:154.1986: CERN-EP-85-115:

A Search for Glueballs and a Study of Double Pomeron Exchange at the CERN Intersecting Storage Rings

Z. Phys. C30:27.1986: CERN-EP-85-164:

Dijet Production Cross Section and Fragmentation of Jets Produced in p p Collisions at $63 \mathrm{GeV}$

Phys. Rev. Lett. 55:2535,1985:

Search for Quark Deconflnement: Strangeness Production in $p p$, deuteron deuteron, $p$ He, and He He Collisions at $\sqrt{s}=31.5$ and $44 \mathrm{GeV}$

Phys. Let . 158B:282.1985: CERN-EP-85-55:

A Comparison of Direct Photon, $\pi^{0}$, and $\eta$ Production in $\bar{p} p$ and $p p$ Interactions at the CERN ISR

Z. Phys. C32:317.1986: C.ERN-EP-86-52:

Three Jet Production in p p Collisions at $63 \mathrm{GeV}$ and a Determination of os

2. Phys. C32:491.1986: C'ERN-EP-86-37;

A Study of the Production of Two Direct Photons in $p$ pollisions at the CERN ISR

Phys. Let. 178B:447.1986: CERN-EP-86-84:

Inclusive $\eta$ Production at Low Transverse Moment um in $63 \mathrm{GeV} p$ p Collisions at the CERN ISR

2. Phys, C34:293.1987: CERN-EP-86-195:

Direct Photon Plus A way Side Jet Production in $p$ Collisions at $E_{\mathrm{cm}}=63 \mathrm{GeV}$ and a Determination of the Gluon Distribution

Phys. Scr. 34:106.:986:

A Comparison of Particles Recoiling from High $p_{T}$ Direct Photons and $\pi^{0}$ 's at the ISR

Phys Lett. 187B:420.1987: CERN-EP-86-215

Evidence for a Directional Dependence of Bose-Einstein Correlations at the CERN Intersecting Storage Rings

Ż. Phys. C34:163,1987: CERN-EP-86-216:

Double Parton Scattering in $p$ Collisions at $E_{\mathrm{cm}}=63 \mathrm{GeV}$

Phys. Lett. 192B:-163.1987: CERN-EP-87-16:

Correlations Between the Production of Prompt Positrons at Low Transverse Momentum and the Asgociated Charged Multiplicity

Phys. Rev. D36:2615.1987: CERN-EP-87-85:

Search for Direct $\gamma$ Production at Low Transverse Momentum in 63 GeV p $p$ Collisions

2. Phys. C36:517.1987: CERN-EP-8T-142:

Pion Interferometry in Jet Events at the CERN Intersecting Storage Rings

2. Phys C38:383.1988:

The Transverse Energy Distribution in ${ }^{16} \mathrm{O}$ - Nucleus Collisions at 60 and $200 \mathrm{GeV}$ per Nucleon

Z. Phys. C38:397,1988:

The Transverse Energy Distribution in Hadron-Lead Collisions

Phys. Lett. 214B:295,1988;

The Transverse-Energy Digtribution of ${ }^{32} \mathrm{~S}$ - Nucleus Collisions at $200 \mathrm{GeV}$ per Nucleon

Phys. Rev. D38:2687,1988:

Comparison of Low $p_{T}$ Photon Production in High-and Low-multiplicity Colligions at the CERN-ISR

Phys. Lett. 231 B:359.1989:

Measurement $d \sigma / d E_{t}$ in Collisions of Light Nuclei at $\sqrt{\theta_{11 n}}=31.5 \mathrm{GeV}$

Nucl. Phyt. B333:48.1990: CERN-EP-89-97:

Charged Particle Multiplicity Distributions in Oxygen - Nucleus Collisions at $60 \mathrm{GeV}$ and $200 \mathrm{GeV}$ per Nucleon

CERN-EP-89-113:

Inclusive Photon Production in $p$ nucleus and nucleus-nucleus Collisions at $200 \mathrm{GeV} / \mathrm{Nucleon}$

CERN-EP-89-111:

Inclusive Negative Particle pr Spectra in p-nucleus and nucleusanucleus Collisions at $200 \mathrm{GeV}$ per Nucleon

Nucl. Phys. B342:279.1990: CERN-EP.90-42:

An Emulsion Study of ${ }^{16} \mathrm{O}$ and ${ }^{32} \mathrm{~S}$ Interactions at $200 \mathrm{GeV}$ per Nucleon Selected by Transveise Energy

Phys. Lett 238B:442,1990: CERN-EP-89-172.

Search for Scalar Electrons and Winos at the CERN $\vec{p} p$ Collider

$Z$ Phys. CH6:179.1990:

Search for Top Quark Production at the CERN $\bar{p} p$ Collider

JINR-P1-85-639.

Two-Proton Correlation in He C Interactions at $4.2 \mathrm{GeV} / \mathrm{c}$ per Nucleon

Izv. Akad. Nauk SSSR. Fiz. 51:140,1987

Scattering of Flectrons on ${ }^{3}$ He under 127 Degrees

Phys. Rev. D32:2911.J985; KEK-85-20:

A Study of the Radiative Decay $\boldsymbol{K}^{+} \rightarrow \mu^{+} \nu_{\mu}+$

Yarl. Phys 43:615.19k1i; Sov. J. Nucl. Phys. 43:392.1966; IFVF-45-43;

Relative Yields of $\eta$ Mesons Produced by $10 \mathrm{GeV}$ Pions and Kaous

Yard. Phy, 51.437.1949: IFV'E-89-95:

Study of Exclusive Processes $\pi^{+} \rightarrow \eta$ and $\pi^{+} \rightarrow \pi^{0} \pi^{0}$ on Nuclei at $10.5 \mathrm{GeV} / \mathrm{c}$

IF IF-90-10]

Experimental St udy of $K^{\circ}$-Meson Inclusive Production in $K^{+}$nucleus Interaction at $11.2 \mathrm{GeV}$ 
Akimenko 90C

Akimov 89

Akopova 86

Akrawy 89

Akrawy $89 \mathrm{~B}$

Akrawy $89 \mathrm{C}$

Akrawy $89 \mathrm{D}$

Akrawy $89 \mathrm{E}$

Akrawy 90

Akrawy $90 \mathrm{~B}$

Akrawy $90 \mathrm{C}$

Akrawy 90D

Akrawy 90E

Akrawy 90F

Akrawy $90 G$

Akrawy $90 \mathrm{H}$

Akrawy 90

Akrawy $90 \mathrm{~J}$

Akrawy $90 \mathrm{k}$

Akrawy $90 \mathrm{~L}$

Akrawy $90 \mathrm{M}$

Akrawy 90N

$A \operatorname{lam} 86$

$A \operatorname{lam} 8 \pi$

Alam $87 B$

Alam 89

Alam 89B

Alanckyan 84

Alanakyan 87

Alanakyan 88

Albajar 86

Albajar 86B

Albajar 86C

Altuajar 8 ?

Albajar $87 \mathrm{~B}$

Albajar $87 C$

Albajar $87 D$

Altojar $87 \mathrm{E}$
IFVE-90-36:

Experimental Study of Inchusive $K^{*}(892)^{\circ}$ Meson Production in $K^{+}$nucleus Interactions at 11.2 GeV Kr. Soul, JINR 35.11,1!)

Investigation of Anomalous Pion Production in the Reaction $\mathrm{p} C u \rightarrow \pi^{+} \mathbf{X}$ by 350 MeV Protons

Yiad. Phys H.16i2,1986;

Time Averaged Charge and Energy Spectra of Galactic Nuclei with $Z \geq 50$

Phys. Lett. 23! B-530.19k!, (EIRN-EP-89-133:

Measurement of the $Z^{\circ}$ Mass and Width with the OPAL Detector at LEP

Phy's. Lett. 23613:3164.198!4; C'ERN-EP-89-154:

$A$ Search for the Top and $b^{\prime}$ Quarks in Hadronic $Z^{0}$ Decays

Phys. Let 1. 2:35B:389 1989: (ERN-EP-R!-153:

A Study of Jet Production Rates and a Test of QCD on the $Z^{\circ}$ Resonance

CERN-EP-89-175:

A Search for New Charged Heavy Leptons with the OPAL Detectors at LEP

CERN-EP-89-176:

A Search for Acoplanar Pairs of Leptons or Jets in $Z^{0}$ Decays: Mass Limits on Supersymmetric Particles

Phys. Lett. 235B:379.1990; CERN.EP-89-147:

Measurement of the Decay of the $Z^{0}$ into Lepton Pairs

CERN-EP-!O-00:

A Direct Search for New Charged Heavy Leptons at LEP

Z. Phys. C47:505.1990: CERN-EP-90-48:

A Measurement of Global Event Shape Distributions in the Hadronic Decays of the $Z^{\circ}$

Phys. Lett. 242B:299.1990: C'ERN-EP-90-38:

A Search for Technipions and Charged Higgs Bosons at LEP

Phys. Let. 240B:497.1990: CERN-EP-90-27:

A Combined Analysis of the Hadronic and Leptonic Decays of the $Z^{0}$

Phys. Let t. 2418:133.1990: CERN-EP-90-29:

A Study of the Reaction $e^{t} e^{-} \rightarrow 2 \gamma$ at LEP

Phys. Lett. 244B:135.1990; CERN-EP-90.49

Search for Excited Leptons at LEP

CERN-EP-90-5.5:

Evidence for Final State Photons in Multihadronic Decays of the $Z^{0}$

CERN-EP-90-72:

Limits on Neutral Heavy Lepton Production from $Z^{\mathbf{0}}$ Decay

Phys. Lett. 236B:224.1990: CERN-EP-89-174:

Mass Limits for Standard Model Higgs Boson in $e^{+} e^{-}$Collisions at LEP

CERN-EP-90-81

Analysis of $Z^{\circ}$ Couplings to Charged Leptons

Phys. Lett. 247B:617.1990: CERN-EP-90-94:

A Study of Coherence of Soft Gluons in Hadron Jets

Phys. Lett. 248B:211.1990: CERN-PPE-90-95:

A Direct Search for Neutralino Production at LEP

CERN-EP-90-100:

Search for Neutral Higgs Bosons in $e^{t} e^{-}$Collisions at LEP

Phys. Rev. D34:3279.1986:

Study of the Decay $B \rightarrow J / \psi(1 S) \times$

Phys. Rev. Lett. 58:1814.1987:

Branching Ratios of $B$ (unspec) Mesons to $K^{+}, K^{-}$, and $K^{0} / \bar{K}^{\circ}$

Phys. Rev. Lett. 59:22.1987:

Evidence for Charmed Baryons in $\boldsymbol{B}$ Meson Decay

Phys. Rev. D40:712.1989: Phy's. Rev. D40:3790.1989: ('LNS-89-88B: ('1.EO-89-4:

Search for a Neutral Higgs Boson in $B$ Meson Decay

Fhys. Lett. 226B:401.1985: CLNS-89-921: CLEO-8!1-9:

Measurement of the Isospin Mass Splitting $\Xi_{r}(2460)+\Xi_{r}(\mathbf{2 4 8 0})^{0}$

Yad. Phys, $11+353.1985:$ YERE-707(22)-84:

Structure Functions of the Nuclei ${ }^{12} \mathrm{C},{ }^{63} \mathrm{Cu},{ }^{208} \mathrm{~Pb}$ in the Cumulative Proton Photoproduction

Yad. Phys. 45:751.1987; YERE-889(40)-80:

Correlations in Photoproduction of Cumulative Protons

Yad. Phys. 47:1653.1988: YERE-979(29)-87:

Different Features of Spectra and Strusture Functions of Nuclei from Inclusive and Backward-Forward Correlation Production of Cumulative Photoprotons

Phys. Lett. 185B:233.1987: CERN-EP-86-81

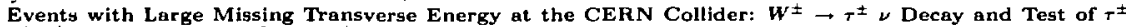
$\mu^{ \pm} e^{ \pm}$Universality at $Q^{2}=m\left(W^{ \pm}\right)^{2}$. Paper 1

Phys Lett. 185B:241.1987: CERN-EP-86-82:

Events with Large Missing Transverse Energy at the CERN Collider. Search for the Decays of W into Heavy Leptons and $Z^{0}$ into Non Interacting Particles. Paper 2

Phys. Lett 186B:237.1987: CERN-EP-86-208:

Beauty Production at the CERN Proton Antiproton Collider. Paper

Phys. Lett. 198B:271.1487; CERN-EP-87-149:

Intermediate Vecta." Boson Cross Sections at the CERN Super Proton Synchrotron Collider and the Number of Neutrino Types

Phys. Lett. 198B:261.1987: C.ERN-EP-87.148: Events with Large Missing Transverse Energy at the CERN Collider: III. Mnss Limits on Supersymmetric Particles

Phys. Lett. 186B:245.I\$67; ('ERN-EP-86-209. Search for $B^{0}-\bar{B}^{0}$ Oscillations at the CERlv Proton Antiproton Collider. Paper 2

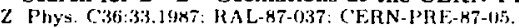

Analysis of the Highest Trangverse Energy Events Seen in the UA1 Detector at the $\bar{p} p$ Collider

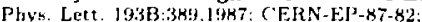

Production of $W^{ \pm}$'s with Large Transverse Montentun a: the CERN Proton Antiproton Collider 
Albajar 88

Albajar 88

Albajar knkb

Albajar 88 C $^{\circ}$

Albajar 88D

Albajat $88 E$

Albajar XNF

Aitrajar xec;

Albajar ksh

Aluajal 89

Abajar 89B

Altrajar $89 \mathrm{C}^{\circ}$

Albajar 90

Albajar $90 \mathrm{~B}$

Albajar $900^{\circ}$

Allajar 900

Albajar $90 \mathrm{E}$

Albanese 85

Albini 85

Albrecht 85

Albrecht $85 \mathrm{~B}$

Altoredht 85.5

Albrecht R.5D

Albreche 85E

Albrecint $85 \mathrm{~F}$

Albrecht $\times 5$;

Albrecht $\times 5.5$

Albrecht 855

Albrecht 4.5.J

Aibrecht $45 \mathrm{~K}$

Albrecht $\times 51$.

Albrechi $\times 5: 9$

Albrecint $45 \mathrm{~N}$

Albrecht $x 6$

Albrecin K6B

Allireche $\mathrm{kfiC}$.

Albrechn Kf;D

Allireriat kfiE:

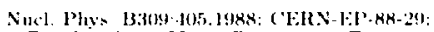

Production of Low Transverse Energy Cluster in $\bar{p} p$ Collisions at $\sqrt{s}=0.2 \cdot 0.8$ TeV and their Interpretation in Terms of QCD Jets

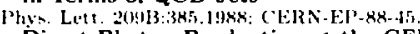

Direct Photon Production at the CERN Proton-Antiproton Collider

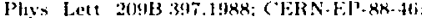

Low Mass Dimuon Production at the CERN Proton-Antiproton Collider

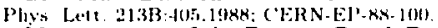

Measurement of the Bottom Quark Production Cross Section in Proton-Antiproton Collisions at $\sqrt{s}=0.63$ TeV

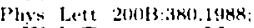

High Transverse Momentum $J / w$ (1S) Production at the CERN Proton-Antiproton Collider

Z Phys. ('37:4k:1.19kx:

Study Heavy Flavor Production in Events with a Muon Accompanied by Jets at the CERN ProtonAntiproton Collider

2. Phys. (37:505.198k:

Search for New Heavy Quarks at CERN Proton-Antiproton Collider

Phys. Let t. 20913 127.1988: (CERN-EP-XK-5)-4: Two Jet Mass Distributions at the CERN Proton - Antiproton Collider

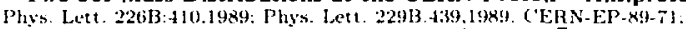
Bose-Einstein Correlation in $\bar{p} p$ Interactions at $\sqrt{s}=0.2$ to $0.9 \mathrm{TeV}$

2. Plys ('+1.15.1!k9: CERN-EP-88-168:

Studies of Intermediate Vector Boson Production and Decay in UA 1 at the CERN Proton-Antiproton Collider

CERN-EP-N9-13\%:

Comparison of $W^{ \pm}$and Direct Photon Cross Sections at Large Transverse Momenta

CERN-EP-90-56:

Intermittency Studies in $p \bar{p}$ Collisions at $\sqrt{s}=630 \mathrm{GeV}$

Nucl. Phys. B335:261.1990; CERN-EP-K4-45:

A Study of the General Characteristics of Proton Antiproton Collisions at $\sqrt{9}=0.2$ TeV to 0.9 TeV

Phys. Lett. 241B:283.1990; CERN-EP-90)-35:

Experimental Limit on the Decay $W^{ \pm} \rightarrow \pi^{ \pm}$个 at the CERIN Proton-Antiproton Collider

PhYs. Lett. 244B:566.1990: CERN-EP-90-61:

$A$ Study of the $D^{*}(2010)$ Content of Jets at the CERN $p \bar{p}$ Collider

CERN-EP-G(1)-5i:

Search for New Heavy Quarks in Proton-Antiproton Collisions at $\sqrt{8}=0.63 \mathrm{TeV}$

Phy's. Lett. 15KB:1 1 66.19*5: CERN-EP-N5-76:

Direct Observation of the Decay of Beauty Particles into Charm Particles

Sov. J. Nucl. Plyys. 43:585.1986: Yad. Phys. 43:917.11866; JNNi-Pl-8.5-144:

Investigation of Inclusive Processes $\pi^{-}$nucleus $\rightarrow p X$ at $40 \mathrm{GeV} / c$

Phys Let t. 150B:235.1965:

Production and Decay of the Charged $D^{*}(2010)$ Meson in $e^{+} e^{-}$Annihilation at $10 \mathrm{GeV}$ Centre of Mass Energy

Phys Lett. 14tiB:111,1985:

Evidence for $D_{S}^{*}$ Meson Production in $e^{+} e^{-}$Annihilation at $10 \mathrm{GeV}$ Centre of Mass Energy

Phy' Lett. 154B:452.1985:

Search for Narrow States Coupling to Tau Pairs in Radiative $\mathbf{Y}$ Decays

Phys Lett. 1538:343.1985:

Production and Decay of the $D_{S}^{ \pm}$Meson in $e^{+} e^{-}$Annihilation at $10 \mathrm{GeV}$ Centre of Mass Energy

Phys. Lett. 157B:326.1985:

Observation of Antideuteron Production in Electron Positron Annihilation at $10 \mathrm{GeV}$ Center of Mass Energy

Phys Lett 15tiB3:13-1985:

Search for Fractionally Charged Particles Produced in $e^{+} e^{-}$Annihilation

Pliys Lett. 15kB:5:25.1965:

Direct Evidence for $W^{ \pm}$Exchenge in Charmed Meson Decay

Plys J.ett. 16013:331.19k5: DESY'-65-1)(6x:

Radiative Decays of the $\Upsilon(2 S)$ into this Three $x_{b}$ (unspec) States

Z. Phys. ('26:45.1985;

A Determination of the Muon Pair Branching Ratio of the $\Upsilon$ (1S) Meson

Phys. Lett. 1633:404.1965:

An Upper Limit on the Mass of the Tau Neutrino

Plist. lett 1628:34:5.1985:

Observation of $B$ Meson Decay into $J / \psi(1 S)$

Z. Phiss. ("29:1677.1965:

Upper Limit for the Emission of Monoenergetic Photons in $\mathbf{Y}(1 S)$ and $\mathbf{r}(2 S)$ Meson Decays

P'hys lett 17913 348.194.5:

Observation of $D_{S}^{ \pm}$Meson Decay into $K *(892) K$

Phys Let1. 1N2B:35, I985:

Determination of the Branching Ratio for the Decay $B \rightarrow D^{*}(2010)^{-} \pi^{+}$

Phys. Let1. 16713:360.1986:

Search for Gluinos in Decays of the $x_{t_{1}}(1 P)$ Meson

Pliys. Rev. leet1 56i:549.1986:

Observation of a New Charmed Meson

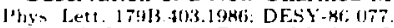

Search for Exotic Decay Modes of the $\mathrm{Y}(1 \mathrm{~S})$

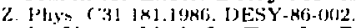

An Upper Limit for Two Jet Production in Direct $\boldsymbol{r}_{\mathfrak{l}}$, , Decnys

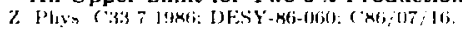

Measurement of Tau Decays into Three Charged Pions 
Albreche $86 \mathrm{~F}$

Albrecht $86 \mathrm{G}$

Albrecht 87

Albrecht $87 \mathrm{~B}$

Albrecht $87 \mathrm{C}$

Albrecht 87D

Aibrecht BAE

Albrecht $87 \mathrm{~F}$

Albrecht $87 \mathrm{G}$

Albrecht $87 \mathrm{H}$

Albrecht $87 \mathrm{I}$

Albrech $87 \mathrm{~J}$

Albrecht $87 \mathrm{~K}$

Albrecht $87 \mathrm{~L}$

Albrecht $87 \mathrm{M}$

Albrecht $87 \mathrm{~N}$

Albrecht 870

Albrecht $87 \mathrm{P}$

A'brecht $87 Q$

Albrecht $87 \mathrm{R}$

Albrecht $87 \mathrm{~S}$

Albrecht $\mathrm{kTT}$

Albrecht 88

Albrecht $88 \mathrm{~B}$

Al'recht $880^{\circ}$

Allorecht $48 D$

Albrerht 88E

Albrecht \&8F

Altrerht $88 C^{\circ}$

Altrecht $8 x \mathrm{H}$

Alturecht 861

Altureche $48 \mathrm{~J}$

Albrecht HoK

Albrecht $8 \mathrm{XL}$

Albrecht $48 \$$

Albrecht $\mathrm{HBN}$

A fbrecht 880

Altrecht KAP

Altsrecht $88 Q$
DESY-86-06il:

Observation of $D_{S}^{+}$Decays into $K^{\cdot(892)} K^{*}$

Phys. Let1, 18:3B:419,1!8R: DESY'-N(i-130:

Observation of Octet and Decuplet Hyperons in $e^{+} e^{-}$Annihilation at $10 \mathrm{GeV}$ Centre-of-Mass Energy

Phys. Lett 1!!9B:297 1!167:

Forward and Trassverse Energy Distribution in Oxygen-Induced Rcaction at 60 A GeV and 200 A GeV

Phys. Lett. 1H51 218 . lont:

Reconstruction of $B$ Mesons

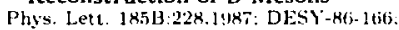

Search for Lepton Nurnber and Lepton Flavour Violation in Tau Decays

Phys. Letl. 147B:425.1987: DESY'-8T-(101:

Observation of Inclusive $D_{S}^{ \pm}$Production in $B$ Meson Decay

Z. Phys C $33: 359.1947$.

The Decay $D^{\circ} \rightarrow K^{0} \phi$

Phis. Let1. 199B:447.1967: DESY-

An Upper Limit on $D^{0}-\bar{D}^{\circ}$ Mixing

Phys. Lett. 199B:451.1987: DESY-87-111:

$B$ Meson Decays into Charmonium States

Phys. Lett. 199B:291.1987: DESY-87.0187:

Determination of as from a Measurement of the Direct Photon Spectrum in $\mathrm{r}(1 S)$ Decays

Plys. Let1. 185B:223.1987: DESY-K6-142:

Evidence for the Decay $\tau^{-} \rightarrow \omega \pi^{-} \nu_{\tau}$

Phys. Lett. 196B:101.1987: DESY'-87-1iak: First Observation of $\gamma \gamma \rightarrow \omega \rho^{0}$

Phys. Lett, 198B:577.1987; DESY-87-(5): First Observation of $\gamma \gamma \rightarrow 2 \omega$

Phys. Let1. 199B:580.1987: DESY-87-128: A Measurement of the Tau Lifetime

Phys. Let 1. 199B:457.1987: DESY- $87+(1633$ :

Measurement of $\eta^{t} \rightarrow \pi^{+} \pi^{-} \gamma$ in $\gamma \gamma$ Collisions

Phys. Lett 267B:349.1988: DESY-87-156):

Measurement of $D_{S}^{*}-D_{S}^{ \pm}$Mass Difference

Phys. Let1, 197B:452.1987, DESY-87-1799;

Measurement of the Decay $B^{0} \rightarrow D^{*}(2010)^{-} \ell^{+} \nu$

Phys. Lett 192B:245.1987: DESY-87-025:

Observation of $B^{0}-\overparen{B}^{0}$ Mixing

Z. Phys. C35:28:3.1987: DESY-86-104:

The Hadronic Transitions from $\Upsilon(2 S)$ to $\Upsilon(1 S)$

Phys. Lett. 195B:102.1987: DESY-87-04:5:

Search for the Decay $D_{S}^{ \pm} \rightarrow \rho^{0} \pi$ as Evidence for Quark Annihilation

Phys. Lett 198B:255.1987: DESY-87-095:

First Observation of $\gamma \gamma \rightarrow K^{*}(\mathbf{8 9 2})^{0} \bar{K}^{*}(892)^{0}$

Phys. Lett. 195B:307.1987: DESY'-87-055:

Search for the Decay $\tau^{-} \rightarrow \nu_{\tau} \nu \pi^{-}$

Phys. Lett. 202B:596.1988:

Charge Particles Distributions in ${ }^{10} \mathrm{O}$ Induced Nuclear Reactions at 80 and $200 \mathrm{GeV}$

Phys. Lett. 201 B:390.1988:

Photon and Neutral Pion Distribution in 60 and $200 \mathrm{~A} \mathrm{GeV}{ }^{16} \mathrm{O}+$ Nucleus and Proton + Nucleus Reactions

Phys. Lett. 202B:149.1988: DESY-87-148:

An Improved Upper Limit on the $\nu_{r}$ Mass from the Decay $\tau^{-} \rightarrow 3 \pi^{-} 2 \pi^{+} \nu_{\tau}$

Phys. Lett. 207B:109.1988: DESY. KS-01I:

Observation of the Charmed Baryon $\Lambda_{r}^{+}$in $e^{+} e^{-}$Annihilation at $10 \mathrm{GeV}$

Phys. Lett. 2108:258.1988: DESY-kR-062:

Search for Decay $B \rightarrow K^{*}$ ?

Phys. Lett. 20913:380.i988: DESY-88-0)53: Search for $D^{0}$ Decays into Lepton Pairs

Phy's. Lett 210B:263.1986: DESY-\$8-1)12:

Observation of Inclusive Beauty-Meson Decays into $\Lambda_{r}^{+}$Baryons

Phys. Lett. 211B:489.1988: DESY-8x-0:58:

Observation of the Charmed Baryon $\Sigma_{r}(\mathbf{2 4 5 5})$ in $e^{+} e^{-}$Annihilations

Z. Phys. C39:177.198\%; DESY'-87-141:

Hyperon Production in $e^{+} e^{-}$Annihilation at $10 \mathrm{GeV}$ Centre of Mass Energy

Phys. Lett. 210B:267.1988: DESY-8X-0377:

Lifetimes of Charmed Mesons

2. Phys. CA1:55T.1984: DESY-84-079

Inclusive $\phi-M e s o n$ Production in Electron-Pasitron Interactions in the Energy Region of the $\mathbf{\Upsilon}$ Resonances

Phys. Lett. 2101:273.1984: DESY'84-016.

$A$ Search for $\omega \phi$ and $\phi \phi$ Production in the Reactions $\gamma \gamma \rightarrow K^{+} K^{-} \pi^{+} \pi^{-} \pi^{\theta}$ and $\gamma \rightarrow \rightarrow 2 K^{+} 2 K^{-}$

Phys. Lett. 215B:A24.1988. DESY-HK-131. $B$ Meson Decays to $D \pi$ and $D \rho$

Phys. Lett. 212B:52א.1948: DESY-8X-0.1:

Firgt Observation of $\gamma \gamma \rightarrow K^{*}(892)+K^{*}(892)$

Z. Phys. (4]:1.1968: DESY-48-(1868:

Measurement of the Decays $\tau^{-} \rightarrow K^{*}(802)^{-} \nu_{x}$ and $\tau^{-} \rightarrow \rho^{-} \nu_{\tau}$

2. Phys ( A1)-40i 1!ks: DESY-8k-113:

Upper Limit for the Decay of $\tau^{ \pm}$Leptons into $\eta$ Mesons

Plyy Lett. 215B3:-12010.1989: JESY-86-072:

Observation of the Orbitally Excited $\Lambda\left(1520 D_{03}\right)$ Bnryon in ${ }^{+}+$Annihilations

See the legersd ron pagr 19 
Albrecht 8XR

Albrecht $\mathrm{x} 8 \mathrm{~s}$

Albrecht xh'?

Albreclit 8:

Albrecht $89 \mathrm{~B}$

Albrecht $\mathrm{BQC}$

Albrecht $\mathrm{k9D}$

Albrecht $39 \mathrm{E}$

Albrecht $\mathrm{kgF}$

Albrecht $69 \mathrm{G}$

Athrecht $89 \mathrm{H}$

Albrechx X!̣I

Albrecht 89.J

Albrecht $89 \mathrm{k}$

Albrecht $89 \mathrm{~L}$

Albrecht $89 \mathrm{M}$

Albrecht $89 \mathrm{~N}$

Albrecht 890

Albrecht $89 \mathrm{P}$

Albrecht $89 Q$

Albrecht 89R

Albrecht 895

Albrecht 89T

Albrecht $89 \mathrm{C}^{\circ}$

Albrecht $\mathrm{N9V}$

Albrecht 891

Albreche $89 \mathrm{X}$

Albrecht 90

Albrecht 90B

Albrecht $900^{\circ}$

Albrecht 90D

Albrecht 90F:

Albrow $8 \mathrm{x}$

Alcie 86

Alde $x$ ti $B$

Alde $8 t^{\circ} \mathrm{C}^{\circ}$

Alde 860

Alde $\mathrm{A}$ t $\mathrm{F}$.

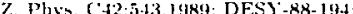

Two-Photon Production of Final States with a $p \bar{p}$ Pair

Z. Plyss (43:181.1984: DESY-H8-16ix:

Measurement of $D^{\circ}$ Decays into $\bar{K}^{0} \omega, \bar{K}^{0} \eta$ and $\bar{K}^{*}(892)^{0} \eta$

Phys. Lett. 209B:11!1988: DESY-AX-1)5(i:

Observation of the Charmless $B$ Meson Decays

Phy, Lelt 2:21B:422.1989: DESY-88-179: Observation of $\mathrm{D}^{*}(\mathbf{2 4 5 9}) 0$ in $e^{+} e^{-}$Anuihilation

Z. Phys. C 13:45.1984: DESY-89-013:

Results on Baryon Antibaryon Correlations in $e^{+} c^{-}$Annihilation

Phys. Lett. 219B:121.1989; DESY'-H8-178: Measurement of $D^{*}(2010)^{+}$Polarization in the Decay $\bar{B}^{0} \rightarrow D^{-}(2010)^{+} e^{-} \bar{D}$

Phys. Lett. 221B:427.1989: GSI-49.24: Fluctuation and Intermittency in $200 \mathrm{~A} \mathrm{GeV}{ }^{10} \mathrm{O}+(\mathrm{C}, \mathrm{Au})$ Feaction

Z. Phys. C42:519.1989: DESY-BR-1.45: Measurement of Inclusive B-Meson Decays into Baryons

Phys. Lett. 217B:205.1989: DESY-84-150:

A Measurement of $\gamma \gamma \rightarrow \rho^{+} \rho^{-}$

Z. Phys. C46:15.1990: DESY-89-066:

Inclusive $\pi^{0}$ and $\eta$ Production in Electron Positron Interactions at $\sqrt{8}=10 \mathrm{GeV}$

2. Phys. C4t:547.1989: DESY-89-(114: Inclusive Production of Charged Pions, Charged and Neutral Kaons and Antiprotons in $e^{+} e^{-}$Annihilation at $10 \mathrm{GeV}$ and in Direct $\mathbf{Y}(2 S)$ Decays

Phys Lett. 230B:1693.1989: DESY-89-061: Observation of $\Delta\left(1232 P_{33}\right)^{++}$Production in $e^{+} e^{-}$Annihilations around $10 \mathrm{GeV}$

Z. Phys. C42:3+19.1989: DESY-88-11ti: Search for Exclusive Radiative Decays of $\mathbf{Y}(1 S)$ and $\boldsymbol{\Upsilon}(2 S)$ Mesons

DESY-S9-179;

Measurement of $\mathrm{K}^{+} \mathrm{K}^{-}$Production in $>$Y Collisions

Phys. Lett. 229B:304,1989: DESY-89-086:

Search for $b \rightarrow a ?$ in Exclusive Decays of $B$ Mesons

2. Phys. C4531. 1989: LLIP-8903:

Global and Local Fluct uations in Multiplicity and Transverse Energy for Cent ral Ultra-Relativistic HeavyIon Interactions

Plys. Lett. 229B:175.1989: DESY-89-082:

Measurement of the Decay $B^{0} \rightarrow D^{-} i^{+} \nu$

Phys. Lett. 236B:102.1990: DESY-89.144:

Study of Antideuteron Production in $e^{+} e^{-}$Annihilation at $10 \mathrm{GeV}$ Center of Mass Energy

Phys. Lett. 230B:162.1989: DESY-89-119: Observation of a New Charmed-Strange Meson

Phys. Let1. 232B:554,1989; DESY-89-117:

Measurement of the Lifetime Ratio $\tau\left(B^{+}\right) / \tau\left(B^{0}\right)$

Z. Phys. C46:9.1090: DESY-89-102:

A Study of Cabibbo-Suppressed $D^{0}$ Decays

DESY-89-096:

Search for $b \rightarrow s$ gluon in $B$ Meson Decays

DESY-89-163:

Search for Rare Semileptonic B-Meson Decays

DESY-89-166:

Search for $B \rightarrow$ strange charged ${ }^{+}$charged $^{-}$in Exclusive Decays of $B$ Mesans

Phy's. Lett. 232B:398,1989: DESY.89-103:

Resonance Decomposition of the $D_{1}(\mathbf{2 4 2 0})^{0}$ Through a Decay Angular Analysis

DESY-B9-164:

Evidence for a Higher Twist Effect in Electron Positron Annihilation into Hadrons at $10 \mathrm{GeV}$ Centre of Mass Energy

Phys. Let. 231B:208,1989: DESY-89-085:

Observation of the Charged Isospin Partner of the $D_{2}^{*}(2460)^{0}$

DESY-90-033:

Observation of the Decay $D_{S}^{+} \rightarrow \eta^{\prime} \pi^{+}$

DESY+00-033;

Study of $p p$ and $\Lambda \Lambda$ Production in $e^{+} e^{-}$Annihilation at $10 \mathrm{GeV}$ Center of Mass Energy

Z. Phys. C:45:529.1990: GS1-89-59:

Transverse Energy Production in the Target Fragmentation Region in ${ }^{16} \mathrm{O}$ Nucleus Reactions at $60 \mathrm{~A}$ $\mathrm{GeV}$ and $200 \mathrm{~A}$ GeV

Phys. Lett. 234B:409.1990: DESY'-89-152:

Observation of Semileptonic Charmless $B$ Meson Decays

Phys. Lett. 241B:278.1990: DESY-90-0)18.

Search for Hadronic $b \rightarrow u$ Decays

RAL-HX-(J5T:

Summary Talk: 23rd Rencontre De Moriond, Mar $13-18,1988$

Nucl Phys. 1326!t 485.1946; Yad. Phys, 44:120.19N6: Sow. J. Nucl. Phys. 44:75.1986: CERN-EP-85-153; JFVE-86-18:

Production of $f_{0}(1590)$ and Other Mesons Decaying into $\eta$ Pairs by $100 \mathrm{GeV} / \mathrm{c} \pi^{-}$on Protons

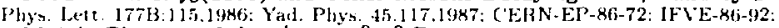

Matrix Element of the $\eta^{\prime} \rightarrow \eta \pi^{\circ} \pi^{\circ}$ Decay

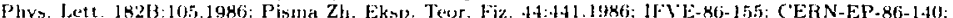

Observation of a Narrow Meson with a Mass of 1750 MeV Decaying into $\eta \eta$ Pair

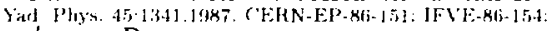

$\eta^{\prime} \rightarrow \infty$; Decay

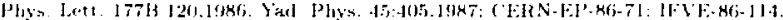

$2.22 \mathrm{GeV} \eta \eta^{\prime}$ Structure Observed in $38 \mathrm{GeV} / \mathrm{c}$ and $100 \mathrm{GeV} / \mathrm{c} \pi^{-} p$ Collisions 
Alde 87

Alde $87 \mathrm{~B}$

Alde $87 \mathrm{C}$

Alde $87 \mathrm{D}$

Alde $88 \mathrm{~B}$

Alde $88 \mathrm{C}$

Alde $88 \mathrm{D}$

Alde $88 \mathrm{E}$

Alde 89

Alde 90

Alder 89

Aleev $84 \mathrm{C}$

Aleev 85

Aleev $85 B$

Aleev 86

Aleev 86B

Aleev $86 \mathrm{C}$

Aleev 87

Aleev $87 \mathrm{~B}$

Aleev 88

Aleev $88 \mathrm{~B}$

Aleev $88 \mathrm{C}$

Aleev 88D

Aleev $88 \mathrm{~F}$

Aleev $88 \mathrm{G}$

Aleev 89

Aleev $89 B$

Aleev $89 \mathrm{C}$

Aleklett 87

Aleksan 86

Aleksandrov $87 \mathrm{~B}$

Aleksandrov 89

A leksanyan 86

Alekseenko 86

Alekseev 87

Alekseev 87B

A lekseev 88

A lekseev 88B
Phys. Lett. 201B:160,1988: Yad. Plyss. 47:1639.1988: Sov. J. Nucl. Phys, 47:1038.1988: 1FVE-87-163: CERN-EP-87-197: Production of $f_{0}(1590)$ Meson in $300 \mathrm{GeV}$ Central $\pi^{-}$Nuclei Collisions

Z. Phys. C36:603.1987: Yad. Phys. 47:385.1988: C'ERN-EP-87-196: IFVE-87-88: St udy of Neutral Decays of the $\eta^{\prime}$ Meson

Yad. Phys 47:1273.1988: IFIE-87-171: Observation of the Tensor $X(1810)$ Meson in $300 \mathrm{GeV}$ Central $\pi^{-} n$ Collisions

Phys. Lett 198B:286.1987: Yad. Phys. 47:997.1988: CER' EP-87-161: 1FVE-87-100:

Neutral Mesons Decaying into $4 \pi^{\circ}$

Z. Phys. C43:541.1989; Yad. Phys, 49:712.1989: Sov. J. Nucl. Phys. 49:440.1989: IFVE-84-15-1: CERN-EP-88-170: Pseudoscalar Meson Production in Central $\pi^{-} n$ Interactions at $300 \mathrm{GeV}$

Yad. Phys. 49:1021.1989: Sov. J. Nucl. Phys. 49:6;36.1989; IFVE-\$8-163;

Study of $\omega-\omega$ Systems Produced in $38 \mathrm{GeV} / c \pi^{-}$p Collisions

Yad. Phys. 48:1724,1986: Phys. Lett. 216B:477.1989: Yad. Phys. 48:1724.198x: Physi. Lett. 216B:447.1989: Sov. J. Nucl. Phys. 48:1035,1988: [FVE-88-153:

Evidence for a 1.9 GeV Meson Decaying into $\pi^{\prime} \eta$

Phys. Lett. 205B:397,1988:

Evidence for a $1^{-+}$Exotic Meson

Phys. Lett. 216B:451,1989:

Study of $\omega-\omega-S y s t e m s$ Produced in $38 \mathrm{GeV} / \mathrm{c}^{-}{ }^{-} p$ Collisions

Phys. Letr. 241B:600.1190; CERN-EP-90-39: IFVE-90-9:

Further Study of Mesons which Decay into $\omega$

SLAC-PLB-5044:

Upper Limit on the Absolute Branching Fraction for $D_{S}^{+} \rightarrow \phi \pi^{+}$

Yad. Phys, 43:619.1986: Sov. J. Nucl. Phys. 43:395.1986; JINR-D1-84-859:

Polarization of Charmed $\boldsymbol{A}_{\mathrm{c}}$ Baryons Produced in Neutron-Carbon lnteractions

Czech. J. Phys. B36:1303.1986: JINR-E1-85-662:

Observation of $D$ Mesons in $n$ C Interactions at $40-70 \mathrm{GeV} / c$

Yad. Phys. 44:1010,1986: Sov. J. Nucl. Phys 44:652,1986; JINR-P 1-85-948;

A-dependence of Narrow Baryon Resonance Production Cross Section

Yad. Phys. 44:661,1986; Sov. J. Nucl. Phys. 44:429.1986;

Inclusive Production of Hyperons and Antihyperons in Neutron Carbon Collisions at $n$ Energy of about $40 \mathrm{GeV}$

Kr. Soob. JINR 19:6.1986:

Polarization of $\Xi^{-}$Produced Inclusively by Neutrons

Kr. Soob. JINR 19:16,1986:

Observation of $X(3100)$ in BIS-2 Experiments

Z. Phys. C.36:27,1987; JJNR-D1-86-550:

A-dependence of Polarization of $\Lambda$ Produced Inclusively in Neutron-Nucleus snteractions

Yad. Phys. 46:1127.1987; JJNR-D1-86-122:

A-dependence of the Production Cross Section of $A_{r}^{+}$Charmed Baryons in Neutron-Nucleus Interactions

Z. Phys. C37:243,1988: JINR-El-87-265;

Production of $\bar{D}^{\circ}$ and $D^{-}$Mcsons in Neutron-Carbon Interactions at $40-70 \mathrm{GeV}$

JINR-P1-88-51:

Diffraction Dissociation of Neutrons in $\Lambda K^{0}$ on Hydrogen at about $40 \mathrm{GeV}$ Energy

JINR-P1-88-397:

Production of Charmed Baryons $\Lambda_{c}^{+}$in $n$ p Interactions at 40 - $70 \mathrm{GeV}$ Neutron Energy

JINR-D1-88-368:

Observation of Narrow Baryoniums in the Experiment BIS-2. Strange Baryoniums

IINR-D 1-88.369:

Observation of Narrow Baryoniums at the Experiment BIS-2. Baryoniums with Hidden Strangeness

JINR-D1-88-194:

Search for Nonstrange Baryonjum Decaying into Strange Particles

JINR-D1-89-624:

Search for Narrow Baryonjum Resonance with Hidden Strangeness

JINR-D1-89-701:

Observation of $\Sigma_{c}(2455)$ Charmed Baryon in $n p$ Interaction at the Serpukhov Accelerator

JINR-DI-89-345;

Inclusive Production of $\$$-Mesons in Neutrina-Proton Interactions at $30-70 \mathrm{GeV}$

Phys. Lett. 197B:34,1987;

Production of Intermediate Mass Fragments in Ultrarelativistic Nuclear Collisions

SACLAY-DPHPE-86-08:

Search for Heavy Neutral Leptons at CELLO

LENI-87-1339:

Production of ${ }^{149} \mathrm{~Tb}$ from $\mathrm{Gd}, \mathrm{Tb}, \mathrm{Ho}, \mathrm{Tm}$, Ta, Au by $1 \mathrm{GeV}$ Protons

LENI-89-1561:

Measurement of Spallation Cross Sections at the Synchrocyclotron Internal Beam

Yad. Phys, 45:1015.1987; YERE-902(53)-86

Measurement of Compton Forward Cross Section on ${ }^{4}$ He Nuclei in the Energy Range of $E=1.8-3.8 \mathrm{GeV}$

Pisma Zh. Eksp. Teor. Fiz. 44:202.1986:

On the Probable Flash of Cygnus X-3 Source in the Energy Range of $E>10^{14} \mathrm{eV}$

Pisma Zh. Eksp. Teor. Fiz. 45:461,1987.

A bout Possible Registration of Neutrino Signal at Baksan Underground Telescope of MINR

Yad. Phys. 46:1360,1987; .IINR-P1-87-72;

Observation of Inverse Electroproduction of Pions on ${ }^{17} \mathrm{C}$ Nucleus at $164 \mathrm{MeV}$ Pion Energy and Determination of lscrector $F_{3}$ Nucleon Form Factor

[TEP-88-23;

Measurement of $\Lambda$-Hyperon Polarization Produced by Neutrons with Momenta $4-10 \mathrm{GeV} / \mathrm{c}$ on Carbon Nucleus

ITEP-88-185:

Measurement of Relative Differential Cross Section of the Elastic $\pi^{-} p$ Scattering in the Backward Hemisphere at $1.43-2.07 \mathrm{GeV} / c$ 
Alckseev $88 \mathrm{C}$

Alekseev 89

Alekseeva $\mathbf{8 8}$

Aleshin 84

Aleshin 85

Aleshin 86

Aleshin $86 \mathrm{~B}$

Aleshin 87

Aleshin $87 \mathrm{~B}$

Aleshin $87 C^{\circ}$

Aleshin 87D

Aleshin $87 \mathrm{E}$

Aleshin 90

Alexander 86

Alexander 89

Alexander 90

Alexopoulos $88 \mathrm{~B}$

Alexopoulos 90

A]fimenkov 88

Alibekux 85

Aljev 89

Alimov 85

Alimov 88

Alimov 89

Alirrov $89 \mathrm{~B}$

Alitti 89

Alitti 90

Aitti 90B

Alitti 9uC

Alitti $40 D$

Alkhazov 85

Alkhazov 85B

Allaterdin 87

Allaby 86

Allaby $86 \mathrm{~B}$

Allaby 87

Allaby 88
Phys. Lett. 205B:200.1088:

Detection of the Neutrino Signal from SN1987 A in the LMC Using the INR Baksan Underground Scintillation Telescope

Yad. Phys. 51:425. I989: ITEP-89-111:

Asymmetry in $\pi^{-}$p (polarized) Elastic Scattering in Momentum Range $1.4-2.1 \mathrm{GeV} / \mathrm{c}$

Vopr. At. Nauki i Techn. ser. Obsch. 2-42:5.19148;

The Influence of the Fragment Production Angle on the Anomalon Effect

Yad. Phys. 15:1358.1987; ITEP-84-117:

Measurement of Protons Polarization at $\pi^{-} p \rightarrow p \pi^{+} \pi^{-} \pi^{-}$Reaction at $4.35 \mathrm{GeV} / c$ and $4.85 \mathrm{GeV} / c$ Momenta

[TEP-85-67:

Varjaus Channel Cross Sections of Strange Particle Production Reactions in $\pi^{-}{ }^{-}$Interactions at $4.5 \mathrm{GeV} / c$ Moment um

Pisma Zh. Eksp. Teror. Fiz. 43:159.1986: Jetp Leut t3:200,1986i: ITEP-\$2-103:

Search for Events Containing Six Quarks in the Final State

Yad. Phys. 46:1701.1987; ITEP-86-122:

Production of $\pi^{\circ}$ Mesons in the $\pi^{-} p \rightarrow \pi^{0} \pi^{+} \pi^{-} \pi^{-} p$ at $4.5 \mathrm{GeV} / \mathrm{c}$

ITEP-87-40:

Production of Heavy Isobars in the $\pi^{-} p \rightarrow \pi^{0} \pi^{+} \pi^{-} \pi^{-} p$ Reaction at $4.5 \mathrm{GeV} / \mathrm{c}$ Moment um

LEN1-87-1259:

Study of Polarization in Inclusive Proton-Deuteron Breakup Reaction p deuteron $\rightarrow p \mu n$ at $1.0 \mathrm{GeV}$

Yad. Phys. 45:1800,1987; ITEP-86i-123; Study of $b_{1}(1235)^{-}$Meson Production at $4.5 \mathrm{GeV} / \mathrm{c}$ in the Reaction of $\pi^{-} p \rightarrow b_{1}(1235)^{-} p$

Yad. Phys. 48:148.1988; Sov. J. Nincl. Phys. 48:92.1!387: 1TEP-87-192:

Nonconservation of Helicity at the Diffraction Production of $b_{1}(1235)$ Mesons in the $\pi^{-} p \rightarrow b_{1}(1235)^{-} p$

KFKI-1987-47-A:

Study of Proton-Deuteron Break-up Reaction in Exclusive Experiment at $1 \mathrm{GeV}$

Phys. Lett. 237B:29,1990:

Study Proton-Deuteron Break-up Reaction in Complete Kinematics at $1 \mathrm{GeV}$

Phys. Rev. D34:315.1986:

Longitudinal Photon Polarization in Muon Pair Production at High $x$.

CLNS-89-940: CLEO-89-12;

The Decay $D^{0} \rightarrow K^{0} \bar{K}^{0}$

CLEO-90-2:

Observation of $\Upsilon(4 S)$ Decays into non $B \bar{B}$ Final States Containing $\psi$ (unspec) Mesons

Phys. Rev. Lett. 60:1622.1988; DUKHEP-88-1:

Multiplicity Dependence of the Transverse-Momentum Spectrum for Centrally Produced Hadrons in Antiproton-Proton Collisions at $\sqrt{s}=1.8 \mathrm{TeV}$

CND-HEP-02-02-90:

Mass Identifed Particle Yields in Antipraton - Proton Collisions at $\sqrt{s}=1.8 \mathrm{TeV}$

LENI-88-1392;

On Neutron Lifetime Measurement with Ultracold Neutrons

tzv. Akad. Nauk SSSR. Fiz. 4!9:1268.1985:

On the Possibility to Isolate Pionic Interactions at the Group Hadron Fall at Mountain Level

Yad. Phys. 51:1597.1990; PTIU-89-103:

Forward-Backward Correlation in Hadron-Nucleus Interactions

Yad. Phys. 41.1553.1985:

Production of Protons in Single and Multinucleon $p{ }^{20} \mathrm{Ne}$ Interactions at $300 \mathrm{GeV} / \mathrm{c}$

PTIL-88-66:

Observation of the Cumulative Isobars in $p^{20} \mathrm{Ne}$ Interactions at $300 \mathrm{GeV} / \mathrm{c}$

PTIL-89-105:

Cumulative Nucleons and Processes of Intranucleus Absorbtion of Pions by Two-nucleon Systems in $p$ ${ }^{20}$ Ne Interactions at $300 \mathrm{GeV}$

Yad. Phys. 51:1600,1990: FVE-89-106:

Correlation between $\gamma$-Quanta and Charged Particles Multiplicities is $p{ }^{20}$ Ne and p nucleon Interactiong $300 \mathrm{GeV}$

Phys. Lett. 235B:363,1989; CERN-EP-80-151:

$A$ Search for the Squark and Gluino Production at the CERN $\bar{p} p$ Collider

2. Phys. C47:11.1990: CERN-EP-9()-20;

Measurement of $W^{ \pm}$and $Z^{0}$ Production Cross Section at the CERN $\bar{p} p$ Collider

Z. Pinys. C47:523.1990: CERN-EP.90-52:

Measurement of the Transverse Momentum Distributions of $W^{ \pm}$and $Z^{0}$ Bosans at the CERN $\bar{p} p$ Collider

Phys. Lett. 241B:150,1990; CERN-EP-90-22;

$A$ Precise Determination of the $W^{ \pm}$and $Z^{0}$ Masses at the CERN $\bar{p} p$ Collider

CERN-PPE-90-105:

A Measurement of Two-Jet Decays of the $W^{ \pm}$and $Z^{0}$ Bosons at the CERN $\bar{p} p$ Collider

Yad. Phys 41:561.1985: Sov J. Nucl. Phys. 41:357.1985:

Elastic Scattering of $1 \mathrm{GeV}$ Protons by He lsotopes

Yad. Phys. 42:8,1985; Sov. 1. Nucl. Phys. 42:1.1985:

Elastic Scattering of $1 \mathrm{GeV}$ Protons and Matter Distributions in 1p Shel] Nuclei

Yad. Phys. 46:1785.1987: JINR-P2-86-731:

Enhancement of Pairing Correlations of Nucleons in Hadron-Nucleus Interactions

Phys. Lett. 179B:301.1986: CERN-EP-86-125.

Test of the Universality of the Electron Neutrino and Muon Neutrino Coupling to the Charged Weak Current

Phys. Lett. 177B:4t6.1986: CEHN-EP-Rf-94:

A Precise Determination of the Electroweak Mixing Angle from Semileptonic Neutrino Scattering

2. Phys CB6:ti11.1987: CEREN-EP-87-140:

A Precise Determination of the Electroweak Mixing Angle from Semileptonic Neutrino Scattering

Z. Phys. (3H:403.1984: CERN-EP'-RT-225:

Total Cross Sections of Charged-Current Neutrino and Antineutrino Internctions cn Isoscnlar Nuclei 
Allaby $88 \mathrm{C}$

Allaby 89

Allasia 85

Allasia 85B

Allasia $85 \mathrm{C}$

Allasia $85 \mathrm{D}$

Allasia 86

Allasia 88

Allasia $88 \mathrm{~B}$

Allasia $88 \mathrm{C}$

Allday 88

Allen 85

Allen $85 B$

Allen 89

Allison 89

Allison $89 \mathrm{~B}$

Allkofer 85

Allkofer 85B

Alport 89

Ainer 84B

Alner 85

Alner $85 \mathrm{~B}$

Alner $85 \mathrm{C}$

Alner 85D

Alner 86

Alner 86B

Alner $86 \mathrm{C}$

Alstongarnjo 88

Alstongarnjo 89

Altarev 86

Althoff 84R

Althoff 85

Althoff $85 \mathrm{~B}$

Althoff $85 \mathrm{C}$

Althoff $85 \mathrm{D}$

Althoff 85E

Althoff 85F
Pliys. Lett, 213B:554.1988; C'ERN-EP-88-81:

Experimental Study of $x$-Distributions in Semileptonic Neutral-Current Neutrino and Antineutrino Reactions

Phys. Lett. 231B:317.1989; CERN-EP-89-79:

Evidence for Neutral Neutrino Current Coupling to Right Handed Quarks

Phys. Rev. Dis1:2996.J985:

Search for $\mu^{ \pm} \pi^{ \pm}$Mass Enhancements in Noutrino and Antineutrino Deuterium Charged Current Interactions

Nucl. Phys. B268: 1.15186: DFUB-10.85;

Inclusive $\rho^{0}$ Production in $\bar{\nu}_{\mu}$ deuteron and $v_{\mu}$ deuteron Charged Current Internctions

Z. Phys. C28:321,1985;

$Q^{2}$ Dependence of the Proton and Neutron Structure Functions from Neut rino and Ant ineutrino Scattering in Deuterium

Phys. Lett. 154B:231,1985:

Fragmentation into Strange Particles in High Energy $\nu p, \nu n, \bar{\nu}$ and $\bar{\nu}$ Interactions

Phys. Lett. 174B:450.1986;

Search for a $\Delta\left(1232 P_{33}\right)-\Delta\left(1232 P_{33}\right)$ Structure of the Deuteron

Z. Phys. C37:527,1988;

Bose-Einstein Correlations in Neutrino and Antineutrino Interactions in Deuterium

Phys. Rev. D.37:219.1988;

Search for Fractionally Charged Particles in (Anti)neutrino-Deuterium Interackions

Nucl. Phys. B307:1,1988; DPHPE-88-013;

Determination of the Neutral Current Chiral Coupling Constants U(I), U(r), D(I) and D(r) from. Neutrino and Antineutrino Deuterium Experiment

Z. Phys. C40:29,1988:

Annihilation and Topological Cross Sections for $p p$ and $\bar{p} p$ Interactions at $200 \mathrm{GeV} / c$

Nucl. Phys. B264:221,1986; CERN-EP-85-33;

A Study of Single Meson Production in Neutrino and Antineutrino Charged Current Interactiong on Protons

Phys. Rev. Lett. 55:2401.1985:

First Observation and Cross Section Measurement of $v_{t} e^{-} \rightarrow \nu_{r} e^{-}$

Phys. Rev. Lett. 6.4 1330.1990: LA-UR-89-3723:

Measurement c* trierference Between $W^{ \pm}$and $Z^{\circ}$ Exchange in Electron-Neutrino Electron Scattering

ANL-HEP.CP-89.i, DK-411:

Underground Muon Observations in the SOUDAN-2 Detector

ANL-HEP-CP-89-92: PDK-402;

Contained events in SOUDAN-2

Phys, Rev, D31:1557.1985;

Primary Cosmic Ray Energy Spectrum up to 50 TeV Derived from Sea-Level Muon Measurenents

Nucl. Phys. B259:1.1985;

Cosmic Ray Energy Spectra at Sea-Level up to $10 \mathrm{TeV}$

Phys. Lett. 232B:417,1989: CERN-EP-89-127

Observation of Shadowing of Neutrino and Antineutrino - Nucleus Interactions and Comparison with PCAC Predictions

Phys. Lett. 151B:309.1985: CERN-EP-84-111:

Observation of $\Xi^{-}$Production in $\bar{p} p$ Interactions at $540 \mathrm{GeV}$ CMS Energy

Phys. Letน. 160B:199.1985; CERN-EP-85-62:

New Empirical Regularity for Multiplicity Diatributions in Place of KNO Scaling

Nucl. Phys. 13258:505.1985; CERN-EP-85-81:

Kaon Production in $\bar{p} p$ Reactions at a Centre of Mass Energy of $540 \mathrm{GeV}$

Phys. Lett. 160B:193,1985: CERN-EP-85-61:

Multiplicity Distributions in Different Pseudorapidity Intervals at CMS Energy of 540 GeV

Phys. Lett. 167B:476.1986: CERN-EP-85-197:

Scaling Violations in Multiplicity Distributions at 200 and $900 \mathrm{GeV}$

Z. Phys. C32:153.1986: CERN-EP-86-57;

Antiproton Praton Cross Sections at 200 and $900 \mathrm{GeV} \mathrm{cm}$ Energy

Z. P'ly Ci3:1.1986. C'ERN-EP-86-126:

Scaling of Pseudorapidity Distributions at c.m. Energies up to $0.9 \mathrm{TeV}$

Phys. Lett. 180B:415,1986: CERN-EP-86-127:

An Accelerator Search at $900 \mathrm{GcV}$ c.m. Energy for the Centauro Phenomenon

Phys. Rev. Lett. 60:1928,1988:

Limit on Majoron Emisgion in $\beta \rho$ Decay of ${ }^{100} \mathrm{Mo}$

Phys. Fev. Letı. 63:1671,1989.

Search for Neutrinaless Double-beta Decay of ${ }^{100} \mathrm{Mo}$

Pisma Zh. Eksp. Teor. Fiz. 44:360.1986:

Search for the Neutron Electric Dipale Moment

Z. Phys. C26:521,1985; OLiNP-65-84: DESY-84-091:

The Production and Decay of $\tau^{ \pm}$Leptons in $e^{+} e^{-}$Annihilation at PETRA Energieg

Phys. Lett. 154B:236.1985:

Search for Spinless Bosons in $e^{+} e^{-}$Annihilation

Z. Phys. C27:27,1985:

A Detailed Study of Strange Particle Production in $e^{+} e^{-}$Annihilation at High Energy

Z. Phys. C29:29,1985: DESY-85-06:3:

A Study of 3 Jet Events in $e^{+} e^{-}$Annihilation into Hadrons at 34.6 GeV CMS Energy

Z. Phys. C29:189.1985; DESY-85-093;

Search for Two-Photon Production of Resonances Decaying into $K \bar{K}$ and $K \bar{K} \pi$

2. Phys. C30:355.1986; OXFORD-NP-89-85; DESY-85-126;

Bose-Eingtein Correlations Observed in $e^{+} e^{-}$Annihilation at a Centre of Mass Energy of 34 GeV

2. Phys. ('29:347.1985: DESY-85-077; Particle Correlation Observed in $e^{+} e^{-}$Annihilation into Hadrons at CMS Energieg between 29 and 37
GeV 
Althoff 86

Althoff $86 \mathrm{~B}$

Althoff $86 \mathrm{C}$

Althoff $86 \mathrm{D}$

Althoff 89

Altzizoglow 85

Alvarez 90

Alvarez $90 \mathrm{~B}$

Alvarez $90 \mathrm{C}$

A maglobeli 87

A maglobeli 89

Amako 87

Amaldi 85

Ameev 85

Arneeva 87

Aneeva 89

Arnelin 86

Amelin 87

Amelin $87 \mathrm{~B}$

Amelin 90

Amendolia 85

Amendolia 86

Amendolia $86 \mathrm{~B}$

Amendolia 87

Amendolia 87B

Amidei 88

Ammar $86 \mathrm{~B}$

Ammar 87

Ammar 88

Ammar $88 \mathrm{~B}$

Ammar 89

Ammar $89 \mathrm{~B}$

Ammosov $84 \mathrm{G}$

Ammosov $84 \mathrm{H}$

Ammosov 85

Ammosov 85B

Ammosov $8.5 \mathrm{C}$

Ammosoy $85 \mathrm{D}$

Ammosov 86
Z. Phys C $31: 537.1986$ : DESY -86i-028:

Measurement of the Radiative Width of the $a_{2}(1320)$ in Two Photon Interactions

2. Phys. C31:527,1986: DESY-86.026:

Measurement of the Photon Structure Function $F_{2}$ at $Q^{2}$ from 7 to $70(\mathrm{GeV} / \mathrm{c})^{2}$

Z. Phys. C 32:343.1986; DESY'-86-027;

A Measurement of the $D^{\mathbf{0}}$ Lifetime

2. Phys. Cu2:11.1986: DESY'-86-025:

Vector Meson Production in the Final State $K^{+} K^{-} \pi^{+} \pi^{-}$of Photon Photon Collisions

2. Phys. C43:375,1989; BONN-ME-89-01:

Photodisintegration of Polarized Deuterons - Measurement of Angular Distributions at $E(\Upsilon)=450,550$ and $650 \mathrm{MeV}$

Plyys. Rey. Lett. 55:799,1985:

Experimental Search for Heavy Neutrino in the $A$ Spectrum of ${ }^{35} \mathrm{~S}$

2. Phys. C47:539.1990; CERN-EP-90-26:

Lifetime Measurements of the $D^{+}, D^{0}, D_{S}^{+}$, and $\Lambda_{c}^{+}$Charmed Particles

CERN.EP-90-66:

Photoproduction of the $\boldsymbol{\Lambda}_{c}$ Charmed Baryon

CERN-EP-90-65;

Measurement of $D_{S}^{ \pm}$and Cabibbo-Suppressed $D^{ \pm}$Decays

Yad. Phys. 45:1020.1987: Sov. J. Nucl. Phys, 45:632.1987:

Baryon State with Hidden Strangeness

Yad. Phys. 50:695,1989:

Asymmetry Measurement in $\pi^{0}$ - and $\eta$ - Meson Inclusive Production in Central Region at $40 \mathrm{GeV} / \mathrm{c}$ KEK-87-106:

New Results from VENUS at TRISTAN

Phys. Lett. 153B:444,1985; CERN-EP-85-10;

New Limits on the Single-Gamma Decay of Orthopositronium

Yad. Phys. 11:938.1985; ALNA-84-14:

Investigation of the Mechanism of Relativistic Nucleus Interaction from Analysis of C C Collisions

ALAIA-87-10:

Exotic Baryon Systems in Multiparticle Interactions

Yad. Phys. 51:1047.1990; JINR-P1-89.560;

Inelastic Interaction of Silicon Nuclei with Nuclear Emulsion at $4.6 \mathrm{GeV} / \mathrm{c}$

P.sma Zh. Eksp. Teor. Fiz. 43:455.1986:

Possible Observation of Cumulative Dibaryon Resonance

Atom. NucL. Elem. Particles, p.36:

The Baryon Resonances in $\pi^{-}$Ne Interactions at $8.2 \mathrm{GeV} / \mathrm{c}$

Atom. NucL. Elem. Particles, p.40;

The Search of the Narrow Dibaryon Resonances in Pion-Nucleus Interactions

Pisma Zh. Eksp. Teor. Fiz. 51:607.1990;

Production of the Superheavy Hydrogen Isotopes in the $\pi^{-}$Capture by ${ }^{0} \mathrm{Li},{ }^{7} \mathrm{Li}$ Nuclei

Phys. Lett. 155B:457,1985; CERN-EP-85-14:

First Measurement of the Reaction $\pi^{-} e^{-} \rightarrow \pi^{-} \pi^{0} e^{-}$

Nucl. Phys. B277:168,1986; CERN-EP-86-34:

A Measurement of the Space Like Electromagnetic Form Factor

Phys. Lett. 178B. .35.1986; CERN-EP-86-I8:

A Measurement of the Kaon Charge Radius

CERN-EP-87-20;

A Measurement of $D^{0}$ Lifetime

Z. Phys. C36:513.1987:

$\Lambda_{c}^{+}$Photoproduction and Lifetime Measurement

Phys. Rev. D37:1750.1988: SLAC-PI:B-4362: L13L-23707:

Measurement of the $\tau^{ \pm}$Lifetime

Phys. Lett. 178B:124.1986: CERN-EP-86-85;

Multiplicity of Charged Particles in $800 \mathrm{GeV} p$ p Interactions

Phys. Lett. 183B:110,1987: CEFN-EP-86-122; FERMILAB-PUB-86-112-E:

Inclusive Charm Cross Sections in $800 \mathrm{GeV} / c p p$ Interactions

LEBD-88-48:

The Study of Cumulative Effects in the Neutrino Emulsion Collisions

Pliys. Rev. Lett. 61:2185.1988:

D-Meson Production in $800 \mathrm{GeV} / \mathrm{c} p$ p Interactions

Pisma Zh. Eksp. Teor. Fiz. 49:189,1989:

Cumulative Proton Production in the Interaction of Neutrino with Photoemulsion Nuclei

Pisma Zh. Eksp. Teor. Fiz. 49:421,1989); IFVE-89-71:

Nuclear Effects on the Average Multiplicity of Charged Particles in High Energy Neutrino Interactions

Yad. Phys. 12:374,1985; IFVE-84-191:

A Study of Reaction $\bar{\nu}$ nucleon $\overrightarrow{\mu^{+}} \boldsymbol{p}^{*}$ nucleon at High Energy

Yad. Phys. 42:664.1985; Sov. J. Nucl. Phys. 42:421.1985: IFVE-84-179:

Limitations for Parton Momentum in Nucleon from Charged Current $\bar{\nu} n$ Interactions

JFVE-85-110:

Neutrino (Antineutrino) Interaction Cross Section Ratio in Neutral and Charged Current Channels up to $30 \mathrm{GeV}$

2. Phys. C $30: 175.1986$ : IFVE-85-107.

Study of $\nu(\vec{\nu})$ Interactions with Nuclei at $3-30 \mathrm{GeV}$

Yad. Phys. 43:1186.1986; IFVE-85-109:

Study of Cumulative Proton Production in Neutrino-Nucleus Interactions

2. Phys. C30:183.1986; IFVE-85-188:

Neutral Strange Particle Production in Neutrino Charged Current Interactions at 3 - $30 \mathrm{GeV}$

Pisma Zh. Eksp. Teor. Fiz, 43:502,10k6:

Observation of the Pseudovect or Charm-Strange Meson Cascade Radiative Decay 
Ammosov $86 \mathrm{~B}$

Ammiosov $86 \mathrm{C}$

Ammoson $86 \mathrm{D}$

Animosor 66E

Ammosov 86F

Ammosov $86 \mathrm{C}$

Ammosov $86 \mathrm{H}$

A תimosov 96I

Ammosov 87

Ammosov 87E

Ammosav $87 \mathrm{C}$

Ainmusuv $87 \mathrm{D}$

Ammosov $87 \mathrm{E}$

Ammosov $87 \mathrm{~F}$

Ammosov 88

Ammosov 88B

Ammosov $88 \mathrm{C}$

Ammosov $88 \mathrm{D}$

Ammosov 88E

Ammosov 88G

Anos 85

A mos 88

Amos 89

Amos 90

Amos 90B

Amroyan 88

Aniroyan 89

Anada 88

Artaniev 83

Ananieva 86

Ananikyan 87

Ananin 85

Anassontzis 85

Anassontzis 87

Anassontzis 90

Ander 39

Andersen 89

Pisma Zh. Ěksp. Teur. Fiz, 43:554,1486;

Quasielastic Production of $\Lambda$ Hyperon in the Antineutrino Interactions at High Energy

Yad. Phys, ti: 16602,1987 ; IFVE-86-160:

Charged Current Antineutrino-induced Coherent Negative Pion Production off Neon

Yad. Plys 45: 533.1987 ; IFVE-86-135:

Study of Inclusive Characteristics of $\rho^{0}$ and $K^{*}(892)^{0}$ Mesons in Antineutrino Charged Current Induced Quark Jet

IFVE-86-94:

Incluaive $\rho^{0}$ and $K^{*}(892)^{\circ}$ Production in $\nu_{\mu} n$ Charged Current Interactions

Z. Phys. C36:377,19Eg; IFVE-86-95;

Neutral Strange Particle Exclusive Production in Charged Current High Energy Antineutrino Interactions IFVE-86-244:

Study of Palarization Effects in $\rho^{0}$ Meson Production in the (Anti)Neutrino Charged Current Induced Quark Jet

Yad. Phys. 16:130,1987; Sov. J. Nucl. Phys. 46:80.1987; PHE-86-10: IFVE-86-133;

Inclusive $\rho^{\mathrm{O}}$ Meson Productions in Neutrino Interactions at SKAT Chamber

Z. Phys. C30:569,1986:

Results for Neutral to Charged Current Cross Section Ratios from $\nu$ and $\vec{\nabla}$ nucleus Interactions below 30 Gev

Yad. Phys. 45:1346.1987:

Invest igation of Inclusive Characteristics of $\rho^{0}$ and $K^{*}(892)^{0}$ Mesons in Quark Jers from Charged Current $\ddot{n} n$ Interactions

Pisma Zh. Eksp. Teor. Fiz. 46:52,1987; IFVE-87-82:

Infuence of Nonperturbative Effects on the Scaling Violation in Neutrino Interactions at $Q^{2}=2-100$ GeV

$12 \cdot 3-87-17$ :

Study of Charged Hadrons Inclusive Characteristics in Neutrino Interactions with Photoemulsion and Nern Nuclei

Z. Phys. C35:329,1987: IFVE-86-222;

Production of $\mu^{-} e^{+}$Pairs in Neutrino Interactions in the SKAT Bubble Chamber Experiment

Phys. Lett. 184B:245,1987; IFVE-86-223:

Observation of $\mu^{-} e^{-}$Events in Neutrino Interactions at $3-30 \mathrm{GeV}$ Neutrino Energy

Pisma Zh. Eksp. Teor. Fiz. 45:453,1987;

The Antineutrino Production of the Charmed Charged Vector Mesons

Yad. Phys. 47:1015,1988; Sov. J. Nucl. Plhys. 47:646,1988; 1FVE-87-81;

Study of Nucleon Structure Function in Neutrino Interactions at $10-200 \mathrm{GeV}$

Yad. Phys. 50:106.1989; Sov. J. Nucl. Phys. 50:67.1989: IFVE-88-122;

Study of $\nu p \rightarrow \mu^{-} \Delta\left(1232 P_{93}\right)^{++}$Reaction at Fnergy $3-30 \mathrm{GeV}$

Pisma Zh. Eksp. Teor. Fiz. 47:555,1988; IFVE-88-77;

Observation of $a_{1}(1260)^{-}$Coherent Production in Antineutrino Neon Charged Current Interactions

Z. Phys, C40:487,1988; IFVE-88-81.

$\nu_{e}-\nu_{\mu}$ Universality Check and Search for Neutrino Oscillations

Z. Phy's. C40:493,1988; IFVE-88-82:

Final Result on $\mu^{-} e^{+}-$Pair Production in Neutrino Interactions with Chamber SKAT Filled wish Freon

Yad. Phys. 47:113.1988;

Observation of Muon Internal Bremsstrahlung in $\nu_{\mu}$ nucleus $\rightarrow \mu^{-} \mathbf{X}$ Reaction

Nucl. Phys. 13262:689,1985; CERN-EP-85-94:

Measurement of Small-Angle Antiproton Proton and Proton Proton Elastic Scat tering at the CERN Intersecting Storage Rings

Plyys. Rev, Lett. 61:525,1988; FERMILAB-PUB-88-38-E:

Measurement of the Nuclear Slope Parameter of the $p \bar{p}$ Elastic Scattering Distribution at $\sqrt{8}=1800$ GeV

Phys. Rev. Lett. 63:2784,1990; FERMILAB-PUB-89-176-E; Measurement of the $\bar{p} p$ Total Cross Section at $\sqrt{s}=1.8 \mathrm{TeV}$

Phys. Lett. 2435:158,1990: CLNS-90-981;

A Luminosity-Independent Measurement of the $\bar{p} p$ Total Cross Sections at $\sqrt{8}=1.8$ TeV

Phys. Let t. 247B:127,1990; FERMIL 1 B-PUB-90-96-E; Antiproton-Proton Elastic Scattering at $\sqrt{8}=1.8 \mathrm{TeV}$ from $|t|=.034$ to $.65(\mathrm{GeV} / \mathrm{c})^{2}$

Y'ad. Phys. 48:461,1988;

Analysis of Charge Distribution of $A_{B}$ Photospallation Products at $E<4.5 \mathrm{GeV}$

YERE-1 193(70)-89:

Investigation of Some Characteristics of Photoproduction of ${ }^{7}$ Be and ${ }^{24} \mathrm{Na}$

Phys. Rev. D37:552,1988;

Neutrino Vacuum Oscillation and Neutrino Burst from SN1987A

Yad. Phys. 41:912,1985; Sov. J. Nucl. Phys. 41:585.1985; J[NR-P1-83-709:

Search for axion at the Impulsive Feactor IBR-2

JINR-E1-86-523;

Double Charge Exchange of Negative Pions in Inclusive Reactions on Nuclei at $40 \mathrm{GeV} / \mathrm{c}$

Yad. Phys. 46:401.1987:

Hadronic Photcabsorption and the Coherent Photoproduction of $\pi^{0}$ Mesons on Be and $C$ Nuclei in the Resonance Energy Region

Yad. Phys. 41:1393.1085:

Elastic Photoproduction of Neutral Pjons on Helium Nucleus near the Resonance $\Delta$ (1232 Pss)

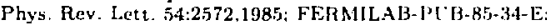
Continuum Dimuon Production in $\bar{p}$ Wt Collisions at $125 \mathrm{GeV} / \mathrm{c}$

Phys. Rev. 1)34:1377.1988: FERMILAB-P[1-B-87-217-E:

High Mass Dimuon Production in $\bar{p} n$ and $\pi^{-} n$ Interactions at $125 \mathrm{GeV} / \mathrm{c}$

Yad. Phys. 51:1314.19501: CERN-EP-89-9K:

High $p_{T} \gamma$ and $\pi^{\circ}$ Production, Inclusive and with a Recoil Hadranic Jet, in $p$ Collisions at $\sqrt{8}=63$ GeV

Phys. Rev. l.te1.62:985. I989.

Test of Newton's Inverse-Squared Low in the Greenland Ice Cap

Phys. Lett 22013:328.14R9: CERN-EP-8!3-35:

A Measurement of Cross Sections for ${ }^{32}$ S Interactions with Al, Fe, Cu, Ag and Pb at 200 GeV/c per

See the legend on page 19. 


\section{Andersen 89 (cont'd) Nucleon}

Anderson 55B Phys. Rev C31:1161.1985:

Particle Hole Strength Excited in the ${ }^{48} \mathrm{Ca}(p, n){ }^{48} \mathrm{Sc}$ Reaction at $134 \mathrm{MeV}$ and $160 \mathrm{MeV}: \mathrm{Gamow}-\mathrm{Teller}$ Strength

Ando 86 KEK-86-8:

Evidence for Two Pseudoscalar Resonances of $\eta^{+} \pi^{-}$System in the $f_{1}(1285)$ and $\eta(1295)$ Regions

Andreer. 94

Yad. Phys. 42:1420.1985: Sov. J. Nuc! Phys. 42:899.1985: LEN1-84-1011:

Correlation Measurements of the Spectra of Backward Protons in the Reaction $p$ deuteron $\rightarrow 2 p n$ at Incident Momenta $1.2-1.7 \mathrm{GeV} / \mathrm{c}$

Andreer 85

LENI-85-1055:

Search of Massive Neut rino in $\pi^{+} \rightarrow \mu^{+} \bar{F}_{\mu}$

Andreer 86

Pisma Zh. Eksp. Teor. Fiz. 44:401,1986:

Is the Cygnus $X-3$ Source Observed at Underground Experiments ?

Andieer 87

Andreev 87B

isma Zh Eksp. Teor Fiz. 45:519.1987:

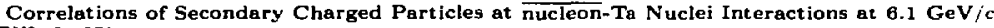

LEXI-8T-1271:

The Reaction $p$ deutercn $\rightarrow p p n$ and the Narrow Dibaryon Resonances

Andreev $87 \mathrm{C}$

Pisma Zh. Eksp. Teor. Fiz. 45:508.1987.

Observation of Invariant Mass Structure of Two Nucleons from the Reaction of Splitting Deuteron by Protons

sindreev 88

Vopr. At. Nauki i Techn. ser. Obsch, 1-41:29.1988:

The Possible Observation of the Dibaryon Resonances in the Reaction $p$ deuteron $\rightarrow p p n$

Andreev $88 \mathrm{~B}$

Andreev $90 B$

ENI-88-1453

Experimental Study of the Reaction $p p \rightarrow p p \pi^{\circ}$ in the Energy Region $600-900 \mathrm{MeV}$

Nuovo Cim. 103A:1163.1990: Yad. Phys. 51:112.1990:

Multiplicities and Correlations of Secondary Charged Particles in the Interactions of Antineutrons and Antideuterons with a Momentum of $6.1 \mathrm{GeV} / \mathrm{c}$ per Nucleon with Tantalum Nuclei

Andreeva $85 B$

ALMA-85-13:

Study of General Characteristics of Interactions of Protong with Light and Heavy Photoemulsion Nuclei at Energies of 200 and $400 \mathrm{GeV}$

Andreera $85 \mathrm{C}$

JINR-P1-85-692:

Topological Characteristics of the $4.1 \mathrm{GeV} / \mathrm{c}^{\mathrm{A}}{ }^{22} \mathrm{Ne}$ Nuclei Fragmentation Process on Pholoemulsion Nuclei

Andreeva 86

Yad. Phys. 45:123.1987: JINR-P1-86-8:

Multiplicities and Angular Distributions of Charged Particles in the Interactions of 22 Ne Nuclei in the Photoemulsion at $4.1 \mathrm{~A}$ GeV/C

Andreete $86 \mathrm{~B}$

Andreeva 88

Andreera $88 \mathrm{~B}$

Search for Exotic Baryon Systems at the Relativistic Nuclear Separative Reactions

Yad. Phy's. 47:157,1988; JINR-P1-86-828:

Fragmentation of ${ }^{22}$ Ne Relativistic Nuclei on Photoemulsion Nuclei

Pisma Zh. Eksp. Teor. Fiz, 47:20.1988:

Transverse Momentum Alpha-Fragmente from Collisions ${ }^{22} \mathrm{Ne}$ with Emulsion Nucleus at tre 4.1 A GeV/c Momentum

Andreera $88 \mathrm{C}$

Yad. Phys. 47:949.1988:

Correlation Phenomena in Fragmentation of Relativistic Nucleus ${ }^{22} \mathrm{Ne}$ at $P=40 \mathrm{GeV} / c$

Andreera 89

Andronenko 86

IINR-P1-89-213

Central Interactions of ${ }^{22} \mathrm{Ne} N u c l e i$ with Heavy Photoemulsion Nuclei at $P=4.1 \mathrm{~A}$ GeV/c

LENI-86-1169:

Measurament of Multiplicity Correlations in Cumulative Feactions

Andryakor 87

Yad. Phrs. 47:1268.1988: 1TEP-87-83:

Interference of Pions, Observed in $\pi^{-} p$ Interactions at $4 \mathrm{GeV} / \mathrm{c}$ and Influence of the Resonances on Space-Time Characteristics

Andryakov 88

Andryakov 89

ITEP-88-37:

The Interference of the Pairs of Pions. Discovering of the Positive Correlations in ( $\pi^{0} \pi^{ \pm}$) Systems

ITEP-89-6T:

Interference of Identical Pions in $\pi^{+} p$ Interactions at $4.5 \mathrm{GeV} / \mathrm{c}$

Angelescu 90

Angelini 86

Angelis 85

Angelis 86

Angelis 87

Angelis 90

Angelopoulos 85

Angelopoulos 86

uovo Cim. 103A:93.1990.

Pion Absorbtion in ${ }^{3} \mathrm{He}$ at 100,120 , and $145 \mathrm{MeV}$

Phys. Lett. 179B:307.1986:

New Experimental Limits on $\nu_{\mu} \rightarrow \nu_{c}$ Oscillations

Nucl. Phys. B263:228.1985: CERN-EP-85-101:

High Transverse Energy and High Transverse Momentum Events in $p \bar{p}$ and $p$ Interactions at $t$ he CERN Intersecting Storage Fings

Phys. Letr. 168B:158.1986: CERN-EP-86-18: BNL-38470:

Observation of KNO Scaling in the Neutral Energy Spectra from He He and p $p$ Collisions at ISR Energies

Phy's. Lett 185B:213.1987: BNL-39112: CERN-PRE-87-026: Large Transverse Momentum $\pi^{0}$ Production in He He, deuteron deuteron cnd $p$ Collisions at the CERN ISR

Nucl. Phys. B327:541.1990: PRINT-89-0666-NICHIGAN-STATE

Direct Phrson Production at the CERN ISR

Phys. Lett i. is $92 \cdot 210.1985$ :

A Search for Structlo e in Charged Meson Spectra from Proton-Antiproton Annihilations at Rest

Phys. Let:. 178B:4t1.1986:

A Search for Narrow Lines in $\gamma$ Spectra from Proton Antiproton Annihilations at Rest

Angelopoulos 88

Angelopoulos $88 \mathrm{~B}$

Phys. Lett. 205B:590.1988:

iteutron Emission from Antiproton Annihilation at Rest in Uranium

Phys. Lett. 212B:129.1988:

A Measurement of the $S$ - and P-Wave Content of Antiproton Annihilation at Fest into Two Pions in Liquid Deuterium

Angelov 88

jIXR-P1-88-905:

The Correlation of Secondary Particles in p C, deuteron C, ${ }^{4} \mathrm{He} C, \mathrm{C}$ C Interactions at $4.2 \mathrm{GeV} / \mathrm{C}$ per Nucleon Momentum 
Angelov 89

Anikina 85

Anikina 858

Anikina $85 \mathrm{C}$

Anikina 8613

Anikina $86 \mathrm{C}$

Anikina 8GD

Anikina 89

Aniol 85

Anjos 86

Anjos 87

Anjos 87B

Anjos $87 \mathrm{C}$

Anjos 87D

Anjos 88

Anjos 88B

Anjos 88C

Anjos 88D

Anjos 88E

Anjos 88F

Anjos 88G

Anjos 89

Anjos 89B

Anjos $89 \mathrm{C}$

Anjos 90

Anjos 90C

Anoshin 87

Ansari 87

Ansari B7B

Ansari $87 \mathrm{C}$

Ansari $8 T D$

Ansari $87 F$

Ansari $\mathbf{R B}$

Ansari 88B

Ansorge 86

Ansorge 87

Ansorge 88
Kr. Soob. JINR 38:11.198::

Clusterization in Processes of Multiple Particle Production by Nuclei and Its Connection with Cumulative Creation of Hadrons

rad. Phys. $11: 711.1985 ;$

Associative Production of Backward Emitted Fast Fragments in Nucleus-Nucleus Interactions

JINR-EI-R5-578:

Study of the Polerization for $\wedge$ Produced in nucleus nucleus Interactions

Yad. Phys. 43:1217,1986: JIN R-p'1-85-208:

The Spectra of $p$, deuteron, ${ }^{3} \mathrm{H}$ Enitted at 160 Degrees in $3.6 \mathrm{GeV}$ per Nucleon ${ }^{12} \mathrm{C}$ Collisions with Nuclei

$Y$. Phys. 45:1680.1987: JINR-PI-86-4iT

Experimental Data on Average Number of Interacted Protons in Inelastic and Central nucleus nucleus Collisions at 3.6 GeV/Nucleon

J1NA-Pl-86-733:

Investigation of Central and Peripheral Interactions of ${ }^{12} \mathrm{C}$ (3.6 GeV/Nucleon) with $\mathrm{Cu}, \mathrm{Pb}$ Nuctei

Phys. Rev. C33:805.1986:

Pion Production in Inelastic and Central Nuclear Collisions at High Energy

Kir. Soob. JINR 34:12.1989:

Experimental Data on Multiplicities in Central Collisions

Phys. Rev. C33:1714.1986: TRI-PP-85-99:

Pion Absorption on ${ }^{3} \mathrm{He}$ at $T(\pi)=62.5 \mathrm{MeV}$ and $82.8 \mathrm{MeV}$

Phys. Rev. Lett. 58:311.1987: FERMILAB-PLB-86-155-E:

Measurement of the $D^{+}$and $D^{0}$ Lifetimes

FERMILAB-CONF-87-3i-E:

Charm Photoproduction Results from the Fermilab Tagged Photon Spectrometer

Phys. Rev. Lett. 60:1379.19R8: FERAILAB-Pl'B-87-218-E: Measurements of the $\Lambda_{\mathrm{c}}^{+}$Lifetime

Phys. Rev. Lett, 58:1818.1987; FERMILAB-PL'B-87-2!-E: Measurement of the $D_{S}^{+}$Lifetime

FERMIILAB-CONF-8T-14i-E:

A Search for Flavor Changing Neutral Current Processes in Decays of Charmed Mesons

Phys. Rev. Lett, t50:1239.1988: FERMILAB-P('B-87-219-E: Study of $D^{\circ}-\bar{D}^{0}$ Mixing

Phys. Rev Lett 62:1587,1989: FERMILAB-PI'B-88-141-E: FEBMILAB-CONF-87-142-E: A Study of the Semileptonic Decay Mode $D^{0} \rightarrow K^{-} e^{+} \nu_{k}$

Phys. Rev. Lett. 62:513.1989: FERMILAB-PIB-88-125-E: Charm Photoproduction Results from E691

Phys. Rev. Iett. 62:125.1989: FERMILAB-PL'B-88-90-E: Measurement of $D_{S}^{ \pm}$Decays to Non-strange States

Phys. Rey. Lett. 62:722.1989: FERMILAB-PUB-88-143-E: Experimental Study of the Semileptonic Decay $D^{+}-\bar{K}^{*}(892)^{0} e^{+} \nu_{e}$

Phys. Rev. Lett. 62:1717.1989: FERMIILAB-PCB-88-155-E: Observation of Excited Charmed Mesons

Phys. Rev. Let . 60:897.1988: FERMILA1B-P'B-87-203-E:

Measurement of $D_{S}^{+}$Decays and Cabibbo-suppressed $D^{+}$Decays

Phys. Rev. Lett. 62:1721.1989; FERAILAB-P'LB-89.24-E:

Observation of $\Sigma_{c}(2455)^{0} \rightarrow \Lambda_{c}^{+} \pi^{-}$Decays

Phys. Lett. 223B:267.1989: FERMILAB-PUB-89-23-E:

A Study of $D_{S}^{ \pm}$and $D^{ \pm}$Decays into Four-Body Final States, Including $\eta \pi^{ \pm}$and $\pi^{ \pm}$

Phys. Rev. D41:801.1990, FERMILAB-PLB-69-144-E: A Study of Decays of the $\Lambda_{r}^{+}$

FERMILAB-PL'B-90-106-E:

Experimental Results on the Decays $D \rightarrow K 4 \pi$

FERMILAB-P[B-90-82-E:

Study of $D_{S}^{+} \rightarrow \phi e^{+} \nu_{r}$ and the Absolute $D_{S}^{+} \rightarrow \phi \pi^{+}$Branching Function

JINR-P1-87-439:

The Charge Dependence of the Effective Mass Spectra of $\pi^{ \pm} p$ Pairs in $\pi^{-}$C Cumulative Interactions at $P=40 \mathrm{GeV} / \mathrm{c}$ with a Total Nuclear Breakup

2. Phys, C36:175.1987: CERN-EP-87-74:

Jet Measures and Hadronic Event Shapes at the CERN $\bar{p} p$ Collider

Phys. Let $186 \mathrm{~B}: 452.1987$ : CERN-EP-87-04:

Search for Decays of the $W^{ \pm}$and $Z^{0}$ Bosons into $q \bar{q}$ Pairs

Phys. Lett. 194B:158.1987: CERN-EP-87-48:

Measurement of $W^{ \pm}$and $Z^{0}$ Production Properties at the CERN $\bar{p} p$ Collider

Phys. Lett. 195B:613.1987: CERN-EP-87-117:

Search for Exotic Processes at the CERN $\bar{p} p$ Collider

Phys. Lett, 186B:440,1987: (CERN-EP-87-155:

Measurement of the Standard Model Parameters from a Study of $\boldsymbol{N}^{ \pm}$and $Z^{0}$ Bosons

Phys. Lete. 21583:175.1984: CERN-EP-88-134:

Measurement of the Strong Coupling Constant as from a Study of $w^{ \pm}$Bosons Produced in Association with Jets

2. Plys C $41: 395,1988$

Direct Photon Production in $\bar{p} p$ at $E_{\mathrm{cm}}=830 \mathrm{GeV}$

2. Phys. (333:175.1980): BONN-11F-R6-19:

Diffraction Dissociation at the CERN Pulsed $p \bar{p}$ Collider at c.m. Energies of 900 and $200 \mathrm{GeV}$

Phis. Lett. 199B:311.14RT: CERX-PRE-H7-0125:

Kaon Production at 200 and $900 \mathrm{GeV}$ c.m. Energy

Z. Phys. ('41:179.198x.

Kaon Production in $\bar{p} p$ Interactions at c.m. Energies from 200 to $800 \mathrm{GeV}$ 
Ansorge 89

Ansorge $89 B$

Ansorge $89 \mathrm{C}$

Antille 87

Antipor 85

Antipor 85B

Antipov $85 \mathrm{C}$

Antipov 86

Antipov 86B

Antipov $86 \mathrm{C}$

Antipov $86 \mathrm{D}$

Antipov 87

A ntipov 87B

Antipov 88

Antipov 88B

Antipov 89

Antipov $89 B$

Antipos $89 \mathrm{C}$

Antonchik 85

Antonchik 87

Antonchik 90

Antonchik $90 \mathrm{~B}$

Antonelli 88

Antos 87

Antos 88

Antreasyan 86

Antreasyan $86 B$

Antreasyan 87

Aoki 87

Aoki 88

Aokı 89

Apalikov 85

Apel 85,

Apel $8.5 \mathrm{~B}$

Apokin 45B

A pokin 86
2. Phys. (43:75.1989

Photon Production at c.m. Energies of 200 and $900 \mathrm{GeV}$

[SIP-89-02: C'ERN-EP-89-41:

Hyperon Production at $200 \mathrm{GeV}$ and $900 \mathrm{GeV}$ c.m. Energy

2. Phys C Ci3:257 1989: ('ENN-EP-88-172:

Charged Particle Multiplicity Distributions at $200 \mathrm{GeV}$ and $900 \mathrm{GeV}$ cm. Energy

Phys Lett. 194B:568.1987: ('ERN-EP-87-121:

$A$ Measurement of the Inclusive $\pi^{\circ}$ and $\eta$ Production Cross Sections at High pr in $\bar{p} p$ Collisions at $E_{\mathrm{cm}}=24.3 \mathrm{GeV}$

2. Phys. C26:495.1985:

Experimental Estimation of the Sum of Pion Electrical and Magnetic Palarizabilities

2. Phys. C27:21,1985: J1NA-E1-84-514:

Study of $\pi \pi^{0}$ Production by Pions in the Nuclear Coulomb Field at Threshold

Kr. Soob. JINR $11: 11.198,5$ :

Measurement of Coupling Constant $\gamma \rightarrow 3 \pi$ in the Process of Pion Pair Production by Pions in the Nuclear Coulomb Field

Phys. Rev. Lett, 56:796.1486:

Experimental Measurement of the $\rightarrow-3 \pi$ Coupling Constant

Phys. Rev. D36:21.1987: JINR-P1-86-498:

Investigation of $\gamma \rightarrow 3 \pi$ Chiral Anomaly During Pion Pair Production by Pions in the Nuclear Coulomb Field

Yad. Phys. 45:1041.1987: IFYE-8ti-134:

Observation of the $\rho^{\circ}$ and $\pi^{-}$Mesong Resonant State in the $\pi_{2}(1670)$ Region

JINR-P1-86-710:

Investigation of Compton Effect on $\pi^{-}$Meson and Charged Pion Polarizability

Yad. Phys. 48-471.1988: [FVE-87-153:

Inclusive Cross Section Measurement of Cumulative Protong Production in $\pi^{-}\left(K^{-}, \bar{p}\right)$ Be Interaction at $40 \mathrm{GeV} / \mathrm{c}$

Yad. Phys 48:138.1988: Suv. J. Nucl. Plys. 48:85.1988: J1NR-P1-87-534:

Elastic Scattering of $\pi^{-}$and $K^{-}$Mesons on Protons at $43 \mathrm{GeV} / \mathrm{c}$ Moment um

IFVE-88-17\%:

On Measurement of $\rho^{0} \rightarrow \mu^{+} \mu^{-}$Decay Branching Ratio in Coherent Dissociation Processes $\pi^{-} \rightarrow \mu^{+} \mu^{-}$ $\pi^{-}$and $\pi^{-} \rightarrow \pi^{+} \pi^{-} \pi^{-}$

Pisma Zh. Eksp. Teor. Fiz, f8:519.1988:

Measurement of $\rho^{\circ} \rightarrow \mu^{+} \mu^{-}$Decay Branching Rat io

Z. Phys. C42:185.1989:

Determination of the Branching Ratio of $\rho^{0} \rightarrow \mu^{+} \mu^{-}$in the Coherent Dissociation $\pi^{-}+\mu^{+} \mu^{-} \pi^{-}$and $\pi^{-} \rightarrow \pi^{+} \pi^{-} \pi^{-}$

Yad. Phys. 51:705.1990: Eur. Lett. 11:725.1990; JINR. P1-89-367:

The Investigation of Radiative Scattering $\pi^{-} p \rightarrow \pi^{-} p$ at $43 \mathrm{GeV}$

JINR-P1-89-282:

The Difractive Production of $\mathrm{K}^{-} \pi^{-} \pi^{+}$System on Nuclei at $40 \mathrm{GeV}$

Yad. Phys, 42:289.1985:

Inelastic Collisions of ${ }^{50}$ Fe Nuclei at $1.8 \mathrm{GeV} / \mathrm{Nucleon}$ Energy with Ag and Br Nuclei and Cascade Model of Nucleus Nucleus Interactions

Yad. Phys, $16: 1344.1987$ :

Fast Fragment in Interactions of Relativistic Nuclei with Photoemulsion

Yad. Phys. 51:765.1990:

Interaction of Relativistic Nuclei ${ }^{40} \mathrm{Ar}$ and Cascade-Evaporation Model

Yad. Phys, 51:936.1990:

The Effect of Bombarding Nucleus Mass upon Light Charged Particles Emiasion with Energy below 400 MeV/Nucleon

Phys. Lett. 212B:133.1988: LAL-88-11

Measurement of the Reaction $e^{+} e^{-} \rightarrow \eta \pi^{+} \pi^{-}$in the Center of Mass Energy Interval 1350 - 2400 MeV

Czech. J. Pliys. B36:1347.19k7:

A Study of the $\rho^{\mathrm{D}}$ Meson Polerization in $\pi^{-}$p Reaction at $5 \mathrm{GeV} / \mathrm{c}$

Yad. Phys. 48:723.1988

$\Delta\left(1232 P_{9 g}\right)^{++}$Inclusive Production in $\pi^{-} p$ Interactions at $5 \mathrm{GeV} / c$

Phys Rev. D33: 1847.1986: SLAC.PL'B-3761: DESY-85-97 Formation of $a_{0}(980)$ and $a_{2}(1320)$ in Photon Photon Collisions

Nuovo Cim. 99A:595.1988: 1NFN-Pl-AE-86-9: Associated Multiplicities in $\mu^{ \pm}$Pair Events at the ISR

Plyy. Rev. D36:2633.1487: SLAC-PLB-4305: DESY+87-05t: Measurement of the Properties of $\eta^{\prime}$ and Search for Other Resonances in $\gamma \uparrow \rightarrow \eta 2 \pi^{0}$

Phys. Letl. 187B-185.1947; C'ERN-EP-86-97:

The Double Associated Production of Charmed Particles by the Interaction of $350 \mathrm{GeV} / \mathrm{c} \pi^{-} \mathrm{Mesons}$ with Emulsion Nuclei

Phys Lett - 209B: 113.1988: C'ERN-EP-8K-52 Some Properties of Charmed Particles Produced in Nucleus Interactiong

Phys Lett. 224B:441,19K9; ('EIRN-EP-89-67

The Production of Charmed Particles in High Energy ${ }^{10}$ O-Emulsion Central Interactions

Pistua Zh. Eksp. Teor. Fiz. 12:2333.1985: ITEP-k5-114: Searches for Heavy Neutrino in $\beta$-Decay

Yad Phys 41:126.1985. Nucl. Phys. B193:269.1981: Sor J. Nucl. Phys 4L:R0.19k5 Analysis of the Reaction $\pi^{-} p \rightarrow \pi^{0} \eta n$ at $40 \mathrm{GeV} / \mathrm{c}$ Beam Momentum

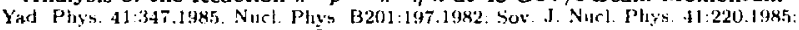
Measurement of $\pi^{-} p \rightarrow 2 \pi^{\circ} n$ at $25 \mathrm{GeV} / \mathrm{c}$

Z. Plys C:15:173.i487: IFVE-8ti-2

Polarization Parameters in the Reactions $\pi^{-} p$ (polarized) $\rightarrow \eta n$ and $\left.\pi^{*} p(p o l a r i z e d) \rightarrow \eta\right)^{\prime} n$ at 40 GeV/c

Yad. Phys 45:1355,1987, IFVF-86-132 Polarization Asymmetry Oscillations in Reaction $\pi^{-} p \rightarrow \pi^{0} n$ at $-t<3(\mathrm{GeV} / \mathrm{c})^{2}$ 
Apokin $86 \mathrm{~B}$

A pokin 86C

A pakin $86 \mathrm{D}$

Apokin 88

Apokin $88 B$

A pokin $88 \mathrm{C}$

Apokin 89

Apokin $89 \mathrm{~B}$

Appel 85

Appel 85B

Appel $85 \mathrm{C}$

Appel 86

Appel $86 \mathrm{~B}$

Aprile 86

Apsimon 89

Apsimon 90

Arakelyan 85

Arakelyan 86

Arakelyan 87

Arakelyan 89

Arakelyan $89 \mathrm{C}$

Arakelyan $89 \mathrm{D}$

Arakelyan 89E

Arakelyan 90

Ardito 87

Arefiev 85

Arefier 86

Ar tev $86 \mathrm{~B}$

Arefey 87

Arefier 90

A refiev $90 \mathrm{~B}$

Arends 85

Arends 88

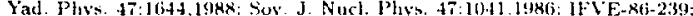

$\pi^{-} p \rightarrow f_{2}(1270) n$ Reaction in the Region-t<2.0 at $40 \mathrm{GeV} / \mathrm{c}$

Yad. Phys. 16:1108.1947: IFVE- $\times 10-240$ :

Quasielastic Charge Exchange of Negative Pions on Carbon into $\pi^{0}, \eta, \eta^{\prime}, \omega$ and $f_{2}(1270)$ Mesons at 39.1 GeV/c

Yad. Phys, 46:1182.19\%7: IFYE-86-241

Z-dependence of Differential and Total Cross Sections in Quasibinary Exchange Reactions on Nucle: $\pi$ $A(Z) \rightarrow\left(\pi^{0}, \pi, \omega, f_{2}(1270)\right) A(Z-1)$ at $39.1 \mathrm{GeV} / c$

Yad. Plyss. 47:727.1988: IFlE- $47 \cdot 44$ :

Asymmetry Observation in the Reaction $\pi^{-} p \rightarrow \pi^{0} \pi^{0} n$

Yad Phys 49:156 1989: Sov J Nucl Phys. 49:97.1989: [FVE-88-78

Asymmetry Measurement of $\pi^{0}$ Mesons Produced in Beam Fragmentation Region on Polarized Protons

Yad, Phys. 49:165.1989: Sov. J Nucl. Phys. 49:103.1989: IFVE-84-79:

Result on Azimuthal Asymmetry Measurement of $\pi^{0}$ - Mesons Produced in Beam Fragmentation Region on Polarized Deuterons

1FVE-89-37:

Measurement of One-Spin Asymmetries in Inclusive $\pi^{0}$ and $\eta$ Production at 90 Degrees CMS in the Reactions $\pi^{-} p$ (polarized) $\rightarrow \pi^{\circ}(\eta) \mathrm{X}$ at $40 \mathrm{GeV} / \mathrm{c}$

Yad. Phys. 49:445.1989: Sov. J. Nucl. Phys. 49:278.1989; JFVE-88-76:

$\pi^{+} \pi^{-} \rightarrow 2 \pi^{0}$ Cross Section at c.m. Energies $0.55 \mathrm{GeV}<m<2 \mathrm{GeV}$ from the Reaction $\pi^{-} p \rightarrow 2 \pi^{0} n$ at $39.1 \mathrm{GeV} / \mathrm{c}$

Phys. Lett. 160B:349.1085: CERN-EP-85-111.

Measurement of the $\sqrt{s}$ Dependence of Jet Production at the CERN $\bar{p} p$ Collider

Phys. Lett. 105B:441.1985: (ERN-EP-85-136:

Experimental Study of the Emergence of Two-Jet Dominance in $\bar{p} p$ Collisions at $630 \mathrm{GeV}$

Z. Phys. C30:341.1986: C'ERN-EP-85-189.

A Study of Three Jet Events at the CERN $\bar{p} p$ Collider

Z. Phys. C30:1,1986; CERN-EP-85-166:

Measurement of $W^{ \pm}$and $Z^{\circ}$ Properties at the CERN $\bar{p} p$ Collider

Phys. Lett. 176B:239.1986: CEAN-EP-86-b4:

Direct Photon Production at the CERN $\bar{p} p$ Collider

Phy's. Rev. D34:2566,1986;

Measurements of Double- and Triple-spin Parameters in p p Elastic Scattering between 440 and 580 MeV

Z. Phys. C43:63,1989: CERN-EP-89-31:

Inclusive Photoproduction of Single Charged Particles at High P-transverse

Z. Phys, C46:35.1990:

A Study of the Point-Like Interactions of the Photon Using Energy-Flows in Photon- and HadroProduction for Incident Energies between 65 and $170 \mathrm{GeV}$

Yad. Phys. 42:3.1985:

Total Cross Section of $\pi^{\alpha}$ Meson and Hadron Photoproduction on Be, C, $O$ and Al Nuclei in the Energy Range 200 - $900 \mathrm{MeV}$

Yad. Phys. $44: 10.1986$ :

Spallation of Nickel Isotopes Induced by Photong of $4.5 \mathrm{GeV}$ Maximum Energy

Yad. Phys. 46:1706,1987:

Estimate of Contribution Introduced by Secondary Reactions to Cumulative Proton Production in $\pi^{-} C^{-}$ Interactions at $5 \mathrm{GeV} / \mathrm{c}$

Yad. Phys. 49:1253.1989:

Electrofission Cross Section and Fission Yield Measurement for ${ }^{235} \mathrm{U}$ ams ${ }^{238} \mathrm{U}$ Nuclei at Energies 1.33 $4.32 \mathrm{GeV}$

YERE-1104(67)-88;

Measurement of the Cross Sectios of Photo and Electrofusion of ${ }^{238} U$ and ${ }^{298} U$ in the Energy Range 1.33 $4.32 \mathrm{GeV}$

Y'ERE-1J65(42)-89:

Measurement of Charged Hadron Production Multiplicity at Interaction of Photons with E-.5 - 3.3 GeV with $A l, C u$ and $P b$ Nuclei

Yad. Phys. 49:1651.1989. Y'ERE-1103(66)-88:

Experimental Study of Shedowing in the Electrofusion of ${ }^{238} U$ Nuclei at $E(\gamma)=1-3.6 \mathrm{GeV}$ and Low $Q^{2}$

Yad Phys. 51 1582,1990:

Investigation of the $(\gamma, \pi)$ and $(\gamma, \pi \mathrm{Xn})$ Renctions on Medium Weight and Medium Heavy Nuclei

CERN-EP.87-162.

Interactions of 80 and 200 A GeV ${ }^{16} \mathrm{O}$ Ions in Nuclear Emulsion

ITEP-85-25:

Measurement of $\pi^{+}, p$, deuteron, ${ }^{3} \mathrm{H},{ }^{3} \mathrm{He}$ Inclusive Cross Sections at 3.5 Degrees in Protons Interactions with Be, Al, Cu, Ta Nuclei at 6.37 and $8.08 \mathrm{GeV} / c$

ITEP-86-150

Search for Exotic Baryon Resonances with $I=5 / 2$ at $\pi^{+} p \rightarrow p \pi^{+} \pi^{+} \pi^{-}$at $3.94 \mathrm{GeV} / \mathrm{c}$

1TEP-86.76:

Study of $\pi^{+} p \rightarrow \Delta\left(1232 P_{33}\right)^{++} \rho^{0}$ Reaction of Baryon Exchange at $3.94 \mathrm{GeV} / c$

ITEP-87-54:

Search for the Baryon Resonances with $I=5 / 2$ in the $\pi^{+} p \rightarrow p \pi^{+} \pi^{+} \pi^{-} \pi^{0}$ Reaction at the Momentum of $3.94 \mathrm{GeV} / \mathrm{c}$

Yad. Plyss. $51: 406.1990$ :

Reaction $\pi^{+} p \rightarrow p \pi^{+} \pi^{+} \pi^{-}\left(\pi^{0}\right)$ at $3.94 \mathrm{GeV} / c$ Incident Momentum: Analysis in the OPER Model

Yad. Plyys $51: 414.1990$ :

Reaction $\pi^{+} p \rightarrow p \pi^{+} \pi^{+} \pi^{-}\left(\pi^{0}\right)$ at $3.94 \mathrm{GeV} / c$ Incident Moment um: Production of Resonances, Including Those with $f=5 / 2$ in Baryon Exchange Processes

Nuel. Phys. A454:579.1446;: BONN-HE-85-14:

Measurement of Total Cross Sections for $\pi^{\circ}$ Photoproduction on Nuclei in the $\Delta$ (1232 $\left.P_{33}\right)$ Resonance Region

BONN-IR-88-0E

Photonuclear Reactions in the $\Delta\left(1232 P_{33}\right)$ Resonance Region 
Areuton 85

Arenton 85B

Arenton 86

Argän 88

Arkhipov 85

Arkhipov 87

Arkhipov 88

Arinstroi:g 8.5

A rinstrong 86

Arustrong $86 \mathrm{~B}$

Armstrong $86 C^{\circ}$

Armstrong 86D

Arinstrong $86 \mathrm{E}$

Armstrong $86 \mathrm{~F}$

Arinstrong 87

Arnistrong $87 \mathrm{~B}$

Arnstrong $87 C^{\circ}$

Armstrong 88

Armst rong 89

Arnustrong $89 B$

Armitrong $89 C^{\circ}$

Arinstrong $89 \mathrm{D}$

Armstrong 89E

Armstrong $89 \mathrm{~F}$

Armstrong 90

Armutlijsky 64

Armut!ijoky 8.5

A rmutlijsky 85B

Armutligsto: kij

Artnut lijsky $86 \mathrm{BB}$

Armutlijsks $\$ 60$

Armutlijiky $* 7$

Arrmutlijky $67 \mathrm{H}$

Phys. Rov Di1:984.1945.

Measurement of the Dijet Cross Section in $\mathbf{4 0 0}$ GeV/r p p Interactions

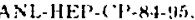

Results from a Hadron Jet Experiment at $E_{\mathrm{cm}}=19.4$ and $27.4 \mathrm{GeV}$

Nucl Plys. B274:707.14H6: PHINT-8ti-1158-AlALZON.

Observation of Massive $\Lambda K_{5} \pi^{+} \pi^{+} \pi^{-} \pi^{-}$Events above $5 \mathrm{GeV} / \mathrm{c}^{2}$

Plits. Lett. 206B:4 1988.

The Threshold Photoproduction of $\pi^{0}$ on Nucleons and on Few-nucleon Systems

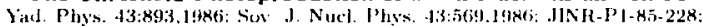

Differential Cross Section of the Renction. $\pi^{-} \beta \rightarrow \eta \Delta\left(1232 P_{33}\right)^{0}$ for a Momentum of $3.3 \mathrm{GeV} / \mathrm{c}$

JiR-Pl-87-271

Differential $C \cdot$. oss Section of the $\pi^{-} p \rightarrow \eta \Delta\left(1232 P_{33}\right)^{0}$ Reaction at a Momenta of $3.3 \mathrm{GeV} / \mathrm{c}$ and 4.75 GeV/c

Yad. Phys. t7:1649.1988: Sos. J. Nuci. Phys. Hi:1044.14hs:

$\pi^{-} p \rightarrow \eta \Delta\left(1232 P_{33}\right)^{0}$ Differential Cross Section at 3.3 and $4.75 \mathrm{GeV} / c$

Nucl. Phys, B262:356.1985: CERN-EP-85-83;

$A$ Polarization in $K^{-}$Fragmentation Region

Phys. Lett. 16tiB:245.1986: CERN-PRE-R6i-03:

Observation of Double $\phi$ Meson Production in the Central Region for the Reactions $\pi^{+} p \rightarrow \pi^{+} 2 K^{+} 2 K^{-}$ $p$ and $p p \rightarrow p 2 K^{+} 2 K^{-} p$ at $85 \mathrm{GeV}$

Phys. Lett. 167B:133.1986; CERN-EP-85.179:

Evidence for Structure in the $1.7 \mathrm{GeV}$ Mass Region of the $K^{+} K^{-}$Final State Centrally Produced in the Reactions $\pi^{+} p \rightarrow \pi^{+} K^{+} K^{-} p$ and $p p \rightarrow p K^{+} K^{-} p$ at $85 \mathrm{GeV}$

Phys. Lett. 17513:383.1986:

A Search for Narrow States in Antineutron Proton Total and Annihilation Cross Sections near $\bar{\pi} n$ Threshold

Z. Phys. C3-1:33.1987: CERN-EP-86-138;

Study of the $K^{+} K^{-} \pi^{+} \pi^{-}$System Centrally Produced in the Reactions $\pi^{+} p \rightarrow \pi^{+}\left(K^{+} K^{-} \pi^{+} \pi^{-}\right) p$ and $p p \rightarrow p\left(K^{+} K^{-} \pi^{+} \pi^{-}\right) p$ at $85 \mathrm{GeV} / c$

Z. Phys. (34:23,1987: CERN-EP-86-139:

Study of the Centrally Produced $F_{1}, K^{2} \pi^{ \pm}$System at $85 \mathrm{GeV} / \mathrm{c}$

C'ERN-EP-86-83:

Backward Scattering in $\pi^{-} p \rightarrow p \pi^{-}, \bar{p} p \rightarrow \pi^{+} \pi^{-}, K^{-} p \rightarrow p K^{-}$and $\bar{p} p \rightarrow p \bar{p}$ at $8 \mathrm{GeV} / \mathrm{c}$ and $12 \mathrm{GeV} / \mathrm{c}$

Z. Phys. ( $35: 167.1987$ : CERN-EP-87-32;

Baryon Antibaryon Production in the Central Region at $85 \mathrm{GeV} / \mathrm{c}$

Phys. Rev. D36:659.1987:

Measurement of Antineutron Proton Total and Annihilation Cross Sections from 100 to 500 MeV/c

Nucl. Phys. B284:643.1987:

Backward Scattering in $\pi^{-} p \rightarrow p \pi^{-}, \bar{p} p \rightarrow \pi^{+} \pi^{-}, K^{-} p \rightarrow p K^{-}$and $\bar{p} p \rightarrow p \bar{p}$ at 8 and $12 \mathrm{GeV} / \mathrm{C}$

C'ERN-EP-88-124.

A Search for Glueballs in the Central Region in the Reaction $p p \rightarrow p(f \times p(s)$ at $300 \mathrm{GeV} / \mathrm{c} \mathrm{Using}$ the CERN OMEG A Spectrometer

Phys. Lett. 221B:216.1989:

A Spin-Parity Analysis of the $f_{1}(1285)$ and $f_{1}(1420)$ Mesons Centrally Produced in the Reaction $p \rightarrow p$ $\left(K_{S} K^{ \pm} \pi^{ \pm}\right.$) p at $300 \mathrm{GeV} / \mathrm{c}$

Phys. Lett. 221B:221.1989:

Observation of Double $\phi$-Meson Production in the Central Region for the Reaction $p p^{\rightarrow} p\left(K^{+} K^{-} K^{+}\right.$ $\left.\mathrm{K}^{-}\right)$at $300 \mathrm{GeV} / \mathrm{c}$

2. Phys. C43:55.1989: CERN-EP-89.24:

Study of the $\pi^{+} \pi^{+} \pi^{-} \pi^{-}$System Centrally Produced by Incident $\pi^{+}$and $p$ Beams at $85 \mathrm{GeV} / \mathrm{c}$

Phys. Lett. 227B:186.1989: (CERN-EP-89-70):

Observation of Centrally Produced $f_{2}(1720)$ in the Reaction $p p \rightarrow p(\Omega)(K \bar{K}) p(s)$ at $300 \mathrm{GeV} / \mathrm{c}$

Phys. Let1. 22k13:536.1949: C'ERN-EP-k9-81:

Evidence for New States Produced in the Central Region in the Reaction $p p \rightarrow p\left(\Omega \pi^{+} \pi^{-} \pi^{+} \pi^{-} p(s p e c t)\right.$ at $300 \mathrm{GeV} / \mathrm{c}$

CERN-EP-89-144:

Recent WA76 Results on Central Production

Z. Phys. C $46 ; 405,1950(1:$

A Study of the Centrally Produced $K^{*}(892)^{0} \bar{K}^{*}(892)^{0}$ Final State in the Reaction $p p \rightarrow p(\Omega)\left(K^{+} K^{-} \pi^{+}\right.$ $\left.\pi^{-}\right) p(s)$ at $300 \mathrm{GeV} / c$

Yind Phys. 41:1235.1985: J1NA-P1-44-328.

Momentum and Angular Characteristics of $\pi^{-}$Mesons and Protons Generated in Nucleus-Nucleus Interactions at $4.2 \mathrm{GeV} / c$ per Nucleon as Functions of Minimum Mass of a Target

Yad. Phys. 42:200.1985: JNR-P1-84-629:

Behaviour of $\pi^{-}$Meson Production Jnvariant Differential Cross Section in Nuclear Interactions at 4.2 GeV/c per Nucleon

Yart. Phys 44:1495. luxt: JINR-131-85-933\%:

Properties of $p \mathrm{C}$ Interactions at $10 \mathrm{GeV} / r$ with the Emission of Cumulative Pions and Protons

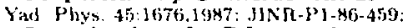

Investigation of $p C$ Interaction at $P=10 \mathrm{GeV} / c$ with the Emission of Cumulative Neutral Pions

JINR-P1-86-765.

Two Proton Correlations in $\mu \mathrm{C}$, deuteron $\mathrm{C}, \mathrm{He} \mathrm{C}$ and $\mathrm{C} \mathrm{C}$ Interactions at $4.2 \mathrm{GeV} / \mathrm{c}$ per Nucleon

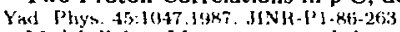

Multiplicity, Momentum and Angular Distributions of Protons in the Interactions of Light Nuclei with Carbon Nuclei at $4.2 \mathrm{GeV} / \mathrm{c}$ per Nucleon

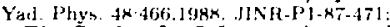

The Study of $p C$ Interactions at $P=10 \mathrm{GeV} / \mathrm{c}$ with the Emission of Cumulative Deuterons

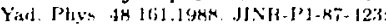

Inclusive Characteristics of $\pi^{-}$Mesons Produced in $p \mathrm{C}$ and $p$ Ta Interactions at $10 \mathrm{GeV} / \mathrm{c}$ Proton Momentum 
A romutlijskv 87C

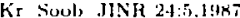

Universality of $4^{-}$Dimensional Baryonic Cluster Properties in Hadron-Nuclear and Nucleus-Nuclear lnceractions within the $4-40 \mathrm{GeV} / \mathrm{c}$ Energy Range

Artnut lijsky $87 \mathrm{D}$

Yad Phys 16:1712 likT JINR.Pl-H7-27.

Experimental Estimation of Secondary Absorbtion Contribution to Proton Production into Backward Herrisphere in Nucleon Carbon Interaction at 4.2 and $10 \mathrm{GeV} / \mathrm{c}$

Aruut lijsky 88

Armutlijsky 89

Arndt 84

hrndt 85

Arndt 87

Arneodo 85

Arneodo $85 \mathrm{~B}$

Arneodo $86 C$

Arzeodo $86 \mathrm{D}$

Arneudo $86 \mathrm{E}$

Arneado 86F

Arneodo 86h

Arneodo 87

Arneodo $8 \% \mathrm{C}$

Arncodo 88

Arneodo $88 \mathrm{~B}$

Arneodo 89

Arneodo $69 \mathrm{~B}$

Arnete 87

Arnison 85

Arnition 85B

Arnison $85 \mathrm{C}$

Arnison B5D

Arnison 6.5E

Arnison 8f

Arnison 86B

Arnison $86 \mathrm{C}$

Arnison 86D

Arnold 86

Arnold 67

Arnolel $87 \mathrm{~B}$

Arnold 88

Arnold 89

Aronson 86

Arpeselliz KKB

Yad Phys. 47:739.1986: JiN12.P1-87.97: Production of $\Lambda$-Hyperons and $K$-Mesons in Cumulative $p C$ Interactions at $10 \mathrm{GeV} / c$

Yad Phys. 49:182,1989: Yat. P'hys. 49:1k2.19k8, JINR-Pl-k7-905: Angular and Momentum Characteristics of Proton from deuteron Ta and C Ta Interactions at $4.2 \mathrm{GeV} / \mathrm{C}$ per Nucleon

Phys, Rev. D31:2230.1985: VPISA-2-85: VPISA-1-N4:

$K^{+}$Proton Partial Wave Analysis to $3 \mathrm{GeV} / \mathrm{c}$

Phys Rev. D32:1085.1985:

Pion-Nucleon Partial Wive Analysis to $1100 \mathrm{MeV}$

Phys. Rex. D35:128.1987. Nucleon-Nucleon Partial-Wave Analysis to $1100 \mathrm{Mev}$.

Nucl. Phys. B258:249.1945: ('ERN-EP-8.5-26: Multiplicities of Charged Hadrons in $280 \mathrm{GeV} / \mathrm{c}$ Muon Proton Scattering

Phys. Lett. ]65B:222.1985: ('ERN-EP-85-14:3; Hadron Multiplicity Variation with $Q^{2}$ and Scale Breaking of the Hadron Distributions in Deep Inelastic Muon-Proton Scattering

Z. Phys. C32:1 1986: CERN-EP-86-42:

The Bose-Einstein Correlations in Deep Inelastic $\mu^{ \pm} p$ Interactions at $280 \mathrm{GeV}$

2. Phys. C33:167.1987: CERN-EP-86-99;

$\rho^{0}$ and $w$ Production in Deep Inelastic $\mu^{+} p$ Interactions at $280 \mathrm{GeV} / c$

2. Phys. C35:417,1987: CERN-EP-86-119:

Comparison Between Hadronic Fina! States Produced in $\mu^{+} p$ and $e^{+} e^{-}$Interactions

2. Phys. C35:1,1987: ('ERN-EP-86-88:

Charm Production in Deep Inelastic Muon-Iron Interactions at $200 \mathrm{GeV} / \mathrm{c}$

2. Phys. C34:283.1987: CERN-EP-86-16u;

Strangeness Production in Deep Inelastic Muon Nucleon Scattering at $280 \mathrm{GeV}$

2. Phys. C36:527.1987: CEHN-EP-87-112:

Jet Production and Fragmentation Properties in Deep Inelastic Muon Scattering

Z. Phys. C35:433.1987: CERN-EP-87-74:

Proton and Antiproton Production in Deep Inelastic Muon Nucleon Scattering at 280 Ge:'

Phys. Lett. 211 B:493.1988:

Shadowing in Deep-Inelastic Muon Scattering from Nuclear Targets

Z. Phys. C40:347.1988:

Charged Structure of the Hadronic Final State in Deep-Inelastic Muon-Nucleon Scattering

Nucl. Plyss. B321:541.1989: CERN-EP-89-25:

Measurements of the $u$ Valence Quark Distribution Function in the Proton and $u$ Quark Fragmentation Functions

Nucl. Phys. B333:1.1990: CERN-EP-89-121:

Mensurement of the Nucleon Structure Function in the Range $0.002<x<0.17$ and $0.2<Q^{2}<8$ GeV in Deuterium, Carbon and Calcium

Phy. Rev. Lett $5 \times: 1906.1987$.

Neutrino Mass Limits from SN 1987 A

Phys. Lett. 155B:442.1985: (ERN-EP-85.19;

Intermediate Mass Dimuon Events at the CERN $p \bar{p}$ Collider at $E_{c i n}=540 \mathrm{GeV}$

Lelt. Nuovo Cim. 41:1.1985: ('ERN-EP-85-108:

$W^{ \pm}$Production Properties at the CERN $p \bar{p}$ Collider

Phys. Lett 15813:494.1985:

Comparison of Three-Jet and Two-Jet Cross Sections in $p \bar{p}$ Collisions at the CERN SPS $p \bar{p}$ Collider

Phys. Lett. 166B:484.1986. CF.KN-EP-B5-145:

Intermediate Vector Boson Properties at the CERN Super Proton Synchrotron Collider

('ERN-EP-85-116:

Inclusive Jet Production at $E_{\mathrm{cm}}=546 \mathrm{GeV}$

Phys. Lett. 172B 461.1986. ('ERN-EP-s6-29:

Measurement of the Inclusive Jet Cross Section at the CERN $p \ddot{p}$ Collider

Phys. Lett. 177B:244.1946; FAN-EP-86-92:

Angular Distribution, for High Mass Jet Pairs and a Limit on the Energy Scale of Compositeness for Quarks from the CER $N \bar{p}$ Collider

CERN-EP-86-60:

Results on $W^{ \pm}$and $Z^{\prime}$ Physics from the UAI Collaboration

Nuct. Phys. B276:253.1'int, CERN-EP-86-55:

Analysis of the Fragmentation Properties of Quark and Gluon Jets at the CERN SPS $p \bar{p}$ Collider

Phys. Rov. Lott. 57:174.1!k6i; SLAC.PLB-3810:

Mensurement of Elastic Electron Scattering from the Proton at High Momentum Trangfer

Phys. Rev. Lett. 5R:1723.19R7: SLAC-PUB-4145:

Deuteron Magnetic Form Factor Measurements of High Momentum Transfer

Phys. Lett. 186B:435.19k7. ('FHN-EP-86-207:

Experimental Search for Assaciated Gluino Production and Decay in 350 GeV/c $\pi^{-}$Emulsion Interactions

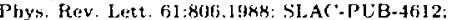

Measurements of Transverse Quasielastic Electron Scattering from the Deuteron at High Momentum Trangfers

SLAK'Pl:B-4918:

Trangverse Electrodisintegration of the Deuteron in the Threshold Region at $\mathrm{High}^{2}$

Phys. If(uv. D3:3:318(1).19ki

Measurement of the Rate of Formation of $\pi_{\mu}^{ \pm}$Atoms in $K_{L}$. Decay

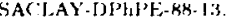

A Review of Running Underground Experiments

See the legetud on page I! 
Artemiev 89

Artuso 89

Artuso 89B

Artykov 86

Artykov $86 \mathrm{~B}$

Artykov 90

Arutyunyants 85

Arvieux $84 \mathrm{C}$

Asad 85

Asai 84

Asai 87

Asai 89

Asai $89 \mathrm{C}$

Asariuna 90

Asatiani 85

Asaturyan 86

Asaturyan $86 \mathrm{C}$

Ash $85 \mathrm{~B}$

Ash $85 \mathrm{C}$

Ash 85D

Ash 87

Ash $87 \mathrm{~B}$

Ashery 88

Ashford 85

Ashford $85 \mathrm{~B}$

Ashitkov 85

Ashman $8 \mathrm{~B}$

Ashman 88B

Ashman $880^{\circ}$

Asbman 89

Asmanl 58

Asratyan 85

Astatyan $8.5 \mathrm{~B}$

Asratyan 86

Asratyan $86 \mathrm{~B}$

Asratyan 87

Asratyan $87 \mathrm{~B}$
[TEP'-X9-186.

The ${ }^{136}$ Xe Double a Decay Track Experiment

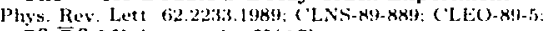
$B^{0} \overparen{B}^{0}$ Mixing at the $\mathbf{T}(\mathbf{4 S})$

CLNS-89-926: What Did We Learn about $V(u b)$ from $B$ Decays?

Yad. Phys. 43:1472.1986: Sov. 1 Nucl. Phys. 43:949.1946:

$\rightarrow$ and $\pi^{\circ}$ Multiplicities in Proton Inelastic Interactions with Neon Nuclei and Nucleons at $300 \mathrm{GeV}$

Yad. Phys 44:400.1966: Inclusive Characteristics of Gamma Quanta in $\pi^{-12} \mathrm{C}$ Interactions at $4 \mathrm{GeV} / \mathrm{c}$

Yad. Pliys. 51:744.1990: FVE-89-99:

Transverse Momenta of $\gamma$ Quanta in $p{ }^{20} \mathrm{Ne}$ and $p$ nucleon Interactions at $300 \mathrm{GeV}$

ITEP-85-119:

Angular Correlations of Particles Produced in $\pi^{-} p \rightarrow K^{0} \pi^{ \pm} X, \pi^{-} p \rightarrow K^{0} \bar{K}^{0} \mathrm{X}, \pi^{-} p \rightarrow K^{0} \Lambda \times$ at 4.5 GeV/c Momentum

Nucl. Phys. A444:579.1985: SACLAY'DPH-N-2104:

A Study of the Reaction deuteron $\left(\pi^{ \pm}, p\right) \mathbf{X}$

Nucl. Phys. B255:273.1985: CERN-EP-8-4-14:

Elastic Scattering of Charged Mesons, Antiprotons and Protons on Protons at Incident Momenta of 20 , 30 and $50 \mathrm{GeV} / \mathrm{c}$ in the Moment um Transfer Range $0.5<-t<8 \mathrm{GeV} / c^{2}$

2. Pliys. C27;11,1985: CERN-EP-84-81: IFVE-85-65:

Inclusive $K_{S}, \Lambda$ and $\bar{\Lambda}$ Production in $380 \mathrm{GeV} / \boldsymbol{c} p$ p Interactions Using the European Hybrid Spectrometer

Phys. Lett. 187B:249.1987:

Measurement of the Cross Section for 7 deuteron $\rightarrow \pi^{\circ}$ deuteron at $140 \leq \theta \leq 156$ Degrees

INS-REP-775:

Search for $\Delta\left(1232 P_{33}\right)-\Delta\left(1232 P_{33}\right)$ Component in Deuteron

2. Phys. C46:593.1990: CERN-EP-89-180:

Experimental Results on Proton Diffractive Dissociation - Study of the Quark-Diquark Pomeron Coupling Phys. Lett. 237 B:588.1990;

A Search for Correlated $e^{+} e^{-}$Pairs in the Decay of ${ }^{241} \mathrm{Am}$

Izv. Akad. Nauk SSSR. Fiz. 49:1377.1985:

The Results of Muon Investigation at Aragats

Yad. Phys. 14:1006.1986:

St udy of Correlation Properties of Processeg with Cumulative Protons in $\pi^{-} \mathrm{C}$ Interactions at $5 \mathrm{GeV} / \mathrm{c}$

Pisna 2h. Eksp. Teor. Fiz. 44:266,1986:

Polarization Parameters $\Sigma, T, P$ for the $\gamma p \rightarrow p \pi^{0}$ in the Energies of $0.9-1.5 \mathrm{GeV}$ at 120 Degrees

Phys. Rev. Lett. 55:2118.1985:

Precise Measurement of the Leptonic Branching Ratios of the Teu Lepton

Phy. Rev. Lett. 55:1831,1985;

Precision Measurement of Electroweak Effects in $e^{+} e^{-} \rightarrow \mu^{+} \mu^{-}$at $E_{c m}=29 \mathrm{GeV}$

Phys. Rev. Lett. 54:2477,1985: SLAC-PUB-3591:

Search for Monojet Production in $e^{+} e^{-}$Annihilation

Phys. Rev. Let t. 58:1080,1987: SLAC-PC B-40.40:

Observation of Charge Asymmetry in Hadron Jets from $e^{+} e^{-}$Annihilation at $E_{\mathrm{cm}}=2 \theta \mathrm{GeV}$

Phys. Rev. Lett. 58:640.1987: SLAC-PLB-4123:

Determination of the Lifetime of Bottom Hadrons

Phys. Lett. 215B:41.1988:

A Search for $\pi n n$ Bound Systems

Phys. Rev. C'31:663,1985;

Low Energy $\bar{p}$ Nuclear Absorption Cross Sections

Phys. Rev. Lett. 54:538.1985:

Measurement of the Real to Imaginary Ratio of the $\bar{p} p$ Forward Scattering Amplitudes

[zv. Akad. Nauk SSSR. Fiz. 49:1396.19x-i.

Study of Outstripped Particles in Frame of Tachyon's Hypothesis

Phys. Lett, 202B:603.1988: (:ERN-EP-88-1)ti:

Measurement of the Ratios of Deep Inelastic Muon-Nucleus Cross Sections on Various Nuclei Compared to Deuterium

Phys. Lett. 206B:364,1988: CERN-EP-87-230;

A Measurement of the Spin Asymmetry and Determination of the Structure Function $G_{1}$ in Deep Inelestic Muon-Proton Scattering

2. Phys. ('39:169.1988:

Exclusive $\rho^{0}$ and $\phi$ Production in Deep Inelastic Muon Scattering

Nucl. Phys. B328:1.1969: CERN-EP-89-73:

An Investigation of the Spin Structure of the Proton in Deep Inelastic Scattering of Polarized Muons on Polarized Protons

2. Phys. C37:191.1988:

Charge Particles Correlation in $\bar{p} p$ at c.m. Energies of 200, 546 and $900 \mathrm{GeV}$

Yad. Phys. 43:598.1986: Sov. J. Nuc1. Phys 13:380.1986) I7EP-85-115:

The Experimental Comparison of EMC Effect in the $\bar{\nu} n$ and $\bar{\nu} p$ Interactions in Neon Nucleus

Yad. Phys 41:1193.19\$5: Sov. J. Nucl. Phys 11:763.1985:

The Distribution of Quarks and Antiquarks in the Proton from the Analysis of $\bar{\nu} n$ and $\bar{\nu}$ Interactions in the Neon Nucleus

Phy:s. Lett. 1.5613.4.41.j98ti:

Charmed Strange Vector Meson Production in Antineutrino-Nucleon Interactions

ITEP-B6-77:

Observation of the Charmed Strange Pseudovector Meson Cascade Radiative Decay

Pisma Zh. Eksp, Teur. Fiz. 46i:413.1987: ITEP'-k7-189:

The $D_{S}^{-}$Meson Production in the Antineutrino Interaction

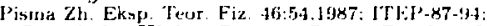

Search for Higher Strange States Produced in $\bar{\nu} n$ and $\nu n$ Interactions 
Asratyan $R 7^{\circ}$

Astbury 85

Astier 89

Astier $89 B$

Aston 85

Aston 85B

Aston 86

Aston $86 \mathrm{~B}$

Aston 87

Aston $87 \mathrm{~B}$

Aston 88

Aston 83B

Aston $88 \mathrm{C}$

Aston 88D

Aston $88 E$

Astun $88 \mathrm{~F}$

Aston $88 \mathrm{C}$

Aston $88 \mathrm{H}$

Aston 881

Astou 88J

Aston 89

Ataton $89 \mathrm{~B}$

Atageldieva 88

Atherton 85

Atiya 89

Atiya 90

Atkinson $84 F$

A2kinson 85

Atkinson 85B

Atkinson $85 \mathrm{C}$

Atkinson $85 \mathrm{D}$

Atkinson $85 E$

Atkinson $85 \mathrm{~F}$

Atkinson 86

Atkinson $86 \mathrm{~B}$

Atkinson 88

Atoyan 89

Aloyall 90

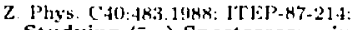
Studying $(\bar{c} \theta)$ Spectroscopy in $\bar{\nu} \mathbf{n}$ Collisions

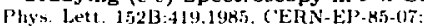
Measurement of Deep Inelastic Compton Scattering of High Energy Photons

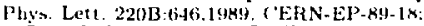
Search for Neutrino Oscillations

CERN-EP.A! -124:

A Search for Neutrino Oscillations

Phy's. Rev. D32:2255.1435: Analysis of Inclusive $K \bar{K} \pi$ from $11 \mathrm{GeV} / c K^{-} p$ Jnteractions

Jhys. Rev. D32:2270.1945:

Inclusive Production of Multistrange Hyperons from $11 \mathrm{GeV} / \mathrm{c} K^{-}$p lnteractions

Phys. Let1. $180 \mathrm{~B}: 308,19 \mathrm{Ab}$ :

Observation of the Leading $\bar{K}^{*}$ (unspec) $L$-excitation Series from $J^{P}=1^{-}$through $5^{-}$in the Reaction $K^{-}$ $p \rightarrow K^{-} \pi^{+} n$ at $11 \mathrm{GeV} / \mathrm{c}$

SLAC-PLB-4054:

A Study of Strange and Strangeonium States Produced in LASS

Phys. Lett. 19-B:579.1947: SIAC-PUB-1132; DPNL-87-16:

Observation of the $\Omega^{*}$ (unspec) $^{-}$Production in $K^{-} p$ Intercetions at $11 \mathrm{GeV} / \mathrm{c}$

Nucl. Phys. B292:693,1987:

The Strange Meson Resonances Observed in the Reaction $K^{-} p-\bar{K}_{\pi}^{+} \pi^{-} n$ at $11 \mathrm{GeV} / \mathrm{c}$

Phys. Lett. 201B:573.1988:

Evidence for Two Strangeonium Resonances with $J^{P C}=1^{++}$and $1^{+-}$in $K^{-} p$ Interactions at 11 GeV/c Nucl. Phy' B296:193.1988: SLAC-PLB-1260; DPNC'-N7-25:

A Study of $K^{-} \pi^{+}$Scattering in the Reaction $K^{-} p \rightarrow K^{-} \pi^{+} n$ at $11 \mathrm{GeV} / c$

Nucl. Phy's. B301:525.1988: SLAC-PUB-4279: DPNL-87-15:

A Study of the $K_{S} K_{S}$ System in the Reaction $K^{-} p \rightarrow K_{S} K_{S} \wedge$ at $11 \mathrm{GeV} / \mathrm{c}$

Phys. Let t. 208B:324.1988: SLAC-PUB-4489:

Spin Parity Determination of the $\phi_{3}(1850)$ from $K^{-}$Interactions at $1 \mathrm{GeV} / \mathrm{c}$

Phys. Let . 215B:799.1988: SLAC-PUB-4657.

Observation of a New $\Omega^{*}$ (unspec) ${ }^{-}$at $2.47 \mathrm{GeV} / \mathrm{c}^{2}$ in $\mathrm{K}^{-} p$ Interactions at $11 \mathrm{GeV} / \mathrm{c}$

Phys. Lett. 215B:199.1988: SLAC-PUB-46til; DPNL-8k-24:

Evidence for a $J^{P C}=4^{++} K \bar{K}$ State at $2.2 \mathrm{GeV} / \mathrm{c}^{2}$ from $K^{-} p$ Interaction at $11 \mathrm{GeV} / \mathrm{c}$

Phys. Lett. 201B:169.1988: SLAC.PUB-4393: DPNL:-87-45:

Observation of the Selective Coupling of $K^{*}$ States to the $K^{-} \eta$ Channel

SLAC-PLB-4773: C $88 / 0 R / 15$;

Recent Result in Strangeonium Spectroscopy

SLAC-PLB-ATOS:

The Spectroscopy of Mesons Containing Strange Quarks

SLAC-PUB-4768:

The Strangeonium Spectrum Seen in LASS: Implications for Glueball Spectroscopy

SLAC-PUB-4821: DPNC'-88-50;

Recent Results from $S=-3$ Baryon Spectroscopy from the LASS Spectrometer

SLAC-PLB-5155:

Update on $K^{*}$ Studies at SLAC

Yad. Phys. 48: 1 729,1988:

Deuteron-Triton Component in Energy Spectra of Slow Particles

Phys. Lett. 158B:81,1985:

Direct Mensurement of the Lifetime of the Neutral Pion

Phys. Rev, Lett. 63:2177.1989; BNL-43212; PRINCE'TON-HEP-89-01; TRI-PP-89-70:

Search for a Light Higgs Boson in the Decay $\boldsymbol{K}^{+} \rightarrow \pi^{+}$higgs, higgs $\rightarrow \mu^{+} \mu^{-}$

Phys. Rev. Lett. 64:21.1990:

Search for the Decay $K^{+} \rightarrow \pi^{+} \nu \bar{\nu}$

Z. Phys. C26:199.1965: CERN-EP-84-80;

The $\rho(1700)$ in the Reaction $\gamma p \rightarrow \pi^{+} \pi^{-} \pi^{0} \pi^{0}$ p at Photon Energies of $20-70 \mathrm{GeV}$

Z. Phys. C29:333.1985: CERN-EP-85-89:

Evidence for a $\omega \rho^{ \pm} \pi^{ \pm}$State in Difractive Photoproduction

Z. Phys. C30:521.1986: CERN-EP-85-170;

Inclusive Photoproduction of $\phi, K^{*}(892)$ and $K_{2}^{*}(1430)$ in the Photon Energy Range 20 to $70 \mathrm{GeV}$

2. Phys. C30:531.1986; ('ERN-EP-85-171:

Study of $\eta \pi^{+} \pi^{-}$States in the $\rho(1700)$ Mass Region Photoproduced in the Reaction $\gamma p \rightarrow \eta \pi^{+} \pi^{-} p$ at Photon Energies of 20 to $70 \mathrm{GeV}$

Z. Phys. C27:233.1985: CERN-EP-84-106:

Photoproduction of $\Xi$ Mesons by Linearly Polarized Photons of Energy 20 - 40 GeV and Further Evidence for a Photoproduced High Mass $(K \bar{K})$ Enhancement

Z. Phys. C26:19.1985: CERN-EP-84-124:

Photoproduction of Multiparticle States in the Beam Fragmentation Region for Photon Energies in the Range 50 - $70 \mathrm{GeV}$

Z. Phys. C:30:541.19R5, CERN-EP-85.194

Search for a $\phi(1680)$ Vector Meson in Diffactive Photopraduction of $\mathrm{K}^{+} \mathrm{K}^{-} \pi^{+} \pi^{-}$

2. Phys. C34:303.19\$7: C.EIRN-EP-86-199:

A Peak in the $\eta \omega$ Mass Spect.rum from Diffractive Photoproduction

Z. Phys. C'34:157.1987: CERN-EP-86-185:

Diffractive Photoproduction of a $b_{1}(1235) \pi$ System

Z. Phys. C:38:535, 19k8:

Photon Difractive Dissociation to $2 \rho \pi$ and $\rho 3 \pi$ States

Phys. Lett. 220B:317.1!ky;

A Search for Photonless Annihilation of Orthopositronium

MINR-90-P-(1659:

A Search for a Light Pseudoscalar Particle in One Photon Orthopositronium Decay: $e^{+} e^{-} \rightarrow$ positronium 
Atuban : th|cont id

Atrashkevich wit

stwater 87

Aubert $\mathrm{HAC}$

A ubert 8.5

Aubert $85 \mathrm{~B}$

Aubert 850

Aubert $85 \mathrm{D}$

Aubert 8 .5

Aubert nit

Aubert $86 \mathrm{~B}$

Aubert $86^{\circ}$

Aubert $x^{7}$

Aubert 89

Audit 89

A uer 85

Auer 86

Auer 868

Auer 88

Auffet $x=$

Auffet $8.5 B$

Auge $\mathrm{sti}$

Auge 8tiB

Auge 89

Auge 89B

Augustin 8 A $^{-}$

Augustin $65 \mathrm{~B}$

Augustin $85, C^{\circ}$

Augustin $65 \mathrm{D}$

Augustin KiF.

Augustin $8 r$

Augustin $8 B$

Augustun 6 HB

Augustarl 8inc

Aulchenkes of

Aulchunks, stils

Aulcherno sfje

Aulcherses $\rightarrow$ ) axion

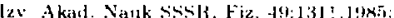

The Energy Spectra and Space Distributions of Muons in Extensive Atmospheric showers

Phys. Let . 19!13 30.1!ki:

Transverse Momentum Spectrum of Mesons Produced in High Energy

Phys. Lett 15213:+333 J1955: ('ElRN-El'-X.1-16.1:

A Measurement of the Difference between the Single Nucleon Crosg Section for J/W(1S) Muoproduction in Iron and in $H_{2}, D_{2}$ Targets

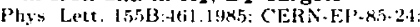

A Search for the Decay $D^{\circ} \rightarrow \mu^{+} \mu^{-}$

Z. Phy' C $30: 23$. J986: ('ERN-El'-45-72:

A Search for Higher I wist Effects in the Hadronic Distributions in Deep Inelastic Muon Proton Scattering

Phys. Let $161 \mathrm{~B}: 203.1465$ : CERN-EP-S5-1119: Exclusive $\rho^{0}$ Production in Deep Inelastic Muon Proton Scattering

Niucl. Phys. B259:169.3985: CERN-EP-85-34:

A Detailed Study of the Proton Structure Functions in Deep Inelastic Muon Proton Scattering

Phys. Let t $160 \mathrm{~B}: 417.1965$ : CERN-EP-kit-197:

A Determination of the Fragmentation Functions of u-quarks into Charged Pions

Z Phys. C:31:175.19k6: CERN-EP.K(j-1)9.

A Comparison of the Energy Distributions of Hadrons Produced in Deep Inelastic Scattering of Muons on Hydrogen and Deuterium Targets

Nucl. Phys. B272:158.1986; CERN-EP-86-05:

A Detailed Study of the Nucleon Structure Functions in Deep Inelastic Muon Scattering in Iron

Fhys. Lett. 167B:127.1986: CERN-EP-85-kr:

$D^{\circ}$ Production in Deep Inelastic Muon Scattering on Hydrogen and Deuterium

Nucl. Phys. B293:T10.1987: CERN-EP-87-(j6:

Measurement of the Nucleon Structure Functions $F_{2}$ in Deep Inelastic Muon Scatteriag from Deuterium and Comparison with Those from Hydrogen and Iron

Phys. Let 1. 21813:248.1989: CERN-EP-88-33: Evidence for Anomalous Prompt Photons in Deep Inelastic Muon Scattering at $200 \mathrm{GeV}$

Phys. Let 1. 227 B:331,1989:

First Measurement of the Reaction ${ }^{3} \mathrm{He} \rightarrow p \mathrm{p}$

Phis. Hev D32:1609.1985:

Measurement of Triple and Double Spin Parameters in Elastic Proton Proton Scattering at 6 GeV/c

Phys. Rey. D3 $1: 1.1980$ :

Measurement of Spin Spin Correlation Parameters in the p p System at $11.75 \mathrm{GeV} / \mathrm{c}$

Phys. Fev. Di3-t:2581.1986: ANL-HEP-PR-8ti-3n:

Observation of a New Structure in the Difference between the p p Total Cross Sections for Antiparallel and Parallel Longitudinal Spin States

Phys. Rev. Lett. 62:2649.1989: ANL-HEP-PR-8x-9ti:

Observation of Structures in the Mass Range of 2700 to $2900 \mathrm{MeV}$ in t2e Difference between the $p$ Total Cross Sections for Pure Helicity States

Phys. Rev. Lett 55.1362.1985:

Evidence for Nonnucleonic Effects in the Threshold Electrodisintegration of the Deuteron at High Moment um Transfer

Phys. Rev. Lett. 5it:649.1985:

Magnetic Formfactor of the Deuteron

Phys. Lett. If6B:163.1986: CERN-EP-86-08:

High Energy Photoproduction of Large Transverse Momentum $\pi^{\circ}$ Meson: A Quantitative Test of QCD

Phy. Leth. 182B:409 1986: CERN-EP-86-176: LAL-N6-32:

Topological Isolation of the Deep Inelastic QED Compton Scattering

LA L- $89-20$ :

Present Status of the NA31 Experiment

LAL-Bit-2ti:

Study of Some $K^{0}$ Rare Decay Processes in NA31

L.A I- $-4+2-22$ :

Results on $J / w(1 S)$ Radiative Decays from the DM2 Experiment at DCI

LAL-Xij-17:

DM2 Results on $J / \psi(1 S)$ Decays

LAL-XIT-3T:

Selected Results on $J / \psi(1 S)$ Hadronic Decays from DM2 and MARK-III

I.A1-85-2?:

DM2 Results on Hadronic and Radiative $J / w\langle 1 S\}$ Decays

1.AI,- $4.5-3,3$ :

Quark Spect roscopy

2. Phys. ('36:369.1987. LAL-K7-10.

Radiative Decay of $J / \psi(1 S)$ into,$\pi^{+} \pi^{-}$

Phys. Hev. Let fio (1:22,34.198k:

Measurement of Radiative $J / v(1 S)$ Decays in $K \bar{K}$ States

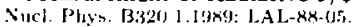

Study of the $J / w(1 S)$ Decay into Five Pions

1.AI. $-8 x-31$

Spectroscopy Related to Two Pholon Physics

NOS:0-Xi-17:3.

The $e^{+} e^{*} \rightarrow \gamma \gamma \gamma, e^{+} e^{-}>\gamma, e^{+} e^{+} e^{-}$Processes in VEPP-2M

Novio-xi-10i):

Study of Decuys of $\rho$ and $د$-Mesons with the Neutral Detector at the VEPP-2M Collider

volio-kfi.lifi:

Experiments with the Neutral Detector at the VEPP-2M in the Energy Range 1.0 - 1.4 GeV

flay Lett lafis 132.tikt.

The Width of the Meson 
Aulchenko 87B

A ulchenko $87 \mathrm{C}$

A vakyan 85

A vakyan $85 \mathrm{H}$

A vakyan $85 \mathrm{C}$

Avakyan 85D

A wakyan $85 \mathrm{E}$

Avakyan $85 \mathrm{~F}$

Avakyan 86

Avakyan $87 \mathrm{~B}$

Avakyan $88 B$

A rakjan $88 \mathrm{C}$

Avakyan 89

Arakyan $89 \mathrm{~B}$

Avakyar: 890

A vakyan 90

Avdejchikov 85

Avdejchikov 86

Avdejchikov 8 ?

Avdejchikov $87 \mathrm{~B}$

Avdejchikov $87 \mathrm{C}^{\circ}$

Avdejchikov $87 \mathrm{E}$

Avdejchikov $87 \mathrm{~F}$

Avdejchikov $87 \mathrm{G}$

Avdejchikor $47 \mathrm{H}$

Avdejchikov 87I

Avdejchikov 88

A venier 45

Averchikov 87

Avericher 89

Averill 89

Avery 8:5

Avery 87

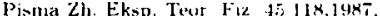

The Search for Possible Exotic State $C(1480)$ at VEPP-2M

Novo-8i-90.

Search for Rare Processes with the Neutral Detector at the VEPP-2M Collider

Vopr. At Nauki i Techn. 4-25:-7T,19kĩ.

Search for the Large Mass Particles in Cosmic Rays

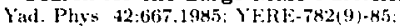

Measurement of the Polarization of Cumulative Protons in \& nucleus $\rightarrow p \mathbf{X}$ Reaction

lav. Akad. Nauk SSSR. Fiz 4!: 1254.1945:

The Single Protons Flux Intensity at the Height of Mouritnins and the Cross Sections of Protons - Air Nuclei Inelastic Interactions

Nucl Phys B259:156.1965:

Determination of Inelasticity Partial Coefficients of Pions and Protons in Iron in the Energy Range 0.5 5.0 TeV

Nucl. Phys. B259:163.1985.

Determination of the Crass Section of the Pion and Nucleon Interaction with Iron Nuclei in the Energy Range 0.5 - 5.0 TeV

Yad. Phys. 41:163.1985:

Composition of the Flux of Cosmic Ray Hadrous at Mountain Altitude at Energies E>0.5 TeV

Yad. Phys. 41:1224.1986: Suv. J. Nucl. Phys. 4t:795.1!126; Study of Characteristics of Albedo Particles Generated in Interactions of Cosmic Hadrons

Yad. Phys. Ab:14ti.1987:

Measurement of Proton Polarization in $\pi^{\circ}$ Production at $\theta=70$ Degrees in Linearly Polarized Photon Beam

Pisma Zh. Eksp. Teor. Fiz. 4\$:235.1948:

About Possible Excess of the Hadronic Groups in the Direction on Cygnus X-3, observed by the PION Detector

Jad. Phys 18.1716.1948: Sun J Nucl. Phys tr:103011988:

Measurement of Polarization of Protons in the Reaction,$p \rightarrow p \pi^{0}$ at $\theta\left(\pi^{0}\right)=80$ Degrees in a Photon Linearly Polarized Beam

Yad. Phys 49:468.1949:

Polarization of Cumulative Protons in $q$ nucleus $\rightarrow p$ X Reaction

Yad. Phys. 50:13:4.1989:

Diferential Energy Spectrum and Angular Distribution of Hadrons at Mountain Altitude at Energies $E$ $>10^{3} \mathrm{GeV}$

Yad. Phis. 50:1348.1989:

The Study of the Dependence of Hadron Inelastic Interaction Cross Section on Energy and Atomic Number of Target in the Range 0.5 - $10 \mathrm{TeV}$

Yad. Phys. 51:1585.1990:

Polarization of Cumulative Protons in r nucleus $\rightarrow p$ X Reaction

Yad. Phys. 44:440.1986: JINR-P1-85-640:

Search for Nuclei with A nomalons and Fractionally Charged Fragments of ${ }^{16} \mathrm{O}$ at $4.5 \mathrm{GeV} / c$ per Nucleon

JINR-P1-86-664:

Emission of ${ }^{8} \mathrm{He}$ and ${ }^{8} \mathrm{Li},{ }^{7} \mathrm{Li},{ }^{8} \mathrm{Li}$ Fragments in ${ }^{4} \mathrm{He}$ Collisions with ${ }^{10 \mathrm{H}} \mathrm{Ag}$ and ${ }^{187} \mathrm{Au}$ at $3.33 \mathrm{GeV} / \mathrm{Nucleon}$ JINR-P1-87-509:

Formation of 5 - 12 Charge Fragments in the $p$ Au Reaction at 2.6 - 7.5 GeV Proton Energy

Yad. Phys. 48: $1736.1988:$ JINR-P1-87-872:

Experimental Study of Intermediate Mass Fragment Formation Mechanism in $p$ Au and ${ }^{4}$ He Au Interactions

JINR-P1-87-42.

Inclusive Differential Cross Sections for the Formation of the Middle Mass Fragments $(Z=5-12)$ Produced by the Protons (2.6 - 7.5 GeV) Interacting with Au Nuclei

Pisma Zl. Eksp. Teor. Fiz. 46:141.1487:

Production of Intermediate Mass Fragments in $p$ Au Reaction at 2.6 - 7.5 GeV Protons Energy Interval

IINR.P1-87-fiots:

Inclusive Differential Cross Sections for the Formation of the Fragments with Charges 5 - 12 Produced by the ${ }^{a} \mathrm{He}$ Nuclei at $1.3-13.5 \mathrm{GeV}$ Energy with Au Nuclei

INR-P1-87-7109:

Inclusive Differential Cross Sections for the Formation of the Intermediate Mass Fragments $(Z=5-12)$ Produced by the He Nuclei (13.5 GeV) Interacting with Au Nuclei

Pisma Zh. Eksp. Teor. Fiz, 47:131.1988: Jetp L.ett. 47:157.1988: JINR-PI-87-830

Observation of Minimum in Energy Dependence of Tau Parameter of Charge Fragment Yield in Interaction of ${ }^{4}$ He Relativistic Nuclei with Gold Nuclei

JJNA-PI-RT-369:

Inclusive Differentia) Cross Sections for the Formation of the Intermediate Mass Fragments $(Z=5-10)$ Produced by the Pratons with Momentum of $2.55 \mathrm{GeV} / \mathrm{c}$ Interacting with Nuclei

Yarl. Phys. 50:409.1989; JINR-P1-88-796:

Analyzing Power Measuremait of deuteron (polarized) p Elastic Scattering at $2-12 \mathrm{GeV} / c$ and in deuteron (polarized) $\mathrm{C} \rightarrow p \mathrm{X}$ Reaction at $2.38 \mathrm{GeV} / \mathrm{c}$

Nucl. PJjis. A 436:8:3,1945:

Parity Nonconservation in the Radiative Capture of Polarized Neutrons by ${ }^{95} \mathrm{Cl}$

Kr. Soot. JINR 25:4,1!967.

Cumulative Antiproton Observation

Kr. Soots JINR 37:5.1989:

Experimental Data on the Investigation of Two-Particle Pion-Proton, Proton-Proton and Deuteron-Proton Correlations in the Cumulative Particle Production in Proton, Detiteron and ${ }^{4}$ He-Nuclei Interactions with Nuclei

Phys. Kev. Di39: 123.14k41.

Measurement of the $D^{0}, D^{+}$, and $D_{S}^{+}$Meson Lifetime

Phys Rev. D32:22:1.1965:

Bose- Eingtein Correlations in $e^{+} \epsilon^{-}$Annihilations in the $\Upsilon(1 S)$ Region

Phy, Lett IA3] 429.1987.

Limits on Rare Exclusive Decays of $B$ Mesons 
Avery 88

Avery 89

Avery 89B

Avery 90

Avignone 65

Avignone 86

Avignune $86 \mathrm{~B}$

Avignone 87

Avignone $87 \mathrm{~B}$

Avignone 88

Avrannenko 87

Avramenko 88

Avvakumov 84

Avvakumov 86

Avvakumov $86 \mathrm{~B}$

Azhgirej 85

Azhgirej 86

Azhgirej 87

Azhgirej 88B

Azimov $84 \mathrm{~B}$

Azimov $84 \mathrm{C}$

Azimov 85

Azinov $45 \mathrm{~B}$

Aziniov $85 \mathrm{D}$

Azimov $85 \mathrm{E}$

Azimov $85 \mathrm{~F}$

Azimov $85 \mathrm{C}$

Azimov 86

Aziz 85

Aziz 8.5B

Aziz $850^{\circ}$

Aziz 68

Azuelos 86

Baatar 85

Baatar 87

Bantar 8713

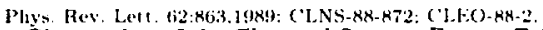

Observation of the Charmed Strange Baryon $\Xi_{r}(\mathbf{2 4 6 0})^{0}$

L.BL-20593:

Bose-Einstein Correlations of Pions in $e^{+} c^{-}$Annihilation at 20 GeV Center-of-Mass Energy

Phys. Lett. 223B:470.1989; C'LNS-89-886; CLEO-8!-2:

A Search for Exclusive Penguin Decays of $B$ Mesons

Phys. Rev. D41:774,1990: CLNS-89-939: (L.EO-89-11;

$P$-wave Charmed Mesons in $e^{+} e^{-}$Annihilation

Phrs. Rev. Lett. 54:2309.1985:

Ultralow Background Study of Neutrinoless Double $\beta$ Decay of ${ }^{70} \mathrm{Gr}$. New Limit on the Majorana Mass of $\nu_{e}$

Phys. Rey. Di3-4:97.198i;

New Experimental Limit on the Stability of the Electron

Pliys. Rev. C34:066.1986: Search for the Double- $\beta$ Decay of ${ }^{76} \mathrm{Ge}$

Phys. Rev. D35:1713,19K7:

Analysis and Interpretation of a Large Body of ${ }^{76}$ Ge Zero Neutrino Double $\beta$ Decay Data

Phys. Rev. D35:2752.1987:

Laboratory Limits on Solar Axions at Ultralow-Background Germanium Spectrometer

Phys. Res. D37:618,1988:

Search for Axions from $1115 \mathrm{KeV}$ Transition of ${ }^{\mathrm{as}} \mathrm{Cu}$

JINR-El-8T-337:

The Observation of ${ }^{4} \mathrm{H}_{S}$ Relativistic Hypernuclei Produced in ${ }^{4} \mathrm{He}$ Colligions with Light Nuclei at 18 GeV/c

Pisma Zh. Eksp. Teor. Fiz. 48:-14,1988- JINR-Dl-88-6i!l:

Production and Decay of Relativistic Hydrogen Hypernuclei

Nucl. Phys. B255:253.1985: JFVE-84-187;

Polarization in the $\pi^{-} p \rightarrow \pi^{0} n$ Reaction at $40 \mathrm{GeV} / \mathrm{c}$

Yad. Phys. 42:1152,1985:

Meagurement of Asymmetry in $\pi^{-} p \rightarrow K^{0} \wedge$ Reaction at $40 \mathrm{GeV} / \mathrm{c}$

Yad. Phys. 42:1146,1985: Suv. J. Nucl. Phys. 42:725.1985: Observation of Asymmetry in $\pi^{-} p$ (polarized) $\rightarrow n$ Reaction at $40 \mathrm{GeV} / \mathrm{c}$

JINR-D1-85-618;

Scattering of Relativistic Protons and Deuterong from Atomic Nuclei

JINR-PI-86-728:

Investigation of a deuteron $p \rightarrow p X$ Reactions at $\theta \mathrm{GeV} / c$ in the Region of Large Transversal Momenta of Protons

Yad. Phys. 46:1134.1987:

Study of deuteron nucleus $\rightarrow p$ X Reactions at $9 \mathrm{GeV} / \mathrm{c}$ at Proton Large Transverse Momenta

Yad. Phys. 48:1758,1988: JINR-P1-88-23;

Spectra of Deuterons Emitted with Large Transversal Momenta in Collisions of $\theta$ GeV/c Deuterons with Hydrogen, Deuterium and Carbon Nuclei

Yad. Phys. 12:913.1985; Sov. J. Nucl. Phys. 42:579.1985: PTIU-84-27-FVE: Search of Dibaryon Resonances in Hadron Hadron Interaction

Pisma Zh. Eksp. Teor. Fiz. 40:316,1984; Jetp Lett. 40:1109,1988:

The Possible Observation of Narrow Diproton Resonances in Hadrons-Nuclei Interactione

Yad. Phys. 11:149.1985:

Angular Correlations between Charged Particles in Hadron-Nucleus Interactions in the Energy Range 20 - $400 \mathrm{GeV}$

[zy. Akad. Nauk SSSR. Fiz. 49:1275,1985:

Study of Space Characteristics of $\gamma$ Families, Produced at the Super High Energy E=10 ${ }^{16}$ eV

Czech. J. Phys. B35:832,1985: PTIU-20-84-FVE:

Multiplicity of Charged Particles in Interactions of Neutrons with Protons, Carbon and Neon Nuclei in the Momentum Range $1-200 \mathrm{GeV} / \mathrm{c}$

PTIL-29-85-FVE:

Production of $\gamma$ Quanta at Inelastic $p{ }^{20} \mathrm{Ne}$ and $p n$ Interactions at $300 \mathrm{GeV}$

Czech. J. Phys. B35:920.1985;

A Study of Fast Neutron Production in $p$ Ne and $p p$ Interactions at $300 \mathrm{GeV} / c$

2. Phys. A322:677.198.5; LLIP-8513:

Evidence for Azimuthai Correlations in Inelastic Interactions of ${ }^{66}$ Fe Nuclei in Emulaion at 2.5 GeV/c A

PTIL-86-36-FVE:

Searcis for Resonances with $B=3$ and $B=4$

Z. Phys. (27:325.19R5: TIFR-BC:-8:-7:

Charged Charm Production in Proton Emulsion Interactions at $400 \mathrm{GeV} / \mathrm{c}$

2. Phy' C29:339.1985: Pisma Zh. Eksp. Teur. Fiz. 44:407.1986: [FVE-86-174: CERN-PRE-85-059: TIFR-85-4; Study of $A$ Production in Target Fragmentation Region from $p$ Interactions at $360 \mathrm{GeV} / \mathrm{c}$ in the Triple Regge Framework

Yad. Phy's. 46:136.1987: Z. Phys. C30:381.1986: IFVE-86-158: (ERN-EP-85-120.

Inclusive $K^{*}(\mathbf{8 9 2})$ and $\Sigma\left(1385 P_{13}\right)$ Production in $360 \mathrm{GeV} / c p p$ Interactions Using the European Hybrid Spectrometer

Yad. Phys. 47:1035.1986: Z. Phys. $934: 429.1987$ : IEV'E- $77-(i 2$; ('ERN-EP-86-166:

Neutral Strange Particles Correlation in $360 \mathrm{GeV} / \mathrm{c} p$ Interactions

Phys. Rev. Lett. 56:2241,1946: TIRI-PP-k6;-s

Constraints on Massive Neutrinos in $\pi^{+} \rightarrow e^{+} \nu_{r}$ Decay

JINR-P1-B5-6998:

Mean Characteristics of Secondary Particles in Cumulative $\pi^{-} \mathrm{C}$ Interactions at 40 GeV/c

Yad. Phy's. 46:146.1.19k7: J1NR-P1-46-721:

Characteristics of Secondaries Produced in $\pi^{-} C$ Interactions at $40 \mathrm{GeV} / \mathrm{C}$ as a Function of the Target Mass

J1NI-P1-87-8IJ7:

Analysis of Hadron-Nuclear Interactions at $4-40 \mathrm{GeV} / r$ as a Function of Target Mass 
Baatar 88

Baatar 88B

Baat ar 89

Baatar 89B

Baatar 90

Baatar $90 B$

Baba 86

Babrev 90

Babecki 85

Babintsev 85

Babintsev 86

Babintsev 8CB

Babintsev 88

Bacala 88

Bacala $88 B$

Bachnaal 86

Backenstoss 85

Backenstoss $85 \mathrm{~B}$

Baden 86

Badier 85

Badier 85B

Badier $85 \mathrm{C}$

Badier 85D

Badier $85 \mathrm{E}$

Badier 85F

Badier 86

Bagdasaryan 8.5

Bagdasaryan 85B

Bagdasaryan 88

Bagdasaryan 90

Bagheri 87

Bagheri 87B

Baglin 85

Baglin 86

Yad. Phys. 48:764.1988:

Analysis of Cumulative Hadron Nuclear Interactions at $4-40 \mathrm{GeV} / c$ as a Function of Target Mass JINR.P1-88-469:

The Dependence of Characteristics of $\pi^{ \pm}$Mesons Produced in $\pi^{-} \mathrm{C}$ Interactions at $40 \mathrm{GeV} / c$ on Total Energy in the c.m.s. and QCD of Semihard Process

JINR-P1-89-46:

Dependence of Characteristics of Hadron-Carbon Interactions at $4-40 \mathrm{GeV} / \mathrm{c}$ with Cumulative $\pi^{-}$Meson and Proton Emission on Target Mass and their Connection with Generation of Particles with Large Transverse Momentum

INR-P1-89-424:

Inclusive Cross Section of Cumulative $\pi^{-}$Meson Production in $\pi^{-}$C Interactions of $40 \mathrm{GeV} / \mathrm{c}$ as Function of Kinetic and Trangverse Energies

JINR-P1-90-26;

Inclusive $\pi^{-}$Meson Spectra with Cumulative Number $N(K)>0.35$ Produced in deuteron $C$, He $C$ and $C$ C - Interactions at $4.2 \mathrm{GeV}$ per Nucleon

JINR-P1-90-202:

Cumulative Production of $\pi^{-}$Mesons in $\pi^{-} \mathrm{C}$ Interaction at $40 \mathrm{GeV} / \mathrm{c}$

Phys. Lett. 180B:406.1986:

Search for a Light Neutral Particle in the Decay of the $3.68 \mathrm{MeV}$ State in ${ }^{23} \mathrm{C}$

Yad. Phys. 51:524,1990:

Aximuthal Correlations between Secondary Charged Particles from Inelastic Interactions of Relativistics Nuclei

Acta Phys. Polon. B16:323.1985:

Pion-Nucleus Interactions in Emulsion at $300 \mathrm{GeV} / \mathrm{c}$

Yad. Phys, 42:1157,1985: Sov, J. Nucl. Phys, 42:733,1985: IFVE-85-64:

Inclusive Production of $\Delta\left(1232 P_{33}\right)^{++}$and $\Delta\left(1232 P_{33}\right)^{\circ}$ Isobars in $\bar{p} p$ Interactions at $32 \mathrm{GeV} / c$

Yad. Phys. 44:637.1986: Sov. J. Nucl. Phys. 44:412.1986: Inclusive Production of $\Sigma\left(1385 P_{13}\right)$ Hyperons in $\bar{p} p$ Interactions at $32 \mathrm{GeV} / c$

Yad. Phys. 45:102.1986:

Investigation of Jet Structures in $K^{-} p$ and $\bar{p} p$ Interactions at Incident Momentum of $32 \mathrm{GeV} / \mathrm{c}$

Yad. Phys. 50:1013.1989: IFVE-89-4:

Study of Strange Boson Resonance Production in $\bar{p} p$ Interactions at $32 \mathrm{Ge} r / c$

KEK-88-44: AMY 88-07:

Measurement of Cross Sections and Charge $A: y$ mmetry for $e^{+} e^{-} \rightarrow \tau^{+} \tau^{-}$and $e^{+} e^{-} \rightarrow \mu^{+} \mu^{-}$for $\sqrt{s}=52$, 55 and $58 \mathrm{GeV}$

Phys. Lett. 218B:112.1989; AMY-88-15; KEK-88-81: Measurement of Cross Sections and Charge Asymmetry for $e^{+} e^{-} \rightarrow \tau^{+} \tau^{-}$and $e^{+} e^{-} \rightarrow \mu^{+} \mu^{-}$for $\sqrt{s}$
from 52 to $57 \mathrm{GeV}$

Nucl. Phys. B263:458.1986;

Large Angle $\Lambda$ Production in Exclusive Reactions between 3 and $12 \mathrm{GeV} / c$

Nucl. Phys. A448:567.1986; SIN-PR-85-01:

Measurement of the Ratio $\left(\pi^{-3} \mathrm{He} \rightarrow \pi^{0}{ }^{9} \mathrm{H}\right) /\left(\pi^{-{ }^{3}} \mathrm{He} \rightarrow\right.$ deuteron $\left.n\right)$ and of the Respective Partial $K$ X-ray Yields

Phys. Rev. Lett. 55:2782.1985:

Evidence for a Direct Three Nucleon Pion Absorption Process

LBL-22046:

$\Lambda$ Production in Electron - Positron Annihilation at $29 \mathrm{GeV}$

CERN-EP-85-107:

Search for Longlived and Penetrating New Particles in $300 \mathrm{GeV} / \mathrm{c} \boldsymbol{\pi}^{-}$Interactions

2. Phys. C26:489,1985; CERN-EP-84-16;

Drell-Yan Events from $400 \mathrm{GeV} / \mathrm{c}$ Protons Determination of the $K$-factor in a Large Kinematical Domain

Phys. Let1. 164B:184,1985; CERN-EP-85-145:

Direct Photon Pair Production from Pions and Protons at $200 \mathrm{GeV} / \mathrm{c}$

Phys. Lett. 158B:85.1985: CERN-EP-85-59;

$2 J / \psi(1 S)$ Production and Limits on Beauty Meson Production from $400 \mathrm{GeV} / c$ Protons

Z. Phys. C.30:45,1986: CERN-EP-85-112:

Inclusive High $p_{T} \pi^{0}$ Production from $\pi^{ \pm}$and Protons at $200 \mathrm{GeV} / c$

2. Phys. C31:341.1986: CERN-EP-85-200;

Direct Photon Production from Pions and Protons at $200 \mathrm{GeV} / \mathrm{c}$

2. Phys. C31:21.1986: CERN-EP-86-22:

Mass and Lifetime Limits on New Longlived Particles in $300 \mathrm{GeV} / \mathrm{c}^{-}$Interactions

YERE-822(49)-85:

Interactions of Electrons with ${ }^{\circ} \mathrm{Li},{ }^{8} \mathrm{Be},{ }^{2} \mathrm{C},{ }^{28} \mathrm{Si}$ Nuclei in the Region of Quasi-Elastic Peak and $\Delta\left(1232 P_{3 s}\right)$ Resonance

YERE-8.38(65)-85:

Quasielastic Electron Scattering $\left(e^{-}, e^{-} p\right)$ on ${ }^{9} \mathrm{Be}$ and ${ }^{28} \mathrm{Si}$ Nuclei

YERE-1077(40)-88:

Measurement of the Spectra of $\left(e^{-}, e^{-}\right)$Scattering from ${ }^{9} \mathrm{Be}$ and ${ }^{12} \mathrm{C}$ Nuclei in the lnelastic Region at $Q^{2}<0.4 \mathrm{GeV} / c$

YERE-1216(2)-90:

Phenomenological Analysis of Experimental Data on Photoproduction of $\pi^{0}$ Mesons

TR1-PP-87-90:

The Reaction $\pi^{-} p \rightarrow \pi^{0} n$ below the $\Delta\left(1232 P_{33}\right)$ Resonance

TRI-PP-87-89:

The Reaction $\pi^{-} p \rightarrow q n$ below the $\Delta\left(1232 P_{39}\right)^{\circ}$ Resonance

Phys. Lett. I63B:400.1985; CEKN-EP-85-146:

Upper Limits of the Proton Magnetic Formfactor in the Timelike Region from $\bar{p} p \rightarrow e^{+} e^{-}$at the CERN ISR

Phys. Lets. 172B:455.1986: CERN-EP-86-53:

Formation of the $\chi_{c 1}(1 P)$ and $\chi_{c 2}(1 P)$ Charmonium Resonances in Artiproton-Proton Annihilation and Measurement of their Masses and Total Width

See the legend on page 19 . 
Baglin $86 \mathrm{~B}$

Baglin 87

Baglin 87B

Baglin 87C

Baglin 89

Baglin 89B

Baglin $89 \mathrm{C}$

Bagnaja 84E

Bai 90

Bailey 85

Bailey $85 \mathrm{~B}$

Bailey $35 \mathrm{C}$

Bailly 86

Bailly $86 \mathrm{~B}$

Bailly 86D

Bailly 87B

Bailly $87 \mathrm{C}$

Bailly 87D

Bailly $87 \mathrm{~F}$

Bailly 87G

Bailly $87 \mathrm{H}$

Bailly 88

Bailly 88B

Bailly $88 \mathrm{C}$

Bailly $88 \mathrm{E}$

Bajramov 86

Bajranov 89

Bakatanov 85

Bakatanov 88

Baker 8.5

Baker 85B

Baker $85 \mathrm{C}$

Baker 86

Baker 87

Baker 89

Balandin 85

Balats 87
Phys, Lett. 171B. 135.1986: ('ERN-EP'-8t-17:

Search for the $1 p$ Charmonium State in $\bar{p} p$ Annihilations at the CERN Intersecting Storage Rings

Phys. Lett. 187B:191.198T ('ERN-EP-8T-02:

Direct Observation and Partial Width Measurement of $\gamma \gamma$ Decay of Charmonium States

Phys. Lett. 195B:85.1987: ('ERN-EP.87-94;

Angular Distribution in the Reactions $\left.\bar{p} p \rightarrow \chi_{c}(1) P\right),\left(\chi_{12}(1 P)\right) \rightarrow \gamma J / \psi(1 S) \rightarrow \gamma e^{+} e^{-}$

Nucl. Phys. B280:592.1987 CERN-EP-87-30;

$J / \psi(25)$ Resonance Formation Masses and Total Width

Phys. Leti. 220B:471,1989:

The Production of $J / 2 \cdot(1 S)$ in $200 \mathrm{GeV} /$ Nucleon Oxygen-Uranium Interactions

Phys. Lett. 225B.296.1989: ('ERN-EP-89-60:

Precision Measurements of $\bar{p} p$ Elastic Scattering Cross Section at 90 Degrees in Incident Momentum Range between $3.5 \mathrm{GeV} / c$ and $5.7 \mathrm{GeV} / \mathrm{c}$

Phys. Lett. 231 B:5.57.1989:

Measurement of the $\phi \phi$ Cross Section in $\bar{p} p$ Annihilation at $E_{\mathrm{ern}}=3 \mathrm{GeV}$

Phys. Let L. 154B:338,1985: CEFN-EP-84-166:

Study of Electron Pairs below the $Z^{0}$ Mass Produced in $\bar{p} p$ Collisions at $540 \mathrm{GeV}$

SLAC-PUB-5191:

Measurement of the A bsolute Inclusive Leptonic Decay Branching Fraction of the $D_{S}^{+}$Meson

Z. Phys. C28:357,1985:

Measurement of the Lifetime of Charged and Neutral $D$ Mesons with High Resolution Silicon Strip Detectors

Z. Phys. C29:1.1985;

Leading Proton and Antiproton Distributions in Proton Nucleus and Antiproton Nucleus Interactions

Z. Phys. C30:51.1986: MPI-PAE-EXP-EL-1.54:

iveasurement of $D \mathrm{Mc}$ on Production in $200 \mathrm{GeV} \pi^{-}$Be Interactions

Jetp Lett. 44:530,1986, Z. 1 . tys. C31:367,1986: Pisma Zlı. Eksp. Teor. Fiz. 44:411.1986; IFVE-86-167: CERN-EP-86-32: Test of a Quark Diquark Fragmentation Mechanism in Proton Proton Interactions at $380 \mathrm{GeV} / \mathrm{c}$

Z. Phys. C35:295.1087: CE' N-EP-87-42:

Analysis of Transvers. Momentum Distributions for $p$ p Interactions at $360 \mathrm{GeV} / \mathrm{c}$ in the Framework of a Quark Diquark Fragn ntation Model

2. Phys. C35:309.1987: CE. N-EP-87-59;

Inclusive Pion Product ion in $360 \mathrm{GeV} / c$ p p Interactions

Phys. Let t. 195B:609,1987: 'ERN-EP-87-99:

Strangeness and Diquerk Suppression Factors in $360 \mathrm{GeV} / \mathrm{c} p$ p Interactions

Z. Phys. C36:545.1987: Yal Phys. 49:1014,1989: Sov. J. Nucl. Phys. 49:631.1989: IFVE-88-64: CERN-EP-87-108: Inclusive Production i $\rho^{\circ}$ and $f_{2}(1270)$ Mesons in $\pi^{-} p$ Interactions at $360 \mathrm{GeV} / \mathrm{c}$

Z. Phys. C35:301.1987: CEH N-EP-87-53:

Multiparticle Production in Proton Nucleus Collisions at $360 \mathrm{GeV} / \mathrm{c}$ Uring the European Hybrid Spectrometer

Z. Phys. C40:13.1988; CERX-EP-87-113-Rev:

Two Particle Correlations in $360 \mathrm{GeV} / \mathrm{c}$ p p Interactions

Eur. Lett. 4:1261.1987; CE! Ү-EP-87-133:

Longitudinal Distribus in of $\pi^{ \pm}, K^{ \pm}, p$ and $\bar{p}$ Produced in $360 \mathrm{Gev} / \mathrm{c}^{-} p$ Interactions

Yad. Phys. 48:1333.1988; $S$, J. Nucl. Plysis. 48:848.1988; IFVE-87-188:

Longitudinal Distribut :on of Charged Particles Produced in $360 \mathrm{GeV} / c \pi^{-}$p Interactions on EHS

Z. Phys. C40:215,1988: CEF N-EP-88-10:

Rapidity Dependence if Multiplicity Distributions in Proton-Nucleus Collisions at $360 \mathrm{GeV} / \mathrm{c}$

Phys. Lett. 206B:371.1988:

Event Shape in Mome tum Space of Proton-Proton Interaction at $360 \mathrm{GeV} / \mathrm{c}$ and Comparison to $e^{+} e^{-}$ Data

2. Phys. C37:7,1988; IFVE-i-7.80: CERN-EP-87-118:

The Impact Parameter A nalysis of Proton-Proton Elastic and Inelast ic Interactions at $380 \mathrm{GeV} / \mathrm{c}$

Z. Plys. C43:341,1989: CER V-EP-88-177;

Bose-Einstein Correlation for Pions Produced in p p Collisions at $360 \mathrm{GeV} / \mathrm{c}$

Yad. Phys. 44:1219,1986: Sor J. Nucl. Phys. 44:792.1986:

On Scaling on the Average for Charged Pions in Pion-Carbon Interactions at $5 \mathrm{GeV} / \mathrm{c}$

Yad. Phys. 49:1337.1989:

Study of Partial Inelas icity Coefficients of Charged Secondary Pions in Hadron-Nucleus Interactions at High Energies

Pisna Zh. Eksp. Teor. Fiz. +307.1985:

Cascades in Muon Underground Groups. New Methods of Determination of Primary Cosmic Rays Composition

Pisma Zh. Eksp. Teor. Fiz. Hh:121.1988:

Cross Section of the Photoproduction at the Photon Energy from 0.9 to $10 \mathrm{TeV}$

BNL-36658;

Limits on Likesign Dil, 'tons Production in $\nu_{\mu}$ Interactions

Phys. Rev. D32:531.j98.5:

Opposite-Sign Dilepton Production in $\nu_{\mu}$ Interaction

BNL-36996: CONF-8.506194-5:

Limits on Like-Sign Dilepton Production in $\nu_{\mu}$ Interactions

Phys. Rev. Di34:1251.1986:

Strange Particle Produ tion in Neutrino Neon Charged Current Interactions

Phys. Rev. Lett. 51:28:32.19.

Search for Short-lived Neutral Particles Emitted in $K^{+}$Decay

Phys. Rev. D40:2753.1989: Plys. Rev. D41:1715.1990:

Measurement of Muon- Veutrino-Electron Elastic Scattering in the FERMILAB 15 Foot Bubble Chamber JINR-P1-85-388:

Helicity Polar Angular Distributions in the Reaction $\pi^{-} p-\pi^{+} \pi^{-n}$ at $338 \mathrm{MeV}$ Kinetic Energy of Primary $\pi^{-}$Mesons

Yard. Phys. 49:1332.1989: S Search for $K_{S}-2 \gamma$ D. 
Baldin 85

Baldin 85B

Baldin 86

Baldin 86B

Baldin 87

Baldin 87B

Baldin 88

Baldin 88B

Baldin $88 \mathrm{C}$

Balea 85

Balea 86

Balest ra 84

Balestra 85

Balestra 85B

Balestra 86

Balestra 86B

Balestra $8 T$

Balestra $87 \mathrm{~B}$

Balestra $87 \mathrm{C}$

Balestra 88

Balestra 89

Balestra $89 \mathrm{~B}$

Balgansuren 88

Balke 88

Ball 87

Ball 88

Ballagh 86

Ballagh 89

Baller 88

Baloshin 84

Baloshin 87

Baltay 85

Baltay 86

Baltrusaitis 85

Baltrusaitis 85B

Baltrusaitis $85 \mathrm{C}$

Baltrusaitis 85D

Baltrusaitis $85 \mathrm{E}$

Baltrusajtis $8.5 \mathrm{~F}$
JINR-EI-85-675; JINR-P1-85-820;

Four-dimensional Jets as Universal Characteristics of Multiple Particle Production

2. Phys. C33:363,1987; JINR-E1-85-415:

Universality of Hadron Jets in Soft and Hard Particle Interactions at High Energies

Kr. Soob. JINR 16:24.1986;

Four-Dimensional Jets as Universal Characteristics of Multiple Particle Production

Yad. Phys. 44:1209.1986: Sov. J. Nucl. Phys. 44:785.1986;

Four-Dimensional Jets of Hadrons: Universal Characteristics of Multiple Production of Particles

JINR-El-87-142:

Universal Four-dimensional Hadron Jets and the Observability of Colour Charges

Kr. SOob. JINR 21:17,1987;

Hadron Jets in Deep Inelastic $\bar{\nu} n$ Interactions and Universality of the Jet Properties in Relative Four

Velocity Space

Pisma Zh. Eksp. Teor. Fiz. 48:127,1988:

Measurement of the Antiproton Yield in the Collisions of $\mathrm{C}$ with $\mathrm{Cu}$ at the Energy $3.65 \mathrm{GeV} / \mathrm{N}$

Yad. Phys. 48:995,1988:

Clusierization in Four-dimensional Relative Velocity Space and Invariant Distribution of Hadron Jets

Yad. Phys. 49:1304.1989; JINR-P1-88-331:

Automodel Properties of Baryon Clusters in Interactions of $p$, deuteron, He, $\mathbf{C}$ and $\pi^{-}$Particles with

Carbon Nuclei within $4-40 \mathrm{GeV} / \mathrm{c}$ per Nucleon Momentum interval

JINR-P1-85-132:

Methodical Analysis of Inelastic deuteron ${ }^{3} \mathrm{C}^{\theta} \mathrm{H}$ Interactions at $P=8.2 \mathrm{GeV} / \mathrm{C}$ with Maximum Momenta of Secondary Particles

Yad. Phys. 44:434.1986:

Two Proton Correlations in He C Interactions at $4.2 \mathrm{GeV} / \mathrm{c}$ per Nucleon

Kr. Soob. JINR 6:11.1985; CFRN-EP-84-108:

Restriction on Amount of Antimatter in Early Universe from $\bar{p}$ "He Reaction Data

Phys. Lett. 165B:265.1985; CERN-EP-85-92;

Inelastic Interaction of Antiprotons with ${ }^{4} \mathrm{He}$ Nuclei between 200 and $600 \mathrm{MeV} / \mathrm{c}$

Eur. Lett. 2:115.1986: CERN-EP-85-122;

Antiproton Annihilation on $\mathrm{Ag} / \mathrm{Br}$ Nuclei

Nuovo Cim. 92 A:139,1986:

Inelastic Reactions Induced by Pions on ${ }^{4} \mathrm{He}$ at $68 \mathrm{MeV}, 120 \mathrm{MeV}, 135 \mathrm{McV}, 56 \mathrm{MeV}, 174 \mathrm{MeV}$ and 208 $\mathrm{MeV}$

Czech. J. Phys. B36:340.1986: CERN-EP-85-79; C85/03/18

Low Energy Antiproton Annihilation on Nuclei

Phys. Lett. 194B:343,1987: CERN-EP-87-43:

$\overline{\bar{p}}$ - ${ }^{4}$ He Breakup Cross Section at $180 \mathrm{MeV}$

CERN-EP-87-65:

Annihilation of Antiprotons at Rest in ${ }^{3} \mathrm{He}$ and ${ }^{4} \mathrm{He}$

Phys. Lett. 194B:192,1987:

Neutral Strange Particle Production in $\bar{p}{ }^{20}$ Ne Reactions at $607 \mathrm{MeV} / \mathrm{c}$

Phys. Lett. 215B:247,1988:

$\bar{p}{ }^{3}$ He Reaction Cross Section at $200 \mathrm{MeV} / \mathrm{c}$

Phys. Lett. 217B:43,1989;

An Observation of a Leading Meson in $\bar{p}$ Ne Reaction at $600 \mathrm{MeV} / \mathrm{c}$ Incident Momentum

Phys. Lett. 230B:36.1989; CERN-EP-89-03;

Antiproton - Helium Annihilation around $45 \mathrm{MeV} / c$

JINR-P1-88-503:

The Study of Dibaryon States in deuteron $p$ Interactions

Phys. Rev. D37:587,1988:

Precise Measurement of the Asymmetry Paramıter $\Delta$ in Muon Decay

Nucl. Phys. B286:635.1987;

Measurement of $n p$ Analyzing Power $A_{00 n o}$ Using the Deuteron Polarized Beam of SATURNE-II

Z. Phys. C40:193.1988;

Measurement of the Spin Correlation $A_{00 k k}$ in $n$ p Elastic Scattering between 0.63 and $1.08 \mathrm{GeV}$

Phys. Rev, D37:1744.1988: UCPPG-86-7-17:

Observation of Coherent $\rho^{+}$Production in Neutrino-Neon Interactions

Phys. Rev D40:2764.1989:

Left-Right Asymmetry in Neutrino Produced Hadron Jets

Phys. Rev. Lett. 60:1118.1988:

Comparison of Exclusive Reactions at Large $T$

Yad. Phys. 43:1487.1986: Sov. J. Nucl. Phys. 43:959.1986: JT'EP-84-136:

The Amplitude Analysis of $K_{S} K_{S}$ System from the Reaction of $\pi^{-} p \rightarrow K_{S} K_{S}$ nucleon at $40 \mathrm{GeV} / \mathrm{c}$

Sov. J. Nucl. Phys, 48:770.1988: Yad. Phys. 48:1213.1988: JTEP-87-137:

Evidence of Resonance with Mass of $3075 \mathrm{MeV} / c^{2}$ and Spin 4 is $\pi^{-} p \rightarrow K_{S} K_{S} n$ at $40 \mathrm{GeV} / c$

Phys. Rev. Lett. 55:2543,1985:

Limits on Like-Sign Dilepton Production in $\nu_{b l}$ Interactions

Phys. Rev, Lett 57:2629.1986;

Evidence for Coherent Neutral Pion Production by High Energy Neutrinos

Phys. Rev. D31:2192.1985:

Limits on Deeply Penetrating Particles in the $>10^{17}$ eV Cosmic Ray Flux

Phys. Rev. Lett. 55:150,1985:

Measurements of Cabibbo-Suppressed Decays of Charmed $D^{+}$and $D^{0}$ Mesons

Phys. Rev. Lett. 54:1875.1985;

Evidence for a High Energy Cosmic Ray Spectrum Cutoff

Phys. Rev. Lett. 54:1976.1985; Phys. Rev. Lett. 55:638.1985:

Direct Measurement of Charmed $D^{+}$and $D^{0}$ Semileptonic Branching Ratios

Phys. Rev. D32:566.1985:

Decays of the $J / \psi(1 S)$ into Two Pseudoscalar Mesons

Phys Rev D32:2883.1985;

$J / \psi(1 S)$ Decays into a Vector and Pseudoscalar Meson and the Quark Content of the $\eta$ and $\eta^{\prime}$ 
Baltrusait is $\mathbf{8 5 G}$

Baltrusaitis $85 \mathrm{~J}$

Balt rusait is 86

Balt tusait is $86 \mathrm{~B}$

Baltrusaitis $86 \mathrm{C}$

Baltrusait is $86 \mathrm{D}$

Balt rusait is $86 \mathrm{E}$

Beiltrusait is 87

Bamberger 86

Bamberger 88

Bamberger 88B

Bamberger 89

Banaigs 86

Banaigs 86B

Banaigs 87

Band 85

Band 88

Band 89

Banerjee 85

Banerjee 85B

Banerjee 86

Banerjee $86 \mathrm{~B}$

Banerjee 86C

Banerjee 89

Banner 85

Banner 85B

Bannik 8:

Bannik 87B

Bannikov 88

Bannikov 89

Bannikov 89B

Bano 86

Bano 87

Barabanov 86

Barabash 87

Barauvash 87B

Barabash 88

Barabash 89
Phys Rev Lett 55:1723.1985.

Observation of $J / L$ (iS) Radiative Decay to Pseudoscalar w

Phys. Rer. Lett 55:1842.194:s. SLAC C PLB-3732.

Tau Leptonic Branching Ratios and a Search for Goldstone Decay

Phys. Rev. D33629.1981:

Hadronic Decay of the $\eta_{c}(1 S)$

Phes Rev. D:33: 1222.1986;

Study of the Radiative Decuy $J / v(1 S) \rightarrow \gamma \rho \rho$

Phis. Rev. Let t 56:107.1986:

Observation of a Narrow $K \bar{K}$ State in $J / v(1 S)$ Radiative Decays

Phys, Rev. Lett 56:2136.1986i

Search for Nonspectato: Decays of the $D^{0}$

Phys. Rev. Lett. 56:2140.1986:

Direct Measurement of Charmed $D$ Meson Hadronic Branching Fractions

Phys. Rev. D35:2077.1987: SLAC-PLB.3720:

Fadiative Decays of the $J / u^{\prime}(1 S)$ into $\gamma \pi^{+} \pi^{-}$and $\rightarrow K^{+} K^{-}$

Phis. Lett. 184B:271.198-: CERN-EP.86-194:

Multiplicity and Transverse Energy Flux in ${ }^{16} \mathrm{O}+\mathrm{Pb}$ at $200 \mathrm{GeV}$ per Nucleon

Phy's. Lett. 203B:320.1988:

Probing the Space-Time Geometry of Ultrarelativistic Heavy-Ion Collision

Phrs. Lett. 205B:583.1988:

Charge Particles Multiplicities and Inelastic Cross Sections in High Energy Nuclear Collisions

Z. Phys. C43:25.1989: MPI-P.AE-EXP-EL-198:

A Study of $K^{0}, \Lambda$ and $\Lambda$ Production in $60 \mathrm{GeV}$ and $200 \mathrm{GeV}$ per Nucleon $O$ Au and $p$ Au Collisions with a Streamer Chamber Detector at the CERN SPS

Nucl. Phys. A445:737,1985:

Diffractive One Pion Production in the He $p \rightarrow$ He X Process at $p(\mathrm{He})=7.0 \mathrm{GeV} / \mathrm{c}$

Phrs. Rex. C32:1448.1985:

First Observation of the deuteron deuteron $\rightarrow{ }^{4}$ He $\eta$ Reaction

Phys. Rev. Lett. 58:1922.195:

Search for the Charge Symmetry Breaking Reaction deuteron deuteron $\rightarrow{ }^{4} \mathrm{He} \pi^{0}$ at $0.8 \mathrm{GeV}$

Fhy's. Rev. Let1. 59:415.198- iLAC-PL'B-4294: COLO-HEP-147:

Precise Measurement o- the Lifetime of the Tau Lepton

Phys Lett. 200B:221,1988:

Additional Evidence for $B^{0}-\bar{B}^{0}$ Mixing

Phys. Lett. 218B:369.1989: SLAC-PLB-48T1:

A Measurement of the $\epsilon^{-} e^{-}-B \bar{B}$ Forward - Backward Charge Asymmetry at $\sqrt{s}=29 \mathrm{GeV}$

Z. Phys. C28:!63.1985:

Studies of $\bar{p} p \rightarrow \bar{n} n$ and $\bar{n} p$ Annihilation at Incident Momenta of 700 and $760 \mathrm{MeV} / c$

Jour. of Phys. G 11:80T,1985. i'RINT-85-0237-TATA-1NST:

Test of the Hypothesis if Scaling in the Mean in Inclusive Photon Production in Antiproton Proton Interactions

Z. Phys. C31:401.1986: CERN PRE-85-058; TJFR-BC-85-5:

Inclusive $\phi$ Production in $K^{-} p$ Interactions at $110 \mathrm{GeV} / c$ and Search for Structure in $0 \pi^{-}$

Z. Phys. C31:409.1986: CERN-PRE-86-048: TIFA-BC-85-7:

Inclusive $\Sigma\left(1385 P_{13}\right)$ Production in $K^{-}$p Interactions at $110 \mathrm{GeV} / \mathrm{c}$

Z. Phys. C 32:163.1986:

A Study of $\bar{n} p$ Annihilations between 0.5 and $0.8 \mathrm{GeV} / c$

Phys. Rev. Lett. 62:12.1989:

$\hat{\Lambda}$ and $\bar{\Lambda}$ Production frum Proton-Antiproton Collisions at $\sqrt{6}=1.8 \mathrm{TeV}$

Phys. Lett. 156B:129.1985: CER V-EP-85-42;

A New Search for Relativistic Particles with Fractional Electric Charge at the CERN $\tilde{p} p$ Collider

Z. Phys. C27:329.1985: CERN-PRE-84-091:

Inclusive Particle Production in the Transverse Momentum Range between 0.25 and $40 \mathrm{GeV} / \mathrm{c}$ at the CERN $\bar{p} p$ Collider

JINR-P1-8T-546:

Observation of Particle Collective Flux in the Collisions of $4.1 \mathrm{~A} \mathrm{GeV} / \mathrm{c}^{22} \mathrm{Ne}$ with $\mathrm{Ag}, \mathrm{Br}$ Emulsion Nuclei

Yad. Phys 45:431,1987: Sov. J. N . l. Phys. 45:272.1987; JINR-P1-86-117;

Emission of Fast Deuterons at the Proton Interaction with Emulsion Heavy Nuclei

JINR-E1-88-476:

Evidence for High Twist Mechanisms in High $p_{T} \pi^{-} p$ Events with Prompt $\rho^{0}$ Production at $38 \mathrm{GeV} / \mathrm{c}$

Yad. Phys. 51:749.1990: JINR-P1-s'-308:

Production of Backward Protons with Momenta 0.2 - $0.5 \mathrm{GeV} / \mathrm{c}$ in Lab. System which are Geierated in $\pi^{-}$nucleus Interactions at $40 \mathrm{GeV} / \mathrm{c}$

JINR-E1-89-486:

Resonance Pattern of Low Muon Pairs Produced in $38 \mathrm{GeV} / \mathrm{c} \pi^{-} \mathrm{C}$ Interactions

Phys. Lett. 166B:453.1986:

Evidence for the Anomalous B haviour of $Z=2$ Secondary Nuclei in Hydrogen

Phys. Lett. 196B:255.1987:

Further Evidence for the Anon.alous Interaction of ${ }^{3}$ He Secondary Nuclei with Protons

Pisma Zh. Eksp Teor. Fiz. 43:166.1 tunt

Study of the Double 3 -Decay of ${ }^{130} \mathrm{Xe}$

Pisma Zh. Eksp Teor Fiz 45:171.19k:

Study of the Double 3 -Decay of $130 \mathrm{Xe}$

ITEP-87-165:

Limit on the 3+3 Decay with Ma oron Emission of ${ }^{46} \mathrm{Ca}$

ITEP-8R-104:

Serch for $2 B(2 \mu)$ Decay of ${ }^{\circ 0} Z_{r}$ by Nuclear Emulsion

Phy. Lett. 223B 273,1989

Fresult of the Experiment on Search for Double 3 Decay of ${ }^{196} \mathrm{Xe},{ }^{194} \mathrm{Xe}$ and ${ }^{124} \mathrm{Xe}$ 
Barabash 89B

Barabash 89C

Barabash 89D

Barabash 90

Baran 88B

Baranov N5

Barasch 85

Barate 80

Barate $86 \mathrm{~B}$

Barate $96 \mathrm{C}$

Barbarogalti 89

Barbarogalt 190

Barbier 88

Barbier $88 \mathrm{~B}$

Barbierı 88B

Bardadinotui $\$ 5$

Bardin s:-

Bardin RTB

Bareyre 86

Bari N5

Baringer 86

Baringer 8 -

Baringer 88

Barish 8T

Barish 88

Barkalox $\mathrm{RT}$

Barklow 90

Barkov 8.5

Barkov $8.5 B$

Barkov $850^{\circ}$

Barkov 87

Barkov BTB

Barkov kiC

Barkov ix

Barkov 89

Barlag 86

Barlag 87
TEP-R!-130.

Status Report on Double 3 Decay Experiment in the USSR

ITEP-Bu-125:

Search for Double is Decay of ${ }^{1: 0} \mathrm{Cd}$ to First Exited State of ${ }^{116} \mathrm{Sn}$

ITEP-R4-184:

Search for Double 3 Decay of ${ }^{300}$ Mo to the Exited States of ${ }^{100} R \mathrm{U}$

Yad. Phy' $51: 3.1 ! 900$ :

Results of ${ }^{136}$ Xe Double 3 Decay Experinent

Phys. Rev Lett 61:400.10Ns:

$\ddot{\Delta}\left(1232 P_{33}\right)$ Electroproduction and Inelastic Charge Scattering from Carbon and Iron

Yad. Phys 41 1520.1985: Sor .I Nucl. Phys 41:963.1965. PHE-k4t-17:

? Quanta and $\pi^{\circ}$ Mesons Inclusive Production in $\nu$ Nuclei and $\bar{\nu}$ Nuclei Interaction at Ene:gy up to 30 GeV

Phy.s. Le.t. L61 B:265. 1985: LBL-17065:

Energy Spectrum of Subthreshold $\boldsymbol{K}^{-}$Produced in Relativistic Nuclear Collisions. (Revised Version)

Phys. Lett $17+\mathrm{B}: 458,198 \mathrm{~s}$ :

Photoproduction of Charged Hadrons at Large Transverse Momenta

$Z$ Phys C33.505.1986. CERN-EP.86-180:

Measurement of $J / v(1 S)$ and $b(2 S)$ Real Photoproduction on ${ }^{8} \mathrm{Li}$ at a Mean Energy of $90 \mathrm{GeV}$

CERN-EP-86-27.

Heavy Flavor Photoptoduction and Decay

LBL-26917: C88 10,19 :

The Search for the Top Quark

LBL-28673: FERMILAB-CONF-90-42-E:

Search for the Top Quark in Electron-Muon Events with CDF

Phys Rev. D3i:1113.1988:

Correlation among the Particles Produced in Proton Interactions with Emulsion Nuclei at $800 \mathrm{GeV} / \mathrm{c}$

Phys Rev. Lett. 60:405.198:

Central Collisions of 14.6, 60, and $200 \mathrm{GeV} / \mathrm{Nucleon}{ }^{10} \mathrm{O}$ Nuclei in Nuclear Emulsion

Phys. Rev. Lett. 61:27.1988: L M1-PP-88-143;

Limit on the Magnetic Moment of the Neutrino from Supernova SN1987A Observations

CERN-EP-85-168:

Direct Photon Production from Pions and Protons at $200 \mathrm{GeV} / \mathrm{c}$

Phys. Lett. 1950:292.1987: CERN-EP-87-21:

Search for a Narrow Resonance about the $f_{+}(2220)$ in the Formation Channel $\bar{p} p \rightarrow K^{+} K^{-}$

Phys. Let1. 1928:471.1987: CERN-EP-87-90:

A Measurement of the $\bar{p} p \rightarrow \pi^{+} \pi^{-}$Reaction for $158 \leq p(\bar{p}) \leq 275 \mathrm{MeV} / \mathrm{c}$

Nuovo Cim 9C:159.1986i:

Status of the FREJUS Experiment and Preliminary Results on Contained Events.

Phys. Lett. 163B:282.1985: CERN-EP-85-132: JINP-E1-85-747;

A Measurement of Nuclear Effects in Deep Inelastic Muon Scattering on Deuterium, Nitrogen and Iron Targets

Phys. Pev. Lett. 56:1346.1986:

A Production in $e^{+} e^{-}$Annihilations at $28 \mathrm{GeV}:$ A Comparison with Lund Model Predictions

Phys. Rev. Lett. 59:1993,1987.

Production of $\eta$ and $\omega$ Mesons in $r^{ \pm}$Decay and a Search for Second-Class Currents

Phys Lett 206B:551.1988: CLEO-87-8: CLNS-87-105.

Production Cross Section and Electroweak Asymmetry of $D^{*}(2010)$ and $D$ Mesons Produced in $e^{+} e^{-}$ Annihilation at $29 \mathrm{GeV}$

Phys Rey D36:2641.198\%:

Search for Grand Unification Monopoles and Other Ionizing Heavy Particles Using a Scintillation at the Earth's Surface

Phys Pept. 157:1.1988:

The Physics of the Tau Lepton

Kr Soub. Fiz. 8i-12:25.1987:

A bout Rise with Energy the Hadron-Air Nucleus Cross Section on the Basis of Data on Spread Increase of WAS with Height at the Energies of $>10^{16} \mathrm{eV}$

SLAC-PL'B-5196: LBL-28581:

Searches for Supersymmetric Particles Produced in $Z^{0}$ Boson Decay

Nucl Phys. B256:365,1985:

Electromagnet ic Pion Form Factor in the Timelike Region

Plsma Zh Eksp. Teor. Fiz. 12:113.1985: Jetp Lett. 42:138.19Rk:

Measurement of the Neutral Kaon Mass

NOVO-85-129:

Production of Light Nuclei at Small Momenta in Proton Nuclei Interactions at $70 \mathrm{GeV}$

Pisma Zh Eksp. Teor. Fiz. 46:132.1987:

Measurement of $\boldsymbol{\omega}$ Meson Parameter by Cryogenic Magnetic Detector

Yad. Phys 46:108k.19k7

The Precision Neutral Kaon Mass Measurement

NOYO- $\mathrm{nT}-1$

Measure ,ent of $\omega$ Meson Parameters by Means of Cryogenic Detector

Yad. Phys ; 63.1968 :

The Investigation of Multipion Creation with the Cryogenic Magnetic Detector at the VEPP-2M Storage Ring

NOVO-89-15.

Investigation of the Process $e^{+} c^{-} \rightarrow \pi^{+} \pi^{-} \pi^{0}$ at the Energy Range 2.420 - 2.510 MeV on VEPP-2M with the Help of Cryogenic Magnetic Detector

Phys Lett 1841 283.1947. CERN-EP-86i-173:

Measuremen of the Lifetime of the Charmed Baryon $\Lambda_{c}^{+}$

$Z$ Phys. (37.17 of7: CERN.E.P. B7-72:

Lifetimes of ( harged and Neutral $D$ Mesons 
Barlag 88

Barlag $8 B$

Barlag KRB

Barlag $\mathrm{NBC}$

Barlag $x \times D$

Barlag 89

Barlag 49B

Barlag $\times 9 C^{\circ}$

Barlag 90

Barlag 90B

Barlag 900

Barlag 90D

Barlet1 8.5

Barluestaud Ki

Barloutad so

Barlow NT

Barlow 88

Barlow kg

Barmun 85

Barmin 86

Barmin $86 \mathrm{~B}$

Barmin $86 \mathrm{C}$

Barmin 87

Barmin $88 B$

Barmin 89

Barmun א9B

Barnabei $\times 6$

Barnes 85

Barnes 87

Barnes $\times 7 \mathrm{~B}$

Barnes $8 x$

Barnes 89

Barnes 89B

Barnes 90

Baron 90

Barr 88

Barr 90

Barr 90B

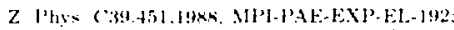

Production of $D, D^{*}(2010)$ and $D^{*}$ Mesons in $200 \mathrm{GeV} / c \pi^{-}, K^{-}$and $p$ Si Internctions

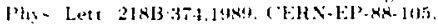

Precise Determination of the Lifetime of the Charmed Baryon $\Lambda^{+}$

('ERN-EP.RK-1133:

Results on $\Lambda_{c}^{+}, D_{s}^{+}, D^{0}$ and $D^{+}$Decny Properties from the NA32 Experiment

('EHN-EP'PR-104:

Results on $A_{i}^{+}, D_{j}^{+}, D^{0}$ and $D^{+}$Production Properties in $230 \mathrm{GeV} \pi \quad \mathrm{Cu}$ Interactions from the NA32 Experiment

CERN-FIS-89-43:

Measurement of the Mass and Lifetime of the Charmed Strange Baryons $\Xi_{c}(2460)^{+}$in Two New Decay Modes

Phy: Lett. 23213:561.1989.

Measurement of Branching Ratios and Branching Fractions of the Charmed Meson $D^{0}$

P'hys. Lett. 233B-522.1989: ('F:RN-EP-R9- 145:

Measurement of the Mass and Lifetime of the Charmed Strange Baryon

Plys. Lett 236B: 195.1440: CERN-EP-190-0N.

First Measurement of the Lifetime of the Charmed Strange Baryon

2. Phys C46:5633.1990: MPI-P'AE-EXP-EL-220.

Measurement of the Masses and Lifetimes of the Charmed Mesons $D^{0}, D^{+}$and $D_{.5}^{-}$

CER.N-EP-90-4T

Measurement of frequencies of various decay mades of charmed particles $D^{0}, D^{+}, D_{S}^{+}$and $\Lambda_{r}^{+}$including the observation of new channels

CERN-EP-901-T-

Production of the Charmed Baryon $\Lambda_{t}^{+}$in $\pi^{-} \mathrm{Cu}$ and $K^{-} \mathrm{Cu}$ Interactions at $230 \mathrm{GeV}$

Phys. Rev. (32:234,1455:

Proton Nucleon Spin Rotation and Depolarization Parameters at $800 \mathrm{MeV}$

DPHPE- 7 7-04:

Results from Nucleon Decay Experiments

OPHPE-8R-15:

Review of Nucleon Decay Experiments

DESY:-8T-009:

Results from PETRA (n Review of Results from the Five PETRA Experiment During the Past Year)

Nucl. Instr. and Meth. A $271.471 .198 \times$ :

Production of High Momentum Negative Pions by $800 \mathrm{MeV}$ Protons at 0 to 20 Degrees

Phys. Rev. Lett. 02:10019.1989:

Measurement of the Spin-Fatation Paraneters $A$ and $R$ for $\pi^{+} p \rightarrow \pi^{+} p$ and $\pi^{-} p \rightarrow \pi^{-} p$ Scattering from 471 to $625 \mathrm{MeV} / \mathrm{c}$

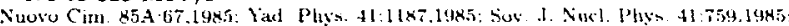

Search for $C P$ Violation in the $K^{0} \rightarrow \pi^{+} \pi^{-} \pi^{0}$ Decay

Yad Phys 45:97.1986;

Measurement of the Ratio of Decay Probabilities for $\mathrm{K}^{+} \rightarrow \mathrm{e}^{+} \pi^{\mathrm{O}}$, and $\mathrm{K}^{+} \rightarrow \pi^{+} \pi^{+} \pi^{-}$

Yad Phys. 4t:965.1986: Suv. J Nigcl. Phys. 41:622.1986:

Search for Decay $K_{S} \rightarrow e^{+} e^{-}$

Nueru Cim $96 \mathrm{~A} \cdot 15 \%$.1986) JTEP-ki-74:

Search for $K_{s}-2$; Decay

Yad. Phys 47:1011.1988: ITEP-87-10N

Measurement of the Probabilities of $\mathrm{K}^{+} \rightarrow \mu^{+} \nu \rightarrow$ Decay

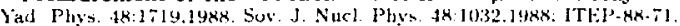

The Measurement of the Probability of $\mathrm{K}^{+} \rightarrow \pi^{0} \pi^{0} e^{+} \nu$ Decay

Yad. Physs. $50: 3.1959$.

Radiative Capture of Stopped $\pi^{-}$Mesons by Xenon Nuclei

Yad. Phys 50:6-19.1984:

The Measurement of the $\mathrm{K}^{+} \rightarrow \pi^{+} \pi^{+} \pi^{-}$, Decay Probability

Phys Fev Lett ist:15+2.198ti:

Total Cross Section for Deuteron Photodisintegration between 15 and $75 \mathrm{MeV}$

CERN-EP-K5-65.

Status and Future of Experiment PS-185 ( $\bar{p} p-$ hyperon hyperon $)$ at CERN

Phys Leet, 189B3:249.1947: ('ERN-EP-N7-22.

Study of the Reaction $\bar{p} p \rightarrow \bar{\Lambda} \wedge$ near Threshold

Phym Lett. 19913 147.1987. ('ERT-EP-87-15:

$C P$ Invariance in the Reaction $\bar{p} p \rightarrow \bar{\Lambda} \Lambda \rightarrow \bar{p} \pi^{+} p \pi^{-}$at $1.546 \mathrm{GeV} / c$

Phis Lett. 206B 146.19k8: CF.AN.EP-87-209:

A Measurement of Cross Sections for ${ }^{10} \mathrm{O} \mathrm{Al}$ and ${ }^{10} \mathrm{O} \mathrm{Pb}$ Interactions at 60 and $200 \mathrm{GeV} / \mathrm{c}$ per Nucleon Nirovo ('111. 102A:541.19k!9.

Study of the Reaction $\bar{p} p \rightarrow \bar{\lambda} \wedge$ Close to Threshold at LEAR

Phys Lett 229B:432.1989: CERN-EP-89-78:

Threshold Measurement of the Reaction $\bar{p} p \rightarrow \bar{\Lambda} \Lambda$ at Lear

CERN-FP.90-40.

Measurement of the Reaction $\bar{p} p \rightarrow \bar{\Lambda} \Sigma^{0}+\operatorname{cc}$ at $1.695 \mathrm{GeV} / \mathrm{r}$

CERN-EP-90-05:

Electromagnetic Dissociation of $200 \mathrm{GeV} / \mathrm{Nucleon}{ }^{16} \mathrm{O}$ and ${ }^{32} \mathrm{~S}$ Ions in Nuclear Emulsions

Phys. Lett 21413:303.19k4. 11Z-ETAP-84-18:

Search for the Decay $K_{L} \rightarrow \pi^{0} e^{+} e^{-}$

CERN-EI'-90-01

NA31 Results on CP Violation in $K$ Decays, and a Test of $C P T$

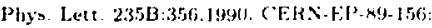

Search for a Neutral Higgs Particle in the Decay Sequence $k_{L} \rightarrow \pi^{\circ}$ higgs and higgs $\rightarrow e^{+} e^{-}$ 
Bart 9015

Barre'au 86 ;

Barretito 8513

Bartel 840

Bartel 85

Barte' 8513

Bartel $85 \mathrm{C}$

Bartel $85 \mathrm{D}$

Bartel 8.5E

Bartel $85 \mathrm{~F}$

Bartel N5C

Bartel 85H

Bartel 85J

Bartel $85 \mathrm{~K}$

Bartel 85L

Bartel 85M

Bartel 86

Bartel BGB

Bartel $86 \mathrm{C}$

Bartel 861

Bartel $86 \mathrm{E}$

Bartel 86F

Bartel 86G

Bartel 86H

Bartel b7

Bartel $87 \mathrm{~B}$

Burtel $87 \mathrm{C}$

Bartels 88

Bartelt 8.5

Bartelt 87

Bartha 86

Bartke 85

Bartke 86

Bartke $k 9$

Bartoletto 86

Bartoletto 87

Baru $\times .5$

Baria R6
CER V-FI-Allig:

Observation of the Decay $K_{L} \rightarrow \pi^{0} 2 \gamma$

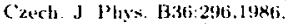

Deep Inelastic Electron Scattering on Light Nuclei

DESY.k5-1)8ti: FT! IM-k5-3:

A Study of Energy-Energy Correlations with TASSO and PLUTO

2. Plys. (26:507.1985; DESY'-8.4078:

New Resules on $e^{+} e^{-} \rightarrow \mu^{+} \mu^{-}$from the JADE Detector at PETRA

Z Phys. (28:343.1985: DESY'-85-1929:

A Study of Photon Production in Hadronic e+ $e^{-}$Annihilation

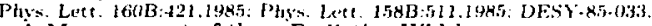

A Measurement of the $\eta$ Radiative Width $\eta \rightarrow \gamma$ ?

Phys. Let t. 15213:385.1985:

A Search for the Supersymmetric Partner of Electrons

Phys. Lett. 15213:392.1985:

A Search for Scalar Muons

Phys. Lett. 155B:28R,1985: DES Y - Y i -022 :

A Search for Monojet Events Produced by Virtual $Z^{0}$ Bosons in $e^{+} e^{-}$Annihilation at PETRA

2. Phys. C29:505,1985: DESY-k5-(6ti0;

A Search for the Supersymmetric Chargino in $e^{+} e^{-}$Annihilation at PETRA

Phys. Lett 161B:197.1965: DESY- -5.5 -069:

Inclusive Neutral $D^{*}(\mathbf{2 0 1 0})$ Production and Limits on $D_{S}^{*}$ Production in $e^{+} e^{-}$Annihilation at PETRA

Phys. Lett. 157B:340.1985; DESY-K5-036:

Comparison of 'Three-Jet Events with QCD Shower Models

DESY-8.5-0R1:

Evidence for $n(1440)$ Production in High Energy $e^{+} e^{-}$Annihilation

2. Phys. C 30:371.j986: DESY-45-131:

Tests of the Standard Model in Leptonic Reactions at PETRA Energies

Phys. Lett. 161B:188.1985; DESY'-45-065:

Tau Lepton Production and Decay at PETRA Energies

Phys. Lett. 160B:337.1985: DESY-X5-057:

A Measurement of the Total Cross Section and a Study of Inclusive Muon Production for the Process $e^{+}$ $e^{-} \rightarrow$ hadrons in the Energy Range between $39.70 \mathrm{GeV}$ and $46.78 \mathrm{GeV}$

2. Phys. C31:349.1986: DESY-K6-001:

Determination of the $B$ Lifetime

DESY-BG-0.3:

Exclusive Production of Proton Antiproton Pairs in Photon Photon Collisions

2. Phys. C30:545.1986: DESY'-86-1005:

Lepton Pair Production in Double Tagged Two-Photon Interactions

2. Phys C31:359.1986: DESY-86-023:

Radiative Tau Pair Production and Search for New Particles Decaying into Tau

Phys. Lett. 174B:350.1986: IJESY-8fi-045:

Exclusive Production of Proton-Antiproton Pairs in Photon-Photon Collisions

Phys Let1. 182B:216.1986: DESY-Kfi-091:

Measurement of the Branching Fractions for $\tau^{ \pm} \rightarrow e^{ \pm} \nu_{e} \nu_{\tau}, \tau^{ \pm} \rightarrow \mu^{ \pm} \nu_{\mu} \nu_{\tau}$ and $\tau^{ \pm} \rightarrow \pi^{ \pm} \nu_{\tau}$

Phys. Let1. 163B:277,1986: DESY-85-1171:

A Measurememt of Mean Semi-Muonic Branching Ratio of Beauty Hadrons Produced at PETRA

2. Phys. (333:23,1986: DESY-X6-086:

Experimental Studies on Multijet Production in $e^{+} e^{-}$Annihilation at PETRA Energies

2. Plyy's. (36:15.1987. DESY-87-1331:

Search for Leptoquarks and Other New Particles with Lepton-Hadron Signature in $e^{+}:^{-}$Interactions

Phys. Lett. 184B:288.1947: DESY-84i-125:

Observation of Charmed Mesons in Photon Photon Colligions

2. Phys (33:334.1987: DESY-86-129:

Determination of Semi-muonic Branching Ratios and Fragmentations Functions of Heavy Quarks in $e^{+}$ $e^{-}$Annihilation at $E_{\mathrm{cm}}=34.6 \mathrm{GeV}$

Z. Phys C $36: 85.1988$.

Spectra of Negative Particles and Photons in Collisio:ss of $p-W t$ and ${ }^{16} \mathrm{O}-\mathrm{W}$ at $200 \mathrm{GeV} / \mathrm{N}$

Phys. Rev. D32:1630.1985:

Evidence for Time and Directional Enhancements of Multimuon Cosmic-Ray Events

Phys. Rev. D36: 1990.19k7: Phys. Rew. D40:1701.1986\%:

Monopole-Flux and Proton-Decay Limits from the SOUDAN-1 Detector

Phys. Rev. Lett. 56:685.1966:

Search for A nomalous Single Photon Production in $e^{+} e^{-}$Annihilation at $E_{\mathrm{cm}}=29 \mathrm{GeV}$

2. Phys. (29:9.1985.

Asymmetry of $\pi^{-}$Meson Emission in Nucleus Nucleus Collisions as a Measure of the Target-to-Projectile Ratio of the Numbers of Interacting Nucleons

JINR-E1-86-3:32:

Size of the Proton Emission Region in Pion Xenon Interactions at $3.5 \mathrm{GeV} / \mathrm{c}$ from Two-Particle Correlations

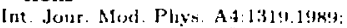

Relativistic Heavy Ion Reactions

Phys Rev Lett. 565080$)(19 k 6$.

Inclusive $\phi$ Production in Beauty Meson Decay

Phys. Rev D35:19.1987.

Inclusive B-meson Decays into Charm

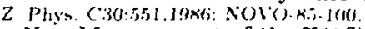

New Measurement of the $r(1 S)$ Meson Mass

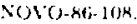

Measurement of the Total Cross Section of Two Photon Production of Hadrons 
Baru $86 \mathrm{~B}$

Baru 87

Baru 89

Barwolff 85

Barwolf 88

Basdevant 90

Baskov 88

Bastid 89

Bat on 85

Batskurich $k 8$

Bat istum 85

Battistuni 86

Battistoni $85 \mathrm{~B}$

Battistoni $86 \mathrm{C}$

Baturin 85

Baturin 80

Baturin 87

Baturin 87B

Baturin 88

Bazusov 85

Bat usov 85.5

Bat usuv $4.5 \mathrm{C}$

Bat usov 87

Batusov $87 \mathrm{~B}$

Batusov $87 \mathrm{C}^{\circ}$

Batusov 88

Batusov 88B

Batusor $88 C$

Bat usov s9B

Batusov 890 :

Batyuuya Hu $^{-1}$

Bat yunya 45

Batyunya K5B

Bat vurtya $\mathrm{HSC}$

Batyunaca $8.5 \mathrm{D}$

Batyunya 86

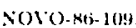

A Search for $f_{4}(2220)$ in Radiative Decays of the $Y(1 S)$ Meson

NOYO-RT-31:

Results of MD- 1 Detector on the Search of $X(2200)$ in the Decays $Y(1 S) \rightarrow \neg K^{+} K^{-}$and $Y(1 S) \rightarrow \gamma \phi \phi$

Z. Phys (-12:505.1936):

A Search for $f_{4}(2220)$ and $X(2200)$ in Radiative Decays of the $r(15)$ Meson

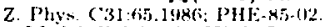

Multiplicities in High P-transverse Madron Nucleus Interactions

Z. Phys. C37:337. 1985:

Neutral Strange Particle Production in High Transverse Moment urn $\pi^{-}$nucleus Interactions at $40 \mathrm{GeV} / \mathrm{c}$ Phys. Lett 234B:3455.1940:

Is there Room for Charged Dark Matter?

LEBD-88-143:

Electron-Positron Pair Production by ? Quanta with Energies from 13 to $25 \mathrm{GeV}$ in Oriented Tungsten Crystals

P(C.F-RI-89-02:

Exclusive Mensurements of Light Fragment Production at Forward Angley in Ne - Pb and Ne - NaF Collisions at $E / A=400 \mathrm{MeV}$ and $800 \mathrm{MeV}$

Z Phys. C29:15.1985:

Dilepton and Trilepton Production by Antineutrinos and Neutrinos in Neon

Yad. Phys 50:1613.1989: J1NR-P1-88-858;

Correlation over Multiplicity between Negative Pions and Protons-Participants in C Ta Interactions at 4.2 GeV/c per Nucleon Moment um

Phys. Lett. 15.5B:465.1985: ('ERN-PRE-45-05.5:

Observation of a Time Modulated Muon Flux in the Direction of Cygnus X-3

Nuovo Cim. 90: 182.1986 :

Contained Events in the Mont Blanc Nucleon Stability Experiment

Nuovo Cim. 9C:196.1986:

Cosmic Muon Results from the NUSEX Experiment

Nuovo Cim 9C:551.1946:

Limit on Monopole Flux in the Mont Blanc NUSEX Experiment

LENI- 65 , 1093:

A-dependence of Cumulative Neutron and Proton Yieldb at 140 Degrees from Proton Nucleon Interactions at $1 \mathrm{GeV}$

LEN]-86-1167:

Study or Two-Particle Energy Correlations in Proton-Nuclei Interactions with the Cumulative Protons Production

LENI-8T-1322:

Experimental Data on Charge-Exchange $(p, n)$ Reaction at Proton Energy $1 \mathrm{GeV}$

LENI-8T-1302:

Measurement of Neutron and Proton Spectra at 94 Degrees and 120 Degrees Produced by 1 GeV Protong on $\mathrm{C}$ and $\mathrm{Pb}$

Yarl. Phys. 47:70s,196r: JiNR-P1-ki-71:

Determination of Nucleon Form Factor on the Basis of Date from $\pi^{+{ }^{7} \mathrm{Li}} \rightarrow e^{+} e^{-} \mathrm{X}$ Reaction at $380 \mathrm{MeV}$ Kinetic Pion Energy

Yad. Phys. 42:1165.1985: Suv. J. Nucl. Phys. 12:736.1985: JINR-P1-85-11:

Production of Hypernuclei in Interactions of Protons with Photoemulsion Nuclei at 250 and $70 \mathrm{GeV}$

JlNR-Pl-85-495:

Search for Supernuclei in Interactions of Protons with Photoemulsion Nuclei at 250 and 70 GeV

K'r. Suob. JINR 12:6.1985.

Suppression of the Annihilationless Break-up Processes at $\bar{p}$ He Interaction

Yad. Physs. 47:1004.1988; JINR-P1-87-308:

Observation of the Decays of Charmed $D$-mesons and $\Sigma_{r}(\mathbf{2 4 5 5})^{++}$Baryon Produced in Neutrino Emulsion Interactions

Pismia Zh. Eksp. Teor. Fiz. 46:213.1987: JINR-P1-47-5!1

Resonance Production of $\Lambda_{r}^{+}$in Neutrino Interactions with Nucleus in Photoemulsion

Kr Soub. JINR $21 \cdot 5,1987$ :

Annihilation of Stopping Antiprotons in ${ }^{4} \mathrm{He}$ and ${ }^{3} \mathrm{He}$

Kr. Souib. JINR 33:15.1988.

Antiproton - He Elastic Scattering at $600 \mathrm{MeV} / \mathrm{c}$

Kr. Sout JINR 333 23.198R:

Production of Neutral Strange Particles in $\bar{p}{ }^{2} \mathrm{He}$ Annihilation at $600 \mathrm{MeV} / \mathrm{c}$

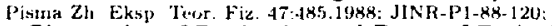

Observation of Production and Decay of Excited $(c)$ B) State with Approximately $27 \theta 0 \mathrm{MeV} / \mathrm{c}^{2}$ Mass in Nuclear Photoemulsion

JINR-P!-89- -475 :

Estimation of $\nu_{b} \rightarrow \nu_{\tau}$ Oscillation Parameters in Hybrid Experiment E-564

Yad. Phys. 30:1524.1989; JiNR-E1-89-222:

Production of $\Lambda$-Hyperons and $K_{S}-$ Mesons in Annihilation of Antiproton in the Nuclei at $600 \mathrm{MeV} / \mathrm{c}$

Sov J. Nucl. Phys 12:5733.1965: Yad. Phys .12:903.1985: JINR-E1-84-7\$(1;

Study of the Reaction deuteron $p \rightarrow \bar{p} p \bar{n}$ at $12 \mathrm{GeV} / \mathrm{c}$ and $\bar{n} p$ Elastic Scattering at $6 \mathrm{GeV} / \mathrm{c}$

Z. Phys ('25:21:3.1!35:;

Inclusive $K_{S}, \Lambda$ and $\bar{\lambda}$ Production in $\tilde{p} p$ Interactions at $22.4 \mathrm{GeV} / c$

Pliys. He. Let $5.5: 562.1965$ :

Polarization Effects in $\rho^{\circ}$ Meson Production in Antiproton Proton Interactions at 22.4, 12 and 5.7 GeV/c

Yad. Plys 4. 1489,1986; JINH-P!-85-85,4:

Study of $\bar{p} p$ Elastic Scattering at $22.4 \mathrm{GeV} / \mathrm{c}$

C:zech. J Phys. B3:36:1273.1486;. JINR-PI-H5-86.4:

Characteristics of Charged Particle Multiplicity in $\bar{n} n$ and $n n$ Interactions and $\bar{n} n$ Annihilation Processes at 6.1 GeV/c

(zedi. J. Phys. 1334:257.198\%, JINR-P1-86-79:3:

Cross Section of Two Prong Exclusive Reactions in $\bar{p} p$ Interactions at $22.4 \mathrm{GeV} / r$ 
Balyunya $86 \mathrm{BL}$

Batyuma $86 C^{\circ}$

Batrunya nit

Batsurya 87

Batyunya 87B

Bastmmy $87 \mathrm{D}$

Batyunya 87E

Batyunya 8TF

Batyunya $87 \mathrm{C}$

Batyunya 87H

Batyunya 87]

Batyunga หร J

Batyinga 88

Batyunya $88 \mathrm{~B}$

Batyunza 89

Batyurya 90

Bauer 85,

Batmann $8 x$

Bay 86

Bavman $8 T$

Bayukov 85

Bayukov 85B

Bayukor $8.5 \mathrm{C}$

Bayukoy 8.5D

Bayukov ose

Bayukov 85F

Bayukov xi

Bayukov 58

Bayukov 89

Bryukor K9B

Bayukov $89 C^{\circ}$

Bazarov 85B

Bazhasion us

Bazhatiav KAB

Bean 86

Be:an 47

BHan $47 \mathrm{~B}$

Brard Xis

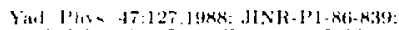

Multiplicity Distribution of Charged Purticles in $\bar{n} p$ and $n p$ Interactiong and $\bar{n} p$ Annitilation at 6.1 GeV/r

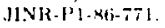

The Main Properties of the $\bar{p} p \rightarrow \bar{p} y \pi^{+} \pi$ Reaction at $22.4 \mathrm{GeV} / \mathrm{s}$

JINT.Pl-ki-8ish.

Resonence Production Cross Sections in the $\bar{p} p \rightarrow \bar{p} p \pi^{+} \pi^{*} \pi^{0}$ Reaction at $22.4 \mathrm{GeV} / \mathrm{c}$

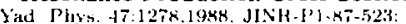

Determination of the Topological Cross Sections of Inelastic deuteron deuteron Interaction at 12 GeV/c

Yad. Phys +ti:1+49.1487: J1NH-I'l-kT-22:

General Features of Multiparticle Production in lnelastic $\bar{n} p$ Interactions at $6.1 \mathrm{GeV} / \mathrm{c}$

JIXR-P1-87-3.40

Determination of the Topological Cross Sections of deuteron deuteron Interactions at $12 \mathrm{GeV} / \mathrm{c}$

Yad Plys. 4T:413.19kB: JINR-P1+ki-8:3:

The Study of the Diffraction Processes in the $\tilde{p} p \rightarrow \bar{p} p \pi^{+} \pi^{-} \pi^{0}$ Reaction at $22.4 \mathrm{GeV} / \mathrm{c}$

Yad. Plis. 46:1117.19k7:

General Properties of the Reaction $\bar{p} p \rightarrow \bar{p} p \pi^{+} \pi^{-}$at $22.4 \mathrm{GeV} / c$

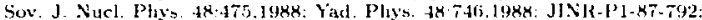

The Study of $\bar{n}$ Interactions at $\boldsymbol{\theta} .1 \mathrm{GeV} / c$

JISR-P]-87-8.9;

The Study of Inclusive Characteristics of deuteron deuteron Interactions at $12 \mathrm{GeV} / c$

JlNR-P1-BT- Bon.

The Study of Neutral Strange Particle and Gamma-Qunturn Production in deuteron deuteron Interaction at $12 \mathrm{GeV} / \mathrm{c}$

Nucl Plys B294 J103i.1967

Study of $\rho^{0}$ Meson Spin Alignment in $\bar{p} p$ and $p p$ Interactions

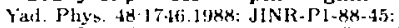

The Multiscatteriog Process Part in deuteron deuteron Interactions at 12 GeV/c Determination

Yaul. Phys. 47:731.1988: JINR-P1-87-67:

Study of the Resonance Production in $\bar{n} p$ Interactions at $6.1 \mathrm{GeV} / \mathrm{c}$

JINR-1-89-26:

Difference of Nonannihilation $\bar{p} p$ Interactions at $22.4 \mathrm{GeV} / \mathrm{c}$ from $p$ Interactions at Close Energy

Yad. Phys. 51.1573.1990: JINR-P1-89-5.56:

Peculiarities of Nonannihilation of $\bar{p} p$ Interartions at $22.4 \mathrm{GeV} / c$

Phys. Rev. Lett, 5.1:753.1985:

Differences between Proton and $\pi^{-}$Induced Production of the Charmonium $\times$States

Phys Rov. D:37:3107. I948:

Experimental Limit for Charge of the Free Neutron

Phys. Lett. 174B:445.1986:

Measurement of the Pion Axial Form Factor from Radiative Decay

Phys. Rept. i47:155.1987:

A nomalons in Relativistic Heavy Ion Collisions

Yad. Phys, 44:412.1986: ITEP-85-53:

Correlations of Cumulative Hadrons in Hadron-Nuclei Interactions

Phys. Lett 189B:291.1987: ITEP-85-118:

Correlations between Neutrons with Small Relative Momenta Produced in p Pb Interactions at 7.5 GeV/c

Yad. Plyss. 42.145.1985: Sov. J. Nucl. Phys. 42:23k.1945: Suv. J. Nucl. Phys. 12:116.1585: Yad. Phys. 42:377.1985: Angular Dependences of Inclusive Nucleon Production in Nuclear Reactions at High Energies and Separation of Contributions from Quasi-free and Deep-inelastic Nuclear Procesges

ITEP.K5-.5:

Protons, Neutrons and Deuterons Leaking out from Different Nuclei

Yad. Phys. 42:1114.1985: Suv. J. Nucl. Phys. 12:K95.1985: 1TEP-N4.98:

Production of Pions in Hadron-Nucleus Interactions at the Initial Momenta from 1.0 up to 0.0 GeV/c

Yad. Phys 41:15k.19k5: Suv. J. Nucl. Phys 41:101.19k5: ITEP-8;-192:

Neutron to Proton Ratios in $p$ Nuclei and $\pi^{ \pm}$Nuclei Interactions

ITEP-86-99:

Correlation of Cumulative Protons at Hadron Nuclei Interactions

1TEP-s女-2:

The Size and Form of the Region of Hadron-Nucleus Feactions

rad. Plive 50:719.1!k9:

Measurement of the Correlation Function of the Curnulative Protons in Proton-Nuclear Interactions at $7.5 \mathrm{GeV} / \mathrm{c}$

Yad Phy, 50:102:3.14h9:

The Size and Shape of the Region of Emission of Secondaries in Hadron-Nucleus Reactions

Yad. Jihys 52.440.1900: [TEP-X?-[37

Correlation Functions of Cumulative Baryons in $p$ nucleus and $\pi$ nucleus Interactions at 3 GeV/c Initia] Momentum

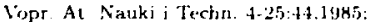

Study of Muon Pairs of Direct Generation in Extensiv 4 ir Showert

Pistin Zh Eksp Tiur. Fiz. 47:-135.leks:

Measurement of Polarization Parameter $M_{n o k n}$ in $p$ Scattering at $950 \mathrm{MeV}$

LENI-Xs-1315\%:

Polarization in the Scatcering of Polarized Protons on the Polarized Proton Target at $950 \mathrm{MeV}$

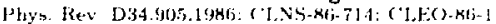

Exclusive Radiative $\mathbf{Y}(1 S)$ Decays

Plyy. Raev D:35:3533.1987.

Improved Upper Limit on Flavor-Changing Neutral Current Decays of the $b$ Quark

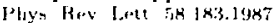

Limits on $B^{0} \bar{B}^{0}$ Mixing and $\tau\left(B^{0}\right) / \tau\left(B^{+}\right)$

Plyts. Hex ( $\$ 2$ 1111.1!6s:

Observation of High Energy $\gamma$ Rays in Intermediate Energy uucleus nucleus Collisions 
Bebek 86

Bebek 86

Bebek $8 T$

Bebek $87 \mathrm{~B}$

Bebek 89

Beck 87

Becker 87

Becket 87B

Becker $87 \mathrm{C}$

Beer 86

Behrend 65

Behrend $65 \mathrm{~B}$

Behrend 86

Behrend $86 \mathrm{~F}$

Behrend 860

Behreisd B6D

Behrend 8:

Behrend 87B

Behrend $\mathrm{HTC}$

Behrend $87 \mathrm{D}$

Behrend 87E

Bebrend 88

Behrend 88B

Behrend $88 \mathrm{C}$

Bei,rend 88D

Behrend $8 \% E$

Behrend $88 \mathrm{~F}$

Behrend $88 \mathrm{C}$

Behrend 89

Behrend $89 \mathrm{~B}$

Behrend 890

Behrend 89D

Behrend 895

Behrend $89 \mathrm{~F}$

Bchrend gef;

Behrend 89H

Brohrend 89J

Beitrend 89.]

Beturends 85

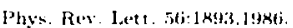

Decay $D^{0} \rightarrow \Phi \bar{K}^{0}$

Phis Rev D36tisno.ishi.

Measurement of the $T^{ \pm}$Lifetime

Phys. Rev. D36:12K!1 1!KT. CLNS-K6-742:

Exclusive Decays and Masses of the B-mesons

Phys. Rev. Lett. 6:2:8,14k!:

Search for the Charmless Decays $B \rightarrow p \bar{p} \pi$ and $p \bar{p} \pi \pi$

Phys. Hev. Lett. 59:15:37.1987

Isoscalar and lsovector Form Factors of ${ }^{3} \mathrm{H}$ and ${ }^{3}$ He for $Q$ below $2.91 /$ fm from Electron-Scattering Measurements

Phys. Lett, 184B:277.1987: CERN-EP-K6-172 Lifetime Measurement of $D_{S}^{ \pm}$Mesons

Phy's. Let1. 193B:117.1987: SLAC-PL'B-A1!4:

$A$ Search for the Lepton Family Number Violating Decay $D \rightarrow \mu^{ \pm} e^{ \pm}$

Pbis. Rev. Lett. 59:] Bti.1987: SLAC.P(13-41.49:

Study of the $K \bar{K} \pi$ Final State in $J / \psi(1 S)$ Hadronic Decays

Phys. Rev. Lett $57: 671.1586$ :

Emission of Muonium into Vacuum from a Silica-Powder Layer

Phys. Lett 161B:182,198.5:

Experimental Limit on Monojet Production in $e^{+} e^{-}$Annibilation

Phys. Let1, 158B:5.36.198.5:

An Investigation of the Processes $e^{+} e^{-} \rightarrow \mu^{+} \mu^{-} \gamma$ and $e^{+} e^{-} \rightarrow e^{+} e^{-} \gamma$

Phys. Lett. 168B:420.1986: LAL-85-50:

Excited Lepton Search

Phyi, Lett 178B:452.1986: SACLAY-DPHPE-X6-16: DESY-46-1)63: Search for Light Leptoquark Bosons

Phys. Lett. $181 \mathrm{~B}: 178.1986$; DESY-86-100:

Search for Excited Quarks in $e^{+} e^{-}$Interactions with CELLO Detector

Phys. Lett. 176B:247.1986; LA L-86-11: DESY-8tj-0511.

A Search for Single Photons at PETRA

7. Phys. C35:181.1987; DESY-87-013:

Searches for Supersymmetric Particles with the CELLO Detector at PETRA

Phys. Lett. 19:3B:157.1987: DESY-87-016:

A Search for Hadronic Events with Low Thrust and an Isolated Lepton

Phys. Lett. 193B:376.1987: DESY'-87-030: Search for Production of Charged Higgs Particles

Phys. Let 183B:400.1987: DESY-86-133:

Determination of as and $\sin ^{2} \theta_{14}$ from Measurements of the Total Hadronic Cross Sections in $e^{+} e^{-}$ Annihilation

Phys. Lett. 19] B:209.1987: DPHPE-87-02: DESY-57-005:

A Measurement of the Muon Pair Production in $e^{+} e^{-}$Annihilation at $38.3 \leq E_{c m} \leq 46.8$ GeV

Plys: Lett 200B:226.1988: DESY-87-127. Upper Limit for the Decay $\tau^{-} \rightarrow \eta \pi^{-} \nu$

Phys. Lett. 202B:154,1988: DESY-87-150:

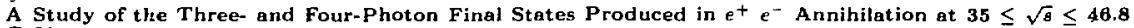
GeV

2. Pliys CAl:7.5988: DESY-88-060:

A Search for New Leptons

Phys. Lett. 215B:186.1988: DESY-88-052:

Neutrino Counting with the CELLO Detector and Search for Supersymmetric Particle

Z. Phys. C43:91.1989: DESY+88-193:

The $K_{S} K_{S}$ Final State in $\gamma \gamma$ Interactions

Phys. Lett. 212B:515.1988: DESY-88-086:

An A nalysis of Multihadronic Events Produced with Two Energetic Leptons in $e^{+} e^{-}$Annihilation

Z. Phys. C 43:1 1989: DESY-88-192: LAL-R6-61:

An Experimental Study of $e^{+} e^{-}$Annihilation into Four Leptons at $\sqrt{8} \geq 35 \mathrm{GeV}$

Phys. Let t. 218B:403.1989: DESY-88-185:

Measurement of the Reaction $\gamma \gamma \rightarrow \rho^{+} \rho^{-}$with the CELLO Detector

2. Phys. C47: 1.1990: DESY-89-008:

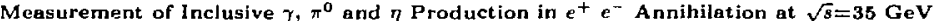

Z. Phys. C44:63.1989: DESY-89-019:

Model Dependent Limits on $\Lambda_{S}$ from $e^{+} e^{-}$Annihilation in the Energy Range from 14 to $48 \mathrm{GeV}$

Pliys. Lett 222B:16j3.1989: DESY-89-1)16;

$\tau^{ \pm}$Production and Decay with the CELLO Detector at PETRA

Z. Phys. C $12: 367.1989:$ DESY-84-149:

$K K_{K} \pi$ Production in Tagged and Untagged $\gamma \gamma$ Interactions

$Z$ Phys. Ca5:397.1900: IJESY-89.140:

Inclusive Strange Particle Production in $e^{+} e^{-}$Anrihilation

2. Phys. (46:583.1996). DES)'-89-177: $a_{2}(1320)$ and $\pi_{2}(1870)$ Formation in the feaction $\gamma+\rightarrow \pi^{+} \pi^{-} \pi^{0}$

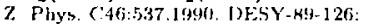

Exclusive $T^{ \pm}$Decay with the CELLO Detector at PETRA

DESY -84.176

Limits on Electron Compositeness from Bhaba Scattering

2. Phys (47:333.1900: DESY'-89-125:

Heavy Quark Charre Asymmetries with the CELLO Detector

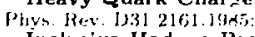

Inclusive Hedron Production in $Y$ Decays and in Nonresonant Electron-Positron Annihilation at 10.40 GeV 
Behrends 85B

Behrends 87

Beise 89

Bekmirzaev 85

Bekmirzaev 86

Bekmirzaev 87

Bekmirzaev 87B

Bekmirzaev $87 \mathrm{C}$

Bekmirzaev 88

Bekmirzaev 88B

Bekmirzaev 88C

Bekmirzaev 89

Bekrenev 86

Bekrenev 86B

Belenky 85

Belikov 83B

Belikov 85

Belikov 89

Belkacern 85

Bell 85

Bell $85 \mathrm{~B}$

Bell $85 \mathrm{C}$

Bell 85 D

Bell 86

Bell 86B

Beligardt $k$ it

Betlini 84

Bellotti 86

Bellotti 89

Bellotti $89 \mathrm{C}$

Bellotti $89 \mathrm{E}$

Bellotti 89F

Bellotti 89G:

Bellotti $89 \mathrm{H}$

Bekmyttsev $\mathrm{RH}$

Belestatsky $\mathrm{RA}$

Belousars $R H$
Phys Rev. 1D322468,1945:

New Determination of the Michel Parameter in $\tau^{ \pm}$Decay

Phys. Rev. Let1. 59:4117.1957.

$\Gamma(b \rightarrow u \ell v) / \Gamma(b \rightarrow c t v)$ from the End Point of the Lepton Momentum Spectrum in Semileptonic B Decays

Phys. Rev. Lett. 62:25933.1984t

Measurement of $\gamma^{18} \mathrm{O} \rightarrow{ }^{15} \mathrm{O} n$ at Medium Energies

JINR-P1-85-680;

Investigation of Multiplicity of $\pi$ Mesons, Produced in $n \mathbf{C}$ and $n$ Ta Interactions $₫$ it $P=4.2 \mathrm{GeV} / \mathrm{c}$

Yad. Phys. 44:405.1986: Investigation of Multiplicity of $\pi^{-}$Mesons Produced in $n \mathrm{C}$ and $n$ Ta Interactions at $4.2 \mathrm{GeV} / \mathrm{c}$

J1NR-PI-87.443:

Characteristics of Fast Secondary Neutrons in $p p$, and $p^{12} \mathrm{C}$ Interactions at $10 \mathrm{GeV} / \mathrm{c}$ Moment um

JINR-P1-87-652:

The Study of Fast Neutron Production $(p>1 \mathrm{Gev} / \mathrm{c})$ in $p \bar{p}$ and ${ }^{12} \mathrm{C}$ Interactions at $4.2 \mathrm{GeV} / \mathrm{c}$

Yad. Phys. 17: 1284.1988: JINR-P1-87-311:

Momentum and Angular Characteristics of $\pi^{-}$Mesons Produced in $n p, p p, n C$ and $n$ Ta Interactions at 4.2 GeV/c Momentum

Yad. Phys. 49:488.1989; JINR.E1-88-196:

Investigation of Momentum and Angular Characteristics of Protons Produced in $n \mathrm{C}$ and $n$ Ta Interaction at $4.2 \mathrm{GeV} / \mathrm{c}$ Momentum

JINR-P1-88-6]7.

The Study of Neutron Interactions with Protons and Carbon Nuclei at $4.2 \mathrm{GeV} / \mathrm{c}$

JINR-E1-88-192:

The Study of Fast Neutron Production $(p(n)>1 \mathrm{GeV} / \mathrm{c})$ in ${ }^{12} \mathrm{C} p$ and ${ }^{12} \mathrm{C}{ }^{12} \mathrm{C}$ Interactions at $P=4.2 \mathrm{GeV} / \mathrm{c}$ per Nucleon

Yad. Phys. 19:1030.1989: Sov. J. Nucl. Phys. 4y:637.1989:

Fast Neutron Production $(p(n)>1 \mathrm{GeV} / \mathrm{c})$ in $p p$ and $p^{12} \mathrm{C}$ Interactions at $P=4.2$ and $10 \mathrm{GeV} / \mathrm{c}$

LENI-86-1215:

Measurement of the Spin Rotation Parameters $R$ and $A$ in $\pi^{-} p$ Elastic Scattering at Energy $450 \mathrm{MeV}$

Pisma Zh. Eksp. Tuor. Fiz. 44·203.1986:

First Measurement of the Spin and a Revolving Parameter in Elastic $\pi p$ Scattering at the Low Energies of $\pi$ nucleon Resonances

Yad. Phys, 42:894.1985: Sov. J. Nucl. Phys. 42:567.1945:

Measurement of the Total Cross Section of $\bar{\nu}_{e} p \rightarrow n e^{+}$Reaction by Means of Detection of Neutrons at the Reactor of Rovno Atomic Power Plants

2. Phys, A320:625.1985: JFVE-83-156:

Quasielastic Neutrino and Intineutrino Scattering: Total Cross Sections, Axial Vector Forinfactor

Yad. Phys. 41:919.1985: Sov. J. Nucl. Phys. 41:589.1985:

Restrictions on Parameters of Oscillations of Muon Neutrinos from Quasielastic Scattering Data

IFVE-B9-167:

Preliminary Results of the 1989 Experiment on Prompt Muon Production in $70 \mathrm{GeV} / \mathrm{cp}$ nucleus Interactions

Phys Rev. Lett. 54:2667.1985: CERN-EP-85-18:

Measurement of the Total Energy Radiated by $150 \mathrm{GeV}$ Electrons in a Ge Crysta

Nucl. Phys. B254:475.1985; C'ERN-EP-84-131:

Momentum Distributions of Nuclear Fragments in He He Collisions at $E_{c m}=125 \mathrm{GeV}$

Z. Phys. C27:191.1985: CERN-EP-84-133:

Charged Particle Spectra in He He and He p Collisions at CERN ISR

Z. Phys. C.32:335.1986: CERN-EP-85-188:

Many-Particle Rapidity Correlations in Light Ion Interactions at the CERN ISR

Z Phys. A324:67.1985: C.ERN-EP-85-199:

Evidence for Direct $p p$ Interactions in the Fragmentation Channel $p \mathrm{He} \rightarrow p p \mathrm{X}$ at $E_{\mathrm{cm}}=88 \mathrm{GeV}$

Nucl Phyi. A325:7.1986: CERN-EP-86-15: CERN-EP-86-15-rev:

Nuclear Stopping Power in He He and deuteron deuteron Collisions

Z. Phys. C30:513.1986: CERN-EP-85-186:

High $p_{T}$ He He and He $p$ Interactions

Nucl. Phys. B299: 1.1988: SIN-PR-87-09; PR-87-09;

Search for the Decay $\mu^{+} \rightarrow e^{+} e^{+} e^{-}$

Sov J. Nucl. Phys A1:781.1945: Yad. Phys. 41:1223.1945; JlNR-El-84-280:

2- Resonances in the $\pi^{+} \pi^{-} \pi^{-}$Systems

Nuovo Cim. $95 \mathrm{~A}: 1.1986$

The Milano Experiment on Lepton Number Nonconservation in Double $\beta$ Decay of ${ }^{78}$ Ge

Phys. Lett. 221 B:209.1989:

A Search for Lepton Number Nonconservation in Double $\beta$ Decay of ${ }^{139} \mathrm{Xe}$

BL'HEP-89-29g:

Search for Stellar Collapse with the MACRO Detector at Gran Sasso

BLHEP-89-29e.

Study of the Primary Cosmic Rays at $E=10^{13} \mathrm{eV}$ to $10^{26} \mathrm{eV}$ by Simultaneous Observation of Extensive

Air Showers and Underground Muons at the Gran Sasso Laboratory

BL.HEP. $89-29 \mathrm{~d}$.

Multiple Muon Physics with the MACRO Detector at Gran Sasso

BL'HEP-89-29c:

Single Muon Physics with the MACRO Detector

BL'HEP-89-291;.

A Search for Magnetic Monopoles with the MACRO Detector at Gran Sasso

LEN I-8H-13:31.

On Measurement of the Correlation Coefficients in Neutron $\beta$ Decay with Ultracold Neutrons

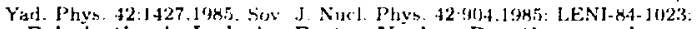

Polarization in Inclusive Proton-Nucleus Reaction $p$ nucleus $\rightarrow$ p $X$ at $1 \mathrm{GeV}$

Kr. Suctb. Fiz RH-7:21,194k.

Estimation of Resonance Parameters of $\Delta\left(1232 P_{33}\right)$ from the Data on Photoproduction of $\pi^{\circ}$ Mesons on 
Belousov 88 (cont i)

Beltrami 85

Beltrami 850

Belt rami 87

Beltrami 873

Beltramin 85

Belyaev 85

Belyaev 86

Belyaev 86B

Belyaev 88

Belyaey $88 B$

Belyare

Belyaev 88D

Belyate 89

Belyaer 89B

Belyaev 89(

Benayoun 86

Benayoun 87

Benayoun 87B

Bender $44 C^{\circ}$

Bensinger 8.5

Bensinger 88

Benvenuti 85

Benvenuti b6

Benvenuti 87

Benvenuti 87B

Benvenisti $87 \mathrm{C}$

Benvenuti $87 \mathrm{D}$

Benvenusi 89

Benvenuti $\mathrm{KgB}$

Benvenuti sge:

Berdnikov xis

Berdniken Hf; the ${ }^{12} \mathrm{C}$ Nuclei

Phys lin lett 5.1:1735.1965:

Öbservation of Tau Leptons Decay to Five Charged Particles

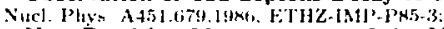

New Precision Measurenents of the Muonic $3 D(5 / 2) \rightarrow 2 P(3 / 2) X-$ Ray Transition in ${ }^{24} \mathrm{Mg}$ and ${ }^{24} \mathrm{Si}$ : Vacuum Polarization Test and Search for Muon-Hadron Interactions Beyond QED

Ply.i. Lett. 194B:326.1987:

Muon Decay: Measurement of the Integral Asymmetry Parameter

Helv. Phys. Acta (j):6ll.19hi:

Measurement of the Integral Asymmetry in $\mu$ Decay and Implication for the wino Mass

Nucl. Phys. A442:266.1985: DPPD-24-K4:

Proton ${ }^{3}$ He Elastic Scattering: A Phase Shift Analysis by a Separable Potentinl Model

Kir Soub. JINR 8:29.1985.

Ratio of Cumulative Pion Yields of Different Sign in Proton Nuclear Interactions at 17.5 up to $63 \mathrm{GeV}$ Proton Energy

Yad. Phys. 43:1469.14ktj: Sov. J. Nucl. Phys. 43:44.17.1986;

Measurement of the Polarization $H$ Parameter in Reaction $\gamma p \rightarrow \pi^{+} n$ with $320 \mathrm{MeV}$ Photons

Yád. Phys 14:289.1986:

Cross Section Asymmetry in the ${ }^{3} \mathrm{He}$ Two Particle Disintegration by Linearly Polarized Photons

Yad. Phys. 49:473,1989; JINR-P1-88-33;

Energy Deperdence of Pions Yield in Reaction $p \mathrm{C} \rightarrow \pi^{ \pm}$X for Proton with Energy from 15 to $67 \mathrm{GeV}$

JINR.Pl-88-34:

The Cross Section of Pions Production at an Angle of 159 Degrees for Interactions of Protons Having Energy from 15 to $61 \mathrm{GeV}$ with Carbon Nuclei

Kr. Soob. JINR 28:5,1988:

Cun.alative Proton Polarization in $p^{12} \mathrm{C} \rightarrow p \mathrm{X}$ Reaction for Incident Protons in 17 to $62 \mathrm{GeV}$ Energy Region

Kr. Soub. JINR 33:3K.1988:

A-dependence of Cumulative Pion Production Cross Sections in Proton Nucleus iateractions at High Energies

Kr. Soub. JINR 3.1:5,1984.

Measurement of Scattering Asynmetry of Cumulative Protons and Deuterons Emitted at Angle of 95 Degrees l.s. in $p$ C Interactions at Energies of Incident Protons from 17 to 62 GeV. Cumulative Proton Polarization

JINR-P1-89-112:

The Cross Section of $\pi^{+}$and $\pi^{-}$Production at an Angle of $15 \theta$ Degrees I.s. in Proton-Nuclear Interactions at the Energy of the Incident Proton from 15 to $65 \mathrm{GeV}$

JINR-P1-89-40:3:

Spin Effects in Cumulative Production of Protons and Deuterons in Proton-Nucleus Interactions at 18 $64 \mathrm{GeV}$

Phys. Lett. 183B:412.1967: CERN-EP-86-164:

Evidence for Higher Twist Mechanisms in Prompt $\rho^{0}$ Meson Production at $p_{\Gamma}>2 \mathrm{GeV} / \mathrm{c}$ in $300 \mathrm{GeV} / \mathrm{c} \pi^{-}$ n Interactions

DESY-K7-025: CDF-LPC'-87-099

Evidence for Prompt High pT $\eta$ Mesons at ISR

Phys. Lett. 198B:2א1.1987: CERN-EP-87-156:

Search for Glueballs at High $p$ in $300 \mathrm{GeV} / c \pi^{-} n$ Interactions

Phys. Rev. B31:1.1945: P(-B4-510: ILHEE-53: CM1-HF-B4-43: ANL-HEP-P'R-M4-08:

Study of Quark Fragmentation at 29 GeV: Global Jet Parameters and Single Particle Distributions

Nucl. Phys. B252:561.1985:

Measurement of Decay Parameters and Polarization in Inclusive $\Xi^{-}$Production from $K^{-} p$ Interactions

Phys. Lett. 215B:195.1984;

Upper Limit for the $\Xi^{0} \rightarrow \Sigma^{0} \gamma$ Radiative Decay

Phys. Lett. 158B:531.1985; CERN-EP-85-43:

Upper Limits on $D^{0} \bar{D}^{0}$ Mixing and bottom bottom Production from Muon-Nucleon Scattering

JINR-E1-86-591:

New Results on Nuclear Effects in Deep Inelastic Muon Scattering on Deuterium and Iron Targets

Phys. Lett. 195B:97.1987; CERN-EP-47-101. JINR-E1-87-699;

Test of QCD and a Measurement of Lambda from Scaling Violations in the Nucleon Struct ure Function $F_{2}$ at High $Q^{2}$

Plys. Lett. 1898:483.1987; JINR-E1-87-99; CERN-EP-87-13:

Nuclear Effects in Deep inelastic Muon Scattering on Deuterium and Iron Targets

Phys. Lett. 195B:01.1987: C'ERN-EP-87-100: JINR-E1-87-549:

A High Statistics Measurement of the Nucleon Structure Function from Deep Inelastic Muon Carbon Scattering at High $Q^{2}$

JINR-E1-87-689:

A High Statistics Measurement of the Proton Structure Function and Tests of QCD from Deep Inelastic Muon Scattering at High $Q^{2}$

Phys. Lett. 223B:485.19K9: JINR-E1-89-540: ('ERN-EP-89-106:

A High Statistics Measurement of the Proton Structure Function $F_{2}\left(r, Q^{2}\right)$ and $R$ from Deep Inelastic Muon Scattering at High $Q^{2}$

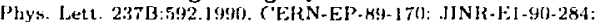

A High Statistics Measurement of the Deuteron Structure Functions $F_{2}\left(x, Q^{2}\right)$ and $R$ from Deep Inelastic Muon Scattering at High $Q^{2}$

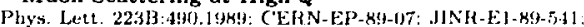

Test of QCD and a Measurement of $\Lambda_{S}$ from Scaling Violations in the Proton Structure Function $F_{2}(x$, $Q^{2}$ ) at High $Q^{2}$

Yad. Phys, 12:561.198:

Inelastic Scattering of $130 \mathrm{MeV} K^{+}$Mesons on Light Nuclei with Emission of Proton

Yard Plhys. $\{4: 272.1964:$ :

The $\left(\boldsymbol{K}^{+}, \boldsymbol{K}^{+} \boldsymbol{p}\right)$ Small Recoil Momentunı Transfer Reaction on Light Photoemutsiun Nuclei at $\boldsymbol{K}^{+} \mathrm{Mo}$ menta of $0.6 \mathrm{GeV} / r$ 
Berdzenishvi 85

Beretras 86

Berezhnoj 85

Berezin 86

Berge RT

Berge 89

Berger 85

Berger $85 \mathrm{~B}$

Berger $85 \mathrm{C}$

Berger 85D

Berger $85 \mathrm{E}$

Berger $85 F$

Berger 85G

Berger $85 \mathbf{H}$

Berger 86

Berger 86B

Berger $86 \mathrm{C}$

Berger 86D

Berger 87

Berger 87B

Berger $87 \mathrm{C}$

Berger 88

Berger 88B

Berger $88 \mathrm{C}$

Berger 89

Berger 89B

Berger 89C:

Bergsina $84 \mathrm{C}$

Bergsma 85

Bergsma 85B

Bergsma $85 C$

Bergsma 88

Bermon 86

Bermon 90

Bernard 85

Bernard 86

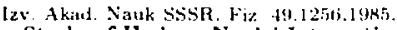

Study of Hadron Nuclei Interactions at Energy E>1 TeV

Phys Rev. D34 5i3.1986:

Praduction of the $\Xi^{0}$ and $\Xi^{0}$ Hyperons by $400 \mathrm{GeV}$ Protons

Yad. Phys. 41:1420,1985.

Spin Rotation Function of $500 \mathrm{MeV}$ Protons Scnttered by ${ }^{40} \mathrm{Cn} N$ uclei

yind Phys. $43: 358,1486$ :

Measurement of $K^{0}$ Total Cross Sections on Nuclei

2. Phys. Ci35:43.1987: CERN-EP-AT-0y:

Total Neutrino and Antineutrino Charged Current Cross Section Measurement in 100 , 160 and 200 GeV Narrow Band Beams

CERN-EP-89-103:

A Measurement of Differential Cross Sections and Nucleon Structure Functions in Charged-Current Neutrino Interactions on Iron

Z. Phys. C27:167.1985:

Sterman-Weinberg Jets and Energy Flow in $e^{+} e^{-}$Annihilation at c.m. Energies between 9.4 and 35 GeV

Z. Phys. C27:249.1985: DESY-84-098:

Tagged Two Photon Production of Muon Pairs

Z. Phys. C26:199.1985: DESY-84-074:

Pion Pair Production in Photon-Photon Interactions

Z. Phys. C.29:183.1985:

Study of the Reaction $\gamma \gamma \rightarrow 2 \pi^{+} 2 \pi^{-} \pi^{0}$ and Upper Limits on the Production of $\gamma \rightarrow 2 \omega$ and $\gamma \rightarrow \rho^{0} \omega$

Z. Phys. C27:341.1985:

Tests of the Standard Model with Lepton Pair Production in $e^{+} e^{-}$Reactions

Z. Phys. C28:1.1985:

Measurement of $\tau^{ \pm}-$Lepton Production and Decay

Z. Phys. C28:365.1985:

A Study of Energy Energy Correlations in $e^{+} e^{-}$Annihilations at $E=34.6 \mathrm{GeV}$

Z. Phys. C29:499,1985:

Jet Production at High Transverse Momenta by Interactions of Two Quasi-Real Photons

Phys. Lett. 167B:120.1986:

Evidence for Exclusive $\eta_{c}(15)$ Production in $\gamma \gamma$ Interactions

ANL-HEP-PR-86-17:

Deep Inelastic Lepton Scattering from Nucleons and Nuclei

Phys. Lett. 174B:118,1986:

Search for Muons from the Direction of Cygnus X-3

Nuovo Cim. 9C:350.1986:

Preliminary Results on Underground Muon Bundles Observed in the FREJUS Proton Decay Detector

Z. Phys. C33:351.1987. DESY-86-102:

Jet Production in Photon Photon Interactions

Phys. Rept . 146:1.1987:

Photon Photon Reactions

Nurl. Phys. B281:365.1987: DESY-86-068;

Measurement and QCD Analysis of the Photon Structure Function $F_{3}\left(x, Q^{3}\right)$

Z. Phys. C37:329.1988: DESY-87-104:

Tensor Meson Excitation in the Reaction $\gamma \rightarrow K_{S} K_{S}$

Z. Phys, C38:521.1988: DESY-87-173:

Measurement and Analysis of the Reaction $\gamma \gamma \rightarrow 2 \pi^{+} 2 \pi^{-}$

Phys. Rev. Lett. 61:919.1988:

Identifcation of deuteron $p \rightarrow{ }^{3}$ He $\eta$ Reaction very near Threshold: Cross Section and Deuteron Tensor Analyzing Power

Nucl. Phys. B313:509.1989: LAL-88-28:

Result from the FREJUS Experiment for Nucleon Decay Modes into $\bar{\nu}+$ megon

Pnys. Lett. 227B:489.1989: LAL-89-2:3:

Study of Atmospheric Neutrino Interactions with the FREJUS Detector

DPHPE-89-21

Search for Neutron-Antineutron Oscillations in the FREJUS Detector

Phys. Let t. 153B:111.1985: CERN-EP-84-143:

Evolution of Nucleon Structure Functions in the $Q^{2}$ Range between 20 and $10000 \mathrm{GeV}^{2}$ from a New QCD A nalysis of Neutrino Data

Phys. Lett. 157B:458.1985: CERN-EP-85-38:

Search for Axion-Like Particle Produc;ion in $400 \mathrm{GeV}$ Proton Copper Interactions

Phys. Lett. 157B:469.1985: CERN-EP-85-37:

Measurement of the Cross Section of Coherent $\pi^{\circ}$ Production by Muon Neutrino and Antineutrino Neutral-Current Interactions on Nuclei

C'EHN-EP-85-113:

A Precision Measurement of the Ratio of Neutrino Induced Neutral-Current and Charged Current Total Cross Sections

Z. Phys. C40:171,1988: CERN-EP-88.51:

A Search for Neutrino Oscillations

Phys. Rev. Lett. 55:1850.1986:

Flux Limit of Cosmic-Ray Magnetic Monopoles from a Fully Coincident Superconducting Induction Detector

Phys. Rev. Lett. 64:839.1990:

New Limit Set on Cosmic-Ray Monopole Flux by a Large-Area Superconducting Magnetic-Induction Detector

Phys. Lett. 169B:459,1986: ('ERN-EP-85-155:

Pseudorapidity Distribution of Charged Particles in Diffaction Dissociation Events at the CERN SPS Collider

Plyss. Lett. 171B:142.1986: ('ERN-EP-86-21.

Large-t Elastic Scattering at the CERN SPS Collider at $E_{c m}=630 \mathrm{GeV}$ 
Bernard N6B

Bernarij 87

Bertiardi 8.5

Bermatio 86

Bernardi ks

Bernasconi 87

Bernasconi 88

Bernstein 85

Berustein 84

Berrada 85

Berthet 8.5

Bertin 86

Bertin $\times 8$

Bertini Bt

Bertini 8.5

Bertini 88

Bertini $88 \mathrm{~B}$

Bertini $88 \mathrm{C}$

Bertini 89

Bertl 85

Beshliu 85

Beshliu 86

Besson 8.5

Besson 86

Beter 85

Betev 85B

Bethke 88

Bethke $88 \mathrm{~B}$

Bethke xy

Bethke 89B

Beusch 86

Beznogikh $x$

Bhadra 85

Bhanjá 85

Bhat tacharje $8 !$

Bhat tacharjo K! KJ

Bhat tacharge $x ! c^{\circ}$

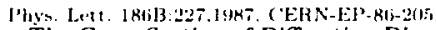

The Cross Section of Diffraction Dissocintion at the CERN SPS Collider

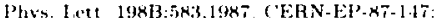

The Real Part of $\ddot{p} p$ Elastic Scattering Amplitude at Centre of Mass Energy of 546 GeV

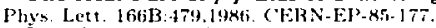
Search for Neutrino Decay

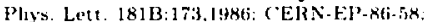

Anomalous Electron Production Observed in the CERN PS Neutrino Beam

Phys, Lett. 203B:3332.1988:

Further Limit on Heavy Neutrino Coupling

(CEN-EP-87-120):

Preliminary Results on Inclusive $\gamma$ Cross Section in $\bar{p} p$ Collisions at $E_{\mathrm{c} m}=24.3 \mathrm{GeV}$ from UA Experiment

Phys. Lett. 206B:16.3.1988:

Direct Photon Production in Proton-Antiproton Interactions at $\sqrt{8}=24.3 \mathrm{GeV}$

Phys. Rev. Letl 54:16:31.198:

Measurement of $\epsilon^{\prime} / \epsilon$ in the Neutral Kaon System

Phys. Rev. D:37 310:3.1988.

Search for New, Long-Lived, Neutral Particles

CERN-EP- $+55-47$

A Search for Hypernuclei Formation in $\ddot{p}$ Annihilation on Heavy Nuclei

Nucl. Phys. A4t3:589.1985

Very Backward $\pi^{0}$ and $n$ Production by Proton Projectiles on a Deuterium Target at Intermediate Energies CERN-EP-X6i-196:

Very Low Energy Pion Production at a $300 \mathrm{GeV}$ Proton Beam

Nuow C'im lout 305 .1988:

Low Energy Pion Yield at High Energy Accelerntors as a Function of the Primary Proton Beam Momentum

Phys. Lett. 15KB:19.1985: C.ERN-EP-4-159:

The $\left(K^{-}, \pi^{+}\right)$Strangeness Exchange Reaction on ${ }^{10} \mathrm{O}$

Phys. Lett. 16:2B:-7it.1985:

A Strong Energy Dependence of the Analyzing Power in the $p p \rightarrow$ deuteron $\pi^{+}$Reactions and the Question of an Isovector Dibaryon Resonance

Phys. Lett. 203B:18.1985:

Strong Energy Dependence of the Analysing Power in the $p p \rightarrow$ deuteron $\pi^{+}$Reaction and the Question of an Isovector Dibaryon Resonance. II.

DPH-N-SACLAY-2531B:

Spin Observables in $\bar{p} p$ Elastic and $p$ Inelastic Scattering

DPH-N-SACLAY-25.3TT:

Spin Observables in $\bar{p} \boldsymbol{n}$ Elastic Scattering

Phys. Let t. 228B:531.1959: CERN-EP-89-83:

Full Angular Distribution of the Analyzing Power in $\bar{p} p$ Elastic Scattering at $6 \theta 7 \mathrm{MeV} / c$

Niuct. Phys. B260:1.1985:

Search for the Decay $\mu^{+} \rightarrow e^{+} e^{+} e^{-}$

JINR-D]-85-4i33:

About the Nature of Narrow Structures in Effective Masses of Two Protons

Yad. Phys. 43:888.1986: Sov. J. Nucl. Phys. 43:565.1986:

Cross Sections of Neutron-Proton Interactions Channelg at Momenta of $1-5 \mathrm{GeV} / \mathrm{c}$

Phys. Rev. Lett. 5.t:381,1985:

Observation of New Structure in the $e^{+} e^{-}$Cross Section above the $\mathrm{Y}(4 S)$

Phys. Rev. Di3:300.1986:

Search for Monaenergetic Photons from $\mathrm{Y}(1 S) \rightarrow \gamma \mathrm{X}$

2. Phys ("24:9.1985: CEFN-EP-85-03:

Diferential Cross Section of High Mass Muon Pairs Produced by a $194 \mathrm{GeV} / \mathrm{c} \pi^{-}$Beam an a Tungsten Target

2 Phys. (28.15.1985: CERN-EP-85-04:

Observation of Anomalous Scaling Violation in Muon Pairs Production by $184 \mathrm{GeV}^{-c} \pi^{-}$Tungsten Inter. actions

Plys. Let1. 2]3B:235.1988: DESY-88-105:

Experimental Investigation of the Energy Dependence of the Strong Coupling Strength

L.BL.25247:

Jet Physics in $e^{+} e^{-}$Annihilation: Evidence for the Running of $\alpha_{5}$

Z. Phys C43.325 1989: SLAC-PLB-4944: LI3L-26957

Studies of Jet Production Rates in $e^{+} e^{-}$Annihilation at $E_{\mathrm{cm}}=29 \mathrm{GeV}$

Z. Phys. (43:331.1989:

An Experimental Approach to Optimize and 'I est Perturbative QCD to $Q\left(\alpha_{5}^{2}\right)$

C.ERT-EP-HG-68:

Praton-Antiproton Pair Production in $\pi^{ \pm}$Hydrogen and Nucleus Interactions at $30 \mathrm{GeV} / c$ and the Frmation Zone of Hadrons

JJNR-E2-8x-609:

Measurement of Vector Analyzing Power of Reactions deuteron (polarized) $\mathbf{C} \rightarrow \boldsymbol{P}$ and deuteron (polarized) $\mathrm{C} \rightarrow$ deuteron $\mathrm{X}$ at $800 \mathrm{MeV} / \mathrm{Nucleon}$

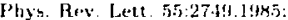

Study of Inclusive $K_{s,}, \Lambda$ and $\bar{\Lambda}$ Production in Diffractive $\gamma p$ Interactions

Phy's Rev. Latt. 54:Tit.19x:

Search for Anomalous Fragments in $1.8 \mathrm{~A} \mathrm{GeV}{ }^{40} \mathrm{~A}_{\mathrm{r}}$ Reactions in Nuclear Emulsions

Czech. I. Phys. Bi39.12,1939:

The Study of Cluster Phenomenon at Accelerator Energies

Phys Lett 225,B:t59.19Ko:

Empirical Regularity for Multiplicity Distribution in $\boldsymbol{\nu} n$ Interactions

Acti Ply Polon. B20:\$11 1:189

Scaling of Muitiplicity Distribution in $p n$ Interactions at High Energy 
Bhattacharje 90

Biagi 84

Biagi 85

Biagi 85B

Biagi 86

Biagi 86B

Biagi 87

Biagi 87B

Biagi $87 \mathrm{C}$

Biagi 87D

Bialkowska 8

Bienlein 88

Bigi 84

Biino 87

Bimbot 85

Bini 89B

Binkley 90

Bintinger 85

Binz 89

Binz 89B

Biunta $87 \mathrm{~B}$

Bionta $87 \mathrm{C}$

Bionta 88

Bird 88

Birman 88

Birsa 85

Bisello 86

Bisello 86B

Bisella 87

Bisello 88

Bisello $88 \mathrm{~B}$

Bisello 89

Bisello $89 \mathrm{~B}$

Bisello 90

Biswas 86

Bitsadze 85

Bitsadze 85B
Phys. Rev. D.419.1990: Multiplicity Distribution in $p$ n Interaction at $400 \mathrm{GeV} / \mathrm{c}$ and Its Empirical Regularity

Phys. Lett. I50B:230.1985: (CERN-EP-84-i6. Measurements of the Lifetime of the Chamed Strange Baryon $\Xi_{c}(2460)^{+}$

Z. Plys. (28:175.1985: (CERN-EP-84-154:

Properties of the Charmed Strange Baryon $\Xi_{r}(2460)^{+}$and Evidence for the Charmed Doubly Strange Baryon $\Omega_{r}$ at $2.74 \mathrm{GeV} / c^{2}$

Z. Phys. C28:495.1985: RAl-85-048:

A Measurement of the Branching Ratio $\Sigma^{+} \rightarrow p \gamma, \Sigma^{+} \rightarrow p \pi^{0}$

Z. Phys. C30:201.1980: C'ERN-PRE-85-07it:

First Measurement of the $\boldsymbol{A} \rightarrow n>$ Branching Ratio

Z. Phys. C31:33.1986: LGVA-DPNC-85-12-113: ('ERN-PRE-85-(1)5: First Observation of $\boldsymbol{\Omega}^{*}$ (unspec) Resonances

Z. Phys. C34:15.1987: CERN-PRE-810-095:

$\Xi^{-}$Resonances in $\Xi^{-}$Be Interactions. 1. Diffractive Production in the $\Lambda K^{-}$and $\Xi^{-} \pi^{+} \pi^{-}$Channels Z. Phys. C34:175.1987:

$\Xi^{*}$ Resonances in $\Xi^{-}$Be Interactions. 2. Properties of $\Xi\left(1820 D_{13}\right)$ and $\Xi(1950)$ in the $\Lambda \bar{K}^{0}$ and $\Sigma^{0} \bar{K}^{0}$ Channels

Z. Phys. C $34: 187.1987$

$\Xi^{-}$and $\Omega^{-}$Inclusive Production in $\Xi^{-}$Be Interactions at $116 \mathrm{GeV} / \mathrm{c}$

Z. Phys. C35:143.1987: CERN-PRE-86-112: First Measurement of the $\left(\Xi^{-} \rightarrow \Sigma^{-} \gamma\right) /\left(\Xi^{-} \rightarrow \Lambda \pi^{-}\right)$Branching Ratio

Phys. Let t. 17:3B:349.1986:

Transverse Momentum Correlations in $\mathrm{C} \mathrm{C}$ and $\mathrm{C}$ Ta Interactions at Momentum $4.2 \mathrm{GeV} / \mathrm{c}$ A

DESY- $88-165:$ : $C 88 / 08 / 29.2$ :

Observed and Unobserved States - Crystal Ball Results on Two-Photon Physics

Z. Phys. C27:303.1985: PITHA-84-19

Possible Implications of a Very Small Value of $\epsilon^{\prime} / \epsilon$

Phys. Rev. Let t. 58:2523.1987:

$J / \psi(1 S)$ Longitudinal Polarization from $\pi$ nucleon Interactions

Nucl. Phys. A $440: 636.1985$;

Inclusive $\left(p, \pi^{ \pm}\right.$) Reactions at $201 \mathrm{MeV}$ and $180 \mathrm{MeV}$

Phys. Lett. 221 B:99.1989:

A Sensitive Search for the Emission of a Neutral Particle in the Decay of the First Excited State in ${ }^{16} \mathrm{O}$

FERMILAB-CONF-90-28-E:

Log(s) Physics Results from CDF

Phys. Rev, Lett. 54:763.1985:

Measurement of the Total Hadronic Cross Section in Virtual Photon Photon Interactions

Phys. Lett. 231B:323.1989: FRE1-N1EP-89-02:

Measurement of the $\left(p(p o l), n\left(p a^{\prime}\right)\right)$ Inclusive Spin Transfer Parameters $K_{0 n n o}$ and $K_{0 k k 0}$ on Carbon with $590 \mathrm{MeV}$ Protons

FREI-MEP-89-03:

Measurement of the Total Cross Section Differences $\sigma_{L}(n p)$ and $\sigma_{T}(n p)$ in the Energy Range from 140 to $590 \mathrm{MeV}$

Phys. Rev. D36:30.1987

Underground Search for Muons Correlated with Cygnus X-3

Phys. Rev. Lett. 58:1494,1987;

Observation of a Neutrino Burst in Coincidence with Supernova 1987A in the Large Magellanic Cloud

Phys. Rev. D38: 768,1988 :

Contained Neutrino Interactions in an Underground Water Detector

SLAC-332;

Cerenkov Ring Imaging and Spectroscopy of Charged $K^{*}$ (unspec) Interactions at $11 \mathrm{GeV} / c$

Phys. Rev. Lett 61:1557,1988; BNL-41406: IUHEE-88-5:

Partial Wave Analysis of the $K^{+} \bar{K}^{0} \pi^{-}$System

Phys. Lett. 155B:437.1985: CERN-EP-85-28;

Polarization at Small Angles in Elastic $\bar{p} p$ and $\bar{p} \mathrm{C}$ Scattering at $550 \mathrm{MeV} / \mathrm{c}$

Phys. Lett. 179B:289.1986: LAL-86-18:

A Measurement of $\eta_{c}(1 S) \rightarrow \phi \phi$ in the Radiative Decay of the $J / \psi(1 S)$

Phys. Lett. 179B:294.1986: LAL-86-20:

Search of Glueballs in the $J / \psi(1 S)-\gamma \phi \phi$ Decay

Phys. Lett. 192B:239,1987: LAL-87-01;

Pseudoscalar $\omega \omega$ Production at Threshold in $J / \psi(1 S) \rightarrow \gamma \omega$ Decay

Phys. Let t. 200B:215,1988: LAL-87-34;

First Observation of the $\eta_{r}(15) \rightarrow 2 \rho^{0}$ Decay

Z. Phys. C39:13,1988

Study of Reaction $e^{+} e^{-} \rightarrow K^{+} K^{-}$in the Energy Range $1350<\sqrt{a}<2400 \mathrm{MeV}$

Phys. Rev. D39:701.1989; LAL-88-12;

First Observation of 'Three Pseudoscalar States in the $J / \psi(1 S) \rightarrow \gamma 2 \rho$ Decay

Phys. Lett. 2208:321.1989; LA L-88-47;

The Pion Electromagnetic Formfactor in the Time-Like Energy Range $1.35<\sqrt{s}<2.4 \mathrm{GeV}$

Phys. Let 241 B:617.1990; LAL-90-12:

Study of the $J / w(1 S) \rightarrow \gamma \phi \phi$ Decay

Pliys. Rev. D33:3167.1986:

Observation of $A$-dependence in Koba-Nielsen-Olesen Scaling Distributions for High Energy hadron nucleus Interactions

Phys. Lett. 167B:138.1986: J1NR-E1-85-6111.

New Upper Limit for the Branching Ratio of the $K_{S} \rightarrow e^{+} e^{-}$Decay

Nucl. Pbys. B260:497.1985:

Study of the Hypercharge Exchange Reactions $\pi^{+} p \rightarrow K^{+} \Sigma^{+}$and $\pi^{+} p \rightarrow K^{+} \Sigma\left(1385 P_{13}\right)^{+}$Reaction at $12 \mathrm{GeV} / c$ 
Bitsidze Hij

Bitsadze mob

Bitter 89

Bityukov 65

Bityukov $85 C^{\circ}$

Bityukov 86

Bityukov 86B

Bityukov 87

Bityukov 88

Bityukor 89

Bun ukov 90

Bizzeti 89

Bjorken 88

Blair 89

Blankleider 84

Blait 8.5

Blaylock 87

Blazey 85

Blecher 87

Blewitt 85

Blewitt 85 D

Blinov 84B

Blinov 85

Blinov 85B

Blinov $85 C^{\circ}$

Blinov 85D

Blinov $85 \mathrm{E}$

Blinoy 86

Blinov $86 \mathrm{~B}$

Blinov $86^{\circ}$

Blinov 47

Blinov $47 \mathrm{~B}$

Blinov n7s:

Blinov 88

Blinov KKH

Block 84

Blonitel an
Nincl. Phys. B270.7751.1987: JINR-E1-8(i-129:

$A$-dependence of $\eta$ Meson Inclusive Production at $10.5 \mathrm{GeV} / \mathrm{c}$

JINR-EI-86-7R0:

Differential Cross Sections for Reactions $\pi^{+} n \rightarrow k^{+} \Sigma^{0}$ and $\pi^{+} n \rightarrow K^{+} \Lambda$ and Ratio of Differential Cross Sections for Quasi-Binary Processes $\pi^{+}$nucleus $\rightarrow K^{+}$hyperon nucleus on Carbon and Deuterium Nuclei at $10.3 \mathrm{GeV} / \mathrm{c}$

Phys. Lett. 236B:951.1990: PD-1989-97.

A New Experimental Limit on Neutron-Antineutron Transitions

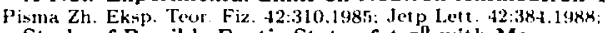
Study of Possible Exotic State of $\phi \pi^{0}$ with Mass near $1.5 \mathrm{GeV}$

IFVE-85-19:

Study of $f_{1}(1285) \rightarrow K^{+} K^{-} \pi^{0}$ Decay and $f_{1}(1285)$ and $f_{1}(1420)$ Mesons Production in Exclusive Reactions Induced by $\pi^{-}$and $K^{-}$Mesons at $32.5 \mathrm{GeV} / c$

Phys. Le :t. 188B:383.1987; IFVE-86-110; Study of a Possible Exotic $\phi \pi^{0}$ State with a Mass of abont $1.5 \mathrm{GeV} / \mathrm{c}^{2}$

Yad. Phys. 46:506.1986: IFVE-86-242: Observation and Study of Vector $C(1480)$ Meson Decaying into $\phi \pi^{0}$

Yad. Phys. 17:1258.1988; IFVE-87-155: Study of Light Mesons Radiative Decays

Phys. Lett. 203B:327.1988: Pisma 2 h. Eksp. Teor. Fiz. 45:368.1987: IFVE-ki-35: Observation of $f_{3}(\mathbf{1 2 8 5}) \rightarrow \phi \rightarrow$ Radiative Decay

IFIE-89-192:

Search for Rare Radiative Decay $f_{1}(1285) \rightarrow \rho \gamma$

IFV'E-90.'2:2.

Study of the Radiative Decay $\eta^{\prime} \rightarrow \pi^{+} \pi^{-} \gamma$

Phys. Rev: Lett 62:2901.1989: Senrch for a Composition-Dependent Fifth Force

Phys Rev. D38:3375.1988; FERM11LAB-PL:B-88.14 Search for Neutral Metastable Penetrating Particles Praduced in the SLAC Beam Dump

ANL-HEP-CP-H9-07: The CDF Direct Photon A nalysis

Plys. Rev. C31:1380.1985: FIAS-R-145: PRINT-84-0969-INDLANA: The Relationship between Partial Wave Ansplitudes and Polarization Observables in $p p \rightarrow$ deuteron $\pi^{+}$ and $\pi$ deuteron $\rightarrow \pi$ deuteron

Phys. Rev. Lett. 54:1628.1985;

Measurement of the $C^{P}$ Nonconservation Parameter

Phys. Rev. Letl. 58:2171.1987: SLAC-PlB-1158:

Observation of $e^{+} e^{-} \rightarrow D_{S}^{ \pm} \mathrm{X}, D_{S}^{*} \mathrm{X}$ at $E_{\mathrm{cm}}=4.14 \mathrm{GeV}$

Phys. Rev. Lett. 55: 1820.1985:

Hard Scattering with Exclusive Reactions: $\pi^{-} p$ Elastic Scattering and $\rho$ Meson Production

TRI-PP-87-23:

Search for Muon Positron Conversion

Phys. Rev. Lett. 55:2114,1985:

Experimental Limits of the Free Proton Lifetime for Two- and Three-body Decay Modes

CALT-68-1327:

A Search for Free Proton Decay, and Nuclcon Decay in ${ }^{t} \mathrm{O}$, Using the Invariant Mass and Moment um of Exclusive Final States

Jour. of Pliys. G 11:623,1985; ITEP-84-40:

Study of Nuclear Vertex Function and Nuclear Vertex Constant of Decay ${ }^{3} \mathrm{He} \rightarrow p$ deuteron from Data on ${ }^{3}$ He $p \rightarrow p p$ deuteron Reaction at the 2.5 and $5 \mathrm{GeV} / \mathrm{c}^{3} \mathrm{He}$ Nucleus Moment um

Yad. Phys. 41:719.1985: Sov. J. Nucl. Phys. 41:457.1985: ITEP-84-93:

Main Characteristics of the Reaction ${ }^{3} \mathrm{He} p \rightarrow 3 p n$ ot the Incident ${ }^{3} \mathrm{He}$ Moment um of $2.5 \mathrm{GeV} / \mathrm{c}$

NOVO-8, 59 -99:

Search for Narrow Resonances in $e^{+} e^{-}$Collisions in the Mass Region $7.2-10 \mathrm{GeV}$

Yad. Phys. 44:626,1986: Sov. J. Nucl. Phys. 44:405.1986; NOVO-\$5-96:

Two Photon Production of $e^{+} e^{-}$Pairs with Small Invariant Massea

Yad. Phys. 41:1440,1985: Sov. J. Nucl. Phys. 41:913.1985: ITEP-85-60:

Study of Mechanism of the ${ }^{3} \mathrm{He} p \rightarrow 2 p$ deuteron Reaction at ${ }^{3} \mathrm{He}$ Momentum of $5 \mathrm{GeV} / c$

NOVO-85-95:

Preliminary Results of the Experiment with MD-1 Detector on Two-Photon Production of Muon Pairs and Hadrons

Yad. Phys. 45:619.1987: ITEP-86-65:

Measurement of Nuclear Vertex Function of ${ }^{3} \mathrm{He} \rightarrow p p \mathrm{n}$ from the Reaction ${ }^{3} \mathrm{He} p \rightarrow 3 p \mathrm{n}$ at the Incident ${ }^{3}$ He Moment um of 2.5 and $5 \mathrm{GeV}$

NOVO-86-110:

Upper Limit for the Two Photon Width of a Neutral Particle with the Mass of $1.8 \mathrm{MeV}$

NOVO- $66-107$ :

Upper Limit for a Two-Photon Width of $\eta_{c}(15)$

Yad. Phys. 45:10138, 1987

$e^{+} e^{-}$Pairs Production by a Synchrotron Radiation Photon on a Counter Beam Electron

ITEP-87-76:

Charged Exchange Quasielagtic React ion $p^{3} \mathrm{He} \rightarrow n p p p$ at the Medium Energies

NOVO-87-92:

An Experiment on Measurement of Two Photon Width of the $\eta^{\prime}$ and $a_{2}(1320)$

Yad. Phys. 47:93:3.1!58:

Quasielastic Charge-Exchange Reaction $p^{3} \mathrm{He} \rightarrow n p p$ at Intermediate Energies

Yad. Phys. 47:889.1948:

Limit on the Two Photon Width of the Particles with the Mass of $1.8 \mathrm{MeV} / \mathrm{c}^{2}$

Kev. of Mlorl. Phys. 57:563.1985: LB1-17522:

High Energy $p \bar{p}$ and $p p$ Forward Elastic Scattering and Total Cross Sections

2. Phys ('.15:361 19901:

Electroweak Parameters from a High Statistics Neutrino Nucleon Scattering Experiment 
Bloom 8.5C

Biumenfeld 89

Blumer 85

Bock 85B

Bock 89

Buck 89B

Bocquet 86

Bocquet 87

Bodek 89

Bodenkamp 8.5

Boehm 86

Bofill 87

Bagdanov 88

Bagert 85

Bogen 85B

Bogerz 86

Bogolyubsky $84 \mathrm{~B}$

Bogolyubsky 84D

Bogolyubsky 86

Bogolyubsky 86B

Bogolyubsky 86C

Bogolyubsky 86D

Bogolyubsky $86 \mathrm{E}$

Bogolyubsky 86F

Bogolyubsky 86C

Bogolyubsky $86 \mathrm{H}$

Bogolyubsky 87

Bogolyubsky 87B

Bugolyubsky $87 \mathrm{C}$

Bogolyubsky 87D

Bogolyubsky $87 \mathrm{E}$

Bugolyubsty 88

Bogolyubsky 8RB

Bogolyubsky 88C:

Bugolyubsky 88E

Bogolyubsky $88 \mathrm{~F}$
SIAC-PCB-386.4: CN5: (17:26):

Recent resuits from DORIS-II

Phys. Rev. Let1. 62:2237.1984: Search for $\nu_{\mu} \rightarrow \nu_{\mathrm{r}}$ Oscillutions

Phy's Let 1. 161 B:407.1985.

Experimental Constraints on Lepton Mixing Angles and Neittrino Mass Differences for Threc Simultaneously Oscillating Neutrino Flnvors

BONN-IR-85-14:

Untersuchung der Reaktion ๆ deuteron $\rightarrow p p \pi^{-}$nuf Mogliche Dibaryon-Zwischenzustande

GSI-89-12:

Source Parameters Deduced from Bose-Einstein Correlations of Two and Three Soft Pions in Symmetric Heavy Ion Interactions et $\mathrm{B50} \mathrm{A} \mathrm{MeV}$

Mod. Phys. Lett. A3:1745.1989;

Bose-Einstein Correlations of Positive Pions in Col'isions of $\mathrm{Nb}+\mathrm{Nb}$ and $\mathrm{Au}+\mathrm{Au}$ at $650 \mathrm{~A} \mathrm{MeV}$

Phys. Lett. 182B:146.1986: CERN-EP-86-95:

Observation of the Decay of Heavy Hypernuclei

Phys. Lett. 192B:312.1987: CEAN-EP-B7-11;

Delayed Fission from the Antiproton Annihilation in ${ }^{200} \mathrm{Bi}$, Evidence for Hypernuclear Decay

LR-1120: ER-13065-587:

A Comparison of Quark and Gluon Jets Produced in 3 Jet $e^{+} e^{-}$Annihilation Events

Nucl. Phys. B255:717.1985;

Measurement of the Reaction $\neg p \rightarrow p \bar{p} p$ at $4.7 \leq E \leq 6.6 \mathrm{GeV}$

Nucl. Phys. A434:451C.1985;

Introduction to Neutrino Mass

Phys. Rev. D36:3309.1987: FERMIILAB-PLB-87-33-E:

Limits on $\nu_{\mu} \rightarrow \nu_{\mathrm{r}}$ and $\nu_{\mu} \rightarrow \nu_{e}$ Oscillations

Yad. Phys. 47:316.1988:

Topological Characteristics of ${ }^{12} \mathrm{C}$ and ${ }^{22} \mathrm{Ne}$ Fragmentation on Nucleons

Phys. Rev. Lett. 55:574.1985:

Determination of the Nuc. . Structure by Means of the Weak Neutral Current

Phys. Rev. Lett. 55:1969.1985:

Determination of $\sin ^{2} \theta_{W}$ and $\rho$ in Deep Inelastic Neutrino Nucleon Scattering

FERMILAB-CONF-85-108-E:

Determination of the Nucleon Structure Using the Weak Neutral Current

Yad. Phys. 41:1210.1985; Sov. J. Nucl. Phys. 41 773.1985: IFVE-84-140:

Study of Elastic $\bar{p} p$ Scattering at $32 \mathrm{GeV} / c$

Yad. Phys. 41:105.1985: Sov. J. Nucl. Phys. 41:66.J985; JFVE-84-52;

Separation of Clusters in Multidimensional Phase Space for the Reaction $\mathrm{K}^{-} \mathrm{p} \rightarrow \mathrm{K}^{-} \mathrm{p} \pi^{+} \pi^{-}$at $32 \mathrm{GeV} / \mathrm{c}$

Yad. Phys. 44:979,1986; Sov. J. Nucl. Phys. 44:631,1986; 1FVE-86.53;

Study of Correlation Between the Transverse Momenta of Secondary Hadrons in $\bar{p} p$ Interactions at 32 $\mathrm{GeV} / \mathrm{c}$

Yad. Phys. 43:1 199.1986: Sov. J. Nucl. Phys. 43:768,1986:

One-particle Inclusive and Semi-inclusive Spectra of $\Lambda$ Hyperons in $\ddot{p} p$ Interactions at $32 \mathrm{GeV} / \mathrm{c}$

Yad. Phys. 43:350,1986;

Cross Sections of Exclugive Reactions in $\bar{p} p$ Interaction, at $32 \mathrm{GeV} / c$

Yad. Phys. 45:1667.1987: IFVE-86-156:

Charge Priperties of Annihilation Exclusive Reactions in $\bar{p} p$ Interactions at $32 \mathrm{GeV} / \mathrm{c}$

Yad. Phys. 47:1027.1988; IFVE-86-219;

Study of Diffractive Processes in Exclusive Channel $\bar{p} p \rightarrow \bar{p} p 2 \pi^{+} 2 \pi^{-}$at $32 \mathrm{GeV} / \mathrm{c}$

Yad. Phy's. 43:907.1986:

Analysis of Elastic $\vec{p} p$ Scattering in the Impact Parameter Representation for the Energy Interval of 10 $\mathrm{GeV}-100 \mathrm{GeV}$ and the Comparison with the $p$ p Data

Yad. Phys. 41:1201.1986: Sov. J. Nucl. Phys. 4-1:780,1986:

Study of Leading Prr perties of Protons and Antiprotons from $\ddot{p} p$ Exclusive Reactions at $32 \mathrm{GeV} / \mathrm{c}$

IFVE-86-220:

Study of Inclusive and Semi-inclusive Transverse Momentum Distributions of the Charged Hadrons in $\bar{p}$ $p$ Interactions at $32 \mathrm{GeV} / c$

Yad. Phys. 46:522.1987;

Cross Sections of Particle Diffractive Production in $\bar{p} p$ Interactions at $32 \mathrm{GeV} / c$

Yad. Phys. 48:448.1988: Sov. J. Nucl. Phys. 48:282,1988: IFVE-87-154:

A Study of the Proton Diffractive Structure in Reaction $\bar{p} p \rightarrow \bar{p}\left(p 2 \pi^{+} 2 \pi^{-}\right)$at $32 \mathrm{GeV} / c$

Sov. J. Nucl. Phys. 48:467.1988; Yad. Phys. 48:733.1988: IFVE-87-167;

Study of $K_{S}$-mesons Inclusive Production in $\bar{p} p$ Interactions at $32 \mathrm{GeV} / \mathrm{c}$

Yad. Phys. 46:811.1987:

Analysio of Multiparticle Correlations in $\bar{p} p$ Interactions at $32 \mathrm{GeV} / \mathrm{c}$ by Filled Rapidity-interval Method

Yad. Phvs. 46:1680.1987:

Study of Multiplicity of Charged Particles in $p p$ and $\bar{p} p$ Interactions at $32 \mathrm{GeV} / c$

Yad. Phys. 17:142.1988:

Dependence of Characteristics of $\bar{p} p$ Interactions at $32 \mathrm{GeV} / \mathrm{c}$ upon Nature and Energy of the Leading Particles

Yad. Plyys. 47:401.1988:

Baryon Number, Strangeness and Electric Charge Distrib tion in $\bar{p} p$ Interactions at 32 GeV/c in Event with Leading Baryons

Yard Phys 47:712.1:34k:

Study of r.telusive and Semi-inclusive Transverse Moment um Distrihutions of the Charged Hadrons in $\bar{p}$ $p$ Interactions at $32 \mathrm{GeV} / c$

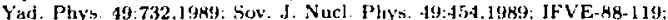
Inclusive Production of $\gamma$ Quanta and $\pi^{\circ}$ Mesons in $\bar{p} p$ Interactions at $32 \mathrm{GeV} / c$

Yad. Phys. 50:6\$3.1989: IFVE-89.5:

Study of Neutral Strange Particle Production in $p$ Interactions at $32 \mathrm{GeV} / c$ and Comparison with $\bar{p} p$ Data 
Bogulyubsky \&\&G

Bogulyubsky :9B

Bogolizubsky 89D

Bohm \&4E

Bokeneyer ss

Bokemeyer 89

Boldea 85

Bologna 85

Bolonkin 86

Bolonkin 87

Bolonkia 88

Bolonkin 89

Bolotor 85

Bolotov 85B

Belotor 85C

Bolotor $\$ 6$

Boloto: B6B

Bolotov 87

Bolotov 88

Bolton 86

Bolton 68

Bond $28 \mathrm{~B}$

Bond 89

Bondar 84

Bondarenko 37

Bondarenko $87 \mathrm{~B}$

Bopesinı 8\%

Bonesin: 68

Bonesin! $88 B$

Bonesini 89

Bonesini 89B

Bonin s6

Bonino 88

Bonneaud 86

Bonner 87

Bonner 88

Bonner 88B

Bonner 89

Bonnetbidaud 88

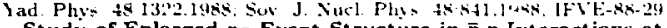

Study of Enlarged $p_{T}$ Event Struct ure in $\bar{p} p$ Interactions at $32 \mathrm{GeV} / \mathrm{c}$

Yad Phys. 49:436.1989. Sov J. Nucl Phy: 49.272.19\%,

Mean Multiplicities and Mean Energies of Particles in Diffraction Clusters in 32 GeV $\bar{p} p$ Interartions

rad. Phys 50:115.1989. Sov. J. Sucl Phys int-3.19k9.

Angular Distributions of Secondaries in Diffractively Produced Systems in $32 \mathrm{GeV} / c \bar{p} p$ Interactions

Phys. Res. D31:3005.1955. DOE-ER-0309:-5:-2.

The New Value for the Electron Asymmetry in $\Sigma^{-}-n c^{-} \bar{\nu}_{c}$ and Hyperon Semileptonic Decays

GSI-88-68.A:

Narrow Positron Line Emission in Collisions of Very Heavy lons

GSI-89-49:

Gorrelated Flectron-Pasitron Emission in Heavy-lon Collisions

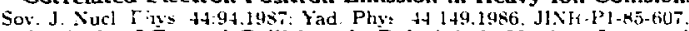

A nalysis of Central Collisions in Relativistic Nuclear Interactions

Nuovo Cin. BC: 76.1985 .

Primary Cosmic Ray Spectrum at Energies Approximately $10^{13} \mathrm{eV}-10^{10} \mathrm{eV}$ from Multiple Muon Events in NUSEX Experiment

Yad. Phys. 43:1211.1986: Sov. J. Nucl. Phys 43:-76.19s6.

Study of the Reaction $K^{-} p-K_{S} K_{S}$ strange at $40 \mathrm{G} \cdot \mathrm{V} / \mathrm{C}$

ITEP-ST-52:

Study of the $f_{2}(1720)$ and $f_{2}(2300)$ in the Reaction $\pi^{-}, h_{S} K_{S} n$ at the $40 \mathrm{GeV} / c$

Tucl. Phys. B309:426.198s. Yad. Phys 46:-99.1987:

$f_{2}(1 ; 20)$ and $f_{2}(2300)$ Resonances in the $\pi^{-} p \rightarrow K_{5} K_{V}$, Reaction at Momentum $40 \mathrm{GeV} / \mathrm{c}$

ITEP-R9-149:

$T=$ Study of the Reaction $K^{-} p \rightarrow \Lambda \bar{\Lambda} \gamma^{*}$ (unspec) at the $40 \mathrm{GeV} / c$ Mlomentum

Yad. Phys. 4t:117.1986: MINR-85-P-0441.

Investigation of $\mathrm{K}^{-} \rightarrow \pi^{-} \pi^{0} \pi^{\circ}$ Decay

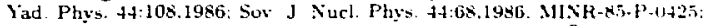

Experimental Investigation of Rare Mode $K^{-} \rightarrow \pi^{0}>e^{-} \nu_{e}$. and $K^{-} \rightarrow \pi^{0} \pi^{0} e^{-} \bar{\nu}_{c}$

Pisnaa Zh. Ekop Teor. Fiz 42:390.1985. Jetp Lett 42:4k1,19k5.

Observation of $\mathrm{K}^{-} \rightarrow \pi^{-} \pi^{0} \pi^{0}$, Decay

Pisma Zh. Ehsp. Teor. Fiz, 43 405.19s6:

Experimental Limitation of the Probability of $\pi^{0} \rightarrow$ 4) Decay

Yad. Phys 45:1652.1987: MINR-86-P-0486:

Experimental Investigation of Structure Radiation in $\boldsymbol{K}^{-} \rightarrow \pi^{-} \pi^{0}$, Decay

MINR-8T-P-0510:

About Improvement of Experimental Limitation on the Probability of $K^{-}-\pi^{-}$?

Pisma Zh. Eksp. Teor. Fiz. ti *i.1988:

Measurement of Decay's Formfactor for $K^{-}-\pi^{0} e^{-}$

Phys. Rev. Lett. 56:2461.1986: L.A-L'R-86-446:

Search for the Decay $\mu^{+}-e^{+}$,

Phys. Rev. D3s:2077.1988:

Search for Rare Muon Decays with the Crystal Box Detector

Phys. Rev. Lett. 60:1110.1988: KEK-8T-11ki ICR-REPORT-154-8i-8:

Search for Gamma Rays from Supernova 1987A at Energies Greater than $100 \mathrm{TeV}$

Phy = Rev Lett 61:2292.1989: ICR-REPORT-161-88-T: KEK-h8-i:

Search for TeV Gamma Rays from SN 1987 A December 1987 and January 1988

Pisma Zh Eksp Teor. Fiz. 40.40.1984: Jetp Lett 40:1265.1488:

Search of Light Particles with Charge $=2 / 3$ in $t^{+} e^{-}$Annihilation

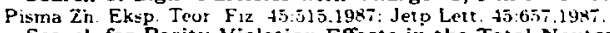

Search for Parity Violation Efects in the Total Neutron Cross Section of ${ }^{293} \mathrm{U}$ Nuclei

Jetp Lett 46:279.1987. Pisma Zh Eksp Teor. Fiz. 46.222.198-

Violation of Spatial Parity in the Total Cross Section for Interaction of Thermal Neutrons with ${ }^{250} \mathrm{Pu}$ Nuclei

2. Phys. C3T 39.19\%T: CERT.EP-8T-164

High Transverse Momentum $\pi^{\circ}$-production by $\pi^{-}$and $\pi^{+}$on Protons at $280 \mathrm{GeV} / \mathrm{c}$

2. Phys C $35.535 .1988:$ CERN-EP-85-185.

High Transverse Momentum Prompt Photon Production by $\pi^{-}$and $\pi^{-}$on Protons at $280 \mathrm{GeV} / \mathrm{C}$

Z. Phys. C38:3i1 1.198s.

Production of High Trarsverse Momentum Prompt Photons and Neutral Pions in Proton-Proton Interactions at $280 \mathrm{GeV} / \mathrm{c}$

2. Phvs. C42:527.1989: C'ERN-EP-8x-17*.

High Transverse Moment um $\eta$ Production in $\pi^{-} p, \pi^{+} p$ and $p p$ Interactions at $280 \mathrm{GeV} / c$

Z Phys. C:1:11.1989.

The Structure of Events Triggered by Direct Photons in $\pi^{-} p, \pi^{+} p$ and $p$ p Collisions at $280 \mathrm{GeV} / c$

Nucl. Phys A+45:341.1985.

He Nucleus Elastic Scattering at Intermediate Energies

Phys. Lett. 211B:239.198B.

Evidence for Transverse Jets in High-Mass Diffraction

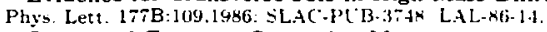

Quasi-real Compton Scatering Measurement and $e^{*=}$ Search with DELCO at PEP

Phvs. Rev. Lett. 58:447.1987.

Spin Transfer in Hyperon Production

Why's. Rev. D38:729.1988:

Spin-Parameter Measurement in $\Lambda$ and $\kappa_{;}$Production

Phy. Rev. Lett. 61.191木.19*\$. ANL-HFP.PH-Mg-11.

Analyzing Power Measurement in Inclusive $\pi^{0}$ Production at $H$ igh ${ }_{F} F$

Phys. Rev Lett. 62.1591.19k!

Spin-Parameter Measurement in Inclusive $\Sigma^{0}$ Production

Phys. Rept. 170:325.19ks.

Cygnus X-3, a Critical Review 
Bonvin 89

Bonvin 90

Boos 85

Boos 86

Boos $86 \mathrm{~B}$

Bous 86C

Boos 87

Boos $87 B$

Boos 88

Boos 88B

Boos 88C

Boos 89

Booth 85

Booth 86

Bop: 86

Bordalo 87

Bordalo 87B

Bordalo 88

Boris 85

Boris 87

Boris $87 \mathrm{~B}$

Borisov 84

Borisov 85

Borisov 858

Borisov $85 \mathrm{C}$

Borisov 85D

Burisoy 86

Borisov 87

Borisov $87 \mathrm{~B}$

Borkursky 84

Bortuletto 88

Bortoletto 89

Burtoletto 69B

Burtoletto 90

Burzakov 8 i

Borzatov 90
Z. Phys. C41:59I.1989;

Double Prompt Photon Production at High Transverse Momentum by $\pi^{-}$on Protons at $280 \mathrm{GeV} / \mathrm{c}$

Phys. Lett. 236B:523.1990: CERN-EP-140-07.

Intrinsic Transverse Momente in the $\pi^{-} p \rightarrow q, \times$ Reaction at $280 \mathrm{GeV} / \mathrm{c}$

ALMA-85-04:

Angular and Energy Characteristics of Secondary Charge Particles from $\ddot{p} p$ Interactions at $22.4 \mathrm{GeV} / \mathrm{c}$

Yad. Phys. +3:105,1986: Compatible Analysis of Antiproton and Proton-Nucleon Interactions

Yad. Phys. 43:116.198ti: Sov. J. Nucl. Phys. 43:74,1986:

Hadron Deuteron Double Scattering Effects and Their Dependence on Incident Energy

ALMA-86-11:

Specifics of Angular Distributions of Secondary Particles in Proton Interactions, Accompanied by Total Breakdown of Nuclear Targets

Yad. Phys, 46:540,1987: JINR-P1-86-520

Production of Backward Protons with Momenta 0.2 - $0.5 \mathrm{GeV} / \mathrm{c}$ in Lab System Which Are Generated in $\bar{p}$ nucleus Interactions at $\mathbf{4 0} \mathrm{GeV} / \mathrm{c}$

JINR-E1-8T-398:

Charged Particle Spectra in $\pi^{-} p, \pi^{-}$deuteron and $\pi^{-}$C Interactions at $38 \mathrm{GeV} / \mathrm{C}$ with Single-Particle High $p_{T}$ Trigger

ALMA-88-04;

Investigation of Total Destruction of the Atomic Nuclei in the Inelastic Hadron Nucleus Interactions at the $40 \mathrm{GeV} / \mathrm{c}$

ALMA-88-16:

Investigation of Multiparticle Correlations in the Range $200-400 \mathrm{GeV}$

Yad. Phys 48:1005.1988:

Study of Diffrective Production of Hadron Systems in $p$ Interactions at $69 \mathrm{GeV} / \mathrm{c}$

ALMA-89-01:

Investigation of Exclusive Reaction with Six Charged Particles in $\vec{p} p$ Interactions at $22.4 \mathrm{GeV} / \mathrm{c} \mathrm{Momen-}$ tum

Nucl. Phys. B273:67T.1986: CERN-EP-85-138

A High Statistics Study of the $\phi \phi$ Mass Spectrum

Nucl. Phys. B273:689.1986:

Anmular Correlations in the $\phi \phi$ System and Evidence for Hadronic $\eta_{c}(1 S)$ Production

Phys. Kev. Lett. 56:919,1986: Phys. Rev. Lett. 57:1192,1986; HD-P'-86-04:

The $\beta$ Decay Asymmetry of the Neutron and Ga/Gv

Phys. Lett. 193B:368.1987: CERN-EP-87-67:

Nuclear Effects on the Nucleon Structure Functions in Hadronic High-Mass Dimuon Production

Phys. Lett. 193B:373.1987: CERN-EP-87-68:

Observation of a Nuclear Dependence of the Transverse Momentum Distribution of Mussive Muon Pairs Produced in Hadronic Collisions

2. Phys. C $39: 7,1988$ :

Open Besuty Production in High Energy $\pi^{-}$Tungsten Interactions

Pisma Zh. Eksp. Teor. Fiz. 42:107.1985: Jetp Lett, 42:130.1985: Phys. Lett. 159B:217.1985:

The Neutrino Mass from the Tritjum $\mathcal{A}$ Spectrum in Valine (ITEP-84)

Pisma Zh. Eksp. Teor. Fiz. 45:267.1987:

The Neutrino Mass from the Tritium $\beta$ Spectrum (ITEP-86)

Phys. Rev. Lett. 58:2019.198ĩ : Phys. Rev. Lett. 61:245.1988:

Neutrino Mass from the $\beta$ Spectrum in the Decay of Tritium

Sov. J. Nucl. Phys 41:74,1985; Yad. Phy's. 41:116.1985: JINR-P1-84-3!i

Measurement of Polarization in the $\pi^{-} p \rightarrow \pi^{0} n, \pi^{0} \rightarrow 2 \gamma$ Reaction at $40 \mathrm{GeV} / \mathrm{c}$ with the Registration of All Reaction Products

Izv. Akad. Nauk SSSR. Fiz. 49:1278,1985:

Jet Phenomenon in Giant Superfanily

Izv. Akad. Nauk SSSR. Fiz. 49:1282, 1985:

General Properties of Hadronic Families

lav. Akad. Nauk SSSR, Fiz. 49:1285.1985:

On Halo's Struct ure of Great Gamma-Families

Bull. of Ac. of Sci. LSSR Phys. Ser. 49:42.1985: Izv. Akad. Nauk SSSR. Fiz. 49:1288.1985:

Interaction Range of Hadrons with Energies Above $20 \mathrm{TeV}$ in Lead. Pamir Collaboration

Pisma Zh. Eksp. Teor. Fiz. 43:559.1986:

The Measurements of the Transmission's Polarization Parameter $K_{\text {noon }}$ in $p$ Scattering for Energies of $800-970 \mathrm{MeV}$

Kr. Soob. JINR 21:40.1987:

Investigation of the Possibility of Neutron Electric Charge Measurement by Means of Ultracold Neutrons

Phys. Lett. 190B:226,198T:

Observation of a High Energy Cosmic Ray Family Caused by a Centauro Type Nuclear Interaction in the Joint Emulsion Chamber Experiment at the Pamir

Jour. of Phys. G 11:69.1985; LENI-84-918;

Measurement of the Differential Cross Sections of the $\pi^{+}$deuteron $\rightarrow p$ Reaction at 280, 300, 330, 357, 390, 420 and $450 \mathrm{MeV}$ Pion Energies

Phys. Rev. D37:1719.1988: Phys. Rev. D39:1471.1989:

Charm Production Nonresonant $e^{+} e^{-}$Annihilation at $\sqrt{s}=10.55 \mathrm{GeV}$

Plyst. Rev Letr. 62:2430.1989: CLNS-89-687: CLEO-89-3:

A Search for $b \rightarrow$ u Transitions in Exclusive Hadronic B Meson Decays

Phys. Rev Lett 133:1607.1989:

Study of the Decays $\bar{B}^{0} \rightarrow D^{*}(2010)+\ell^{-}$

('LEO-90-1:

Exclusive and Inclusive Decays of $B$ Mesons into $D_{S}^{ \pm}$Mesons

Yad Phys $76: 16011.19 \times 7$

Megsurement of $e^{+} e^{-}$Pair Emission Probability in Thernal Neutron Capture

JINR.P15-90-60

i Search for 180 Degrees Correlated Pairs of Gamma-Quanta in ${ }^{4}$ He-Decay of ${ }^{238} \mathrm{Pu}$ 
Boschitz 86

Buschitz 86

Busenti 90

Bussingham 89

Bosted 89

Boswell \&6

Botnet 89

Boucher $8 x$

Bouchez $8 \mathrm{H}$

Boudard 88

Bougault 90

Bourdarios 88

Bourquin 86i

Buttemeur $8 \mathrm{H}$

bouteneur 89

Bovet 44

Bowruck 85

Bowcuck $x$

Bowrock 87

Boweork x

Bowcock $\times 9$

Bon'cuck R9B

Buwcock 90)

Bryarinue $\mathrm{Bt}$;

Buyarinor \&7

Boyarnov x:B

Boyarinow

Boyarinov

Boyarnov RKC

Boyarinov R9

Boyer 86

Boyentesn RT

Bozzo 85

Brack 88

Brach 89

Brady ke

Bratabletroks $x$,

Bratashevity $x, B$

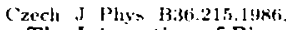

The Interaction of Pions with Deuterons

Z. Phys. ( 4 ti:377.1!)+1).

A nalysis of Transverse Moment un and Event Shape in $\nu$ it Scat tering

LBL-27:30.

Measureinent of the $\eta$ Parameter in $\mu^{+}$decay

SLAC.P['B-513]:

Measurement of the Deuteron and Proton Magnetic Form Factors at Lasge Momentum Transfers

Phys. Rev C:32:1289.19k5.

Inelastic Scattering of $\pi^{+}$and $\pi^{-}$Mesons from ${ }^{3}$ He arid ${ }^{4}$ He at Energies of $350 \mathrm{MeV}, 400 \mathrm{MeV}$, and 475 MeV

Phys. Lett. 236B:4KR,1400: (ERN-EP-89-80).

Production of Prompt Electrons in the Charm $p_{T}$ Region at $\sqrt{s}=630 \mathrm{GeV}$

Phys. Lett. 207B:217.1988:

New Experimenta! Limits on Radiative Neutrino Decay

DPHPE-88-14:

Neutrino Oscillations at Reactors

Plivs. Lett. 21+B:6,1948:

The $T_{20}$ of the deuteron $p \rightarrow{ }^{3}$ He $\pi^{\circ}$ Reaction at Threshold and $\pi$ Absorbtion on a Pair of Nucleus

LPCC-90-02:

Sidewards Flow Effect in $\mathrm{Kr}+\mathrm{Au}$ Central Collisions at $43 \mathrm{MeV} / \mathrm{u}$

L.AL-88-34:

Results of the FREJUS Experiment on Nucleon Decay

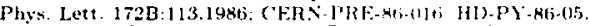

Evidence for Narrow States Decaying into $\Lambda \bar{p} \pi$ 'b at $3.1 \mathrm{GeV} / c^{2}$ with Charges +1 , 0 and -1

LAPP-EXP-88.20:

A nalysis of the Reaction $\pi^{-} p \rightarrow \pi^{0} \eta n$ at $100 \mathrm{GeV}$

LAPP-EXP-89-17

A New Scalar Meson Decaying into $\eta \pi^{0}$ and Results of $\eta^{\prime} \pi^{0}$ A nalysis at $100 \mathrm{GeV} / \mathrm{c}$

Phys. Lett 153B:231.1485: S1X-PR-\$4-1T:

A New Determination of the $\pi^{-} p$ and $\pi^{-}$deuteron $2 P$-1 A Strong Interaction Shifts Using Crystal Difraction

Phys Rev Lett 55.423.1985. C1.EO-85-6: CLNS-85-651)

$\Lambda_{f}^{+}$Production from $e^{+} e^{-}$Annibilation in the $\boldsymbol{Y}$ Energy Region

Phys. Hes Lett. 56:2676.1984.

Upper Limits for the Production of Light Short Lived Neutral Particles in Radiative r(1S) Decay

Phys. Rey Lett 58:307.1987:

Study of $\pi^{+} \pi^{-}$Transitions from the $\Upsilon(3 S)$

Phy. Rev Dis:2679.1988. Phys. Rev. D40.1701.j989

Investigation of the Total Charm-Pair Cross Section in Nonresonant $\epsilon^{+} e^{-}$Annihilation at $\sqrt{b}=10.5$ GeV

Phys. Rev. Lett 62 1240.1949: C'LNS-88-476: ('LEO-88-3:

$\Sigma_{r}(2455)^{++}$and $\Sigma_{r}(2455)^{\circ}$ Production from $e^{+} e^{-}$Annihilation in the $r$ Energy Region

Phys. Rev. D40:263.1989: CLNS-89-895: C'LEO-69-6:

Search for the Production of Fractionally Charged Particles in $e^{+} e^{-}$Annibilations at $\sqrt{s}=10.5$ GeV

Plivs. Ray. D41:805.1990; ("LNS-49-950; CLEO-89-13:

Search for Neutrinolss Decays of the $T$ thepton

ITEP-86i-1:30.

Production of Cumulative Protons with the Momentum of $0.6-1.83 \mathrm{GeV} / \mathrm{c}$

ITEP-8T-5.

Production of Cumulative Pions with the Momentum of $0.6-1.62 \mathrm{GeV} / r$ in the $p$ nucleus $\rightarrow \pi \pm \mathrm{X}$ by Protons of $10.14 \mathrm{GeV}$

Yad Pliss 46:1472.14kT:

Production of Cumulative Protons and Pions with the Momentum of 0.6 - 1.83 GeV/c by Protons of 10.14 GeV

Yind. Phys 77:942.1!kx: ITFP-46-16i5

Production of Deuterons and Tritium Nuclei with the Momentum of $0.6-1.83 \mathrm{GeV} / c$ on the Be, Al, Cu, Ta at 119 Degrees by Protons of $10 \mathrm{GeV}$

$1 T$ EP.

The Cumulative $K^{+}$Mesons Production by Proton with Energy $10 \mathrm{GeV}$

ITEP-KR- ]3R

The Cumulative $K^{-}$Mesons Production by Proton with Energy $10 \mathrm{GeV}$

Yad Ply 501605 14k9.

The Cumulative Kaon Production by $10 \mathrm{GeV}$ Protons

Phys. Rav Lett 56; 2077.198t;

Charged Meson Pair Production in $\%$ Interactions

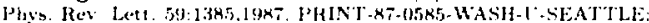
Search for an Imtermediate Range Composition Dependent Force

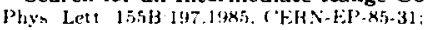

Elastic Scattering at the CERN SPS Collider up to a Four Monientum Transfer of $1.65 \mathrm{GeV}^{2}$

TRI-PI'-NX-24.

Large Angle $\pi^{+} p$ Elastic Scattering at $66.8 \mathrm{MeV}$

TKI-PIP-K(1-11)2

Absolute Differential Cross Section for $\pi^{2}$ p Elastic Scattering at $30 \mathrm{MeV}<T\left(\pi^{ \pm}\right)<67 \mathrm{MeV}$

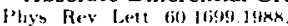

Large pt from the Fragmentation of $1.2 \mathrm{GeV} / \mathrm{Nucleon}{ }^{130} \mathrm{La}$ Nuclei

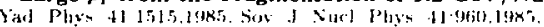

Measurement of Polarization of Protony from Reaction $\gamma p \rightarrow p \pi^{0}$ in the Third Resonance

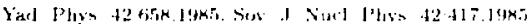

Measurement of Polarization of Protony from Reaction $p \rightarrow p \pi^{0}$ at 110 Degrees in the Range of the Second and Third Resonunces 
Brat isherstis 8ti

Bratashersky 8t,r3

Bratashersy atice

Bratalhessky 87

Bratakhes sky 87I)

Brau 88

Briun 8!

Braunmumzing 88

Braunschweig xti

Braunschueig तi

Braunschweig $47 B$

Braunschueig a

Braunschueig 988B

Braumschueig KR(

Braunschueng RRD

Braunschweig $88 E$

Braunschweeg REF

Braunsthweig knC;

Braunchwetg 89

Braunschweig 89B

Braunscluwe'g $\times 9$

Brannschueig 845

Braunschweeg $89 \mathrm{E}$

Braumacliweig 89F

Braunsclateg K9G;

Braumschu'vig $89 \mathrm{H}$

Braunselinesg 8:

Braucustiueg 895

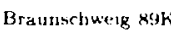

Braunschwerg 89L.

Braustoluweig 90]

Braunscisweig G(j)

Bravila ki:

Bravina $x: 4$

Breakstorie 8:

Isteaketure sins

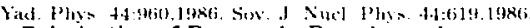

Polarization of Protons in Reaction , deuteron - n at $E(\gamma)=0.7 \quad 1$ GeV for $\theta(p)=00$ Degrees

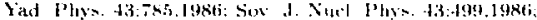

Investigation of Polarization Paraneters in Deuteron Photofusion Induced by Linearly Polarized Photons

lkr. Fiz. ZJurs $31 \cdot 1306.1986$ :

Measurements of Polarization of Secondary Protons in the $\gamma p \rightarrow p \pi^{0}$ Reaction at $\theta(\pi)=150$ Degrees CMS within the Range of $E(\gamma)=0.450 \mathrm{GeV} .0 .700 \mathrm{GeV}$.

Yad Phys. 46 i 11195.1987 :

Polarizetion of Proton in the Reaction $p p \rightarrow p \pi^{0}$ under an Angle of $\theta\left(\pi^{\circ}\right)=120$ Degrees in CMS at the Thisd Maximum of the Total Cross Section

Yad. Phys. $45: 1672.1987$ :

Polarization of Cumulative Protons from the Reaction 7 nucleus $\rightarrow p$ X at a Maximum Energy of 1.6 GeV for the Brensstrablung Photon Spectrum

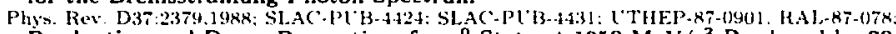

Production and Decay Properties of $\omega \pi^{0}$ State at $1250 \mathrm{MeV} / \mathrm{c}^{2}$ Produced by $20 \mathrm{GeV}$ Polarized Photons on Hydrogen

(izech. J. Phys B39) 126; 1989:

Cross Section of the Interactions of ${ }^{4} \mathrm{He}$ Nuclei with Protons at B.6 and $13.4 \mathrm{GeV} / \mathrm{c}$

2. Phys Ci3:45.1988: BNL-40838:

Transverse Energy Distributions in Si-Nucleus Collisions at $10 \mathrm{GeV} / \mathrm{Nacleon}$

2. Phys. Ci33:13.1986: DEsi'-86i-0Ti

Inclusive $\pi^{\circ}$ Production by $e^{+} e^{-}$Annihilation at $34 \mathrm{GeV}$ Center of Masy Energy

Z. Phys. Ci36:349.1987: F'l'AN-EJ-N7-04: DESY'-8T-081:

A Study of Energy-Energy Correlations between 12 and $46.8 \mathrm{GeV}$ c.m. Energies

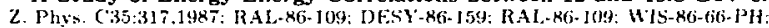

Measurement of the $D_{S}^{ \pm}$Lifetime

2. Phys. ('38:543.1988: DES'Y'kH-005.

A Search for Particles with Magnetic Charge Produced in $e^{+} e^{-}$Annihilations at $\sqrt{s}=35 \mathrm{GeV}$

2. Phys. C.37:171.1988: FTI'A

A Study of Bhabha Scattering at PETRA Energies

2. Phys. (41:359.1488: DESY-88.107.

Jet Fragmentation and QCD Models in $e^{+} e^{-}$Annihilation at c.sn. Energies between 12.0 and 41.5 GeV

2. Phys. C $39: 331$ 14k8; DESY.88-0:34.

A Measurement of the $\tau^{ \pm}$Lifetime

2. Phys. C40:16.1988: Z. Phys. ( $42: 348.1988:$ DESY-88-059:

A Measurement of Muon Pair Production in $e^{+} e^{-}$Annihilations at Centre of Mass Energies $35.0<\sqrt{8}<$ 46.8 GeV

Z. Phys. C41:353.1488: DESY-88-091.

Measurement of the Two-Photon Reaction $\gamma \rightarrow \rightarrow \pi^{+} \pi^{+} \pi^{-} \pi^{-}$at Large Values of $Q^{2}$

Z. Phys. C41:385.1988: DESY-88-100:

Evidence for Direct Photons from Quarks in Electron-Positron Annihilation

2 Phys. C+11533.1989: DESY'-88-050: W'1S-88-15-PH:

Study of $\eta_{r}(1 S)$ Production in Two-Photon Collisions

2 Phys. (12:189.1989: DESY-88-164:

Pion, Kaon and Proton Cross Sectiong in $e^{+} e^{-}$Annihilation at 34 and 44 GeV c.m. Energy

2 Phys. C42:17.1989: DESY-88-112:

A Study of Jets from $b$ Quarks Produced in $e^{+} e^{-}$Annihilations at $\sqrt{s}=35-46 \mathrm{GeV}$

$Z$ Phys C45.193.1989: DESY-89-038:

Charged Multiplicity Distributions and Correlations in $e^{+} e^{-}$Annihilation at PETRA Energies

Z Phys C45:1.1989: DESY-89-032: WIS-89-7-PH:

Comparison of Inclusive Fractional Monentum Distributions of Quark and Gluon Jeta Produced in $e^{+} e^{-}$ Annihilation

2. Phys. ('43:549.1989: DES'Y-89-1335.

A Measurement of Electroweak Effects in the Reaction $e^{+} e^{-} \tau^{+} \tau^{-}$at $35 \mathrm{GeV}$ and $42.4 \mathrm{GeV}$

Z Phys. ( $44: 365.1989:$ DESY'-89-1)5:3:

Production and Decay of Charmed Mesons in $e^{+} t^{-}$Annihilation at $\sqrt{s}>28 \mathrm{GeV}$

$Z$ fliys C45:1 1.1989: DESY-89-069; FTLAA-EP-BS-03:

Experimental Study,$f$ Jet Masses in $e^{+} e^{-}$Annihilation at c.m. Energies Between 12 and $43.5 \mathrm{GeV}$

z. Phys. C45:204.1989: D2, T'-88-173: OI'NP-84-3

Strange Haryon Production in $e^{+} e^{-}$Annibilation

Phys Lett 231B:548.1989: DESY'-81-1092:

Study of Intermittency in Electron-Positron Annihilation into Hadrons

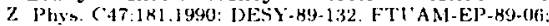

Experimental Study of the Orientation of Three-Jet Events in $e^{+} e^{-}$Annihilation at Petra

2 Phys. ( $44: 1.1989$ : DESY-88-154:

Measucement of the A verage Lifetime of $B$ Hadrons

2 Phys. ( $+7: 1 \times 7.1990)$ DESYY-190-(1):3.

Global Jet Properties at 14 - 44 GeV Center of Mass Energy in $e^{+} e^{-}$Annihilation

DESY-(10-1)]2:

Production of Charmed Mesons in ? Interactions

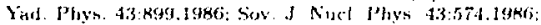

Reconstructed Inclusive and Semi-inclusive $\boldsymbol{x}^{+}$Meson Rapidity Differential Cross Sections in the $\bar{p} p$ Annihilation at $32 \mathrm{GeV} / \mathrm{c}$

Yid. Phys. 501:392,1489.

Multiplicity Correlations in Forward and Backward Hemispheres in CMS in $\bar{p} p$ and $p p$ lnteractions at $32 \mathrm{GeV} / \mathrm{c}$

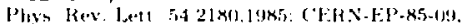

A Measurement of $\bar{p} p$ and $p p$ Elastic Scattering in the Dip Region at $E_{c m}=53 \mathrm{GeV}$

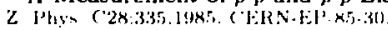

A Diquark Scattering Model for High Proton Production in $p$ p Collisions at the ISR 
Breakstune- $\mathrm{k} 5 \mathrm{~s}$

Brenkstolle X.5

Breaksture R.5E

Breakstun: xit

Breaksturle HEB

Breakstore $\mathrm{KGC}$

Breakstork RGD

Breakstume RGE

Breakstume 86F

Breakst one $\$ 66$;

Breakstone $\mathrm{xi}$

Breakstole $k \mathrm{k}$

Brtakstunt $K K B$

Breakstone $48 \mathrm{C}$ :

Breakstune NG

Breakstune SyB

Breakstone 90

Brechtmann kx

Brechtmann $\times \times B$

Brerdon 89

Brefeld kit

Brtas 89

Breuker 8 s

Brick NG

Brick w9

Brick yo

Bridges $k 6$,

Bridges 4fiB

Bridges hlic:

Bridges 865

Brient 87

Britur, $\$ B$

Brym 47

Bress ky

Brovkin 4

Browder xy

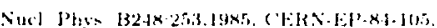

A Measurement of $\tilde{p} p$ and $p p$ Elastic Scatering at ISIR Energies

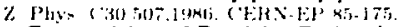

Composition of Particles Enited nt Large $r$ and Medium Angles in $p$, denteron deuteron, and He He Collisions at the CERN Intersecting Storage Ring:

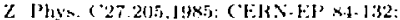

Gluon Tagging in Hard Proton Proton Interections bt the ISR

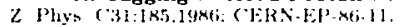

Production of the $f_{2}(1270)$ Meson in the Double Poneron Exchange Reaction $p p \rightarrow \mu p \pi^{+} \pi^{-}$at $E_{c m i}=62$ GeV

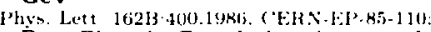

Bose-Einstein Correlations in $p$ and $\bar{p} p$ Interactions at $\sqrt{s}=63 \mathrm{GeV}$

('ERN-El'-86-124

Pomeron Pomerou Collisions at the CERN ISR

2 I'hys C35:151] 1987. CERA-EP-KT-23:

Inclusive Cross Section Ratios in High pr. Proton Proton Scattering at ISR Energies

Z. Ihy. ('33:-475.1987: CERN-EY-x6-J8it:

A Study of Gluon Scattering and Gluon Fragmentation in High $p_{I}$ Interactions at the the ISR

Phys. Lett. 18313:227.1987; ('EKN-EL-86-123:

Multiplicity Dependence of Transverse Momentum Spectra in $p p$, $\bar{p} p$, deuteron deuteron and He He Collisions at ISR Energies

z Phys. C33:333.19k7: C'ERN-JPP-66-132:

Multiplicity Dependence of the Average Transverse Momentum and of the Particle Source Size in p Interactions at $E_{\mathrm{cm}} 62,44$ and $31 \mathrm{GeV}$

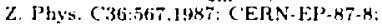

Tagging Diquarks by Protons of High Transverse Monentum in $p$ Collisions at the ISR

Z. Phys. C'40:2017.19kx: C'ERN-E.P-86-2L:

Contribution of Single Difiraction Dissociation to High $y_{T}$ Production in Proton Proton Collisions at $\sqrt{s}=62 \mathrm{GeV}$ at the CERN ISP

Nuovo C.In. 102A:1199.1!39: C'ERN-EP-86-133.3

Charged Multiplicity Distributions in Rapidity Bins for $p$ p Collisions at $\sqrt{\mathbf{s}}=31,44$ and $62 \mathrm{GeV}$

2 Phys. ('40:-11.1984: (ERN-EP-84-13:

Transverse Momentum Distribution in Pomeron-Pomeron Collisions at CER ISR

Z. Phys ( $+3: 165.1989$ : ('ERN-EP-H9-21:

Production of Meson Resonances as Leading Particles in Jets in Proton-Proton Collisions at $\sqrt{8}=62$ GeV at the CERN ISR

Z. Phys. C.ง2:387,1989: 15-J-3226:

Inclusive Pomeron-Pomeron Interactions at the CERN ISA

(ERN-EP-90-74

A Sensitive Test of QCD from Parton-Parton Scattering of the 1SR

il $-8 B-16$.

Fragmentation Cross Sections of ${ }^{32} \mathrm{~S}$ at $0.7,1.2$ and $200 \mathrm{GeV} / \mathrm{Nucleon}$

Si- $48-08:$

Fragmentation Cross Sections of ${ }^{16} \mathrm{O}$ at 60 and $200 \mathrm{GeV} / \mathrm{Nucleon}$

Phys. Lett. $216 \mathrm{~B}:+59.1989$.

Precise Comparison of Antiproton-Proton and Proton-Proton Forward Elastic Scattering at $\sqrt{8}=24.3$ GeV

Viucl lustr and Meth. A22N22\%.19Ni) BONN-81-2:3.

Measurement of the Polarization Degrees of Accelerated Polarized Electrons at the 2.5 GeV Synchrotron in Bonn for Energies between $0.85 \mathrm{GeV}$ and $2 \mathrm{GeV}$

Z. Phys ( $43: 175.1989$ :

Search for Free Neutron-Antineutron Oscillations

Nuc! Phys A.155:641.1960. BOONN-HE-6.5-3]:

Deuteron Electrodisintegration in the $\Delta\left(1232 P_{33}\right)$ Fesonance Region at a Four Momentum Transfer $Q^{2}=0.23(\mathrm{GeV} / \mathrm{c})^{2}$

2 Plive ( $31,59,19, \mathrm{Ht}$.

Scaling in Soft Hadroproduction by Poyitive Projectiles in the Range of $32 \mathrm{GeV} / \mathrm{c}-147 \mathrm{GeV} / \mathrm{c}$

Plys R.y 1)39.248.1.1989.

Multiparticle Production by $200 \mathrm{GeV} / \mathrm{c}$ Hadrons on Gold, Silver and Magnesium Targets

Phys Rev DA1:765.1!6):

Rapidities of Produced Particles in $200 \mathrm{GeV} / \mathrm{c} \pi^{+} / p / K^{+}$Interactions on $\mathrm{Au}, \mathrm{Ag}$. And $\mathrm{Mg}$

Plyy. Rer Lett 56:211.19k6:

Evidence for a New State Produced in Antiprotons at Rest in Liquid Deuterium

Phys. Let 1 MOB:313.19\%6:

Antiproton Annihiletions in Deuterium at Rest into Two Pions, Evidence for a $\bar{p} n$ Bound States near Threshold

Jhys Rev lett. $56: 215.1986$ :

Diference Spectra: Dominance of Two-Body Cascades in Antiproton-Neutron Annihilation at Rest

Piju's. Rev 1,ett is $1534.19 \mathrm{kf}$,

Properties of the $\zeta(1480)$

SI.AC:PLis-45):

Recent Results for MARK-III

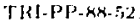

A Study of the Decay $\pi^{+}+\ldots+\nu$,

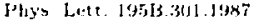

Measurcment of the Lifetine of Hadrons Containing the b-Quark

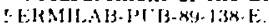

Search for Short-lived Particles Produced in an Electron Bcam Dump

[TEP.S4-13!

About Possible Existence of Strange Exotic Baryon Resonance in $p 3 \pi^{+} \pi$ System

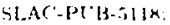

Recent Results on Weak Decays of Charmed Mesons from the MARK-III Experiment 
Browil $\times 6$

Brou'n $86 \mathrm{~B}$

Browil 89

Brucker 85

Brucker 86

Bruckner 85

Bruckner 85B

Bruckner 86

Bruckner 8-

Bruerkner 90

Bรusner 89

Bryman 85

Bryman 86

Bryman 86B

Buchle 88

Buchle 89

Budd 85

Budilov 90

Budko 85B

Budkovsky 8;

Bugg 47

Bujak 8.5

Bukin 89

Buklej 86

Bumazhnov 86

Burchat 85

Burchat 86

Burchat $\mathrm{B} 6 \mathrm{~B}$

Burchat 89

Burchat 90

Burgov 85

Burgov 86

Burgey 87

Burkhardt 85

Burkhardt 45B

Burkhardt $85,{ }^{\circ}$
FERMIILAL-CONF-RI3-3;-E:

Production of Hadrons and Leptons at High pr and Pairs at High Mass

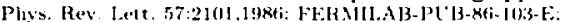

A New Limit on axion Production in $800 \mathrm{GeV}$ Hadronic Showers

Plys. Rev. l.ett. 63:26337.1!3k!t.

Dimuon Production in $800 \mathrm{GeV}$ Proton-Nucleus Collisions

Plys. Rey. Di32:16i15.1985.

Int ranuclear Cascade in $\nu_{\mu}$ Ne Interactions

Phys. Rev. Di3:2183.1986: BNL-38.1157:

Limits on Neutrino Oscillations in the Fermilab Narrow-Band Beam

Phys Lett. 166B:113.198ti: CERN-E1'-R5-141:

Antiproton-Proton Elastic Cross Sections in the Momentum Range between 180 and $600 \mathrm{MeV} / \mathrm{c}$

Phys Lett. 15813:160.1985: CERN-EP-85-74

Real-to-Imaginary Ratio of the $\bar{p} p$ Forward Elastic Scattering Amplitude in the Momentum Range between 180 and $590 \mathrm{MeV} / \mathrm{c}$

Phys. Lett. I6!1B:302.1986: CERN-EP-XF-202:

Measurement of the $\overline{\mathbf{p}} \boldsymbol{p} \rightarrow \bar{n} n$ Cross Section at Low $\bar{p}$ Momenta

Phys. Lett. 197B:463.19k7; CERN-EP-H7-15t:

Search for a Narrow Resonance and Antiproton-Proton Annihilation Cross Sections in the Beam Momentum Range between 400 and $600 \mathrm{MeV} / \mathrm{c}$

Z. Phy's. A352:217.1990: CERN-EP-89-105:

Measurement of the Antiproton-Proton Annihilation Cross Section in the Beam Momentum Range between 180 and $600 \mathrm{MeV}$

2. Phys. C42:361.1989: PHE-87-05: IFVE-89-94:

Neutron to Proton Cross Section Ratios in Neutrino and Antineutrino Charged Current Reactions for $E<30 \mathrm{GeV}$

Phys. Rev. Lett 55:465.1985:

Search for $\mu^{-} e^{-}$Conversion in $T i$

Phys. Rev. Di3:1211.1986;

Measurement of the $\pi^{+} \rightarrow e^{\dot{*}} \nu$ Branching Ratio

Phys. Rev. Lett. 57:2787.1986: TRJ.PP-86-73:

Exotic Muon Decay $\mu^{+} \rightarrow e^{+} \mathbf{X}$

Phys. Lett. 213B:125.1968:

Pion Production on Carbon by Medium Energy Neutrons

FREI-MEP-89-01:

Neutron Induced Pion Production on Carbon, Copper and Bismuth at Intermediate Energies

Phys. Rev. D31:1132.1985:

Charge Distributions of Hadrons Associated with Hadronic $J / \psi(1 S)$ Production

Phys. Lett. 243B:341.1990:

Small Angle Proton-Proton Correlations in Collisions of High Energy Light Ions with Carbon and Gold Nuclei

IzV. Akad Nauk SSSR. Fiz. 49:1373.1985;

Clusters of High Energy Muons and Chemical Composition of the Primary Cosmic Rays

ITEP.85-40:

Measurement of Piou Proton Scattering Asymmetry at $1.4-2.1 \mathrm{GeV} / \mathrm{c}$

Phys. Lett. 194B:563. I 947 :

$\bar{p} p$ Total Cross Sections below $420 \mathrm{MeV} / \mathrm{c}$

Phys. Rev C:32:620.10N5:

Mass Yield Distribution for the Interaction of Silver with $300 \mathrm{GeV}$ Protons

Yad. Phys 50:909.1949:

The Process $e^{+} e^{-} \rightarrow \pi^{+} \pi^{-} \pi^{0}$ in Nonresonance Energy Region $2 E$ up to $1.4 \mathrm{GeV}$

Yad. Phys. 44:671.1946):

Laboratory Backward Emission of Pions in Reactions $\pi^{ \pm}$nucleus $\rightarrow \pi^{+} \mathrm{X}$ at $1.5 \mathrm{GeV} / \mathrm{c}$

Yad. Phys 46:531.1966; IFVE-86-159:

Study of Correlations between Rapidities of Two Charged $\pi$ Mesons in $\bar{p} p$ Interactions at $32 \mathrm{GeV} / c$

Phys. Rev. Lett. 54:2449.1985: SLAC-PUB-3602: LBL-19347:

Measurement of the Branching Fraction for $\tau^{\rightarrow} \rightarrow 5 \pi^{ \pm}\left(\pi^{0}\right) \nu_{\tau}$ and an Upper Limit on the Tau-Neutrino Mass

SLAC-292:

Decays of the $\tau^{ \pm}$Lepton

Phys. Rev. D35:27.19K7. SLAC-PLB-4006: LBL-21915:

Measurement of the Branching Fractions of the $\tau^{ \pm}$Leptor Using a Tagged Sample of $\tau^{ \pm}$Decays

SCIPP-89-26:

First Resuits from the SLAC Linear Collider and MARK-II Detector

Phys. Rev. D41:3542.1990: SLAC-PCB-5172: LBL-28396:

A Search for Decays of the $Z^{\circ}$ to Unstable Neutral Leptons with Mass between 2.5 and $22 \mathrm{GeV}$

ITEP-B.5-24:

5. Idy of Singularities in Momentum Spectrum of $\pi^{-} \mathrm{C} \rightarrow \pi^{-}$X Feaction at the $1.5 \mathrm{GeV} / c$

ITEP.86.R:

Protons and Deuterons Production at $135 \pm 6$ Degrees in the Inclugive Reaction on the C Nuclei under $\pi^{-}$ Mesons at $0.85-2.5 \mathrm{GeV} / \mathrm{c}$ Moment um

Yad. Phys. 45:743.1987:

Production of Protons and Deuterons under Angles near 100 and 130 Degrees in Inclusive Feactions on Nuclei Exposed to $\pi^{+}$and $\pi^{-}$Mesons with Incident Momenta of $1.5 \mathrm{GeV} / \mathrm{c}$

2. Phys ("31:39.1946: ('EHN-EP'K5-19):

A re Three "Prompt" Like-Sign Dimuons?

Phys. Lett. 150B:242.1985:

Muon Decay: Meagurement of the Positron Polarization and Implications for the Spectrum Shape Parameter $\eta, V-A$ and $T$ Invariance

Phys. Lat. I $160103: 3433.1985$ :

Muon Decay: Measurement of the Transverse Positron Polarization at General Analysis 
Burkhardt 87

Burkhardt bis

Burnets 85,

Burnett 85D

Burjett 86

Burnett 87

Burnham 87

Burow 87

Buschbeck 89

Busentitz 89

Bussiere 88

Butler 86

Butsev 85

Bylsma 87

Bystricky 85

Bystricky $85 \mathrm{~B}$

Bystricky $85 \mathrm{C}$

Byst ricky $8.5 \mathrm{D}$

Bystricky $86 \mathrm{D}$

Bystricky 87

Cai 87

Cai $87 \mathrm{~B}$

Caldwell 85

Caldwell 86

Caldwell 87

Caldwell as

Caldwell asB

Calicchio 87

Calloway 89

Cameron $85 \mathrm{~B}$

Camilleri 87

Campagnari 88

Campagnolle 89

Camporesi 87

Canklin 88

Capraro st

Carbon b5

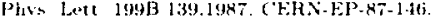
Observation of the Decay $K_{S} \rightarrow 2 \gamma$ and Measurement of the Decay Rate $K_{L} \rightarrow 2 \gamma$ and $K_{S} \rightarrow 2 \gamma$

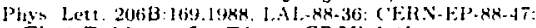

First Evidence for Direct $C P$ Violation

K'R-121-R2-2:

Composition and Energy Spectra of Cosmic Ray Nuclei Above $500 \mathrm{GeV} / \mathrm{Nucleon}$ from the JACEE Emulsion Chamber Experiments

IC R R-121-45-2:

Cosmic Ray Results on Ultra-relativistic Nucleus Nucleus Interaction by Balloon Emulsion Experiments

I'hyss Rev. Letl $57: 3249.1986$.

Average Transverse Momentum and Energy Density in High Energy Nucleus Nucleus Collisions

P'is: Rev. D35̃: 824.1987 :

Nucleus Nucleus Interactions Between 20 and $65 \mathrm{GeV}$ per Nucleon

TRI-PP-87.22:

Search for $\mu^{-} e^{-}$Conversion on $T i$

BONN-IR-87-25:

Study of Baryon Production in $p-\bar{p}$ Interactions at Center-of-Mass Energies from $200 \mathrm{GeV}$ to $900 \mathrm{GeV}$

Mod. Phys. Lett. A4:1871.1!s69.

Intermittency in Multiparticle Production - a Brief Experimental Survey

Phys. Rev. D40: I.1989:

High Energy Photoproduction of $\pi^{+} \pi^{-} \pi^{0}, K^{+} K^{-}$, and $p \bar{p}$ States

Z. Phys. C38:117.1988: CERN-EP-88-25:

The Production of $J / \psi(1 S)$ in $200 \mathrm{GeV} / \mathrm{A}$ Oxygen-Uranium Interactions

SLAC-290:

Measurement of the Charged and Neutral $D$ Meson Lifetimes

Yad. Phys, 44:425.1986: JINR.P1+85-590:

The Study of $7.3 \mathrm{GeV}$ Deuteron Jnteraction with ${ }^{83} \mathrm{Nb},{ }^{168} \mathrm{~Tb},{ }^{207} \mathrm{~Pb}$ and ${ }^{208} \mathrm{Bi}$

Phy's. Rev. D35:2269.1987:

Limit on Tau Decay to Seven Charged Particles

Nucl. Phys. A44t:597,1985;

Measurement of $n p$ and $p p$ Asymmetry with an Accelerated Polarized deuteron Beam from $725 \mathrm{MeV}$ to $1000 \mathrm{MeV}$ per Nucleon

Nucl. Phys. B258:483.1985:

Measurement of the Spin Correlation Parameter $A_{\text {ookk }}$ for $p$ Elastic Scattering in the Energy Range 0.72 $-1.1 \mathrm{GeV}$

Nucl. Phys. B262:715.1985:

Measurement of the Spin Correlation Parameter $A_{00 m}$ for $p p$ Elastic Scattering in the Energy Range from 0.83 to $1.1 \mathrm{GeV}$

Nucl. Phys. B262:727.1985:

Measurement of the Spin Correlation Parameter $A_{00 n n}$ and of the Analyzing Power for $p$ plastic Scattering in the Energy Range from 0.5 to $0.8 \mathrm{GeV}$

SACLAY-DPHPE-86-13:

Nucleon-Nucleon Phase Shift Analy sis

DPHPE-87-03:

Energy Dependence Nucleon-Nucleon Inelastic Total Cross Sections

HZPP-87-10:

Interaction Free Path and Projectile Alpha-Fragment in Kr-Emulsion Collisions at $1.4 \mathrm{~A}$ GeV

HZPP-87-2:

A High Multiplicity Cosmic Ray Event Observed in Chinese Satellite Emulsion

Phys. Rev. Lett. $54: 281.1985$ :

Limit on Lepton Noncongervation and Neutrino Mass from Double $\beta$ Decay

Phys. Rev. D33:2737,1986:

Half-life Limits on the Zero Neutrino and Two Neutrino Double $\beta$ Decay of ${ }^{70} \mathrm{Ge}$

Phys. Rev. Lett. 59:419.1987:

Limits on Neutrinoless $\beta \beta$ Decay Including that with Majoron Emission

Int. Jour Mlod. Phys. A4:1851.1989: ('C'SB-HEP-8x-8:

Review of Double $B$ Decay Experiments

LCSB-HEP-88-10.

Experimental Search for Dark Matter - an Update

Phys. Lett. 19:3B:131.1987:

A Direct Measurement of the Energy Spectrum of Cosmic Ray Muons in the Mont Blanc Underground Laboratory

Phys. Lett. 232B:549.1989:

$A$ Search for Free Quarks in Heavy-Ions Collisions at 80 and $200 \mathrm{GeV} / \mathrm{Nucleon}$

Yhys. Rev. D32:3070.1985:

Measurement of the Analyzing Power for $p p \rightarrow p$ at $m T^{2}=6.5(\mathrm{GeV} / c)^{2}$

Pliys. Rept. 144:51,1987:

Proton-Antiproton Physics at the CERN Intersecting Storage Rings

Phys. Rev. Lett 61:2062.1988:

Search for the Decay $\mathrm{K}^{+} \rightarrow \pi^{+}{ }_{2}^{+} e^{-}$

Nuovo C'im. 102A:653.1989: CERN-EP-84-13:

Production of Heavy Hypernuclei with Antiproton

SLAC:-PI:B-4332:

Recent Measurement of the Average Lifetime of Hadrons Containing b-Quarkj from PEP Experiments at SLAC

Nucl. Phys. B311:613.1484: HAL-8K-1356:

Measurement of the Spin-Rotation Parameter $\beta$, in the Reaction $\pi^{+} p \rightarrow K^{+} \Sigma^{+}$at 1.69 and $1.88 \mathrm{GeV} / \mathrm{c}$

Nucl. Phys. B2x\&:659.1967: ('ERN-EP-87-37:

The $\rho$ Radiative Decay Width: A Measurement at $200 \mathrm{GeV}$

Nucl. Phys B25.4697.16\%5) ('ERN-E1)-84-1633:

Precise Measurements of Proton-Antiproton and Proton-Proton Total Cross Sections at the CERN Intersecting Storage Rings 
Cardello 84

Carlsmith 86

Carbmith 87

Carosi 90

Carroll 88

Carroll 89

Cason 89

Cassata 88

Cassel 85

Cásiday 89

Cassiday $89 \mathrm{~B}$

Castellina 8.5

Castro 88

Calanesi 86

Catanesi 88

Catanesi 89

Cavasinni 85

Cebra 89

Cenci 87

Cerradini 8.5

Chacon 88

Chakrabarti 85

Chalners 85

Chan 88

Chang 85

Chapin 85

Chauvat 85

Chauvat 87

Chekulacy 88B

Chen 85

Chen 89

Chen $89 \mathrm{~B}$

Chen $89 \mathrm{C}$

Cheplakov 85

Chernavskaya 87

Chestnov 87

Chiang 86

Chiba $k 7$
Phys. Rev. D32:1.1985: FERA1ILAB.PL'B-H4.122.1: Charg ad Hyperon Production by $400 \mathrm{GeV}$ Protons

Phys. Rev. Lett 56:18.1986; Measurement of the $K^{*}(892)^{0}$ Radiative $W^{\text {idth }}$

Phys. Rev. D36:3502.19k7,

Limit on the Radiative Width of the $K_{2}^{*}(1430)^{\text {a }}$

Phys. Lett. 237B:303.1990: ('ERN-EP.90-06:

$A$ Measurement of the Phases of the CP-Violating Amplitudes in $K^{0} \rightarrow 2 \pi$ Decays and a Test of $C P T$ Invariance

Phys. Rev. Lett. 61:1698.19k8:

Nuclear Transparency to Large Angle p p Elastic Scattering

Phys. Rev. Lett. 62:1829.1989.

Subthreshold Antiproton Production in ${ }^{28} \mathrm{Si}{ }^{28} \mathrm{Si}$ Collisions at $2.1 \mathrm{GeV} / \mathrm{Nucleon}$

PRINT-89-0374-NOTRE-DAME:

Search for Exotic Mesons in the $K \bar{K} \pi$ Final State

IFYE-88-178:

On Measuring $\pi_{2}(\mathbf{1 6 7 0}) \rightarrow \rho^{0} \pi^{-}$Decay Probability in $\pi^{-} n-\pi^{+} \pi^{-} \pi^{-} n$ at $40 \mathrm{GeV} / \mathrm{c}$

CLNS-85-644

$B$ Meson Results from the $\mathbf{T}$ Region

Phys. Rev. Lett. 62:383.1989:

Evidence for $10^{18} \mathrm{eV}$ Neutral Particles from the Direction of Cygnus X-3

Phys. Fev. Lett. 63:2329.1989.

Search for a Rays above $10^{\mathrm{d}} \mathrm{eV}$ from Cygnus $\mathrm{X}-3$ during the June and July 1989 Radio Outbursts

Nuovo Cim. 8C:93,1985;

Charm Hadroproduction Cross Section up to $100 \mathrm{TeV}$ from Measurements of the Cosmic Ray Muon Angular Distribution. Results of the Mont Blanc Experiment

LAL-88-58:

The $\pi, K$, Proton Electromagnetic Form Factors and New Related DM2 Results

Phys. Lett. 187B:431.1967: CERN-EP-86-177:

The Production of Beauty Particles in $\pi^{-}$U Interactions at $320 \mathrm{GeV}$ Energy

Phys. Lett. 202B:453.1988: C'ERN-EP-87-216: Experimental Study of $B \bar{B}$ Production in $\pi^{-}$U Intersaction at $320 \mathrm{GeV}$ Energy

Phys. Let t. 231B:328.1989: C'ERN-EP-89-118;

$\ddot{B} \vec{B}$ Inclusive Cross Section in $320 \mathrm{GeV} \pi^{-}$U Interactions

Z. Phys C28:487,1985: CERN-EP-85-44:

Observation of Double Pomeron Exchange in He He Collisions at the CERN Intersecting Storage Fing

Phys. Lett. 227B:336.1989:

Two-Particle Correlations from $500 \mathrm{MeV} p \mathrm{Ag}$ and $p$ Be

DFCPG-5-87:

Latest Result on Experimental Tests of the Electroweak Theory at the CERN $\bar{p} p$ Collider

CERN-EP-85-196:

Study of Minimum Bias Trigger Events at $E_{\mathrm{cm}}=0.2-0.8 \mathrm{TeV}$ with Magnetic and Calorimetric Analysis at the CERN Proton Antipraton Collider

Phys. Rev. Lett. 60:780.1988:

Observation of a Nonspherical Pion Source in Relativistic Heavy-Ion Collisions

Z. Phys C27:1,1985:

Inclusive $\gamma$ Production in $\bar{p} p$ Interactions at $12 \mathrm{GeV} / \mathrm{c}$

Phys. Lett. 153B:235.1985;

Measurement of $K(1,1)$ and $K(n, n)$ in $p$ deuteron $\rightarrow n p p$ at 600,650 and $800 \mathrm{MeV}$

BNL-42223:

Production and Phenomenology of Clueballs

Phys. Lett. 157B:357.1985:

ᄀ Ray Energy Spectrum from Orthopositronium Three-Gemma Decay

Phys. Rev. D31:17.1985:

Diffraction Dissociation of Photons on Hydrogen

Phys. Lett 1638.263.1986: CERN-EP-85-128:

Test of $C P$ Invariance in $A$ Decay

Phys. Let t. 199L-304.1987; CERN-EP-87-184;

Production of $\Lambda^{+}$with Large $x_{F}$ at the ISR

Sov. J. Nucl. Phys. 411:282.1989: Yad. Phys. 49:452.1989: IFYE-88-31:

Study of Meson System Seructure in Events with Two Leading Particles in $\bar{p} p$ Interactions at $32 \mathrm{GeV} / \mathrm{c}$ Phys. Rev. D.31:23Rs,1985:

Observation of the Decay $\vec{B} \rightarrow D^{*}(2010)^{+} \rho^{-}$

Phys. Rev. D39:3528.1989: (LNS-89-885: CLEO-89-1

Measurement of the Muonic Branching Fractions of the $\Upsilon(1 S)$ and $\Upsilon(3 S)$

Phys. Lett. 226B:192.1989: CLNS-89-920: CLEO-89-8:

Measuring of $D_{S}^{ \pm}$Decay Modes

Phys. Let . 2438:169.1990: CLEO-89-15:

Measurement of $\gamma \sim$ Widths of Charmonium States

JINR-E1-85-861:

$\gamma$ Production in in Pe-jpheral Interactions of Relativistic Carbon Nuclei in Propane and Anomalon Problem

LEBD-87-345

Unique Event of Central Interaction of Calcium Nucleus ( $\left.E=1.2 \cdot 10^{19} \mathrm{eV}\right)$ with Heavy Nucleus of Photoemulsion

Yad. Phys. 45:19.1987

Nucleon Emission Effect on Kinematical Characteristics of Fragments from 1 GeV Proton Induced Nuclear Fission

Phys. Fev. D34:1619.1986; B3Nl-33347:

Search for Exclusive $J / \psi(15)$ Production

Phys. Rev, 036:3321.1947: P1,5s. Lete. 1773:217,1946; KEK-47-117: KEK-8i-10:

Search for Narrow States by Detection of Monochronatic $\gamma$ Rays in $\bar{p} p$ Annihilation at Rest 
Chiba $87 \mathrm{~B}$

Chiba $8 \mathrm{~s}$

Chiba 89

Childers 85

Chilingarian $x x$

Chliapnikov 90

Chrien 86

Chrien 88

Christenson 85

Chung 85

Chupp 89

Chuvila 86

Claesson 8.5

Clark 85

Clarke 86

Cobbaert 87

Cotbaert $87 \mathrm{~B}$

Cobbaert 88

Cobbaert 88B

Coffman 87

Coffman $8 x$

Coffman 89

Cole 88

Collins $85 \mathrm{E}$

Connell 88

Conway 89

Coopersarkar 85

Coopersarkar 85.5

Corriveau 88

Coteus 87

Coteus 87B

Couchut 87

Courau 86

Court 86

Cousins x

Cowan 86

Cowan $8 x$

Coward 85

Cowley $x 8$

Cowsik $x$
Phy Lett. 202B:447.198\%: KEK-s7-141

Search for Narrow Peaks in Inclusive $\pi^{0}$ Spectra from $\bar{p} p$ Annihilation at Rest

Phys. Rev. D38:2021.14HX; KLK.KH-25:

Antiproton-Proton Annihilation at Rest into $\pi^{0}$ Meson and $\gamma$ Meson with Meson $=0, \pi^{\prime}, \rho^{\circ}, \pi$ and $\pi^{0}$

Phy Rev. D39:3227,1989: KEK-89-6: $\eta$ and $\eta^{\prime}$ Production in $\bar{p} p$ Annihilation at Rest

Phys. Rev. Lett, 55:19621.1985:

Production Dynamics of the $\boldsymbol{Y}$ in Proton - Nuclean Interactions

LEBD-88-75:

Upper Boundary of Iron Nuclei Fraction in Primary Cosmic Rays at $E>10^{10} \mathrm{eV}$

Phys. Let 1. 240B:519.1990.

Production Ratio of Pseudoscalar to Vector Mesons

Czech. J. Phys. B36:4I0.1986:

Recent Hypernuclear Research at the Brookhaven AGS

Phiv's. Rev. Lett, 60:2595.1988: Search for Bound States of the $n$ Meson in Light Nuclei

Phys. Rev. Lett. 55.154.1985;

Limits on Charm Production in Hadronic Interactions near Threshold

Phys. Rev. Lett. 55:779.1985:

Spin and Parity Analysis of $K \bar{K} \pi$ System in the $f_{1}(1285)$ and $f_{2}(1420) / \eta(1440)$ Regions

Phvs. Rev. Lett. 62:505.1980:

Experimental Limits on the Radiative Decay of SN1887A Neutrinos

ITEP-86-164:

Dibaryon Resonance $2.9 \mathrm{GeV}$ in Elastic Backward $\pi^{-}$deuteron Scottering

Phys. Rev. D33:2729.1986: LLIP.8514:

Alpha Particle Interactions with Nuclei at $12 \mathrm{~A} \mathrm{GeV/c}$

Phys. Rev. D32:1061.1985:

Triggered Bubble Chamber Study of the Reaction $\pi^{+} p \rightarrow \Delta\left(1232 P_{33}\right)^{++} \pi^{0} \pi^{0}$ at $16 \mathrm{GeV} / c$

Phys. Rev. D33:19.1986:

New Limits on the Production of A nomalous Nuclear Fragments in Deuteron-Deuteron Collisions

Z. Phys. C36:577.1987: CERN-EP-87-77;

The Holographic Bubble Chamber Experiment and the Determination of the Effective Charmed Quark Mass and the $K$-Factor for Hadronic Charm Production

Phys. Lett. 191B:456.1987: CERN-EP-86-212: $A$-dependence of the Charm Production Cross Section in $320 \mathrm{GeV} / c \pi^{-}$Interactions

Phys. Lett. 206B:546.1988: CERN-EP-88-34: $A$-dependence of the Charm Production Cross Section in $300 \mathrm{GeV} / c$ Proton Interaction

Phys. Lett. 213B:395.1984: $A$-dependence of Low Mass Muon Pair Production in $300 \mathrm{GeV} / \mathrm{c} p$ and $320 \mathrm{GeV} / \mathrm{c} \pi^{-}$Interactions

Phys. Rev. D36:2185.1987; SLAF.PUB-4314; Upper Limit on $\operatorname{Br}\left(\tau^{ \pm} \rightarrow \eta \pi \nu\right)$

Phys. Rev. D38:2695.1988: Pbys. Rev. D40:3788.1989: Measurement of $J / \psi(1 S)$ Decays into a Vector and a Pseudoscalar Meson

Phys. Rev. D41:1410.1990: SLAC-PLB-5104: Study of the Doubly Radiative Decay $J / \psi(1 S) \rightarrow \gamma \gamma \rho^{0}$

Phys. Rev, D37:1 1 1u5.1988:

Nonsinglet Valence-Quark Distribution from Neutrino-Deuterium Deep-inelastic Scattering

ANL-HEP-PR-85.92:

The Two Particle Inclusive Cross Section in $e^{+} e^{-}$Annihilation at PETRA, PEP and LEP Energies

Phys. Rev. Lett. 60:2242 1988:

Search iur Low-Energy Resonances in the Electron-Positron Annihilation-in-Flight Photon Spectrum

Phys. Rev. D39:42.1989: Experimental Study of Muon Pairs Produced by $252 \mathrm{GeV}$ Pions on Tungsten

Phys. Let. 160B:207.1985: CERN-EP-85-104:

Search for Heavy Neutrina Decays in the BEBC Beam Dump Experiment

Phys. Let1. 160B:212.1985: CERN-EP-85-97:

Bounds on Light Gluinos from the BEBC Beam Dump Experiment

2. Phys. C38:15.1988:

Transverse Energy Distribution in ${ }^{10}$ O-Nucleus Collisions

FERMILAB-CONF-87-147-E:

Charm Hadroproduction Results from Fermilab E-400

Phys. Rev. Lett. 59:1530.1987: FERMILAB-PLB-87-146-E:

Production of the Charmed Strange Baryon $\Xi_{r}(2460)^{+}$by Neutrons

LAL-א?-40:

Experimental Search for Gluonic Mesons

Nucl. Phys. B271:1.1986:

Lepton and Pion Pair Praduction in $\gamma \gamma$ Collisions Measured near the Threshold at DCI

Phys. Rev, Lett. 87:507.1986:

Energy Dependence of Spin Effects in $p p \rightarrow p p$

Phys. Rev. D3s:2914.19k8. Search for tt. Decay $K_{L} \rightarrow \mu^{+} e^{-}, K_{L} \rightarrow \mu^{-} e^{+}$and $K_{L} \rightarrow e^{+} e^{-}$

Phys. Rev. Let: 50:444.1986:

Observation of Correlated Narrow Peak Structures in Positron and Electron Spectra from Superheavy Collision Systems

LBL-24715.

Inclusive $\pi^{ \pm}, K^{ \pm}$and $p \bar{p}$ Production in $e^{+} e^{-}$Annihilation at $\sqrt{s}=29 \mathrm{GeV}$

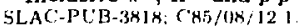

Recent Results on $D$ Meson Decays from the MARK-JII

Phys. Letl 2011 B:196.1988:

Coincident Proton Emission from the Continuum Induced by $200 \mathrm{MeV}$ Proton on ${ }^{12} \mathrm{C}$

Phys. Rev Let1, 61:2179.198\%.

Limit on the Strength of Intermediate-Range Forces Coupling to Isospin 
Cowsik 90

Crabb $8 B$

Cramer 85

Crawford 86

Crawford 88

Cresti 86

Cribjer 87

Crittenden 86

Cromar 86

Csorna 85

Csorna 86

Csorna 87

Csorna $87 \mathrm{~B}$

Cumalat 87

Cumalat $87 \mathrm{~B}$

Cupal 85

Dabrowski 86

Dadykin 87

Daftari 87

Daintan 85

Dalitz 90

Damdinsuren 87

Damdinsuren 88

Damdinsuren 88B

Damdinsuren 89

Damdinsuren 89B

Danevich 89

Daniloy 87

Danilov 88

Danilov 89

Danzmann 89

Dasu 87

Dasu 87B

Dasu 88

Daum 87

Daveriport 86

Davier 86

Davier 87

Davier 89
Phys. Rev. Lett. 64:336.1990:

Strength of Intermediate-Range Forces Coupling to Isospin

Phiss. Ret Let: 60:2351.1988: 1 N-HE-88-1:

Measurement of Spin Effects in $p p \rightarrow p p$ at $18.5 \mathrm{GeV} / \mathrm{c}$

Z. Phys. C29:513.1985:

Measurement of the Magnetic Formfactor of the Deuteron for $Q^{2}=0.5$ to $1.3(\mathrm{GeV} / \mathrm{c})^{2}$ by a Coincidence Experiment

Phys. Rev. Let'. 56:1043.1986:

Precision Measurement of the Mass Difference Mass $\left(\pi^{-}\right)-\operatorname{Mass}\left(\pi^{0}\right)$

Phys. Lett. 213B:391.1988: SIN-PR-48-05:

Precision Measurement of the Mass Difference Mass $\left(\pi^{-}\right)-\operatorname{Mass}\left(\pi^{\circ}\right)$

Phys. Let 1, 177[ [:206.1986:

Measurement of the Antineutron Mass

Phys. Lett. 188B:168.1987:

Limits on Neutrino Oscillation Parameters from the Chlorine Solar-Neutrino Experiment

Phys. Rev. D31:2584.1986: (:ERN-PRE-86-049: FERMII.A [3-PLB-86-31-E:

inclusive*Hadronic Production Cross Sections Measured in Proton Nucleus Collisions at $E_{c m}=27.4 \mathrm{GeV}$

Phys. Rev. Lett. 56:2561.1986:

Flux Limit of Cosmic Ray Magnetic Monopoles from a Multiply Discriminating Superconducting Detector

Phys. Rev. Lett. 54:1894.1985;

Inclusive Decay of Beauty Mesons into Charged $D^{*}(2 \mathrm{C10})$

Phys. Rev. Lett. 56:1222.1986:

Measurement of the Direct Photon Spectrum from the $\Upsilon(1 S)$

Phys. Rev. D35:2747.1987:

Limit on the Mass of the Tau Neutrino

Phys. Lett. 191B:318.1987:

Measurement of the $D^{\circ}, D^{+}$and $D_{S}^{+}$Meson Lifetimes at $\sqrt{8}=10.68 \mathrm{GeV}$

Phys. Lett. 210B:253.1988: FERMILAB-PL'B-B7-192-E:

Observation of $D^{0} \rightarrow K^{0} \bar{K}^{\circ}$

FERAILAB-CONF-87-197-E:

Neutron Production of Charm Particles in Fermilab E-400

Phys. Rev. Lett. 55:566,1985;

Measurement of the Ratio $\Gamma\left(K_{L} \rightarrow \pi^{+} \pi^{-}\right) / \Gamma\left(K_{L} \rightarrow \pi \ell \nu\right)$ for $K_{L}$ with $65 \mathrm{GeV} / c$ Laboratory Momentum

Nucl. Phys. A434:373C, 1985:

Sigma Hypernuclei and Their Lifetimes

Pisma Zh. Eksp. Jeor. Fiz. 45:464.1987:

About Registration of Rare Event by Neutrino Detector at Mont Blanc on 23 Feb 1987

Phys. Kev. Lett. 58:859.1987;

Evidence for a New Meson: A Quasinuclear $n \bar{n}$ Bound State?

RAL-B5-025:

Hadron Photoproduction at Medium Energy

Phys. Lett 236B:76.1990:

Is There a Bound ${ }^{4} \mathrm{H}_{S}$

JINR-P1-B7-932;

Total Cross Section Measurement of the Reaction ${ }^{27} \mathrm{Al}{ }^{12} \mathrm{C} \rightarrow{ }^{24} \mathrm{Na} \mathrm{X}$ at $3.6 \mathrm{GeV} / \mathrm{Nucleon}$

JINR-P1-88-135:

Yield Determination of Radionuclides Formed in Interactions of Relativistic Nuclei with Complex Nuclei

JINR-P1-88-312:

Application of Robust Fitting to Determination of Isobaric Cross Sections of Residual Nuclei in Relativistic Interactions

JINR-E1-89-481:

Yield of Radionuclides Formed in the Interaction of 3.65 a GeV ${ }^{12}$ C-Ions and Protons wixh Pb

Yad. Phy's. 52:330.1990: J[NR-P1-89-757:

Target Residues from the Reactions of $3.85 \mathrm{~A} \mathrm{GeV}$ Deuterons with ${ }^{83} \mathrm{Nb},{ }^{109} \mathrm{Ag},{ }^{159} \mathrm{~Tb},{ }^{107} \mathrm{Au}$, and ${ }^{207} \mathrm{~Pb}$

Pisma Zh. Eksp. Teor. Fiz. 49:417.1989:

Search of Double $\beta$ Decay of ${ }^{116} \mathrm{Cd}$ with the Help of Scintillator ${ }^{210} \mathrm{Cd}$ WO4

ITEP-B7+213:

$B$ Mesons

ITEP-di-180:

Recent Argus Result on B-Meson Decays

DESY-49-147:

Recent ARGUS Results on $B$ Meson Decays

Phys. Rev. Lett. 62:2353.1989:

Correlated Two-Photon Lines from $6 \mathrm{MeV} /$ Nucleon $\mathrm{U} T \mathrm{Th}, \mathrm{U} \mathrm{U}$, and $\mathrm{Th}$ Th Collisions

Phys. Rev Lett. 61:1061.1988: ROCH-LR-391-87: Precision Measurement of $R=\sigma_{L} / \sigma_{T}$ in Deep Inelastic Electron Scattering

ROCH-1:R-998-87:

A Study of the Kinematic and Nuclear Dependence of $R=\sigma_{L} / \sigma_{T}$ in Deep Inelastic Electron Scattering

Phys. Rev. Lett. 60:2591.1988:

Measurement of the Difference in $R=\sigma_{L} / \sigma_{T}$ and $\sigma_{A} / \sigma_{P}$ in Deep-Inelastic $e^{-}$-deuteron, $e^{-}-$Fe and $e^{-}$-Au Scattering

Phys. Rev. D36:2624.1987:

Search for Admixture of Massive Neutrinos in the Decay $\pi^{+} \rightarrow{ }^{+}{ }_{\nu}$

Phys. Rev. D33:2519.1986:

Observation of Double $\varphi$ Meson Production in $400 \mathrm{GeV} / \mathrm{c}$ Proton Nucleon Interactions

Phys. Lett. 180B:295.1986: LA L- $-86-25$ :

Search for Axion Like Particles in Electron Bremsstrahlung.

LAL-87.27:

Axions: A Review

Phys. Lett. 229B:150.1989; L.AL-89-24;

An Unambiguous Search for a Light Higgs Boson 
Davis 85

Davis 88

Dawson 86

Day 87

De 89

Debebe 85

Deboer 85

Decanp 89

Decamp 89B

Decamp $89 \mathrm{C}$

Decarnp 89D

Decamp 89E

Decamp 89G

Decamp $\mathbf{8 9 H}$

Decamp 90

Decamp 90B

Decamp 90C

Decamp 90D

Decamp 90E

Decamp 90F

Decamp 90G

Decamp $90 \mathrm{H}$

Decamp 901

Degtyarenko 89

Degtyarenko $9 r$

Dk ? agh 89

Delaat 85

Delavaissier 85

Deleenerrosi 86

Delesquen 85

Delesquen 88

Delesquen 89

Deliná 89

Delima 90B

Dernarzo 47

Demarzo 87B

Dernesty 88
TRI-PP-85-75:

A Test of Time Reversal Invariance in $p p$ Elastic Scattering at $200 \mathrm{MeV}$

TRI-PP-88-72:

Measurements of the $n p$ Observables at TRIUMF

Nuov Cim. 9C:1125.1986:

The Proton - Air Cross-Section at $10^{10} \mathrm{eV}$

Phys. Rev. Lett. 59:427.1587:

i Scaling in Electron Nucleus Scattering

FERMIILAB-CONF-81-151-E:

The Production of $J / \psi(1 S)$ and Associated Particles in the Collision of $530 \mathrm{GeV} / \mathrm{c}$ Protons and Pions with Nuclear Targets

Phys. Rev. C31:1841.1985:

Reaction deuteron ( $p$, deuteron $\pi^{+}$) $n$ at $506 \mathrm{MeV}$

Nucl. Phys. A444:589,1985:

Precision Measurement of the $2 P$-1s Transition Wavelength in Muonic ${ }^{13} \mathrm{C}$

Phys. Lett. 231B:519.1989: CERN-EP-89-132:

Determination of the Number of Light Neutrina Species

Phys. Lett. 235B:399.1930: CERN-EP-89-169:

A Precise Determination of the Number of Families with Light Neutrinos and of the $Z^{\circ}$ Boson Partial Widths

Phys. Lett. 236B:233.1990: CERN-EP-89-15ĩ:

Search for the Neutral Higgs Boson from $Z^{\circ}$ Decay

Phys. Lett. 236B:86,1990: CERN-EP-89-158;

Search for Supersymmetric Particles Using Acoplanar Charged-Particle Pairs from $Z^{0}$ Decays

Phy's. Lett. 236B:51 1.1990; CERN-E!-89-165;

A Search for New Quarks and Leptons from $Z^{\circ}$ Decay

Phys. Lett. 234B:399.1990; CERN-EP-89-141:

Determination of the Leptonic Branching Ratios of the $Z^{0}$

Phys. Lett. 237B:291.1990: CERN-EP-89-168:

Search for Neutral Higgs Bosons from Supersymmetry in $Z^{\circ}$ Decays

Phys. Lett. 241B:623.1990: CERN-EP-90-34:

A Search for Pair-Produced Charged Higgs Boson in $Z^{0}$ Decays

Phys. Lett. 241B:635.1990: CERN-EP-100-23:

Search for Decays of the $Z^{0}$ into a Photon and a Pseudoscalar Meson

Phys. Lett. 244B:551.1990: CERN-EP-90-54;

Heavy Flayour Production in $Z^{0}$ Decays

Phys. Lett. 244B:541.1990: CERN-EP-90-63:

Search for Neutralino Production in $Z^{\circ}$ Decays

Phys. Lett. 241B:141.1990; CERN-EP-90-16:

Search for the Neutral Higgs Boson from $Z^{0}$ Decays in the Higgs Mass Range between 11 and $24 \mathrm{GeV}$

Phys. Lett. 234B:209.1990; CERN-EP-89-139;

Properties of Hadronic Events in $e^{+} e^{-}$Annihilation at $\sqrt{s}=91 \mathrm{GeV}$

Phys. Lett. 236B:501,1990: CERN-EP-89-167:

Search for Exited Leptons in $Z^{0}$ Decay

CERN-EP-90-70;

Search for a Very Light Higgs Boson in $Z^{0}$ Decays

CERN-PPE-90-107:

Search for Excited Neutrinos in $Z^{0}$ Decay

JTEP-89-62;

Inelastic Electron-Nucleus Interactions at $5 \mathrm{GeV}$ Detected by AFGUS

1TEP-90-12:

Production of Cumulative $\Delta\left(1232 P_{\mathbf{a 3}}\right)$ in $e^{ \pm}$nucleus Interactions at $5 \mathrm{GeV}$

CALT-68-1578:

Resonant Substructure in $K^{-} \pi^{+} \pi^{+} \pi^{-}$and $\widetilde{K}^{0} \pi^{+} \pi^{+} \pi^{-}$Decays of Charmed $D$ Mesons

Phys. Lett. 162B:81,1985:

Strong Interaction Effects in Pionic ${ }^{205} \mathrm{~Pb}$

Phys. Rev. Lett. 54:2071,1985:

$\Lambda$ Production in $e^{+} e^{-}$Annihilation at $29 \mathrm{GeV}$

Phys. Lett. 1778:228.1986:

New Limits on Generation Mixing for Massive Neutrinos from $\pi^{+} \rightarrow \mathrm{e}^{+} \nu_{\mathrm{e}}$ Decay

Phys. Rev. D32:21.1985:

Measurement of the Reaction $\pi^{+} n \rightarrow \pi^{+} \pi^{-} p$ at 5.98 and $11.85 \mathrm{GeV} / \mathrm{C}$ Using a Transversely Polarized Deuteron Target

Nucl. Phys. B304:673.1988:

Measurement of Analyzing Power and Spin Correlations in n $p$ Elastic Scattering at .744 and $.794 \mathrm{GeV}$ Using a Deuteron Polarized Beam

Phys. Rev. D39:21,1989:

Measurement of the Reaction $K^{+} n \rightarrow K^{+} \pi^{-}$p at 5.88 and $11.85 \mathrm{GeV} / c$ Using a Transversely Polarized Deuteron Target

Nuove Cim. 101 A:975,198!:

Cross Section of Ternary Fission of Al, Ti, Co and Zr Nuclei Induced by $(0.8-1.8)$ GeV Photons

Nuovo Cin. J03A: 701,1990: Fission of Al, Ti, Co, $\mathrm{Zr}, \mathrm{Nb}, \mathrm{Ag}, \mathrm{In}, \mathrm{Nd}, \mathrm{Sm}$ and $\mathrm{Ta}$ Nuclei Induced by $(0.8-1.8) \mathrm{GeV}$ Photons

Phys. Rev. DS6:8.1987:

Measurement of Direct Photon Production at Large Transverse Momentum in $\pi^{-} p, \pi^{+} p$, and $p p$ Collisions at $30 \mathrm{C} \mathrm{GeV} / c$

Phys. Rev. D36:16.1987: MPI-PAE-EXP-EL-169:

Measurement of $\pi^{\circ}$ Production at Large Transverge Momentum in $\pi^{-} p, \pi^{+} p$ and $p p$ Collisions at 300 $\mathrm{GeV} / c$

Yad. Phys. 48:609.1988:

Experimental Study of Inclusive Quasielastic Electron Scattering on ${ }^{4} \mathrm{He}$ 
Denidor 89

Dengler $86 \mathrm{C}$

Depantilis 87

Derado 88

Derado 90

Derbin 86

Derrick 85

Derrick 85B

Derrick $85 \mathrm{C}$

Derrick 85D

Derrick 8.5E

Derrick 85F

Derrick 85G

Derrick 86

Derrick 86B

Derrick $86 \mathrm{C}$

Derrick 86D

Derrick 87

Derrick 87B

Derrick $87 \mathrm{C}$

Dersch 85

Desantics 86

Desantics 88

Deuzet 85

Dewalf 86

Dick 86

Dickey 85

Diekmann 88

Diemoz 86

Diesburg 87

Dieter 89

Digiacomo 85

Dijkstra 86

Dijkstra 86C

Dijkstra 86D

Dingus 88

Dingus 88B

Ditzlex 87
I'TEP-S4-103:

Measurement of the $\mathrm{K}^{+} \rightarrow \mu^{+} \nu$, Decay Probability

Z. Phys ( $33: 187.11) \times 6$ : MPI-P'AF-EXY-EL-165:

Multiplicity Distributions in Rapidity Intervals for $p$ p and $p$ nucleus Interactions at $200 \mathrm{GeV}$

Pliys. Re: Le'te. 59: H39.1947:

Limits on the Abundance and Coupling of Cosmic Axions at $4.5<m<5.0 \mathrm{MeV}$

Z. Phys. ( $40: 25,1988$

Long Range Correlations in Hadron Nucleus Interactions

2. Phys. C+7:23.1990: API-PAE-LXP-EL-221:

Investigation of Intermittency in Muon-Proton Scattering at $280 \mathrm{GeV}$

Pismá Zh. Eksp. Teor. Fiz. 43:164.1986:

New Experiment on Study of Reactor's Neutrino Elastic Scattering on Electron

Phys. Rev. D31:2352,1985:

New Results on the Reaction $e^{+} e^{-} \rightarrow \mu^{+} \mu^{-}$at $E_{\mathrm{cm}}=29 \mathrm{GeV}$

Phys. Rev. Let1. 33:1971.1985: A.NL-HEP-PR-8-46: HRS-PR-8.4-1: PL-84-517: IL'HEE-57. 1'M1-HE-87-13:

Inclusive $D^{0}$ and $D^{+}$Production in $e^{+} e^{-}$Annihilation at $29 \mathrm{GeV}$

Phys. Rev. Lett, 54:2568.1985: Production of $\phi$ and $D_{S}^{ \pm} \rightarrow \phi \pi^{ \pm}$in $e^{+} e^{-}$Annihilation at $29 \mathrm{GeV}$

Phys. Lett. 164B:199.1985:

Inclusive Charged Particle Production near the Kinematic Limit in $e^{+} e^{-}$Annihilation at $29 \mathrm{GeV}$

Phys. Lett. 166B:468.1986; ANL-HEP-PR-85-113: ILHEE-72: EN1-HE-85-21: Pl'-85-5-13: Precision Test of QED by Direct Comparison of $e^{+} e^{-} \rightarrow \gamma \rightarrow$ and $e^{+} e^{-} \rightarrow e^{+} e^{-}$at $29 \mathrm{GeV}$

Phys. Leti. 158B:519.1985: Neutral $K^{*}(892)$ and $\rho^{0}$ Meson Production in $e^{+} e^{-}$Annihilation at $29 \mathrm{GeV}$

Phys. Lett. 165B:444.1985. Comparison of Charged Particle Muitiplicities in Quark and Gluon Jets Produced in $e^{t} e^{-}$Annihilaion at $29 \mathrm{GeV}$

Phys. Lett. 168B:299.1986 Rapidity Dependence of the Charged Particle Multiplicities Distritutions in $e^{+} e^{-}$Annihilation at $29 \mathrm{GeV}$

Phys. Lett 166B:463.1986;

New Results from Bhabha Scattering at $\$ 9 \mathrm{GeV}$

Phys. Rev. D34:3304,1986:

Study of Quark Fragmentation in $e^{+} e^{-}$Anrihilation at 29 Gev: Charged Particle Multiplicits a, d ingle Part icle Rapidity Distributions

Phys. Rev. D34:3286.1986: ANL-HEP-PR-86-33; JLHEE-84: CNL-HE-86-7: PL-86-563: C86i/6i/16:

Experimental Study of the Reactions $e^{+} e^{-} \rightarrow e^{+} e^{-}$and $e^{+} e^{-} \rightarrow \gamma \gamma$ at $28 \mathrm{GeV}$

Phys. Rev. D35:2639.1987:

Hadron Production in $e^{+} e^{-}$Annihilation at $\Sigma_{\mathrm{cn},}=28 \mathrm{GeV}$

Phys. Lett. 189日:260.1987:

Evidence for the Decay $\tau^{+} \rightarrow \pi^{+} \eta \nu_{\tau}$

Z. Phys. C35:323.1987:

Charged Particle Multiplicity Distributions in $e^{+} e^{-}$Annibjlations at $29 \mathrm{GeV}$ : A Comparison with Hadronic Data

Phys. Rev. Lett. 55:1176,1485: Unusual Behavior of Projectile Fragments from the Interaction of Copper with Relativistic Ar Ions

Czech. J. Phys. B36:290.1986;

Deuteron Photodisintegration between $100 \mathrm{MeV}$ and $225 \mathrm{MeV}$

LNF-88-5.:

A. Critical Review of Deuteron Photodisintegration Data

LAL-85-20:

Search for Proton Decay in the FREJUS Experiment

Z. Phys. C31:13.1986:

$K^{+}$Fragraentation and Prompt Kaon Production in $K^{+}{ }_{p}$ Collisions at $70 \mathrm{GeV} / \mathrm{c}$

Phys. Rev. D33:32.1986:

Search for Possible Superheavy Particles in Sodium Nuclei

Nucl. Phys. A 441:189.1985:

The ${ }^{84} \mathrm{Fe}$ ( $p$ (polarized), deuteron) ${ }^{53} \mathrm{Fe}$ and ${ }^{140} \mathrm{Ce}$ ( $p$ (polarized), deuteron) ${ }^{139} \mathrm{Ce}$ : eactions at $122 \mathrm{MeV}$

Phys. Rept. 159:99.1988:

Spectroscopy of Mesons Containing Light Quarks $(u, d, s)$ or Gluons

Phys. Rept. 130:293.1986:

Nucleon Structure Functions from Nectrino Scattering

Phys. Rev. Lett. 59:2711.1987:

Measurement of the $\Sigma_{c}(2455)^{0}-\Lambda_{c}^{+}$and $\Sigma_{c}(2455)++-\Lambda_{c}^{+}$Mass Differences

BON"- [R-89-07:

Comparison between Photoproduction and Hadroproduction of $\rho^{0}$ Mesons Usink the CERN Omega Spectrometer

Phys. Rev. C31:292,1985:

Inclusive Pion Production in $330 \mathrm{MeV}, 400 \mathrm{MeV}$, and $500 \mathrm{MeV}$ Proton Nur uus Collisions

2. Phys. C31:375.1986: NIKHEF-H-86-1:

High Statistics Inclusive $\phi$ Meson Production at SPS Energy

Z. Phys. C32:353.1986:

Joint Production of $\phi$ Mesons and $\pi^{ \pm}, \pi^{0}, p, \bar{p}, K_{S}$ and $K^{ \pm}$in Hadronic Interactions

2. Pluys. C32:349,1986:

Hadronic $\phi$ Production and the Lund Model for Low pr Interactions

Phys. Rev. Lett. 60:1785.196x:

Search for Signal from Cygnus X-3 at Energ'cs above $50 \mathrm{TeV}$

Phys. Rev. Lett. 61:1906.1948

Ultrahigh-Energy Pulsed Emission from Hercules X-1 with Anomalou Air-Shower Muon Production

Phys. Rov. Lett. 50:I1545.19N7:

Measurement of Cll and Csl in $n$ p Elastic Scattering at 484 and $634 \mathrm{MeV}$ 
Dmitrier $k 5$

Dobrot in 85

Dobrowolsky kx

Dudge 85

Duers 86

Dolidze 86

Dolinsky 8.5

Dolinsky 86

Dolinst.y 88

Dolinsky 8 \&B

Dolinsky 89

Dolinsky 89B

Dombeck 87

Dombsky H.5

Domogatsk! 89

Donughue $84 \mathrm{D}$

Dorentrosch 86

Dorenbusch $86 \mathrm{~B}$

Dorentosch ơ

Dr mutusach 89

Doser $\mathrm{kH}$

Doss $x f$

Doss 87

Doss 88

Dougherty $\mathrm{xH}$

Doweli 86

Drechsel 85;

Drell 89

Dropenky 8f;

Drutskery ${ }^{4}$

Drutskoy n7 I3

Drutskes kh

Drutskoy s:

Drazhunin 44

Druzhinat

Druzhisus $x$;

Drischusus ok
Plys Le.1t. 15:13 1.4:3.14k!s.

First Meakurement of the Asyntnetry in Electron Scattering by a Jet Target of Polarized Deuterium Atoms

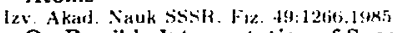

On Possible Interpretation of Special Type Events

LENI-8\%-1454:

Experiment al Data on Elastic $p p, n p, p$ deuteron, and $p$ He Forward Scattering at Intermediate Energies Phys. Rey (32:781.1!14:5.

$\left(e^{-}, p\right)$ and $\left(e^{-}, \mathrm{He}\right)$ Reactions in ${ }^{80} \mathrm{Z} r$ and ${ }^{92} \mathrm{Zr}$

Nucl Phis. A4t5:55\%.lesis:

Composite Particle Emission Following :- Absorption in ${ }^{8} \mathrm{Li}$ Test of Reaction Mechenisms

JIXR-E1-86-58:

Enhancements Observed in the Two Proton Invariant Mass Digtribution in the Pionless Deuteron Breakup at $3.3 \mathrm{GeV} / \mathrm{c}$

NoVO- -5.98 :

Results of the Experiments from the Neutral Detector at VEPP-2M in the Energy Region $\sqrt{s}=1.05-1.40$ GeV

Phy: Let 17+B:-153.1966: NOYO-8ti-6:\%: Reaction $e^{+} e^{-}-\pi^{0}$ in the c.m. Fnergy Range from 1.0 to $1.4 \mathrm{GeV}$

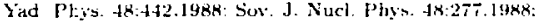
Observation of the Decay $\omega \rightarrow \pi^{0} e^{+} e^{-}$

2. Phy C42:511.19k9: NOV'O- $4 \mathrm{k}-89$ :

Radiative Decays of $\rho$ and $\lrcorner$ Mesons

NONO-89-68:

Revi w of $e^{-} e^{-}$Experiments with Neutral Detector on VEPP-2M. Part 1

NOVO- is 104: Review of $e^{+} e^{-}$Experiments with Neutral Detector on VEPP-2M. Part 2

Phys. Let1, 194B:591.1987:

Search for Neutrino Oscillations in the Appearance Mode $\nu_{\mu}-\nu_{t}$ for Neutrino Energies near the Muon Threshold

TRI-PP-85-1:

Inclusive Measurement of $\left(p, \pi^{-} \times n\right)$ Double Charge Exchange Reactions on Bistnuth from Threshold to $800 \mathrm{MeV}$

Arta Phys. Hung 65:323.1989:

Searching for Monopoles in Lake Baikal

Phys. Rev. D31:70.1985: CMHEP-201:

Measurement of the Weak Neutral Current via Nuclear Parity Violation

Phys. Let 180B:303.1986: CERN-EP-86-93:

Experimental Veriflcation of the Universality of Electron Neucrino and Muon Netitrino Coupling to the Neutral Weak Current

Phys. Lett 166B:-473.1986: CEIRN-EP-85-190:

A Search for Decays of Heavy Nelutrinos in the Mass Range of $0.5-2.8 \mathrm{GeV}$

Z. Phys. C 40:497.1988: CERN-EP-87-167:

Prompt Neutrino Production in $400 \mathrm{Gel}$ ?roton-Copper Interactions

Z. Phys. C41:567.1989; CERN-EP-88-136;

Experimental Results on Neutrino - Electron Scattering

Phys. Lett. 215B 792.1988:

$\bar{p}$ p Annihilation into $K^{0} \bar{K}^{0}$ in Hydrogen Gas

Phys. Rev Lett. $57: 302.1986$ :

Nuclear Cellective Flow as a Function of Projectile Energy and Mass

Phys Rev Lett. 59:2720.1987.

Fragment Flow in Nuclear Collisions

Phys. Rev. ( $37: 1633.1988$ :

Multiplicity and Bombarc ng Energy Dependence of the Entropy in Relativistic Heavy-lon Reactions

L.i3L-26303:

An Experimental Investigation of Double $\beta$ Decay of ${ }^{100} \mathrm{Mo}$

('ERN-EP-SW-152:

Recent Results from the UA1 Experiment

Phys. Rev Lett. 54:30.1945:

Search for A nomalous Fragments of ${ }^{\mathrm{B} 0} \mathrm{Fe}$ Using Plastic Nuclear F'rack Detectors

(I.NS-89-432.

$B^{0} \mathscr{B}^{\circ}$ Mixing and Charmless $B^{*}$ Decays: Recent CLEO Results

Phys Rev (:32:1305.1985)

Excitation Functions for the Production of ${ }^{18} \mathrm{Fl}$ and ${ }^{24} \mathrm{Na}$ from $\mathrm{Al}$ and Si with Fast Pions

ITEP-87.112:

Cross Sections of the Strange Particles Production C zuatiels in $\tau^{+} p$ Interactions at $4.23 \mathrm{GeV} / \mathrm{c}$

ITEP-sT- 113 .

Observation of Strange Exotic Baryons at $\Lambda \pi^{+} \pi^{+}$System

ITEP-ss-1.

Exotic Baryons with Isotop Spin $J=5 / 2$ in $\pi^{+} p$ Interactions

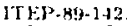

Search for Strange Exotic Baryon Resonances at the $4.23 \mathrm{GeV} / \mathrm{c}$ Momentum

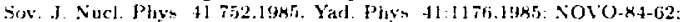

Test of Quantum Electrodynamics in the Campton Scattering of Quasireal Photons by Electrons and Positrons at the VEPP-2M Storage Ring

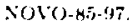

Study of 0 Meson Decays with the Neutral Detector at the VEPP-2M Collider

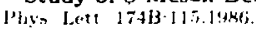

investigation of the Reaction $e^{+} \epsilon^{\prime \prime} \rightarrow \eta \pi^{+} \pi^{-}$in the Energy Range up to $1.4 \mathrm{GeV}$

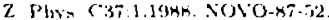

Search for Rare Radiative Decays of o Meson nt VEPP-2M 
Dubar 89

Dulisina $\mathbf{3 8}$

Dubovy 88

Duch 89

Dufy 85

Duffy 86

Duffy 88

Dugan 85B

Dukes 87

Dukhovskoy 87

Durkin 88

Dworkin 90

Dyakonov 89

Dyck 85

Dzhaoshvili 87

Dzhaoshvili 88

Dzhaoshvili 88B

Dzhaoshvili 90

Dzhincharadz $8 \hat{0}$

Ebisu 84B

Ebisu 87

Eckart 88

Eckhardt 88

Edberg 88

Edwards 89

Efendiev 89

Eñov 89

Eggert 89

Egli 86

Eoli 89

Eichler 86

Ejiri 89

Ellegard 85,

Ellegaard 89

Elliott 86

Elliote 87

Ellott $87 \mathrm{~B}$
Yark, Phys. 40.1239.1949:

Parametrixation of Total Cross Sections at Intermediate Energies

Pisma Zh. Eksp. Teur. Fia 14.233 .1986 :

Observation of the Slow Pion Production in the Nucleus Nucleus Internctions

Yad. Phys. 77.1663.1488: Soy .J Nucl. Phy: 17:105.1.1968:

Nuclear Composition of 300 - 500 TeV Cosmic Rays from EAS Hadronic Component

Z. Phy's. C45:223.1989: M2-1:TAl'89-5:

Observation and Amalysis of $E$ Mesons in $\bar{\rho} p$ Annihilation at Rest in $H_{y} G_{a s}$

Pliys. Rev. Lett. 55: 1816i.1945:

A-dependence of Charm Production

Phys. Rev. I ett. 57:1522.1446.

Characteristics of Charm Production by $400 \mathrm{GeV}$ Protons

Phys. Rev. D38:2032.1988:

Neutrino Production by $400 \mathrm{GeV} / c$ Protons in Beam-Dump Experiment

Phys. Rev. Lett. 55:170.1985: HL'TP-85-A033:

New Neutrino Constraints on Majorana Mass Matrices

Phys. Lett. 193B:135.1987:

Polarization of $\Sigma^{\mathbf{0}}$ Hyperons in Inclusive Procuction from $28.5 \mathrm{GeV} / \mathrm{c}$ Protons on Beryllium

Yad. Phys. 47:1816.1988; ITEP-87-198:

Meacurement of the Total Cross Sections of the Proton Interactions with $\mathrm{Nuclei}^{8} \mathrm{Li}$, ${ }^{7} \mathrm{Li}$, and ${ }^{\theta} \mathrm{Be}$ at 2 $\mathrm{GeV} / \mathrm{c}$

Phys Rev Lett 61:1811.1988:

Limit on $\bar{\nu}_{\mu} \rightarrow \bar{\nu}_{e}$ Oscillations

Phys. Rev. DHl:780.1990:

High Statistics Measurement of Ga/Gv in $\Lambda \rightarrow p \ell^{-} \bar{\nu}$

Pisma Zh. Eksp. Teor. Fiz. 50:408,1989:

On Change of Nuclear Composition of Primary Cosmic Radiation in the Energy Range $10^{17}-10^{18}$ eV

Phys. Lett. 157B:139.1985:

Atomic Mass Differences in Gadolinium and Terbium atd the $K$ Capture of ${ }^{168}$ Tb as an Indicator of Neut riro Mass

Pisma Zh. Eksp. Teor. Fiz. 45:264.1987:

Evidence of Narrow Jets in Hadron-Carbon Interactions at Average Energy of $0.4 \mathrm{TeV}$

Yad. Phys. 18:299.1988:

Particle Distribution at the Target Fragmentation Region in Hadron-Carbon Interactions at the Average Energy .4 TeV

Yad. Phys 48:1853.1988:

A bont Fluctuations of Transverse Encrgy and Multiplicity Distributions on Pseudorapidity in the Intaractions of Hadrons with Carbon Nuclei at Average Energy of .4 TeV

Nucl. Phys. B336:86, 1990 :

Dependence of Inelasticity Coefficients of Charged rarticles on the Atomic Number of a Target Nucleus in the Energy Range 0.2 - $2.0 \mathrm{TeV}$

Y'ad. Phys. 44:991.1986: Sov. J. N_cl. Phys. 44:639.1986:

Correlations of Identical $\pi^{ \pm}$Mesons and Characteristics of their Emisgion Region in $\pi^{-} p$ Interactions at $5 \mathrm{GeV} / \mathrm{c}$

Jour. of Phys. G 11:883.1985: KOBE-84-08:

New Limit on the Magnetic Monopole Density in Old Iron Ore

Phys Rev. D36:3359.1987.

Search for Magnetic Monopoles Trapped in Old Iron Ores Using a Superconducting Detector

BONIV-IR-88-21:

Two-Particle Correlations and Density Fluctuations in Proton-Antiproton Interactions at c.m. Energies from 200 to $800 \mathrm{GeV}$

Phys. Rev. Lett . 60:2567.1988:

Tower Gravity Experiment: Evidence for Nonnewtonian Gravity

LBL-25652:

Inclusive Production of Vector Mesons in $e^{+} e^{-}$Annihilation at $\sqrt{s}=29 \mathrm{GeV}$

SLAC-PLB-5160;

Hadron Production in $e^{+} e^{-}$Annihilation from $\sqrt{s}=5.0$ to $7.4 \mathrm{GeV}$

Kr. Soob. JINR 36:9.1989:

The Partial Wave Analysis of $K^{-} \pi^{-} \pi^{+}$System Coherently Produced on Nuclei at $40 \mathrm{GeV}$

Yad Phys. 49:900.1989:

About Cross Section of the Interaction of Protons with Air Nuclei at the Energies $2 \cdot 10^{10}$ eV

CERN-EP-89 42

Experimental Situation of Beauty Oscillations

Phys. Let2. 175B:97,1986:

First Observation of the Decay $\pi^{+}-e^{+} \nu_{r} e^{+} e^{-}$and a Determination of the Formfactors $F_{\nu}, F_{a}, R$

Phys. Lett. 222B:533.1989: PSI-PR-89-02:

Measurement of the Decay $\pi^{+}-e^{+} \nu_{e} e^{+} e^{-}$and Search for a Light Higgo Boson

Phys. Lett. 175B:101.1986:

Limits for Short Lived Neutral Particle Emitted in $\mu+$ or $\pi+$ Decay

Phys. Lett. 2281:24,1989.

Search for the Dihyperon by Double Weak Decay of Nuclei

Phys. Lett. 1543:110.1985:

The $\boldsymbol{p}\left({ }^{3} \mathrm{He},{ }^{3} \mathrm{H}\right) \Delta\left(1232 P_{33}\right)++$ Reaction

Phys. Lett. 2:31 B:365.1989:

Spin Structure of the $\Delta\left(1232 P_{33}\right)$ Excitation

Phys. Rev. Lett, 56:2582.1986:

Experimental Investigation of Double $\beta$ Decay in ${ }^{82} \mathrm{Se}$

Phys Rev. Lett. 59:16413.1087:

Limit on Neutrinoless Double- $\beta$ Decay with Majoron Emission in ${ }^{2} \mathrm{Se}$

Phys Rey la't i. 59:20201.1687:

Direct Evidence for Two-Neutrino Double- $\$ 3$ Decay in ${ }^{82}$ Se 
Elnadi 88

Elnad, sk

Elnagly 87

Elmaghy $\& 7 \mathrm{~B}$

Elsen 90

Endo 88

Enghard: 8i

Eno 88

Eino 89

Eno 89B

Eno 89C

Enyo 85

Epstein 85

Ereditato 85

Ergakov 86

Erhan 85

Ermakov 86

Ermakov 86B

Ermakov 86C

Etnuein 85

Ero 87

Erriquez 85

Esaulor 86

Esaulov 8T

Etkia 85

Etkin 98

Fabbri 88

Fairfield 88

Faissner 88

Faissner 89

Falciano 85

Falciano 86

Falk 83

Falvard 88

Tayard 89

Fayet 89

Fearing 86

Feindt 89

Felcini 89

Ply. Rer. Lett (i) 1.271.14ssi:

Production of a New Light Neutral Boson in High Energy Collisions

JINR-E1-NT-4T2.

Particularities of ${ }^{22} \mathrm{Ne}$ Nuclei Inelastic Interactions with Emulsion at $4.1 \mathrm{~A}$ GeV/c

JINR-E1-RT-54T:

Fragmentation of ${ }^{2} \mathrm{Ne}$ in Emulsion at $4.1 \mathrm{~A} \mathrm{GeV} / \mathrm{c}$

2. Pl:y' ( $46: 349.1490$ : DEsi:-89-127:

A Measurement of the Weak Axial Couplings of the $b$ and $c$ Quarks

INS-724:

Reaction Mechanism of ${ }^{4} \mathrm{He}(\gamma, p \mathrm{n}) \times$ at $E(\gamma)=0.17 \mathrm{GeV}$ to $0.27 \mathrm{GeV}$

2. Phys C $35: 511,198 ;: 2 F K-617$;

Limits on Heavy axion Production from the Reaction $n p \rightarrow$ deuteron axion

KEK-88-4T: ANI - $88-10$ :

A Search for New Heavy Quarks Using Hadronic Events Containing Leptons

KEK-89-4: ANY'-89-01

Recent Resules from AMY Experiment

Phys. Rev. Lett . 63:1910.1989; KEK-89-46: AMY'-89-08:

Search for a Fourth-Generation Charge $-1 / 3$ quark

l'R-1132:

A Search for a Fourth Generation Charge $-1 / 3$ Quark

Phys. Lett 159B: 1.1985: KEK-85-23:

Analyses of Particle Production in Hadron Nucleus Reactions at Several GeV with Two Moving Source Model

Phys. Rev, C32:967.1485: T'R1-PP-85-20:

Study of the ${ }^{3}$ He $(p, 2 p)$ deuteron and ${ }^{3}$ He ( $p, p$ deuteron) $p$ Reactions at Large Recoil Momenta

Phys. Lett. 157B:463.j985: CERN-EP-85-11

Upper Limits for botton bottom Production in $\pi^{-}$Tungsten Interactions at $194 \mathrm{GeV} / \mathrm{c}$

Czech. J. Phw a $36: 985,1946$ :

Investigation of High Energy Lightest Nuclei Produced in the Interaction of Protons with Incident Momenta from 2 to $10 \mathrm{GeV} / c$ with Be, Al, Cu, Ta

Phys. Lett. 152B:131.1985; CERN-EP-84-147; Comparison of $\ddot{p} p$ and $p p$ Elastic Scattering with $0.6<-t<2.1 \mathrm{GeV}^{2}$ at the CERN ISR

Yad. Phys. 44:143.1986: Observation of Low Lying Dibaryon Resonances in the ${ }^{40} \mathrm{Ar}$ Reaction at $1 \mathrm{GeV}$

LENI-86-1158:

Investigation of Final States of the $p p \pi^{+}$System in the Reaction $p{ }^{40} \mathrm{Ar}$

Yad. Phys. 43:1359.1986:

Investigntion of the Parity Nonconservation in the Reaction ${ }^{10} \mathrm{Bor}\left(n,{ }^{4} \mathrm{He}{ }^{7} \mathrm{Li}\right.$ )

SACLAY-DPHPE-85-06:

Early Results from the FREJUS Experiment

Nucl. Phys. A472:733,1987; KFKI-1987-23-A:

Production of Protons, Deuterons and Tritons on Carbon by Intermediate Energy Neutrons

Phys. Scr. 33:202,1986: CERN-EP-85-165;

A Measurement of the Total Charm Cross Section in $200 \mathrm{GeV} / c$ and $360 \mathrm{GeV} / \mathrm{c} p \mathrm{n}$ Interactions Using a Holographic Bubble Chamber

Yad. Phys. 44:1187.1986: Sov. J. Nucl. Phys. 44:770.1986;

Scaling of Inclusive $e^{-}$deuteron Spectra and of Seructure Functions in the Quasielastic Fegion

Yad. Phys. 45:410,1987:

Measurement of the Neutron Magnetic Form Factor in the deuteron ( $e^{-}, e^{-}$) $n p$ Reaction for Momentum Transfers $0.48<Q^{2}<0.83 \mathrm{GeV}^{2}$

Phys. Lett. 165B:21 $7,198 \overline{5}$ :

Observation of Three $2^{++}$Resonances in the Glueball-Enhanced Channel $\pi^{-} p \rightarrow \phi \phi n$

Phys. Lett. 201B:568,1988; BNL-40200: BNL-40716; Increased Statistics and Observation of $g_{T}, g_{T}^{\prime}$ and $g_{T}^{\prime \prime} 2^{++}$Resonances in Glueball Enhanced Channel $\pi^{-}$ $p \rightarrow \phi \phi$

DFUB-88-20:

Maximum Charged Particle Densities in Small Intervals of Rapidity at ISR Energies

SLAC-PUB-466T:

Search for axion Production in $\mathbf{r}(1 S)$ Decays

Z. Phys. C37:231.1988:

Search for Two-Photon Decay of a Light Penetrating Particle from 590 MeV Proton Beam Dump

2. Phys. C44:55i.1989:

Search for the Electron-Positron Decay of an Axionlike Particle from a 590 MeV Proton Beam Dump

Phys. Letl. 158B:92,1985; CERN-EP-85-22:

Production of $\boldsymbol{T}$ by $194 \mathrm{GeV} / \mathrm{c}$ Negative Pions on Tungsten

2. Phys. C31:513.1986: CERN-EP-86-35:

Angular Distributions of Muon Pairs Produced by $194 \mathrm{GeV} / c$ Negative Pions

Phy's. Rev. C33:988.1986: TH1-PP-83-12T:

Pion Production Cross Sections and Analyzing Powers in the Inclusive ${ }^{12} \mathrm{C}\left(p, \pi^{+}\right) \times \mathrm{Reaction}$ at 400 $\mathrm{MeV}$ and $450 \mathrm{MeV}$

Phys. Rev. D38:2706.1988: LAL-88-04;

Study of Hadronic $J / \psi(1 S)$ Decays Involving $\phi$ and $\omega$ Production

LAL-89-40:

Stat us in CP Violation

Phys. Lett. 219B:521.1989: LPTENS-8k-33:

Flux Limit for High Energy Cosmic Photinos from Underground Experiments

Czech. J. Phys. B.36:263,1986:

Radiative Reactions in Two and Three Nucleon Sytems

DESY-K9-142:

Light Hadrons as Seen via Two Photons by CELLO

CERN-EP-89-173:

Search for $t$-Quark Decay into Charged Higgs with the UA1 Experiment 
Foldman 85

Fidiuas 8513

Fridman R9

Fesdmal

Felipric 89

Ferbel dit

Ferguson हi

Fernandez $84 C$

Fernandez 85

Fernande'z $85 \mathrm{~B} 3$

Fernande'2 850

Firnandez 850

Fernandez Bi

Fermandez $87 \mathrm{~B}$

Fermandez BTC

Fertaroto ge

Fickunger $86 \mathrm{~B}$

Fidecaro $\mathbf{8 5}$

Filaseta $47 \mathrm{~B}$

Filator $8 x$

Finley

Fisilyer $\mathrm{k}$

Fisher kT

Fistuer 89

Fitch 8.5

Fitch 86

Fitch 88

Fitzgerald n6

Fontane $\mathrm{RS}$

Ford 86

Ford 87

Ford $87 \mathrm{~B}$

Furd $87 C$

Ford 89

Forden 8513

Furino 87

Foudas 88

Foudas 8HB

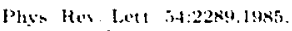

Search for Monojet Production in $f^{+} 6^{-}$Annihilation

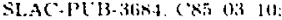

Search for Heavy Neutrinos Produced in $e^{+} e^{-}$Annihilation

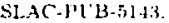

Measurement of the $Z^{\circ}$ Boson Resonance Parameters

SLAC-PI'H-5I:S:

Production and Decay of $Z^{\circ}$ Bosonts at the SLC

('FHN-EP-8(1-11:

$\pi^{-} p$ Elastic Scattering from $295 \mathrm{ta} 450 \mathrm{MeV} / \mathrm{c}^{+}$

Arta Phys Pelon. Bl T:435.198ti.

Direct Photon Production

Pliys. Fe'v Diti 19lil.1987

$\rho$ and $\nu$ Production in $\pi^{+} p$ Interactions at $15.7 \mathrm{GeV} / \mathrm{c}$

Phys. Fe'v Lett. 5.t:1118.1985: SLAC'I'1'13-3520:

Search for Single Photons from Supersymmetric Particle Production

Phys Rev, Di3 $1: 2724.1985$ :

Measurement of Energy Energy Correlations in $e^{+} e^{-}-$hadrons at a Center of Mass Energy of 29 GeV

Phys. Fev. D31:1537.1985. SL.AC-P!'B-34T!t.

Precision Measurement of Total Cross Section for $e^{+} t^{-} \rightarrow$ hadrous at a Center of Mass Energy of 29 GeV

Phys. Fer. Letr. 54:1620.1985:

Electroweak Effects in $e^{+} e^{-} \rightarrow \tau^{+} \tau^{-}$at $28 \mathrm{GeV}$

Plivs. Rev. Lett. it:1624.1985.

Measurement of Tau Lifetime and Branching Ratios

Phys. Fur D35:10.1987; SLAC-PlB-1005:

Electroweak Effects in $\epsilon^{+} c^{-} \rightarrow e^{+} c^{-}$at $E_{\mathrm{m} m} 29 \mathrm{GeV}$

Phys. Rev D35:1.1987, SL.AC-Pl'B-4(I)t:

Tests of Quantum Electrodynamics with Two, Three, and Four-Photon Final States from $e^{t} e^{-}$Annihilation at $E_{\text {tm }}=29 \mathrm{GeV}$

Phys. Rev. [35:374,1987: \$1.' -Pli-402i:

Search for Single Electruis from Supersymmetric Particle Production

2 Plys, (44:541.1989: INFN-88-923: ROAE-923-1988:

Search for the Inclusive Production of $J / \psi(1 S)$ in $e^{+} e^{-}$Collisions Uaing PLUTO Data

Phis. Rev. D34:3332.1986.

Search for the $X(1936)$ Meson in Antiproton Proton Interactions

Phys. Lett. 156l3:122.1985: ('ERN-PRE-R5-0135:

Experimental Search for Neutron-Antineutron Transitions with Free Neutrons

AX-1220-ILLINOIS:

Hadroproduction of $\Lambda_{c}^{+} \rightarrow p K \pi$

LENI-88-14014:

Ternary Nuclear Fission and Fission with a Large Multiplicity of Charged Particles in ${ }^{230} \mathrm{U}$ Nuclei by 1 GeV Protons

Phys. Rev. D33:2528,1946: ANL-HEP-Pll-85-74:

Study of Polarized Proton Diffraction Dissociation in the Reaction $p p \rightarrow p \pi^{+} \pi^{-} p a t 11.75$ GeV/c

2. Plivs. C 3 s: 105,1988:

Transverse Momentum Systematics in Proton Proton and Light lon Collisions at the ISR

Phys. Lett. 192B:460,1987: Limit on Double $\beta$ Decay with Majoron Emission

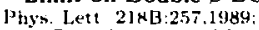

A Search for Double $\beta$ Decay in ${ }^{76} \mathrm{Ge}$

2. Phys. C31:51.1986: CERN-EP-85-195;

Evidence for Higher Twist Effects in Fast $\pi^{-}$Production by Antineutrinos in Neon

Phys. Rev. D3:3.1486, 1986:

Search for $D^{*}(2010)$ Production in $\pi$ nucleon Interaction

Phys. Rev. Leth 60:1401,1948:

Limits on the Existence of a Material-Dependent Intermediate-Range Force

Phys. Rev Cat fil9.1940:

Forward-Angle Cross Sections for Pion-Nucleon Charge Exchange between 100 and $150 \mathrm{MeV} / \mathrm{C}$

Nucl. Plys. 13:321:299.198!

Measurement of the Spin Correlation Parameters $A_{00 k k}$ and $A_{00 k k}$ in $p$ Elastic Scattering from .88 to 2.4 GeV

Phys Hev Di3:3:3472.1986: SLAC:PLB-4003:

Search for Single Photons from Radiative Neutrino or Supersymmetric Particle Production

Plivs. Lett. 19KB:247,1947: SLAC-PLB-4333: COLO-HEP.14k; Measurements of Tau Decays to Three Pions

Phys Rev. D361:1971.1987: SLAC-PL'B-4066; COLO-HEP-13:; Measurement of the Polarization of Tau Leptons Produced in $e^{+} e^{-}$Annihilation at $\sqrt{s}=29$ GeV

Phy Rev. D35.40k.19K7. SLAC-P('B-4036; COLO-HEP.123: SLAC-PCB-4636; COLO-HEP-123; Preciae Measurement of the Branching Fraction for the Decay $\tau^{ \pm} \rightarrow \nu_{\tau} \pi$

Phys. Rev. D40 1385.1949: SLAC-PL13-434X.

Measurement of as from Hadron Jetr in $e^{+} e^{-}$Annibilation at $\sqrt{s}$ of $29 \mathrm{GeV}$

RAL-85-076;

Lifetime Measurements of Hadrons Containing Heavy Quarks at PETRA and PEP

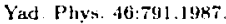

Associative Photoproduction of Charned $\Lambda_{r}^{+}$Baryons and $\bar{D}$-Mesons and $\Lambda_{r}^{+}$Weak Decays

CR-1057:

Neutrino Production of Opposite-Sign Dimuons at the Tevatron

FERMILAB-C'ONF-HA- I61)-E:

Neutrino Production of Charm at FNAL E744 
Fratue do

Frauklin

Franklin 8 R

Fransson 10

Franz 45

Franz 88

Franz 88B

Franz $\times 9$

Franzini 87

Franzini 89

Frascaria 87

Frascaria 89

Fredrikston 87

Freeman 89

Friednan 89

Friedman 90

Fritschi 86

Fry 89

Fuess 87

Fujisaki $8 \mathbf{8}$

Fukui 88

Fulton 89

Fuiton 89B

Gabriclse 90

Gabunia 89

Gabuma 90

Gachura 85

Gagarin 89

Ciniser 85

Galsser 49

Gijewski 89

Gal $86 \mathrm{~B}$

Gall 98

Galutuyald 88

Gan ks

Gan 8.53

Gan 87

Gan $87 B$

Gan 88

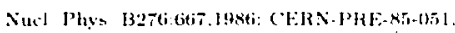
A Spin-Parity Analysis of the $o h^{+}$System Produced in the Reaction $K^{+} p-\phi K^{+} p, b^{-} K^{+} K^{-}$at 13 GeV/r

Phys Let: 1:413.111.1985

Evidence for a Narrow $S$ Resonance in $\bar{p} p$ Annihilation at $1937 \mathrm{MeV}$

$\mathrm{Tl} H \mathrm{HE} \cdot 8 \mathrm{~B} \cdot 1 \mathrm{112}$.

Experimental and Theoretical Status of Buryon Magnetic Momenty

THESIS:

A Search for Prompt Electrons with Low Transverse Moment um in 630 GeV Proton-Antiproton Collisions

Physi. Let1 153B:3*2.1985

Charged Particle Production on ${ }^{12} \mathrm{C}$ by Intermediate Energy Neutrons

Nucl Phys At9u:titi. 198R.

Total Neut ron-Nucleus Cross Sections at Intermediate Energies

LBL-25705.

Study of High Energy Nucleus Nucleus Collisions

FREI-AIEP. \&!\}-04:

Neutron Induced Production of Protons, Deuterons and Tritons on Copper and Bismuth

Phys. Rev. D35:248.1987:

Limits on Higge Bosons, Scalnr-Quarkonia, and $\eta_{b}$ from Radiative $\Upsilon$ (1S) Decays

Phys. Rept. 173:1.1989: CEIRN-TH-50N3:

$B \bar{B}$ Mixing: A Review of Recent Progress

INPO-DRE-87-31:

Search for Strange Sixquark States in $p \rightarrow K+X$ Missing Mass Spectra

Nuovo Cim. 102A 561.1989 .

Hyperon-Nucleon Final-State Interaction in $p p \rightarrow K^{+} X$ Experiment and $t h e \Lambda N\left(2130^{3} S_{1}\right)^{+} S=-1$ Strange Dibaryon

Phys. Rept. 14t.184.1987: THITA-THY-Bh-06:

High-Energy Collisions with Atomic Nuclei: The Experimental Results

FERMILAB-CONF-89-1+4-E:

A Missing Transverse Energy Analysis of $1.8 \mathrm{TeV} \vec{p} p$ Collisions Observed at CDF

Phys. Let t. 231B:39.1989: TRI-PP-89-49:

Integral Cross Sections for $\pi^{+} p$ Scattering between 52 and $128 \mathrm{MeV}$

TRI-PP-90-9: Integral Cross Sections for $\pi^{+} p$ Interactions at Low Energies

Phys. Lett. 173B:485.1986:

An Upper Limit for the Mass of $\bar{\nu}_{c}$ from Tritium $\beta$ Decay

KEK-89-30: AMY'-89-02:

Tests of QCD at the TRISTAN $e^{+} e^{-}$Collider

Phys. Rev. D35:3297.1987: Search for Anomalous Particles in High Energy Hadron Proton Interactions

Nuovo Cin. 99A:395.1988:

Polarization Meagurement in Pion-Nucleon Charge Exchange $\pi^{+} n \rightarrow \pi^{0} p$ at $6 \mathrm{GeV} / \mathrm{c}$

Phys. Lett. 202B:441,1988: KEK-87-152: Vector Resonances Around $1.6 \mathrm{GeV}$ of the $\eta \pi^{+} \pi^{-}$System in the $\pi^{-} p$ Charge Exchange Reaction at 8.95 $\mathrm{GeV} / \mathrm{c}$

Phys. Lett. 22.1B:445.1989; CLNS-88-877: C'LEO-88-4:

First Observation of Inclusive $J / \psi(1 S)$ Production in UPSILON Decays

Phys. Rev. D41:1401.1990; CLNS-89.913; C'LEO-89.7:

Radiative $\mathbf{Y}(1 S)$ Decays

(CERN-PPE-90-98:

A 1000-Fold Improvement in the Measured Antiproton Mass

Yad. Phys. 50:1035.1989:

Yields of Neutral Strange Particles in Pion Nuclear Interactions at $40 \mathrm{GeV} / \mathrm{c}$

Yad. Phys. $51: 1607.1990$ :

Associative Multiplicity in $\pi^{-}$A Interactions with Strange Particle Production

ITEP-85-59:

Measurement of Total Cross Sections of Protons and $\pi^{+}$Mesons with Nuclei at $1.35-3.75$ GeV/c

Pisma Zh. Eksp. Teor Fiz 49:424.1984:

Nuclear Scaling Destruction in the Heavy Nucleus Interactions

Phys. Rev. D34:T11.1986: SLAC-PL'B-2899: Charmonium Spectroscopy from Inclusive $\psi(3770)$ and $J / w(1 S)$ Radiative Decays

Phys. Rev. Lett. 62:1425.1989:

Search for Photon of Energy > 50 TeV from SN 1987A in Early 1988

Phys. Rev. Let1. 62:2069.1989: Experimental Upper Limit to the Galactic Stellar-Collapse Rate

TRI-PP-86-43: Hypernuclear Interactions

Phy's Rev. Let1. 60:186,1988:

Precision Measurement of $K^{-}$and $\Sigma^{-}$Masses

YERE-1084 147$\}-88$

Polarization Investigations of Photoproduction on Deuteron in the Dibaryon Resonance Excitation Region

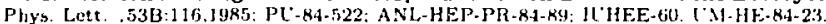

Measurement of the Reaction $e^{+} e^{-} \rightarrow \tau^{+} T^{-}$at $29 \mathrm{GeV}$

PL-85-539:

New Fesults on Tau Lepton from PEP

Phys. Rev. Lett 51):411.1987: LBS-22362: SLAC:P('B-4110.

Study of Tau Decay Modes with Multiple Neutral Mesons in the Final States

Phys. Lett. 1973:561.1967: SLAC-P('U-4365:

Upper Limit on the Branching Ratio for the Decay $\tau^{-} \rightarrow \pi^{-} \eta \nu_{\tau}$

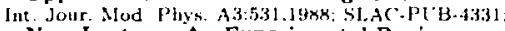
New Leptons: An Experimental Review 
Gan $88 B$

Ganenko RB

Ganenko R!

Garcon 80

Gascun 861

Garcon $87 B$

Garcun 84

Garuet ! H9

Garreta 87

Gatreta 85

Garutchatiz 87

Garutchava 87B

Gasparyan 84B

Gasparyan 85

Gavrilos 85

Gavrilov 85B

Gavrin 89

Gazdzicki 85

Gazzaly 87

Geer 89

Geesaman 89

Geichgimbel 85

Geiregat 89

Geiregat 90

Geist 89

Gelphman 85

Germer 89

Gentile 87

Georgiopoulo 84

Gerbier 87

Gerdyukov 86

Gerdyukov 86B

Gerdyukov 87

Germand 85C

Ghosits 85

Ghosh 86

Ghosh 87

SLAC:-PT'13-46004

Review of Recent Results on the $\tau^{t}$ Lepton

Pisina Zh. Fksp Tior. Fiz. $1743 \times .1$ thk

Asymmetry in Cross Section of Inclusive Reaction $(\gamma, p)$ and $(\gamma, \pi)$ an the ${ }^{3} \mathrm{He}$ and ${ }^{4} \mathrm{He} N$ uclei

Jisma Zh. Eksp. Tior. Fiz. 50:220.14kil

The Mensurenent of Deuteron Spliting Cross Section by Photons Polarized Parallel and Transversed with Respect to Reaction Plane

Nucl. Phys. Att5ititio.19k5:

The Continuous Energy Uependence of $\mu$ p Differential Elastic Cruss Sections between $500 \mathrm{MeV}$ and 1200 MeV

Ceach. J. Phys. B3ti 955.1981;.

The Experimental Search for Narrow Dibaryons at SATURNE

Phys Let1. 183B:273.19RT: Search for Narrow Structures in the Analyzing Power for $p p$ Elastic Scattering (655 - 1017 MeV)

SACLAY-DPhN-25199:

Polarization Measurements in Elastic Electron - Deuteron Scattering

Phys. Rev. D40:170\%.1989: ANL-HEP-PR-8(1-21

Measurement of a Mixed Spin-Spin Correlation Parameter for $n$ p Elastic Scattering

Phys, Let t. 1501:05.1955: ('ERN-EP-84-92.

Search for Antiproton Nucleus States with $\bar{p} p$ Reactions

CERN-EP-85-69:

Search for Antiproton Nucleus States

Pistma Zh. Eksp Jeor. Fjz, 47.121,1984: IFlE-8ï-184:

An Estimate of the Central $\bar{\Lambda}$ Production in Cross Section of the Reaction $K+p \rightarrow \bar{\Lambda} \times$ at 32 GeV/c

Yad. Phys. 48:1142.1988: IFVE-8T-110:

The Triple Regge Analysis of Inclusive Production of $\Lambda$ and $\bar{\Lambda}$ Interactions at $32 \mathrm{GeV} / c$

Yad. Phys. 41:1229.1985: JINR-P]-84-32T:

Proton Characteristics as a Function of Cumulative Variable in C Ta Collisions at 4.2 GeV/c per Nucleon

JINR-PI-85-14:

Some Characteristics of Protons Emitted in Backward Hemisphere in deuteron Ta and C Ta Interactions at $P=4.2 \mathrm{GeV} / \mathrm{CA}$

Yad. Phys. $41: 843,1985$ :

Analysis of the Deuterons Production in hadron nucleus $\rightarrow$ deuteron $X$ Interactions

ITEP-85-121:

Isotope and Isotone Effects in the Inclusive Formatiun of Nucleons at High Energy

Yad. Phys 49:321.1989:

Pion Charge-Exchange Reaction $\pi^{+37} \mathrm{Cl} \rightarrow{ }^{37} \mathrm{Ar} \pi^{0}$. Total Cross Section Meast!rement at 380 , 335, and 390 MeY

Z. Phys. C31:549.1986: J[NR-E1-85-949

Peculiarities of $\Lambda$ Hyperon and $\pi^{-}$Meson Production in Nucleus-Nucleus Colligions at High Energies

Phys. Rev. Lett. 58:1084.1987: KEK-87-13:

First Measurement of the Real Part of $p$ Double Spin Flip Amplitude

FERMILAB-CONF-89-207-E:

Recent Results from the CDF Experiment at the Tevatron Proton-Antiproton Collider

Phy's. Rev. Lett. 63.734.1989.

Proton Propagation in Nuclear Studied in the A-dependence of the ( $e^{-}, e^{-} p$ ) Reaction in the Quasifree Region

BONN-HE-85-36: C85/09/26

New Results from the UA5/2 Experiment

Phys. Lett. 232B:539.1989:

A New Determination of the Electroweak Mixing Angle from $\nu_{\mu}-\epsilon^{\rightarrow}$ Scattering

C'ERN-EP-90-75:

First Observation of Neutrino Trident Production

CERN-EP-89-166

Non-Trivial Dynamical Features of Parton-Parton Scattering at the ISR

Phys. Few. D32:2893,1985; SLAC-286:

Measurement of the Decay $\mathbf{r}(2 S) \rightarrow \pi \pi \mathbf{r}(1 S)$

DESY-F1-d9-01

Jet Properties in $e^{+} e^{-}$Annihilation at the Center-of-Mass Energies between $14 \mathrm{GeV}$ and $44 \mathrm{GeV}$

Phys. Rev. D35:1011,1987.

Search for Magnetically Charged Particles Produced in $e^{+} e^{-}$Annihilations at $E_{\mathrm{cm}}=10.6 \mathrm{GeV}$

Phys. Lett. 152B:428,1985: FE.RMILAB-PLB-84-113-E:

Observation of the Cabibbo Suppressed Decay $D^{x} \rightarrow \phi \pi^{ \pm}$

Phys. Rev. Lett. 59:2535,1987:

Charges and Angular Distributione of Fast Fragments Produced in 3.2 TeV ${ }^{16} \mathrm{O}$ Collisions with Pb

Yad. Phys. 46:144.1987: I1 VE-86-162:

Study of Three-body Reactions $K^{+} p \rightarrow K^{+} K^{+} \Lambda$ and $K^{+} p \rightarrow p$ o $\bar{\Lambda}$ et $32 \mathrm{GeV} / \mathrm{c}$

Yád. Phys. 45:1350.1987: IFVE-86-116:

Study of Quagi-Two-Body Channels of the Reaction $K^{+} p \rightarrow K^{0} p \pi^{+}$at $32 \mathrm{GeV} / \mathrm{c}$

IFVE-87-60:

Study of Reactions $K^{+} p \rightarrow K^{0} 2 \pi^{-} p, K^{+} p \rightarrow K^{U} 3 \pi^{+} 2 \pi^{-} p$ and their Diffractive Dissociation Channels at $32 \mathrm{GeV} / \mathrm{c}$

Phys. Rev. C32:1087,1985

Difraction Analyois of Pion Elastic Scattering from ${ }^{10} \mathrm{Ar}$

Phys. Rev. Lett. 54:396.1985.

Evidence of Anomalous Behavior of Relativistic Alpha Particles Emitted in ${ }^{12} \mathrm{C}$ Emulsion Interaction at 4.5 GeV/c per Nucleon

Czech. J. Phys. B36:1358,1986:

A Comparative Study of $p(e m)$ and ${ }^{12} \mathrm{C}$ (em) Interactions at $4 \mathrm{GeV} / c-5 \mathrm{GeV} / c$ per Nucleon

Nuovo Ciri. 97A:24.1987

Directional Coherence of Medium Energy Protons Emitted in ${ }^{52} \mathrm{C}$ Emulsion Interaction at 4.5 Gev/c/N

See the legend on page 19 
(ibush HG

Ghosh nyl

Ghosh $89 C^{\circ}$

Ghosh 89D

Ghosh 90

Ghosh 90B

Ghosh 90C

Gibbons $\mathrm{RH}$

Gibsun 89

Gidal 85

Gidal BK

Gidel $88 B$

Gidal $88 C^{\circ}$

Gilbert $\$ 5$

Gilbert $86 \mathrm{~B}$

Gill 90

Gilman $\mathrm{kg}$

Gilman 90

Ginther 87

Gituelman 87

Gladney 85

Gladney $86 \mathrm{~B}$

Gladney 90

Gladyazdziad 88

Glagolev 85

Glagoler 86

Glagoler $86 \mathrm{~B}$

Glagoler 87

Glagule: 88

Glagolev 88B

Glagolex 89

Glagolev 89B

Cilagolev 89C

Glagolev 90

Class 958

Cilavanakov 86

Glavanakov 87

Glavanakov $x$

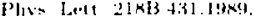

The Validity of the Negative Binomial Multiplicity Distribution in the Case of the Relativistic Nucleus Nucleus Interaction

Nuavo Cim $101 \mathrm{~A}$ 129.14k9:

Many Pion Correlation in ${ }^{1 \mathrm{H}} \mathrm{O} \mathrm{Ag} / \mathrm{Br}$ Interaction at $2.1 \mathrm{GeV} / \mathrm{c} / \mathrm{Nucleon}$

Nuovo Cim 101A t6it.liks

Strong Pion Correlation in Target Fragmentation Region Observed in "Cold" Events Produced in ${ }^{12}$ CEmulsion Interactions at $4.5 \mathrm{GeV} / \mathrm{c}$

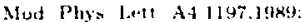
Zonal Poissonian Pion Multiplicity in Central ${ }^{24} \mathrm{Mg} \mathrm{Ag} \mathrm{Br}$ Collisions at Dubna Energies

Nuoviu Cim 103A:423.1943:

Study of Transverse Momentum Spectrum of Proton Projectile Fragrnents in $4.5 \mathrm{~A}$ GeV/c ${ }^{12} \mathrm{C}$ Emulsion Interactions. Evidence of a Single Temperature

Eur. Let1. 1 i 535.1490 : Multiplicity Characteristics of Symmetric and Asymmetric Heavy-Ion Interactions at 4.5 GeV/C

Eur. Lett 12:25. 14940: Supermassive Magnetic Monopoles Flux from the Oldest Mica Samples

Phys. Rer. Lett 61 26fil.1986: PRINI-RK.0T34-CHICACiO New Limits on $K_{L}, K_{S} \rightarrow \pi^{\circ} e^{+} e^{-}$

C'ERN-EP.8S-67:

An Experimental Test of CPT Invariance in the Neutral Kaon System

LBL- 19992, C85/06;00

Two Photon Physics with the MARK-Il at PEP

Plys Rev. Let 59:2616.14kK: SLA('-PLB-4275: LBL-264)1. Evidence for a Spin-1 Resonance in the Reaction $q \gamma^{*} \rightarrow \boldsymbol{K}^{0} \boldsymbol{K}^{ \pm} \pi^{ \pm}$

Phys. Rev. Lett 5t:2012.19kK. SL.AC-P1:B-4274: LBL-22694. Observation of Spin- $f_{1}(1285)$ in the Reaction $\gamma \gamma^{\circ} \rightarrow \eta \pi^{+} \pi^{-}$

L.BL-26172:

Resonance Formation in $\rightarrow$ Collisions

Phys. Rev. Lett. 55:2680.1985:

Measurement of Parity Nonconservation in Atomic Cesium

Phy Rev. A34:792.1986;

Atomic-beam Measurement of Parity Nonconservation in Cesium

Int Jour. Mod. Phys. A5:T55.1990: Study of the Characteristics of ${ }^{139}$ La Emulsion Interactions at $1.2 \mathrm{GeV}$

SLAC-PLB-4984: Rare Decays

Phys Rev. Lett. 64:622.194u Forward-Angle Charged-Pion Electroproduction in the Deuteron

Phys. Rev. D35:1541.1987: Search for Charmed Mesons Produced in Hadronic Interactions

CLNS-87-81: $B$ Meson Decay

SLAC-279: [MI1-85-1]313

Measurement of the Lifetimes of the Neutral and Charged $D$ Mesons

Phys. Rer, D34:2601.19k6. SLAC - P ['B-3947: LBL-21.50:3

Measurement of the $D^{0}$ and $D^{+}$Lifetimes

FERMILAB-CONF-UU-15-E:

A Study of $T \pm$ Decays of the $W=$ Boson at CDF

INP-1432-PH:

Intermittency in Super-High Energy Cosmic Ray Events

Yad. Phys. 42:181.1985: JINR-P1-81-98:

Evaluation of the Motion Effect on Space Dimensions of the Interaction Region for $\pi^{-} p$ Multiple Production Reactions

Nucl. Phys. A445:572.194.5

Alpha Particle Breakup in the Interaction with Protons at $8.6 \mathrm{GeV} / \mathrm{c} \mathbf{H e}$ Momentum

JINR-E1-86-78:

Peculiarities of Deuteron Production in ${ }^{4}$ He Proton Interactions

JINR-P1-87-61.

Azimuthal Correlations and Deuteron Production in Proton Interactions with Light Nuclei

JNR-P1-88-6.

Break up of the Polarized Deuteron deuteron $p \rightarrow p p n$

JINR-P1-88-592:

Total Disintegration of Helium Nuclei in ${ }^{3} \mathrm{He}$-Interaction at $13.5 \mathrm{GeV} / \mathrm{c}$

JINR-Pl-89-2!\&:

Multiplicity of Charged Particles in Interactions of Oxygen Nuclei with Hydrogen at 3.1 GeV/c Momentum

JINR-EL-89-246:

Further Evidence for Narrow Dibaryon States in deuteron $p$ Interactions

JINF-PI-89-5H4:

Characteristics of deuteron $\rightarrow p p p \pi^{-}$and $n p \rightarrow p p \pi^{-}$Reactions and deuteron Wave Function

Yad. Phus. 51:73ri.!9g0:

Narrow Dibaryon States in deuteron $p$ Interactions

Phys. Rev [:31:288.1985;

Measurements of Spin Correlation Parameters A(Il) and A(sl) for $p$ (polarized) $p$ (polarized) $\rightarrow \pi$ deuteron between $500 \mathrm{MeV}$ and $800 \mathrm{MeV}$

Phys. Letc. 178B:155.]1986:

Photoproduction of Neutral Pion on ${ }^{12} \mathrm{C}$ and ${ }^{6} \mathrm{Li}$ Nuclei at Threshold

Yad Phys. 45:3.1947.

Measurement of Total $\pi^{0}$ Meson Photoproduction Cross Sections on ${ }^{\theta}$ Li and ${ }^{12} \mathrm{C}$ Nuclei near the Threshold

Yad Phys $5(1: 1316.19 k ! 9$

Photoproduction of Neutral Pions on Light Nuclei near Threshold 
Glover 85B

Guimenky 89

Goetz 15

Gold 46

Goldhaber $85 C$

Goldman 87

Goldman $\mathrm{N}_{\mathrm{T}} \mathrm{C}$

Golovin $\mathrm{kB}$

Golubey 85

Golubev 86

Golubev 87

Golubeva 89

Golubeva 90

Gornez 85

Gumez 86

Gomez 86B

Goodman 85

Gorbenko 85

Gornov 86B

Gornov 87

Gornov 87B

Gornov 88

Gorringe 85

Gorshkova 85

Gosset 89

Gourlay 86

Grab 87

Grab 88

Grabez 88

Graboseh $x 6$

Grabusch 86B

Grabosch 86D

Grabusch 89

Grace 85

Gram 89

Grassler 85:

Grassler 86

Grassiler 84

Gray 87
Pliys. Rev. (31 1.1485:

Optical Model Analysis of $200 \mathrm{MeV} p$ (polarized) ${ }^{16} \mathrm{O}$ Elastic Scattering Data Measured to Large Momentum Transfers

Phys. Lett. 23713.287.1984:

A Search for a KeV Peeudoscalar in the Two Body Decay of Orthopositronium

Phys. Rev. C31.1563.1945:

Measurement of Pion Induced Deuteron Breakup at $150 \mathrm{MeV}$

LBL-224:33:

Hard Photon Processes in Electron Positron Annihilation at $29 \mathrm{GeV}$

LBL-20445:

Lifetime Measurements at PEP and PETRA

Phys. Re'v. D36:1543.1987:

Light-Boson Emission in the Decay of $\mu^{+}$

Pliys. Ret. Lete 60:1789,1988: TAL1'-1543-87:

Implications of the Supernova SN1987 A Neutrino Signals

JINR-E1- $88-175$ :

Fragmentation Cross Section of ${ }^{18} \mathrm{Fl}$ at $4 \mathrm{~A} \mathrm{GeV}$

Yad. Phys 11:1183.1985:

Observation of $\phi \rightarrow \eta e^{+} e^{-}$decay

Yad. Phys. 44:633.1986: Sov. J. Nucl. Phy's. 44:404.198t;

The $\phi \rightarrow \pi^{+} \pi^{-}$Decay

Yad. Phis. 45:J004.1987:

Upper Limit on Neutral Pion Polarizability

Plys. Lett. 221 B:238,1!889:

Inclusive Proton Spectra from 0.6 - 1 GeV/c Pions Interactions with Nuclei

Pisma Zh. Eksp. Teur. Fiz. Fi:298.19!)(1):

Excitation of $\Delta$-Isobars in Nuclei in the Single Charge-Exchange Pion Reaction at the Energy of 1 GeV

SLAC-PLB-3552:

Possible Indication of an A-dependence of $\boldsymbol{R}$ in Deep Inelastic Electron Scattering fron: Nuclei

Plys. Rev. D35:2736.1987: FERMILAB-PUB-86-160-E:

A Measurement of the Nuclear Enhancement in High $E_{t}$ and Jet Event Production

FERMIILAB-CONF-H6-65-E:

Hadron-Nucleus Interactions at High Energy

Phy's. Rev. Lett. 54:877.1985:

Measurement of Gamow-Teller Strength Distributions in Masses 13 and 15

Pisina Zh. Eksp. Teor. Fiz. 42:348.1985:

Polarization of Proton at ${ }^{3}$ He Fission by Linearly Polarized Photons

LENI-86-1185;

A-dependence of Charged Particles Yields followed 1 " apped Pion Absorbtion in Nuclei

Pisind Zh. Eksp. Teor, Fiz. 45:205.1987;

Evidence of Superheavy Isotopes of $\mathrm{H}_{2}$ in the Absorbtion Reaction of $\pi^{-}$Mesons by ${ }^{\theta} B e N^{\prime}$ clei

Atom. NucL. Elen. Particles. P. 18:

A-dependence of the Yield of the Charged Particles in the Capture Reaction of the Stopping $\pi{ }^{*}$ Mesons with Nuclei

Yad. Phys. 47:959.1988:

Emission of Protons in Absorbtion of Stopped Negative Pions by Be, C, Si, Cu and Ge Nuclei

Phys. Let1. 16213:71.1985;

X-Rays from Antiprotonic Hydrogen and Deuterium

Z. Phys. A320:301,1985:

${ }^{4}$ He Fragmentation Induced by $46 \mathrm{GeV}-400 \mathrm{GeV}$ Protons

Phys. Rev. Lett. 62:1251,19k9:

Nuclear Collective Flow and Charged Pion Emission in Ne-Nucleus Collisions at $E / A=800 \mathrm{MeV}$

Phis. Rev. Lett 56:2244.1986:

Polarization of $\Lambda^{\prime} s$ and $\bar{\Lambda}$ 's in $p p, \bar{p} p$, and $K^{-} p$ Interaction at $176 \mathrm{GeV} / c$

SLAC-PUB-4372:

Limits on Rare $D$ - Meson Decays

SLAC-PUB-4809:

Rare Decays of Charmed $D$ Mesons

Phys. Lett. 207B:27,1988:

Mechanisms of Light Fragment Production in the Interactions of $8.8 \mathrm{GeV}{ }^{4} \mathrm{He}{ }^{w i t h}{ }^{208} \mathrm{~Pb}$

z. Phys. C31:203.1986: IFVE-86-117; PHE-B5-12:

Coherent $\pi$ Meson Production in Neutrino and Antineutrino Interactions on Freon Nuclei

Yad. Phys. 46:1673,1987: IFV'E-B6-224

Observation of Internal Muon Bremsstrahlung in $\nu_{\mu}$ nucleus $\rightarrow \mu^{-} \mathrm{X}$

Yad. Phys. 47:1630.1986: ]FVE-86-221:

Study of Quasielagtic Reactions in $\nu_{\mu} n \rightarrow \mu^{-} p$ and $\bar{\nu}_{\mu} p \rightarrow \mu^{+} n$ in Bubble Chamber SCAT at $3-20$ GeV

Z. Phys. C41:527.1989;

Cross Section Measurement of Single Pion Production in Charged Current Neutrino and Antineutrino Interactions

Phys. Rev. Let1. 55:1055.1985:

Weak Decay of ${ }^{12} \mathrm{C}$ and ${ }^{11}$ Bor Hypernuclei

Phys. Rev. , i. 62:1837,1969:

Dependence of the Cross Section for Inclusive Pion Double Charge Exchange on Nuclear Mass and Charge

Nucl. Phys. B272:253.1966: 'EHN-EH'-85-41:

Inclusive $\rho^{0}$ Production in $\bar{\nu}_{\mu} p$ Charged Current Interactions

Nucl. Phys. E2T3:2ri3.19thi,

Prompt Neutrino Production in $400 \mathrm{GeV}$ Proton Copper Interactions

Yad. Phys. 17:722,1988.

Study of $\pi^{+} p, K^{+} p$ and $p$ Elastic Scattering at $250 \mathrm{GeV} / c$

('INi-87-107.

Topics and Results from CESR 
Green 80

Green k1j13

Girene sti

Greenlet $\times 5$

Greenles w

Greenshaw xg

Grifuls 8.5B

Grigalashel 88

Grigoron $\mathrm{n}+\mathrm{H}$

Grigorov kic

Gingurov 90

Gristin $\mathrm{Mis}$

Cisistin 85B

Gishun 85C

(i5)-h11) Xtils

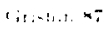

lis:ilit, $\mathrm{kK}$

cistima $\times \times 13$

Cirstraenkos $\times 4$

Riving $\times$

Girourn 45,

Grumutek $H 6$

fiomstuan $\times 7$

Cironsmanhand 86 ;

Cisotz HE

Ginadies 85

Gianzoroli $x$

Giaraldo xy

Ginaraldo xists

Ginlkaryan $x_{i}^{-}$

Gulkanyan $47 \mathrm{~B}$

Gulkasyan $\mathrm{RT}$ (

(julksiyas 87D

Gulkanyan BA

Ciulkanyan мнВ

Gulkityan MAC

(itilkinyan shi)

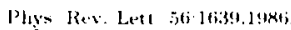

Observation of a Narrow Enhancement in $\phi K K$ and $\phi \pi \pi$ Final States Produced in $400 \mathrm{GeV} p n$ Interactions

TRI-I'P'.86-74:

Light Fragment Spectra to Upper Kinematic Limits for $300 \mathrm{MeV}$ Proton Reactions with Be and $\mathrm{Ag}_{\mathrm{g}}$

Phys. Re. Lett 56:819.198ti.

New Determination of the Deuteron Binding Energy and the Neutron Mass

Phys. Re. Lett 55:1555.19K5. FFI-X5-44-CHIC A(50):

Production of Massive Muon Pairs in $\pi^{-}$nucleus Collisions

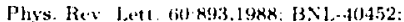

Search for $K_{L} \rightarrow \mu^{+} e^{-}, K_{L} \rightarrow \mu^{-} e^{+}$and $K_{L} \rightarrow e^{-} e^{+}$

Z. Plys (42:1.16K9: DESY'-8\&-154:

A Measurement of the Charge Asymmetry of Hadronic Events in Electron Positron Annihilation

Pliys. Lett. 168B:264.1986; ( $1 \mathrm{AB}-\mathrm{FT}-137$ :

Limits on Masses of Excited Electrons and Muons from Neutrino Scat tering off Electrons

Yad. Phys 48:476.1988:

Inelastic Cross Sections and Multiplicities of Secondaries Produced in Collisions of Relativistic Nuclei with Carbon and Tantalum at $2.3 \mathrm{~A}$ and $4.2 \mathrm{~A} \mathrm{GeV} / \mathrm{c}$

Doklady Akid Niak SSSR 30 K $: 50.1989$ :

Part of Protons in the Cosmic Ray Flux at the Energies $>25 \mathrm{TeV}$

Pjstua Zh. Eksp. Teur Fiz. 419:2415.1989:

Charge Distribution of Cosmic Rays at the Energy $>1 \mathrm{TeV}$

Yat. Phys. 51.157 .11490 :

Satellite Studies of High and Superhigh Energy Cosmic Rays

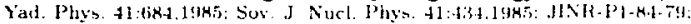

Fragmentation of Quarks and Diquarks into Strange Particles in $r^{-}$p Interactions at $P=40 \mathrm{GeV} / \mathrm{c}$

Yad. Phys. 41.371 .19145

Hadron Jets with Strange Particle Production in Cumulative $\pi$ C Interactions at $P=40 \mathrm{GeV} / c$

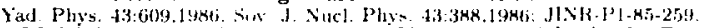

Multiplicity Correlations between Charged Particles in the Forward and Backward Hemispheres in c.m.s. of $\pi^{-} p$ Interactions at $40 \mathrm{GeV} / \mathrm{c}$

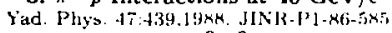

Observation of $\pi^{0} \pi^{0}$ [nterference Effect and Size of Neutral Pion Emission Volume in $\pi^{-}$Xe Interaction at $3.5 \mathrm{GeV} / \mathrm{c}$

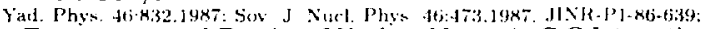

Temperature and Density of Nuclear Matter in $C \mathrm{C}$ Interactions at $P=4.2 \mathrm{GeV} / c$ per Nucleon

JINH-Pl-Nx-ij20.

Neutral Star Characteristics in ( $\pi$ Xe)-Interactions at $3.5 \mathrm{GeV} / \mathrm{c}$ Momentum

JINR-PI-XX-K2!

Investigation of $\Delta\left(1232 P_{33}\right)^{++}$Isobar Generation in Light Nucleus ( $p$, deuteron, ${ }^{4} \mathrm{He},{ }^{1 \text { in) }}$ Interactions with Carbon and Hydrogen Nuclei at $4.2 \mathrm{GeV} / \mathrm{c}$ Momentum

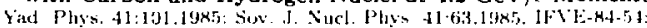

Charged Particle Multiplicity in $K^{+} p$ Interactions at $70 \mathrm{GeV} / c$

LAL-BH-14:

Neutrino Counting in Single Photon Experiments

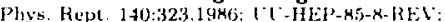

In Search of the Supermassive Magnetic monopole

Pliys. Rev Lett. 57:3241.1986;

Search for the Rare Decay $\mu^{+} \rightarrow e^{+} \gamma$ ?

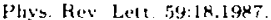

Measurement of the Lifetime of $K_{S}$ Mesons in the Momentum Range 100 to $350 \mathrm{GeV} / \mathrm{c}$

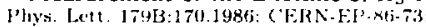

A High Statistics Study of $\Upsilon$ Mesons Production in $\pi^{-}$Wt Reactions at $286 \mathrm{GeV} / \mathrm{c}$

Phys. Lett. 153B: I 1985:

The Neutrino Mass from Double \& Decay

Phys. Lett 158B:15.1985:

Neutron-Proton Total Cross Section between 120 and $580 \mathrm{MeV}$

2 Phy: ('37:545.1948: CERN-EP-H7-194:

Angular Distributions of Muon Pairs Produced by Negative Pions on Deuterium and Tungsten

Nuove Cin. 102A:1137.1989: C'ERN-EP-BY-NT

Antiproton - Nucleus Interaction at Intermediate Energies

TORINO-89:

Antiproton - Nucleus Physics: Experimental Overview

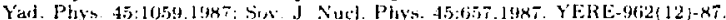

Experimental Estimate of $S$ ondary Absorption Contribution to Cumulative Proton Praduction

Yad. Phys 45:1065,14347.

Mult iplicity Correlations between $\pi^{0}$ Mesons and Nonrelativistic Protons in deuteron and ${ }^{12} \mathrm{C}$ Interactions with Tentalum Nuclei at $4.2 \mathrm{GeV} / \mathrm{c}$ per Nucleon

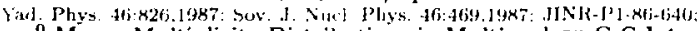

$\pi^{\circ}$ Meson Multiplicity Distributions in Multinucleon C C Interactions at $4.2 \mathrm{GeV} / \mathrm{C}$ per Nucleon

YFRE-97:3(2:3)-Xit:

Pion Multiplicity Distribution Moments in Nucleus-Nucleus Collisions and Experimental Verification of he Independent Interactions Model

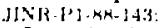

Investigation of Correlations between Protons in Nuclear-Nuclear Interactions at $4.2 \mathrm{GeV} / \mathrm{c}$ per Nucleon

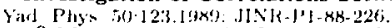

$\pi^{\circ}$-Meson Spectra in $\mathrm{C}$ Ta and Multinucleon C C-Interactions at $4.2 \mathrm{GeV} / \mathrm{c}$ per Nucleon

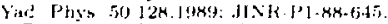

Summary Characteristics of Pions in Nucleus-Nucleus Interactions at $4.2 \mathrm{GeV} / \boldsymbol{c}$ per Nucleon

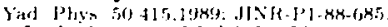

Inclusive and Multiple Characteristics of Cumulative Protons in Nucleus-Nucleus Interactions at 4.2 GeV/c per Nucteon 
Gulkanyan 89

Guo 89

Gustafssun 88

Gutbrod 89

Guy 87

Guy 89

Haas 85

Haas 86

Haas 88

Haba 88

Haine: 86

Haines 90

Haissinski 85

Hallin 86

Halling 89

Hallman 85

Hamagaki 85

Hamann 90

$\operatorname{Han} 85$

Hanlon 85

Hanni 8.5

Hara 86

Harder 89

Hardy 89

Harper 55

Harris 87

Harris 90

Hartouni $x .5$

Hasell 85

Hasinoff 88

Hasinot 89

Haupt 8.5

Hausamnamn 89

Hawkins 89

Hawkins ngP

Hayes 89

Hayes $89 \mathrm{~B}$

Haysak s.5

Hearty 87
VERE-1150)(27)-80

The Measurement of Inelastic and Topological Cross Section of Deuteron Carbon Interactions at the Energy $1 \mathrm{GeV} / \mathrm{Nucleon}$

FERMIILAB-PLB-89-234-E:

Improved Limit on axion Production in $800 \mathrm{GeV}$ Hadronic Showers

Mod. Phys. Let1. A3:1323.1988.

Energy and Multiplicity Dependence of Fragment Flow in High Energy Nuclear Collisions

Phys. Lett. 216B:267,1989:

A New Component of the Collective Flow in Relativistic Heavy-Ion Collisions

2. Phys, C36:337.1987: CERN-ËP-86-217:

A Study of the EMC Effect Using Neutrino and Antineutrino Interactions in Neon and Deuterium

Phys. Lett. 229B:421.1989: CERN-EP-89-76:

Neutrino Interactions, Proton Production and a Nuclear Effect

Phys. Rev. Lett. 55:1248.1985:

Decay $B \rightarrow J / \psi(1 S) \mathrm{X}$

Phys. Rev, Lett. 56:2781.1986: Observation of the Decay $B \rightarrow D_{s}^{ \pm} \mathrm{X}$

Phis. Rev I att. 60:1614.1988:

Upper Limits on Charm-Changing Neutral Current Interactions

Nucl. Phys's. B299:627.1988:

A Precision Measurement of Polarization Parameters for the $\pi^{+} p \rightarrow K^{+} \Sigma^{+}$Reaction at 13 Laboratory Momenta 1490 and $2069 \mathrm{MeV} / \mathrm{c}$

Phys. Rev. Lett. 57:1986.1986:

Calculation of Atmospheric Neutrino Induced Backgrounds in a Nucleon Decay Search

Phys Rev. D41:692,1990: LA-L'R.89.1 105:

A Limit on Possible Energy Dependent Velocities for Massless Particles

LAL-85-32:

New Particle Searches at $e^{+} e^{-}$Machines

Phys. Rev. Lett. 57:2105.1986:

Reatrictions on a $1.7 \mathrm{MeV}$ Axion from Nuclear Pair Transitions

CLNS-89-910.

Recent Results from CLEO with Fully Reconstructed $B$ Mesans

Nuci. Plys. A440:697.1985:

Neutral Pion Production in Central Collisions of Relativistic ${ }^{12} \mathrm{C}$ and ${ }^{80} \mathrm{Ar}$ on ${ }^{200} \mathrm{~Pb}$

Phys. Lett. 150B:-116.1985

Measurement of High pt Light Fragments at c.m, 90 Degrees in $800 \mathrm{MeV} / \mathrm{A} \mathrm{C} \mathrm{C} C o l l i s i o n$

C'ERN-PPE-90.108:

In-Flight Annihilation $\bar{p} p \rightarrow \varphi \phi$ and $\bar{p} p \rightarrow \bar{K} K$ with JETSET at LEAR

Phys. Rev. Lett. 55:36,1985:

Observation of $B^{*}$ Production in $e^{+} e^{-}$Interactions above the $b$ Flavor Threshold

Phys. Rev. D32:2441.1985: FERMILAB-PLB-85-101-E:

A Comparison of Cross Sections from Deep Inelastic Neutrino Scattering on Neon and Deuterium

Acta Phys. Poion. B17:795.1986: CERA-EP-85-87:

Search for Monojet and Multi-Jet Events with Large Missing $p_{T}$ in the UA2 Experiment

Phys. Res. Lett. 56:553.1986:

Slow-monopole Search with Large Area Helium Gas Proportional Counter Array

DESY-F15-89-01:

Measurement of Inclusive Production of $D$ Mesons in B Decays with the ARGUS Detector

TASC'C.P-89-4:

Superallowed $0^{+} \rightarrow 0^{+}$Nuclear $\beta$ Decays: A Critical Survey with Tests of CVC and the Standard Model

Phys. Rev. Di31:1151,19ki:

Parity Nonconservation in Proton Water Scattering at $1.5 \mathrm{GeV} / \mathrm{c}$

Phys. Rev. Let 58:463.1987:

Pion Production in High Energy Nucleus-Nucleus Collisions

FERMILAB-C'ONF-90-118-E:

Recent Results on Direct Photons from CDF

Phys. Rev. Iett 54:628.1985:

Inclusive Production of $\Omega^{-}$and $\bar{\Omega}+$ by $K_{L}$ Carbou Interactions in the Energy Range $80-280$ GeV/c

TAI-PP.85.50:

Elastic Scattering of Polarized Protons from ${ }^{3} \mathrm{He}$ at Intermediate Energies

THI-PP.88-82.

Radiative Muon Capture with the TRIUMF TPC

TRI.PP-89-54:

Radiative Muon Capture in Light Nuclei

Z. Phys. C28:ij7.1985: (ERN-PRE-85-05T

Polarization of $A$ Hyperons in $K^{-} p$ Interactions

Pliys, Rev. D40:22,1989.

Direct Experimental Reconstruction of the p p Elastic Scattering Amplitude between 447 and 579 MeV

SLAC'-337.

Tests of QED to Fourth Order in $\alpha_{s}$ in Electron - Positron Collisions at $20 \mathrm{GeV}$

SLAC'PL'B-4949:

Search for a Charged Lctoton Specific Force in Electron - Posit ron Collisions

Phys. Rev Di38:3351.1983

Seatistical Study of Tau-Decay Data

SLAC.PL'B-5061:

Statis of the Tau One Prong Problem

Kr. Sixob. IINR 8.39 .1985$.

On a Rescnant Structure in the $p \mathrm{Cu} \rightarrow \pi^{+} \mathrm{X}$ at $350 \mathrm{MeV}$

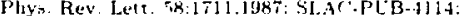

New Results ol. Single-Photon Production at $E_{\mathrm{cm}}=29 \mathrm{GeV}$ 
Hearty 88

Hearty 88

Hearty 89

Heck 88

Hegner 89

Heinrich 89

Heltsiey 86

Heningway 84

Henrard 87

Heppelmanu 85

Heppelmann 89

Hertzog 88

Hessey 89

Hessing 90

Hewett 85

Hicks Xis

Hicks 86

Hiel 89

Hill 88

Hill 89

Hirata $87 \mathrm{~B}$

Hirata $87 \mathrm{C}$

Hirata $88 \mathrm{~B}$

Hurata $88 C^{\circ}$

Hirata $88 D$

Hirata $88 \mathrm{E}$

Hirata $88 \mathrm{~F}$

Hırata 89

Hurata $89 \mathrm{~B}$

Hirata $89 \mathrm{C}$

Hurata $89 \mathrm{D}$

Hurata 90)

Hurat is 90 T

Huruahige n4C

Hoin 88

Ho 89

Hesfman 8 s

Hoffinatin 88

Hofrnasin $\mathrm{K5}$
Phys. Rey D39:3207.19K9: SLAC'-1Pl'B-46K4: TWSEA-PL'B-KB-2:

Search for the Anomalous Production of Single Photons in $e^{+} e^{-}$Annihiciation at $\sqrt{s}=28 \mathrm{GeV}$

LBL.285-4T.

Measurement of the $Z^{\circ}$ Boson Resonance Parameters at SLC

Z. Phys. C38:19,1988:

Study of the Energy Flow in ${ }^{16} \mathrm{O}-\mathrm{Nucleus}$ Collisions at 60 and $200 \mathrm{GeV} /$ Nucleon

Z. Phys. C $46: 547.1090:$ DESY-xy-17\%:

Final Results on $\mu^{ \pm}$and $\tau^{ \pm}$Pair Production by the JADE Collaboration at PETRA

Phys. Rev. Let t. 63:356.198:)

Measurement of the Ratio of Sea to Valence Quarks in the Nucleon

CLNS-86-728:

Measurement of the $r^{ \pm}$and $D^{\circ}$ Lifetimes

Nucl. Phys. B253:742.1985: CERN-EP-84-113:

Production of $\Lambda\left(1405 S_{01}\right)$ in $K^{-}$p Reactions at $4.2 \mathrm{GeV} / \mathrm{c}$

Nucl. Phys. B292-670.1987: I.A L-87-04:

Study of SU(2) and SU(3) Violations in $J / w(1 S)$ Baryonic Decays

Plyys. Rev. Lett . 55:1824.1985:

Decay Distribution of High Transverse Momentum $\rho$ Mesons

Phys. Lett. 232B:167.1989:

Momentum Dependence of Nuclear Spectral Functions as observed in $(p, 2 p)$ Quasielastic Scattering at Large $Q^{2}$

Phys. Rev. D37: 1142.1988:

Exotic-Atom Measurement of the Magnetic Dipole Moment of the $\Sigma^{-}$Hyperon

2. Phys. C42:175.1989:

A Measurement of the Branching Ratio for $\Sigma^{+} \rightarrow p$, Decay

FERMILAB-CONF-90-91-E:

Inclusive Jet Cross Section at $\sqrt{s}=1.8 \mathrm{TeV}$

Phys. Rev. D33:1519.1986: Phys. Rev. D34:298.1986: IS-J-1905:

Neutrino Mass Limits from the Fritzsch Mass Matrix

Phys. Rev. C31:1323.1985:

Fission of Heavy Nuclei Induced by Energetic Pions

Niucl. Phys. A434:97C.1985:

Studies of $p$ Shell Nuclei by Large Moment um Transfer Electron Scattering

Nucl. Phys. B327:1.1989:

Improved Limits on the Weak, Neutral, Hadronic Axial Vector Coupling Constant from Quasielastic Scattering of Polarized Electron

Phys. Rev. Lett. 60:999.1988:

Electromagnetic Dissociation for High-Z Projectiles and at Ultrarelativistic Energies

Z. Phy's. C42:355.1989: DESY'-88-166:

Observation of Spin-1 Resonance Formation in the Final State $K K \pi$ Produced in Tagged Two-Photon Collisions

[TT-ICEPP-87-04: LPR-0]44E: Search for 88 Solar Neutrinos at KAMIOKANDE-II

Phys. Fiev. Lett, 58:1490.1987:

Observation of a Neutrino Burst from the Supernova SN 1987 A

Phys. Lett. 205B:+16.1988:

Experimental Study of the Atmospheric Neutrino Flux

Phys. Rev. Lett. 61 2653.1988: Phys. Rev. Lett. 6. 694.1989: ICR-177-88-23: KEli-88-3i. Search for Correlation of Neutrino Events with Solar Fluxes in Kamiokande

Phys. Lett. 22UB:308,1989. KEK-88-116:

Experimental Limits on Nucleon Lifetime for Lepton + Meson Decay Modes

KEK-R3-131:

Search for "Solar-flare Neutrino" Events in KAMIOKANDE

Phys. Ker. D38:448.1988:

Observation in the KAMIOKANDE-II Detector of the Neutrino Burst from Supernova SN1987A

Phys. Rev Lett. 53:16.1989: ICR-REPORT-184-89 5: KEK-84-2: Observation of Solar Neutrinos in the KAMIOKANDE-II Detector

KEK-B9.4.5.

Recent Results and Status of the KAMIOKANDE Project

IC:R-Repurt-195-89-12:

Recent Solar Neutrino Data from KAMIOKANDE-II Detector

[CR-2U1-89-18; KEK-89-15] KOBE-AP-89-06: OCLNS-89-1]: ['PR-178E:

Search for Neutrino Events in the KAMIOKANDE-II Detector in Correlation with the Solar-flare Activity in March 1980

ICR-213-90-6: KFK-90-43. KOBE-AP-90-02: NLIG-DP-90-02: OLLNS-90-03: TIT-HPE-90-2: ('PR-0189E:

Results from One Thousand Days of Real-time Directional Solar Neutrino Data

IC R-214-90-7, KEK-90-3- TOKAI-1-KAK1-90-02: KOBE-AP-90-03: NIG-DF-90-3: OCLNS-90-04, TIT-HPE-90-3. LPRU] $92 \mathrm{~F}$.

Constrainte on Neutrino Oscillation Parameters from the KAMIOKANDE-II Solar Neutrino Data

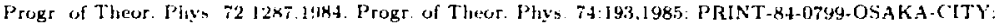
Analysis of the Dibaryon Resonances with $J^{P}=2^{+}$and $3^{-}$by a Three Channel $\left(p, n, \Delta\left(1232 P_{3 s}\right), \pi\right.$ deuteron) $K$ Matrix Method

('ALT-68-15.33:

Status of the $\theta / f_{2}(1720)$

Phys. Let 2.44B:573.1950: KEK-X9-19is: ANY'-89-22:

Observation of Anomalous Production of Muon Pairs in $e^{+} e^{-}$Annihilation into Four-lepton Final Stateg

Phys. Lett 208B 1-19.198\%. L.A-1'R-88-426. The New Limit for the Branching Ratio for the Decay $x^{0} \rightarrow \nu \nu$ from Beam Dump Experiments

Phys. Lett 20013-583.1988:

Search for Projectile Fragments with Fractional Charge in Relativistic Heavy Ion Collisions

LBL-20117. ('45, (1)6/09:

The Investigation of Parton Fragmentation with the TPC Detector at PEP; Recent Results 
Hofmann 87

Hofmann $87 \mathrm{~B}$

Hogan 80

Hohler 89

Hoistad 86

Holder 89

Holl 86

Hollas 85

Hollebeek 85

Hollebeek 86

Holt kamp 85

Holynski 86

Holynski B6B

Holynski 89

Holynski 898

Holzkamp 88

Hsiung 8.5

Hsiung 88

Hsueh 85

Hsueh 88

Hubbard 898

Huber $\mathbf{8 8}$

Huber 89

Huber 90

Hufnes 85

Hughes 89

Humanic 88

Hurst 89

Huston 86

Hutcheon 89

Iddir 88

Igarashi 87

Irnanish, 8.5

Imanishi $x H$

Inagaki 88

lnagaki $\mathbf{\theta 9}$

Inagaki 893

Iragaki 8gC
LBL-23922:

The Physics of Jets

LBL-23921:

Production of Strange Particles in Hadronization Processes

Nucl. Phys. A.43.4:-475C.1985:

A Progress Report on Recent Rare Muon Decay Experiments at the Los Alamos Meson Physics Facility TKP-89-3:

Methods and Results of Pion Nucleon Partial Wave Analysis

Phys. Lett. 17\% B:299.1986:

The ( $p$ (polarized), $\pi^{ \pm}$) Reactions on Be at $650 \mathrm{MeV}$

SI-89-11:

Experimental Status of $C P$ Violation

BONN-1R-86-15:

Charged Hadron Production in Proton Antiproton Interactions at $200 \mathrm{GeV}$ to $900 \mathrm{GeV}$ Center-of-Mass Energy in Comparison with Predictions of the Dual Parton Model

Phys. Rev. Lett. 55:29,1985:

Wolfenstein Polarization Observables for the Reaction $p-p \pi^{+} n$ at $800 \mathrm{MeV}$

SLAC-PCB-3816: C85/07/29:

Single Photon Searches at PEP

SLAC-PLB-3904:

ASP: A Search for Single Photon Events at PEP

Phys. Rev. C31:957.1985:

Pion Scattering to $4^{-}$States in ${ }^{19} \mathrm{C}$

Acta Phys. Polon. B17:201.1986:

Analysis of Angular Distributions of Charged Particles Produced by Negative Pions on Nuclei at $300 \mathrm{GeV}$

Z. Phys. C31.467.1986:

Pseudorapidity Distribution from Pion-Emulsion Interactions in the Energy Range $60 \mathrm{GeV}$ to $300 \mathrm{GeV}$

INS-1.456-PH:

One- and Two-Dimensional Analysig of the Factorial Moments in $200 \mathrm{GeV} /$ Nucleon $p{ }^{10} \mathrm{O}$ and ${ }^{32} \mathrm{~S}$ Interactions with Ag/Br Nuclei

Phys. Rev. Lett. 62:733.1989:

Evidence for Intermittent Patterns of Fluctuations in Particle Production in High-Energy Interactions in Nuclear Emulsion

BONN-IR-88-40:

Inclusive Photoproduction of Kaons and Pions in the Moment um Range $100 \mathrm{GeV} / \mathrm{c}-170 \mathrm{GeV} / \mathrm{c}$

Phys. Rev. Lett 55:457.1985: FERMILAB-PUB-85-103-E:

A-dependence of the Inclusive Production of Hadrons with High Transverse Momenta

FERMIILAB-CONF-88-164-E:

Measurement of $\epsilon^{\prime} / \epsilon$ at Fermilab

Phys. Rev. Lett. 54:2399.1985: FERMILAB-PUB-85-21-E:

Measurement of the Electron Asymmetry in the $\beta$ Decay of Polarized $\Sigma^{-}$Hyperons

Phys. Rev. D38:2056.1988:

High-Precision Measurement of Polarized $\Sigma^{-} \beta$ Decay

LBL-27687:

Fragmentation Properties of Jets Production in Proton-Antiproton Collisions at $\sqrt{s}=1.8$ TeV

Phys. Rev. Let t. 61:2189.1988: TRI-PP-88-30:

Search for Mixing of $\left(\mu^{+} e^{-}\right)$and $\left(\mu^{-} e^{+}\right)$with Fermi Coupling Strength

TRI-PP.89-64:

Search for Mixing of Muonium ( $\mu^{+} e^{-}$) and Antimuonium ( $\mu^{-} e^{+}$)

Phys. Rev. Let:. 64:835.1990:

Limit on the Flux of Cosmic-Ray Magnetic Monopoles from Operation of an Eight-Laop Superconducting Detector

Phys Rept. 125:129.1985:

Heavy Fragments Produced in p nucleus and nucleus nucleus Collisions at Relativistic Energiea

LA-LR.89-1011:

Non-Newtonian Gravitational Forces and the Greenland Ice-Sheet Experiment

Z. Phys, C38:79.1988:

Pion Interferometry with Ultrarelativiatic Heavy-Ion Collisions from the NA35 Experiment

Eur. Lett. 10:213.1989:

A Single PEP Upper Limit on $B^{0} \bar{B}^{0}$ Mixing

Phys. Rev. D33:3199.1986.

Measurement of the Resonance Parameters and Radiative Width of the $\rho^{+}$

TRI-PP-89-55;

Measurement of $n p \rightarrow$ deuteron $\pi^{0}$ Cross Sections very neur Threshold

Phys. Lett. 205B:564.1988. LPTHE-ORSAY'88-03;

$\left(q, \bar{q}\right.$, gluon) Hybrid and $(2 \eta, 2 \bar{q})$ Diquonium Interpretation of GAMS $1^{-+}$Resonance

Phys. Rev Lett. 60:2359.1988: KEK-87-112: AMY'-87-U6:

Search for Isolated Leptons in Low Thrust $e^{+} e^{-}$Annihilation Events at $\sqrt{8}=50$ and $52 \mathrm{GeV}$

Piyys. Rev. Lett. 54:2497.1985:

Neutral Pion Photoproduction on the Deuteron

Nuovo C'in. IOOA 735.1988:

Meagurement of Large Angle Cross Sections of Coherent Pion Photoproduction on Deuteron

KEK-H8-20:

Search for Rare Decays $K_{L} \rightarrow \mu^{+} e^{-}, K_{L} \rightarrow \mu^{-} e^{+}$and $K_{L} \rightarrow e^{+} c^{-}$

Hhys. Rev, D40:1712.1989. KEK-K9.25: ('T-HE-89-0)3:

Search for $K_{L} \rightarrow \mu^{+} e^{-}, K_{L} \rightarrow \mu^{-} e^{+}$and $K_{L} \rightarrow e^{+} e^{-}$Decays

KEK-89-3:

A Series of Experiments at KEK for Light Quark Meson Spectroscopy, E64/121/135

KEK-89-12:

The Experimental Status of E137 at KEK (Search for Rare Decay $K_{L} \rightarrow \mu^{+} e^{-}$and $K_{L} \rightarrow \mu^{-} e^{+}$) 
Incandela 86

Inoun 85

Inoue $85 \mathrm{~B}$

Inzhechik 90

Iovcliev 87

lrion 85

Irom 85

Ishij 85

Istmatova 85

Lstmatova $85 \mathrm{~B}$

Itep 89

Ivanenko 86

lvanenko 87

Ivanenko $88 \mathrm{~B}$

Ivanenko 89

Ivanov 87

Iwasaki 85B

Iwasaki 89

Izen 88

Jaffe 88

Jaffe 89

Jain 85

Jain 86

Jain 87

Jain 87B

Jain $87 \mathrm{C}$

Jain 88

Jain 88B

Jain 90

Jain 90B

Jakubowski BS

Jammes 89

Jani 87

Janissen 89

Janbsen 89

Janssetו 9f)

Jastrzembsti 48
Phys Hex. D34:26:37.1986

First Result from a 1.1 n Diameter Superconducting Monopole Detector

Jour, of Phys. G 11.657 .1985 .

Longitudinal Development of Air Shower Electrons Studied from the Arrival Time Distributions of Atmospheric Cerenkov Light Measured at $900 \mathrm{~m}$ Above Sea Level

Jour. of Phys. G 11:669,1985:

Longitudinal Development of Air Shower Electrons Studied from the Arrival Time Distributions of Atmospheric Cerenkov Light Measured at $5200 \mathrm{~m}$ Above Sea Level

Yad. Phys, 51:11.1990:

Spatial Parity Violation on E1 Transition of ${ }^{23{ }^{7}} \mathrm{~Np}$ Nucleus

Yad. Phys 47:451.1988: JINR-E1-87-166:

Study of the Polarization for $\Lambda$ Hyperon Produced in $C^{12} \mathrm{C}$ and $\mathrm{C}$ Ta Interactions at $4.2 \mathrm{GeV} / \mathrm{c}$ per Nucleon

SLAC-PLB-3643: C85/04/20:

Spectroscopy of the $\boldsymbol{r}(2 S)$ with the Crystal Ball

Phys. Rev. C31:1464,1985:

Pion Single Charge Exchange on ${ }^{7} \mathrm{Li}$ at Low Energies

Nucl. Phys. B254:458,1985:

Differential Cross Sections for Proton Compton Scattering at Incident Photon Energies between 900 MeV and $1150 \mathrm{MeV}$

Yad. Phys. 41:935,1985:

Characteristics of Cumulative $q$ Quanta in $\pi^{-12} \mathrm{C}$ Interactions at $4 \mathrm{GeV} / \mathrm{c}$

Yad. Phys. 43:924.1986: PTIU-28-85-FVE:

Multiplicity of Charged and Neutral Particles in $\pi^{-12} \mathrm{C}$ Interactions at $4 \mathrm{GeV} / \mathrm{c}$

Lsp. Fiz. Nauk 157:369.1989: Oscillation of $B$ Mesons

Pisma Zh. Eksp. Teor. Fiz. 44:200,1986:

Multicharged Primary Cosmic Ray Particles with the Energy of more then 2 TeV

Yad. Phys. 45:1069.1987;

Energy Spectrum of Helium Nuclei in Primary Cosmic Rays in the Energy Range from 1 up to 10 Tev/Nucleon

Pisma Zh. Eksp. Teor. Fiz. 48:468.1988:

Energy Spectrum and Charge Component of the Primary Cosmic Reys with the Energy Greater 2 TeV

Pisma Zh. Eksp. Teor. Fiz. 49:192.1989:

Energy Spectrum of the Primary Cosmic Rays at the Energy 1 - 100 TeV from the SOKOL Data

Kr. Soob. JINR 26:4.1987:

Measurement of Electron Mass by Means of Narrow Component of Annihilation Fadiation Peak

Nucl. Phys. A433:580 1985:

Small Angle $\bar{p} p$ Elastic Scattering and the Real to Intaginary Rat's of the Forward Amplitude between $413 \mathrm{MeV} / \mathrm{c}$ and $715 \mathrm{MeV} / \mathrm{c}$

KEK-89-163:

Recent Results on QCD from TRISTAN

SLAC-PUB-4753:

Semileptonic Charm Decay

Phys. Rev. Das:1016.1988:

High Transverse-Momentum Hadron-Hadron Correlations in $\sqrt{8}=38.8$ GeV Proton Proton Interactions

Phys. Rev. D40:2777,198, TERMILAB-PLB-89-69-E:

High Transverse Moment um Single Hadron Production in $p p$ and $p$ deuteron Collisions at $\sqrt{s}=27.4$ GeV and $\sqrt{8}=38.8 \mathrm{GeV}$

Phys, Lett. 154B:252.1985: Investigation of Anomalons in He Fragments

Phys. jiev. D34:2886.1986: Short- and Long-Range Correlations of Produced Furticles at Very High Energies

Phys. Rev. Lett. 59:2531.1987: Search for Quark-Gluon Plasma in Nucleus-Nucleus Collisions at 3.2 TeV

Phys. Lett. 187B:175.1987:

Multiparticle Production in Proton-Nucleus Collisions at Tevatron Energy

Z. Phys. C36:45.1987:

High Energy Muon Nucleon Inelastic Scattering at Low $Q^{2}$

Nucl. Phys. B301:517.1988:

Hadron Production by $150 \mathrm{GeV}$ Muons in Nuclear Emulsion

Nuovo Cim. 99A:9.1988:

Multiplicity Dependence of the Number of Projectile Collisions in Hadron-Nucleus Interactions

Phys. Lett. 235̄B:351.1990.

Observation of Scaling in Nucleus-Nucleus Interactions at Ultra-Relativistic Energies

Phys. Lett. 24]B:273.194i):

Multifractality of Inelastic Nuclear Interactions at 1 . B TeV

Z. Phys. C40:49.1988: DESY-K8-032: SLAC-PL'B-4567

Determination of $\Gamma(e e)$ of the $\boldsymbol{T}(1 S)$ and $\boldsymbol{T}(2 S)$ Resonances, and Measurement of $R$ at $W=8.39$ GeV

Phys. Lett. 22:B:21.1989: SACLAY-DPH-N-25,50:

Total Cross Section Measurement of $\pi^{\circ}$ Photoproduction near Threshold on Complex Nuclei

JINR-E1-8T-39T:

Hadrons and $\gamma$ 's Associated to Dimuons and $J / w(1 S)$ Particles in $\pi$ - C Interactions at 38 GeV/c

TIR. PP-89- 89 :

Muonium Production fra Fine Silica Powder

Phys. Let 22813:273,1989. DES '-81'054: SLAC'-PI'B-495\%: HEN-317:

The Michel Parameter for the Decay $\tau^{ \pm} \rightarrow e^{ \pm} \nu \bar{\nu}$

THES

Leptonic Decay Modes of the $T^{\mp}$ Lepton

Phys. Res. Lett 61:2300.19k6. BNL-41507:

Limit on $K_{L} \rightarrow \pi^{0} e^{+} e^{-}$and $K_{L} \rightarrow e^{+} e^{-}$ 
Jawahery 85

Jeanmatie 85

Jeckelmann $86 \mathrm{~B}$

Jenni 89

Jensen 89

Jodidio 86

Johns 89

Johnson 86

Johnson 87

J'nes 65

Jones $85 \mathrm{~B}$

Jones 86

Jones 87

Jones $87 \mathrm{~B}$

Jones 89

Jones $89 \mathrm{~B}$

Jones 89C

Jones 90

Jongejans 89

Jousset 88

Jovchev 85

Joyner 89

Judek 86

Jung 86

Jung 89

Juric 86

Juricic 88

Juster 8.5

Kaarsberg 87

Kaarsberg 84

Kageyama $n$ ?

Kalantarnaye 59

Kamat as

Kanıи 89

Kanarek 88

Kanazirsk 87
Phys. Rev. D31:961.1985:

Production of the $\bar{K}^{*}(892)^{0}$ Resonance in $\pi^{-}$p Interactions at $16 \mathrm{GeV} / \mathrm{c}$

LAL-65-34: DM2 Regults on $J / \psi(1 S)$ Decays

Plys. Rev. Lett. 56:1444,1986: Nucl. Phys. A457:71,1966; ETHZ-[MP-P86-2:

New Precision Determination of the $\pi^{-}$Mass from Pionic X-Rays

CERN-EP-89-51:

Collider Experiments

DOE-ER-01545-429:

Two-Photon Production of the $\eta_{c}(1 S)$

Phys. Rev. D31:1967.1986: Phys. Rev. D37:237.1988: LBL-21616:

Search for Right Handed Currents in Muon Decay

ANL-HEP-CP-89-99:

Underground Muons from Direction of Cygnus X-3

SLAC-294:

Measurements of Charged Two Particle Exclusive States in Photon-Photon Interactions

BUHEP-87-12;

Recent Results on Single Photon Production from ASP and MAC

2. Phys. C27:43.1985: MPI-PAE-EXP-EL- 1:11;

The $K^{\mathbf{0}} / \pi^{-}$Ratio and Strangeness Suppression in $\nu p$ and $\bar{\nu}$ Charged Current Interactions

2. Phys. C28:23,1985: PRE-85-009: MPI-PAE-EXP-EL-146:

Polarization of $\Lambda$ Hyperon Produced Inclusively in $\nu p$ and $\bar{\nu} p$ Charged Current Interactions

Phys. Lett. 178B:329.1986:

Measurement of the Neutral to Charged Current Cross Sections Ratios for Neutrino and Antineutrino Interactions on Protons

Z. Phys. C37:25.1987: MPI-PAE-EXP-EL-182:

Experimental Test of the PCAC-Hyporhesis in Charged Current Neutrino and Antineutrino Interactions on Protons

Z. Phys. C36:593.1987: MPI-PAE-EXP-EL-183:

Production of Charmed Hadrons in Neutrino Praton and Antineutrino Proton Charged Current Interactions

Z. Phys. C43:349,1989:

Search for Fractiona] Electric Charge in Meteorite Samples

Z. Phys. C43:527.1989; MPI-PAE-EXP-EL-199:

Experimental Test of the PCAC Hypothesis in the Reactions $\nu_{\mu} p \rightarrow \mu^{-} p \pi^{+}$and $\bar{\nu}_{\mu} p \rightarrow \mu^{+} p \pi^{-}$in the $\Delta\left(1232 P_{33}\right)$ Fegion

2. Phys. C44:379.1989:

A Measurement of the Proton Structure Functions from Neutrino Hydrogen and Antineutrino Hydrogen Charged Current Interactions

Z. Phy's. C46:25,1990:

$W^{Z}$ and $Q^{2}$ Dependence of Charged Hadron and Pion Multiplicities in $\nu p$ and $\bar{\nu}$ Charged Current Interactions

Nuovo Cin. 101 A:135.1989:

Multiplicity Distributions of Charged Hadrons Produced in (Anti)Neutrino - Deuterium Charged- and Neutral-Current Interactions

Phys. Rev. D41:1389.1190: LAL-88-25:

The $J / \psi($ iS $) \rightarrow$ Vector + Pseudoscalar Decays and the $\eta, \eta^{\prime}$ Quark Content

Yad. Phys. 42:194,1985: JINR-P1-84-279;

Characteristics of $C$ Ta Jnteractions with $\Lambda$ and $K^{0}$ Production at $4.2 \mathrm{GeV} / c$ per Nucleon

Phys. Rev. D39:1865.1989;

Diffractive Production of $\pi^{-} \pi^{-} \pi^{+}$in $200 \mathrm{GeV} / c \pi^{-} n$ Interam lons

Phys. Rev. C34:890.1986:

Ánomalous Behavior of Singly Charged Relativistic Secondary Particles Produced in Collisions of ${ }^{16} \mathrm{O}$ Ions with Emulsion Nuclei at 2 A GeV

Phy. Rev. Lett. 56:1775.1986:

Measurement of $D_{s}^{ \pm}$Meson Lifetime

Phys. Rev. Lett. 64:1041.1990: SLAC-PLB-5136: LBL-27998:

Search for Long-Lived Massive Neutrinos in $Z^{\circ}$ decays

Nuovo Cim. 92A:181,1986:

Angular Distributions of Secondary Relativistic Charged Particles Produced in Interactions of Negative Pions in Emulsion at $300 \mathrm{GeV} / \mathrm{c}$

Phys. Rev. D39: 1,1989: LBL-25018:

Bose-Einstein Correlations in $e^{+} e^{-}$Collisions

Phys. Rev. Let. 55:2261.1985:

Tritium Electromagnet ic Formfuctors

Phys. Rev, D35:2265.1987:

Measurement of the Branching Ratio for $\mathrm{Y}(3 S) \rightarrow \mathrm{\mu}^{-} \mathrm{\mu}^{+}$

Phys. Rev. Lett. 62:2077.1980:

Measurement of the Branching Ratio for Decay of $\boldsymbol{\Upsilon}$ States to $\mu^{-} \mu^{+}$

Phys. Rev. D35:265.5.1987;

Measurement of $\ddot{p} p$ Elastic Scattering at Beam Momenta becween 390 and $780 \mathrm{MeV} / c$

Phys. Rev Lett. 63:2032.1989

Energy Dependence of Dispersive Effects in ${ }^{12} \mathrm{C}$

UT-HE-88-05:

New Reaults from $e^{+} e^{-}$Collisions

FERMIILA': CONF-89-246-E.

A Study, of Weak Boson Pruduction with $W^{ \pm} \rightarrow e^{ \pm} \nu$ and $Z^{0} \rightarrow e^{+} e^{-}$at CDF

Yad. Phys. 48:1752.1988: JINR-P1-8K-30:

Proton and $\pi^{-}$Meson Spectra in C C - Interaction at $4.2 \mathrm{GeV} / c$ per Nucleon

JINR.PI.87-750:

Determination of Double Pomeron Exchange Contribution to the Cross Section of $\bar{p} p \rightarrow \bar{p} p \pi^{+} \pi^{-}$Reaction

See the legend on page 19 
Kanazirski 87 (cont'd) at $22.4 \mathrm{GeV} / \mathrm{c}$

Kanazirski 84 Czech. J. Plits. B39:(223.19k!).

Kanevsky 85

The Interactions $\bar{p} p \rightarrow \bar{n} \pi^{-} p$ at $22.4 \mathrm{GeV} / \mathrm{c}$

Vest n. of Moscow l'niv. Fiz.. Astr. 26:27.1985:

The Application of Multilayer X-Ray Film Chambers for Determination of Hadron Interacticn Length in Lead

Kaplan 89

Phys Rew DA1:2334.1990: FERAILAB-PLB-89-26i3-E:

Test of Scaling of the Massive-Dihadron Cross Section

Karev 88

Yad Phys. 50:727.1989: JINR-El-88-110:

Anomalous Component of Absorbtion Function of Relativistic Fragments Produced in Mg Nuclear Interactions with Plexigtas at $4.5 \mathrm{~A} \mathrm{GeV} / \mathrm{c}$

Karlen $88 \mathrm{~B}$

Phy. Rev. D39:1861,1989: SLAC-PLB-4560: LBL-249-82: Measurement of Single and Double Radiative Low $Q^{2}$ Bhabha Scattering at $E_{\mathrm{en}}=2 \mathrm{~g} \mathrm{GeV}$

Karlen $88 \mathrm{C}$

Káarnaukhov 86 SLAC-325:

A Study of Low $Q^{7}$ Radintive Bhabha Scattering

JINR-P1-86-373:

$\Sigma\left(1385 P_{23}\right)^{ \pm}, K^{*}(892)^{0}$ Resonances in $\pi^{-} p$ Interactions at $16 \mathrm{GeV} / c$

Karnaukhov 87

INR-PI-87-559:

Observations of $K(1629)$ Mesons

Kartik 90

Phys. Rev. D41:1.1990:

A-dependence of $J / \psi(1 S)$ Production in $\pi^{-}$Nucleus Collisions at $530 \mathrm{GeV} / \mathrm{c}$

Kass 89

Káabama $8 \overline{7}$

DOE-ER-01545-430:

Precision Measurement of the $\tau^{ \pm}$Lifetime

Nucl. Phys. A438:685.1985:

Measurement of deuteron $p$ Cross Sections in the Momentum Range $2 \mathrm{GeV} / \mathrm{c}-3.7 \mathrm{GeV} / \mathrm{c}$

Katsanevas 8

Phys. Rev Lett. 60:2121,1987: FERMILAB-PLB-87-57-E:

Nuclear Target Effects in $J / \psi(1 S)$ Production in $125 \mathrm{GeV} / c$ p and $\pi^{-}$Interactions

Karakami 8 i

Kawakami 89

Phy5. Let?. 187B:198.1987

An Upper Limit for the Mass of the Electron Antineutrino from the INS Experiment

INS-758: KEK-89-60:

Third Measurement of Anti-Electron-Neutrino Mass at INS

Kawamura 89

Kazarinov 85

Kechechyan 89

Keh 88B

Keizer 85

Kennett 87

Kennett $87 \mathrm{~B}$

Kernel 89

Phys. Rev. D40:729,1989: ICR-172-88-18:

"Quasidirect" Observations of Cosmic-Ray Primaries in the Energy Region $10^{12}-10^{14} \mathrm{eV}^{2}$

JINR-P1-85-426:

Amplitude Analysis of $\pi n$ Scattering at $40 \mathrm{GeV} / \mathrm{c}$

Kr. Soob. JINR 35:5.1989:

Scaling of Proton Cluster Distributions in the Range of the First Intermediate Asymptotics

Phys. Lett. 212B:123.1988: DESY-88-065: SLAC-PL:B-4634; HEN-295: Search for Exotic $T^{ \pm}$Decays

Phys. Lett. 157B:255.1985:

Deuteron Knockout from ${ }^{3} \mathrm{He}$ with the $\left(e^{-}, e^{-}\right.$deuteron) Feaction

Vucl. Phys. B28-653.1987; CALT-68-1367:

The Production of Neutral Pions from $200 \mathrm{GeV} \pi^{-} p$ Collisions in the High $x$ Region

Nucl. Phys. B282:626.1987

A Comparison of Diffractive $\overline{\boldsymbol{A}}$ and $\bar{\Xi}$ Photoproduction

Fhys. Letน. 225B:198.1989:

Measurement of $\pi^{-} p \rightarrow \pi^{-} p \pi^{0}$ Reaction near Threshold and Breaking of Chiral Symmetry

Kernel $89 B$

CERN-EP-89-104:

Measurement of $\pi^{-} p \rightarrow \pi^{-} p \pi^{0}$ and $\pi^{-} p \rightarrow \pi^{+} \pi^{-}$n near Threshold and Breaking of Chiral Symmetry

Kesten 8.5

Ketor 86

Phys. Let . 16]B:-112,1985: ANL-HEP-PR-85--77: IUHEE-67: PU-85-533: UN-HE-85-12; Comparison of Light Quark and Charm Quark Fragmentation

Pisma Zh. Eksp. Teor. Fiz. 44:114,1986:

Strange Phenomena Searching in Reactor Antineutrino Flux

Kelov $86 \mathrm{~B}$

Ketov 88

KIAE-86-4266-2;

Limitations on the Electrombgnetic Characteristics of Antineutrino at Reactor Experiment

Pisma Zh. Eksp. Teor. Fiz. 47:177,1988:

The Spectra of $\bar{\nu}_{e}$ from the Atomic Reactor and the Test of the Electroweak Theory

Khan 88

Khan 89

Khiari 89

uovo Cim. 99A:417.1988:

Interactions of Relativistic Carbon Nuclei in Nuclear Emulsion

Nuovo Cim. 101A:93.1989:

Interaction Mean Free Path of Projectile Fragments from ${ }^{12} \mathrm{C}$ Emulaion Colligions at $4.5 \mathrm{GeV} / \mathrm{c}$

Phys Rev. D39:45,1989; LNI-HE-87-36;

Acceleration of Polarized Protons to $22 \mathrm{GEV} / \mathrm{c}$ and the Measurement of Spin-Spin Effects in p (polarized) $p$ (polarized) $\rightarrow p p$

Kichimi $8 \mathbf{8}$

Kicsling 8:

KEK-88-yf:

Search or New Particles at TRISTAN

MPJ-PAE-EXP-EL-155:

Resules from PETRA on Elcctrowesk Effects and Tests of QED

Kim 85

Phys. Rev. D31:513.1985:

Angular Distribution of Sh r Particles Produced in the Collisions of $30 \mathrm{GeV}-400 \mathrm{GeV}$ Protons with Emulsion Nuclei

Kin s6 Plys. Rev. Lett. 56:1779.1986

Left-Right Asymmetry in Inverse $\pi^{-}$Photoproduction from a Transversely Polarized Proton Target

Kın

Phys. Rev. C.32:1454,1985:

Inclusive Angular Distribution of He and Li Fragments Produced in Fe C and Fe Pb Collisions at 1.88 GeV/Nucleon

Kin 88

Kim S\$B

A Search for Charged and Neutral Heavy Leptons in $e^{+} e^{-}$Annibilations at $\sqrt{8}=58 \mathrm{GeV}$

KEK-XY-61.

Asymmetry in Lepton Pair Production from $e^{+} e^{-}$at $\mathrm{KEK}$ 
Kim $88 C$

Kim B8C

Kim 88D

Kin 89

Kim $89 \mathrm{~B}$

Kim $89 \mathrm{C}$

Kim 89D

Kim 89E

Kim 89F

Kim 89G

Kim 90

Kinney 86

Kinoshita $88 \mathrm{~B}$

Kinoshita $\angle B C$

Kinoshita $89 \mathrm{~B}$

Kirov 85

Kirpichnikov 89

Kishida 89

Kistryn 87

Kistryn 89

Kitagaki 86

iKitagaki 88

Kitching 86

KJar 84

Klein 84B

K]:In 87

Klein $87 \mathrm{~B}$

Klein 88

Klein 89

Klein $80 \mathrm{~B}$

Klein $89 C$

Kleinwort 89

Klem 86

Kleme sts

Kfimenko 89

Klinkern 86

Kriyazey 85

Ko 84
KEK-88-49. AMY-88-12

A Study of QED Processes in the Electron-Positron Interactions and Search for E: to $58 \mathrm{GeV}$

Phys. Res. Lett, 61:011.1!88; KEK-88-8; AMY-Ks.(11. KNIHEP-Bt-1)1.

Experimental Mass Limit for a Forth Generation Sequential Lepton from $e e^{-}$Anrihilaticns at $\sqrt{s}=56$ GeV

Phys. Lett. 219B:62.1989.

Test on $\pi$ Partial Wave Analysis at Onset of the Roper Resonance

Phys. Lett. 223B:476.1989; KEK-88-125: AMY- $\times x+18$ :

Search for the Substructure of Leptons in High Energy QED Processes at TRISTAN

Phys. Rev. Lett. 63:1772.1989: Phys. Rev. Lett 63:1772.1990): KEK-69-44; AMY-89-07.

A Comparison of Quark and Gluon Jets Prodl ed in Higk Energy $e^{+} e^{-}$Annihilations

Phys. Rev. D40:244.1989:

Differential Cross Section for $\pi^{-} p \rightarrow \gamma n$ from 427 to $625 \mathrm{MeV} / c$

KEK-89-5i: AMY-89-13:

Recant Results from AMY

KEK-89-52: AMY'-89-11:

Measurement of the Cross Section for $e^{+} e^{-} \rightarrow \gamma \gamma$ at TRISTAN

KEK-89-177: AMY-89-20:

A Search for Leptoquark and Colored Lepton Pair Production in $e^{+} e^{-}$Annihilations at TRISTAN

Phys. Rev. DA1:732.1990:

Analyzing Power for $\pi^{-} p$ Charge Exchange in the Backward Hemisphere from 301 to $625 \mathrm{MeV} / c$ and a Test of Pion Nucleon Partial-Wave Analyses

Phys. Rev. Letl. 57:3152.1986:

Inclusive Pion Double Charge Exchange in ${ }^{4} \mathrm{He}$

Phys. Rev. Letl. 60:1610.1988: KEK-87-149:

Search for Highly Ionizing Particles in $e^{+}$- Annihilations at $\sqrt{\varepsilon}=50-52 \mathrm{GeV}$

KFK-88-32:

Recent Results from NIKKO at TRISTAN

Phys. Let2. 228B:543.1989: KEK-89-50:

Search for Highly Ionizing Particles in $e^{+} e^{-}$Annihilation at $\sqrt{8}=50-60.8$ GeV

Izv. A kad. Nauk SSSR. Fiz. 49:1352.1985:

Search of Shower Excess from Cu $3 \mathrm{~b}$ Nebula and Swan X-3

ITEP-89-177:

Double $\beta$ Decay Experiment

CT-HE-89-05:

Menaurement of Deuteron-Deuteron Total Cross Sections in the Incident Moment um Range 1.5 - 4.0 $\mathrm{GeV} / \mathrm{c}$

Phys. Rev. Let1. 58:1616.1987:

Precision Measurement of Parity Nonconservation in Proton Proton Scattering at $45 \mathrm{MeV}$

Phys. Lett. 219B:58.1989:

Measurement of Parity Nonconservation in Proton-Deuteron Scattering at $43 \mathrm{MeV}$

Phys. Rev. D34:2554,1986:

Charged Current Exclusive Pion Production in Neutrino-Deuterium Interactions

Phys. Lett. 214B:281.1988: FERMILAB-PLB-88-59-E:

A New Method to Investigate the Nuclear Effect in Leptonic Interactions

Phys. Rev. Lett, 57:2363,1986;

Polarized Proton Proton Bremsstrahlung

Phys. Rev. D31:491.1985; PRINT-84-0530-HEIDELBERG:

Multiparticle Production in Proton - Nucleus Collisions at $200 \mathrm{GeV}$

Forts. d. Phys. 33:375,1985; PHE-84-01:

Interference of Electromagnetic and Weak Interactions at High Energies and Neutral Current Muon Electron Universality

Phys. Rev. Lett. 58:644.1987: SLAC-PUB-4082: LBL-22408:

Observation of $\Xi^{-}$Production in $e^{+} e^{-}$Annihilation at $28 \mathrm{GeV}$

Phys. Rev. Lett. 59:2412.1987; SLAC-PUB-4338; LBL-23539:

Observation of $\Omega^{-}$Production in $e^{+} e^{-}$A nnihilation at $29 \mathrm{GeV}$

SLAC-330;

Charmed and Strange Baryon Production in 29 GeV Electron - Positron Annihilation

Phys. Rev, Lett. 62:2444,1989; SLAC-PUB-4696: LBL-25827:

$\Lambda_{c}^{+}$Production and Semileptonic Decay in $28 \mathrm{GeV} e^{+} e^{-}$Annibilation

BUHEP-89-30:

First Results from MACRO

Int. Jour. Mod. Phys. A5:1457.1990: BUHEP-89-16 Charmed Baryons: A New Laboratory for Charm Studies

Z. Phys. C42:7.1989: DESY.88.155:

A Messurement of the $\tau^{ \pm}$Lepton Lifetime

Phys. Rev. D37:41.1988: SLAC-PU13-4025: SLAC-300:

Meagurement of the Average Lifetime of Hadrons Containing Bottom Quarks

Z. Phys. C37:179.1988:

The $\beta$-Decay Asymmetry of the Neutron

MINR-89-I1:

Baksan Exp. . int on the Search of $8 \&$ Decay of ${ }^{100} \mathrm{M}$

Phys. Lett. 205, .1988.

Search for 5 ,onnnt Bhabha Scattering arcust an Invariant Mags of $1.8 \mathrm{MeV}$

Sov. J Nucl. F an 1:361.1986: Yad. Phys, 43:25.1981j. IF1'E-85-90:

Assaciated Production of the $K^{-}(892)^{ \pm}$with Other Particles and of the $\rho^{0}$ with Other Particles in Inclusive $K^{+} p$ Reactions at $32 \mathrm{GeV} / \mathrm{c}$

KEK-BS-30. ANY-B8-103:

$e^{+} e^{-}$Phymica at TulstaN - the First Year and a Half of AMY 
Kobayashi 87

Kobayashi 88

Kobayashi 88B

Kobayashi 89

Kobayashi 89B

Kobayashi 89C

Koch 86

Koch 89

Kochocki 89

Koenig 89

Koenigsmann 85

Koiso 84

Kolanoski 85

Kolaroski 86

Kolanoski 87

Kolb 89

Koltick 85B

Komamiya 85

Komamiya 89

Komamiya 89B

Komamiya $89 \mathrm{C}$

Komamiya 90

Konaka 86

Konigsmann 86

Kopeikin 90

Kopke 89

Kopp 85

Koptyev 88

Kopylova $\mathbf{8 6}$

Kopylova 86B

Kopylova 86E

Kopylova 87

Korenchenko 87

Korolev 85

Korsgen 88

Koshiba $85 \mathrm{~B}$

Kosvintsev 86

Kozhuharov 88
Phys. Rev. Lett. 59:868,1987;

New Measurement of the Asymmetry Parameter for the $\mathbf{\Sigma}^{+} \rightarrow p \gamma$ Decay

Phys. Rev. Lett. 60:2599.1988; KEK-87-78;

Projectile Fragmentation of the Extremely Neutron-rich Nucleus ${ }^{11} \mathrm{Li}$ at $0.79 \mathrm{GeV} / \mathrm{Nucleon}$ KEK-88-134:

$\pi^{+}$Induced Quasifree Double-Charge-Exchange Reaction on ${ }^{\circ} \mathrm{Li}$

Phys. Lett. 232B:51,1989; KEK-89-27;

Electromagnetic Dissociation and Soft Giant Dipole Resonance of the Neutron-Dripline Nucleus ${ }^{11} \mathrm{Li}$

KEK-89-169.

Projectile Fragmentation of a Weakly-bound ${ }^{11}$ Be Nucleus at $0.8 \mathrm{GeV} /$ nucleon

KEK-89-157:

Electromagnetic Dissociation of Exotic Projectiles at $0.8 \mathrm{GeV} / \mathrm{Nucleon}$

Nuovo Cim. 96A:182.1986:

Search for Axions at the Nuclear Power Reactor Biblis-A

Phys. Rev. Lett. 63:498,1989;

Differential Cross Sections for Coherent and Incoherent Neutral-Pion Photoproduction from Calcium

ANL-HEP-CP-89-93; PDK-403; Studies of $\mu^{\prime} \mathrm{s}$ Underground with the SOUDAN-2 Tracker

Phys. Let t. 218B:12,1989;

On the Momentum Correlation of $\left(e^{+} e^{-}\right)$Pairs Observed in $U$ U and $\mathbf{U} P b$ Collisions

DESY-85-089; C85/07/03:

Y Spectroscopy

Nucl. Phys. A 433:619.1985; UT-HE-84-10:

Search for the $P_{11} \Sigma(1660)$ Resonance in the Reactions $K^{-} p \rightarrow \Sigma^{-} \pi^{+}$and $K^{-} p \rightarrow \Sigma^{+} \pi^{-}$in the Momentum Range $597 \mathrm{MeV} / \mathrm{c}$ to $888 \mathrm{MeV} / \mathrm{c}$

BONN-HE-85-34; C85/08/19;

Two Photon Processes

BONN-HE-86-08: C86/03/10;

Recent Results on Two Photon Processes from DESY

DESY-87-175,

Two-Photon Physics

Phys. Rev. Lett. 62:509,1989:

Limits to the Radintive Decays of Neutrinos and Axions from Gamma-Ray Observations of SN1987A

PRINT-86-0057; PU-85-532;

Limits on the Tau Neutrino Mass

HD-PY-86-01:

Search for New Particles in $e^{+} e^{-}$Annihilation

Phys. Rev. D40:721,1989; SLAC-PUB-4771; LBL-26186;

Searches for Nonminimal Higgs Bosons from a Virtual $Z^{0}$ Decaying into a Muon Pair at PEP

Phys. Rev. Lett. 64:987,1989; SLAC-PUB-5137: 1.BL-27999:

Determination of $\alpha_{S}$ from a Differential Jet Multiplicity Distribution at SLC and PEP

SLAC-PUB-5154;

Study of Hadronic Final States of $Z^{\circ}$ Boson Decays

SLAC-PUB-5175; LBL-28457;

Search for Non-minimal Higgs Bosons from $Z^{0}$ Boson Decay

Phys. Rev. Lett. 57:659,1986; KEK-86-9; KEK-86-9;

Search for Neutral Particles in Electron Beam Dump Experiment

Phys. Rept. 139:243,1986: DESY-86-009;

Radiative Decays in the $\phi$ Family

Pisma Zh. Eksp. Teor. Fiz. 51:75,1990;

A Search for Massive Neutrino on the Reactor of Rovno AES

Phys. Rept. 174:67,1989; CERN-EP-88-93; $J / \psi(1 S)$ Decays

Z. Phys. C28:171,1985; CERN-EP-85-08;

A Mensurement of Energy Loss Distributions of Energetic Muons in Iron

Zh. Eksp. Teor. Fiz. 94(1):1,1988;

Subthreshold Production of $\boldsymbol{K}^{+}$Mesons in Proton-Nuclear Interactions

JINR-P1-86-141.

Investigation of a Possibility of Cumulative Hadron Produ ion as a Tesult of Isobar State Decay

JINR-P1-86-109

Angular Dependence of Slope Parameters of Invariant Inclusive Cumulative Hadron Production Cross Sections in $p C$ Interactions at $p(p)=10 \mathrm{GeV} / c$ and $\pi^{-}$Interactions at $40 \mathrm{GeV} / c$

JINR-P1-86-251:

Aximuthal Correlations of Cumulative Hadrons with Secondary Particles in $p$ C Interactions at $10 \mathrm{GeV} / \mathrm{c}$

JINR-P1-87-941;

Observation of Nucleon-Carbon Interactions at $P=4.2$ and $10 \mathrm{GeV} / c$ with the Summary Kinetic Energy of Secondaries Higher that Incident One

Yad. Phys. 46:313,1987; Sov. J. Nucl. Phys. 46:192,1987: JINR-P1-86-720:

On Decays $\pi^{+} \rightarrow e^{+} \nu$ axion $\left\langle e^{+} e^{-}>, \pi^{+} \rightarrow \mu^{-} e^{+} e^{+} \nu\right.$

Phys. Lett. 165B:262.1985:

Analysing Powers in Free $n$ p Forward Elastic Scattering at Energies at 630 to $1000 \mathrm{MeV}$

BONN-JR-88-47;

Research on the Charge Asymmetry in Inclusive Photoproduction of Mesons in the Forward Direction in the Momentum Range of $60 \mathrm{GeV} / \mathrm{c}-170 \mathrm{GeV} / \mathrm{c}$ in the CERN OMEGA Spectrometer (WA-69)

UT-ICEPP-85-02;

Kamioka Nucleon Decay Experiment

Pisma Zh. Eksp. Teor. Fiz. 44:444,1986;

The Measurement of the Neutron's Life Time by Ultracold Neutron Save Method

GSI-88-68B:

Experiments on Low Energy Bhabha Scattering. Search for Neutral Resonances in the MeV/ $\mathrm{c}^{2} \mathrm{Mass}$ Region 
Kozlovsky 86

Kozma 86

Kozma 86B

Kozma 87

Kozma 88

Kozma 88B

Kozma 89

Kozma 89B

Kozma $89 \mathrm{C}$

Kozma 90

Kozma 90B

Kral 89

Krasitnikov 85

Krasilnikov 85B

Krasnov 86

Krasnov 87

Krasnov 88

Krasnov $88 B$

Krastev 88

Krauss 89

Krebs 86

Kreinick 89

Kreiss! 87

Krishnaswamy 86

Kristiansson 85

Krivicich 88

Krivoruchenk 87

Krizmanic 89

Krofcheck 85

Krofcheck 87

Kroha 89B

Kubic 85

Kuhlen 86B

Kuhlen 90

Kulikov 87

Kumar 89

Kumita 89
Yad. Phys. 44:968,1986; Sov. J. Nucl. Plıys. 44:624.198G; 1FVE-86-52;

Boson Resonances Production in $\bar{p} p$ Interactions at $32 \mathrm{GeV} / \mathrm{c}$

Czecil. J. Phys, B38:1317,1988; JINR-E1-86-606:

Spallation of Copper by $9 \mathrm{GeV} / \mathrm{c}$ Protons and Deuterons

JINR-E1-86-607;

Spallation of Nickel by $9 \mathrm{GeV} / \mathrm{c}$ Protons and Deuterons

JINR-E1-87-350;

Target Residues from the Reactions of $\theta \mathrm{GeV} / c$ Protons at deuteron with ${ }^{181} \mathrm{Ta}$

JINR-E1-88-245;

Nuclear Reaction of Medium and Heavy Target Nuclei with High Energy Prijectiles. Fragmentation of ${ }^{107}$ Au by 3.65 A GeV ${ }^{12}$ C-lons and 3.65 GeV Protons

Czech. J. Phys. B40:29,1989; JINR-E1-88-244;

Nuclear Reactions of Medjum and Heavy Target Nuclei with High-Energy Projectiles. Spallation of ${ }^{85} \mathrm{Mn}$, ${ }^{\circ} \mathrm{Co}$, Ni and Cu by 3.65 A GeV ${ }^{12}$ C-Ions and 3.85 GeV Protons

JINR-E1-89-252;

Nuclear Reactions of Tantalum with 3.65 A GeV ${ }^{12} \mathrm{C}$-Ions and 3.65 GeV Protons

Nucl. Instr. and Meth. A291:662,1990; JINR-E1-89-745: Cross Sections for the Production of ${ }^{11} \mathrm{C}$ in $\mathrm{C}$ Targets by 3.65 A GeV Projectiles

JJNR-E1-89-482:

Target Residues from Reaction of $3.65 \mathrm{~A} \mathrm{GeV}{ }^{12} \mathrm{C}$ with ${ }^{232} \mathrm{Th}$ and ${ }^{238} \mathrm{U}$

Czech. J. Phys. B40:38,1990;

Nuclear Reaction of Medium and Heavy Target Nuclei with High Energy Projectiles. Fragmentation of $\mathrm{Ag}$ and ${ }^{10 T} \mathrm{Au}$ by $3.65 \mathrm{~A} \mathrm{GeV}{ }^{12} \mathrm{C}$-Ions and $3.65 \mathrm{GeV}$ Protons

JINR-E1-90-185;

Systematics of Target Recoil Properties of Intermediate Fragments Produced in the Interaction of 3.65 A GeV ${ }^{12} \mathrm{C}-$ Ions and Protons with Complex Nuclei

Phys. Rev. Lett. 64:1211,1990; SLAC-PUB-5147; LBL-28099; Measurement of the $b \bar{b}$ Fraction in Hadronic $Z^{\circ}$ Decays

Izv. A kad. Nauk SSSR, Fiz. 49:1321,1985;

The Spectra and Non-regularity of Super High Energy Extensive Atmospheric Showerg at E>10 ${ }^{10} \mathrm{eV}$

Izv. Akad. Nauk SSSR, Fiz. 49:1325,1985; Anisotropy of Intensity and Energy Spectra of High Energy Cosmic Ray

Kr. Soob. JINR 16:11,1986;

On Some Peculiarities of 4.1 A GeV/c Momentum ${ }^{22}$ Ne Nucleus Collisions with Nuclei in Photoemulsion

Yad, Phys. 47:1309,1988; JINR-P1-87-348; The Study of Two-Particle Correlations in Inelastic Interactions of ${ }^{20}$ Ne Nucleus with Emulsion at 4.1 GeV/c per Nucleon

JINR-P I-88-389;

The Study of Total Disintegration of Lead Nuclei with ${ }^{24} \mathrm{Mg}$ Nuclei at 4.5 a GeV/c

JINR-P1-88-252;

The Topological Characteristics of Silicon Nuclei Fragmentation at $4.5 \mathrm{GeV} / \mathrm{c}$

JINR-P1-88-31

$\Lambda\left(1520 D_{0 s}\right)$ Production in Neutron-Nucleon Interactions at $40 \mathrm{GeV}$ Neutron Energy

Phys. Rev. Lett. 64:999,1989; YCTP-P14-89:

$Z^{0}$ Decay and the Search for Dark Matter

Phys. Lett. 171B:37,1986; Subthreshold Negative Pions and Energetic Protons Produced at $\theta(\mathrm{c} . \mathrm{m})=$.90 Degrees in $246 \mathrm{MeV} / \mathrm{s}$. $1 \mathrm{cleon}$ ${ }^{130} \mathrm{La}{ }^{230} \mathrm{La}$ Collisions

CLNS-89-956; $B$ Physics from CLEO

Z. Phys. C37:557,1987; CERN-EP-87-183;

Remeasurement of the Magnetic Moment of the Antiproton

Nuovo Cim. 9C:167,1986;

Results from the KGF Proton Decay Experiment

LUIP-8505;

Large Angle Correlationg Observed in Intermediate Energy Heavy Ion Collisions

LBL-25233;

A New Limit on the Neutrinoless Double $\beta$ Decay of ${ }^{100} \mathrm{Mo}$

ITEP-87-155;

Neutrino Masses from the Data on Second Neutrino Burst Observed from Supernova SN987A

THESIS;

A Search for the Oscillation of Muon Antineutrinos to Electron Antineutrinos using the AGS Wide Band Beam

Phys. Rev. Lett. 55:1051,1985;

Gamow-Teller Strength Function in ${ }^{71} \mathrm{Ge}$ via the $(p, n)$ Reaction at Medium Energies

Phys. Lett. 189B:299,1987;

Gamow-Teller Strength Distribution in ${ }^{81} \mathrm{Kr}$ and the Consequences for a ${ }^{\mathrm{d}} \mathrm{Br}$ Solar Neutrino Detector

MPI-PAE-EXP-EL-215-89;

Measurement of Heavy Quark Charge Asymmetries at $35 \mathrm{GeV}$ and $43 \mathrm{GeV}$ and Heavy Flavor Physica with CELLO

Yad. Phys. 43:89,1986; Sov. J. Nucl. Phys. 43:57,1986; IFVE-85-63;

Charged Multiplicities in Forward and Backward Hemispheres in CMS in $K^{+} p$ Interactions at $70 \mathrm{GeV} / \mathrm{c}$

DESY-86-052:

Hadronic Events with Low Thrust Containing Isolated Leptons

CALT-68-1619;

$Z^{0}$ Decays into Lepton Pairs

Pisma Zh. Eksp. Teor. Fiz. 46:249,1987:

Registration of Super High Energy Cosmic Rays from Binary Systems Cygnus X-1 and Cygnus X-3

Mod. Phys. Lett. A4:115,1989;

Multiparticle Production in Hadron Nucleus Collision and the Negative Binomial Distribution

KEK-89-48; AMY-89-10

Measurement of $\boldsymbol{R}$ for $e^{+} e^{-}$Annihilation at TRISTAN 
Kumita 89B

Kunne 88

Kunne 88B

Kunne $88 \mathrm{C}$

Kuplennikov 90

Kurdadze 86

Kurdadze 88

Kurepin 88

Kurihara 89

Kutsidi $\mathbf{8 6}$

Kuzichev 88

Kuzichev 89

Kvatadze 88

Lac 88

Lac 89

Lac 89B

Lac $89 \mathrm{C}$

Lac 89D

Lach 89

Lamm 87

Lancon 86B

Landsberg 85

Lan:Jsberg 86

Landsberg 87

Landsberg 88

Landsberg 89

Lang $85 \mathrm{~B}$

Lang 87

Last 88

Lattimer $\mathbf{8 8}$

Lawrence 89

Lebedev 88

Lechanoinele 86

Leclaire 87

Lee $85 \mathrm{~B}$

Lee 88

Leffler 86
KEK-89-188; AMY-89-21; Measurement of $R$ for $e^{+} e^{-}$Annihilation at TRISTAN

Phys. Lett, 206B:557,1988: CERN-EP-88-22: Asymmetry in $\bar{p} p$ Elastic Scattering

Nucl. Phys. B323:1,1989; CERN-EP-88-171; Measurement of $d \sigma / d \omega$ and $A_{0 n}$ in $\bar{p} p$ Elastic Scat tering between 497 and $1550 \mathrm{MeV} / \mathrm{c}$ THESIS:

Measurement of Asymmetries and Differential Cross Sections in Antiproton-Proton Elastic Scattering at Momenta between 497 and $1550 \mathrm{MeV} / \mathrm{c}$

Yad. Plyys. 51:1210,1990; Study of the Quasifree Peak Shift in the $e^{-}{ }^{4} \mathrm{He} \rightarrow e^{-} \mathbf{X}$ Reaction

Pisma Zh. Eksp. Teor. Fiz. 43:497,1986;

Study of the Reaction $e^{+} e^{-} \rightarrow \pi^{+} \pi^{-} 2 \pi^{0}$ up to $1.4 \mathrm{GeV} E_{\mathrm{cm}}$

Pisma Zh. Eksp. Teor. Fiz. 47:432,1088:

Investigation of the Reaction $e^{+} e^{-} \rightarrow \pi^{+} \pi^{-} \pi^{+} \pi^{-}$for $2 E$ up to $1.4 \mathrm{GeV}$

Pisma Zh. Eksp. Teor. Fiz. 47:16.1988;

Yield of Pions and Kaons in the Collisions of Relativistic Nucleus with Energy 3.65 GeV/N

KEK-89-64; AMY-89-16;

Study of $e^{+} e^{-}$Annihilation into Four-Lepton Final State

Yad. Phys. 43:931,1986;

Some Correlation Features of Multiplicity Distributions for Charged Secondaries Produced in Collisions of Relativistic Nuclei

ITEP-88-112;

Detector for the Antiproton Annihilation

ITEP-89-6G;

Antiproton-Nuclei Annihilation Cross Section on Be, C, Al, Fe, Cu, Cd and Pb at the Momenta 1.28, $1.53,1.76$, and $2.5 \mathrm{GeV} / \mathrm{c}$

Z Phys. C38:551,1988:

On the Directional Dependence of Bose-Einstein Correlations

Nucl. Phys. B297:653.1988:

Meagurement of the Spin Correlation Parameters $A_{00 s k}$ and $A_{00 k k}$ in $p p$ Elastic Scattering at .84 and 1.0 GeV

Nucl. Phys. B315:269,1989:

Measurement of the Parameters $N_{0 n k k}, D_{0.05}$ and $K_{0, k_{0}}$ in $p$ Elastic Scattering between 0.84 and 2.7 GeV

Nucl. Phys. B315:284,1989;

Measurement of the Parameters $D_{0 n o n}$ and $K_{0 m o}$ in p $p$ Elastic Scattering between 0.84 and $2.7 \mathrm{GeV}$

Nucl. Phys. B321:269.1989;

Measurement of the Parameters $N_{\text {osko }}, D_{0 \text { onon }}$ and $K_{\text {osko }}$ in $p p$ Elastic Scattering between .84 and $2.1 \mathrm{GeV}$

Nucl. Phys. B321:284,1989;

Measurement of the Parameters $N_{0 a n k}, K_{0 s s 0}, K_{0 n n o}$ and $D_{0 s 0 k}$ in $p p$ Elastic Scattering between .84 and 2.7 GeV

FERMIILAB-CONF-89-19-E;

Magnetic Moments of the Hyperons: A Short Experimental Feview

Phys. Rev. D36:3341,1987;

High-Energy Diffraction Dissociation of $K_{L}$ into Exclusive Final State

SACLAY-DPHPE-86-12;

Recent Results on Direct Photon Production at CERN

Usp. Fiz. Nauk 146:185,1985:

Electromagnetic Leptonic Decays and the Structure of Light Mesons

Phys. Rept. 128:301,1986;

Electromagnetic Decays of Light Mesons

IFVE-87-83;

Search for Exotic Mesons and Study of Rare Mesonic Radiative Decays at "LEPTON" Spectrometer

IFVE-88-143:

Study of Meson Spectroscopy on "LEPTON" Apparatus

IFVE-89-54:

Exotic Hadrons

Phys. Rev. Lett. 54:170,1985:

Parity Nonconservation in Elastic $p$ He Scattering and the Determination of the Weak Meson - Nucleon Coupling Constants

Z. Phys. C33:483,1987:

Neutrino Production of Dimuons

Phys. Rev. Lett. 60:995,1988;

Pulsed-Beam Neutron-Lifetime Measurement

Phys. Rev, Lett. 61:23.1988;

Limits on the Neutrino Magnetic Moment From SN1987A

Phys. Rev. Lett. 63:1121,1989; OG-4-1-22;

Upper Limit to the Flux of Neutral Particles from Cygnus X-3 above $5 \cdot 10^{17} \mathrm{eV}$

Vopr. At. Nauki i Techn. ser. Obsch. 2-42:12.1988,

The Analysis of the Two-Particles Inclusive Cross Section of Protons Production in the Interaction of 3.6 $\mathrm{GeV} /$ Nucleon ${ }^{12} \mathrm{C}$ with $\mathrm{Pb}$ in the $B(i k)$ Variables

DPHPE-86-18;

Critical Review of the Present Status of Neutron - Proton Scattering up to $1 \mathrm{GeV}$

SLAC-321;

A Search for Supersymmetric Electrons with the MARK-II Detector at PEP

SLAC-282; SLAC-PUB-3676; C:85/03/10;

Radiative Decays of the $\psi(25)$ to All Photon Final States

Phys. Lett. 205B:219,1988:

Intermediate Energy Proton Scattering from ${ }^{40} \mathrm{Ca},{ }^{80} \mathrm{Zr}$ and ${ }^{208} \mathrm{~Pb}$

SLAC-293;

A Search for Primarily Noninteracting Decay Modes of the $\boldsymbol{r}$ 
Lehar 86

Lehar 87

Lehar 87B

Lehar 88

Leitch 85

Lepekhin 89

Lepikhin 87

Leskin 86

Letessiersel 89

Letessiersel 8:5

Levi 85

Levy 88

Lhote 87

Lhote 89

Li 89

Li $89 \mathrm{~B}$

Lichtenstadt 85

Lile 89

Lim 90

Linsley 84

Linssen 87

Lippman 85

Liss 90

Liltenberg 89

Liu 88

Liu 88B

Lloydowen 86

Lockman 89

Lohner 88

London 89

Longacre 86

Longacre 86B

Longacre 87

Longuemare 88

Longuemare 89

Lorenz 88

Losecco 85
DPHPE-86-28;

An Energy Dependent Phase Shift Analysis of Proton-Proton Scattering between 700 and $1300 \mathrm{MeV}$

Phy: Lett. 189B:241,1987;

Messurement of the Spir Dependent Neutron-Proton Total Cross Section Difference $\sigma_{T}$ and $\sigma_{\mathcal{L}}$ between 0.63 and $1.08 \mathrm{GeV}$

Nucl. Phys. B294:1013,1987;

Measurement of the Spin Correlation Parameter $A_{00 n n}$ of $p p$ in a Large Angular Region between 0.88 $\mathrm{GeV}$ and $2.7 \mathrm{GeV}$

Nucl. Phys. B296:535,1988;

Measurement of the Spin Correlation Parameter $A_{00 t 5}$ in $p$ Elastic Scattering from 0.88 to $2.7 \mathrm{GeV}$

Phys. Rev. Lett. 54:1482,1985:

Double Analog Transition ${ }^{14} \mathrm{C}\left(\pi^{+}, \pi^{-}\right){ }^{14} \mathrm{O}$ at $50 \mathrm{MeV}$

LENI-89-1557

The Search for Correlated Groups of Particles Produced in the Interactions of ${ }^{22}$ Ne Nucleus with Nuclei of Photoemulsion

Jetp Lett. 46:275,1987; Pisma Zh. Eksp. Teor. Fiz. 46:219,1987;

Below Threshold and near Threshold Production of Antiprotons in Colligions with Nuclei

Kr. Soob. JINR 17:5,1986;

Momentum Spectra of Pions and Protons in $p,{ }^{12} \mathrm{C}$ and ${ }^{22} \mathrm{Ne}$ Collisions with Emulsion Nuclei at 4.5 GeV/c/Nucleon

LBL-26802;

Mass and Transverse Moment um Dependence of the Dielectron Yield in $p$ Be Collisions at $4.9 \mathrm{GeV}$

LBL-27080;

Mass and Transverse Momentum Dependence of the Dielectron Yield in $p$ Be Collisions at $4.9 \mathrm{GeV}$

CERN-EP-85-172; C85/0T/18;

UA 1 Results on $W^{ \pm}$and $Z^{\circ}$ Production

DESY-88-115;

Two Photon Production of Exclusive Final States

Phys. Lett. 198B:139,1987;

Exclusive Measurements of Mean Pion Multiplicities in ${ }^{4}$ He nucleus Reactions from 200 to 800 MeV/Nucleon

SACLAY-DPH-N-2553B;

Inclusive Experiments, Correlation and Pion Photoproduction Data

KEK-89-34; AMY-89-03:

Multihadronic Event Properties in $e^{+} e^{-}$Annihilation at $\sqrt{8}=52$ to $57 \mathrm{GeV}$

KEK-89-149; ANY-89-19;

Multi-hadron Event Properties in $e^{+} e^{-}$Annihilation at $\sqrt{8}=52 \mathrm{GeV}$ to $67 \mathrm{GeV}$

Phys. Rev. C32:1096,1985;

Blackbody Description of Antiproton Nucleus Scattering

THESIS;

Neutrino Oscillations: A Search for Electron Neutrino Appearance in a Wide Band Muon Neutrino Beam KEK-90-9; CERN-TH-5680-90;

Correlation between Solar Neutrino Flux and Solar Magnetic Activity for Majorana Neutrinos

Lett. Nuovo Cim. 42:403,1985: UNML-5-4-84-2;

$p$ p Total Cross Section at $E_{\mathrm{cm}}=15$ TeV to $150 \mathrm{TeV}$ from Cosmic Ray Data

CERN-EP-87-36;

Messurement of Antiproton Small Angle Elastic Scattering at Low Momentum

Phys. Rev. Lett. 54:285,1985;

Precise ${ }^{3} \mathbf{H}-{ }^{3}$ He Mass Difference for Neutrino Mass Determination

FERMILAB-CONF-90-17-E;

$J / \psi(1 S)$ and $r(1 S)$ Production at CDF

BNL-43660;

Rare Kanon Decays

Phys. Rev. Lett. 58:2288,1988:

Evidence of $2 q 2 \bar{q}$ Mesong in $\bar{p} n$ Annihilations and $\gamma \gamma$ Reactions

Phys. Rev. Letl. 61:271,1988;

Nuclearite Flux Limit from Gravitational-Wave Detectors

Nucl. Phys. B274:685,1986; CERN-EP-86-03;

Total, Elastic and Inclusive Single Diffractive Cross Sections in He He Collisions at the CERN Intersecting Storage Rings

SLAC-PUB-5139;

Production of the $f_{0}(\theta 75)$ Meson in $J / \psi(1 S)$ Decays

Z. Phys. C38:97,1988;

Neutral Transverse Momentum Spectra in 60 and $200 \mathrm{~A} \mathrm{GeV}{ }^{20} \mathrm{O}+\mathrm{Nucleus}$ and Proton + Nucleus Reactions

SACLAY-DPHPE-89-03.

Results from the CERN Pilot Study of Ultrarelativistic Nucleus-Nucleus Interactions

Phys. Lett. 177B:223,1986; BNL-38257;

A Measurement of $\pi^{-} p \rightarrow K_{S} K_{S} n$ at $22 \mathrm{GeV} / c$ and a Systematic Study of the $2^{++}$Meson Spectrum

BNL-38729;

The Results of Two Scattering Processes: $\pi \pi \rightarrow \phi \phi$ and $\pi \pi \rightarrow K \bar{K}$

BNL-39954;

The Argand Amplitudes for $\phi \phi$ Event from $\pi^{-} p \rightarrow \phi \phi n$ at $22 \mathrm{GeV} / \mathrm{c}$

LAL-88-33;

Study of Atmospheric Neutrino Interactions with the FREJUS Detector

LAL-89-35.

Study of Neutrino Oscillations in the FREJUS Experiment

Phys. Lett. 214B:10,1988; MPI-PAE-EXP-EL-190;

Search for Narrow Resonance Production in Bhabha Scattering at Centre-of-Mass Energies near $1.8 \mathrm{MeV}$

Phys. Lett. 184B:305,1985;

Investigation of Matter Enhanced Neutrino Oscillations Relevant to the Solar Neutrino Problem 
Losecco $85 \mathrm{~B}$

Losecco 87

Losecco B7B

Losecco 89

Louis 86

Lovelock 85

Low 87

Low 89

Love 85

Lowe 86

Lowe $86 \mathrm{~B}$

Lowe $86 \mathrm{C}$

Lubat:i 89

Luca 85

Luk 88

Lund 88

Lund 89

Lund berg 89

Lurz 87

Luth 87

Lyons 87

Lyubinoy 88

Lyukoy 89

Ma 86

Machner 85

Madaras 86

Madey 85

Madigan 85

Mageras 86

Mahi 88

Maki 88

Maki 88B

Maki 89

Mallik $89 \mathrm{~B}$

Mampe 89

Manabe 89

Mandelli 88

Mangotra 85

Mann 86
Phys. Rev. Lett. 54:2299,1985:

Tege of Neutrino Oscillations Using Atmospheric Neutrinos

Phys. Lett. 188B:388.1987:

Limits on the Flux of Energetic Neutrinos from the Sun

Phys. Rev. D35:2073,1987;

Limits on the Neutrino Lifetime

UND-PDK-89-4:

Search for Dark Matter with IMB-1

Phys. Rev. Lett. 56:1027.1986:

Upper Limitg on the Decay $D^{0} \rightarrow \mu^{+} \mu^{-}$and on $D^{0} \bar{D}^{0}$ Mixing

Phys. Rev, Lett. 54:377,1985:

Masses, Widths and Leptonic Widths of the Higher $\mathbf{Y}$ Resunances

Phys. Lett. 1831:232,1987:

Production and Fragmentation of the $D^{\cdot}(2010)^{0}$ Meson in $e^{+} e^{-}$Annihilations at $29 \mathrm{GeV}$

Phys. Lett. 228B:548,1989; KEK-89-47; AMY-89-09:

Search for Non-Minimal Higgs Production in $e^{+} e^{-}$Annihilations at $\sqrt{s}=56 \mathrm{GeV}$

SLAC-PUB-3683; C85/03/10:

The Status of the $\zeta(8.3)$

SLAC- $-30 \overline{7}^{-}$

A Search for Narrow States in Radiative $\mathbf{Y}$ Decays

SLAC-PUB-4151:

Recent Results from the Crystal Ball Experiment

SLAC-PUB-4449;

Crystal Ball Regults on $\tau^{ \pm}$Decays

LWSEA-PUB-89-12:

Search for $\boldsymbol{K}^{+} \rightarrow \pi^{+} \mu^{+} e^{-}$and Study of $\boldsymbol{K}^{+} \rightarrow \pi^{+} e^{+} e^{-}$at BNL

LAL-85-15;

The Pion Formfactor in the Time-Like Region $1.38 \mathrm{GeV}<E_{\mathrm{cm}}<2.28 \mathrm{GeV}$

Phys. Rev, D38:19.1988:

New Measurement of Properties of $\Omega^{-}$Hyperon

2. Phys. C38:51,1988;

Charged Particle Spectra in ${ }^{10}$ O-Induced Nuclear Collisions at the CERN SPS

GSI-89-04:

Fluctuation in Multi-Particle Production and $\gamma / \pi^{0}$ Ratios in $200 \mathrm{GeV} \mathrm{A}{ }^{16} \mathrm{O}+\mathrm{Au}$ Collisions

Phys. Rev. D40:355T,1989:

Polarization in Inclusive $\boldsymbol{\Lambda}$ and $\overline{\boldsymbol{\Lambda}}$ Production at Large $\mathrm{pT}_{\mathrm{T}}$

2. Phys. C36:383.1987: SLAC-PCB-4302: DESY-87-038:

Experimental Upper Limite for the Hadronic Transitions $\mathbf{Y}(2 S) \rightarrow \eta \Upsilon \Upsilon(1 S)$ and $\Upsilon(2 S) \rightarrow \pi^{0} \Upsilon(1 S)$

SLAC-PUB-4222:

Lifetimes of Heavy Leptons and Hadrons

2. Phys. C36:363,1987;

Search for Free Quark Production at the CERN SPS Collider

Kr. Soob. JINR 33:5,1988;

Properties of Four-dimensional Baryon Clusters in Cumulative Nucleus-Nucleus Interactions

Nuovo Cim. 102A:583.1989:

Study of Hypernuclear Production and Search for Supernuclei in Proton-Nuclear Interactions in Photoemulsion at 70 and $230 \mathrm{GeV}$

2. Phys. C30:191.1986;

Some Tests of Fragmentation Models in Exclusive Channels in $\boldsymbol{K}^{-}$p Reactions at $32 \mathrm{GeV} / \mathrm{c}$

Phys. Rept. 12\%:309.1985;

Fast Particle Emission from Nuclear Reactions

LBL-21537:

Studies of Parton Fragmentation and Baryon Production with the TPC at PEP

Phys. Rev. Lett. 55:1453.1985:

Inclusive Neutron Spectra at 0 Degrees from the Reactions P.b (Ne, $n$ ) $X$ and Na (Ne, $n$ ) $X$ at 390 $\mathrm{MeV} /$ Nucleon and $790 \mathrm{MeV} / \mathrm{Nucleon}$

Phys. Rev. D31:966,1985:

Transverse Spin Dependence of the $p$ p Total Cross Section from 0.8 to $2.5 \mathrm{GeV} / \mathrm{c}$

Phys. Rev. Lett. 56:2672,1986;

Search for Light Short Lived Particles in Radintive $\mathbf{Y}$ Decays

Phys. Rev. Leit. 60:1936.1988;

Energy Dependence of Proton-Induced Xenon Fragmentation and the Approach to Liquid Gas Criticality in Nuclear Matter

KEK-88-112;

Recent Results from TRISTAN

KEK-88-52;

Recent Resulte from TRISTAN

KEK-89-145:

Results from TRISTAN

SLAC-PUB-4828;

Results from MARK-III on the $J / \psi(1 S)$ Decays

Phys, Rev. Lett. 63:593,1989:

Neutron Lifetime Measured with Stored UItracold Neutrons

Phys. Rev. Lert. 63:490.1989:

Polarizationg and Cross Sections of $\Lambda$ Hyperons Produced at Backward Angles in the Reaction $\pi+{ }^{12} \mathrm{C} \rightarrow$

$A x$ at $4 \mathrm{GeV} / c$

CERN-EP-88-182:

UA2 Results from the 1987 Run

Nuovo $\mathrm{Cim}$. 87A:279.1985:

Characteristics of ${ }^{36} \mathrm{Fe}$ Emulsion Interactions at $1.7 \mathrm{GeV} / \mathrm{A}$

Plys. He.. D34:2545.1986:

$\boldsymbol{K}$-Meson Production by $\nu_{11}$ Deuterium Reactions near Threshold: Implications for Nucleon-Decay Searches 
Marage 86

Marage 87

Marage 89

Marchand 85

Markytan 89

Marshak 85

Marshak 85B

Marshall 85

Marshall 88

Marshall 89

Marsiske 90

Marti 85

Maruyama 89

Marx 86

Maschnann 89

Masek 87

Masuda 88

Mathiazhagan 89

Mathiazhagan $89 \mathrm{~B}$

Mathie 85

Mathis $\mathbf{8 8}$

Matis 86

Matis 88

Natsinos 89

Matsuda 86

Matteuzzi 85

Mattig 89

May 89

May $89 B$

Mayer 86

Mayer 89

Mayle 87

Mazzucato 86

Medonough 88

Mcfarlane 85

Mcgaughey 86

Mcnaughton 86

Mcneil 88

Meparland 85
2. Plyys, C31:191.1986: CERN-PRE-80-042:

Coherent Single Pion Production by Antineutrino Charged Current Interactions and Test of PCAC

2. Phys. C35:275,1987; CERN-PRE-87-058:

Coherent Pion Production of $\rho^{-}$Mesons in Charged Current Antineutrino Neon Interactions in BEBC

Z. Phys. C43:523.1989;

Coherent Production of $\pi^{+}$Mesons in $\nu$ Ne Interactions

Pliys. Lett. 153B:29.1985:

Transverse and Longitudinal Response Function in Deep Inelastic Electron Scattering from ${ }^{3} \mathrm{He}$

z. Phys. C43:557.1989; Studies in $\bar{p} p$ Annihilation

Phys. Rev. Lett. 54:2079.1985: Evidence for Muon Production by Particles from Cygnus X-3

Plys. Rev. Lett. 55:1965,1985: Time Distributions for Underground Muons from the Direction of Cygnus X-3

RAL-85-078;

Electroweak Effects in $e^{+} e^{-}$Annihilation

TRI-PP-88-53;

An Improved Limit for Conversion of $\left(\mu^{+} e^{-}\right)$to $\left(\mu^{-} e^{+}\right)$

RAL-89-021: $e^{+} e^{-}$Annihilation at High Energies

Phys. Rev. D41:3324.1990: SLAC-PUB-5163: DESY-90-002: A Measurement of $\pi^{\circ} \pi^{0}$ Production in Two-Photon Collisions

Phys. Leit. 163B:71,1985;

Double $\beta$ Decay Half Life of ${ }^{82} \mathrm{Se}$

INS-REP-76I:

Exclusive Photoreactions on Light Nuclei

Fiz. Elem. Chastits At. Yadra 17:1231,1986;

Interaction of Pjons with ${ }^{3} \mathrm{He}$ and ${ }^{4} \mathrm{He}$ Nuclei

Z. Phys. C46:555,1990: DESY-89-141: SLAC-PUB-5133: Inclusive $J / \psi(15)$ Production in Decays of $B$ Mesons

Phys. Rev. D35:2758,1987:

Results from a Magnetic Monopole Search Utilizing He Proportional Tubes

DPNU-88-52;

A Study of Multihadron Production at TRISTAN

Phys. Rev. Lett. 63:2181.1989: (:CI-89-10:

New Experimental Limits on $K_{L} \rightarrow \mu^{-} e^{+}, K_{L} \rightarrow \mu^{+} e^{-}$and $K_{L} \rightarrow e^{+} e^{-}$Branching Ratios

Phys. Rev. Lett. 63:2185,1989; LCI-89-41: KL-245: Measurement of the Branching Ratio for the Decay $K_{L} \rightarrow \mu^{+} \mu^{-}$

Plys. Lett. 154B:28,1985;

First Measurements of $i T(11)$ in the Pion Deuteron Breakup Reaction

LBL-25261:

A Search for New Leptons with Heavy Neutrinos in $e^{+} e^{-}$Annihilation at $\sqrt{8}=29 \mathrm{GeV}$

LBL-21670;

A Search for Stable Quarks Produced by the Tevatron

Phys. Rey. D39:1851,1989: LBL-25680: Search for Free Quarks Produced at Bo0 Ge $/ \mathrm{c}$ Using a New Concentration Technique

Z. Phys. C44:79,1989; CERN-EP-89-57:

Backward Particle Production in Neutrino Neon Interactions

Phys. Rev. D33:2563.1986: Elastic $p$ scattering Amplitudes at $6 \mathrm{GeV} / c$

Phys. Rev. D32:800.1985:

Improved Upper Limit on $\nu_{\tau}$ Mass

Phys. Rept. 177:141,1989;

The Structure of Jets in $e^{+} e^{-}$Collisions

Phys. Lett. 225B:450,1989: Observation of an Isovector Meson AX(1585) in Annihilation of the $\bar{p} p$-Atom from $P$ State

Nuovo Cim. 102A:401.1989:

Strangeness-2 Hypernuclei

Phys. Lett. 181B:25,1986: Large Variation in the Backward Angles A nalyzing Power of the $p$ (polarized) deuteron $\rightarrow{ }^{3} \mathrm{H} \pi^{+}$Reaction around $1 \mathrm{GeV}$

SACLAY-DPhN-2565:

$\eta$ (and $\pi$ ) Production and Decay at SATURNE

Phys. Lett. 203B:188.1988; EF1-87-104-CHICAGO; UNN-TH-87-837; CERN-TH-4887; FERMILAB-PUB-87-225-A: Constraints on Axions from SN1987A

Phys. Rev. Lett. 57:3144.1986: Precise Measurement of Neutral Pion Photoproduction on the Proton near Threshold

Phys. Rev. D38:2121.1988:

New Search for the $C$-Noninvariant Decay $\pi^{0} \rightarrow 3 \gamma$ and the Rare Decay $\pi^{0} \rightarrow 4 \gamma$

Phys. Rev. D32:547,1985;

Measurement of the Rate for Pion $\beta$ Decay

Phys. Rev. Lett. 56:2156,1986;

Dynamics of Low-Energy Antiproton Annihilation in Nuclei as Inferred from Inclusive Proton and Pion Mensurements

Nucl. Jnstr. and Moth. A241:435.1985:

The $p \mathrm{C}$ Analyzing Power between $100 \mathrm{MeV}$ and $750 \mathrm{MeV}$

KEK-88-128: C'88/11/18:

Recent Resuits from TRISTAN

Phys. Lett. 156B:47.j985: TRI-PP-85-6;

Angular Dependence of the ${ }^{6} \mathrm{Li}\left(\pi^{+},{ }^{3} \mathrm{He}\right){ }^{3} \mathrm{He}$ Reaction at $60 \mathrm{MeV}$ and $80 \mathrm{MeV}$ 
Mcparland $85 \mathrm{~B}$

Meicr 89

Meklttiev 88

Melnikov 89

Mermaz 86

Merritt 87

Merritt 87B

Mestayer 85

Metcalf 89

Meyer 85D

Meyer $85 \mathrm{~F}$

Meyer 88

Meyer $88 \mathrm{~B}$

Meyers 86

Meziani 85

Miake 84

Miake 88

Michel 85

Miettinen 88

Mikaclyan 88

Mikhailov 85

Mikhajlichen 87

Mikucki 86

Miller 87

Miller 87B

Miller $87 \mathrm{C}$

Miller 89

Mills 85

Mills 87

Milner 85

Milner 85B

Milner 87

Mincer 85

Minowa 87

Minowa 89

Mir 88

Mir 89

Mishra 8.5
Nucl. Phys. A 456:629.1986: TRI-PP-85-105:

A Study of the ${ }^{6} \mathrm{Li}\left(\pi^{+},{ }^{3} \mathrm{He}\right){ }^{3} \mathrm{He}$ Reaction at $60 \mathrm{MeV}, 80 \mathrm{MeV}, 100 \mathrm{MeV}$ and $140 \mathrm{MeV}$ Incident Pion Beam Energies

CERN-EP-89-178:

Physics with Jets - Recent Results from UA2

JINR-P1-88-760;

Analysis of Transverse Energy Spectra in Collisions of Light Relativistic Nuclei

Vopr. At. Nauki i Techn. ser. Yad, 2:11.1989:

Spatial Parity Nonconservation in $\gamma$-Decay of ${ }^{237} \mathrm{~Np}$

SACLAY-DPH-N-2366:

Phase Shift Analysis of ${ }^{12} \mathrm{C}$ Ion Elastic Scattering Measured at Very High Energy

UR-999;

2 Froduction of Same-Sign Dimuons at the Tevatron

FERMILAB-CONF-87-150-E:

Measturement of Same-Sign Dimuon Production in High Energy Neutrino Interactions

CLNS-85-705:

Baryon Production at the CLEO Detector

KEK-89-21:

Recent Results from TRISTAN

Phys. Rev. C31:309.1985:

Netitron Proton Radiative Capture Cross Section at $T(n)=185 \mathrm{MeV}$

Nucl. Phys. B252:27i,1985;

Search for Nucleon Decay with Iron Calorimeters

Phys. Rev. D38:754,1988:

Mensurement of Pion-Proton Bremsstrahlung for Pions at $299 \mathrm{MeV}$

BONN-1R-88-60;

Polarized Target Physics at the Bonn Electron Accelerators

Phys. Rev. D3.1:1265.1986: LBL-20047:

Measurement of the Nucleon Structure Function in lron Using 2.5 and $93 \mathrm{GeV}$ Muons

Phys. Rev. Lett. 54:1233.1985: CEA-CONF-7571:

Transverse Response Furictions in Deep Inelastic Electron Scat cering for ${ }^{40} \mathrm{Ca},{ }^{48} \mathrm{Ca}$, and ${ }^{58} \mathrm{Fe}$

Phys. Rev. C31:2168,1985:

Production Mechanism of Backward Energetic Protons Studied from Two Particle Correlations in 800 MeV p nucleus Collisions

Z. Phys. C38:135.1988:

Preliminary Spectrometer Zesults from EB02

Nucl. Phys. A441:617.1985;

Measurement and Hybrid Model Analysis of Integral Excitation Functions for Proton Induced Reactions on Vanadium, Manganese and Cobalt up to $200 \mathrm{MeV}$

Phys. Let ${ }^{+}$zar B:222.1988;

Jet Production from Nuclei at $400 \mathrm{GeV} / \mathrm{c}$

Pisma Zh. Eksp. Teor. Fiz. 47:124,1988;

Cross Section of the Reactior $\vec{\nu}_{e} p \rightarrow n e^{+}$and Fundamental Characteristics of the Weak Interaction

ALMA-85-05;

Observed Effects of Magnetic Charge in Experiments with Ferromagnetic Aerosols

ITEP-87-114:

Search for the $p \pi^{+} \pi^{+}$Exotic Baryon State in $\pi^{+} p \rightarrow p \pi^{+} \pi^{+} \pi^{-}$Reaction

Phys. Rev. D34:42,1986:

Inclusive Strange Particle Production in Single-Vee Events in $200 \mathrm{GeV} / \mathrm{c} \pi^{-} n$ Interactions

Phy's. Rev. Lett. 58:2408,1987:

Subthreshold Pion Production with Associated Multiplicity Selection in the Reaction ${ }^{190}$ La ${ }^{139} \mathrm{La} \rightarrow \pi^{ \pm}$ $\mathbf{X}$

JINR-E1-87-362

Losses and Dissipation of Pion Energy in $\pi^{-}$Xenon Collisions at $3.5 \mathrm{GeV} / \mathrm{c}$

JINR-P1-87-179:

Multiplicity Characteristics of Particles Emitted in $\pi^{ \pm}$Xe Collisions at $(2.34 \mathrm{GeV} / \mathrm{c}-\mathrm{g} \mathrm{GeV} / \mathrm{c})$

PU-89-643:

Recent Results from CLEO on $B$ Physics at the $\mathbf{Y}(4 S)$

Phys. Rev. Lett. 54:624,1985:

Upper Bound on the Tau Neutrino Mass from the Previously Unobserved Decay Mode $\tau^{ \pm} \rightarrow K K \pi \nu_{r}$

Phys. Rev. D36:707,1987:

Search for a Bhabha Scattering Resonance near $1.8 \mathrm{MeV} / \mathrm{c}^{2}$

Phys. Rev. Lett. 54:1472,1985:

Search for Fractional Charges in Niobium and Tungsten

Phys. Rev. Let1. 54:1237,1985:

Observation of $\Lambda$ Hypernuclei in the Reaction ${ }^{12} \mathrm{C}\left(\pi^{+}, K^{+}\right)(\Lambda)^{12} \mathrm{C}$

Phys. Rev. D36:37.1987:

Search for Fractionally Charged Particles

Phys. Rev. 1)32:541,1985;

Search for Heavy Longlived Particles in High Energy Cosnic Rays

Nucl. Phys. B'294:979,1987;

Measurement of Polarization Parameters for the $\pi^{-} p \rightarrow \pi^{0} n$ Charge Exchange Scattering from 1965 $\mathrm{MeV} / \mathrm{c}$ to $4220 \mathrm{MeV} / \mathrm{c}$

Phys. Rev. Lett. 62:1091,1989; UT-IC'EPP-88.05:

Search for Resonances in the $e^{+} e^{-} \rightarrow \gamma \gamma$ Process in the Mass Region around $1.082 \mathrm{MeV} / \mathrm{c}^{2}$

SLAC-PUB-465]; [WSEA-88-01:

$J / \psi(1 S)$ Results from MARK-III

SLAC-PUB-5114:

Recent $\psi(2 S)$ Results from MARK-III

Phys. Rev. C32:995.1985:

Isospin Effect in $\pi^{ \pm 14} \mathrm{C}$ Elastic Scattering at $50 \mathrm{MeV}$ 
Mishra 87

Mishra 89

Mishra 89B

Mishra 90

Mitropolskii 87

Miyamoto 87

Mjyano 88

Mokhtari 85

Mokhtari 86

Mokhtari 86B

Mooney 89

Moore 90

Morales 88

Mordechai 85

Mori 88

Mori 89

Mori 89B

Morsch 85

Mostovoj $8 \bar{t}$

Mukherjee 86

Mukhopadhyay 86

Muller 89

Muraki 84

Murtagh 85B

Murtagh 89

Musset 86

Mutchler 88

Myung 88

Myung 89

Nachimann 8.5

Nagae 87

Nagy 89

Nakai 89

Nakamura 85

Nitamura $85 B$

Nakamura 87

Nakamura 88

Nakamura 89
Phys. Rev. Lett. 59:1397,1987;

Search for Neutral Heavy Leptons from $\nu$ nucleus Scattering

Z. Phys. C44:187,1989;

A Study of Wrong-Sign Muon Production in $v_{\mu}$ Nucleon Interaction

Phys. Rev. Lett. 63:132,1989;

Measurement of Inverse Muon Decay $\nu_{\mu} e^{-} \rightarrow \mu^{-} \nu_{e}$ at Fermilab Tevatron Energies 15 - 600 GeV

FERMIILAB-CONF-90-100-E;

Dilepton and Dihadron Production in Proton-Nucleus Collisions at $800 \mathrm{GeV}$

LEN1-87-1250;

Monopole Excitation of Nuclei in the Bound Muon Decay

KEK-87-118;

New Results from TOPAZ at TRISTAN

Z. Phys. C38:2788,1988; KEK-87-160;

Neutral Strange Particle Productions and Inelastic Cross Section in $\bar{p}$ Ta Reaction at $4 \mathrm{GeV} / \mathrm{c}$

Phys. Rev. Lett. 55:359.1985;

Analyring Powers in $\pi^{+} p$ Elastic Scattering at Intermediate Energies

Phys. Rev. D35:810,1986;

Analyzing Powers and Transversity Cross Sections for $\pi^{+} p$ and $\pi^{-} p$ Elastic Scattering from 471 to 687 $\mathrm{MeV} / \mathrm{c}$

Phys. Rev. D33:296,1986:

Analyzing Powers for $\pi^{-} p$ Elestic Scattering in the Energy Region of the Roper Resonance

Phys. Rev. D39:2494.1989:

Inclusive Charged- $D^{*}(2010)$ Production in $205 \mathrm{GeV} / \mathrm{c} \pi^{-}$Be Interactions

Phys. Lett. 244B:347,1990;

Longitudinal Energy Flow in Hard Proton-Nucleus Collisions

Nuovo Cim. 100A:525.1988;

Result of a Search on the Neutrinoless Double $\theta$ Decay of ${ }^{76}$ Ge to the Excited States of ${ }^{7 a}$ Se

Phys. Rev. C32:999,1985;

Systematics of Continuum Pion Double Charge Exchange on $T=0$ Nuclei

KEK-38-43; AMY-88-06;

Measurement: of $\boldsymbol{R}$ and a Search for Heavy Quark Production in $e^{+} e^{-}$Annihilation for $\sqrt{5}$ from 50 to 56 GeV

Phys. Lett. 218B:499,1989: AMY-88-16: KEK-88-109:

Measurement of the $e^{+} e^{-}$Total Hadronic Cross Section and Determination of $m\left(Z^{0}\right)$ and $\Lambda_{M t S}$

UR-1104; ER-13065-571;

Total Hadronic Cross Section in Electron - Positron Annihilation at Center-of-Mass Energies from 50 GeV to $57 \mathrm{GeV}$

Phys. Rev. C31:1715,1985:

Analysis of Giant Resonances in Proton, ${ }^{3} \mathrm{He}$, and Alpha Scattering and the Spin Flip Strength in ${ }^{208} \mathrm{pb}$

Phys, Let. 188B:181,1987;

The Measurement of the Asymmetry of Tensor-polarized Deuteron Electrodisintegration at $180 \mathrm{MeV}$ Electron Energy

Phys. Rev. Lett. 60:991,1988; FERMILAB-CONF-86-102-E; FERMILAB-PUB-87-200-E:

Azimuthal Energy Flow in Deep Inelastic Neutrino Scattering

Phys. Rev. Lett. 56:206,1986; SIN-PR-86-01:

Are there "Visible" Axions?

Phys. Rev. Lett. 63:2621,1989;

Determination of the Gravitational Constant by an Experiment at a Pumped-Storage Reservoir

Nucl. Instr. and Meth. A236:47.1985; ICR-117-84-6:

Fadial and Longitudinal Behavior of Nuclear Electromagnetic Cascade Showers Induced by $300 \mathrm{GeV}$ Protons in Lead and Iron Absorbers

BNL-37294; CONF-850869-13:

Same Sign Dilepton Production by Neutrinos

BNL-39661;

Review of the Brookhaven Neutrino Workshop

Nuovo Cim. 9C:559.1986;

Magnetic Monopoles

Phys. Rev. D38:742,1988;

Measurement of Imaginary Part of the $I=1 \bar{n} n S$-Wave Scattering Length

KEK-88-46; AMYY-88-09;

A Search for MARK-J Events at TRISTAN

KEK-89-62; AMY-89-15;

A Study of Multihadron Events with Isolated Leptons Produced in $e^{+} e^{-}$Annihilation at $\sqrt{s}=50$ to 61.4 GeV

Nucl. Phys. B254:19,1985;

Deep Inelast ic Scattering and Asymptotic Freedom

Phys. Let t. 191B:31,1987: KEK-86-74;

Quasifree Production of $\Delta\left(1232 P_{33}\right)$ Isobars in Proton-Nucleus Reactions at $3.88 \mathrm{GeV} / \mathrm{c}$

Acta Phys. Hung. 64:177,1989:

Recent Results of the European Muon Collaboration

KEK-88-21;

Quasifree Hadron Productions in $3.9 \mathrm{GeV} / \mathrm{c}$ Nuclear Reactions

Phys. Lett. 1618:417,1985;

Search for Cosmic Nuclearites at Sea Level

Phys. Rev. C31:1853,1985;

Reaction $\bar{p}{ }^{12} \mathrm{C} \rightarrow \bar{n} \mathbf{X}$ at $590 \mathrm{MeV} / \mathrm{c}$

Phys. Lett. 183B:395,1987;

Search for Supermassive Relics

ICR-182-88-28:

Results in Nonaccelerator Particle Physics

Plyys. Rev. D39:1261.1989:

Search for Long Lived Exotic Particles in $12 \mathrm{GeV}$ Proton-Nucleus Interactions 
Napolitano 88

Naroska 85

Naroska 87

Nash 89

Nash 90

Nath 89

Naudet 86

Nauder 88

Naudet 88B

Naudet $88 \mathrm{C}$

Naumenko 89

Navia 88

Nelson 87

Nernst 85

Ni 87

Niebuhr 89

Nieminen 85

Nieto 89

Nikolsky 85

Norman 87

Norman $87 \mathrm{~B}$

Norman 89

Novikov 90

Nozaki 89

Numao 86

Numao 86B

Numao 89

Oberauer 87

Odaka 89

Odell 86

Odian 85

Odyniec 89

Ogawa 89

Ohashi 86

Ohi 85

Ohkubo 85

Ohl 90

Ohmori 88

Ohmori 89

Othshima 86
Phys. Rev. Lett. 61:2530,1988;

Measurement of the Differential Cross Section for the Reaction $r$ deuteron $\rightarrow n$ at High Energies and $\theta(\mathrm{c.m})=.\theta 0$ Degrees

DESY-85-090; C85/07/03; C85/06/10.1;

Electroweak Interference Effects in $e^{+} e^{-}$Interactions and Selected Results on $\tau^{ \pm}$Decays

Phys. Rept. 148:67,1987; DESY-86-113; $e^{+} e^{-}$Physics with the JADE Detector at PETRA

SLAC-PUB-5141:

A Mensurement of the $Z^{0}$ Boson Resonance Parameters at the SLC

SLAC-356;

A Measurement of the Resonance Parameters of the Neutral Intermediate Vector Boson

Phys. Rev. D39:3520,1989, ANL-HEP-PR-89-20; Spin Correlation Parameter Ann for $n \mathrm{p}$ Elastic Scattering at $780 \mathrm{MeV}$

Phys. Rev. Lett. 56:808,1986;

Evidence for Higher Twist Effects in Hard $\pi$ p Collisions at $200 \mathrm{GeV}$ 'c

LBL-25353;

Threshold Behavior of Direct Electron Production in Proton Beryllium Collisions

Plyys. Rev. Lett. 62:2652,1989; LBL-26461:

Threshold Behavior of Electron Pair Production in $p$-Be Collisions

LBL-25660;

Electron Pair Production in p Be and $\mathrm{Ca} \mathrm{Ca}$ Collisions at the BEVALAC

Pisma Zh. Eksp. Teor. Fiz. 50:226,1989

Direct Measurement of $\pi^{\circ}$ Meson Photoproduction on the Light Nuclei

Nuovo Cim. 99A:619,1988;

Hadron Produced in Atmospheric Interactions

Nucl. Phys. B294:1022,1987; FERM1lLAB-PUB-87-73-A: ANL-HEP-PR-87-91: Invariant Cross Section at $\sqrt{s}=28$ GeV for Coplanar High $p_{T}$ Clusters Selected by a Hardware Trigger

Phys. Rev. Lett. 54:2195,1985; Observation of Three $P$ States in the Radiative Decay of $\mathbf{r}(2 S)$

Phys. Rev. Lett. 59:2716,1987; Search for Spontaneous Conversion of Muonium to Antirnuonium

Phys. Rev. D40:2796,1989; PSI-PR-89-04:

Search for the Decay $\pi^{0} \rightarrow e^{+} e^{-}$

Jour. of Phys. G 11:421,1985;

Composition and Spectra of Cosmic Ray Hadrons at Sea Level

LA-UR-89-239;

Analysis of the Greenlend Ice Sheet Experiment in Terms of New Gravitational Forces

Izv. Akad. Nauk SSSR, Fiz. 49:1260.1985;

Hadrons at E>5 TeV and Photon-Electronic Shower at the Atmospheric Depth

Phys. Lett. 195B:126.1987; LBL-21834:

New Limits on the Double Decay Half-Lives of ${ }^{94} \mathrm{Zr},{ }^{96} \mathrm{Zr},{ }^{116} \mathrm{Cd},{ }^{124} \mathrm{Sn}$

Phys. Rev. Lett. 58:1403,1987; Phys. Rev. Lett. 58:2609, 1987: LBL-22165:

Searches for Supermassive X-Particles in Iron

Phys. Rev. D39:2499,1989; LB1,-22884:

Search for Supermassive Chan-Glashow Particles in Lead

Pisma Zh. Eksp. Teor. Fiz. $51: 3,1990$

Test of the Pauli Principle

KEK-89-19;

Tests of the Standard Model of Electroweak Interactions at the $e^{+} e^{-}$Collider TRISTAN

TRI-PP-86-34;

Rare Decays

Phys. Rev. D34:2900,1986: TR1-PP-86-38;

Bounds for Masgive Neutrinos in the Region $1 \mathrm{GeV}$ to $50 \mathrm{GeV}$ from the $\mu^{-} e^{-}$Conversion Experiment TRI-PP-89-52

Lepton Flavor Conserving Rare Pion and Kaon Decayo

Phys. Lett. 198B:113,1987;

Experimental Limits on the Decay of Reactor Neutrinos

KEK-89-49;

New Limits on Neutral Scalar Bosons

Phys. Rev. D36:1,1987; SLAC-PUB-3923;

Forward Charge Asymmetry in $20 \mathrm{GeV} \gamma p$ Reactions

SLAC-PUB-3589; C84/10/31:

Selected Topics from $J / \psi(1 S)$ Decays

LBL-27926;

Uttrarelativistic Nucleus-Nucleus Collisions at CERN: The NA35 Experiment

KEK-89-51:

Results from VENUS

Phys. Rev. C34:764.1986;

Behavior of Projectile Fragments of $1.88 \mathrm{GeV} /$ Nucleon ${ }^{58} \mathrm{Fe}$

Pliys. Lett. 160B:322.1985; KEK-85-17:

Search for Heavy Neutrinos in the $\beta$ Decay of ${ }^{36} \mathrm{~S}$ an Evidence Against the $17 \mathrm{KeV}$ Heavy Neutrino

Phys. Rev. C31:510,1985;

$(\pi, \pi n)$ Reactions in ${ }^{48} \mathrm{Ca}$

YAUG-A-90-2;

Improved Experimental Limit on $K_{L} \rightarrow \pi^{0} e^{+} e^{-}$

Phys. Lett. 230B:27,1989; KEK-88-117: DPNU-89-1:

Measurement of Analyzing Power in Forward Angle for Elastic p deuteron Scattering at $3.5 \mathrm{GeV}$ KEK-89-104: DPNL-89-43:

Analyzing Powers of Quasi-Elastic $C(p, 2 p)$ and $C u(p, 2 p)$ Scattering at $3.5 \mathrm{GeV}$ and Relativistic Effects INS-598;

Review on Neutrino Mass Measurement 
Okhrimenko 87

Okonov 88

Okusawa 88

Olsen 88

Omori 89

Onel 89

Ong 87

Ong 88

Ong 88B

Ong 83

Orito 89

Osterheld 86

Otterlund 88

Otterlund 88B

Ottermann 85

Ottermann 85B

Ouldsaada 88

Ouldsaada 88B

Ouldsaada 89

Oyama 86

Oyama 87

Oyama 87B

Oyama 88

Oyama 88B

Pa] 86

Palestini 85

Palka 87

Palka 87B

Pallin 87

Panagiotou 89

Paoletti 89

Papadimitrio 89

Pare 90

Park 85

Park 85B

Park 88

Park 89

Park 89B
Kr. Soob. J1NR 22:12,1987;

Relative Four-Velocity Distributions of $\pi^{\circ}$ und $\eta$ Mesons Produced in the Reaction $\pi^{-}$Xe at $3.5 \mathrm{GeV} / \mathrm{c}$ JINR-P1-88-54G;

The Pion Production Multiplicity as a Meanure of Excitation Degrees in Nucleus-Nucleus Collisions at 3.66 GeV/A

KEK-87-146;

Study of Baryon Production and Its Features in $405 \mathrm{GeV} / \mathrm{c}$ Proton-Proton Interactions

ROCH-UR-1089-88;

Test of the Standard Model in High Energy $e^{+} e^{-}$Reactions at TRISTAN

KEK-89-1;

A Search for a Baryonium in $\bar{p} p$ Annihilation at Rest

Phys. Rev. D40:35,1989;

Elastic p (polarized) p (polarized) Scattering between 240 and $470 \mathrm{MeV}$

SLAC-320;

Measurement of the $B$ Hadron Lifetime

Phys. Rev. Lett. 60:2587,1988; SLAC-PUB-4550; LBL-24908: CALT-68-148i;

Inclusive Lepton Production in $e^{+} e^{-}$Annihilation at $29 \mathrm{GeV}$

SL.AC.PUB-4511;

Measurement of the $B$ Hadron Lifetime from MARK-II at PEP

Phys. Rev. Lett. 62:1236.1989; SLAC-PUB-4559; LBL-24937;

A Refined Measurement of the $B$ Hadron Lifetime

Phys. Rev. Lett. 63:597.1989; UT-1CEPP-89-01;

New Limits on Exotic Two-Body Decay of Orthopositronium

SLAC-PUB-4160;

Measurements of the Total Hadronic and Inclusive $D^{*}(2010)$ Cross Sections in $e^{+} e^{-}$Annihilations between 3.87 and $4.5 \mathrm{GeV}$

2. Phys. C38:65,1988;

Review of High Energy Heavy Ion Reactions in Emulsion

LUIP-8806;

Multiplicities and Rapidity Densities in Nucleus-Nucleus Collisions at Ultracelativistic Energies

Nuct. Phys. A436:688, 1985:

Elastic Electron Scattering from ${ }^{3} \mathrm{He}$ and ${ }^{4} \mathrm{He}$

Phys. Rev. C32:928,1985;

Large Angle $\pi$ deuteron Scattering in the Region of the $(3,3)$ Resonance

Z. Phys. C39:1,1988; DESY-88-015:

Comparison of the Particle Flow in $q \bar{q} \gamma$ and $q \bar{q}$ gluon Events from $e^{+} e^{-}$Annihilation at PETRA

DESY-88-177;

Recent Results from PETRA

Z. Phys. C44:567,1989; DESY-89-063;

A Measurement of the Charmed Quark Asymmetry in $e^{+} e^{-}$Annihilation

Phys. Rev. Lett. 56:991,1986;

Search for High Energy Muons from Cygnus X-3

Phys. Rev. D36:3537,1987;

Search for High Energy Muons from Cygnus X-3 During the Radio Outburst in 1983 and 1985

Phys. Rev. Lett. 59:2604,1987;

Search for High-Energy Neutrinos from SN1987A: First Six Months

Phys. Rev. D39:1481,1989; KEK-88-51;

Experimental Study of Upward-Going Muons in KAMIOKANDE

KEK-88-16;

Search for High-Energy Neutrinos from SN1987A

Phys. Rev. D33:2708,1986;

Prompt Electron Production from the DELCO Detector at the SLAC Storage Ring PEP

Phys. Rev. Let L. 55:2649,1985;

Pion Structure as Observed in the Reaction $\pi^{-}$nucleon $\rightarrow \mu^{+} \mu^{-} \mathbf{X}$ at $80 \mathrm{GeV} / c$

Phys. Lett. 189B:238,1987; CERN-EP-87-10;

Experimental Limit on the Decay $D^{0} \rightarrow e^{+} \mu^{-}$and $D^{0} \rightarrow e^{-} \mu^{+}$

2. Phys. C35:151.1987; Z. Phys. C35:151,1987; MPI-PAE-EXP-EL-172;

Semileptonic Decays of Charmed $D^{ \pm}$Meson: Measurement of the Lifetime and the Cross Section

Nucl. Phys. B292:653,1987; LAL-8T-03;

Baryon Pair Production in $J / \psi(1 S)$ Decays

Int. Jour. Mod. Phys. A5:1 197,1990; CERN-EP-89-131:

$\Lambda$ Polarization in Hadron-Nucleon, Hadron-Nucleus and Nucleus-Nucleus Interactions

FERMILAB-CONF-89-264-E:

Small Angle Physics at CDF: A Progress Report

Phys. Rev. Lett. 63:28,1989: EFI-89-24:

A Search for $K_{L} \rightarrow \pi^{\circ} \gamma_{\gamma}$

Phys. Lett. 242B:512,1990; CERN-EP-89-181:

Inciusive Production of $\pi^{0}$ 's and Feynman Scaling Test in the Fragmentation Region at the SppS Collider

Plys. Rev. Lett. 54:22.1985:

Experimental Limits on the Nucleon Lifetime for Two- and Three-Body Decay Modes

Nucl. Phys. B252:261.1985:

Experimental Limits on Monopole Catalysis, $n \bar{n}$ Oscillations, and Nucleon Lifetime

KEK-88-45: AMYY-88-08;

The Physics of Jets Produced in Electron-Positron Collisions at Center of Mass Energies from 50 to 56 $\mathrm{GeV}$

Phys. Rev. Letz. 62:1713.1989: KEK-88-113: AMY-88-17:

Experimental Evidence for the Non-Abelian Nature of QCD from a Study of Multijet Eventa Produced in $e^{+} e^{-}$Annihilation

KEK-89-53: ANY-89-12:

Determination of the QCD Renormalization Scalr and $\Lambda_{M S}$ from Multi-Jet Events Produced in ElectronPositron Collisions 
Parker 89

Parkin 86

Patalakha 85

Patterson 90

Paub 85

Paul 89

Pauletta 87

Pavlyak 86

Pavlyak 86B

Pelzer 89

Peng 87

Peng 89

Perdereau 89

Perdereau 89B

Perdrisat 84

Perdrisat 87

Perelygin 85

Perepelitsa 87

Perepelitsa 88

Perl 85

Perl 86

Perrot 86

Perrot 87

Perrot 88

Petersen 85

Petersen 86

Petersen 86C

Petersen 88

Petradza 89

Petradza 90

Peyaud 89

Phan 85

Phillips 89

Picciotto 88

Piccolo 89

Pigot 85

Piilonen 86

Pillai 88
Phys. Rev. Lett. 63:1570.1989:

Search for a $T=2$ Dibaryon

Nucl. Phys. B227:634,1986;

Antiproton-Deuteron Annihilution into Neutral Strange Particles at Incident Momenta below 1 GeV/c

Yad. Phys. 42:1403,1985; Jov. J. Nucl. Pliys. 42:887.1985; 1FVE-85-20;

On Study of Pion Multiple Production in Nondifractive Exclusive Reactions in $K^{-} p$ Interactions at 32.1 $\mathrm{GeV} / \mathrm{c}$

Phys. Rev. Lett. 64:1491.1990;

Determination of $\operatorname{Re}\left(\epsilon / e^{\prime}\right)$ by the Simultaneous Detection of the Four $K_{L}, K_{S} \rightarrow 2 \pi$ Decay Modes

Z. Phys. C27:211,1985; CERN-EP-84-98;

Forward Particle Production in $\pi^{-} p$ and $K^{-} p$ Collisions at $58 \mathrm{GeV}$ and Comparison with Quark Models

Z. Phys. C45:25,1989; BONN-HE-89-07: Measurement of the Neutron Lifetime in a Magnetic Storage Ring

Phys. Lett. 211B:19,1988: KEK-87-138;

All at Small Momentum Transfers for the First Complete Determination of the Forward $p$ s Scatiering Amplit ude

JINR-P1-86-238;

Secondary Particle Correlations in $\pi^{-}$Xe Interaction: Secondary Particle Multiplicitieg

JINR-P1-86-239;

Secondary Particle Correlations in $\pi^{-}$Xe Interaction: Multiplicity Distributions

BONN-IR-89-05;

Production of Rare Particles in Proton - Antiproton Interactions at Center-of-Mass Energies from 200 GeV to $900 \mathrm{GeV}$

Phys. Rev. Lett. 58:2027,1987:

Observation of $\eta$ Meson Production in the Reaction $\pi^{-{ }^{3}} \mathbf{H e} \rightarrow \eta{ }^{3} \mathbf{H}$

Phys. Rev. Lett. 63:2353.1989;

Coherent $\eta$ Meson Production in the Reaction $\pi^{-{ }^{9}} \mathrm{He} \rightarrow \eta^{3} \mathrm{H}$

LAL-89-06;

New Results from Atmospheric Neutrino Studies in the FREJUS Experiment

LAL-89-11;

Recherche D'Oscillations des Neutrinos Atmospheriques dans L'Experience du FREJUS

Phys. Lett. 156B:38,1985; TRJ-PP-84-114:

Large Angle deuteron $(p, 2 p)$ at $507 \mathrm{MeV}$

Phys. Rev. Lett. 59:2840,1987;

Cross Section and $T_{20}$ in 0 Degrees Deuteron Breakup at $2.1 \mathrm{GeV} / \mathrm{c}$

Kr. Soob. JINR 7:5,1985;

Search for the Tracks of Heavy and Superheavy Cosmic Ray Nuclei in Olivine from Meteorites

ITEP-87-161;

Experiment on Search for Tachyons Production in $\pi^{-} p$ Interactions at $4.6 \mathrm{GeV} / \mathrm{c}$

Phys. Lett. 203B:335,1988;

Events with Apparent Electrical Charge Nonconservation

Phys. Rev. D32:2859,1985;

Searches for Unstable Neutral Leptons in Low Multiplicity Events from Electron-Positron Annihilation

Phys. Rev. D34:3321,1986: SLAC-PUB-3847; LBL-20697;

Study of Noncolinear Two Charged Particle Events Produced in 29 GeV Electron Positron Annihilation

Nucl. Phys. B278:881,1986: SACLAY-DPHPE-86-09;

Measurement of the Total Cross Section Difference in the Energy Range from 0.43 to $2.4 \mathrm{GeV}$

Nucl. Phys. B294: 1001,1987;

Measurement of the $p$ Analysing Power $A_{00 \pi 0}$ in a Large Angular Region between $0.88 \mathrm{GeV}$ and 2.7 GeV

Nucl. Phys. B296:527,1988;

Meagurement of the Spin Correlation Parameter $A_{00 s k}$ in p p Elastic Scattering from 0.88 to $2.7 \mathrm{GeV}$

Phys. Rev. Lett. 55:1954,1985;

Inclusive Charged Particle Distribution in Nearly Threefold-Symmetric Three-Jet Events at $E_{\mathrm{cm}} 28 \mathrm{GeV}$

Phys. Rev. Lett. 57:949,1986;

Measurement of the $\mathbf{\Sigma}^{\circ}$ A Transition Magne.ic Moment

SLAC-PUB-3933;

Inclusive Charged Particle Distribution in Nearly 3 Fold Symmetric 3 Jet Events at $E_{\mathrm{em}}=20 \mathrm{GeV}$

Phys. Rev. D37:1,1988; SLAC-PUB-4290; LBL-23243;

Multihadronic Evens at $E_{\mathrm{cm}}=29 \mathrm{GeV}$ and Prediction of $\mathrm{QCD}$ Models from $E_{\mathrm{cm}}=29 \mathrm{GeV}$ to $E_{\mathrm{cm}}=93 \mathrm{GeV}$

SLAC-347;

Study of Four-Lepton Final States in Electron-Positron Interactions at $29 \mathrm{GeV}$

LBL-27192;

Study of Four-Lepton Final States in $e^{+} e^{-}$Interactions at $E_{\mathrm{cm}}=29 \mathrm{GeV}$

DPHPE-89-22;

Recent Results on $C P$ and $C P T$ Studies from $K_{L}, K_{S} \rightarrow 2 \pi$ Decays

Phys. Rev. C32:609.1985;

Nuclear Polarization in Muonic ${ }^{80} \mathbf{Z r}$

Phys. Lett. 224B:348,1989;

A Searrh for Nucleon Decay with Multiple Muon Decays

Phys. Rev. D37:1131,1988; TRI-PP-87-58:

Search for Majoron Production and Other Process Associated with $\pi^{+} \rightarrow e^{+} \nu$ Decay

LNF-89-019-P;

$B$ Meson Lifetime Measurement

Nucl. Phys. B249:172.1985;

Study of the Production of Strange $S=-1$ Dibaryon in the Interactions of $K^{-}$and $\pi^{+}$on Deuterium below $1.5 \mathrm{GeV} / \mathrm{c}$

Phys. Rev. Lett. 57:1402,1986;

Unique Determination of the Formfactor Ratio in Radiative Pion Decay

Phys. Lett. 207B:389,1988:

A Study of Charge Symmetry Violation in $\pi^{+}$and $\pi^{-}$Elastic Scattering from ${ }^{3} \mathrm{H}$ and ${ }^{3} \mathrm{He}$ 
Piragino 86B

Pitman 89

Pitzl 89

Platchkov 89

Plothowbesch 88

Pluta 88

Pluta 88B

Pniewski 85

Ponting 88

Porter 89

Poth 85

Price 85

Price 86

Price 87

Price 88

Prokoshkin 85

Prokoshkin 87

Prokoshkin 87B

Prokoshkin 87C

Pugh 88

Pugh 89

Punjabi 88

Purohit 88

Raab 87

Rabin $\mathbf{8 5}$

Rabin 86

Rabin $\mathbf{8 8}$

Raffelt 90

Rahbar 87

Ramello 88

Ramm 85

Ransome 90

Rapaport 85

Rath 89

Raymond 85

Redwine 86

Rees 86

CERN-EP-86-75;

Antjproton-Nucleus Interaction: Review of the Experimental Situation

SLAC-PUB-4826; C88/0T/27;

Evidence for $D_{S}^{+} \rightarrow e^{+} \mathrm{X}$

Z. Phys. C46:1,1990; DESY-89-129;

A Study of Photon Production in Hadronic Events from $e^{+} e^{-}$Annihilation

SACLAY-DPHN-2572B

Deuteron $A\left(Q^{2}\right)$ Structure Function and the Neutron Electric Formfactor

IHEP-HD-88-5;

Recent Results and Future Prospects of the UA2 Experiment at the CERN $\bar{p} p$ Collider

JINR-E1-88-450;

Nuclear Matter Excitation in Pion-Xenon Collisions at $3.5 \mathrm{GeV} / c$ Inclusive Spectra of Neutral Pions

JINR-E1-88-754;

Space-Time Characteristics of Secondary Proton Sources in Relativistic Nuclear Collisions from TwoParticle Correlations

Nucl. Phys. A443:685,1985;

Final State Interactions in the Decay of the Hypernucleus ${ }^{\circ} \mathrm{Li} s$ and a Reappraisal of the Binding Energies of $A=\theta$ Hypernuclei

Phys. Rev, Lett. 63:1792,1990; TAI-PP-88-89,

Analyzing Power Measurement in $p$ (polarized) $n \rightarrow \pi^{-} p p$ (singlet $S$ wave): Pion Absorption by Quark Clusters?

SLAC-PUB-5148

Measurement of $B^{0}-\bar{B}^{0}$ Mixing using the MARK-II at PEP

CERN-EP-85-75;

Antiprotonic Atoms at LEAR: Achievements and Perspectives

ANL-HEP.CP.85-117;

Underground Muons from Cygnus X-3

Phys. Rev, Lett. 56:1226,1986

Search for Supermassive Magnetic Monopoles Using MICA Crystals

Phys. Rev, Lett. 59:2523,1987;

Search for Highly lonizing Particles at the Fermilab Proton-Antiproton Collider

Phys, Rev, Lett. 61:2193,1988;

Electromagnetic Spallation of $6.4 \mathrm{TeV}^{32} \mathrm{~S}$ Nuclel

Fiz. Elem. Chastits At. Yadra 16:584,1985;

Exclusive Production and Decay of Glueballs

IFVE-87-99,

Study of Mesons with Enhanced Gluon Component (Gl-aballs Included) and Mesons with High Spins with the Help of Multiphaton $4 \pi$ Spectrometer

IFVE-87-86;

Meson Spectroscopy at Serpukhov Accelerator

Fiz. Elem. Chastits At. Yadra 18:503,1987;

Experimental Studies at the IHEP $70 \mathrm{GeV}$ Accelerator (1983 - 1983, Electronics Setups)

Acta Phys. Polon. B19:307,1988;

Search for the Quark-Gluon Plasma - the NA35 Experiment at the CERN SPS

LBL-27833;

Search for the Quark-Gluon Plasma (1889): The NA35 Experiment at the CERN SPS

TRI-PP-88-85;

The deuteron $(p, 2 p) n$ Reaction at $608 \mathrm{Mev}$. Part I.

FERMILAB-CONF-88-200-E;

Charm Physics

Phys. Rev. D37:2391,1988; FERMILAB-PUB-87-144-E; Measurement of the $D^{0}, D^{+}$, and $D_{S}^{+}$Lifetime

ITEP-85-1 17;

Splitting of Photoemulsion Nuclei at Inelastic Scattering of $32 \mathrm{GeV}$ Muons at Small Angle. Emission of Fast Protons

ITEP-86-114;

Splitting of Photoemulsion Nuclei at Inelastic Scattering of $32 \mathrm{GeV}$ Muons on the Small Angles. Collective Effect:

ITEP-88-34;

The Photoemulsion Nucleus Splitting in Inelastic Muon Scattering at Small Angle with $32 \mathrm{GeV} / \mathrm{c} \mathrm{Mo-}$ mentum. Slow Particles Productions

MPI-PAE-PTH8-90;

What Have We Learned irom SN 1987 A

Phys. Lett. 194B:338,1987;

Some Proton Spin Observables Obtained in p deuteron Elastic Scattering at 500 and $800 \mathrm{MeV}$

Z. Phys. C38:73,1988;

First Result from Hybrid Emulsion Experiment on ${ }^{10} \mathrm{O}-\mathrm{N} u c l e u s$ Colligions at $200 \mathrm{GeV} / \mathrm{N}$

Phys, Rev. D32:123,1985: Another Mode of Decay of $m\left(\mu^{ \pm} \pi^{ \pm}\right)(0.429)$

Phys. Rev. Lett. 64:372,1990; Charged-Particle Multiplicities Following Pion Absorbtion on ${ }^{\circ} \mathrm{Li}$

Phys. Rev. Lett. 54:2325,1985;

Solar Neutrino Detection: Experimental Determination of Gamow-Teller Strengths via the ${ }^{\mathrm{aB}} \mathrm{Mo}$ and 115 In $(p, r)$ Reactions

Phys. Aev. D40:693,1989; PRINT-89-0158-NOTRE-DANE;

The $K_{S} K_{S} \pi^{0}$ System Produced in $\pi^{-} p$ Interactions at $21.4 \mathrm{GeV} / \mathrm{c}$

PRINT+85-0056;

Analyzing Power Measurements for $p$ p Elastic Scattering at High $p T^{2}$

Nucl. Phys. A434:239C,1985;

$\pi$ nucleus Interactions

Phys. Rev. C34:627,1986;

Continuum Polarization Transfer in $500 \mathrm{MeV}$ Proton Scattering and Pionic Collectivity in Nuclei

See the legend on page 19. 
Reesager 90

Reeves 86

Reiner 86

Remsberg 88

Ren 88

Ren 88B

Ren $8 \mathrm{BC}$

Repellin 87

Reutens 85

Reutens 90

Reya 85B

Rich 87

Rich 87B

Richard 87

Richman $\mathbf{8 5}$

Riles 87

Riles 88

Rilea 89

Riles 89B

Riley 87

Riordan 87

Ritter 88

Roberts 86

Roche 84

Roche 87

Roche 88

Roche 88B

Roche $88 \mathrm{C}$

Roche 89

Roe 89

Roe 89B

Roehrich 85

Roepke 85

Rokni 88

Romano 89

Romanowski 85

Ronjin 86
Phys. Lett, 235B:30,1990;

First Observation of $\beta$-Delayed Deuteron Emission

Phys. Rev. D34:1960,1986; Spin Parity Analysis of $\bar{p} \rightarrow f_{1}(1420) X$

Phys. Lett. 176B:233,1986;

3earch for Long Fange Interactions at Highly Relativistic Velocities

Z. Phys. C38:35,1988; BNL-40536;

Meaurement of Enercy and Charged Particle Emission in the Central Rapidity Region from ${ }^{16} \mathrm{O}$ nucleu and $p$ nucleus Colligions at $14.5 \mathrm{GeV} / \mathrm{c}$ per Nucleon and Preliminary Results from Si nucleus Collisions

Phys. Rev. D38:1404,1988; ICR-153-87-7;

Hadronic Interactions and Primary Cosmic Ray Composition at Energies around $10^{16} \mathrm{eV}$ to $10^{16} \mathrm{eV}$ Derived from the Analysil of High-Energy $\gamma$ Families

Phys. Rev. D38:1417,1988;

Properties of Hadron Families Observed with Thick-Type Emulsion Chambers at Mts. Kanbala and Fuji and Search for Centauro Events

Phys. Rev. D38:1426,1988;

Primary Cosmlc Rny Protons above $10^{1 \mathrm{~B}} \mathrm{eV}$ Derived from the Observation of Superhigh Energy Halo Events

LAL-87-53;

Recent Result: from the U.A2 Experiment

Phys. Lett. 152B:404,1985;

Measurement of $\sin ^{2} \theta_{W}$ and $\rho$ in Deep Inelastic Neutrino Nucleon Scattering

Z. Phys. C45:539,1990;

A Measurement of the Neutral Current Electroweak Parameters using the Fermilab Narrow Band Neutrino Beam

Phys. Lett. 166B:223,1986; TIFR-TH-85-18; DO-TH-85-23; Constraints on luino and $\bar{q}$ Masses from the New Missing pr Data at the $p \bar{p}$ Collider

Phys. Lett. 1948:173,1987; DPHPE-87-07;

A Senrch for Strongly Interacting Dark Matter

Phys. Rept. 151:239,1987;

Experimental Particle Physics without Accelerators

LAL-87-48;

Hard Electromernetic Processes

CALT-68-1260; C85/03/10.

Results on the $\eta(1440)$ from MARK-III

Phys. Rev. D35:2914,1987; SLAC-PUB-4156; LBL-2265 I;

Limit on the Decay $D^{0} \rightarrow e^{+} \mu^{-}$and $D^{0} \rightarrow e^{-} \mu^{+}$

SLAC-PUB-4742;

Application of New Technique for Identifying Exotic Events with Low Visible Energy in $e^{+} e^{-}$Annihilation at $29 \mathrm{GeV}$

SLAC-342

A Search for a Close Mass Lepton Doublet

SLAC-PUB-5043; LBL-27555;

Search for Nearly Degenerate Lepton Doublet $\left(\ell^{-}, \ell^{0}\right)$

Phys. Lett. 1978:23,1987;

Polarization Obeervables for the Reaction $p p \rightarrow p p \pi^{\circ}$ at $800 \mathrm{MeV}$

Phys. Rev, Lett. 59:755,1987; SLAC-PUB-4280; UR-993;

Search for Short Lived Axions in an Electron Beam Dump Experiment

LBL-25574;

Transverse Energy and Multiplicity Dlatributions in Collisions at $60 \mathrm{GeV}$ per Nucleon and $200 \mathrm{GeV}$ per Nucleon

RAL-86-058:

Compilation of Data on $\gamma \gamma \rightarrow$ hadrons

Nucl. Phys. A439:721,1985; LBL-18064;

Particle- $\gamma$ Coincidence Meaurements in ${ }^{12} \mathrm{C}+{ }^{12} \mathrm{C}$ and ${ }^{12} \mathrm{C}+\mathrm{Pb}$ Collisions at $2.1 \mathrm{GeV} / \mathrm{Nucleon}$ Incident Ener By

LBL-24449;

First Results on Dilepton Production at the BEVALAC

LBL-25475; LBL-26460;

Dielectron Production in $p$ Be and Ca Ca Collisions at the BEVALAC

LBL-25325:

First Obaervation of Dielectron Production at the BEVALAC

Phys. Rev. Lett. 61:1069,1988;

First Observation of Dielectron Production in Proton-Nucleus Collisions below $10 \mathrm{GeV}$

Puys. Lett. 226B:228,1989; LBL-27050;

Dielectron Production in Ca Ca Collisions at $1 \mathrm{~A} / \mathrm{GeV}$ and $2 \mathrm{~A} / \mathrm{GeV}$

SLAC-338;

Resanance Production in Two Photon Interactions

SLAC-PUB-4931;

A Measurement of the Radiative Width of the $\eta$ and $\eta^{\prime}$ Mesons with the ASP Detector

Phys. Lett. 153B:203,1985;

Pion Photoproduction on ${ }^{14} \mathrm{Nit}$ at $173 \mathrm{MieV}$ Photon Energy

Phys. Rev. C31:1556,1985: Depletion of Light Cluster Production in $1 \mathrm{GeV}$ Proton Nucleus Collisions

Phys. Lett. 202B:35,1988;

Isobaric-Analog-State Transitions in Pion Charge-Exchange Reactions above the $\Delta\left(1232 P_{33}\right)$ Resonance CERN-EP-89-30;

Summary cf Emulsion Data on High Energy Nucleus-Nucleus Interactions

Acta Phys. Polon. B16:179,1985;

Prompt Neutrino Production in a $400 \mathrm{GeV} / \mathrm{c}$ Proton Beam Dump Experiment

Yad. Phys. 46:1693,1987; IFVE-86-157;

Study of Two Particle Inclusive $K^{+} p$ Reactions at $70 \mathrm{GeV}$ 
Rose 90

Rosen 88

Rosenberg 85B

Rosenfeld 88

Rosrer 85E

Rotscheidt 88

Roudeau 88

Rouse 87

Rowson 85

Rowson 85B

Foy 85B

Rubbia 86

Ruckstubl 85B

Ruckstuhl 86

Rublmann 88

Rutherfoord 85

Ryan 85

Rybicki 85

Rybicki 86

Sadler 87

Safronov 88

Safronov 88B

Sagawa 88

Sagawa 89

Saj 86

Saidkhanov 86

Saloaj 87

Salaj 89

Sakaj 90

Sakuda 85

Sakuda 88

Salvini 88

Sangster 87

Sapozhnikov 86

Saroff 90
Phys. Lett. 234B:460,1990;

Polarisebility of the Neutron

Comm. on Nuc, and Part. Phys, 18:31,1988; LA-UR-87-3423:

On Seeing the First Double $\beta$ Decay

SLAC-289; UMI-86-08218;

A Study of Hadronixation Uaing Energy Elow from $e^{+} e^{-}$Annihilation into Quarks and Gluons at $E_{c m}$ of $29 \mathrm{GeV}$

KEK-88-41; AMY-88-04;

Results from the First Year at AMY

EFI-85-63-CHICAGO;

Heavy Quark Spectroscopy

BONN-IR-88-55;

Investication of Inelastic Scattering in $\gamma-p$ Reactions in the Energy Range $65 \mathrm{GeV}$ to $175 \mathrm{GeV}$ Using the CERN OMEGA Spectrometer

LAL-88-37; C88/03/13;

Charm Photoproduction Mechanisms and a Few Preliminary Results from the NA14 Experiment

LBL-24729;

The Inclusive Cross Section of Charged Hadrons in 3 Jet Events at $\sqrt{6}=28 \mathrm{GeV}$

Phys. Rev, Lett. 54:2530,1985;

Charged Multiplicity of Hadronic Event Containing Heavy-Quark Jets

LBL-20463;

Propertiea of Heavy Quark Jets Produced by $e^{+} e^{-}$Annihilation at $29 \mathrm{GeV}$

Nucl. Phys. A442:686,1985;

Deformation and Target Spin Dependent Effects in ${ }^{\circ}$ Be $p$ (polarized) at $220 \mathrm{MeV}$

Usp. Fiz. Nauk 147:371,1986;

Experimental Observation of the Intermediate Vector Bosons $W^{+}, W^{-}$and $Z^{0}$

Nucl. Phys. A433:634,1985;

Measurement of the Hyperfine Splitting of the 1s State in Muonic ${ }^{7}$ Li as a Search for Axial Vector Muon Nucleon Interaction:

Phys. Rev. Lett. 56:2132,1986:

Study of Three-Prong $\tau^{ \pm}$Decays and Determination of the $a_{1}(1260)$ Parameters

SACLAY-DPHPE-88-18;

Measurement of the Strong Coupling Conatant $a_{S}$ from a Study of $W^{ \pm}$Bosons Produced in Areocintion with Jet:

PRINT-86-00E1;

Hadronic Production of Real and Virtual Photons

Phys. Rev. D32:802,1985;

Cryogenic Photon Mass Experiment

2. Pbys. C28:65,1985; CRAC-INP-85-1280;

Indication for a Broad $J^{P C}=2^{++}$Meson at $840 \mathrm{MeV}$ Produced in the Reaction $\pi^{-} p \rightarrow \pi^{+} \pi^{-} n$ at High $|t|$

Acta Phys. Polon. B17:317,1986; INP-1259-PH;

Postible Observation of the Scalar Meson at $1280 \mathrm{MeV}$ in the Reaction $\pi^{-} p \rightarrow \pi^{+} \pi^{-} n$ at $17.2 \mathrm{GeV} / \mathrm{c}$

Phys. Rev. D35:2718,1987;

Difierential Crose Sections for $\pi^{+} p$ and $\pi^{-} p$ Elastic Scattering from 378 to $687 \mathrm{MeV} / \mathrm{c}$

Yad. Phys. 47:1523,1988;

Fant Deuterons from Proton-Nucleus Collisions at $2.5-8.2 \mathrm{GeV}$

ITEP-88-144;

Production of High Energy Nuclei ${ }^{4} \mathrm{He},{ }^{3} \mathrm{He}$, deuteron, ${ }^{3} \mathrm{H}$ in Interaction $10.1 \mathrm{GeV} / \mathrm{c}$ Protons with Be, Al, Cu, Ta

Phys. Rev. Lett. 60:93,1988; KEK-87-84;

Measurements of $\boldsymbol{R}$ and a Search for Heavy-Quark Production in $e^{+} e^{-}$Annihilation at $\sqrt{8}=50$ and 52 GeV

Phys. Rev. Lett. 63:2341,1989; KEK-89-38; AMY-89-04;

Measurement of $e^{+} e^{-} \rightarrow b \bar{b}$ Forward-Backward Charge Anymmetry between $\sqrt{0}=52$ and $67 \mathrm{GeV}$

Phys. Rev. Lett. 55:2668,1985;

Interpretation of the Breakup Reaction deuteron $p \rightarrow p p n$ at the Incident Deuteron Momentum Range $2 \mathrm{GeV} / \mathrm{c}-3.7 \mathrm{GeV} / \mathrm{c}$ in Terms of Free $n \mathrm{p}$ Scattering

Yad. Phys. 44:137,1986;

Analysis of Five-Prong $p n$ Interactions Generated in Nuclear Emuision at $21-25 \mathrm{GeV} / \mathrm{c}$

KEK-87-119; AMY-87-05;

New Reault: from AMY at TRISTAN

KEK-89-42; AMY-89-06;

A Search for Heavy Leptons and New Particles Beyond the Standard Model in $e^{+} e^{-}$Annihilintion at $\sqrt{6}=60-60.8 \mathrm{GeV}$

Phys. Lett. 234B:534,1990; KEK-89-134; AMY-89-18;

$A$ Search for SUSY Particles in $e^{+} e^{-}$Annihilations at $\sqrt{a}=50-60.8 \mathrm{GeV}$

Phys. Lett. 152B:399,1985;

Propertles of Bottom Quark Jeta in $e^{+} e^{-}$Annihilation at $29 \mathrm{GeV} / \mathrm{c}$

KEK-88-88;

Teat: of the Stendard Model at TRISTAN

Phys. Rept. 171:231,1988;

Physice with Matter-Antimatter Colliders

Phvs. Lett. 188B:29,1987;

Light Fragment Production in Proton Xenon Interactions between $1 \mathrm{GeV}$ and $18 \mathrm{GeV}$

JINR-P4-86-695;

Investigationg with Antiprotons at LEAR Facility

Phys. Rev. Lett. 64:995,1990; AZPH-EX-89-01;

Single-Spin Asymmetry in Inclusive Reaction $p(p o i) p \rightarrow \pi^{+} X, p(p o l) p \rightarrow \pi^{-} X, p(p o l) p \rightarrow \pi X$, at I3.3 and $18.5 \mathrm{GeV} / \mathrm{c}$ 
Sasaki 88

Sagaki 80

Sato 87

Savage 86

Savage 86B

Savage $88 \mathrm{C}$

Savoynavarro 85

Saxon 86

Schard 85

Schablitzky 89

Schaeffer 90

Schaffner 89

Schaller 85

Schellman 85

Schiavon 89

Schindler B5

Schindler 86

Schindter 87

Schindler $\mathbf{8 8}$

Schindler 89

Schmickler 86

Schmidke 86

Schmidt 87

Schmidt 88

Schmiedmayer 88

Schmitt 88

Schmitz 88

Schneider 90

Schnetzer 89

Schubert 89

Schukraft 88

Schukraft 88B

Schumm 88

Schurman 87

Schutt 88

Schutte 89

Schwartz 88

Sculli 87
KEK+88-48; AM1Y-88-11

Hadron Production in $\gamma \gamma$ Collisions at Large $Q^{2}$ at AMY

KEK-89-56; ANY-89-14;

A Measurement of the Fhoton Siructure Function $F_{2}$ at an Average $Q^{2}$ of $67(\mathrm{GeV} / \mathrm{c})^{2}$

Phys. Rev. Lett. 58:2722,1987;

Analysis of the Neutrino Burst from Supernova 1987 in the Large Magellanic Cloud

Phys. Lett. 1671:481,1986;

A Search for Fractional Charges in Native Mercury

Phys. Rov. Lett. 57:178.1986;

A Search for a Short Lived Neutral Particle Produced in Nuclear Decay

Phys. Rev. D37:1134,1988;

New Limit on Light Scalar and Psendoscala- Particles Produced in Nuclear Decay

CERN-EP-85-64;

Experimental Evidence for the Decay $w^{ \pm}-\tau^{ \pm} v_{T}$ of the Charged Intermediate Vector Boson at the CERN $\boldsymbol{p} \overline{\boldsymbol{p}}$ Collider

RAL-86-073;

Measurement of Electroweak Effects in $e^{+} e^{-}$Annihilation

Phys. Lett. 160B:188,1985: SLAC-PUB-3696: LBL-19725:

Upper Limit on $B^{0} \bar{B}^{0}$ Mixing in $e^{+} e^{-}$Annihilation at $29 \mathrm{GeV}$

BONN-IR-89-32;

Separation der Elektrischen Formfaktoren $G_{c}$ and $G_{q}$ des Deuterons bei $Q^{2}=0.495(\mathrm{GeV} / \mathrm{c})^{2}$

Acta Phys. Polon. B21:357,1990;

SIJ1987A. A Review

Phys. Rev. D39:990,1989;

Limit on the Decays $K_{L} \rightarrow \mu^{+} e^{-}$and $K_{L} \rightarrow \mu^{-} e^{+}$

Phys. Rev, C31:1007,1985;

Nuclear Charge Radii of the Even Sulfur Isotopeg ${ }^{32} \mathrm{~S},{ }^{34} \mathrm{~S}$, and ${ }^{36} \mathrm{~S}$ and of ${ }^{31} \mathrm{Ph}$ Using Muonic Atoms

Phys. Rev. D31:3013,1985; SLAC-PUB-3521; LBL-18761:

Measurement of $K^{ \pm}$and $K^{0}$ Inclusive Rates in $e^{+} e^{-}$Annihilation at $29 \mathrm{GeV}$

Nucl. Phys. A505:595,1990; CERN-EP-89-38;

Real to Imaginary Ratio of the $\bar{p} p$ Forward Elagt ic Scattering Amplitude at $550 \mathrm{MeV} / \mathrm{c}, 757 \mathrm{MeV} / \mathrm{c}$ and $1077 \mathrm{MeV} / \mathrm{c}$

SLAC-PUB-3799; CALT-68-1307; C85/07/15;

New Results on Charmed $D$ Meson Decay

5LAC-PUB-4055;

New Results on Charmed $D, D_{S}^{ \pm}$and $D_{S}^{*}$ Production and Decay from the MARK-III

SLAC-PUB-4248;

Henvy Quark Spectroscopy and Decay

SLAC-PUB-4694;

Results on Semileptonic $D^{0}$ and $D_{S}^{ \pm}$Decays and Evidence for Non- $D$ Decays of the $\psi(3770)$

SLAC-PUB-4997;

An Experimental Review of the Decays of the $D_{S}^{ \pm}$Meson

BONN-IR-86-34:

Difractive Particle Production in Proton-Antiproton Interactions at Center-of-Mass Energies of goo GeV and $200 \mathrm{GeV}$

Phys. Rev. Lett. 57:527,1986; SLAC.PUB-4031: LBL-21417:

Study of the Decey $\boldsymbol{r}^{-} \rightarrow \pi^{-} \pi^{-} \pi^{+} \nu^{2}$

Actn Phys. Polon. B19:399,1988; GSI-87-64;

Oxygen Induced Reaction at 200 and $60 \mathrm{GeV} /$ Nucleon

2. Phys. C38:109,1988; GSI-88-10;

Target Framentation in Proton-Nucleus and ${ }^{16} \mathrm{O}-\mathrm{Nucleus}$ Reactions at 60 and $200 \mathrm{GeV} / \mathrm{Nuclean}$

Phys. Rev. Lett. 61:1065,1988;

Measurement of the Electric Polarizability of the Neutron

Z. Phys. C40:199,1988; DESY-88-031; SLAC-PUB-4568:

Search for Radiative $\mathbf{Y}(1 S)$ Decays into Light Mesong

MPI-PAE-EXP-EL-193;

Hadron Production in High Energy Neutrino and Antineutrino Collisions

Z. Phys. C46:341,1990; 1PNL-89-8;

Inclusive $\Xi^{0}, \Xi\left(1530 P_{19}\right)^{-}$and $\Xi\left(1530 P_{19}\right)^{0}$ Production in $\Xi^{-}$Be Interactions at $116 \mathrm{GeV} / c$

LBL-26761;

Inclusive Production of $K^{+}$Mesons in $2.1 \mathrm{GeV} / \mathrm{Nucleon}$ Nuclear Collisions

IEKP-KA-89-6;

Review of $B$-Meson Decay Results

Z. Phys. C38:59,1988;

Measurement of Multiplicity Digtributions in Oxygen-Tungaten Collisions at $200 \mathrm{GeV}$ per $\mathbf{N u c l e o n}$

CERN-EP-88-176;

Recent Result from HELIOS (NA34) on Proton-Nucleus and Nucleus-Nucleus Reaction

Phys. Rey. Lett. 60:1618,1988;

Neutrino Production of Same-Sign Dimuons

Phys. Rept. 147:1,1987;

Analytical Treatment of High Energy Nucleus-Nucleus Collisiong

Phys. Lett. 203B:22,1988;

Nucleon Scattering from ${ }^{200} \mathrm{~Pb}$ at Low and Intermediate Energies

DESY-89-007; C88/08/29.2;

Mensurement of the Direct Photon Spectrum from the $\mathbf{Y}(1 S)$

Phys. Rev. D37:1758,1988;

Photino Flux Limits from the Harvard-Purdue-Wisconsin Underground Detector

Phys. Rev. Lett. 58:1715,1987;

Limits on $X(2200)$ Formation in $\ddot{p} P \rightarrow K^{+} K^{-}$ 
Sealock 89

Sediak 88

Seest rommorr 85

Seftor 89

Segel 85

Seidel 88

Selen 89

Sengupta 88

Sengupta 89

Sengupta $89 \mathrm{~B}$

Seth 85

Shabratova 86

Shahbazyan 88

Shahbazyan 90

Shaw 87

Shaw 89

Sheldon 86

Shepko 87

Shibata 86

Shimanyky 88

Shimizu 89

Shin 86

Shipbaugh 87

Shipbaugh 88B

Shirai 88

Shivpuri 86

Shivpuri 87

Shivpuri 87B

Shivpuri 88

Shivpuri 88B

Shklyarevsky 86

Shoemaker 88

Shor 89

Shypit 88

Sibirtsev 88

Sibirtsev 90

Siksin 87

Silverman 85

Silvestrov 86
Phys, Rev, Lett, 62:1350,1989

Electroproduction of the $\Delta\left(1232 P_{33}\right)$ in Nuclei

Fiz. Elem. Chastits At. Yadra 19:445.1988. Antinucleon Nucleon Annihilation at Rest and Flight

Phys. Rev. C31:923,1985: M4 Transitions Observed in Pion Inelastic Scattering on ${ }^{15} \mathrm{Nit}$

Phys. Rev. D39:2457,1989;

Recoil Proton Polarization in $\pi$ p Elastic Scattering at 547 and $625 \mathrm{MeV} / \mathrm{c}$

Pliys. Rev. C32:721,1985: Inclusive $150 \mathrm{MeV}$ Proton Induced Spectra at Forward Angles

Phys. Rev. Lett. 61:2522,1988:

Senrch for Multitrack Nucleon Decay

DOE-ER-3072-49;

Hunting for the Rare Decay $\mathrm{K}^{+} \rightarrow \pi^{+} \mu^{+} \mu^{-}$

Phys. Lett. 213B:548,1988;

Multiplicity Distribution and their Fluctuations in Pseudo-rapidity Windows at Relativistic Heavy-Ion Collisions

Eur. Lett. B:15,1989

Mean Free Paths of He Fragments Produced in Collisions of ${ }^{16} \mathrm{O}$ at $60 \mathrm{GeV} / \mathrm{N}$

Phys. Lett. 222B:301,1989;

On the Production of Helium Fragments in Ultrarelativistic Heavy-Ion Collisions

Phys. Lett. 158B:23,1985;

Polarized Proton Inelastic Scattering from ${ }^{40} \mathrm{Ca}$ and ${ }^{44} \mathrm{Ca}$ at $500 \mathrm{MeV}$

JINR-P1-86-303;

Spectra of Relativistic $\pi^{\ddagger}$ Mesons and $p$ in Inelastic ${ }^{22}$ Ne Interactions with Photoemulsion Nuclei at 4.1 GeV/c per Nucleon

z. Phys. C39:151,1988;

An Evidence for a Possible Stable Dibaryon

Phys. Lett. 235B:208,1990; Phys. Lett. 244B:580,1990; Kr. Sooh. JINR 38:5,1989; The Observation of a Stable Dibaryon

Phys. Rev. D36:3533,1987;

Search for Free Quarks Produced by $14.5 \mathrm{GeV}$ 'Nucleon Oxygen Ions

Phys. Rev. Lett. 63:1342,1989; KEK-89-41; AMY-89-05;

A Search for Unstable Heavy Neutral Leptons in $e^{+} e^{-}$Annihilation at $\sqrt{b}$ from 50 and $60.8 \mathrm{GeV}$

Phys. Rev. Lett. 57:1398,1986; SLAC-PUB-4030; LBL-21872;

Comparison of the Particle Flows in Three Jet and Radiative Two Jet Events from $e^{+} e^{-}$Annihilation at $E_{\mathrm{cm}}=29 \mathrm{GeV}$

Phys. Rev. D35:2917,1987;

Search for Superhenvy Grand Unifled Magnetic Monopoles in Cosmic Rays

Phys. Lett. 172B:283,1986; NUP-A-85-18; Fragmentation of Nucleus at High Energy

JINR-PI-88-443;

Spectra of Protons Flying out at Big Angles in deuteron $p \rightarrow p$ X Reaction at $3.3 \mathrm{GeV} / \mathrm{c}$

KEK-89-146;

Observation of Narrow Structure in the $p p$ Elastic Analyzing Power

Phys. Rev. Lett. 55:2672,1985; TRI-PP-85-58;

$T_{20}$ in $\pi^{+}$deuteron Elastic Scattering between 118 and $148 \mathrm{MeV}$

Phys. Rev. Lett. 60:2117,1987; FERMILAB-PUB-87-220-E;

Production of the $D_{S}^{ \pm}$by High Energy Neutrons

RX-1221-ILLINOIS;

Production of Charm Mesons by High Energy Neutrons

KEK-88-56;

Recent Reault from TRISTAN

Nuovo Cim. 91 A:376,1986:

Interactions of $5 \mathrm{GeV} / \mathrm{c} \overline{\mathbf{p}}$ with Light Emulsion Nuclei

Phys. Rev. D36:3353,1987;

Four-Momentum Transfer between Fireballs in Proton-Nucleus Interactions at $400 \mathrm{GeV}$

Phys. Rev. D35:3508,1987;

Multiplicity, Rapidity, and Rapidity Correlations in $800 \mathrm{GeV}$ Proton Nucleus Interactions

Nuovo Cim. 97A:773,1988;

Fireball Parameters in Proton Nucleus Interactions at $400 \mathrm{GeV}$

Nuovo Cim. 99A:385,1988.

Multiplicity Correlations among the Charged Secondaries in Proton-Nucleus Interactions at $400 \mathrm{GeV}$

Yad. Phys. 47:117,1988; LENI-86-1176;

Phase Shift Analysis of the $p$ Amplitudes in the Energy Range $380-1000$ Mey

Phys. Rev. D37:1120,1988;

Search for Strange Baryonium States in $\tilde{p}$ deuteron Interactions at $8.9 \mathrm{GeV} / \mathrm{c}$

Phys. Rev. Lett. 63:2192,1989;

Subthreshold $\overline{\boldsymbol{p}}, \boldsymbol{K}^{-}, \boldsymbol{K}^{+}$, and Energetic Pion Production in Relativistic Nucleus-Nucleus Collisions

Phys. Rev. Lett. 60:901, 1988;

Evidence Against Broad Dibaryon

Yad. Phys. 47:1040,1988;

$A$-dependence of Proton, $\pi^{+}$and $K^{+}$Meson Production in $10.1 \mathrm{GeV} / c$ Proton Interaction with Nuclei

Yad. Phys. 51:1587,1990;

Study of A Universality in Proton and Pion Production at Intermediate Energies

IFVE-87-64;

Amplitude Analysis of Pion-Nucleon Scattering at $40 \mathrm{GeV} / \mathrm{c}$

Nucl. Phys. A444:621.1985.

Cross Section Measurements of $p$ deuteron $\rightarrow{ }^{3} \mathrm{He} \pi^{0}$ and $p$ deuteron $\rightarrow{ }^{3} \mathrm{H} \pi^{+}$at Intermediate Energies Kr. Soob. JINR 14:9,1986;

Analysis of $e^{+} e^{-}$Pair Production in Experiments with Neutral Kaons 
Silvest rov 87

Simich 86

Simpson 85

Simpson 89

Sim son 89B

Sinervo 86

Sinervo 89

Singh 98

Singh 89B

Singh 8!

Sirunyan $\mathbf{8 8}$

Skarhy 89

Skwarnicki 85B

Skwarnicki 87

Skwarnicki $87 \mathrm{~B}$

Sliwa 83

Smart 86

Smirnn" 35

Smirnova 88

Smith 85

Smith 85B

Smith 85D

Smith 86

Smith 86B

Smith $86 C$

Smith 86D

Smith 86E

Smith $86 F$

Smith $86 G$

Smith 87

Smith 87B

Smith $87 \mathrm{C}$

Smith 89

Smith 90

Snow 85

Snyder 89

Sobchak 88

Soderstrom 90

Soffe: $\mathbf{8 5}$
JINR-P1-87-3;

Analysis of $\mu^{+} \mu^{-}$Pair Production in Experiments with Neutral Kaons

Phys. Rev. D34:692,1986;

Dependence of Average Characteristics of $\pi^{-}$Mesons on Number of Interacting Protons in Nucleu* Nucleus Collisions at $4.2 \mathrm{GeV} / c$ per Nucleon

Phys, Rev. Lett. 54:1891,1985:

Evidence of Heavy-neutrino Emission in $\beta$ Decay

Phys. Rev. D39:1825,1989;

Evidence of the $17 \mathrm{KeV}$ Neutrino in the $\beta$ Spectrum of ${ }^{35} \mathrm{~S}$

Phys. Rev. D39:1837,1989

Evidence of the $17 \mathrm{KeV}$ Neutrino in the $\beta$ Spectrum of ${ }^{3} \mathrm{H}$

SLAC-299;

A Study of the Strange Meson Spectrum as Observed in the Reaction $K^{-} p \rightarrow \bar{K}^{0} \pi^{+} \pi^{-} n$ at 11 GeV/c UPR-0171E;

New Particle Searches at CDF

Phys. Rev. Lett. 61:1073,1988;

Multiplicity Diatribution in Paeudorapidity Intervals with ${ }^{32} \mathrm{~S}$ at $200 \mathrm{GeV} / \mathrm{Nucleon}$ and ${ }^{10} \mathrm{O}$ at 200 and $60 \mathrm{GeV} /$ Nucleon

Phys. Lett. 214B:480,1988;

Interaction Mean Free Paths of He Fragments from Ultrahigh-Energy Nucleug-Nucleus Collisions in Emulsion

Phys. Rev. C39:1835,1989;

Long-Range Correlations in Nucleus-Nucleus Interactions at Ultrahigh Energies

YERE-1083(46)+88

Polarization Investigation of the Photoproduction of $\pi$ and $\eta$ Mesons on Nucleons in the Resonance Enerky Range 1-2 GeV

THESIS;

Forward Muon Production in Proton-Antiproton Collisions at $E_{\mathrm{cm}}=1.8 \mathrm{TeV}$

DESY-85-042; C85/03/10;

Spin Analysia of the $x_{b}$ States

Phys. Rev. Lett. 58:972,1987; SLAC-PUB-4045; DESY-86-087; SLAC-PUB-4045; DESY-86-087: Spin Analyais of the $\chi_{b}$ (unspec) States

SLAC-PUB-4506; DESY-87-166;

Search for $\tau^{ \pm}$Decays to the $\eta$ Meson

Phys. Rev. D32:1053,1985; FERMILAB-PUB-83-96-EXP:

A Study of $D^{*}(\mathbf{2 0 1 0})$ Production in High Energy $\gamma p$ Interactions

Acta Phys. Polon. B17:41,1986;

Neutrino Interactions in Emulsion Stacks inside the Fermilab 15 Foot Bubble Chamber

ITEP-85-30;

$K^{+}$Meeon Inelastic Scattering on the Light Nuclei at $130 \mathrm{MeV}$

Yad. Phys. 47:419,1988;

Dynamical Characteristics of High-Energy $\bar{p} p$ Annihilation

Phys. Lett. 153B:188,1985;

A Search for Fractional Electric Charge on Levitated Niobium Spheres

Phys. Lett. 167B:248,1986; CERN-EP-85-157;

Observation of Longitudinal Event Structure in Proton Diffractive Dissociation at ISR

Phys. Lett. 163B:267,1985; CERN-EP-85-129;

Evidence for Pomeron Single-Quark Interactions in Proton Diffraction at the ISR

Phys. Lett. 171B:129,1986;

A Search for Fractional Charge Changes on Levitated Niobium Spheres

Phys. Lett. 184B:293,1987; CERN-EP-86-158;

Observation of Correlations Between Forward Protons and 90 Degrees Trigger Protons at $E_{\mathrm{cm}}=62 \mathrm{GeV}$

Phys. Rev. Lett. 57:803,1986; TRI-PP-86-13;

Tensor Analyzing Power in $\pi$ deuteron Elastic Scattering

TRI-PP-86-33; C86/05/26;

Teneor Analyzing Power in $\pi$ deuteron Elastic Scattering

TRI-PP-86-110;

First Mensurement of the Tensor Analyzing Power $T(2,1)$ in $\pi$ deuteron Elastic Scattering

TRI-PP-86-80;

Direct Measurement of the Tensor Polarization of a Polarized Deuteron Target

Phys. Lett. 181B:407,1986;

Searches for Fractional Electric Cherge on Niobium Samples Exposed to Liquid Helium

Phys. Lett. 185B:209,1987; CERN-EP-86-182;

$\boldsymbol{\Lambda}$ Polarization in Proton Proton Interactions from $E_{\mathrm{cm}}$ a1 to $\mathrm{B2} \mathrm{GeV}$

Phys. Lett. 197B:447,1987;

Searches for Fractional Electric Charge in Tungsten

TRI-PP-87-69;

Enercy Dependence of the Charge Asymmetry $A(T, \theta)$ in $\pi$ deuteron Elastic Scattering

FERMILAB-CONF-89-275-E;

Intermediate Vector Bosons in the Muon Channel

Phys. Letc. 234B:191,1990;

A Search for the Electric Dipole Moment of the Neutron

Phys. Rev. D32:11,1985

Negative Kaon and Antiproton Production near $700 \mathrm{MeV} / \mathrm{c}$ by $28.4 \mathrm{GeV} / \mathrm{c}$ Protons

Phys. Lett. 229B:169,1989; SLAC-PUB-4986; IUHEE-89-2;

Search for B Decay to Higge Bosons for Higga Boson Masses between $50 \mathrm{MeV} / \mathrm{c}^{2}$ and $210 \mathrm{MeV} / \mathrm{c}^{2}$

JINR-P1-88-393;

Momentum Distributions of Nucleons Flying at Large Angles in ${ }^{4} \mathrm{He} p$ Collisions

SLAC-PUB-5192; LBL-28860; CALT-68-1616;

$A$ Search for Pair Production of Heavy Stable Charged Particles in $Z^{0}$ Decays

CPT-85-P-1809; nucleon nucleon Elastic Polarizationg at High Energieg 
Sokoloff 86

Soldnerrembo 87

Son 88

Sonderegger 88

Sonderegger 89

Sorensen 88

Sowinski 87

Spahn 89

Sphicas 88

Spivak 88

Sromicki 86

Stanco 88

Stanislaus 89

Steele 89

Stenlund 88

Stenz 86

Stewart 90

Stirling 87

Stock 87

Stockdale 85

Stockdale 87

Stockhausen 86

Stockhausen 87

Stockhausen 87B

Stoker 85

Stoker 89

Stopa 87

Strakovsky 86

Streets 89

Strobele 88

Strugalski 85

Strugalski 85B

Strugalski 86

Strugalski 86B

Strugalski 88

Strugalski 88B
Phys. Rev. Lett. 57:3003,1986; FERMILAB-PUB-86-120-E;

Experimental Study of the $A$-dependence of $J / \psi(1 S)$ Photoproduction

BONN+IR-87-31;

Inveatigation of the Reaction $\gamma p \rightarrow \pi^{+} \pi^{-} p$ from Experiment WA69 in the Energy Range from 60 GeV to $170 \mathrm{GeV}$

KEK-88-76; AMY-88-14;

Search for New Heavy Flavore with AMY at TRISTAN

Z. Phys. C38:129,1988; CERN-EP-88-26;

The Study of $\pi$ and $K$ Production in Proton-Uranium and Oxygen-Uranium Interactiona at 200 GeV/Nucleon Using Decay Muon

CERN-EP-89-19

Dimuon and $J / \psi(1 S)$ Production in U1trarelativistic Ion Collisions

Z. Phys. C38:3,1988;

Oxyzen-Induced Reactions at 60 a GeV and 200 a GeV Studied by Calorimetry

Phys. Lett. 199B:341,1987;

A Meawrement of the Spin Correlation Parameter $c_{n n}(\theta)$ in n-p Scattering at $181 \mathrm{MeV}$

Phys. Rev. Lett. 63:1574,1989;

Study of Charge Symmetry in ${ }^{4} \mathrm{He}$ by Simultaneous $e^{-4} \mathrm{He} \rightarrow e^{-} p^{3} \mathrm{H}_{\text {and }} e^{-4}{ }^{4 e} \rightarrow e^{-} n{ }^{3} \mathrm{He} \mathrm{Measure}-$ ments.

CERN-EP-88-184;

High $E_{\ell}$ Jets in UA 1

Zh. Eksp. Teor. Fiz. 94(9):1,1988;

Neutron Lifetime in KIAE Experiment

Phys, Rev. Lett. 57:2359,1986;

Spin Dependence in Low Energy $n$ - p Scattering

LAL-88-51;

New DM2 Reaults on $f_{1}$ (1285) and $\eta(1440)$

Phys. Lett. 219B:237,1989; TRI-PP-88-70;

Search for $T=2$ Dibaryons via $\pi^{-}$deuteron $\rightarrow \gamma X$

SLAC-350;

A Search for Single Electron Production in Electron Pogitron Annihilation at $E=2 \theta \mathrm{GeV}$

LUIP-8807;

Search for Non-gtatistical Particle Density Fluctuations in ${ }^{16} \mathrm{O}+\mathrm{Ag}$ Br and ${ }^{52} \mathrm{~S}+\mathrm{Au}$ Interactions at 200 A GeV

BONN-IR-86-18;

Investigation of Charged Pion and Nuclear Photoproduction in the $\Delta$ (1232 Pss) Resonance Region

FERMILAB-PUB-90-22-E;

Production of High pT Jets in Hadron-Nucleus Collisions

RAL-87-107;

A Compilation of Data on the Energy Energy Correlation and its Asymmetry in $e^{+} e^{-}$Annihilation

Phys. Pept. 135:259,1987;

Particle Production in High Energy Nucleus-Nucleus Collision:

2. Phys. C27:53,1985;

Search for Muon neutrino Oscillations and Antineutrino Oscillations in the Mass Range $16<m^{2}<1000$ $\mathrm{eV}^{2} / \mathrm{c}^{4}$

SLAC-PUB-4467

Obeervation of $D_{S}^{+} \rightarrow \eta \pi^{+}$, and a New Limit on $D^{+} \rightarrow \mu^{+} \nu_{\mu}$ and $F / D$

SLAC-PUB-4026;

$J / \psi(1 S)$ Results from MARK-III

SLAC-PUB-4312,

An Upper Limit on the Decays $D^{0} \rightarrow \mu^{-} e^{+}$and $D^{0} \rightarrow \mu^{+} e^{-}$

SLAC-PUB-4313;

The Decay $\tau^{ \pm} \rightarrow \rho \nu$ (and $\tau^{ \pm} \rightarrow \pi \eta \nu$ ?)

Phys. Rev. Lett. 54:1887,1985;

Search for Right-handed Currents by Means of Muon Spin Rotation

Phys. Rev, D39:1811,1989; SLAC-PUB-4590; JHU-HEP-88-0301;

Limits on New Lepton Pairs $\left(\ell^{-}, \ell^{0}\right)$ with Arbitrary Neutrino Mass

Acta Phys. Polon. B18:429,1987;

Neutral Strange Particle Production in $\pi^{-}$deuteron Interactions at $21 \mathrm{GeV} / \mathrm{c}$

Czech. J. Phys. B36:225,1986;

The $\pi^{+}$deuteron $\rightarrow p p$ Reaction at Intermediate Energie:

FERMIL AB-PUB-89-42-E;

Atomic Weight Dependence of the Production of Hadron Pairs from $800 \mathrm{GeV} / \mathrm{c}$ Protons on Nuclear Targeta

Z. Phys. C38:89,1988;

Negative Particles Productions in Nuclear Collisions at 60 and $200 \mathrm{GeV} / \mathrm{Nucleon}$

JINR-El-85-888;

Experimental Study of the Pion - Xenon Nucleus Collisions without Particle Production at 3.5 GeV/c Momentum: Physical Meaning of the Proton Multiplicity Distribution

JINR-El-85-23I;

Experimental Investigations of the Particle Production Process at the Stage before the Decay of Resonance:

JINR-E1-86-642

The Determination of Matter Densities in ${ }^{131} \mathrm{Xa}(Z=54)$ Nucleus Using Negatively Charged Pions

JINR-E1-86-579;

Nucjeon Emiagion from Target Nuclei which Occurs when Hadrons Traverse Them

JINR-E1-88-211

Experimental Study of Hadron Passage through Intrenuc'ne- $M M_{a t+c r}$

JINR-E1-88-669;

Energy Dependence of the Total Cross Section for Electron-Positron Pair Creation by $\gamma$ Quanta in Liquid

Xenon, Measured in Bubble Chamber 
Strugalski 88C

Stuart 90

Stubbs 87

Stubbs 89

Stubenrauch 86

Stubenrauch 89

Sugahara 88

Sugahara 88B

Sugano 86

Sugimoto 88

Sullivan 87

Sulyaev 88

Sumiyoshi 88

Summers 87

Sun 85

Suzuki 87

Suzuki 88

Suzuki 89

Svec 84

Sviridov 88

Szabelski 56

Szklarz 89

Tacik 86

Takahashi 88

Takamatsu 89

Takibaev 88

Takibaev 90

Takita 86

Takita $89 \mathrm{~B}$

Talebzadeh 87

Tanaka 87

Tanihata 85

Tanihata 86

Tanihata 88

Tanimori 85

Tanimori 89
JINR-E1-88-639;

Retardation of Hadrons in Passing through Intranuclear Matter

Phys. Rev. Lett. 64:983.1990; KEK-89-127; A A1Y-89.17;

Forward-Backward Charge Asymmetry in $\mathrm{e}^{+} \mathrm{e}^{-} \rightarrow$ hadron jet

Phys. Rev. Lett. 58:1070.1987:

Search for an Intermediate Range Interaction

Phys. Rev. Lett. 62:609.1989:

Limits on Composition-Dependent Interactions Using a Laboratory Source: Is There a Fifth Force Coupled to Irospin ?

SACLAY-DPHPE-86-14;

$W^{ \pm}$and $Z^{0}$ Production Properties

Czech. J. Phys. B39:266.1989; SACLAY-DPHPE-87-20: (87/09/14.3:

Test of the Standard Model with Weak Bosons in UA1

KEK-88-10;

New Results from TOPAZ at TRISTAN an Hadron Physics

KEK-88-94; C88/10/:7.2;

Present and I uture of $B$ Physics

ANL-HEP-CP-86-36; C86/03/16;

Charged Particle Multiplicities in $e^{+} e^{-}$Annihilation at $29 \mathrm{GeV}$ : Measurements in the Central Fapidity Regions and in the Gluon Jet

Phys. Rev, D37:583.1988; KEK-87-132;

Search for Structures in the $\bar{p} p \rightarrow \pi^{+} \pi^{-}$and $\bar{p} p \rightarrow K^{+} K^{-}$Cross Sections between 360 and $760 \mathrm{MeV} / \mathrm{c}$

Phys. Rev. D36:674,1987;

Measurement of the Ratio of $\Sigma^{0}$ to $\Lambda$ Inclusive Production from $28.5 \mathrm{GeV} / c$ Protons on Beryllium

IFVE-88-100;

High pT Hadron Production in Hadron-Nuclei Collisions

KEK-88-28;

Recent Results from VENUS at TRISTAN

CERN-EP-87-86;

Beauty Physics at UA 1

Phys. Rev. C31:515.1985;

Measurements of the Spin Rotation Parameters for $p$ (polarized) deuteron $\rightarrow p$ (polarized) deuteron Elastic Scattering at $496 \mathrm{MeV}, 647 \mathrm{MeV}$, and $800 \mathrm{MeV}$

Nucl. Phys. B294:961,1987;

Measurement of Differential Cross Sections for the $\pi^{-} p \rightarrow \pi^{0} n$ Charge Exchange Scattering from 1869 $\mathrm{MeV} / \mathrm{c}$ to $2965 \mathrm{MeV} / \mathrm{c}$

KEK-88-38;

Review of Atmospheric Neutrino Phenomena in Underground Nucleon Decay Detectors

KEK-89-40;

Solar Neutrino Observation at KAMIOKANDE-II

Jour. de Phys. 46:C2-281.1985; PRINT-85-0096:

Observation of a $\mathrm{D}^{++}(750)$ Gluonium Candidate in Measurements of $\pi^{-} p$ (polarized) $\rightarrow \pi^{+} \pi^{-} n$ on Polarized Target at $6 \mathrm{GeV} / c, 12 \mathrm{GeV} / c$ and $17.2 \mathrm{GeV} / c$

Yad. Phys. 49:172,1989; IFVE-88-101;

Analysis of Inclusive Prompt Muon Production in $p$ Fe Interaction at $70 \mathrm{GeV}$

Nuovo Cim. 9C:377,1986:

Relevance of Multiple Muons Detected Underground to the Mass Composition of Primary Cosmic Rays

LAL-89-61;

Partial Wave Analysis of $\eta(1440)$ from DM2

Phys. Rev. C32:1335,1985;

Measurement of Three Protons in Coincidence Following Absorption of $228 \mathrm{MeV} \pi^{+}$in Carbon

DPNU-89-14;

Search for Supersymmetric Particles in $e^{+} e^{-}$Annihilation at TRISTAN

KEK-89-154;

Experimental Studies on Meson Spectroscopy at KEK

Yad. Phys. 47:446,1988;

Azimuthal Asymmetry and Fluctuations in Slow-Particle Emission in High Energy Proton-Nuclear Intteractions

Yad. Phys. 51:925,1990;

Energy Characteriatics of Double Charged Particles from High Energy Hadron-Nucleus Interactions

Phys. Rev. D34:902,1986:

Search for Neutron-Antineutron Oscillation in ${ }^{10} \mathrm{O}$ Nuclei

1CR-186-89-3;

An Experimental Study of Atmospheric Neutrino Flux with the KAMIOKANDE Detector

Nucl. Phys. B291:503,1987; CERN-EP-87-47;

Search for Tau Neutrino Interactions in the BEBC Beam Dump Experiment

KEK-87-139;

On p p Inelasticities at Intermediate Energies

Phys. Lett. 160B:380,1985;

Measurements of Interaction Cross Sections and Radii of the He Isotopes

Phys. Rev. Lett. 55:2676.1985; INS-550;

Measurements of Interaction Cross Sections and Nuclear Radii in the Light $P$ Shell Region

Phys. Lett. 206B:592,1988;

Measurement of Interaction Cross Sections Using Isotope Beams of Be and Bor and Isospin Dependence of the Nuclear Radii

Phys. Rev. Lett. 55:1835,1985;

Observation of an Enhancement in $\bar{p} p \rightarrow \pi^{+} \pi^{-}$and $\bar{p} p \rightarrow K^{+} K^{-}$Cross Sections at $\bar{p}$ Momentum of 500 $\mathrm{MeV} / \mathrm{c}$

KEK-89-35;

JANZOS Results on PeV and TeV Gamma Rays from SN1987 A and the Future Plan for a TeV Collaboration with Adelaide 
Tanimori 89B

Tannenbaum 87

Tannenbaum 88

Tannenbaum 89

Tao 88

Tariq 90

Tauchi $8 \mathcal{E}$

Tenner 88

Terrier 87

Thomas 89

Thorndike 88

Thron 84

Timmers 84

Tixier 88

Tkaczyk 86

Toki 85B

Toki 86

Toki 87

Toki 88

Toki 88B

Toki 89

Toki 89B

Tokushuku 90

Tolstov 87

Tomaradze 86

Tonapetyan 85B

Tonelli 88

Tonelli 89

Toothacker 87

Torres 85

Tosello 89

Totsuka 88

Toteuka 89

Totsuka $89 \mathrm{~B}$

Tretyakova $\mathbf{8 8}$

Trost 89

Trower 85

Troyan 86
Pliys. Rev. D41:744,1990: KEK-89-140: Experimental Study of the Reactions $\bar{p} p \rightarrow \pi^{+} \pi^{-}$and $K^{+} K^{-}$between 360 and $760 \mathrm{MeV} / \mathrm{c}$

BNL-40066: Relativistic Heavy Ion Collisions

BNL-41354: Measurement of Energy Flow from Oxygen, Silicon and Proton Interactions with Nuclei at the BNL TANDEM-AGS

Int. Jour. Mod. Phys. A4:3377,1989; BNL-42802: Transverse Energy Production in Light and Heavy Ion Interactions

Int. Jour. Mod. Phys. A 1:749,1988: The UA1 Experiment

Acta Phys. Polon. B21:215,1990; Some Angle Dependent Characteristics of Charged Shower Particles Produced at High Energies KEK-88-39;

Test of the Standard Model of Electroweak Interactions at TRISTAN

NIK-HEF-H-88-6:

An Estimate of the Rescattering Fraction in Neutrino and Antineutrino Deuteron Interactions

Phys. Rev. Lett. 59:1534,1987: Measurement with a Free Neutron Beam of Absolute Neutron-Proton Forward Elastic-Scattering Differential Cross Section at Intermediate Energies

Phys. Rev. Lett. 63:1902,1989:

Testing the Inverse-Square Law of Gravity on a 465 m Tower

Phys. Rept. 157:183,1988; Decays of the $b$ Quark

Phys. Rev. D31:451,1985; FERMILAB-PUB-84-53-E; Search for Heavy Charged Particles and Light Nuclei and Antinuclei Produced by $400 \mathrm{GeV}$ Protons

Phys. Rev. D31:99,1985; THEF-NYM-84-05: The S(1834)

Phys. Lett. 212B:523,1988; LAl-88-13:

Looking at $C P$ Invariance and Quant um Mechanics in $J / \psi(1 S) \rightarrow \Lambda \bar{\Lambda}$ Decay

2. Phys. C33:33,1986;

Fast Proton Production in $\pi^{ \pm}$Ne at $30 \mathrm{GeV} / \mathrm{c}$

SLAC-PUB-3832; C85/07/29; Recent MARK-1II Results in Radiative $J / \psi(1 S)$ Decays

SLAC-PUB-4153; $D_{S}^{ \pm}$Production Data From MARK-III

SLAC-PUB.4410;

Experimental Review of $J / \psi(1 S)$ Decays

SLAC-PUB-4784; Review of $J / \psi(1 S)$ Decays

SLAC-PUB-4824. BNL Glueball Review

SLAC-PUB-5093;

Study of $\psi(2 S)$ Decays

SLAC-PUB-5087;

Study of $D_{S}^{ \pm}$Decays

Phys. Lett. 235B:245,1990; KEK-89-160;

Anaiysis of Deuteron Spectra from Hadron-Nucleus Reactions at Several GeV/c with the Coalescence Model

Kr. Soob. JINR 21:26,1987;

A bu ut A nomalon Interpretation of ${ }^{40} \mathrm{Ar}$ Cu Collisions at 0.8 and $1.8 \mathrm{GeV}$ per Nucleon Energies

Yad. Phys. 45:110,1987: IFVE-86-54;

Systematics in the Transverse Momentum Distributions and Azimuthal Correlations in the Inclusive $K^{+}$ p Reactions at $32 \mathrm{GeV} / \mathrm{c}$

Ukr. Fiz. Zhurn. 30:679,1985;

Photoproduction of Charged Pions on the Atomic Nuclei and its Absorbtion at the Centre of Nuclei

INFN-PI-AE-88-8; CDF Results and Prospects for Elastic Scattering

INFN-PI-AE-89-7;

Proton-Antiproton Collisions at $\sqrt{s}=1.8$ TeV: Recent Results from the CDF

Phys. Lett. 197B:295,1987;

Measurement of the Stopping Power of Nuclei for $100 \mathrm{GeV} / \mathrm{c}$ Protons and Antiprotons

Phys. Rev. D34:707,1986; FERMJLAB-PUB-85-117-E:

Observation of the $\phi K \pi$ Decay of the $\left.K_{4}^{\prime \prime 2045}\right)$

Nuovo Cim. 102A:663,1989;

Strangeness Production by Antiproton

ICR-REPORT-180-\&\$-26;

Non-Acceler ator Particle Physics

ICR-Report-1'92-89-9;

Search for WIMPS

ICR-Report-194-89-11.

Kamiokande, 1983 - 1989 - 1990's

LEBD-88-244;

Central Interactions of ${ }^{16} \mathrm{O}$ Nuclei with Heavy Nuclei of Photoemulsion at $200 \mathrm{GeV} / \mathrm{Nucleon}$

Phys. Rev. D4D:1703,1989; FERMILAB-PUB-89-145-E;

New Measurement of the Production Polnrization and Magnetic Moment of the Cascade Minus Hyperon Nucl. Phys. B252:285, 1985;

Detection of Free Fractional Electric and Magnetic Charge

Kr. Soob. JINR 13:12,1985:

Narrow Diproton Resonances in the $n p \rightarrow p p \pi^{-}$Reaction at $p(n)=1.257 \mathrm{GeV} / \mathrm{c}$ 
Troyan 88

T9agova 85

Tschirhart 88

Tsertos 88

Tsertos 88B

Tsertos 89

Tsertos 89B

Tsuchiaki 90

Tsukerman 85

Tsukerman 85B

Tsukerman sar

Turkot 88

Turley 85

Turner 88

Tuts 87

Tzeng 85

Ukhanov 86

Ukhanov 86B

Ullmann 85

Underwood 89

Ungricht 85

Unno 88

Ushida 86

Ushida $86 B$

Ushida 86C

Ushida 88

Ushida $88 B$

Valenti 85

Vandervelde 89

Vandyck 86

Vandyck 87

Vanoers 85

Vanpolen 87

Vapenikova 88

Vartapetyan 89

Varvell 87

Vasenko 88

Vasenko 89

Vashkevich 88

Vasserman 86
JINR-D1-88-329;

Narrow Diproton Resonances in the Reaction of $n p \rightarrow p p \pi^{-}$

lzv. Akad. Nauk SSSR, Fiz. 49:1318,1985;

Study of Charge Component of Hadrons in Extensive Atmospheric Showers with $N\left(\right.$ particles) $>10^{5}$

Phys. Lett. 205B:407,1988;

Messurement of the Inclusive $K_{S}$ Branching Fraction in $\tau^{ \pm}$Decay

Phys. Lett. 207B:273,1988;

Sentitive Search for Neutral Resonances in Bhaba Scattering around $1.8 \mathrm{MeV} / \mathrm{c}^{2}$

Z. Phys. A331:103,1988;

New Limits for Resonant Bhaba Scattering around the Invariant Mass of $1.8 \mathrm{MeV} / \mathrm{c}^{2}$

GSI-89-33;

Search for Resonant Bhaba Scattering at MeV Energies

Phys. Rev. D40:1397,1989; GS1-89-21;

High Sensitivity Measurements of the Excitation Function for Bhabha Scattering at MeV Energie*

Phys. Lett. 236B:81,1990 Search for Neutral Boson in Orthopositronium Decay

ITEP-85-112;

Strong Interaction: Experiments of 1982 - 1984. Hadrons

ITEP-85-113;

Strong Interaction: Experiments of $1982-1984$. Glueball

ITEP-85-136;

Strong Interaction: Experiments of 1882 - 1984. Heavy Quarks

FERMILAB-CONF-88-199-E;

Quark GIuon Plasma - Overview and Experimental Results from E-735

Phys. Lett. 157B:19,1985.

The ${ }^{10} \mathrm{O}(\gamma, p(0)){ }^{1 d}$ Nit Reaction at $E(\gamma)=190 \mathrm{MeV}$

Phys. Rev. Lett. 60:1797,1988;

Axion: from SN1987A

Phys. Lett. 186B:233,1987;

Search for Light Gluinos

Phys. Rev. Lett. 55:1172,1985;

Charm Production in Neutron-Nucleon Interactions

Yad. Phys, 45: 1032,1987; IFVE-86-93;

Azimuthal Correlations between Secondary Charged Hadrons in $K^{-} p$ Interactions at $32 \mathrm{GeV} / \mathrm{c}$

IFVE-86-195;

Inclusive $\gamma$ Quanta and $\pi^{0}$ Mesons Productions at $70 \mathrm{GeV} / \mathrm{c}$

Phys. Rev. C31:177,1985;

Pion Inelastic Scattering to Giant Resonances and Low Lying Collective States in ${ }^{119}$ Sn and ${ }^{40} \mathrm{Ca}$

IFVE-89-55;

The First Results of FNAL Polarized Program (Experiment E-5B1/E-704)

Phys. Rev. C31:934,1985;

Teneor Polarization in Pion Deuteron Elastic Scattering

KEK-88-64;

Recent Result from VENUS at TRISTAN

Phys. Rev. Lett. 56:1767,1986;

Lifetimes of the Charmed Particles $D^{ \pm}, D_{S}^{-}$and $\Lambda_{\mathrm{r}}^{+}$Produced by Neutrinos

Phys. Rev. Lett. 56:1771,1986;

Lifetirne of $D^{\circ}$ Charmed Mesons Produced in Neutrino Interactions

Phys. Rev. Lett. 57:2897,1986;

Limits to $\nu_{\mu}, \nu_{e} \rightarrow \nu_{\tau}$ Oseillations and $\nu_{\mu}, \nu_{e} \rightarrow \tau^{-}$Direct Coupling

Phys. Lett. 206B:380,1988;

Production Characteristics of Charmed Particles in Neutrino Interactions

Phys. Lett. 206B:375,1988;

Cross Section for Neutrino Production of Charmed Particles

CERN-EP-85-66:

The UA-06 Experiment

Phys. Rev. D39: 1492,1989;

Ponsible Evidence for a New Particle from SN1987 A

Phys. Rev. D34:722,1986;

Electron Magnetic Moment from Geonium Spectra: Early Experiments and Background Concepts

Phys. Rev. Lett. 59:26,1987;

New High Precision Comparison of Electron and Positron g Factors

TRI-PP-85-7]

Two and Few Nucleon Systems

Phys. Rev. D,36:1983,1987;

Search for Fractional Charges Using Droplet-Jet Techniques

2. Phys. C37:251,1088;

Electroproduction of Single Charged Pione from Deuterium at $Q^{2}=1 \mathrm{GeV}^{2}$ in the Resonance Region YERE-1151(28)-89;

The Longitudinal and Transverse Response Functions of the ${ }^{12} \mathrm{C}$ Nucleus at $Q=600$ and $680 \mathrm{MeV} / \mathrm{c}$

Z. Phys. C36:1,1987; CERN-EP-87-46;

Mensurement of the Structure Functions $F_{2}$ and $F_{9}$ Comparison with QCD Predictions Including Kinematical and Dynamical Higher Twist Effects

ITEP-88-75;

Double- $\beta$ Experiment with Enriched Germanium Detector

Mod. Phys. Lett. A5:1299,1990; ITEP-89-178

New Results in the ITEP/YEPI Double $\beta$ Decay Experiment with Enriched Germanium Detector

Yad. Phys. 47:1054,1988;

Experimental Study of High-Energy EAS Muons

Pisma Zh. Eksp. Teor. Fiz. 43:457,1986;

Search for $K_{S} \rightarrow 2 \gamma$ Decay 
Vesserman 86B

Vasserman 87

Vassernan 87B

Vasserman $87 \mathrm{C}$

Vasserman 88

Vassiliadis 85

Vecko 89

Vegni 86

Velichko 85

Velichko 88

Venkataraman $85 B$

Verbeure 87

Veres 85

Vershinsky 90

Vesna 89

Vesztergombi 88

Vidyakin 87

Vidyakin 89

Vidyakin $89 \mathrm{~B}$

Vidyakin 90

Virodov 89

Viryasov 89

Vishnyakov 85

Vlasov 86

Vlasov 88

Vlasov 89

Vlasov 89B

Vlasov 90

Voitsekhovsk 86

Vukal 88

Vokalova 85

Voloshin 87

Vonfeilitzsc 88

Vonfrankenbe 89

Vorobiev 84C
Pisma Zh. Eksp. Teor. Fiz. 44:493.1986;

Observation of $e^{+} e^{-} \rightarrow \gamma \gamma \gamma \gamma$

Phys. Lett. 198B:302,1987; NOVO-87-117;

Comparison of the Anomalous Magnetic Momentum of Electron and Positron, Experiment 1887

Yad. Phys. 47:1635,1988; Sov. J. Nucl. Phys. 47:1035,1988: NOVO-87-120:

Observation of $\rho^{0} \rightarrow \pi^{+} \pi^{-} \gamma$ Decay

Phys. Lett. 187B:172,1987;

New Experiment on the Precise Comparison of the Anomalous Magnetic Moments of Relativistic Electrons and Powitrons

Yad. Phys. 48:753,1988;

Search for $\rho^{0} \rightarrow \pi^{+} \pi^{-} \pi^{0}$ Decay

CERit-EP-85-96;

Contribution to Meson Spectroscopy from Central Production

Czech. J. Phys. B39:297,1989; FZU-8-88;

Production of the Charmed Baryon $\boldsymbol{\Lambda}_{c}^{+}$in Neutron-Proton Interactiong

Yad. Phys. 43:1480,1986; Sov. J. Nucl. Phys. 43:954,1986; JINR-El-85-498;

A Study of Inelastic Diffraction of $\pi^{-}$Meson in $\pi^{+} \pi^{-} \pi^{-}$System Using a "Live" Targel of Siljicon

Yad. Phys. 42:1325,1985;

Elastic Scattering of Protons on Helium Nuclei in the Energy Range of $700-1000 \mathrm{MeV}$

Yad. Phys. 47:1185,1988;

Elastic $p$ deuteron Scattering at Small Angles in the Energy Range of $700-1000 \mathrm{MeV}$

DESY-85-115; WIS-85-40-PH; C86/07/16;

$B$ Lifetime Menurements from PETRA

IFVE-87-185;

Minimum Bias Events from EHS (How to Extract Phyaics)

Kr. Soob. JINR 9:43,1985;

Search for Anomalous lnteraction of Projectile Fragments of ${ }^{24} \mathrm{Mg}$ Nucleus by UsIng of Cerenkov Spectrometer

Pisma Zh. Eksp. Teor. Fiz. 51:82,1990;

Observation of the Reactor Antineutrino Interaction with Deuteron in the Neutral and Charged Current Channels on the Rovno AES

LENI-89-1560;

The Obeervation and Analysis of P-violating Effects auch as $\Sigma(n)^{*} p(\gamma)$ in the Integral $\gamma-$ Spectra from the Reactions $n^{115} \mathrm{Cd} \rightarrow \gamma^{114} \mathrm{Cd}$, and $n^{86} \mathrm{Fe} \rightarrow \gamma^{87} \mathrm{Fe}$

Z. Phys. C38:125,1988;

First Fesults on Strangenes. Production in 60 and $200 \mathrm{GeV} /$ Nucleon Heavy Ion Reactions from the NA35 Streamer Chamber

Zh. Eksp. Teor. Fiz. 93:424,1987:

Detecting of Antineutrinos in Beam from Two Reactors

Pisma Zh. Eksp. Teor. Fiz. 49:130,1989;

Observation of the Weak Charge Current in the Interaction of the Reactor Antineutrino with Deuteron

Pisma Zh. Eksp. Teor, Fiz. 49:646,1989;

Inveatigation of the Antineutrino-Electron Scattering with the Help of Fluororganic Scintillator Detector

Pisma Zh. Eksp. Teor. Fiz. 51:245,1990;

Observation of the Weak Neutral Current in the Interaction of the Reactor Antineutrino with Deuteron

Vopr. At. Nauki i Techn. ser. Yad. 2:9,1989; Experiment on the Dirac Monopole Search at the CERN Accelerator

JINR-P1-89-511;

Momentum and Angular Distributions of $\pi^{-}$Mesong Produced in deuteron $C$ Interactions at $1 \mathrm{GeV} / \mathrm{c}$

JINR.P1-85-221;

About Angular Dependence of the $\pi^{-}$nucleus $\rightarrow p$ X Reaction Invariant Cross Sections at $40 \mathrm{GeV} / \mathrm{c}$ Pion Momentum

ITEP-86-174;

Correlations of Protons and Deuterons with Deuterons at Proton Nuclei Interactions

Yad. Phys, 52:489,1990; ITEP-88-101; Meaurement of the Correlation Function of the Three Cumulative Baryons

ITEP-89-11;

Mesarement of the Correlation Function of the Cumulative Protons and Neutrons

Yad. Phys. 50:712,1989;

Study of $p$ deuteron, $p{ }^{3} \mathbf{H}$ and deuteron deuteron Correlation Functions

ITEP-90-63;

Investigation of Pion Correlations with Pions and Cumulative Baryons in Proton Nucleus Interactions at $7.5 \mathrm{GeV} / c$

Pisma Zh. Eksp. Teor. Fiz. 43:567,1986;

Asymmetry in the Reaction deuteron $\left(e^{-}, e^{-}\right.$deuteron) at $1-1.5 \mathrm{GeV}$

Yad. Phys. 50:1046,1989; JINR-PI-88-215;

Spectra of $\pi^{ \pm}$Mesons and Protons in Central and Peripheral Collision of $p,{ }^{12} \mathrm{C}$, ${ }^{22} \mathrm{Ne}$ with Photoemulsion at $4.5 \mathrm{GeV} / \mathrm{c}$

Kr. Soob. JINR 12:15,1985

Inelastic Collisions of ${ }^{22}$ Ne Nuclei with Nuclei in Photoernulaion at $90 \mathrm{GeV} / \mathrm{c}$

Usp. Fiz. Nauk 152:361,1987; Sov. Phys. Usp. 30:553,1987

Physics of $\boldsymbol{r}$ Resonances: Ten Years Later

Phys. Let t. 200B:580,1988;

Limita on Neutrino Stability from the Gamma Ray Flux Measured in Coincidence with Neutrinas from SN1987A

CERN-EP-89-155;

Progreas in Hyperon Production at LEAR

Yad. Phys. 43:111,1986; ITEP-84-60;

Measurement of Maximum in the $\pi^{ \pm}$Mesons Distributions from the Reactions $\pi^{+}$nucleus $\rightarrow \pi^{ \pm}$mult [p] nucleus 
Vorobiev 85

Vorobiev $85 \mathrm{~B}$

Vorobiev 86

Vorobiev $86 \mathrm{~B}$

Vorobiev 87

Vorobiev $87 \mathrm{~B}$

Vorobiev $87 \mathrm{C}$

Vorobiev $88 B$

Vorobiev $\mathbf{8 8 C}$

Vorobiev 88D

Vorobiev $88 \mathrm{E}$

Vorobiev 89

Vorobiev $89 \mathrm{~B}$

Vorobiev $89 \mathrm{C}$

Vorobiev 90

Vorobiev $90 \mathrm{~B}$

Voronin 88

Voronko 88

Vorontsov 88B

Vovchenko 85

Vovchenko 86

Vovchenko $86 \mathrm{~B}$

Vovchenko 89

Vovehenko 89B

Voyvodic 85

Voyrodic 86

Voyvodic $86 \mathrm{~B}$

Vuillemin 85

Wachs 89

Waddington 85

Wagner 85

Wagner 87

Wagner 89

Wagner $89 \mathrm{~B}$

Wah 85

Walk 85

Walker 89
ITEP-85-130;

Same Topological Characteristics of $\pi^{-}$Mesons Interactiong with Nuclei

Yad. Phys. 41:1541,1985; Sov. J. Nucl. Phys. 41:976.198::

Measurement of Polarization of Cumulative Protons

ITEP-86-98;

Lambdometer

Yad. Phys. 44:1396,1986;

Irregularities in Angular Distribution of Cumulative Particles near 180 Degrees in 1.s.

NOVO.87-138:

Limit on the Constant of arion Interaction

ITEP-87-126;

Energy and A-Dependence of the Ylelds of Cumulative $\Lambda$ Particles at $\theta$ Degrees in 1.s.

ITEP-87-125;

Search for Diproton Resonances in Proton - Nucleus Interactions at $7.5 \mathrm{GeV} / \mathrm{c}$

Phys. Lett. 208B:146,1988:

New Limit on the arion Interaction Constant

Yad, Phys. 48:436,1988; Sov. J. Nurl. Phys. 48:273,1988:

Upper Limit on the Electron Widths of C-even Mesons: $\eta^{\prime}, f_{0}(\theta 75), f_{2}(1270), f_{0}(1400), a_{0}(980), a_{2}(1320)$

ITEP-88-72;

Soft $\pi^{+}$Mesons in $p$ nucleus and $\pi^{-}$nucleus Interactions

ITEP-88-113;

The Polarization of Cumulative $\Lambda$ 's Flying from C, Al, Cu, Cd, Pb Nucleus at 90 Degrees in 1.8 .

NOVO-89-31:

Preliminary reaults of the Search for Exotic Long Range Interaction with Violation of $T$ Invariance

Pisma Zh. Eksp. Teor. Fiz. 49:584,1989;

Soft Pion Production in p nucieus Interactions

ITEP-89-27;

The Results of Electronic Experiment on the Investigation of the Reaction $\left(\pi^{\ddagger}, p\right) A(C, A l, C u, C d, P b)$ $\rightarrow \mathbf{\Lambda} \mathrm{X}$

ITEP-90-5:

Cumulative $\Delta\left(1232 P_{33}\right)$ Production in $p$ A Interactions at $7.5 \mathrm{GeV} / c$

Yad. Phys. 51:135,1990:

Search for Narrow Diproton Resonance

ITEP-88-117;

The Creation of Antiprotons with Momentum from 1.25 to $5 \mathrm{GeV} / \mathrm{c}$ in: Interactions of Protons with Be, Al, Cu and Th Nucleus at $10.1 \mathrm{GeV} / \mathrm{e}$

JINR-P1-88-294;

Generation of Neutrons in a Lead Target by Protons, Deuterons, Alpha Particles with $4.5 \mathrm{GeV} / \mathrm{c}$ per Nucleon Moment um

ITEP-88-11:

A-dependence of Proton's Fragmentation with Energy $9.2 \mathrm{GeV}$ on Nucleus

LENI-85-1110;

Study of Phase Shift Analysis of $p p$ Scattering at the Energy of $970 \mathrm{MeV}$

Yad. Phys. 44:456,1986;

Search for Solutions of Phase Shift Analysis of p p Internctions at $970 \mathrm{MeV}$

Pisma Zh. Eksp. Teor. Fiz. 44:119,1986;

Angular Dependence of Correlation Parameters Aoonn and Asymmetry $A_{000 n}$ in Elastic $p p$ Scattering in the Enercy Interval 690 - $850 \mathrm{MeV}$

Yad. Phys, 49:720,1989;

Polarization Parameters $A_{000 n}$ and $A_{00 n n}$ in Proton Proton Elastic Scattering at $690-890 \mathrm{MeV}$

Yad. Phys. 50:1005.1989;

Energy Dependence of Polarization Transfer Parameter in $p$ scattering

Yad. Phys. 44:649,1986; Sov. J. Nucl. Phys. 44:421,1986; ITEP-85-97:

Search for Charmed Particles Produced in Neutrino Interactions in Emulsion Placed inside the Fermilab 15 Foot Bubble Chamber

ITEP-86-91;

Mean Multiplicities of Secondary Particles Produced in Neutrino Interactions with Emulsion and Neon

Pisma Zh. Eksp. Teor. Fiz. 43:401,1986; ITEP-86-51;

Observation in Nuclear Emulsion of a Charmed $\Sigma_{c}(2455)^{0}$ Baryon Decay to $\Lambda_{c}^{+} \pi^{-}$with a Subsequent $\Lambda_{c}^{+}$ Decay to $\mathbf{\Sigma}^{+} \pi^{-} \pi^{+}$

CERN-EP-85-29; C85/01/07.2;

$W^{ \pm}$and $Z^{0}$ Production in the UA1 Experiment at the CERN Proton-Antiproton Collider

Z. Phys. C42:33,1989; DESY-88-11 1; SLAC-PUB-4691:

The Electron Spectrum from B Meson Decays

Phys. Rev. C31:888,1985;

Interactions of Energetic Gold Nuclei in Nuclear Emulsions

Phys. Rev. C31:1934,1985;

Radiochemical Measurements of $200 \mathrm{MeV}$ Proton Induced Figsion of ${ }^{139} \mathrm{Cs}$

Phys. Rev. D36:2850,1987; SLAC-PUB-4304; LBL-23321;

Measurement of the $D^{0}$ Lifetime from the Upgrnded MARK-II Detector at PEP

CDF-PUB-CDFR-1033;

Search for Supersymmetric Particles at CDF

Phys. Rev. Lett. 64:1095,1990; SLAC-PUB-5107; LBL-27839; COLO-HEP-198;

Messurement of the $B^{0}$ Meson Lifetime

Phys. Rev. Lett. 55:2551,1985;

Measurement of $\mathbf{\Sigma}^{-}$Production Polarization and Magnetic Moment

Phys. Rev. D34:2611.1986; DESY-85-019; SLAC-PUB-3820;

The $x_{b}$ States in Exclugive Radiative Decay of the $\mathbf{r}(25)$

Phys. Lett. 224B:353,1989; Phys. Lett. 240B:522,1990;

Measurement of the Proton Elastic Form Factors for $Q^{2}=1-3(\mathrm{GeV} / \mathrm{c})^{2}$ 
Waltham 83

Wang $85 \mathrm{D}$

Ward 86B

Warner 85

Wasiliev 90

Wasserbaech 87

Wasserbaech 89

Watts 90

Weber 89

Weinstein 89

Weir 89

Weir 90

Weller 88

Wendt 87

Westbrook 87

Wharton 85

Whitaker 86

Whitehouse 89

Whitlow 90

Wichees $\mathbf{8 7}$

Wicklund 85

Wicklund 87

Wiedner 87

Wiedner 89

Wightman 87

Wightman 88

W:Ikerson 87

Wilkinson 86

Wilkinson 87

Williams 88

Williams 89B

Willis 89

Wimmersperg 87

Winstein 89

Wise 85

Wittek 87

Wittek 88

Wittek 89
Nucl. Phys. A433:649,1985; TRI-PP-83-68; C83/08/21

Spin-Spin Correlations and Spin Asymmetr: for the Resction p p $\rightarrow \boldsymbol{n} \boldsymbol{\pi}^{+}$at Intermediate Energies

Phys. Rev. C31:1662,1985;

${ }^{\mathrm{B} e}\left(p, p\right.$ He) ${ }^{5}$ He Cluster Knockout Reaction with $150 \mathrm{MeV}$ Polarized Protons

CERN-EP-86-80;

Low $p_{T}$ Physics at the $p \vec{p}$ Collider

Nucl. Phys. A443:64,1985:

Comparison of the Noncomplanar ${ }^{\circ} \mathrm{Li}\left(p, p\right.$ deuteron) ${ }^{4} \mathrm{He}$ Reactions at $120 \mathrm{MeV}$ and $200 \mathrm{MeV}$

Pisma Zh. Eksp. Teor. Fiz. 51:550,1990;

Observation of the Events Excesses in the Experiment on the Search for Double $\beta$ Decay of ${ }^{100}$ Mo

SLAC-PUB-4289;

Result: on Charmed Meson Decays from MARK-I I

SLAC-PUB-5012;

Resulta on $D$ and $D_{S}^{ \pm}$Decays from MARK-III

FERMILAB-CONF-90-112-E;

Properties of Inclusive $W^{ \pm}, Z^{0}$ Events in $\bar{p} p$ Collisions at $1.8 \mathrm{TeV}$

Phys. Lett. 230B:31,1989; TRI-PP-89-58;

Multi-Nucleon Pion Absorbtion in the ${ }^{4} \mathrm{He}\left(\pi^{+}, p\right.$ p p $) \pi$ Reaction

CALT-68-1591;

Results from the MARK-II at SLC on Decays of the $Z^{0}$

Phys. Rev. D41:1384,1990; SLAC-PUB-4999; CALT-68-1559;

Upper Limits on $D^{ \pm}$and $B^{ \pm}$Decays to Two Leptons Plus $\pi^{ \pm}$or $K^{ \pm}$

CALT-68-1603;

A Measurement of $B^{\circ} \bar{B}^{\circ}$ Mixing in $e^{+} e^{-}$Annihilation at $29 \mathrm{GeV}$

Phys. Lett. 213B:413,1988;

The EnerE Dependence of Polarization Observables in the deuteron deuteron $\rightarrow{ }^{4}$ He $\gamma$ Renction

Phys. Rev. Lett. 58:1810,1987; SLAC-PUB-4177; LBL-22797;

Search for Heavy Neutrino Production in $e^{+} e^{-}$Annihilation at $29 \mathrm{GeV}$

Phys. Rev. Lett, 58:1328,1987:

New Precision Measuremert of the Orthopositronium Decay Rate: A Discrepancy with Theory

Phys. Rev. C31:526,1985;

Triple Differential Cross Section to Discrete States in the ${ }^{10} \mathrm{O}\left(\pi^{+}, 2 p\right){ }^{14}$ Nit Reaction

BUHEP-86-10;

Single Photon Production in $e^{+} e^{-}$Annihilation

Phys. Rev. Lett. 63:1352,1989;

Radiative Kan Capture at Rest in Hydrogen

SLAC-357;

Deep Inelastic Structure Functions from Electron Scattering on Hydrogen, Deuterium, and Iron at 0.6 $\mathrm{GeV}^{2}<Q^{2}<30.0 \mathrm{GeV}^{2}$

Phys. Rev. Lett. 58:1821,1987.

Bounds on Right-handed Currents from Nuclear $\beta$ Decay

Phys. Rev. D34:19,1986; ANL-HEP-PR-85-81;

Study of the Reaction $p p \rightarrow p \pi^{+} n$ with Polarized Beam from 3 to $12 \mathrm{GeV} / \mathrm{c}$

Phys. Rev. D35:2670,1987;

Study of the Reaction p $p \rightarrow p \pi^{+} n$ wit: Polarized Beam from 1.18 to $1.98 \mathrm{GeV} / \mathrm{c}$

Phys. Rev. Lett. 58:648,1987;

Determination of the Real Part of the Isospin Forward Scattering Amplitude of Pion Nucleon Scattering at $55 \mathrm{MeV}$ as a Test of Low Energy Quantum Chromodynamics

Phys. Rev. D40:3568,1989;

Hidden Strangeness in the Proton? Determination of the Real Part at the Isospin-Even Forward-Scattering Amplitude of Pion-Nucleon Scattering at $5.43 \mathrm{MeV}$

Phys. Rev. D36:3529,1987;

Analyzing Power for $\pi^{-} p$ Charge Exchange and a Test of Isospin Invariance up to $\eta$ Threshold

Phys, Rev. D38:3365,1988;

$\pi^{-} p$ Charge-Exchange Aralyzing Power from 547 to $687 \mathrm{MeV} / \mathrm{c}$

Phys. Rev. Lett. 58:2023,1987;

Limit on $\bar{\nu}_{e}$ Mass from Free Molecular Tritium $\beta$ Decay

Nucl. Phys. A434:573C,1985;

Anomalons?

Phys. Rev. Lett. 58:855.1987

Polarization and Magnetic Moment of the $T^{+}$Hyperon

Phys. Rev. D38:1365, 1988; SLAC-PUB-4573; DESY-88-033:

Formation of Paeudoscalars $\pi^{0}, \eta$ and $\eta^{\prime}$ in the Regction $\gamma \gamma \rightarrow \gamma \gamma$

Phys. Lett. 216B:11,1989;

Pion Double Charge Exchange above the $\Delta\left(1232 P_{3 s}\right)$ Resonance

Phys. Lett. 229B:33,1989;

Experimental Search for $B=2, T=2$ Bound States around the $\pi$ nucleon nucleon Threshold

Phys. Rev. Lett. 59:266,1987;

Observation of Bhabha Scattering in the Centre of Mass Kinetic Energy Range 342 to $845 \mathrm{KeV}$

EFI-89-60;

A Measurement of $\epsilon^{\prime} / \epsilon$ by $\mathbf{E 7 3 1}$

Phys. Rev. C31:1699, 1985;

Inelastic Electron Scattering from ${ }^{48} \mathrm{Ca}$

Phys. Lett. 187B:179,1987;

Spin Alignment of $\rho^{0}$ Mesons Produced in Antineutrino and Neutrino Neon Charged Current Interactions

Z. Phys. C40:231,1988; MPI-PAE-EXP-EL-188;

Production of $\pi^{0}$ Mesons and Charged Hadrons in $\bar{\nu}$ Neon Charged Current Interaction.

Z. Phys. C44:175,1989; MPI-PAE-EXP-EL-201;

Production of $p^{+}, p^{-}, p^{0}, \eta, \omega$ and $f_{2}(1270)$ Mesong in Antinucleon Neon and Neutrino Neon Charged Current Interactions 
Wood 85

Wood 88

Woods 88

Wormser 87

Wormser 88

Wormser 88B

Wormser 89

Wormser 89B

Wu 85

Wu 86

Wu 87

Wu 89

Wuensch 89

Wuensch 89B

Yamamoto 85

Yamamoto $85 \mathrm{~B}$

Yamamoto 85C

Yamamoto 85E

Yamanaka 85

Yamanaka 89

Yamanaka 90

Yamauchi 85

Yamauchi 88

Yamazaki 85

Yamazaki 86

Yasumi 85

Yasumi 86

Yelton 86

Yock 86

Yokosawa 85

Yokosawa B5C

Yoshida 87

Yoshida 87B

Yoshida 89

You 89

Yuan 86

Zacek 85

Zacek 86
Phys. Rev. Lett. 54:635,1985;

Inclubive Pion Double Charge Exchange in ${ }^{10} \mathrm{O}$ and ${ }^{40} \mathrm{Ca}$

Phys. Rev. D37:3091,1988; SLAC-PUB-4374; LBL-23812;

Determination of $\alpha_{S}$ from Energy-Energy Correlation in $e^{+} e^{-}$Annihilation at $29 \mathrm{GeV}$

Phys. Kev. Lett. 60:1695,1988:

First Result on a New Measurement of $\epsilon^{\prime} / \epsilon$ in the Neutral-Kaon System

SLAC-PUB-4466;

Indications for the Decays $D_{S}^{ \pm} \rightarrow \eta \pi^{ \pm}$and $D_{S}^{ \pm} \rightarrow \eta^{\prime} \pi^{ \pm}$

Phys. Rev. D38:1001,1988; LBL-24793; SLAC-PUB-4536;

Observation of $J / \psi(1 S)$ Production in $e^{+} e^{-}$Annihilation at $29 \mathrm{GeV}$

Phys. Rev. Lett. 61:1057,1988; LBL-25072; SLAC-PUB-4554;

$\eta$ and $\eta^{\prime}$ Production in $e^{+} e^{-}$A nnihilation at $29 \mathrm{GeV}$ : Indications for the $D_{S}^{ \pm}$Decays into $\eta \pi^{ \pm}$and $\eta^{\prime} \pi^{ \pm}$

LAL-89-10;

Selected Topics from NA14: Charm Production and New Resulte on $D_{S}^{ \pm}$Decays

LAL-89-27;

NA 14' Regults on $D_{S}^{ \pm}$Decays

Phys. Lett. 162B:227,1985; Print-85-0829;

Majorana Neutrino and Lepton Number Nonconservation in ${ }^{48} \mathrm{Ca}$ Nuclear Double $\beta$ Decay

DESY-86-007;

Search for Supersymmetric Particles at PEP and PETRA

DESY-87-164;

$e^{+} e^{-}$Interaction at High Energies

Phys. Rev, D41:2339,1990; SLAC-PUB-5128; LBL-27924:

Radiative $\tau^{ \pm}$Production and Decay

UR-1107; ER-13065-574;

Laboratory Limits on Axion: and Other Weakly Coupled Nearly Massless Particles

Phys. Rev, D40:3153,1990; FERMILAB-PUB-89-185-E;

Reault: of a Laboratory Search for Cosmic Axions and Other Weakly-Coupled Light Particles

Phys. Rev. Lett. 54:522,1985;

Charged $D^{*}(2010)$ Produ son in $e^{+} e^{-}$Annihilation at $29 \mathrm{GeV}$ and a Limit on $D^{0} \bar{D}^{0}$ Mixing

Phys. Rev. D32:2901,1985;

Measurement of $D^{0}$ Lifetime in $e^{+} e^{-}$Annihilation at High Energy

CALT-68-1318;

A Study of Charged $D^{*}(2010)$ Mesons Produced in $e^{+} e^{-}$Annihilation at $E_{\mathrm{cm}}=29 \mathrm{GeV}$

LBL-20749;

Hadron Production at PEP and PETRA

Phys. Rev. D34:85,1986; KEK-85-72;

Search for Right-handed Currents in the Decay $\boldsymbol{K}^{+} \rightarrow \mu^{+} \nu_{\mu}$

FERMILAB-CONF-89-35-E;

A CP Violntion and Rare Kaon Decay Experiment at Fermilab

FERMILAB-CONF-9n-14-E;

$A$ New Meanurement of $C P$ Violation Parameter $\epsilon^{\prime} / \epsilon$

KEK-85-63;

Messurement of the Vector Asymmetry in $\pi^{+}$deuteron Elastic Scattering at $0.74 \mathrm{GeV} / c$

KEK-88-63;

Recent Result from TOPAZ at TRISTAN

Phys. Rev. Lett. 54:102,1985; KEK-84-10;

Formation of $A=12 \Sigma^{-}$Hypernucleus from $K^{-}$Absorption at Rest, Observation of a $\Sigma^{-}$Spin Orbit Doublet of Narrow Width

Nucl. Phys. A434:363C,1985;

Formation and Structure of $\mathbf{E}$ Hypernuclei

KEK-85-45;

Mensurement of the Mass of the Electron Neutrino Using Electron Capture in ${ }^{103} \mathrm{Ho}$

Phys. Lett. 181B:169,1986; KEK-86-70;

The Mass of the Electron Neutrino Using Electron Capture in ${ }^{109}$ Ho

Phys. Rev. Lett. 56:812,1986;

Measurement of the Branching Fractions $\tau^{-} \rightarrow \rho^{-} \nu_{\tau}$ and $K^{*}(892)^{-} \nu_{\tau}$

Phys. Rev. D34:698,1986;

Heavy Conmic Rays at Ses Level

ANL-HEP-CP-85-18;

Summary of $S=0$ Dibaryon Resonances and Candidates

ANL-HEP-CP-85-93; C85/08/26;

Polarization Phenomena in Nucleon-Nucleon Scattering at Intermediate and High Energies Including the Present Status of Dibaryons

Phys. Rev. Lett. 59:2915,1987; KEK-87-82; KOBE-HEP-87-04; KUNS-894; OULNS-87-6; TMUP-HEL-87-23;

A. Search for Sequential Heavy Leptons at the Energy $\sqrt{8}=52$ GoV at TRISTAN

Phys. Lett. 198B:570,1987; KEK-87-81; KOBE-HEP-87-03; KUNS-893; OULNS-87-05; TMUL-HEL-87-22;

Measurement of $\boldsymbol{R}$ and Search for New Heavy Quarks in $e^{+} e^{-}$Annihilation at 50 and 52 GeV Centre-ofMasa Energies

Phys. Rev, D39:3516,1989;

High Resolution Measurement of Messive Dielectron Production in 800 GeV Proton Beryllium Collisions BIHEP-EP-89-1;

A. Search for Neutrinoless Double $\beta$ Decay of ${ }^{48} \mathrm{C}$

Phys. Rev. Lett. 57:1680,1986;

Measurement of Parity Nonconservation in the Proton Proton Total Cross Section at $800 \mathrm{MeV}$

Phys. Lett. 164B:193,1985;

Improved Limits on Oscillation Parameters from $\bar{\nu}_{q}$ Disappearance Measurements at the Goesgen Power Reactor

Phys. Rev. D34:2621, 1986;

Neutrino Oacillation Experiments at the Goesgen Nuclear Power Reactor 
Zacek 86B

Zajmidoroga 85

Zapalac 86

Zatsepin 89

Zdesenko 85

Zdesenko 86

Zelinski 86

Zelinski 88

Zhang 88

Zheng 90

Zhokin 89

Ziegler 85

Ziegler 88

Zielinsky 86

Zielinsky 88

Zlatanov 89

Zybalov 88

Zybalov 90

Zybalov 90B
Nuovo Cim. 9C:516,1986;

Search for Neutrino Oscillations at the Nuclear Power Reactor in Goesgen

JINR-P1-85-616; The $a_{1}(1260)$ Meson Production in Nuclear Coulomb Field and $a_{1}(1260) \rightarrow r \gamma$ Radiative Decay Width Estimation

Phys. Rev. Lett. 57:1526,1986; FERMILAB-PUB-86-96-E;

$A$ Measurement of the $\Sigma^{-}$Magnetic Moment Using the $\Sigma^{-} \rightarrow n e^{-} \bar{\nu}_{e}$ and $\Sigma^{-} \rightarrow n \pi^{-}$Decay Mode*

Yad. Phys. 49:426,1989;

Study of Muon Inelastic Scattering with 100-ton Scintillation Detector of the INR Artyamovak Scientific Station

Yad. Phys. 43:1065,1986; INRU-85-31;

Results of Underground Experiment on Noutrinoless Double $\beta$ mecay of ${ }^{78} \mathrm{Ge}$

Pisma Zh. Eksp. Teor. Fiz. 43:459,1986;

Experimental Limitations of Double Electron Capture Probability at ${ }^{106} \mathrm{Hg}$

Yad. Phys. 43:791,1986;

Momentum Characteristics of Spectatora in ${ }^{4}$ He-Nucleus Fragmentation Processe.

Yad. Phys. 47:744,1988;

Azimuthal Correlations and Deuteron Production in Proton Interactions with Light Nuclei

Phys, Rev. Lett. 61:385,1988;

Experimental Limit on the Flux of Relic Antineutrinos from Past Supernovae

KEK-90-5; AMY-90-02;

Charged Hadron Multiplicities in $e^{+} e^{-}$Annihilation at $\sqrt{s}=50$ to $61.4 \mathrm{GeV}$

ITEP-89-80;

Binary Reaction of Baryon Exchange

Phys. Rev. C32:301,1985; TRI-PP-85-9;

Polarized Proton Induced Pion Production on ${ }^{10}$ Bor at $200 \mathrm{MeV}, 225 \mathrm{MeV}, 250 \mathrm{MeV}$ and $260 \mathrm{MeV}$ Incident Energies

Phys. Lett. 206B:151,1988;

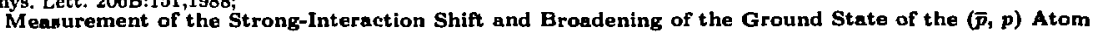

z. Phys. C31:545,1986;

Experimental Constraints on $J^{P C}=1^{-+}, I=1$ Hybrid Mesons

UUPP-TSL-ISV-88-16;

Enhancements in Two-Nucleon Invariant Masses: A Survey of Experimental Data

JINR-PJ-89-64;

An Impact Parameter Analysis of Antiproton-Proton Elastic and Inelastic Scattering at $22.4 \mathrm{GeV} / \mathrm{c}$

Yad. Phys. 47:1505,1988;

Proton Polarization in $\gamma^{5} \mathrm{He}\left({ }^{4} \mathrm{He}\right) \rightarrow p$ (polarized) $\mathrm{X}$ Reactions and Quasideutron Model

Yad. Phys. 51:32,1990;

Proton Polarization in Deuterium Photodisintegration at $E=200-350 \mathrm{MeV}$

Yad. Phys. 51:609,1990

A Measurement of Inclusive Proton Polarization in Reaction $\gamma$ nucleus $\rightarrow p \mathrm{X}$ on Nuclei ${ }^{6} \mathrm{Li},{ }^{9} \mathrm{Be},{ }^{12} \mathrm{C},{ }^{27} \mathrm{Al}$ 
This index lists papers by beam, target, and beam momentum. The ordering is by beam mass, then target mass, then beam momentum. For a given beam momentum, ID's are ordered by year (most recent to oldest). then author name. For the full reference, see the ID/Reference/Title lndex.

In most cases, we give both the equivalent lab momentum for scattering on a fixed target and the c.m. energy $E_{\mathrm{cm}}$, the latter in parentheses. However, for colliding beam experiments, we usually only give $E_{\mathrm{cm}}$ : we also give only $E_{\mathrm{cm}}$ for reactions above $2 \mathrm{TeV} / c$ equivalent lab moment um. When a range of momenta are studied, we list the lower and upper ends of the range, e.g. " $50-70$," ordered by the lower end of the range. For some experiments: such as neutrino experiments, the listed range is only approximate.

A question mark means that the indicated information is nissing from the database. usually because it was not given in the paper.

\section{Illustrative Key}

Beam and Target: see the Particle Vocabulary for nomenclature.

Lab Momentum: in $\mathrm{GeV} / \mathrm{C}$ (not listed for colliding beam experiments).

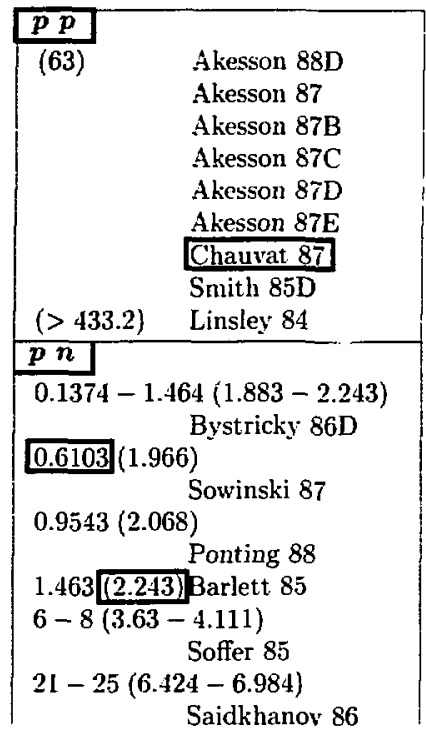

Document ID: see the $I D / R e-$ ference/Title Index for the full reference.

CM Energy $\boldsymbol{E}_{\mathrm{cm}}$ : in $\mathrm{GeV}$, in parentheses. 


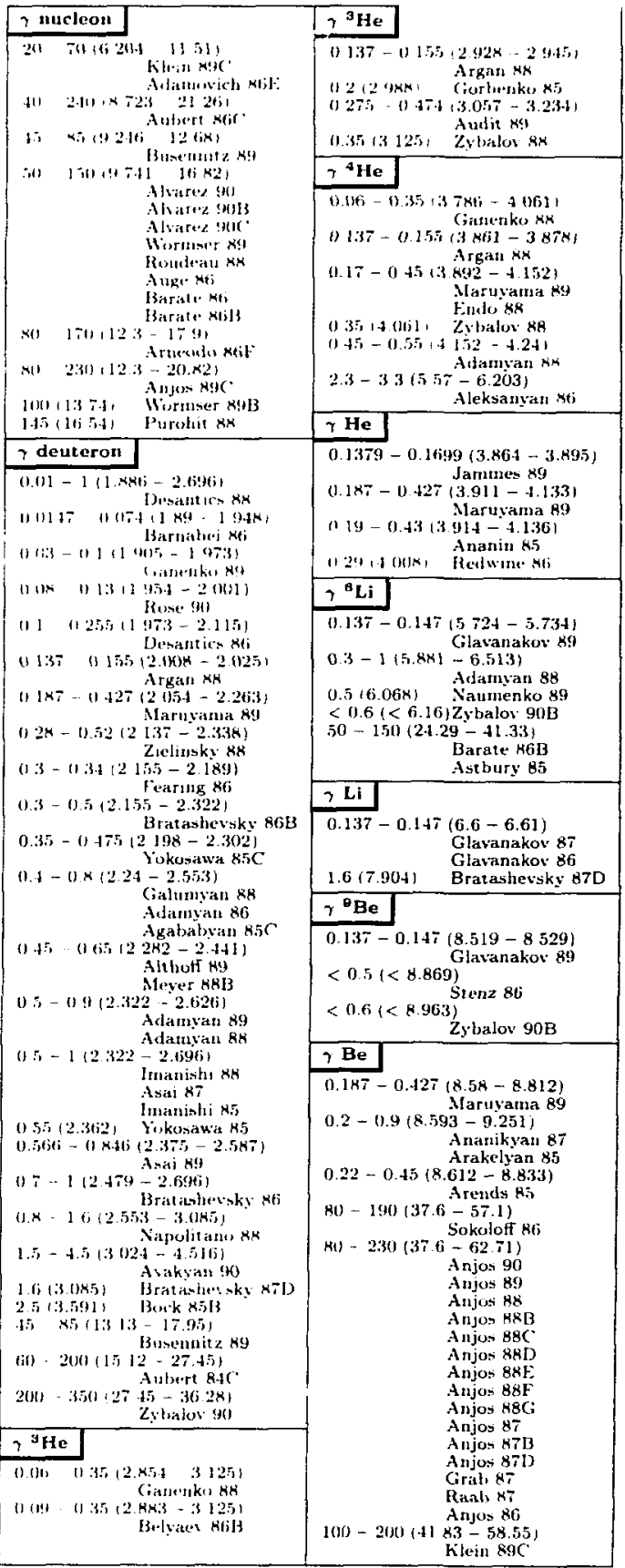




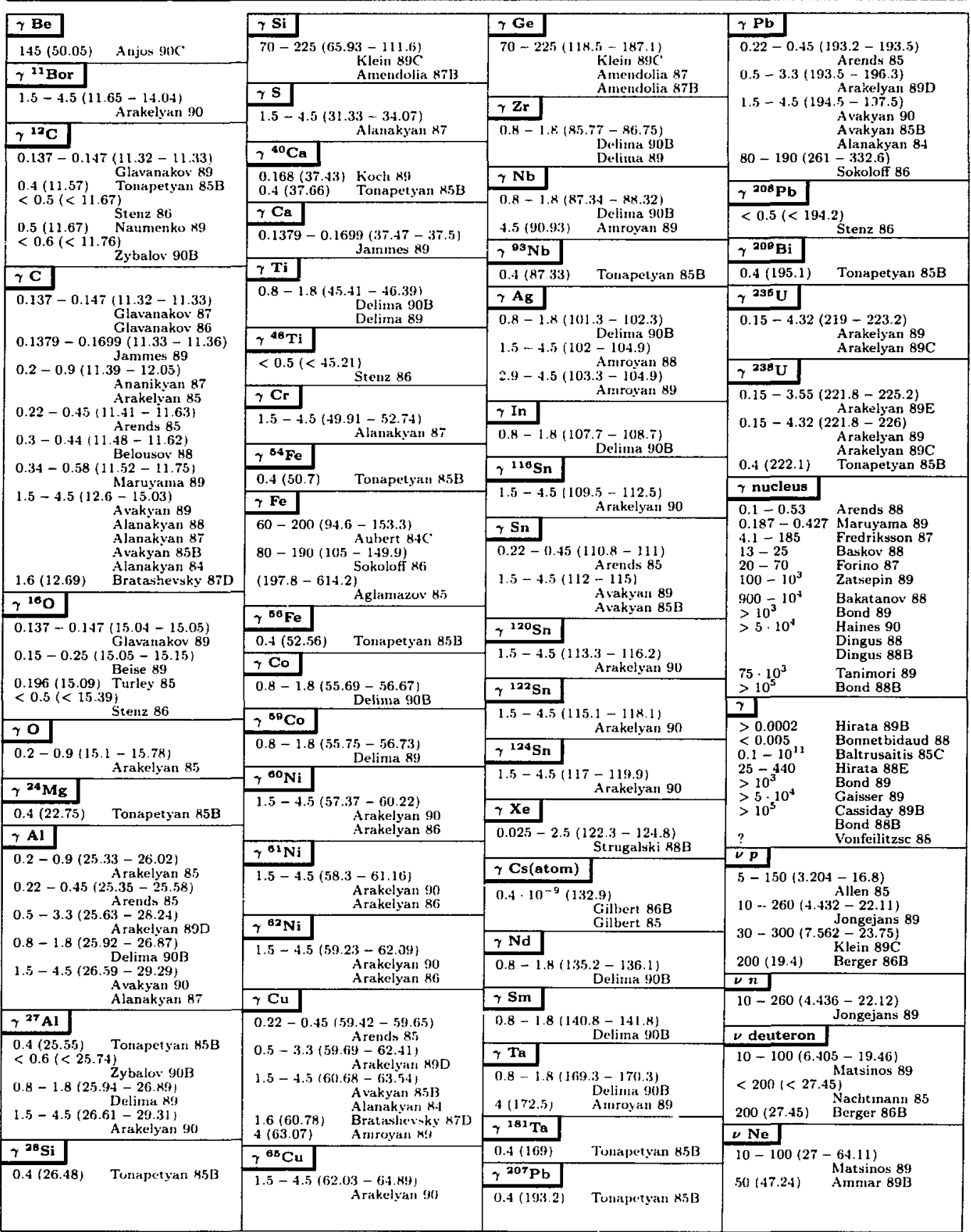

Entries in order of bean mass, then target mans, then bean monent um. ('ertain chemical symbols for nuclei have been changed to ayoid ambiguity with particle names. (See the Particle Vucalsulary.) Bean momeat a are equivaleut pub in GeV/r: then $E_{c m}$ in GeV follows in parentheses. For certain initial states only $E_{t}$ lin parentheses) is gives. See the legend on page 123. 


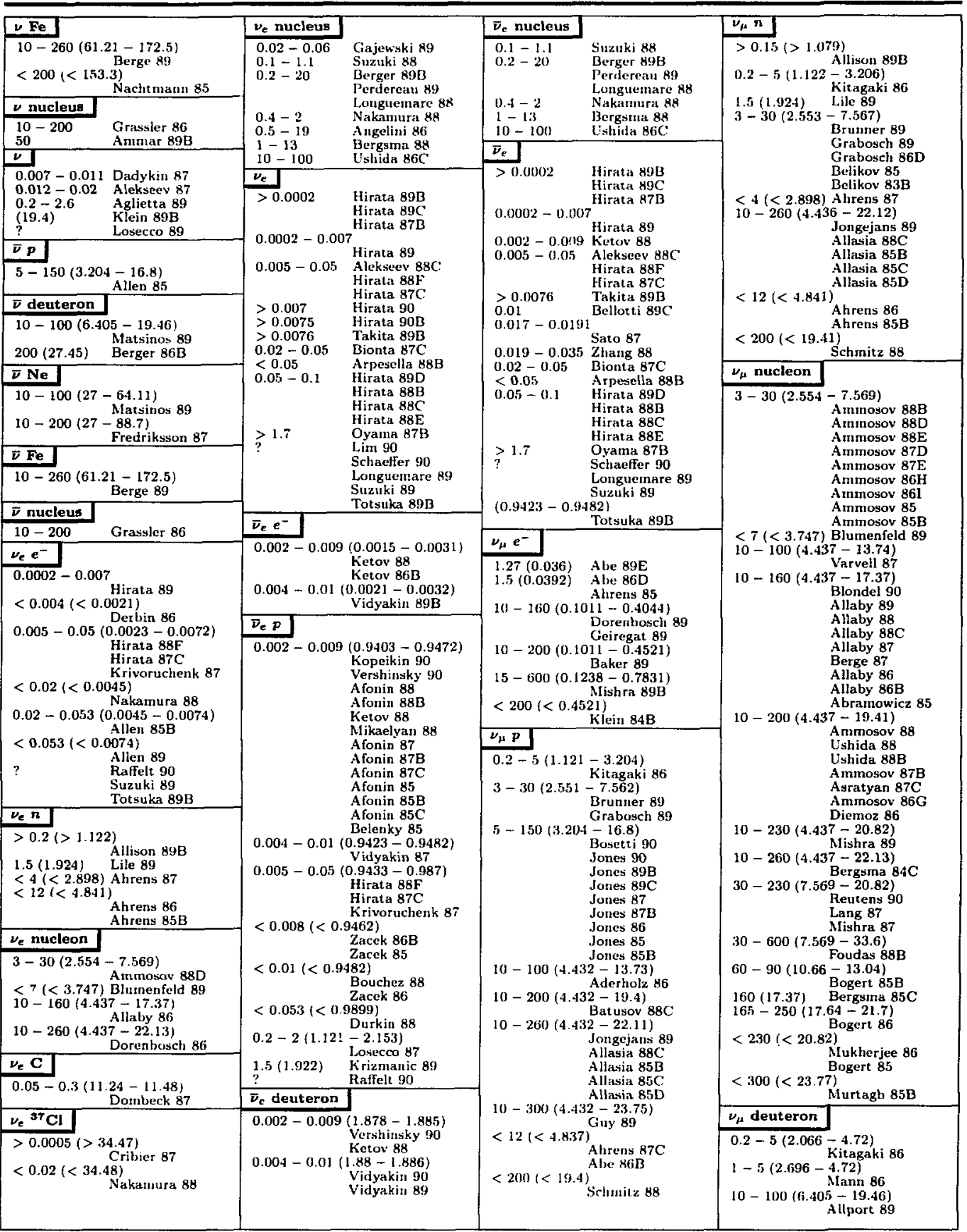




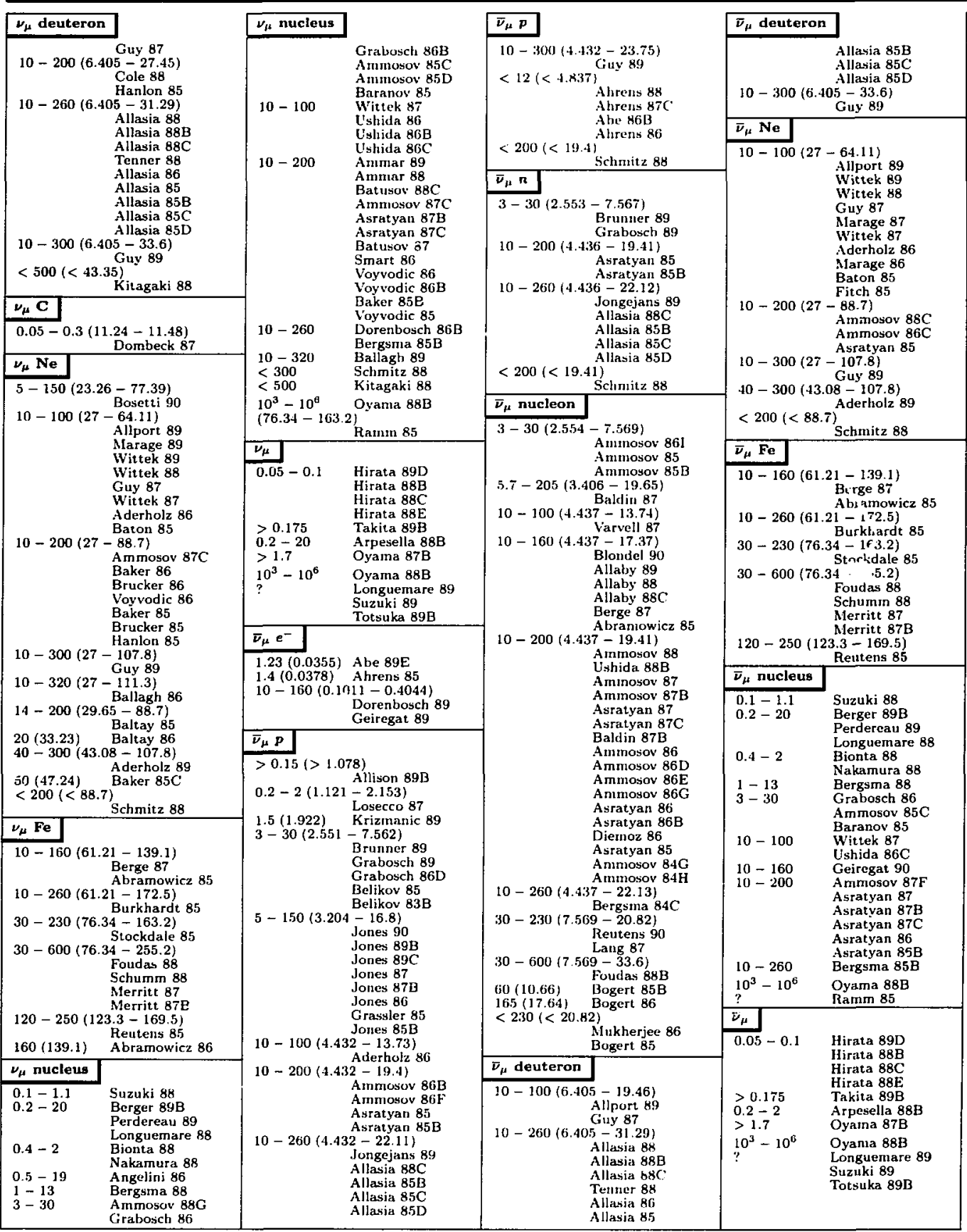

Entrics in order of bearn mass, then target mass. then beam momentum. Certain chemical symbols for nuclei have been changed to avoid ambiguity with particle nanes. (See the Particle Vocabulary.) Beam mornentat are eriuivalent ptub in GeV/ $r$ : then $E_{\mathrm{c} \text { an }}$ in GeV follows in parentheses. For certain initial states only $E_{\mathrm{crn}}$ (in parentheses) is given. See the legernd un page 123 


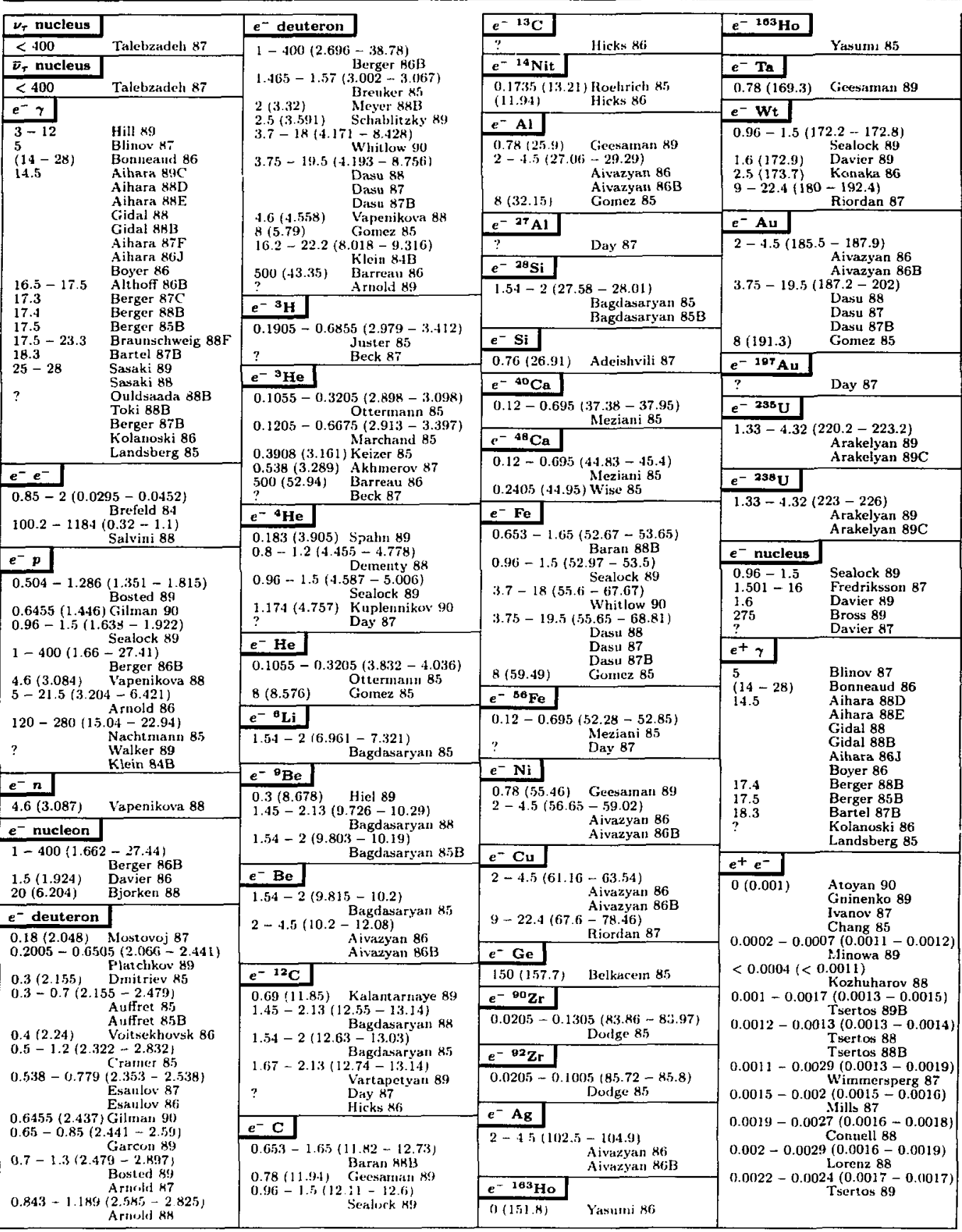




\section{$e^{+} e^{-}$}

$<0.0056$ ( $<0.0025)$ Klinken 88 $0.69-0.94(0.0266-0.031)$ Ajaltouni 85B

$9.784-1075(0.1-1.048)$ Druzhinin 84

$10(0.1011)$ Albrecht 85 औ $15-10^{3}(0.1238-1.011)$

29 (0.1722) Kolanoski 87

$50-5 i(0.2261-0.2414)$ Kim 89B

$126.8-1918(0.36-1.4)$

244.6 - $978.5(0.5-1)$ Dolinsky 88B

244.6 - $1079(0.5-1.05)$ Dolinsky 89

$244.6-1918(0.5-1.4)$ Dolinsky 89B Vorobiev $88 \mathrm{C}$ A ulchenko 86 Vasserman $86 \mathrm{~B}$

$400.8-1918(0.64-1.4)$ Kurdadze 88 Kurdadze 86 $426.2-883.1(0.66-0.95)$

$426.2-1918(0.66-1.4)$ Bukin 89

$535.8-648.3(0.74-0.814)$ Aulchenko 87

$565.2-626.2(0.76-0.8)$ Dolinsky 88 Barkov 87

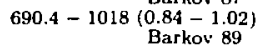

706.9 - $988.3(0.85-1.005)$

$(0.9-38) \quad$ Klein $84 \mathrm{~B}$

$978.5-1079(1-1.05)$

Landsberg 86

Druzhinin 85

Goluber 85

$978.5-1918(1-1.4)$ Golubev 87 Dolinsky 86 Bondar 84

978.5 - $1079(1-1.05)$ Golubev 86 Vasserman 86

1016 - $1926(1.019-1.403)$

Barkov 88

1058 - 1918 (1.04 - 1.4) Aulchenko 86C

$1079-1918$ (1.05 - 1.4) Aulchenko 86B Druzhinin 86 Dolinsky 85

$1603-1918(1.28-1.4)$

1654 (1.3) Aulchenko 87B

(1.35-2.4) Bisello 89B Antonelli 88 Bisello 88B

$(1.4-2) \quad$ Courau 86

$(1.4-2.2) \quad$ Luca 85

$(1.9-2.4) \quad$ Castro 88

$(2.236-44.72)$

(<2.4) Sarshall 89

$(3-3.2) \quad$ Bisello 88
Tixier 88

$\begin{array}{ll}(3-5) & \text { Schindler } 87 \\ (3.095-29) & \text { Juricic } 88\end{array}$

(3.1) Baltrusaitis 85E

(3.45)

Balt rusaitis 8 .
Ajaltouni 87

(3.5-7.2) Barish 88

(3.0) Blinov $88 \mathrm{~B}$

Blinov 8613

Blinov B5C

(3.77)

Coffnan 87

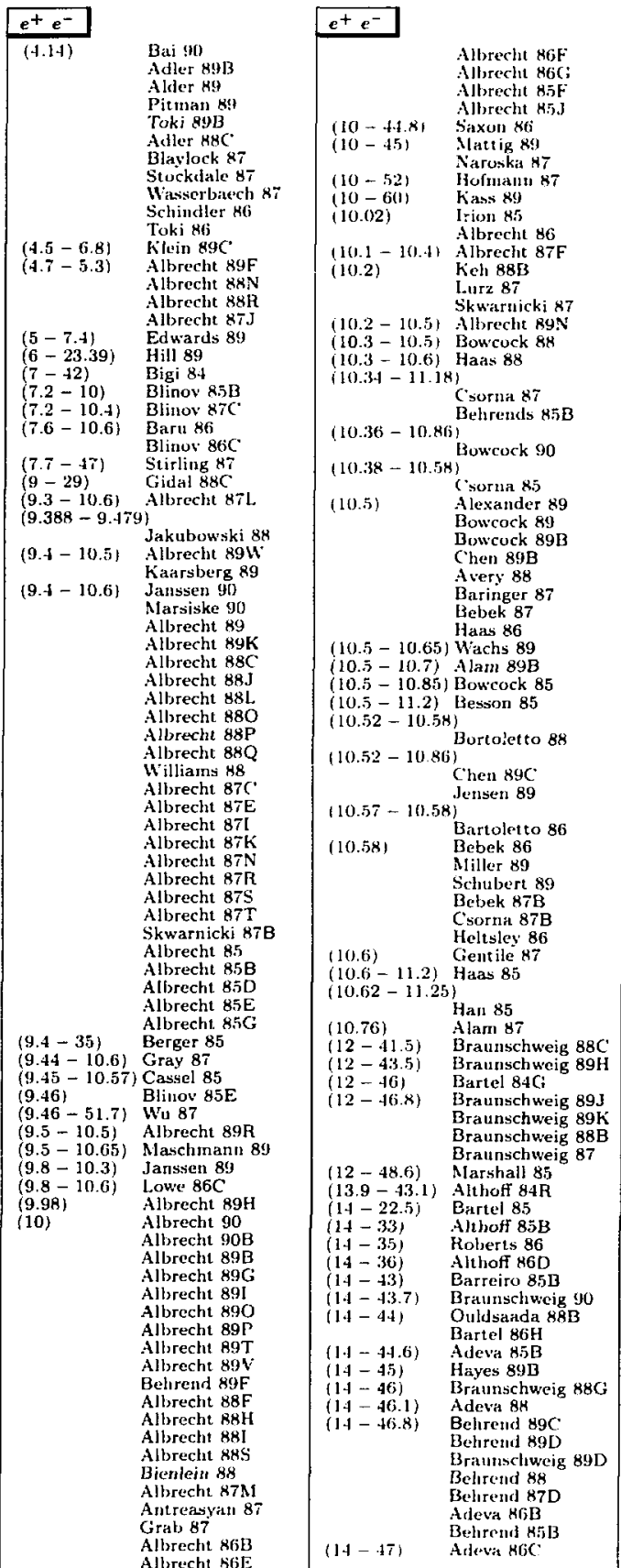

$e^{+} e^{-}$

$(15-45)$
$(17)-17.5)$
$(17-3.7)$
$(17-3)$
$(17.3)$
$(17.5)$
$(22-38)$
$(22-46.7)$
$(22-46.78)$
$(22-56)$
$(27)$
$(27-37)$
$(28-468)$
$(29)$

Bartel $85 \mathrm{~F}$

Gato 88

Althoff 86

Braunschuejg 90B

Collius 85E

Bartel 85B

Behrend $\mathrm{B8E}$

Bthof 85D

Braunschweig 89E

Bethike $8 \mathrm{~s}$

Adeva $85 \mathrm{C}$

Bet like $88 B$

Aihara 86B

Bartel 85E

89G

Petradra 90

Abachi 89

Abachi $89 B$

Abachi 89D

Aihara 89

Aihara $89 \mathrm{~B}$

Aihara 89C

A verill 89

Avery 89

Band 89

Bethke 89

Bethke 89B

Ford 89

Hawkins 89

Haw-kins 89B

Hurst 89

Klein 89

Komamiya 89

Komamiya $89 \mathrm{~B}$

Ong 89

Petradza 89

Porter 89

Riles 89

Riles $89 \mathrm{~B}$

Roe 89

Roe 89B

Snyder 89

Steele 89

Wagner 89B

Weir 89

Wu 89

Abachi 88

Abachi 88B

Abachi $88 \mathrm{C}$

Aihara 88

Aihara $88 \mathrm{~B}$

Aihara 88C

A ihara $88 \mathrm{E}$

Aihara $88 \mathrm{~F}$

A kerlof 88

Amidei 88

Band 88

Baringer 88

Cowan 88

Edberg 88

Gidal 88

Gidal 88B

Hearty 88

Karlen 88B

Karlen 88C

Klein 88

Mathis 88

Oung 88

Ong 88B

Petersen 88

Riles 88

Tschirhart 88

Wood 88

Wormser 88

Wormser 88B

Abachi 87

Abachi 87B

Abachi 87C

Abachi 87D

Abach $\mathrm{B} 7 \mathrm{E}$

Abachi $87 F$

Aihara 87

A ihara $87 \mathrm{C}$

Aibara B7D

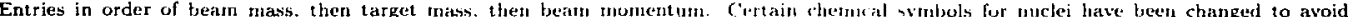

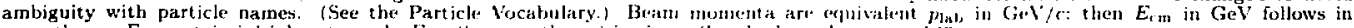

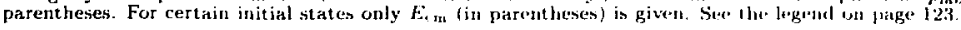


Aihara $87 \mathrm{E}$

Aihara 87E

Aihara 870

Ash 87

Ash 87B

Band 87

Brom 87

Bylsma 87

Camporesi 87

Derrick 87

Derrick 87B

Derrick 87C

Fernandez 87

Fernandez 87B

Fernandez 87C

Ford 87

Ford $87 \mathrm{~B}$

Ford $87 \mathrm{C}$

Gan 87

Gan 87B

Hearty 87

Johnson 87

Klein 87

Klein 87B

Low 8 ?

Ong 87

Riles 87

Rouse 87

Wagner 87

Wendt 87

Wormser 87

Abachi 86

Abachi 8CB

Abachi 86C

A bachi 86D

Aihara 86

Aihara $86 C$

Aihara 86D

Aihara 86E

Aihara $86 \mathrm{~F}$

Aihara 86G

A ihara $86 \mathrm{H}$

A ihara 861

Baden 86

Baringer 86

Bartha 86

Boyer 86

Burchat 86

Burchat 86B

Derrick 86

Derrick 86B

Derrick 86C

Derrick 86D

Ford 86

Gladney 86B

Gold 86

Hollebeek 86

Johnson 86

Jung 86

Klem 86

Madaras 86

Pal 86

Perl 86

Petersen 86C

Ruckstuhl 86

Schmidke 86

Sheldon 86

Sugano 86

$W_{u} 86$

Yelton 86

Aihara 85

Aihara $85 \mathrm{~B}$

Aihara $85 \mathrm{C}$

Aihara 85D

Aihara 85E

Aihara $85 \mathrm{~F}$

I lara $85 \mathrm{C}$

Akerlof 85

Akerlof 85B

Ash 85B

Ash 85C:

Ach $85 \mathrm{D}$

Baitel 85G

Beltrami 85

\begin{tabular}{|c|c|c|c|}
\hline$e^{+} e^{-}$ & & $e^{+} e^{-}$ & \\
\hline $\begin{array}{l}(29-34) \\
29-35) \\
(29-37) \\
(29-37.5) \\
(29-42.6 \\
(29-43.9) \\
(29-44) \\
(29-65) \\
(29.9-46.78) \\
(30-35) \\
(30-40) \\
(30-46.7) \\
(30-46.78) \\
(32-46.78) \\
(33-3.5) \\
(33-36.6) \\
(33-37.5) \\
(33-46.8) \\
(34) \\
(34-35) \\
(34-44) \\
(34.2) \\
(34.5) \\
(34.57-43.71 \\
(34.6)\end{array}$ & 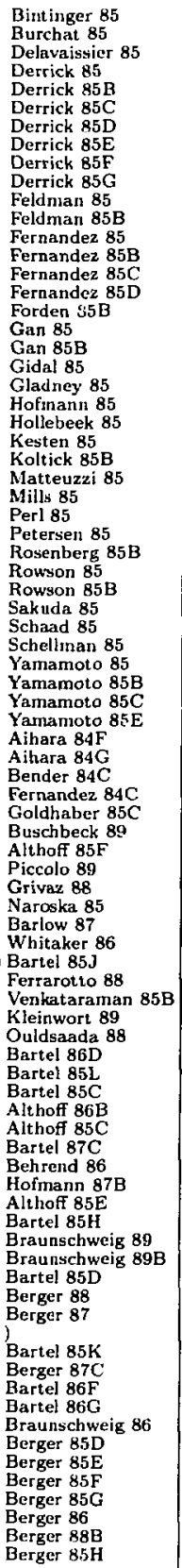 & 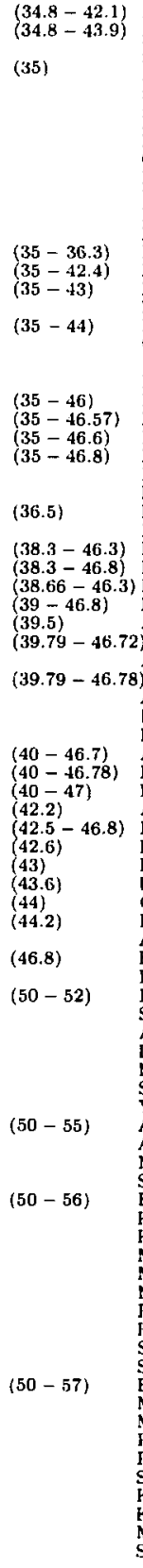 & 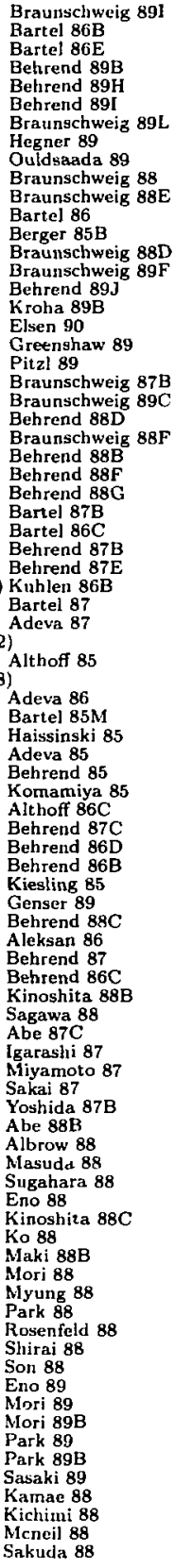 \\
\hline
\end{tabular}

$e^{+} e^{-}$

Yamauchi 88

4heng 90

Sakai 90

Sakai 90

Abe $89 \mathrm{P}$

Bodek 89

Eno 89B

Eno 89C

Kim 89C

Kim $89 \mathrm{E}$

Kim 89G

Kinoshita 89P

Kurihara 89

Low 89

Sakai 89

Shaw 99

$(50-61.4) \quad$ Adachi 90B

Ho 89

Iwasaki 89

Kim $89 \mathrm{~F}$

Kumita 89B

Maki 89

Myung 89

Adachi 88

Adachi 88B

Adachi $88 \mathrm{C}$

A be 87

Adachi 87

Amako 87

Yoshida 87

(52 - 55) Adachi 88D

$(52-56) \quad$ Bacala 88

Kim 88B

Kim 88C

Tauchi 88

(52 - 57) Adachi 89

Li 89

Li $89 \mathrm{~B}$

Metcalf 89

Sagawa 89

Bacala 88B

Takahashi 88

$(52-60.8) \quad$ Adachj 89B

Adachi 89D

Ogawa 89

(52 - 61.4) Adachi 90C

A be $89 \mathrm{~K}$

(53.3 - 59.5) Adachi 89C

$(54-61.4)$ Abe 90

Abe $90 \mathrm{C}$

Abe 891

(54.5) Olsen 88

$(55-56) \quad$ Sumiyoshi 88

$(55-57) \quad$ A.be $89 \mathrm{~F}$

Unno 88

$(55-60.8) \quad$ Adachi 90

Abe 89J

Odaka 89

(56)

Abe 88D

Abe $88 \mathrm{E}$

Kim 88

Kim 88D

Maki 88

$(56-57) \quad$ Abe $88 \mathrm{~F}$

(56.5-60.8) Adachi 89E

$(58.5-61.4)$ Kumita 89

(60.8) Fry 89

(89.2 - 93) Abrams 89D

Jung 89

(91.1) Abrams 89E

Aarnio 90

Aarnio 90B

Abreu 90

Abreu 90B

Abreu $90 \mathrm{C}$

Abreu $90 \mathrm{D}$

Abreu $90 \mathrm{E}$

Abreu 90F

Adeva 90

Adeva 90B

Adeva 90C

Adeva 90D

Akrawy 90 


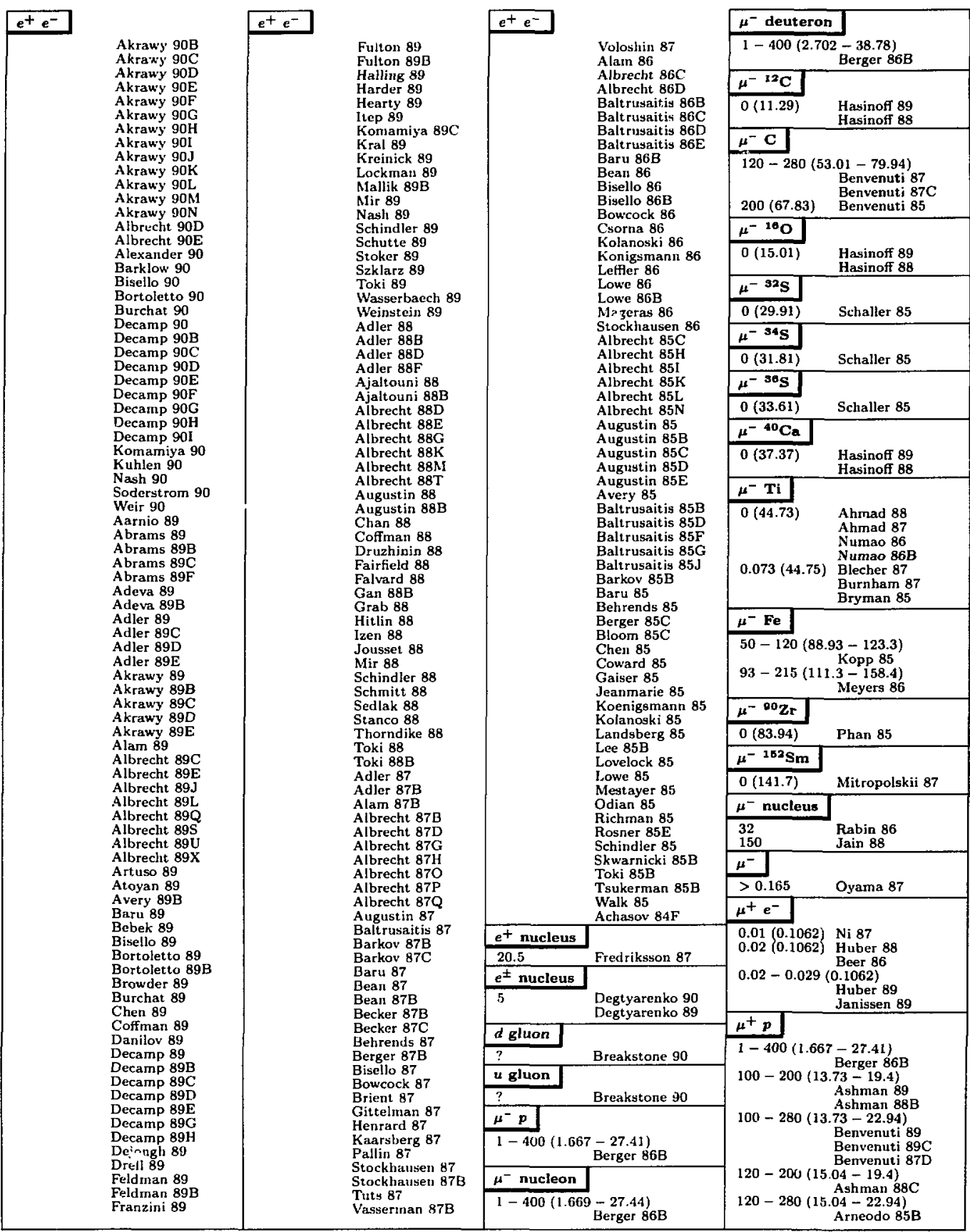




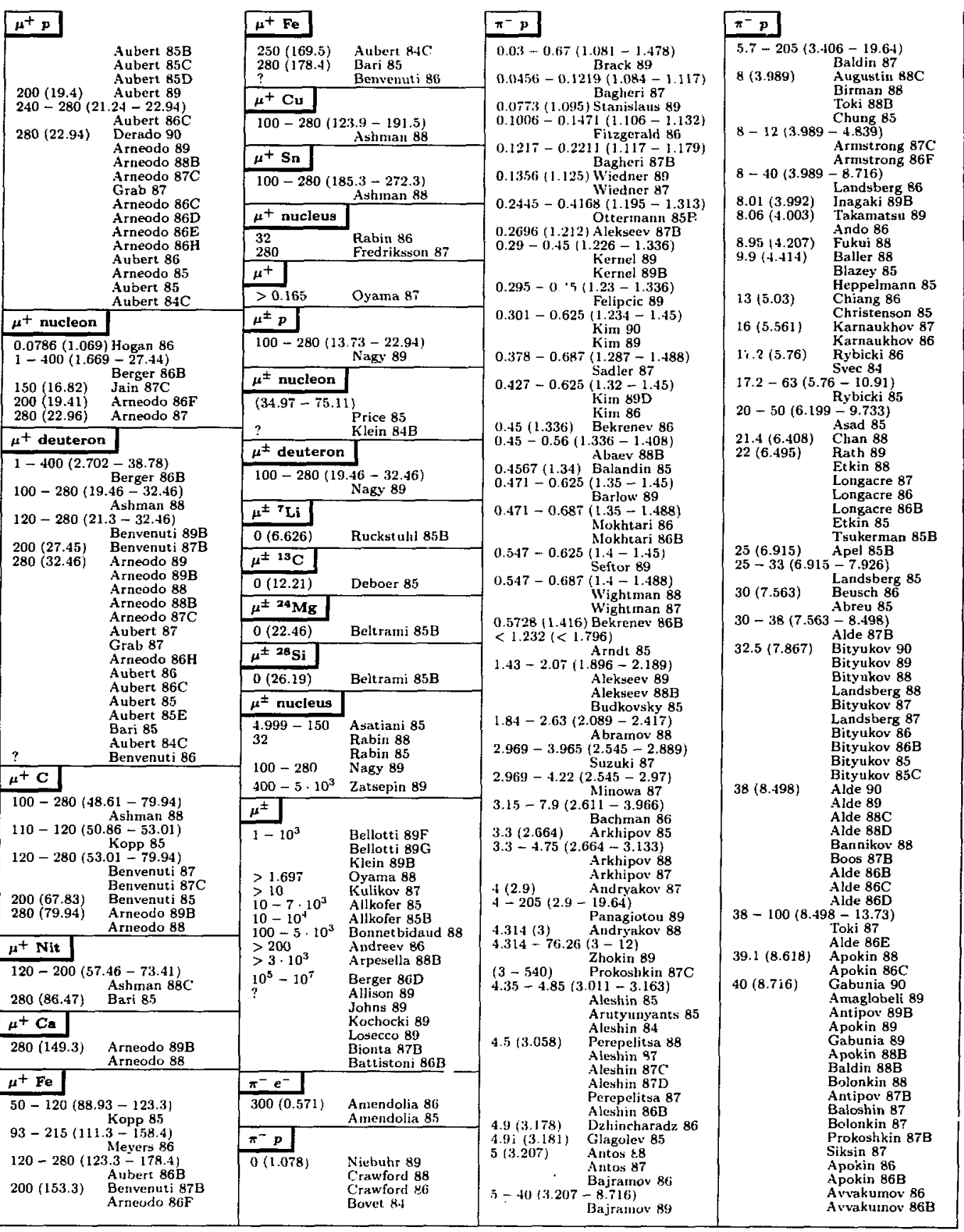




\section{$\pi^{-} p$}

Baldin 86

Baldis $86 \mathrm{~B}$

Apel 85

Apokin $85 \mathrm{~B}$

Baldin 85

Bajdin 85B

Grishin 85

Grishin 85B

Grishin 85C

Kazarinov 85

Prokoshkin 85

Avvakumov 84

Baloshin 84

Borisov 84

$40-100(8.716-13.73)$

Prokoshkin 8\%

$40-225$ (8.716 - 20.57)

Rutherfoord 85

58 (10.48) Paub 85

$80-140(12.29-16.24)$

A psimon 90

100 (13.73) $\quad \begin{aligned} & \text { Apsimon } 89 \\ & \text { Boutemeur } 89\end{aligned}$

Alde $88 \mathrm{E}$

Boutemeur 88

Iddir $\mathbf{8 8}$

Alde $87 \mathrm{D}$

Alde 86

Augustin $85 \mathrm{E}$

Tannenbaum 89

Becker 87

Kennett 87

Arenton 86

Naudet 86

Arenton 85B

250 (21.68) Adamus 878

$280(22.94)$ Bonvin 90

Bonesini 89

Bonesini 89B

Bonvin 89

Bonesini 88

Bonesini 87

$280-300(22.94-23.75)$

Lancon 86B

Demarzo $87 \mathrm{~B}$

Richard 87

Ferbel 86

350 (25.65) Albanese 85

$360(26.01) \quad$ Aguilarbenit 89

Klein $89 \mathrm{C}$

Agularbenit $87 \mathrm{E}$

Aguilarbenit $87 \mathrm{~F}$

Aguilarbenit $87 \mathrm{H}$

Bailly $87 \mathrm{C}$

Bailly $87 \mathrm{G}$

Bailly $87 \mathrm{H}$

Verbeure 87

Aguilarbenit 86

Aguilarbenit $86 \mathrm{~B}$

Aguilarbenit 86C

Aguilarbenit 86D

Bailly 86

Aguilarbenit 85

Aguilarbenit $85 \mathrm{C}$

Aguilarbenit 85D

Aguilarbenit 85E

$\mathrm{Aguilarbenit} 85 \mathrm{~F}$

(10.58) Albrow 88

$\pi-n$

$1.4-5(1.883-3.21)$

Bayukoy $85 \mathrm{~F}$

1. $84-2.63(2.09-2.419)$

A bramov 88

21 (6.353) Stopa 87

$40(8.722) \quad$ Grishin 85B

$<200(<19.41)$

Hohler 89
$<200(<19.4)$

Chapin 85

$300(23.75) \quad$ Demarzo 87

Aguilarbenit 878

Aguilarbenit $87 \mathrm{D}$

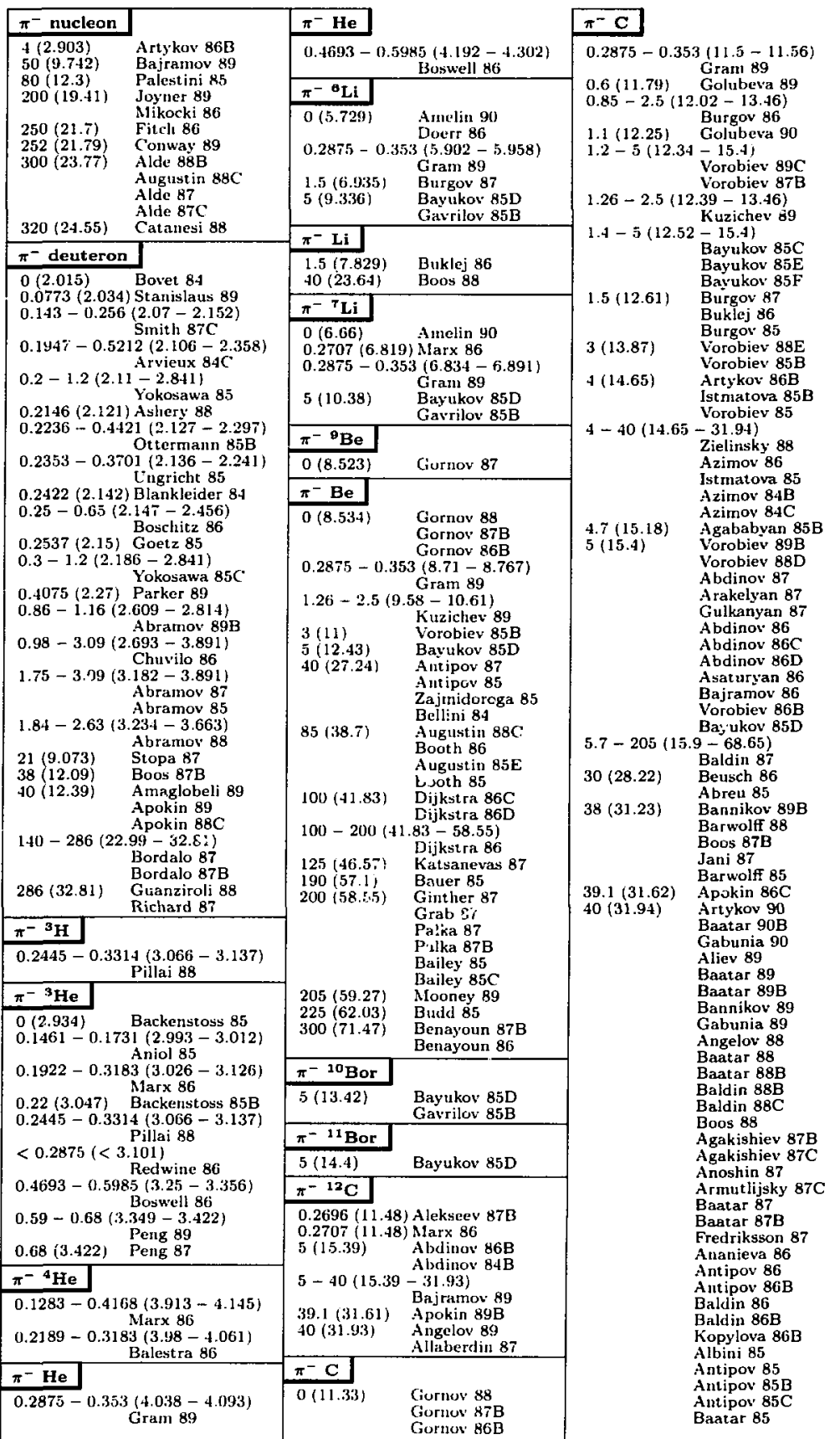

Entries in order of beam mass, then harget mass, then beam momenum. ('ortain clumical symbols for moclei hive been changed to avoid

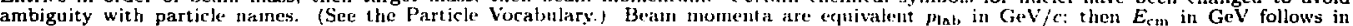
parentheses. For certain initial states only $E_{a t n}$ (in pareutheses) is given. See the legend on page 123. 


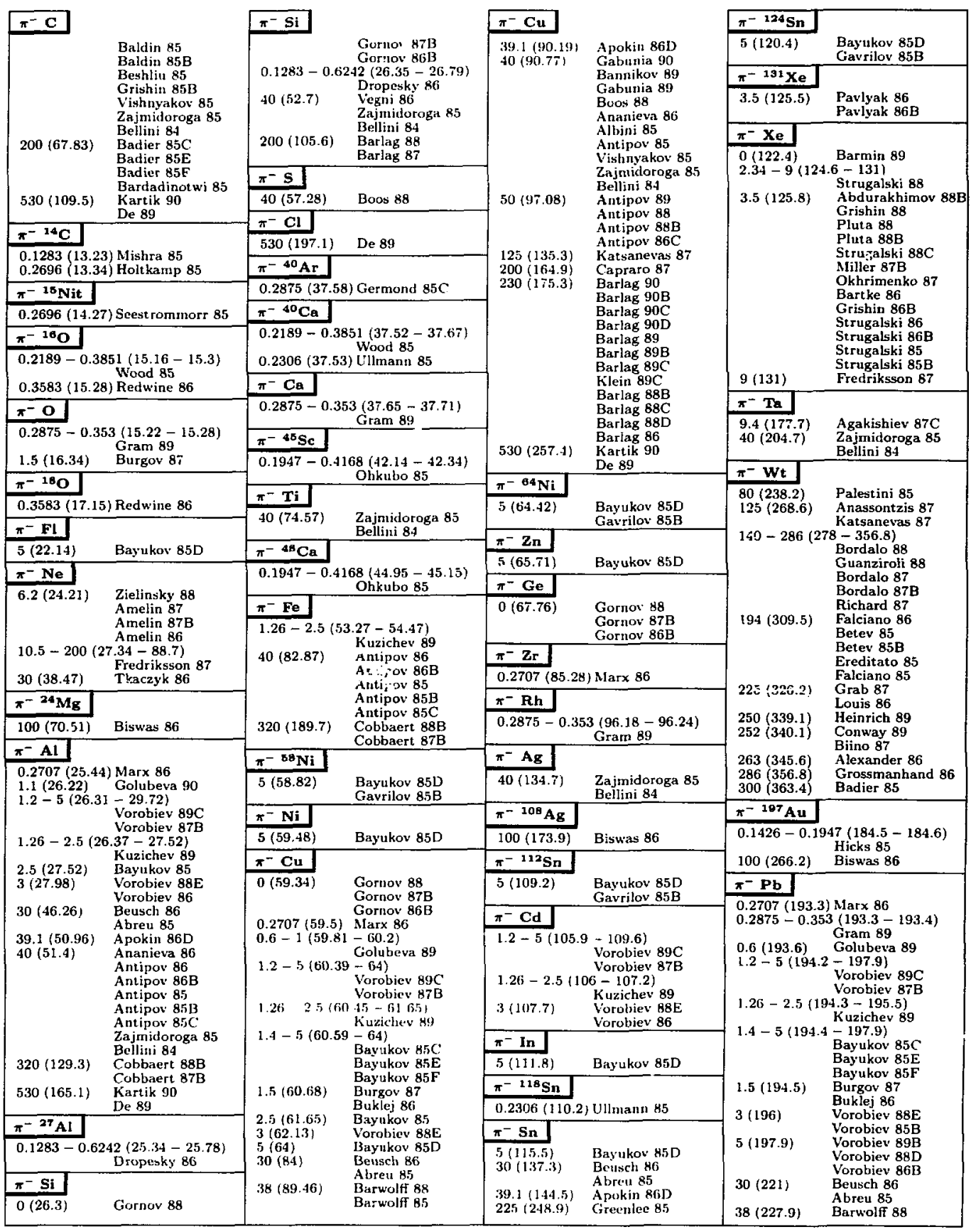




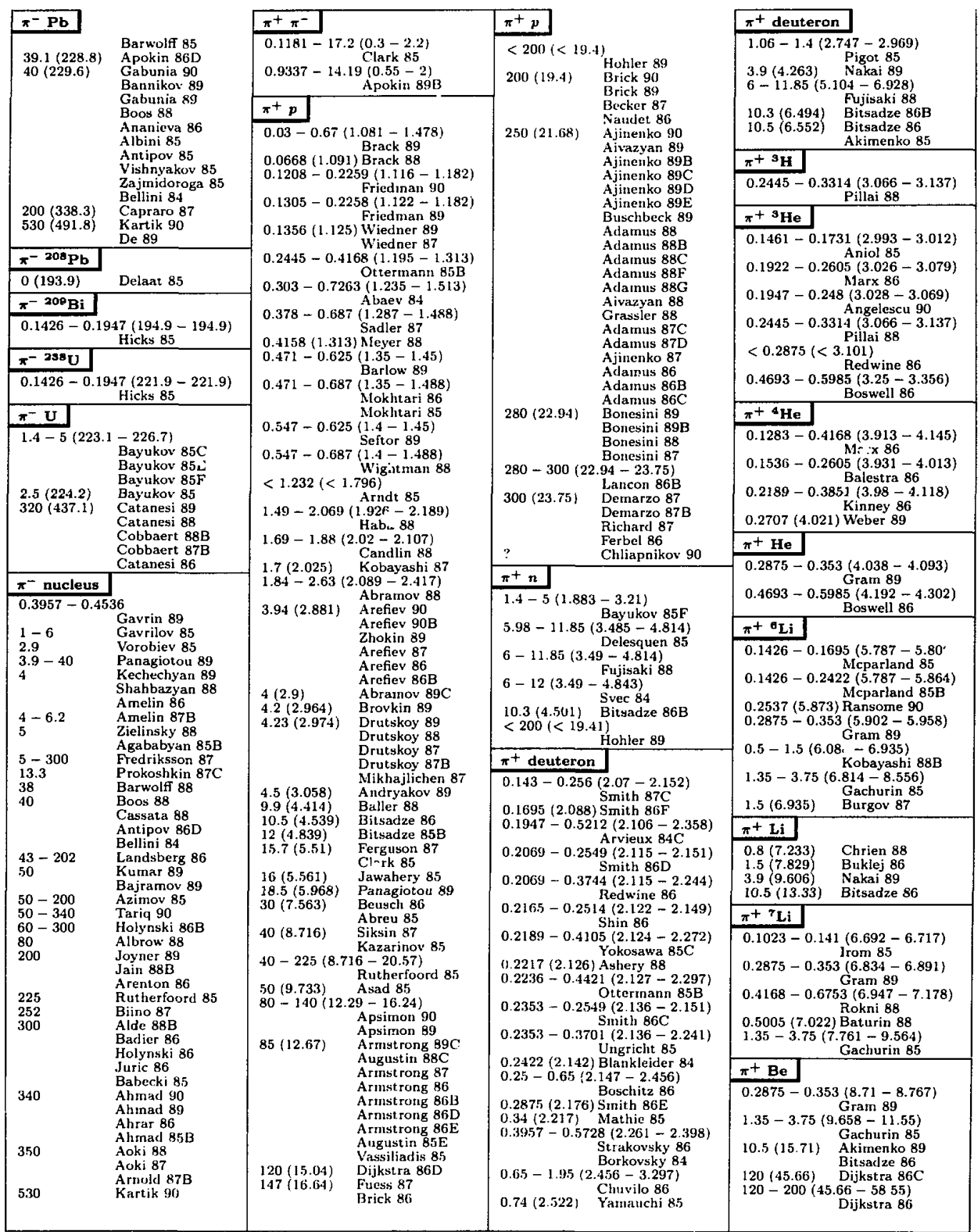

Entries in order of beam tnass, then target mass. then beam moment um. Certain chemical symbols for muclei have been changed to avoid ambiguity with particle names. (See the Particle Vocabulary.) Beam momcuta are equivalent plat in GeV/r: then $E_{c m}$ in GeV follows in parentheses. For certain initial states only $E_{\mathrm{cm}}$ (in parentheses) in given. See the legend on page 123. 


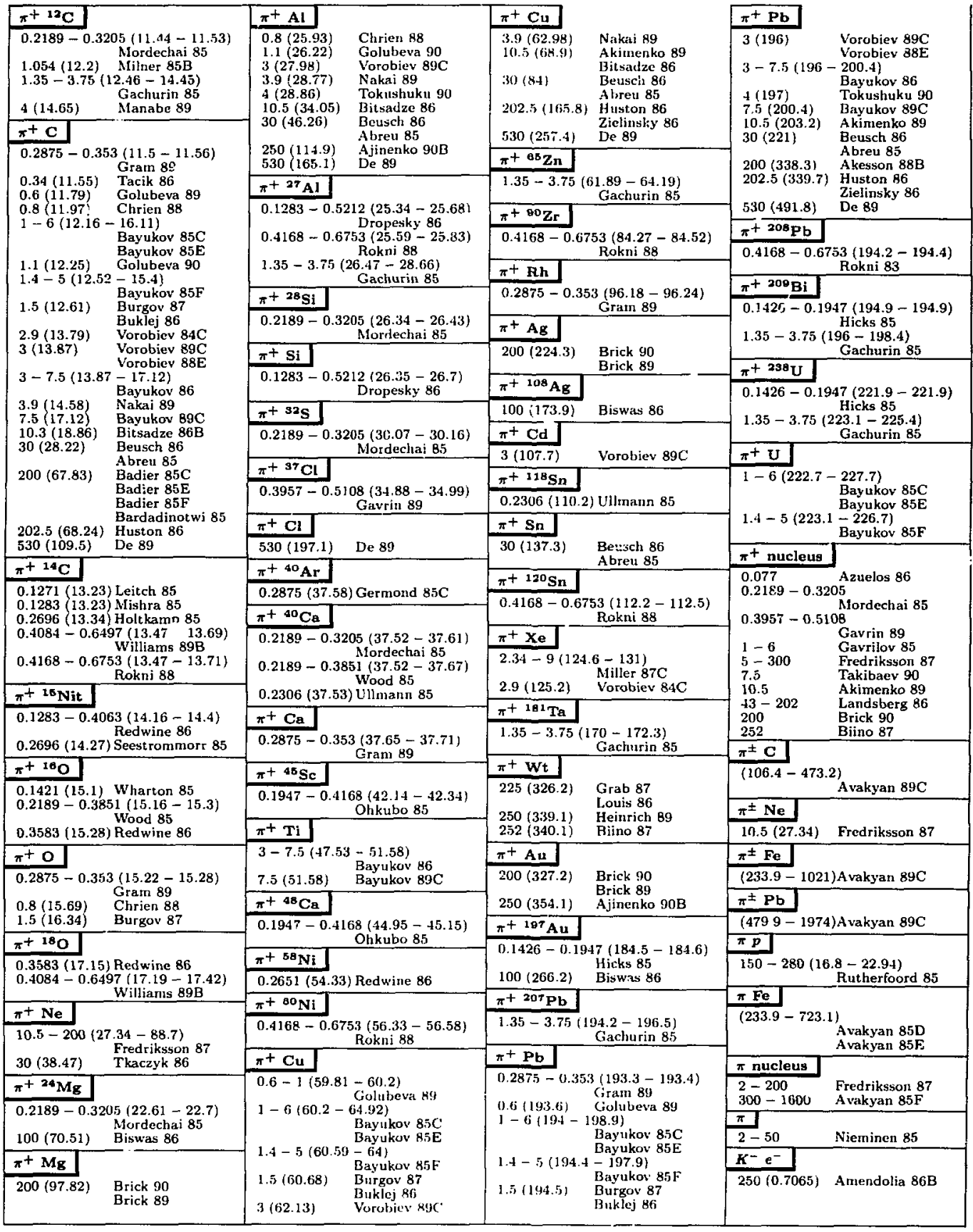




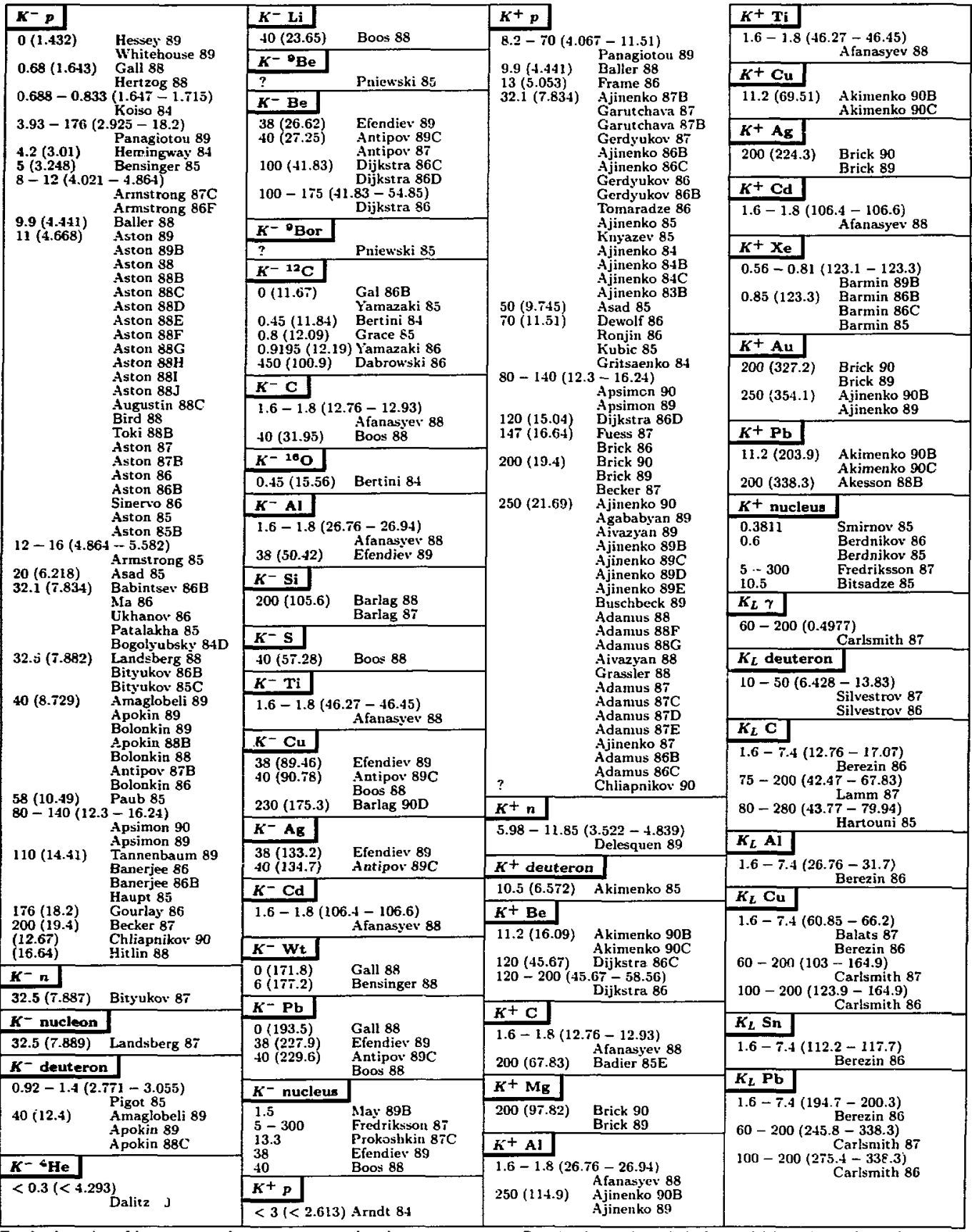

Entries in order of beam mass, then target mass. then beam momentum. Certain chemical symbols for nuclei have been changed to avoid ambiguity with particle names. (See the Particle vocabulary) Beam momenta are equivalent plab in GeV/e; then $E_{\text {crn }}$ in GeV follows in parentheses. For certain initial states only $E_{L \text { ia }}$ 'in rarentheses is given. See the legend on page 123. 


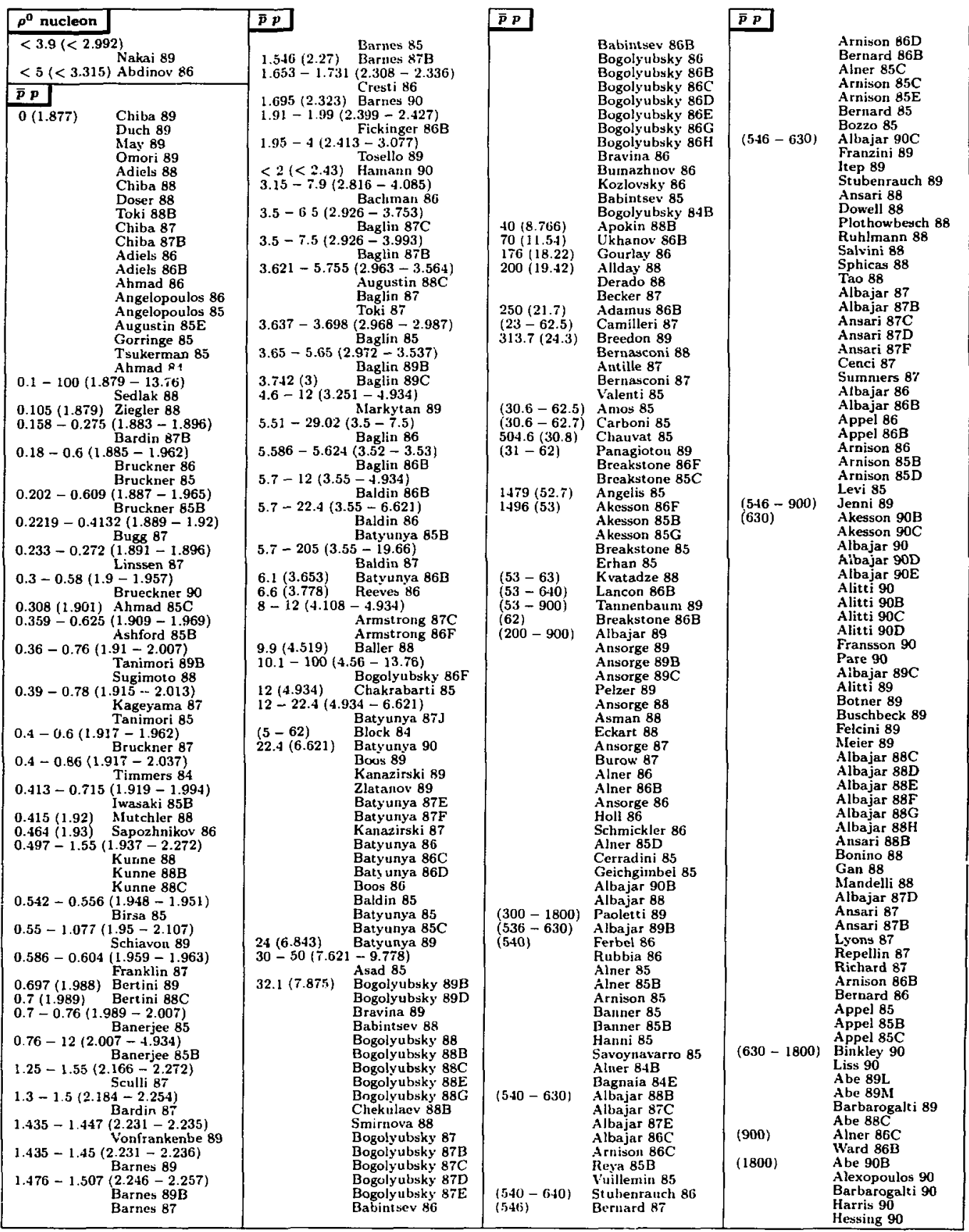




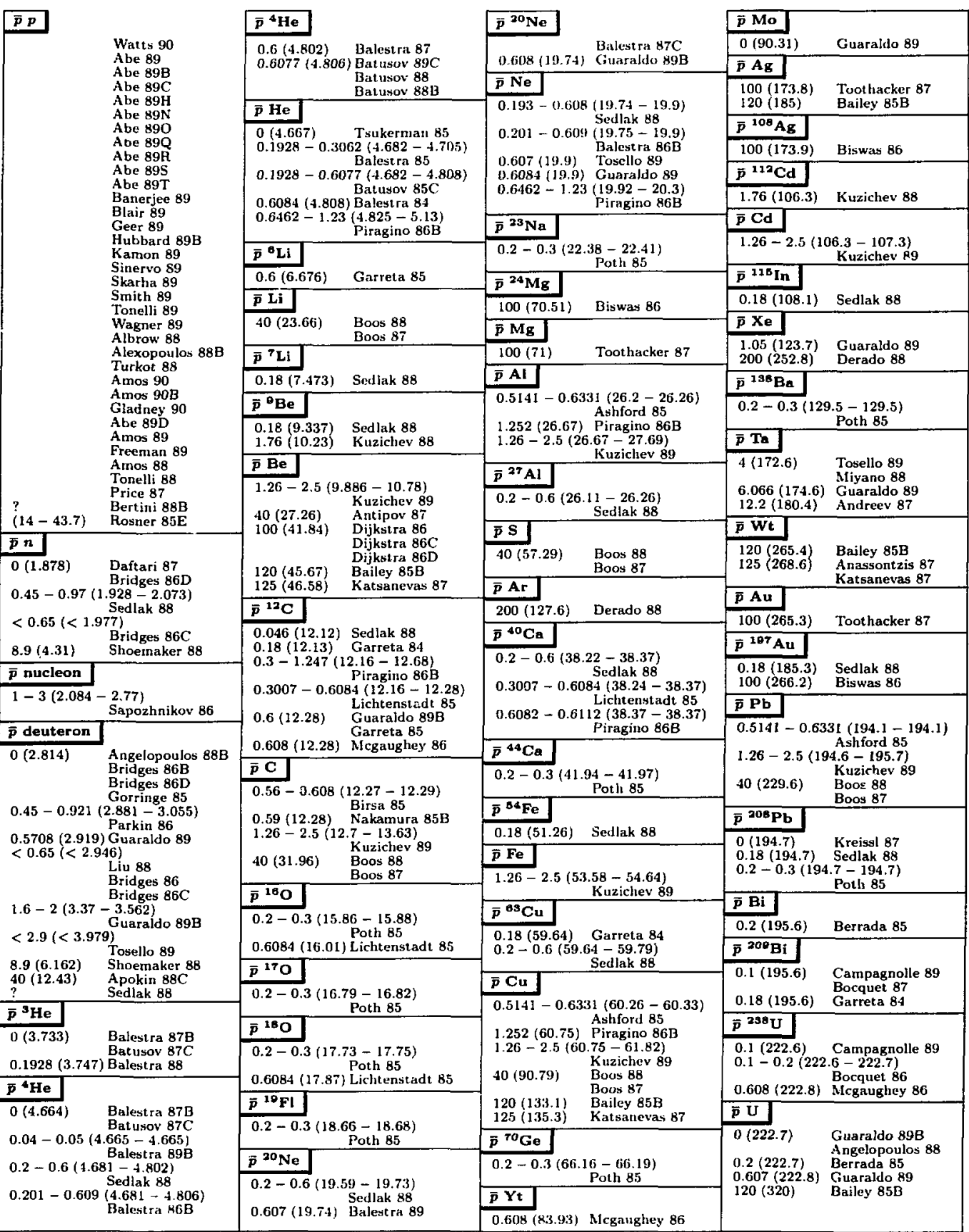

Entries in order of beam mass, then target mass, then beam mometitum. Certain chemical symbols for muclei have been changed to avoid ambiguity with particle names. (See the Partirle Vocabulary.) Biean momenta are equivalent plab in GeV/a: then $E_{\mathrm{rm}}$ in GeV follows in parentheses. For certain initial states only $E_{c \text { cn }}$ (in pitrentheses) is given. See the legend on page 123. 


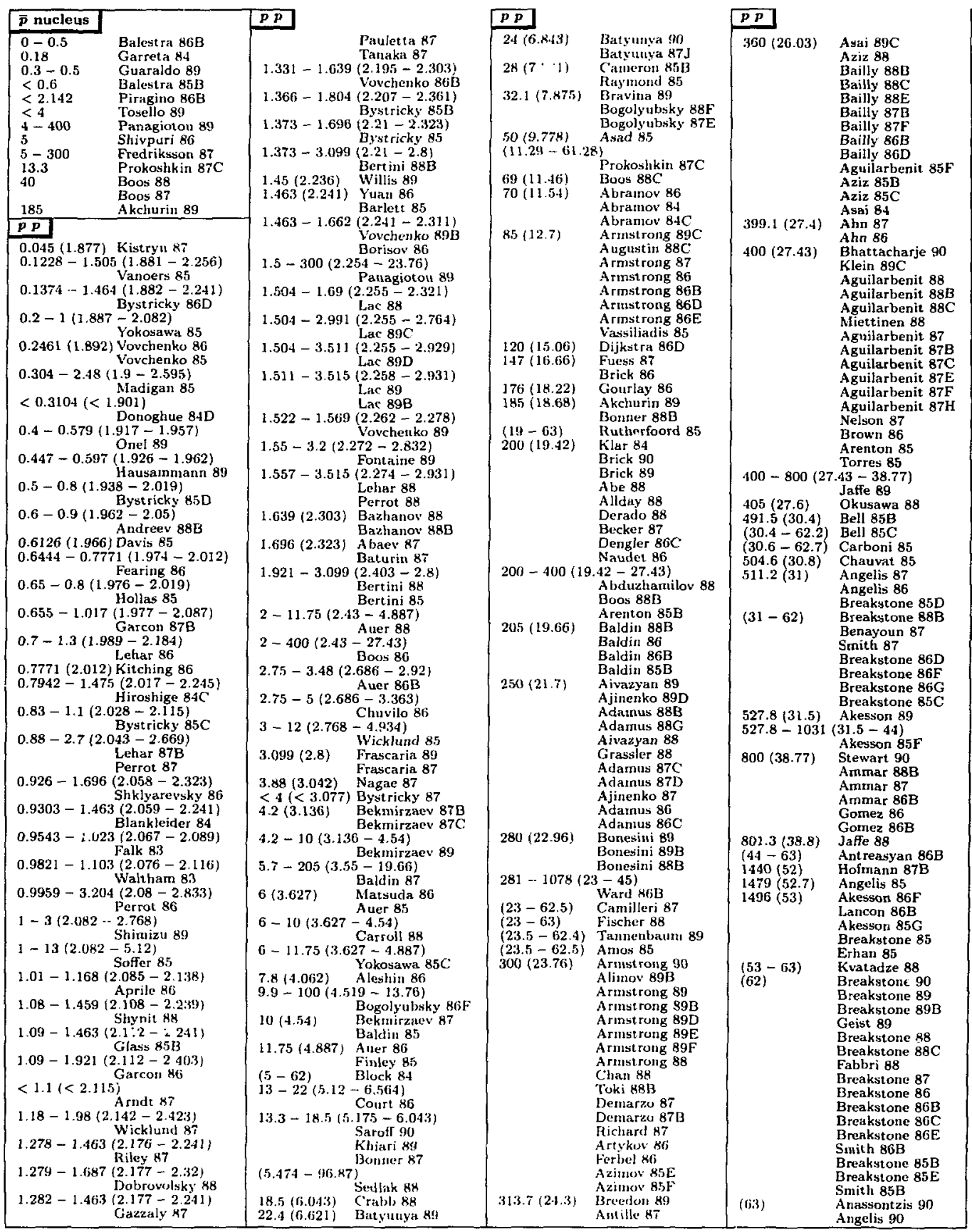




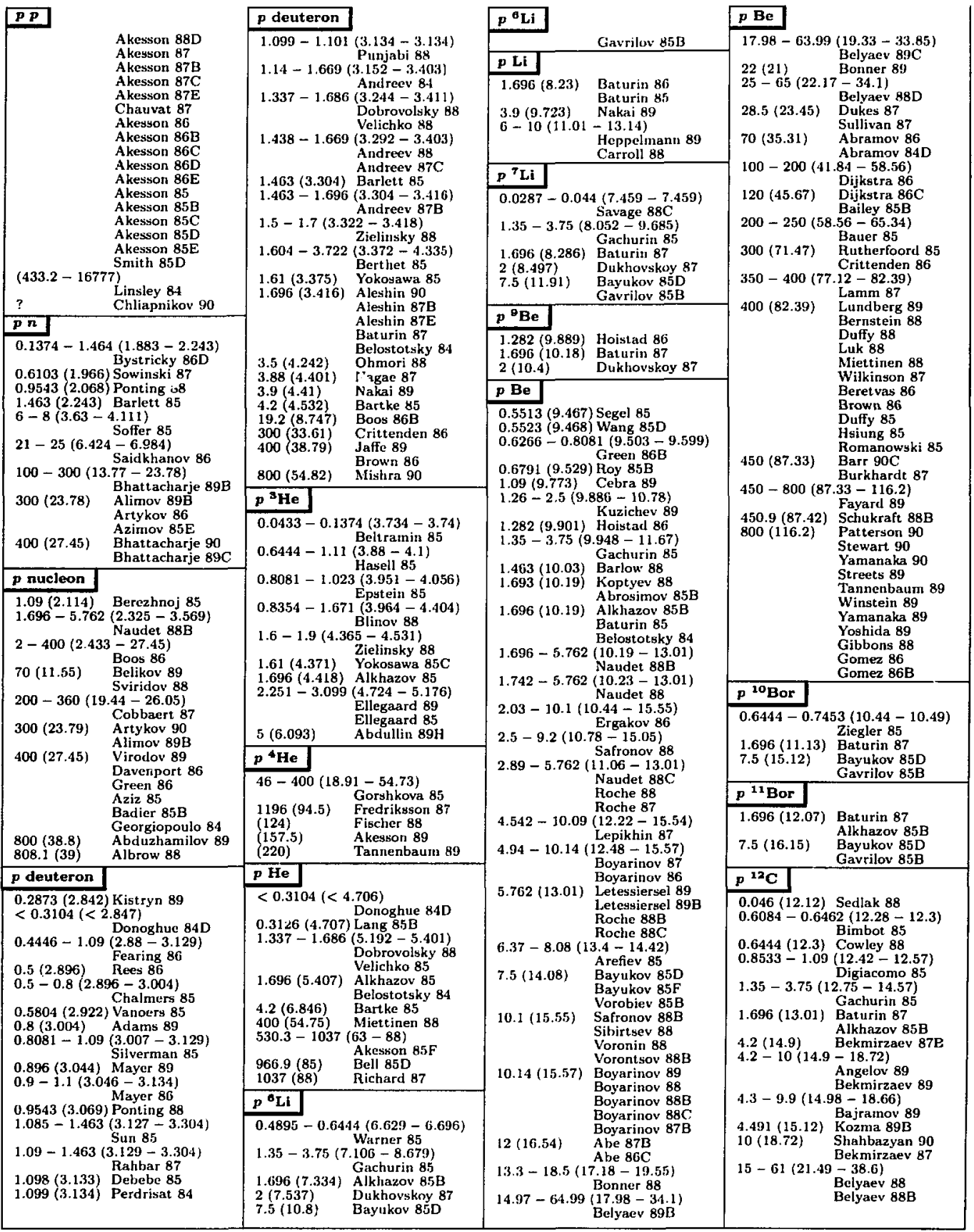

Entries in order of beam mass, then target mass, then beam momentum. Certiin chemical symbols for nuclei have been changed to avoid ambiguity with partirle names. (See the Particle Vocabulary.) Beam momenta are equivalent plab in GeV/ct then $E_{\mathrm{c} n}$ in GeV follows in parentheses. For certain initial states only $E_{\mathrm{cm}}$ (in parentheses) is given. See the legend on page 123. 


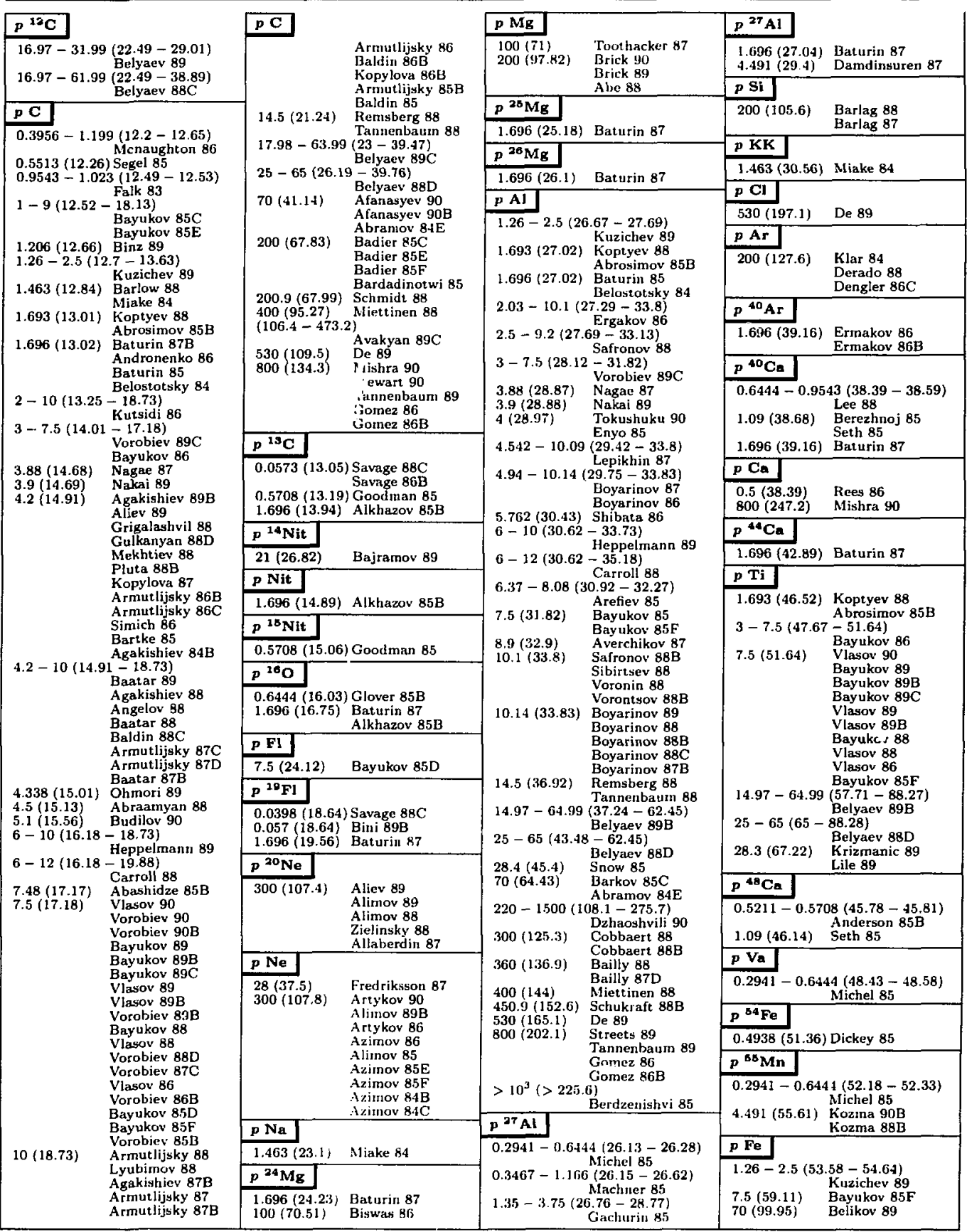




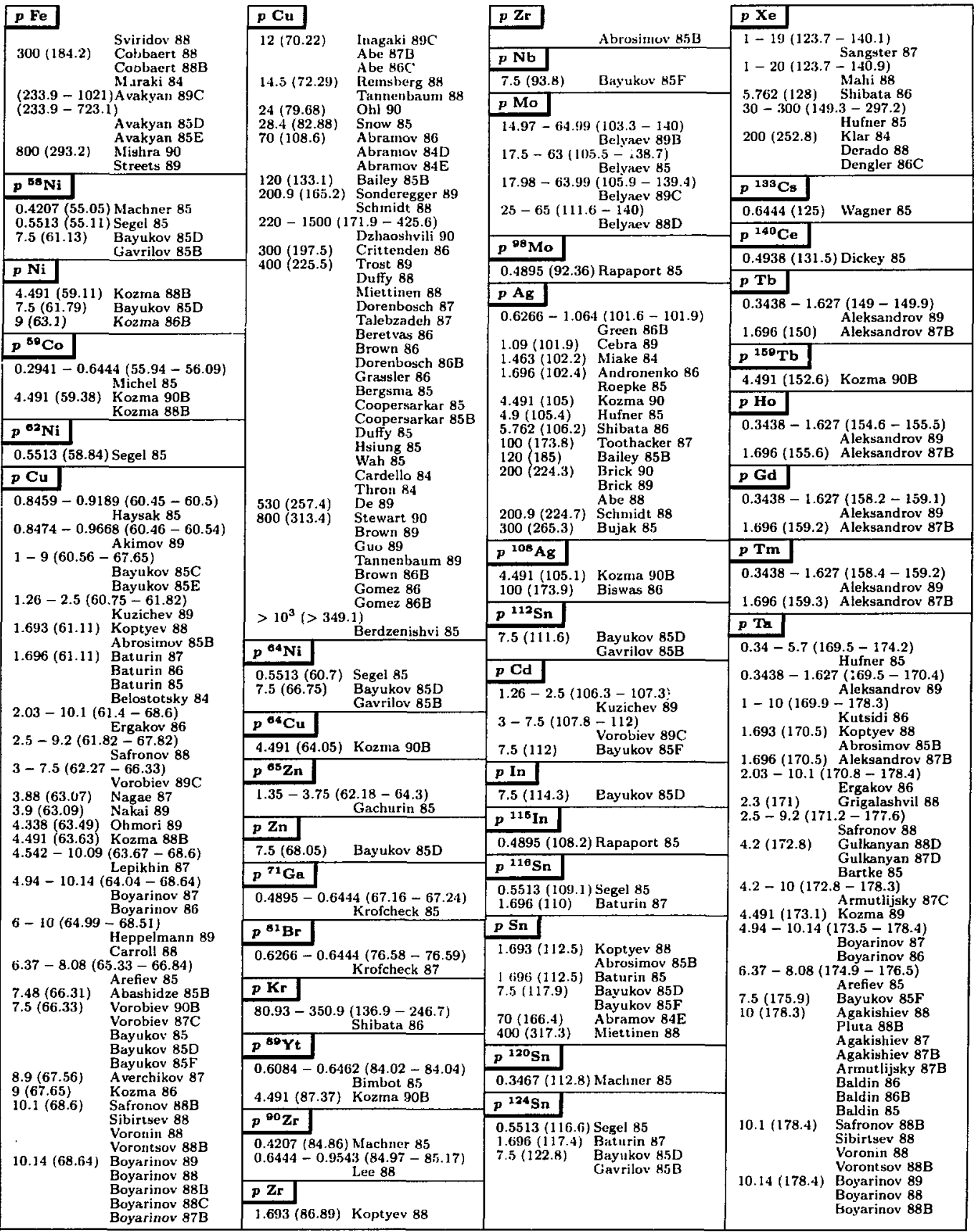

Entries in order of beam mass, then target mess. then bean momentum. Certain clemical symbols lor nuclei have heen changed to avoid ambiguity with particle names. (See the Particle Vocabulary.) Beall monelta are equivalent plab ill GeV/c: then $E_{\text {rin }}$ in GeV follows in parentheses. For certain initial states only $E_{r n}$ (in parentheses) is given. See the legend on page 123 . 


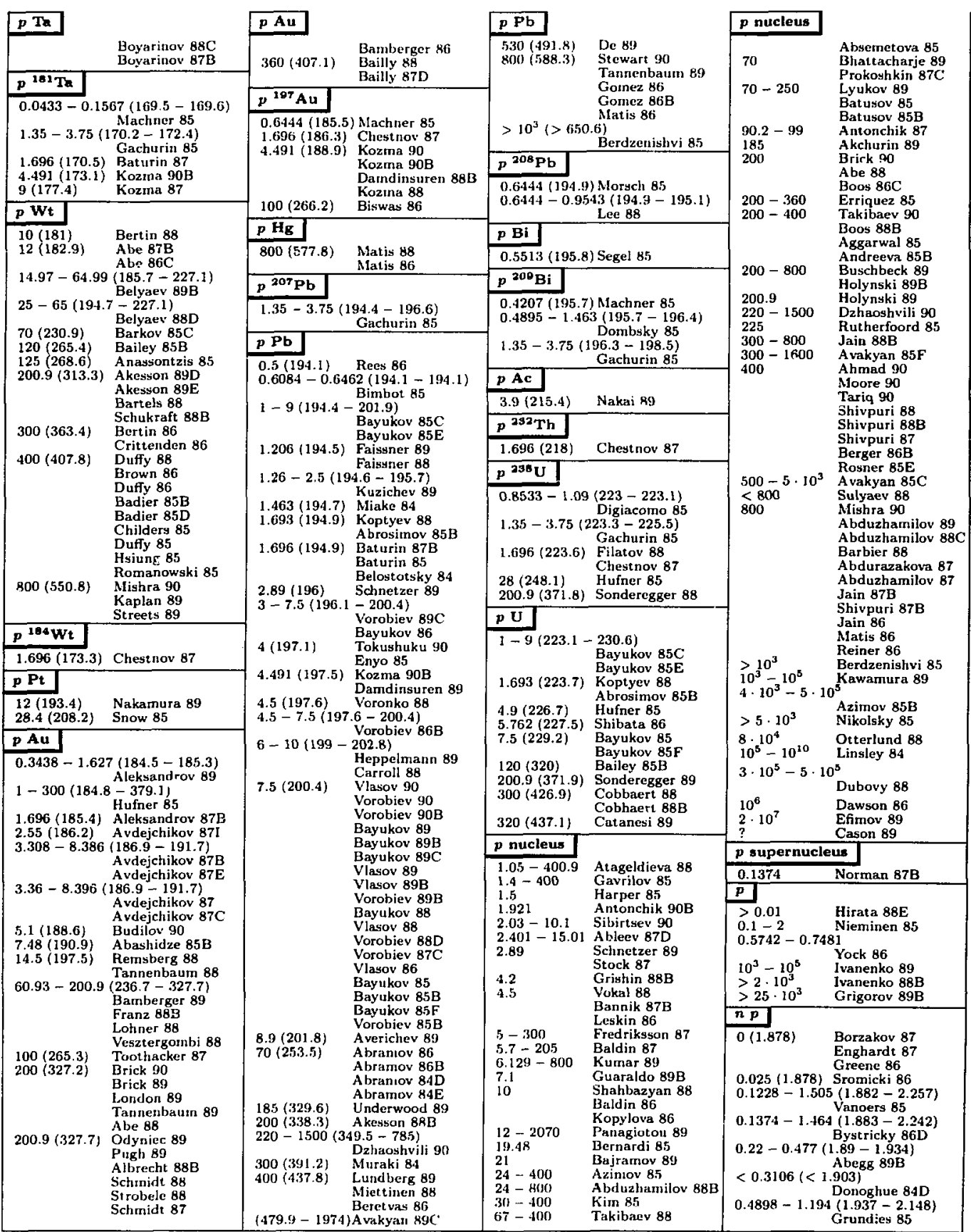




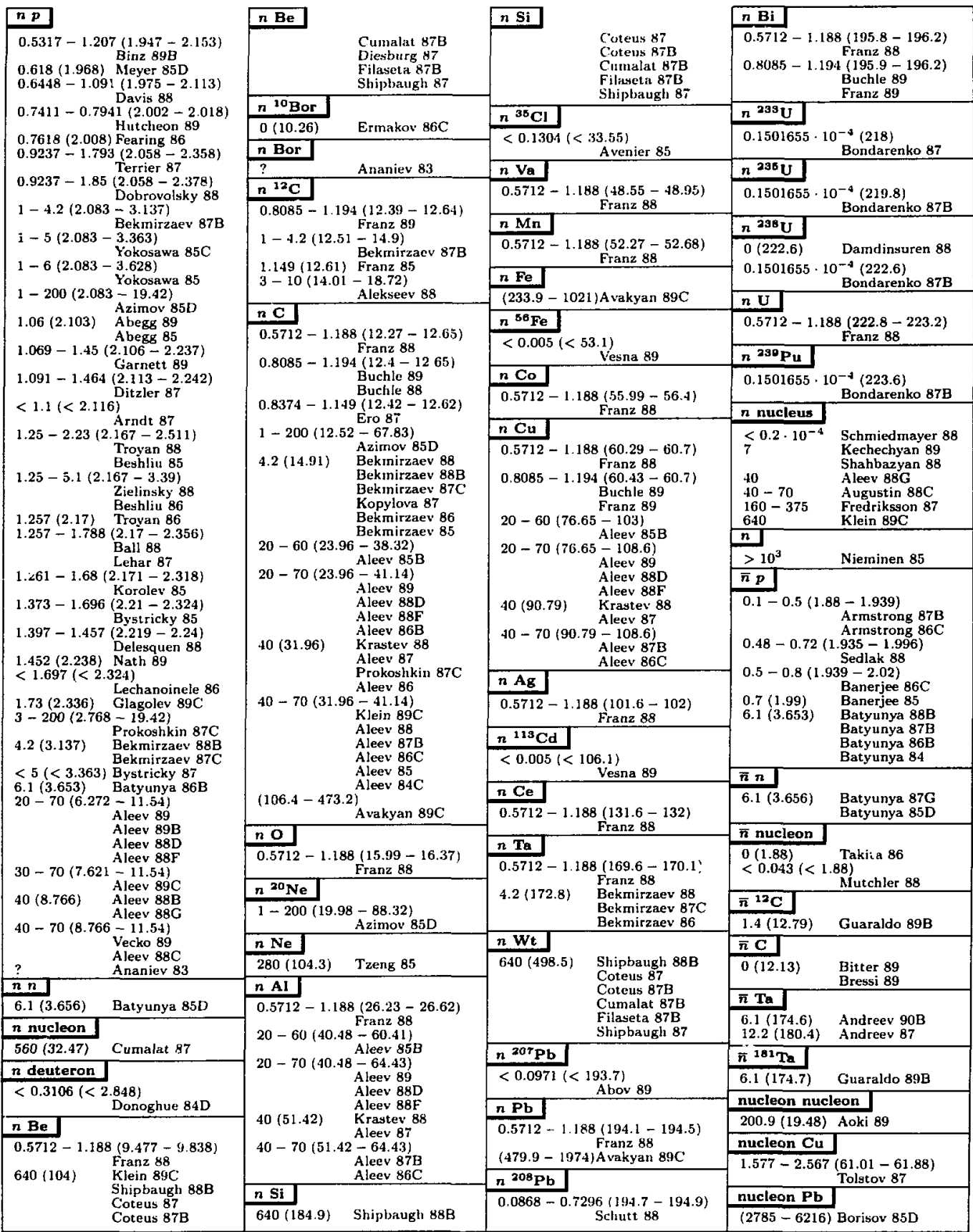

Entries is order of beam mass, then target mass. then beam momemtum. Certain chemical symbols for nuclei have been changed to avoid ambiguity with particle names. (See the Particle Vocabulary.) Beam tnomentat are equivalent plat in GeV/r: then $E_{\mathrm{rm}}$ in $\mathrm{GeV}$ follows in parenthesess. For certain initial states only $E_{r m}$ (in parentheses) is given. See the legend on page 123. 


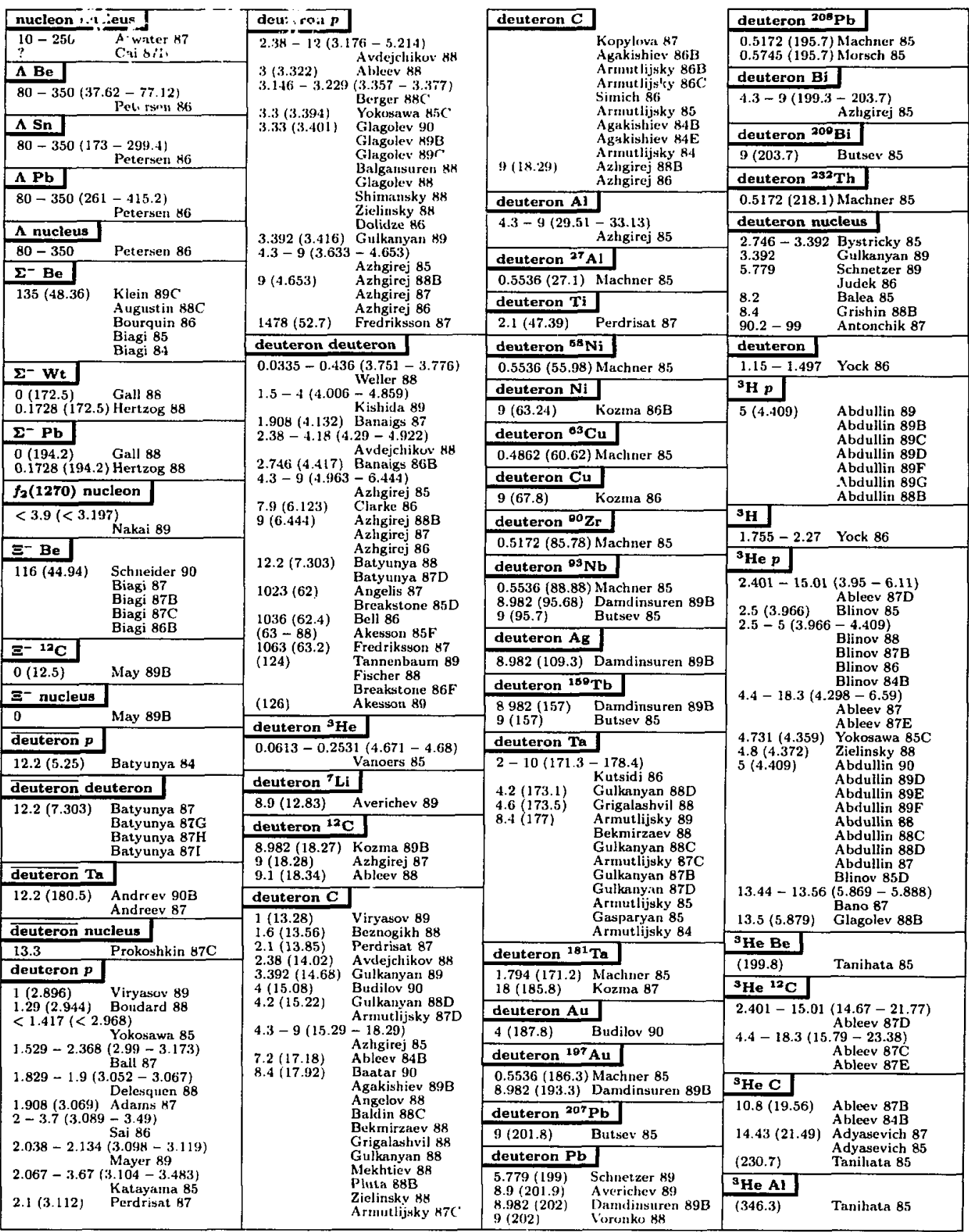




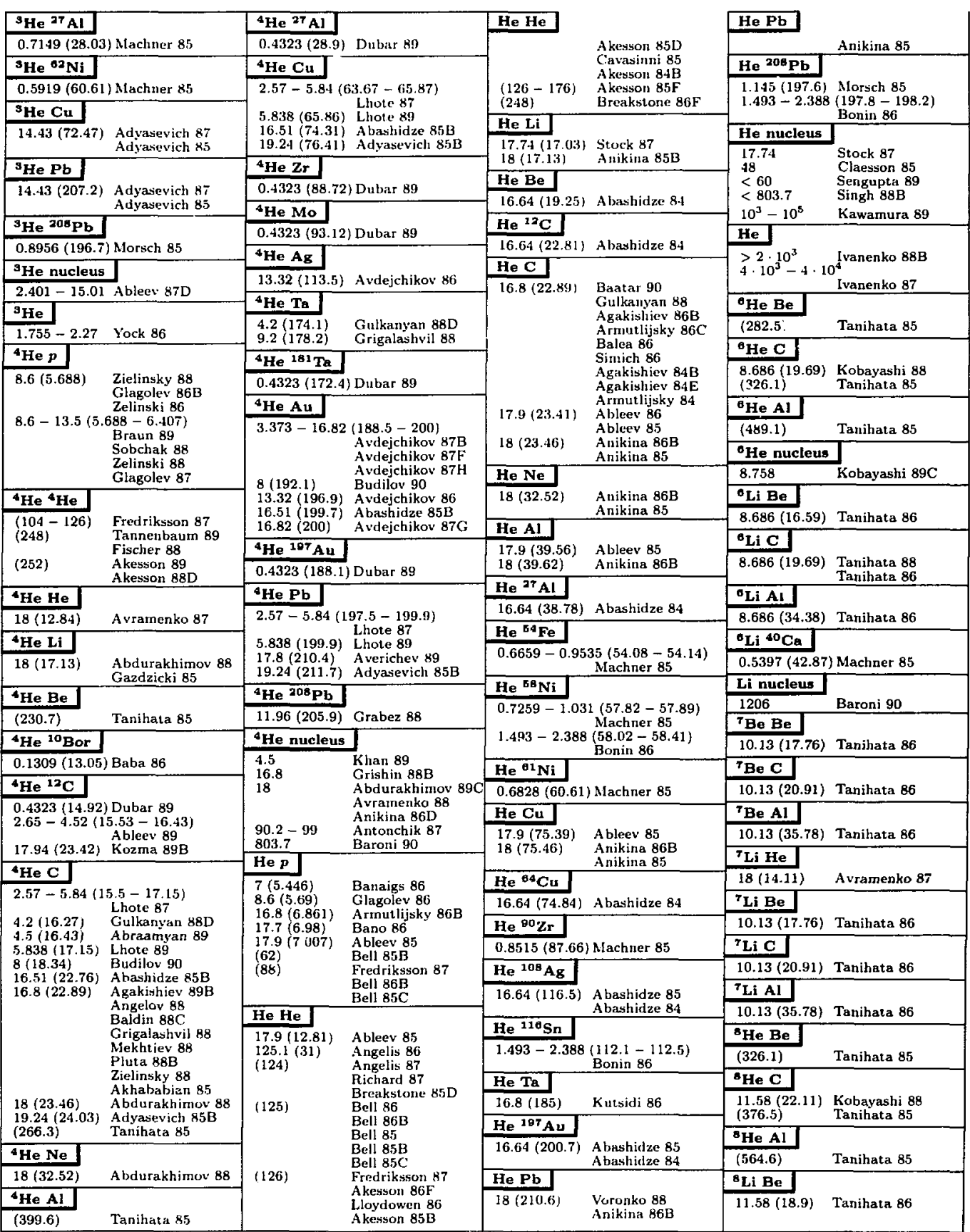

Entries in order of beam mass. then target mass, then beam momentum. Certain clienical symbols for nuclei have been changed to avoid ambiguity with particle names. (See the Particle Vocabulary.) Beatn Homenta are mquivalent plab in GeV/c; then $E_{\mathrm{cth}}$ in $\mathrm{GeV}$ follows in parentheses. For certain initial states only $E_{c m}$ (in parentheses) is given. See the legend on page 123 . 


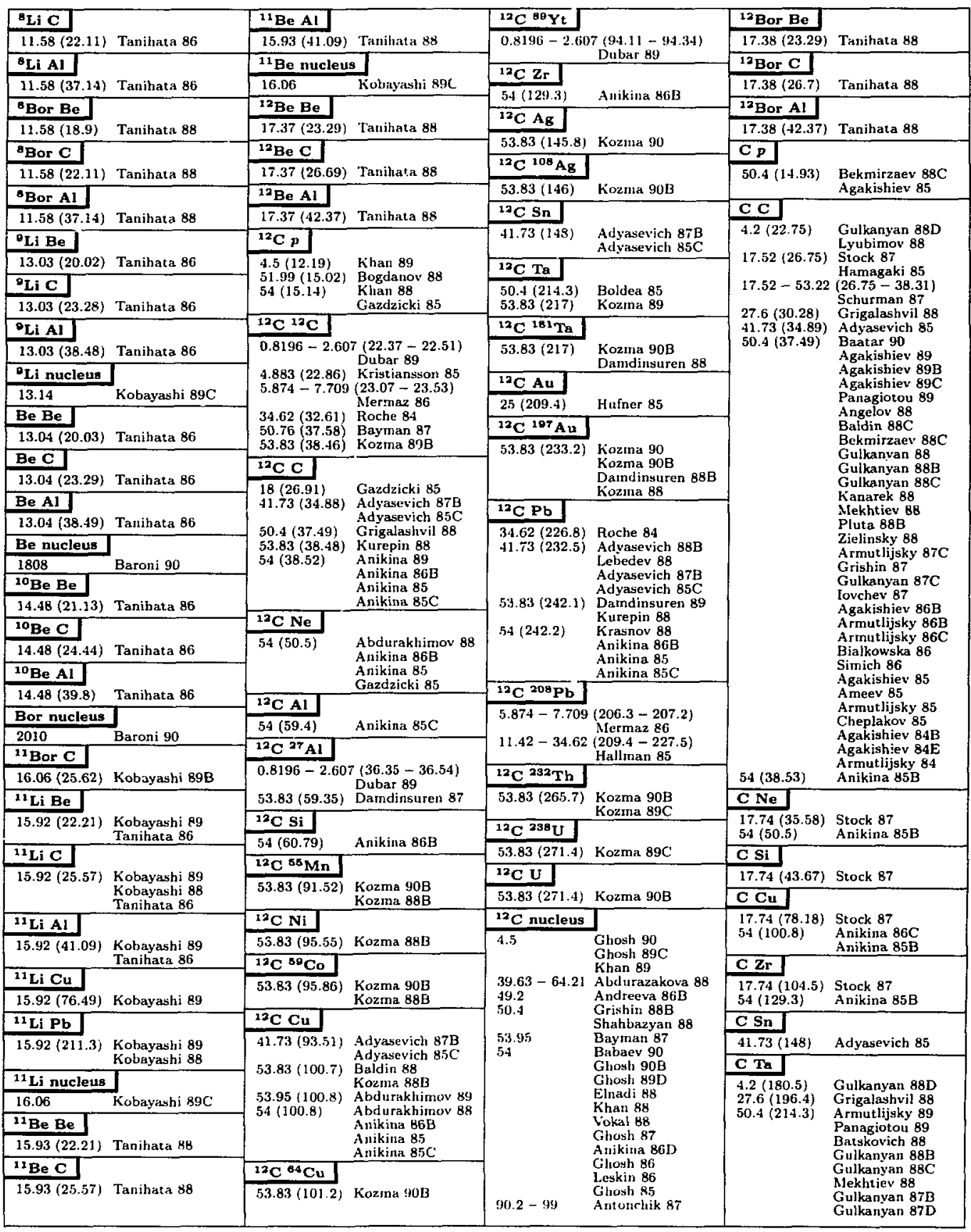




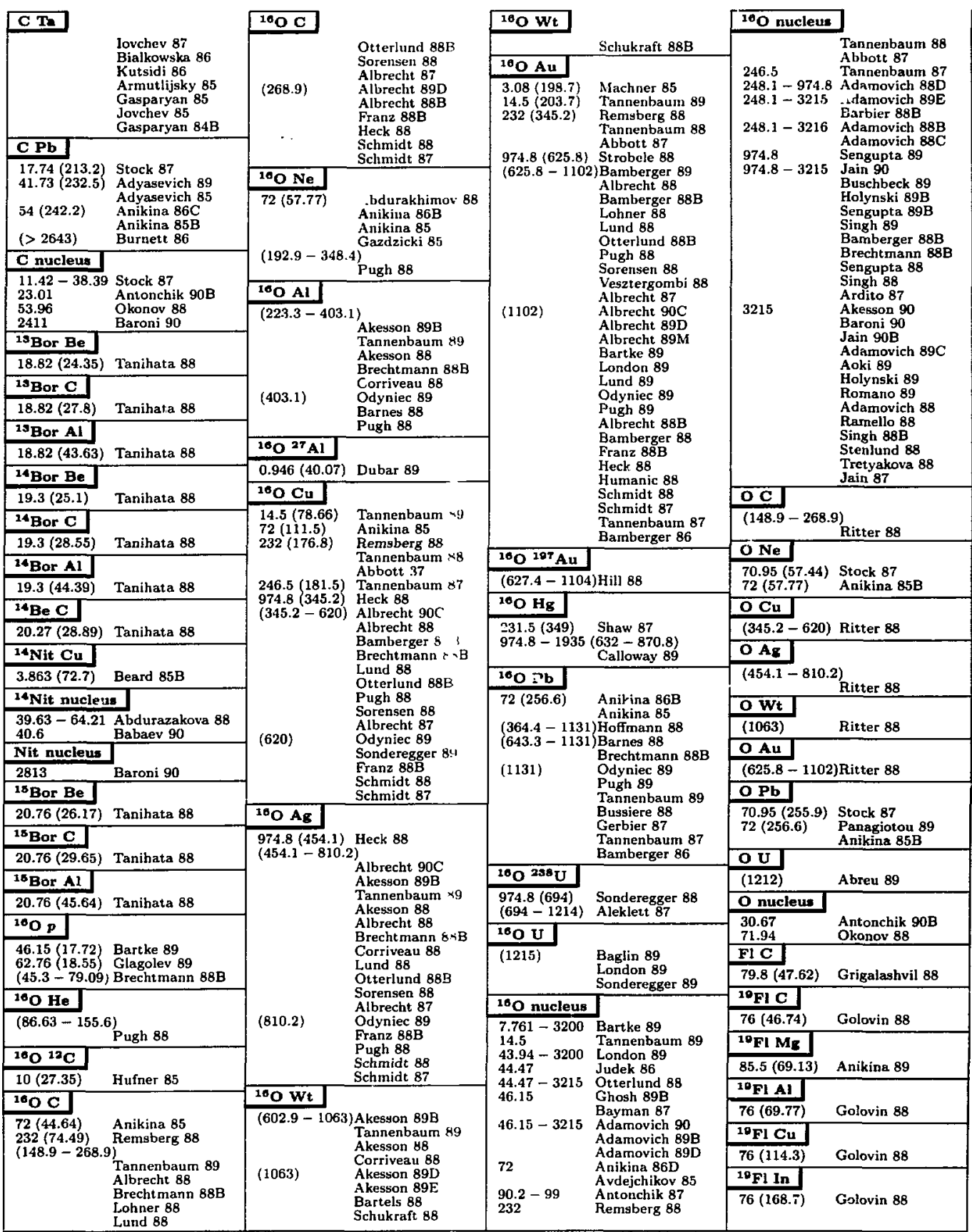

Entries in order of beam mass, then target mass, then beam m inentum. Certain chemical symbols for nuclei have been changed to avcid ambiguity with particle names. (See the Particle Vocabulary.) Beam momenta are equivalent $p_{\mathrm{lab}}$ in $\mathrm{GeV} / \mathrm{c}$ : then $E_{\mathrm{cm}}$ in $\mathrm{GeV}$ follows in parentheseb. For certain initial states only $E_{\mathrm{cm}}$ (In parentheses), given. See the legend on page 123. 


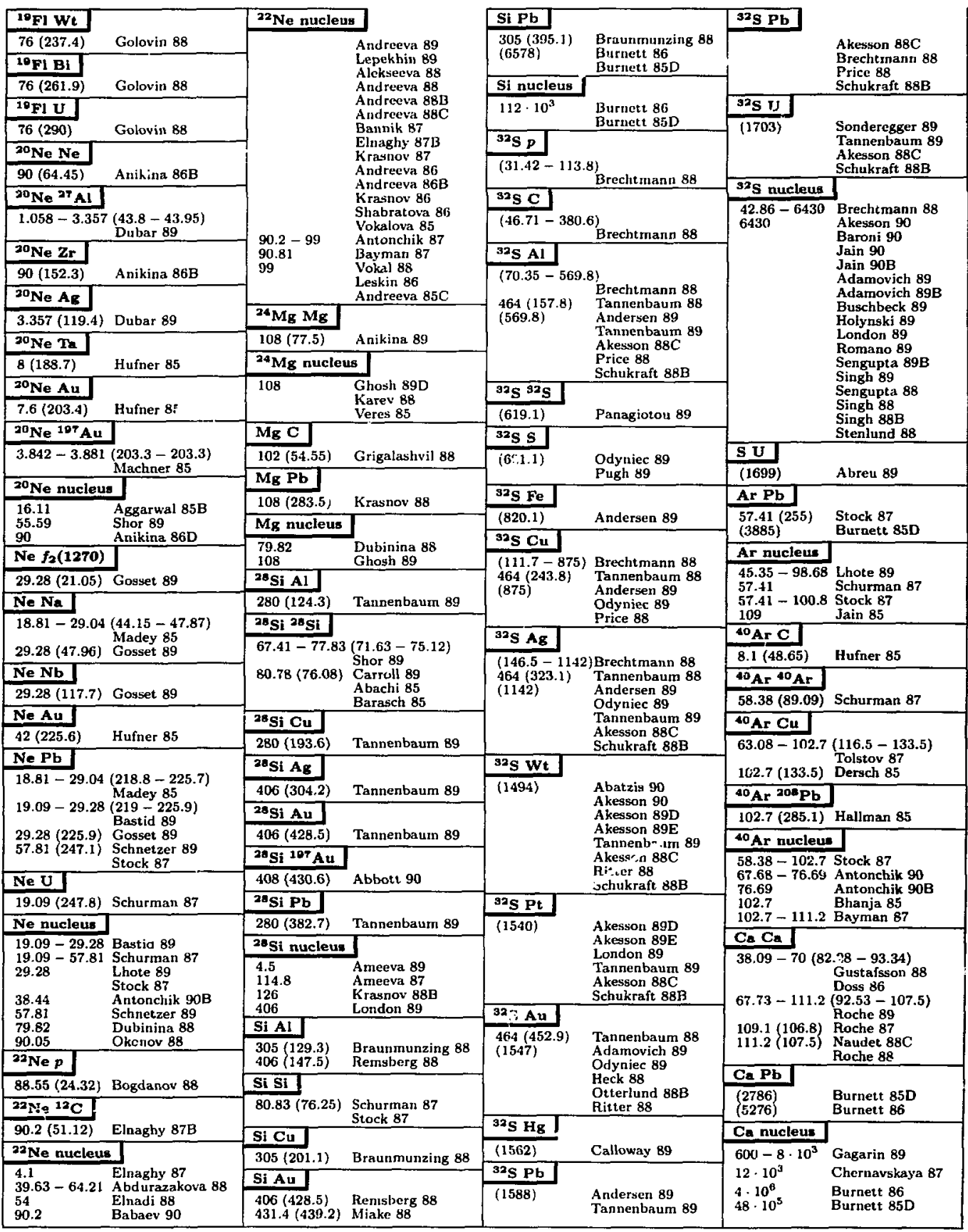




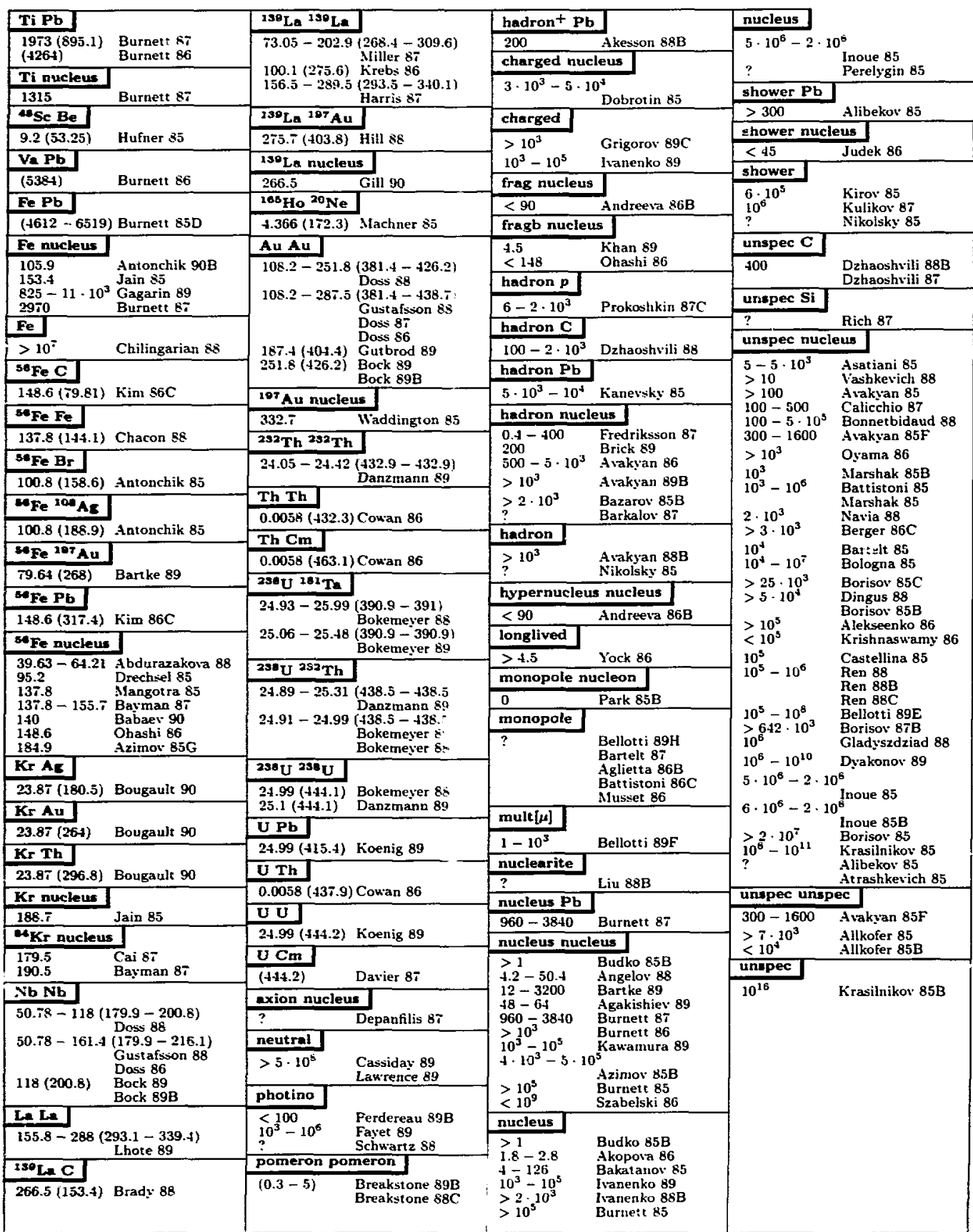

Entries in order of beam mass, then target mass. then beam monent ton. Certain chenical svmbols for nuclei have been changed to avoid ambiguity with particle names. (See the Particle Vocabulary) Beatn romenta are equitalent plat, in GeV/c; then $E_{\mathrm{cm}}$ in GeV follows in parent heses. For certain initial states only $E_{\mathrm{cm}}$ (in parentheses) is given see the legend on page 123 . 
This index lists papers by entire reactions. including beam. target. final state, and beam moment um. The ordering is by beam mass. then target mass. then multiplicity of the final state. By "multiplicity" we mean the number of separate particle names that appear. A name like "vees." " $\mathrm{x}$." or " $\pi^{-}$"s," which can refer to an unspecified number of real particles, only counts as one name. Names like " $0 \wedge$ "s" also count as one name.

The bean noment um is the equivalent lab moment um in GeV/r. For most colliding beanı experiments and for reactions above $2 \mathrm{TeV} / \mathrm{c}$ equivalent lab momentum. we give instead the c.m. energy $E_{\mathrm{cm}}$. in GeV. The c.m. energies are distinguished from lab moment a by being enclosed in parentheses. For a given beam momentum. ID's are ordered by year (nost recent to oldest), then author name.

When a range of moment a were studied. we list the lower and upper ends of the range. e.g. " $50-70 . "$ ordered by the lower end. For some experinents, such as neutrino experiments, the listed range is only approximate.

A question mark means that information is not available. usually because it was not given in the paper.

\section{Illustrative Key}

Initial State of the Reaction: see the Particit locabulary for nomenclature.

Final State of the Reaction: see the Particle locabulary for nomenclature.

Document ID: see the ID/Reference/Title Index for the full reference.

Lab Momentum: in $\mathrm{GeV} / \mathrm{c}$ or, if in parent heses, the c.m. energy $E_{\mathrm{cm}}$. in $\mathrm{GeV}$.

Data Descriptor: brief description of the data neasured: see the Data Descriptor locabulary for nomenclature.

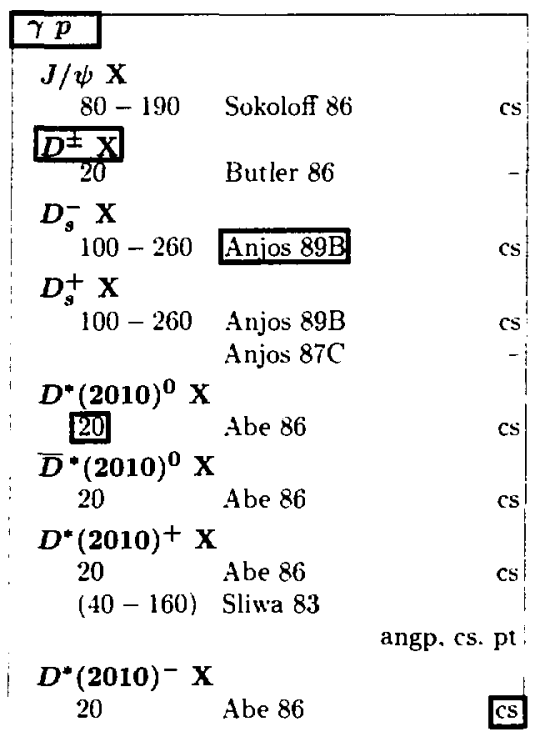




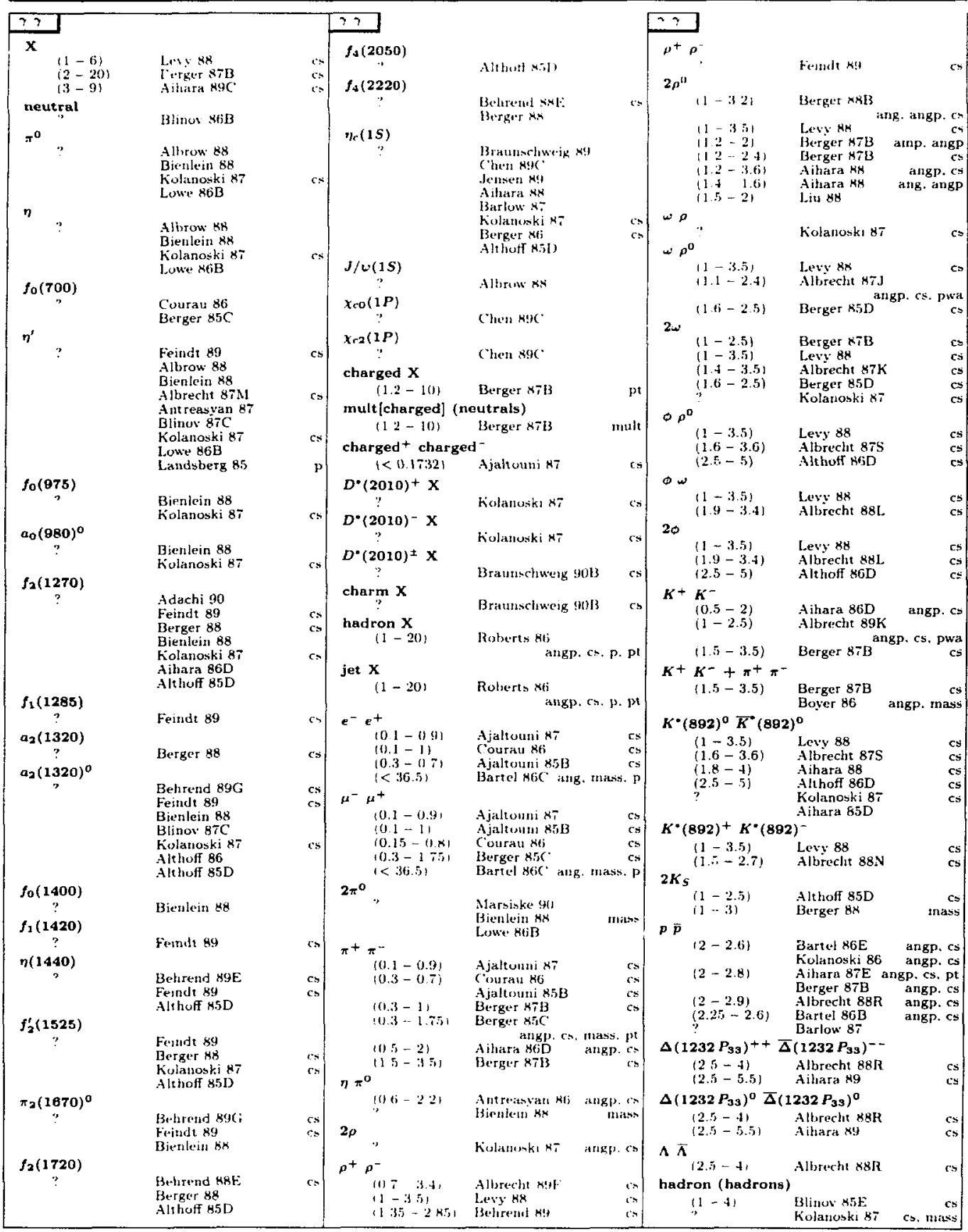




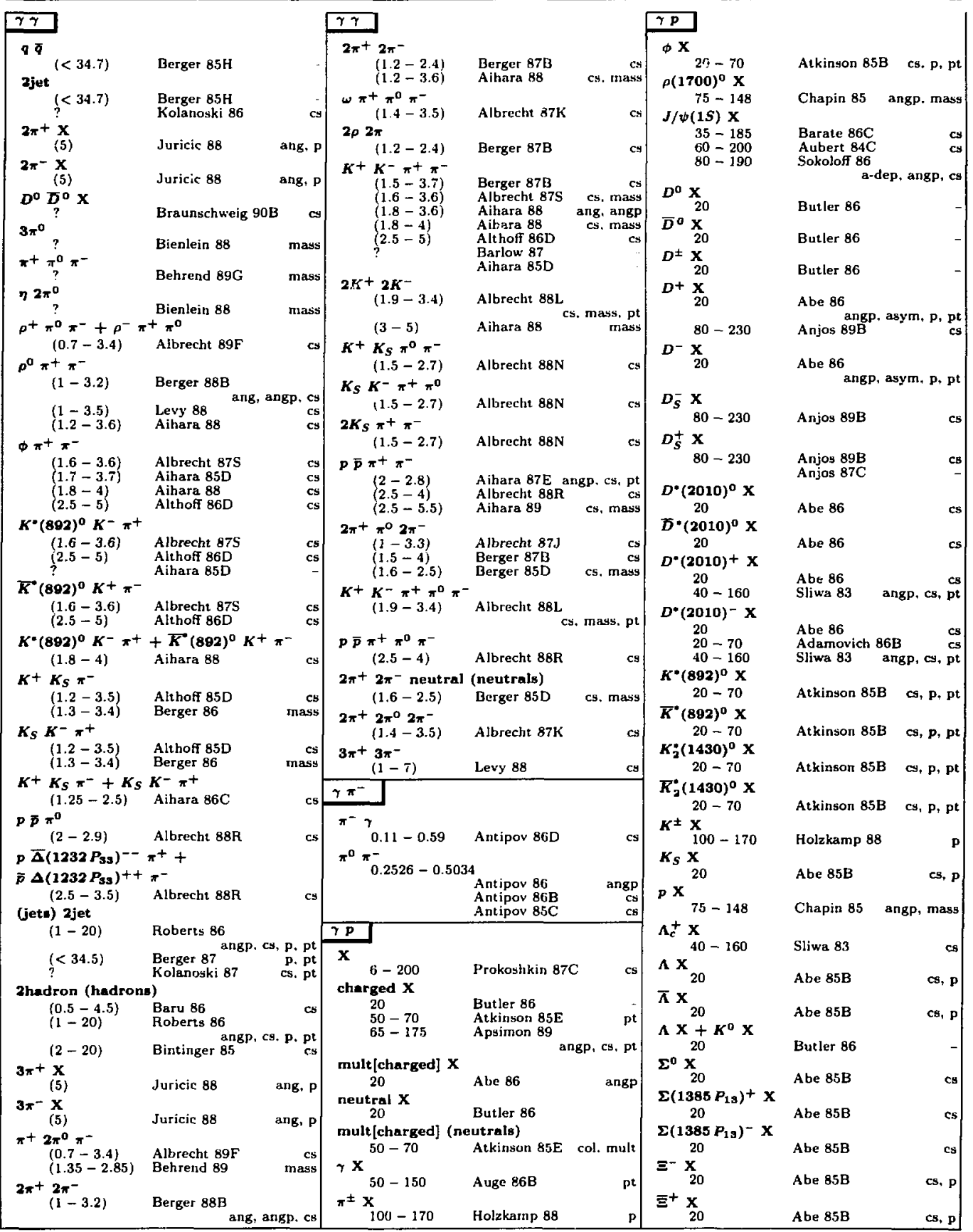

Entries in order of beam mass, then target mass, then unultiplicity of final state. Inclusive reactions have an " $X$ " as the last of the final state particles. Certain chemical symbols for nuclei have been changed to avoid ambiguity with particle names (See the Particle Vocabulary.) Beam momenta are $P_{\mathrm{lat}}$ in $\mathrm{GeV} / c$, Gr in parentheses $E_{\mathrm{cm}}$ in $\mathrm{GeV}$. See the legend on page 15.3 . 


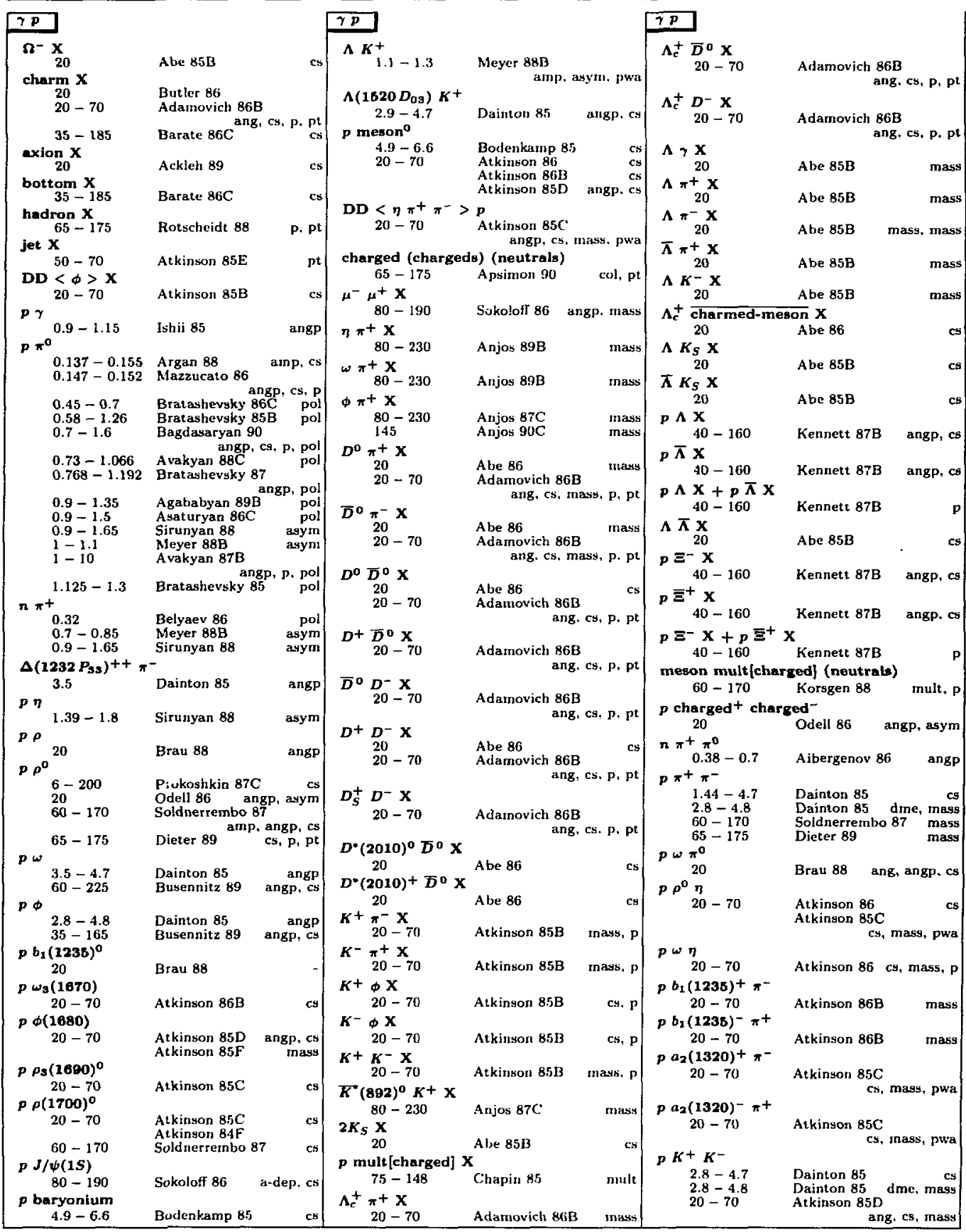




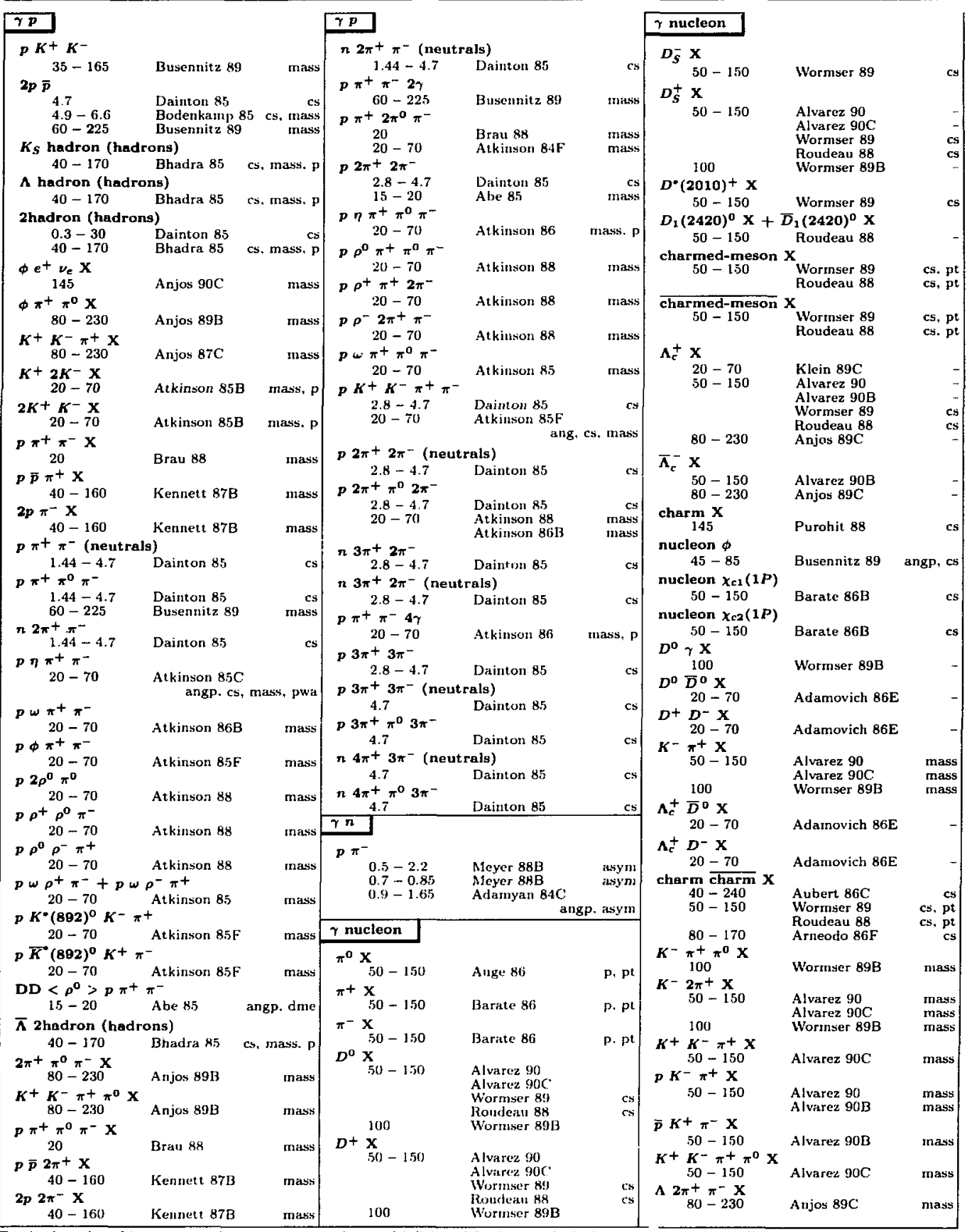

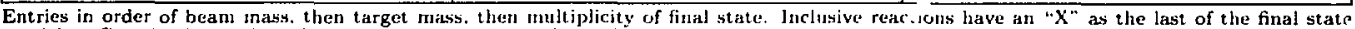

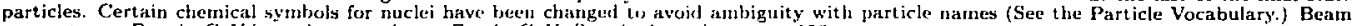
momenta are $P_{\text {lnt }}$ in GeV/c, or in parentheses $E_{r m}$ in GuV. See the legend an page liji3. 


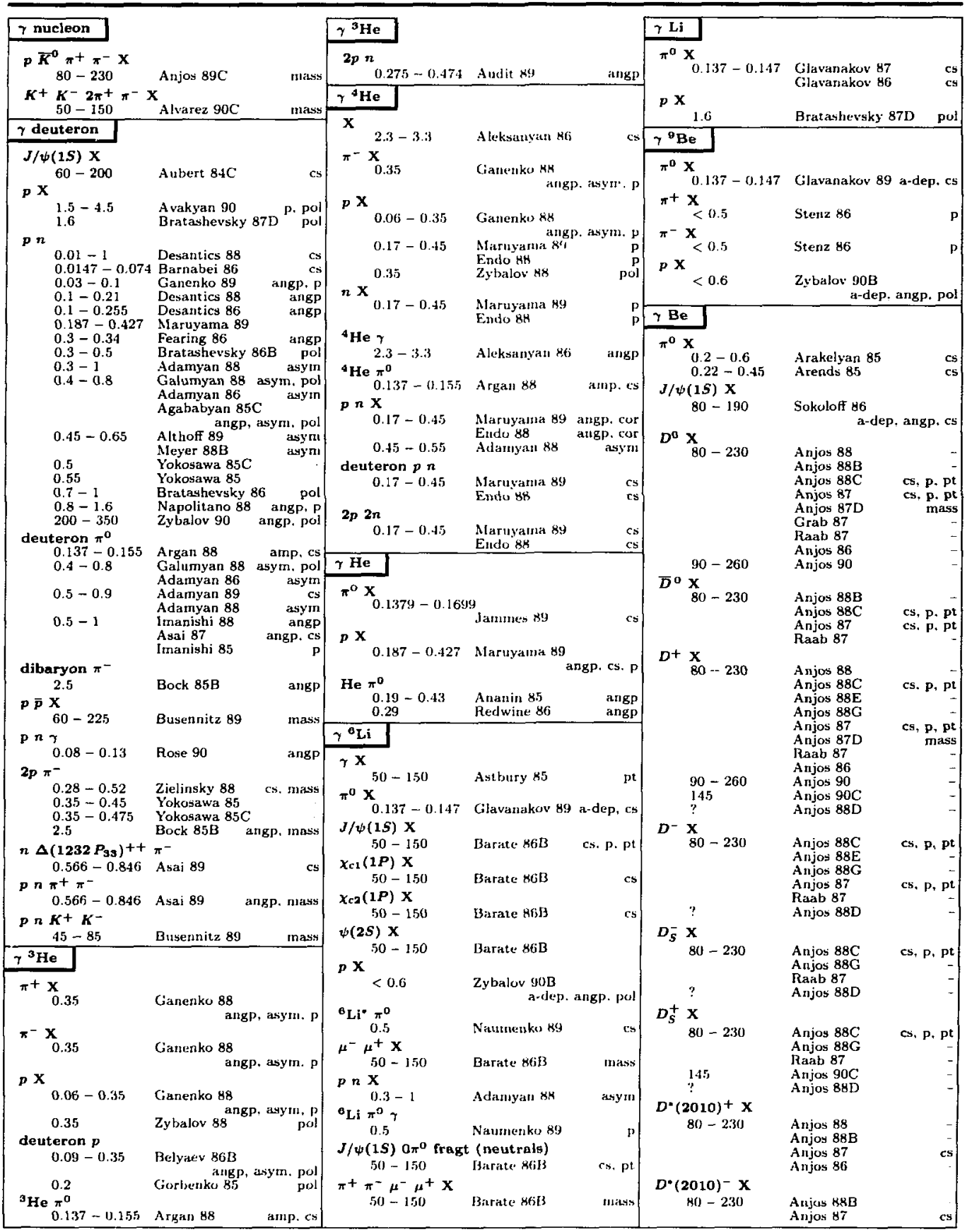




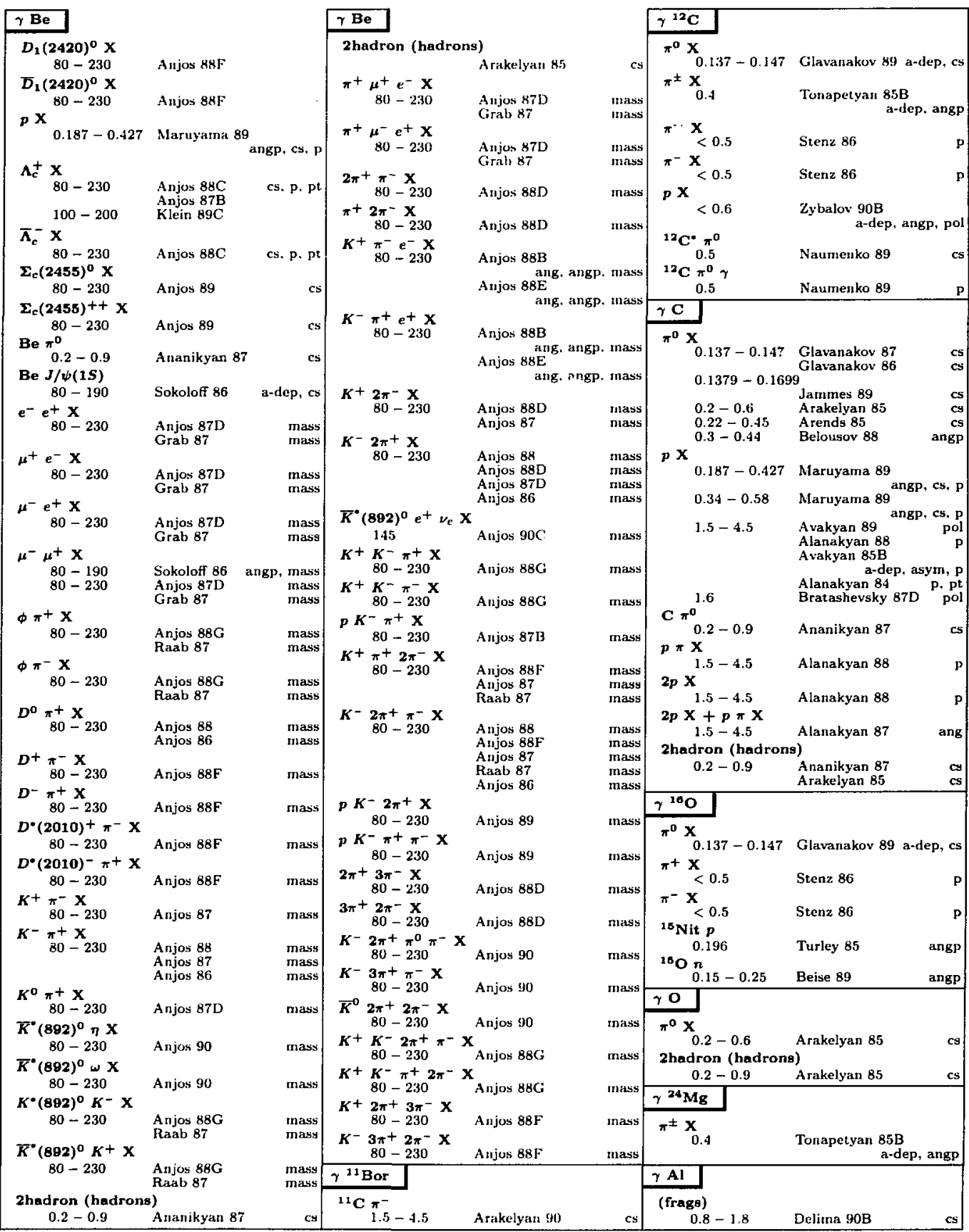

Entries in order of beam mass, then target mass, then multiplicity of hinal state. Inclusive reactions have an " $X$ " as the last of the final state particles. Certain chennical symbols for nuclei have been changed to atvoid ambiguity with particle uames (Sec the Particle Vocabulary.) Beam momenta are $P_{l a b}$ in $\mathrm{GeV} / r$. or in parentheses $E_{r m}$ in $\mathrm{GeV}$. See the legend on page 1.53 


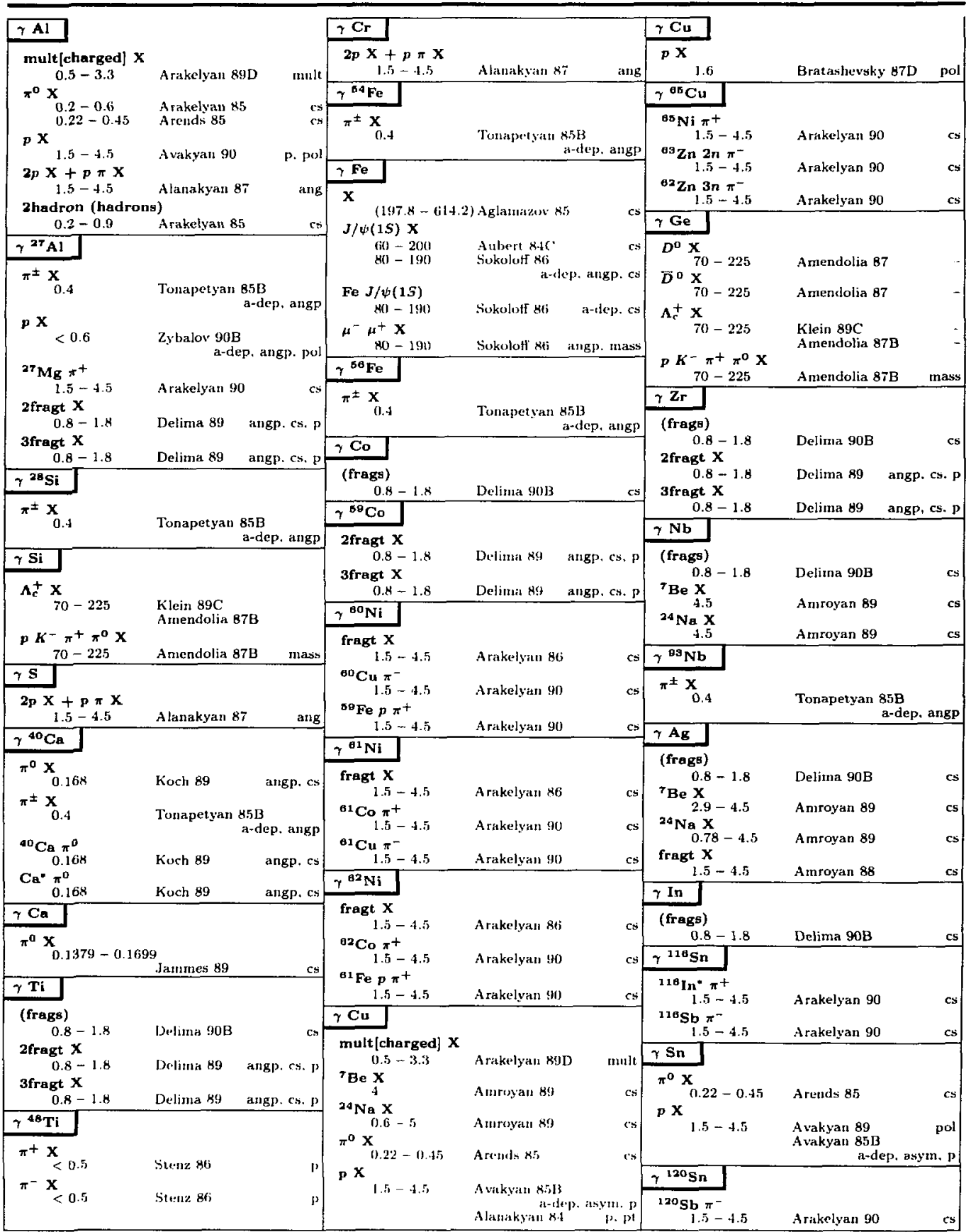




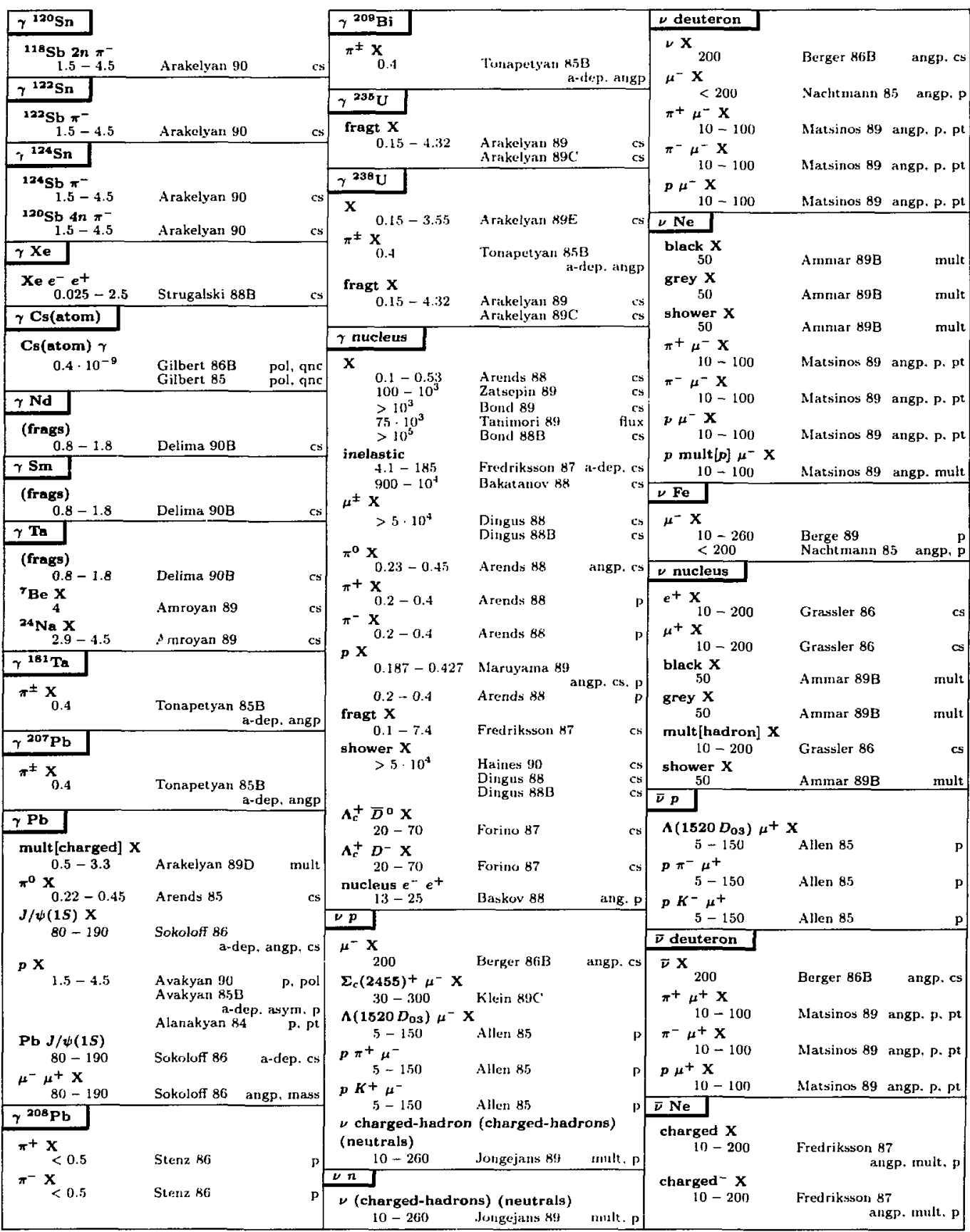

Entries in order of bean mass. then target mass, then multiplicity of final state. Inrisive rcactiuns have an " $X$ " as the last of the final state particles. Certain chemical symbols for nuclei have been changed tos avoid ambiguty with particle names (Sce. (he Particle Vocabulary) Beam

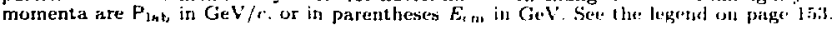




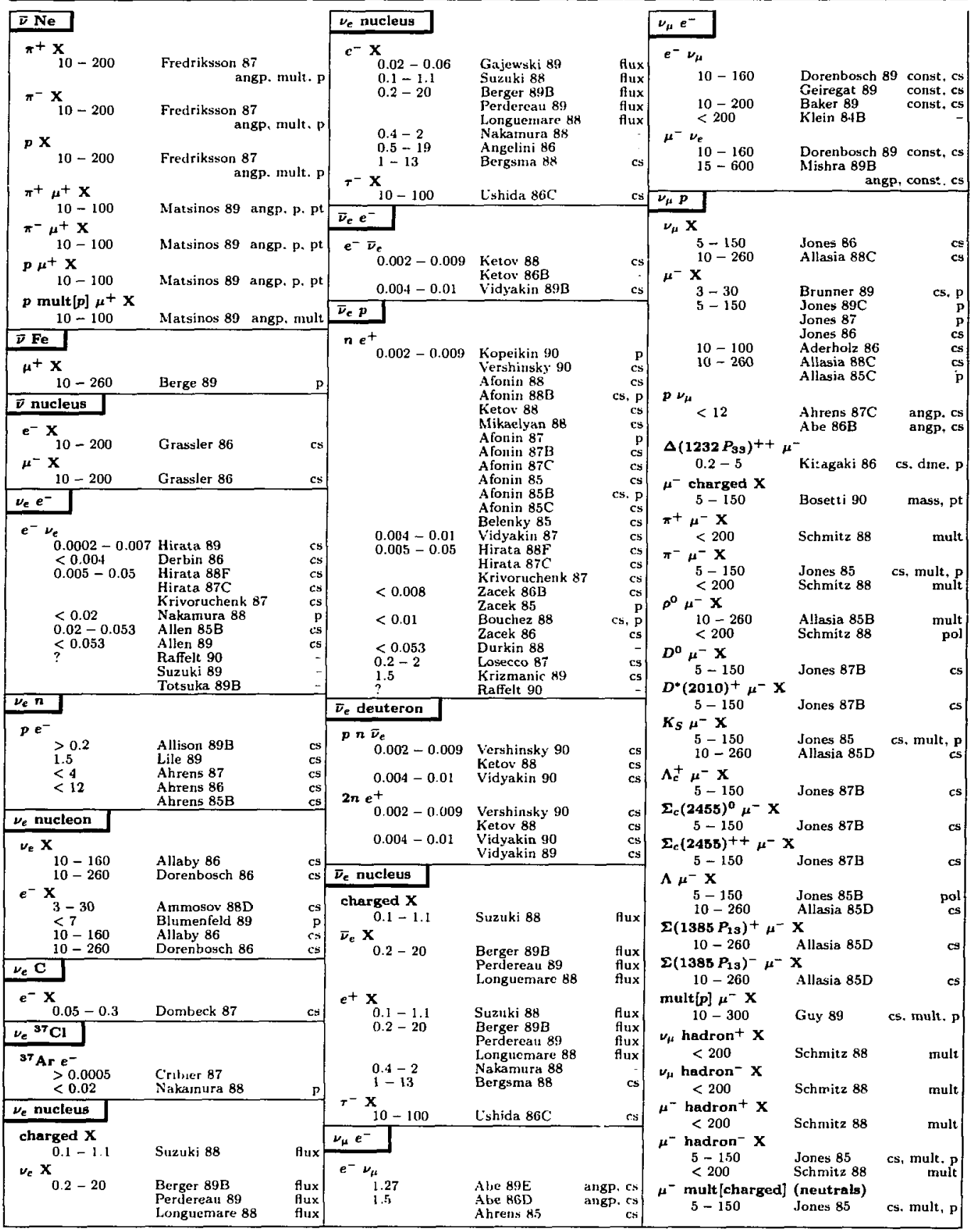




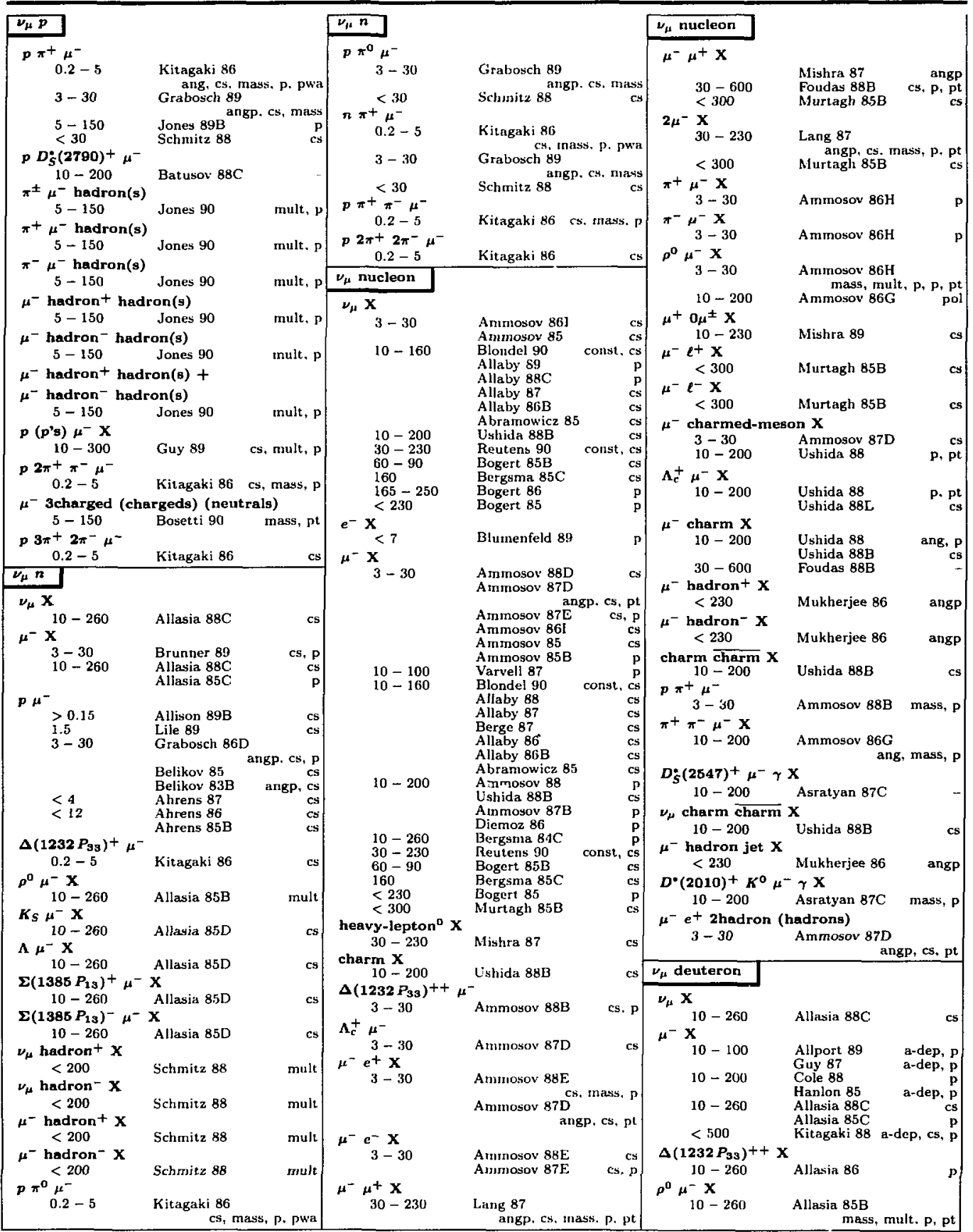

Entries in order of beam mass. then target mass, then multiplicity of final state. Inclusive reactions have an " $X "$ as the l'wst of the final state particles. Certain chemical symbols for nuclei have been changed to ivoid ambiguity with particle nanes (See the Particle Vocabulary.) Beain momenta are $P_{1 n t}$ in $\mathrm{GeV} / c$, or in parentheses $E_{\mathrm{cm}}$ it $\mathrm{GeV}$. See the legend on page 15,3. 


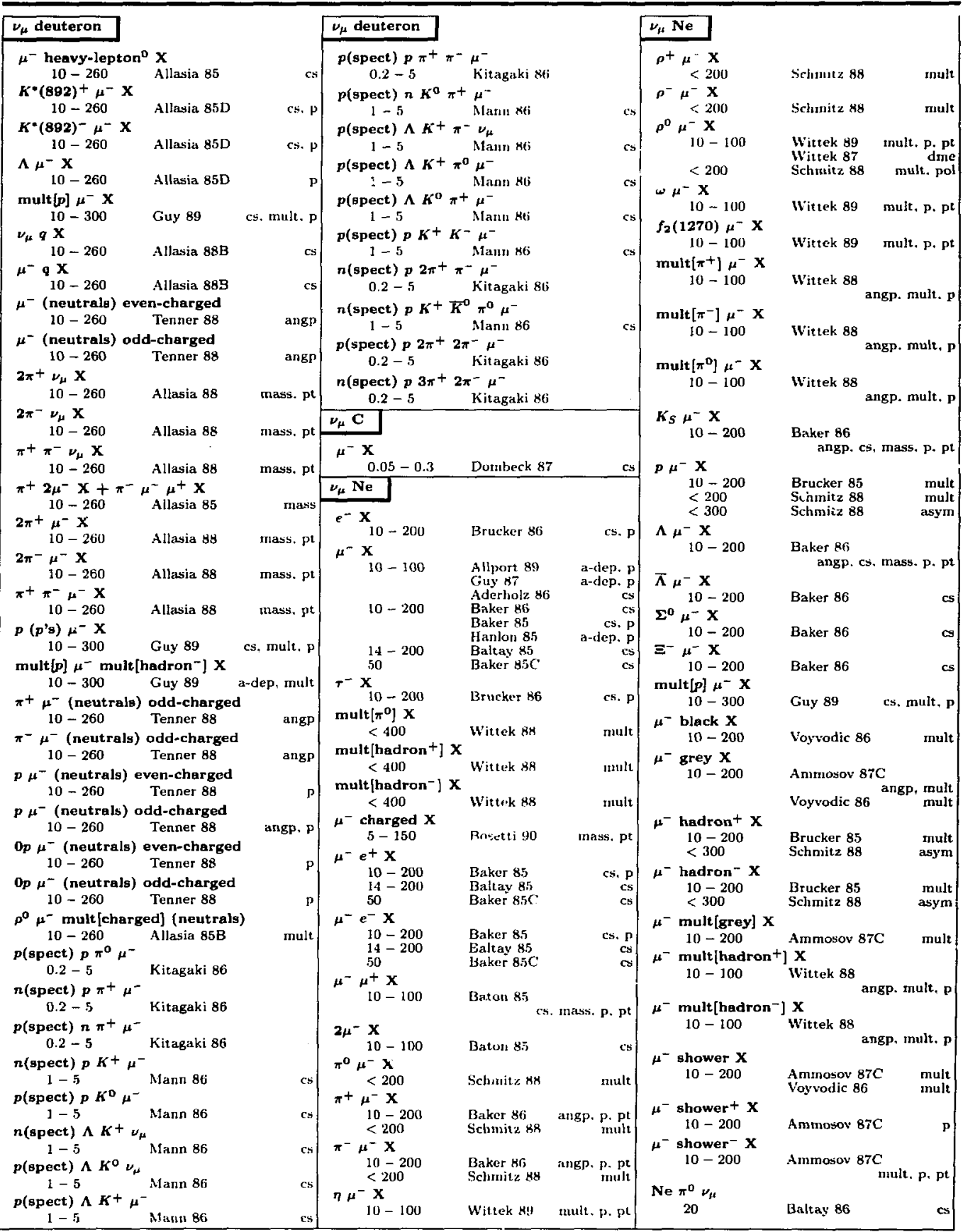




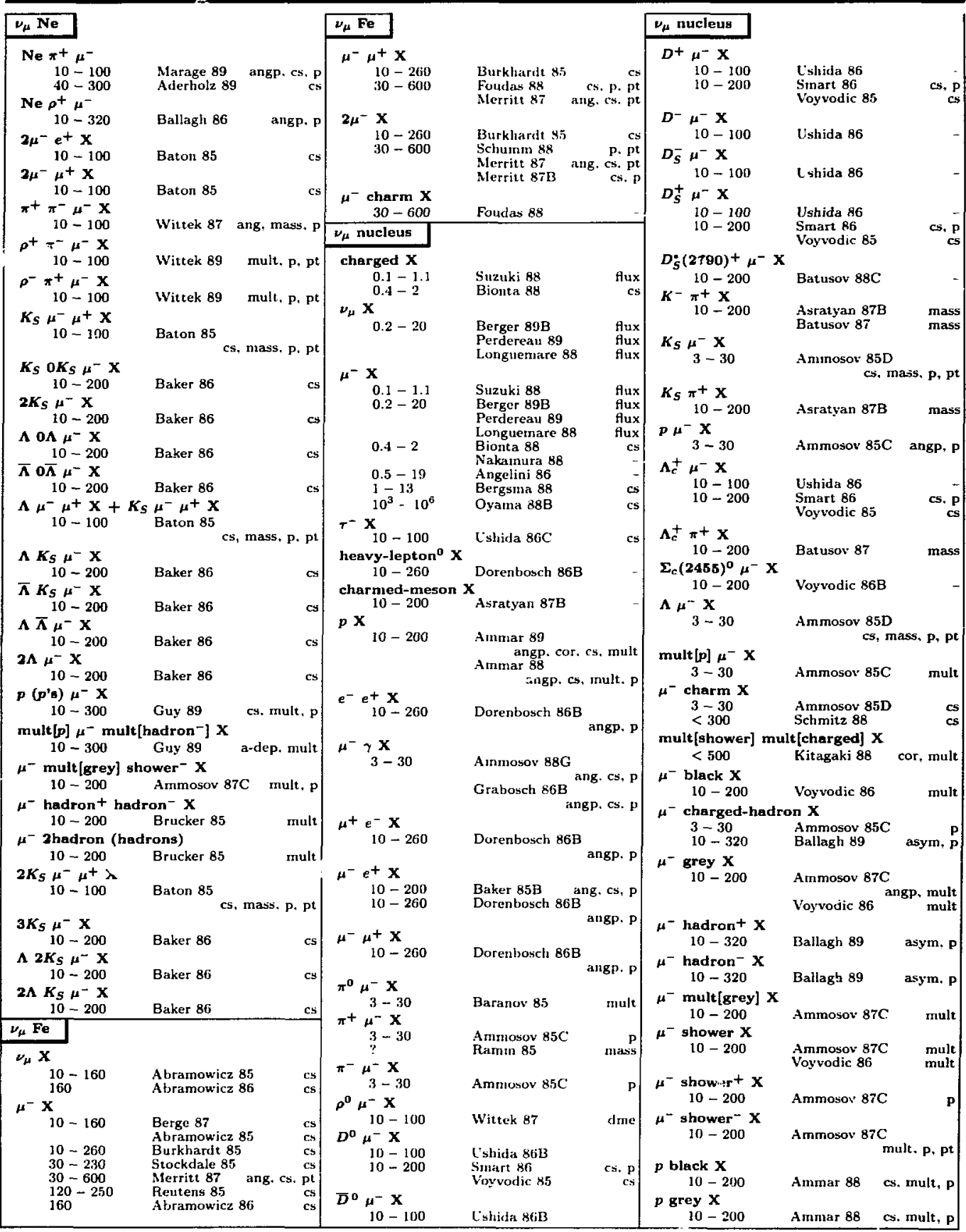




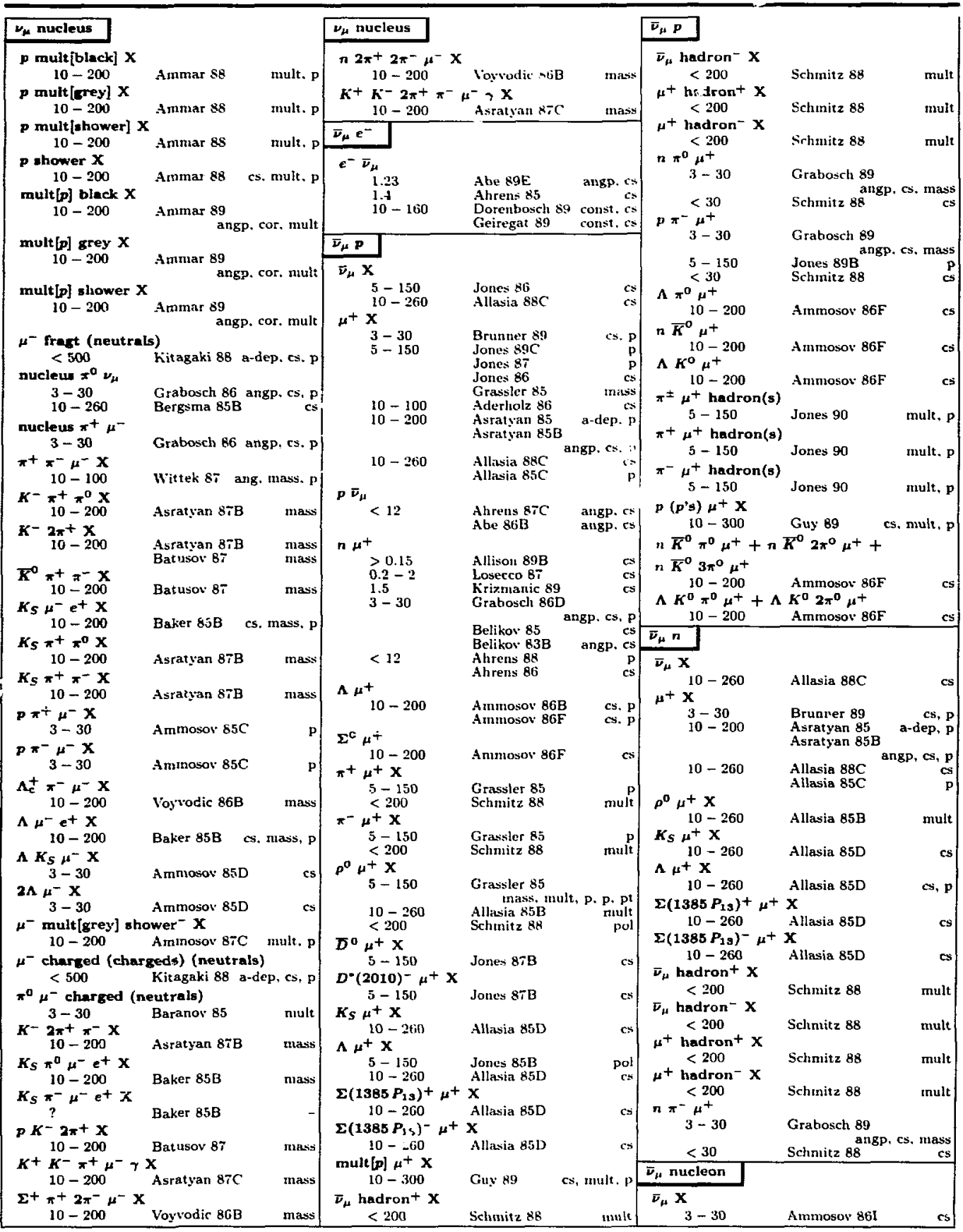




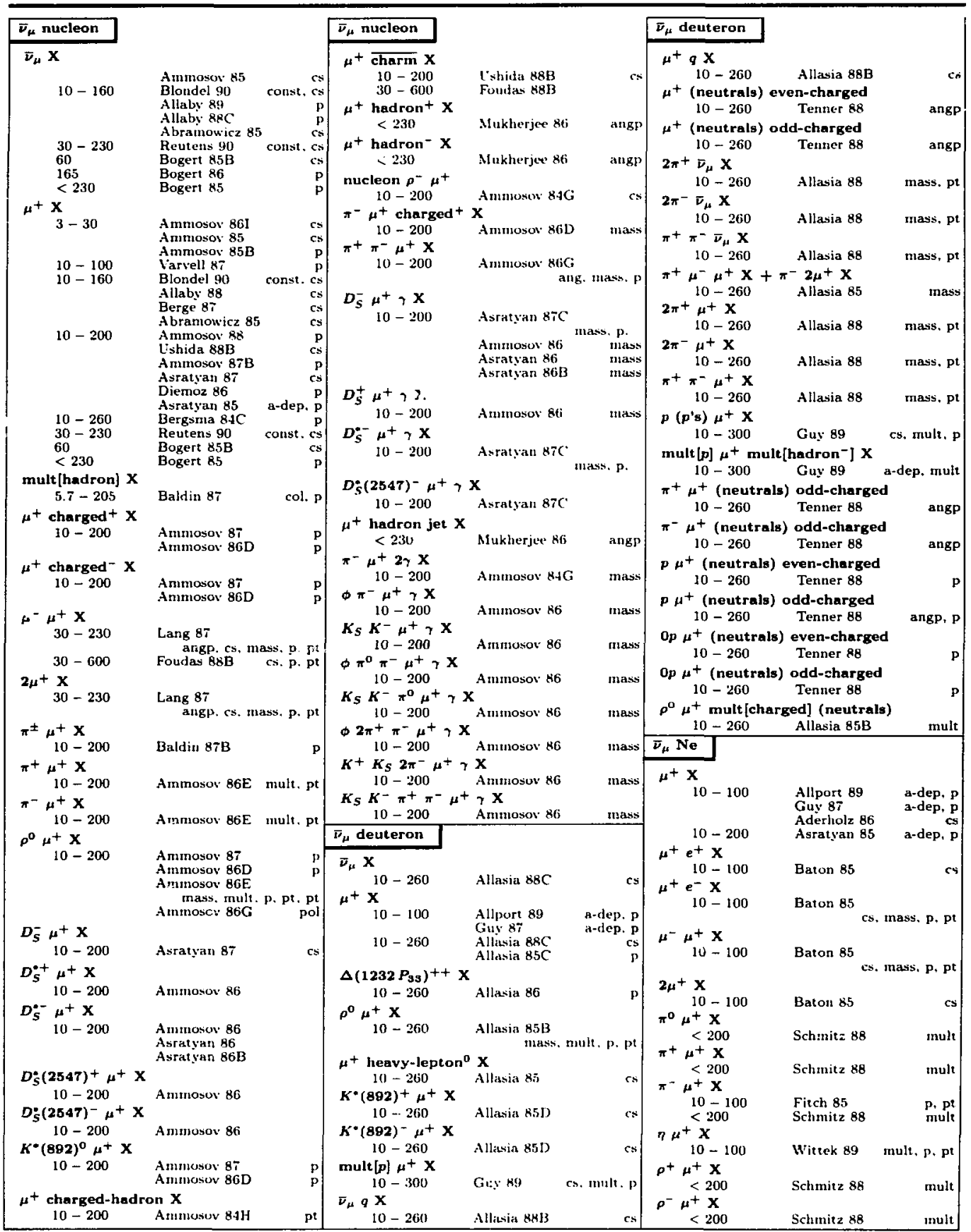

Entries in order of beam mass. then target mass. then multiplicity of final state. Inclusive reactions lave an " $X$ " is the last of the final state particles. Certain chemical symbols for nuclei have been changenl to ivoid anthiguty with pirticle manes (See the Particle Vocabulary.) Beam momenta are $P_{\text {lat }}$ in GeV/c, or in parentheses $E_{1, m}$ in GeV. See 1 ho legend on page 153. 


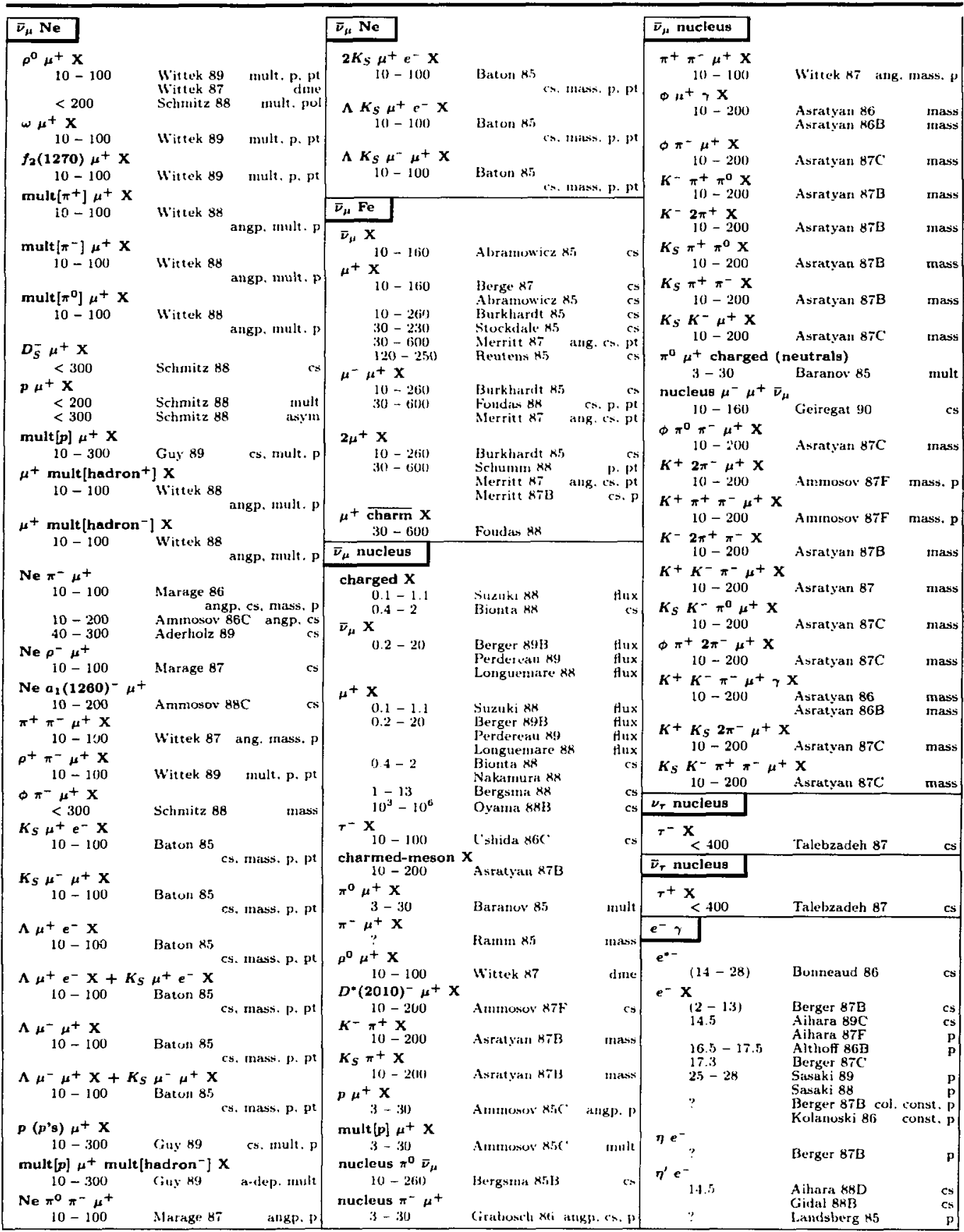




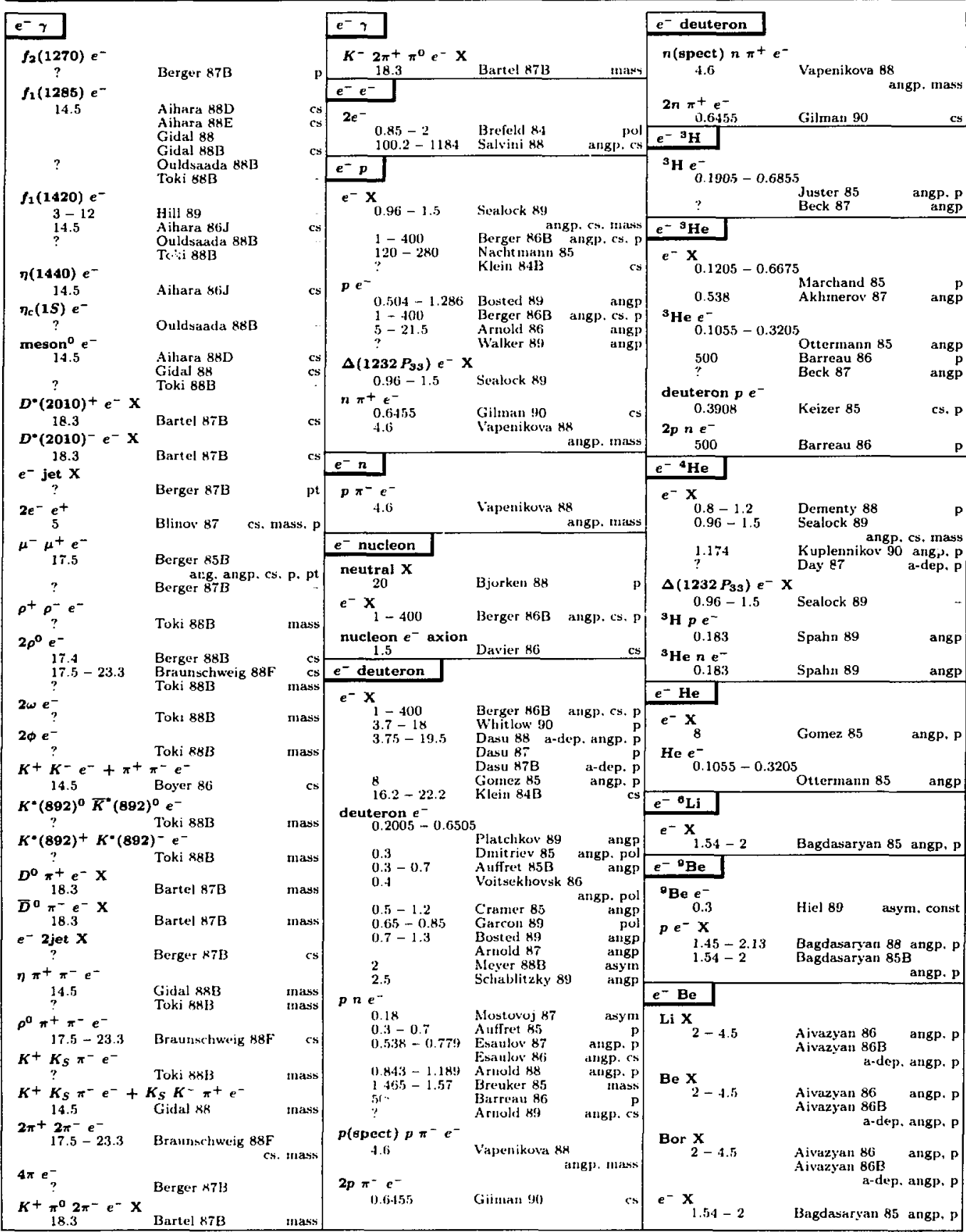

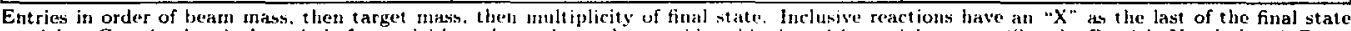
particles. Certain chemical syonbols for anclei have heen changed to avojd andiguity uith particle names (See (he Particle Vocabulary.) Beam

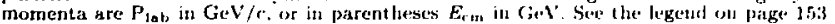




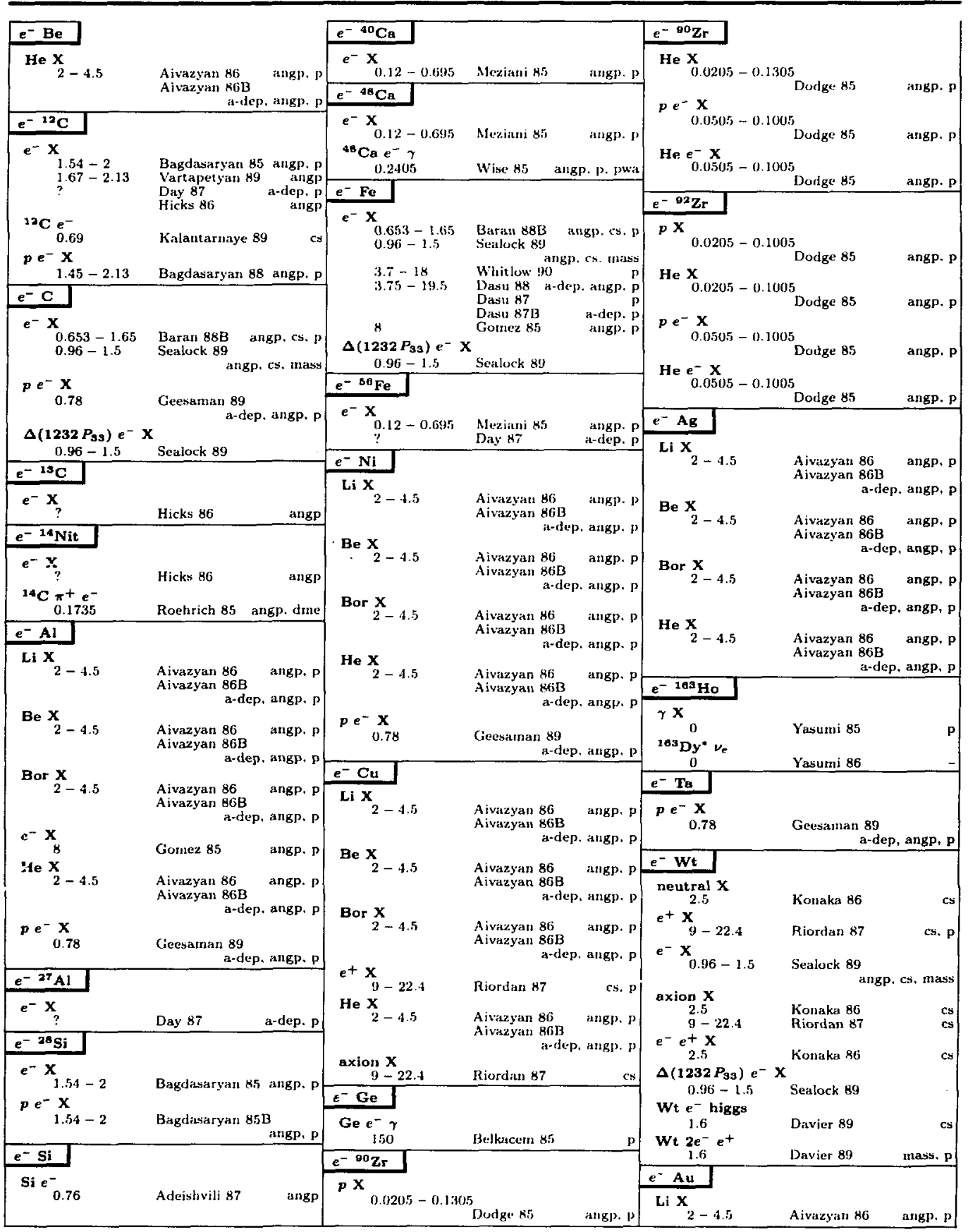




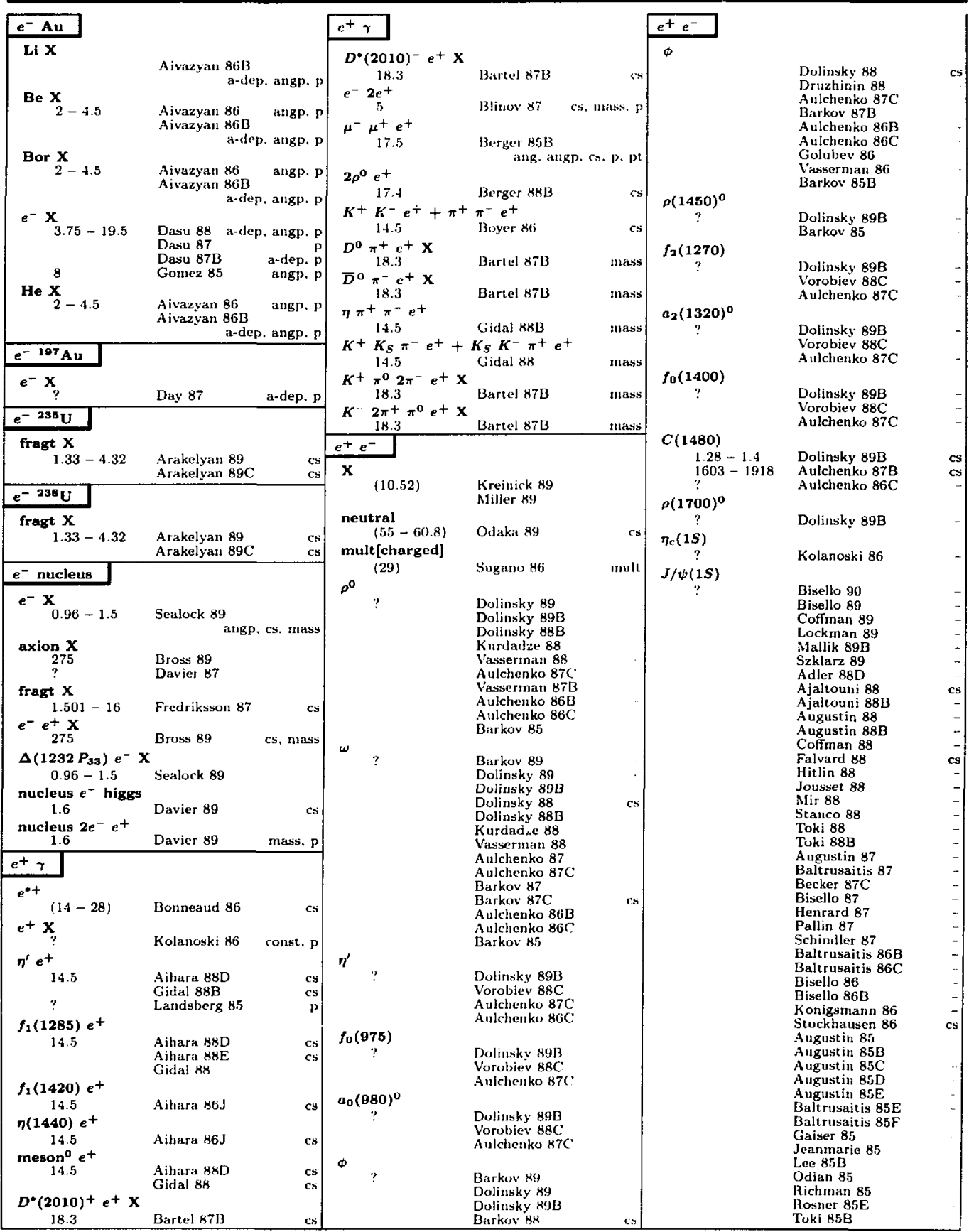

Entries in order of beam mass. then target mass, then multiplicity of timal state. Inclusive reactions have an " $\mathrm{X}^{*}$ is lhe last of the final state

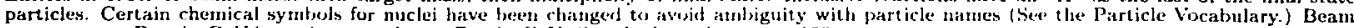

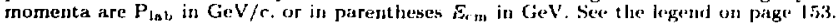




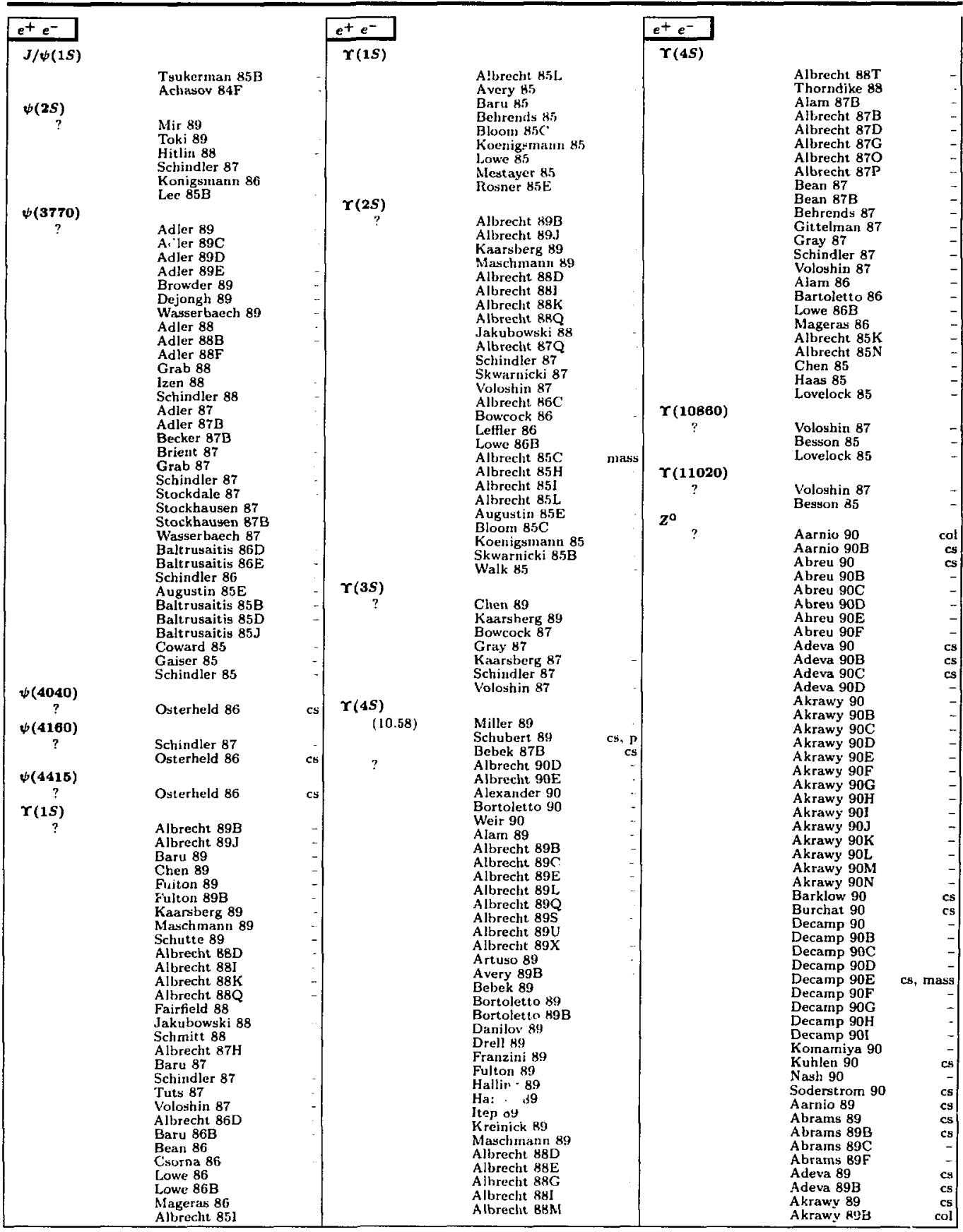




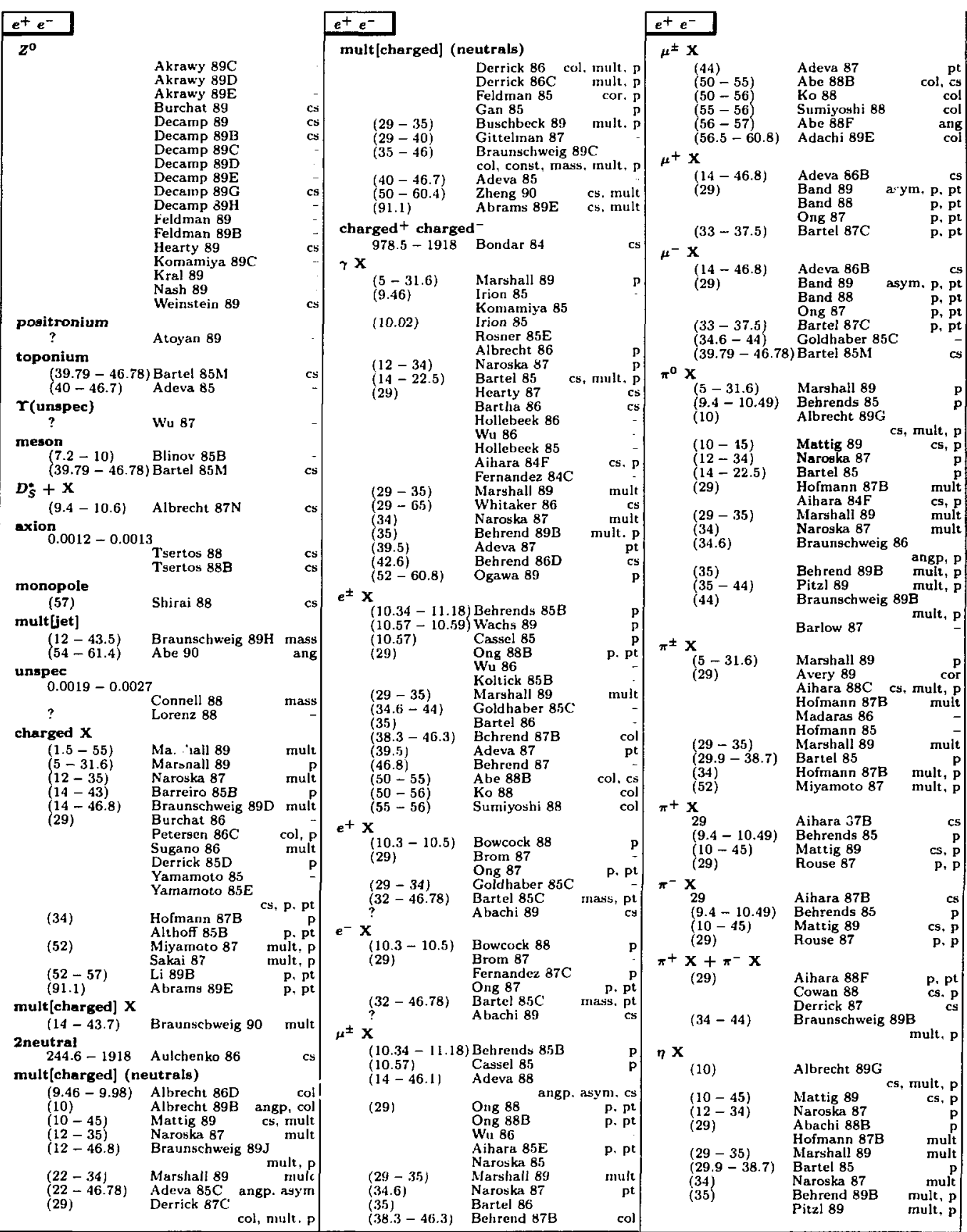

Entries in order of beam mays, then target mass, then multiplicity of final state. Inclusive reactions have an " $X$ " as the last of the final state particles. Certain chemical symbols for nuclei have been changed to avoid ambiguity with particle names (Sec the Particle Vocabulary.) Beam momenta are $\mathrm{P}_{\mathrm{lab}}$ in $\mathrm{GeV} / \mathrm{c}$, or in parentheses $E_{\mathrm{cm}}$ in $\mathrm{GeV}$. See the legend on puge 153. 


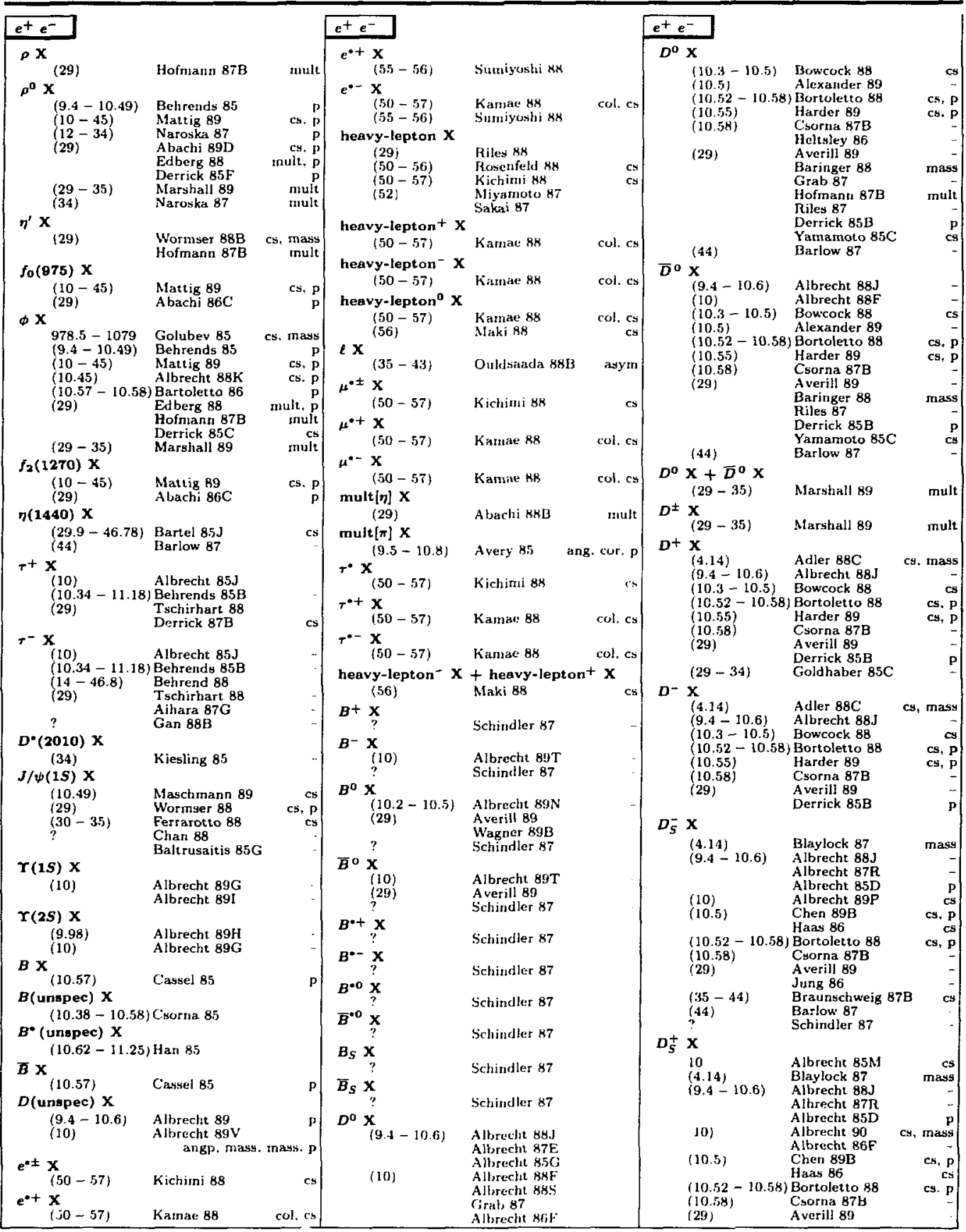




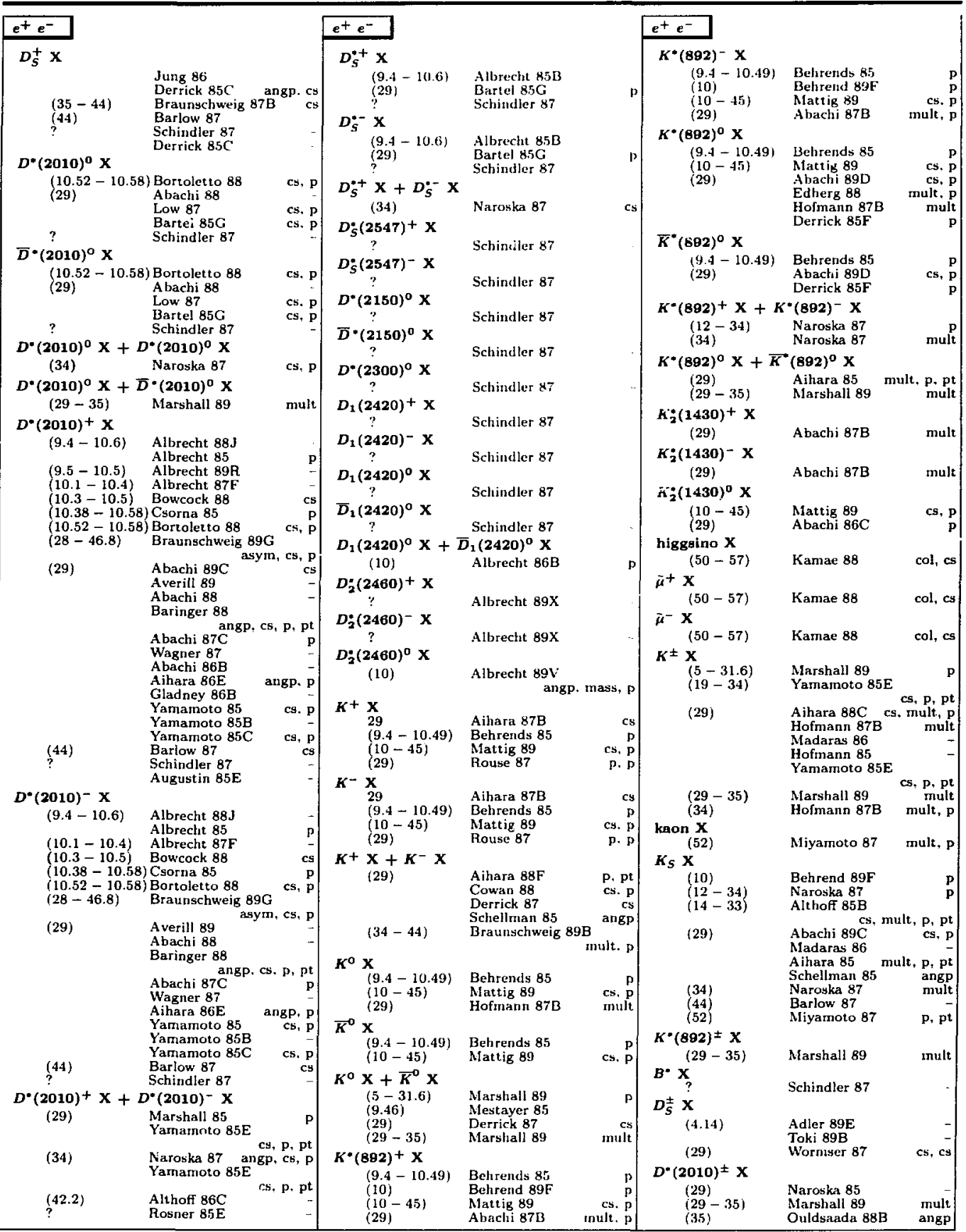

Fntries in order of beam mass, then target mass, then multiplicity of final state. Inclusive reactions have an " $\mathrm{X}$ " as the last of the final state particles. Certain chemical symhols for nuclei have been changed to avoil ambiguity with particle names (See the Particle vocabulary.) Beam momenta are $P_{\text {Isb }}$ in $\mathrm{GeV} / c$. or in parentheses $E_{c \mid 31}$ in $\mathrm{GeV}$. See the Jegend on page 15:3. 


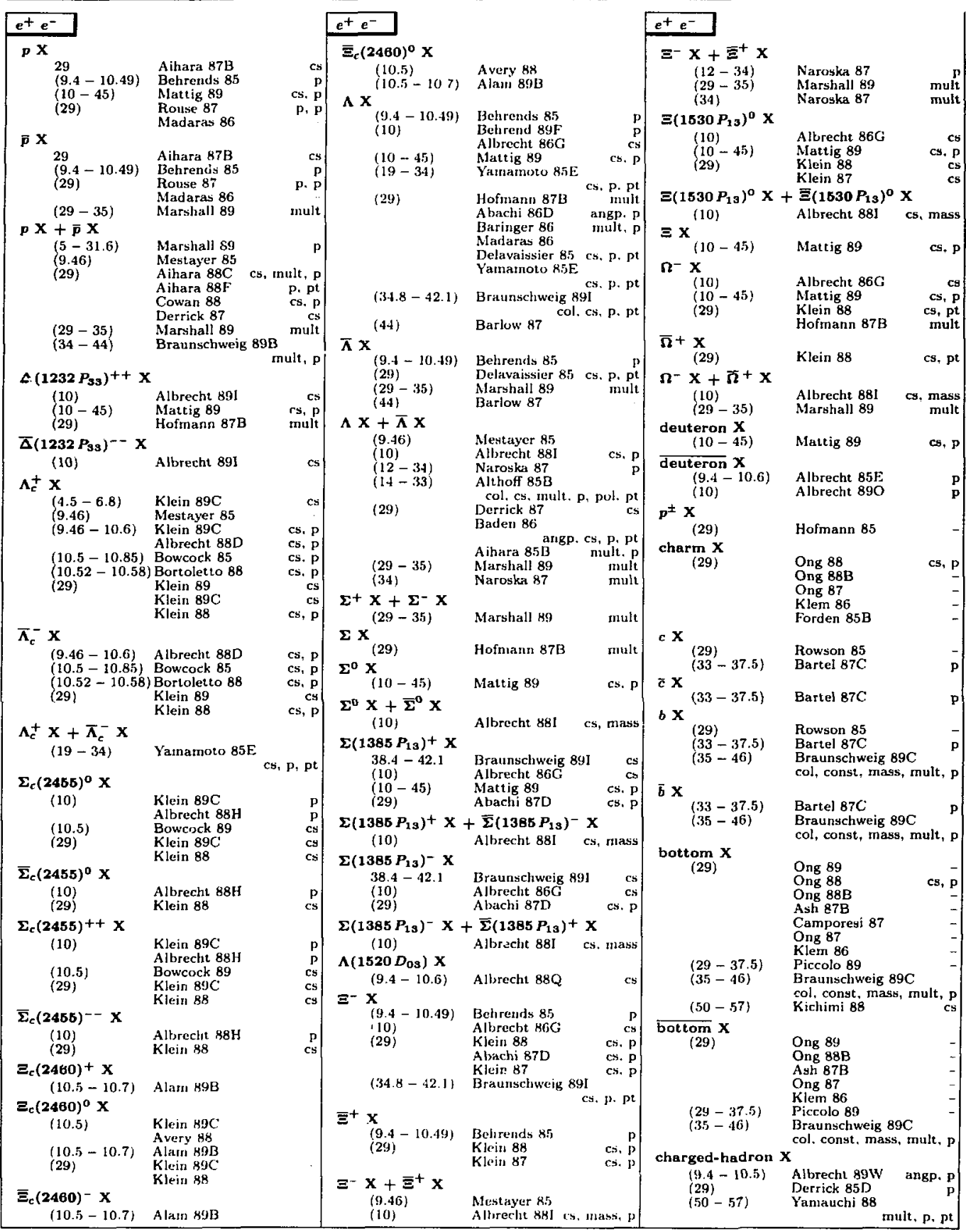




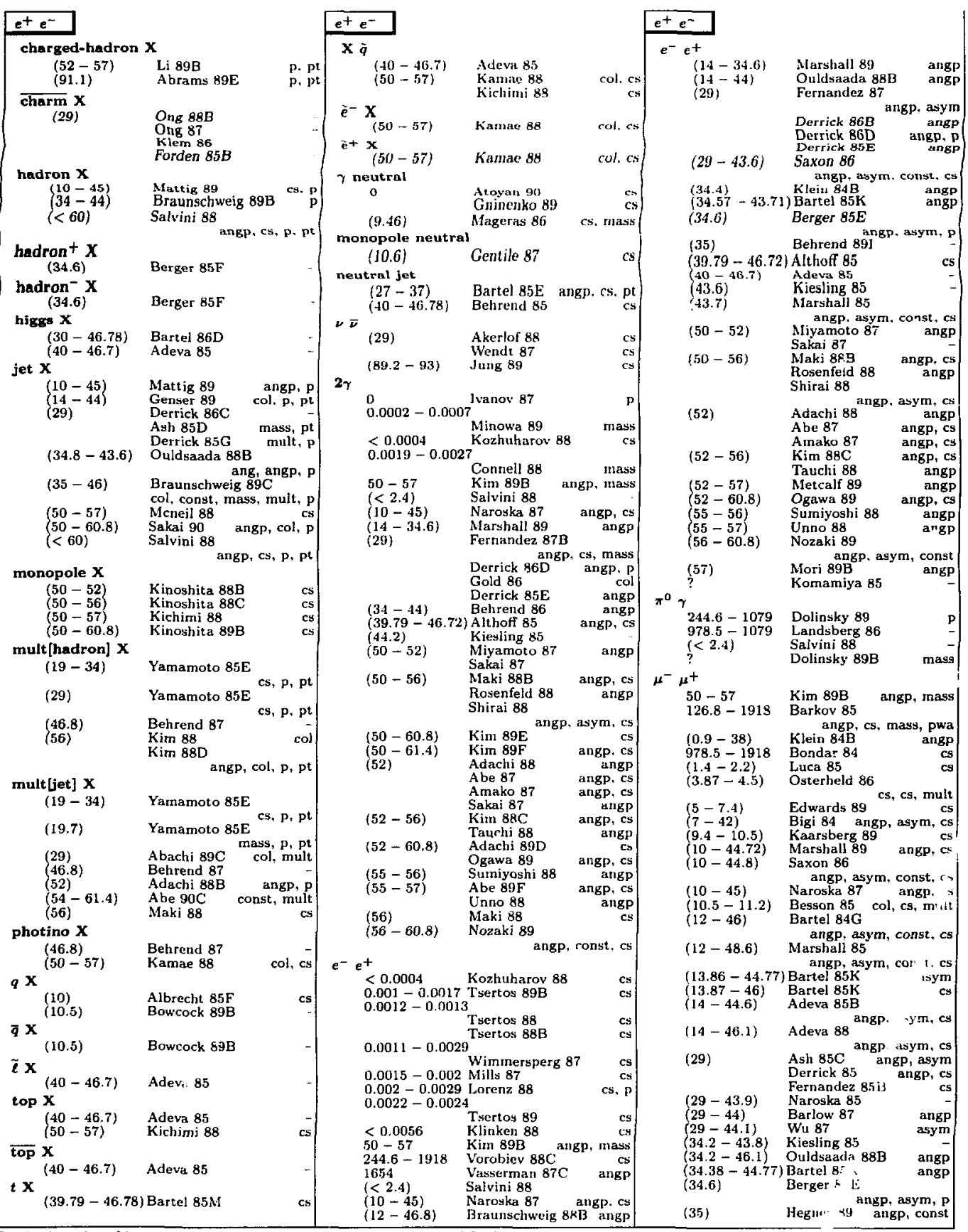

Entries in order of beam mass, then targel mass, then multiplicity of final state. Inclusive reactions have an " $X "$ as the l. $-t$ of $t$ he final state particles. Certain chemical symbols for nuclei have been changed to avoid ambiguity with particle names (See the Particle locabulary.) Beam momenta are $\mathrm{P}_{\mathrm{sb}}$ in $\mathrm{GeV} / \mathrm{c}$. or in parentheses $E_{\mathrm{rm}}$ in $\mathrm{GeV}$. See the legentl on page 153 . 


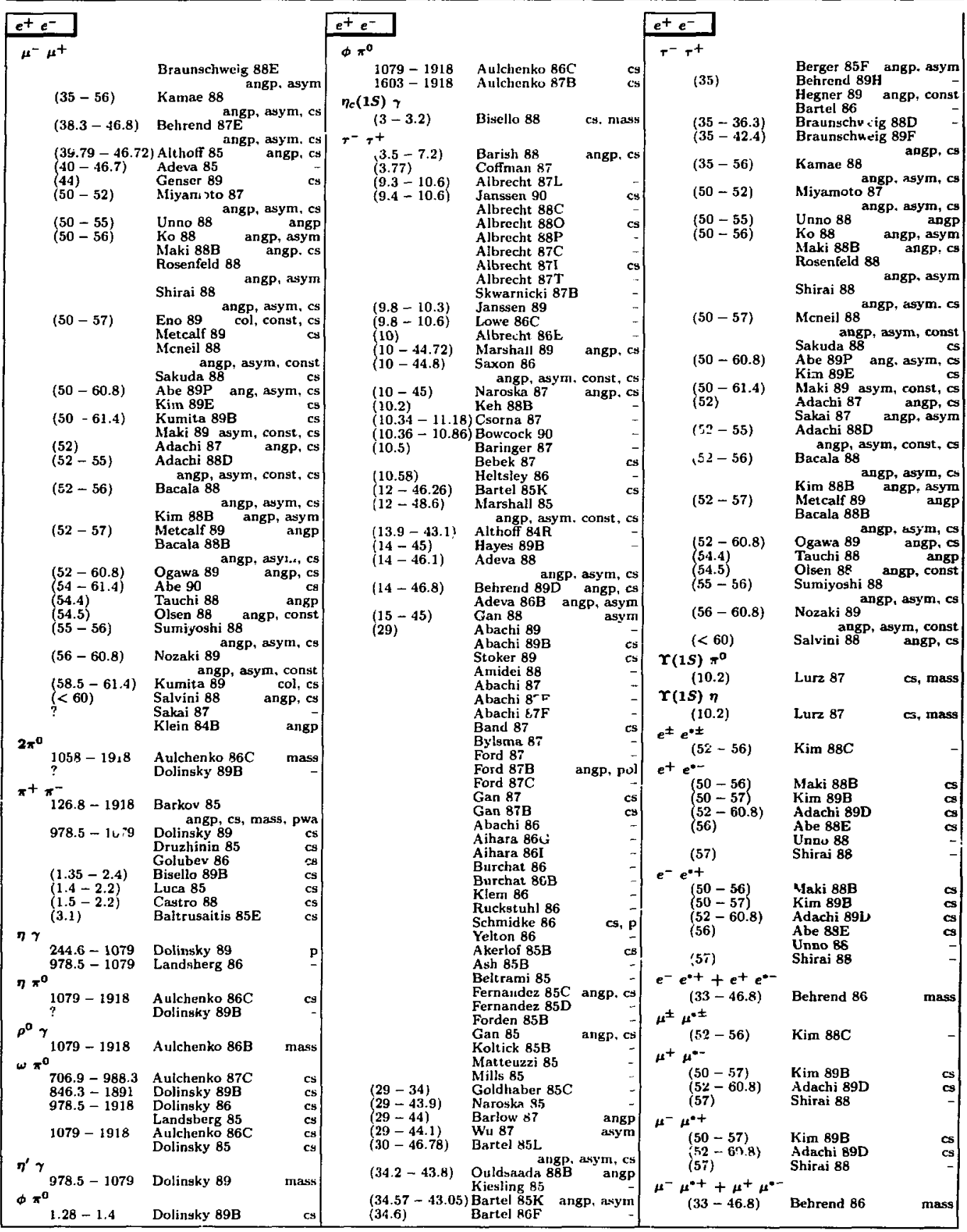




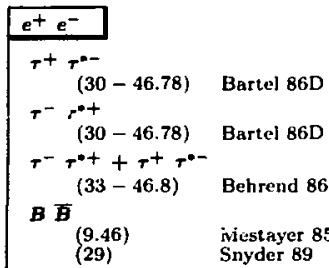

$B$ (unspec) $\bar{B}$ (unspec)

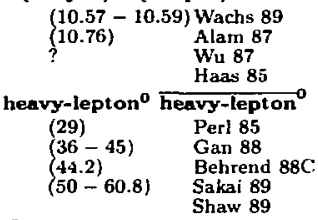

$\bar{\ell}^{0} \mathrm{e}$ ?

$\overline{\boldsymbol{q}}^{*} \boldsymbol{q}^{*}$

(46.8) Behrend 86C

$(52-60.8) \quad$ Ogawa 89

2B (unspec)

$\begin{array}{ll}(35-44) & \text { Elsen 90 } \\ e^{*+} & \\ 244.6-1918 & \text { Aulchenko 86 } \\ (33-46.8) & \text { Behrend 86 } \\ (50-56) & \text { Maki 88B } \\ (50-57) & \text { Kinn 89B } \\ (52-56) & \text { Kirn 88C } \\ (52-60.8) & \text { A jachi 89D } \\ (56) & \text { Abe 88E } \\ (57) & \text { Unno 88 } \\ & \text { Shirai 88 } \\ & \text { Yamauchi 88 }\end{array}$

2heavy-e

? Dolinsky 89B

2heavy-lepton

(56) Kirn 88

heavy-lepton- heavy-lepton ${ }^{-}$

(29) Mathis 88

$(36-45) \quad$ Gan 88

(44.2) Behrend $88 \mathrm{C}$

(50-52) Igarashi 87

$(50-56) \quad$ Ko 88

Maki 88B

Shirai 88

$(50-60.8) \quad$ Kim $89 \mathrm{E}$

(52) Adachi 88B

Amako 87

Yoshida 87

$(52-55) \quad$ Yamauchi 68

$(52-60.8)$ Adachi B9B

$(55-56) \quad$ Sumiyoshi 88

(56) Abe 88D

Kim 88D

Kim 88D

Wu 87

2heavy-lepton 0

$(50-60.8) \quad$ Kim $89 \mathrm{E}$

$2 \ell \quad(9.45-10.57)$ Casisel 85

$e^{+} e^{-}$

$$
\begin{array}{ll}
(40-46.7) & \text { Adeva } 85 \\
(50-60.8) & \text { Sakai } 89
\end{array}
$$

2lepton-colored

$(50-30.8) \quad$ Kim $89 \mathrm{G}$

$\mu^{*+} \mu^{*-}$

$\begin{array}{ll}(33-46.8) & \text { Behrend } 86 \\ (50-56) & \text { Maki } 88 \mathrm{~B}\end{array}$

\begin{tabular}{l}
$(50-57) \quad \mathrm{Kim} 89 \mathrm{~B}$ \\
\hline
\end{tabular}

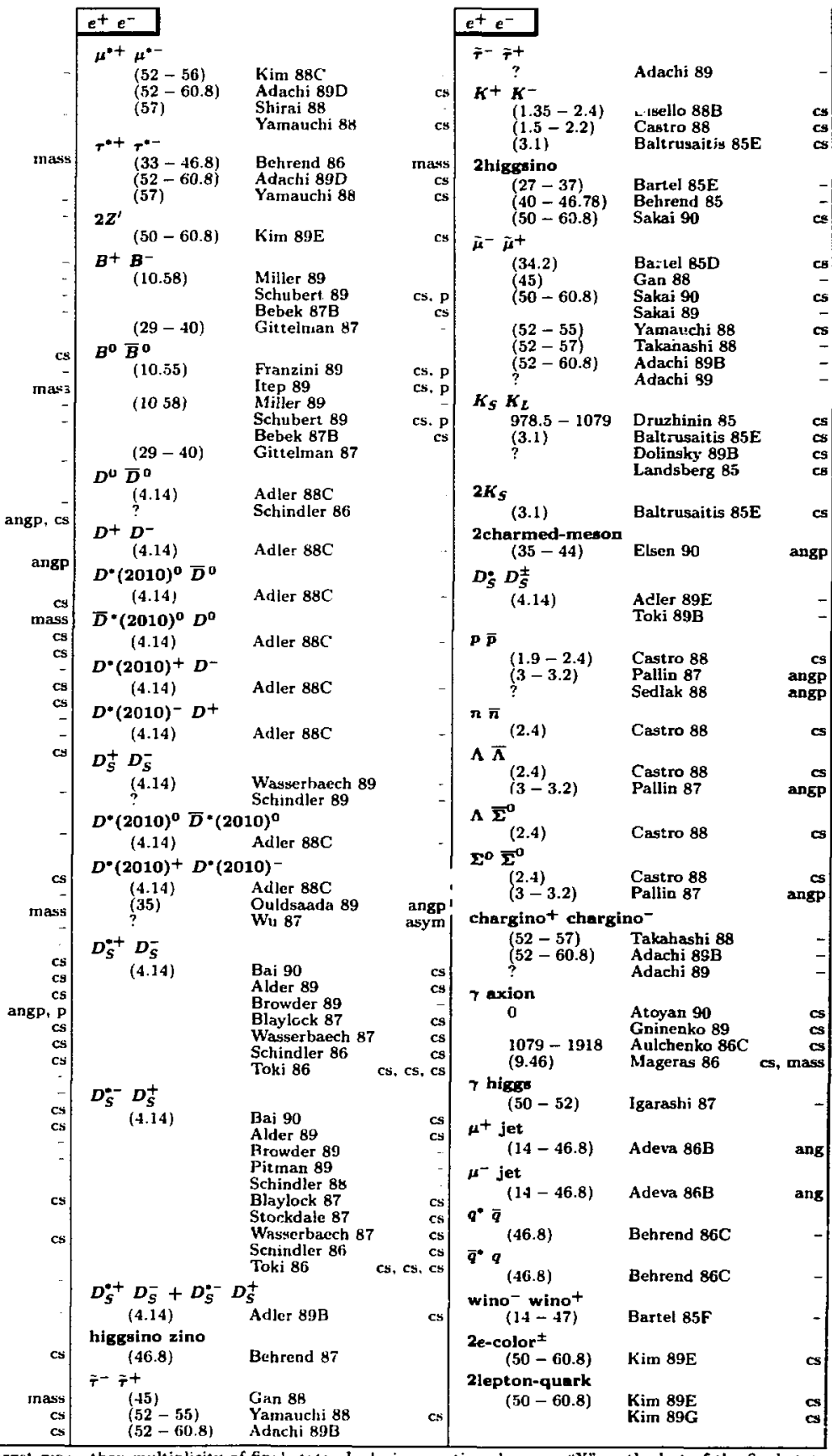

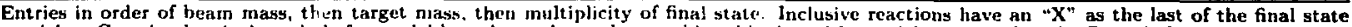

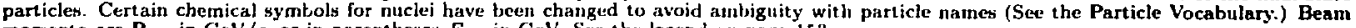
momenta are $P_{1 a t}$ in $G e V / r$, or in parentheses $E_{r m}$ in GeV. See the Irgend on page 15.3. 


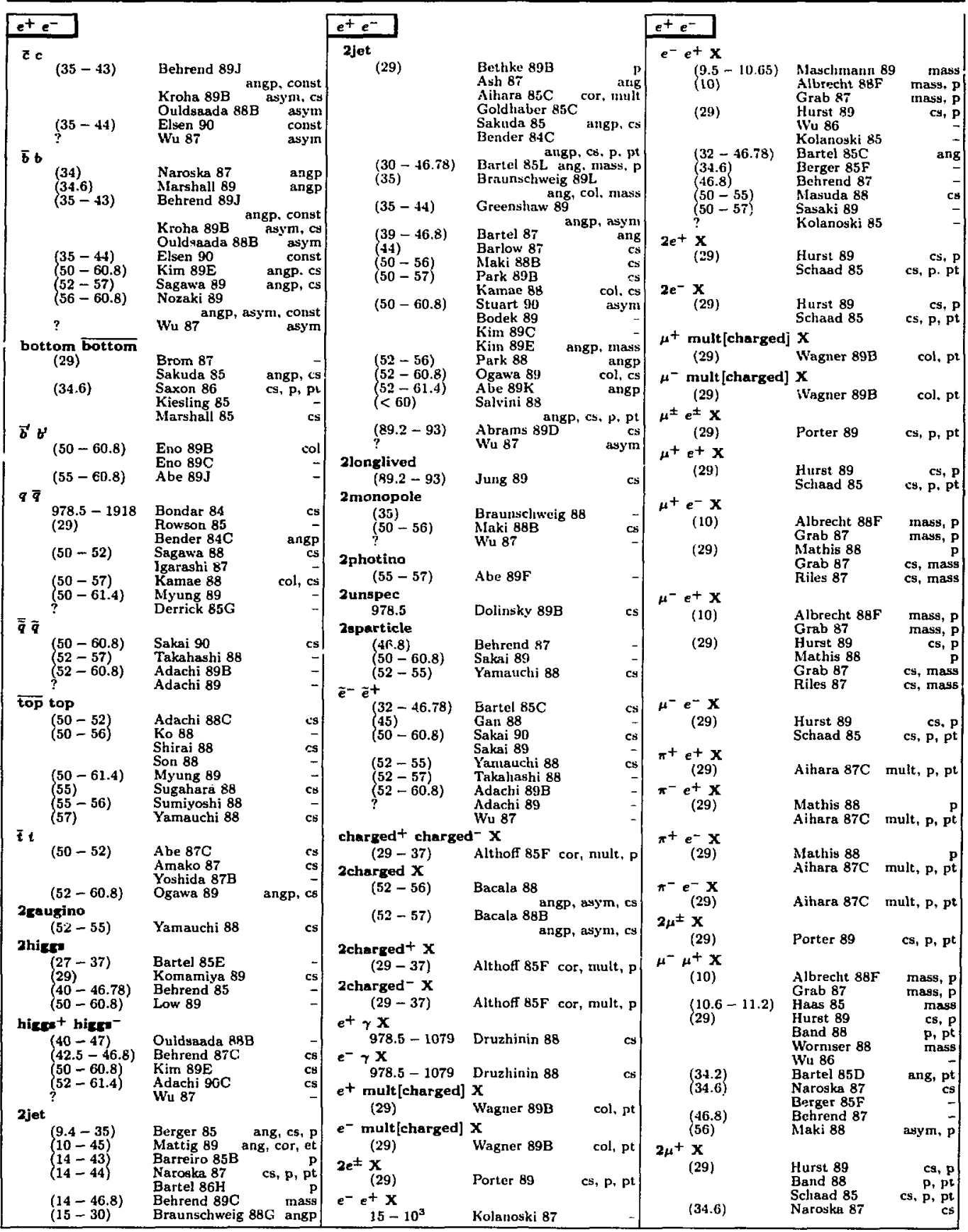




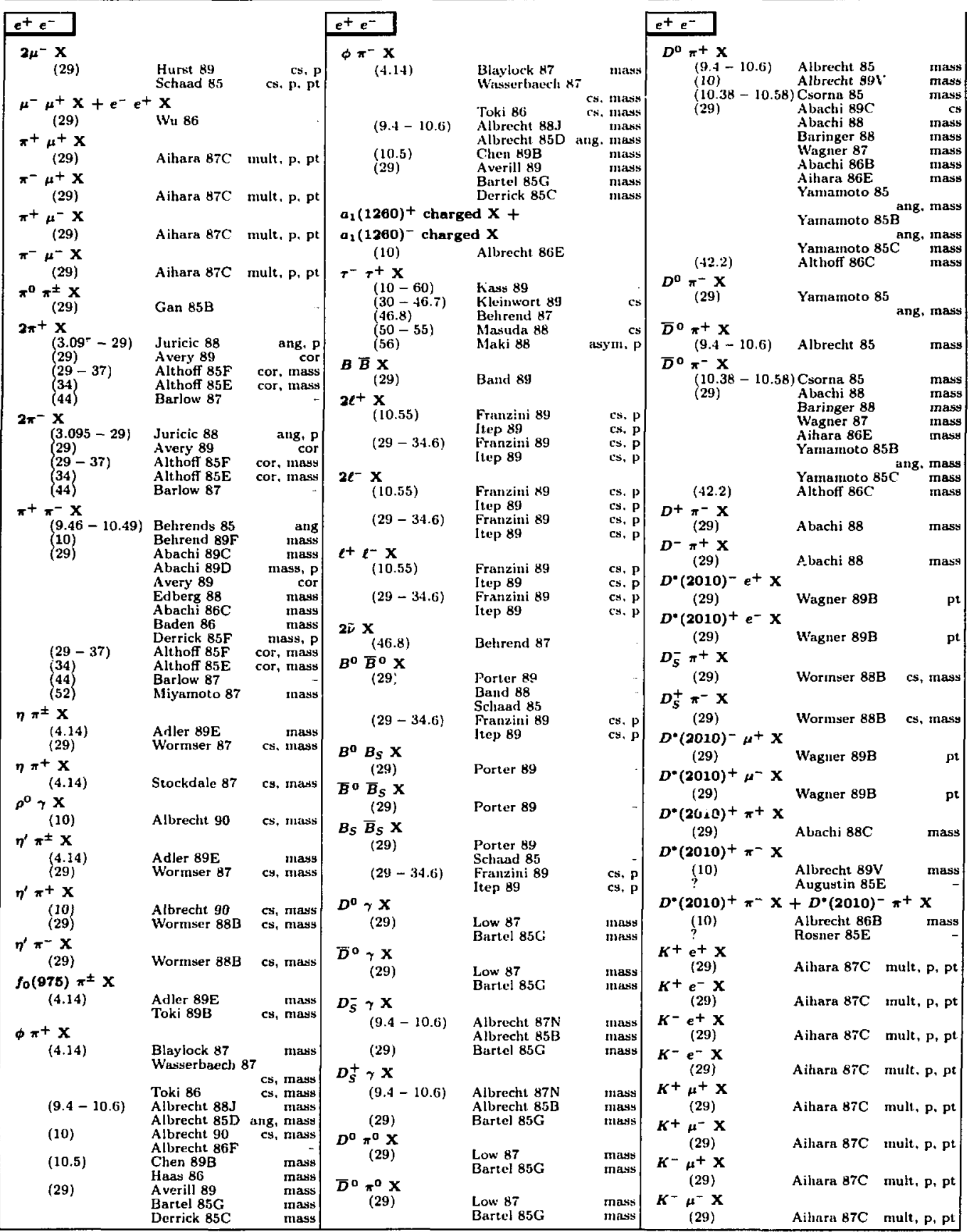

Entries in order of beam masy, then target mass, then multiplicity of final state. Inclusive renctions have an " $X$ " is the last of the final state particley. Certain chemical symbols for nuclei have been changed to nvoid arnbiguity with particle names (See the Particle Vocabulary.) Beam momenta are $P_{\text {lab }}$ in $\mathrm{GeV} / c$. or in parentheses $E_{\mathrm{ctan}}$ in $\mathrm{GeV}$. See the legend on page 153. 


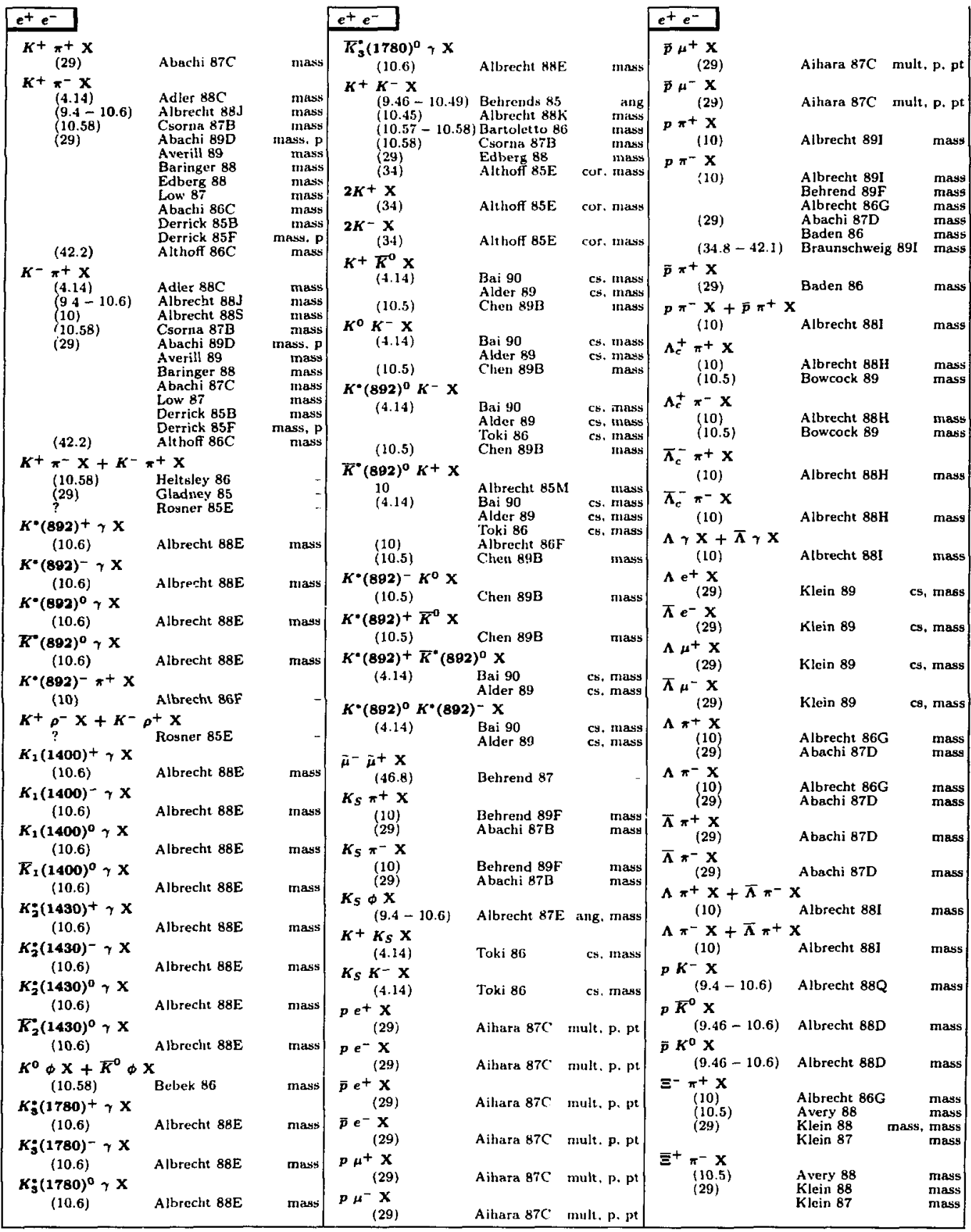




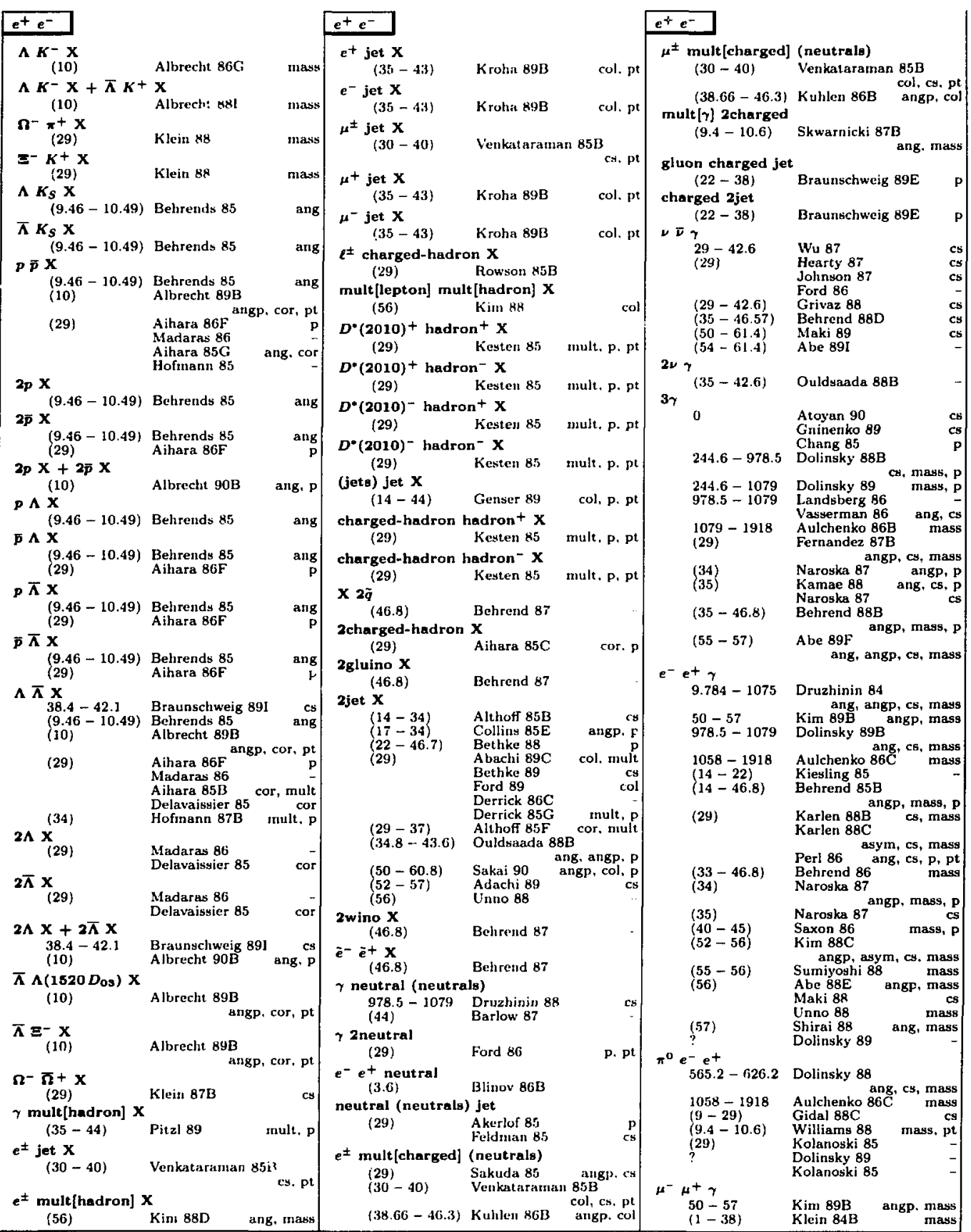

Entries in order of beam inass, then target mass, then multiplicity of final state. Inclusive reactions have an " $\mathrm{X}^{n}$ as the last of the final state particles. Certain chemical symbols for nuclei have beten changed to avoid atnbiguity with jurticle natmes (See the Particle Vocabulary.) Beam momenta are $P_{\text {lab }}$ in $\mathrm{GeV} / \mathrm{c}$, or in parenthesey $E_{\mathrm{cm}}$ in $\mathrm{GeV}$. See the legesd on page 153. 


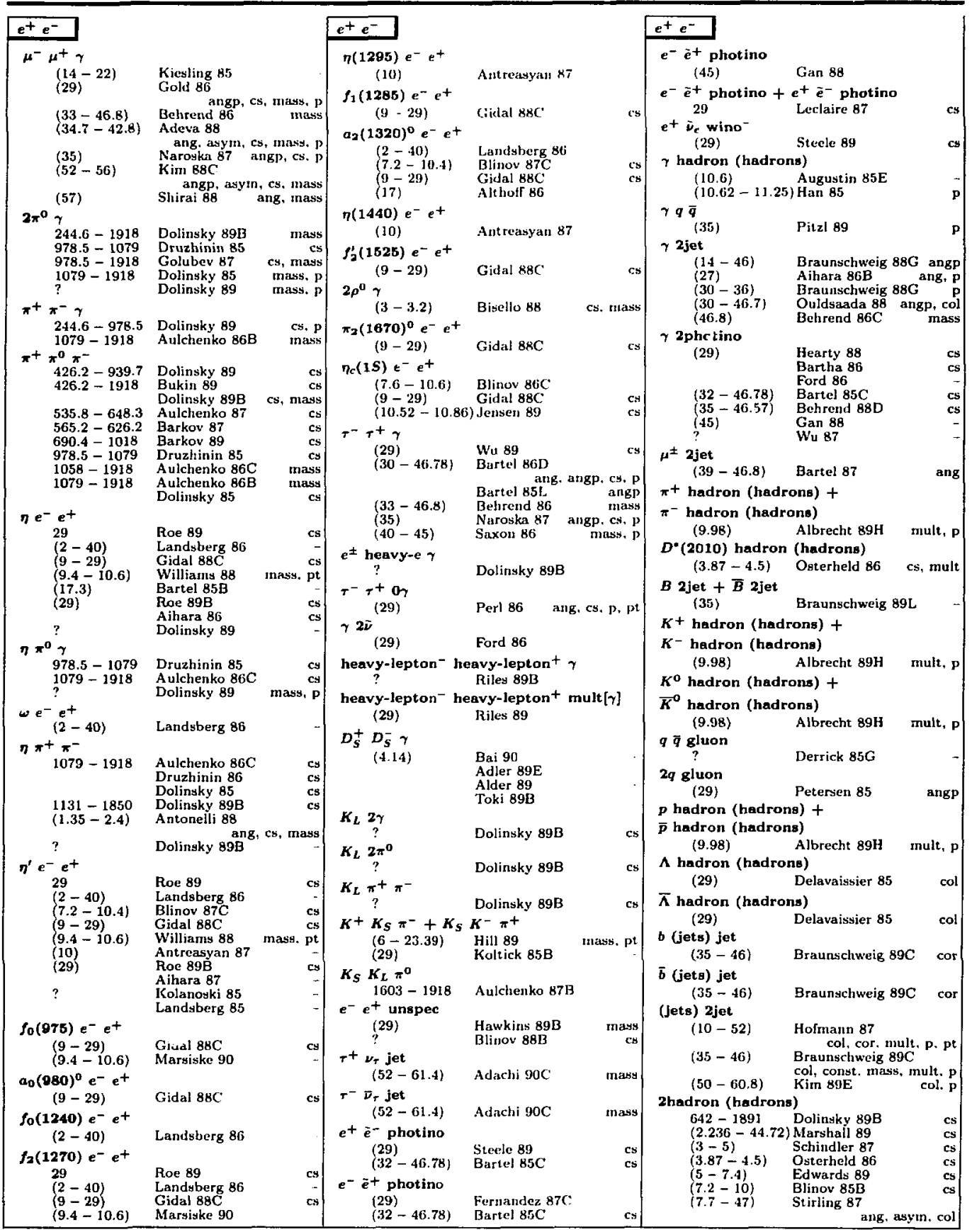




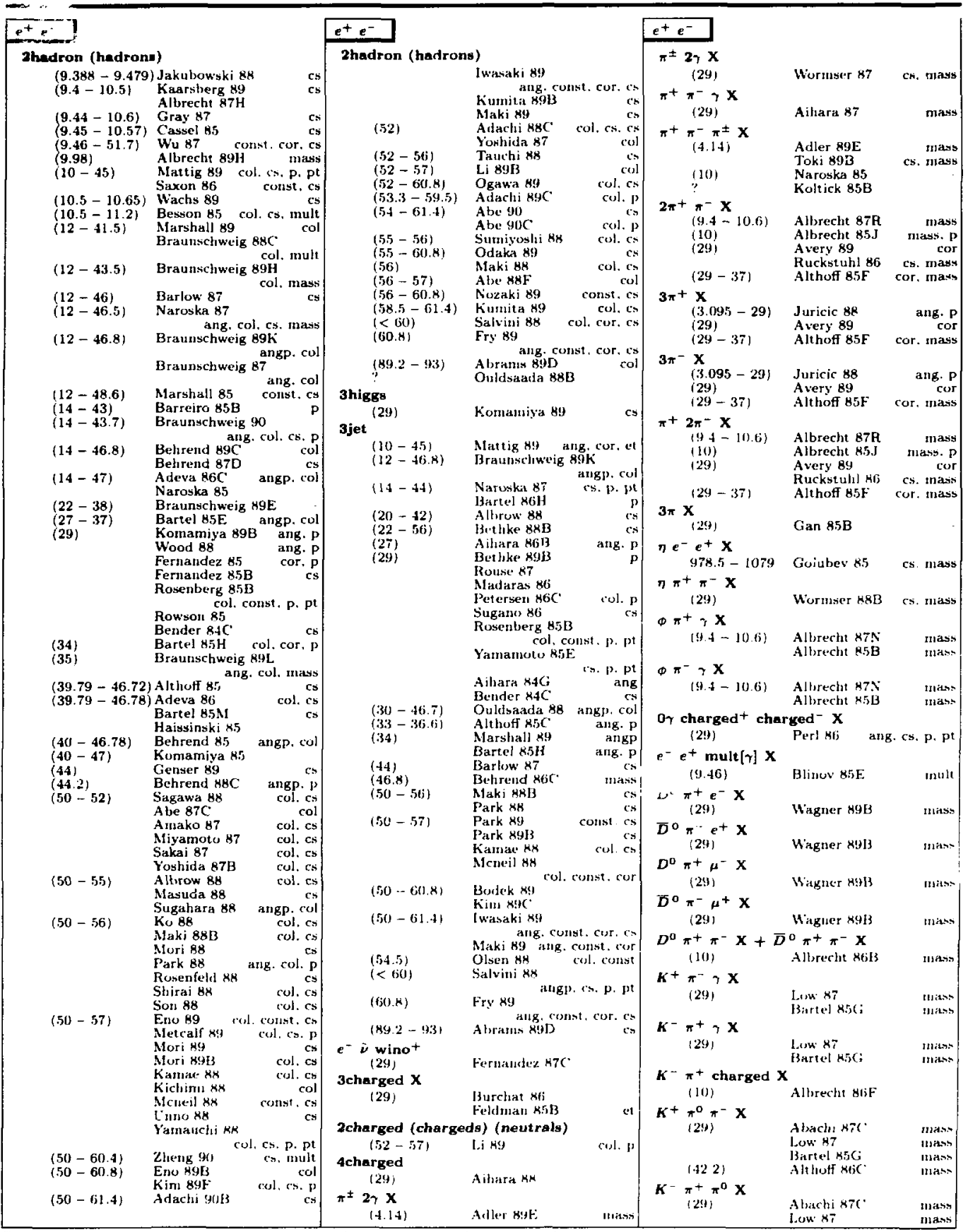

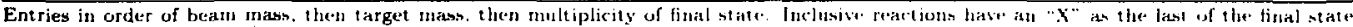

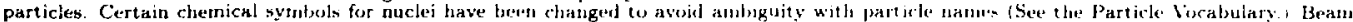

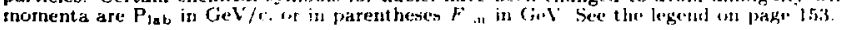




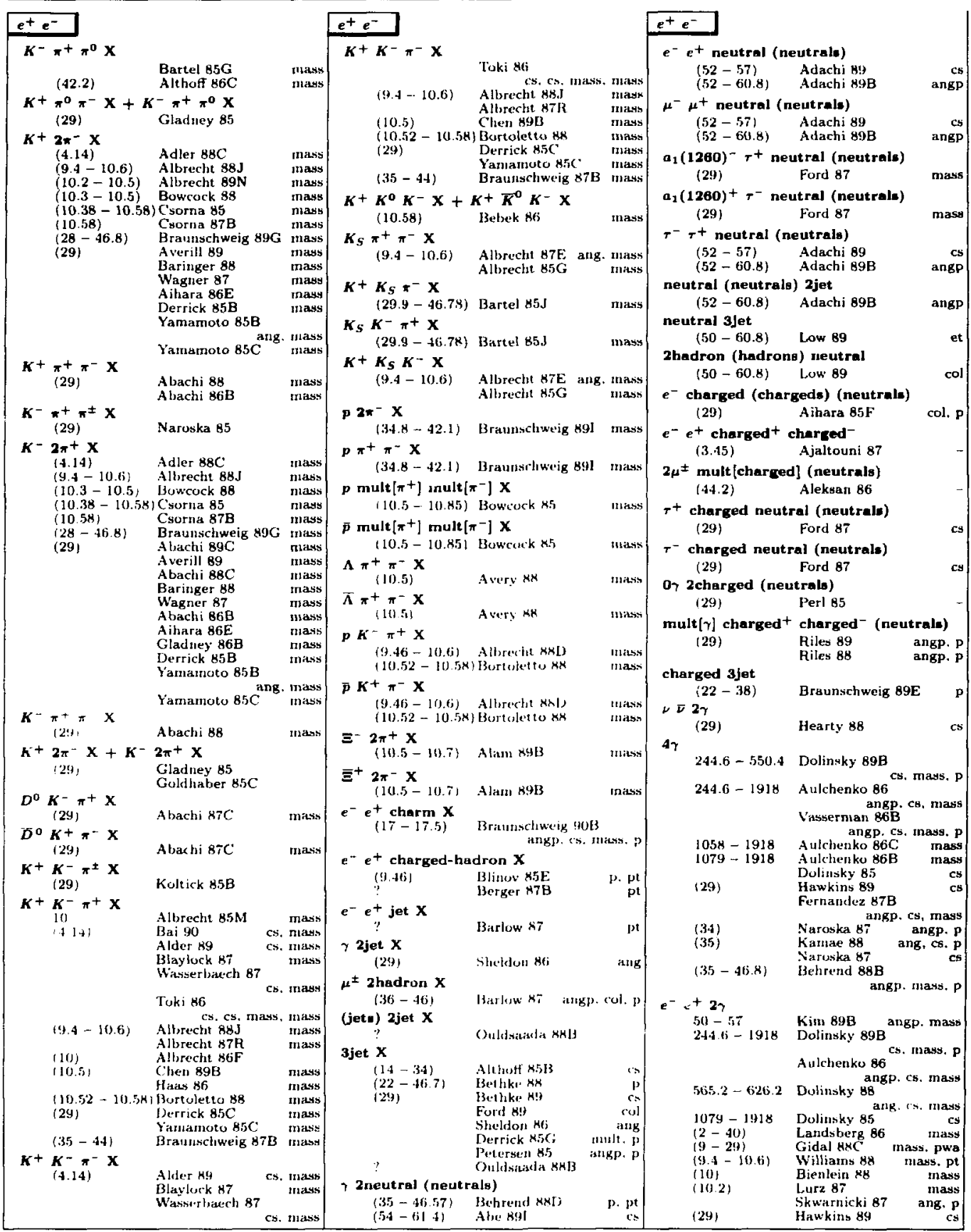




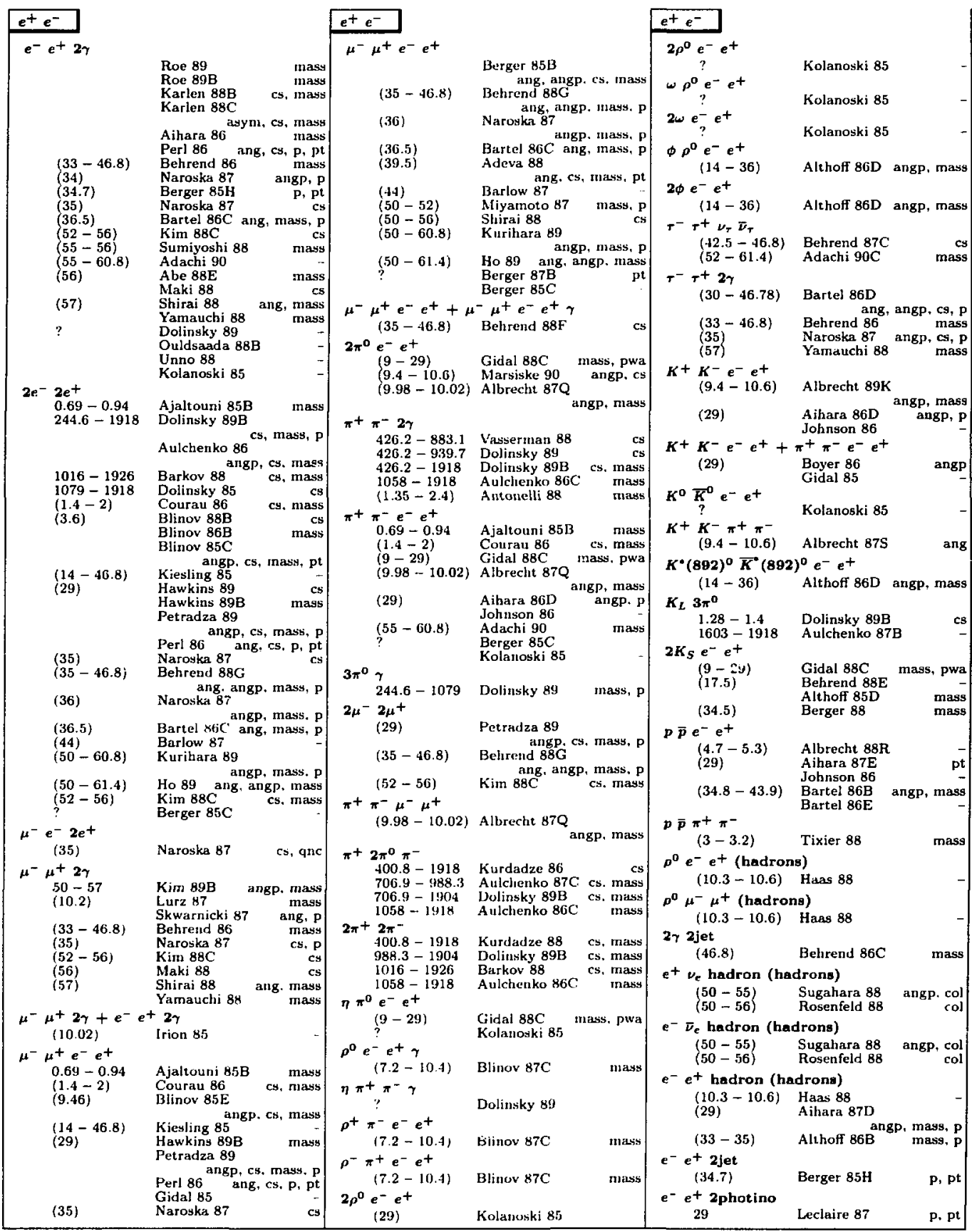

Entries in order of bean mass. then target nass. then multiplicity of fital state. Inclusive reactions have an " $\mathrm{X}$ " as the last of the final state particles. Certain chenical symbols for nuclei have been changed to avoid ambiguity witit particle names (See the Particle Vocabulary.) Beam momenta are $P_{1 a b}$ in $\mathrm{GeV} / \mathrm{c}$, or in parentheses $E_{c m}$ in $\mathrm{GeV}$. See the legend on page 153. 


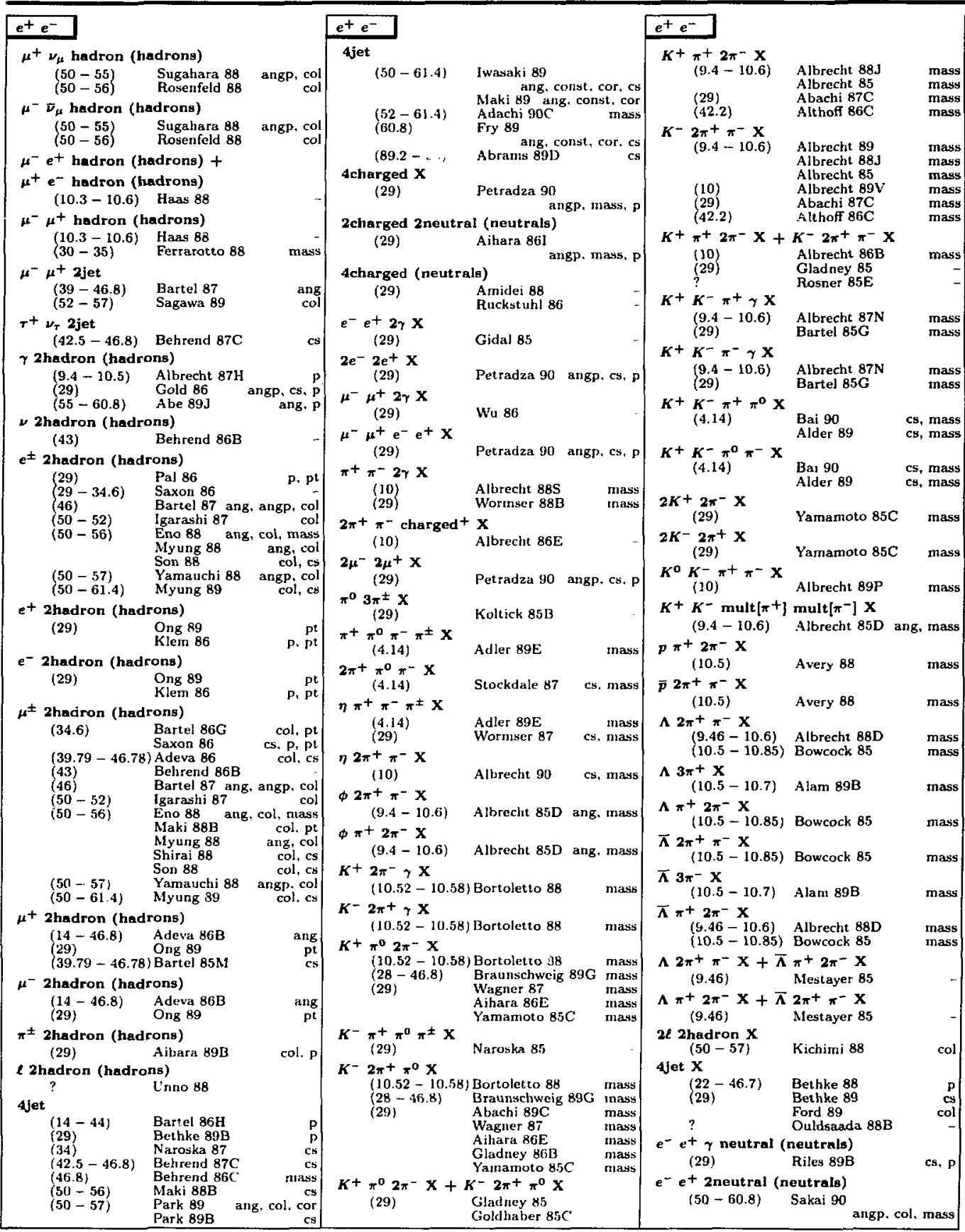




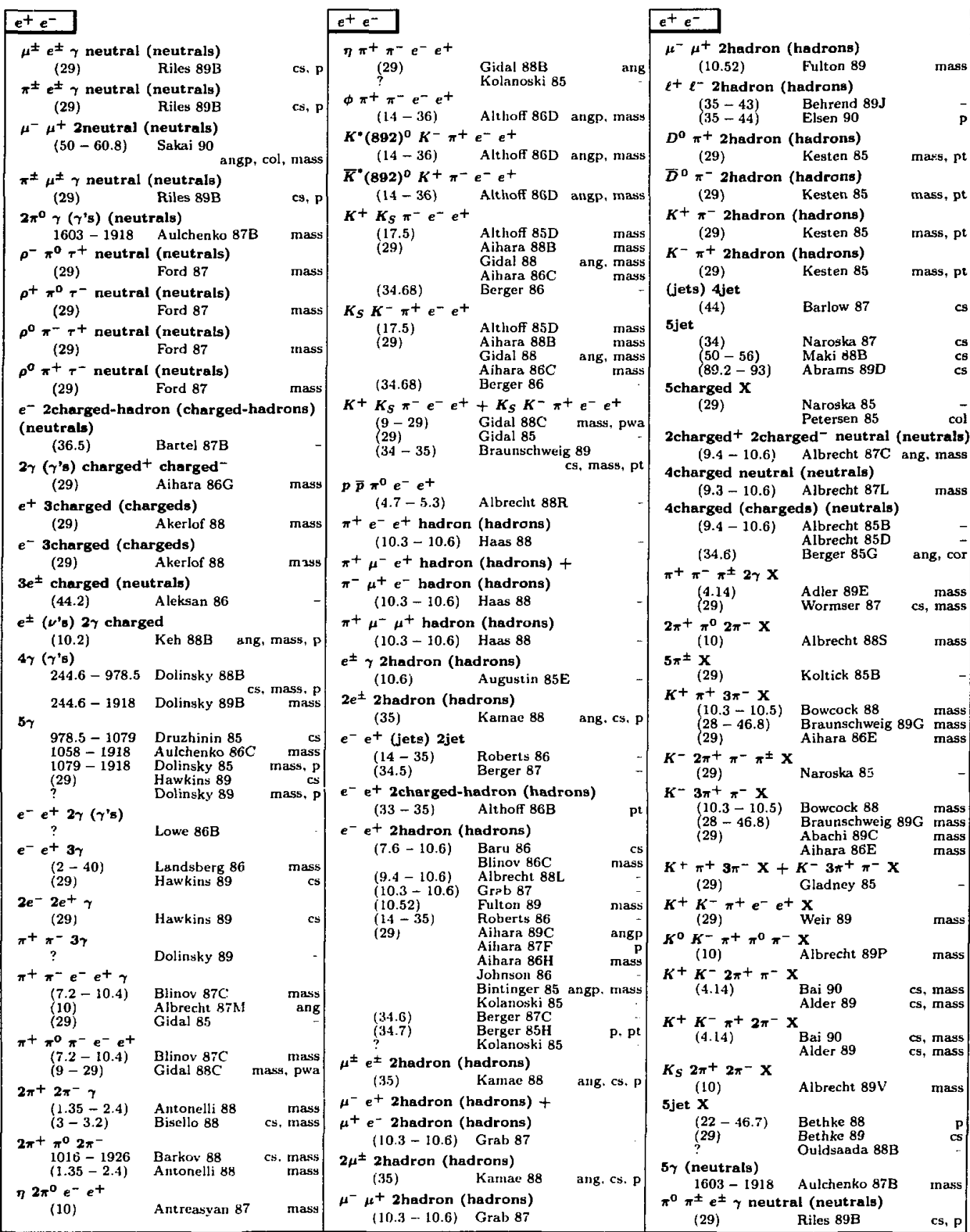

Entries in order of beam mass, then target mass, then inultiplicity of final state. Inclusive reactions have an "X" as the last of the final state particles. Certain chustical symbols for nuclei have heen changed to avoid ambiguity with particle names (See the Particle Vocabulary.) Beam momenta are : lab in $\mathrm{GeV} / \mathrm{c}$, or in parentheses $E_{\mathrm{cm}}$ in $\mathrm{GeV}$. See the legend on page 153. 


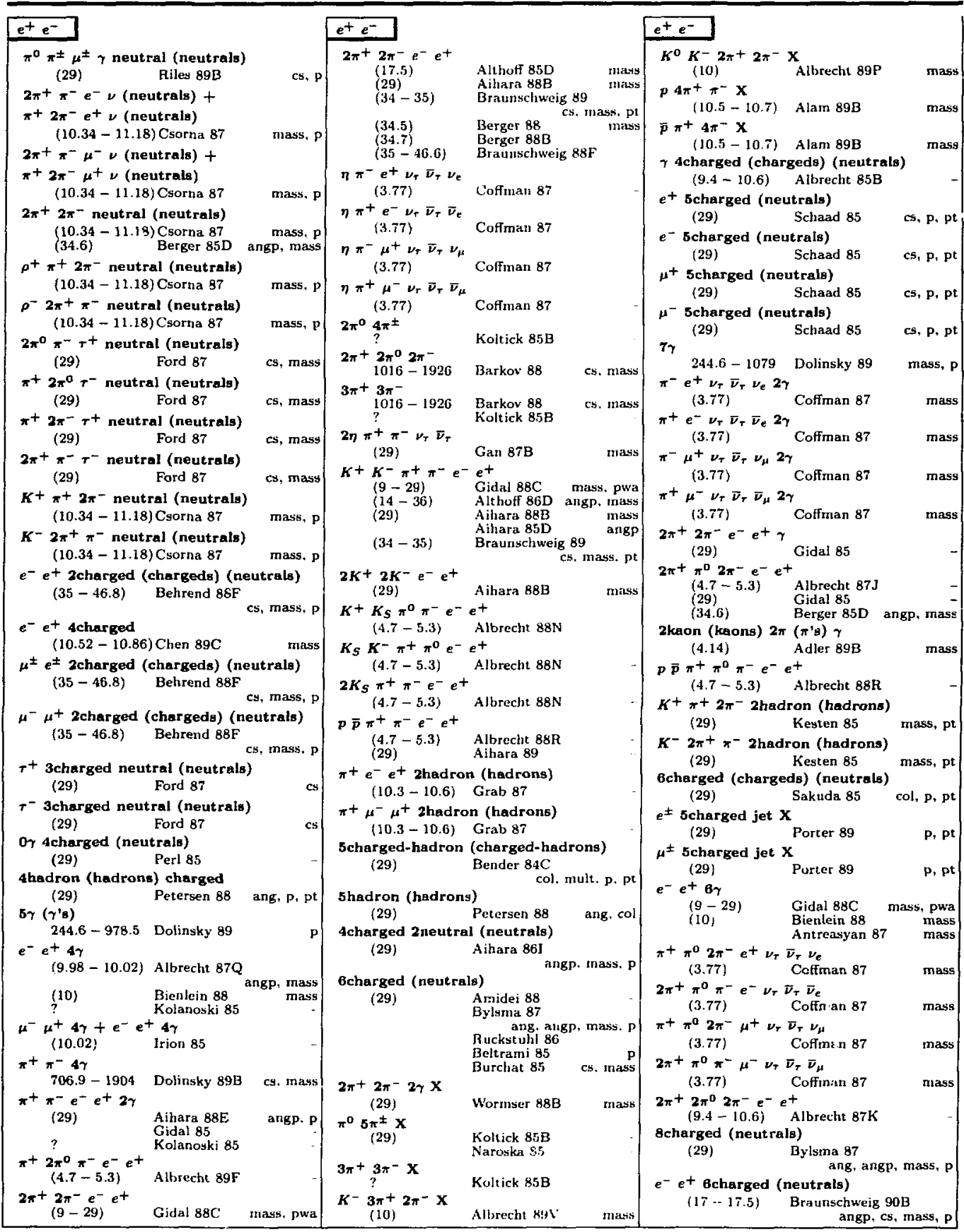




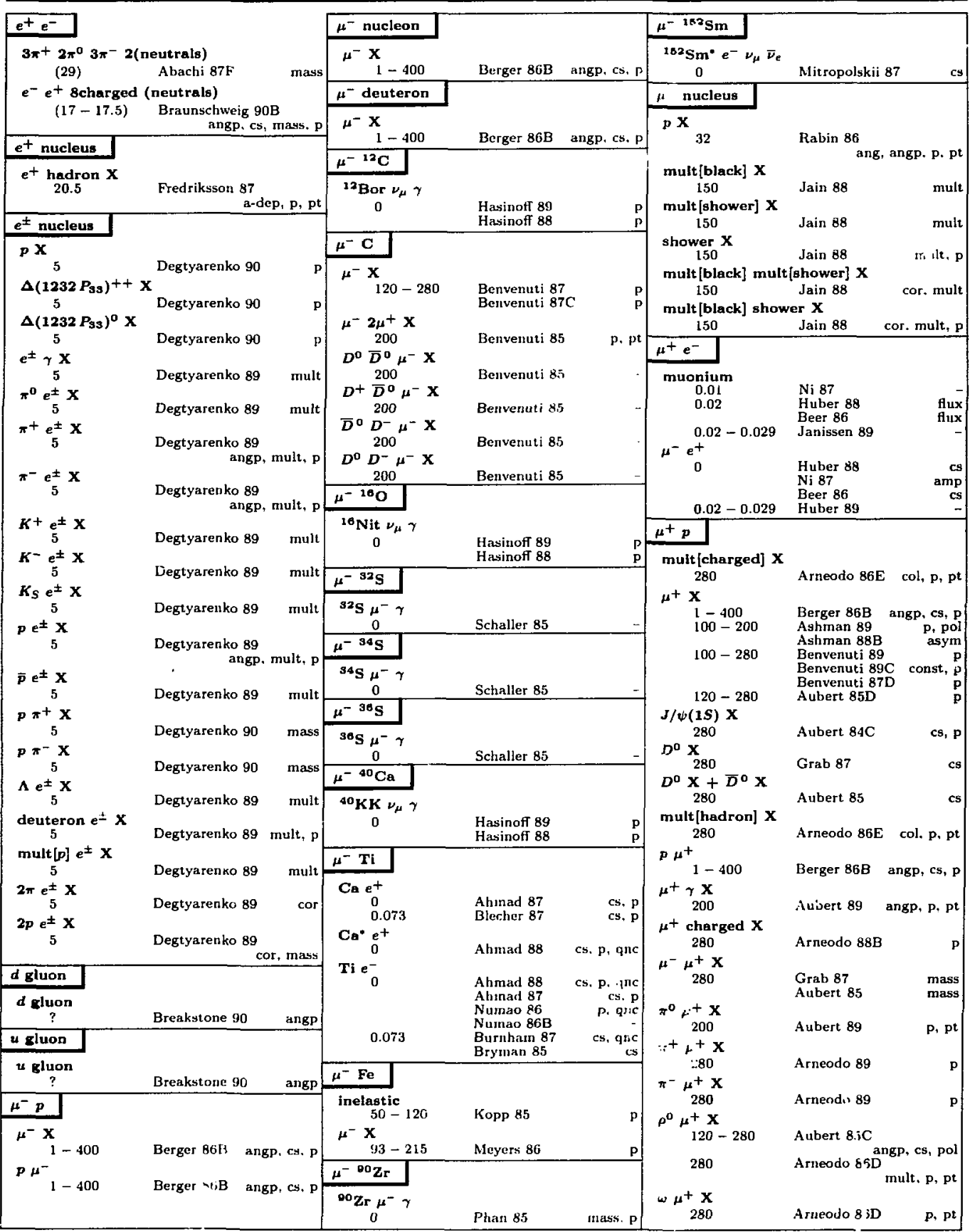

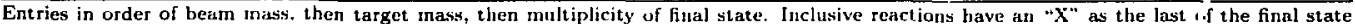

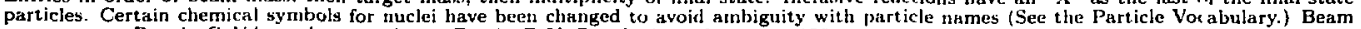
momenta are $P_{\ln b}$ in $\mathrm{Ge} / \mathrm{C}$, or in parentheses $E_{\mathrm{cm}}$ in GeV. See the legend on pagr 153. 


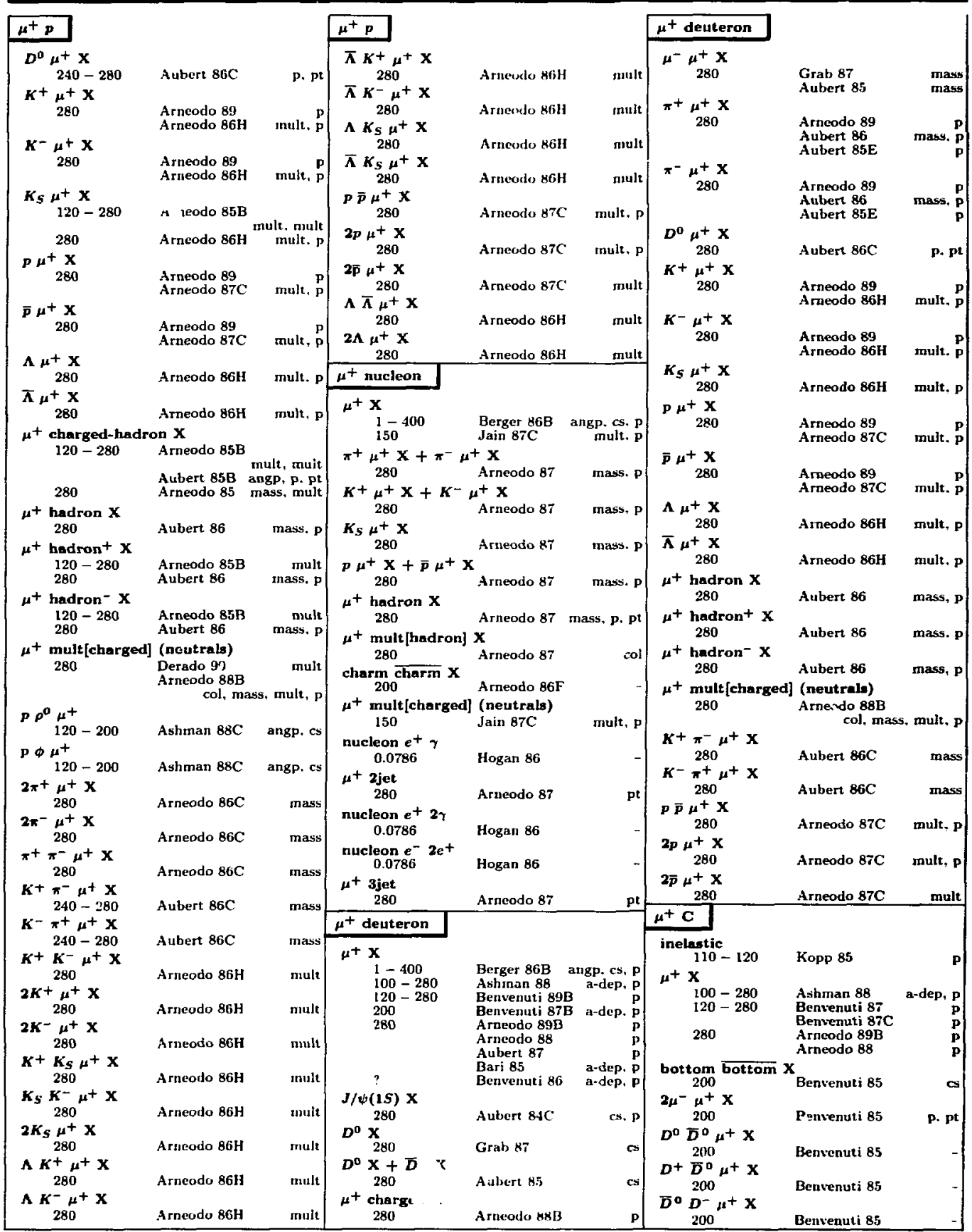




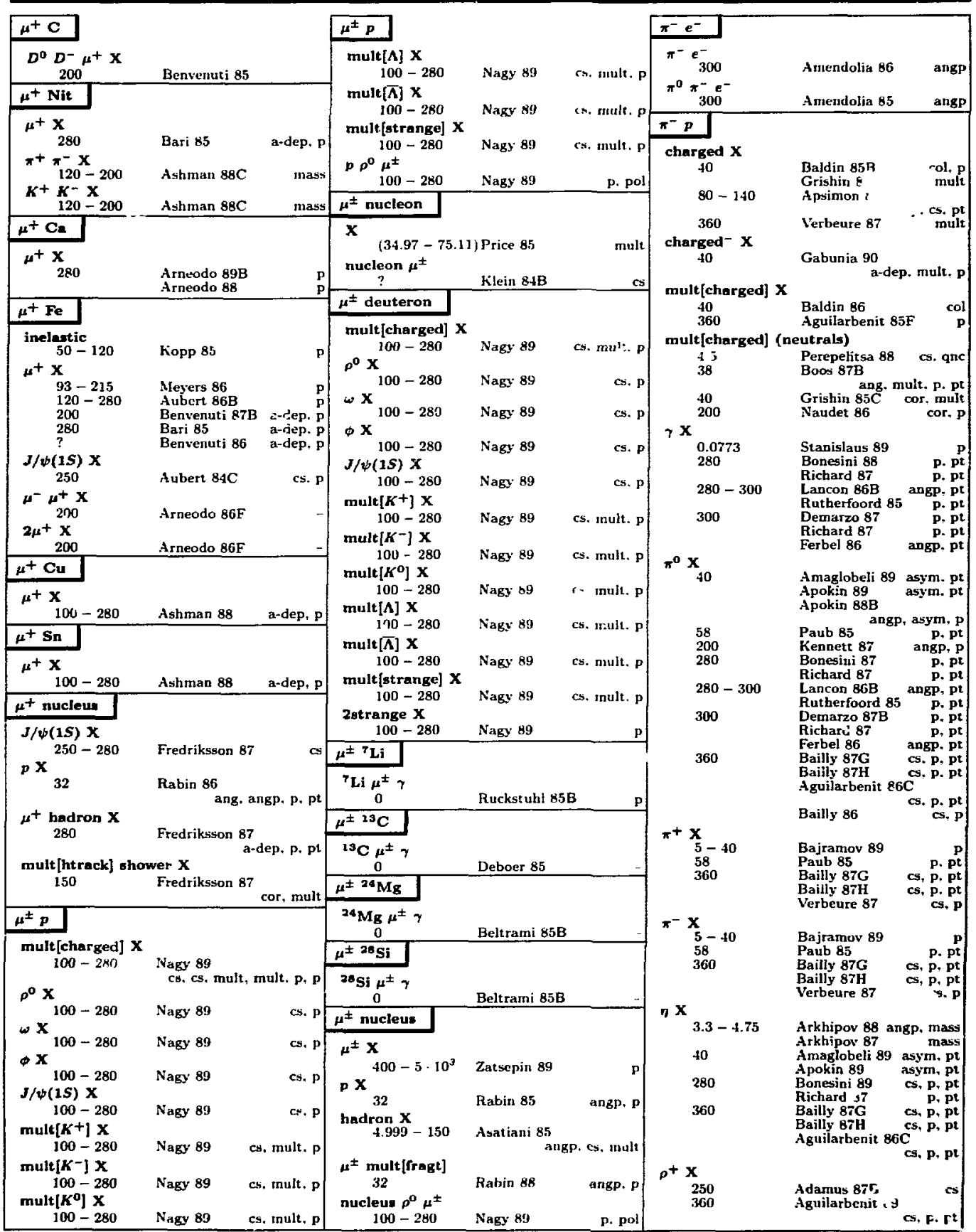

Entries in order of beam mass, then target mass. :hen multiplicity of final state luclusive reactions have an "X" as the last of the final state particles. Certain chemical symbols for nuclei havel clingled to woid ambiguity $w$ ith particle names (Sce the Particle Vocabulary:) Beam moments are $P_{\text {lob }}$ in GeV/c, or in parentheses $E_{1}$. eV. See the legend un page i sos. 


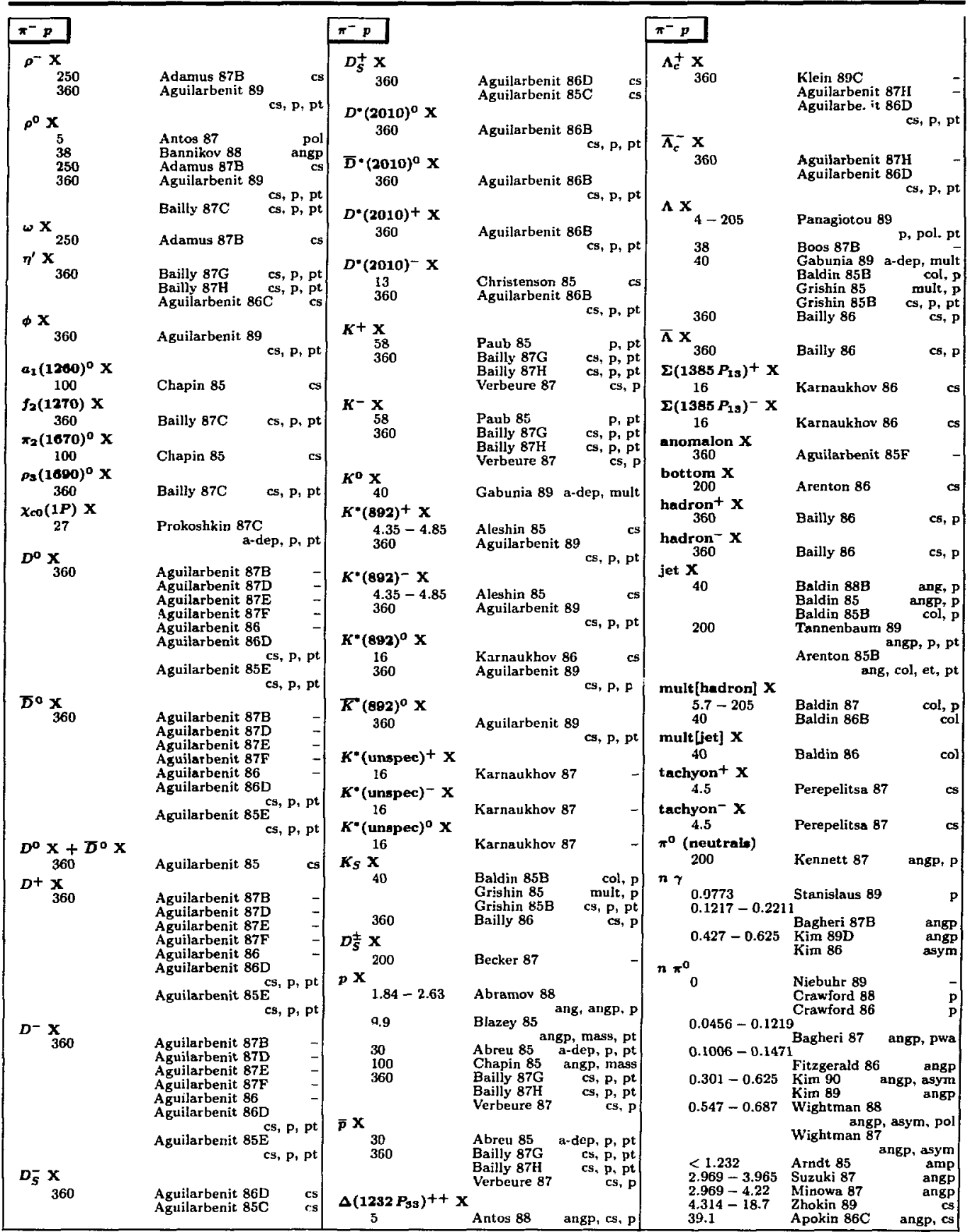




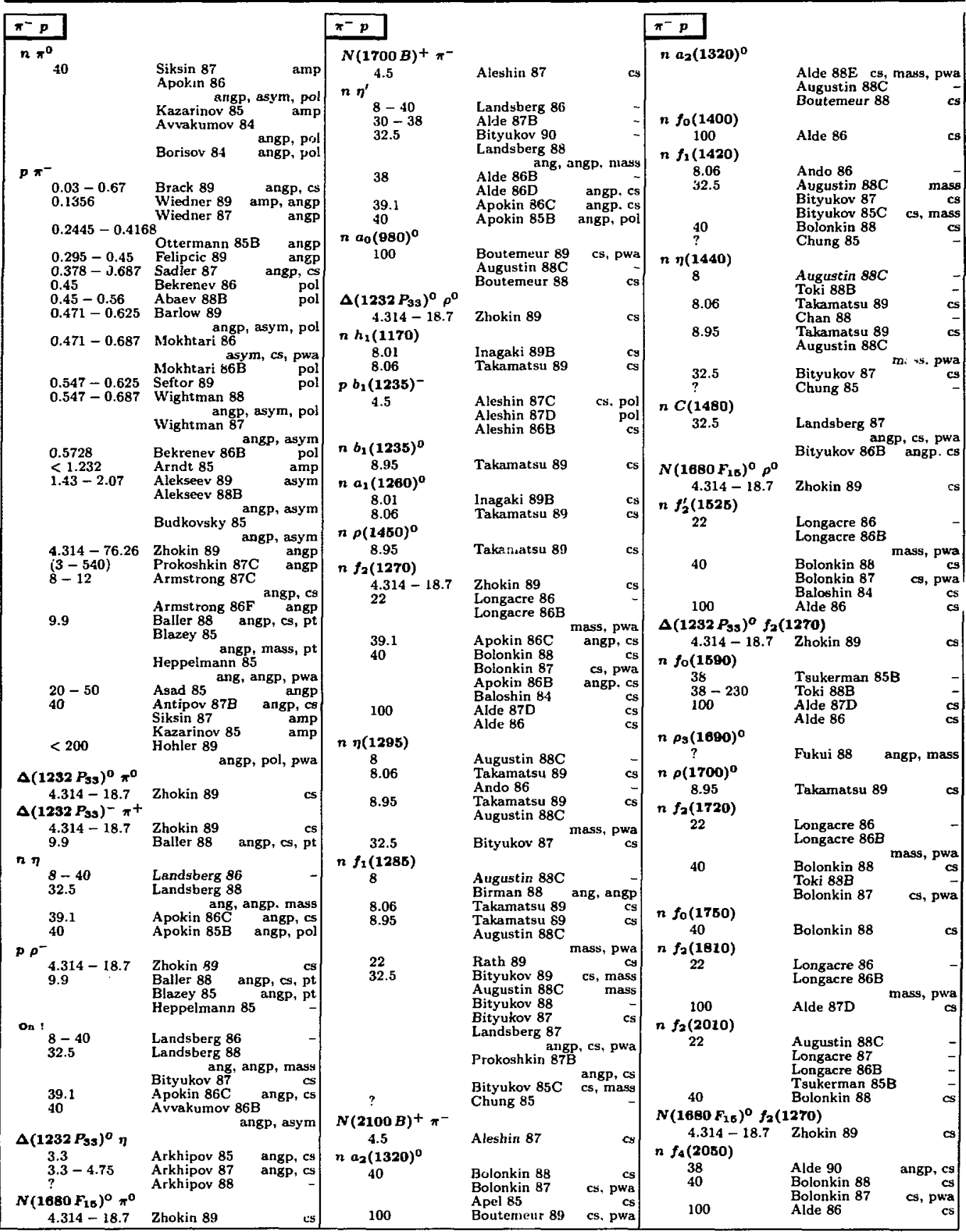




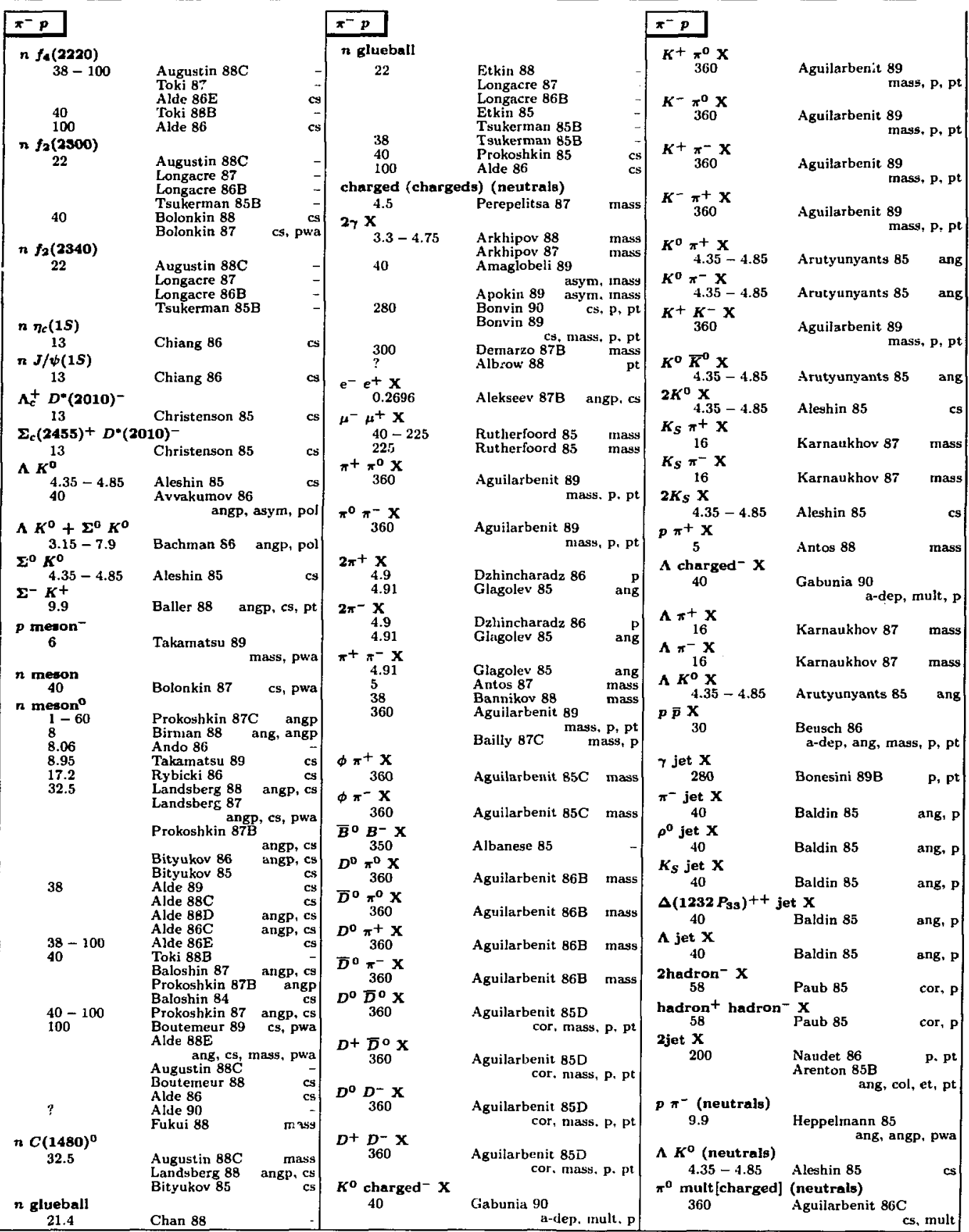




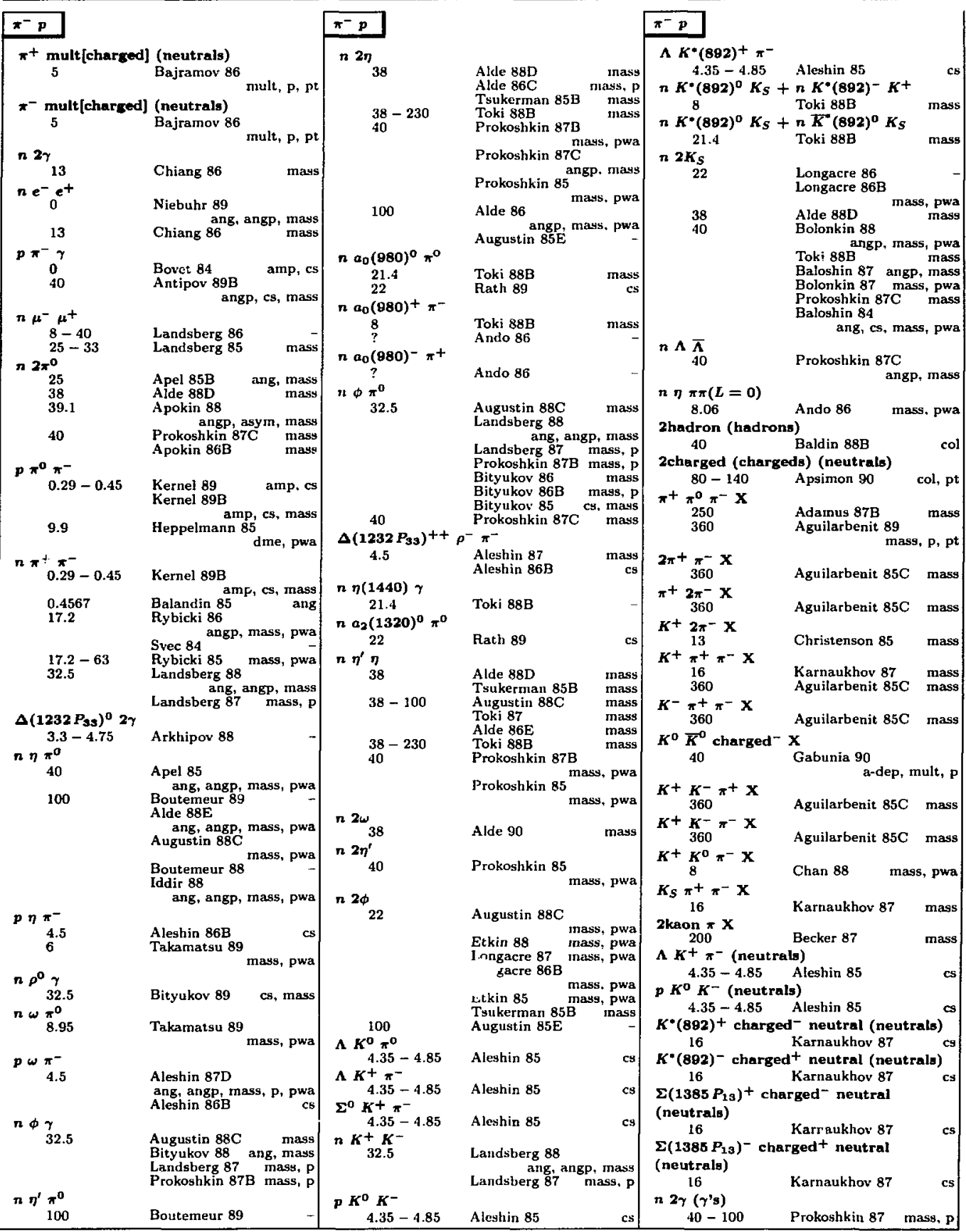




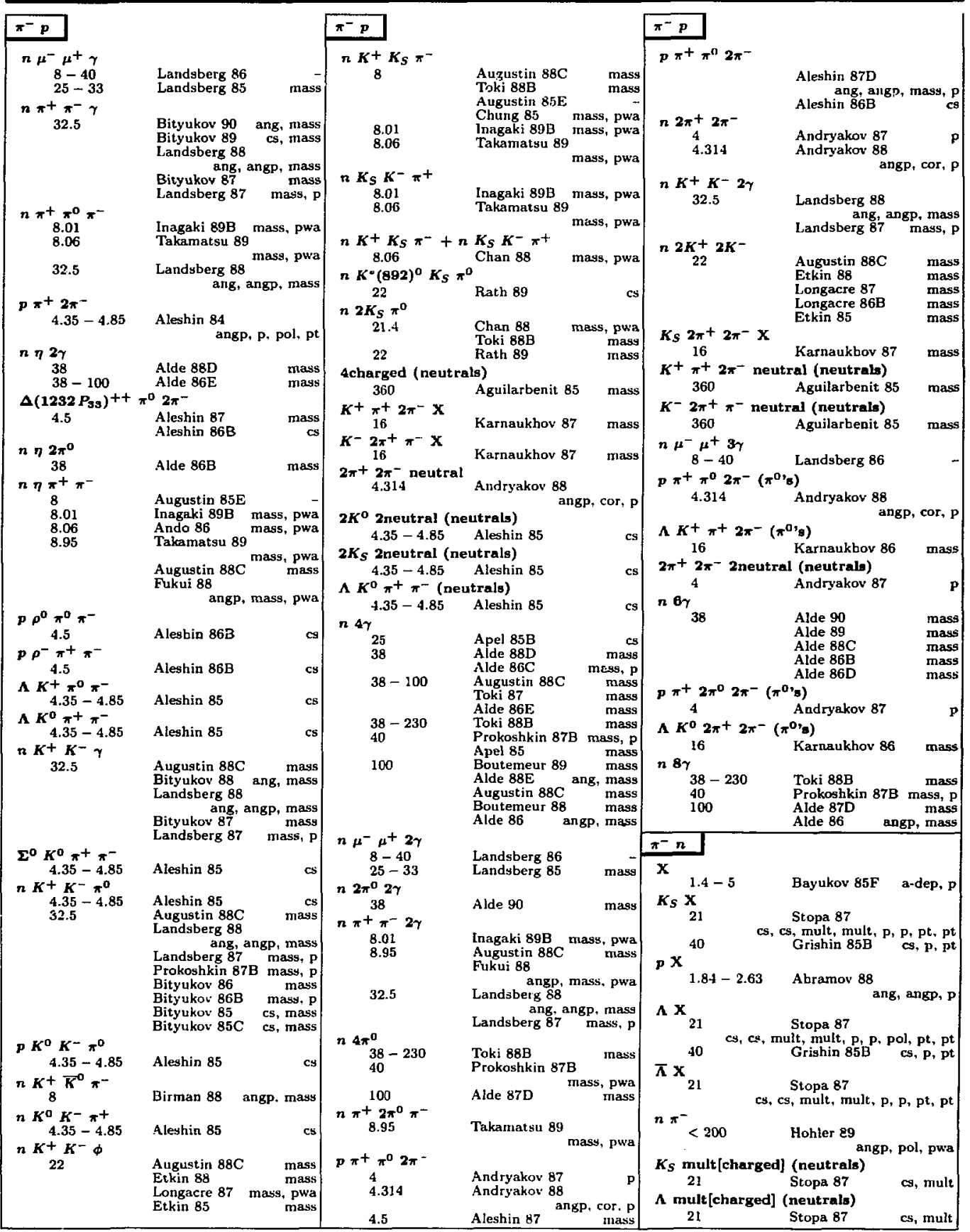




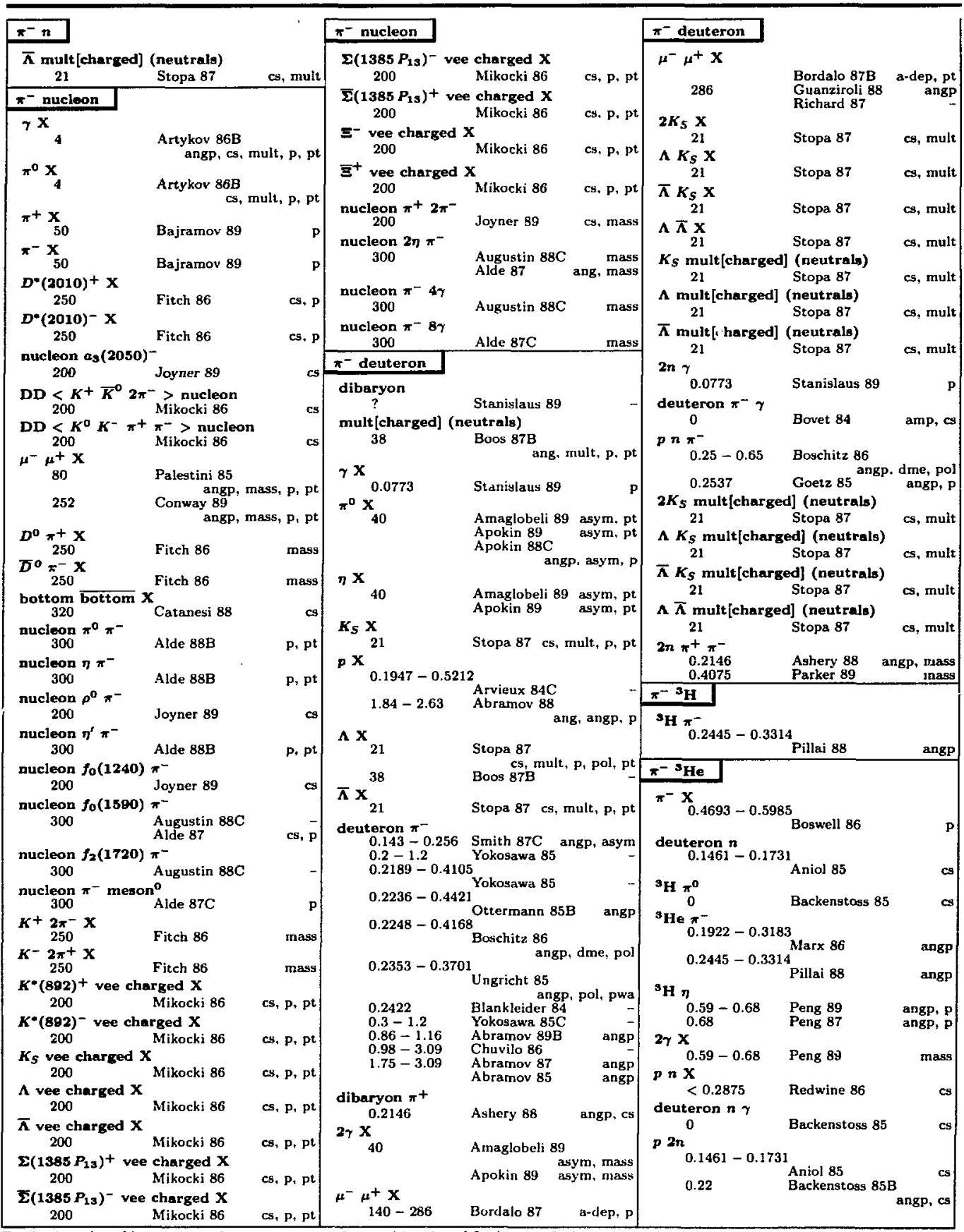

Entries in order of beam mass, then target mass, then mutiplwity of final state. Inclusive reactions have an " $X$ " as the last of the final state particles. Certain chemical symbals for nuclei have beeri changed to avoid imbiguity with particle nannes (See the Particle Vocabulary.) Beam momenta are $\mathrm{P}_{\text {lab }}$ in $\mathrm{GeV} / c$, or in parenthesey $E_{\mathrm{crn}}$ in $\mathrm{GeV}$. See the legend on page 153 . 


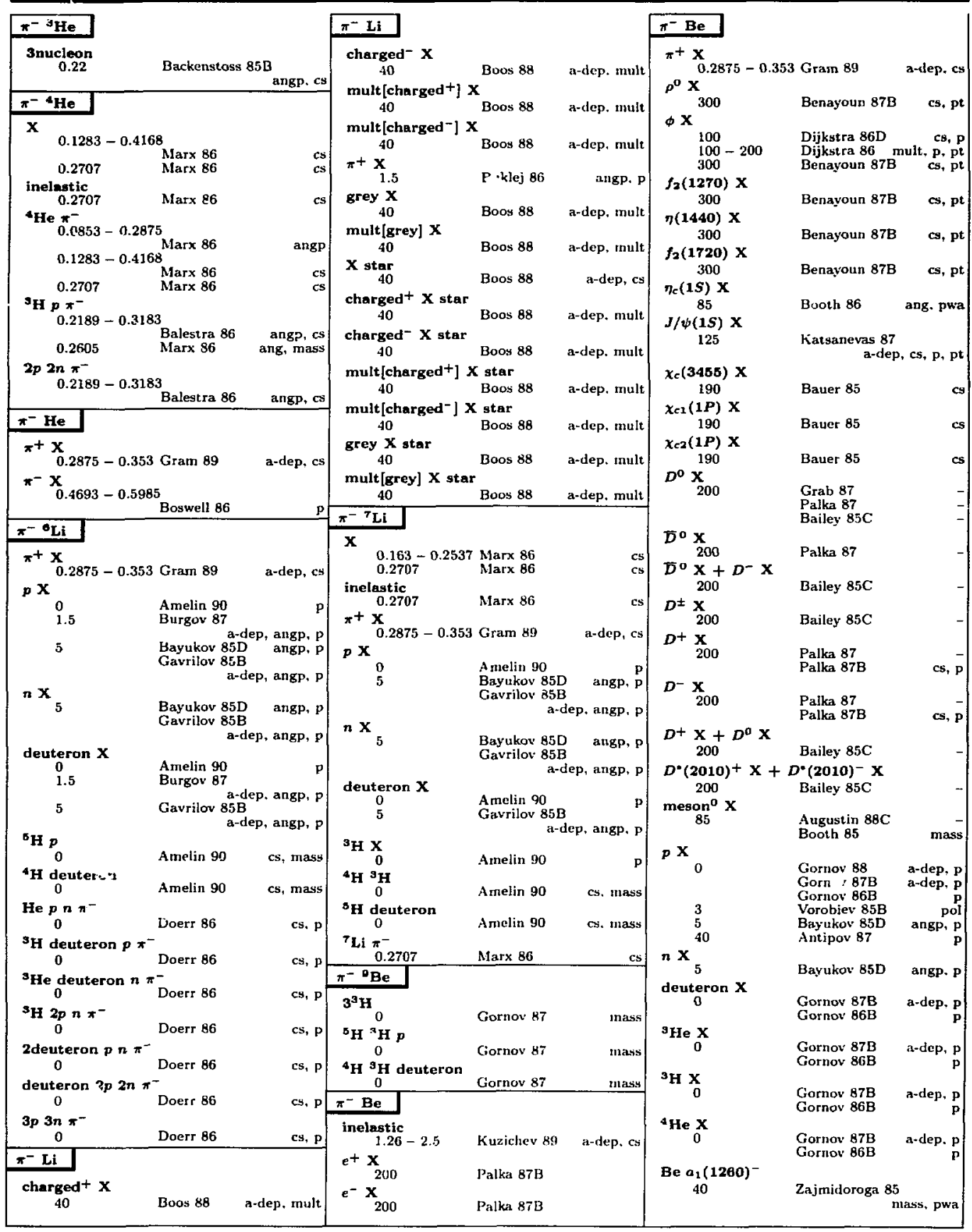




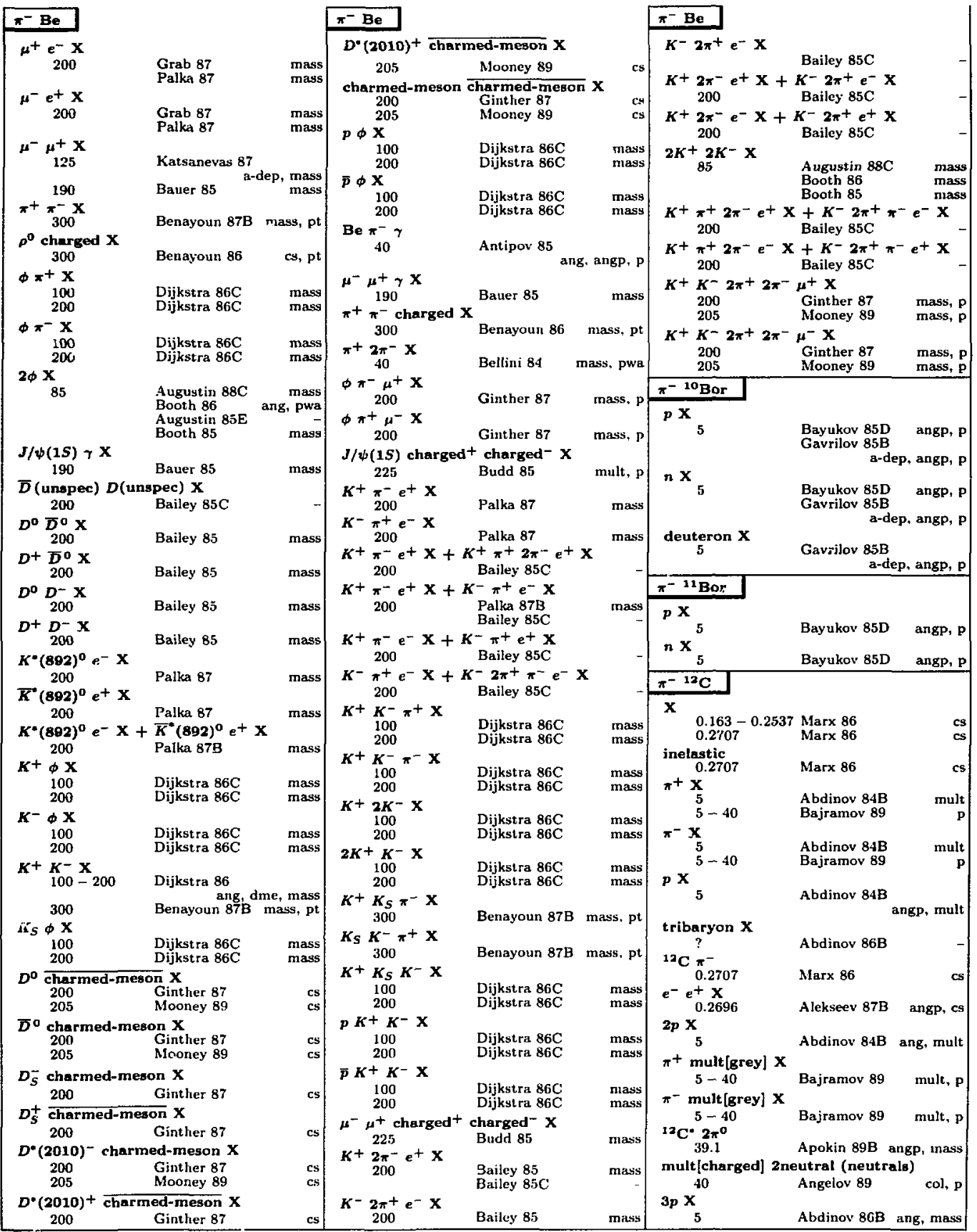

Entries in order of beam mass, then target mass, then multiplicity of final state. Inclusive reactions have an "X" as the last of the final state particles. Certain chemical symbols for nuclei have been changed to avoid ambiguity with particle narnes (See the Particle Vocabulary.) Beam momenta are $P_{1 a b}$ in $\mathrm{GeV} / c$, or in parentheses $E_{\mathrm{c} i t}$ in $\mathrm{GeV}$. See the legend on page 153. 


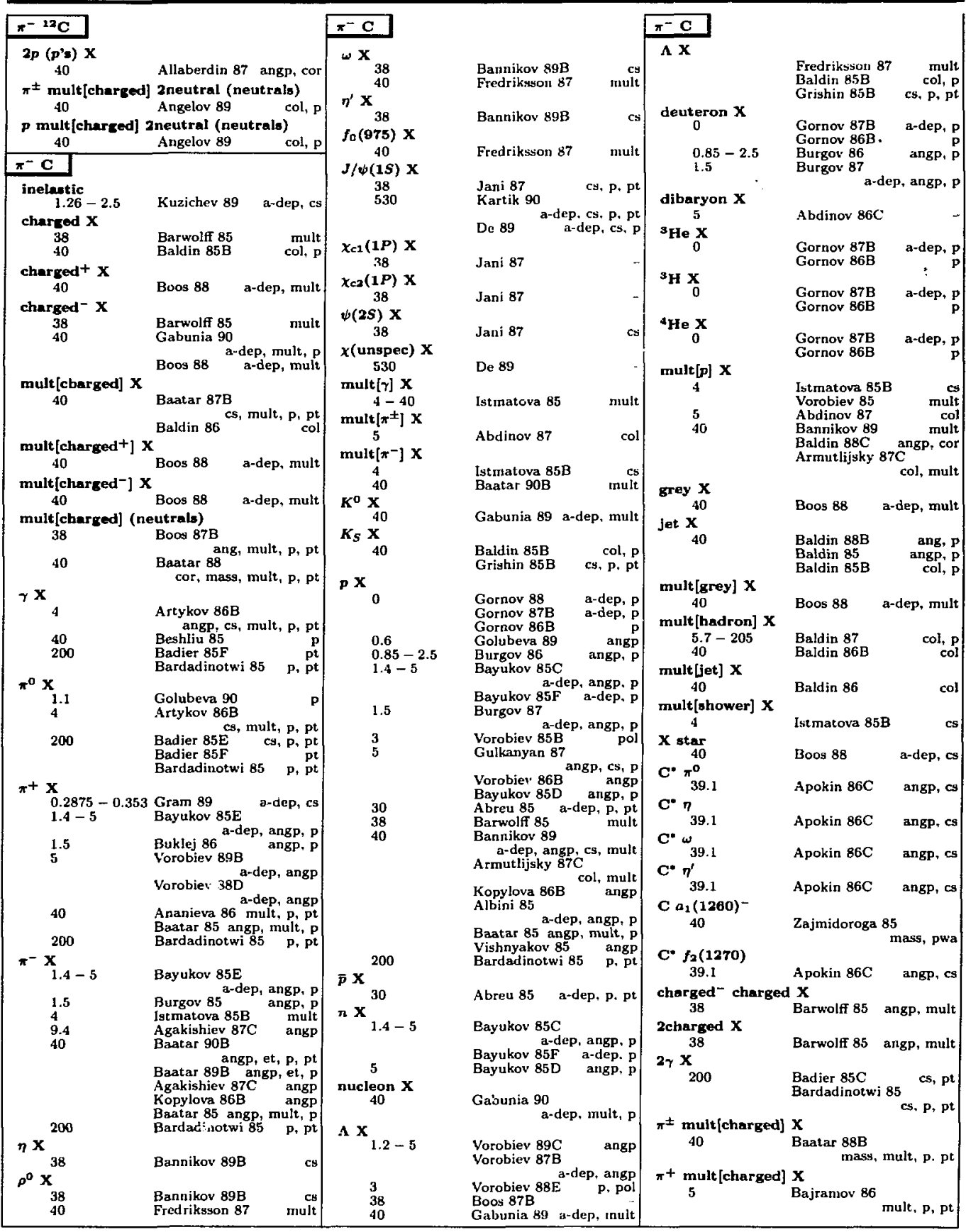




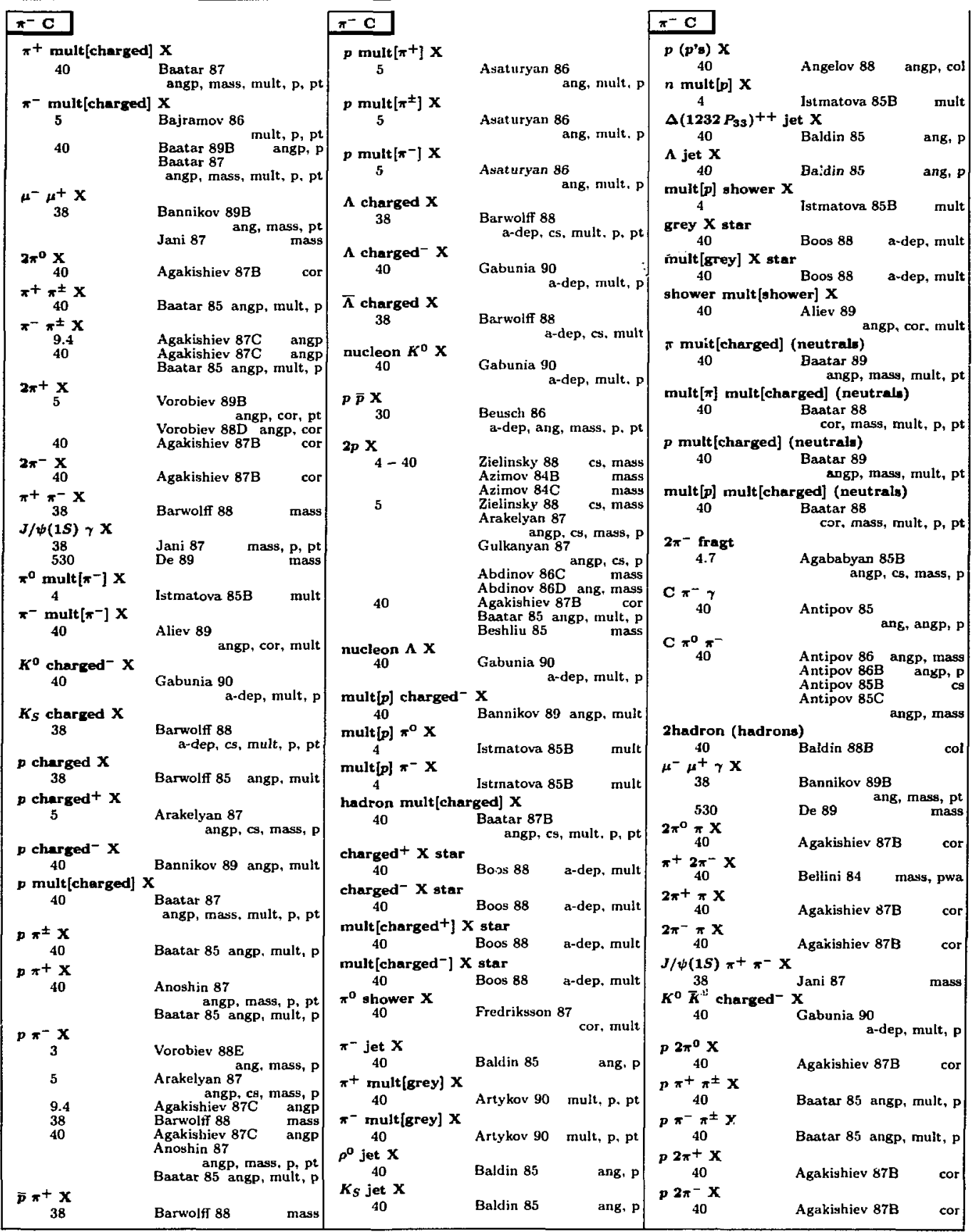




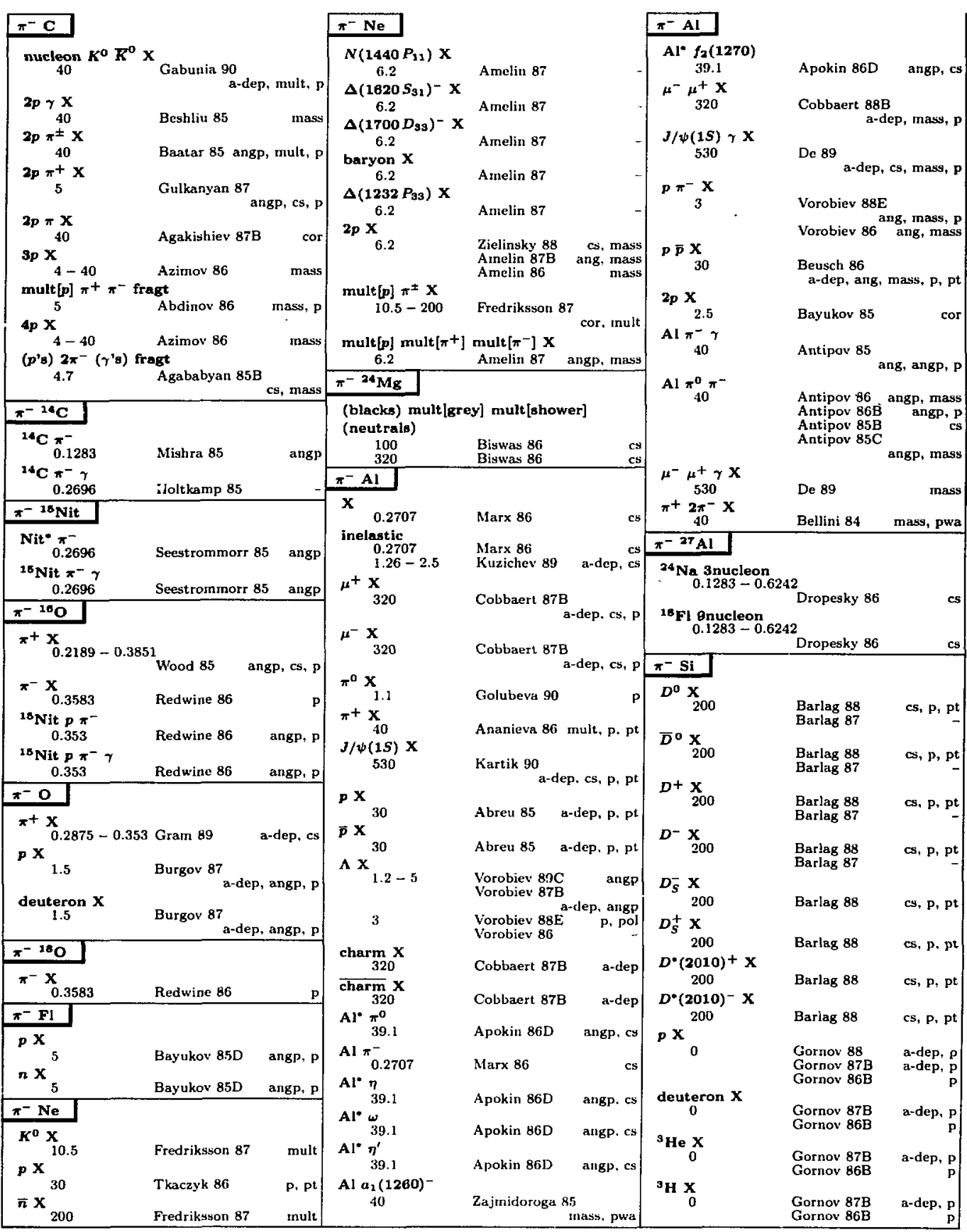




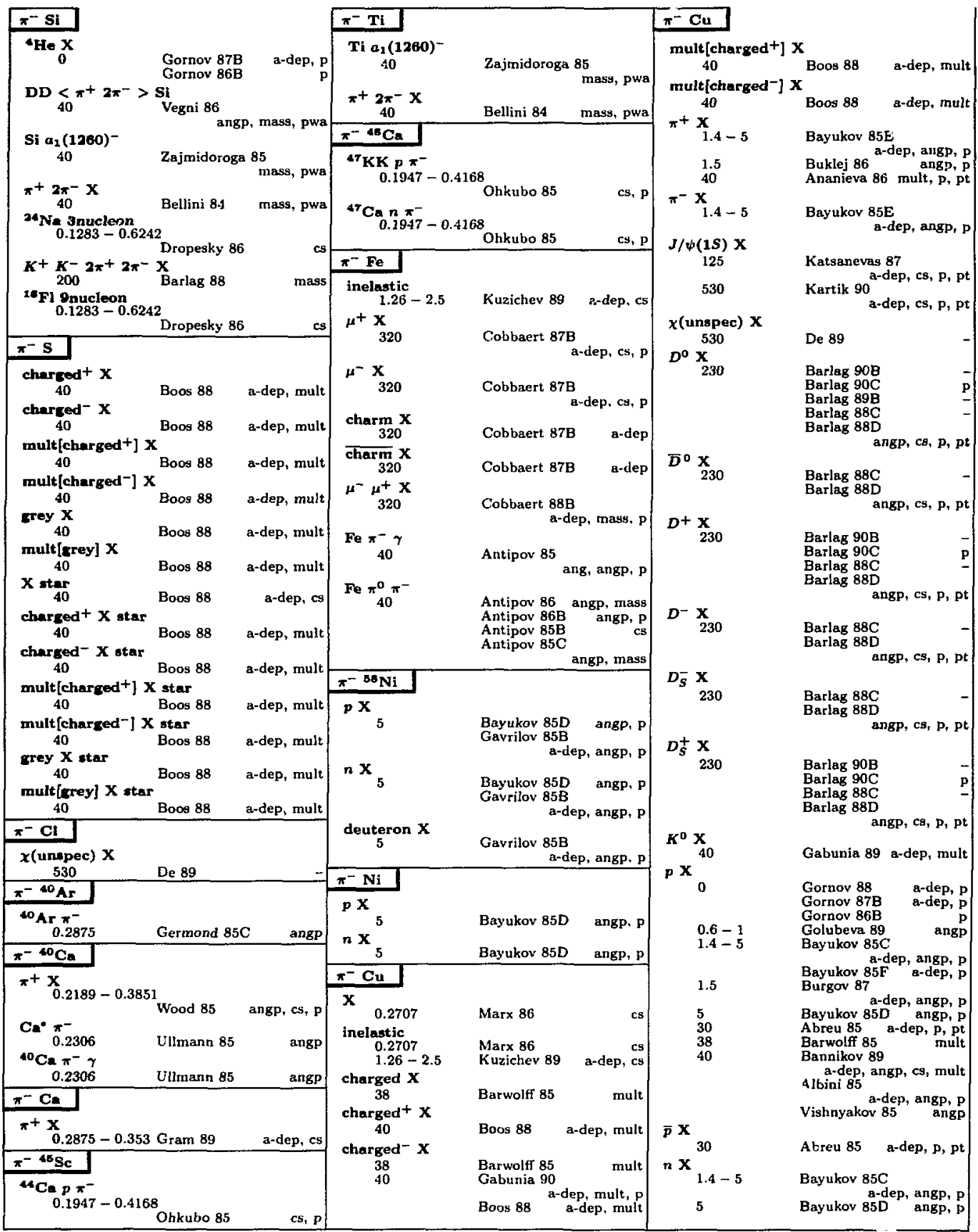

Entries in order of beam mass, then target mass, then multiplicity of final state. Inclusive reactions have an "X" as the last of tite inal state particles. Certain chemical symbols for nuclei have been changed to avoid ambiguity with particle names (See the Particle Vocabulary.) Beam momenta are $P_{\text {lab }}$ in $\mathrm{GeV} / \mathrm{c}$, or in parentheses $E_{\mathrm{cm}}$ in $\mathrm{GeV}$. See the legend on page 153. 


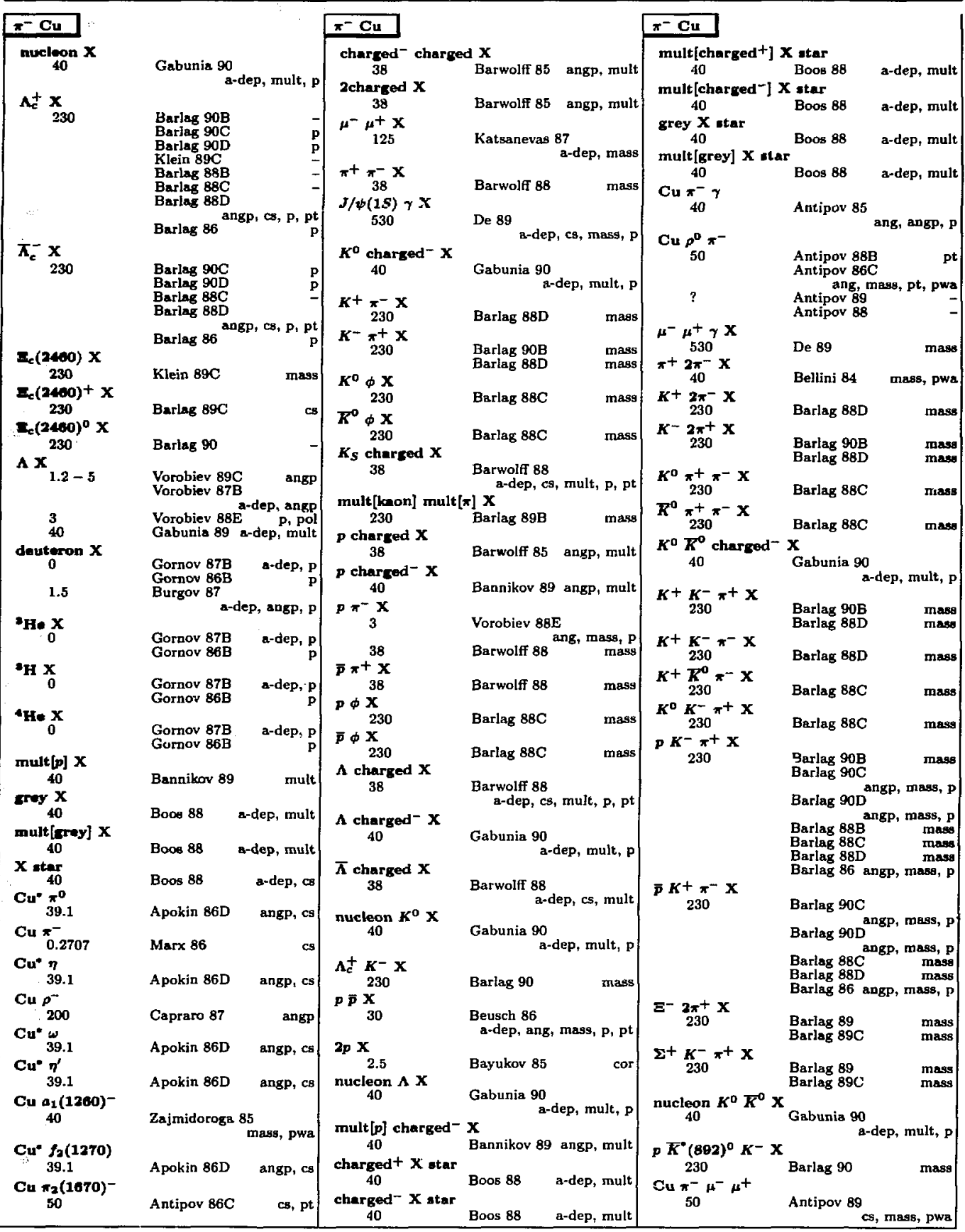




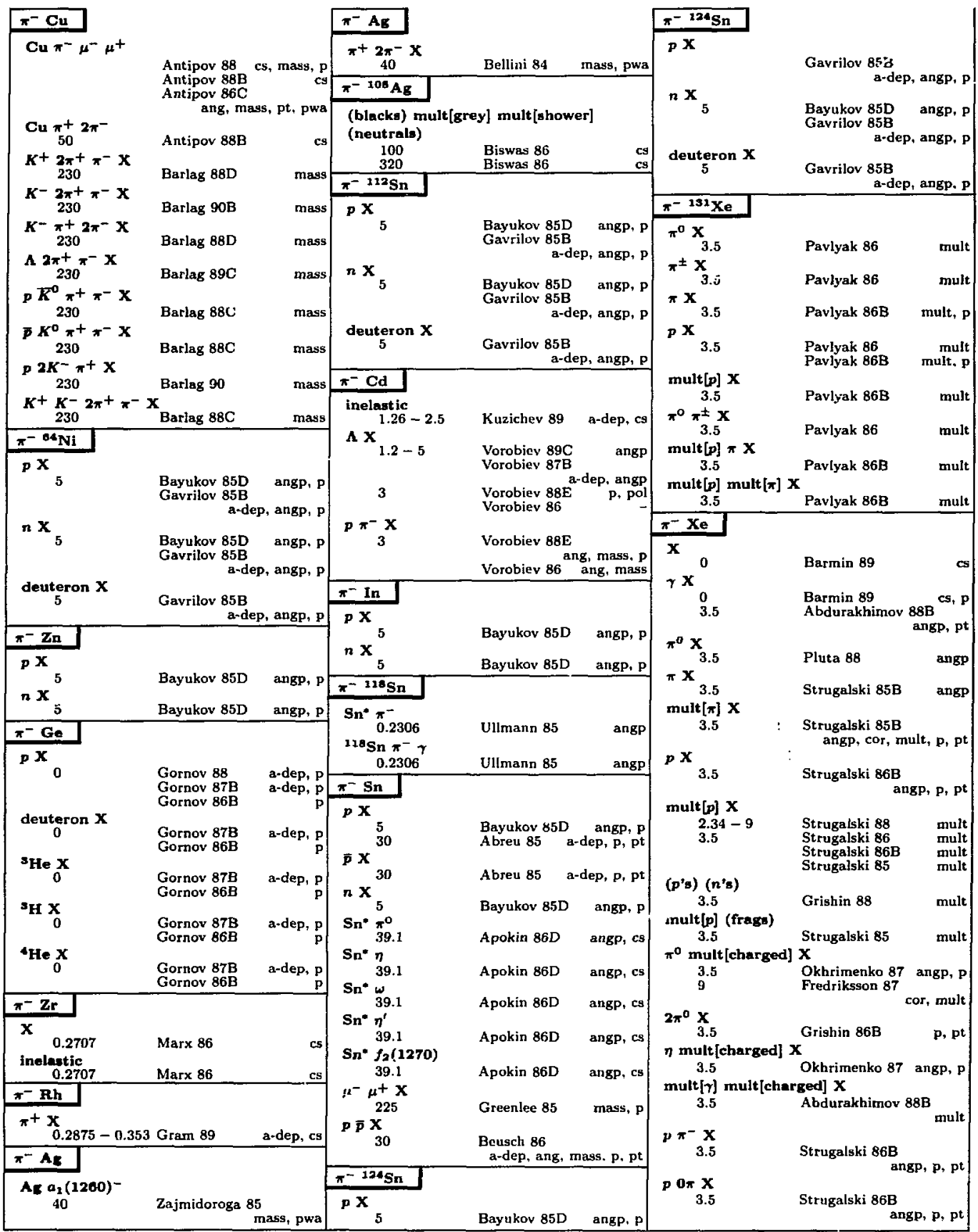

Entries in order of beam mass, then target mass, then multiplicity of final statc. Inclusive reactions have an " $\bar{X}$ " as the last of the final state particles. Certain chemical symbols for nuclei have been changed to avoid ambiguity with particle names (See the Particle Vocabulary.) Beam momenta are $P_{\text {iab }}$ in $\mathrm{GeV} / \dot{\circ}$, or in parentheses $E_{\mathrm{cm}}$ in $\mathrm{GeV}$. See the legend on page 153. 


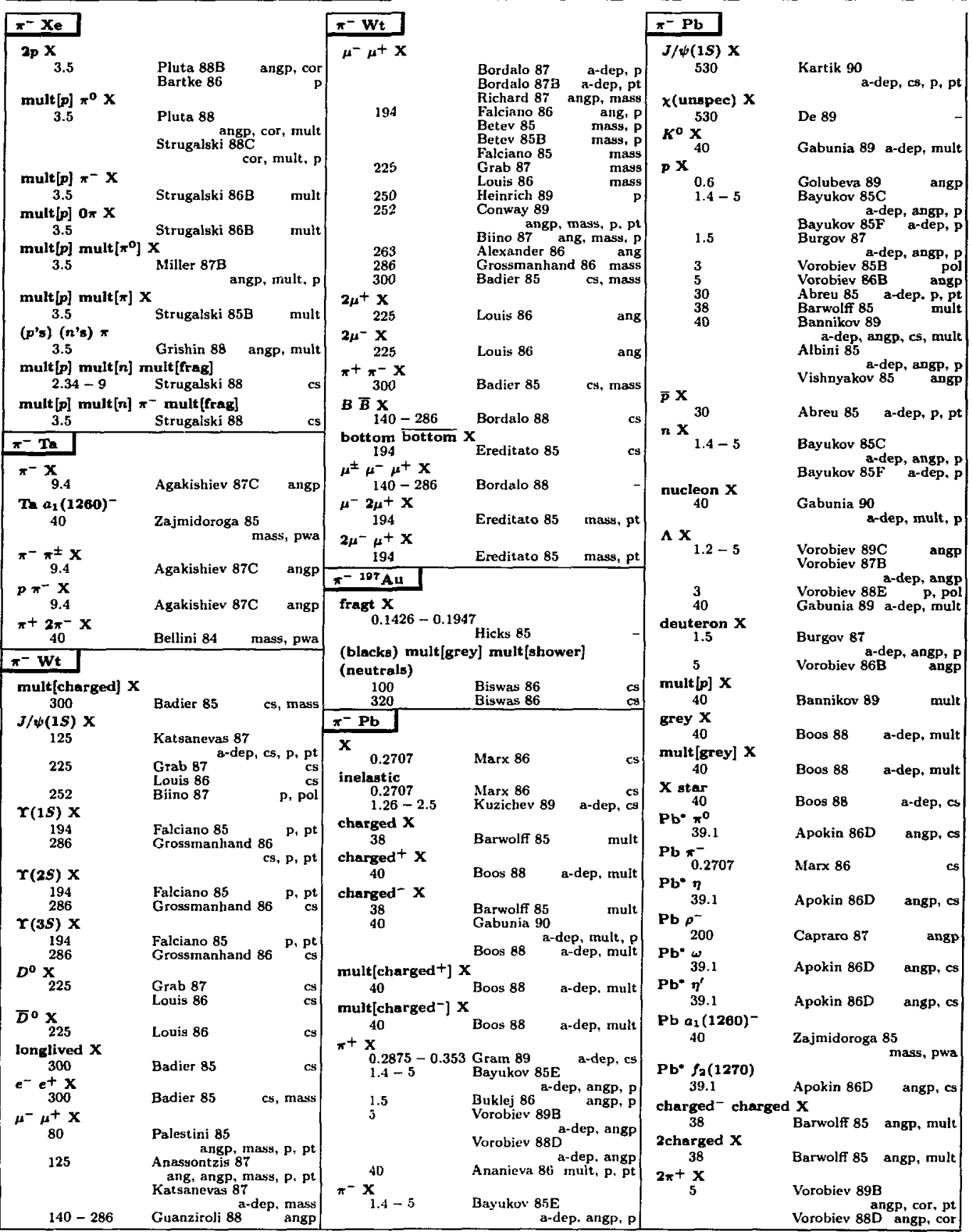




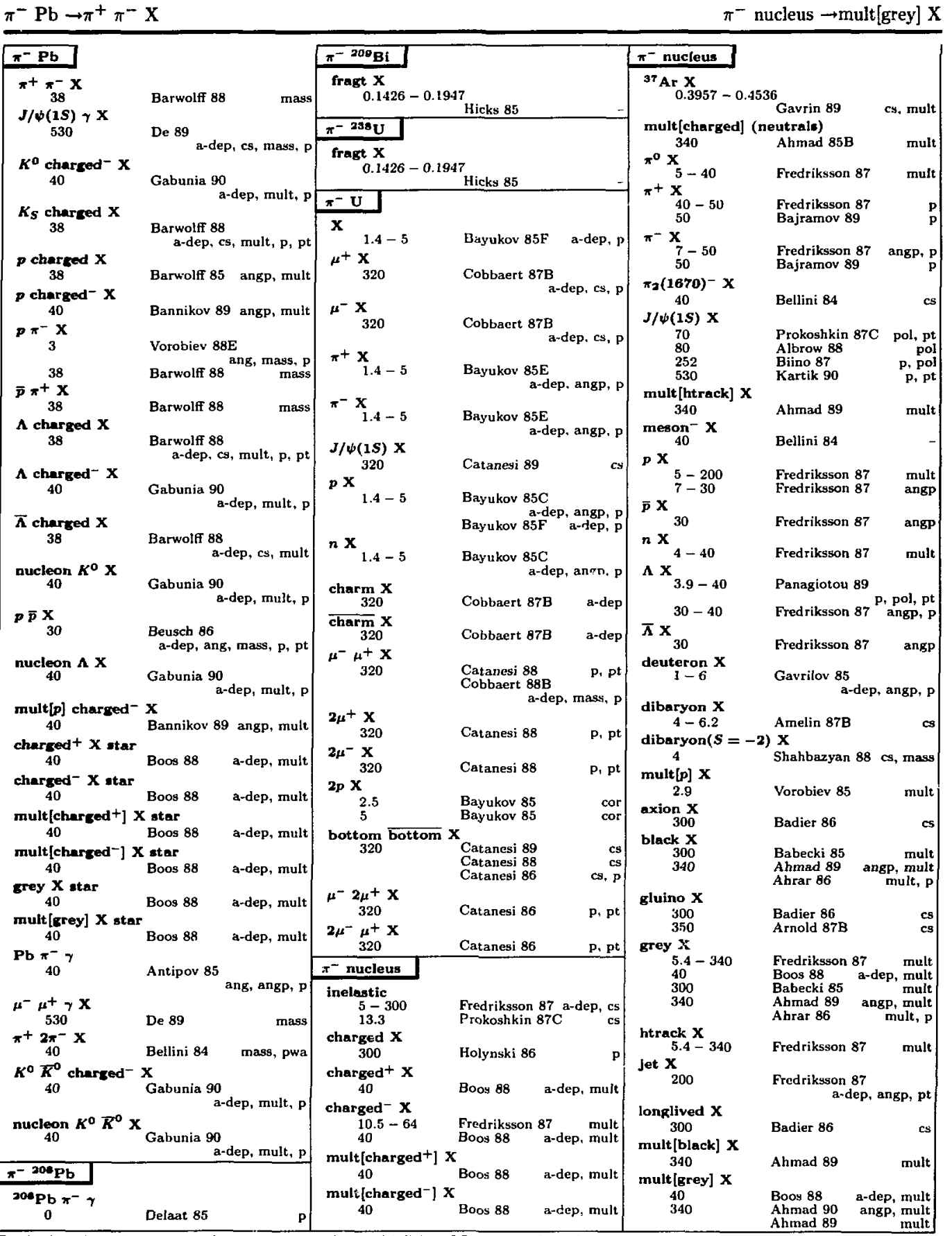




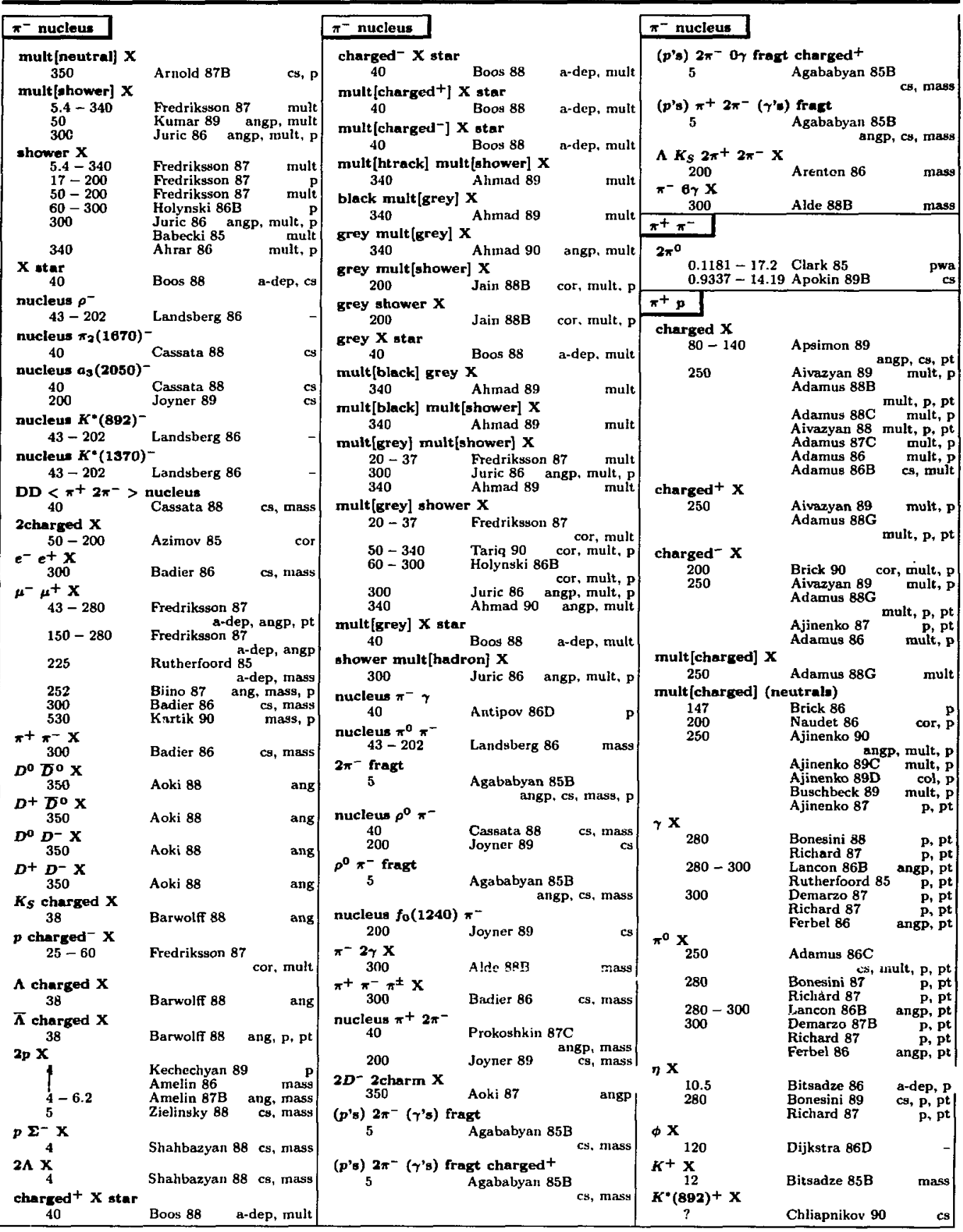




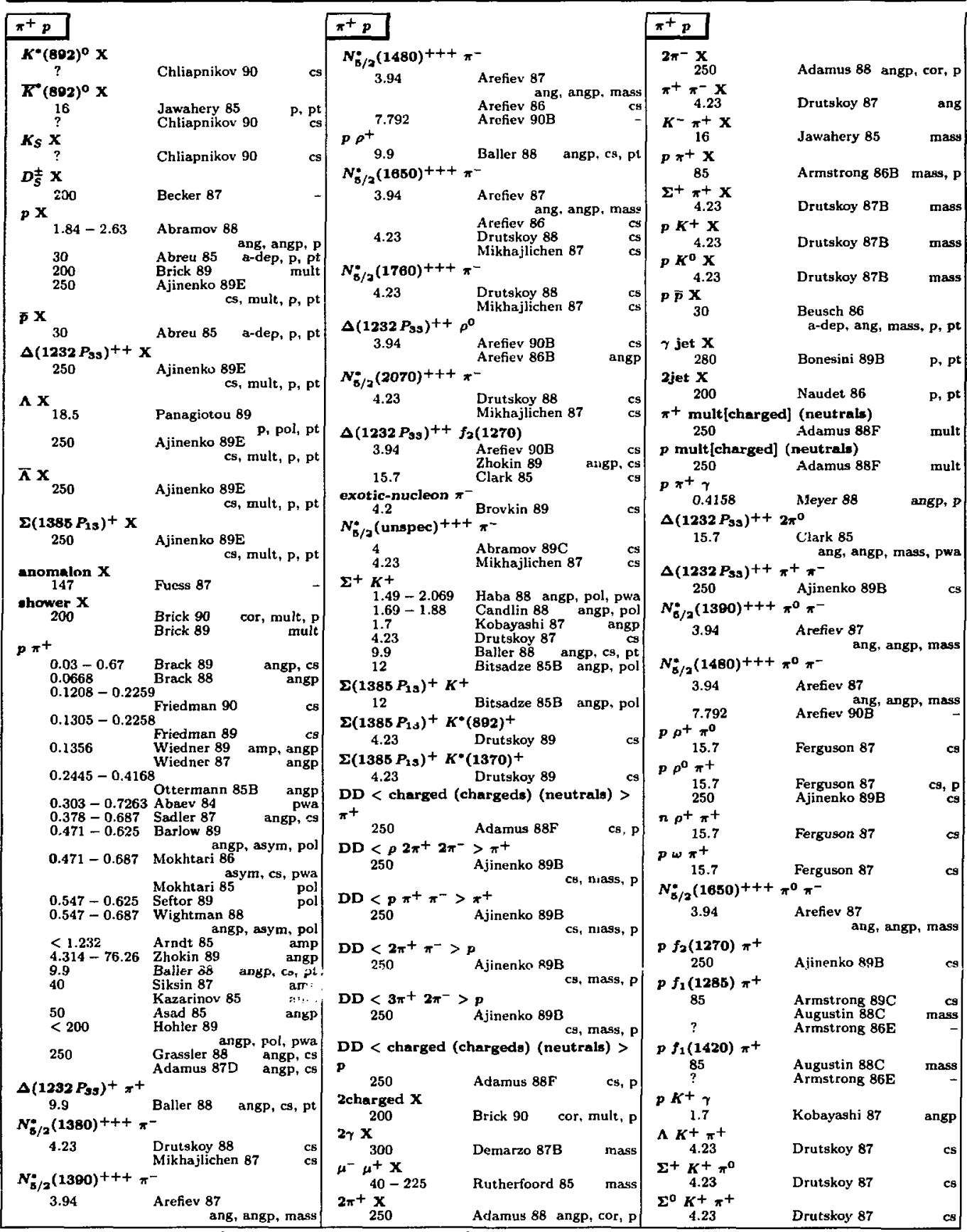




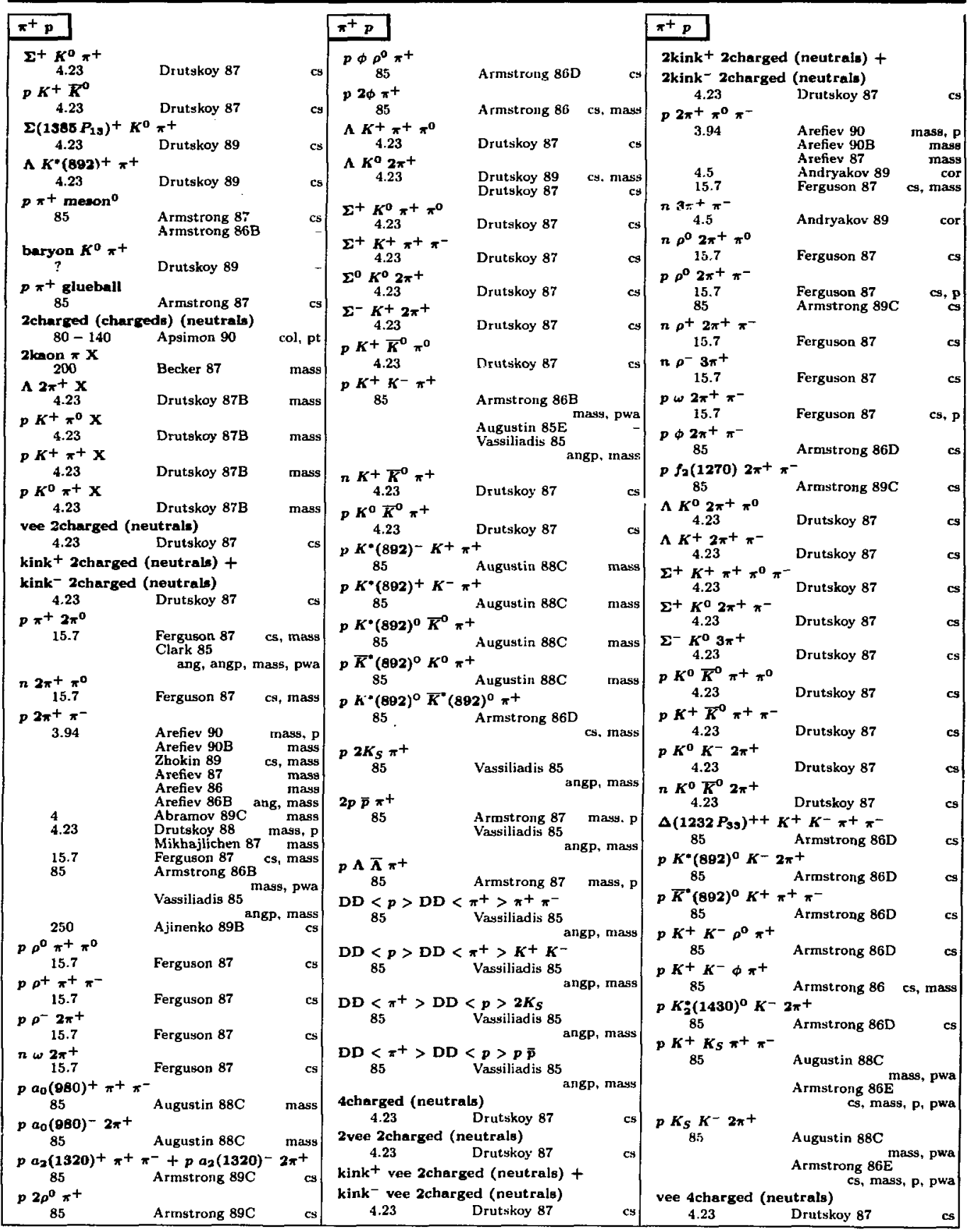




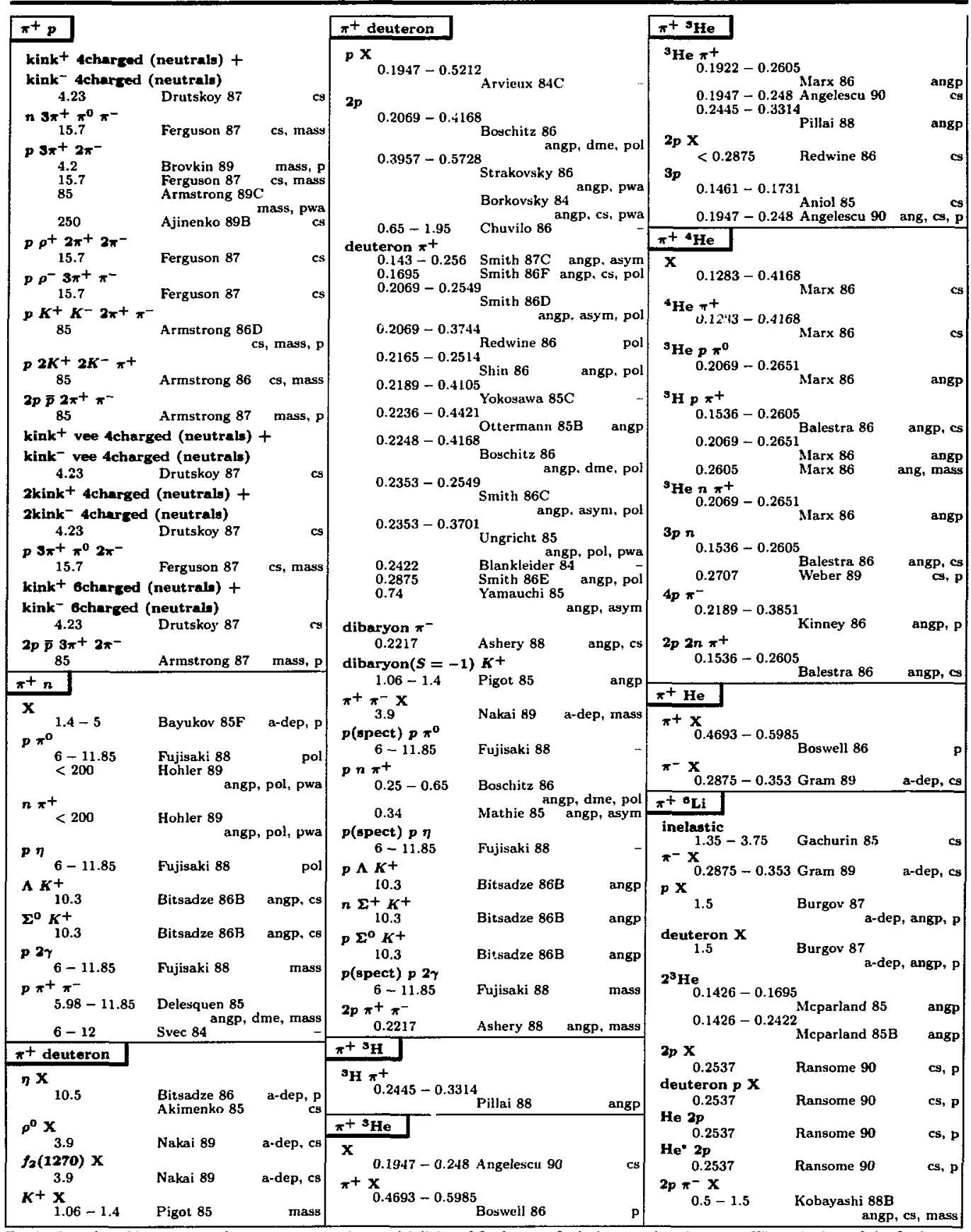

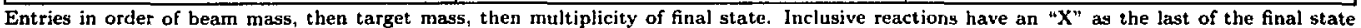

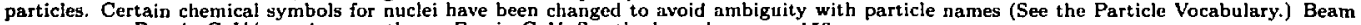
momenta are $\mathrm{P}_{\operatorname{lnb}}$ in $\mathrm{GeV} / c$, or in parentheses $E_{\text {cnn }}$ in $\mathrm{GeV}$. See the legend on page 153. 


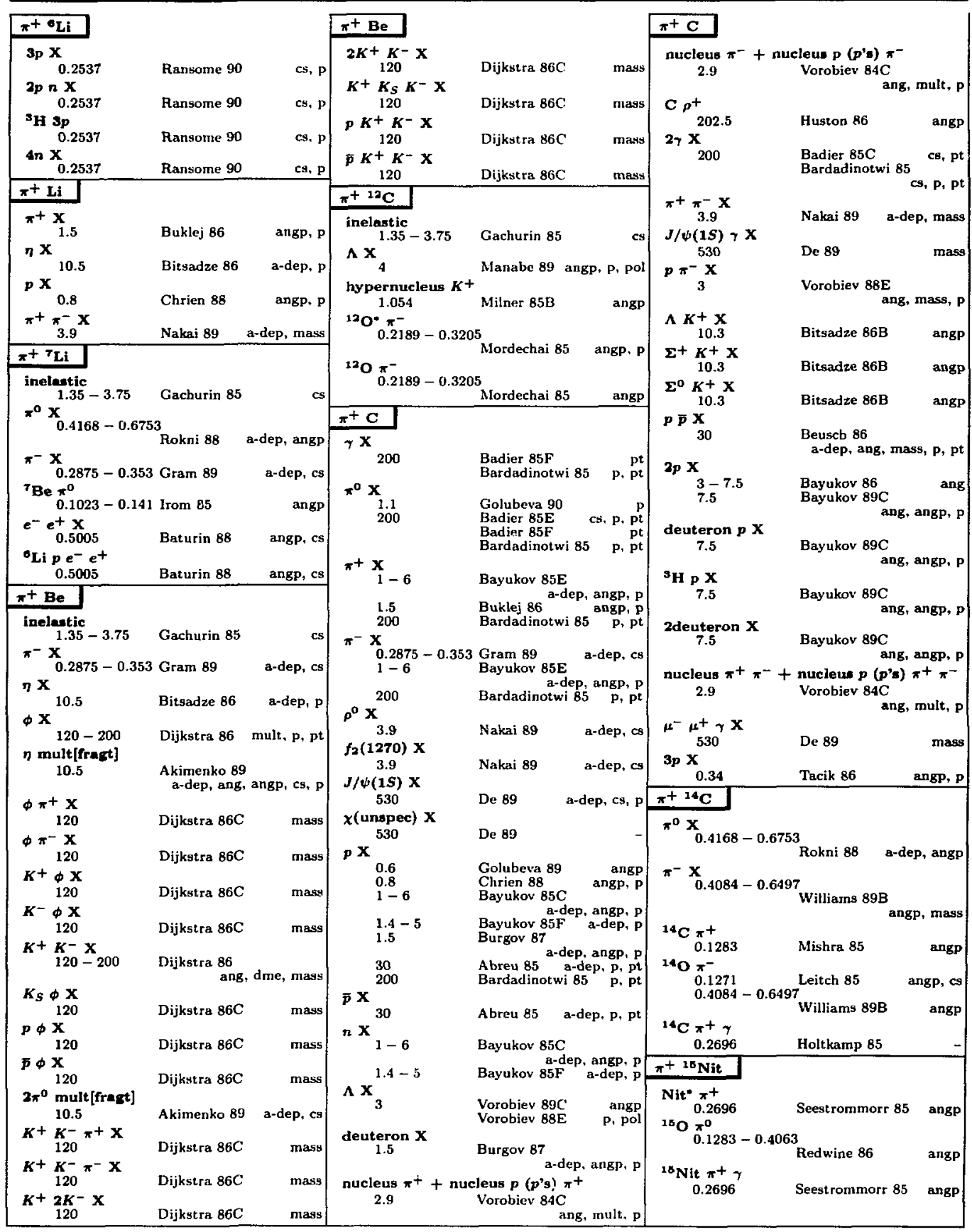




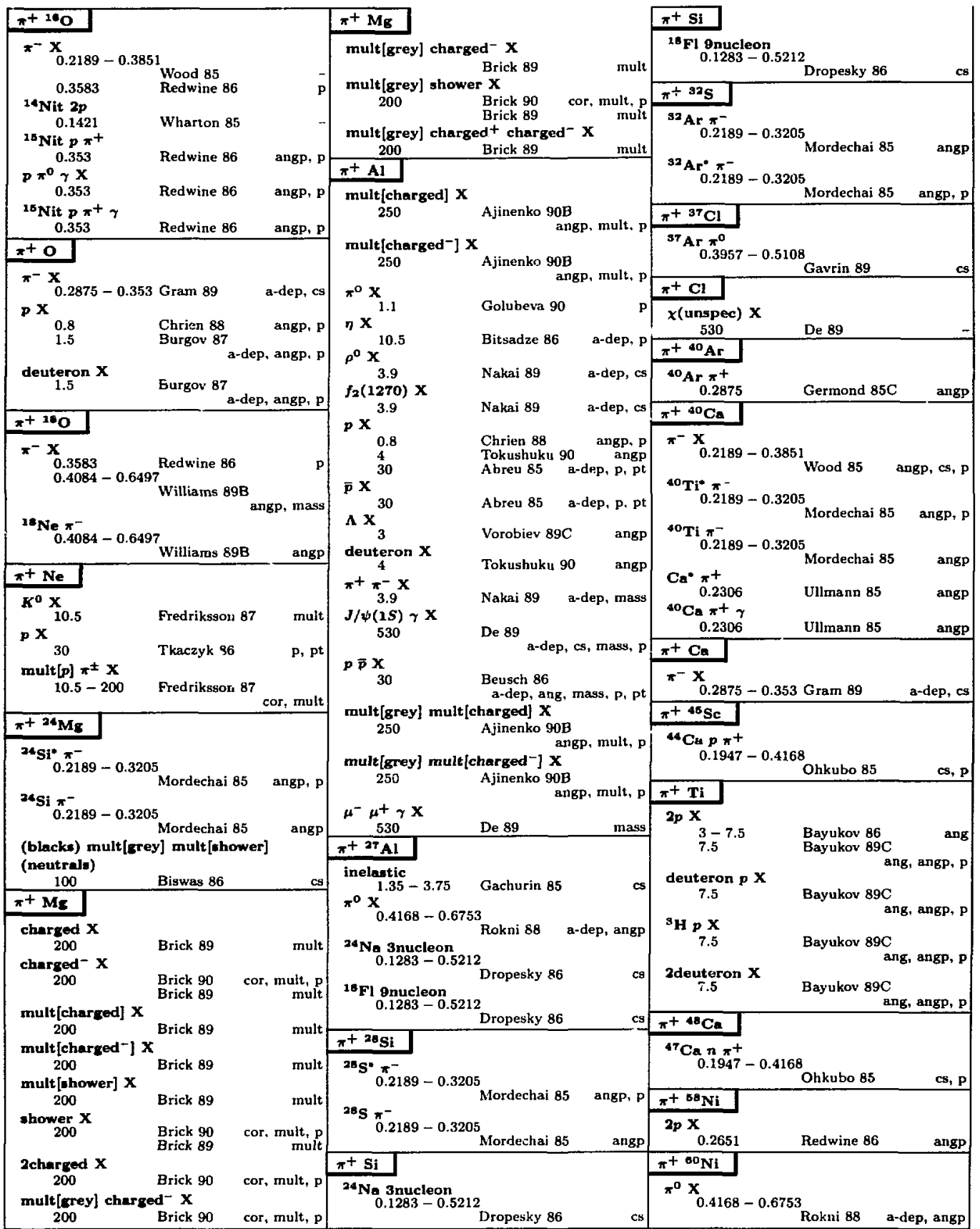

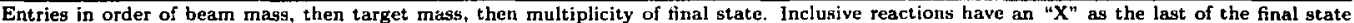

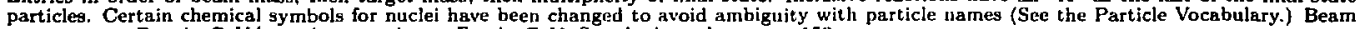
momenta are $P_{1 \star b}$ in $\mathrm{GeV} / \mathrm{c}$, or in parentheses $E_{\mathrm{cm}}$ in GeV. See the legend on page 153. 


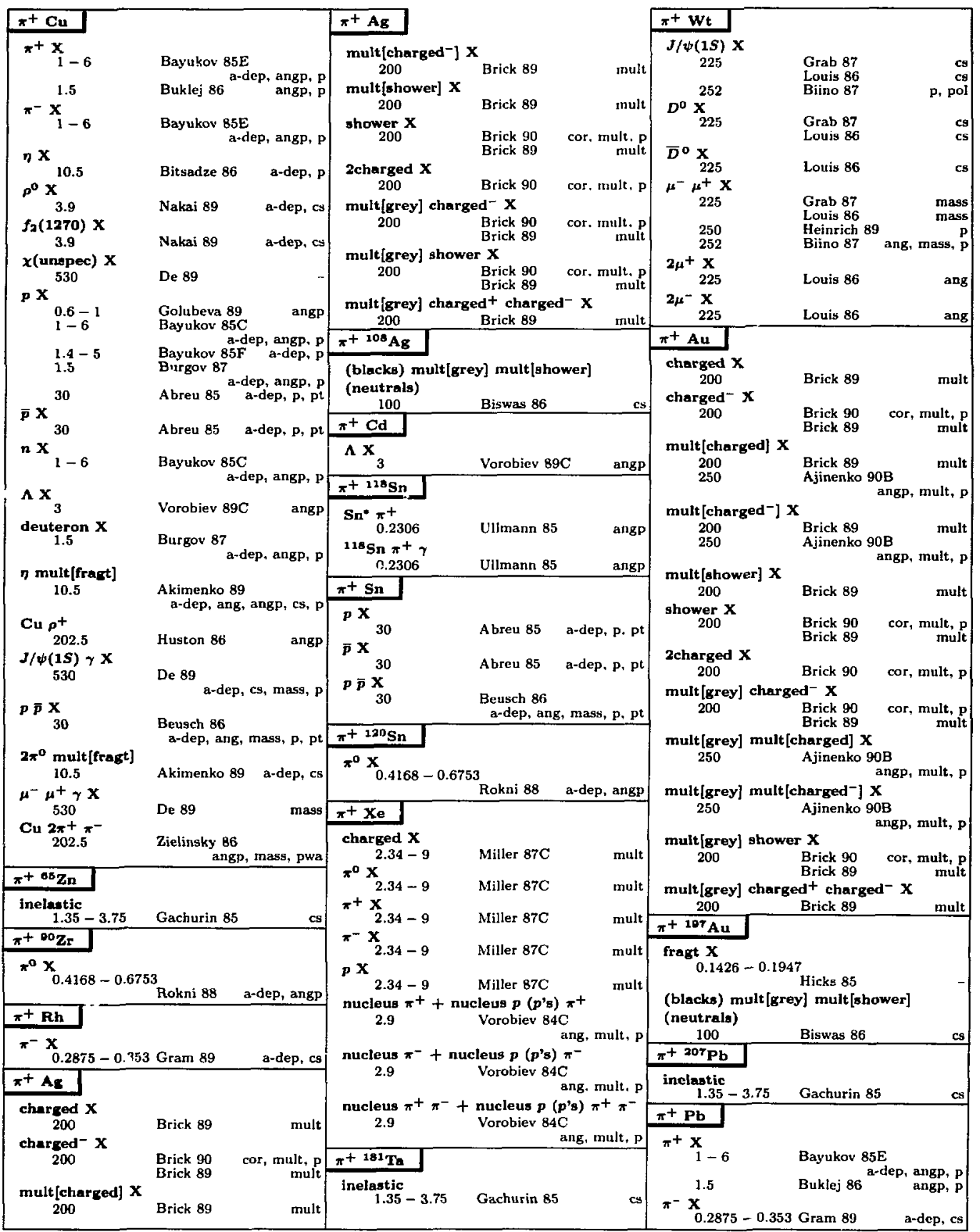




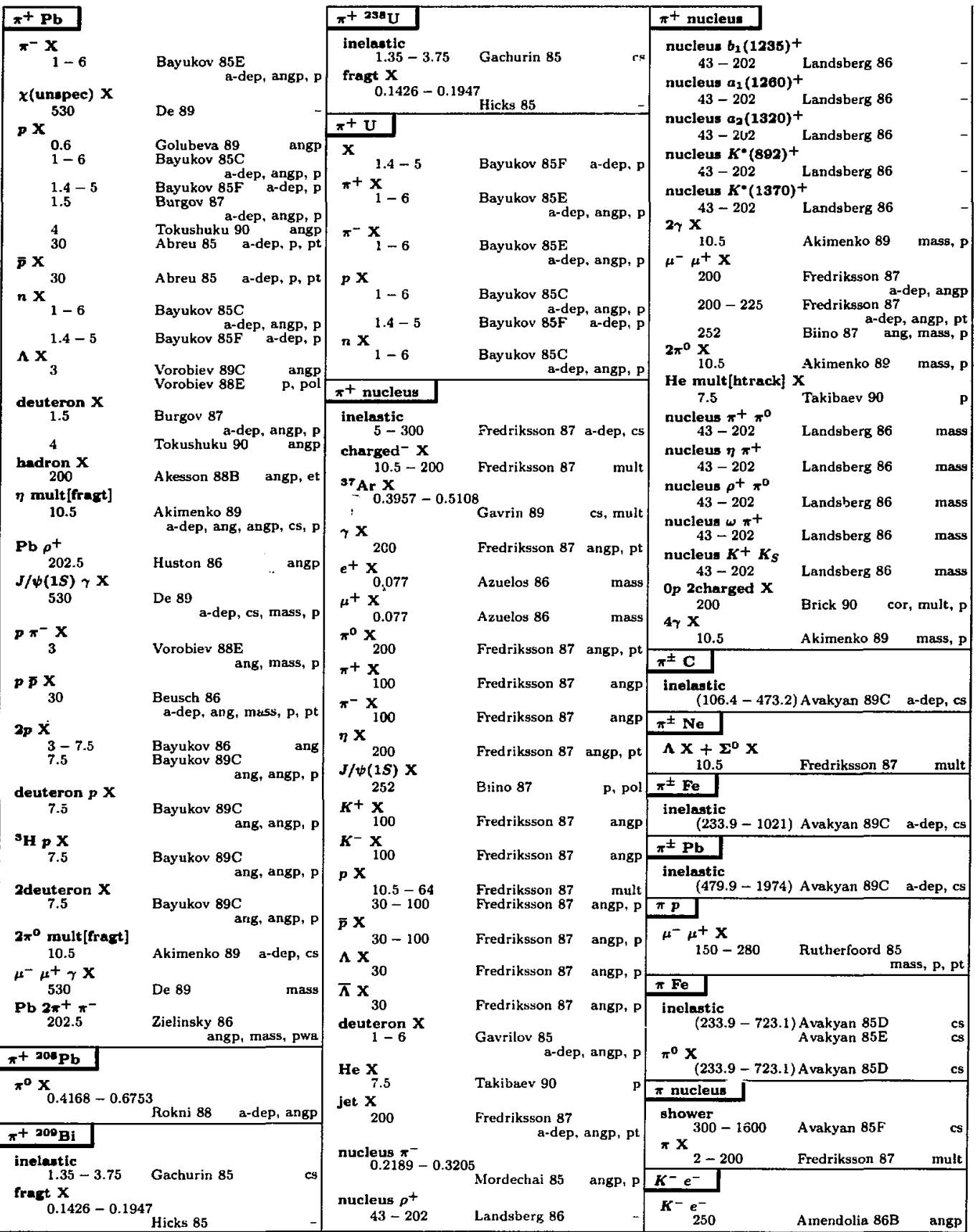




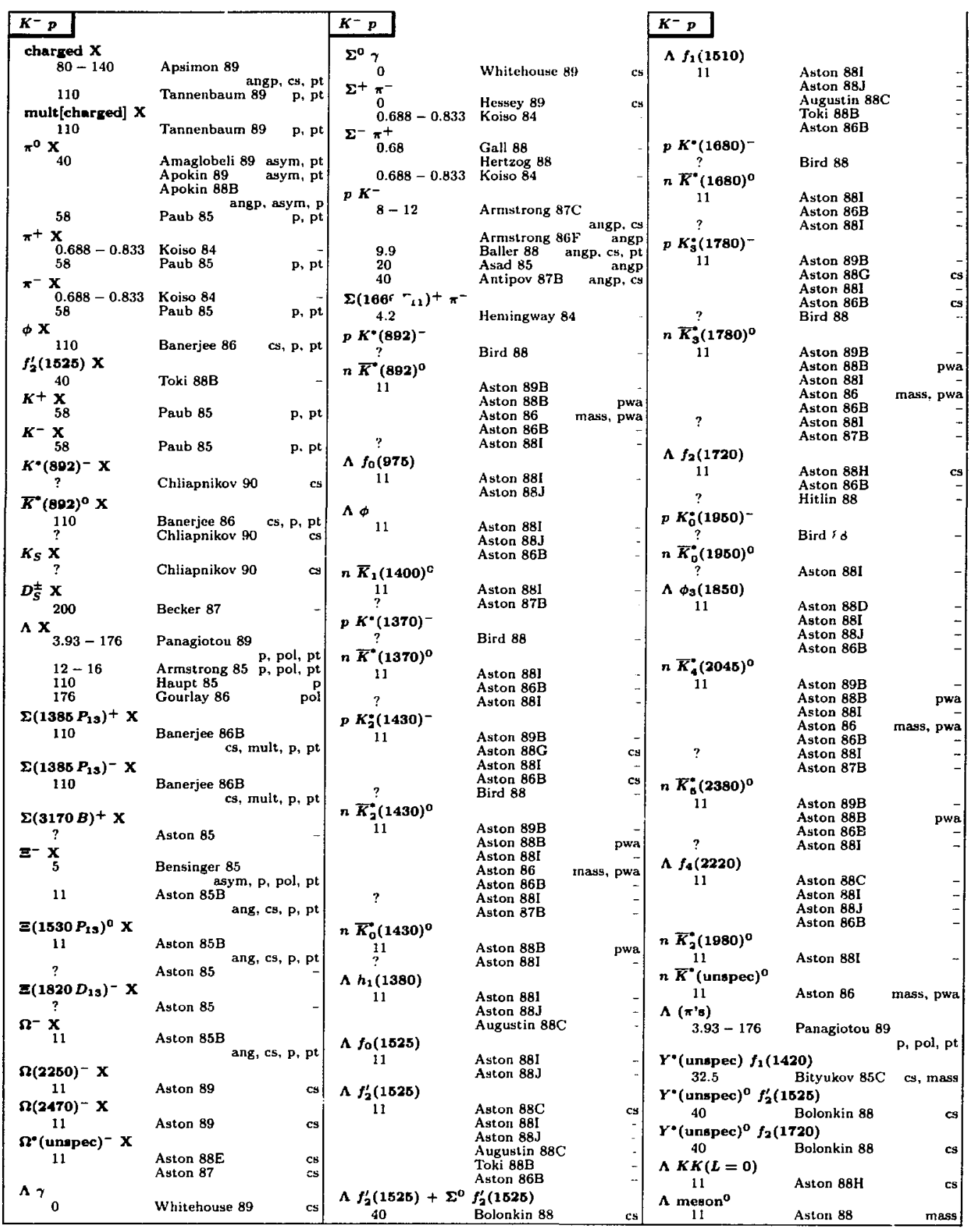




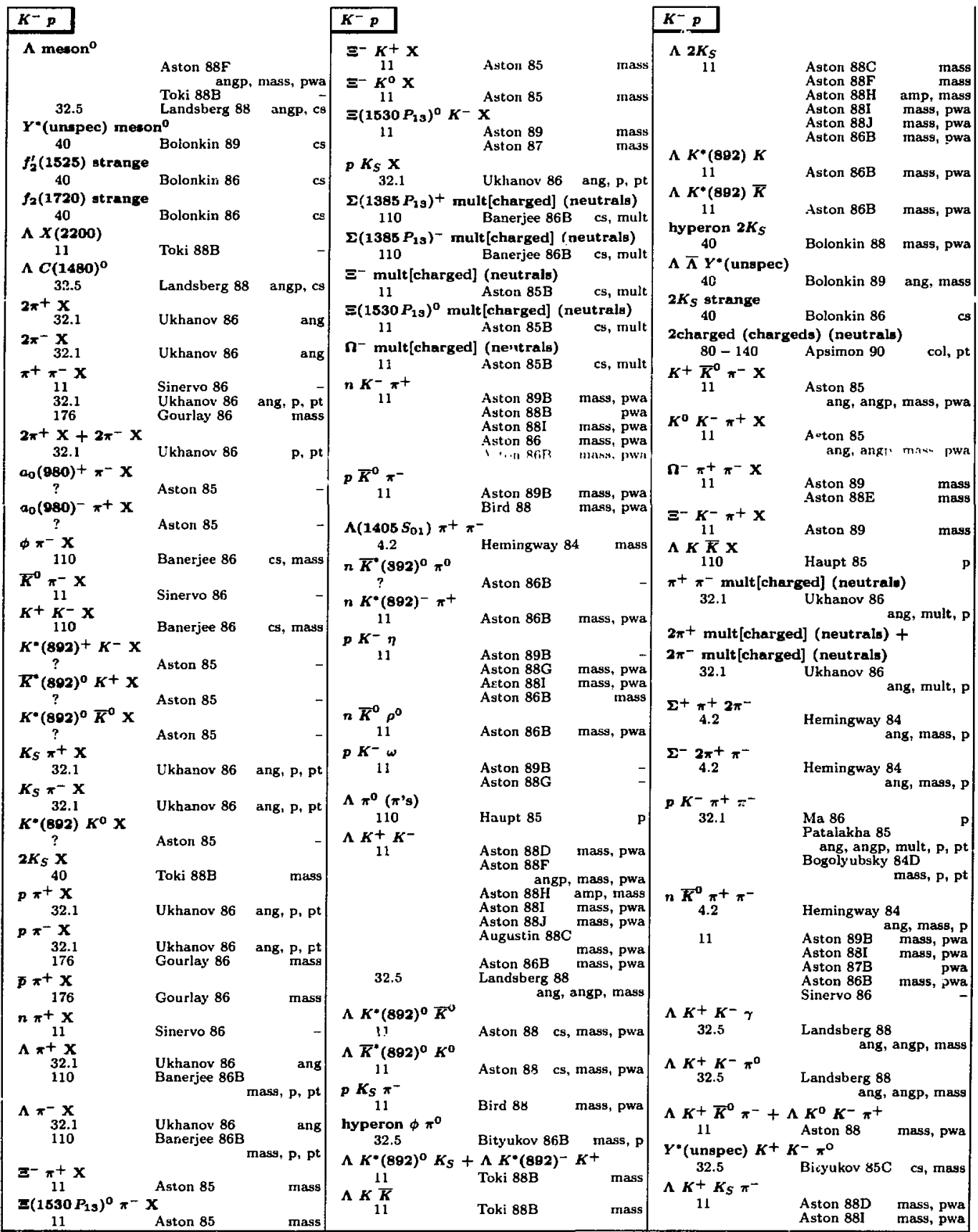




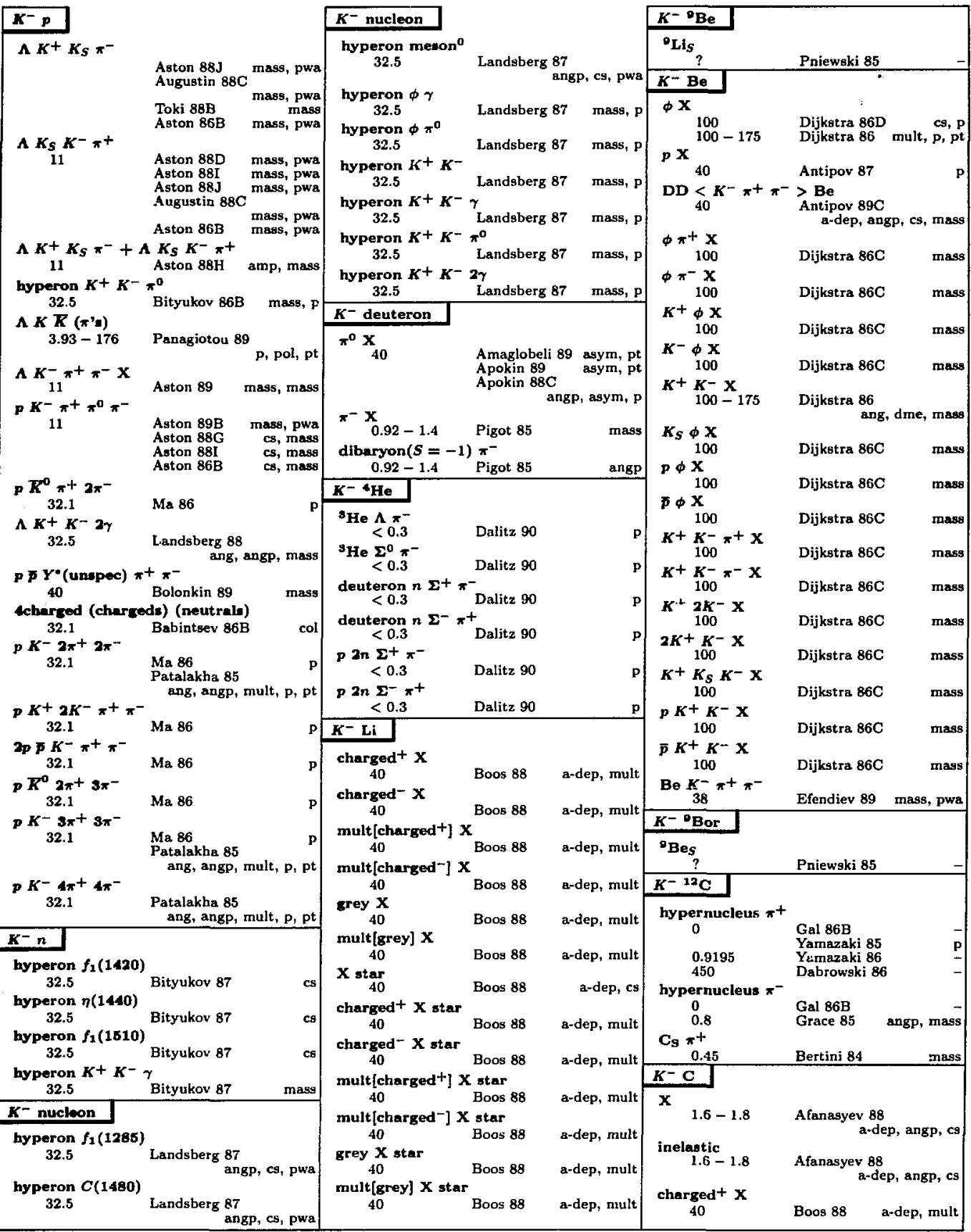




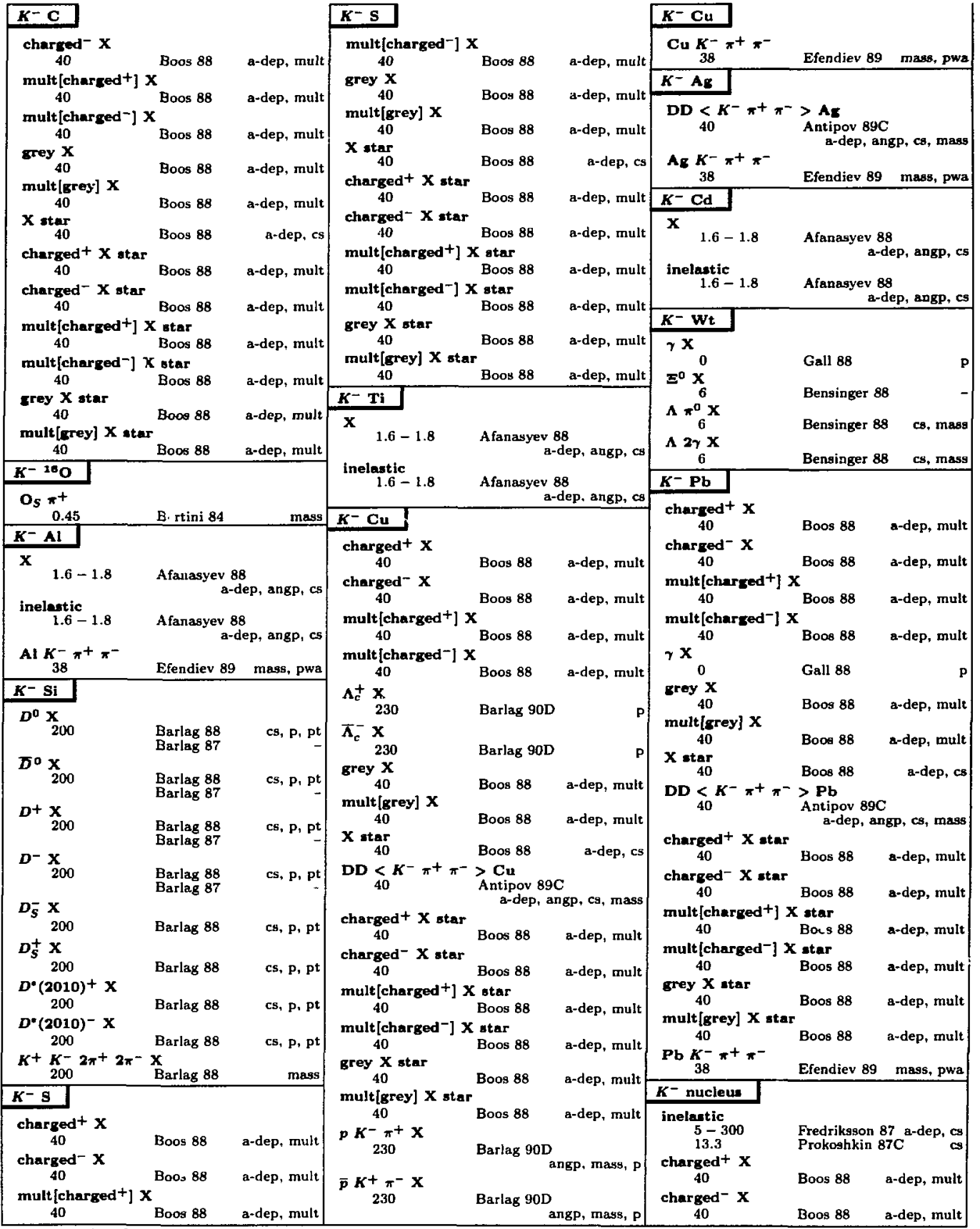

Entries in order of beam mass, then target mass, then multiplicity of final state. Inclusive reactions have an "X" as the last of the final state particles. Certain chemical symbols for nuclei have been changed to avoid ambiguity with particle names (See the Particle Vocabulary.) Beam momenta are $P_{1 a b}$ in $\mathrm{GeV} / c$, or in parentheses $E_{\mathrm{cm}}$ in $\mathrm{GeV}$. See the legend on page 153 . 


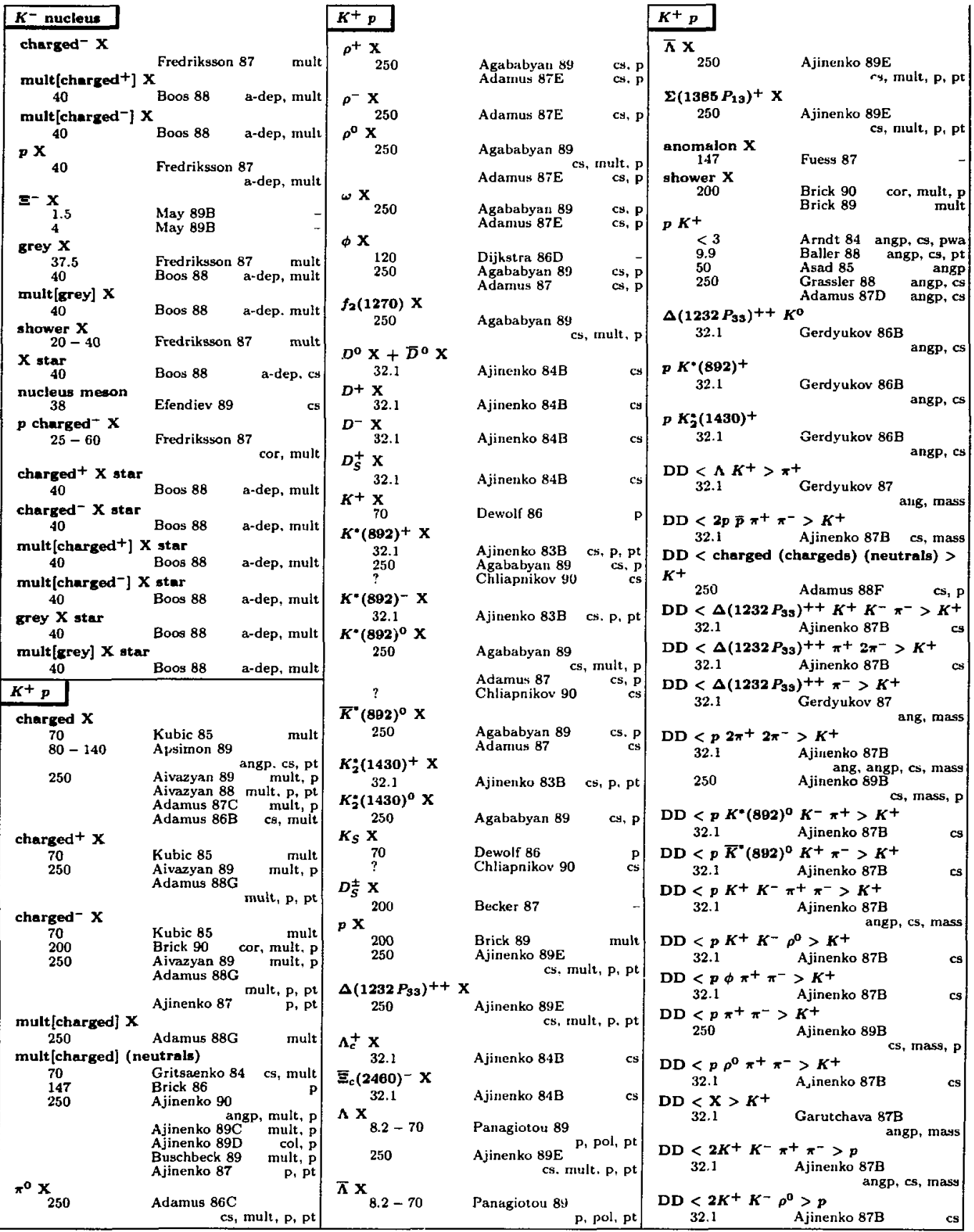




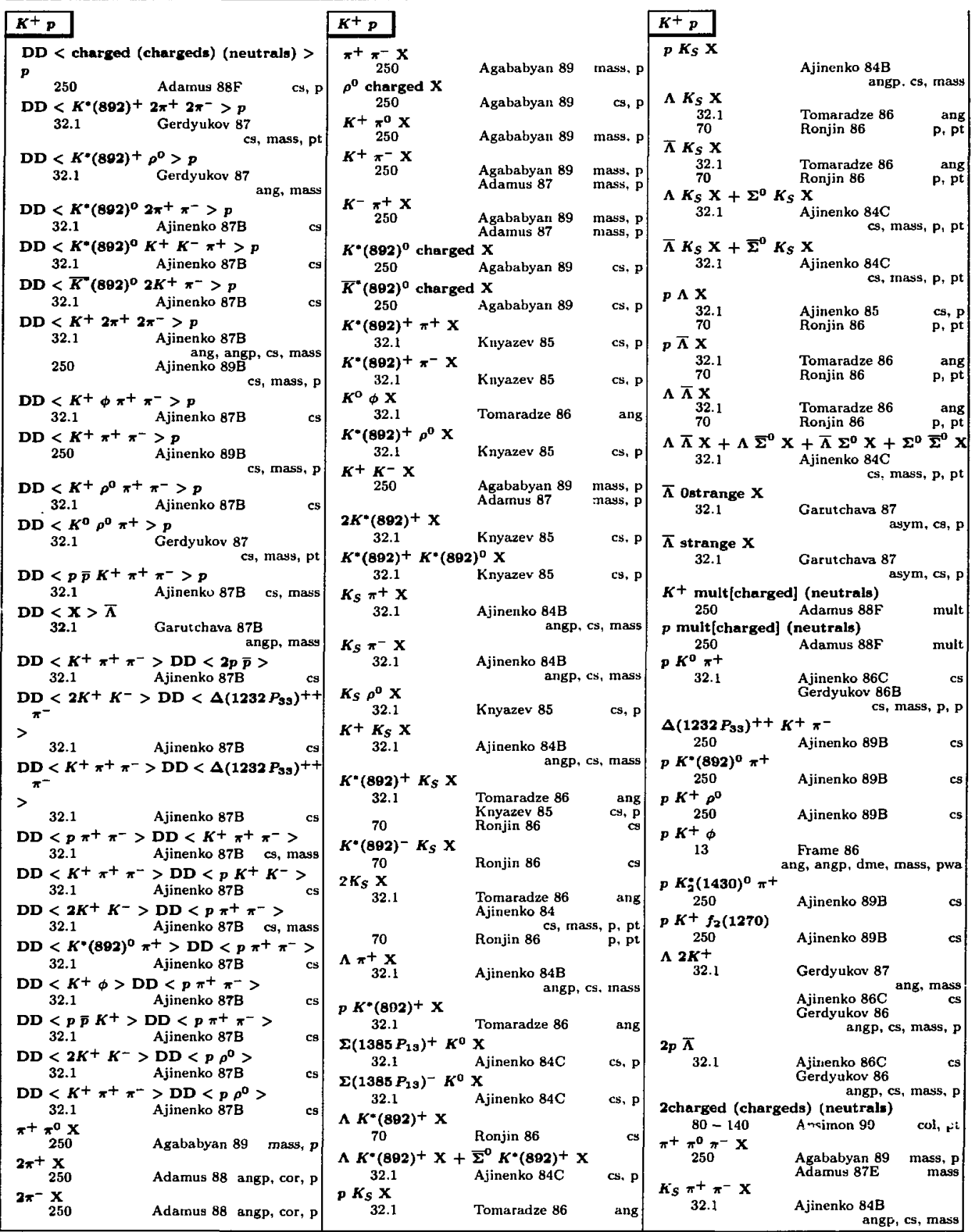




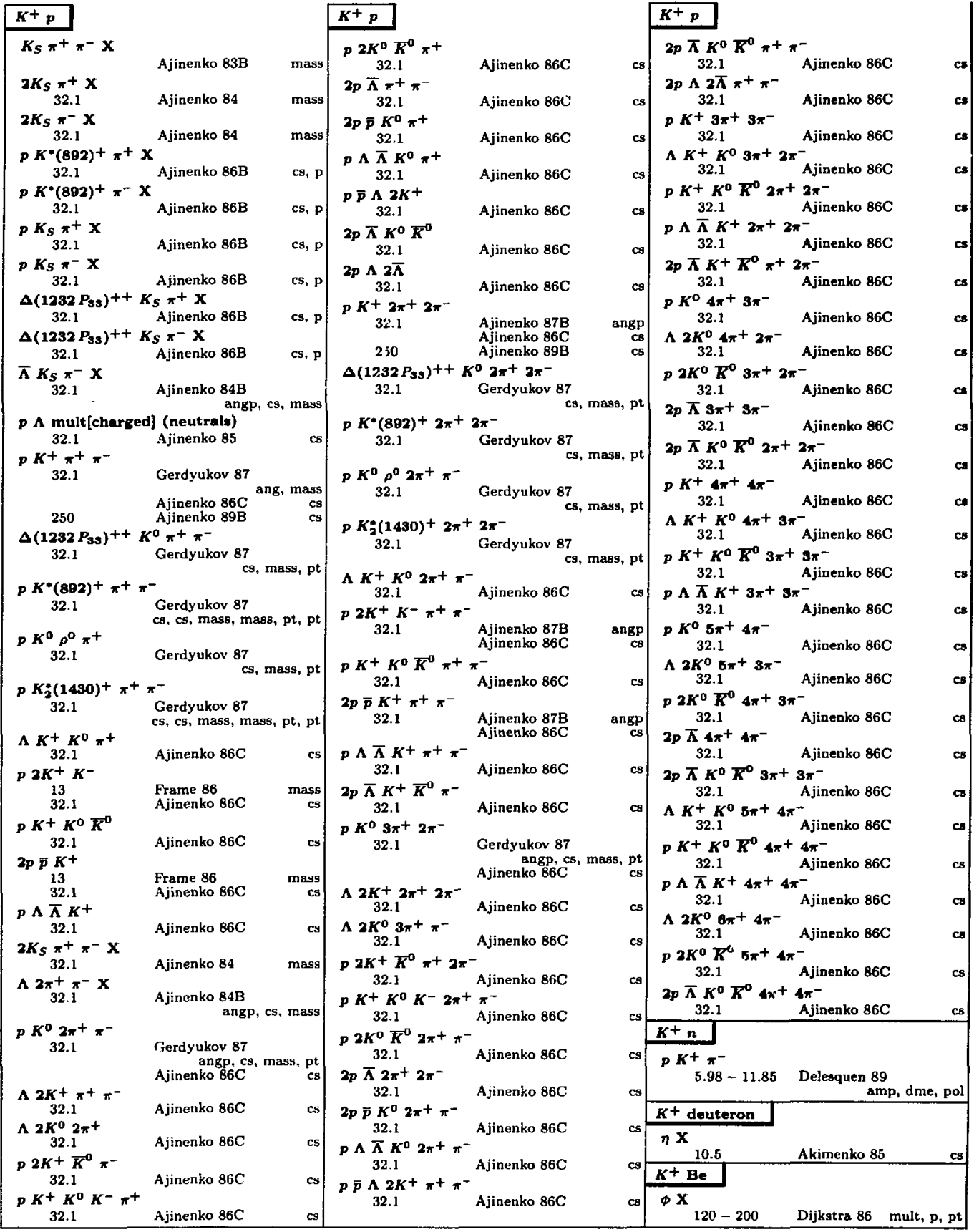




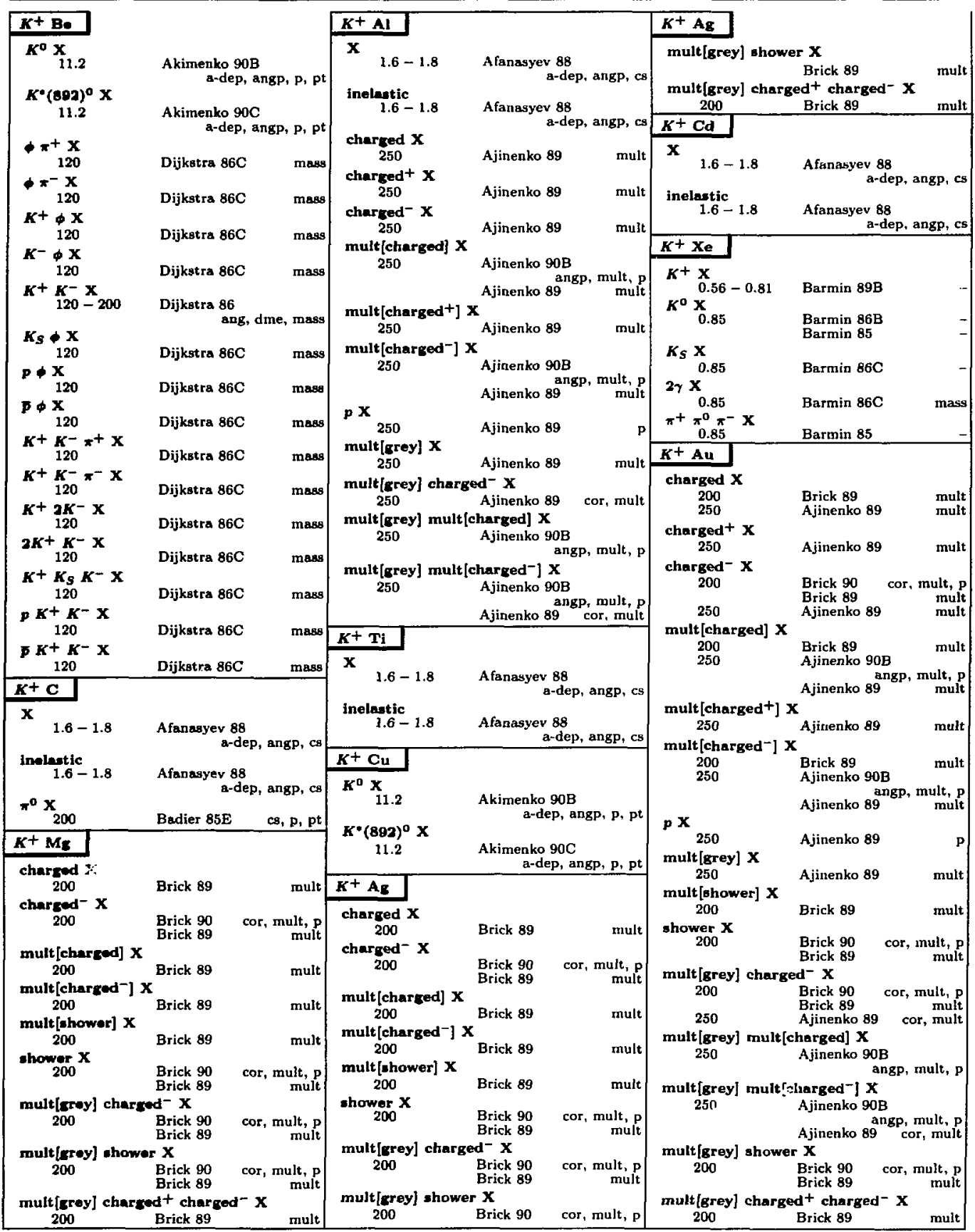

Entries in order of beam mass, then target mass, then multiplicity of final state. Inclusive reactions have an " $X$ " as the last of the final state particles. Certain chemical symbols for nuclei have been changed to avoid ambiguity with particle names (Sce the Particle Vocabulary.) Beam momenta are $P_{\text {lab }}$ in $\mathrm{GeV} / c$, or in parentheses $E_{\mathrm{cm}}$ in $\mathrm{GeV}$. See the legend on page 153 . 


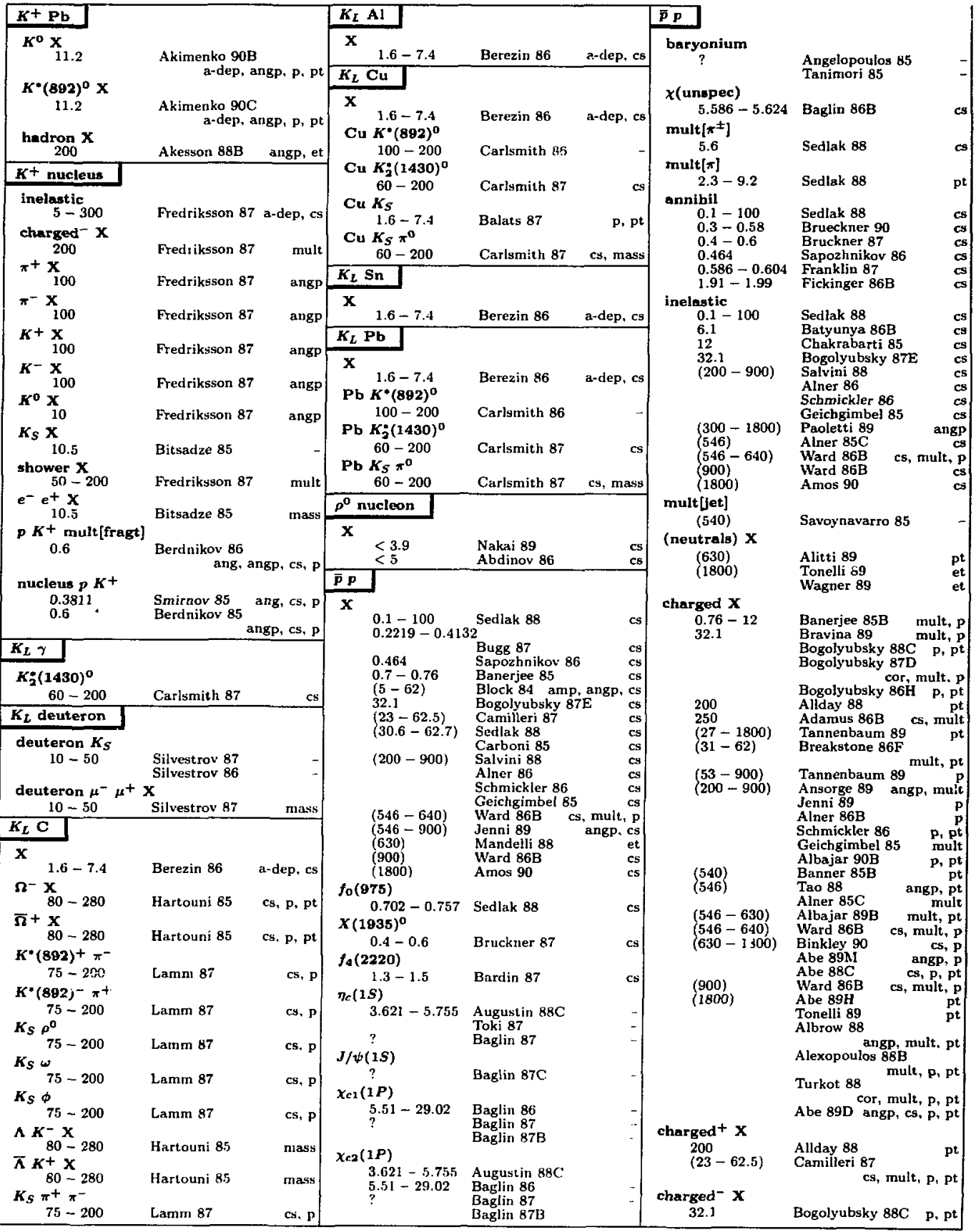




\section{$\overline{\mathbf{p}} \boldsymbol{p}$}

charged $^{-} \mathbf{X}$

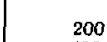

200
$(23-62.5)$

muit [charged] $x$

$0.76-12$

$5.7-22.4$

$(23-62.5)$

$(53-630)$

$(200-900)$

(540)

$\left\{\begin{array}{l}540) \\ 546-640)\end{array}\right.$

$(630)$

(900)

(1800)

(charged

22.4

$\begin{array}{ll}22.4 & \text { Batyunya } 90 \\ 1800) & \text { Freeman } 89\end{array}$

(neutrals)

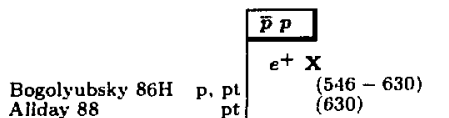

Aliday 88

cs, muit, p, pt

mult [charged] (neut rals)

$0.3-10 \quad$ Sedlak 88

$0.7-0.76 \quad$ Banerjee 85

6.1

12

22.4

32.1

200

(200 - 900)

$(540)$

(630)

(1800)

$\boldsymbol{\gamma} \mathbf{X}$

0

0.105

0.308

$0.76-12$

12

32.1

70

$(23-62.5)$

313.7

1496

$(53-640)$

$(200-900)$

(540)

$(540-630)$

$(546-630)$

$(546-900)$

(630)

$$
e^{ \pm} \mathbf{x}
$$

(1800)

$\begin{array}{ll}(540) & \text { Savoynavarro } 85 \\ (540-630) & \text { Vuillemin } 85\end{array}$

$(546-630)$
Banerjee 85B mult, p

Baldin 86

Camilleri 87

Tannenbaum 89

cs, mult

ang, et, $p$

Ansorge $89 \mathrm{C}$ mult, mult

Eckart 88

col, cor, mult. p

Savoynavarro 85

$\begin{array}{lll}\text { Wavoynavarro } & 85 \\ \text { Ward } 86 \mathrm{~B} & \text { cs, mult, } & -\end{array}$

Appel 85B

col, et

$\begin{array}{lr}\text { Ward } 86 \mathrm{~B} & \mathrm{cs}, \text { mult, } \\ \text { Turkot } 88 & \text { cor, mult, pt }\end{array}$

Freeman 89

Batyunya 86B

Chakrabarti 85

Boos 86

Bogolyubsky 88 cs, mult

Bogolyuhsky 870

Cor, mult, p

Allday 88 cs, mult

Albajar 89 cor, $p$

Tao 88 mult

Holl 86

Alner 850 et, mult, $p$

Cerradini 85

mult

et. mult, $p$, pt

Albajar 906

Alner 85
Alner 85C mult
Albajar 90 mult, p, pt
Buschbeck 89
Hubbard 89B
col, et, mass, mult, p, pt
Alexopoulos 88B

mult, $p, p t$

Omori 89

Adiels 86B

Gorringe 85

Tsukerman 8

Ziegler 88

Ahmad 85C

mult,

Bogolyubsky 88

Bogolyubsky $88 \mathrm{E}$

cs, mult, $p, p t$

Ukhanov 86B cs, p, pt

Camilleri 87

Bernasconi 88

Bernasconi 87

Akesson $85 \mathrm{G}$

Lancon $86 \mathrm{~B}$

Ansorge 89

Ferbel 86

Albajar 88B

Appel $86 \mathrm{~B}$

Salvini 88

Albajar 89C

Albrow 88

Ansari 88B

Richard 87

cs, pt

cs, pt

p, pt

angp, mult

angp, pt
cs, pt

cs, pt

$p, p t$

angp, $p, p t$

p, pt

ang, $p, p t$

p. pt

p, pt

Savoynavarro 85

Appel 86

ang

Albajar 88F

$x^{(630)}$
(630)

$e^{-x}$

$\pi^{ \pm} \mathbf{x}$

(546-630) Albajar 86

(630) Fransson 90

$\mu+\mathbf{x}$

$(546-630)$

(630)

(1800)

$\mu-\mathrm{X}$

$(546-630)$

(630)

$\pi^{0} \mathbf{x}$

5.55

12

32.1

40

70

$(23-62.5)$

313.7

\subsection{6}

$(53-640)$

(540)

(630)

$8.8-9.1$

$(546)$
$(1800)$

$\pi+\mathbf{x}$

0.76

5.56

$12-1078$

32.1

Albajar 86

Frasison 90

Botner 89

Botner 89

Albajar 86

Albajar 88D

Albajar 88F

Skarha 89

Albajar 86

Albajar 88D

Sedlak 88

Chakrabarti 85

Bogoluubsky 87E mult

Apokin 88B

angp, asym, $p$

Ukhanov $86 \mathrm{~B}$

Camilleri 87

Bernasconi 88

Antille 87

Bernasconi 87

\begin{tabular}{r|r}
$\mathbf{p}$ & $\tau^{ \pm} \mathbf{X}$
\end{tabular}

Valenti 85

Akesson 85G

Lancon 86B

Banner 85B

Pare 90

Ansari 88B

Appel $\mathbf{8 6 3}$ angP.

cs, pt

cs, pt

angp

pt

P. pt

Sedlak 88

Ward $86 \mathrm{~B}$

Alexopoulus 90

mult. p. pt

Ahmad 84

Banerjee 85

Sedlak 88

Sedlak 88

Bogolyubsky 88

Smirnova 88 mult, p, pt

Bogolyubsky 87E mult

Bravina 86

Camilleri 87

Ahmad 84

Sedlak 88

Sedlak 88

Sedlak 88

Sedlak 88

Bogolyubsky 88

Bogolyubsky 88C P.

Smirnova 88 mult, $\mathrm{p}, \mathrm{pt}$

Bogolyubsky 87E mult

Bogolyubsky $86 \mathrm{H}$

P, pt

$(23-62.5)$

Bravina 86

Camilleri 87

$\pi^{+} \mathbf{x}+\pi^{-} \mathbf{x}$

$\pi \mathbf{X}$

$(200-900)$

Burow 87

cs, mult

0

12

$(23-62.5)$

313.7

1496

(540)

(630)

$\rho^{0} \mathrm{X}$

$0.7-100$
Botner 89

Chiba 89

Adiels $\mathbf{8 8}$

Chakrabarti 85

Camilleri 87

Bernasconi 88

Antille 87

Bernasconi 87

Akesson 85G

Banner 85B

Albajar $88 \mathrm{C}$ $\mathbf{x}$

12

$\eta^{\prime} \mathbf{X}$

$x^{32.1}$

$\overline{\boldsymbol{p}} \boldsymbol{p}$

$\rho^{0} \mathrm{X}$

$9.1-405$
$12-22.4$

32.1

(630)

(630)

(630)

$f_{0}(975) \times$

(630)

$f_{2}(1270) X$

$0.7-100$

9.1

32.1

$\tau^{ \pm} \times(540)$

$(540-630)$

$J / \psi(15) \times$

5.586 - 5.624 Baglin 86B

$(546-630)$

(630)

$(630-1800)$

Albajar 88C

Albajar 88D

Albajar 88E 


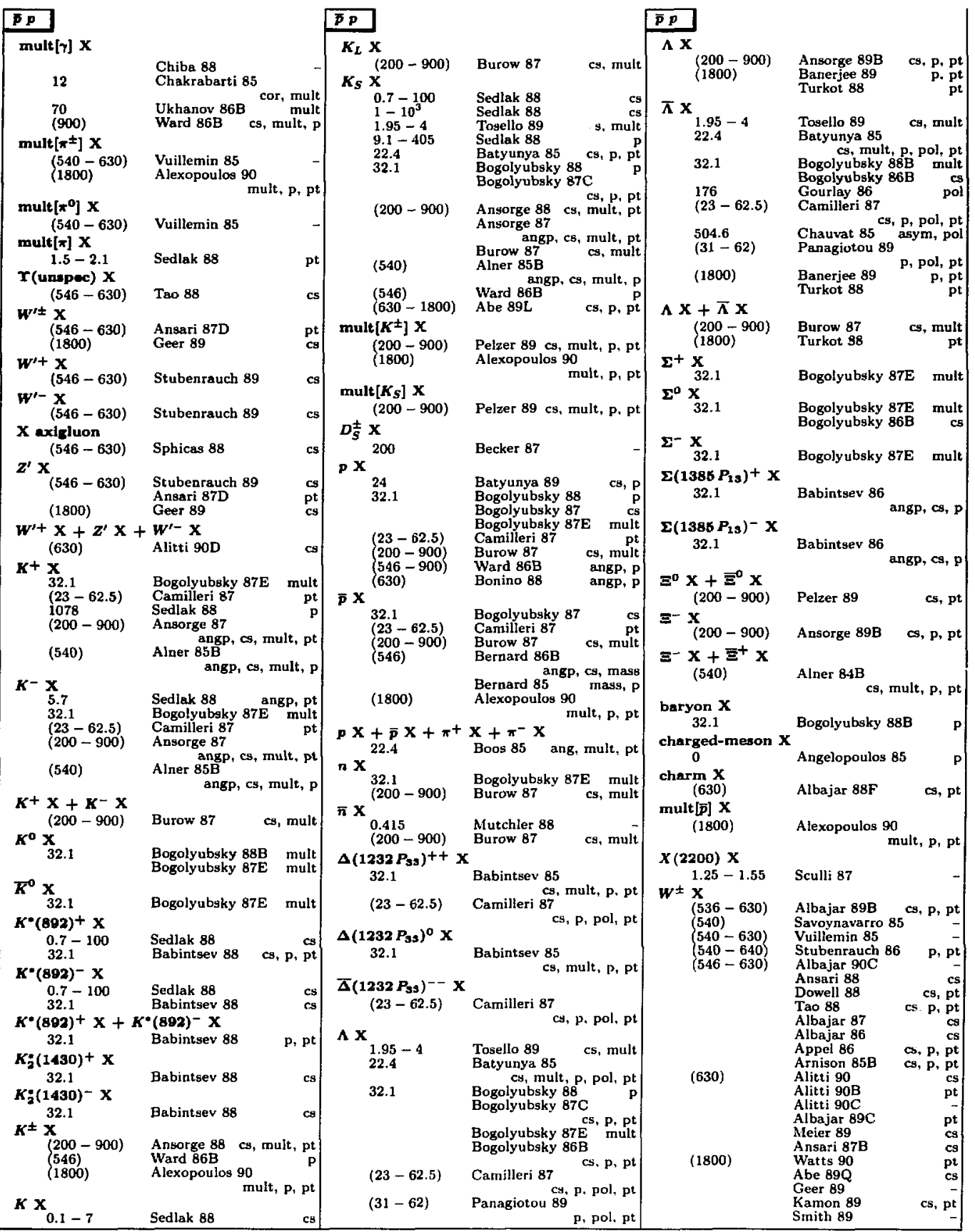




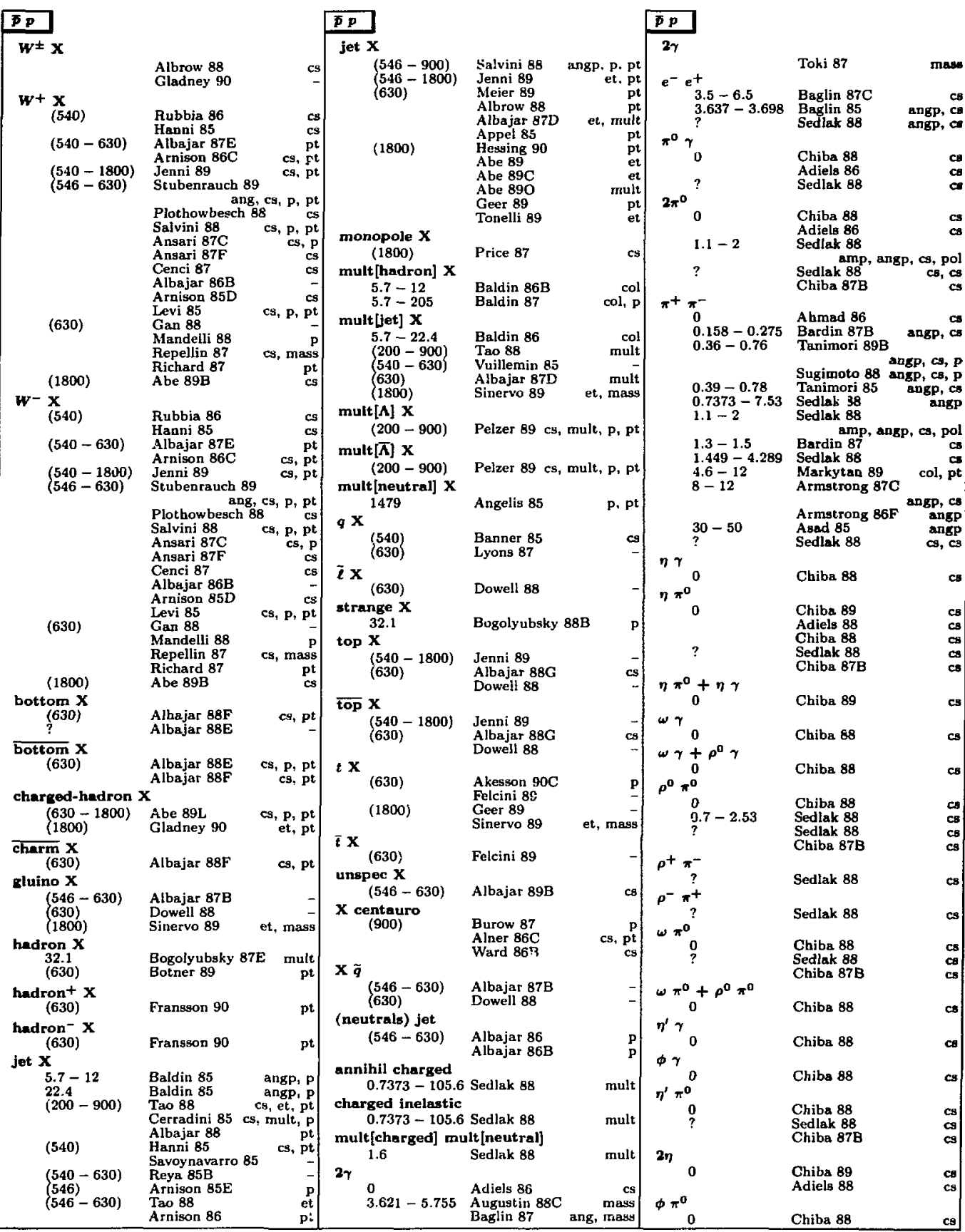




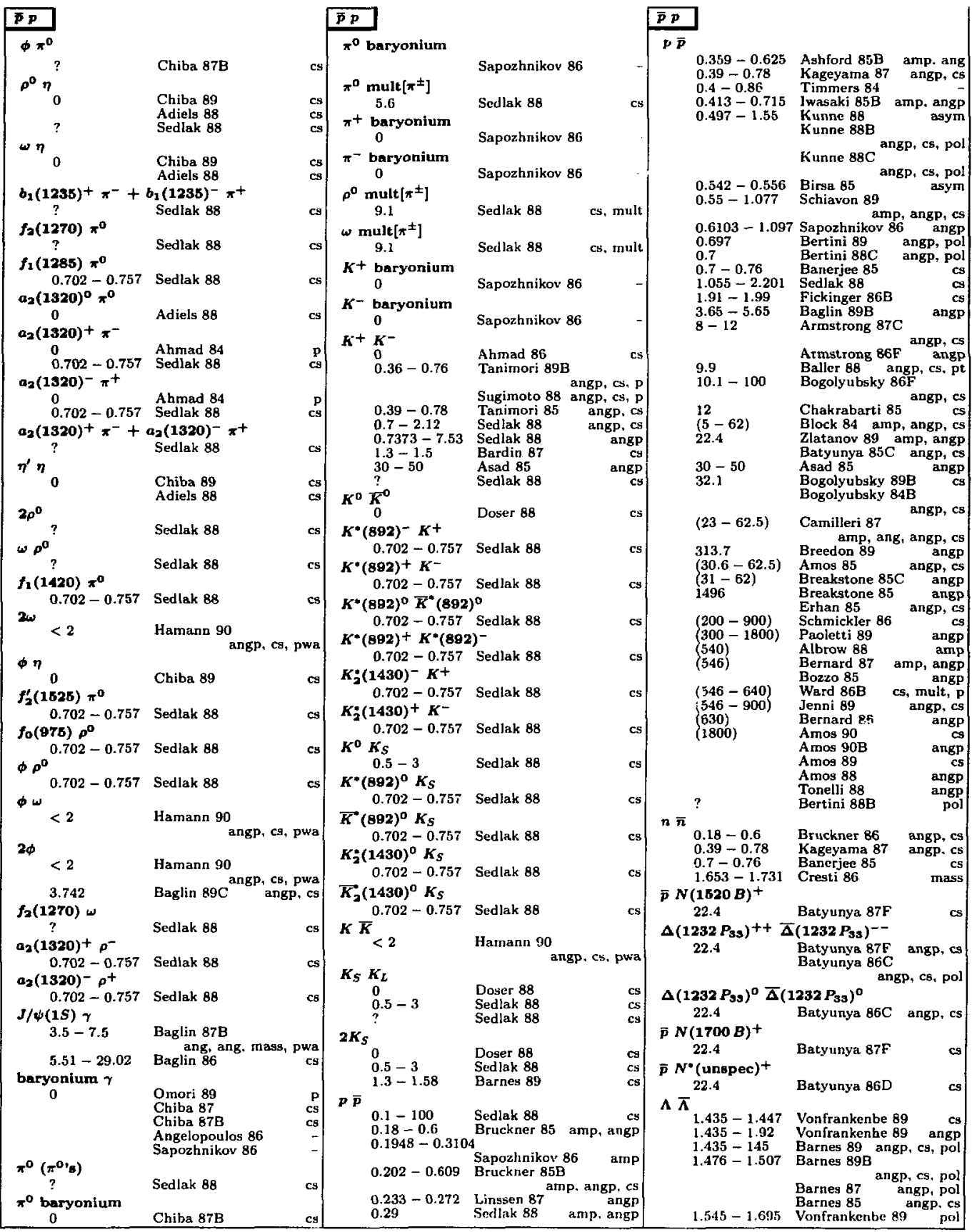




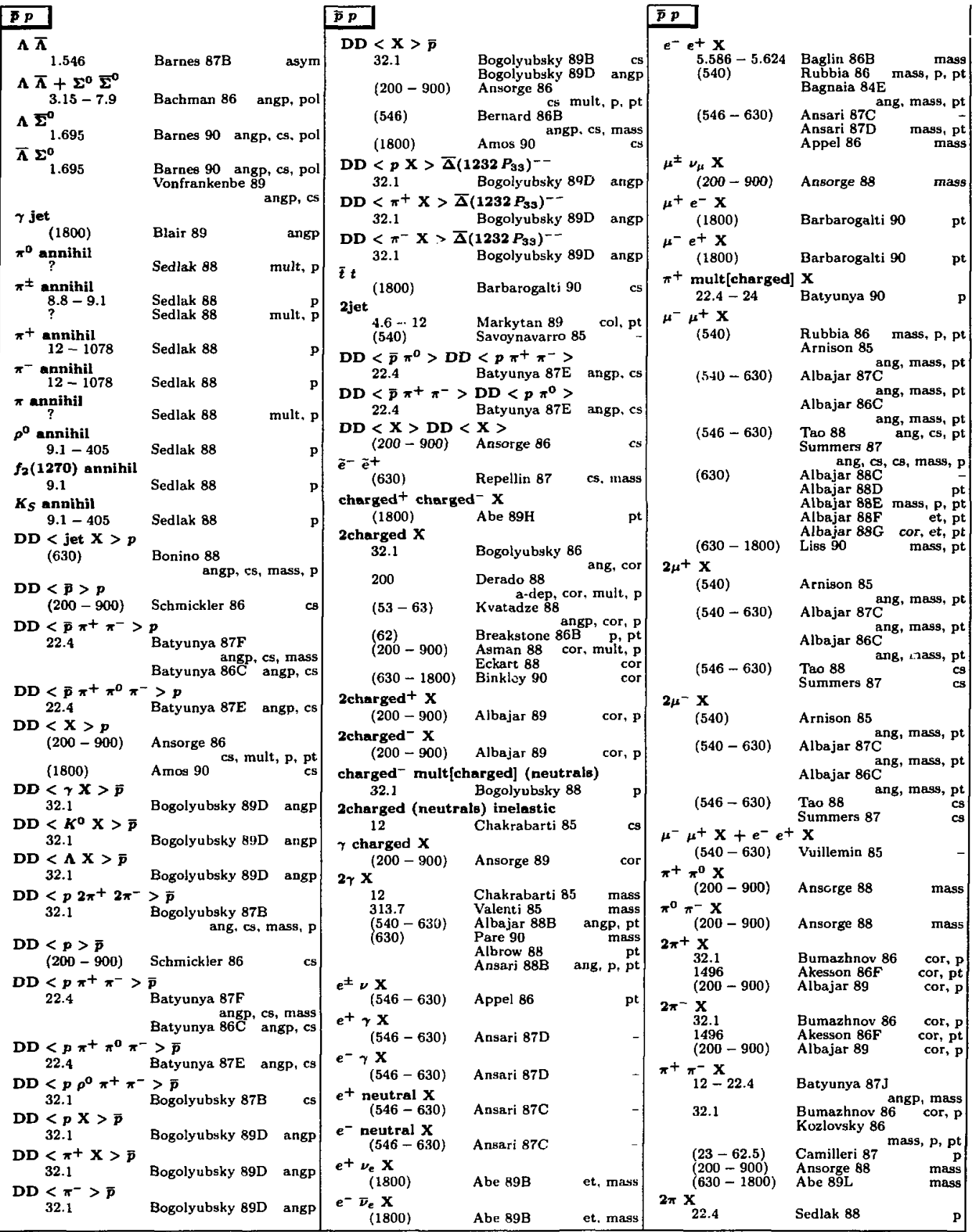




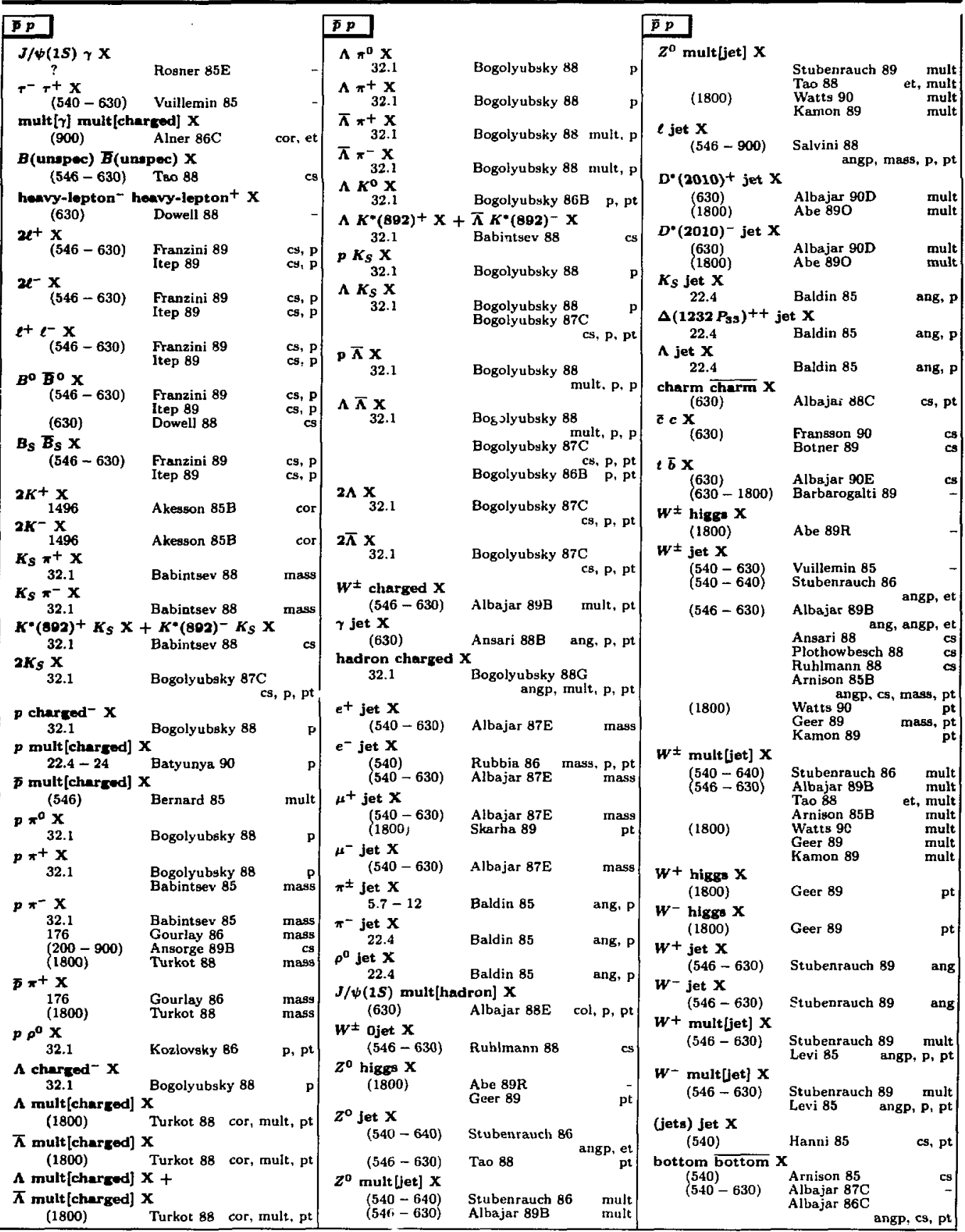




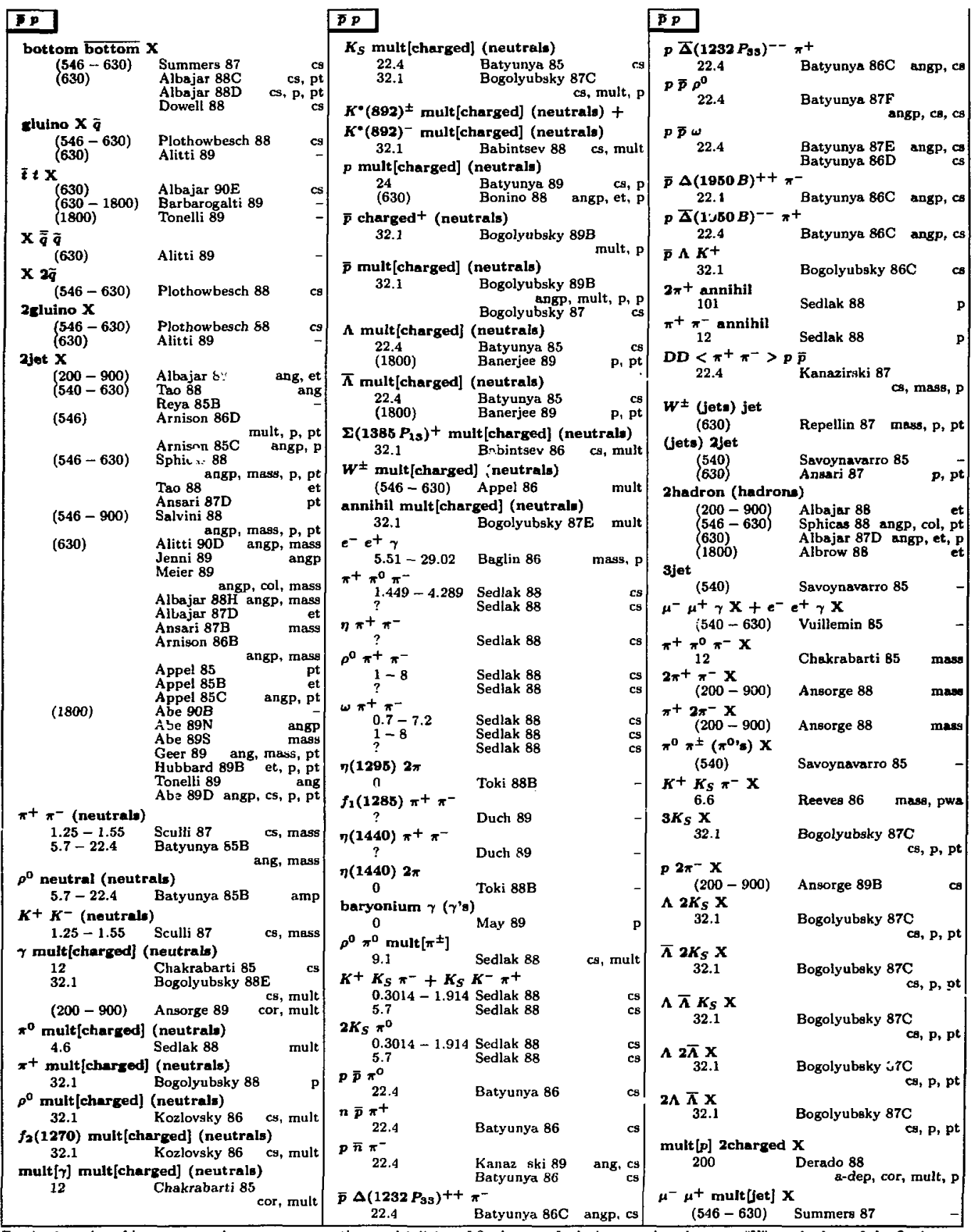




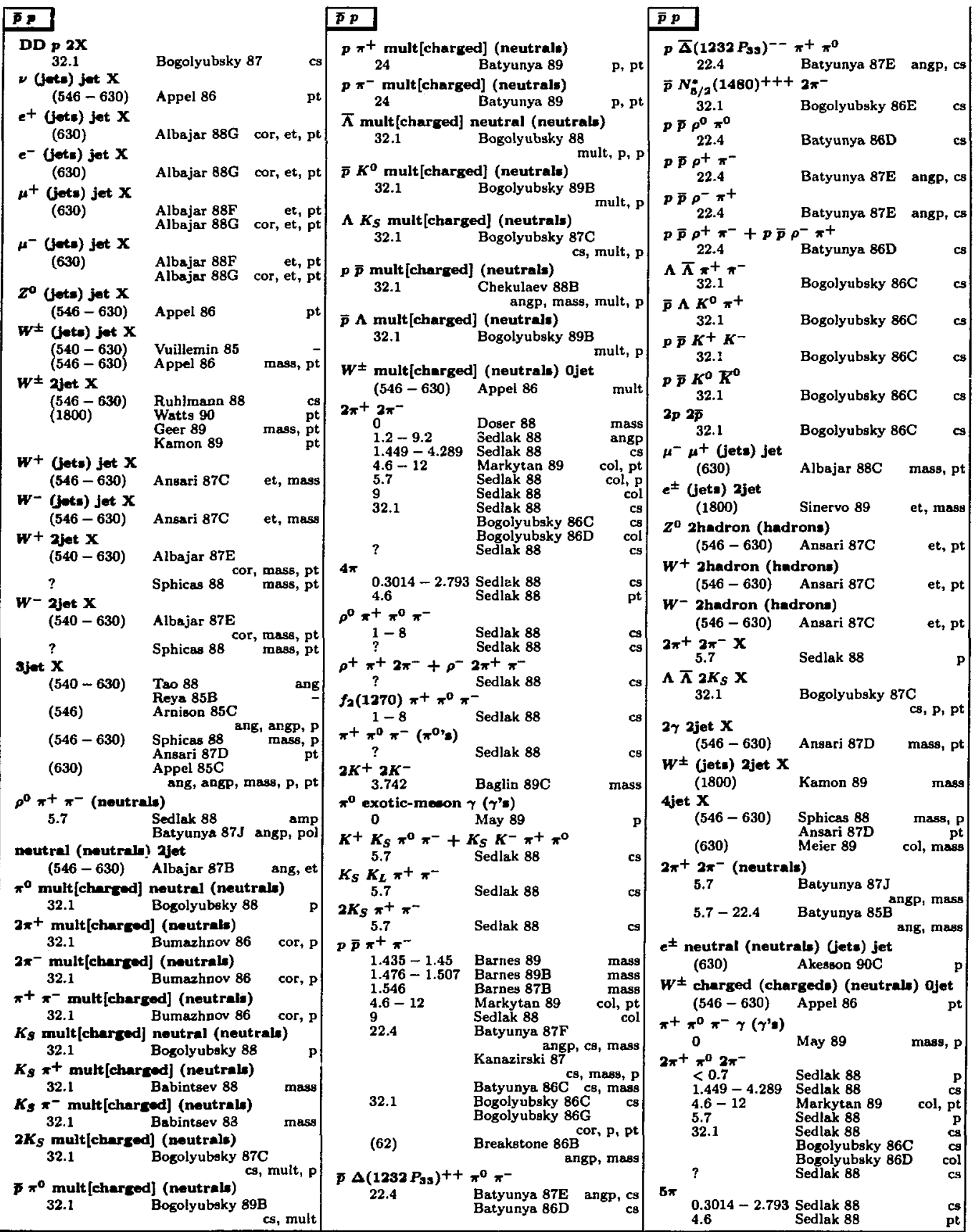




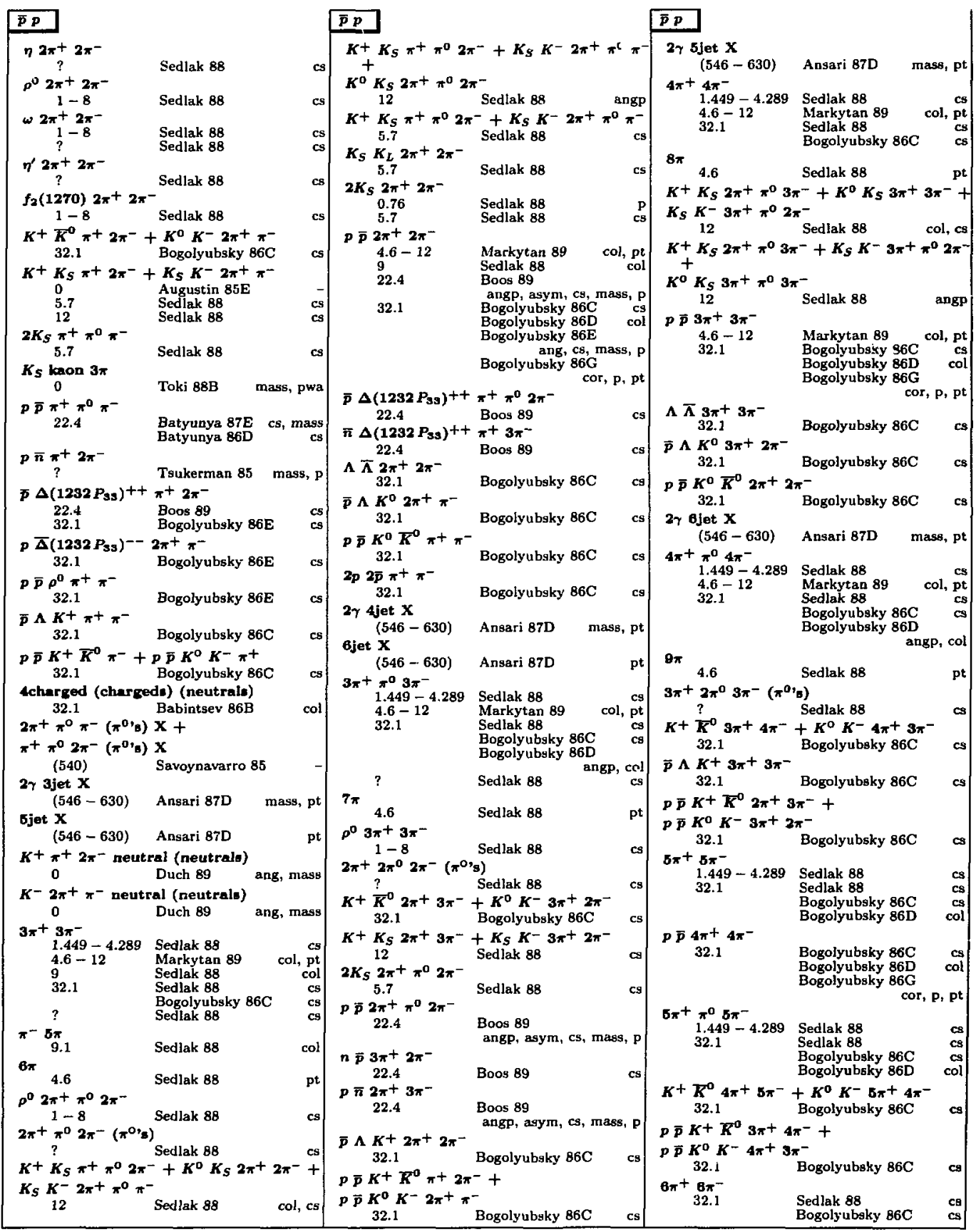




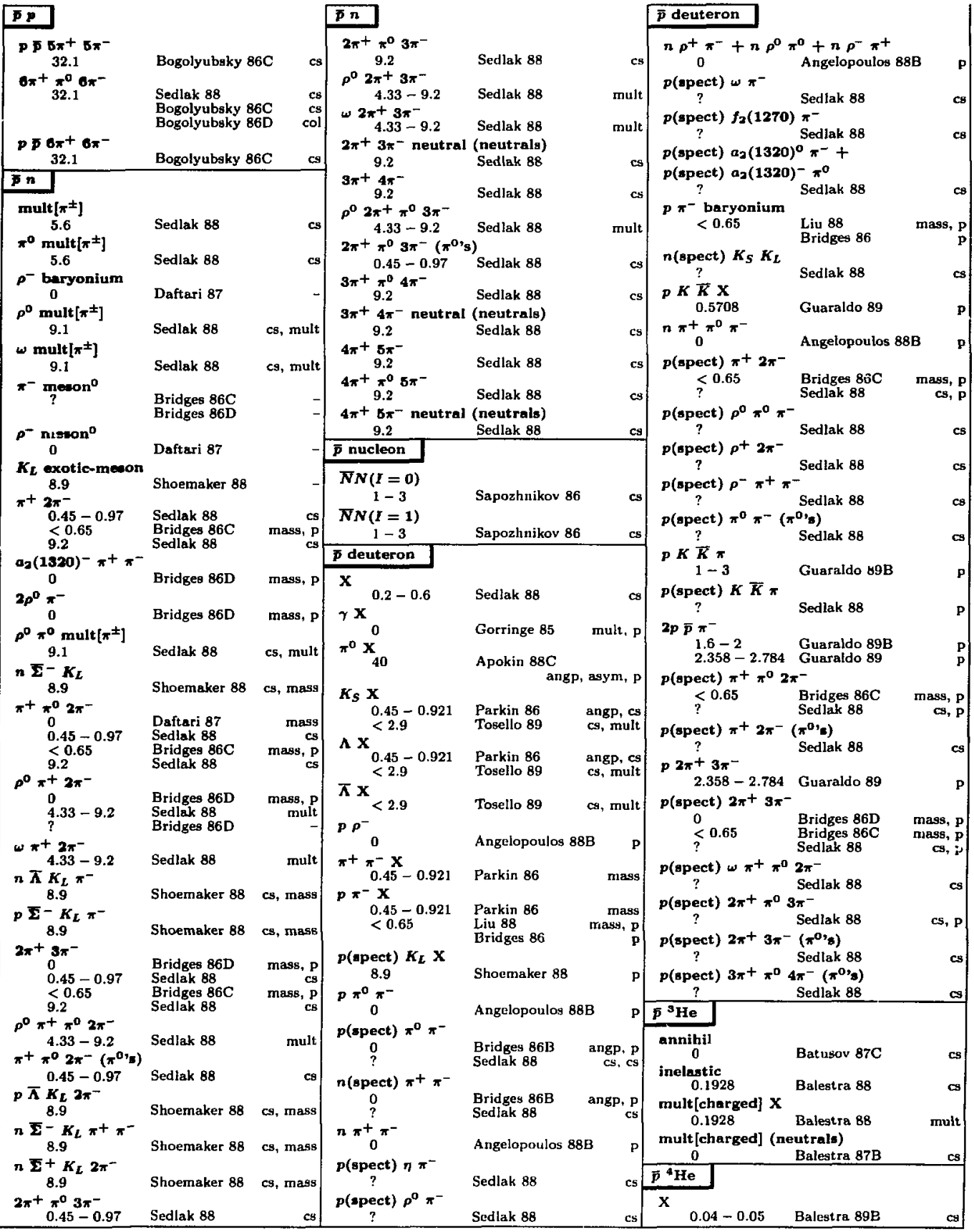




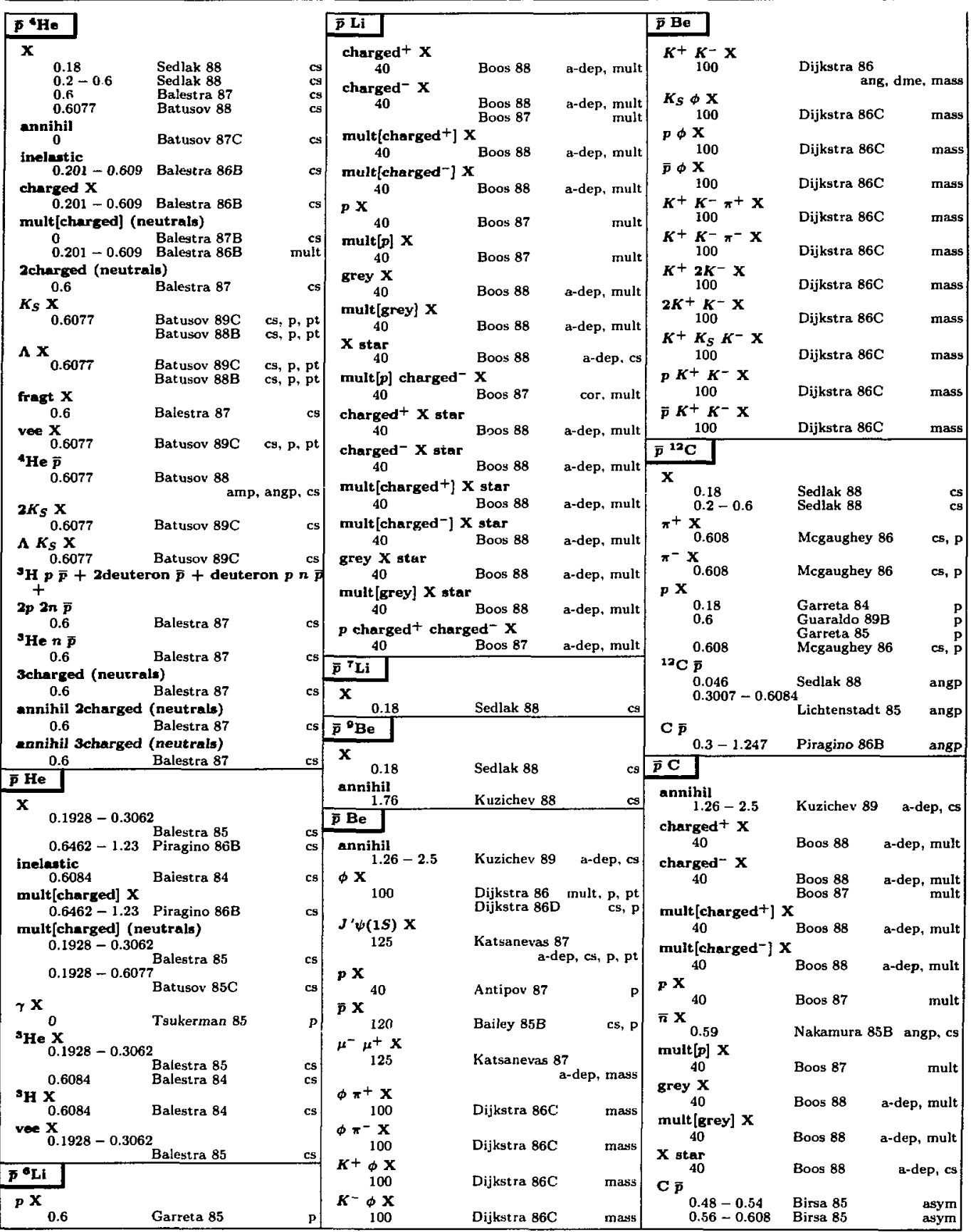

Entries in order of beam mass, then target mass, then multiplicity of fral state. Inclusive reactions have an "X" as the last of the final state particles. Certain chemical symbols for nuclei have been changed to avoid ambiguity with particle names (See the Particle Vocabulary.) Beam momenta are $\mathrm{P}_{\text {lab }}$ in $\mathrm{GeV} / c$, or in parent heses $E_{\mathrm{c} \text { in }}$ in $\mathrm{GeV}$. See the legend on page 153. 


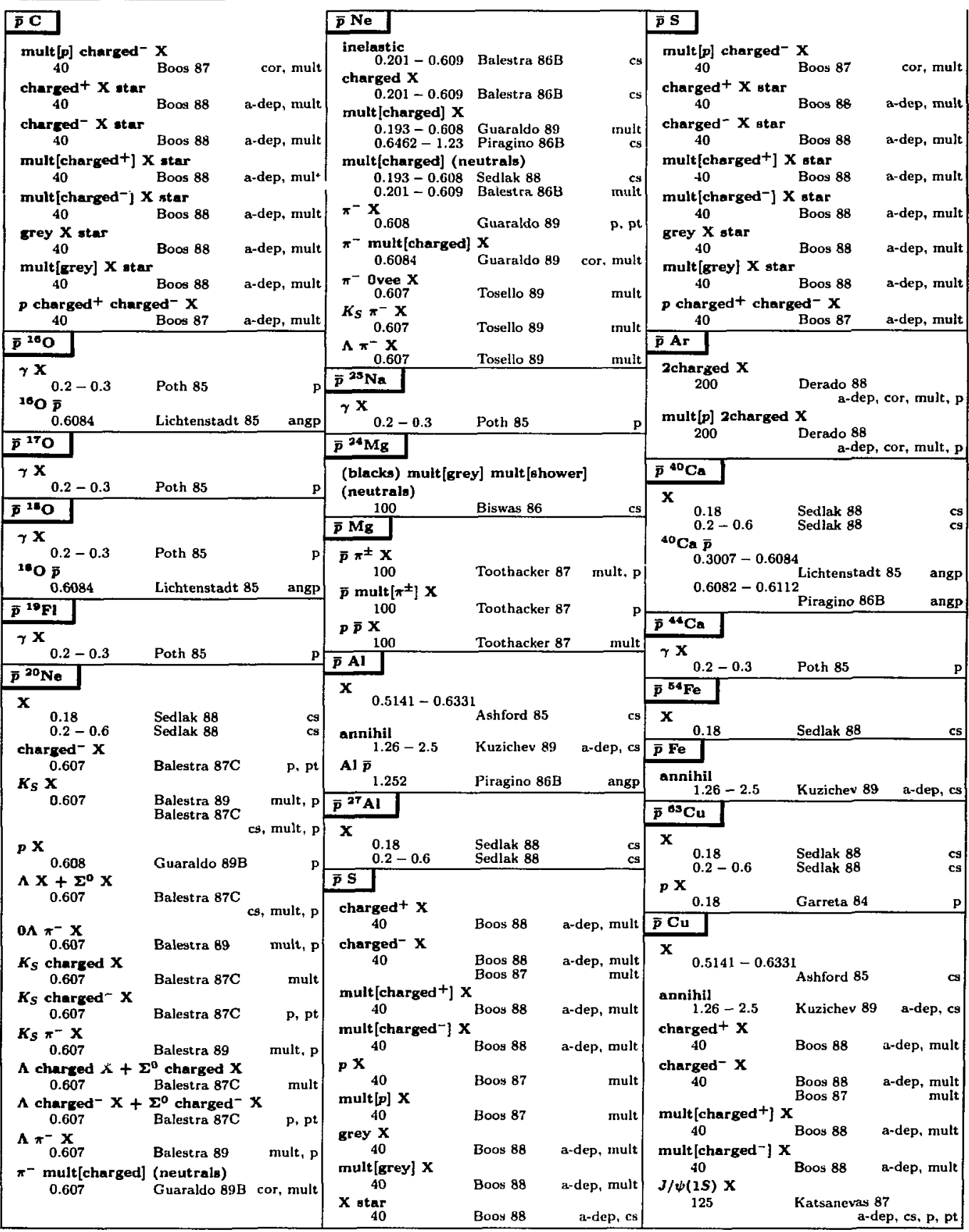




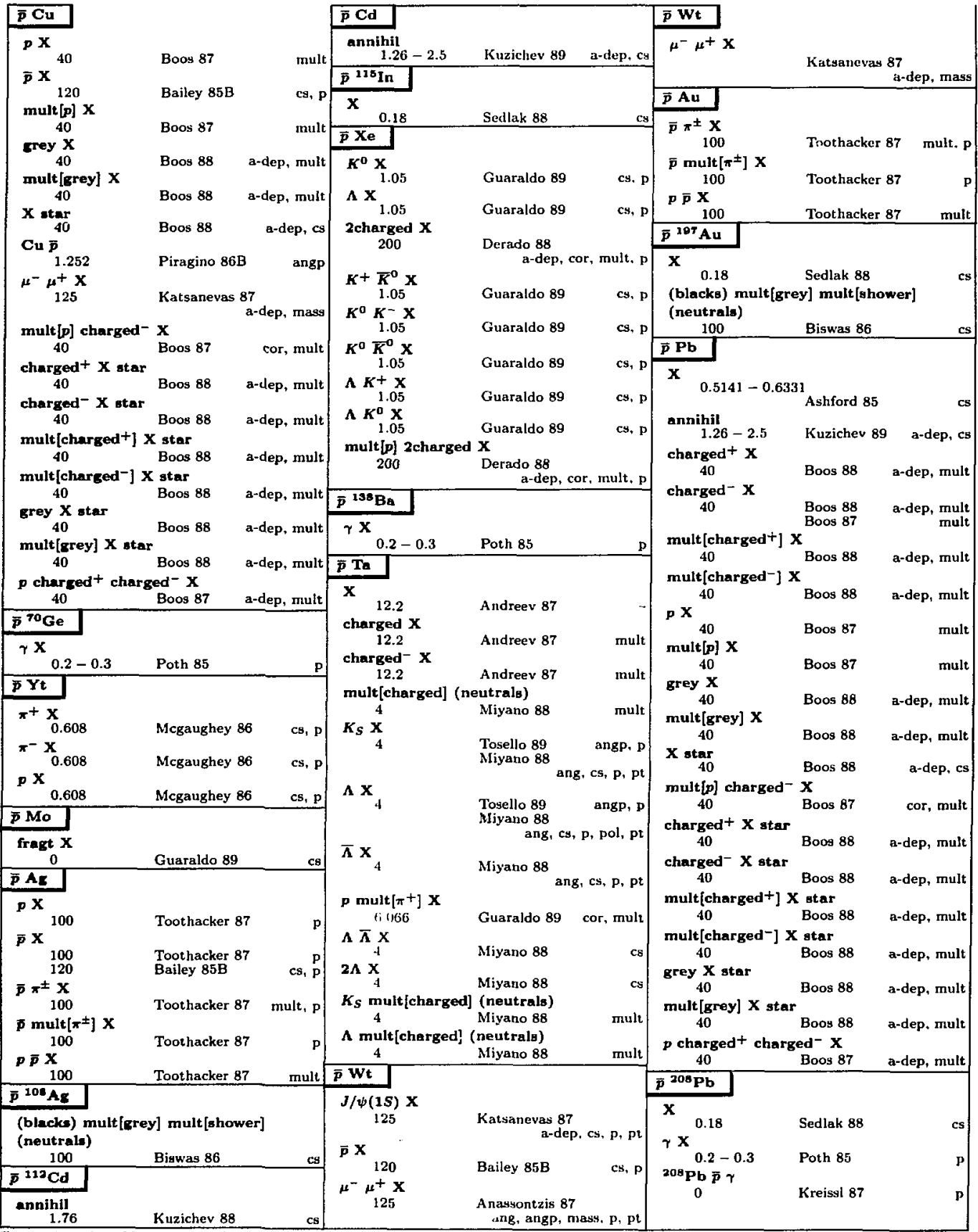

Entries in order of beam mass, then target mass, then multiplicity of fistal state. Inclusive reactions have an "X" as the last of the final state particles. Certain chemical symbols for nuclei have been changed to avoid ambiguity with particle names (See the Particle Vocabulary.) Beam momenta are $\mathrm{P}_{1 \mathrm{sb}}$ in $\mathrm{GeV} / c$, or in parentheses $E_{\mathrm{cm}}$ in $\mathrm{GeV}$. See the legend on page 153 . 


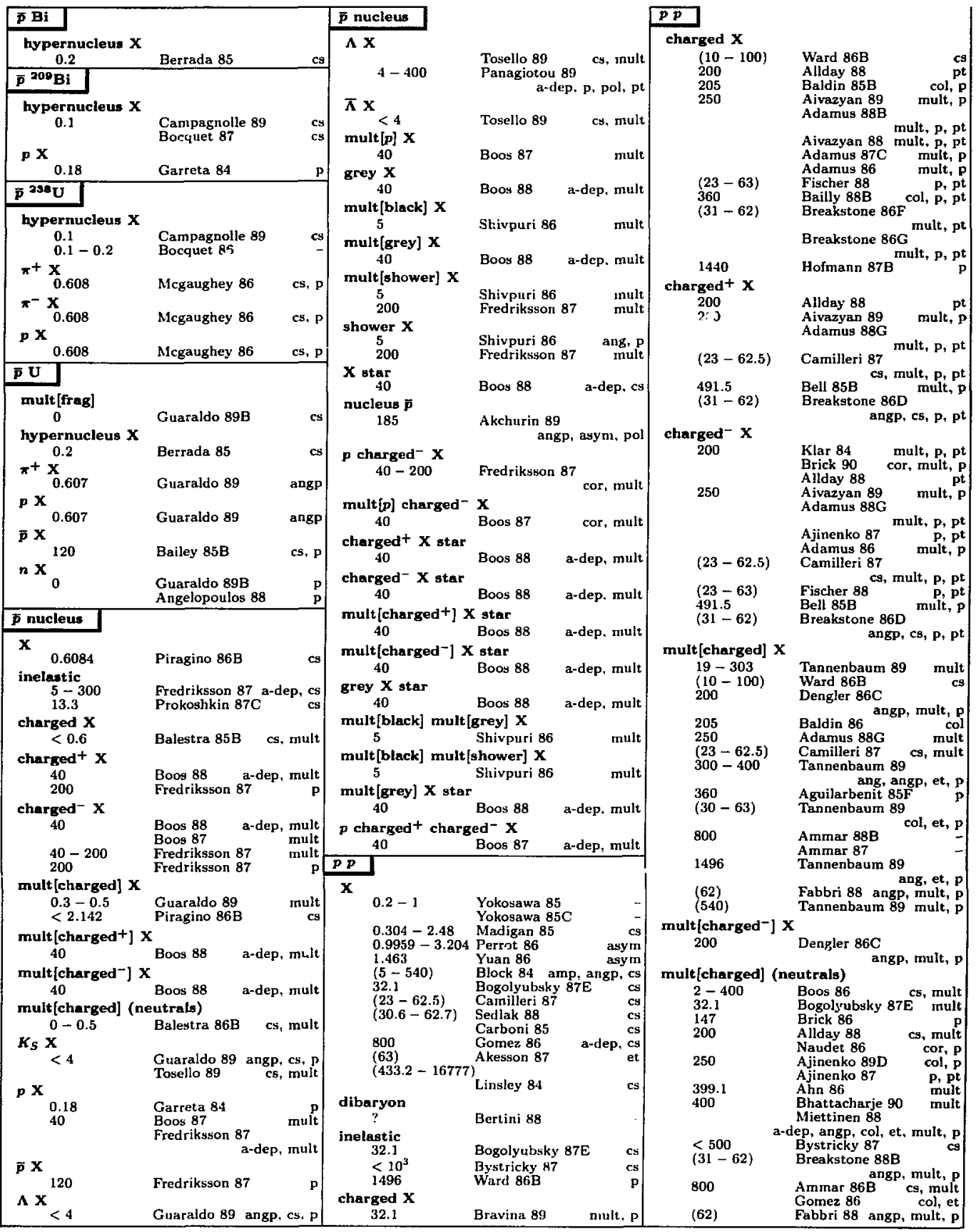




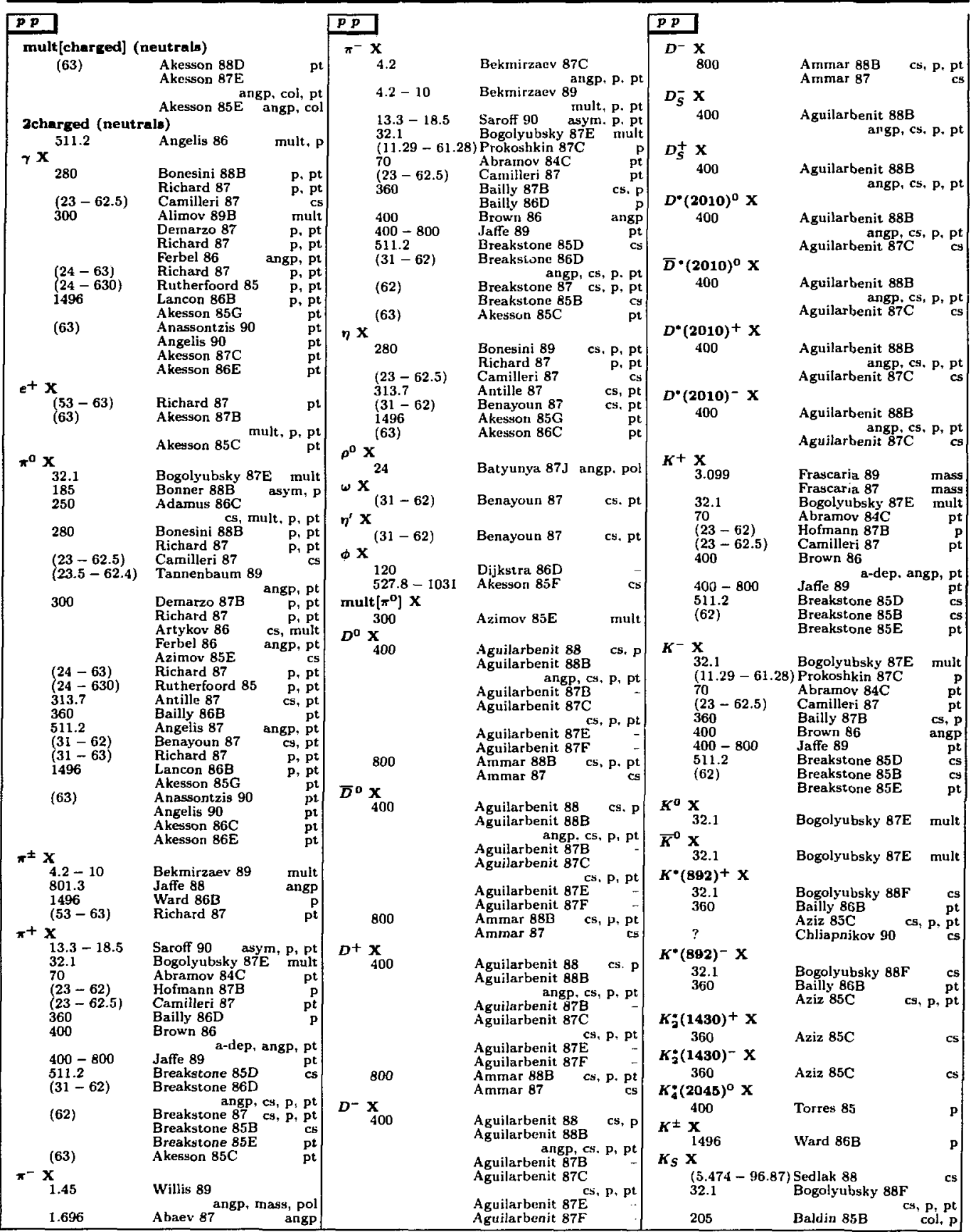




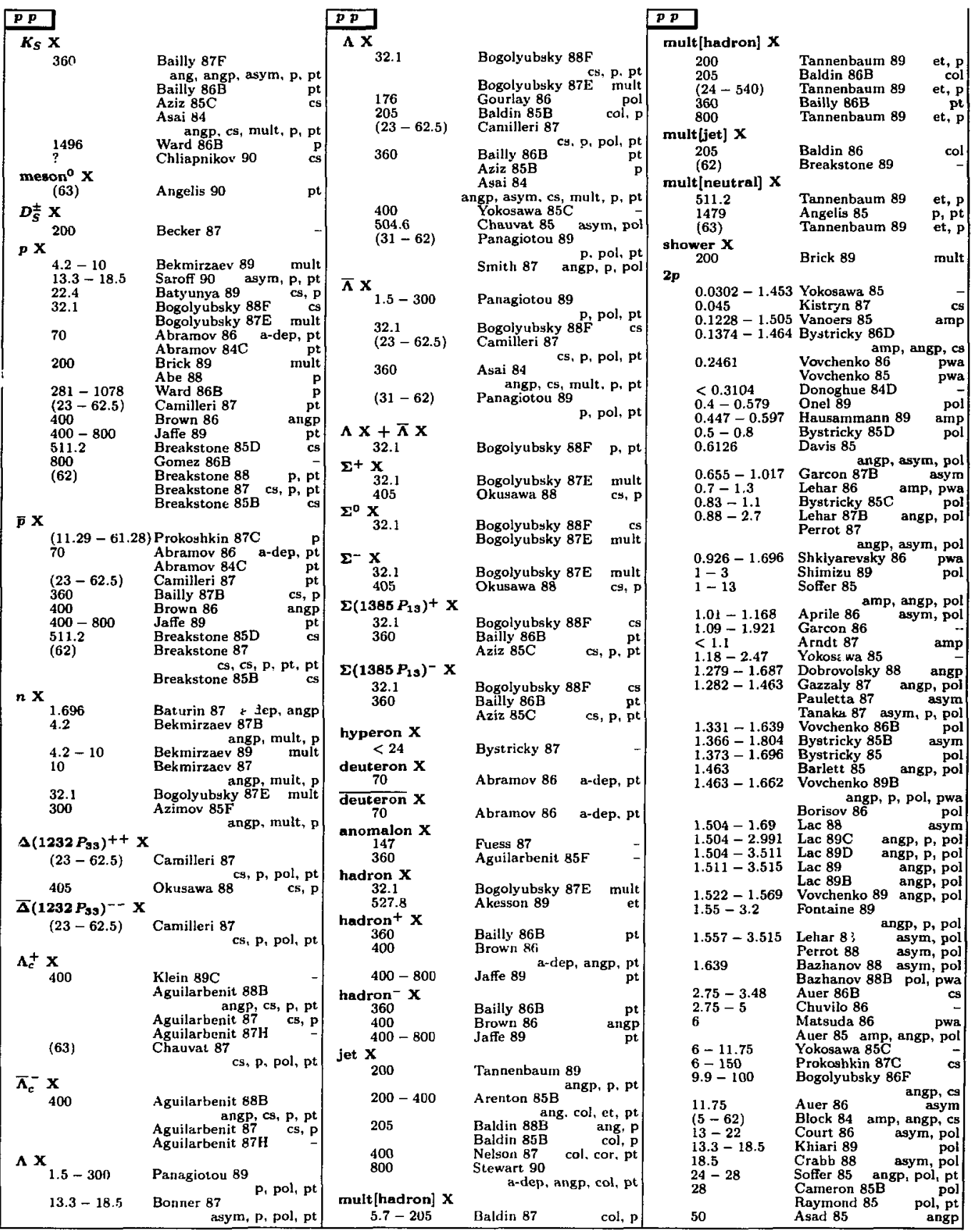


\begin{tabular}{|c|}
\hline $\mathbf{P}$ \\
\hline $\mathbf{3 p}$
\end{tabular}

\begin{tabular}{|c|c|}
\hline $\begin{array}{l}150-300 \\
185\end{array}$ & $\begin{array}{l}\text { Soffer } 85 \\
\text { Akchurin } 89\end{array}$ \\
\hline $\begin{array}{l}250 \\
(23-62.5)\end{array}$ & $\begin{array}{l}\text { Grassler B8 } \\
\text { Adamus } 87 \mathrm{D} \\
\text { Camilleri } 87\end{array}$ \\
\hline $\begin{array}{l}(23.5-62.5) \\
313.7 \\
360 \\
(31-62) \\
1496\end{array}$ & $\begin{array}{l}\quad \text { amp, ang } \\
\text { Amos } 85 \\
\text { Breedon } 89 \\
\text { Bailly 88C } \\
\text { Breakstone } 85 \mathrm{C} \\
\text { Breakstone } 85 \\
\text { Erhan } 85\end{array}$ \\
\hline
\end{tabular}

deuteron $\pi^{+}$

ngp, es

$0.7942-1.475$ Hiroshige $84 \mathrm{C}$

0.9303 - 1.463 Blankleider 84

$0.95-2.5 \quad$ Yokosawa 85

0.9543 - 1.023 Falk 83

$1.09-1.463$ Glass 85B

1.18 Yokosawa 85C

1.373 - 3.099 Bertini 88B

$1.696-3.099$ Yokosawa 85

$1.921-3.099$ Bertini 88

$1.921-3.204$ Yertini 85

$<2 \quad$ Bystricky 87

$2.07-12.3 \quad$ Chuvilo 86

n $\Delta\left(1232 P_{39}\right)^{++}$

$1.18-1.98 \quad$ Wicklusd 87

$3-12$

Wicklusd 87
angp, asym, cs, dme, pwa
Wicklund 85

\section{dibaryon $\pi^{-}$}

1.696 Abaev 87

$\mathrm{AN}\left(2130^{3} \mathrm{~S}_{1}\right)+K^{+}$

$3.099 \quad$ Frascaria 89

dibaryon( $(S=-1) K^{+}$

$3.099 \quad$ Frascaria 87 angp, cs

DD $<2 \pi^{+} \pi^{-}$(neutralg) $>p$

$360 \quad$ Asai 89C mass, p, pt

DD $<K_{S} X>P$ $360 \quad$ Asai 84

DD $\left\langle A K^{+} \phi>p\right.$

(63) Smith 85D

DD $\langle\wedge \mathbf{X}>\boldsymbol{p}$ $360 \quad$ Asai 84

DD $<\boldsymbol{A} x>p$ $360 \quad$ Asai 84

DD $<n 2 \pi^{+} \pi^{-}>p$

$360 \quad$ Asai 89C mass, p, pt

$\mathrm{DD}<\mathrm{p2} 2 \pi^{+} \mathbf{2 \pi ^ { - }}>\mathrm{p}$

(62) Smith 85B ang, mass, $p$

$D D<p \Lambda \bar{\Lambda}>p$

(63) Smith 85D

DD $<p \pi^{+} 2 \pi^{0} \pi^{-}>p$ 360 Asai $89 \mathrm{C}$

ang, mass, $p, p t$

DD $<p \pi^{+} \pi^{-}>p$

360 Asai $89 \mathrm{C}$

DD $<p \pi^{+} \pi^{0} \pi^{-}>p$

ang. mass, $p, p t$

$360 \quad$ Asai $89 \mathrm{C}$

$\mathrm{DD}<\mathrm{X}>\boldsymbol{p}$

ang, mass, $p, p t$

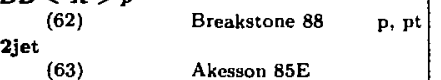

(63) Akesson 85E

charged $^{+}$charged $-\mathbf{X}$ ang, mass, $p, p t$ angp, $P$ $\boldsymbol{p p}$

2charged $X$

200

$(30.4-62.2)$

$(53-63)$

(62)

2charged (neutrals) inelastic

$<500$

$\gamma$ charged $\mathbf{X}$

(63)

$\gamma$ charged $+\mathbf{X}$

(63)

$\gamma$ charged $-x$

(63)

$2 \gamma \mathbf{X}$

300

(63)

$e^{-} e^{+} \mathbf{x}$

(63)

$\pi^{\circ}$ charged X

(62.4)

$\pi^{+}$charged $X$

$527.8-1031$

(62)

$\pi^{+}$charged $+\mathbf{X}$

(62)

$\pi+$ charged $^{-} \mathrm{x}$

(62)

$\pi$ - charged $\mathrm{X}$

$527.8-1031$

$\mu^{-} \mu^{+} \mathbf{X}$

$(19-63)$

$2 \pi+\underset{70}{225}$

360

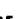

400
1496
$(62)$
$-\mathbf{X}$
70
360

360

1496

(62)

$\pi^{+} \pi^{-} \mathrm{X}$

24

70

$(23-62.5)$

360

(62)

mult [ $\left.\pi^{0}\right]$ mult $\left[\pi^{-}\right]$

$$
D^{0} \stackrel{300}{0}_{400} \mathrm{X}
$$

$$
D^{+} \bar{D}_{400}^{0} \times
$$

$D^{0} D^{-} \mathbf{x}$

400

\section{Bystricky 87}

Angelis 90

A kesson 86E

Angelis 90

Angelis 90

Demarzo 87B

Akesson 86B

Richard 87

Tannenbaum 89

ang. cor, $p$

Akesson $85 \mathrm{~F}$ mult

Breakstone 86E angp, $p$

Breakstone 86E angp, $p$

Breakstone $86 \mathrm{E}$ angp, $\mathrm{p}$

Akesson $85 \mathrm{~F}$

mult

Rutherfoord 85

Rutherfoord 85

Abramov 84

Bailly 88E

ang, angp, asym, p, pt

Bailly $86 \mathrm{D}$

B rown 86

A kesson 86F

mass

Smith 86B

cor, pt

A bratnov 84

Bailly 88E

Bailly $87 \mathrm{~F}$

ang,

Bailly $86 \mathrm{D}$

Akesson 86F

Smith $86 B$

p. pt

p. pt

p. pt

Batyunya 87J

Abramov 84 angp, mass

Camilleri 87

Bailly $87 \mathrm{~F}$

ang, angp, asym, $p, p t$

Smith 86B

cor. pt

p, pt

$\mathbf{x}$

Aximov 85E mult

Aguilarbenit 88B cor, cs, mass, p, pt

Aguilarbenit 87C

Aguilarbenit 88B cor, cs, inass. p. pt

Aguilarbenit 88B

cor, cs, mass, p, pt

p p

$D^{+} \bar{D}^{\mathbf{0}} \mathbf{X}+D^{\circ} D^{-} \mathbf{x}$

$D^{+} D^{-} \mathbf{X}$

Aguilarbenit $87 \mathrm{C}$

Aguilarbenit 88B

cor, cs, mass, p, pt

Aguilarbenit 87C

$K^{+} \boldsymbol{\gamma} \mathbf{X}$

(63)

Akesson 86E

p, pt

$\boldsymbol{K}^{-} \boldsymbol{\gamma} \mathbf{X}$

(63)

A kesson $86 \mathrm{E}$

p, pt

$\boldsymbol{K}^{+}$charged $\mathbf{X}$

527.8- 1031 Akesson 85F

mult

$K^{-}$charged $\mathbf{X}$

527.8 - 1031 Akesson 85F

$K^{+} \pi^{\circ} \mathrm{X}$

(63)

$K^{-} \pi^{0} \mathbf{X}$

(63)

$K^{+} \pi_{70}^{+} \mathbf{X}$

(62)

$K+\pi^{-} \mathbf{x}$

(62)

$K^{-} \pi^{+} \mathrm{X}$

(62)

$K^{-} \pi^{-} \mathbf{X}$

(62)

$K^{*}(\mathbf{8 8 2})^{\circ} \phi X$

400

$\boldsymbol{K}^{+} \boldsymbol{K}^{-} \mathbf{X}$

70

$527.8-1031$

(62)

mult

Akesson $86 \mathrm{E}$

p, pt

A kesson 86E

p, pt

Abramov 84

Smith 86B

p, pt

$p, p t$

Abramov 84

Smith 86B

$\mathrm{p}, \mathrm{pt}$

Abramov 84

Smith 86B

Abramov 84

Smith 86B

Torres 85

Abramov 84

A kesson 85F

Smith 86B

$2 K+\mathbf{x}$

70

527

(63)

Abramov 84

Brown 86

Akesson 85F

Smith 86B

$2 K-x$

527.8 - $1031 \quad$ Akesson 85F

(62)

Smith 86E

(63)

Akesson 85B

$K_{S} \pi^{+} \mathbf{X}$

360

$K_{S} \pi^{-} \mathbf{X}$

360

Aziz 88

p, pt

$P, \mathrm{pt}$

p, pt

p, pt

p, pt

mass

p, pt

p, pt

p, pt

mass

p. pt

cor

cs
p. pt
cor

meson ${ }^{\circ}$ charged $X$

(63) Angelis 90

meson $^{0}$ charged $^{+} \mathbf{X}$

(63) Angelis 90

meson $^{\circ}$ charged $^{-} X$

(63)

Angelis 90

$\boldsymbol{K} \overline{\boldsymbol{K}} \mathbf{X}$

32.1

$\boldsymbol{K}^{ \pm} \boldsymbol{K}_{\boldsymbol{S}} \mathbf{X}$

360

Bogolyubsky 88 F

Bailly $87 \mathrm{~F}$

$2 K_{S} X$

32.1

360

ang, angp, asym, p, pt

Bogolyubsky 88F

Aziz 88

ang, cs, mass, $p, p t$ 


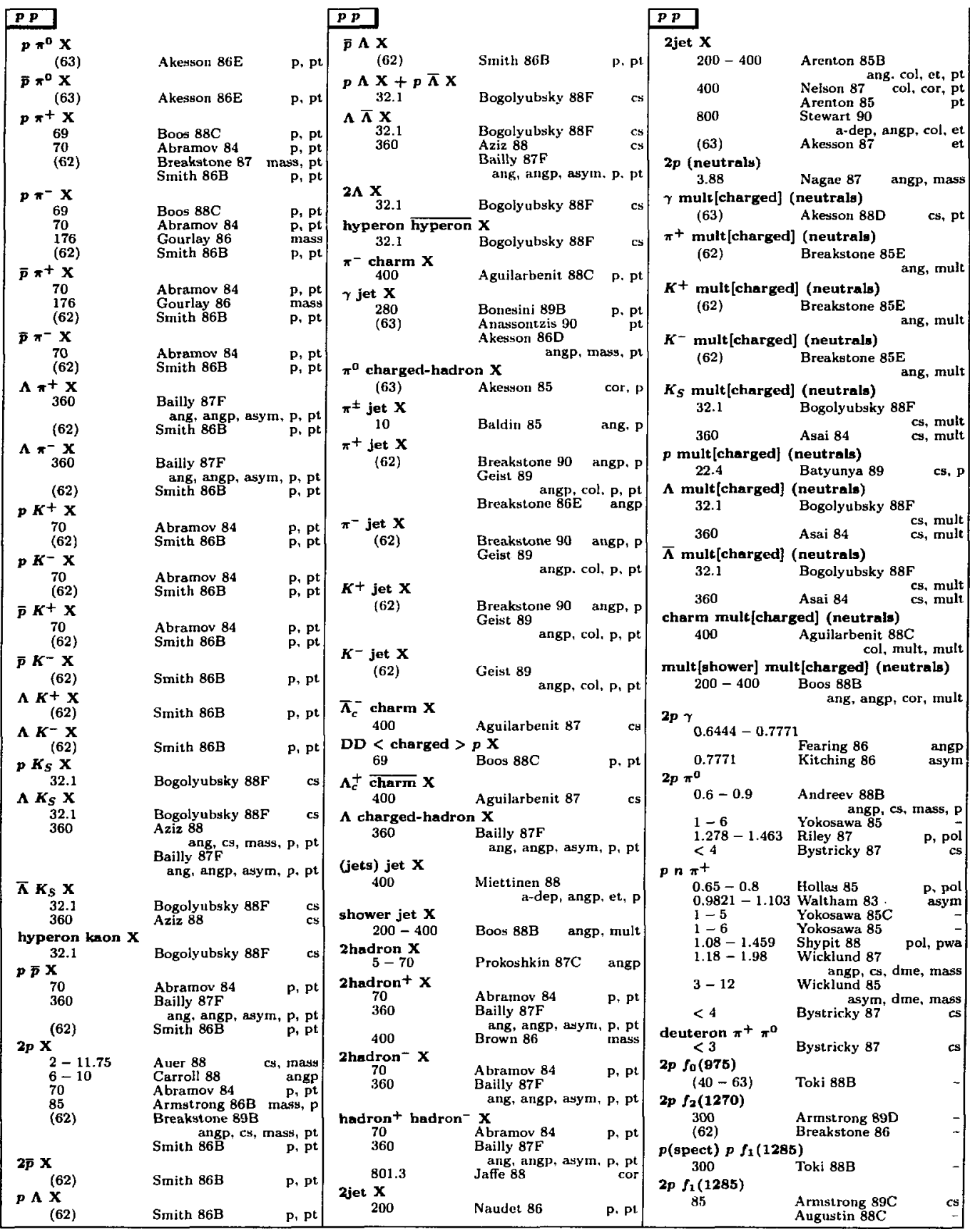




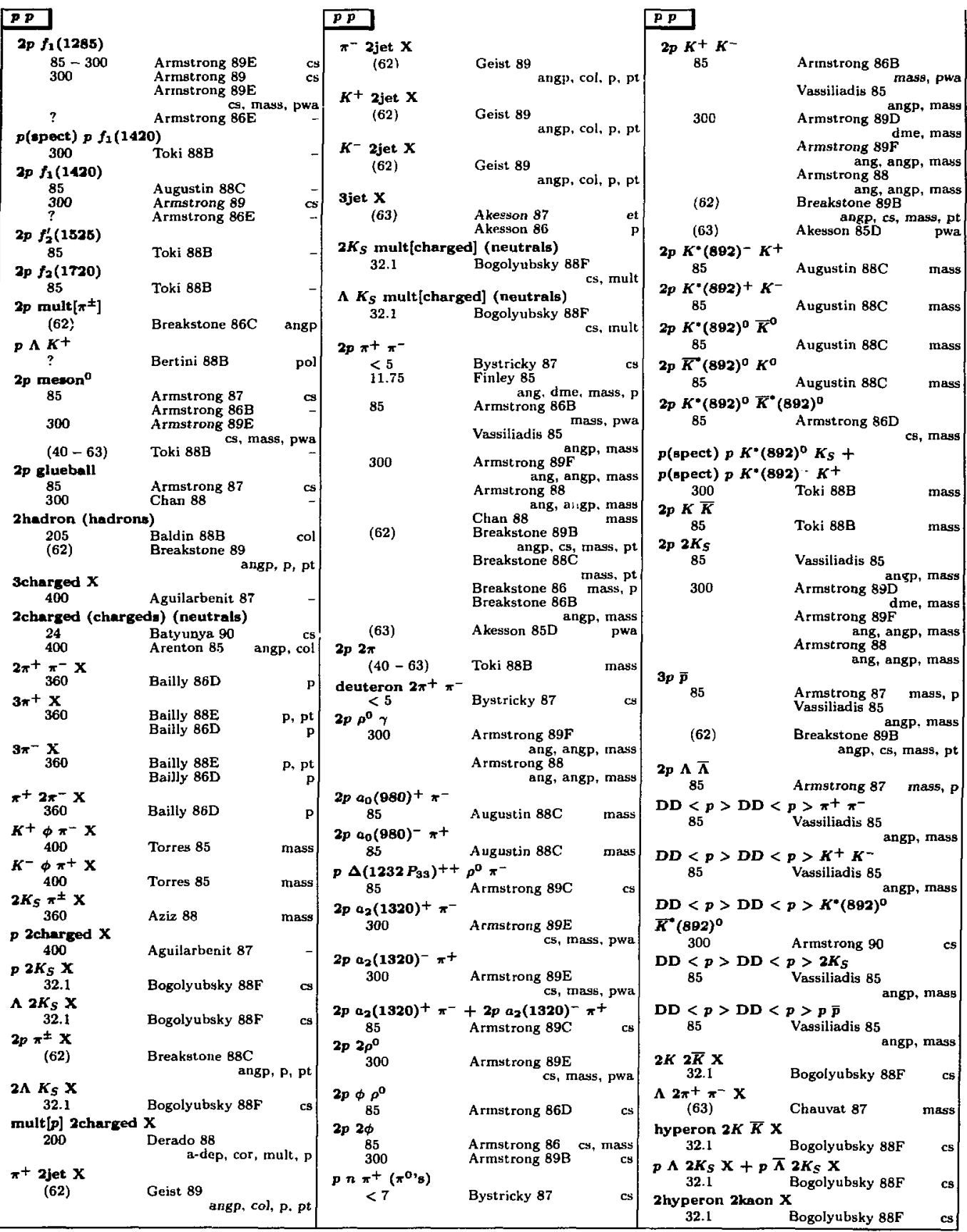




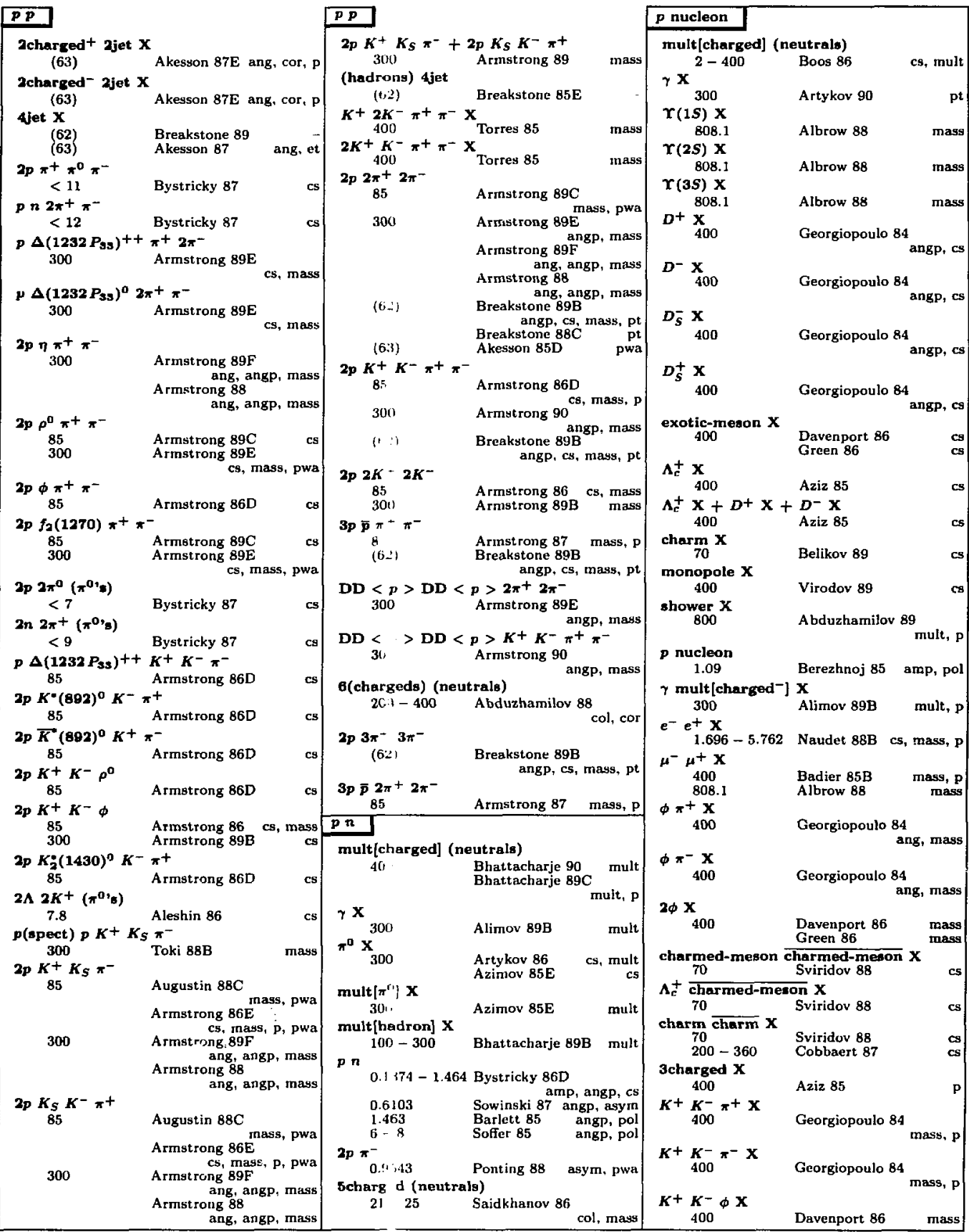




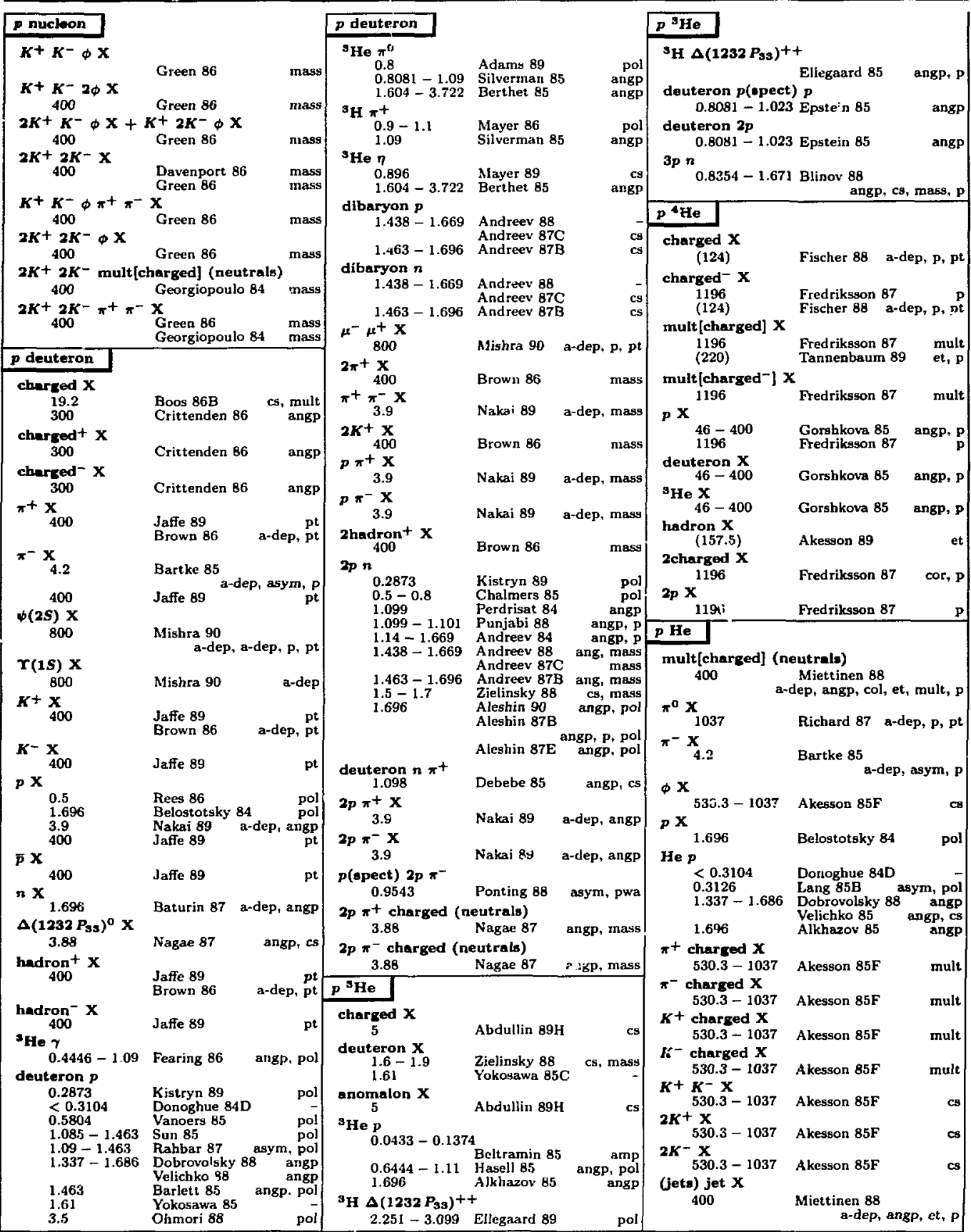

Entries in order of beam mass, then target mass. then multıplicity of final state. Inclusive reactions have an "X" as the last of the final state particles. Certain cliemical symbols for tuclei have been changed to avoid ambiguity with particle names (See the Particle Vocabulary.) Beam momenta are $P_{\text {lab }}$ in GeV/c, or in parentheses $E_{c+n}$ in GeV. See the legend on page 153. 


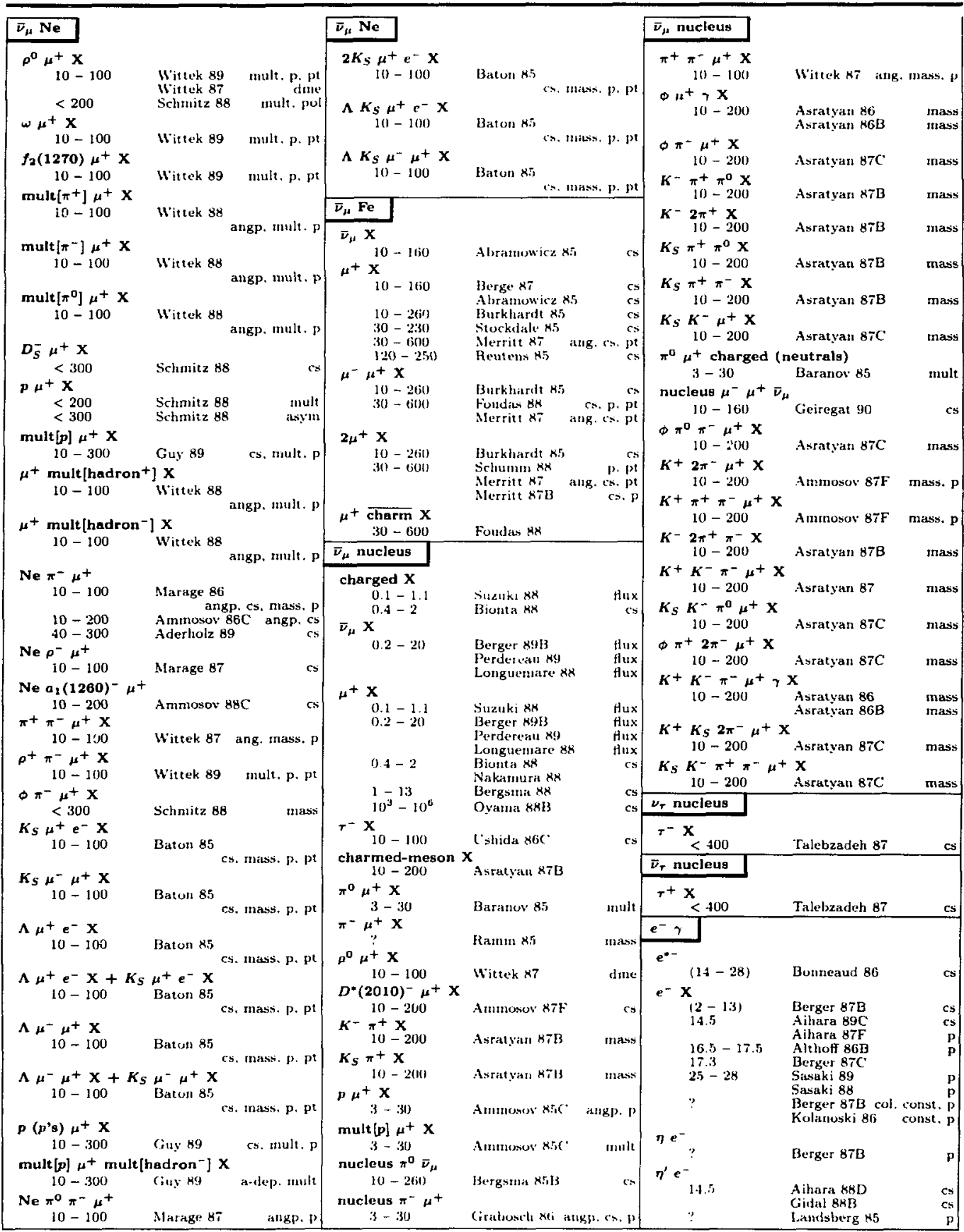




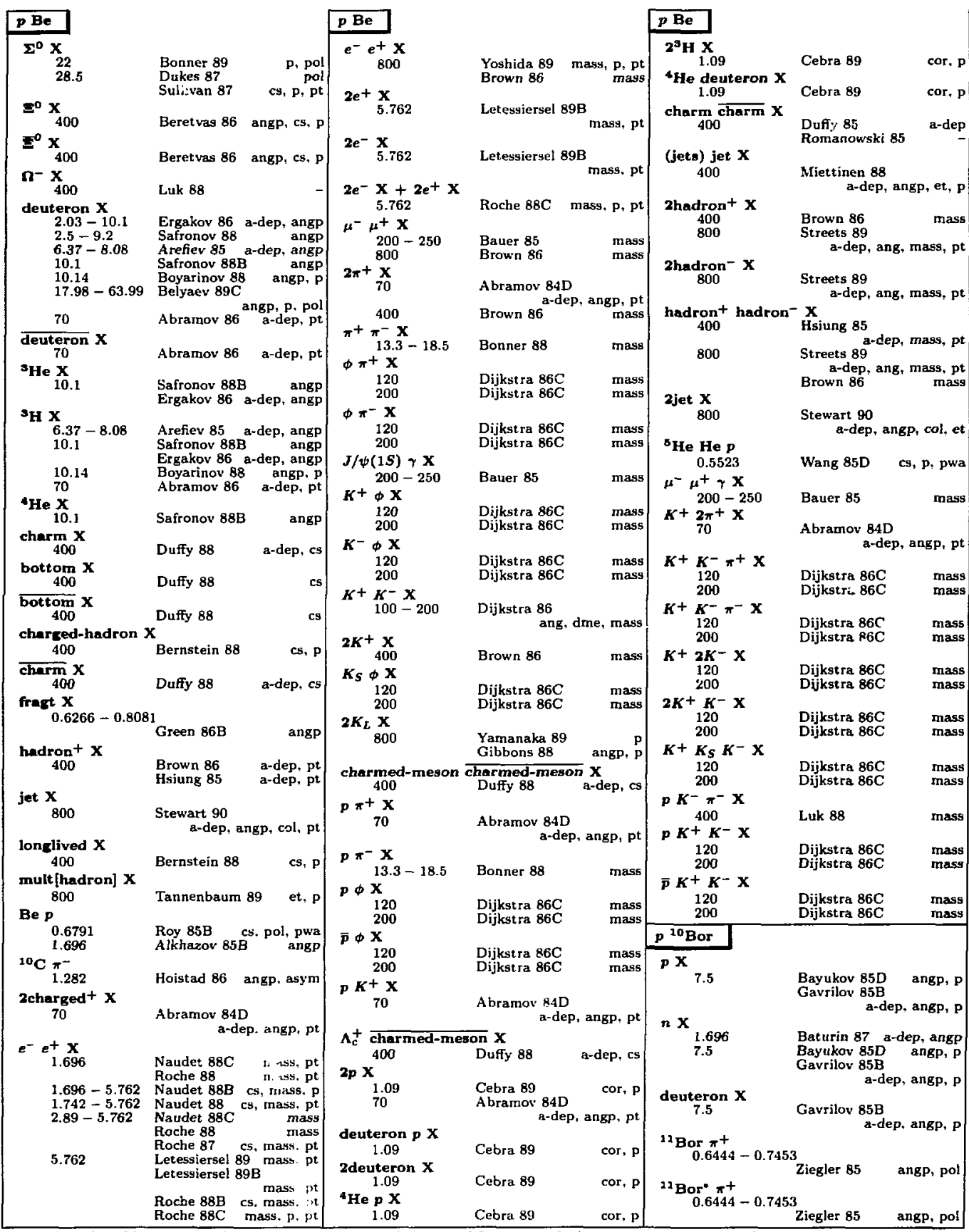




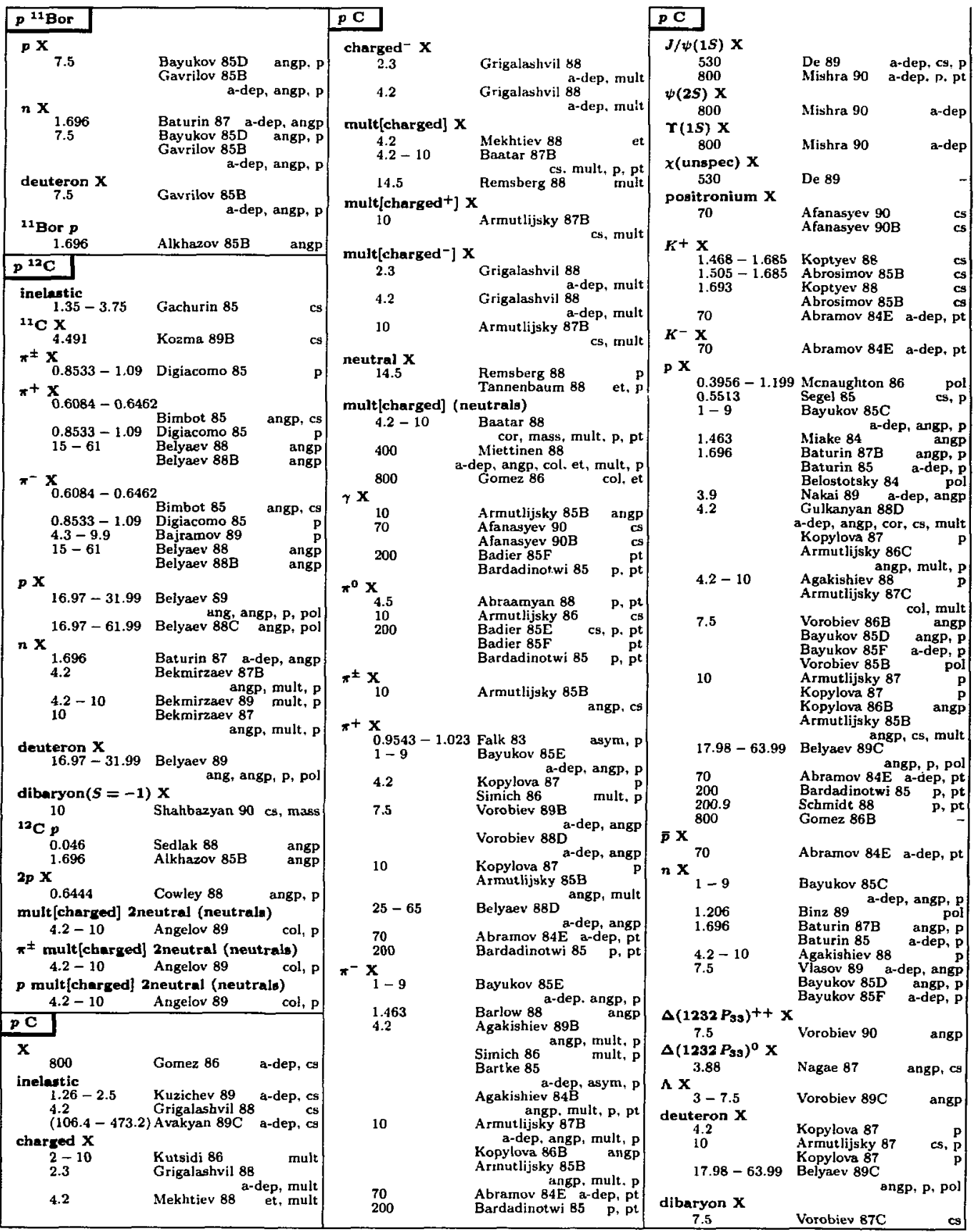




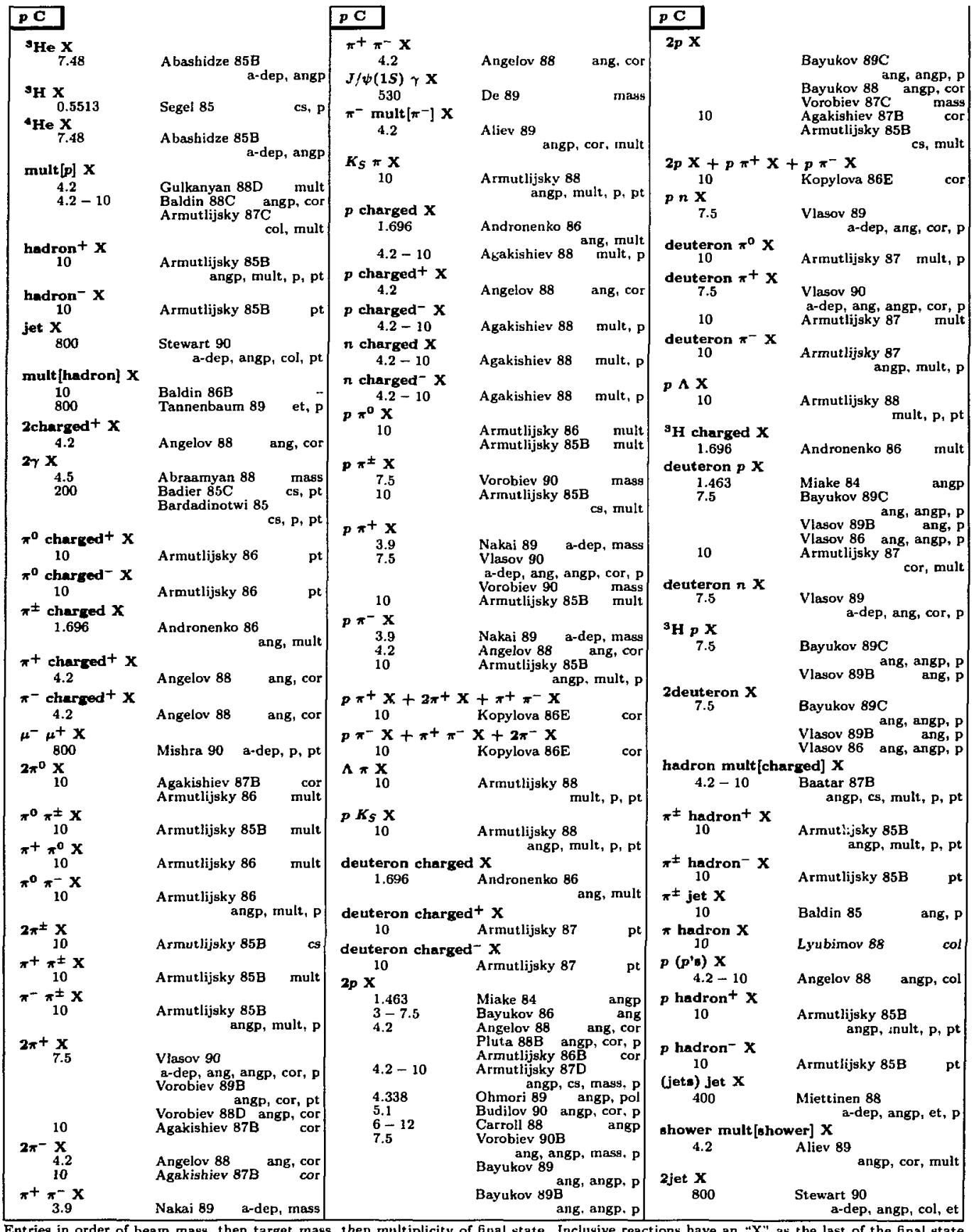




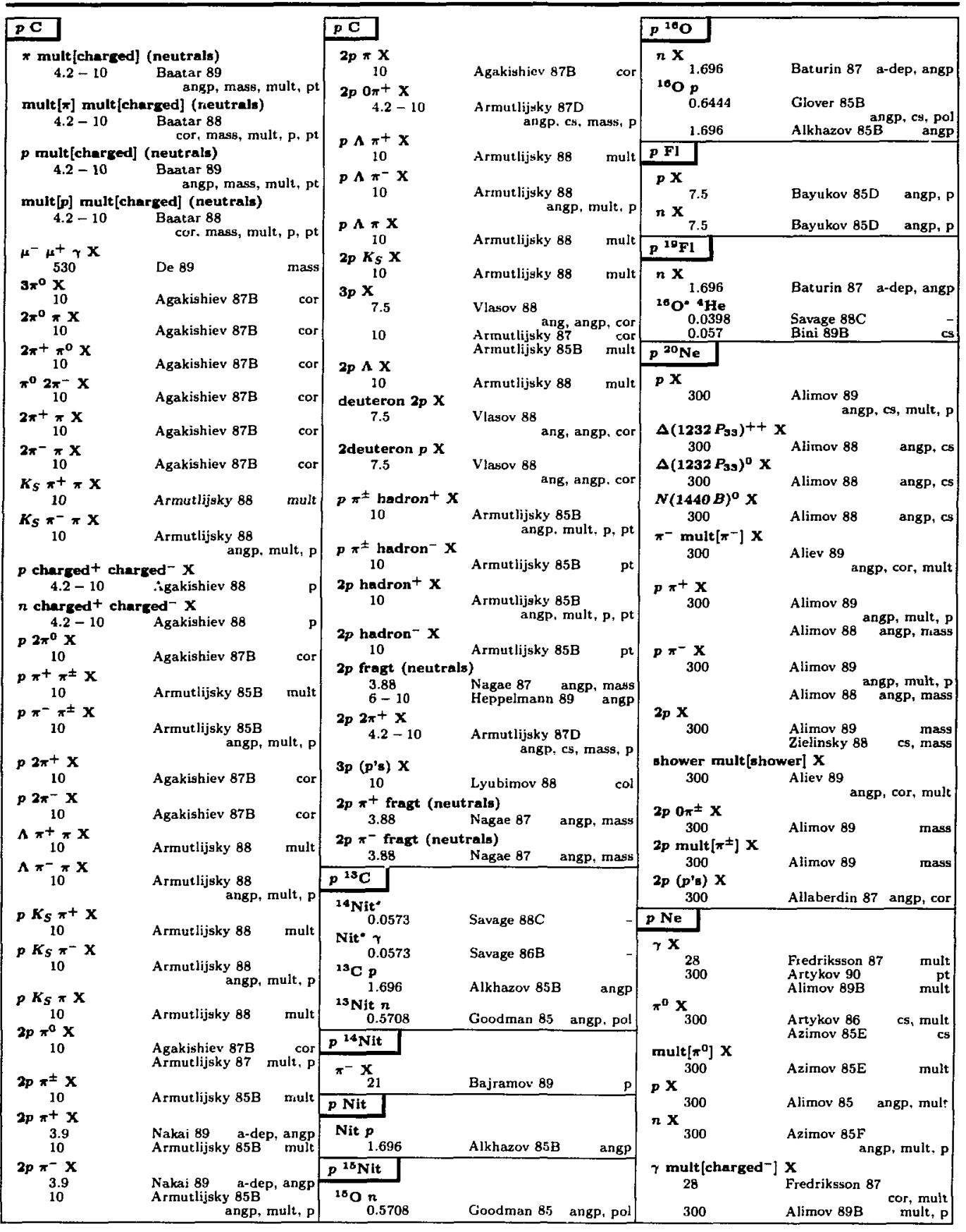




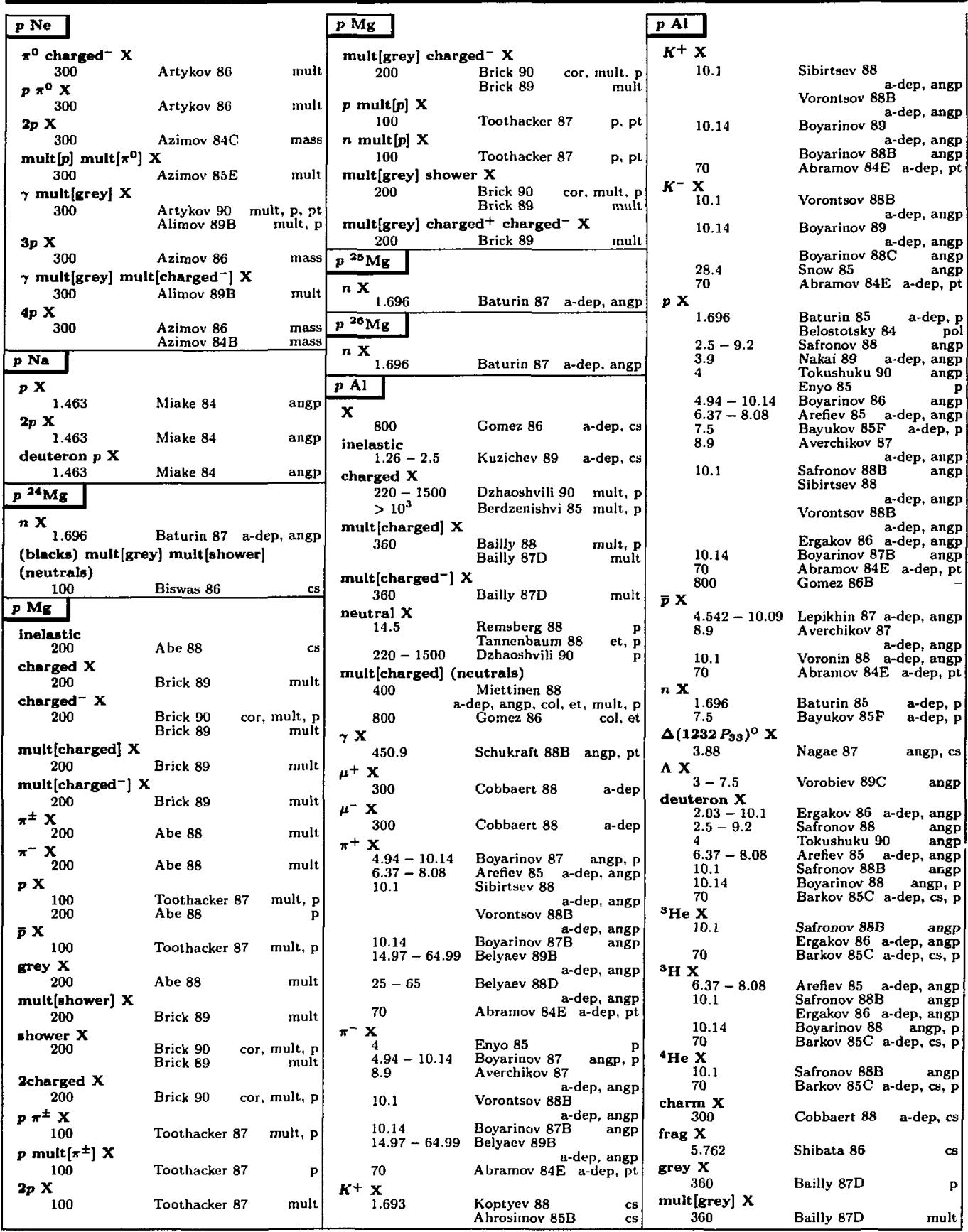




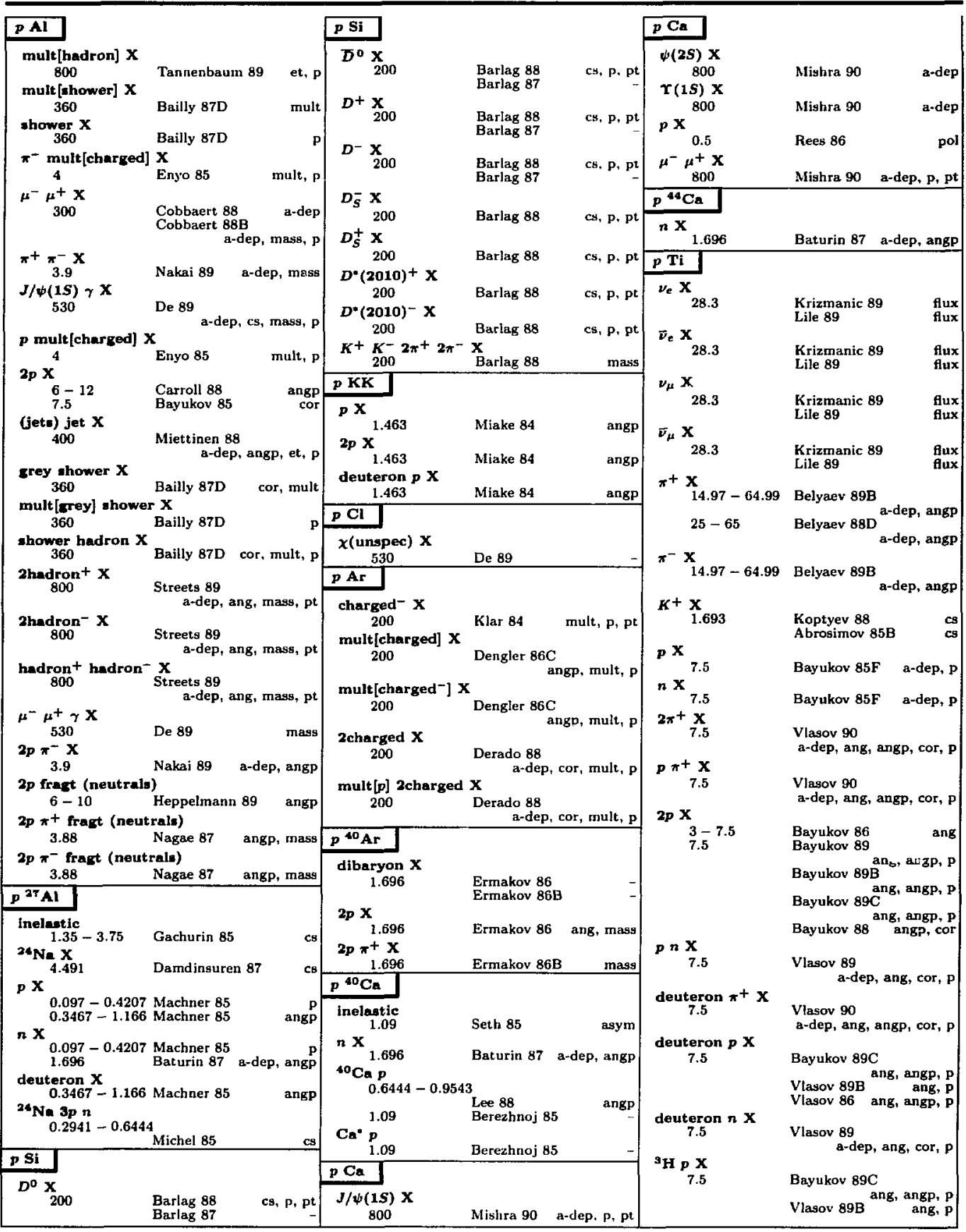




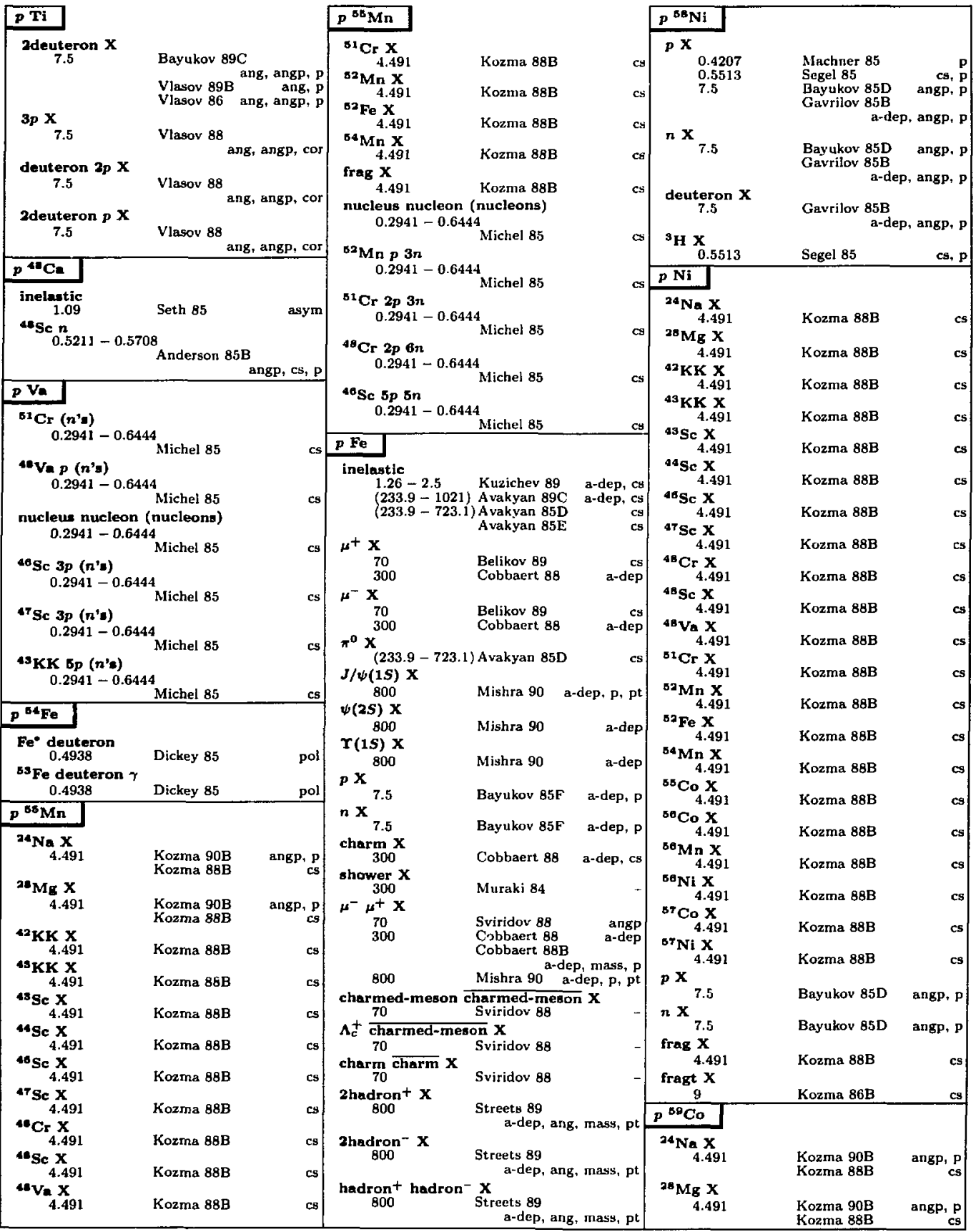




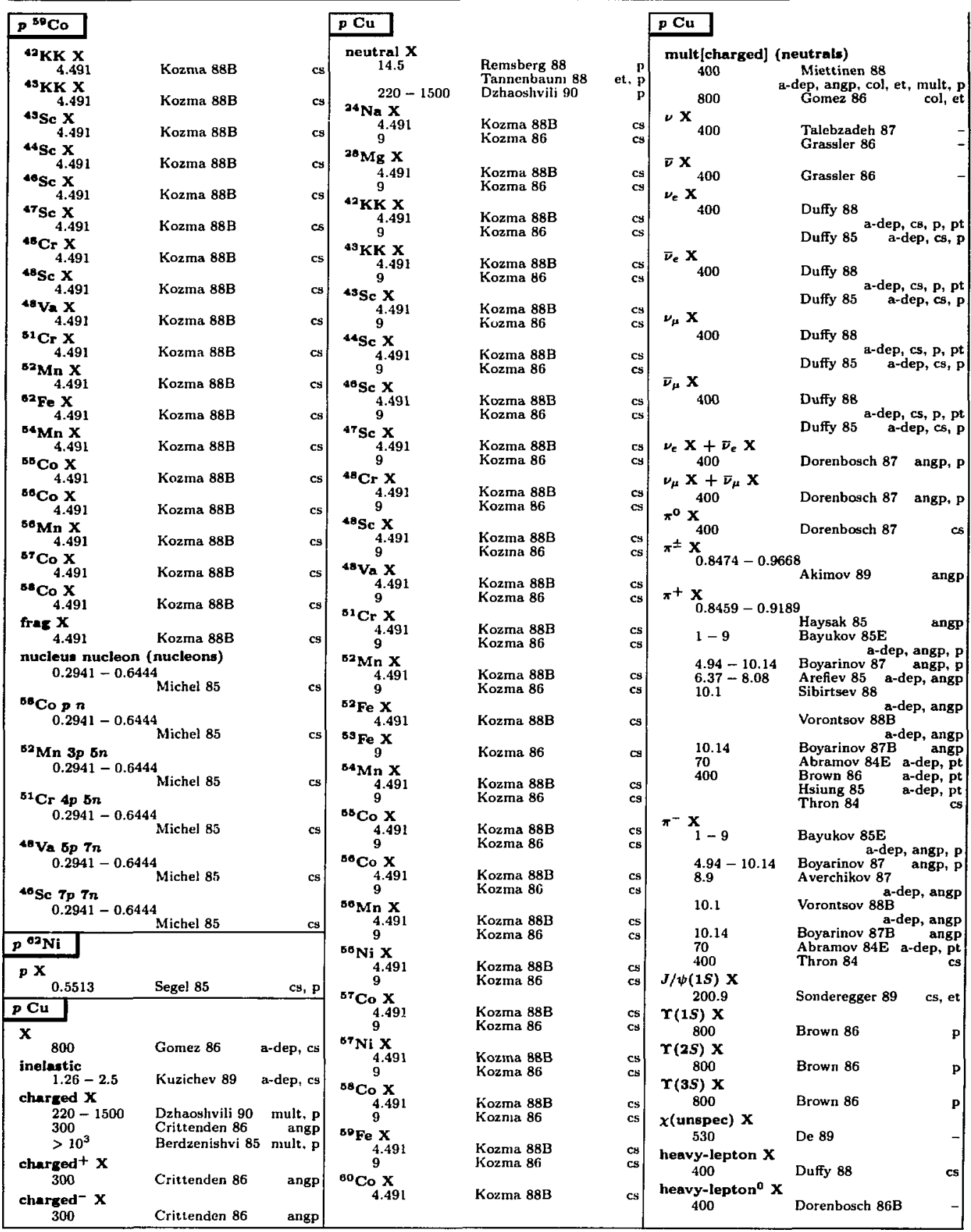




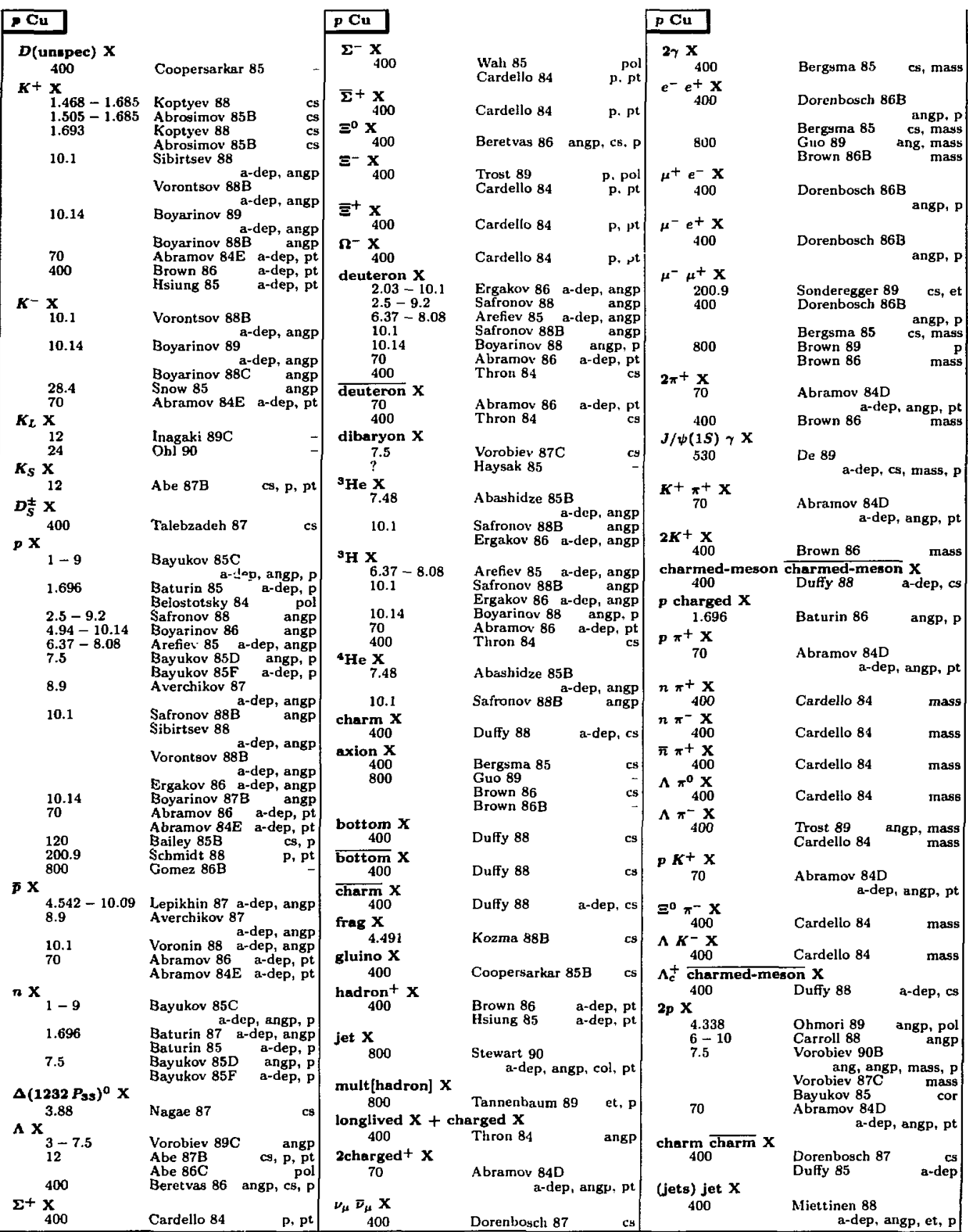




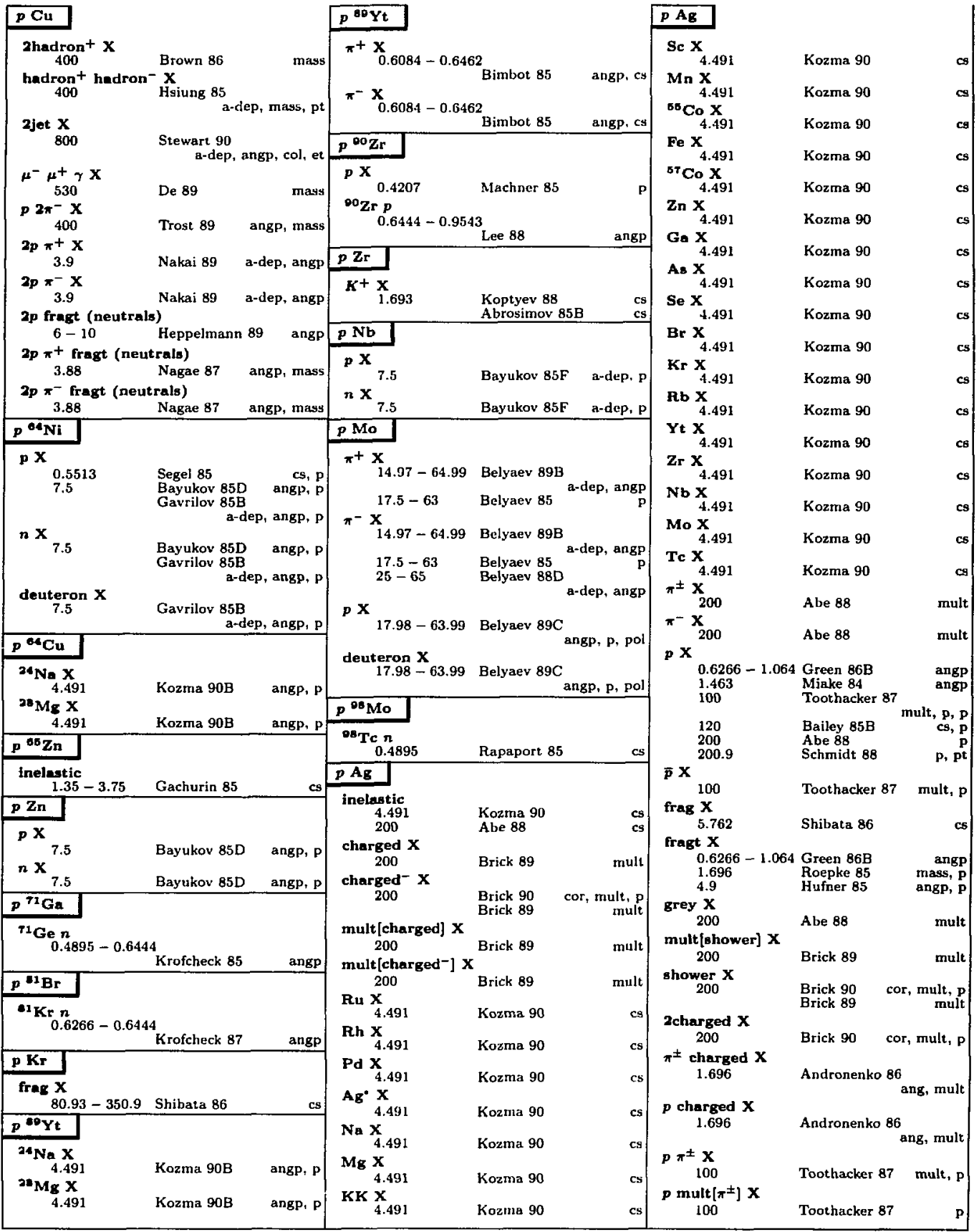




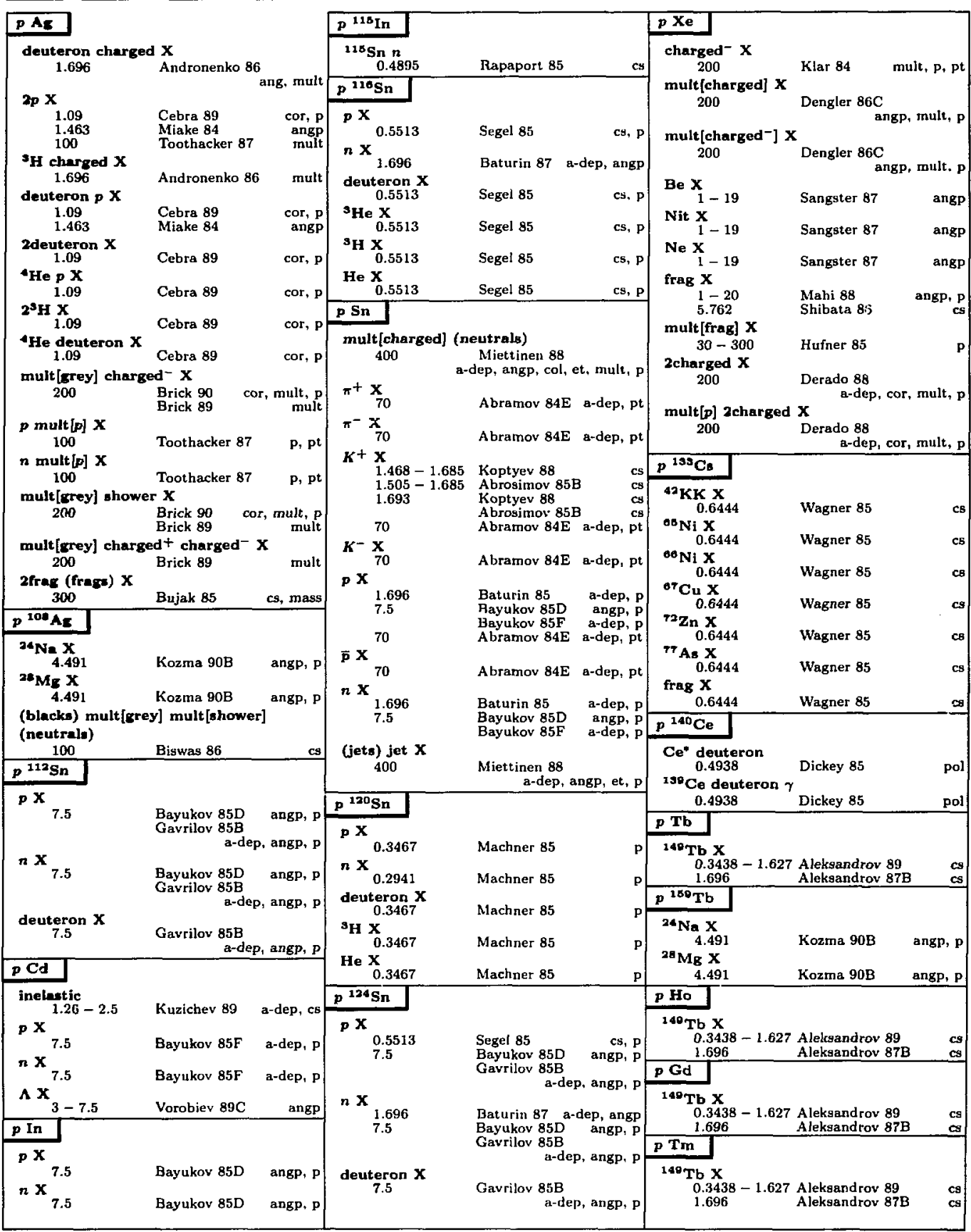

Entries in order of beam mass, then target mass, then multiplicity of final state. Inclusive reactions have an "X" as the last of the final state particles. Certain chemical symbols for nuclei have been changed to avoid ambiguity with particle names (See the Particle Vocabulary.) Beam momenta are Plab in $\mathrm{GeV} / \mathrm{c}$, or in parentheses $E_{\mathrm{cm}}$ in $\mathrm{GeV}$. See the legend on page 15.3. 


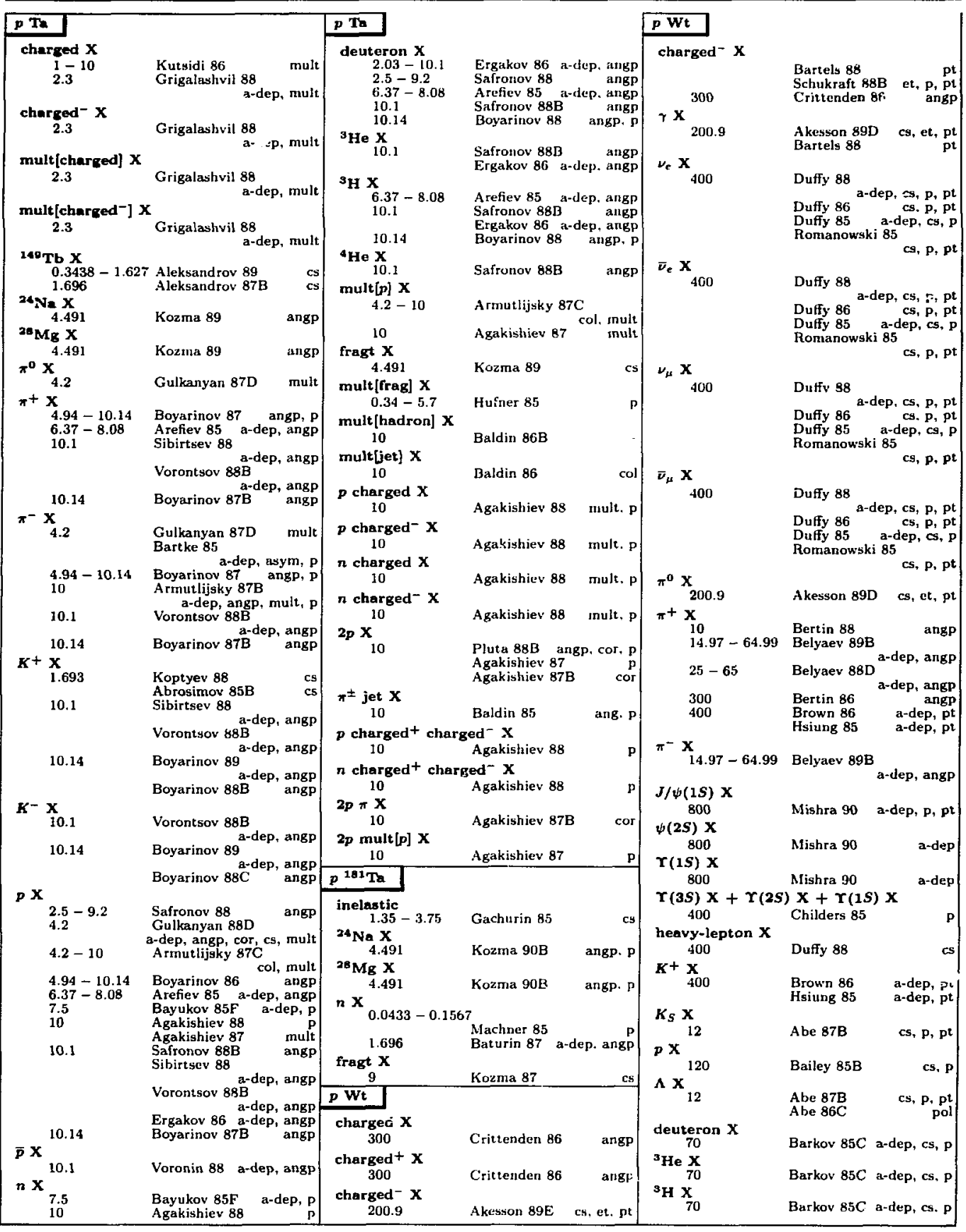




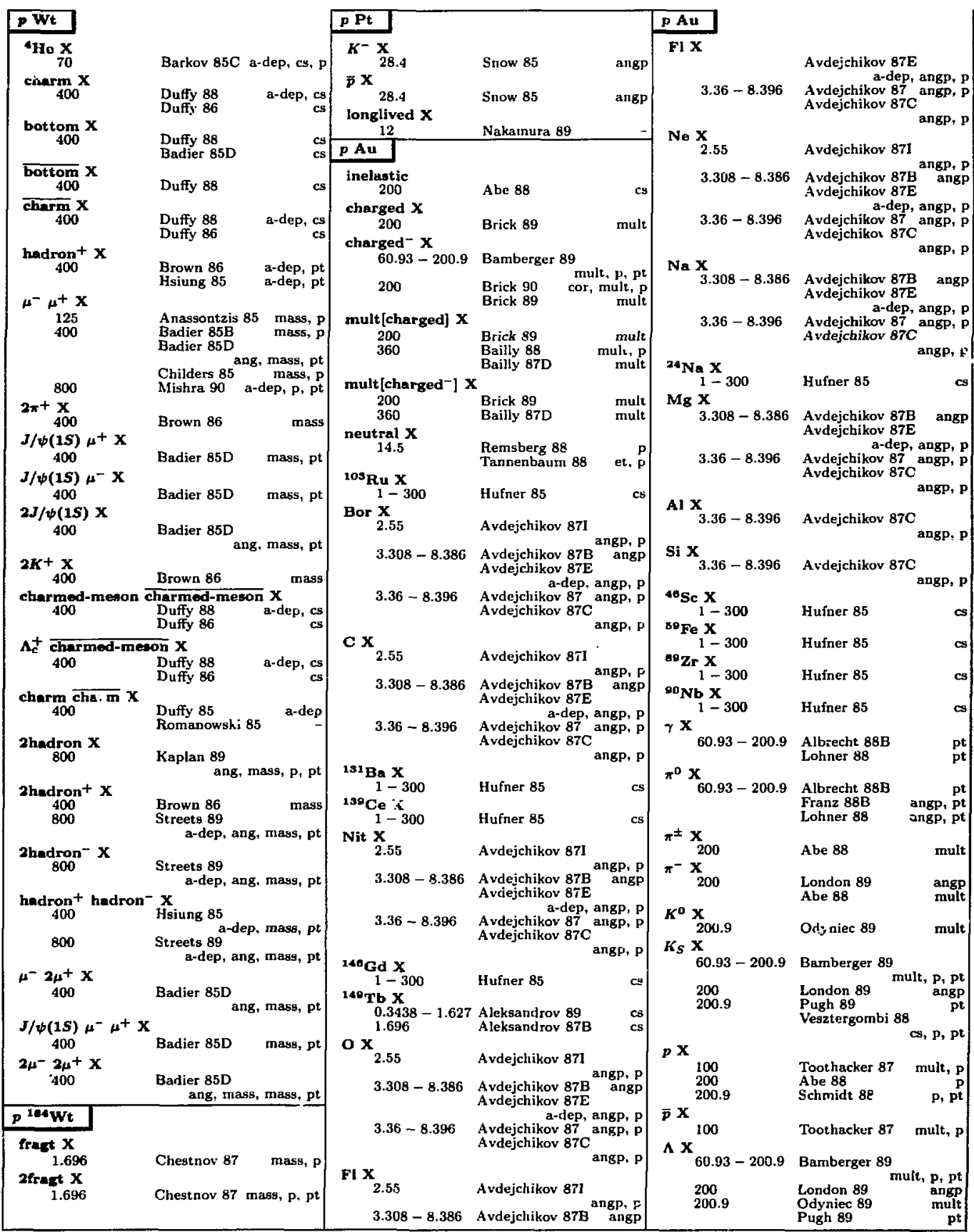




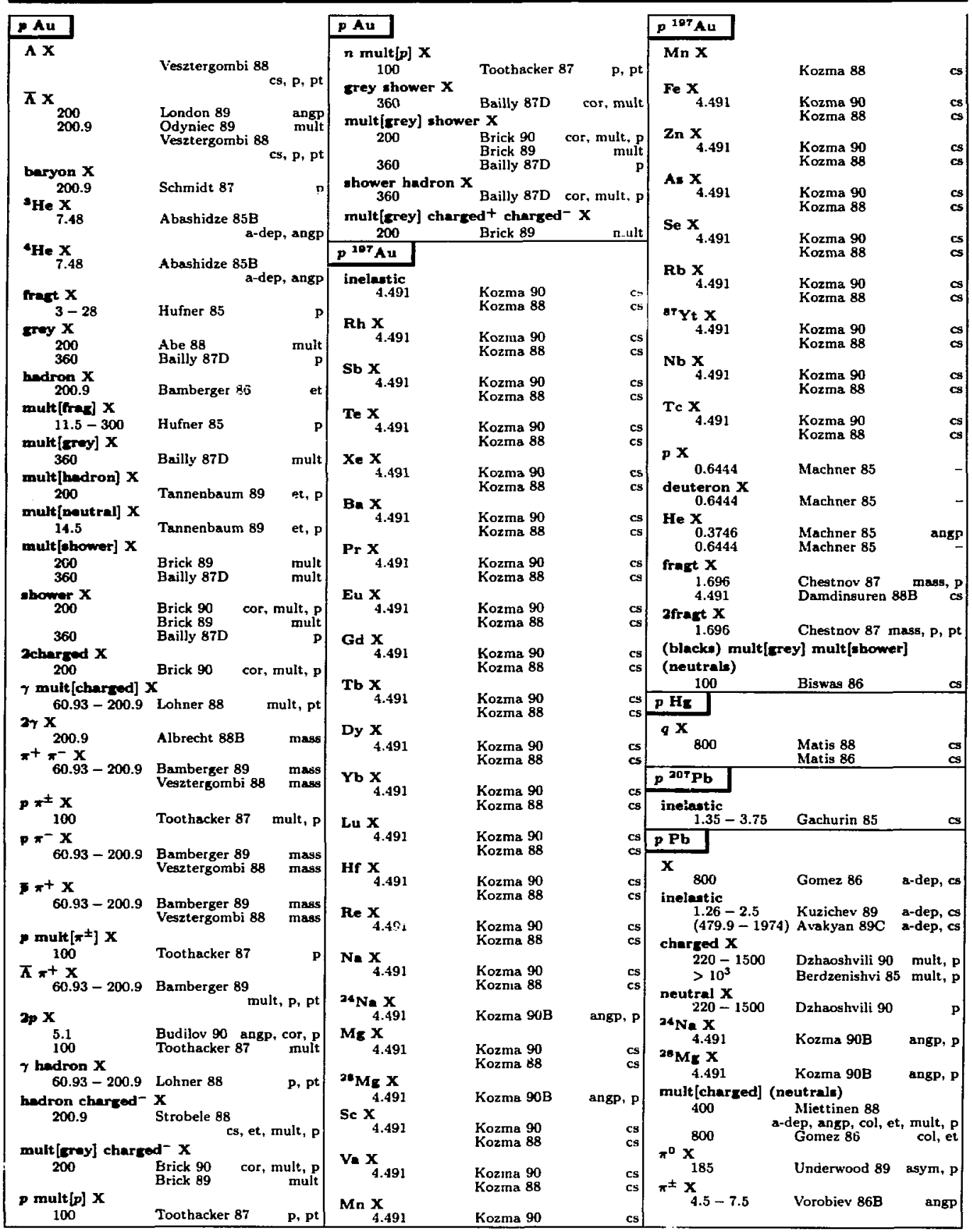




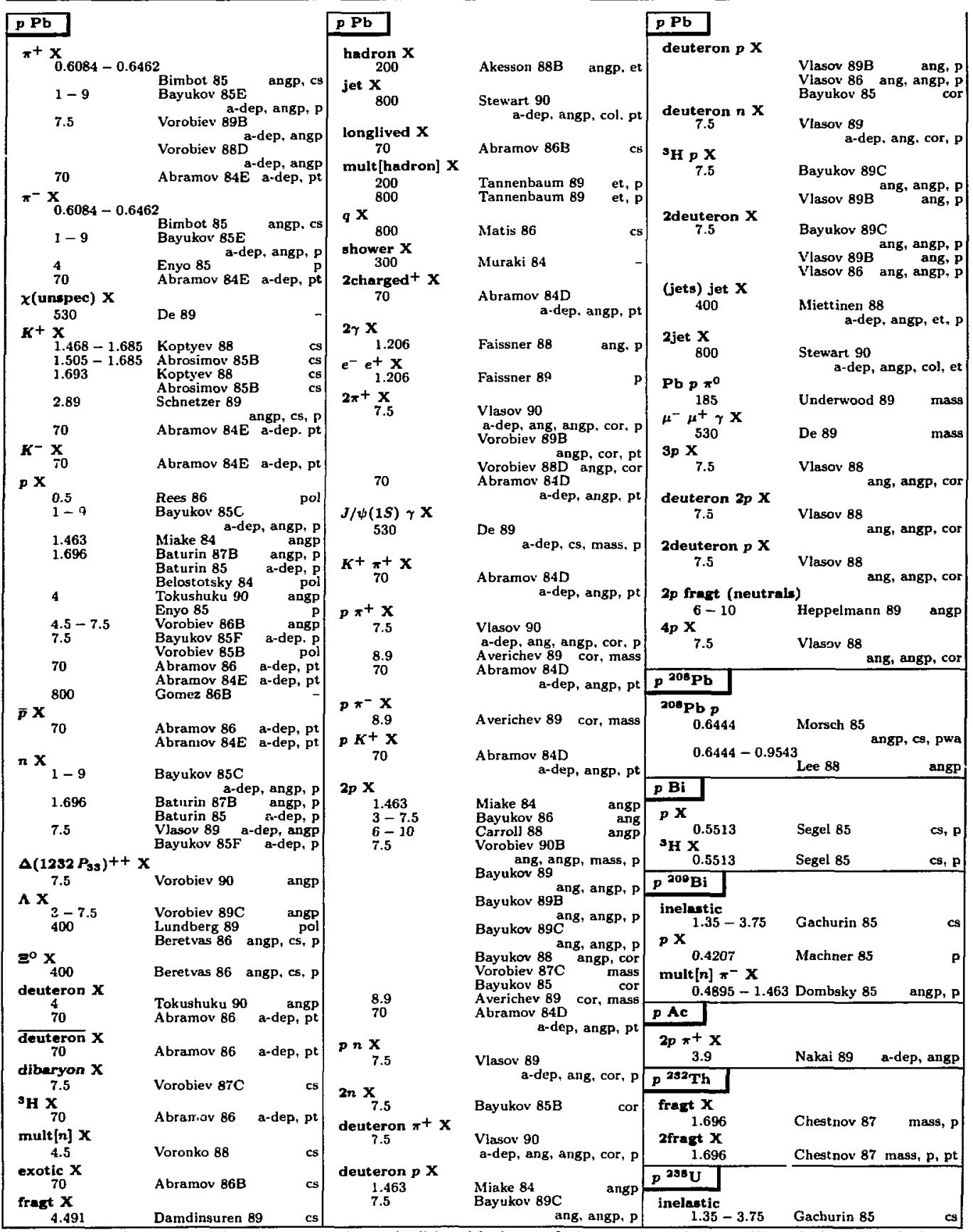




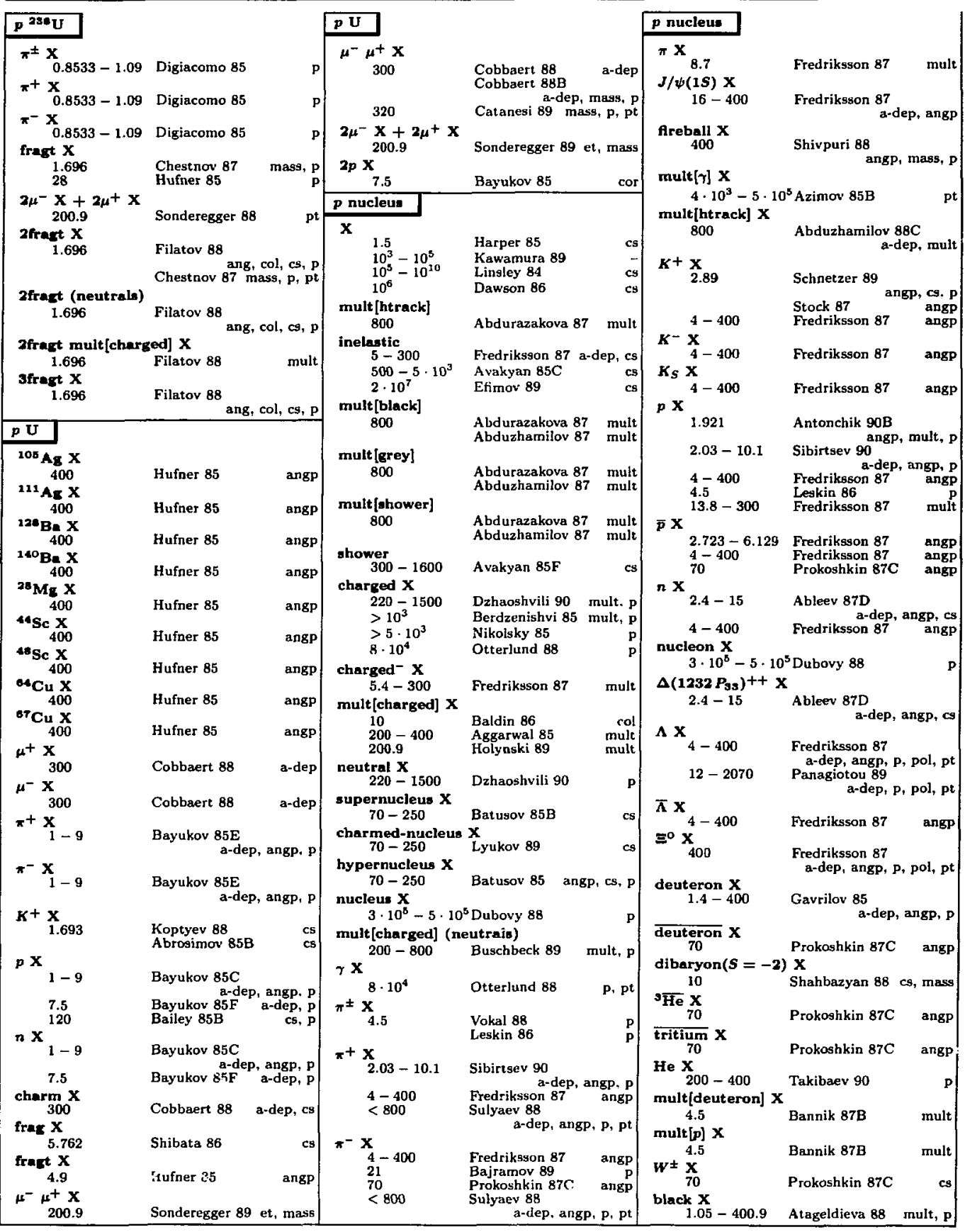




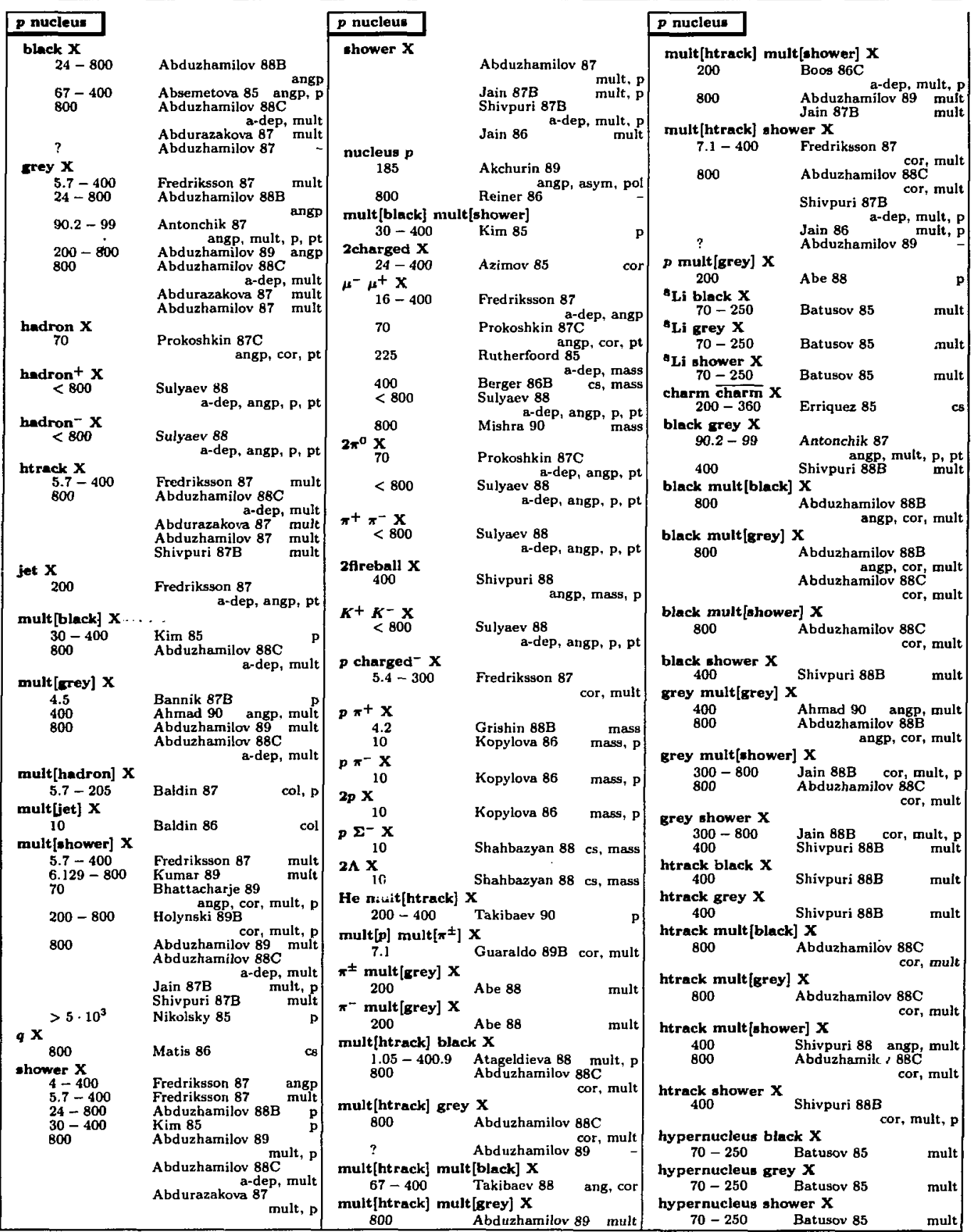




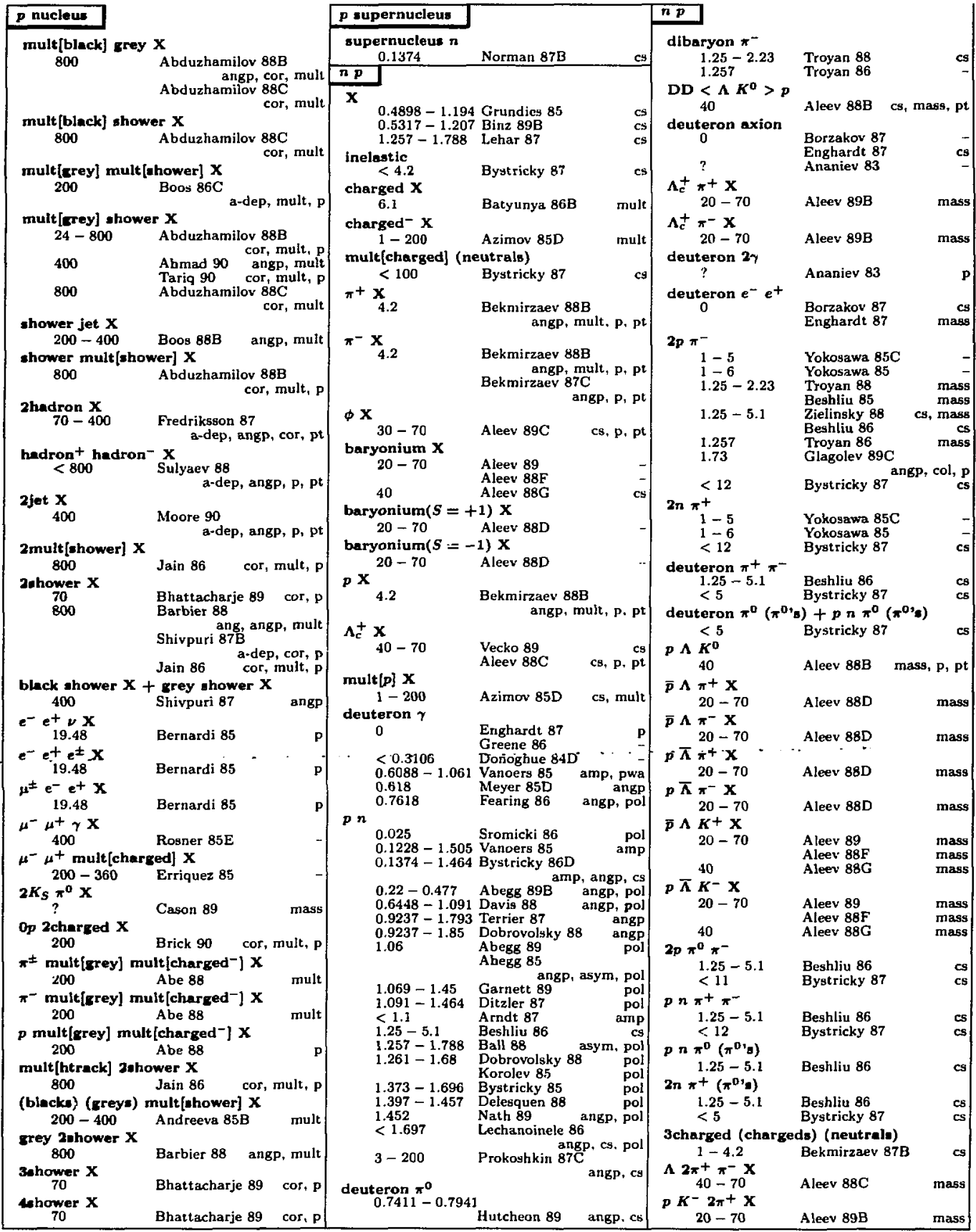




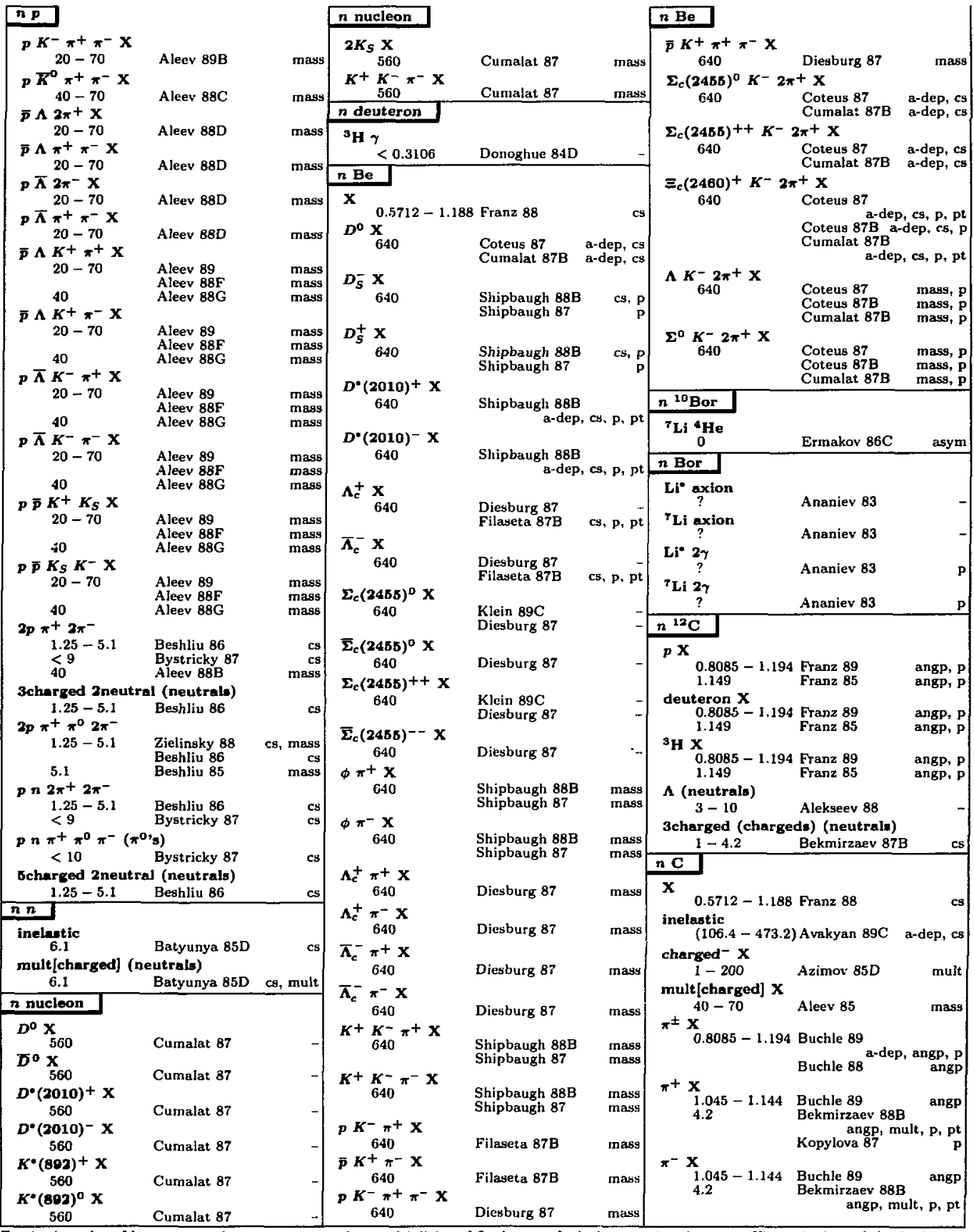

Entries in order of beam mass, then target mass, then multiplicity of final state. Inclusive reactions have an "X" as the last of the final state particles. Certain chemical symbols for nuclei have been changed to avoid ambiguity with particle names (See the Particle Vocabulary.) Beam momenta are $\mathrm{P}_{\mathrm{lab}}$ in $\mathrm{GeV} / \mathrm{c}$, or in parentheses $E_{\mathrm{cm}}$ in $\mathrm{GeV}$. See the legend on page 153 . 


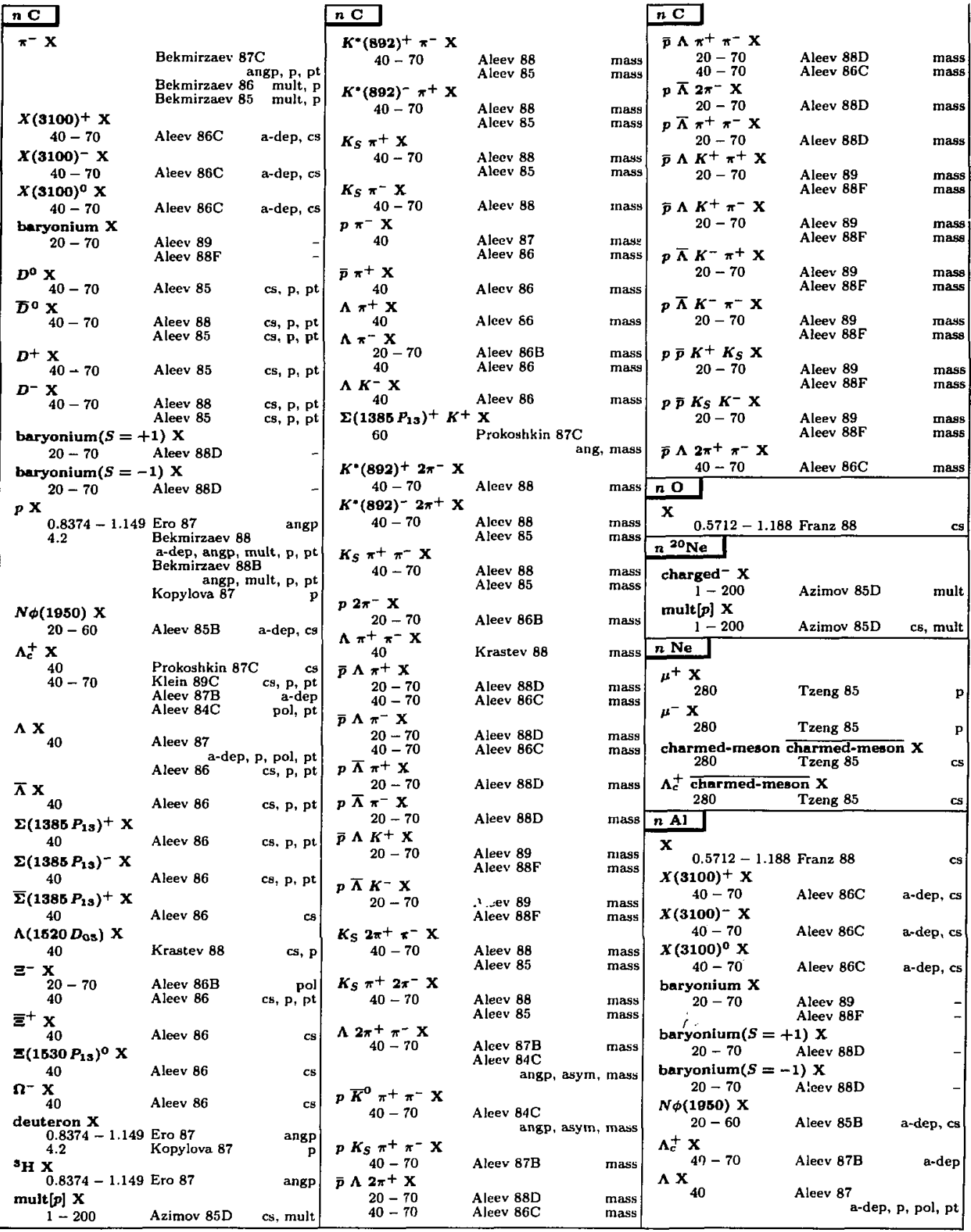




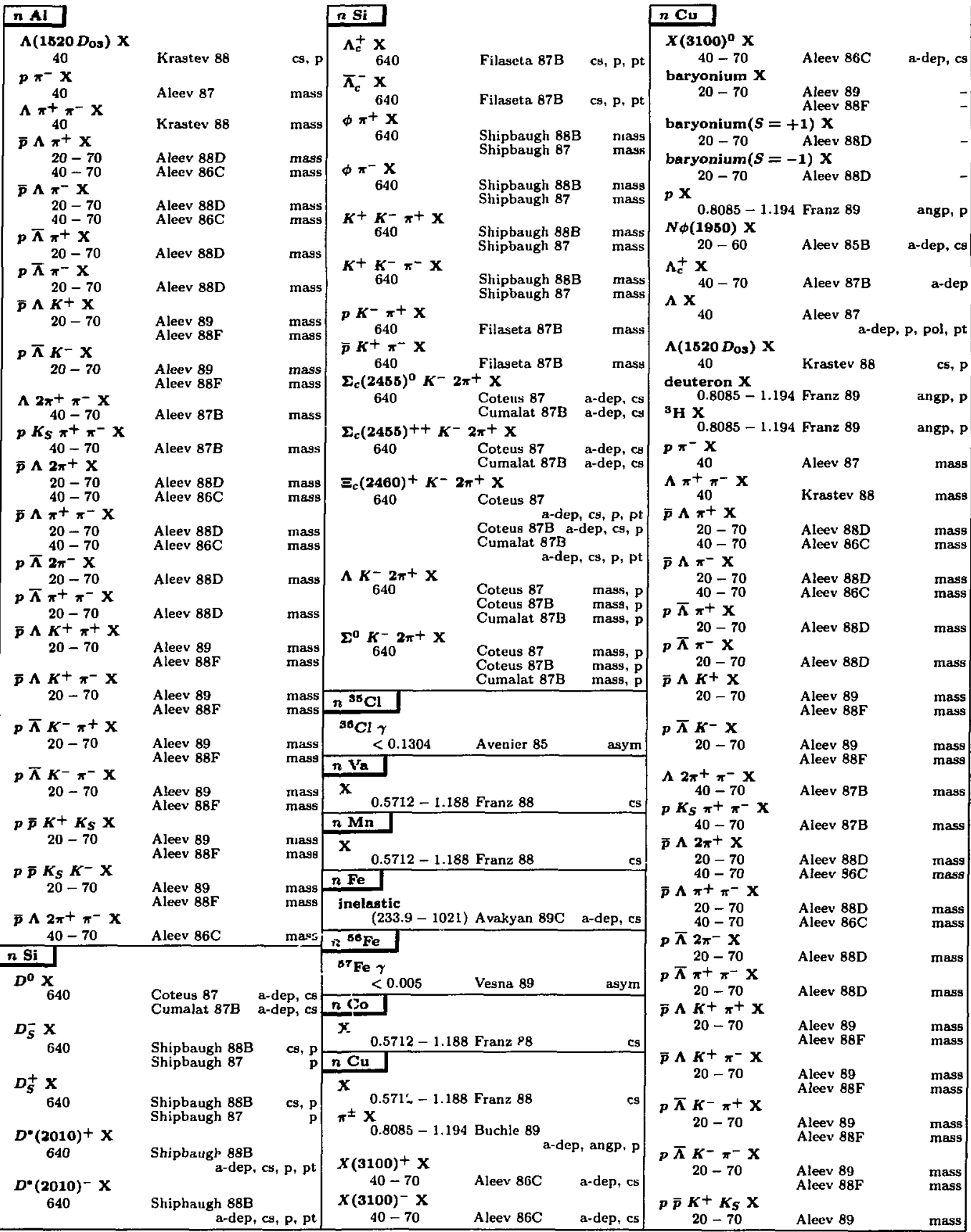




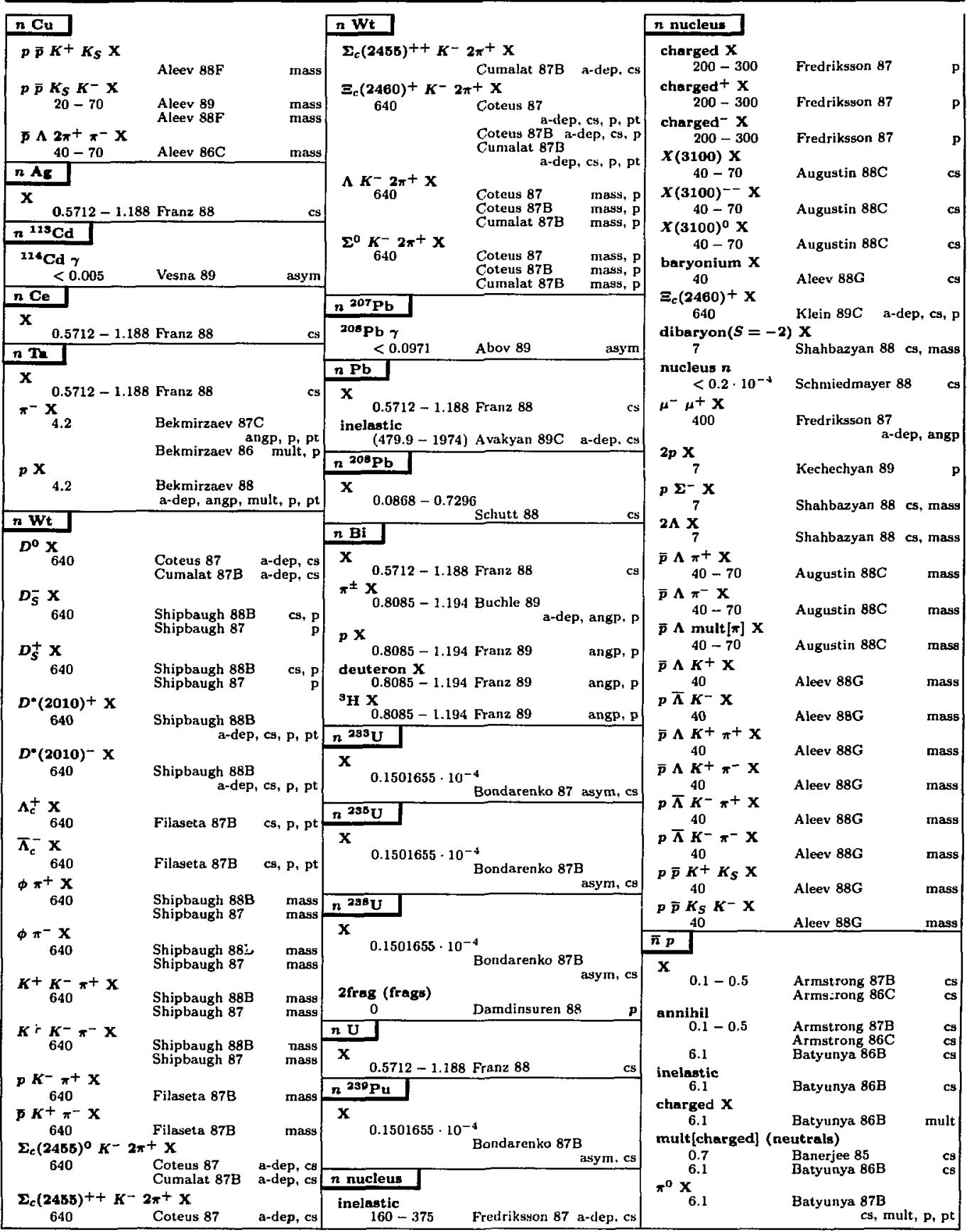




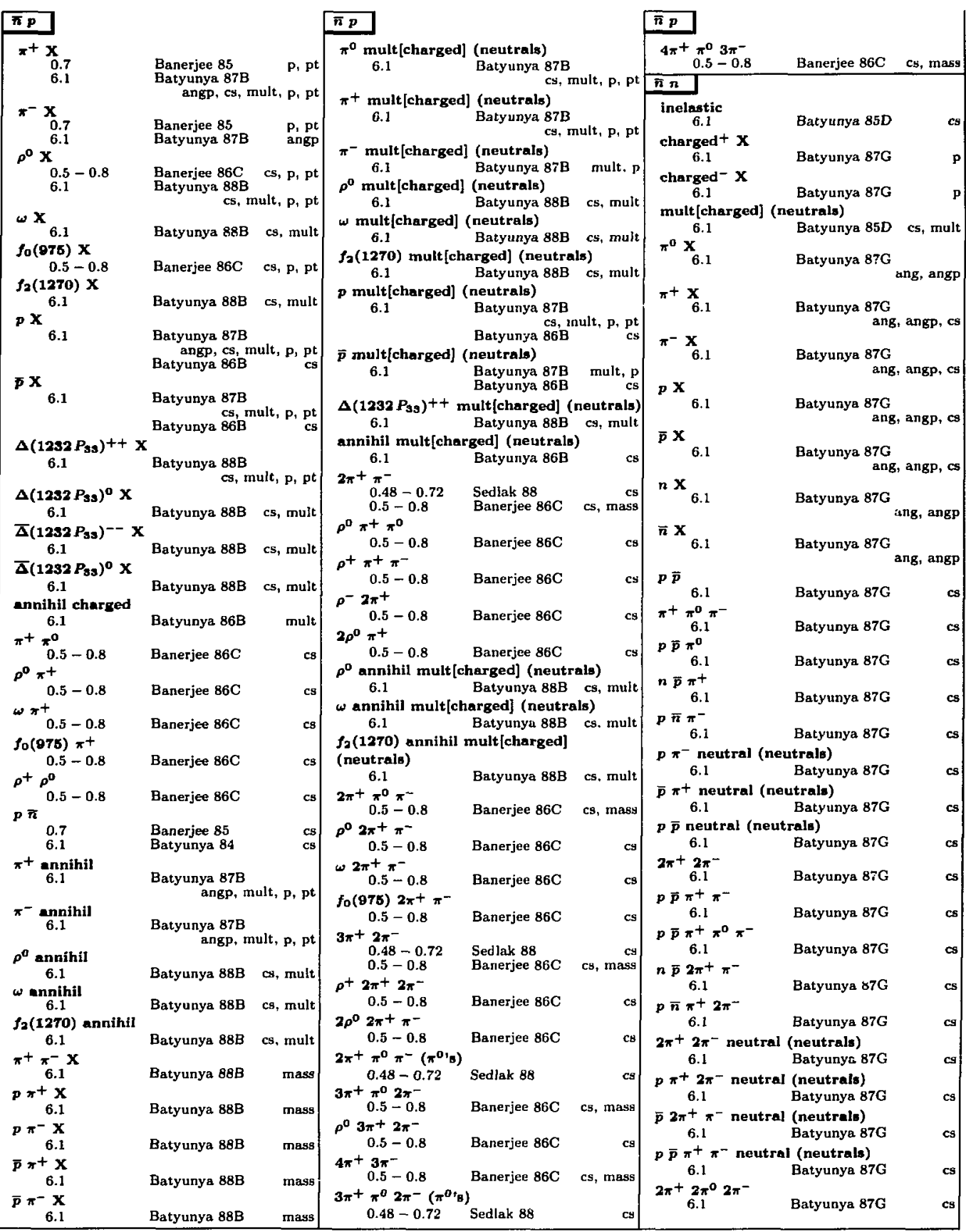




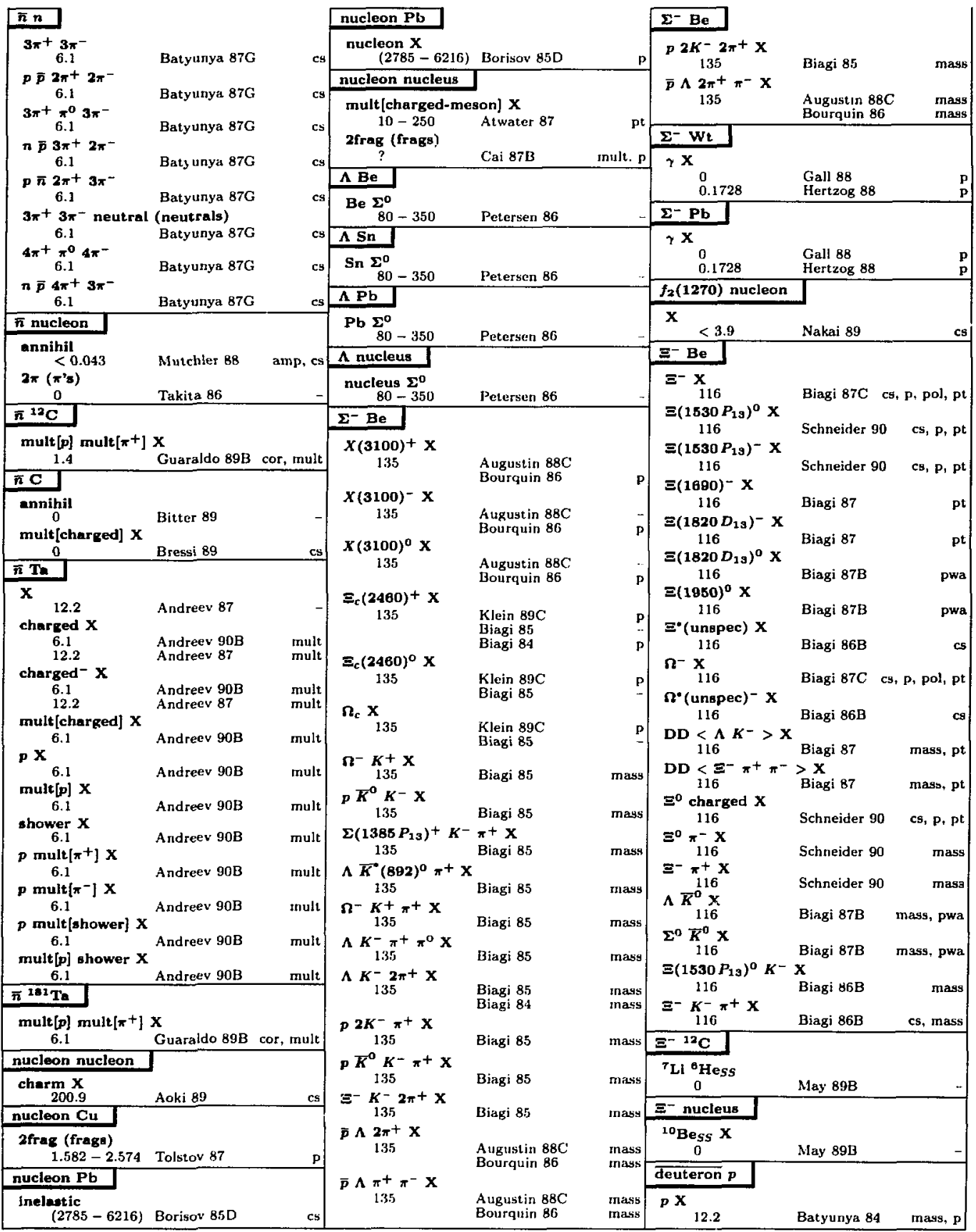




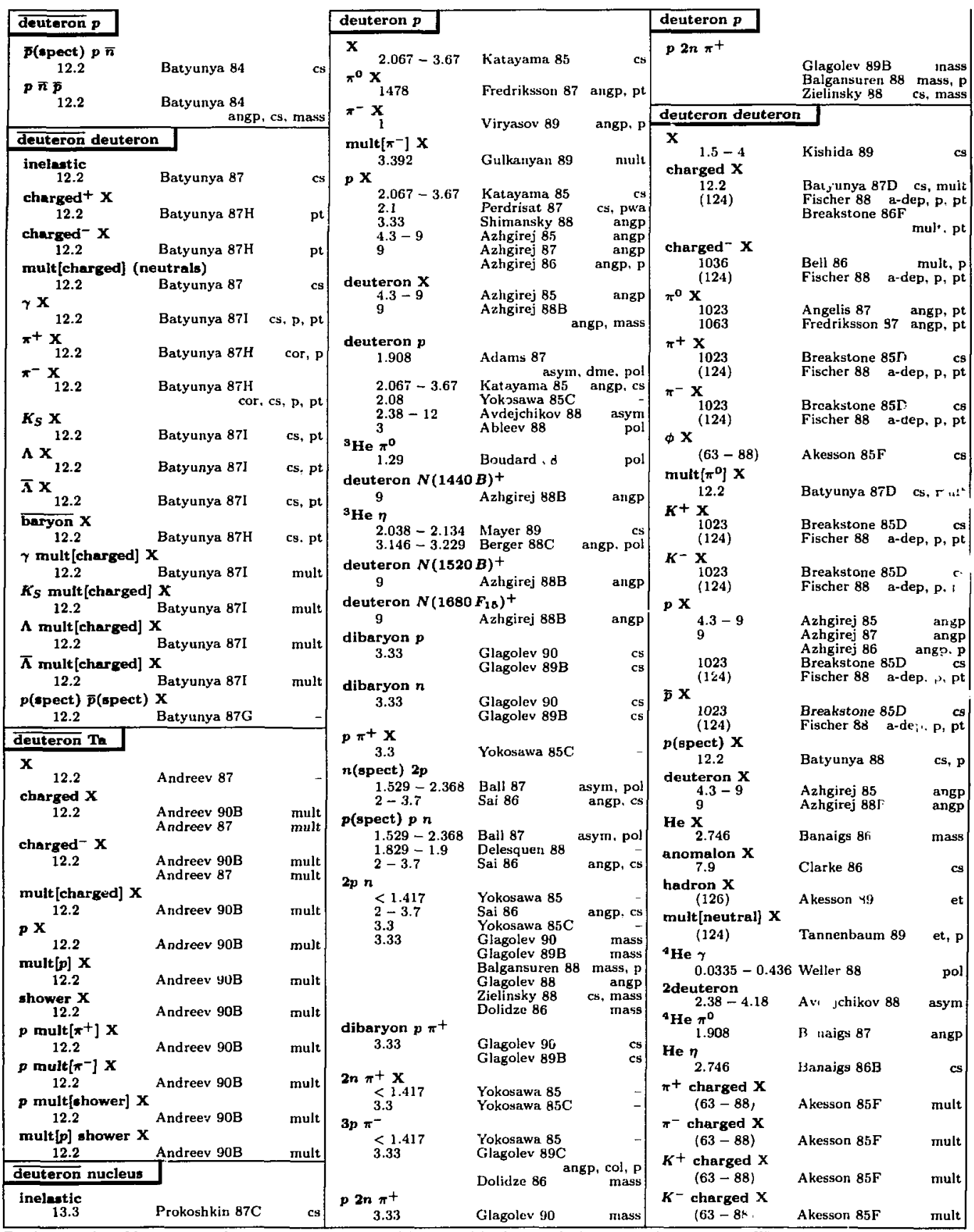

Entries in order of beam mass, then target mass, then multiplicity of final state. Inclusive reactions have an "X" as the last of the final state particles. Certain chemical symbols for nuclei have been changed to avoid ambiguity with particle names (S... the Particle Vocalulary.) Beam momenta are $P_{\text {lab }}$ in $\mathrm{GeV} / c$, or in parentheses $E_{\mathrm{cm}}$ in $\mathrm{GeV}$. Sec the legend on page 153. 


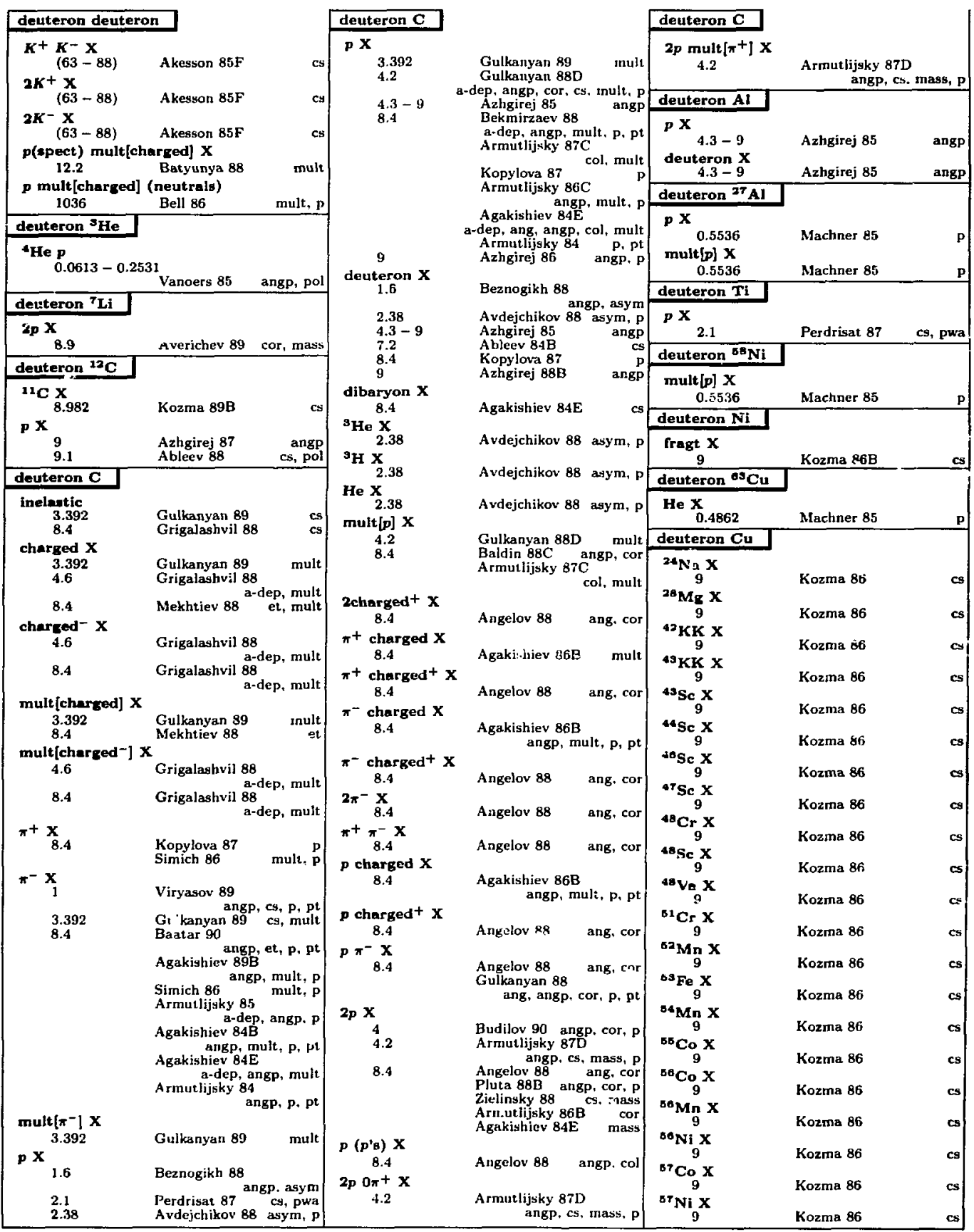




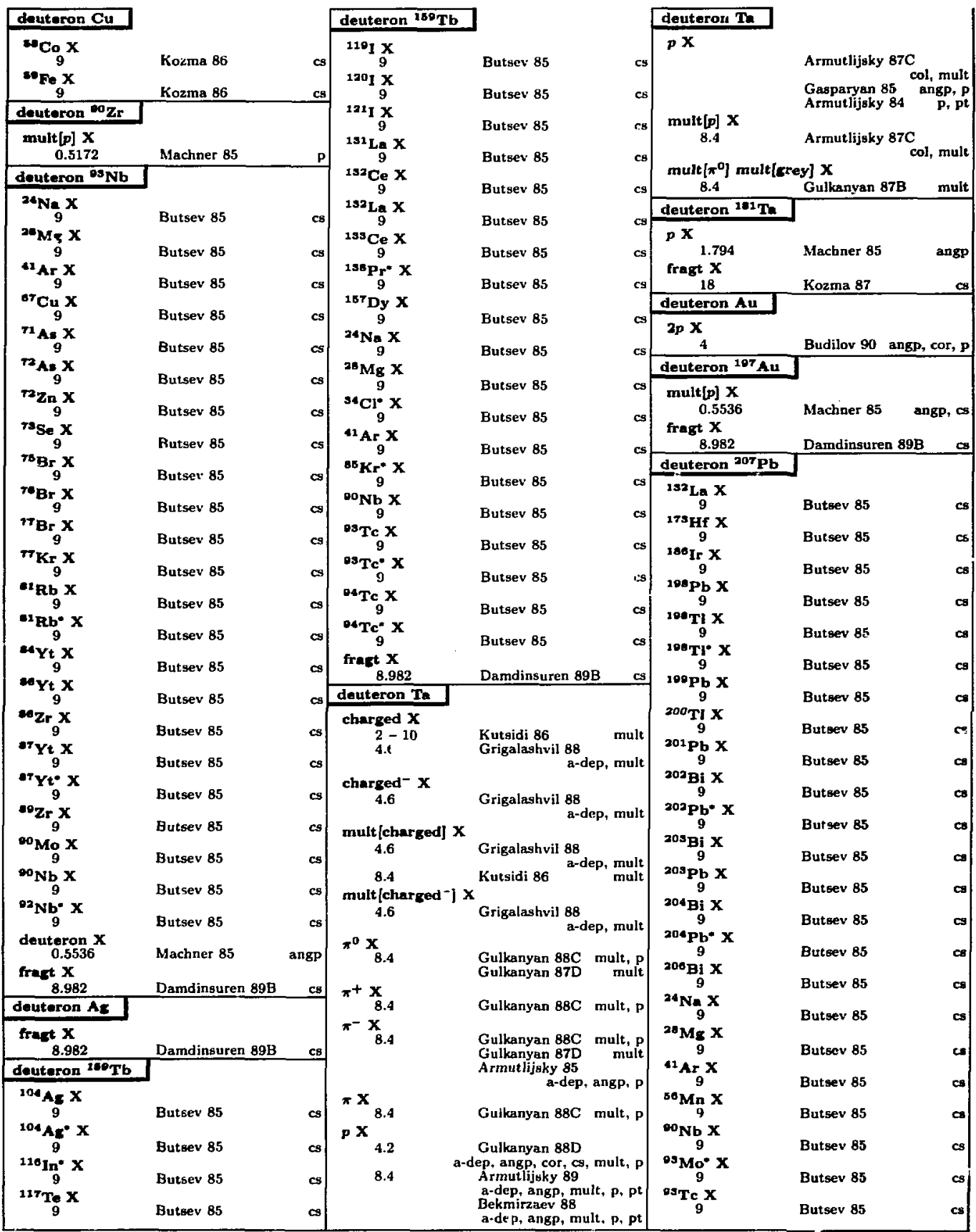




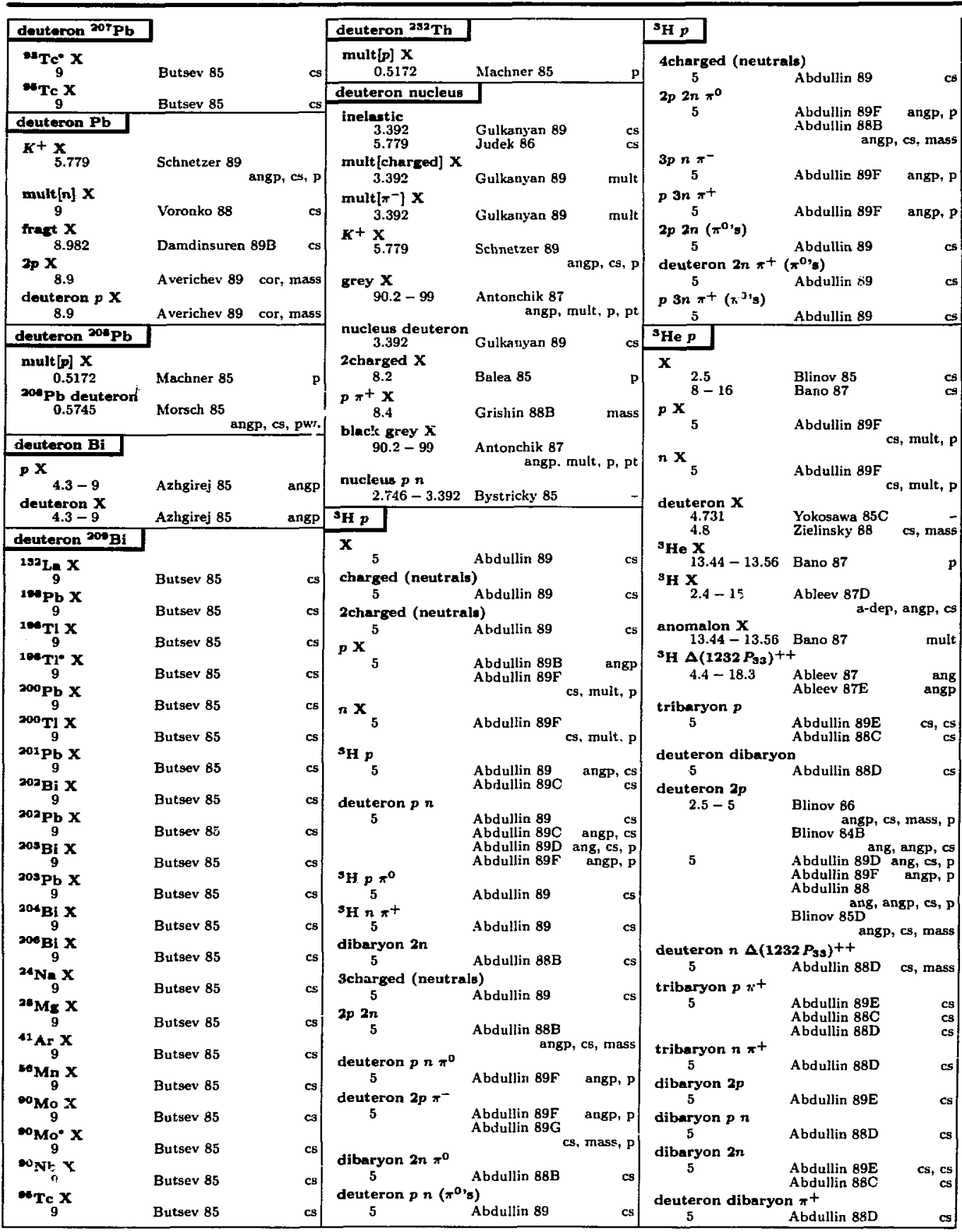




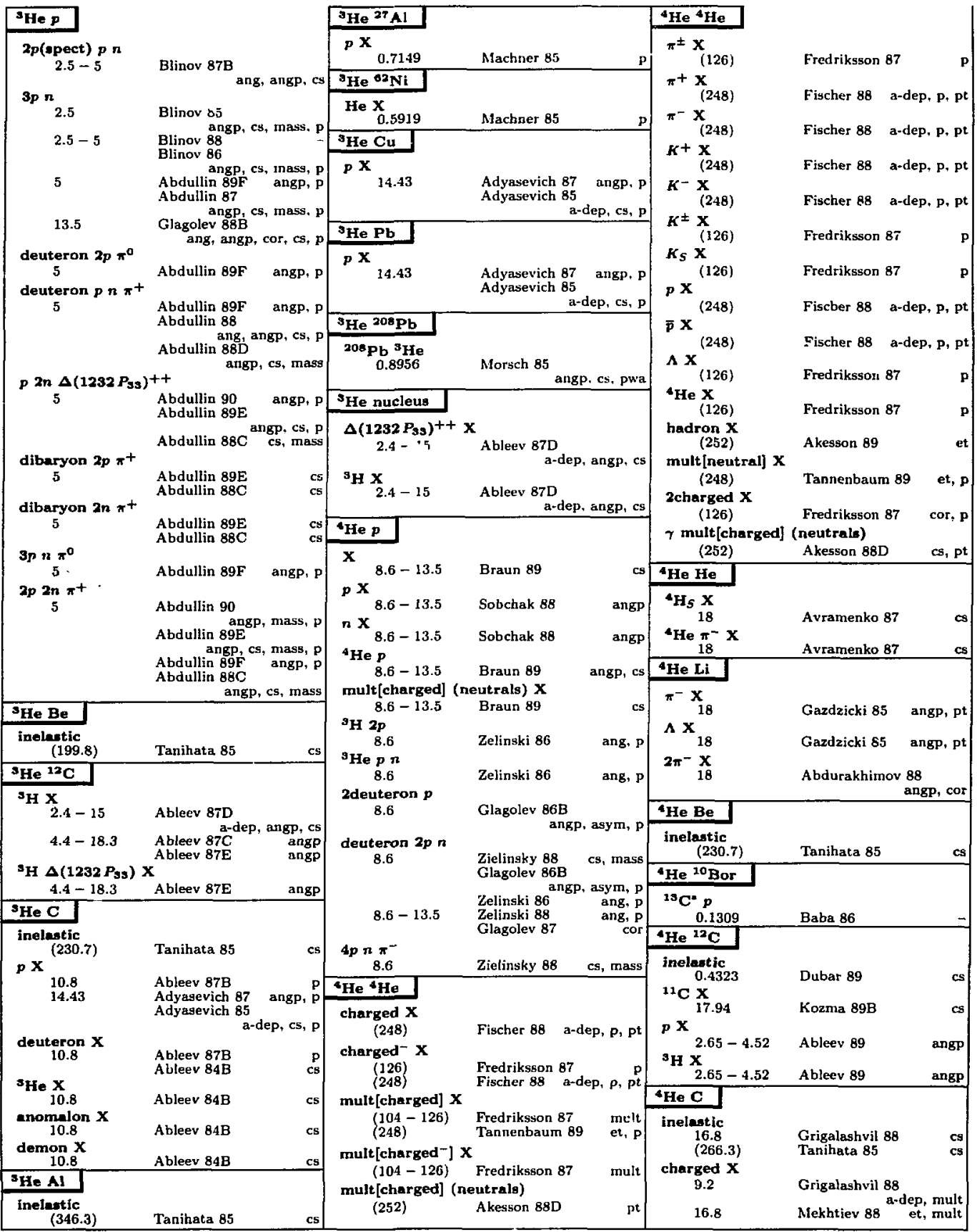

Entries in order of beam mass, then target mass, then multiplicity of final state. Inclusive reactions have an "X" as the last of the final state particles. Certain chenical symbols for nuclei have been changed to avoid ambiguity with particle names (See the Particle Vocabulary.) Beam momenta are $\mathrm{P}_{\text {lab }}$ in $\mathrm{GeV} / c$, or in parentheses $E_{\mathrm{cm}}$ in $\mathrm{GeV}$. See the legend on page 153. 


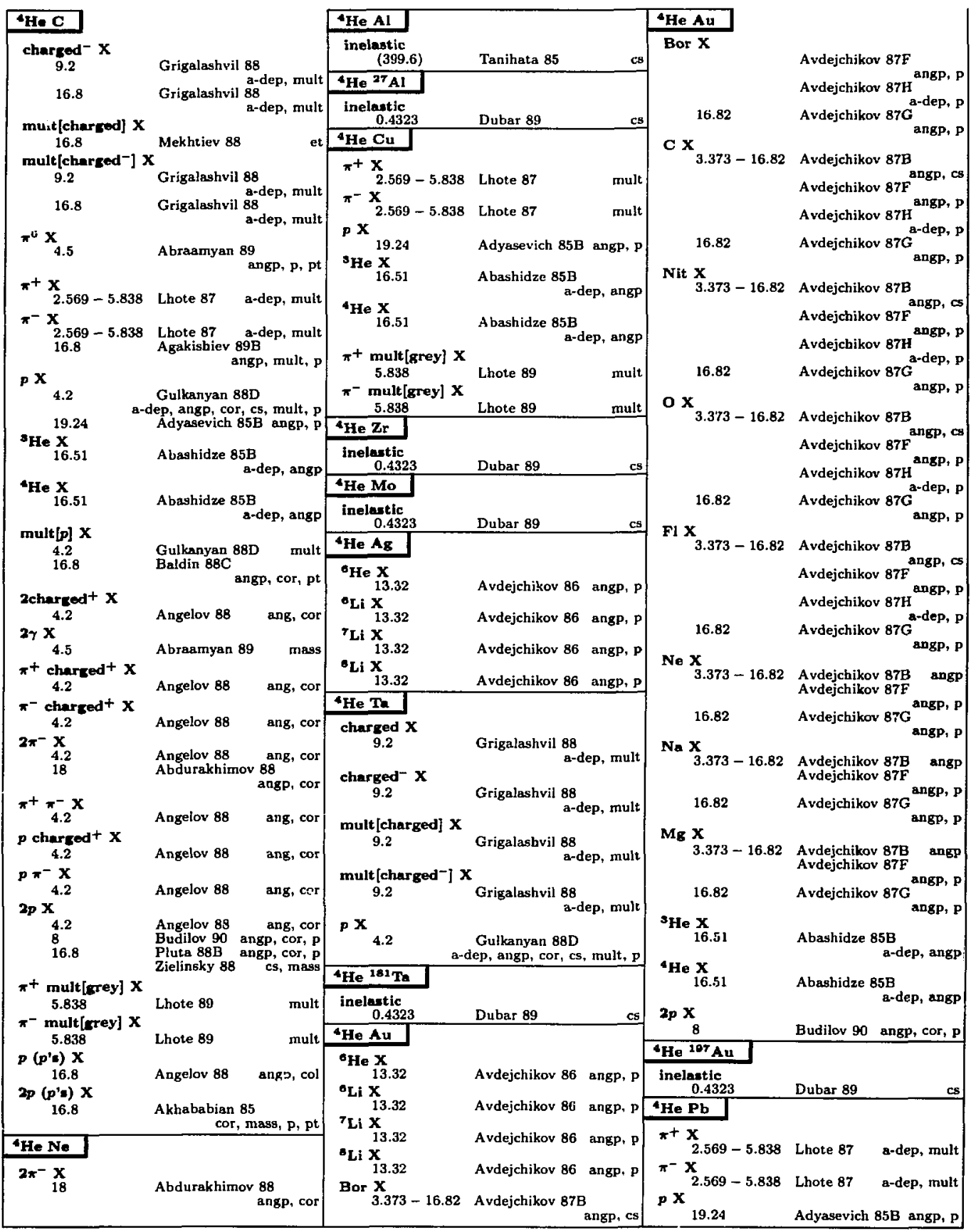




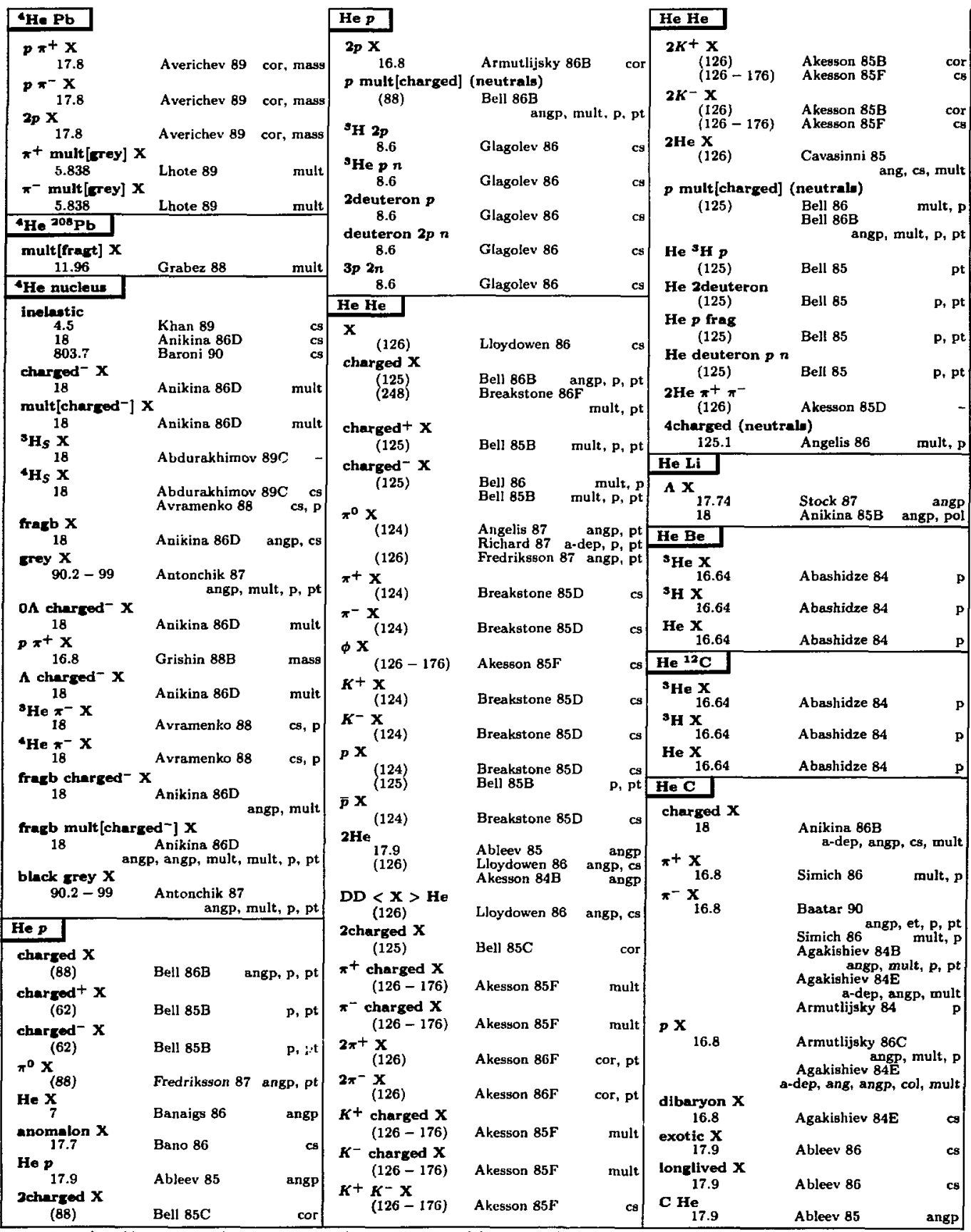


He C $\rightarrow \pi^{+}$charged $\mathrm{X}$

${ }^{8} \mathrm{He} \mathrm{C} \rightarrow$ inelastic

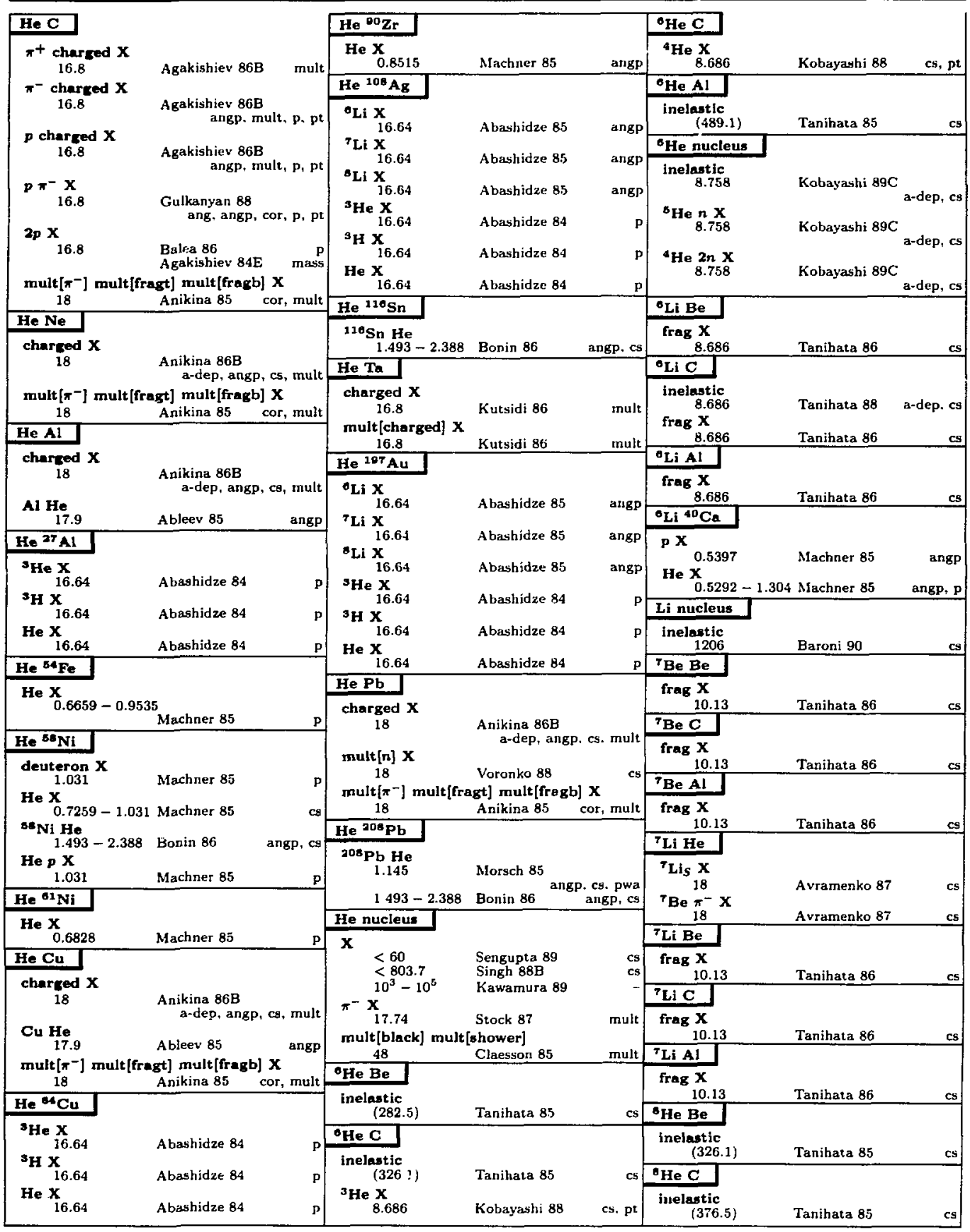




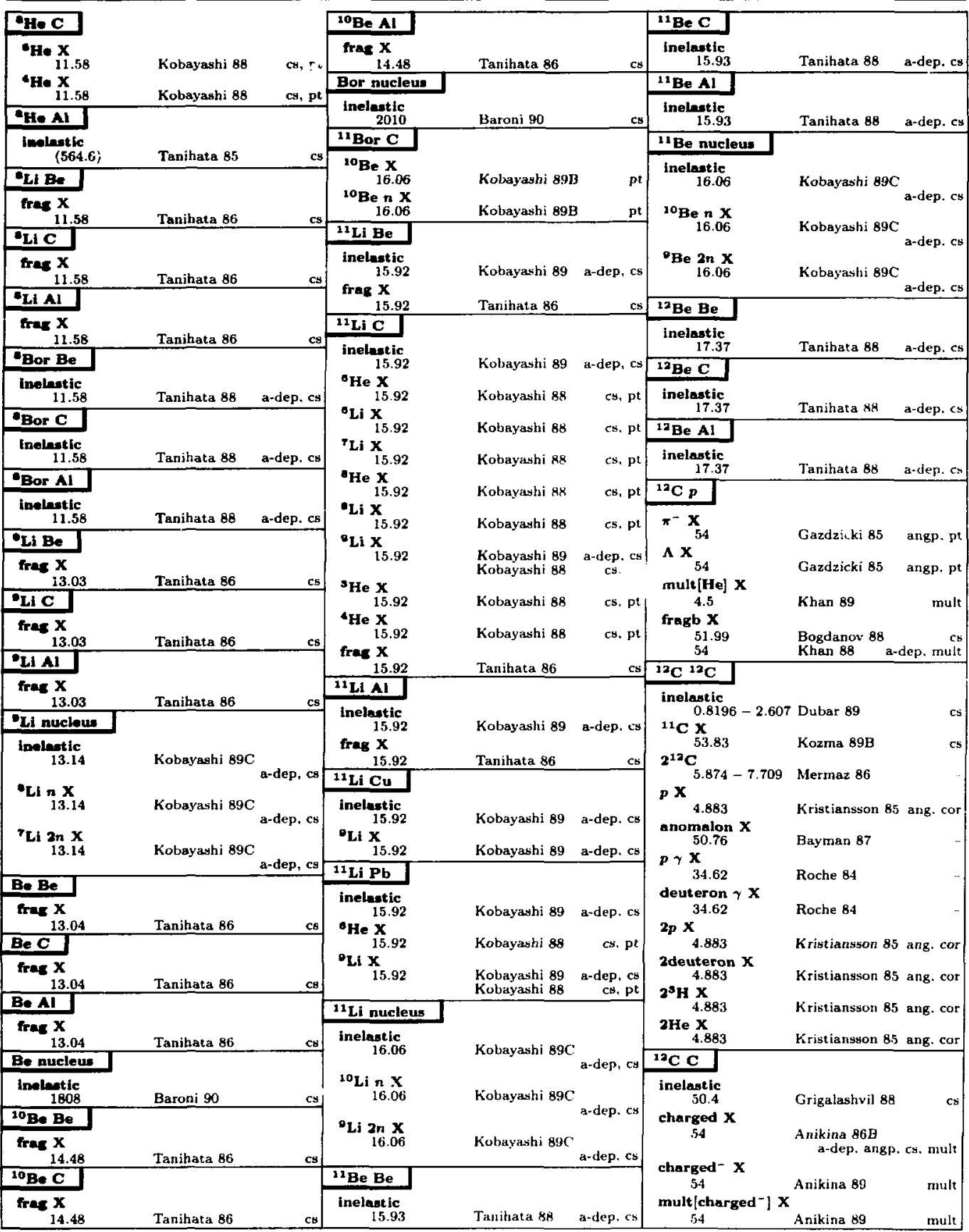

Entries in order of beam mass. then target mass. then multiplicity of final state. Inciusive reartions have an " $X$ " as the last of the final state particles. Certain chemical symbols for nuclej have been changed to avoid ambiguity with particle names (Soe the Particle Vocabulary.) Bean momenta are $P_{1 . t}$ ir. $\mathrm{GeV} / \mathrm{c}$, or in arentheses $E_{\mathrm{cm}}$ in GeV. Sce the logend on page 153 


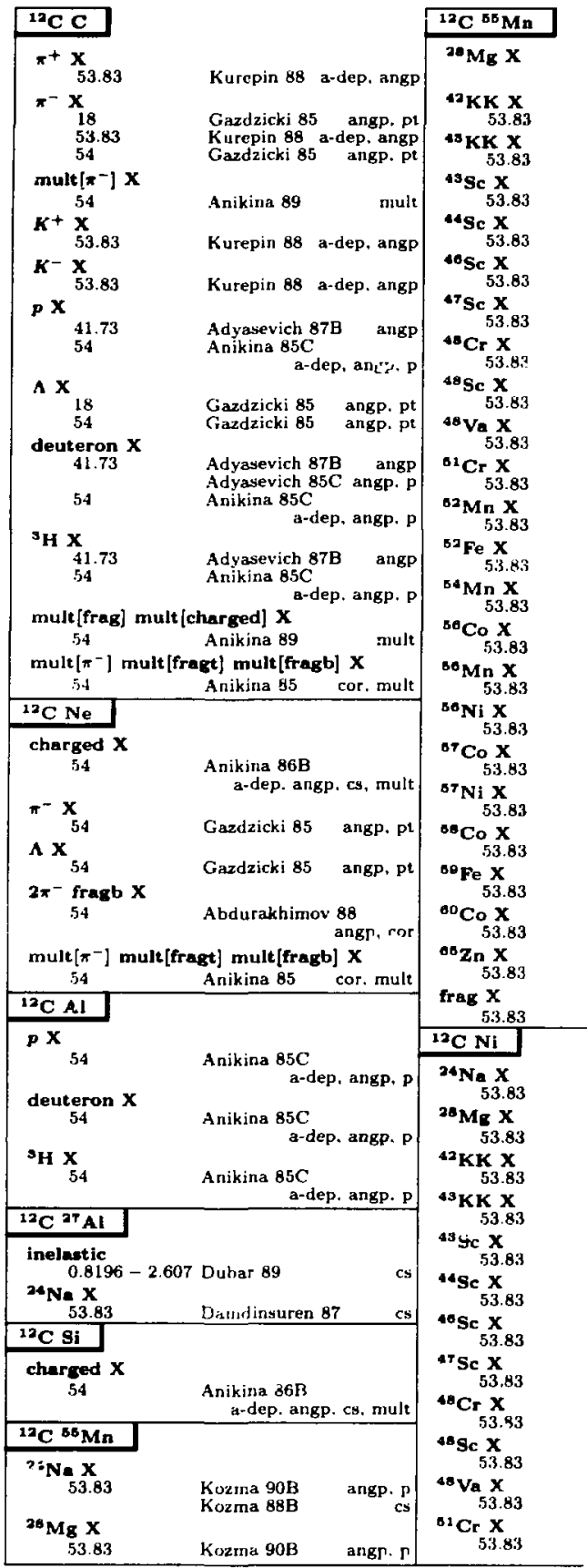

Kozma 88B

Kozma $88 B$

Kozma 88B

Kozma 88B

Kozina 88B

Kozma 88B

Kozma 88B

Kozma 88B

Kozma 88B

Kuzma 88B

Kozma 88B

Kuzma $88 B$

Kozma 88B

Kozma $88 \mathrm{~B}$

Kozma $48 \mathrm{~B}$

Kuzma 88B

Kozma 88B

Kozma 88B

Kozma $88 \mathrm{H}$

Kozma $88 B$

Kozma $88 B$

Kozma 88B

Kozma 88B

Kuzma 88B

Kozma $88 B$

Kuztta 88B

Kuzma 88B

Kozma 88B

Kozma 88B

Kozma 88B

Kozma 88B

Kozma 88B

Kozma $88 \mathrm{~B}$

Kozma 848

Kozma 88B

Kozma 88B

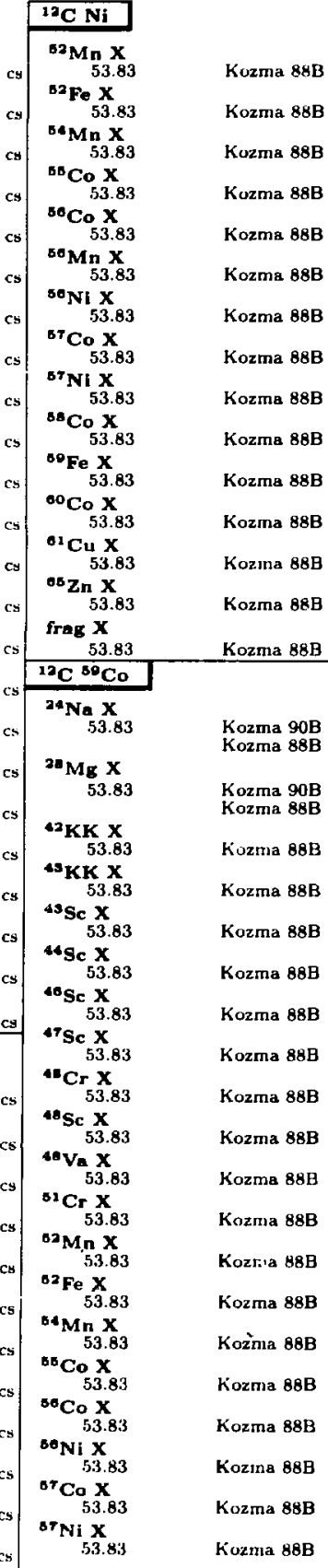




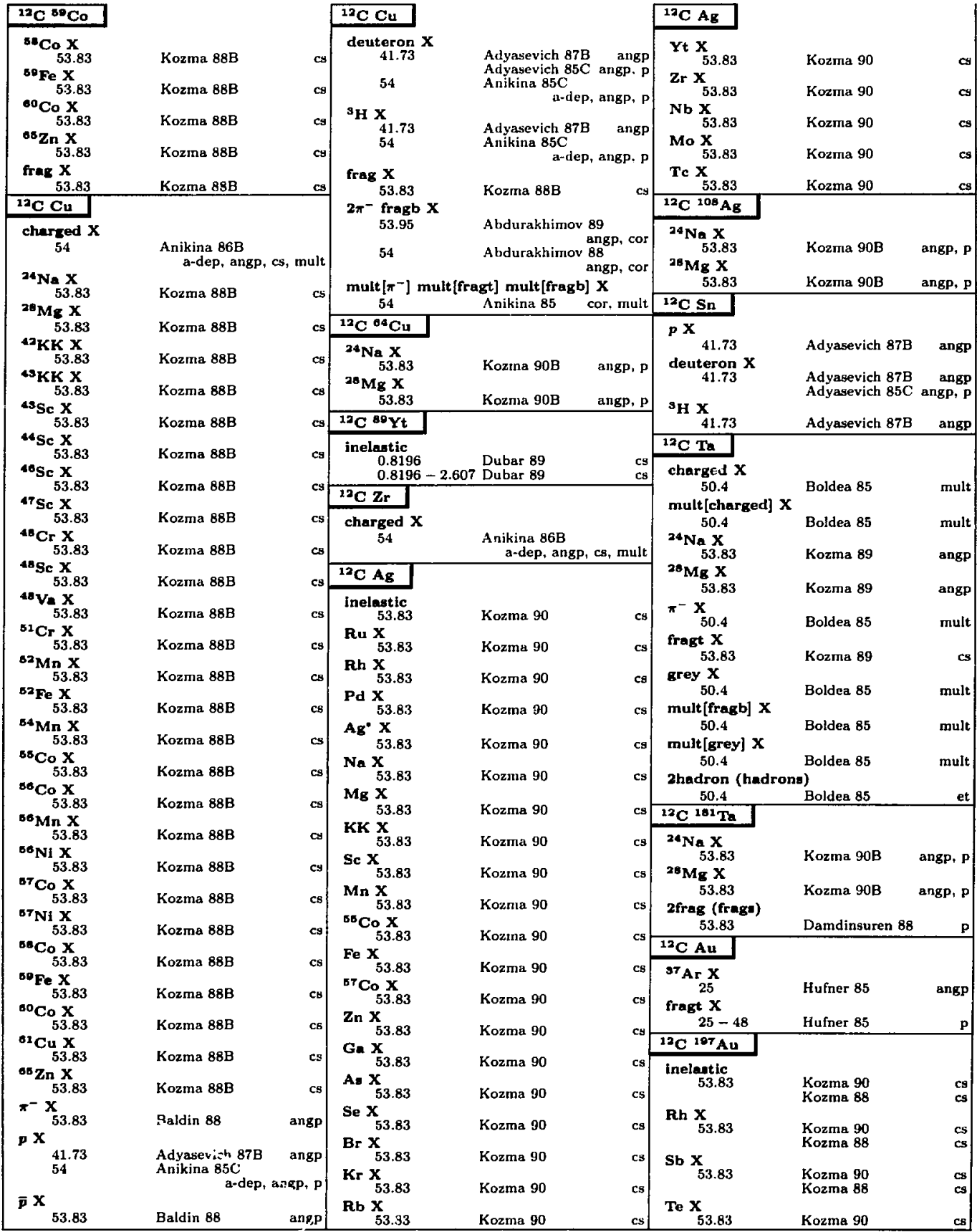

Entries in order of beam mass, then targri mass, then multiplicity of final state. Inclusive reactions lave an " $\mathrm{X}$ " as the last of the final state particles. Certain chemical symbolk for uuclei have been chinged to avoid ambiguity with particle names (See the Particle Vocabulary.) Beam momenta are $P_{\text {iab }}$ in $\mathrm{GeV} / c$, or in parentheses $E_{\mathrm{cm}}$ in GeV. See the legend on page 153. 


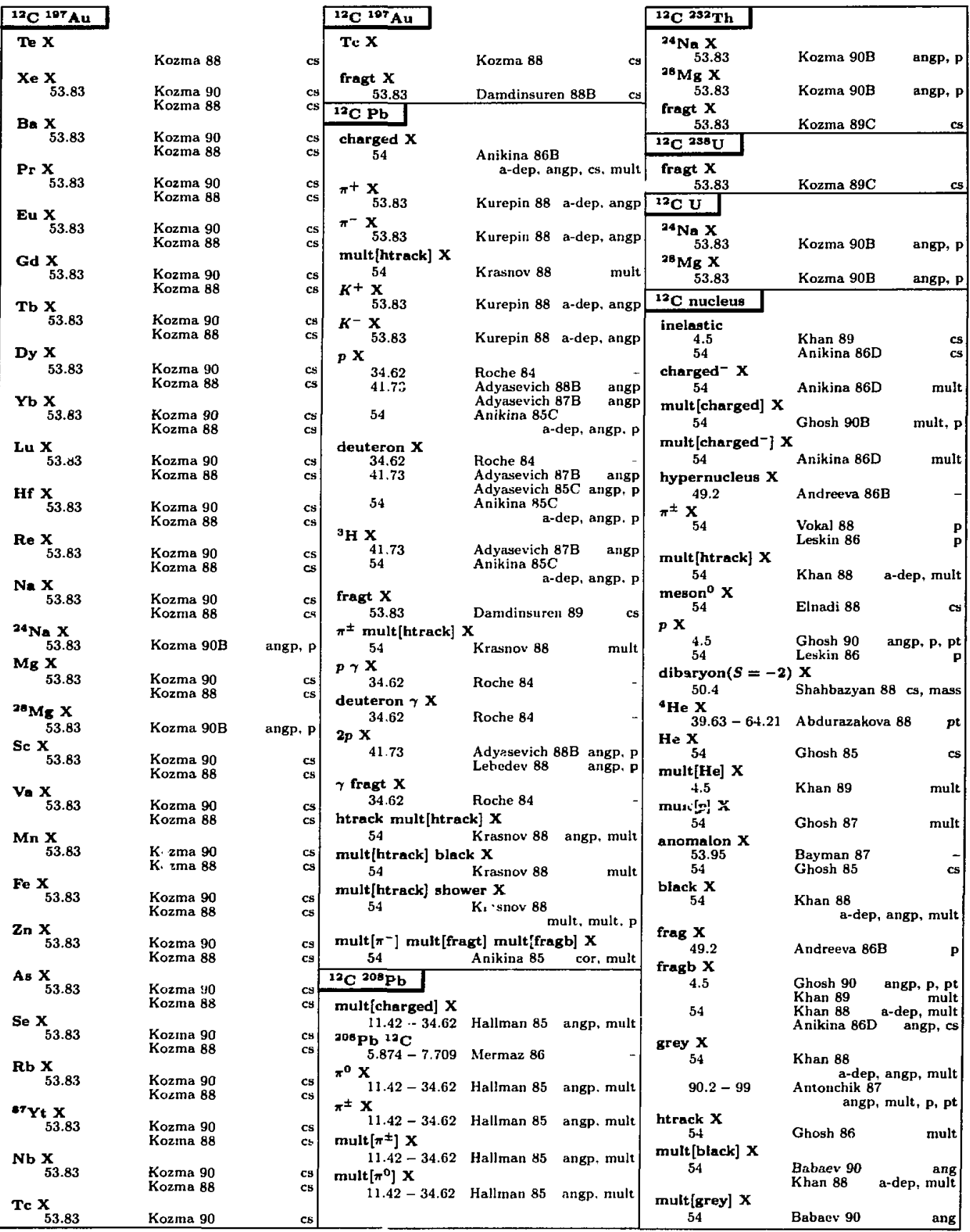




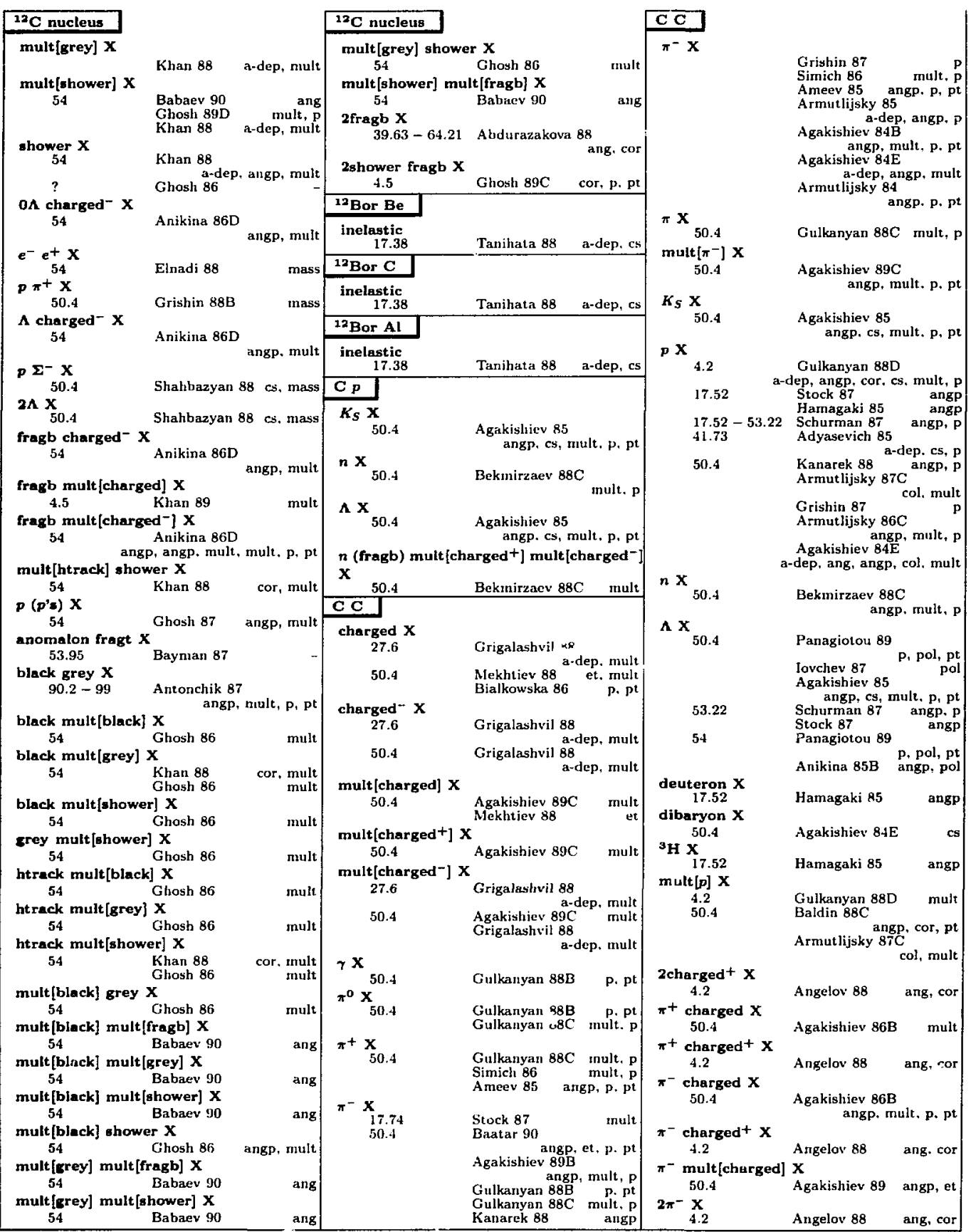




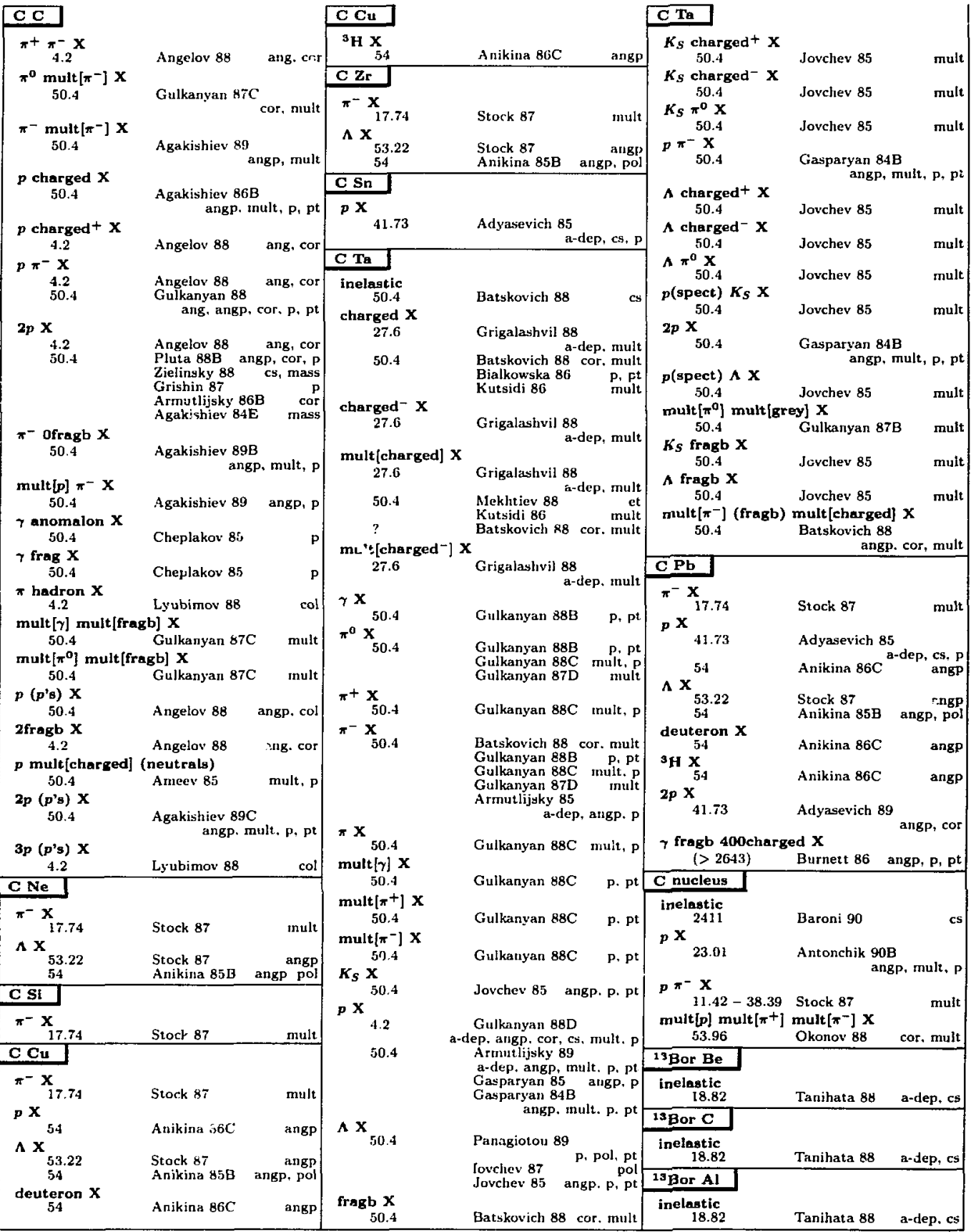


${ }^{14}$ Bor Be $\rightarrow$ inelastic

${ }^{16} \mathrm{O} \mathrm{Cu} \rightarrow$ mult $[$ charged] $\mathrm{X}$

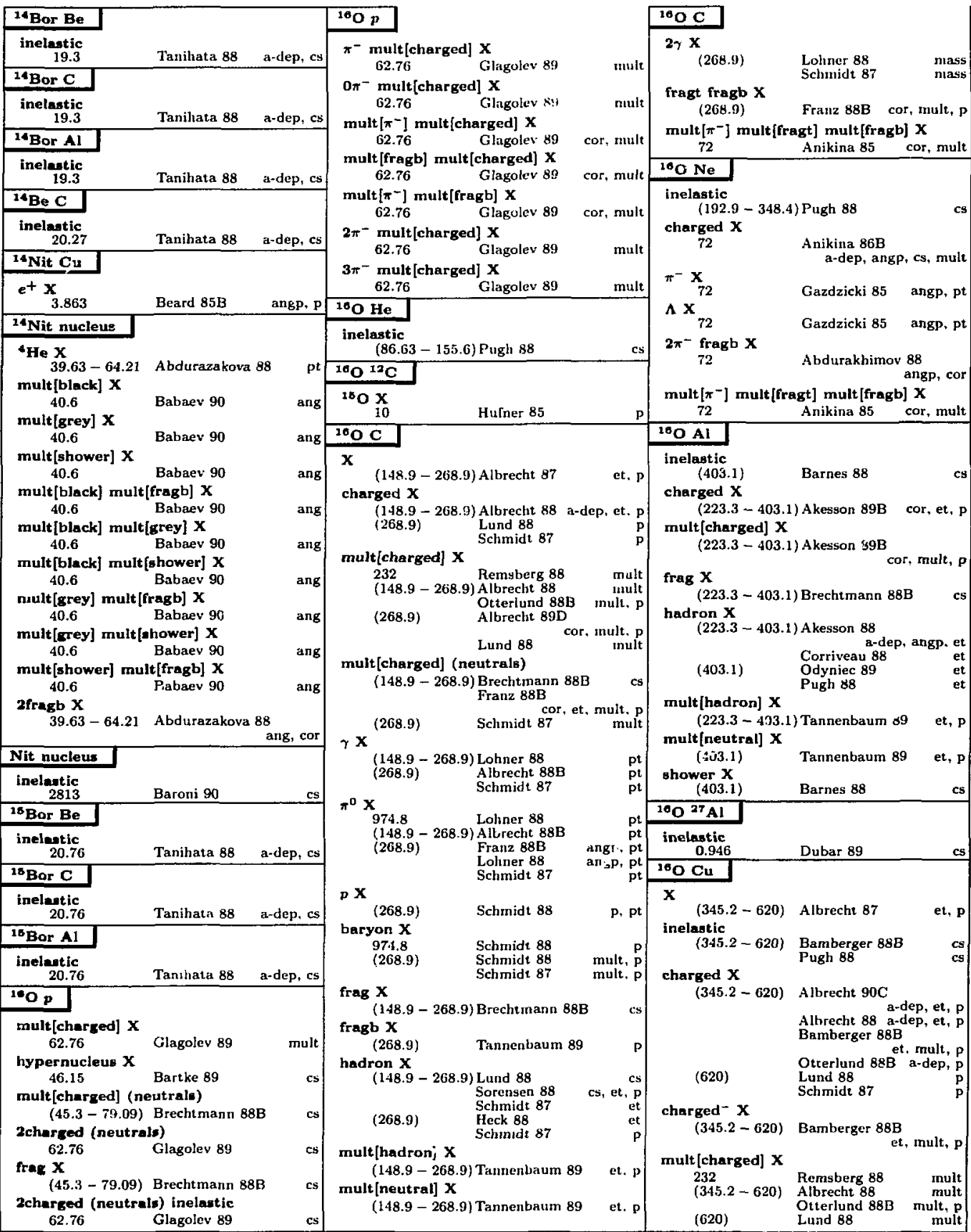

Entries in order of beam mass, then target mass, then multiplicity of final state. Inclusive reactions have an "X" as the last of the final state particles. Certain chemical symbols for nuclei have been changed to avoid ambiguity with particle names (See the Particle Vocabulary.) Beam momenta are $\mathrm{P}_{\mathrm{Iab}}$ in $\mathrm{GeV} / \mathrm{c}$, or in parentheses $E_{\mathrm{cm}}$ in GeV. See the legend on page 153 . 


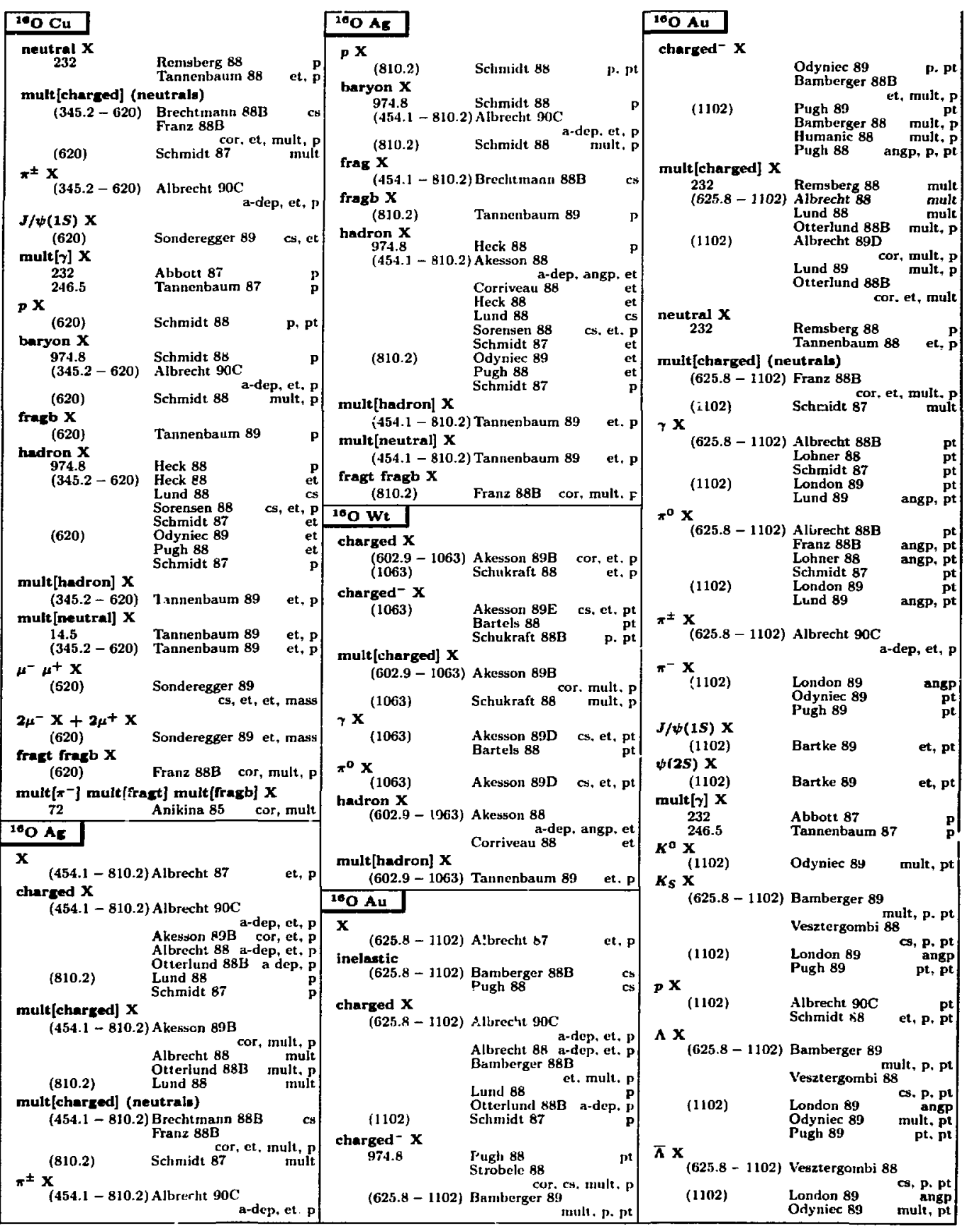




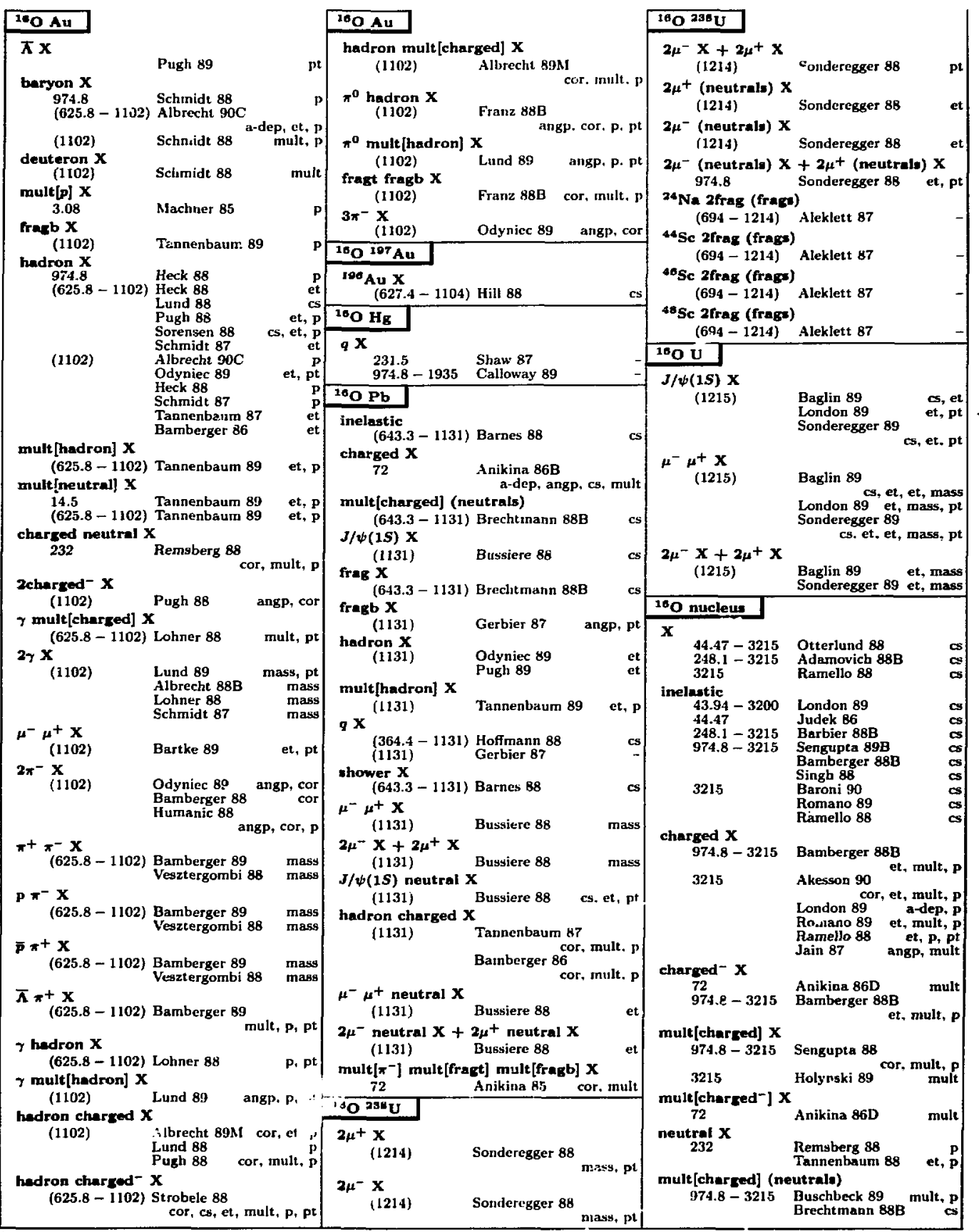




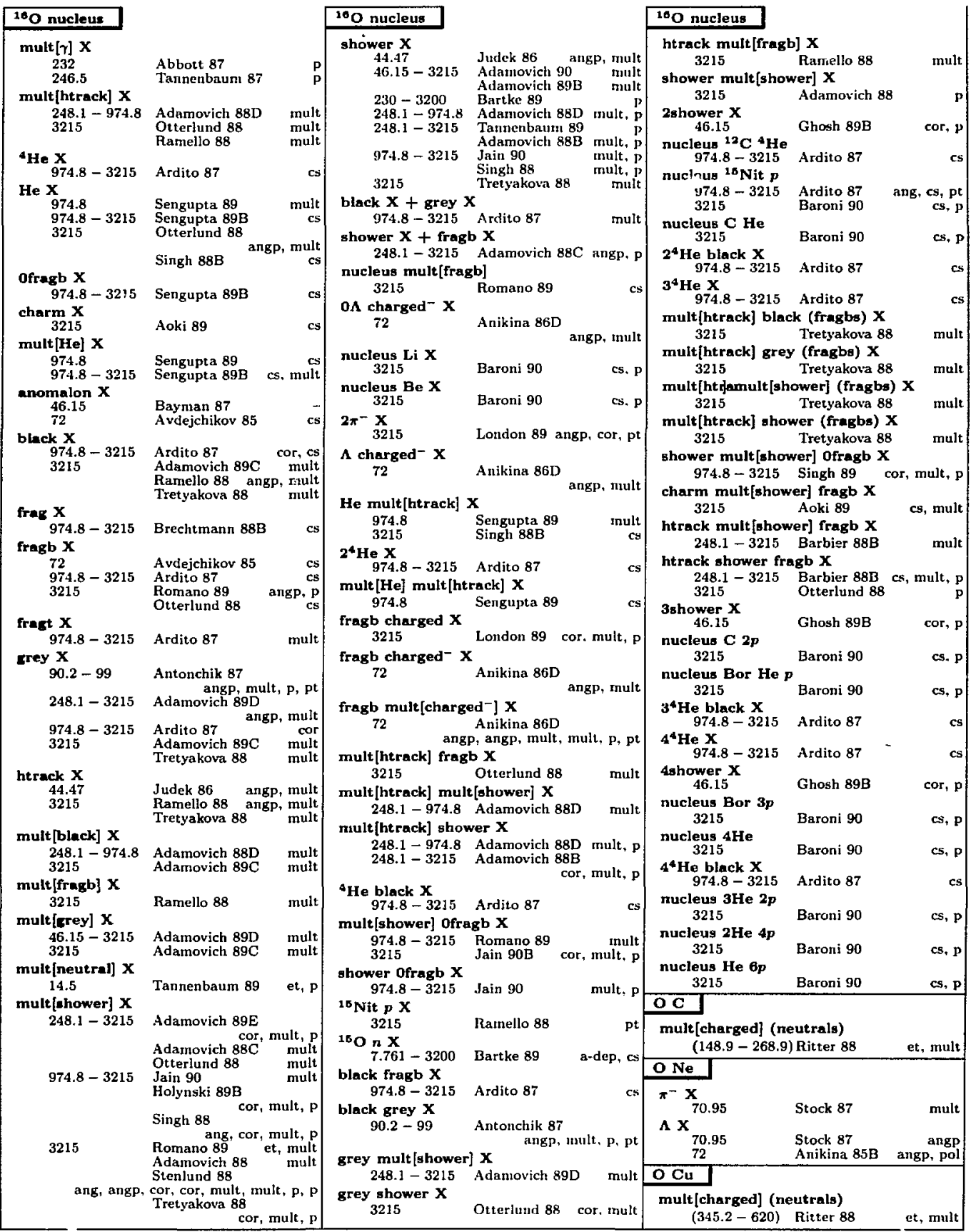




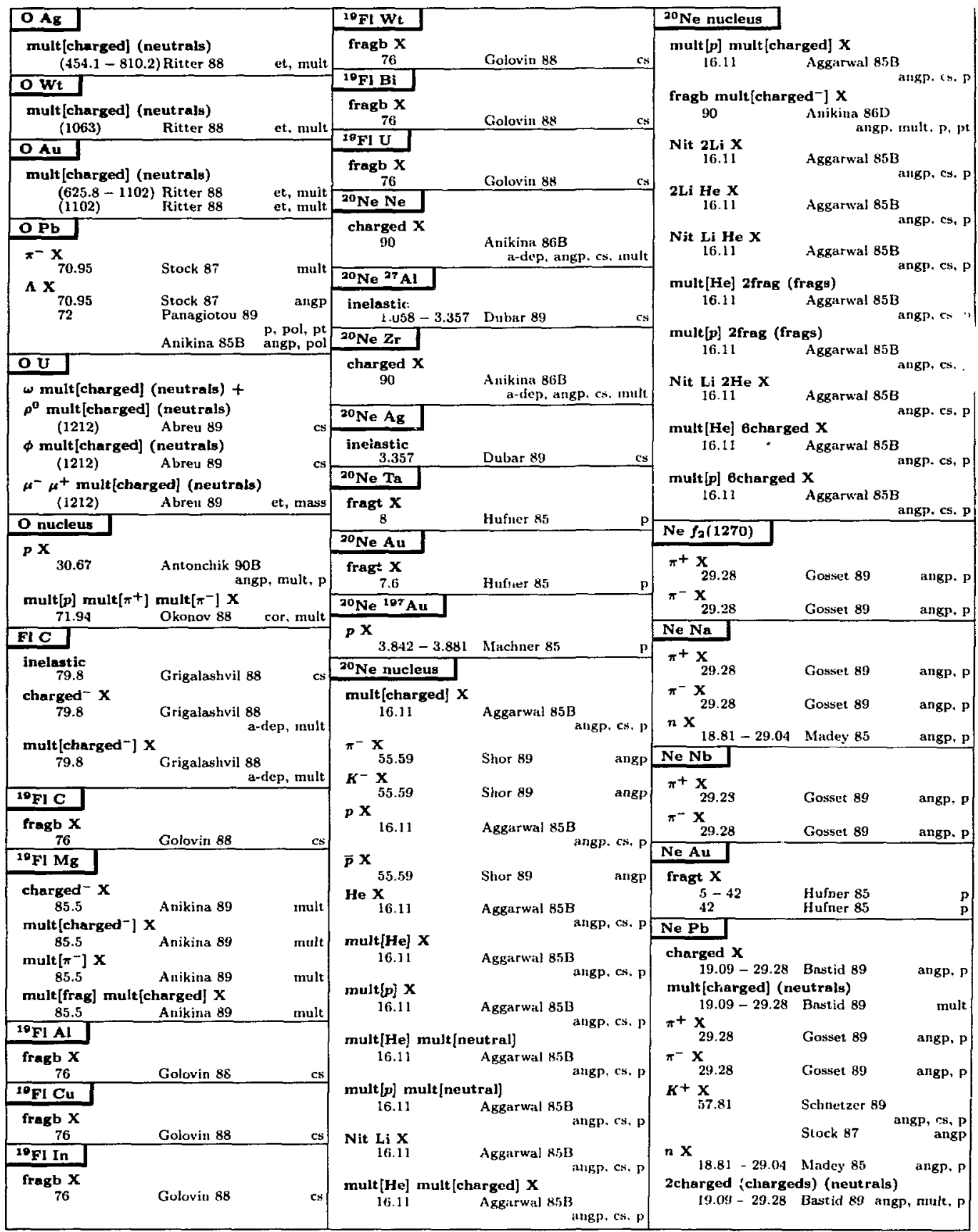

Entries in order of beam mass, then target mass, then miltiplicity of final state. Inclusive reactions have an "X" as the last of the final state particles. Certain chemical symbols for nuclei have heen changer to avoid ambiguity with particlo names (See the Particle vocabulary: Beam momenta are $P_{\text {inb }}$ in GeV/r, or in parentheses $E_{\text {rom }}$ in GeV. See the lenend on page 153. 


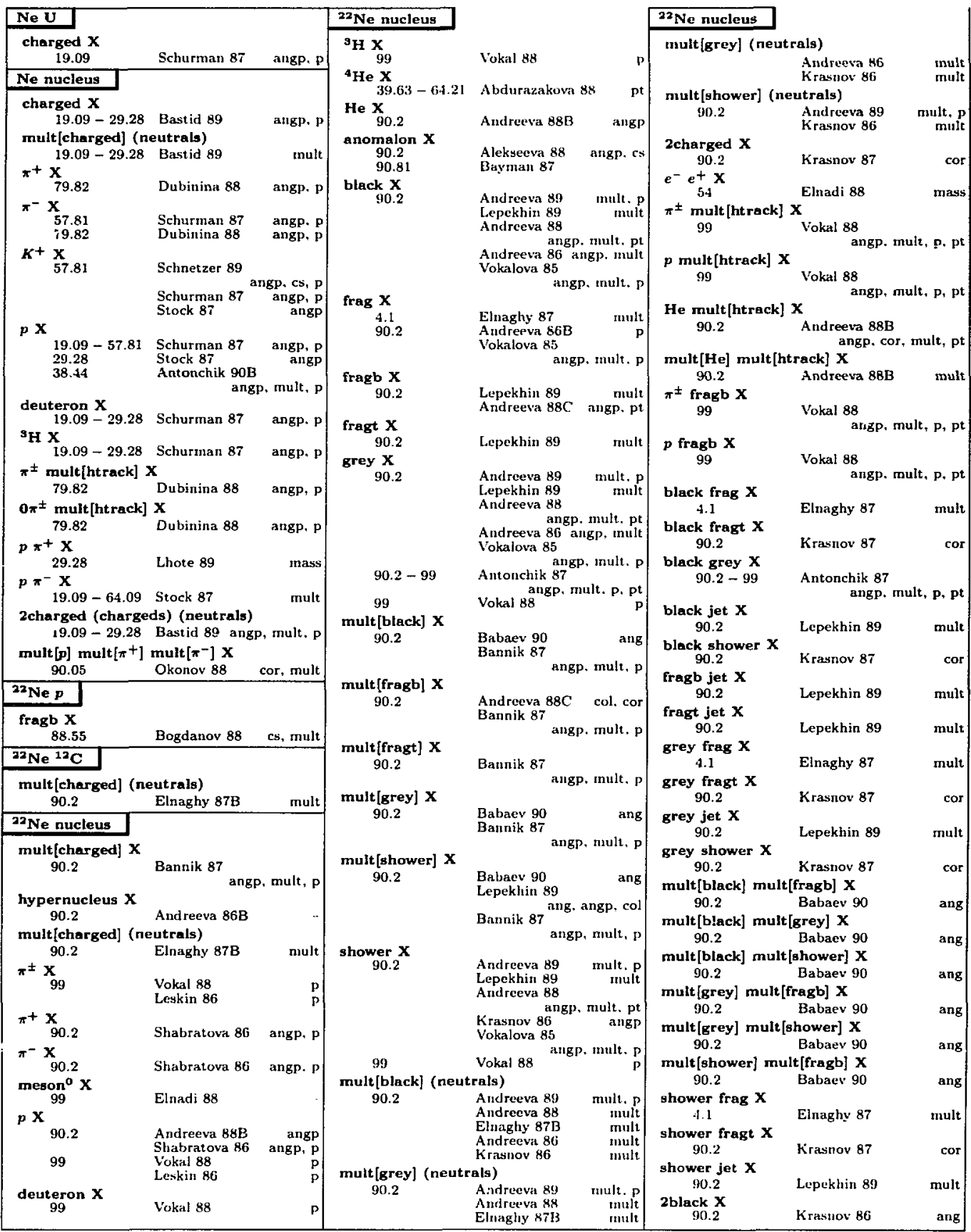




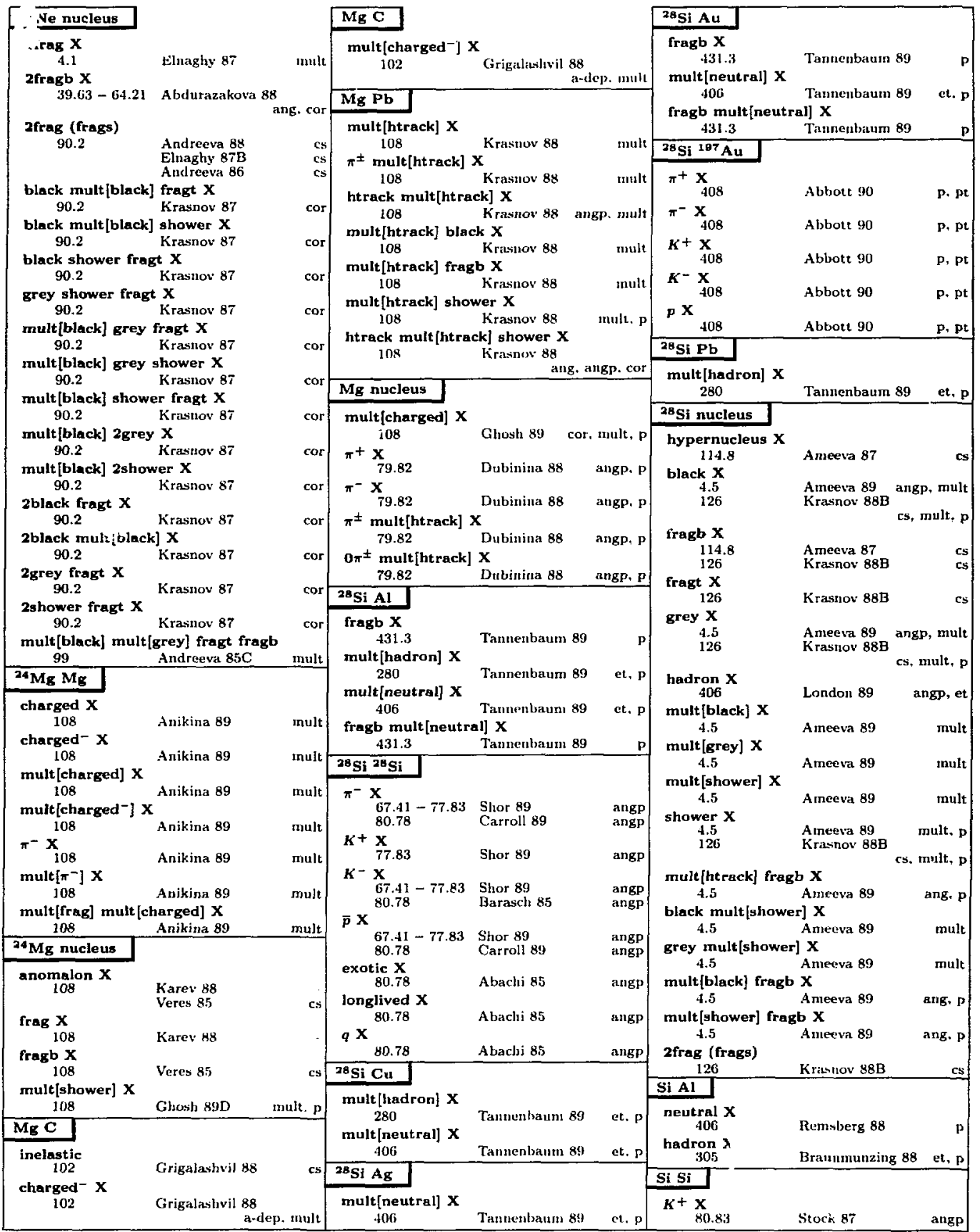




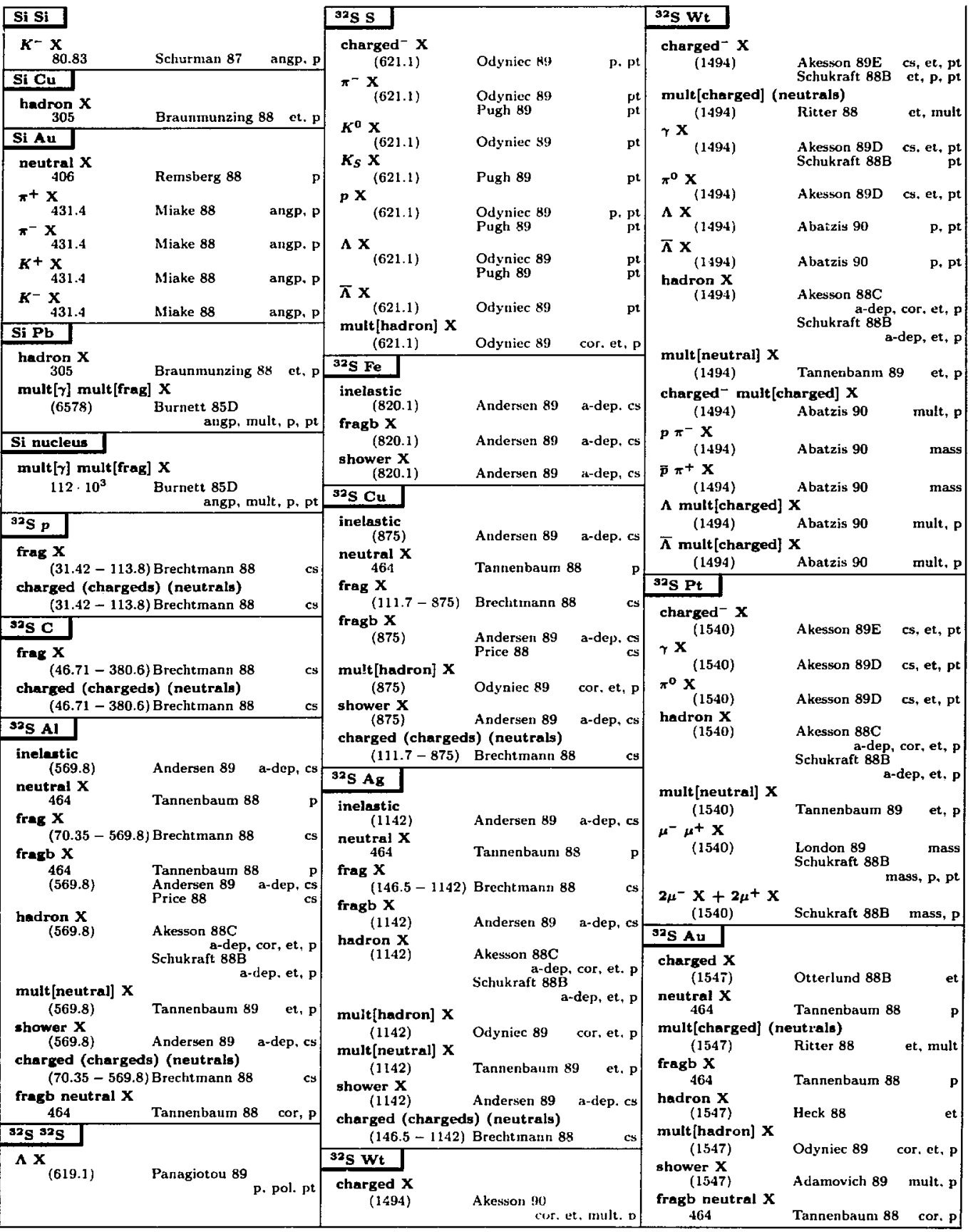




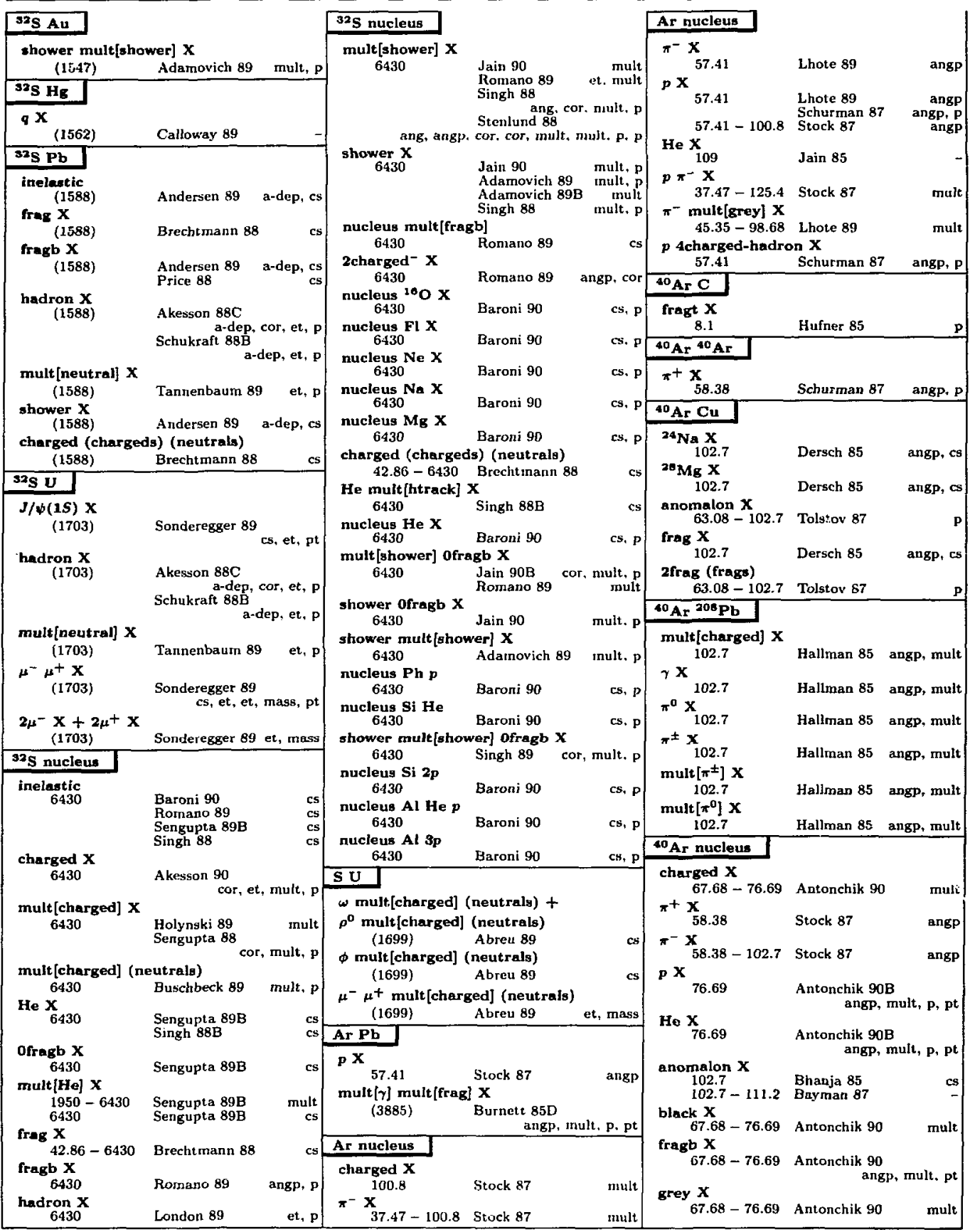

Entries in order of beam mass, then target mass, then multiplicity of final state. Inclusive reactions have an "X" as the last of the final state particles. Certain chemical symbols for nuclei have been changed to avoid ambiguity with particle names (See the Particle vocabulary.) Beam momenta are $P_{\mathrm{lat}}$ in $\mathrm{GeV} / c$, or in parentheses $\boldsymbol{E}_{\mathrm{cm}}$ in GeV. See the legend on page 153. 


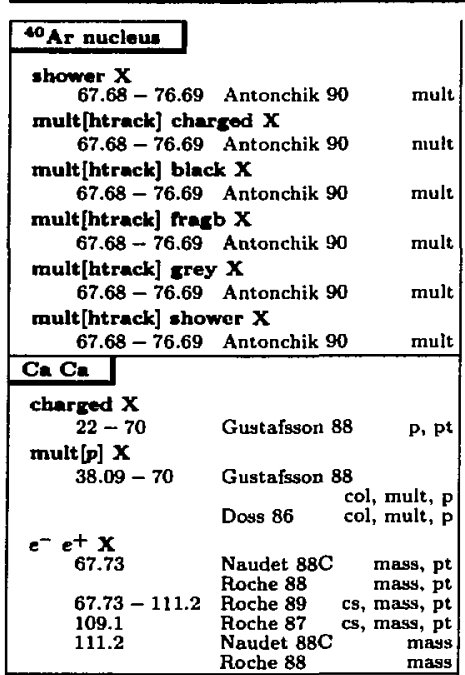

\section{$\mathbf{C a} \mathbf{P b}$}

mult $[\gamma]$ mult [frag] $X$

(2786) Burnett 85D

angp, mult, $p, p t$

$\gamma$ fragb 45lcharged (chargeds) $X$

(5276) Burnett 86 angp, p, pt

$\gamma$ frabb o70charged $X$

(2786) Burnett 86 angp, p.pt

Ce nucleus

charged $\mathbf{X}$

$12 \cdot 10^{3} \quad$ Chernavskaya $87 \quad \mathrm{p}, \mathrm{pt}$

$\mathbf{p} \mathbf{X}$

$600-8 \cdot 10^{3} \quad$ Gagarin $89 \quad$ angp, $P$

$p$ muit [htrack] $X$

$600-8 \cdot 10^{3}$ Gagarin $89 \quad$ angp, $P$ mult [ $\gamma$ ] mult [frag] $X$
$48 \cdot 10^{5}$
Burnett 85D

$\gamma$ fragb roocharged $x$

angp, mult, $p, p t$

\begin{tabular}{|c|c|c|}
\hline $4 \cdot 10^{\theta}$ & Burnett 86 & argp, $p, p t$ \\
\hline $\mathbf{T i} \mathbf{P b}$ & & \\
\hline 207charged $\mathrm{X}$ & & \\
\hline 1973 & Burnett 87 & angP, $P$ \\
\hline p 207charged X & & \\
\hline 1973 & Burnett 87 & ntult \\
\hline \multicolumn{3}{|c|}{$\gamma$ fragb $415 c h a r g e d$ (chargeda) $x$} \\
\hline$(4264)$ & Burnett 86 & angp, p, pt \\
\hline
\end{tabular}

Ti nucleus

2escharged $\mathbf{X}$

1315 Burnett 87 angp, $P$

p 205charged $X$

1315

Burnett 87 mult

$40 \mathrm{Se} B$

frogt $x$ 
This index lists papers by particles produced and their decass. ordered alphabetically by particle, then decay. For a given decay. ID:s are ordered by year (most recent to oldest). then author name. For the full reference, see the 1D/Reference/Title Index.

When no decay mode is given. no mode was given in the original paper.

\section{Illustrative Key}

Particle: see the Particle Focabulary for nomenclature.

Decay: decay mode of the particle.

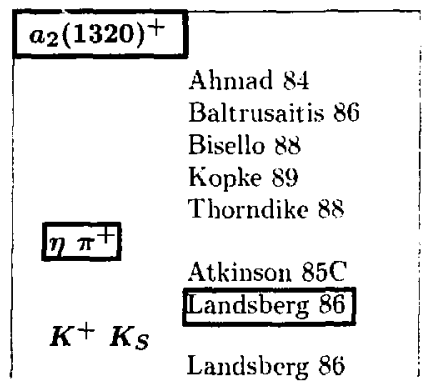

Document ID: see ID/Reference/Title Index for the iull reference. 


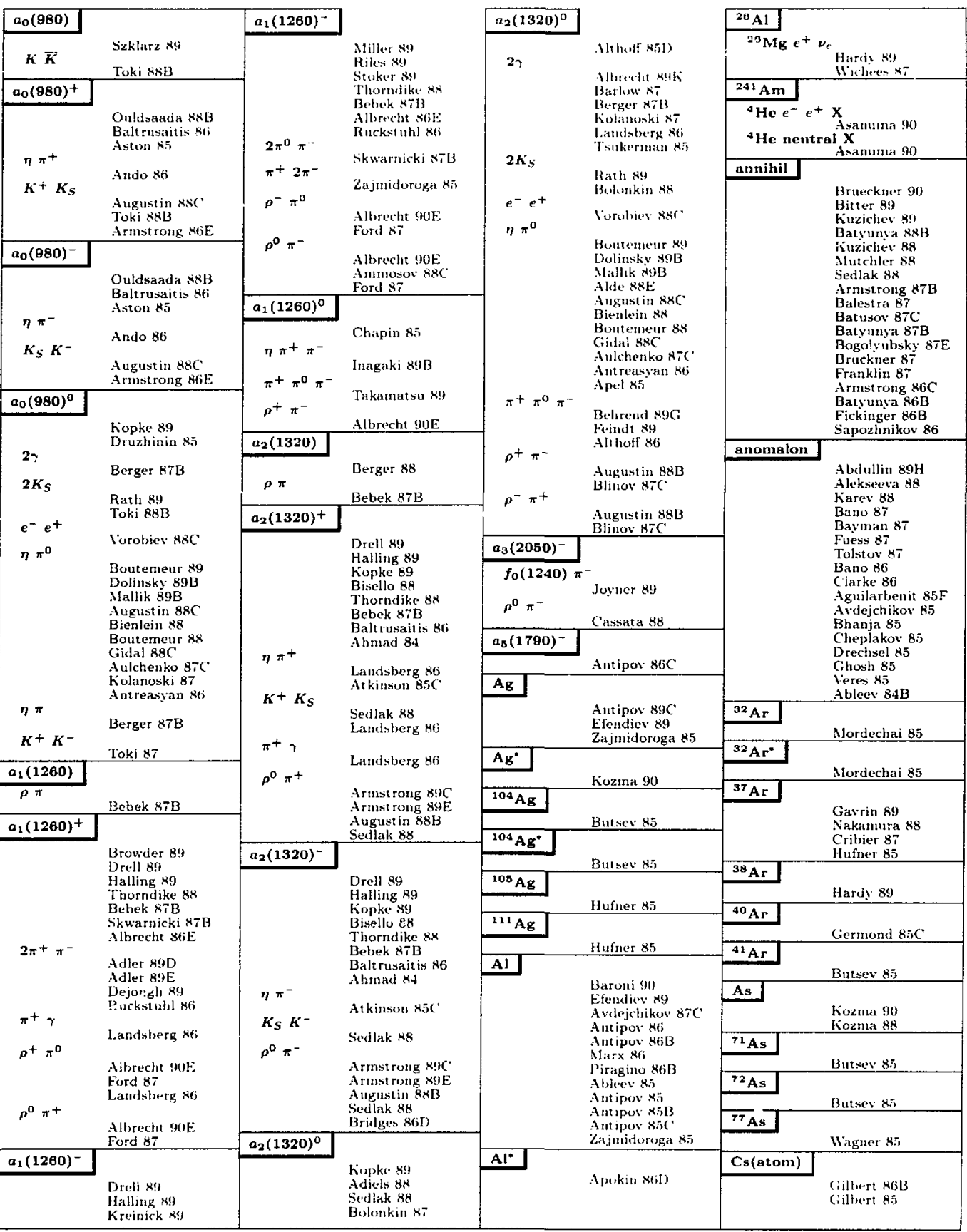




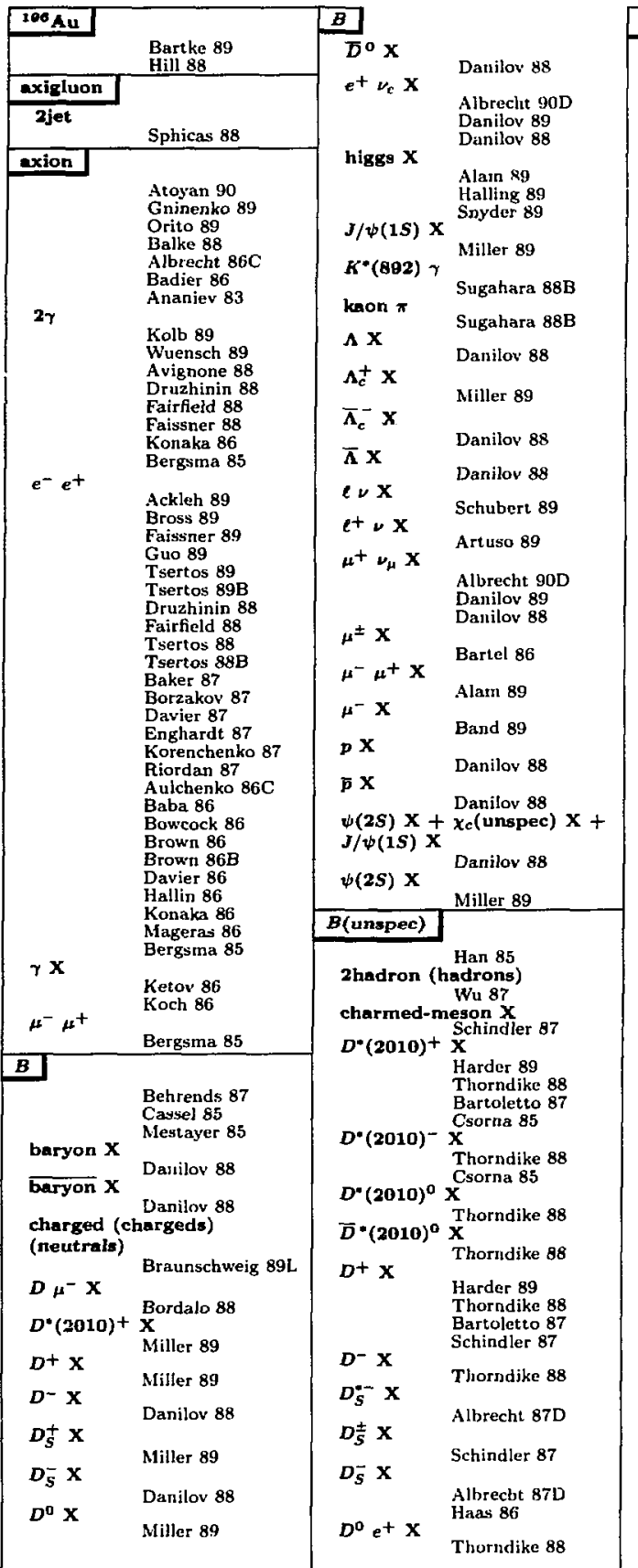

\begin{tabular}{|c|c|}
\hline$B$ (unspec) & \\
\hline$D^{0} e^{-} \mathbf{X}$ & \\
\hline$D^{0} \mu+\mathbf{x}$ & 1 ton \\
\hline$D^{0} \mu^{-X} \mathbf{X}$ & Thorndike \\
\hline$D^{0} \mathbf{X}$ & Thorndike \\
\hline & $\begin{array}{l}\text { Harder } 89 \\
\text { Thorndike } 88 \\
\text { Bartoletto } 87 \\
\text { Schindler } 87\end{array}$ \\
\hline $\bar{D}^{0} \mathbf{X}$ & Thorndi \\
\hline
\end{tabular}

$e^{ \pm} \nu_{e}$ charmed-meson

$e^{ \pm} \mathbf{X} \quad$ Wachs 89

$e^{ \pm} \mathbf{X} \quad$ Wachs 89

Tao 88

$e^{-} e^{+} \mathbf{X} \quad$ Schindler 87

$-x^{-}$Bean 87

$\mathbf{e} \quad$ Thorndike 88

$J / \psi(1 S) \pi^{+} \pi^{-} \mathrm{X}$

Albrecht $87 \mathrm{G}$

$J / \psi(1 S) \times$

Maschmann 89

Thorndike 88

Albrecht 87C

Schindler 87

Alam 86

Albrecht $85 K$

Hais 85

K"(unspec) higgs

Wu 87

$K^{+} e^{-X} \quad$ Tliondike 88

$K^{+} J / \psi(15) 2 \pi$

Albrecht $87 \mathrm{G}$

Albrecht $85 \mathrm{~K}$

$K^{+} J / \psi(1 S) 3 \pi$

Albrecht $85 \mathrm{~K}$

$K^{+} J / \psi(1 S)$

Albrecht $87 \mathrm{G}$

$\boldsymbol{K}^{+} \boldsymbol{\ell}^{-} \mathbf{X} \quad$ Albrecht 85K

$\mathrm{K}^{+} \boldsymbol{\mu}^{-} \mathrm{X} \quad$ Alam 87

Thorndike 88

$K^{-} e^{-} \quad$ Alam 87

Thorndike 88

$K^{-} J / \psi(1 S) 2 \pi$

Albrechn $85 \mathrm{~K}$

$K^{-} J / \psi(1 S) 3 \pi$

$K^{-} J / \psi(1 S)$ Albrecht $85 K$

Albrecht $85 \mathrm{~K}$

$K^{-} Z^{-} \mathrm{X} \quad A \operatorname{lam} 87$

$K^{-} \mu^{-} \mathbf{X}$ Thorndike 88

$\boldsymbol{K}^{-\mathbf{X}} \quad$ Thorndike 88 Alam 87

$K^{0} J / \psi(1 S) 2 \pi$

Albrecht $85 \mathrm{~K}$

$K^{0} J / \psi(1 S) 3 \pi$

$0 \quad s / \psi(1 S)$ Albrecht $85 \mathrm{~K}$

, 0 Albrecht $85 \mathrm{~K}$

Alam 87

$\bar{K}^{0} J / \psi(1 S) 2 \pi$

Albrecht $85 \mathrm{~K}$

K $J / \psi(1 S) 3 \pi$

$\overline{\boldsymbol{K}}^{0} J / \psi(1 S)^{\mathrm{A}} \pi$

$K_{S} e^{-} \mathbf{X}$ Albrecht $85 \mathrm{~K}$

Thorndike 88

\section{$B$ (unspec)}

$K_{S} J / \psi(1 S) 2 \pi$

$K_{S} J / \psi(1 S)$ Albrecht $87 \mathrm{G}$ $\pi$

Albrecht $87 \mathrm{G}$

$K_{S} \mu^{-} X \quad$ Thorndike 88

$\Lambda \bar{\Lambda} \quad$ Alam 87B

$\mathbf{\Lambda} \mathbf{X}$

Albrecht $89 \mathrm{E}$

Thorndike 88

Alam 87B

Schindler 87

$\mathbf{\Lambda}_{c}^{+} \mathbf{X}$

1]brecht $88 \mathrm{G}$

Alam 87B

$\bar{\Lambda} \mathbf{X}$

Thorndike 88

$\ell$ hadron (hadrons)

$\times \quad$ Elsen 90

C Wu 87

$\mu^{-} \mu^{+} X \quad$ Bean 87

$-x \quad$ Haas 85

p $K^{-} \pi^{+} \mathrm{X}^{\text {Thorndike } 88}$

$\mathbf{p} \overline{\mathbf{p}} \mathbf{X}$

Albrecht 88G

p $\mathbf{X}$

Alam $87 \mathrm{~B}$

Albrecht $89 \mathrm{E}$

Thorndike 88

A lam 878

$\overline{\boldsymbol{p}} \mathbf{X}$

Schindle: 87

$\phi \mathbf{X}$

Thorndike 88

$\pi^{+} \pi^{-} e^{-} e^{+} \mathbf{x a r}$

Bartoletto 86

Albrecht $87 \mathrm{G}$

$\pi^{+} \pi^{-} \mu^{-} \mu^{+} \mathrm{X}$

$\psi(2 S) \times \quad$ Albrecht 87G

Albrecht $87 \mathrm{G}$

Schindler 87

$\mathbf{E}^{-} \mathbf{x}$

Albrecht $89 \mathrm{E}$

B* Schindler 87

$B^{*}$ (unspec)

B(unspec) 7

$B^{*+}$ Han 85

\begin{tabular}{|lc|}
\hline & Schindler 87 \\
\hline$B^{*-}$ & \\
\hline & Schindler 87 \\
\hline$B^{* 0}$ & \\
\hline
\end{tabular}

$\boldsymbol{B}^{*}$

Schindler 87

Schindler 87

$B^{+}$

Albrecht 90D

Bortoletto 90

Artuso 89

Bortoletto 89

Bortoletto 89B

Kreinick 89

Miller 89

Albrecht 87P

Bean 87B

Bebek 87B

Schindler 87

$2 K^{+} K^{-}$

Alam 89

Albrecht 890

Entries in order of the equivalem English spe!ling. Certain chemical symbols for nuclei have been changed to avoid ambiguity with particle names (see the Particle Vocabulary). See the legend on page 297. 


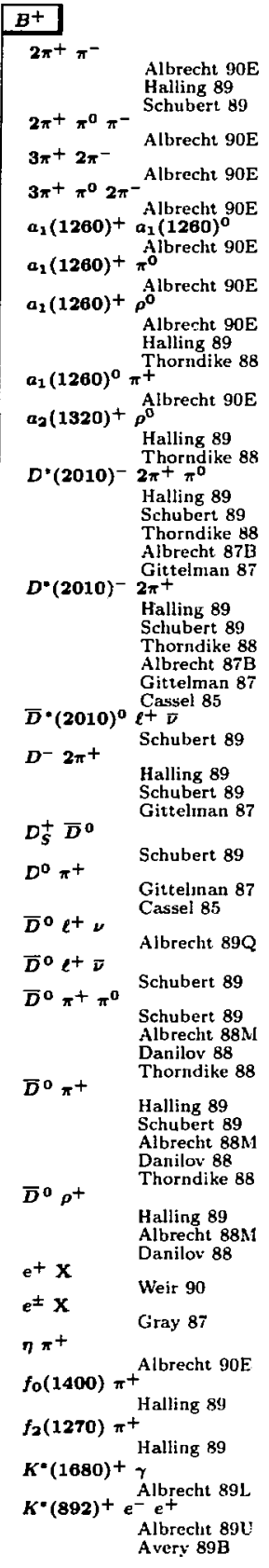

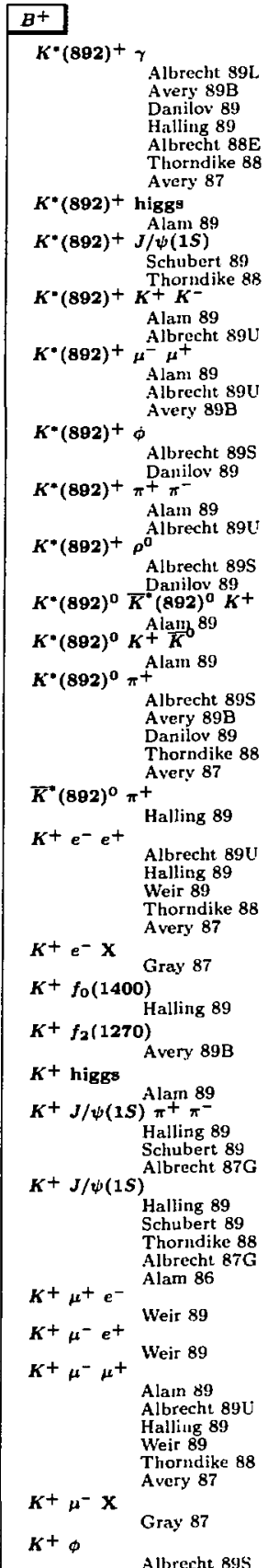

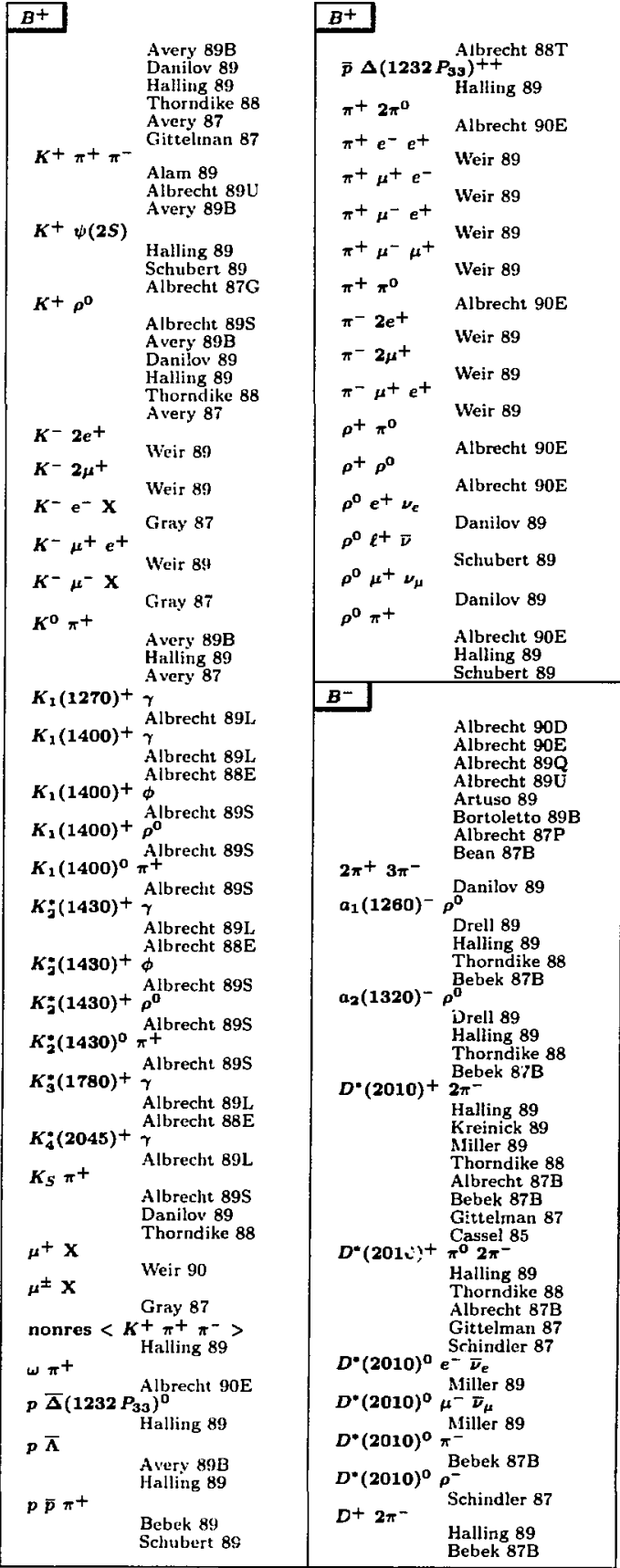




\section{$B^{-}$}

$D_{S}^{-} D^{*}(2010)^{\circ}$

$D_{S}^{-} D^{0}$

Bortoletto 90

Kreinjck 89

$D_{\bar{S}}^{-} \mathbf{x}$

Miller 89

$D^{0} e^{-} \bar{\nu}_{e}$

Bortolet to 90

$D^{\circ} K^{+} K^{-} \pi^{-}$

Bortoletto 90

$D^{0} \mu^{-} \bar{\nabla}_{\mu} \quad$ Bortoletto 90

$D^{0} \mu^{-} \quad$ Miller 89

$D^{0} \pi^{-}$

Albanese 85

Halling 89

Kreinick 89

Miller 89

Albrecht 88M

Danilov 88

Thorndike 88

Bebek 87B

Gittelman 87

Schindier 87

Cassel 85

$D^{0} \pi^{0} \pi^{-}$

Albrecht $88 \mathrm{M}$

Danílov 88

Thorndike 88

$D^{\mathbf{0}} \rho^{-}$

Halling 89

Albrecht $88 \mathrm{M}$

Danilov 88

$e^{ \pm} \mathbf{x}$

Gray 87

$e^{-\mathbf{x}}$

Weir 90

$f_{0}(1400) \pi^{-}$Halling 89

$f_{0}(875) \pi^{-}$

Drell 89

$f_{2}(1270) \pi$

Bortolet to 89

Drell 89

Halling 89

$K^{*}(1880)^{-} \gamma$

Albreche 891 $\mathrm{e}^{+}$

$K^{*}(892)^{-} \gamma$

A very $89 \mathrm{~B}$

Albrecht 89L

A very $89 \mathrm{~B}$

Halling 89

Albrecht 88E

Thorndike 88

A very 87

$K^{*}(892)^{-}$higgs

Alam 89

$K^{*}(892)^{-} J / \psi(1 S)$

Kreinick 89

Miller 89

Thorndike 88

$\boldsymbol{K}^{*}(\mathbf{8 8 2})^{-} \boldsymbol{K}^{+} \boldsymbol{K}^{-}$

Alam 89

$K^{*}(892)-\mu^{-} \mu^{+}$

Alam 89

Avery $89 \mathrm{~B}$

$K^{*}(892)^{-} \phi$

Albrecht 895

$K^{*}(\mathbf{8 9 2})-\pi^{+} \boldsymbol{\pi}^{-}$Alam 89

$K^{*}(892)^{-} \psi(2 S)$

Kreinjck 89

Miller 89

\section{$B^{-}$}

$K^{*}(892)^{-} \rho^{0}$

$K^{\circ}(\mathbf{8 8 2})^{0}$ Albrecht $89 \mathrm{~S}$

$K^{\circ}(\mathbf{8 8 2})^{\circ}{ }_{\text {Alam } 89}$

$K^{*}(\mathbf{8 8 2})^{0} \pi^{-}$

Halling 89

$\bar{K}^{*}(892)^{0} K^{0} K^{-}$

$\bar{K}^{*}(\mathbf{8 9 2})^{\circ} \pi^{-}$Alam 89

Albrecht 895

Avery $89 B$

Thorndike 88

Avery 87

$\boldsymbol{K}^{+} 2 \boldsymbol{e}^{-}$

$K+2 K^{-}$

Weir 89

$\boldsymbol{K}^{+} \mathbf{2 \mu ^ { - }}$

Alam 89

$\boldsymbol{H}^{+} \boldsymbol{\mu}^{-} \mathrm{e}^{-}$

Weir 89

$K^{-} e^{-} e^{+}$

Weir 89

$\begin{array}{lll} & e^{-} & e^{+} \text {Halling } 89\end{array}$

Weir 89

Thorndike 88

$K^{-} f_{0}(1400)$

A very 87

$K^{-} f_{2}(1270)$

Halling 89

$\mathrm{K}^{-}$higgs

Avery $89 \mathrm{~B}$

K $\boldsymbol{J} / \psi(\mathbf{I S )}$ Alam 89

$\pi^{+} \pi^{-}$

Halling 89

Kreinick 80

Miller 89

$K^{-J / \psi(1 S)}$

Albrecht $87 \mathrm{G}$

Halling 89

Kreinick 89

Miller 89

Thorndike 88

Albrecht $87 \mathrm{G}$

Bebek 87B

$K^{-} \mu^{+} e^{-}$

Alam 86

$\boldsymbol{K}^{-} \boldsymbol{\mu}^{-} \mathrm{e}^{+}$

Weir 89

$\boldsymbol{K}^{-} \boldsymbol{\mu}^{-} \boldsymbol{\mu}^{+}$

Weir 89

Alam 89

Halling 89

Weir 89

Thorndike 88

Avery $8 \hat{\mathbf{i}}$

$K^{-} \phi$

Albrecht 89S

Avery 89B

Halling 89

Thorndike 88

Avery 87

Git telman 8 ?

$K^{-} \pi^{+} \pi^{-}$

Alam 89

A very $89 B$

$K^{-} \psi(2 S)$

Halling 89

Kreinick 89

Miller 89

Albrecht 87G

$K^{-} \rho^{\circ}$

Albrecht 89 S

Avery $89 \mathrm{~B}$

Halling 89

Thorndike 88

$A$ very 87

$K_{1}(1270)^{-} \gamma$

Albrecht 89L

$K_{1}(1400)^{-\gamma}$

Albrecht $89 \mathrm{~L}$
$B^{-}$

$K_{1}(1400)^{-} \phi$

$K_{1}(1400)$ - Albreclit 895

$\bar{K}_{1}(1400)^{0} \pi^{-}$

$K_{2}^{*}(1430)^{-}$

Albrecht $89 \mathrm{~L}$
Albrecht $88 \mathrm{E}$

$K_{2}^{*}(1430)^{-} \phi$

$K_{2}^{\prime(1430)}$ - Albrecht 895

Albrecht $89 \mathrm{~S}$

$\bar{K}_{2}^{*}(1430)^{0} \pi^{-}$Albrecht 895

$K_{9}^{*}(1780)^{-}$?

Albrecht 89L

Albrecht 88E

$K_{g}^{*}(2045)^{-} \gamma$ Albrecht $89 \mathrm{~L}$

$\bar{K}^{\mathbf{0}} \boldsymbol{\pi}^{-}$

A very $89 \mathrm{~B}$

Halling 89

Avery 87

$K_{5} \pi^{-}$

Albrecht $8 \mathrm{r}$ -

$\mathbf{A} e^{+} \mathbf{x}$

Thorndike 88

$\Lambda \boldsymbol{\mu}+\mathbf{x}$

Schubert 89

A $\mathbf{X}$

Schubert 89

$\Lambda_{c}^{+} \mathbf{X}$

Schubert 89

$\bar{A} e^{+} \mathbf{X}$

Schubert 89

$\pi_{\mu}+\mathbf{X}$ Schubert 89

$\mathbf{\Lambda} \mathbf{X}$

Schubert 89

$\mu^{ \pm} \mathbf{x}$

Schubert 89

$\mu^{-} \mathbf{X}$

Gray 87

Weir 90

Halling 89

p $\bar{\Delta}\left(1232 P_{33}\right)^{-}$

Halling
Drell 89

$p e^{+} \mathrm{X}$

Halling 89

$p \bar{\Lambda} \mathbf{X}$

Schubert 89

$\boldsymbol{p} \boldsymbol{\mu} \mathbf{x}$

Schubert 89

$p \bar{p} e^{+} \mathbf{X}$

$p \bar{p} \mu+X$

Schubert 89

$p \bar{p}$

Schubert 89

Bebek 89

Danilov 89

Drell 89

Albrecht 88T

Sugahara 88B

$p \bar{p} \mathbf{x}$

Schubert 89

$p \mathbf{X}$

Schubert 89

$\bar{p} \Delta\left(1232 P_{s 3}\right)^{\circ}$

Drell 89

$\bar{p} e^{+} \mathbf{x}$

Halling 89

$\overline{\boldsymbol{p}} \boldsymbol{\Lambda}$.

Schubert 89

$\overline{\mathbf{p}} \boldsymbol{\Lambda}$

Schubert 89

Avery $89 \mathrm{~B}$

Halling 89
$B^{-}$

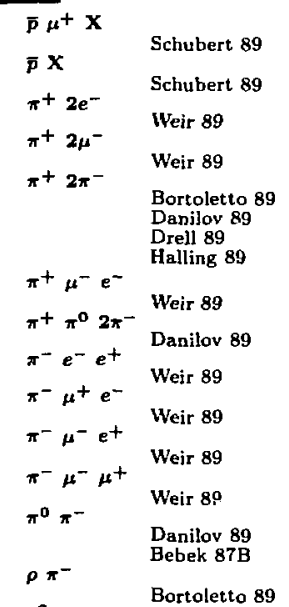

$\rho^{a} \ell^{-}$

$\rho^{0} x^{-}$

Albrecht 89 T

Danilov 89

Drell 89

Halling 89

Bebek 87B

Cassel 85

\begin{tabular}{cc} 
& Cassel 85 \\
\hline$B_{S}$ & \\
$\bar{B}_{S}$ & Schindler 87
\end{tabular}

Eggert 89

Franzini 89

ILep 89

Porter 89

Sugahara 88B

Albajar 87C

Summers 87

Schaad 85

$e^{-x}$

Porter 89

$\mu^{+} \mathrm{X}$

$\mu^{-} \mu^{+}$

Summers 87

$\mu^{-} \mathbf{X}$

Albajar $88 \mathrm{C}$

Porter 89

Albajar 87C

Summers 87

\section{$\overline{\boldsymbol{B}}_{S}$ \\ Franzini 89}

Itep 89

Sugahara 88B

Schindler 87

$\boldsymbol{B}_{\mathbf{S}}$

Exgert 89

Porter 89

Schaad 85

$e^{+} \mathbf{X}$

$\mu+\mathbf{x}$

Porter $\mathbf{8 9}$

Porter 89

Albajar $87 \mathrm{C}$

Summers 87

$\mu^{-} \mu^{+}$

Albajar 88C

$\boldsymbol{\mu}-\mathbf{X}$

Summers 87

Entries in order of the equivalent English spelling. Certain chemical symbols for muclej have been changed to avoid ambiguity with particle names (see the Particle Vocabulary). See the legend on page 297. 
$\boldsymbol{B}^{\circ}$

Bortoletto O Bortoletto 89 Kreinick 89 Miller 89

Bebek 87B

Schindler 87

$2 x^{+} 2 \pi^{-}$ Albrecht 90E
Danilov 89

$2 \pi^{0} \quad$ Albr-iht 90E

Albrecht 90E

Halling 89

Schubert 89

Thorndike 88

$3 \pi^{+} \mathbf{3 \pi}^{-}$Albrecht 90E Danilov 89

$3 \pi^{+} \pi^{0} 3 \pi^{-}$

Albrecht 90E

$a_{1}(1260)+a_{1}(1260)^{-}$

Albrecht 90E

Halling 89

$a_{1}(1260)+x^{-}+$

$a_{1}(1200)^{-} x^{+}$

$a_{1}(1200)+\pi^{-}$

Halling 89

Albrecht 90E

$a_{1}(1260)+p^{-}$

Thorndike 88

Albrecht 90E

Thorndike 88

$a_{1}(1200)^{0} \pi^{0}$

$a_{1}(1200)^{0} \rho^{0}$ Albrecht $90 \mathrm{E}$

Albrecht 90E

$a_{0}(1320)+\pi^{-}+$

$a_{2}(1320)^{-} \pi^{+}$

$\boldsymbol{B}^{0}$

Halling 89

Weir 90

Artuso 89

Danilov 89

Drell 89

Eggert 89

Franzini 89

Hurst 89

Itep 89

Porter 89

Band 98

Dowell 88

Sugahara 88B

Tao 88

Albajar $87 \mathrm{C}$

Albrecht 87P

Bean 87B

Gittelman 87

Summers 87

Schaad 85

$D^{*}(2010)+\ell^{-} \bar{D}$

Halling 89

$D^{*}(2010)+\mu^{-} \bar{\nu}_{\mu}$

$D^{*}(2010)^{+} \mathrm{X}$

Wagner 89B

Averill 89

$2 \pi+\pi^{-}$

Halling 89

Schubert 89

Thorndike 88

Albrecht 87B

Albrecht 87P

$D^{*}(2010)-e^{+} \nu$

Albrecht $89 \mathrm{C}$

Bortolet to 89B

Danilov 89

Wagner 89B

Albrecht 870

Albrecht 87P
$B^{\circ}$

$D^{*}(2010)^{-} \ell^{+}$

Albreclit 890

$D^{*}(2010)-\ell^{+} \bar{D}$

Schubert 89

$D^{*}(2010)^{-} \mu^{+} \nu_{\mu}$

Albrecht $89 \mathrm{C}$

Bortolet to $89 \mathrm{~B}$

Danilov 89

Waguer 89B

Albrecht $87 \mathrm{O}$

Albrecht 87P

$D^{*}(2010)-\pi^{+} \pi^{\circ}$

Halling 89

Schubert 89

Thorndike 88

Albrecht 87B

Albrecht 87P

$D^{*}(2010)^{-} \pi^{+}$

Halling 89

Schubert 89

Thorndike 88

Albrecht 87B

Albrecht 87P

$D^{*}(2010)-\rho^{+}$

Albrecht $85 \mathrm{~N}$

Halling 89

Chen 85

$D^{-} e^{+} \nu_{e}$

$D^{-} C^{+}$

Danilov 89

Albrecht 89N

Albrecht $89 \mathrm{Q}$

$D^{-} \mu^{+} \nu_{\mu}$

$D^{-}+\boldsymbol{\pi}^{0}$

Danilov 89

Schubert 89

Albrecht 88M

Danilov 88

$D^{-} \boldsymbol{x}^{+}$

Thorndike 88

Halling 89

Schubert 89

Albrecht 88M

Danilov 88

$D^{-} p^{+}$

Thorndike 88

Halling 89

Albrecht 88M

Danilov 88

$D_{s}^{+} D^{-}$

Halling 89

Schubert 89

$D^{0} \rho^{0}$

Danilov 98

$\bar{D}^{0} \boldsymbol{\pi}^{+} \boldsymbol{\pi}^{-}$

Thorndike 88

$\bar{D}^{0} \pi^{-} e^{+} \nu_{c}$

Albrecht $89 \mathrm{C}$

Albrecht $89 \mathrm{C}$

$\Delta\left(1232 P_{33}\right)^{++}$Albrecht $88 \mathrm{M}$

$\overline{\mathbf{\Delta}}\left(1232 P_{\mathbf{3 s}}\right)^{--}$

Halling 89

$\Delta\left(1232 P_{33}\right)^{\circ} \bar{\Delta}\left(1232 P_{33}\right)^{0}$

Halling 89

$e^{+} \nu_{e} \mathbf{X}$

$e^{+} \mathbf{x}$

Drell 89

Weir 90

et $x$

Porter 89

$e^{-} e^{+}$

Gray 87

Avery $89 \mathrm{~B}$

Halling 89

Thorndike 88

A very 87

$e^{-x}$

Bean $87 \mathrm{~B}$
$B^{\circ}$

$K^{*}(1680)^{\circ} \gamma_{\text {Albrecht } 89 \mathrm{~L}}$ $K^{*}(\mathbf{8 g 2})+\pi^{-}$

Albrecht 89S

Avery 89B

Danilov 89

Halling 89

Avery 87

$K^{*}(892)^{-} \pi^{+}$

Thorndike 88

$K^{*}(892)^{\circ} 2 K^{*}(892)^{0}$

Alam 89

$K^{*}(\mathbf{8 8 2})^{0} e^{-e^{+}}$

Albrecht 89U

Avery 89B

Halling 89

$K^{*}(892)^{\circ} f_{0}(1400)$

Halling 89

$K^{*}(892)^{0} f_{2}(1270)$

$K^{*}(892)^{\circ}$

Avery 89B

Albrecht 89L

Avery $89 \mathrm{~B}$

Daniov 89

Halling 89

Albrecht 88E

Avery 87

$K^{*}(892)^{0}$ higgs

Alam 89

$K^{*}(892)^{0} J / \psi(1 S)$

Halling 89

Schubert 89

Thorndike 88

Albrecht 87G

Alam 86

$K^{*}(892)^{0} K^{*}(892)^{-} K^{+}$

Alam 89

$K^{*}(892)^{\circ} \bar{K}^{*}(892)^{0} K^{\circ}$

$K^{*}(\mathbf{8 9 2})^{0} K^{+} K^{-}$

Alam 89

Alurecht $89 \mathrm{U}$

$K^{*}(802)^{0} \mu^{-} \mu^{+}$

Alam 89

Albrecht $89 \mathrm{U}$

Avery $89 \mathrm{~B}$

Halling 89

$K^{\bullet}(892)^{\circ}$

Albrecht 895

Avery 89B

Danilov 89

Halling 89

A very 87

$K^{*}(892)^{0} \pi^{+} \pi^{-}$

Alam 89

Albrecht 89U

$K^{*}(892)^{0} \psi(2 S)$

$K^{*}(892)^{0} \rho^{\text {A }}$ Albrecht 87G

Albrecht 89S

Avery 89B

Danilov 89

Halling 89

Avery 87

$\bar{K}^{*}(892)^{\circ}$ higgs

Alam 89

$\bar{K}^{*}(882)^{\circ} \psi(2 S)$

$K^{+} \mathrm{e}^{-} \mathbf{X}$ Schubert 89

Gray 87

$K^{+} K^{0} K^{-}$Alain 89

$K^{+} K_{S} K^{-}$Alain 89

$K^{+} \mu^{-} \mathbf{X}$

Albrecht $89 \mathrm{U}$

$\boldsymbol{K}^{+} \boldsymbol{\pi}$

Gray $\mathbf{8 7}$

Albrecht 89S

A verv 396
$B^{0}$

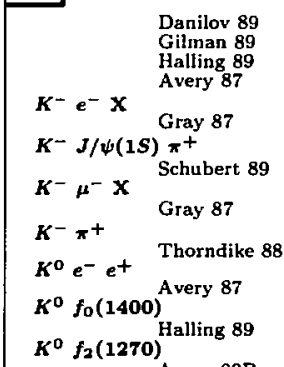

D A very $89 \mathrm{~B}$

$K^{0} J / \psi(1 S){ }^{\text {Alam } 89}$

Halling 89

Schubert 89

Alam 86

$K^{0} \mu^{-} \mu^{+}$

$K^{0} \phi$

Avery 87

Avery $89 \mathrm{~B}$

$K^{0} \pi^{+}$

Halling 89

$K^{0} \rho^{0}$

Alam 89

Avery 89B
Halling 89

Avery 87

$K_{1}(1270)^{\circ} \gamma$

Albrecht $89 \mathrm{~L}$

$K_{1}(1400)^{\circ}$ Albrecht 89S

Albrecht $89 \mathrm{~L}$

$K_{1}(1400)^{\circ} \stackrel{A}{\phi}$

Albrecht $89 \mathrm{~L}$

(1400)0 Albrecht 89S

K(1430)+ Albrecht $89 S$

Albrecht 89 S

$K_{2}^{*}(1430)^{0} \gamma$

Albrecht 89L

$K_{2}^{*}(1430)^{0} \stackrel{A}{\phi}$

$K_{2}(1430)^{0} \rho^{0}$ Albrecht $89 S$

K* (1780) $)^{\circ}$ Albrecht 895

$\mathrm{K}_{\mathrm{g}}\left(\mathbf{1 7 8 0 )} \boldsymbol{\gamma}_{\text {Albrecht }} 89 \mathrm{~L}\right.$

$K_{4}^{*}(2045)^{\circ} \hat{\gamma}$

$K_{S} e^{-} e^{+}$

Albrecht 89L

$K_{S} J / \psi(1 S)$

Albrecht $89 \mathrm{U}$

Albrecht 87G

Thorndike 88

$\boldsymbol{K}_{\boldsymbol{S}} \phi$

Albrecht 895

$\boldsymbol{K}_{S} \pi^{+} \pi^{-}$

Danilov 89

$K_{S} \rho^{0}$

Albrecht $89 \mathrm{U}$

Albrecht 895

Danilov 89

$\mu^{+} e^{-}$

Thorndike 88

Avery $89 \mathrm{~B}$

Thorndike 88

Avery 87 


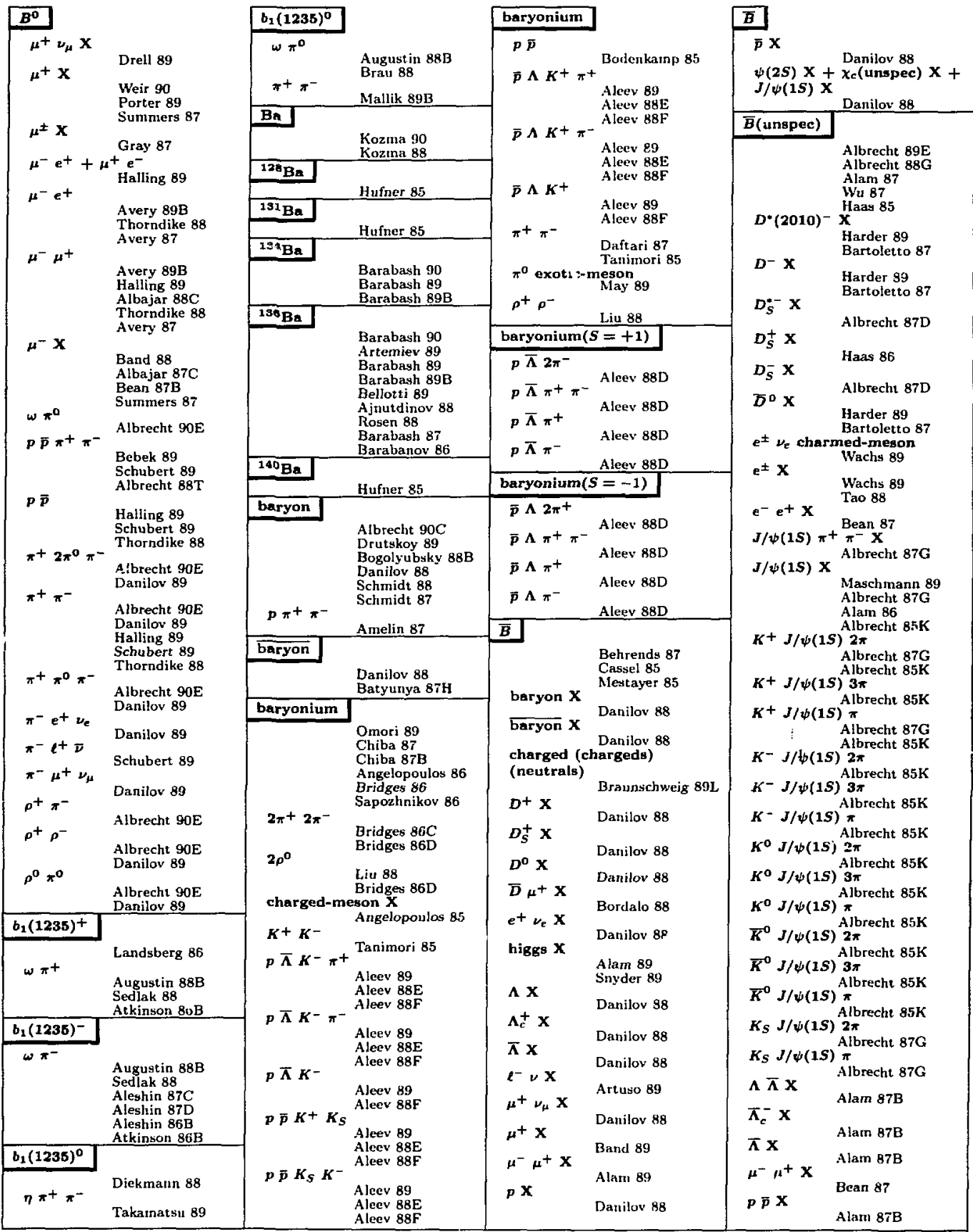

Entries in order of the equivalent English spelling. Certain chemical symbols for duclei have hecn changed to avoid ambiguity with particle names (see the Particle Vocabulary). See the legend on page 297 . 


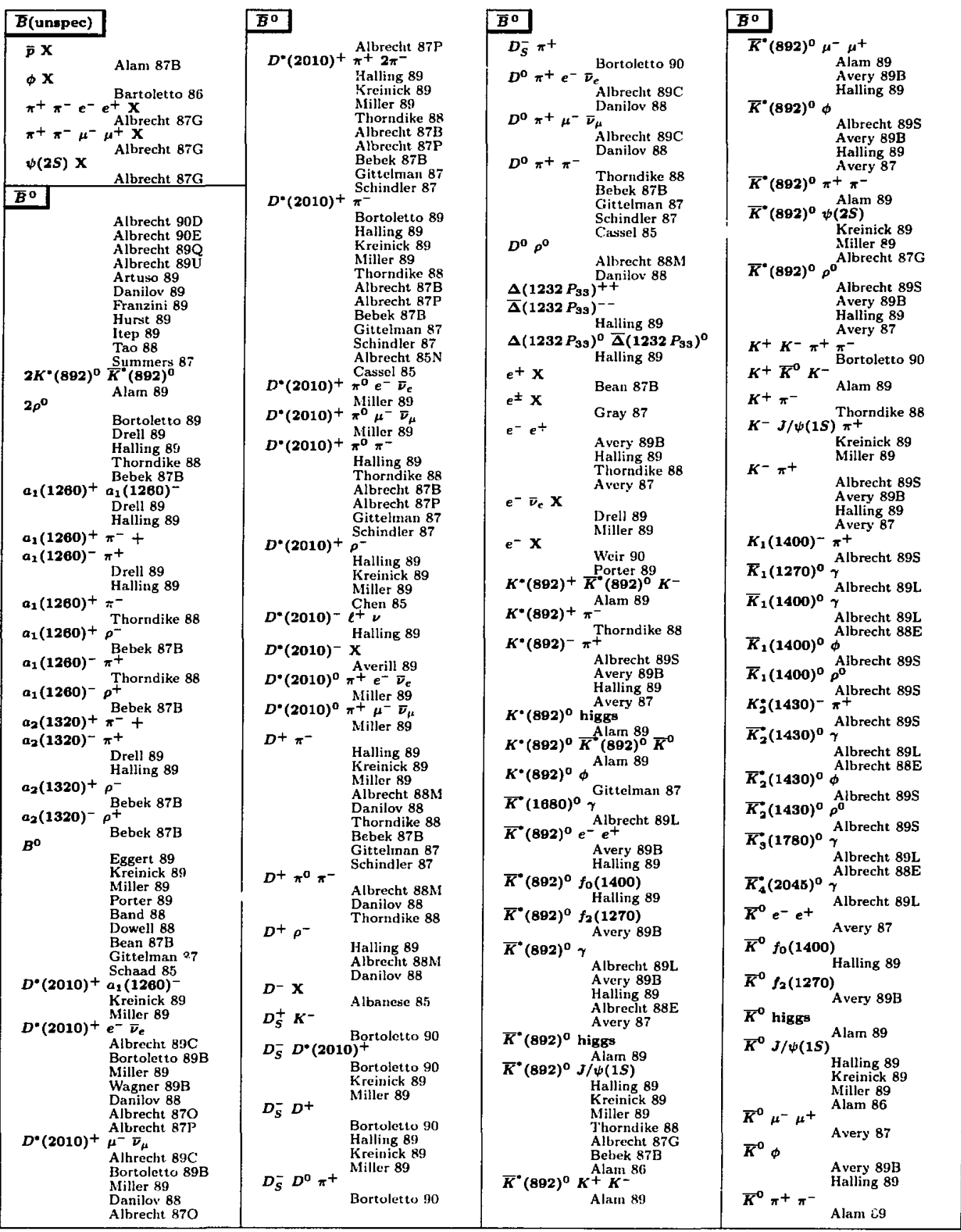




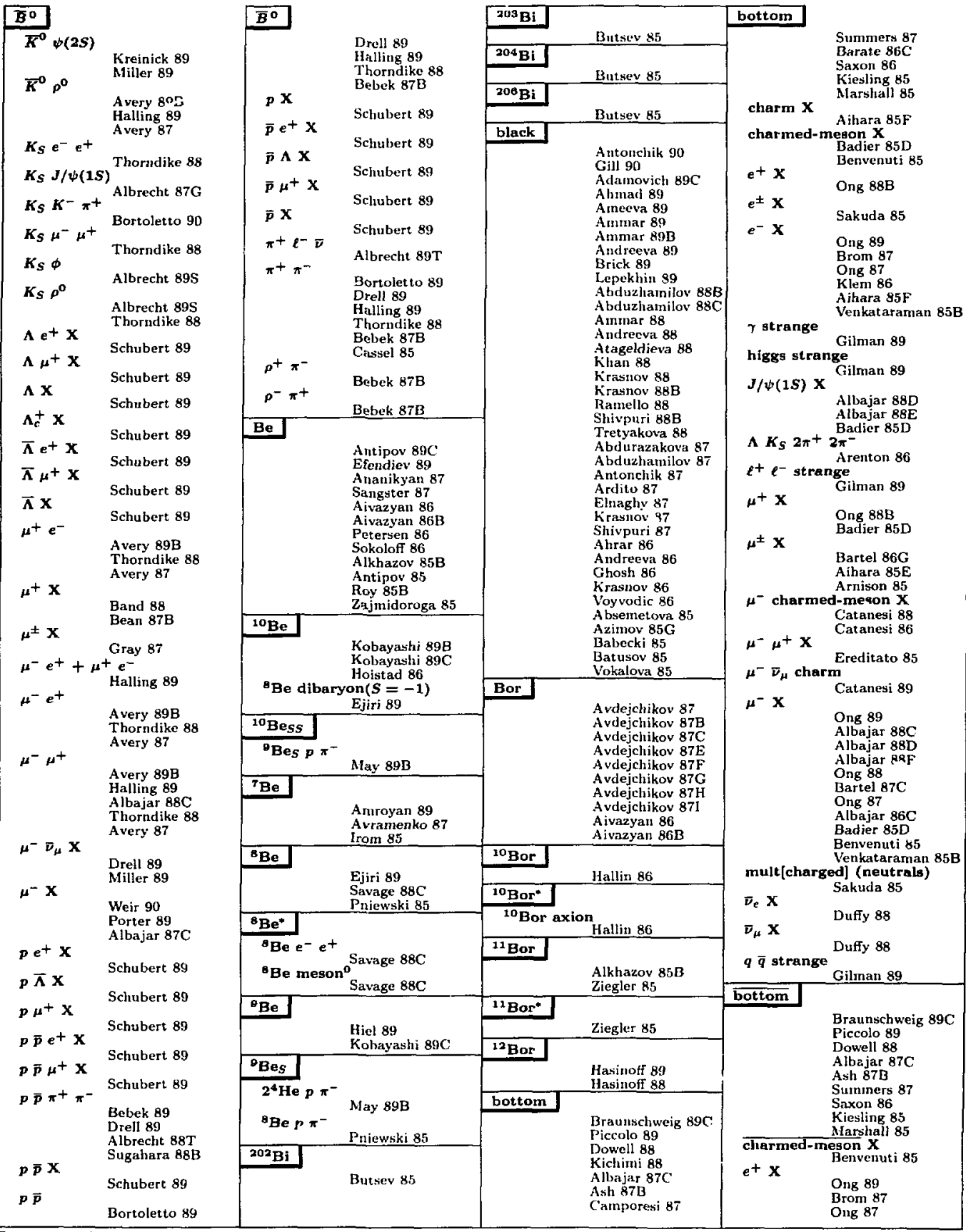

Entries in order of the equivalem English spelling. Certain chemicial symbols for nuclei have loeen changed to avoid ambiguity with particle names (see the Particle Vocabulary). See the legend on page 297. 


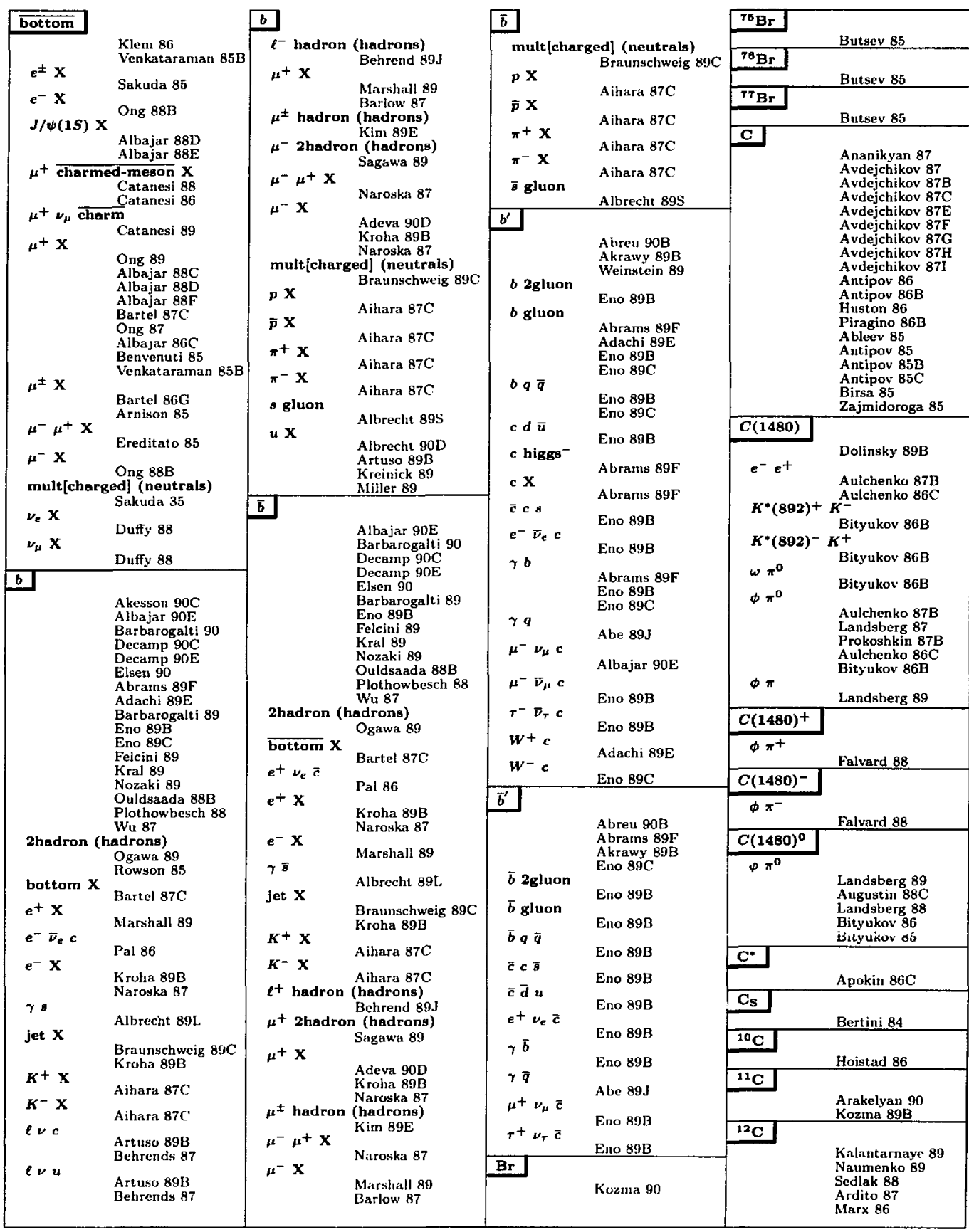




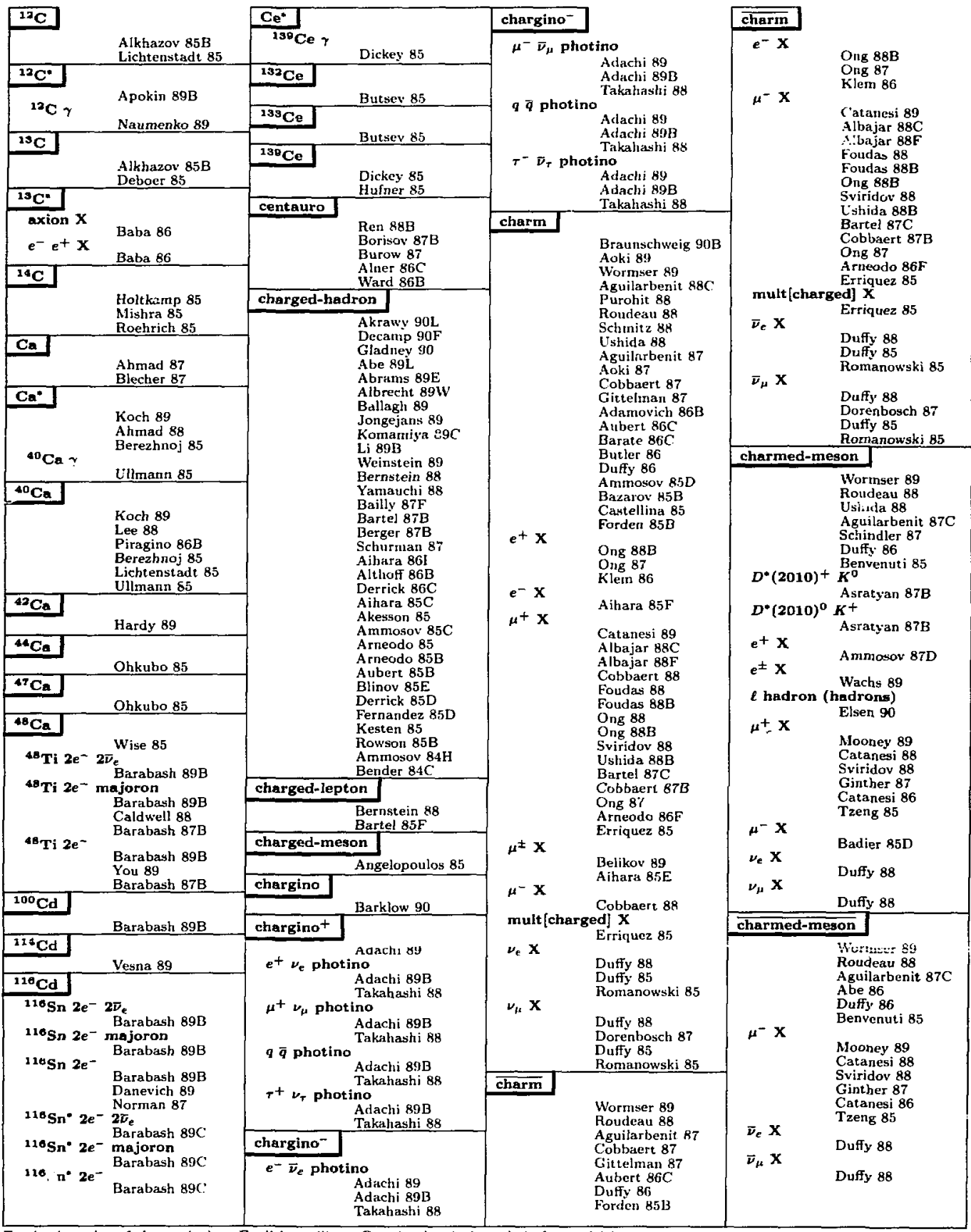

Entries in order of the equivalent English spelling. Certain chemical symbols for nuclei have been changed to avoid ambiguity with particle names (see the Particle Vocabulary). See the legend on page 297 
charmed-nucleus

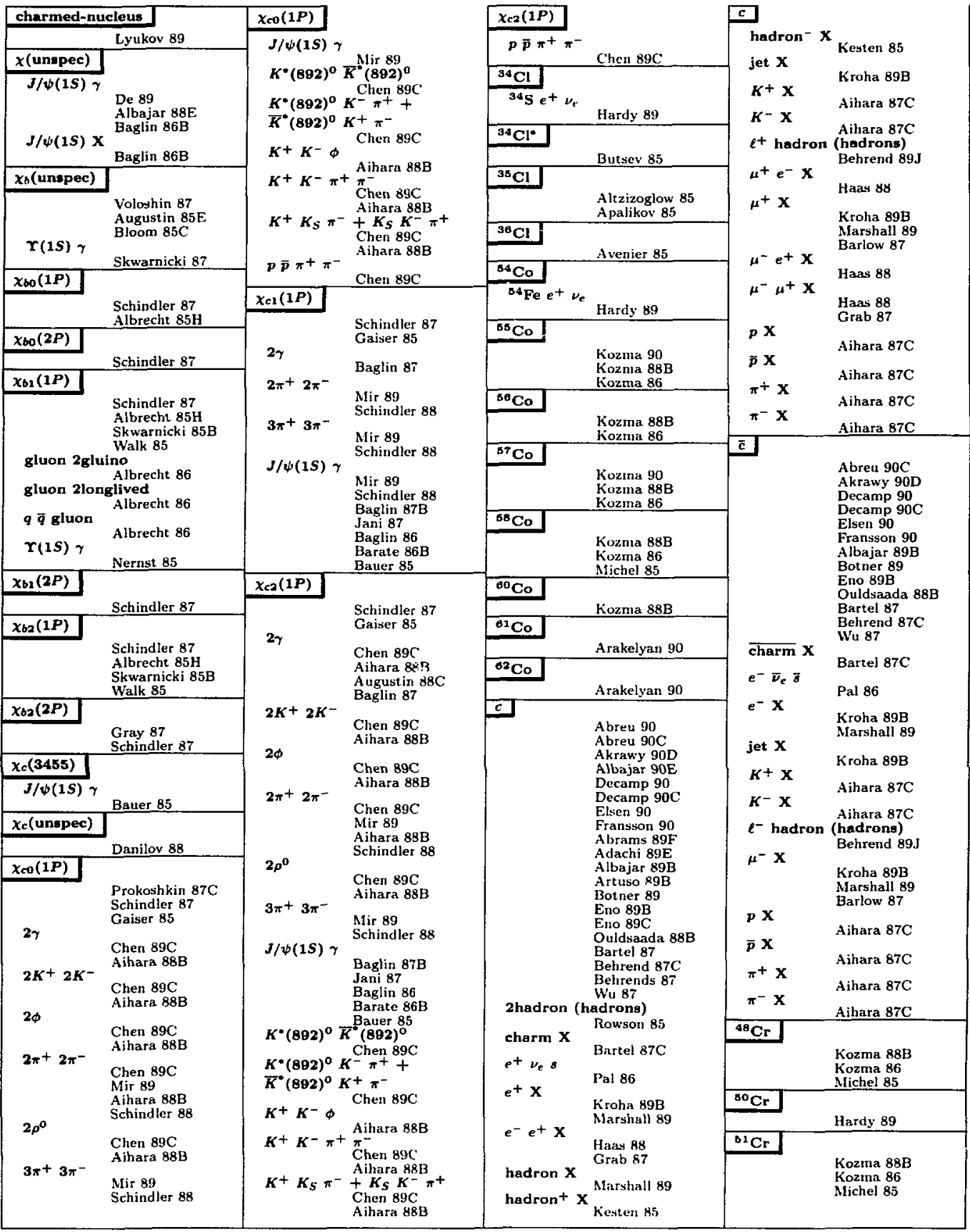




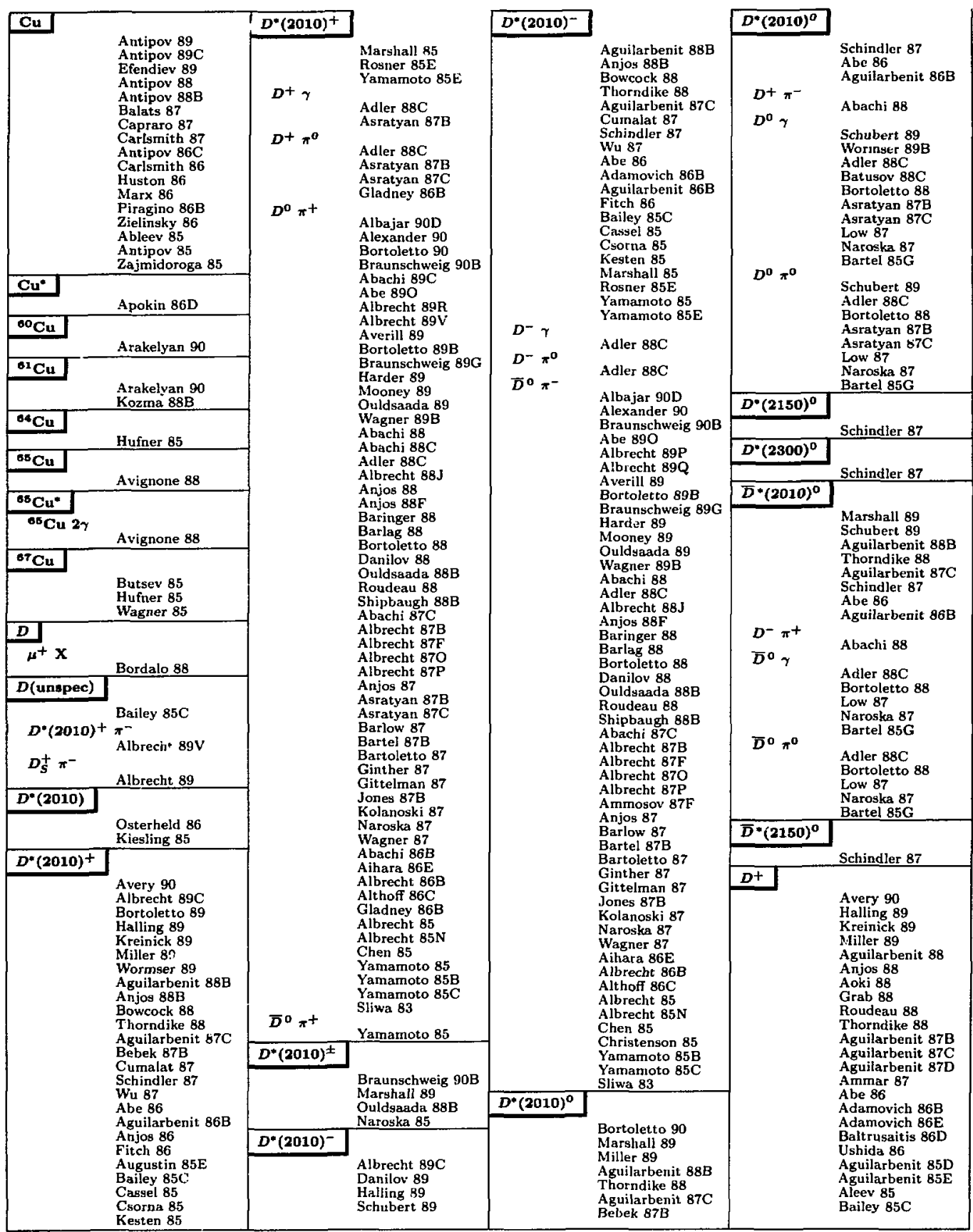


$D^{+}$

Barlag 90C

3charged (neutrals)

Ammar 88B

Aguilarbenit 86

Aguilarbenit 86D

Aziz 85

3charged neutral (neutrals)

Aguilarbenit 88B

Aguilarbenit 87E

$3 \pi^{+} 2 \pi^{-}$

Barlag 90C

Anjos 88D

scharged (neutrals)

Ammar 88B

Aguilarbenit 86

Scharged neutral (neutrals)

Aguilarbenit 88B

A guilarbenit 87E

charged neutral (neutrals)

Aguilarbenit 88B

Ammar 88B

Aguilarbenit $87 \mathrm{E}$

$e^{+}$2charged (neutrala)

Aguilarbenit 86

$e^{+}$4charged (neutrals)

$e^{+} \mathrm{x} \quad$ Aguilarbenit 86

Bowcock 88

Aguilarbenit $87 \mathrm{E}$

Baltrusaitis 85D

$n \pi+$

$\eta^{\prime} \pi^{+}$

Anjos $89 \mathrm{~B}$

$K^{\bullet}(\mathbf{8 9 2})+\bar{K}^{\text {Wormser }}{ }^{89}$

Barlag 90C

$K^{\bullet}(892)^{0} \bar{K}^{0} \pi^{+}$

$\bar{K}^{*}(892)^{\circ}$ Schindler 85

$\bar{K}^{*}(\mathbf{8 9 2})^{0} e^{+\nu_{e}}$

Barlag $88 \mathrm{C}$

Anjos 90B

Anjos 88E

Luth 87

Palka 87B

Schindler 86

$\bar{K}^{*}(892)^{\circ} e^{+}$

Coward 85

$\bar{K}^{*}(\mathbf{8 9 2})^{\circ} K^{+}$Palka 87

Alvarez 90C

Barlag 90C

Adler $88 \mathrm{~F}$

Anjos 88G

Barlag 88C

Brient 87

Baltrusaitis $85 \mathrm{~B}$

$\bar{K}^{*}(802)^{0} \pi^{+}$

Coward 85

Adler 87

Coward 85

Schindler 85

$\overline{K^{*}}(892)^{\circ} \rho^{+}$

$\mathrm{K}^{+} \mathrm{e}^{-} \mathrm{e}^{+}$Schindler 8.5

Weir 89
$D^{+}$

$$
\begin{aligned}
& K^{+} K^{-} 2 \pi^{+} \pi^{-} \\
& \text {Anjos 88G } \\
& K^{+} K^{-} \pi^{+} \pi^{0}
\end{aligned}
$$

$\boldsymbol{K}+\overline{\boldsymbol{K}}^{\mathbf{0}}$

Adler 88F

Brient 87

Schindler 87

Baltrusait is 85B

Coward 85

Schindler 85

$K^{+} \mu^{+} e^{-}$

Weir 89

$K^{+} \mu^{-} e^{+}$

$K^{+} \mu^{-}$Weir 89

$K+\mathbf{X} \quad$ Weir 89

$\mathrm{X}$ Barlag $90 \mathrm{C}$

Aguilarbenit $87 \mathrm{E}$

$K^{-2} e^{+}$

$K^{-2} \mu^{+}$

Weir 89

$K^{-2 \pi}+2 \pi^{0}$

Weir 89

Barlag 90C

$K^{-} 2 \pi^{+} \pi^{0}$ Barlag 90C

Anjos 89B

Aguilarbenit $87 \mathrm{~F}$

Schindler 87

Baltrusaitis $86 \mathrm{E}$

$K^{-} 2 \pi^{+}$

Schindler 85

Alvarez 90

Alvarez 90C

Barlag 90B

Barlag 90C

Bortoletto 90

Anjos 89B

A verill 89

Harder 89

Wormser 89B

Abachi 88

Adler $88 \mathrm{C}$

Adler $\mathbf{8 8 F}$

Albrecht 88J

Albrecht 88M

Anjos 88C

Anjos 88D

Anjos 88F

Barlag 88

Barlag 88C

Barlag 88D

Bortoletto 88

Danilov 88

Izen 88

Adler $\mathbf{8 7}$

Anjos 87

Anjos 87D

Asratyan 87B

Asratyan $87 \mathrm{C}$

Barlag 87

Bartoletto 87

Batusov 87

Bebek 87B

Brient 87

Cyorna 87B

Giutelman 87

Lilth 87

Raab 87
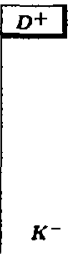

Schindler 87

Anjos 86

Balt rusait is $86 \mathrm{E}$

Gladney 86B

Bailey 85

Ba!trusaitis 85B

Baltrusait is 85D

Derrick 85B

$K^{-} 3 \pi^{+} \pi^{-}$

Schindler 85

Anjos 90

Barlag 90C

Barlag 8BC

$\mathrm{K}^{-} \mathbf{3} \pi^{+} \pi^{\circ} \pi^{-}$

Schindler 87

Barlag 90C

$\boldsymbol{K}^{-} \boldsymbol{\mu}^{+} e^{+}$

$\boldsymbol{K}^{-}+\boldsymbol{T}^{+}$Weir 89

$\nu_{e}$

Izen 88

Aguilarbenit $87 \mathrm{~F}$

Schind ler 87

Schindler 86

Smart 86

Coward 85

Scluindler 85

$\mathrm{K}^{-} \pi^{+} \pi^{\mathrm{o}} e^{+} \nu_{e}$

Aguilarbenit $87 \mathrm{~F}$

$\boldsymbol{K}^{-} \mathbf{X}$

Barlag 90C

Aguilarbenit 88B

Aguilarbenit 87E

Aguilarbenit 86

Georgiopoulo 84

$\boldsymbol{K}^{0} e^{+} \nu_{c}$

$K^{0} K^{-2 \pi+}$

Schind ler 85

$\boldsymbol{K}^{\mathbf{0}} \boldsymbol{\pi}^{+}$

Barlag 90C

Anjos 87D

$\bar{K}_{1}(1270)^{0} \pi^{+}$

Adler 89E

Browder 89

$\bar{K}_{1}(1400)^{n} \pi^{+}$

Dejongh 89

Adler 89E

Browder 89

Dejongh 89

$\bar{K}^{0} \mathbf{2} \pi^{+} \pi^{-}$

Barlag 90C

Adler 89E

Browder 89

Adler $88 \mathrm{~F}$

Izen 88

Aguilarbenit 87F

Brient 87

Schindler 87

Baltrusaitis 85D

Schindler 85

$\bar{K}^{0} 2 \pi+\pi^{0} \pi^{-}$

$\pi^{-}$Aguilarbenit $87 \mathrm{~F}$

$\bar{K}^{0} 3 \pi^{+} 2 \pi^{-}$

$\bar{K}^{0} a_{1}(1260)^{+}$

Adler $89 \mathrm{E}$

$\overline{\boldsymbol{K}}^{0} e^{+} v_{e}$

Browder 89

Izen 88

Schindler 87

$\bar{K}^{0} \mu+\nu_{\mu}$

Coward 85

$\bar{K}^{0} \pi^{+} \pi^{-} e^{+} \nu_{e}$

$\bar{K}^{0} \pi^{+} \pi^{0}$

Aguilarbenit $87 \mathrm{~F}$

Adler 88F

Adler 87

Brient 87

Schindler 87

Coward 85

Schindler 85

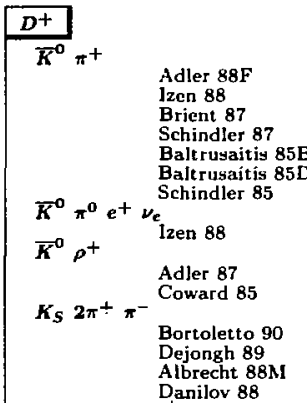

$K_{S} a_{1}(1280)^{+}$

$K_{S} \pi^{+} \boldsymbol{\pi}^{\mathbf{0}}$

Dejongh 89

Asratyan 87B

Asratyan 87C

$K_{s} \pi^{+}$

Baltrusait is $86 \mathrm{E}$

Bortoletto 90

Albrecht $88 \mathrm{M}$

Danilov 88

Asratyan 87B

Asratyan 87C

Baltrusaitis 86E

$\mu+\nu_{\mu}$

Adler 88

Stockdale 87

Wasserbaech 87

Schindler 86

$\mu+\mathbf{x}$

Benvenuti 85

nonres $<K^{+} K^{-}>\pi^{+}$

Brient 87

$\omega \pi^{+}$

Anjos 89B

$\phi 2 \pi^{+} \pi^{-}$

Alvarez 90C

Anjos 88G

$\phi \pi+\pi^{0}$

Alvarez 90C

Anjos 89B

$\phi \pi^{+}$

Alvarez 90C

Barlag $90 \mathrm{C}$

Adler $\mathbf{8 8 F}$

Anjos 88G

Barlag $88 \mathrm{C}$

Biient 87

Schindler 87

Baltrusait is 85B

Coward 85

Schindler 85

Georgiopoulo 84

$\phi \pi^{0} \pi$

Barlag 90C

$\pi^{+} e^{-} e^{+}$

Weir 89

Haas 88

Grab 87

$\pi^{+} \mu^{+} e^{-}$

Weir 89

Anjos 87D

Grab 87

$\pi^{+} \mu^{-} e^{+}$

Weir 89

Haas 88

Anjos 87D

$\pi^{+} \mu^{-} \mu^{+}$

Grab 87

Weir 89

Haas 88

Grab 8 ? 


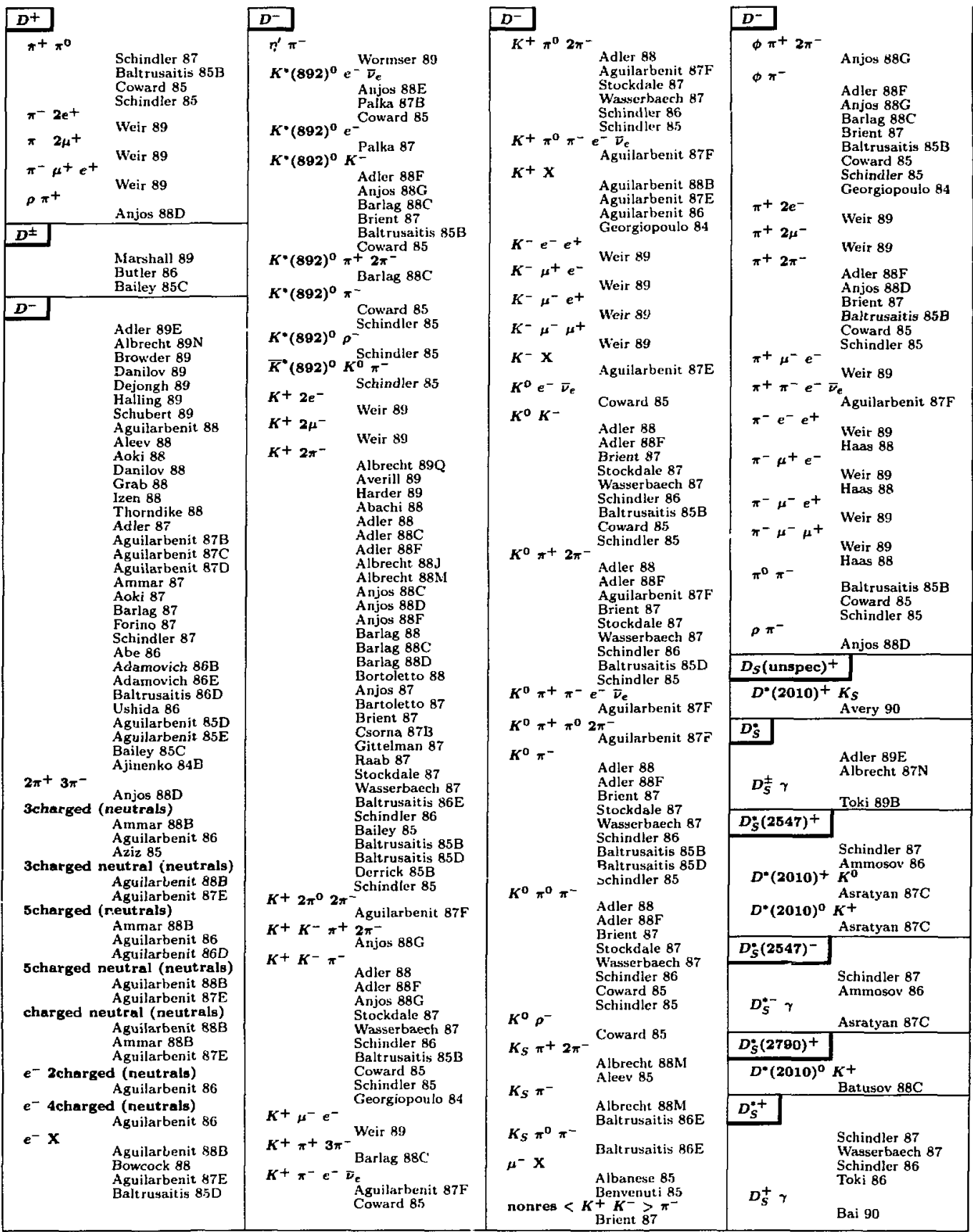




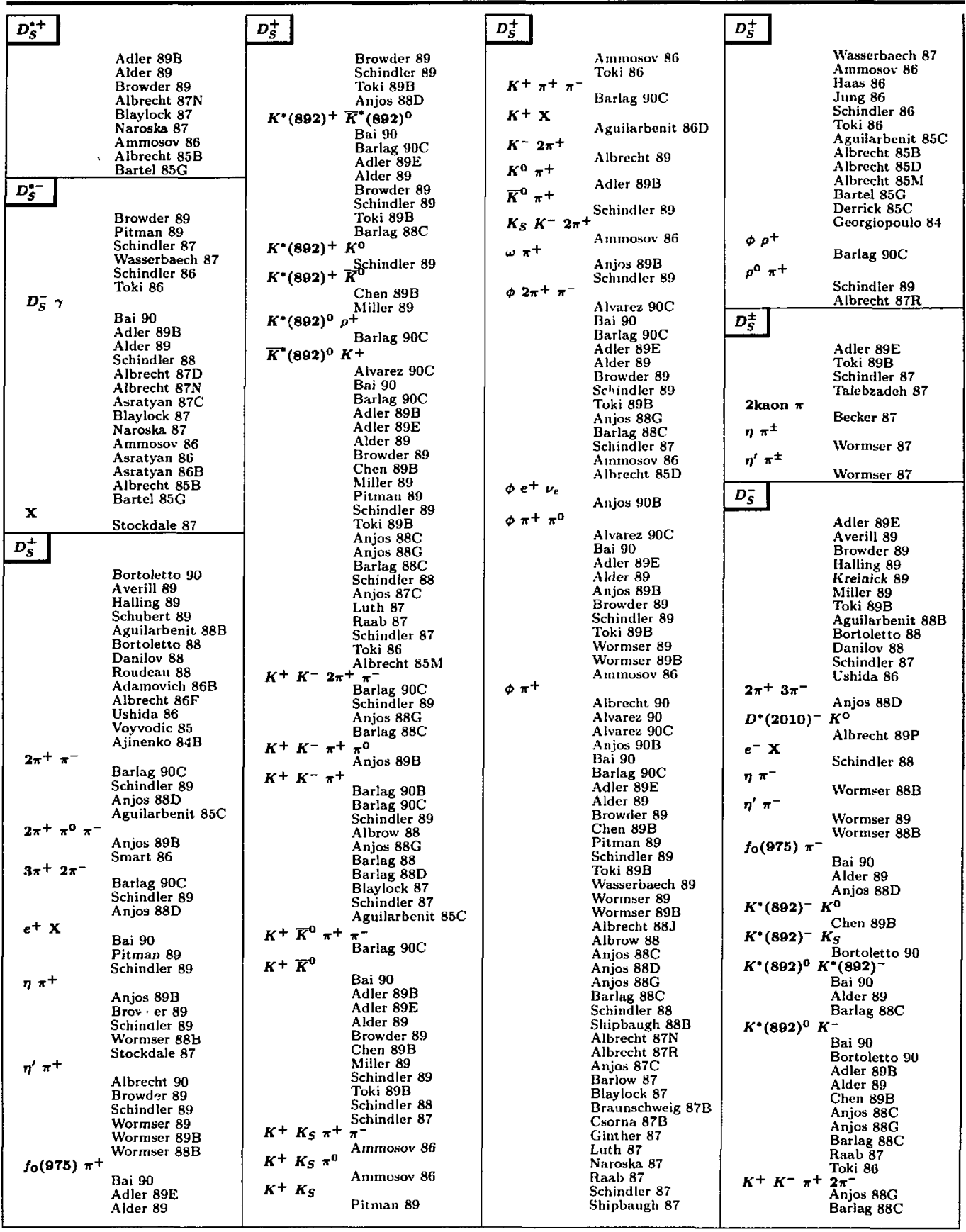




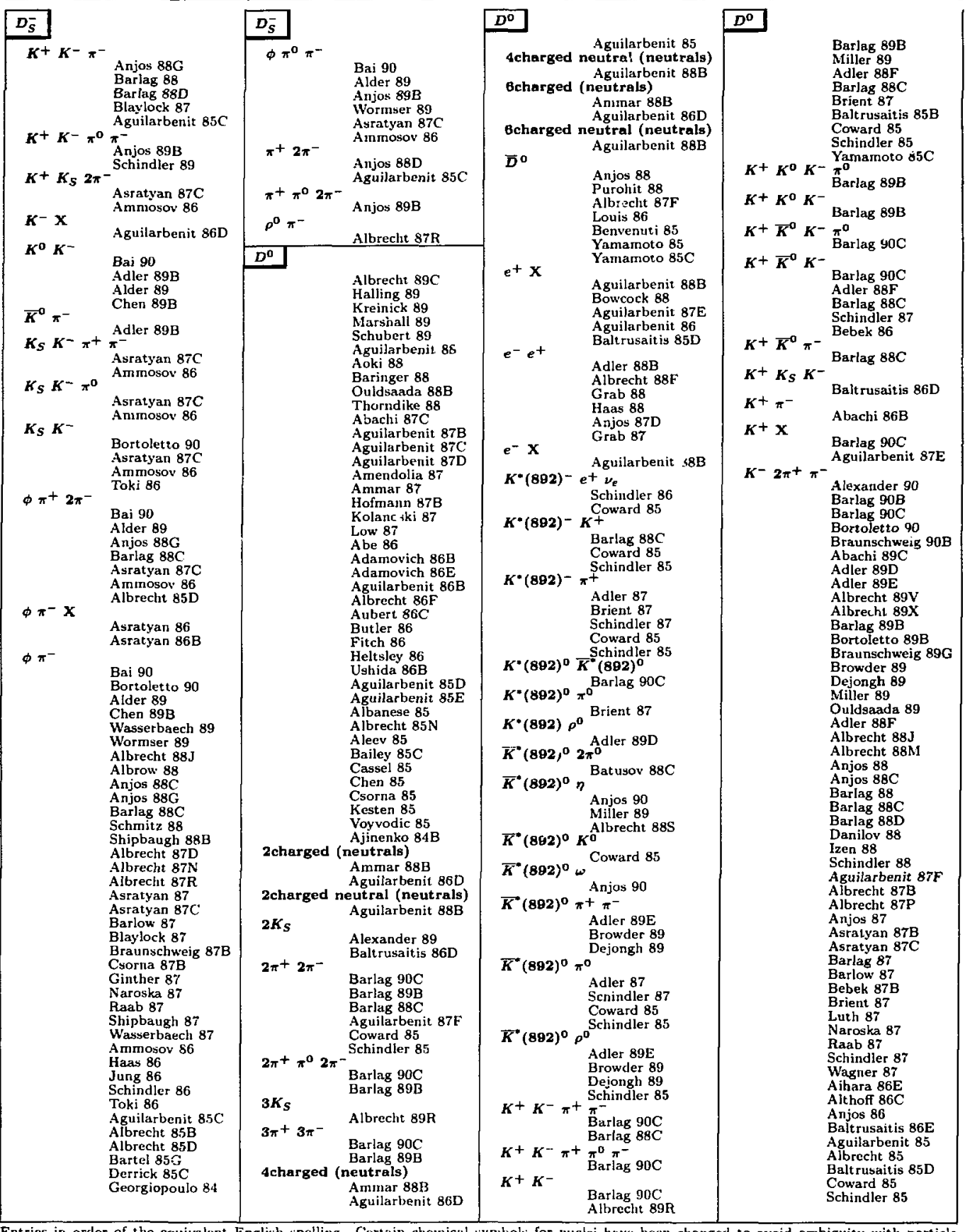




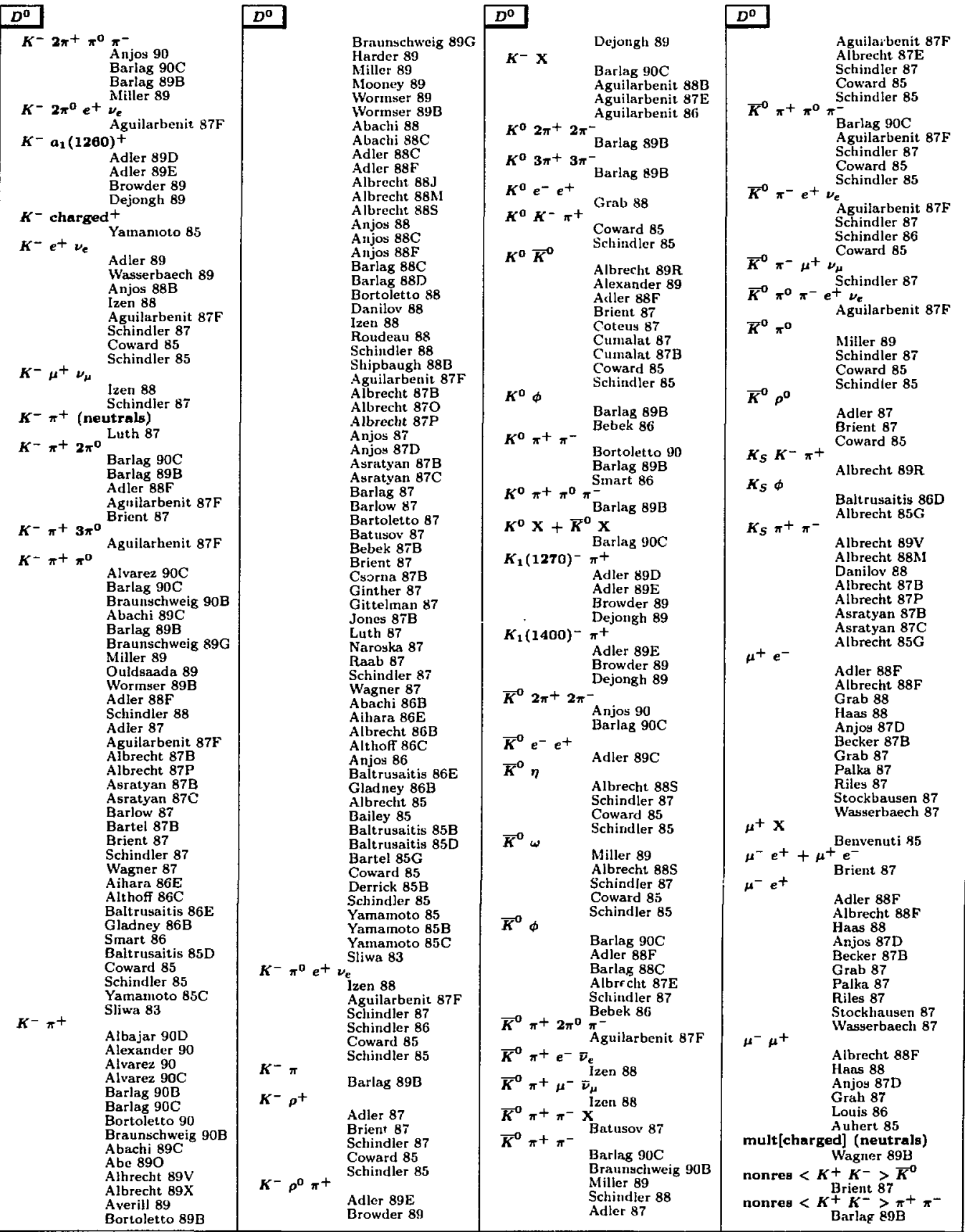




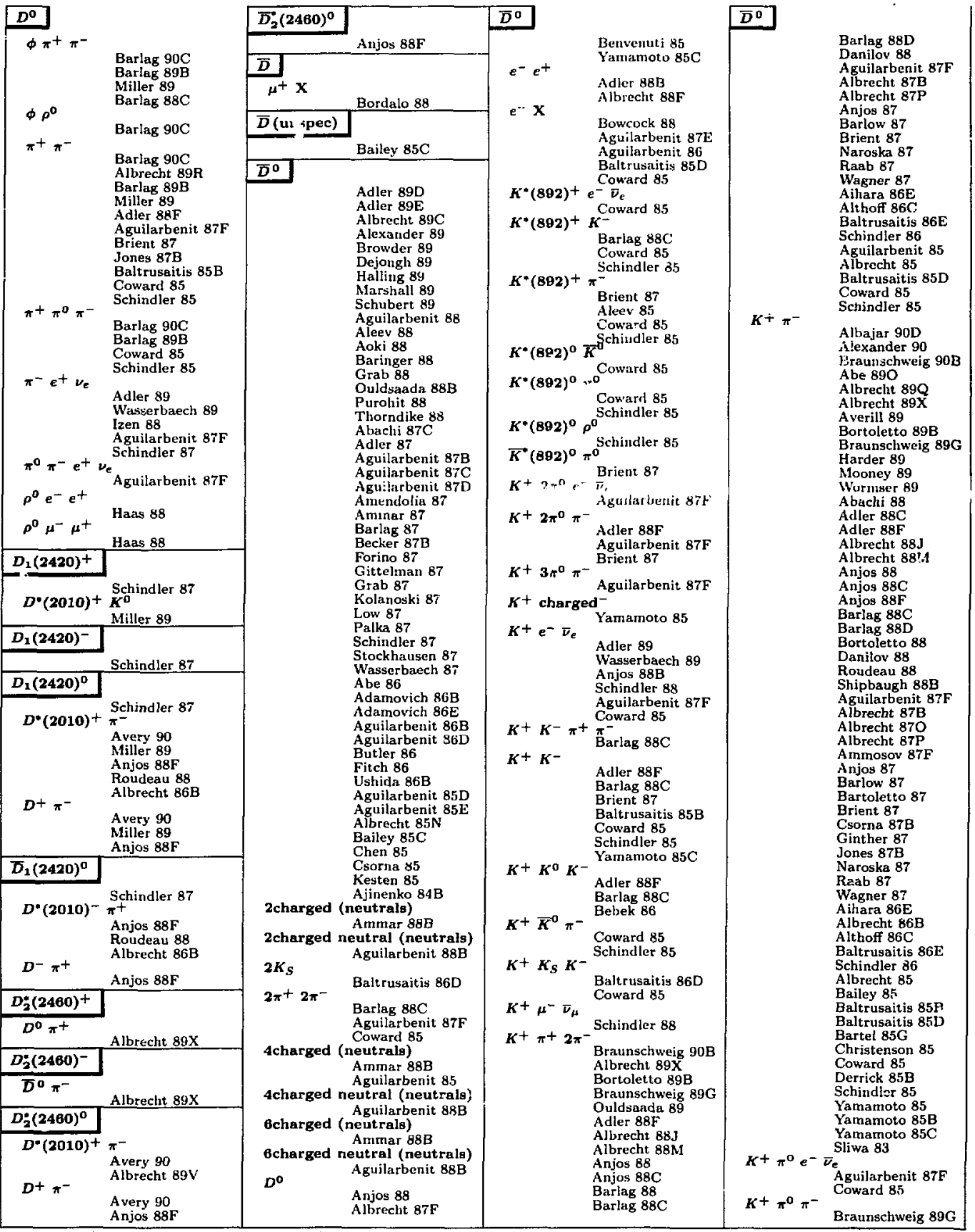

Entries in order of the equivalent English spelling. Certain chemical symbols for nuclei have been changed to avoid ambiguity with particle names (ste the Particle Vocabulary). See the legend on page 297. 


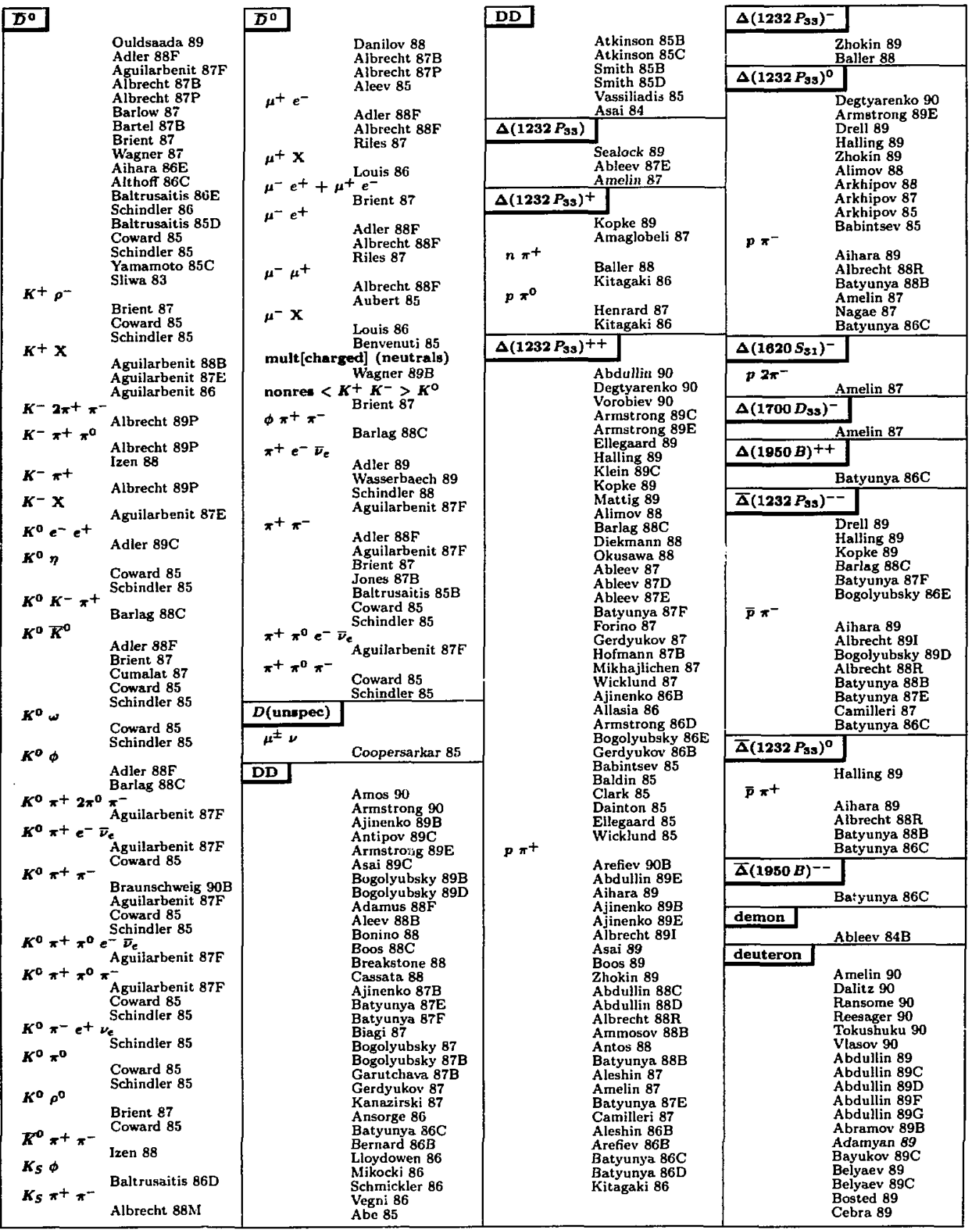




\section{deuteron}

Degtyarenko 89

Franz 89

Garcon 89

Hutcheon 89

Kistryn 89

Maruyama 89

Mattig 89

Platchkov 89

Schablitzky 89

Vlasov 89

Vlasov 89B

Abdullin 88

Abdullin 88D

Adamyan 88

Argan 88

Bertini 88

Bertini 88B

Boyarinov 88

Dobrovolsky 88

Endo 88

Galumyan 88

Imanishi 88

Meyer 88B

Ohnori 88

Safronov 88

Safronov 88 B

Schmidt 88

Velichko 88

Vlasov 88

Voka! 88

Zelinski 88

Ableev 878

Abramov 87

Adyasevich $87 \mathrm{~B}$

Armutlijsky 87

Arnold 87

Asai 87

Balestra 87

Borzakov 87

Burgov 87

Bystricky 87

Enghardt 87

Ero 87

Glagolev 87

Gornov 87

Gornov 87B

Rahbar 87

Schurman 87

Silvestrov 87

Smith 87C

A bramov 86

Adamyan 86

Andronenko 86

Anikina 86C

Belyaev $86 \mathrm{~B}$

Beshliu 86

Blinov 86

Boschitz 86

Eutgov 86

Chuvilo 86

Doerr 86

Ergakov 86

Fearing 86

Glagolev 86

Glagolev 86B

Gornov 86B

Greene 86

Redwine 86

Slin 86

Silvest rov 86

Smith 86C

Smith 86D

Smith 86E

Smith $86 \mathrm{~F}$

Vlasov 86

Voitsekhovsk 86

Vorobiev 86B

Zelinski 86

Abramov 85

Adyasevich $85 \mathrm{C}$

Anikina $85 \mathrm{C}$

Aniol 85

Antonchik 85

Arefiev 85 deuteron

deuteron

Backenatoss 85

Barkov $85 \mathrm{C}$

Barlett 85

Bayukov 85

Bell 85

Bertini 85

Blinov 85D

Cramer 85

Debebe 85

Dickey 85

Dunitriev 85

Epstein 85

Franz 85

Gavrilov 85

Gavrilov 85B

Glass $85 B$

Gorbenko 85

Gorsh'iuva 85

Hamagaki 85

Imanishi 85

Feizer 85

Kristiansson 85

Meyer 85D

Ottermann $85 \mathrm{~B}$

Segel 85

Sun 85

Ungricht 85

Warner 85

Yamauchi 85

Blankleider 84

Blinov 84B

Bovet 84

Donogluue 84D

Hiroshige $84 \mathrm{C}$

Miake 84

Roche 84

Thron 84

Ananiev 83

Falk 83

diberyon $(S=-1) e^{+} \nu_{e}$ Ejiri 89

\section{deuteron}

Albreclit 890

Abramov 86

A Jbreclit $85 \mathrm{E}$

Thron 84

\section{dibaryon}

Abaev 87

Andreev $87 \mathrm{C}$

Bock 85B

$2 \pi \pi^{+}$

$2 n \pi^{-}$

Abdullin 89E

Ashery 88

$2 n$

Giagolev 90

Abdullin $89 \mathrm{E}$

Glagolev 89B

Parker 89

Abdullin $\mathbf{8 8 C}$

Balgansuren 88

$2 p \pi^{+}$

Abdullin $89 \mathrm{E}$

Abdullin 88C

Ashery 88

Ermakov 86B

$2 p$

Glagolev 90

Vorobiev $90 \mathrm{~B}$

Abdullin $89 \mathrm{E}$

Glagolev 89B

Shimizu 89

Abdullin 88B

Abdullin 88C

And reev 88

Balgansuren 88

Troyan 88

Amelin 87B

Andreev 87B

Vorobiev 87C

Abrinov 86C

\section{dibaryon}

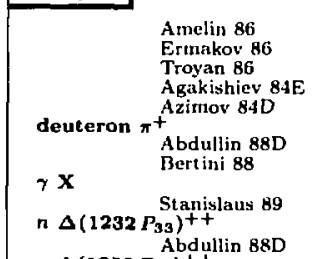

p $\Delta\left(1232 P_{33}\right)^{++}$

Abdullin $89 \mathrm{E}$

Abdullin $88 \mathrm{C}$

p $n \pi^{+}$

Abdullin 88D

p n

Glagolev 90

Glagolev $89 \mathrm{~B}$

Abdullin 88D

Andreev 88

Balgansuren 88

$\pi+\mathbf{x}$

Andreev 87B

Haysak 85
dibaryon( $S=-1)$
Ejiri 89
Pigot 85

n $\Sigma\left(1385 P_{13}\right)^{+}$

Frascaria 87

p $\mathbf{\Sigma}^{-}$

Frascaria 87

dibaryon $(s=-2)$

Ejiri 89

$2 \Lambda \quad$ Shahbazyan 88

Shahbazyan 88

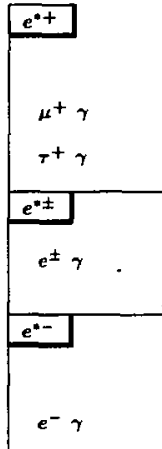

Aulchenko 86

Behrend 86

Bonneaud 86

Decamp 90G

Decamp 90G

Kichimi 88

Kim 88C

Appel 86

Kim 89B

Kamae 88

Kim 88C

Akrawy 90G

Decamp $90 \mathrm{G}$

Adachi $89 \mathrm{D}$

Abe $88 \mathrm{E}$

Gan 88

Maki 88B

Shirai 88

Sumiyoshi 88

Unno 88

Yamauchi 88

Ansari 87D

Repellin 87

Aulchenko 86

Behrend 86

Bonneaud 86

Decamp 90G

Decamp 90G

$7^{-} \gamma$

Avignone 86

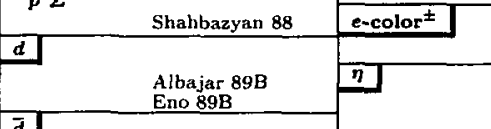

$\bar{d}$

\begin{tabular}{|c|c|}
\hline & $\begin{array}{l}\text { Albajar } 89 \mathrm{~B} \\
\text { Eno } 89 \mathrm{~B}\end{array}$ \\
\hline Dy & \\
\hline
\end{tabular}

Feindt 89

Kopke 89

Landsberg 89

Mallik 89B

Marshall 89

Mattig 89

Miller 89

Schindler 89

Takamatsu 89

Abachi 88B

Aihara 88D

Albajar 88C

Armstrong 88

Aston 88I

Barish 88

Kin $89 B$

Kanlae 88

Kim 88C

Barloutaud 88

Berger 88C

Bernasconi 88

Akrawy 90G

Decamp 900

Adachi 89D

Abe $88 \mathrm{E}$

Gatt 88

Maki 88B

Shirai 88

Sumiyoshi 88

Unno 88

Yamauchi 88

Angari 87D

Repeilin 87
Bourdarios 88

Chiba 88

Coftman 88

Diekmann 88

Fukui 88

Gam 88B

Hitlin 88

Iddir $\mathbf{8 8}$

Mir 88

Ouldsaada 88B

Sedlak 88

Entries in order of the equivalent English spelling Certain chenical symbols for nuclei have been changed to avoid ambiguity with particle names (see the Particle Vocahulary). See the legend on page 297. 
Sirunyan 88

A bachi $87 \mathrm{~F}$

Aihara 87G

Albrecht 870

Antille 87

Aulchenko $87 \mathrm{C}$

Bailly 87G

Bailly $87 \mathrm{H}$

Barloutaud 87

Benayoun 87

Bernasconi 87

Camilleri 87

Chiba 87B

Coftman 87

Derrick 87B

Fredriksson 87

Gan 87B

Hofmann 87B

Kolanoski 87

Lurz 87

Peng 87

Prokoshkin 875

Richard 87

Schindler 87

Stockhausen 87B

Aihara 86G

A kesson $86 \mathrm{C}$

Ando 86

Ant reasyan 86

A pokin $86 \mathrm{C}$

Apokin 86D

Atkinson 86

Aulchenko 86B

Aulchenko 86C

Baltrusaitis 86

Baltrusaitis 86C

Banajgs 86B

Burchat 86B

Druzhinin 86

Haines 86

Konigsmann 86

Krishnaswamy 86

Stockhausen 86

Akesson 85G

Arkhipov 85

Atkinson 85C

Augustin 85C

Augustin 85E

Balt tusaitis $85 \mathrm{~F}$

Banner 85B

Bartel 85

Berthet 85

Blewitt 85

Coward 85

Druzhinin 85

Golubev 85

Kolanoski 85

Lee 85B

Odian 85

Park 85B

Prokoshkin 85

Schindler 85

$2 \gamma$

Tsukerman 85B

Albrecht 90

Adter $89 \mathrm{E}$

Amaglobeli $\mathbf{8 9}$

Apokin 89

Behrend 89B

Berger 89

Bonesini 89

Boutemeur 89

Dolinsky 89

Dolinsky 89B

Hirata 89B

Inagaki 89B

Mir 89

Peng 89

Pitzl 89

Roe 8 ?

Roe 89B

Adiels 88

Aihara $88 \mathrm{E}$

A jaltouni 88
Ajaltouni 88B

Albrecht 885

Albrow 88

Alde 88B

Alde 88D

Alde $88 \mathrm{E}$

Antonelli 88

Arkhipov 88

Augustin 88C

Behrend 88

Bienlein 88

Boutemeur 88

Dolinsky $88 \mathrm{~B}$

Druzhinin 88

Fujisaki 88

Gan 88

Gidal $88 \mathrm{~B}$

Gidal $88 \mathrm{C}$

Jousset 88

Keh 88B

Schnitt 88

Seidel 88

Toki 88

Toki 881

Williams 88

Wormser 88B

Alde 87

Alde 87B

Antreasyan 87

Arkhipov 87

Baringer 87

Berger $87 \mathrm{~B}$

Naroska 87

Okhrimenku 87

Skwarnicki 87B

Toki 87

Wormser 87

Aguilarbenit $86 \mathrm{C}$

Aihara 86

Alde 86

Alde 86B

Alde $86 \mathrm{C}$

Alde $86 \mathrm{D}$

Alde $86 \mathrm{E}$

Bitsadze 86

Landsberg 86

Lowe $86 \mathrm{~B}$

Lowe 86C

Akimenko 85

Apel 85

Apokin 85B

Bartel 85B

Chakrabarti 85

Dolinsky 85

Tsukerman 85

2neutral (neutrals)

$3 \pi^{0}$

Toki 87

Berger 89

Hirata 89B

Alde $88 B$

Bienlein 88

Dolinsky 88B

Schmitt 88

Alde 87B

Alde 86

Lowe 86B

$e^{-} e^{+} \gamma$

Mayer 89

Landsberg 86

Landsberg 85

$e^{-} e^{+}$

Mayer 89

$\mu^{+} e^{-}$

Mayer 89

$\mu^{-} e^{+}$

Nayer 89

$\mu^{-} \mu^{+} \gamma$

Bannikov $89 \mathrm{~B}$

Mayer 80

Prokoshkin 87C

Landsberg 86

Landsberg 85

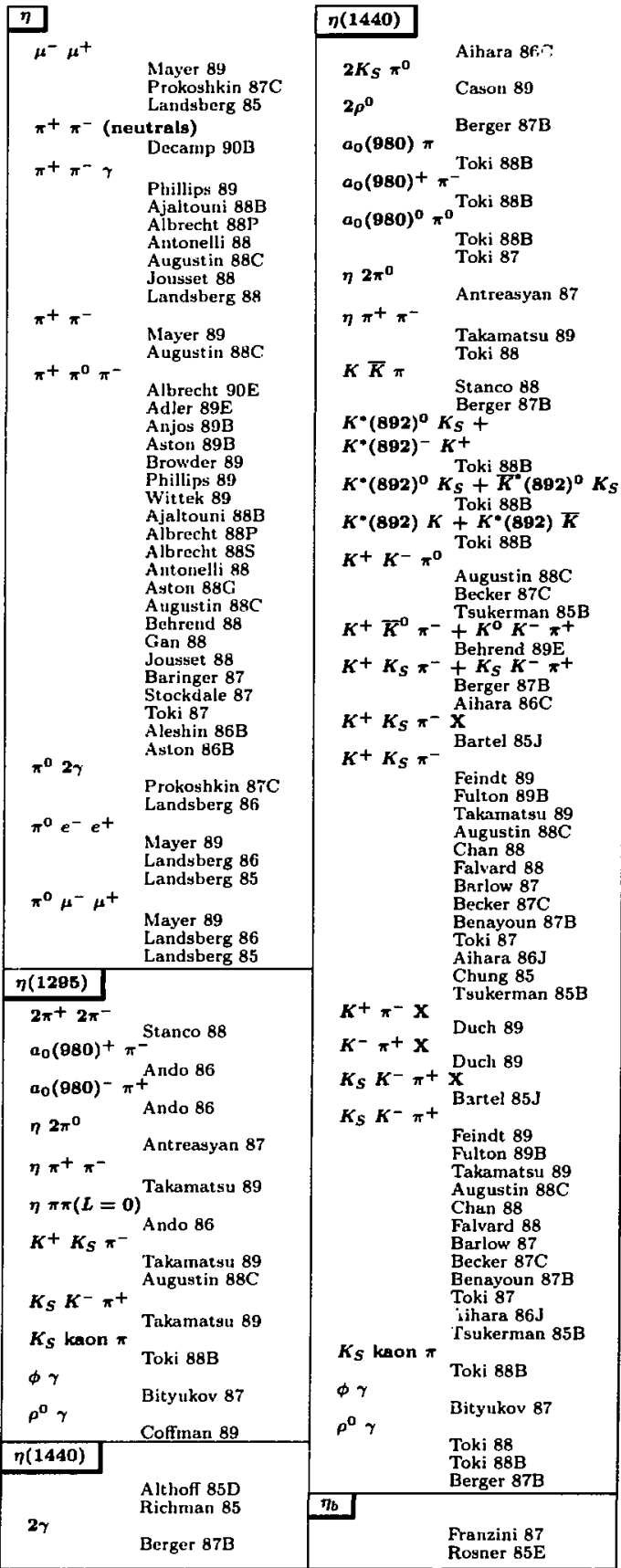


$n_{c}(1 S)$

$2 f_{2}(1270)$

Kopke 89

Althof 85D

Augustin 85D

Gaiser 85

Mallik 89B

Adler 88D

Augustin 88C

Mir 88

$2 \gamma$

Chen 89C

Aihara 88

Aihara 88B

Ajaltouni 88

August in $88 \mathrm{C}$

Baglin 87

Barlow 87

Berger 87B

Toki 87

Blinov 86C

Chiang 86

Kolanoski 86

$2 K^{+}+2 K^{-}$

Chen $89 \mathrm{C}$

Aihara 88B

Bisello 86

2

Mallik 89B

Baltrusaitis 86

$2 \phi$

Chen 89C

Jensen 89

Mallik 89B

Aihara 88

Aihara 88B

Toki 87

Baltrusaitis 86

Bisello 86

Booth 86

$2 \pi+2 \pi^{-}$

Braunschweig 89

Chen $89 \mathrm{C}$

Mallik 89B

Adlet $88 \mathrm{D}$

Aibara 88

Aihara 88B

Bisello 88

Gidal $88 \mathrm{C}$

Ouldsaada 8BB

Baltrusajtis 86

$2 \rho^{0}$

Chen $89 \mathrm{C}$

Jensen 89

Mallik 89B

Adler 88D

Aihara 88B

Bisello 88

Mir 88

Toki 87

Baltrusaitis 86

Baltrusaitis 86

$a_{3}(1320)^{+} \pi^{-}$

Bisello 88

Baltrusaitis 86

$a_{3}(1320)^{-} \pi^{+}$

Bisello 88

Balt rusaitis 86

$\eta \pi^{+} \pi^{-}$

Toki 87

$\eta^{\prime} \pi^{+} \pi^{-}$

Toki 87

Baltrusaitis 86

$f_{2}(1270) \pi$

Baltrusaitis 86

$\boldsymbol{K} \overline{\boldsymbol{K}}$

Berger 87B

$K^{*}(892)^{0} \bar{K}^{*}(882)^{0}$

Chen $89 \mathrm{C}$
Augustin $88 \mathrm{C}$

$a_{0}(980)+\pi$

Baltrusaitis 86

Baltrusaitis 86 $n_{c}(1 S)$

Jensen 89

Mallik $89 \mathrm{~B}$

Schindler 87

Baltrusaitis 80

$K^{*}(892)^{0} K^{+} \pi^{-}+$

$\bar{K}^{*}(892)^{0} K^{-} \pi^{+}$

$K^{*}(\mathbf{8 g 2})^{\circ} K^{-}$Baltrusaitis 86

$\boldsymbol{K}^{*}(\mathbf{8 9 2})^{\circ} K^{+} \pi^{-}$

$K^{*}(892)^{\circ} K^{\text {Chen } 89 \mathrm{C}}$

Jensen 89

$\boldsymbol{K}^{+} \boldsymbol{K}^{-} \boldsymbol{\eta}$

$K^{+} \boldsymbol{K}^{-} \boldsymbol{\phi}$

Baltrnsaitis 86

$K^{+} \boldsymbol{K}^{-} \boldsymbol{\pi}^{+} \boldsymbol{\pi}^{-}$

Aihara 88B

Braunschweis 89

Chen $89 \mathrm{C}$

Aihara 88

Aihara $88 \mathrm{H}$

Gidal $88 \mathrm{C}$

Ouldsaada 88B

Schindler 87

Baltrusaitis 86

$\boldsymbol{K}^{+} \boldsymbol{K}^{-} \boldsymbol{\pi}^{0}$

$K^{+} K_{S} \pi^{-}$Balt rusaitis 86

Chen $89 \mathrm{C}$

Aihara 88B

Gidal 88C

Berger 87 B

$K^{+} K_{s} \pi^{-} e^{-} e^{+}$

$K^{+} K_{S} \pi^{-}$Kolanoski 86

Braunschweig 89

Jensen 89

Ouldsaada 88B

Barlow 87

Kolanoski 87

Toki 87

Baitrusaitis 86

Berger 86

$K_{S} K^{-} \pi^{+} e^{-} e^{+}$

$\boldsymbol{K}_{S} \boldsymbol{K}^{-} \boldsymbol{\pi}^{+}$

Kolanoski 86

Braunschweig 89

Jensen 89

Ouldsaada 88B

Barlow 87

Kolanoski 87

Toki 87

Baltrusaitis 86

Berger 86

$p \bar{p} \pi^{+} \pi^{-}$

$p \bar{p}$

Chen $89 \mathrm{C}$

Berger 87B

Toki 87

Baltrusaitis 86

$\phi \omega$

Mallik 89B

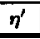

Boutemeur 89

Browder 89

Chiba 89

Dolinsky 80

Kopke 89

Landsberg 89

Schindler 89

Wormser 89

Adiels 88

Albajar 88C

Chiba 88

Coffnan 88

Hitlin $\mathbf{8 8}$

Mir 88

Sedlak 88

Bailly $87 \mathrm{C}$

Bailly 87H

Benayoun 87

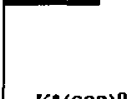

$\eta^{\prime}$

Chiba 87B

Hofmann 87B

Prokoshkin 87B

Bult rusait is 86

Baltrusaitis $85 F$

Druzhistin 85

Kolanoski 85

Lee 85B

Prokoshkin 85

Tsukerman 85B

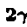

Roe 89

Roe $89 \mathrm{~B}$

Aihara $88 D$

A jaltouni 88

Albrow 88

Alde 88D

Augustin $88 \mathrm{C}$

Bienlein 88

Gidal $88 \mathrm{C}$

Toki 88B

Willinnss 88

Alde 87B

Berger $87 \mathrm{~B}$

Kolanoski 87

Toki 87

Aguilarbenit 86C

Alde $86 \mathrm{E}$

Apokin 86C

Apokin 86D

Lancisberg 86

Lowe $86 B$

Apokin 85B

Tsukerman 85

$2 \pi^{0}$

$3 \gamma$

$3 \pi^{0}$

Alde 87B

Alcle 87B

Alde $87 \mathrm{~B}$

Prokoshkin 87C

$4 \pi^{0}$

$e^{-} e^{t}$

Alde 87B

n $2 \pi^{0}$

Vorobiev $88 \mathrm{C}$

Alde 88B

Bienlein 88

Schunitt 88

Alde 87B

Ant reasyan 87

Alde 86D

Alde 86D

Lowe 86B

$\eta e^{-} e^{t}$

Landsberg 86

Landsberg 85

$\eta$ higgs

$\eta \mu^{-} \mu^{+}$

Prokoshkin 87C

Landsberg 86

Lausdsberg 85

$\pi \pi+$

Adler $89 \mathrm{E}$

Dolinsky $89 \mathrm{~B}$

Ailıara 88D

August in $88 \mathrm{C}$

Druzbinin 88

Gidal $88 B$

Gidal $88 \mathrm{C}$

Jousset 88

Wormser 88B

Aujchenko $87 \mathrm{C}$

Kolanoski 87

Toki 87

Aulchenko $86 \mathrm{~B}$

$\mu^{-} \mu^{+} \gamma$

Aulchenko 86C

Bannikoy $89 \mathrm{~B}$

Prokoshkin: 87C

Landsberg 86
Albrecht 90

Ajaltouni 88B

Wormser 87

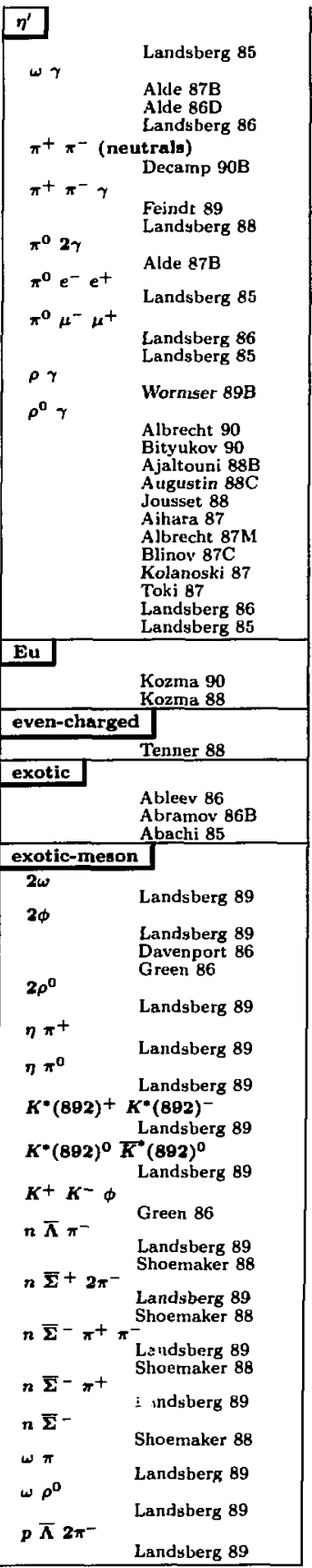

Entries in order of the equivalent English spelling. Certain chemical symbols for nuclei have been changed to avoid ambiguity with particle names (see the Particle Vocabulary). See the legend on page 297. 


\section{exotic-meson}

\begin{tabular}{ll}
$p \bar{\Lambda} \pi^{+} \pi^{-}$ & Shoemaker 88 \\
$p \bar{\Lambda} \pi^{+}$ & Landsberg 89 \\
$p \bar{\Lambda} \pi^{-}$ & Landsberg 89 \\
$p \bar{\Sigma}-\pi^{-}$ & Landsberg 89 \\
$\bar{p} \Lambda 2 \pi^{+}$ & Landsberg 89 \\
$\bar{p} \Lambda \pi^{+} \pi^{-}$ & Landsberg 89 \\
$\bar{p} \Lambda \pi^{+}$ & Landsberg 89 \\
$\bar{p} \Lambda \pi^{-}$ & Landsberg 89 \\
$\phi \pi^{+} \pi^{-}$ & Landsberg 89 \\
$\phi \pi^{0}$ & Green 86 \\
$\phi \pi^{0}$ & Landsberg 89 \\
$\phi \rho^{0}$ & Landsberg 89 \\
$\pi^{+} \pi^{-}$ & Landsberg 89 \\
$\rho^{+} \rho^{-}$ & May 89 \\
$\rho^{0} \pi^{+}$ & Landsberg 89 \\
\hline
\end{tabular}

Landsberg 89

\begin{tabular}{|c|c|}
\hline exatic-nucl & \\
\hline$p 3 \pi^{+} \pi^{-}$ & Brovkin 89 \\
\hline$f_{0}(1240)$ & \\
\hline & Landsberg 86 \\
\hline$\pi^{+} \pi^{-}$ & \\
\hline$f_{0}(1400)$ & \\
\hline
\end{tabular}

\begin{tabular}{|ll|}
\hline $2 \eta$ & Halling 89 \\
$2 \gamma$ & Alde 86 \\
$2 \pi^{\circ}$ & Berger $87 \mathrm{~B}$
\end{tabular}

\begin{tabular}{|c|c|}
\hline $\begin{array}{l}2 \pi \\
e^{-} e^{+}\end{array}$ & $\begin{array}{l}\text { Dolinsky 89B } \\
\text { Bienlein } 88 \\
\text { Aulchenko } 87 \mathrm{C} \\
\text { Berger } 87 \mathrm{~B} \\
\text { Vorobiev } 88 \mathrm{C}\end{array}$ \\
\hline$f_{0}(1525)$ & \\
\hline $2 K_{S}$ & $\begin{array}{l}\text { Aston } 88 \mathrm{I} \\
\text { Aston } 88 \mathrm{~J} \\
\text { Albrecht } 89 \mathrm{~J}\end{array}$ \\
\hline$f_{0}(1590)$ & \\
\hline
\end{tabular}

27

Diekmann 88

Augustin $88 \mathrm{C}$

Toki $88 B$

Alde B?

Prokoshkin 87C

Alde $\mathbf{B 6}$

Tsukerman $85 \mathrm{~B}$

$4 \pi^{\circ} \quad$ Toki $88 \mathrm{~B}$

$\eta^{\prime} \eta$

Alde 67D

Toki 88B

Trukerman 85 B

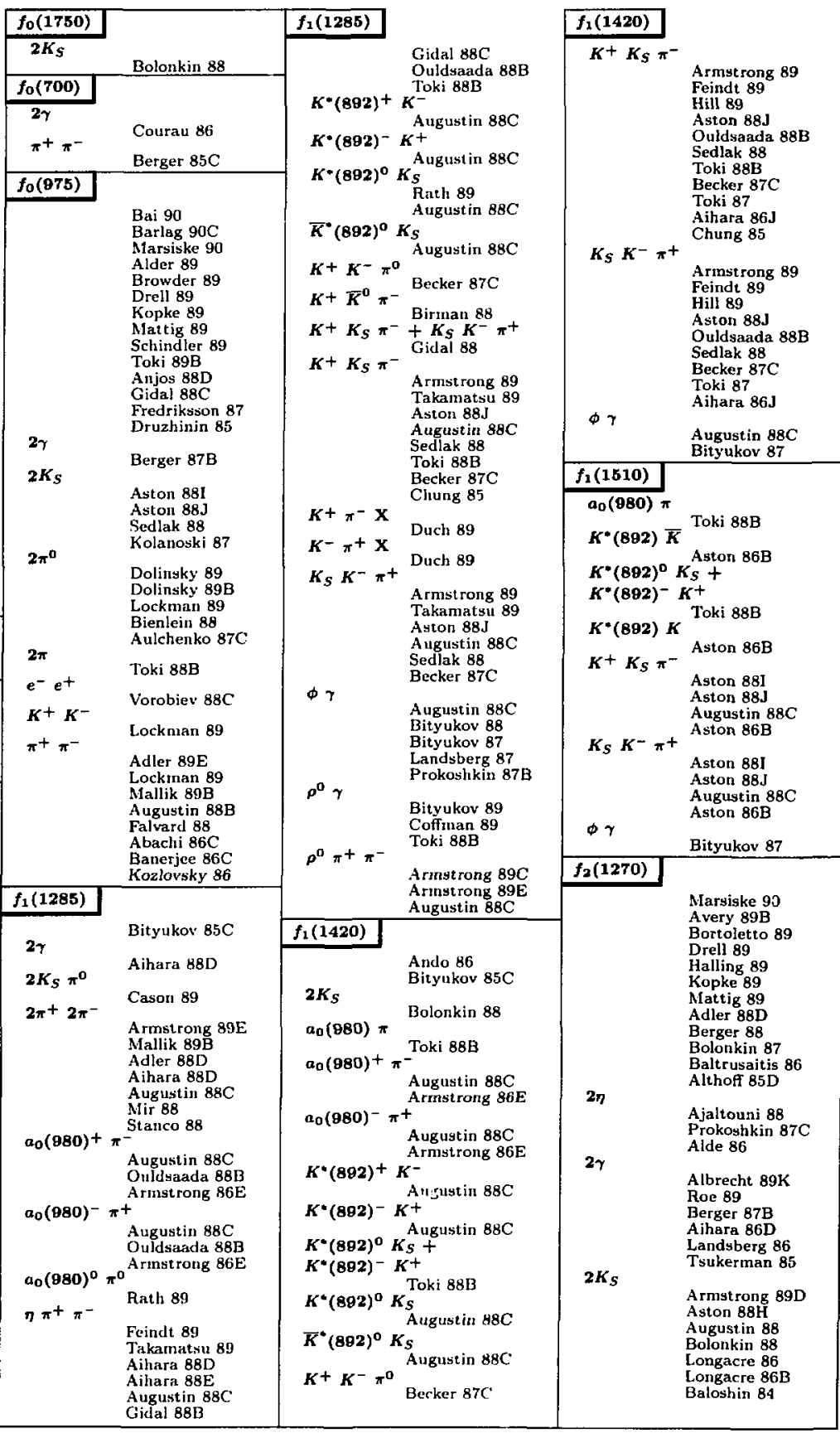




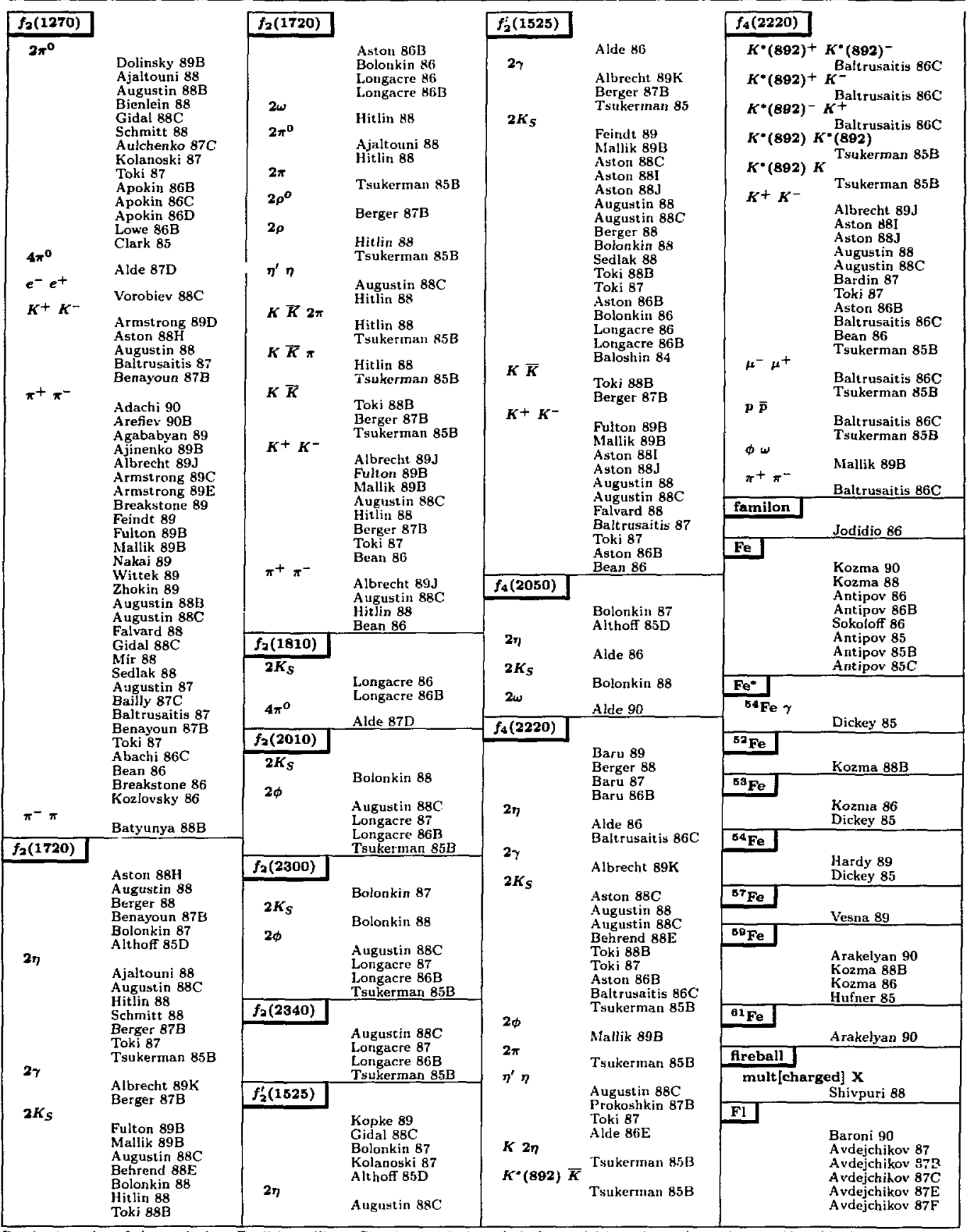




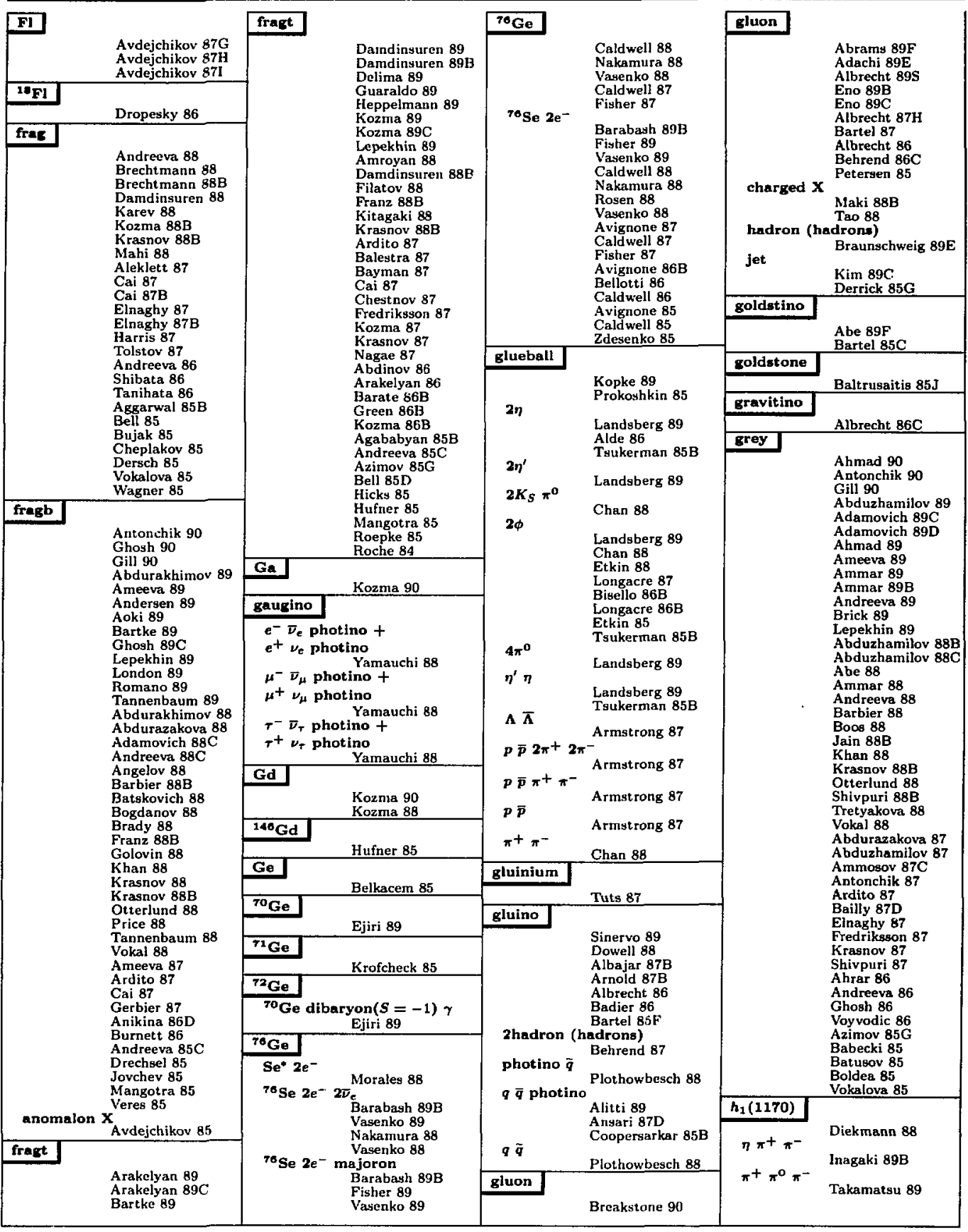




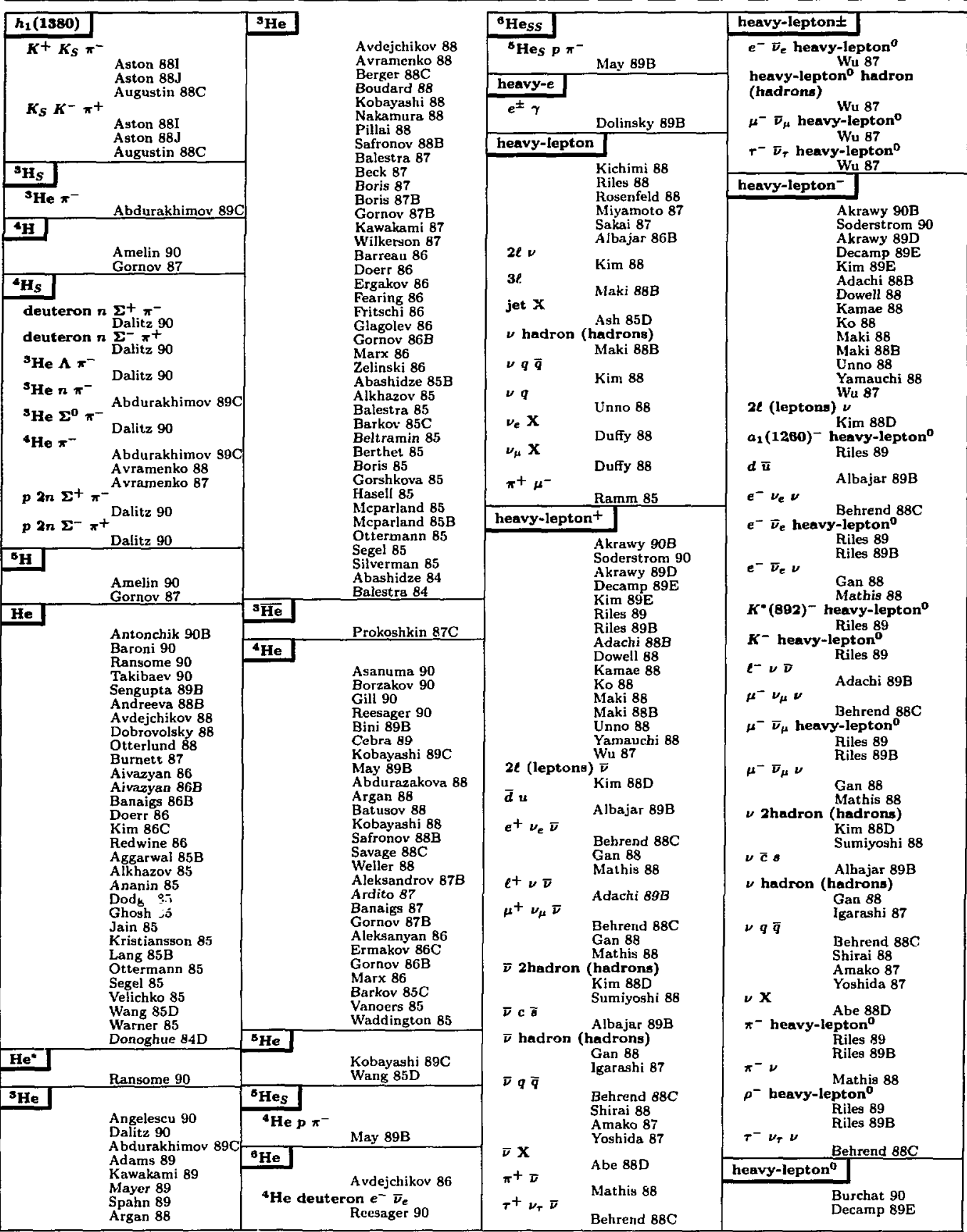

Entries in order of the equivalent English spelling. Certajn chemical symbols for nuclei have been changed to avoid ambiguity with particle names (see the Particle Vocabulary). See the legend on page 297. 


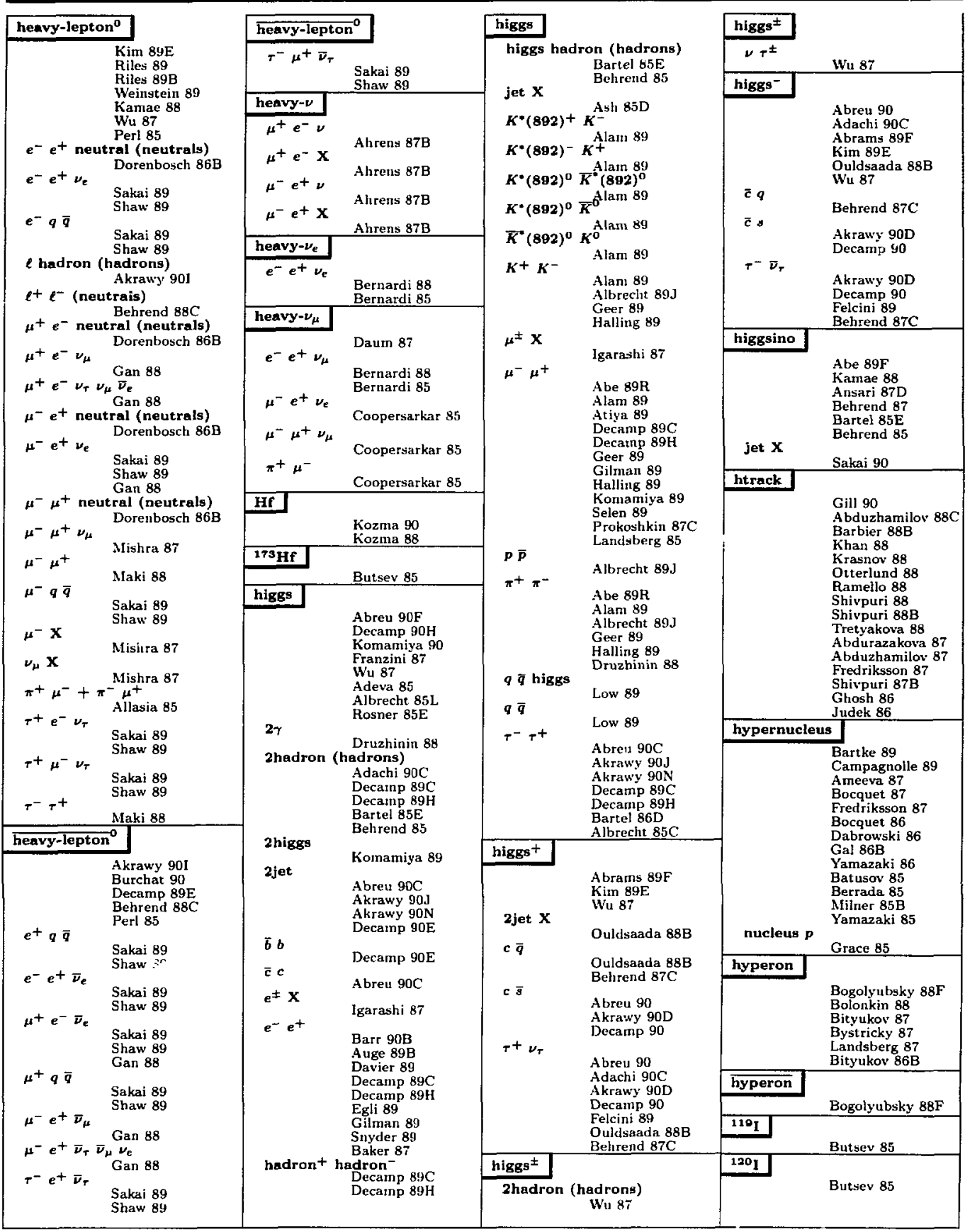




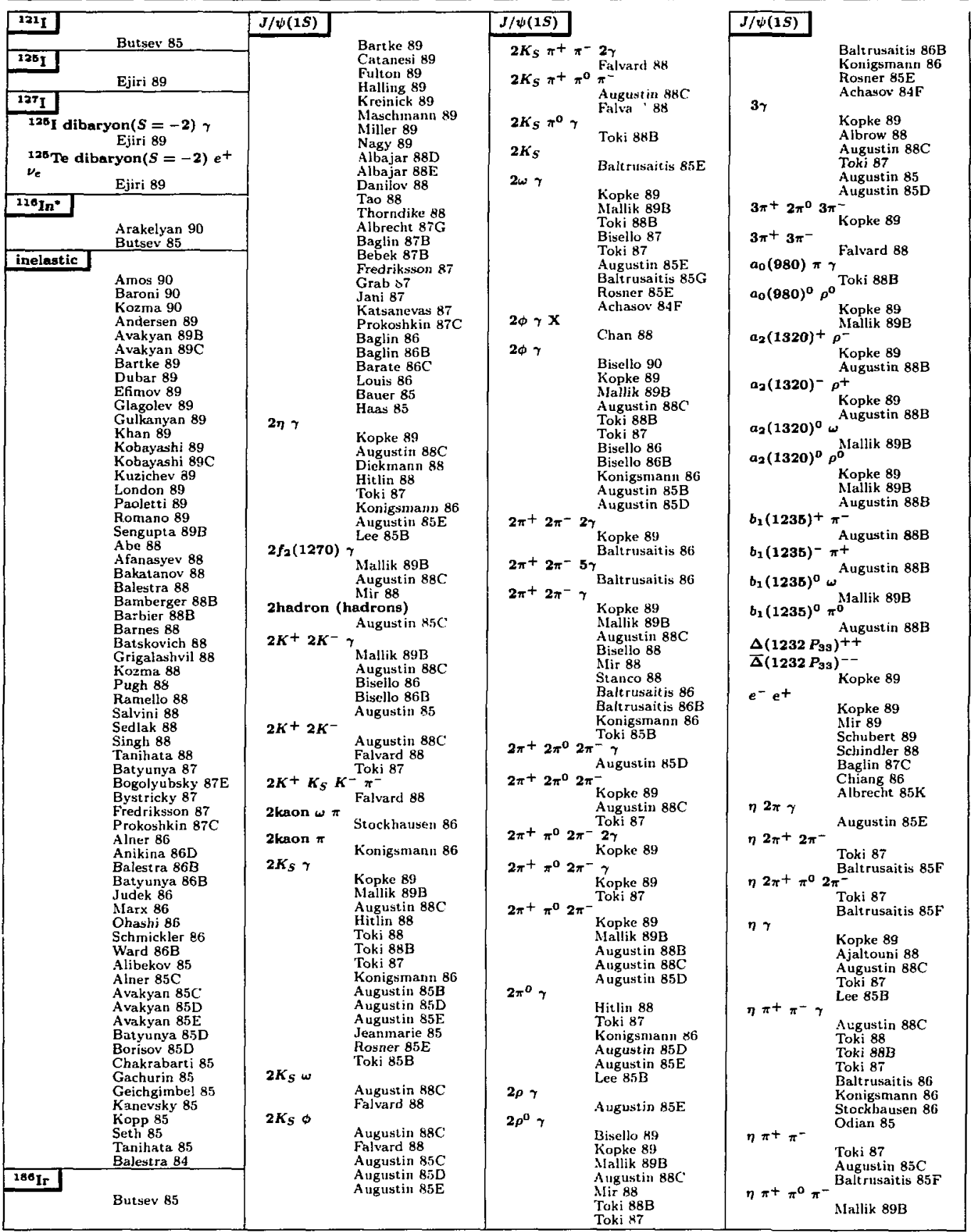




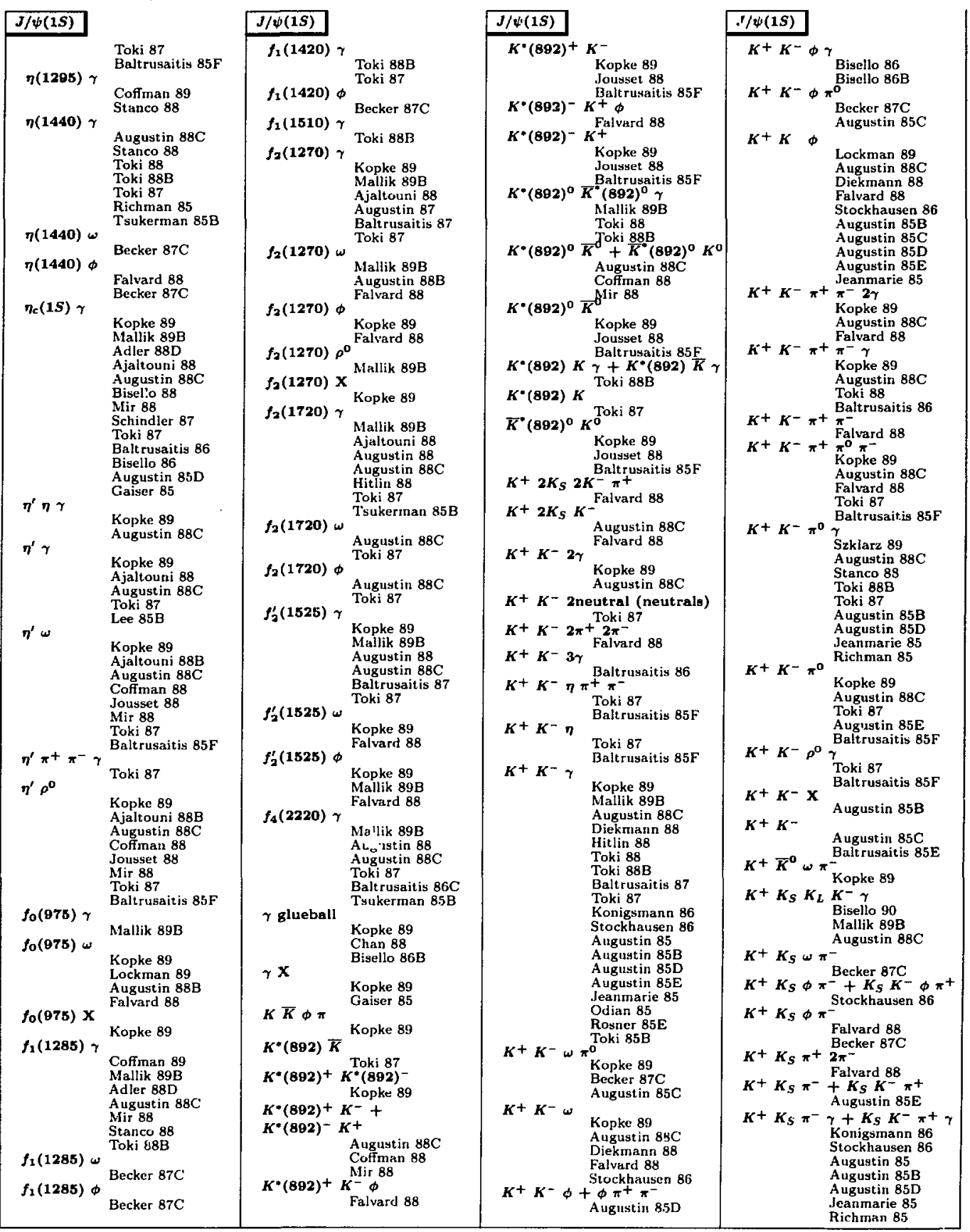




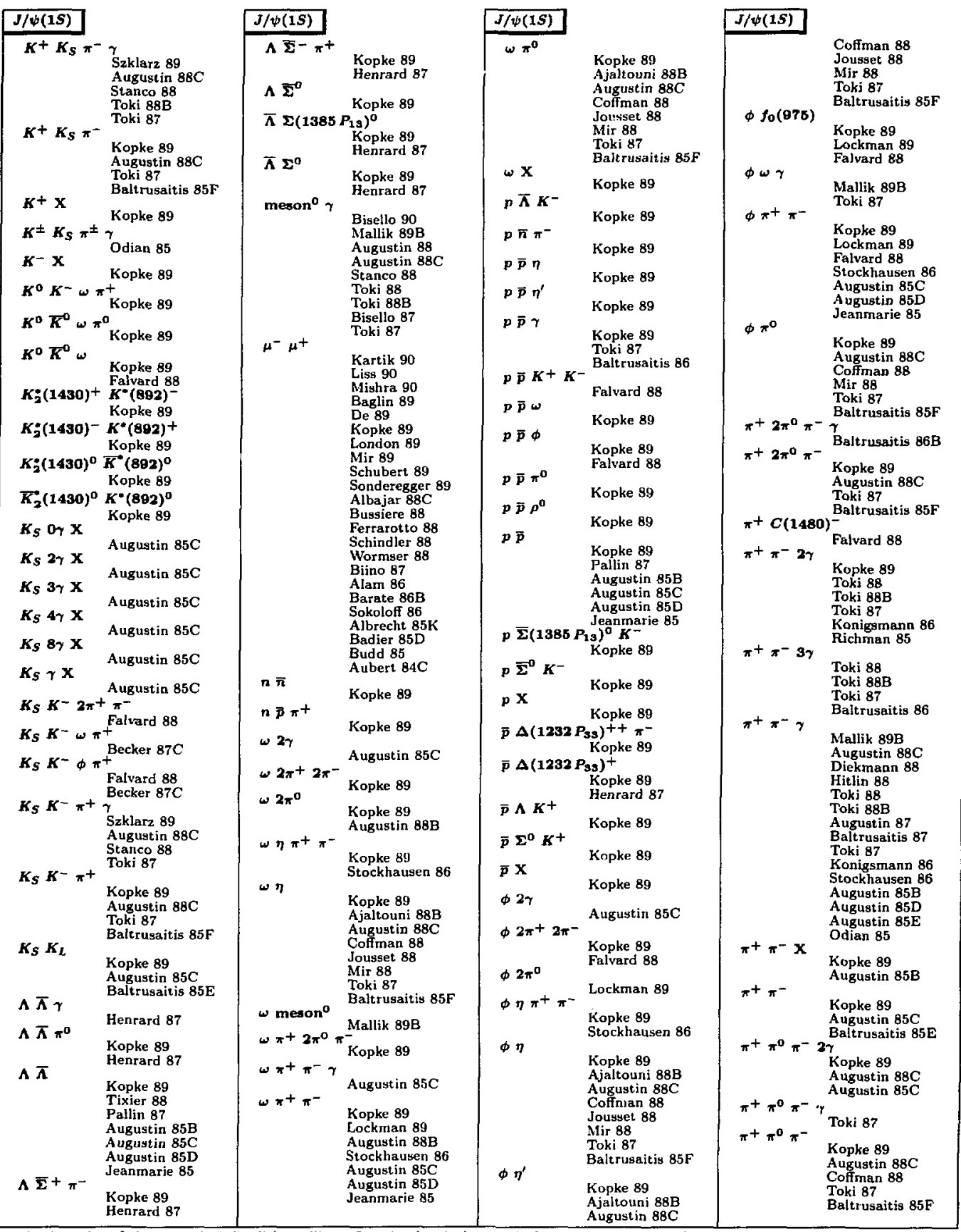

Eutries in order of the equivalent English spelling. Certain chemical symbols for nuclei have been changed to avoid annbiguity with particle names (see the Particle Vocahulary). See the legend on page 297. 


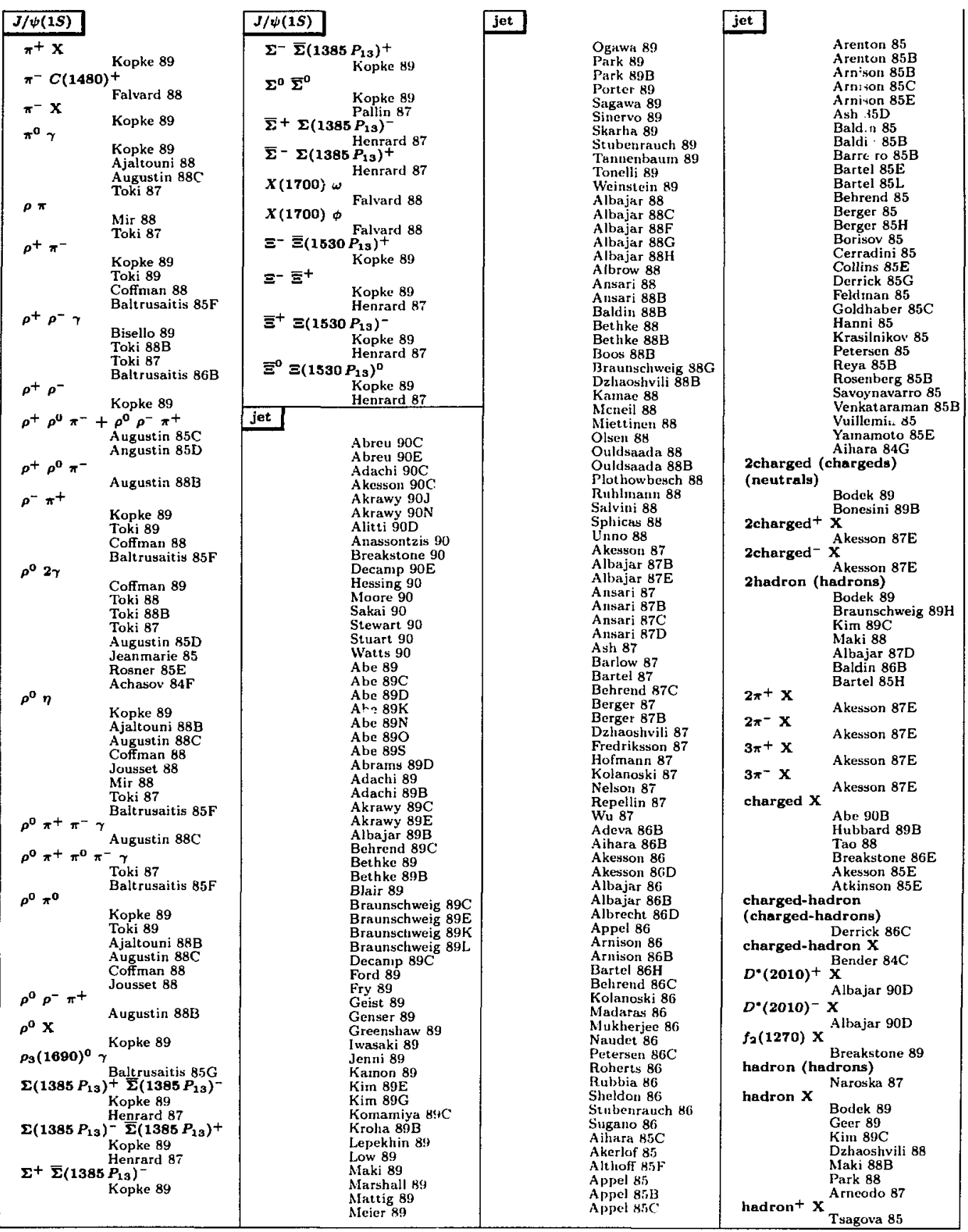




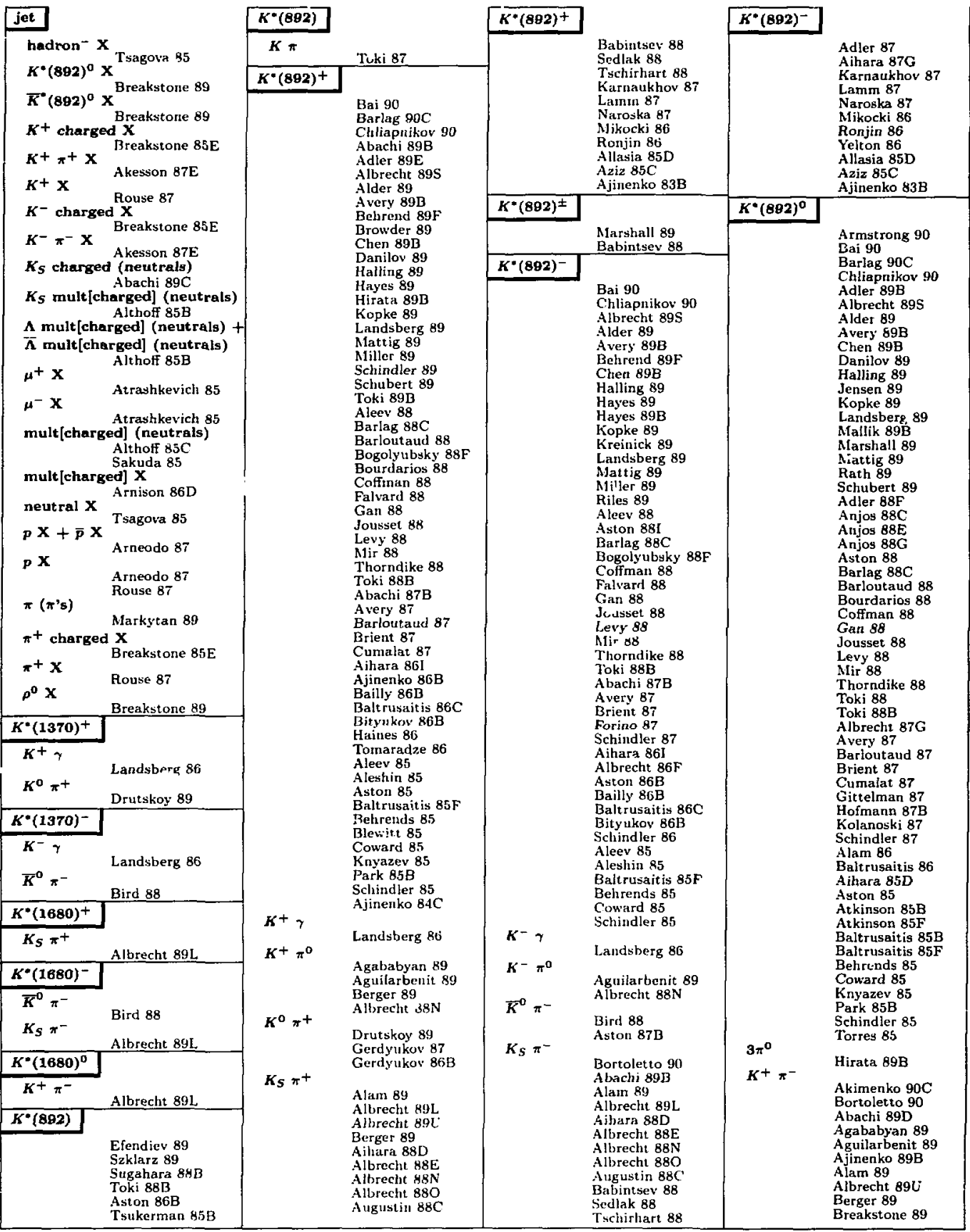




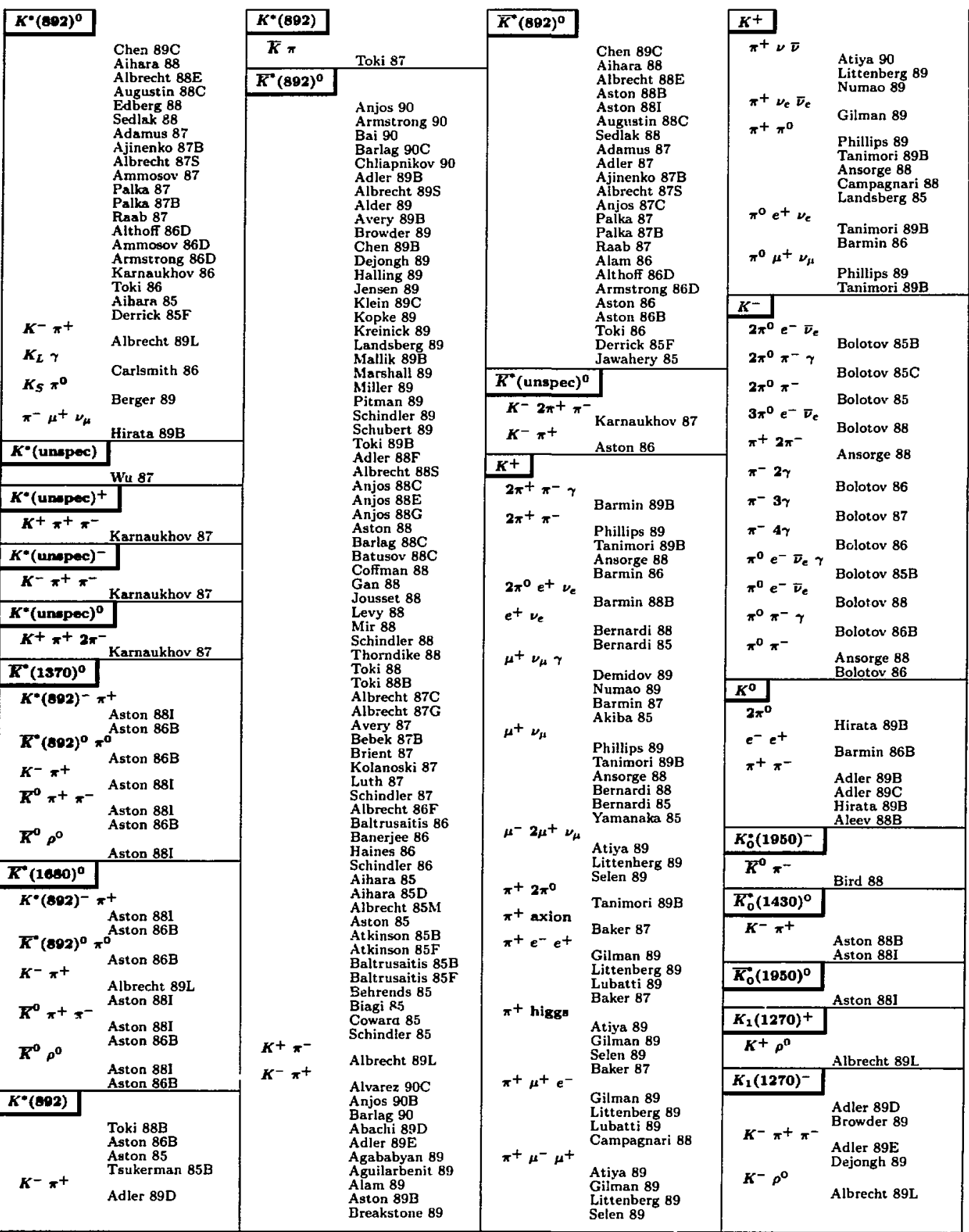




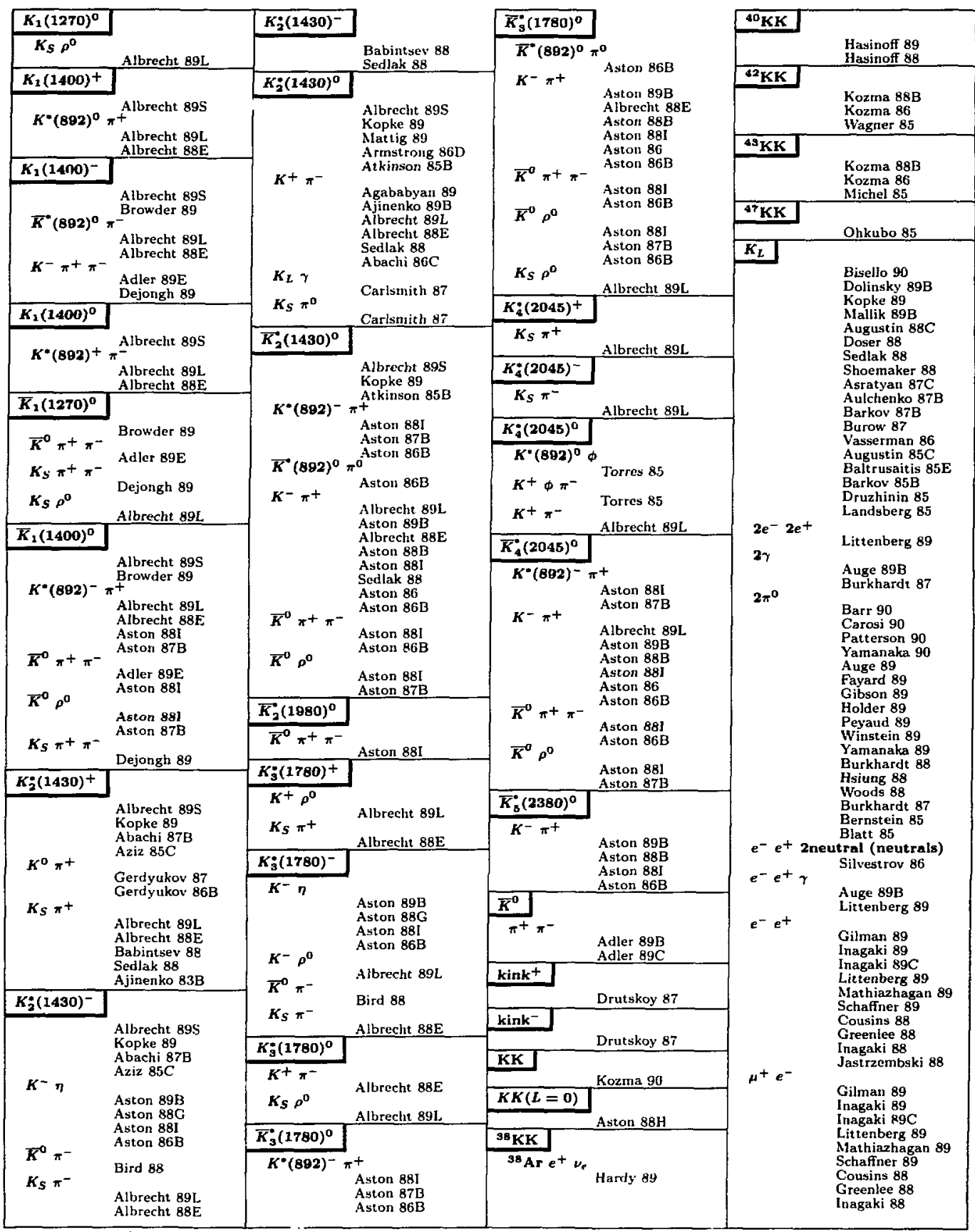




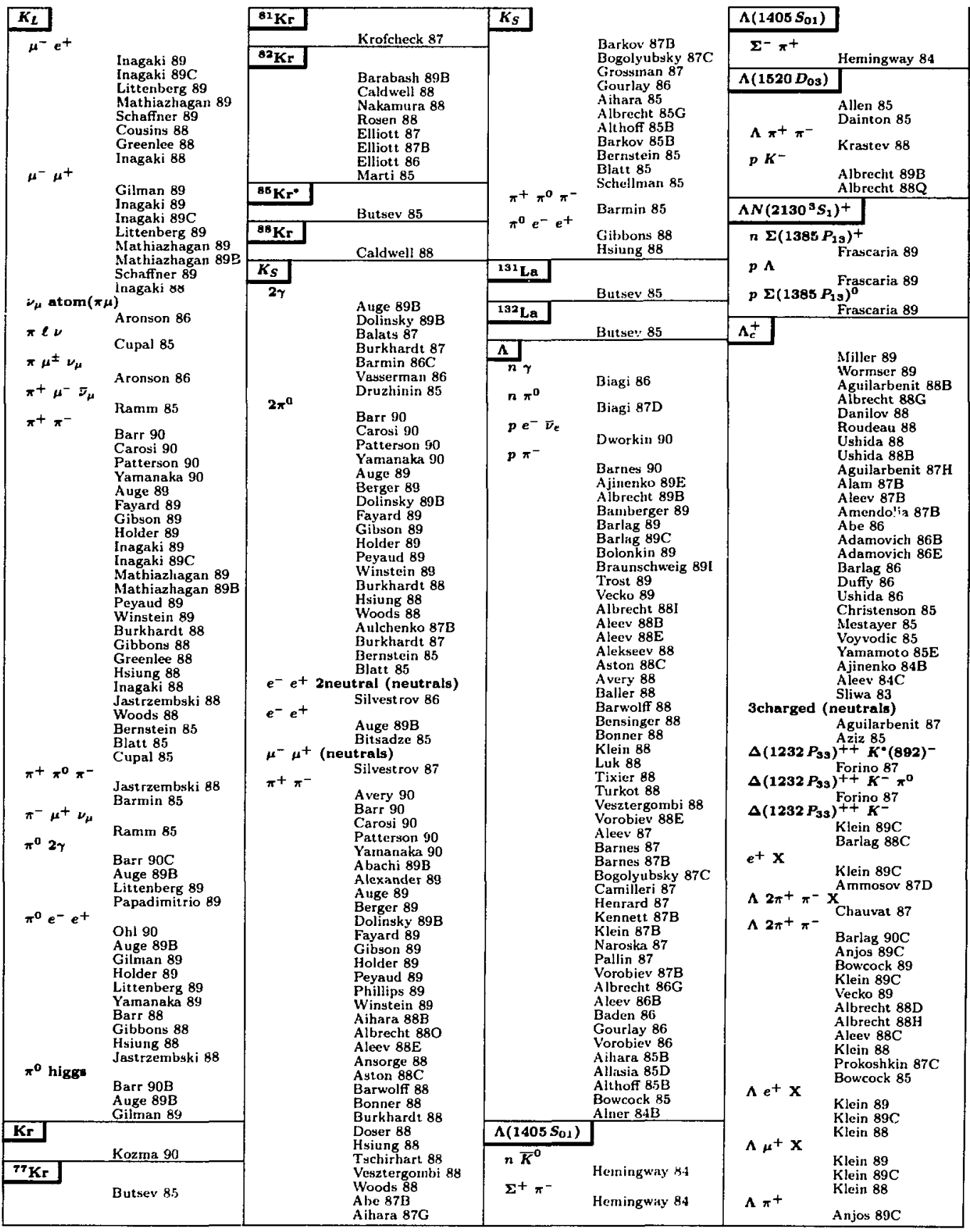




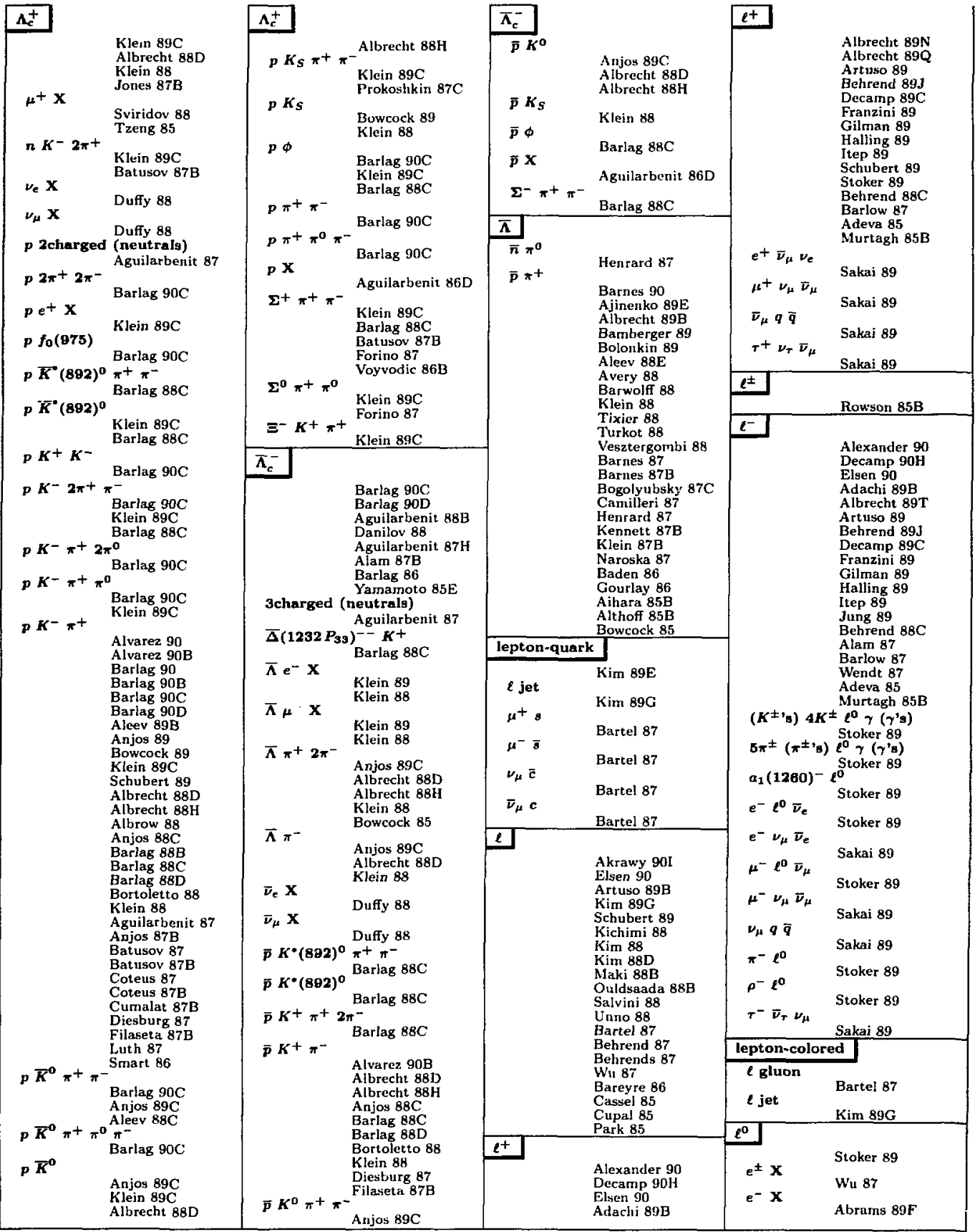




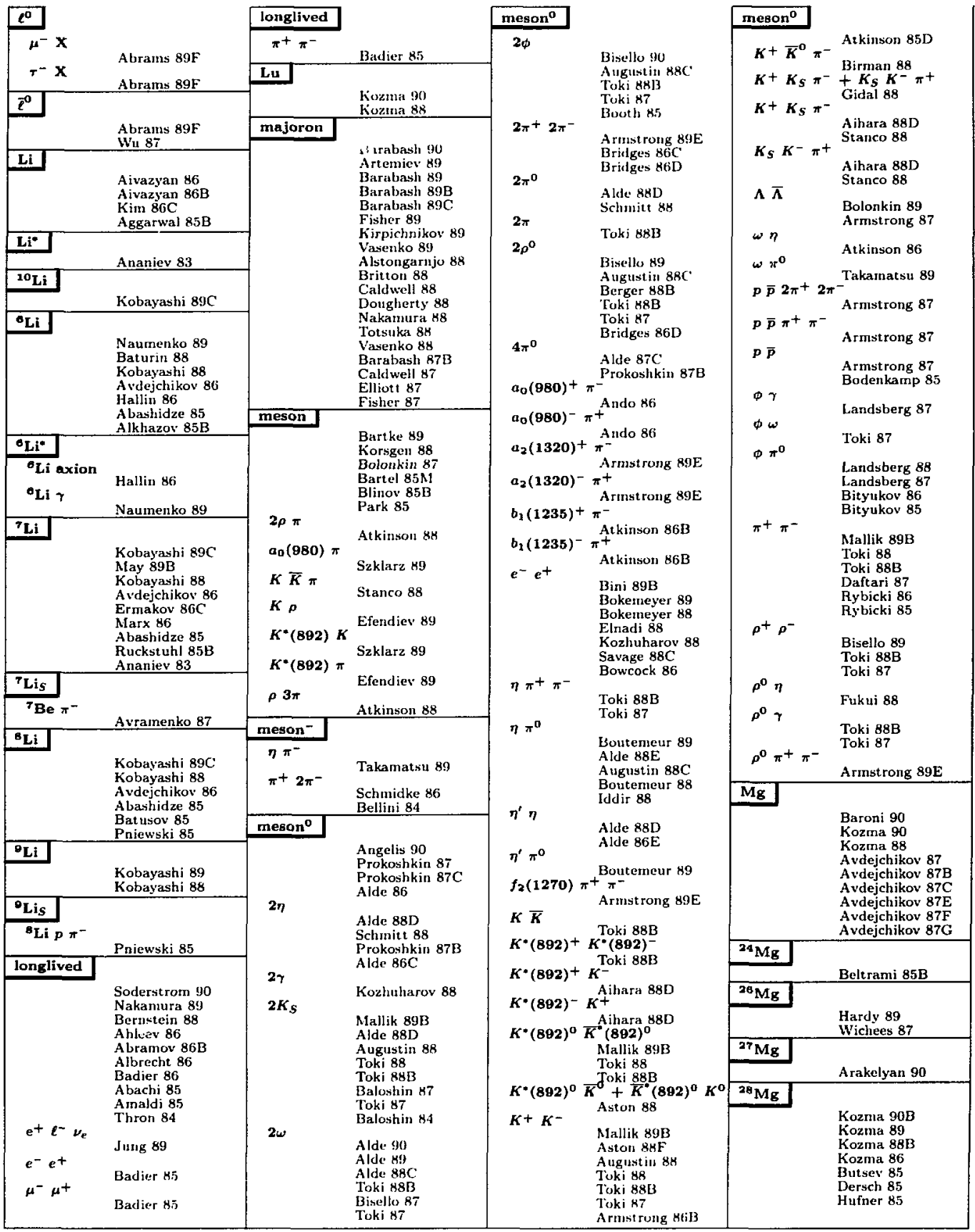




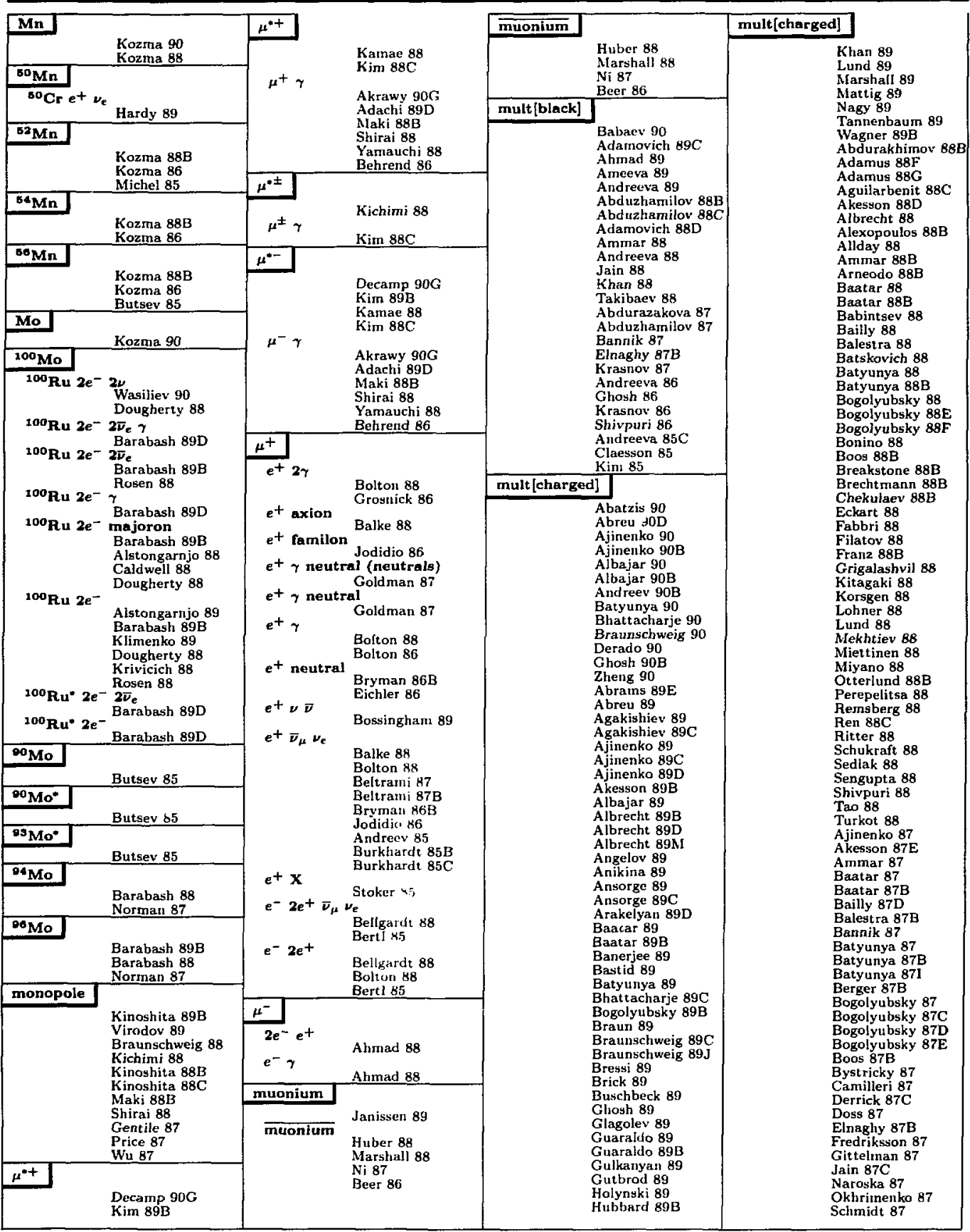

Entries in order of the equivalent English spelling. Certain chemical symbols for nuclei have been changed to avoid ambiguity with particle names (see the Particle Vocabulary). See the legend on page 297. 


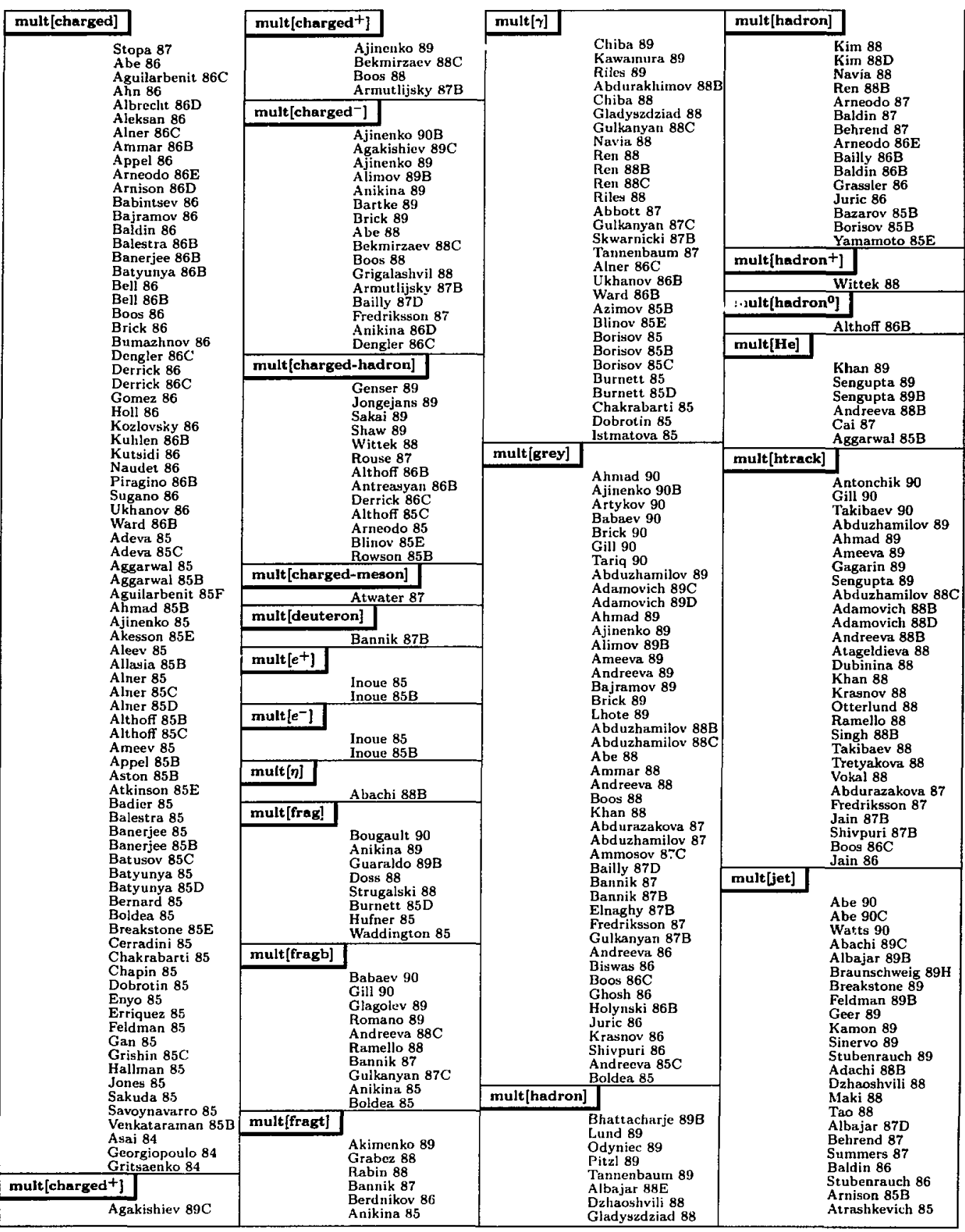




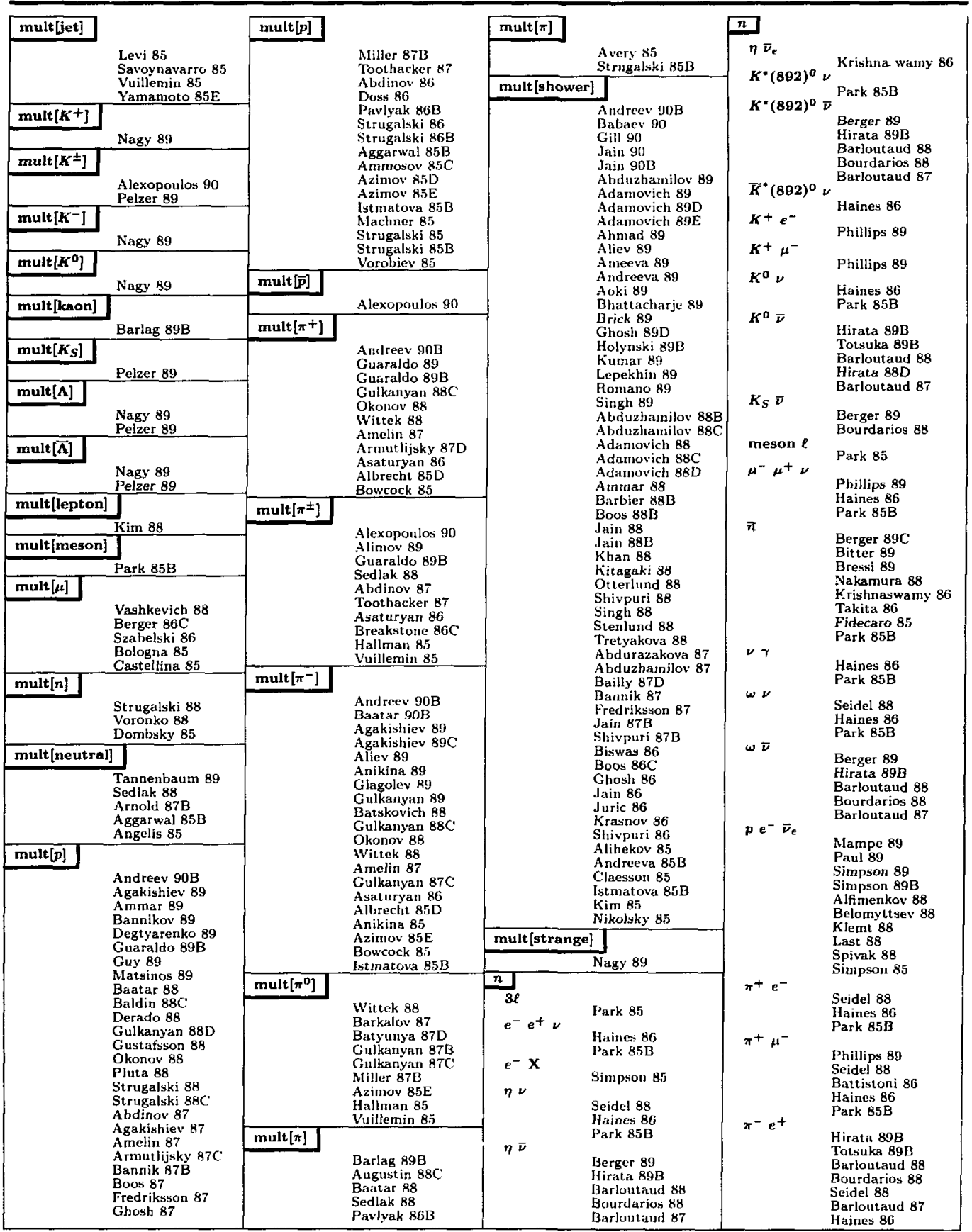

Entries in order of the equivalent English spejling. Certain chemical symbols for nuclei have been changed to avoid ambiguity with particle תames (see the Particle Vocabulary). See the legend on paige 297. 


\begin{tabular}{|c|c|c|c|c|c|c|}
\hline \multicolumn{2}{|l|}{$n$} & \multirow{2}{*}{$\begin{array}{r}N(1700 B)^{+} \\
\Delta\left(1232 P_{\text {ss }}\right)^{++} \\
\text {Aleshin } 87 \\
\pi^{0} \pi^{-}\end{array}$} & \multicolumn{2}{|l|}{$24 \mathrm{Na}$} & \multicolumn{2}{|l|}{ neutral } \\
\hline \multirow[t]{4}{*}{$\pi^{-} \mu^{+}$} & \multirow{4}{*}{$\begin{array}{l}\text { Park 85B } \\
\text { Hirata 89B } \\
\text { Barloutaud } 88 \\
\text { Bourdarios } 88 \\
\text { Scidel 88 } \\
\text { Barloutaud } 87 \\
\text { Battistoni 86 } \\
\text { Haines 86 } \\
\text { Park 85B }\end{array}$} & & \multirow{5}{*}{\multicolumn{2}{|c|}{$\begin{array}{l}\text { Amroyan } 89 \\
\text { Kouma 89 } \\
\text { Kooma 88B } \\
\text { Aleklett 87 } \\
\text { Damdinsuren } 87 \\
\text { Dropesky 86 } \\
\text { Kozma 86 } \\
\text { Dutsev 85 } \\
\text { Dersch 85 } \\
\text { Hufner 85 } \\
\text { Michel 85 } \\
\end{array}$}} & \multirow{2}{*}{\multicolumn{2}{|c|}{$\begin{array}{l}\text { 2hadron (hadrong) } \\
\text { Odaka } 89 \\
\text { charged+ charged } \\
\text { (neutrals) }\end{array}$}} \\
\hline & & $N(2100 B)+$ & & & & \\
\hline & & $\begin{array}{r}\Delta\left(1232 P_{3 s}\right)^{++} \begin{array}{c}\text { Aleshin } \\
\rho^{-} \\
\text {Aleshin } 87\end{array} \\
\end{array}$ & & & $e^{-} e^{+}$ & $\begin{array}{l}\text { Burchat } 90 \\
\text { Asanuma } 90 \\
\text { Aulchenko } 86\end{array}$ \\
\hline & & $n$ (spect) & & & & $\begin{array}{l}\text { Blinov } 86 \mathrm{~B} \\
\text { Eichler } 86 \\
\text { Mageras } 86\end{array}$ \\
\hline$\pi^{0} \nu$ & \multirow{2}{*}{$\begin{array}{l}\text { Haines } 8 C \\
\text { Park } 85 \mathrm{~B}\end{array}$} & $\begin{array}{l}\text { Vapenikova } 88 \\
\text { Ball } 87\end{array}$ & & & & Savage 86B \\
\hline \multirow[t]{3}{*}{$\pi^{0} \bar{v}$} & & $\begin{array}{l}\text { Bridges } 86 \mathrm{~B} \\
\text { Kitagaki } 86\end{array}$ & & $\begin{array}{r}\text { Kozma } 90 \\
\text { Kozma } 88 \\
\end{array}$ & neutralino & \\
\hline & \multirow{3}{*}{$\begin{array}{l}\text { Berger } 89 \\
\text { Hirata } 89 \mathrm{~B} \\
\text { Totsuka } 89 \mathrm{~B} \\
\text { Barloutaud } 88 \\
\text { Bourdarios } 88 \\
\text { Barloutaud } 87\end{array}$} & $\begin{array}{c}\text { Mann } 86 \\
\text { Sai } 86 \\
\end{array}$ & ${ }^{80} \mathrm{Nb}$ & & & $\begin{array}{l}\text { Akrawy 90M } \\
\text { Barklow 90 } \\
\text { Decamp 90D }\end{array}$ \\
\hline & & $N^{*}$ (urspec) + & & Hufner 85 & ${ }^{80} \mathrm{Ni}$ & \\
\hline \multirow[t]{2}{*}{$\rho^{+} e^{-}$} & & Batyunya 86D & ${ }^{92} \mathrm{Nb}^{*}$ & Butsev 85 & & $\begin{array}{l}\text { Kozma 88B } \\
\text { Kozma 86 } \\
\end{array}$ \\
\hline & \multirow{2}{*}{$\begin{array}{l}\text { Seidel } 88 \\
\text { Haines } 86 \\
\text { Park 85B }\end{array}$} & $N_{b / 2}^{*}(1380)^{+++}$ & $\bar{n}$ & & ${ }^{{ }^{57} \mathrm{Ni}}$ & \\
\hline \multirow[t]{2}{*}{$\rho^{+} \mu^{-}$} & & \multirow{2}{*}{ 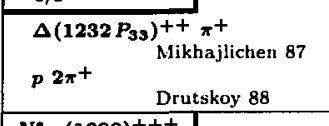 } & \multirow{8}{*}{\multicolumn{2}{|c|}{$\begin{array}{l}\text { Berger 89C } \\
\text { Boos 89 } \\
\text { Kanazirski } 89 \\
\text { kopke 89 } \\
\text { Castro 88 } \\
\text { Nakannura } 88 \\
\text { Burow 87 } \\
\text { Fredrikson } 87 \\
\text { Henrard } 87 \\
\text { Kageyama } 87 \\
\text { Batyunya 86 } \\
\text { Bruckner 86 } \\
\text { Cresti 86 } \\
\text { Krishnaswamy } 86 \\
\text { Fidiecaro 85 } \\
\text { Nakamura 85B } \\
\text { Park 85B } \\
\text { Tsukerman 85 } \\
\text { Cardello 84 } \\
\end{array}$}} & & $\begin{array}{l}\text { Koima 88B } \\
\text { Kozma } 86 \\
\end{array}$ \\
\hline & \multirow{2}{*}{$\begin{array}{l}\text { Phillips } 89 \\
\text { Seidel } 88 \\
\text { Haines } 86 \\
\text { Park 85B }\end{array}$} & & & & ${ }^{88} \mathrm{Ni}$ & Bonin 86 \\
\hline \multirow[t]{4}{*}{$\rho^{-} e^{+}$} & & $N_{s / 2}^{*}(1380)^{+++}$ & & & ${ }^{65} \mathrm{Ni}$ & \\
\hline & \multirow{3}{*}{$\begin{array}{l}\text { Barloutaud } 88 \\
\text { Bourdarios } 88 \\
\text { Seidel } 88 \\
\text { Barloutaud } 87 \\
\text { Haines 86 } \\
\text { Park 85B }\end{array}$} & $\begin{array}{r}\text { Arefiev } 87 \\
\end{array}$ & & & & Wagner 85 \\
\hline & & $N_{8 / 2}^{*}(1480)^{+++}$ & & & ${ }^{86} \mathrm{Ni}$ & \\
\hline & & $\begin{array}{l}\text { Arefiev 90B } \\
\text { Arefiev 87 }\end{array}$ & & & Nit & \\
\hline \multirow{2}{*}{$\rho^{-} \mu^{+}$} & \multirow{3}{*}{$\begin{array}{l}\text { Barloutaud } 88 \\
\text { Bourdarios } 88 \\
\text { Seidel } 88 \\
\text { Barloutaud } 87 \\
\text { Haines } 86 \\
\text { Park } 85 \mathrm{~B}\end{array}$} & $\begin{array}{ll}p 2 \pi^{+} & \begin{array}{l}\text { Bogolyubsky } 86 \mathrm{E} \\
\text { Arefiev } 86\end{array} \\
\end{array}$ & & & & $\begin{array}{l}\text { Avdejchikov } 87 \\
\text { Avdejchikov 87B } \\
\text { Avdejchikov 87C } \\
\text { Avdeichikov } 87 \mathrm{E}\end{array}$ \\
\hline & & $N_{B / 2}^{*}(1650)^{+++}$ & & & & $\begin{array}{l}\text { Avdejchikov 87F } \\
\text { Avdejchikov 87G }\end{array}$ \\
\hline \multirow[t]{5}{*}{$\rho^{0} \nu$} & & \multirow{4}{*}{ 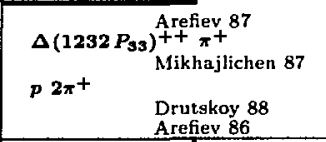 } & $\bar{N} N(I=0)$ & & & $\begin{array}{l}\text { Avdejchikov } 87 \mathrm{H} \\
\text { Avc' jichikgv } 87 \mathrm{I}\end{array}$ \\
\hline & \multirow{4}{*}{$\begin{array}{l}\text { Seidel 88 } \\
\text { Haines 86 } \\
\text { Park 85B } \\
\text { Berger 89 } \\
\text { Hirata 89B } \\
\text { Barloutaud } 88 \\
\text { Bourdarios } 88 \\
\text { Barloutaud } 87 \\
\end{array}$} & & & Sapozhnikov 86 & & Sangster 87 \\
\hline & & & $\bar{N} N(I=1)$ & Sancraikoy 86 & & $\begin{array}{l}\text { Aggarwal 85B } \\
\text { Alkhazov 85B } \\
\end{array}$ \\
\hline & & & ${ }^{150} \mathrm{Nd}$ & Sapczhnikov 80 & Nit* & \\
\hline & & $\frac{N_{8 / 2}(1760)++}{\Delta\left(1232 P_{33}\right)^{++} \pi^{+}}+$ & $\begin{array}{l}{ }^{150} \mathrm{Sm} 2 e^{-} \\
{ }^{150} \mathrm{Sm} 2 e^{-}\end{array}$ & $\begin{array}{l}\text { FFe } \\
\text { Barabash } 89 \mathrm{~B} \\
\text { majoron }\end{array}$ & $\begin{array}{l}{ }^{14} \text { Nit } e^{-} e^{-} \\
{ }^{14} \text { Nit } 1 \text { eut } \\
{ }^{15} \text { Nit }\end{array}$ & $\begin{array}{l}+ \\
\text { Savage } 86 B \\
\text { ral } \\
\text { Savage } 86 B\end{array}$ \\
\hline$N(1440 B)^{+}$ & & p $2 \pi^{+} \quad$ Drutskoy 88 & ${ }^{100} \mathrm{Sm} 2 e^{-}$ & Barabash 89B & & Seestrommorr 85 \\
\hline & Azhgirej 88 & Drutskoy 88 & & Barabash 89B & ${ }^{13} \mathrm{Nit}$ & \\
\hline$N(1440 B)^{0}$ & & $N_{3 / 2}^{*}(2070)^{+++}$ & Ne & & & Goodman 85 \\
\hline & Alimov 88 & $\Delta\left(1232 P_{3 s}\right)^{++} \pi^{+}$ & & $\begin{array}{l}\text { Baroni } 90 \\
\text { Aderholz } 89\end{array}$ & ${ }^{14}$ Nit & \\
\hline$N\left(1440 P_{11}\right)$ & & $p 2 \pi+\quad$ Mikhajlichen 87 & & $\begin{array}{l}\text { Aderholz } 89 \\
\text { Marage } 89\end{array}$ & & $\begin{array}{l}\text { Hardy } 89 \\
\text { Savage } 88 \mathrm{C}\end{array}$ \\
\hline$N\left(1440 P_{11}\right)^{+}$ & & $\begin{array}{r}\text { Drutskoy } 88 \\
\end{array}$ & & Avdejchikov 87 & & $\begin{array}{l}\text { Hallin } 86 \\
\text { Savage } 86 \mathrm{~B}\end{array}$ \\
\hline$p \pi^{+} \pi^{-}$ & & $\mathrm{N}_{\mathrm{s} / \mathrm{2}}^{*}(\text { unspec})^{+++}$ & & $\begin{array}{l}\text { Avdejchikov 87B } \\
\text { Avdejchikov 87C }\end{array}$ & 14Nt+* & Wharton 85 \\
\hline$N\left(1440 P_{11}\right)^{0}$ & Amelin 87 & $\Delta\left(1232 P_{3 s}\right)^{++} \pi^{+}$ & & $\begin{array}{l}\text { Avdejchikov 87E } \\
\text { Avdejchikov 87F }\end{array}$ & $\frac{\left.{ }^{14} \mathrm{Nit}^{*}\right]}{{ }^{14} \mathrm{Nit}_{\text {axion }}}$ & \\
\hline$p \pi^{-}$ & & $\begin{array}{ll}p 2 \pi^{+} & \text {Mikhajlichen 87 } \\
\text { Abramov 89C }\end{array}$ & & $\begin{array}{l}\text { Avuejclikikov } 87 \mathrm{G} \\
\text { Avdejchikov } 871\end{array}$ & ${ }^{14} \mathrm{Nit} e^{-} e^{t}$ & + Hallin 86 \\
\hline$N(1520 B)^{+}$ & Amelin 87 & Na & & $\begin{array}{l}\text { Sangster } 87 \\
\text { Ammosov } 86 \mathrm{C}\end{array}$ & ${ }^{14}$ Nit mes & Savage $88 \mathrm{C}$ \\
\hline & $\begin{array}{l}\text { Azhgirej } 88 \\
\text { Batyunya } 87 \mathrm{~F}\end{array}$ & $\begin{array}{l}\text { Baroni } 90 \\
\text { Kozma 90 } \\
\text { Kozma } 88\end{array}$ & & $\begin{array}{l}\text { Ballagh } 86 \\
\text { Baltay } 86 \\
\text { Marage } 86\end{array}$ & ${ }^{15} \mathrm{Nit}$ & \\
\hline$N\left(1680 F_{16}\right)^{+}$ & $\int_{\text {Azhgirej } 88}$ & $\begin{array}{l}\text { Avdejchikov 87 } \\
\text { Avdejchikov 87B } \\
\text { Avdejchikov 87C }\end{array}$ & ${ }^{18} \mathrm{Ne}$ & Williams 89B & & $\begin{array}{l}\text { Ramello } 88 \\
\text { Ardito } 87 \\
\text { Redwine } 86\end{array}$ \\
\hline$N\left(1880 F_{15}\right)^{0}$ & & $\begin{array}{l}\text { Avdejchikov 87E } \\
\text { Avdejchikav 87F } \\
\text { Avdejchikov 87G }\end{array}$ & $\frac{\text { neutral }}{2 \gamma}$ & & & $\begin{array}{l}\text { Seest rommorr } 85 \\
\text { Turley } 85\end{array}$ \\
\hline & Zhokin 89 & ${ }^{24} \mathrm{Na}$ & & $\begin{array}{l}\text { Thuchiaki } 90 \\
\text { Aulchenko } 86\end{array}$ & ${ }^{10} \mathrm{Nit}$ & \\
\hline$N(1700 B)^{+}$ & Batyunya $87 \mathrm{~F}$ & Kozma 90B & & & & $\begin{array}{l}\text { Hasinoff } 89 \\
\text { Hasinoff } 88\end{array}$ \\
\hline
\end{tabular}




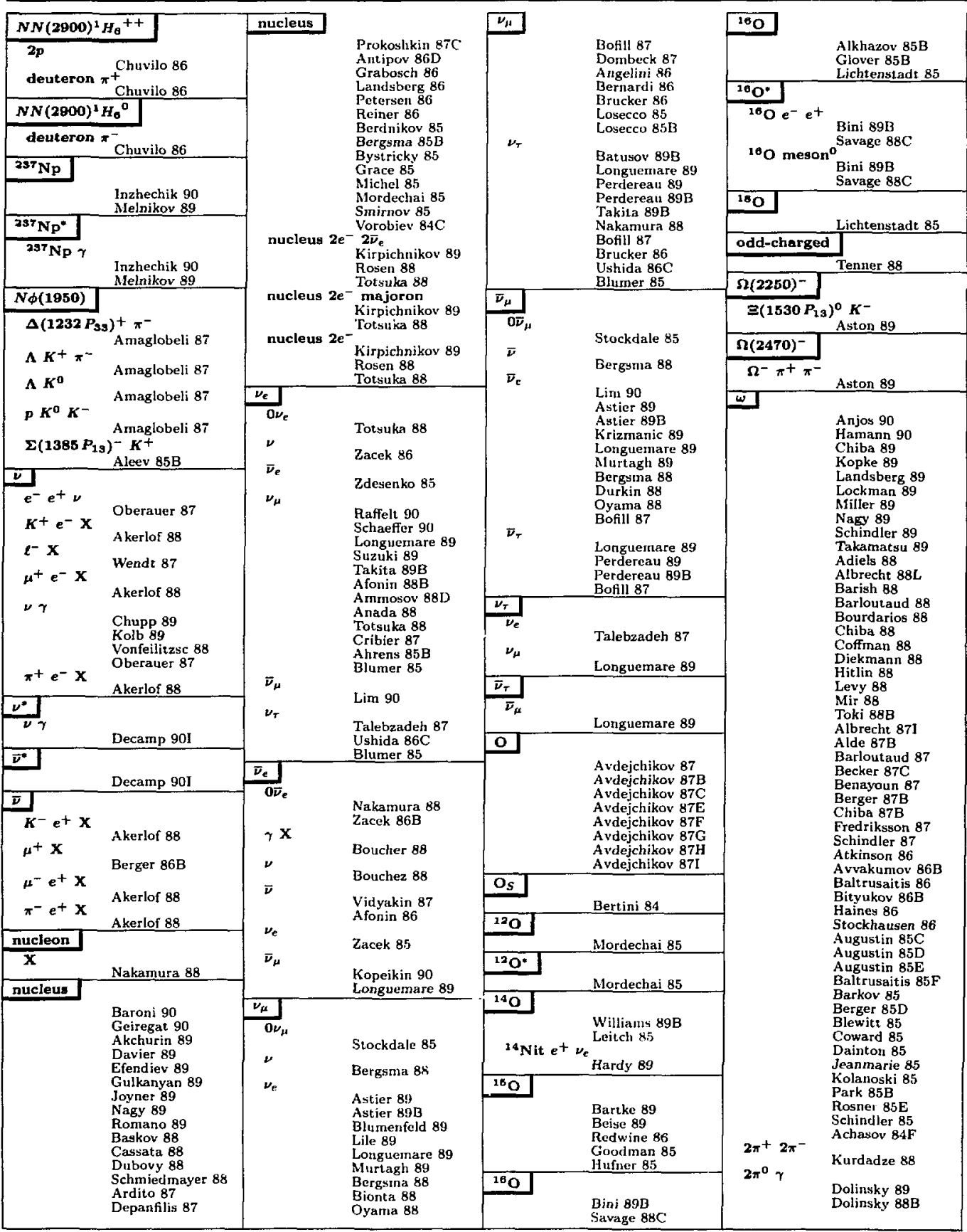

Entries in order of the equivalent English spelling. Certain chemical symbuls for nuclei have been changed to avoid ambiguity with particle names (see the Particle Vocabulary). See the legend on page 297. 


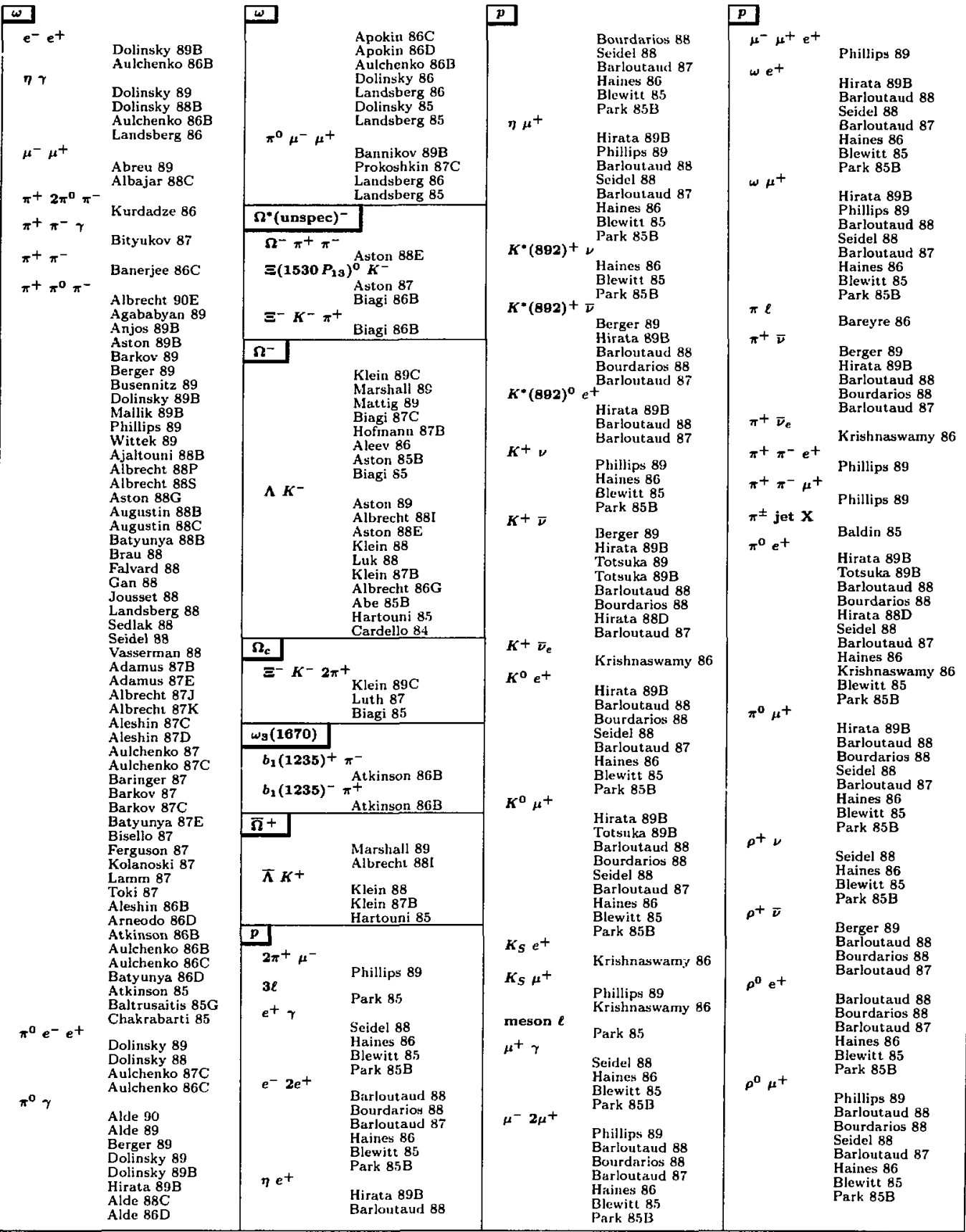




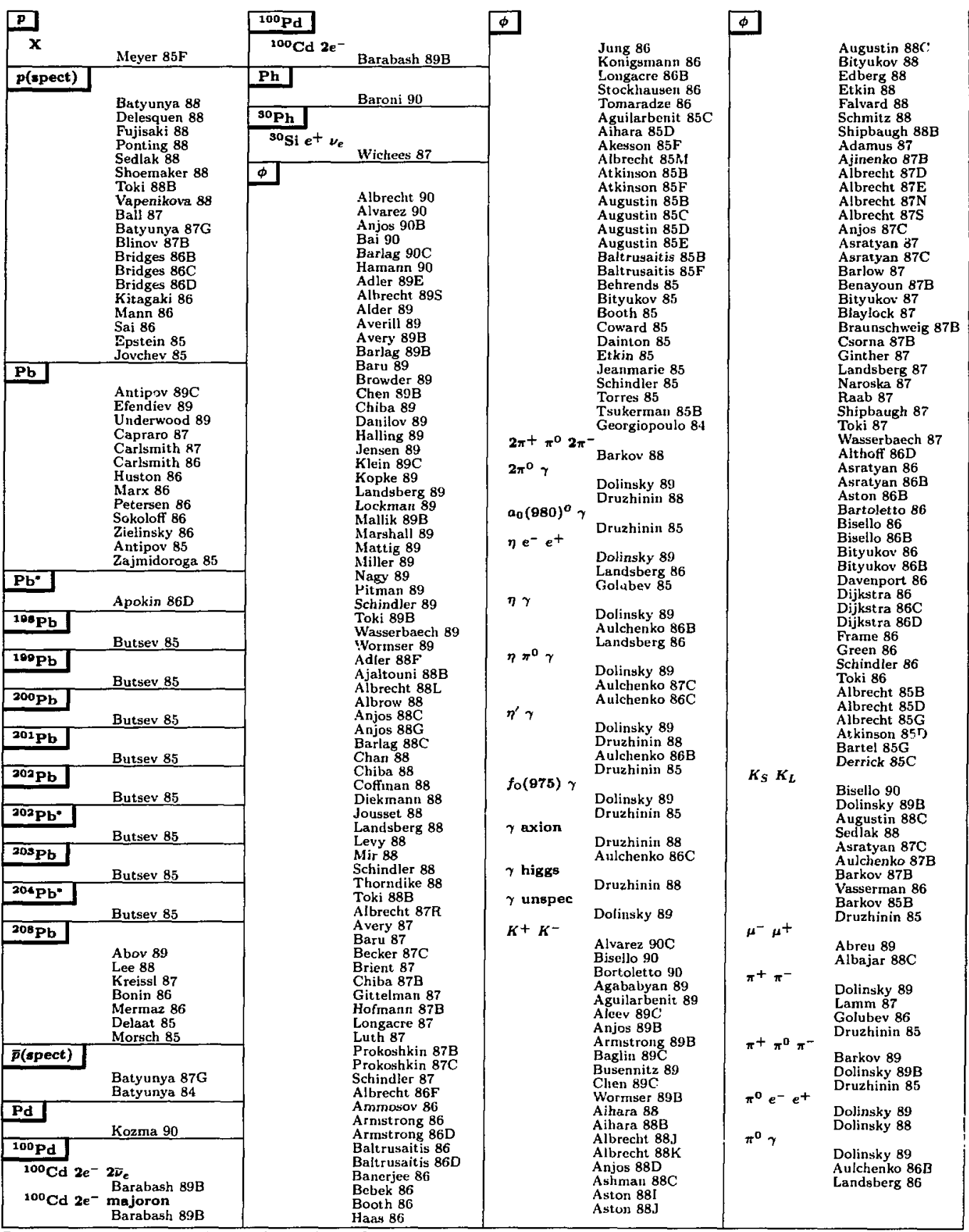

Entries in order of the equivalent English spelling. Certain clemical symbols for nuclei have been clianged to avoid ambiguity with particle names (see the Particle Vocabulary). See the legend oil page 297. 


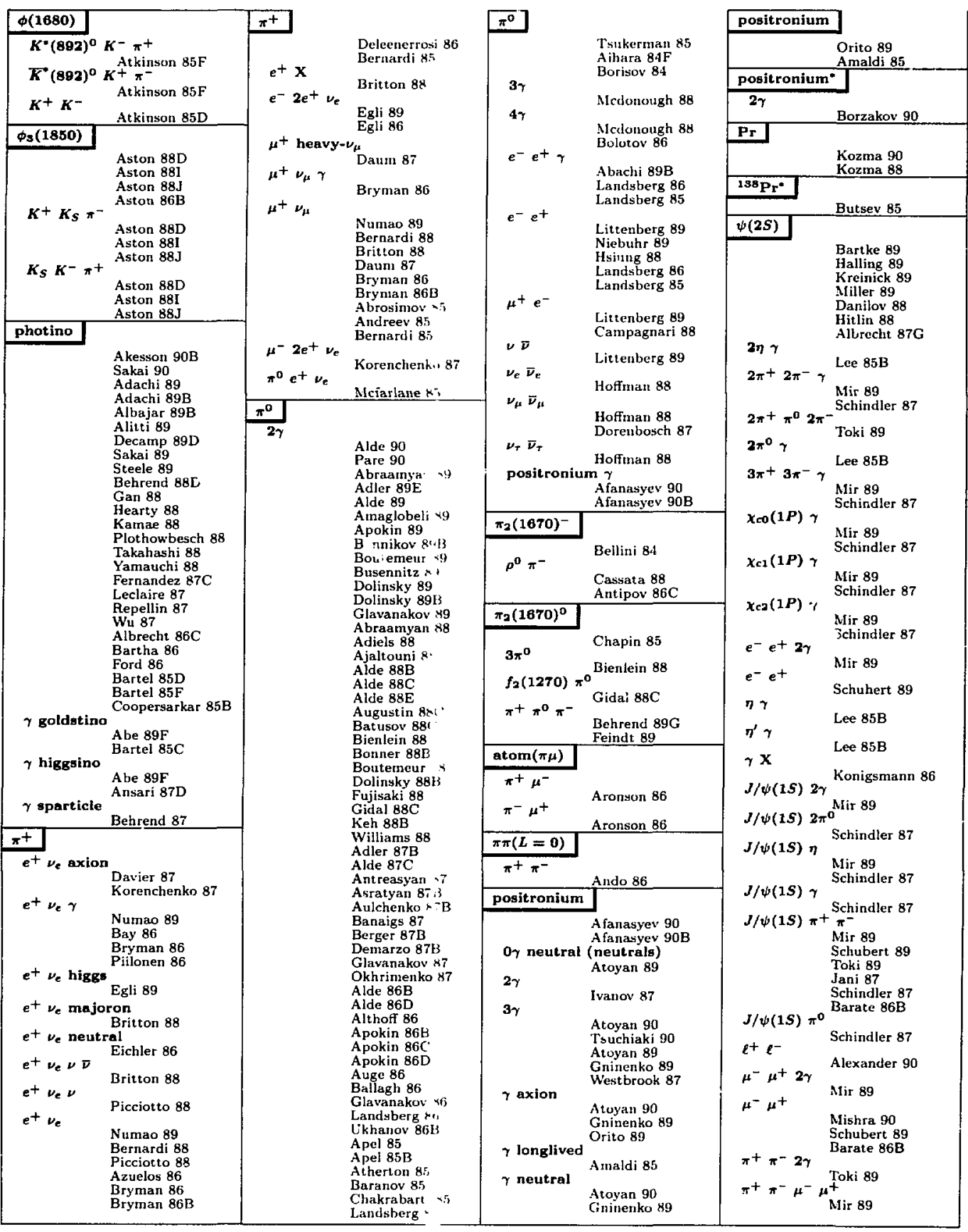




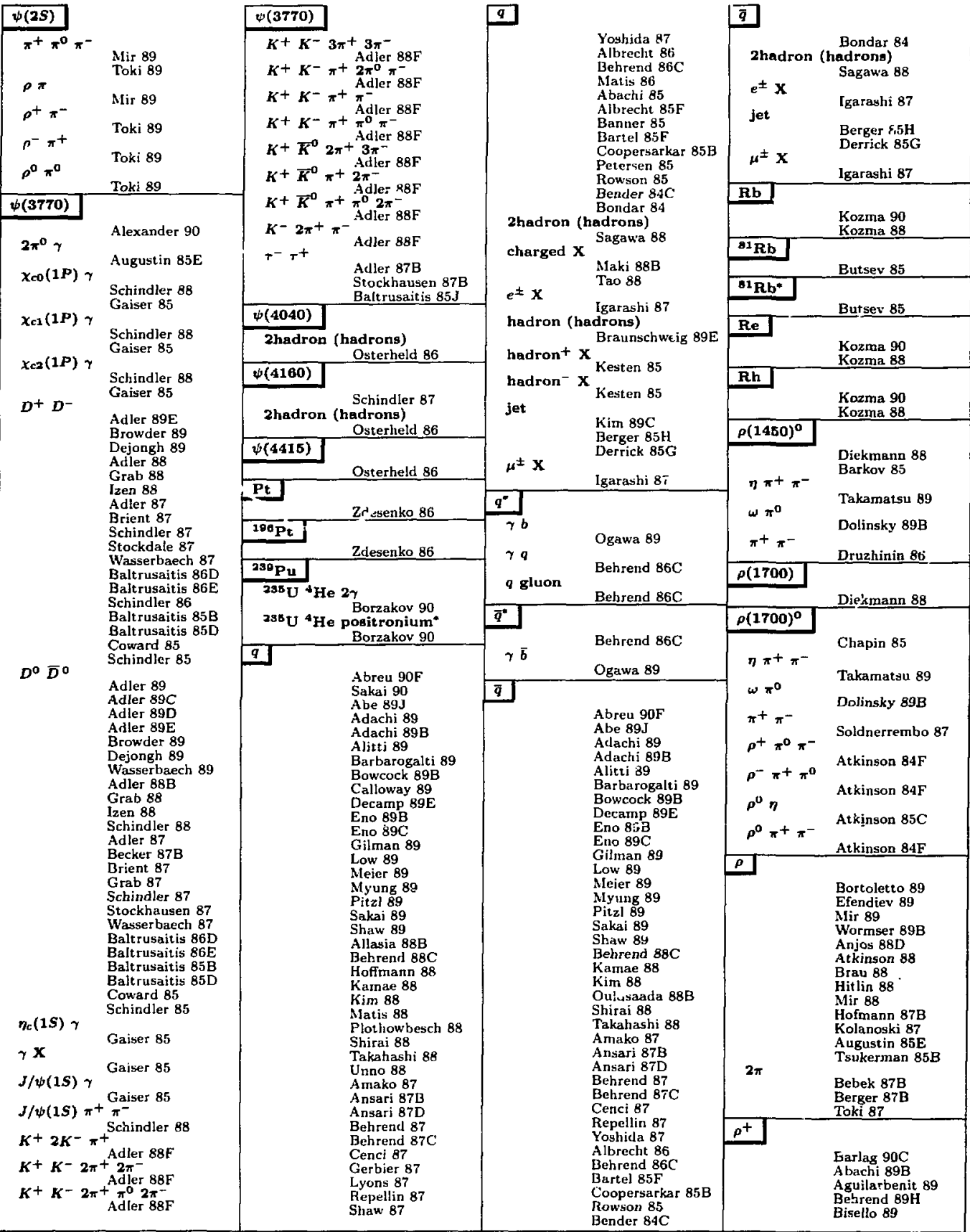




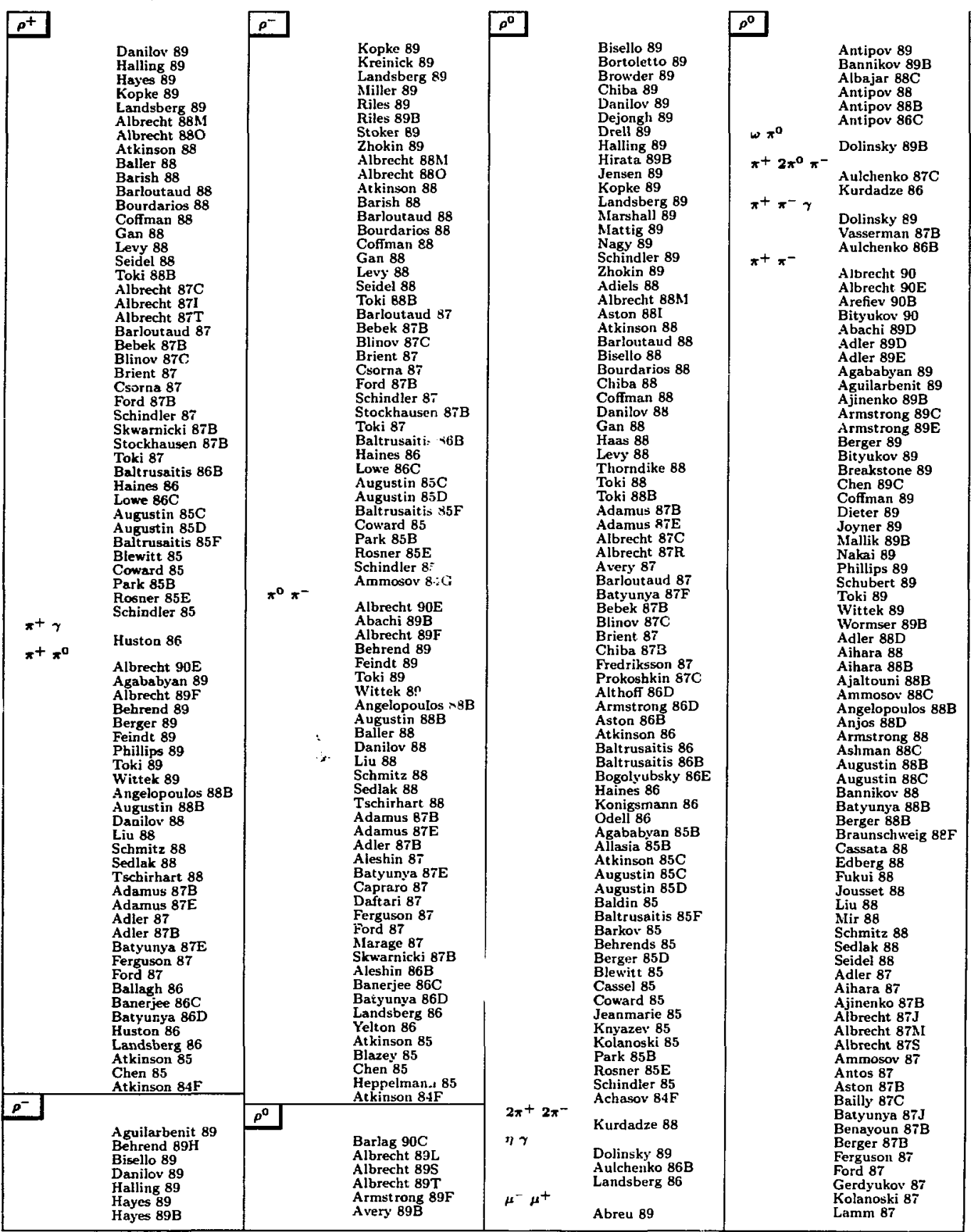




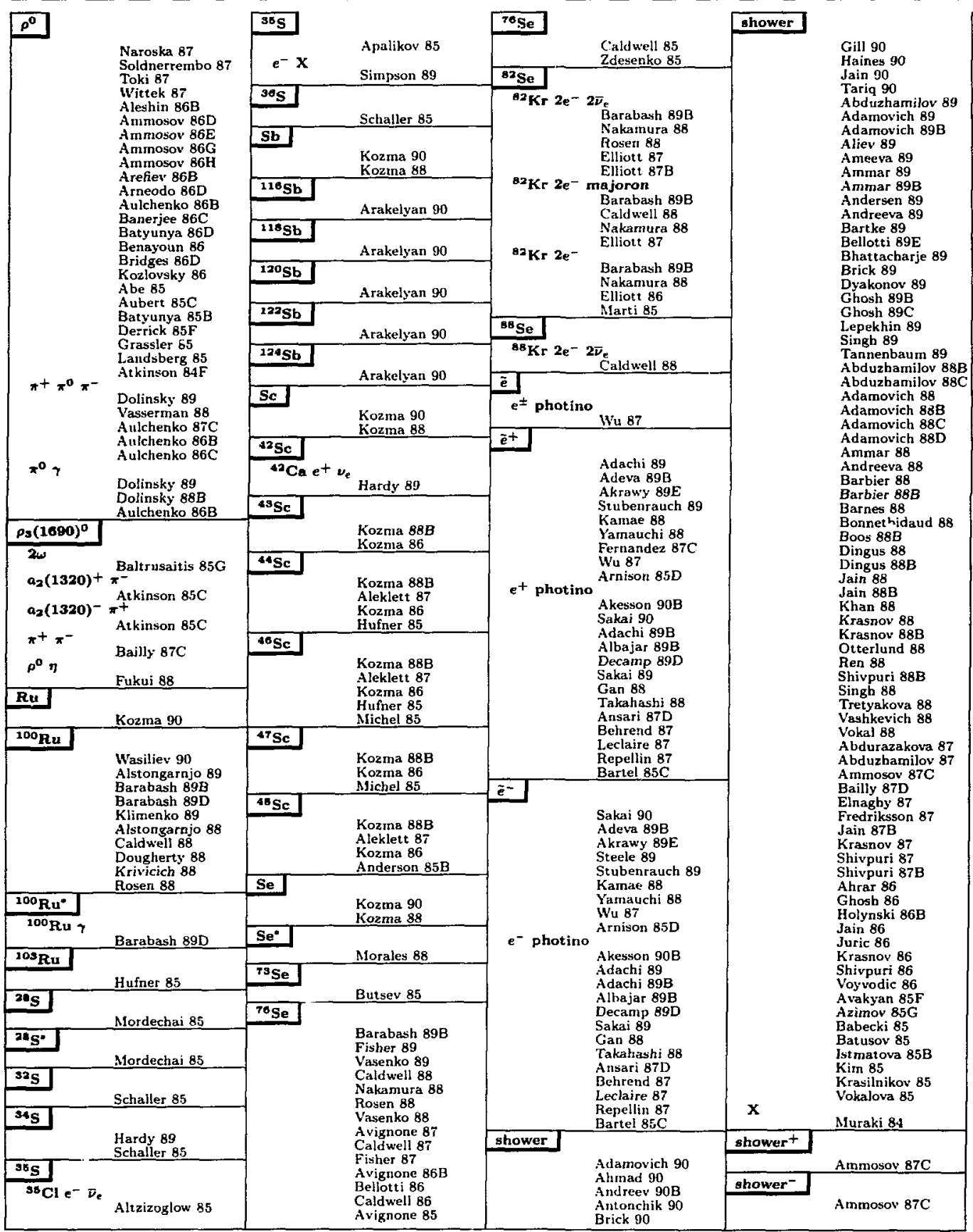

Entries in order of the equivalent English spelling. Certain chenical symbols for nuclei have been changed to avoid ambiguity with particle names (see the Particle Vocabulary). Sec the legend on page 297. 


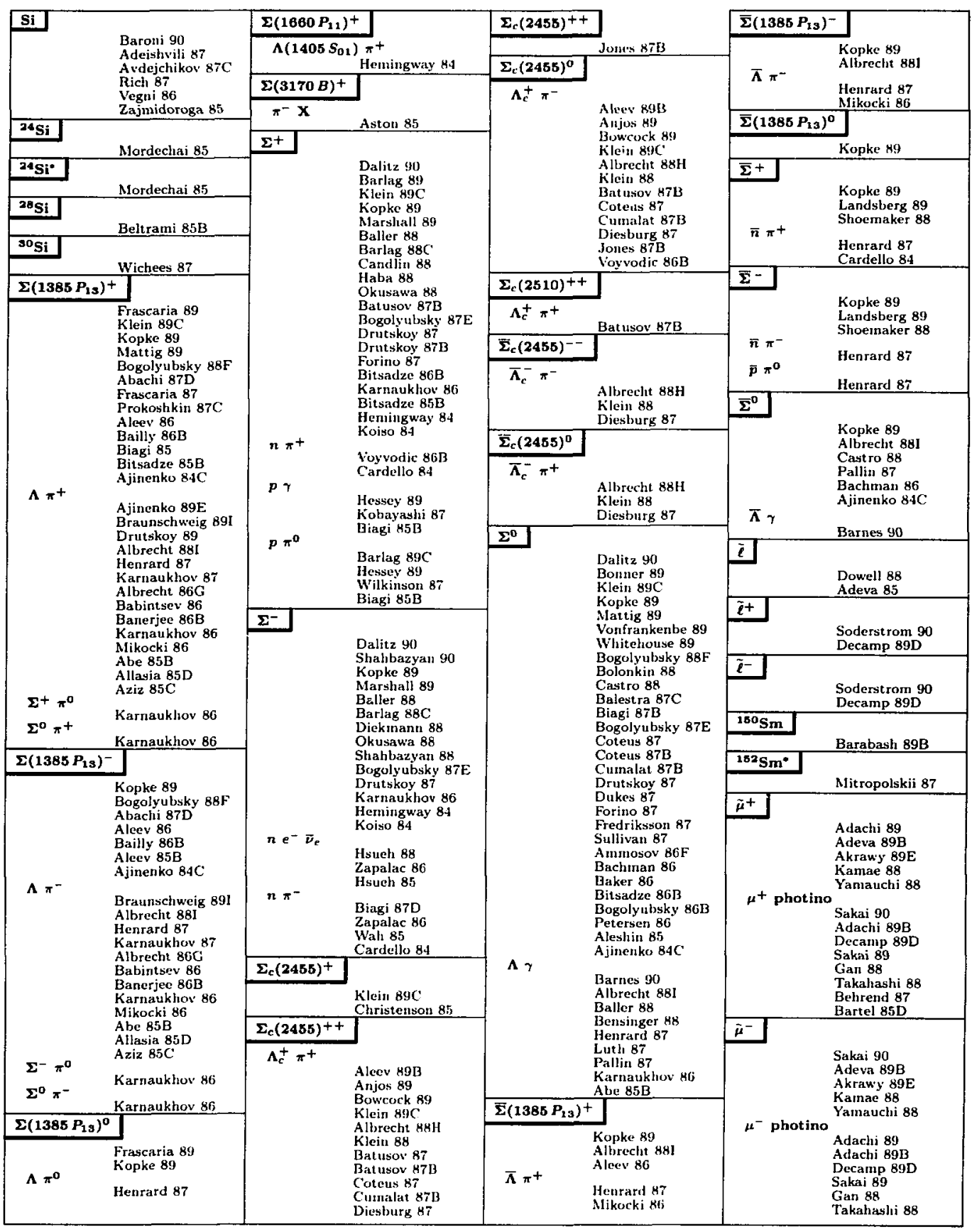




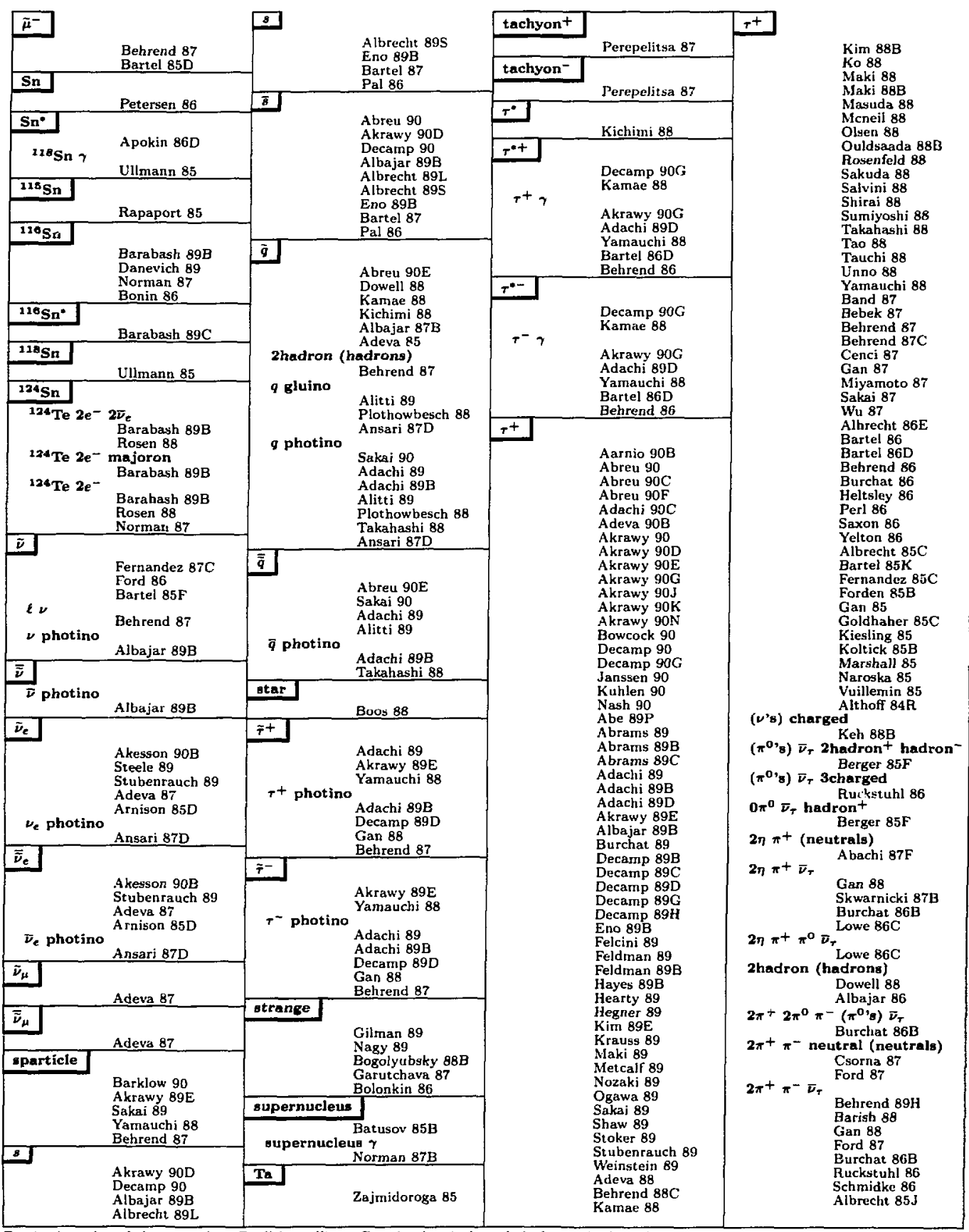




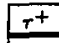

$$
\begin{gathered}
2 \pi^{+} \pi^{-} \pi\left(\pi^{\prime} s\right) \bar{\nu}_{\tau} \\
\text { Barish } 88 \\
2 \pi^{+} \pi^{0} \pi-\begin{array}{c}
\left(\pi^{0} s\right) \bar{\nu}_{\tau} \\
\text { Behrend } 89 \mathrm{H}
\end{array} \\
2 \pi^{+} \pi^{0} \pi^{-} \begin{array}{c}
\text { Gan } 88 \\
\bar{\nu}_{T}
\end{array}
\end{gathered}
$$

3charged (neutrals)

Abachi 89B

Behrend 89D

Braunschweig 89F

Hayes 89

Marshall 89

Adachi 88D

Barish 88

Braunschweig 88D

Gan 88

A tachi 87

Albrecht 87L

Naroska 87

Lowe $86 \mathrm{~B}$

Bartel 85L

3charged neutral (neutrals)

Ford 87

Aihara 861

Akerlof 85B

3charged-hadron neutral (neutralo)

$$
\begin{aligned}
& \text { 37+ Fernandez 85D } \\
& 3 \pi+2 \pi-\bar{\nu}_{T}
\end{aligned}
$$

Bylsma 87

neutrals)

Behrend 89D

Marshall 89

Barish 88

Gan 88

Naroska 87

Bartel 85L

Beltrami 85

scharged-hadron neutra (neutrals)

$5 \pi \bar{\nu}_{\tau} \quad$ Gan 88

7charged (neutrals)

$a_{1}(1260)^{+}$neutral (neutrals) $a_{1}(1260)+\bar{\nu}_{t}$ Ford 87

Ford 87

Skwarnicki 87B

Ruckstuhi 86

charged (neutrals)

$$
\text { Marshall } 89
$$

charged neutral (neutrals)

Abachi 89B

Behrend 89D

Braunschweig $89 \mathrm{~F}$

Hayes 89

Adachi 88D

Albrecht 880

Bacala 88

Bacala $88 \mathrm{~B}$

Barish 88

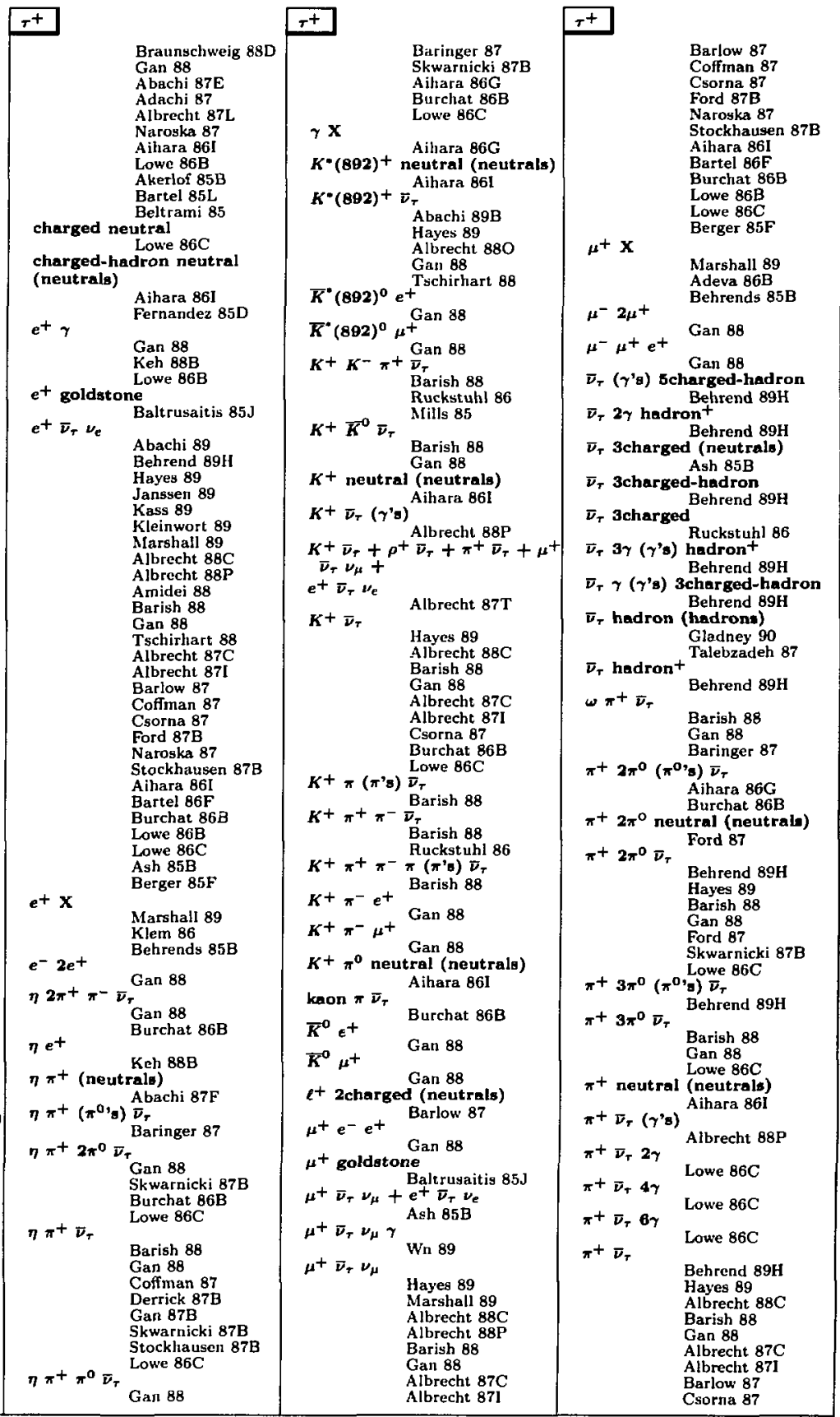




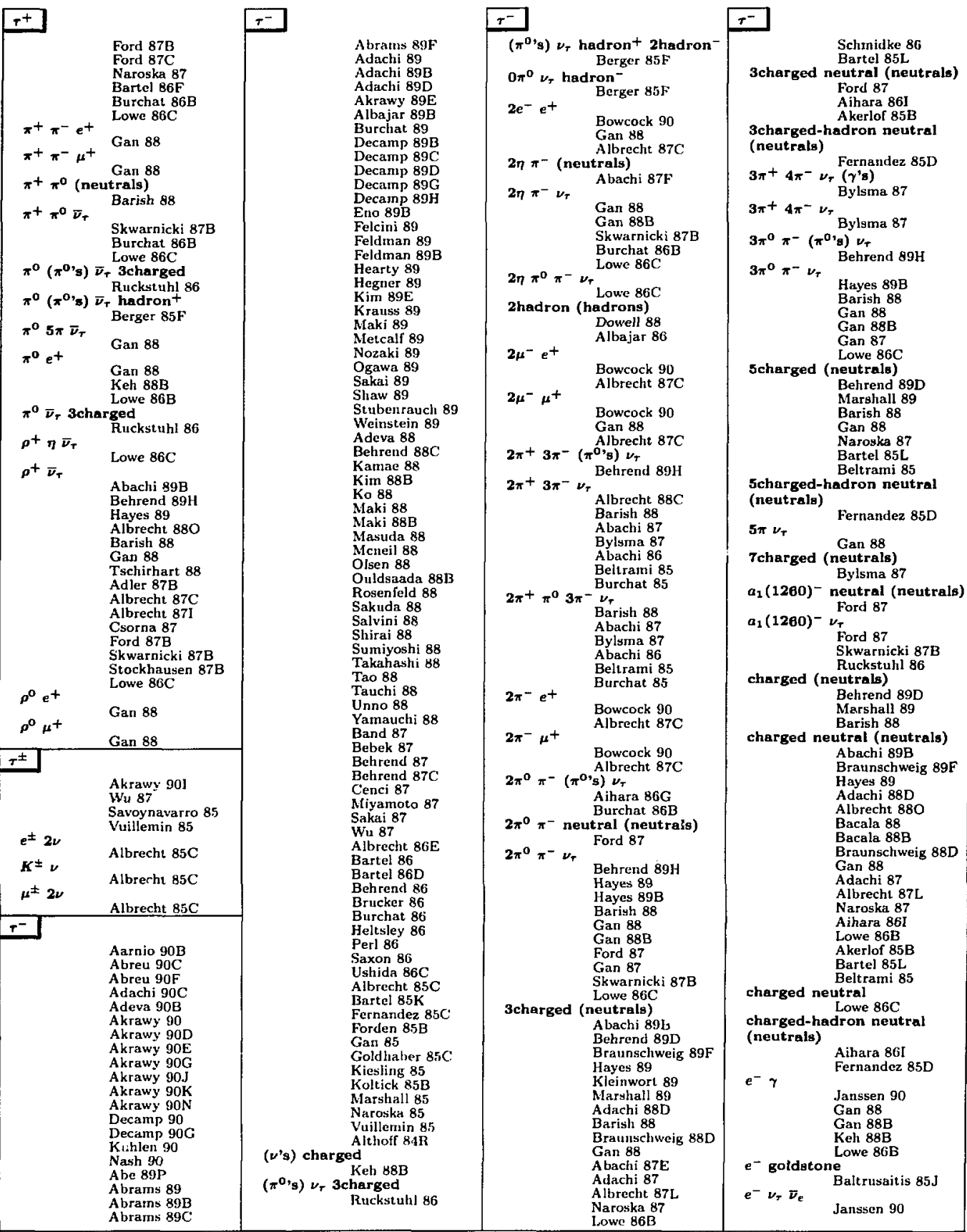




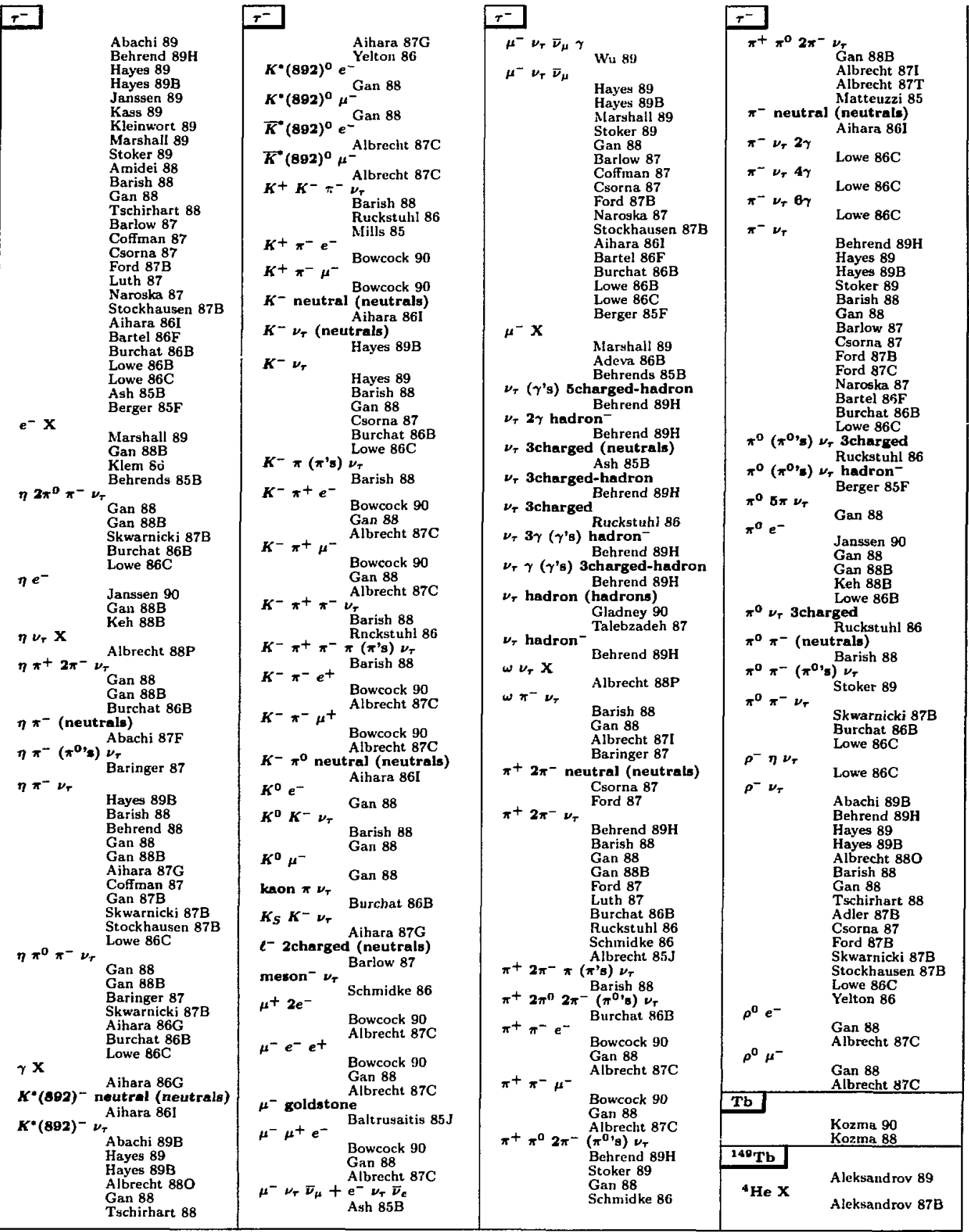




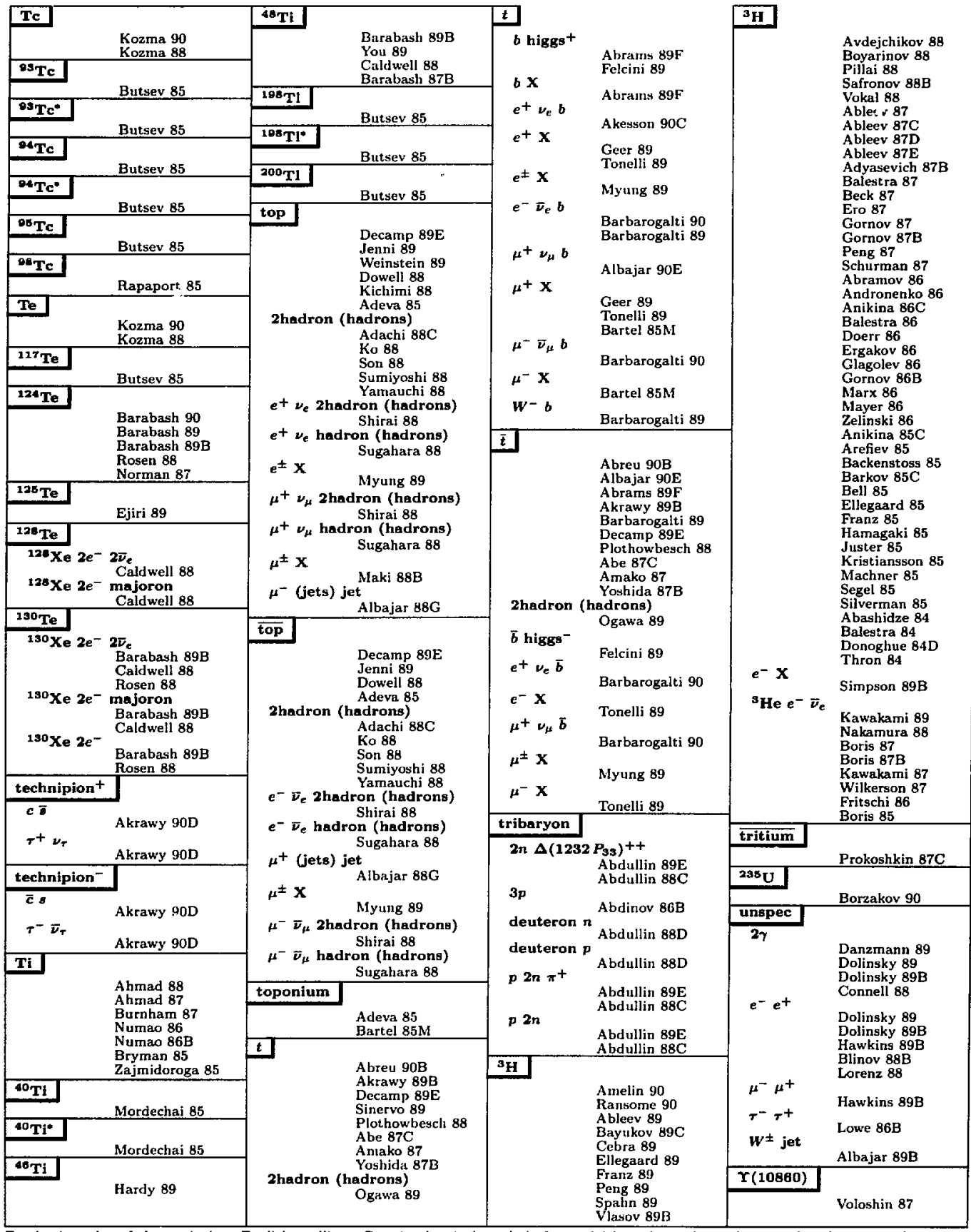




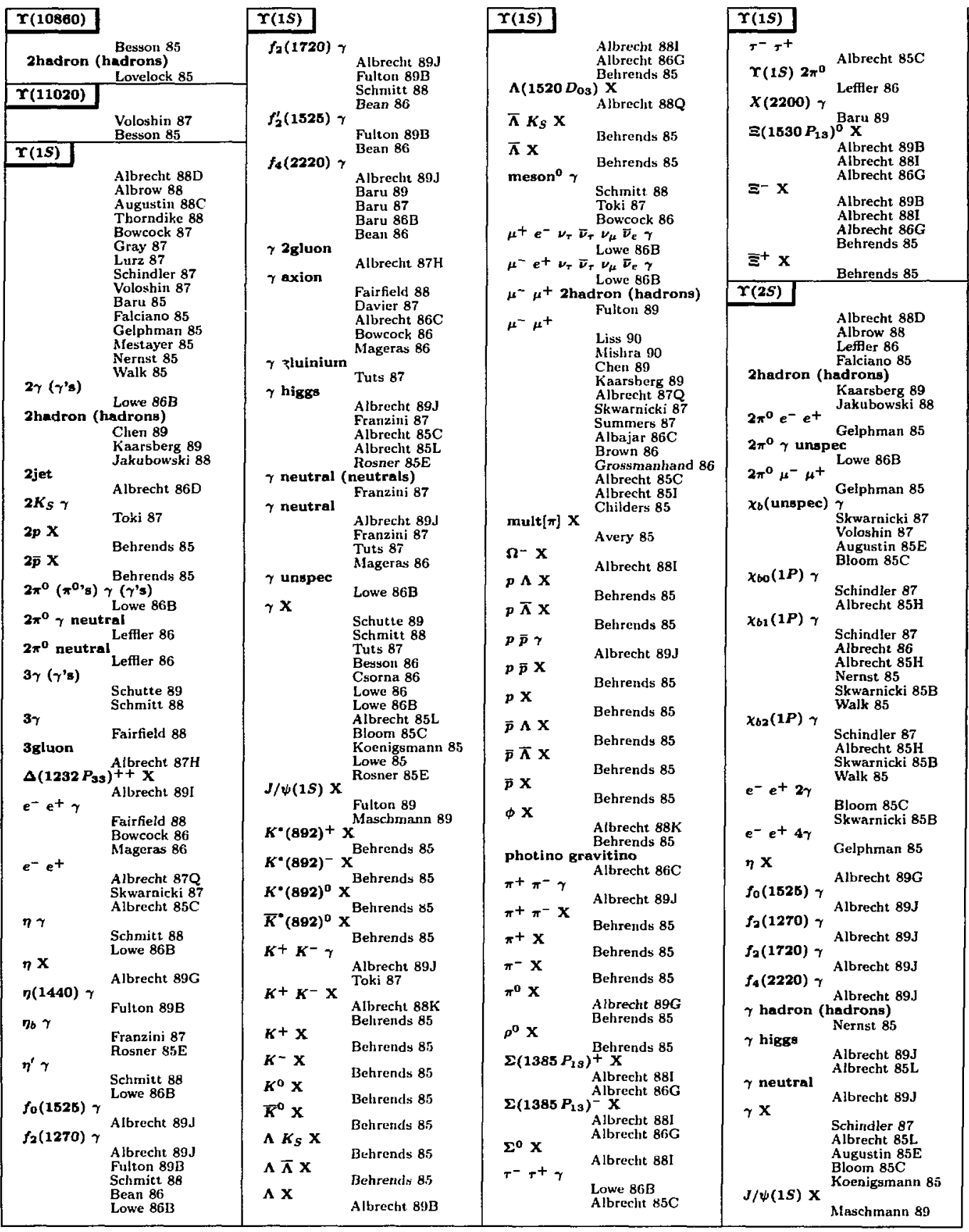




\section{$\mathbf{r}(2 S)$}

$K^{+}$hadron (hadrons)

Albrecht $89 \mathrm{H}$

Albrecht $88 \mathrm{~K}$

$K^{-}$hadron (hadrons) Albrecht 89H

$K^{0}$ hadron (hadrons)

$\bar{K}^{\circ}$ hadron (hadrons) Albrecht 89B
Albrecht 88J

$\Lambda\left(1520 D_{03}\right) \times$

Albrecht $88 \mathrm{Q}$

$\mu^{-} \mu^{+} 2 \gamma+e^{-} e^{+2 \gamma}$

$\mu^{-} \mu^{+} 2 \gamma$

Walk 85

$\mu^{-} \mu^{+} 4 \gamma$

Skwarnicki 85B

$\mu^{-} \mu^{+}$

Gelphman 85

Kaarsberg 89

Summers 87

Albajar 86C

Brown 86

Grosima1 tand 86

Albrecht 85I

$\Omega^{-} \mathbf{X}$

Childers 85

Albrecht 88 hadrons)

$\boldsymbol{p} \overline{\boldsymbol{p}} \boldsymbol{\gamma}$

Albrecht $89 \mathrm{H}$

Albrecht 89J

$\bar{p}$ hadron (hadrons)

$\phi \mathbf{X}$

Albrecht $89 \mathrm{H}$

Albrecht 88K

$\pi^{+}$hadron (hadrons)

$\pi^{+} \pi^{-} e^{-} e^{+} \gamma$

$\pi^{+} \pi^{-} e^{+}$

Bowcock 86

Gelphman 85

$\pi^{+} \pi^{-} \gamma$ axion

Bowcock 86

$\pi^{+} \pi^{-} \mu^{-} \mu^{+}$Bowcock 86

Gejphman 85

$\pi^{-}$hadron (hadrons)

$\pi^{0} \mathrm{X}$

Albrecht $89 \mathrm{H}$

E(1385 $\left.P_{13}\right)^{+}{ }^{\prime}$ ibrecht 89G

E(1385 $\left.P_{13}\right)^{-} X$

$\mathbf{\Sigma}^{0} \mathbf{X}$

Albrectic 88I

(15) $2 \gamma$ Bloom 85C:

Y(1S) $2 \pi^{\circ}$ Walk 8

Walk 85

Albrecht $87 \mathrm{Q}$

Schindler 87

Lowe $86 \mathrm{~B}$

Gelphman 85

$\Upsilon(1 S) \eta$

Lurz 87

$\mathbf{r}(1 S) \pi^{+} \pi^{-}$

Albrecbt 87Q

Bowcock 87

Gray 87
$K+K-\mathbf{X}$ Albrecht 89 J

$x \quad$ Albrecht 89H

$\pi^{+} \pi^{-} \gamma$

Albrecht 89.

$\pi^{+} \pi^{-}$meson $^{\circ} \gamma$

Albrecht 88

r(15) 2r Albrecht 881

Albrecht $87 \mathrm{Q}$

Schindler 87

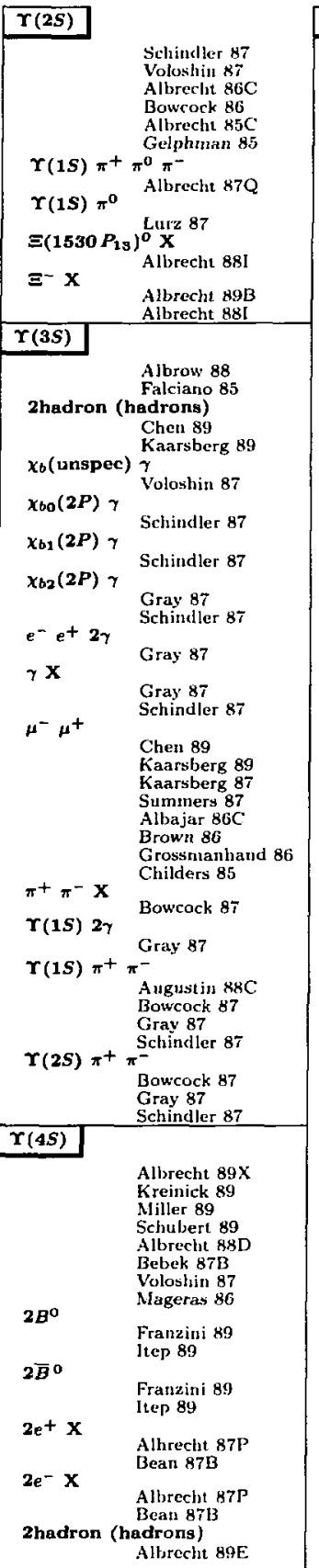

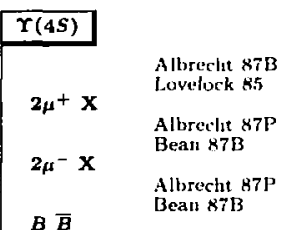

Behrends 87

$B$ (unspec) $\bar{B}$ (unsper)

Albrecht $89 \mathrm{E}$

Harder 89

Mascbmanı 89

Wachs 89

Albrecht 88G

Alam $87 \mathrm{~B}$

Albrecht 87D

Albrecht $87 G$

Bartoletto 87

Bean 87

Alam 86

Bartoletto 96

Haas 86

$\mathrm{B}^{+} \boldsymbol{B}^{-} \quad$ Albrecht 85K

Albrecht 90D

Albrecht 90E

Bortolet to 96

Weir 90

Alam 89

Albrecht 89L

Albrecht 89Q

Albrecht 895

Albrecht 89U

Artuso 89

Avery $89 \mathrm{~B}$

Bebek 89

Bortolet to 89

Bortolet to $89 \mathrm{~B}$

Danilov 89

Halting 89

Albreclit 88E

Albrecht $88 \mathrm{M}$

Albrecht 88T

Daniloy 88

Thorndike 88

Albrecht $87 \mathrm{~B}$

Albrecht 87P

A very 87

Bean 87B

Gittelman 87

Gray 87

Schindler 87

$B^{0} \bar{B}^{0}$

Albrecht 90D

Albrecht 90E

Bortoletto 90

Weir 90

Alam 89

Albrecht $89 \mathrm{C}$

Albrecht 89L

Albrecht $89 \mathrm{Q}$

Albrecht $89 \mathrm{~S}$

Albrecht $89 \mathrm{U}$

Artuso 89

A very 893

Bebek 89

Bortoletta 89

Danilov 89

Drell 89

Halling 89

Albreclit 88M

Albreclat 88T

Thorndike 88

Albrecht 87B

Albrecht 870

Albrecht 87P

Avery 87

Bean 87B

Gittelman 87

Gray 87
Bortolet to $89 \mathrm{~B}$

Albreclit 88E

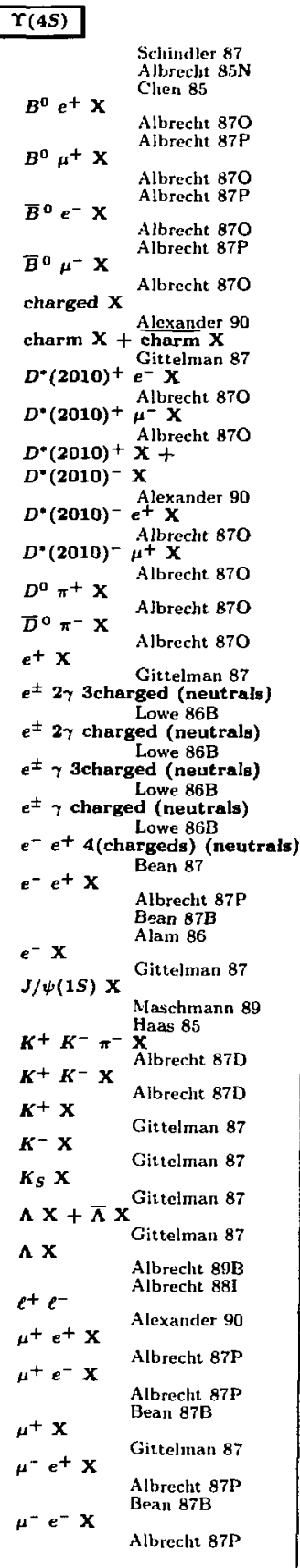

Entries in order of the equivalent English spelling. Certain cliemical symbols for nuclei have been changed to avoid ambiguity with particle rames (see the Particle Vocabulary). See the legrond on page 297 


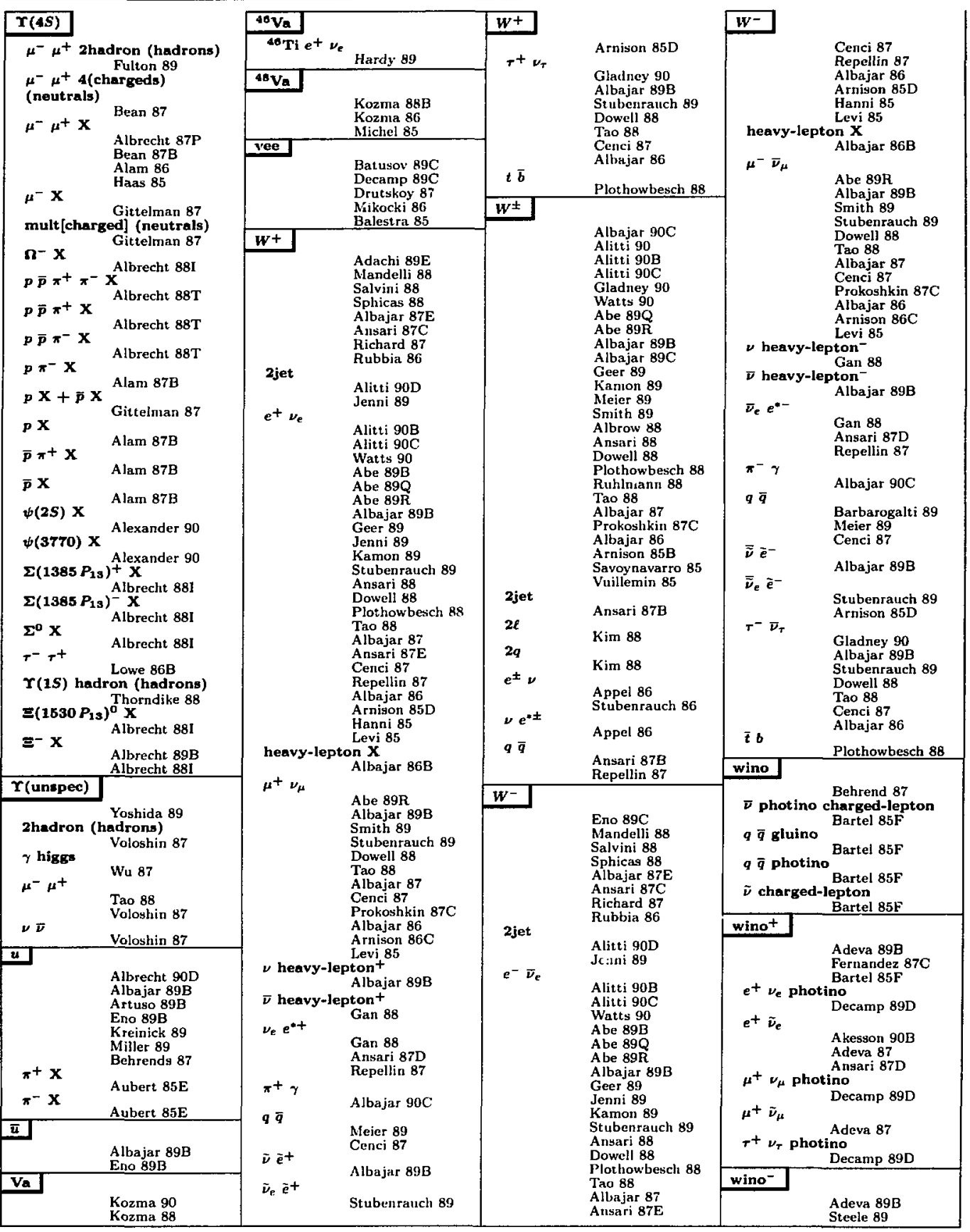




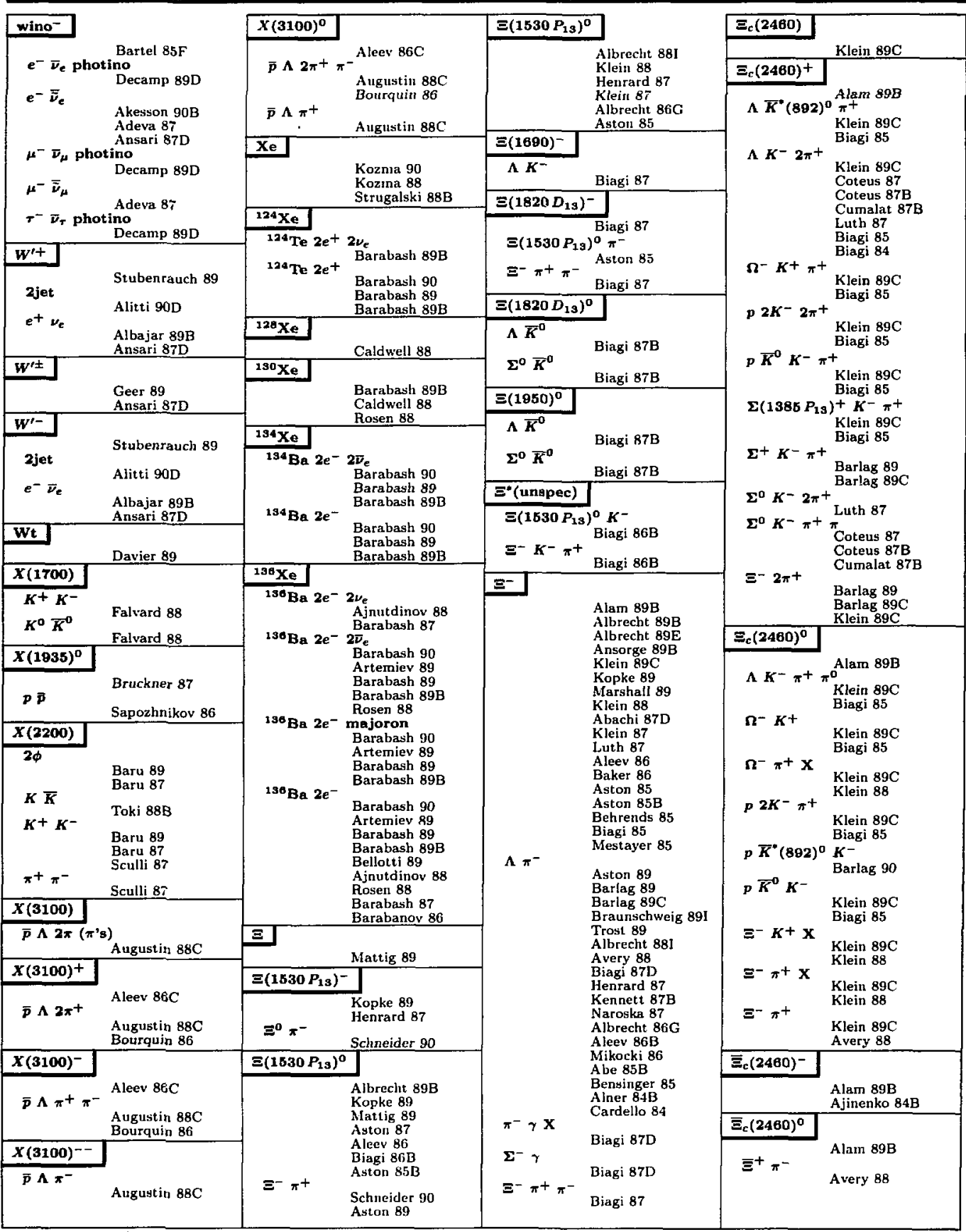




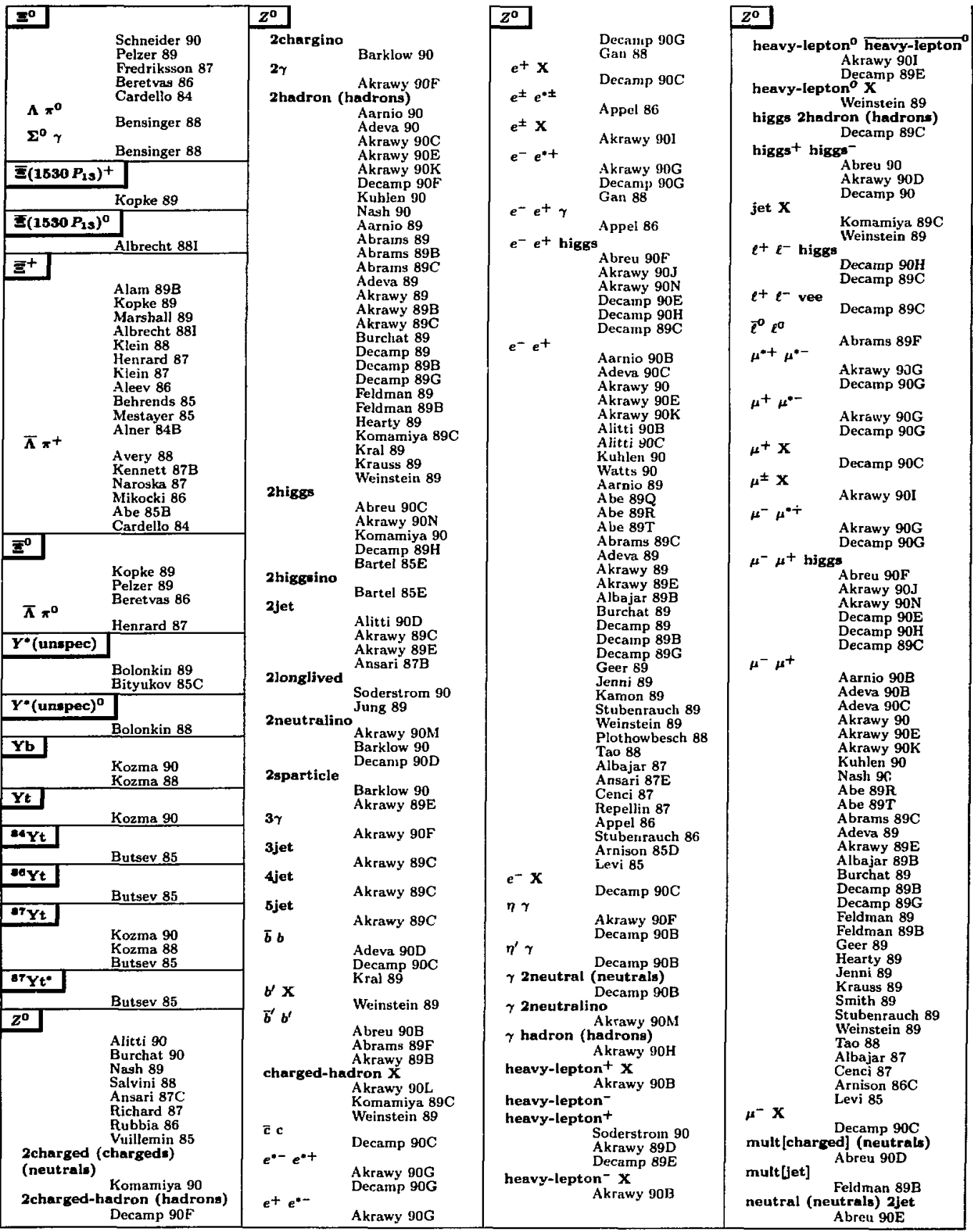




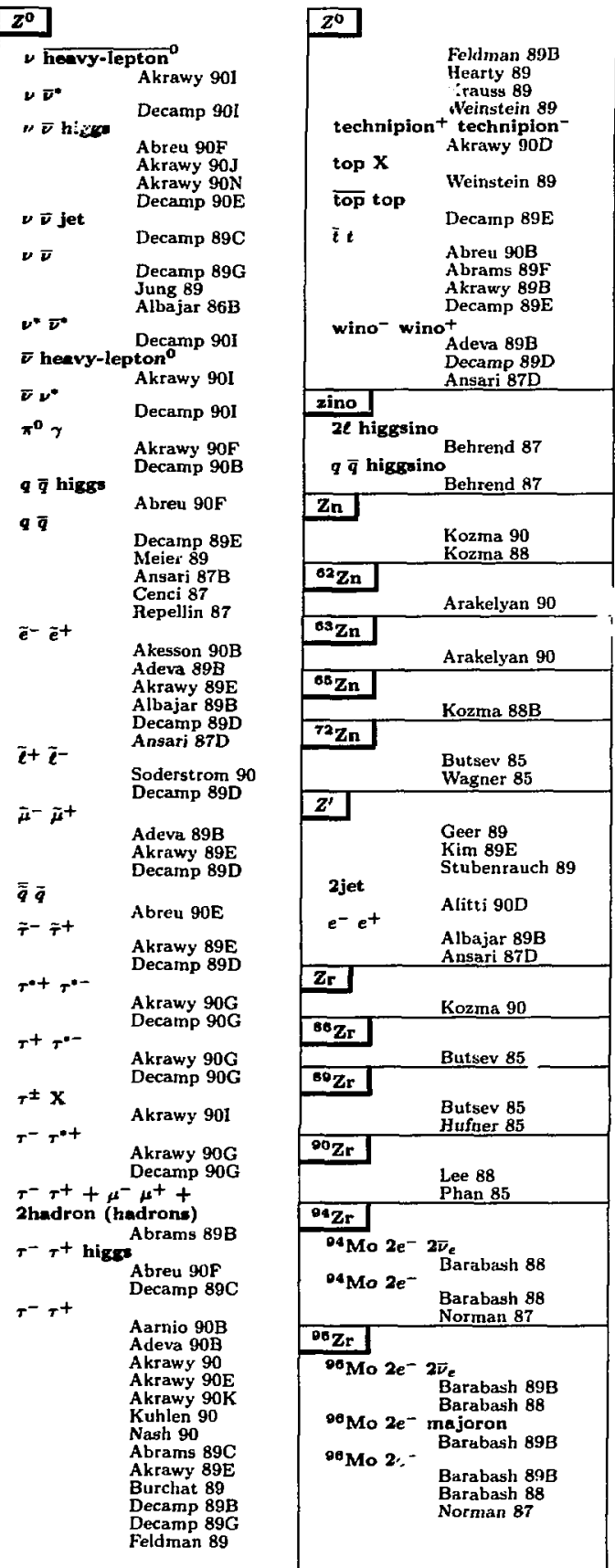

Entries in order of the equivalent English spelling. Certain chemical symbols for nuclei have been changed to avoid atnbiguity with particle names (see the Particle Vocabulary). See the legend on page 297. 
This index lists papers by the accelerator. the experinent number, and the detector used. ordered alphabetically: The Accelerator Vocabulary and the Detector Vocabulary list all the facilities used by papers indexed in this book.

A question mark indicates that the information is missing, usually because it was not given in the paper. A dash mark indicates that the position cannot be filled in.

\section{Illustrative Key}

Accelerator: see the Accelerator locabulary for definitions.

Detector: see the Detector liocabulary for definitions.

\begin{tabular}{|c|c|}
\hline ANI & \\
\hline ANL-E-412 & \\
\hline DBC-12FT & Mann 86 \\
\hline ANL-E-435 & \\
\hline CNTR & Auer 86 \\
\hline SPEC & Auer 86B \\
\hline WIRE & Aver 86 \\
\hline ANL-E-441 & \\
\hline EMS & Finley 85 \\
\hline$\overline{A N L-E}-44$ & \\
\hline SPEC & Auer 88 \\
\hline ANL-E-451 & \\
\hline ENS & Wicklund 85 \\
\hline & Wicklund 87 \\
\hline BNL & \\
\hline BNL-673-593 & \\
\hline $\begin{array}{l}\text { MPS } \\
\text { BNL-701 }\end{array}$ & Bensinger 85 \\
\hline COMB & Franklin 87 \\
\hline $\begin{array}{c}\text { BNL-702 } \\
\text { COMB }\end{array}$ & Snow 85 \\
\hline BNL-723 & \\
\hline SPEC & $\begin{array}{l}\text { Gall } 88 \\
\text { Hertzog } 88\end{array}$ \\
\hline $\begin{array}{c}\text { BNL-726 } \\
\text { WIRE } \\
\text { BNL-732 }\end{array}$ & Christenson 85 \\
\hline CALO & Chiang 86 \\
\hline
\end{tabular}

Document ID: see the ID/Reference/Title Index for the full reference.

Experiment: the experiment's number, where known. 


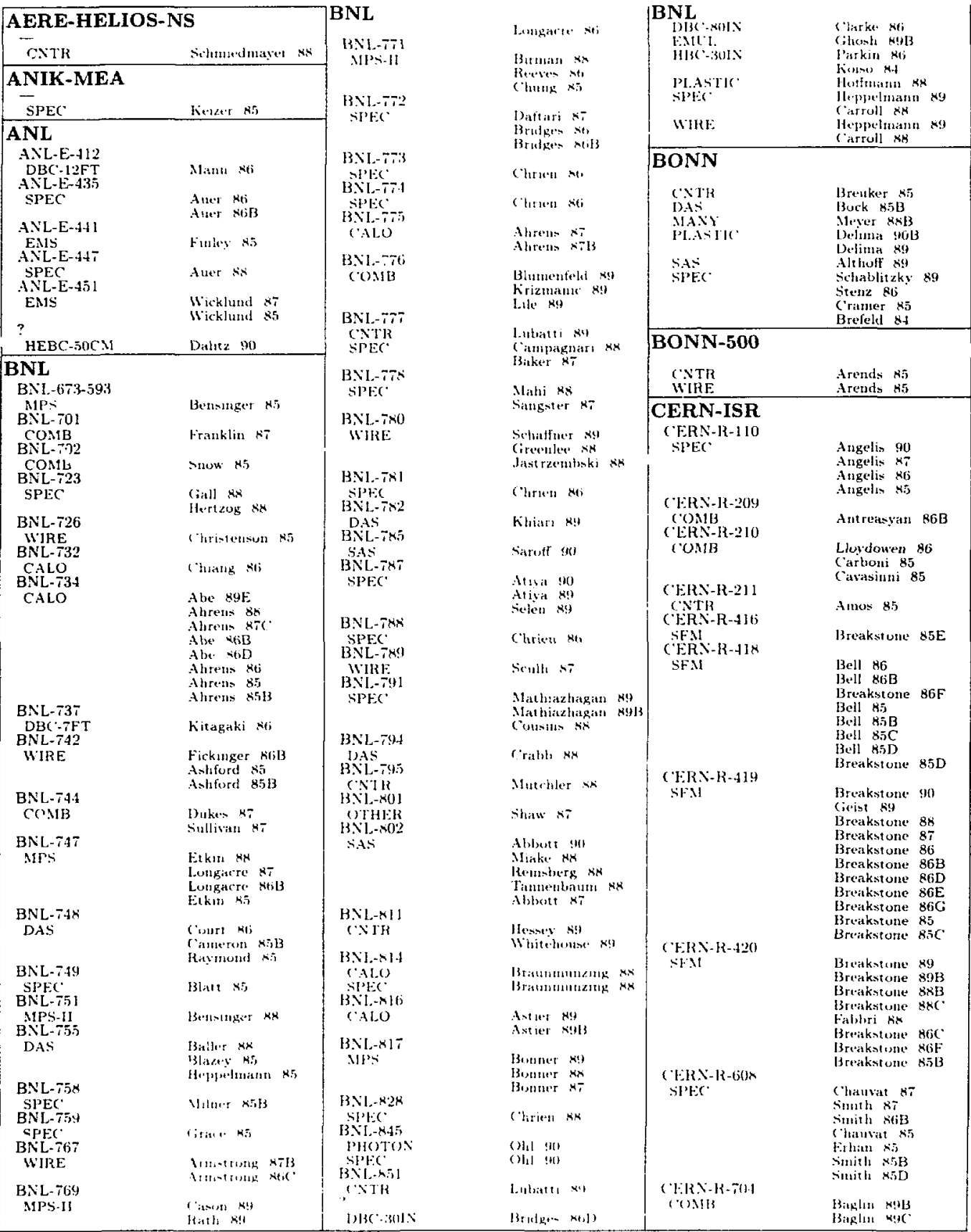




\section{CERN-ISR}

$$
\text { AFS }
$$

CERN-R-B06

AFS

\section{CERN-R-808 \\ AFS \\ CERN-UA-006 DAS}

MANY

CERN-PS-172

\section{WIRE}

CERN-PS-173 COMB

CERN-PS-175 SPEC

CERN-PS-176 PHOTON

CERN-PS- 177 WIRE

CERN-PS-179 STRC
Baglin 87

Baglin 87B

Bagliı 87C

Baglin 86

Baglin 868

Baglit 85

Akesson $86 \mathrm{E}$

Anassontzis 90

Akesion 89

A kesson $88 \mathrm{D}$

Kvatadze 88

A kession 87

A kession $87 \mathrm{C}$

Akesson $87 \mathrm{E}$

Akesso: 86

Akesson 86B

A kesson 86C

Akesson 86D

Akesson 86E

Akesson $86 \mathrm{~F}$

Akesson 85

Akesson 85B

Akesson $85 \mathrm{D}$

Akesson $85 \mathrm{E}$

Akesson $85 \mathrm{~F}$

Akesson 85G

Akession 84B

Akesson 87B

Akession 85C

Akesson $85 \mathrm{G}$

Valemi 85

Fischer 88

Benayoun 87

Camilleri 87

Bardin 87

Bardin 87B

Duch 89

May 89

Doser 88

Ziegler 88

Ahmad 86

Ahmad $85 \mathrm{C}$

Ahinad 84

Schiavon 89

Kunne 88

Kunve 88B

Kunne $88 \mathrm{C}$

Linssen 87

Birsa 85

Brueckner 90

Bruckner 87

Bruckner 86

Bruckuer 85

Bruckıer 85B

Bugg 87

Kreissl 87

Poth 85

Campagnolle 89

Bocquet 87

Bocquet 86

Berada 85

Balestia 89

Balest rá $89 B$

Batusov $89 \mathrm{C}$

Balestra 88

Batusov 88

Batusov 88B

Balestra 87

Balestra 87B

\section{CERN-LEAR}

\begin{tabular}{|ll} 
& Balest ra $87 \mathrm{C}$ \\
& Balest ra 85 \\
& Balest ra 858 \\
CERN-PS-182 & Balusov $85 \mathrm{C}$ \\
SPEC & Adiels 88 \\
& Adiels 86 \\
CERN-PS-183 & Adiels $86 \mathrm{~B}$
\end{tabular}

$$
\text { SPEC }
$$

CERN-PS-184 SPES-11

CERN-PS-185 WIRE

CERN-PS-187

CALLIOPE

CERN-PS-196

OTHER

CERN-PS-198

SPES-II

CERN-PS-20I

SPEC

CERN-PS-202

JETSET

CERN-LEP

CERN-LEP-ALEPH

$$
\text { ALEPH }
$$

CERN-LEP-DELPHI

$$
\text { DELPHI }
$$

Angelopoulos 88 Angelopoulos $88 \mathrm{~B}$ Angelopoutos 86

Angelopoulos 85

Garreta 85

Lichtenstadt 85

Garreta 84

Barnes 90

Barnes 89

Barjes $89 B$

Vonfrankenbe 89

Barnes 8t

Barmes 87B

Barues 85

Mcgaughey 86

Gabrielse 90

Bertini 89

Bertini $88 \mathrm{C}$

Batusov $87 \mathrm{C}$

Hausann 90

Decanı 90

Decamp 90B

Decamp 90C

Decainp 90D

Decamp $90 \mathrm{E}$

Decamp 90F

Decamp $90 \mathrm{G}$

Decamp 90H

Decamp 901

Decamp 89

Decamp $89 \mathrm{~B}$

Decamp $89 \mathrm{C}$

Decanip 890

Decamp $89 \mathrm{E}$

Decamp $89 \mathrm{C}$

Decatnp $89 \mathrm{H}$

Aarnio 90

Aarnio 901

Abreu 90

Abreu 90B

Abreu 90C

Abreu 90D

Abreu 90E

Abreu 90F

Aarnio 89

CERN-LEP-L3

L3

Adeva 90

Adeva $90 \mathrm{~B}$

Adeva 90C

Adeva 90D

Adeva 89

C'ERN-LEP-OPAL

Adeva 89B OPAL
CERN-LEP

Akrawy 90L

Akrawy 90M

Akrauy 90N

Akrawy 89

Akrawy $89 B$

Akrawy 89C

Akrawy 89D

Akrawy 89E

\section{CERN-PBAR/P}

\section{CERN-UA-001}

UAI

Albajar 90

Albajar 90B

Albajar 90C

Albajar 90D

Albajar 90F

Albajar 89

Albajar 89B

Albajar $89 \mathrm{C}$

Felcini 89

Stubenrauch 89

Ajbajar 88

Albajar $88 B$

Albajar $88 C$

Albajar 88D

Albajar 88E

Albajar 88F

Albajar $88 G$

Albajar $88 \mathrm{H}$

Dowell 88

Sphicas 88

Tao 88

Ałbajar 87

Albajar 87B

Albajar $87 \mathrm{C}$

Albajar 87D

Albajar $87 E$

Summers B7

Albajar 86

Albajat $86 \mathrm{~B}$

Albajar $86 \mathrm{C}$

Arnison 86

Arnison $86 \mathrm{~B}$

Arnison $86 \mathrm{C}$

Arnison 86D

Stubenrauch 86

Arnison 85

Arnison 85B

Arnison $85 \mathrm{C}$

Arnison 85D

Arnison 85E

Cerradini 85

Levi 85

Reya 85B

Savoynavarro 85

CERN-UA-002

Vuillemin 85

Akesson 90B

A kesson $90 \mathrm{C}$

Alit t: 90

Alitti $90 \mathrm{~B}$

Alic:i 90C

Alitti 90D

Fransson 90

Alitti 89

Botner 89

Meier 89

Ansari 88

Ansari 88B

Mandelli 88

Plothowbesch 88

Ruhlmann 88

Ansari 87

Ansari 87B

Ansari $87 \mathrm{C}$

Ansari 87D

Ansari 87E

Repellin 87

Appel 86

Appel $86 \mathrm{~B}$

Appel 85

Appel 85B

Appel $85 \mathrm{C}$

Banner 85

Banner B5P

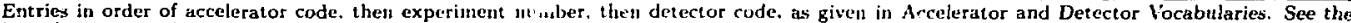
legend on page 359 . 


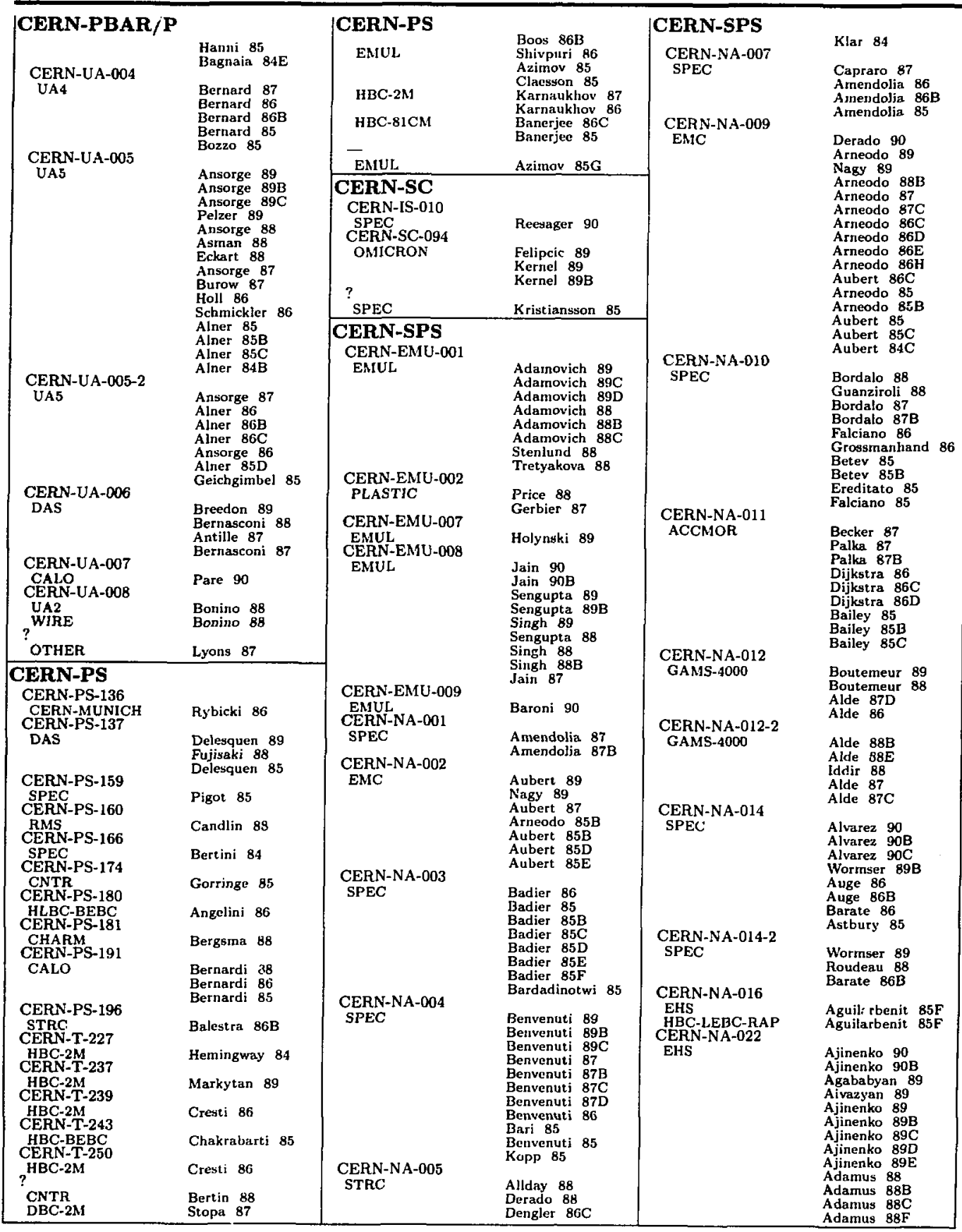




\section{CERN-SPS}

\section{CERN-NA-023 EHS}

\section{CERN-NA-024 \\ CNTK}

CERN-NA-025

HLBC-HOBC-HYB

CERN-NA-027 HBC-LEBC-HYB

\section{CERN-NA-028} EMC

CERN-NA-030

CNTR

CERN-NA-031

CALO

WIRE

CERN-NA-031-2 CALO
Adamus 88G

Ajvazyan 88

Grassler 88

Adamus 87

Adamus 87B

Adamus 87C

Adamus 87D

Adamus 87E

Ajinenko 87

Adamus 86

Adamus $86 \mathrm{~B}$

Adamus 86C

Asai $89 \mathrm{C}$

Aziz 88

Bailly 88

Bailly 88B

Bailly 88E

Bailly 87B

Bailly 87D

Bailly 87F

Bailly 86

Bailly 86B

Bailly $86 \mathrm{D}$

Aziz 85B

Aziz 85C

Asai 84

Demarzo 87

Demarzo 87B

Cobbaert 87

Erriquez 85

Aguilarbenit 89

Aguilarbenit $\mathbf{8 8}$

Aguilarbenit 88B

Aguilarbenit $88 \mathrm{C}$

Bailly 88C

Aguilarbenit 87

Aguilarbenit $87 \mathrm{~B}$

Aguilarbenit $87 \mathrm{C}$

Aguilarbenit 87D

Aguilarbenit 87E

Aguilarbenit 87F

Aguilarbenit $87 \mathbf{H}$

Bailly 87C

Bailly 87G

Bailly 87H

Verbeure 87

Aguilarbenit 86

Aguilarbenit 86B

Aguilarbenit $86 \mathrm{C}$

Aguilarbenit $86 \mathrm{D}$

Aguilarbenit 85

Aguilarbenit $85 \mathrm{C}$

Aguilarbenit $85 \mathrm{D}$

Aguilarbenit $85 E$

Arneodo 89B

Nagy 89

Arneodo 88

Arneodo 86F

Aubert 86

Aubert 86B

Atherton 85

Barr 90

Barr 90C

Carosi 90

Auge $89 \mathrm{~B}$

Barr 88

Burkhardt 87

Barr 90

Barr 90C

Carosi 90

Auge 89B

Barr 88

Burkhardt 87

Barr 90B

Auge 89

Gibson 89

Burkhardt 88
CERN-SPS

WIRE

CERN-NA-032

ACCMOR

\section{CERN-NA-034}

HELIOS

CERN-NA-034-2 HELIOS

\section{CERN-NA-035}

CALO

STRC

\section{CERN-NA-036 \\ CALO \\ WIRE \\ CERN-NA-037 EMC}

\section{CERN-NA-038}

SPEC

\section{CERN-NA-039}

OTHER

CERN-NA-040

PHOTON

CERN-WA-001

\section{CDHS}

CERN-WA-001-2
Barr 90B

Auge 89

Gibson 80

Burkhardt 88

Barlag 90

Barlag 90B

Barlag 90C

Barlag 90D

Barlag 89

Barlag 89B

Barlag 89C

Barlag 88

Barlag 88B

Barlag 88C

Barlag 88D

Barlag 87

Becker 87

Barlag 86

Akesson 88B

Schukraft 88B

A kesson 90

Akesson $89 \mathrm{~B}$

Akesyon 89D

A kesson $89 \mathrm{E}$

Akesson 88

Akesson 88C

Corriveau 88

Ramello 88

Schukraft 88

Schukraft 88B

Bamberger 89

Odyniec 89

Pugh 89

Bamberger 88

Bamberger 88B

Bartely 88

Heck 88

Humanic 88

Pugh 88

Strobele 88

Vesztergombi 88

Bamberger 86

Bamberger 89

Odyniec 89

Pugh 89

Bamberger 88

Bamberger 88B

Bartels 88

Heck 88

Humanic 88

Pugh 88

Strobele 88

Vesztergombi 88

Bamberger 86

Andersen 89

Barnes 88

Andersen 89

Barnes 88

Ashman 89

Ashman 88

Ashman 88B

Ashman 88C

Abren 89

Baglin 89

Sonderegger 89

Bussiere 88

Sonderegger 88

Aleklett $\mathbf{8 7}$

Calloway 89

[jill 88

Berge 89

Burkhardt 85

Blondel 90

\section{CERN-SPS \\ CERN-WA-003 \\ SPEC \\ CERN-WA-007 \\ DAS \\ CERN-WA-013 \\ OMEGA \\ CERN-WA-018 \\ CHARM}

CERN-WA-018-2

$$
\text { CHARM }
$$

CERN-WA-025

DBC-BEBC

CERN-WA-026

SPEC

CERN-WA-027

HBC-BEBC

CERN-WA-028

HBC-BEBC

CERN-WA-038

OTHEF

CERN-WA-042

SPEC

CERN-WA-048

OMEGA

CERN-WA-051

HLBC-BEBC

CERN-WA-057

OMEGAPRIME

Berge 87

Abramowicz 86

Abramowicz 85

Paub 85

Asad 85

Bachman 86

Dorenbosch 89

Geiregat 89

Dorenbosch 87

Dorenbosch 86

Dorenbosch 86B

Bergsma 85

Bergsma 85B

Bergsma 84C

Geiregat 90

Allaby 89

Allaby 88

Allaby 88B

Allaby 87

Allaby 86

Allaby $86 \mathrm{~B}$

Bergsma 85C

Bosetti 90

Jones 90

Jones $89 \mathrm{~B}$

Jones $89 \mathrm{C}$

Jones 87

Jones 87B

Jones 86

Allen 85

Grassler 85

Jones 85

Jones $85 \mathrm{~B}$

Jongejans 89

Allasia 88

Allasia 88B

Allasia $88 \mathrm{C}$

Tenner 88

Allasia 86

Allasia 85

Allasia $85 \mathrm{~B}$

Allasia 85C

Allasia 85D

Biagi 84

Dewolf 86

Ronjin $\mathbf{8 6}$

Ukhanov 86B

Kubic 85

Gritsaenko 84

Banerjee 86 


\section{CERN-SPS}

\section{CERN-WA-058}

EMUL

ONEGAPRINE

CERN-WA-059

HLBC-BEBC

\section{CERN-WA-062} SPEC

CERN-WA-064 SPEC

CERN-WA-066

HLBC-BEBC-HYB

CERN-WA-067

OMEGAPRIME

CERN-WA-069 OMEGA

\section{CERN-WA-070} OMEGA

CERN-WA-071 OMEGAPRIME CERN-WA-072 OMEGAPRIME CERN-WA-074 OMEGA

CERN-WA-075 EMUL
Atkinson $85 \mathrm{C}$ Atkinson $85 \mathrm{D}$ Atkinson $85 \mathrm{E}$ Atkinsoll $85 \mathrm{~F}$

Atkinson B4F

Forino 87

Adamovich $86 \mathrm{E}$

Forino 87

Adanovich $86 \mathrm{E}$

Aliport 89

Guy 89

Marage 89

Matsinos 89

Wittek 89

Wittek 88

Marage 87

Varvell 87

Wittek 87

Marage 86

Baton 85

Fitch 85

Bourquin 86

Biagi 85

Belkacem 85

Talebzadeh 87

Grassler 86

Coopersarkar 85

Coopersarkar 85B

Booth 86

Booth 85

Apsimon 90

Apsimon 89

Dieter 89

Holzkamp 88

Korsgen 88

Rotscheidt $\mathbf{8 8}$

Soldnerrembo 87

Bonvin 90

Bonesini 89

Bonesini 89B

Bonvin 89

Bonesini 88

Bonesini 88B

Bonesini 87

Adamovich $86 \mathrm{~B}$

Abreu 85

Armstrong $87 \mathrm{C}$

Armstrong 86F

Armstrong 85

Aoki 89

Romano 89

Aoki 88

Aoki 87

Arrold 87B

Albanese 85

Arnolid 87B

Armstrong 90

Armstrong 89

Armstrong 89B

Armstrang $89 \mathrm{C}$

Armstrong 89D

Armstrong $89 \mathrm{E}$

Armstrong 89F

Armstrong 88

A rmstrong 87

A rmstrong 86

Armstrong 86B

Armstrong 86D

Armstrong 86E

Vassiliadis $\mathbf{8 5}$

\section{CERN-SPS}

CERN-WA-078 SPEC

CERN-WA-080

PLASTIC-BALL

CERN-WA-085

OMEGAPRIME

CALO

CNTR

EMIUL

PLASTIC

MANY

PLASTIC

\section{CESR}

\section{CESR-CLEO}

\section{CLEO}

Alexander 90

A very 90

Bortolet to 90

Bowcock 90

Alam 89

Alam 89B

Alexander 89

Artuso 89

Avery $89 \mathrm{~B}$

Bebek 89

Bortoletto 89

Bortoletto 89B

Bowcock 89

Bowcock 89B

Chen 89

Chen 89B

Chen 89C

Drell 89

Fulton 89

Fulton 89B

Halling 89

Jensen a9

Kreinick 89

Miller 89

A very 88

Bortoletto 88

Bowcock 88

Haas 88

Alam 87

Alam 87B

Avery 87

Baringer 87

Bartolet to 87

Bean 87

Bean 87B

Bebck 87

Bebek 87B

Behrends 87

Bowcock 87

Csorna 87

Csurna 87B

Gentile 87

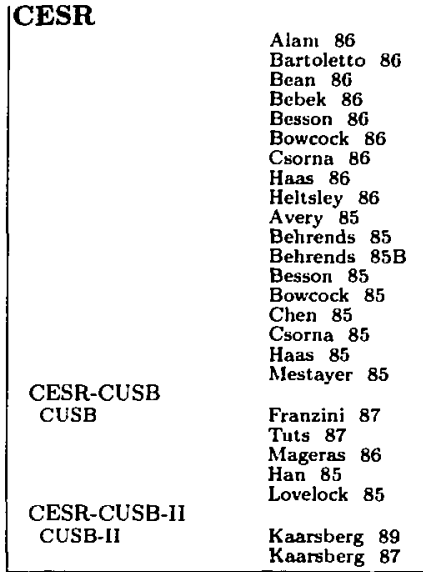

\section{CIT-PELLETRON}

PLASTIC

Savage $88 \mathrm{C}$ Savage $86 \mathrm{~B}$

\section{COSM}

UNDERGROUND-FREJUS

FREJUS

Berger 89B

Longuemare 89

Perdereau 89

Perdereau $89 \mathrm{~B}$

Longuemare 88

Berger $86 \mathrm{C}$

Berger 86D

UNDERGROUND-IMB

IMB

Gajewski 89

Losecco 89

Bionta 88

Bionta 87B

Losecco 87

Losecco 87B

Losecco 85

Losecco 85B

UNDERGROUND-KAMIOKA-I

KAMIOKANDE-I Oyama 86

UNDERGROUND-KAMIOKA-II

KAMIOKANDE-II Hirata 90

Hirata $90 B$

Hirata 89

Hirata $89 \mathrm{~B}$

Hirata 89C

Hirata B9D

Suzuki 89

Takita 89B

Totsuka 89B

Hirata $88 \mathrm{~B}$

Hirata $88 \mathrm{C}$

Hirata $88 \mathrm{D}$

Hirata $88 \mathrm{E}$

UNDERGROUND-LSD

Zhang 88

LSD

Aglietta 86B

UNDERGROUND-MACRO

EAS-TOP Bellotti 89E

MACRO Bellotti 89C

Bellotti $89 \mathrm{E}$

Bellotti 89F

Bellotti $89 \mathrm{H}$

UNDERGROUND-NUSEX

NUSEX

Aglietta 89

Calicchio 87

Dadykin 87

Battistoni 86B

Battistoni 85 


\section{COSM}

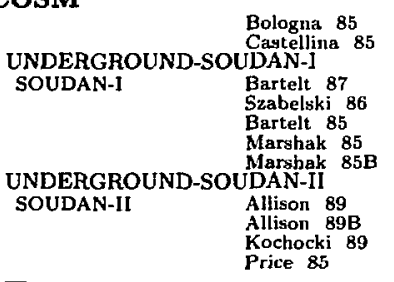

BAKSAN

BBR

CALO

CNTA

COMB

\section{COVER \\ CYGNUS}

DEIS

EMUL

\section{FLYSEYE}

MANY

Bakatanov 88

Alekseev 87

Andreev 86

Bakatanov 85

Budko 85B

Yock 86

Dzhaoshvili 90

Aglamazov 85

Alibekov 85

Avakyan 85

Borisov 85D

Nieminen 85

Domogatsky 89

Efimov 89

Zatsepin 89

Barish 87

Cribier 87

Cromar 86

Dawson 86

Hara 86

Nakamura 85

Nieminen 85

Dzhaoshvili 88

Dzhaoshvilj 88B

Dzhaoshvili 8 ?

Asatiani 85

Azimov 85B

Berdzenishvi 85

Nikolsky 85

Alekseenko 86

Dingus 88

Allkofer 85

Allkofer 85B

Gagarin 89

Kawamura 89

Chilingarian 88

Glady6zdziad 88

Navia 88

Ren 88

Ren 88B

Ren 88C

Atwater 87

Borisov 87B

Cai 87B

Chernavskeaya 87

Burnett 86

Alibekov 85

Borisov 85

Borisov E'5B

Borisov 85C

Borisov 85D

Burnett 85

Bumett 85D

Dobrotin 85

Kanevsky 85

Baltrusaitis 85

Baltrusaitis $85 \mathrm{C}$

Basdevant 90

Fayet 89

Totsuka 89

Arpesella 88B

Nakamura 88

Suzuki 88

Rich 87B

Musset 86

Krasilnikov $85 \mathrm{~B}$

OSPK

OTHER
Burnett 87
CosM

Huber 90

Wuensch 89

Liu $88 \mathrm{~B}$

Depanfilis 87

Bersnon 86

Price 86

Perelygin 85

PHOTON

Inoue 85

PION

lnoue $85 \mathrm{~B}$

Avakyan $89 \mathrm{~B}$

Avakyan $89 \mathrm{C}$

Avakyan 86

Avakyan 85C

Avakyan 85D

Avakyan 85E

Avakyan 85F

PlASTIC Akopova 86

SEMI

Ahlen 88

Ahlen 87

Avignone $87 \mathrm{~B}$

Rich 87

SOKOL Grigorov 90

Grigorov 89B

Grigorov $89 \mathrm{C}$

Ivanenko 89

Ivanenko $88 \mathrm{~B}$

Ivanenko 87

Ivanenko 86

SPEC

Dyakonov 89

Dubovy 88

Vashkevich 88

Barkalov 87

Incandela 86

Atrashkevich 85

Krasilnikov 85

Mincer 85

Tsagova 85

SPRK

WIRE

?

COSM-CYGNUS-X-3

UNDERGROUND-KAMIOKA-I

KAMIOKANDE-I Oyama 87

UNDERGROUND-KAMIOKA-II

KAMIOKANDE-II Oyama 88

UNDERGROUND-MACRO

MACRO Bellotti 89G

UNDERGROUND-NUSEX

NUSEX Battistoni 86B

UNDERGROUND-SOUDAN-I

SOUDAN-I Johns 89

CNTR.

FLYSEYE

Lawrence 89

Cassiday 89

MANY

Cassiday 89B

Arpesella 88B

PION Av': an $88 \mathrm{~B}$

SPEC

K.I: or 87

COSM-HERCULES-X-1

CYGNUS

Haines 90

Dingus 88B

COSM-LMC-X-4

UNDERGROUND-KAMIOKA-II

KAMIOKANDE-II Oyama 88

COSM-SN1987A

MANY

MANY Schaeffer 90

UNDERGROUND-IMB

IMB

Bionta $87 \mathrm{C}$

UNDERGROUND-KAMIOKA-II

KAMIOKANDE-II Hirata 88F

Oyama 88B

Hirata $87 \mathrm{C}$

Oyama 87B

Sato $8 ?$
COSM-SN1987A

BBR

Bond 89

Tanimori 89

Bond 88B

MANY Vandervelde 89

Anada 88

Arpesella 88B

Barbieri 88B

Lattimer 88

Turner 88

Arnett 87

Goldman $87 \mathrm{C}$

Krivoruchenk 87

Mayle 87

Chupp 89

Kolb 89

SMM-GRS

Gaisser 89

SPASE

COSM-SUN

UNDERGROUND-KAMIOKA-II

KAMIOKANDE-II

Hirata 90

Hirata 90B

Hirata 89

Hirata $89 \mathrm{~B}$

Hirata $89 \mathrm{C}$

Hirata 89D

Suzuki 89

Totsuka $89 \mathrm{~B}$

Hirata $88 \mathrm{C}$

Hirat a 88E

Hirata 87B

KAMIOKANDE-II Lim 90

MANY Arpesella 88B

Caldwell 88B

\section{DARE-NINA}

SPEC

Vapenikova 88

DARM-LINAC

EPOS

Bokemeyer 89

Bokemeyer 88

Cowan 86

ORANGE

Bokemeyer 88

\section{DESY}

DESY-094

WAS

Bodenkamp 85

DESY-DORIS-II

DESY-DORIS-ARGUS

ARGUS

Albrecht 90

Albrecht 90B

Albrecht 90D

Albrecht 90E

Degtyarenko 90

Albrecht 89

Albrecht 89B

Albrecht $89 \mathrm{C}$

Albrecht 89E

Albrecht $89 \mathrm{~F}$

Albrecht 896

Albrecht $89 \mathrm{H}$

Albrecht 891

Albrecht $89 \mathrm{~J}$

Albrecht B9K

Albrecht 89L

Albrecht B9N

Albrecht 890

Albrecht 89P

Albrecht B9Q

Albrecht 892

Albrecht 895

Albrecht 89T

Albrecht $89 \mathrm{U}$

Albrecht $89 \mathrm{~V}$

Albrecht $89 \mathrm{~W}$

Albrecht $89 \mathrm{X}$

Danilov 89

Degtyarenko 89

Harder 89

Albrecht $88 \mathrm{C}$

Albrecht $88 \mathrm{D}$ 
|DESY-DORIS-II

Albrecht $88 \mathrm{E}$ Albrecht 88F Albrecht 88G Albrecht $88 \mathrm{H}$ A.lbrecht 881 Albrecht $\mathbf{8 8 J}$

Albrecht $88 \mathrm{~K}$ Albrecht 88M Albrecht $88 \mathrm{~N}$

Albrecht $\mathbf{8 8 O}$ Albrecht $\mathbf{8 8 P}$ Albrecht $\mathbf{8 8 Q}$ Albrecht $88 \mathrm{~S}$ A.lbrecht 88T Danilov 88

Albrecht $87 \mathrm{~B}$

Albrecht 87C

Albrecht 87D

Albrecht 87E

Albrecht 87F

Albrecht 87G

Albrecht $87 \mathrm{H}$

Albrecht 871

Albrecht 87J

Albrecht 87K

Albrecht 87L

Albrecht $87 \mathrm{M}$

Albrecht $87 \mathrm{~N}$

Albrecht $87 \mathrm{O}$

Albrecht 87P

Albrecht $87 \mathrm{Q}$

Albrecht 87R

Albrecht 87S

Albrecht 87T

Albrecht 86

Albrecht 86B

Albrecht $86 \mathrm{C}$

Albrecht 86D

Albrecht 86E

Albrecht $86 \mathrm{~F}$

Albrecht 86G

Albrecht 85

Albrecht $85 \mathrm{~B}$

Albrecht 85C

Albrecht 85D

Albrecht 85E

Albrecht 85F

A]brecht $85 \mathrm{G}$

Albrecht $85 \mathrm{H}$

Albrecht 85I

Albrecht 85J

Albrecht 85K

A]brecht 85L

Albrecht $85 \mathrm{M}$

Albrecht $85 \mathrm{~N}$

DESY-DORIS-CRYS-BALL

CAYS-BALL Janssen 90

DESY-DORIS-CRYSTAL-BALL CRYS.BAL Albrecht 88L Albrecht $88 \mathrm{~A}$

\section{DESY-DORIS-II \\ DESY-PETRA DESY-PETRA-CELLO CELLO}

Behrend 89

Behrend $89 B$

Behrend $89 \mathrm{C}$

Behrend 89D

Behrend 89E

Behrend 89F

Behrend 89G

Behrend 89H

Behrend 891

Behrend 89J

Feindt $b: ?$

Kroha $89 \mathrm{~b}$

Behrend 88

Behrend 88B

Behrend 88C

Behrerid 88D

Behrend 88E

Behrend 88F

Behrend 88G

Behrend 87

Behrend 87B

Behrend 87C

Behrend 87D

Behrend 87E

Aleksan 86

Behrend 86

Behrend 86B

Behrend 86C

Behrend 86D

Behrend 85

DESY-PETRA-JADE JADE

Behrend 85B

Elsen 90

Greenshaw 89

Hegner 89

Hill 89

Kleinwort 89

Pitzl 89

Bethke 88

Ouldsaada 88

Bartel 87

Bartel 87B

Bartel 87C

Naroska 87

Barte] 86

Bartel 86B

Bartel 86C

Bartel 86D

Bartel 86E

Bartel 86F

Bartel $86 \mathrm{G}$

Bartel $86 \mathrm{H}$

Kuhlen 86B

Bartel 85

Bartel 85B

Bartel 85C

Bartel 85D

Bartel 85E

Bartel 85F

Bartel 85G

Bartel $85 \mathrm{H}$

Bartel 85J

Bartel $85 \mathrm{~K}$

Bartel 85L

Bartel 85M

Bartel 84G

Jakubowsk

Keh 88B

Schmitt 88

Williams 88

Antreasyan 87

Lurz 87

Skwarnicki 87

Skwarnicki 87B

Antreasyan 86

Leffler 86

Lowe 86

Lowe $86 \mathrm{~B}$

Lowe 86C

Ge!phman 85

Irion 85

Lowe 85

Nernst 85

Skwarnicki $85 \mathrm{~B}$
Adeva 88

Adeva 87

Adeva 86

Adeva 86B

Adeva 86C

Adeva 85

Adeva 85B

DESY-PETRA-PLUTO

Adeva $85 \mathrm{C}$

\section{PLUTO}

Ouldsaada 89

Berger 88

Berger 88B

Ferrarotto 88

Berger 87
DESY-PETRA-MARK-J

\section{DESY-PETRA \\ Berger $87 \mathrm{C}$ \\ Berger 86 \\ Berger 85 \\ Berger 85B \\ Berger 85C \\ Berger 85D \\ Berger $85 \mathrm{E}$ \\ Berger 85F \\ Berger $85 \mathrm{G}$ \\ DESY-PETRA-TASSO}

TASSO

Braunschweig 90

Braunschweig 90B

Braunschweig 89

Braunschweig 89B

Braunschweig 89C

Braunschweig 89D

Braunschweig 89E

Braunschweig 89F

Braunschweig 89G

Braunschweig $89 \mathrm{H}$

Braunschweig 89I

Braunschweig 89J

Braunschweig 89K

Braunschweig 89L

Genser 89

Braunschweig 88

Braunschweig 88B

Braunschweig 88C

Braunschweis 88D

Braunschweig 88E

Braunschweig 88F

Braunschweig 88G

Braunschweig 87

Braunschweig 87B

Althoff 86

Althoff 86B

Althoff $86 \mathrm{C}$

Althoff 86D

Braunschweig 86

Althoff 85

Althoff $85 \mathrm{~B}$

Althoff $85 \mathrm{C}$

Althoff 85D

Althof 85E

Althof 85F

Althof $84 \mathrm{R}$

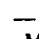

MANY

Ouldsaada 88B

Barlow 87

Kolanoski 86

Kiesling 85

Venkataraman $85 B$

\section{DGSI}

SPEC

Koenig 89

F NAL

FNAL-053A

HLBC-15FT

FNAL-087

SPEC

FNAL-087A

COMB

FNAL-138

HBC-301N

FNAL-180

HLBC-15FT
Baker 89

Baker 86

Baltay 86

Brucket 86

Baker 85

Baker 85B

Baker 85C

Brucker 85

Lamm 87

Hartouni 85

Okusawa 88

Ammosov 88

Ammosov $88 \mathrm{C}$

Ammosov 87

Ammosov 87B

Ammosov 87F

Astatyan 87

Asratyan 87B

Asratyan 87C

A.mmosov 86

Amimosov $86 \mathrm{~B}$ 


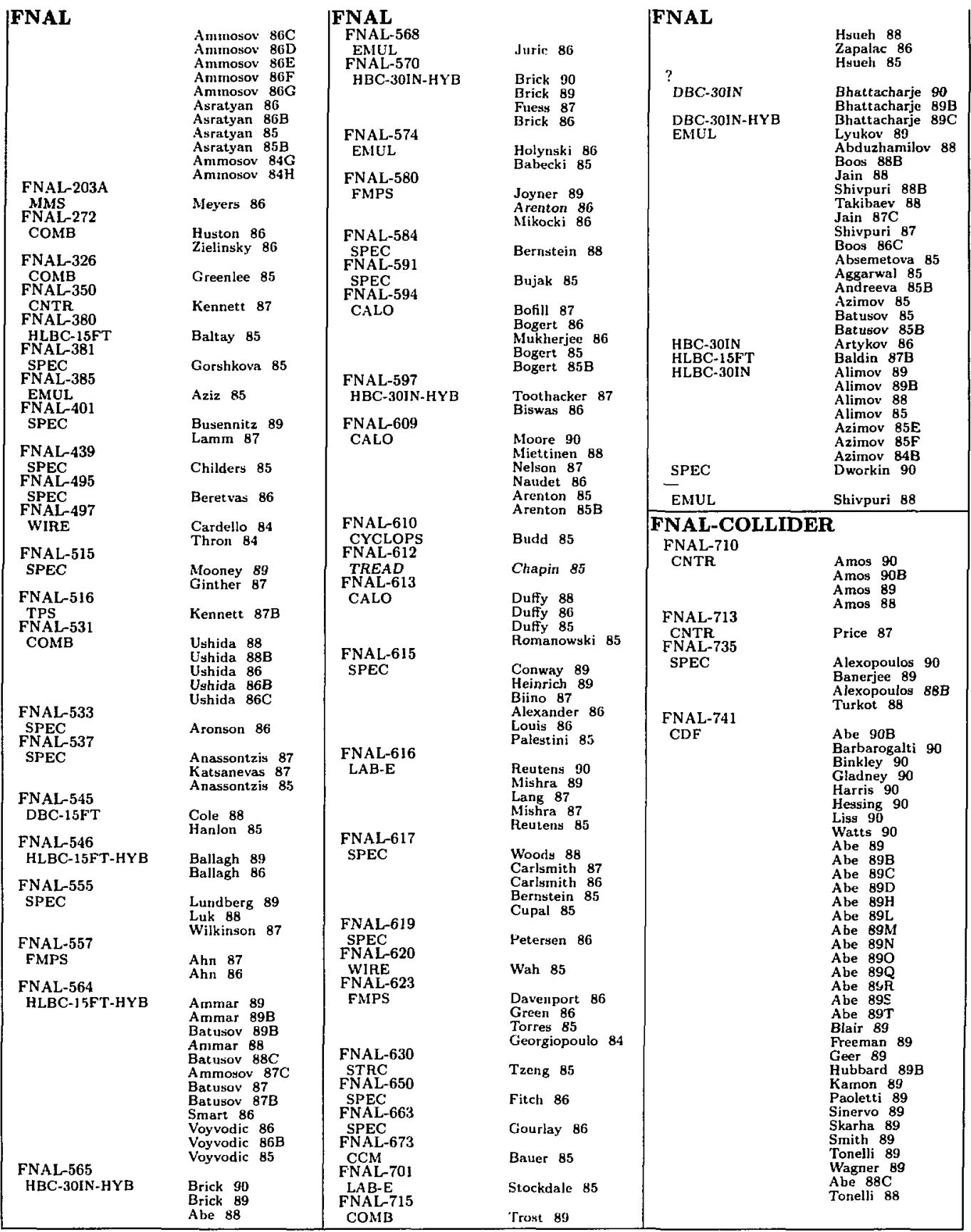




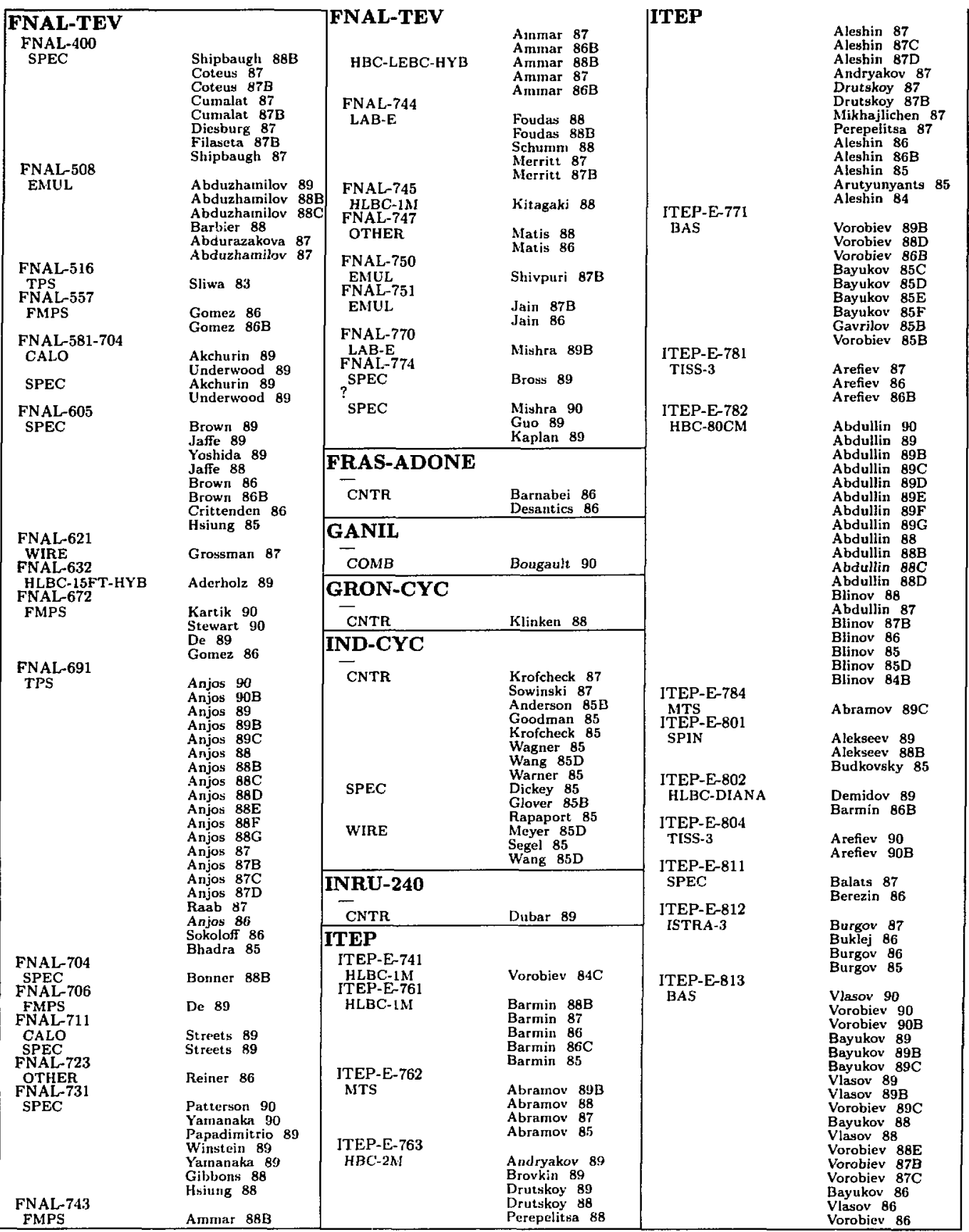




\section{ITEP}

\section{ITEP-E-814}

HLBC-IM

ITEP-E-822

SPEC

ITEP-E-823

CNTR

ITEP-E-831

SPEC

ITEP-E-842

FAS-1

ITEP-E-851

HLBC-1M

CNTR

EMUL

HEBC-80CM

HLEC - 105CM

HLBC-180LIT

OSPK

SPEC

HLBC-180LIT

\section{JINR}

JINR-E-86-01

MASPIC

JINR-E-86-03

ALPHA-POLIS

JINR-E-86-04

GIBS

\section{SEFP-E-017 \\ HLBC-2M}

CNTR
JINR

Bayukov 85

Bayukov 85B

Barmin 89B

Alekseev 88

Afanasyer 88

Sibirtsev 90

Safronoy 88

Safronoy 88B

Sibirtsev 88

Voronin 88

Vorontsov 88

Ergakov 86

Arefiev 85

Boyarinov 89

Boyarinov 88

Boyarinov 88B

Boyarinov $88 \mathrm{C}$

Boyarinov 87

Boyarinov 87B

Boyarinov 86

Barmin 89

Gachurin 85

Berdnikov 86

Berdnikov 85

Smirnov 85

Abdullin $89 \mathrm{H}$

Amelin 87B

Amelin 86

Abdurakhimov $88 \mathrm{~B}$

Grishin 88

Pluta 88

Miller 67B

Okhrimenko 87

Bartke 86

Strugalski 86

Strugalski $86 \mathrm{~B}$

Amelin 87

Amelin 87B

Amelin 86

Kuzichev 89

Kuzichev 88

Lepikhin 87

Dukhovskoy 87

Strugalski 85

Azhgirej 88

Azhgirej 87

Azhgirej 86

Azhgirej 85

Ableev 89

Ableev 88

Ableev 87

Ableev 87B

Ableev 87C

Ableev 87D

Ableev 87E

Ableev 86

Ableev 85

Ableev 84B

Abdurakhimov $89 \mathrm{C}$ Anikina 86C

\section{GIBS}

DAS

DISC

EMUL

Baatar 89

Baatar 88

Baldin 88C

Agakishiev 87B

Agakishiev 87C

Armutlijsky $87 \mathrm{C}$

Baatar $87 \mathrm{~B}$

Kopylova $86 \mathrm{~B}$

Abraamyan 89
Adyasevich 89

Abraamyan 88

Adyasevich 88B

Avdejchikov 88

Avramenko 88

Beznogikh 88

Golovin 88

Karev 88

Lebedev 88

Voronko 88

Adyasevich $\mathbf{8 7}$

Adyasevich 87B

A lekseev 87B

Avdejchikov 87

Avdejchikov 87B

Avdejchikov 87C

Avdejchikov $87 \mathrm{E}$

Avdejchikov 87F

Avdejchikov $87 \mathrm{G}$

Avdejchikov $87 \mathrm{H}$

Avdejchikov 87I

Avdejchikov 86

Abashidze 85

Abashidze 85B

Adyasevich 85

Adyasevich 85B

Adyasevich 85C

Anikina $85 \mathrm{C}$

Avdejchikov 85

Veres 85

Abashidze 84

Budilov 90

Averchikov 87

A verichev $\mathbf{8 9}$

Babaev 90

Ghosh 90

Ghosh 90B

Ameeva 89

Andreevá 89

Ghosh 89

Ghosh 89C

Ghosh 89D

Khan 89

Lepekhin 89

Abdurazakova 88

Alekseeva 88

Andreeva 88

Andreeva $88 \mathrm{~B}$

Andreeva $88 \mathrm{C}$

Bogdanov 88

Dubinina 88

Elnadi 88

Khan 88

Krasnov 88

Krasnov 88B

Vokal 88

Ameeva 87

Antonchik 87

Bannik 87

Bannik 87B

Elnaghy 87

Elnaghy 87B

Ghosh 87

Krasnov 87

Andreeva 86

Andreeva $86 \mathrm{~B}$

Ghosh 86

Krasnov 86

Leskin 86

Shabratova 86

Andreeva $85 \mathrm{C}$

Antonchik 85

Ghosh 85

Vokalova 85

Avramenko 87

Glagolev 90

Braun 89

Glagolev 89

Glagoley 89B

Glagolev 89C

Antos 88

Balgansuren 88

Glagolev 88

Glagolev 88B

Shimansky 88
JINR

HLBC-2N

HLBC- ! M

Troyan $\mathbf{8 8}$

Zelinski 88

Antos 87

Bano 87

Glagolev 87

Bajramov 86

Bano 86

Beshliu 86

Dolidze 86

Dzhincharadz 86

Glagolev 86

Glagolev 86B

Troyan 86

Zelinski 86

Glagolev 85

Bajramov 89

Abdinov 87

Arakelyan 87

Gulkanyan 87

Abdinov 86

Abdinov 86B

Abdinov $86 \mathrm{C}$

Asaturyan 86

Bajramov 86

Pavlyak 86

Pavlyak 86B

Agababyan 85B

Abdinoy 84B

Baatar 90

Shahbazyan 90

Agakishiev 89B

Agakishiev 89C

Angelov 89

Armutlijsky 89

Bajramov 89

Bekmirzaev 89

Gulkanyan 89

Viryasov 89

Agakishiev 88

Angelov 88

Armutlijsky 88

Batskovich 88

Bekmirzaev 88

Bekmirznev 88B

Bekmirzaev 88C

Grigalashvil 88

Grishin 88B

Gulkanyan 88

Gulkanyan 88B

Gulkanyan 88C

Gulkanyan 88D

Kanarek 88

Lyubimov 88

Mekhtiev 88

Shahbazyan 88

Agakishiev 87

Armutlijsky 87

Armutlijsky 87B

Armutlijsky $87 D$

Bekmirzaey 87

Bekmirzaev 87B

Bekmirzaev 87C

Grishin 87

Gulkanyan 87B

Gulkanyan 87C

Gulkanyan 87D

Iovchev 87

Kopylova 87

Agakishiev 86B

Armutlijsky 86

Armutlijsky $86 \mathrm{~B}$

Armutlijsky $86 \mathrm{C}$

Balea 86

Bekmirzaev 86

Bialkowska 86

Grishin 86B

Kopylova 86

Kopylova $86 \mathrm{E}$

Kutsidi 86

Simicl 86

Agakishiev 85

Akhababian 85

Ameev 85

Armutlijsky 85

Entries in order of accelerator code. then experiment number, then detector code, as given in Accelerator and Detector Vocabularies. See the legend on page 359. 


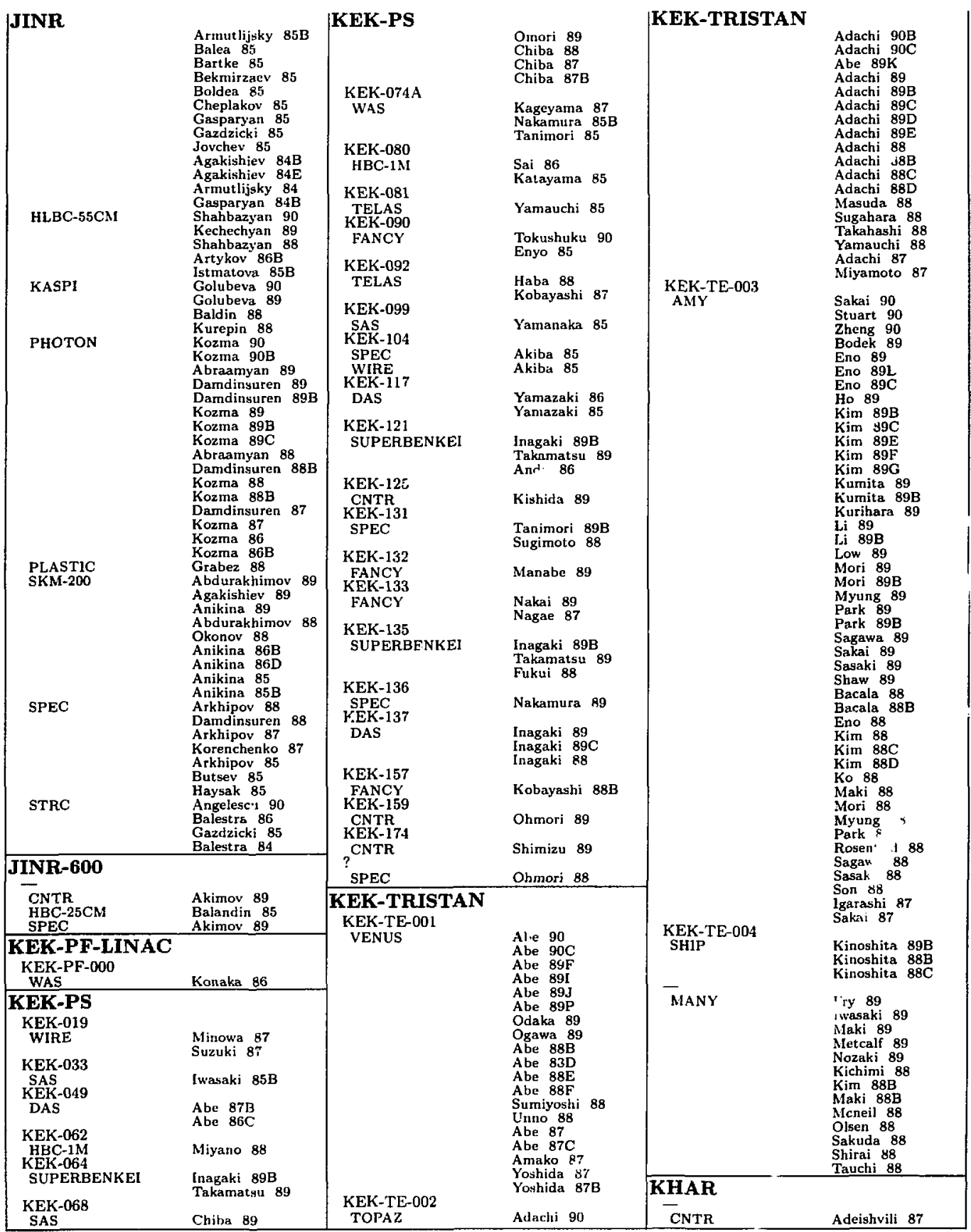




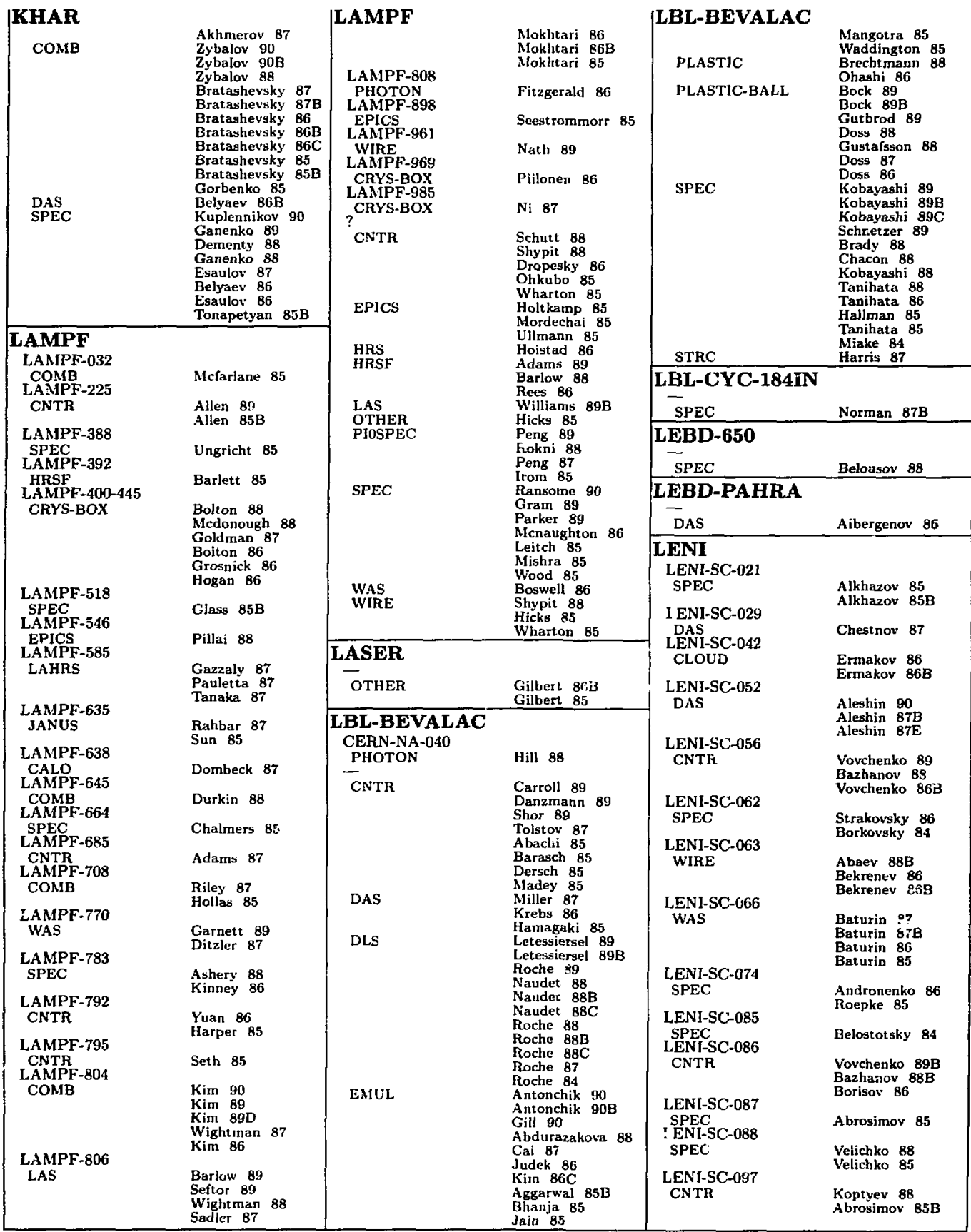


LENI

LENI-SC-108

DBC-35CM

?

CNTR

EMUL

MEGA

SPEC

SPRK

VLN-CYC

OTHER

MANT

CERN-EMU-001

EMUL

?

MANY

EMUL

MANY
MANY

Andreev 88

Andreev 88B

Andreev 87B

Andreev $87 \mathrm{C}$

Andreev 85

Andreev 84

Gavrin 89

Baturin 88

Filatov 88

Mitropolskii 87

Amelin 90

Aleksandrov 89

Gornov 88

Abaev 87

Aleksandrov $87 \mathrm{~B}$

Gornov 87

Gornov 87B

Gornov 86B

Vovchenko 86

Vovchenks 85

Michel 85

Adamovich 90

Landsberg 89

Zielinsky 88

Takibaev 90

Adamovich 89B

Adamovich $89 \mathrm{E}$

Kumar 89

May 89B

Atageldieva 88

Jain 88B

Otterlund 88

Holynskj 86B

Kim 85

Bagdasaryan 90

Chliapnikov 90

Aliev 89

Artuso 89B

Barbarogalti 89

Bartke 89

Bethke 89B

Buschbe:k 89

Eggert 89

Fayard 89

Franzini 89

Gilman 89

Guaraldo 89

Guaraldo 89B

Hayes 89

Hayes 89B

Hohler 89

Holder 89

Itep 89

Jenni 89

Kass 89

Klein $89 \mathrm{C}$

Kopke 89

Krauss 89

Lach 89

Lhote 89

Littenberg 89

London 89

Marshall 89

Maruyama 89

Mattig 89

Murtagh 89

Panagiotoll 89

Preyaud 89

Piccolo 89

Schindler 89

Tarnenbaum 89

Toselio 89

Zhukin 89

Albrow 88

A rendis 88
Augustin $88 \mathrm{C}$

Barish 88

Bertini $88 B$

Bethke 88B

Chan 88

Desanticy 88

Dieksuann 88

Franklin 88

Gan 88

Gan 88B

Gidal $88 \mathrm{C}$

Grivaz 88

Hitlin 88

Hoffman 88

Kamae 88

Levy 88

Liu 88

Purohit 88

Salvini 88

Schmitz 88

Sedlak 88

Sugahara 88B

Sulyaev 88

Thorndike 88

Toki 88B

Totsuka 88

Amaglobeli 87

Arndt 87

Baldin 87

Bayman 87

Berger 87B

Bystricky 87

Couchot 87

Danilov 87

Davier 87

Fredriksson 87

Gittelman 87

Grah 87

Hofmann 87

Hofmann $87 \mathrm{~B}$

Kolanoski 87

Luth 87

Prokoshikin 8:

Prokoshkin 87C

Richard 87

Schindler 87

Schurman 87

Siksin 87

Stirling 87

Stock 87

Tannenbaum 87

Tokj 87

Voloshin 87

Wu 87

Abdinov 86D

Azimov 86

Baldin 86

Baldin $86 \mathrm{~B}$

Barate $86 C$

Be-ọer 86B

Boehm 86

Bogolyubsky 86F

Boos 86

Boschitz 86

Bystrjcky 86D

Chuvilo 86

Dabrowski 86

Dienioz 86

Fearing 86

Ferhel 86

Gal 86B

Hicks 86

Konigsmann 86

Lancon $86 \mathrm{~B}$

Landsberg 86

Lechanoinele 86

Lehar 86

Marx 86

Mermaz 86

Mlukhopadhyay 86

Ohslima 86

Piragino 86B

Reduine 86

Roberts 86

Sapozhnikov BG
MANY

Saxon 86

Shibata 86

Shklyarevsky 86

Smith 86D

Ward $86 \mathrm{~B}$

Whitaker 86

Wilkinson 86

Wu 86

Arndt 85

Augustin $85 \mathrm{E}$

Baldin 85

Banerjee 85B

Beltramin 85

Berezhnoj 85

Blumer 85

Cassel 85

Collins 85E

Dainton 85

Dugan 85B

Forden 85B

Gan 85B

Gavrilov 85

Goldhaber $85 \mathrm{C}$

Grifols 85B

Haissinski 85

Hewett 85

Hufner 85

Kazarinov 85

Koenigsmann 85

Kolanoski 85

Koltick 85B

Komamiya 85

Landsberg 8.5

Machner 85

Marshall 85

Neyer 85F

Morsch 85

Murtagh 85B

Nacht mann B5

Narosken 85

Ramm 85

Rosner 85E

Rutherfoord 85

Soifer 85

Trower 85

Tsukerman 85

Tsukerman 85B

Tsukerman $85 \mathrm{C}$

Vunoers 85

Yamamoto 85E

Yokosawa 85

Yokosawa $85 \mathrm{C}$

Abaev B4

Achasov 84F

Arodt 84

Azimov 840

Bigi 84

Blanklejder 84

Block 84

Bohm 84E

Donogbue 84D

Hiroshige B4C

Klein 84B

Suec 81

Timmers 8

MANZ-LINAC

CNTR

Rose 90

Hiel 89

Spahn 89

SPEC

Koch 89

Ottermann 85

Roehrich 85

MIT-BLA

AHEAD

CNTR

Garcon 89

Beise 89

Turley 85

EL $\backslash Y Y$

SPEC

Kalantarnaye 89

Beck 87

Geesaman 89

Wise 85

Beise 89 


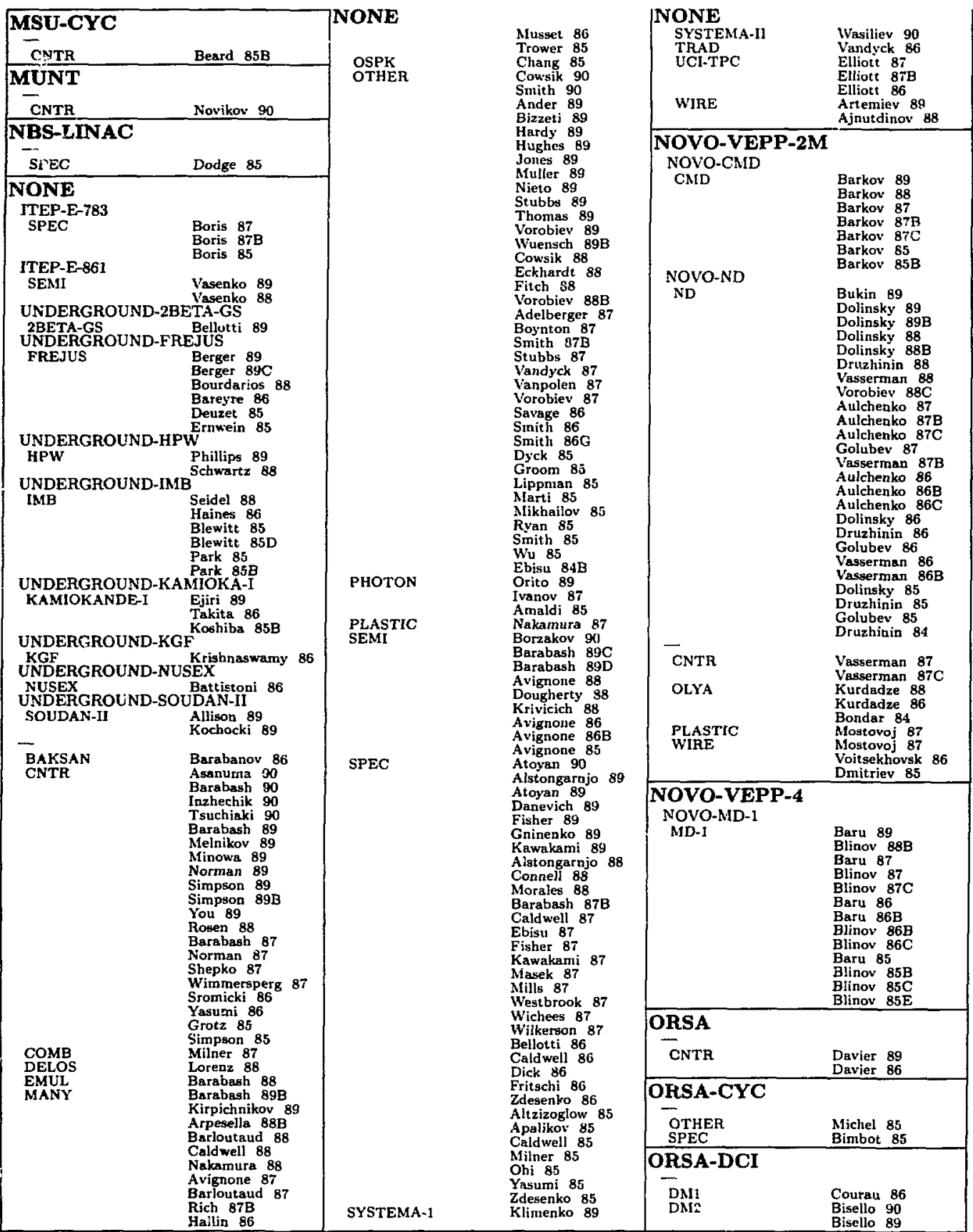




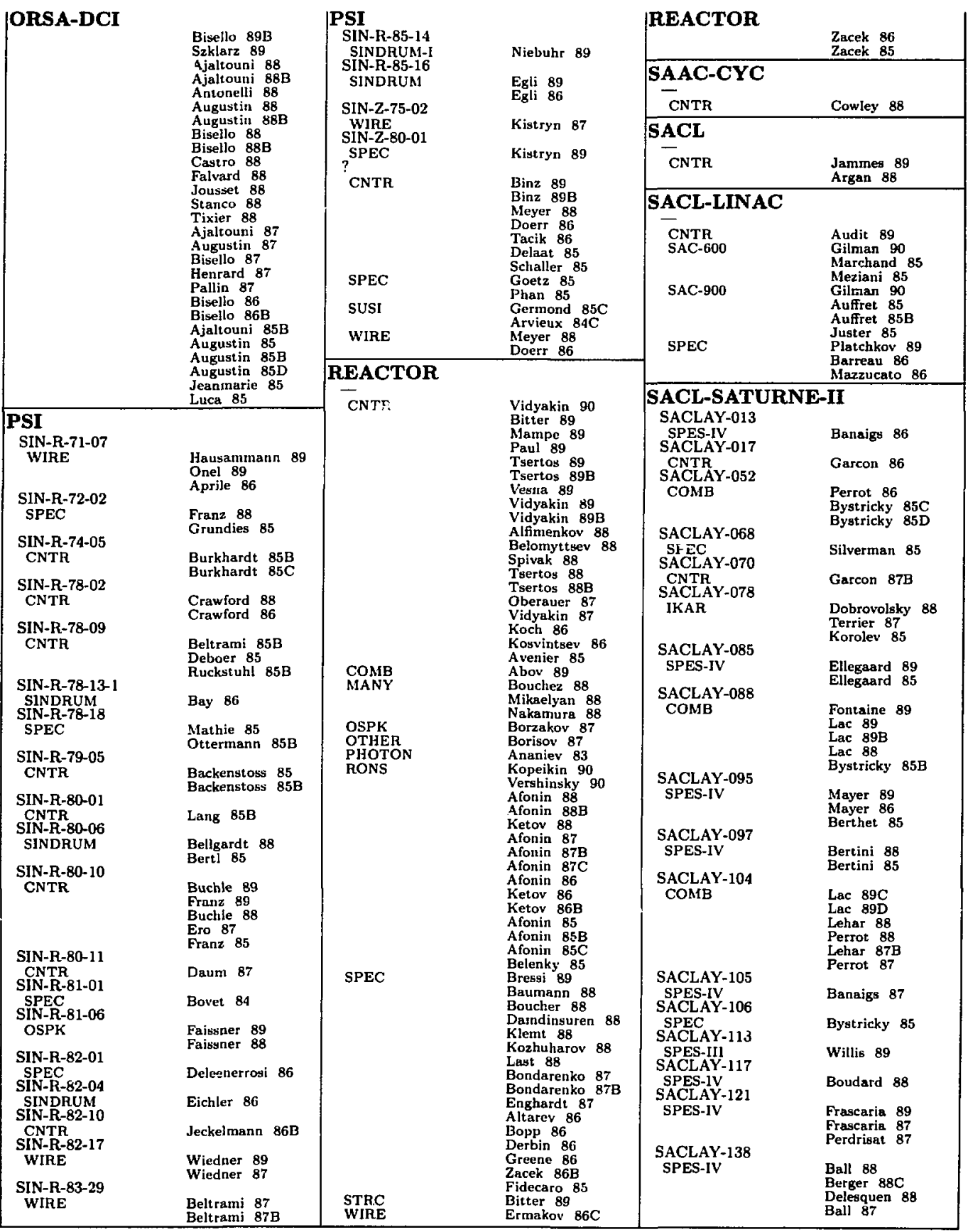


[SACL-SATURNE-II

SACLAY-144

\begin{tabular}{|c|c|}
\hline $\begin{array}{l}\text { COMB } \\
\text { SACLAY-95 } \\
\text { SPES-IV }\end{array}$ & $\begin{array}{l}\text { Leliar } 87 \\
\text { Banaigs } 86 B\end{array}$ \\
\hline DIOGENE & $\begin{array}{l}\text { Bastid } 89 \\
\text { Gosset } 89 \\
\text { Lhote } 87\end{array}$ \\
\hline SPES-I & Bonin 86 \\
\hline
\end{tabular}

MANY

SERP

SERP-E-017

HEBC-2M]

SERP-E-040

SPEC-6M

SERP-E-044

HBC-MIRA

SERP-E-045

OSPK

SERP-E-070

BIS

SERP-E-072

NICE

SERP-E-077

HBC-MIRA

\section{SERP-E-078}

HBC-MIRA

SERP-E-080

MIS

SERP-E-083

HBC-LUDMILA

SERP-E-100

FODS

Garcon 86B

Apel $85 B$
SERP

SERP-E-102

HYPERON

Batar 90B

Batar 89

Batar 89B

Baatar 88

Baatar 88B

Baldin 88C

Agakishiev 87B

Agakishiev 87C

Anoshin 87

Armutlijsky $87 \mathrm{C}$

Baatar 87

Baatar 87B

Kopylova $86 B$

Baatar 85

Grishin 85

Grishin 85B

Grishin 85C

Bolonkin 86

Boos 88C

Belikuv 85

Belikov 83B

Silvestrov 87 Silvestrov 86

Apel 85

Babintsey 86B

Ma 86

Ukhanov 86

Babintsev 85

Patalakha 85

Bogolyubsky 84D

Ajinenko 86C

Antipov $89 \mathrm{C}$

Efendiev 89

Ananieva 86

Vegni 86

Zajmidoroga 85

Bellini 84

Batyunya 89

Booy 89

Kanazirski 89

Zlatanov 89

Andreev 87

Batyunya 87E

Batyunya $87 \mathrm{~F}$

Kanazirski 87

Batyunya 86

Batyunya $86 \mathrm{C}$

Batyunya 86D

Batyunya 85

Batyunya 85C

Batyunya 85D

Boos 85

Abramov 86

Abramov 86B

Abramov 84

Abramov $84 \mathrm{C}$

Abramov 84D

Abramov 84E

SERP-E-104
BIS
SERP-E-105

SERP-E-10T

HLBC-SKAT

SERP-E-112

PROZA

SERP-E-115

ISTRA

SERP-E-119

SPEC

SERP-E-122

HBC-MIRA

SERP-E-133

HBC-MIRA

SERP-E-135

SIGMA

SERP-E-136
SERP-E-105 Aleev 85B

SPEC
Gabunia 90

Banujkov 89

Gabunja 89

Bannikov 88

Barwolff 88

Boos 88

Booy 87

Bocy 87B

Barwolf 85

Brunner 89

Grabosch 89

Ammosov 88B

Aminosov 88D

A rimosov 88E

Ainmosov 88F

Ainmosov 87D

Ainmosov 87E

Ammosov $86 \mathrm{H}$

Ammosov 861

Grabosch 86

Grabosch 86B

Grabosch 86D

Anmosor 85

Ammosov 85B

Ammosor $85 \mathrm{C}$

Ammosov 85D

Baranov 85

Apokin $89 \mathrm{~B}$

Apokin 88

Apokin 86B

Apokin 8BC

Apokin 86D

Avvakumov 86

Avvakumov $86 \mathrm{~B}$

Apokin 85B

Avvakumov 84

Borisov 84

Bolotov 88

Bolotov 87

Bolotov 86

Bolotov 86B

Bolotov 85

Bolotov 85B

Bolotov 85C

Afanasyev 90

Afrnasyev 90B

Bogolyubsky 870

Bravina 86

Ajinenko 87B

Garutchava 87

Garutchava 87B

Gerdyukov 87

Ajinenko $86 \mathrm{~B}$

Gerdyukov 86

Gerdyukov B6B

Tomaradze 86

Ajinenko 85

Knyazev 85

Ajirenko 84

A jinenko 84B

Ajitienko $84 C$

Ajinenko 83B

SERP

SERP-E- 138

HBC-MIRA

SERP-E-139

HBC-LUDMILA

SERP-E-140

GAMS-2000

SERP-E-142

SERP-E-143

SIGMA

SERP-E-144

EMUL

SERP-E-146

BIS-2

Antipov 86C

Sviridov 88

SERP-E-147

MIS
LEPTON-F

Bogolyubsky 89B Bogolyubsky 89D

Bravina 89

Babintser 88

Bogolyubsky 88

Bogolyubsky 88B

Bogolyubsky $88 \mathrm{C}$

Bogolyubsky 88E

Bogolyubsky 88F

Bogolyubsky 88G

Chekulaev 88B

Smirnova 88

Bogolyubsky 87

Bogolyubsky 87B

Bogolyubsky 87C

Bogolyubsky 87D

Bogolyubsky 87E

Babintsey 86

Babintsey 86B

Bogolyubsky 86

Bogolyubsky 86B

Bogolyubsky 86C

Bogolyubsky 86D

Bogolyubsky 86E

Bogolyubsky 86G

Bogolyubsky $86 \mathrm{H}$

Bumazhnov 86

Kozlovsky 86

Bogolyubsky 84B

Batyunya 88

Batyunya 88B

Batyunya 87

Batyunya $87 \mathrm{~B}$

Batyunya 87D

Batyunya 87G

Batyunya 87H

Batyunya 87I

Batyunya 86B

Batyunya 84

Alde 90

Alde 89

Alde $88 \mathrm{C}$

Alde 88D

Alde 87B

Alde $86 \mathrm{C}$

Alde 86D

Prokoshkin 85

Bityukov 90

Bityukov 89

Bityukov 88

Landsberg 88

Bityukov 87

Landsberg 87

Bityukov 86

Bityukov 86B

Bityukov 85

Bityukoy $85 \mathrm{C}$

Antipov 86

Antipov 86B

Antipor $86 D$

Antipov 85

Antipov 85B

Antipov 85C

Barkov $85 \mathrm{C}$

Aleev 89

Aleev 88D

Aleev $88 \mathrm{E}$

Aleev $88 \mathrm{~F}$

Aleev 87

Aleev $86 B$

Aleev 85

Aleev $84 \mathrm{C}$

Bolnnkin 89

Bolonkin 88

Baloghin 87

Bolonkin 87

Baloshin 84 
SERP

SERP-E-148

SIGMA

SERP-E-149

PROZA-M

SERP-E-150

HBC-MIIRA

SERP-E-151

RISK

SERP-E-153

SPEC

SERP-E-157

MIS

SERP-E-159

BIS-2M

SERP-E-163

GAMS-2000

SERP-E-170

CASCADE

SERP-P-156

HLBC-SKAT

EMUL
Antipov 89

Antipov $89 \mathrm{~B}$

Antipov 88

Antipov 88B

Antipov 87

Antipov 87B

Amaglobeli 89

Apokin 89

Apokin 88B

Apokin 88C

Apokin 86

Bogolyubsky $87 \mathrm{E}$

Bannikov $89 \mathrm{~B}$

Janj 87

Belyaev 89

Belyaev 89B

Belyaev 89C

Belyaev 88

Belyaev 88B

Belyaev 88C

Belyaev 85

Cassata 88

Albini 85

Vishnyakov 85

Aleev $89 \mathrm{~B}$

Aleev $89 \mathrm{C}$

Vecko 89

Aleev 88

Aleev 88B

Aleev $88 \mathrm{C}$

Krastev 88

Aleev 87B

Aleev $86 \mathrm{C}$

Alde $86 \mathrm{~B}$

Baskor 88

Belikov 89

Bhattacharje 89

Lyukov 89

Pabin 88

Takibaev 88

Rabin 86

Absemetova 85

Azinov 85

Batusov 85

Rabin 85

HBC-LUDMILA-TST

Andreev 90B

\section{SIEG-BEVALAC}

EMUL

Drech:sel 85

\section{SLAC}

SLAC-BC-067

HBC-40IN-HYB

SLAC-BC-070

DBC-40IN-HYB

SLAC-BC-072

HBC-4OIN-HYB

SLAC-BC-072-073

HBC-4OIN-HYB

SLAC-BC-073

HBC-40IN-HYB

Ferguson 87

Clark 85

Shoemaker 88

Brau 88

Ale 86

Abe 85

Abe $85 \mathrm{~B}$

Ackleh 89

Butler 86

Brau 88
Belyaev 88D

Batusov 85B

SLAC

SLAC-BC-075

HBC-4OIN-HYB

Ackloh 89

Brau 88

Abe 86

Odell 86

SIAC-E-101

SPEC

SLAC-E-131

STRC

SLAC-E-132

LASS

A rnold 87

Jawaliery 85

Aston 88C

Aston 88D

Aston 88E

SLAC-E-135

LASS

Aston 87

Aston 89

Aston 89B

Aston 88

Aston 88B

Aston 88D

Aston $88 \mathrm{~F}$

Aston 88G

Aston 88H

Aston 88I

Aston 88J

Bird 88

Aston 87B

Aston 86

Aston 86B

Sinervo 86

Aston 85

Aston 85B

SLAC-E-136

SLAC-8GEV

SLAC-E-137

CALO

SI,AC-E-140

SLAC-8GEV

Arnold 86

Bjorken 88

Whitlow 90

Aruold 89

Walker 89

Dasu 88

Dasu 87

Dasu 87B

Day 87

Riordan 87

Gomez 85

SLAC-SP-032

MARK-III

?

SPEC

Baltrusaitis 86

\section{SLAC-NPI}

SLAC-NE-4

DAS

SPEC

$A$ be 86

Abe 85

Abe 85B

Cowan 88
SLAC-PEP

Mathis 88

Ajhara 87

Aihara 87B

Aihara $87 \mathrm{C}$

Aihara 87D

Aihara 87E

Aihara $87 \mathrm{~F}$

Aihara $87 \mathrm{G}$

Aihara 86

Ajhara 86B

Aihara 86C

Aihara $86 \mathrm{D}$

Aihara $86 \mathrm{E}$

Aihara $86 \mathbf{F}$

Aihara $86 \mathrm{G}$

Aihara $86 \mathrm{H}$

Aihara 86I

Aihara $86 \mathrm{~J}$

Madaras 86

Hofmann 85

TPC

Aihara 89

Aihara 89B

Aihara 89C

Avery 89

Aihara 88

Aihara $88 \mathrm{~B}$

Aihara 88C

Aihara 88D

Aihara 88E

Aihara 88F

Cowan 88

Edberg 88

Mathis 88

Aihara 87

Aihara $87 \mathrm{~B}$

Aihara $87 \mathrm{C}$

A ihara 87D

Aihara $87 \mathrm{E}$

Aihara 87F

Aihara $87 \mathrm{G}$

Aihara 86

Aihara 86B

Aihara 86C

Aihara 86D

Aihara $86 \mathrm{E}$

Aihara $86 \mathrm{~F}$

Aihara 86G

Ajhara $86 \mathrm{H}$

Ajhara 86I

Aihara 86J

Madaras 86

Hofmann 85

SLAC-PEP-005

Petradza 90

Weir 90

Bethke 89

Hawkins 89B

Klein 89

Komamiya 89

Komamiya 89B

Ong 89

Porter 89

Riles 89

Riles 89B

Snyder 89

Stoker 89

Wagner 89B

Weir 89

Wu 89

Amidei 88

Gidal 88

Gidal 88B

Karlen 88B

Karlen 88C

Klein 88

Ong 88

Ong $88 \mathrm{~B}$

Petersen 88

Riles 88

Wood 88

Wormser 88

Wormser 88B

Gan 87

Gan 87B

Klein 87

Edberg 88 


\section{SLAC-PEP}

\section{SLAC-PEP-006} MAC

SLAC-PEP-009 TPC SLAC-PEP-012 HRS

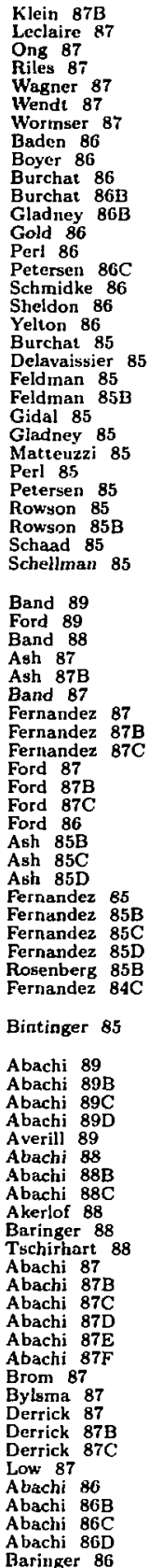

SLAC-PEP

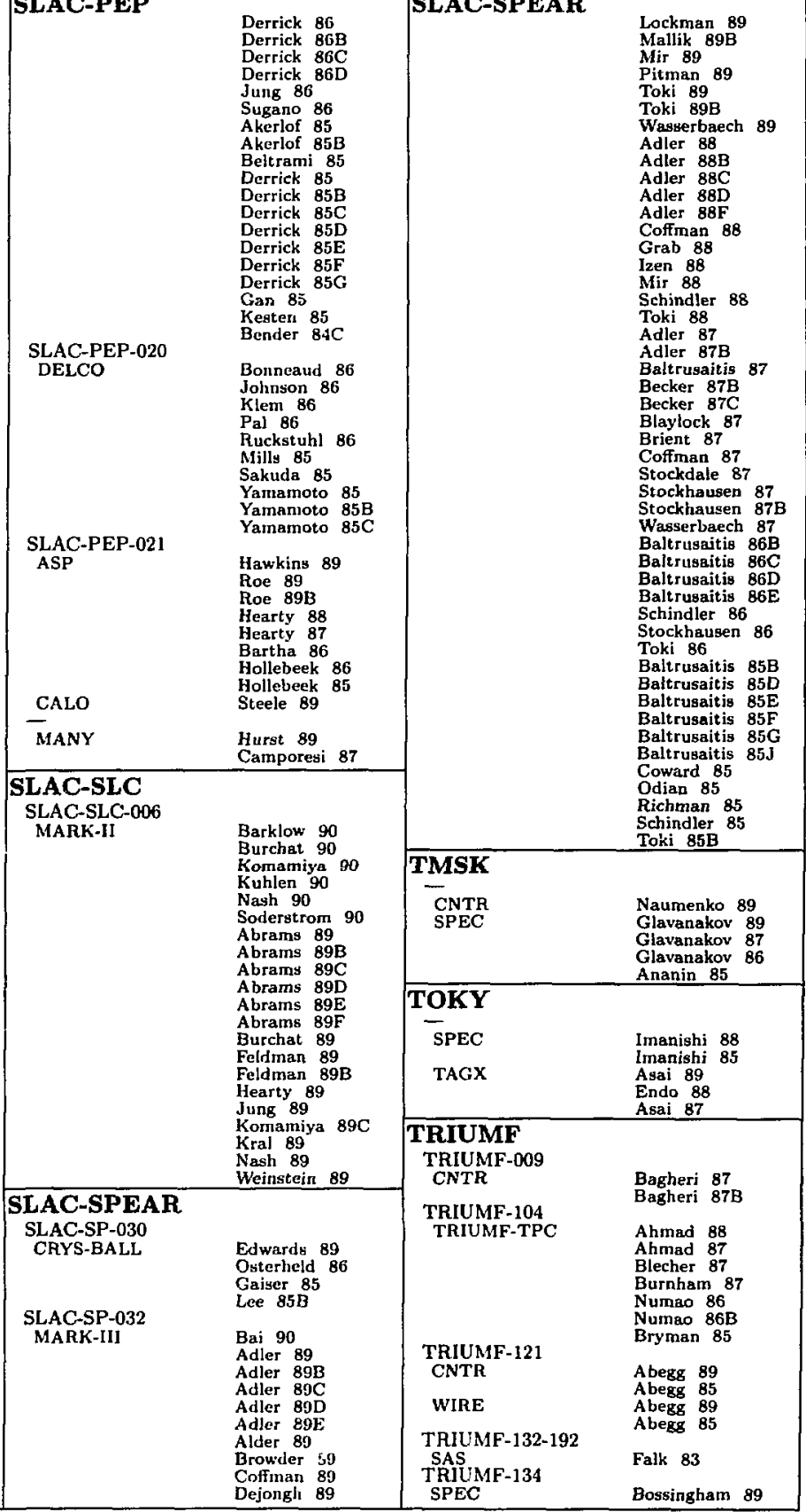


TRIUMF

TRIUMF-171

SPEC

TRIUMF-174

WIRE

TRIUAF-185

CNTR

TRIUMF-205

COMB

TRIUMF-208

SPEC

TAIUMF-247

CNTR

TRILMF-248

PHOTON

TRIUMF-249

WIRE

TRIUMF-304

CNTR

WIRE

SPEC

TRICNF-377

CNTR

TRIUMF-394

CNTR

CALO

CNTR

MANY

MRS

SPEC

VAN-DE-GRAAFF

CNTR

WIRE

Baba 86

YERE-ARUS

CNTR

COMB
Davis 45

Waltham 83

Stoker 85

Shin 86

Kitching 86

Balke 88

Jodidio 86

Britton 88

Picciotta 88

Azuelos 86

Bryman 86

Bryman 86B

Hasinoff 89

Hasinoff 88

Huber 89

Janissen 89

Huber 88

Mtarshalt 88

Beer 86

Huber 89

Janissen 89

Huber 88

Marshall 88

Beer 86

Smith 86C

Sinith 86E

Srmith $86 \mathrm{~F}$

Smith $87 \mathrm{C}$

Brack 89

Brack 88

Stanislaus 89

Friedmasl 90

Abegg 89B

Friedman 89

Weber 89

Green 86B

Mcparland 85

Mcparland A5B

Ponting 88

Davis 88

Hutcheon 89

Lee 88

Punjabi 88

Aniol 85

Debebe 85

Digiacomo 85

Epstein 85

Hasell 85

Roy $85 \mathrm{~B}$

Zjegler 85

Perdrisat 84

Abegg 89B

Cebra 89

Agababyan 895 Bagdasaryan 88 A ivazyan 86 Aivazyajl $86 \mathrm{~B}$

Asaturyan $86 \mathrm{C}$

Bagdasaryan 8.5

Bagdisaryan 8.5B

A vakyars 90

Amrovan 89

Arakelyan 89

Arakelyan $89 \mathrm{~B}$

Arakelyan $\mathrm{HOC}$

Arakelyan H9D

A vakyan 89

Amroyall 88

A vakyan $88 \mathrm{C}$

Sirunyan 88

Ananikyan 87

Avakyan 87B

Aleksanyan 86

Arakelyan 86

Araielyan 85

Avakyan 85B

DAS

Adamyan 89

Aomyan 88

Adamyan 86

Agababyan $85 \mathrm{C}$

Alanakyan 8

Alanakyan 87

Alanakyan 84

SEM1I Arakelyan 90

Agrababyan $89 \mathrm{~B}$

Iartapet van 89

Galuuvan 88

Asaturyan $860^{\circ}$

?

EMLL

Saidkhanov $R 6$

Pniluwisi 85, 


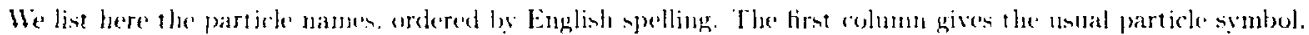
the second gives the linglish spelling. and the thirel gives a hrief definition. The Fuglish spelling in the "computer name." the version to be uned in searching our compue databases.

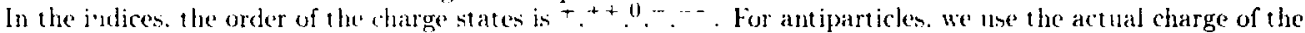
antiparticle. as in $\mathrm{J}^{--}$. All antiparticles are spelled with the suffix "BAR" appenderl to the English portion of the names. as "PBAR" for $\mu$. "DELTABAR (1900S31) 0 " for $\bar{J}\left(1940 . S_{31} l^{\circ}\right.$. and "CHARMBAR" for charm. unless the ant iparticle has a common nane of its unu an in " $h^{-+}$."

We ue the chemical simbuls for mucle except in a fe'w cases where all ambiguity with a particle name exists. For example. we use "KK" for pretassimm to avoid continsion with the $K$ theson. and "Nit" for nitrogen to avoid ruffusion with the mentron and with nickel.

The names we an in writing any reaction are based on those llsed by the anthers of the paper. For example. une piaper might refer to $\pi^{+} . \pi^{-}$. and $\pi^{0}$ partiches while another just uses a $\pi$ to mean all three.

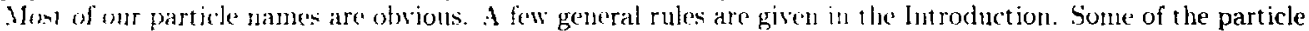
names represent a group of particles whose exact number is not knuwn: for example. " $\pi$ " $"$ " meaning two or more $7^{-}$particlesh "inelastic." ">3 charged." and so ons. Vames like these are treaterl as a single particle name in the Reaction Nomentum ludex. where the reaction final states are ordered by increasing multiplicity. We do give the

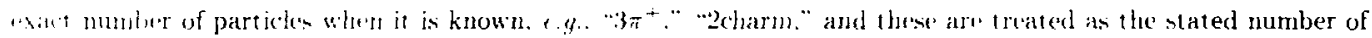
pirticles 


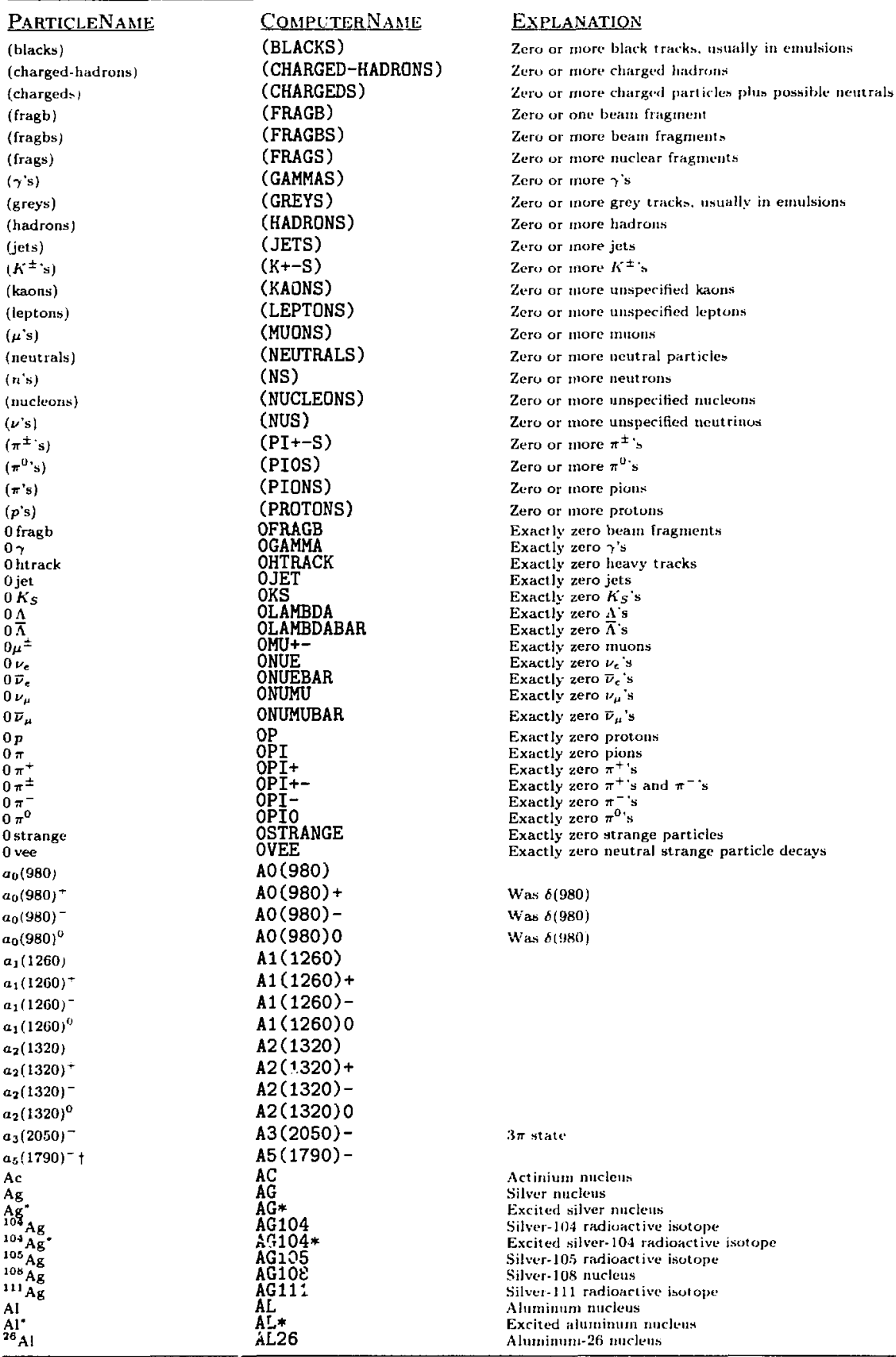




\section{PARTIClENAME}

${ }^{27} \mathrm{Al}$

${ }^{241}$ Am

annihil

anomalon

Ar

${ }_{32} \mathrm{Ar}$

${ }^{37} \mathrm{Ar}$

${ }^{38} \mathrm{Ar}$

${ }^{40} \mathrm{Ar}$

${ }^{4} \mathrm{Ar}$

As

${ }^{72}$ As

Cs(atom)

Ax

${ }_{196}^{A u}$

${ }^{197} \mathrm{Au}$

axigluon

axion

$B$

$B$ (unspec)

$B^{*}$

$B^{*}$ (unspec)

$B^{*+}$

$B *-$

$\boldsymbol{B}^{* 0}$

$\bar{B}^{+0}$

$B^{-}$

$B_{S}$

$\bar{B}_{5}$

$b_{1}(1235)^{+}$

$t_{1}(1235)^{-}$

$b_{1}(1235)^{0}$

Ba

${ }^{128} \mathrm{Ba}$

$23 \mathrm{t} \mathrm{Ba}$

${ }^{134} \mathrm{Ba}$

${ }^{136} \mathrm{Ba}$

$138 \mathrm{Ba}$

${ }^{140} \mathrm{Ba}$

baryon

baryon

baryonium

baryoniam $(S=+1)$

baryonium( $S=-1)$

$\vec{B}$

$\bar{B}$ (unspec)

$\bar{B}^{\circ}$

$\mathrm{Be}$

${ }^{10} \mathrm{Be}$

${ }^{10} \mathrm{Bess}$

$11 \mathrm{Be}$

${ }^{12} \mathrm{Be}$

${ }^{14} \mathrm{Be}$

${ }^{7} \mathrm{Be}$

${ }^{8} \mathrm{Be}$

${ }^{8} \mathrm{Be}^{-}$

${ }^{\mathrm{Be}}$

${ }^{9}$ Bes

Bi

${ }^{302} \mathrm{~B}$;

${ }^{203} \mathrm{Bi}$

${ }^{204} \mathrm{Bj}$

${ }^{206} \mathrm{Bi}$

${ }^{200} \mathrm{Bi}$

black

Bor

${ }^{10}$ Bor

${ }^{10}$ Bor

$11 \mathrm{Bor}$
COMPUTERNAME

AL27

AM241

ANNIHIL

ANOMALON

AR

AR32

AR32*

AR37

AR38

AR40

AR41

AS

AS71

AS72

AS77

ATOM(CS)

AU

AU196

AU197

AXIGLUON

AXIDN

B

$B$ (UNSPEC)

$B *$

B* (UNSPEC)

$B *+$

B*-

$B * 0$

B*BARO

B+

B-

$B / S$

B/SBAR

B1 (1235)+

B1(1235)-

$B 1$ (1235) 0

BA

BA128

BA131

BA134

BA136

138

BA 140

BARYON

BARYONBAR

BARYONIUN

BARYONIUM $(S=+1)$

BARYONIUM $(S=-1)$

BBAR

BBAR(UNSPEC)

BBARO

$\mathrm{BE}$

BE10

BE10/SS

BE11

BE12

BE14

BE7

BE8

BE8*

BE9

BE9/S

$\mathrm{BT}$

BI202

BI203

BI204

BI206

BI209

BLACK

BOR

BOR10

BOR10*

BOR11

\section{EXPLANATION}

Aluminum-27 nucleus

Americium-24l sucleus

Pure annihilation final state in nucleon-antinucleon scattering

Aromalous nuclear fragment

Argon nucleus

Argon-32 nucleus

Excited argon-32 nucleus

Argon-37 nucleus

Argon-38 nucleus

Argon-40 nucleus

Argon- $\$ 1$ radioactive isotope

Arsenic nucleus

Arsenic-71 radioactive isotope

Arsenic-72 radioactive isotope

Arsenic-77 nucleus

Cesium atom

Gold nucleus

Gold-196 nucleus

Gold-197 nucleus

Hypothesized light Higgs scalar boson

$B(5270)$ bottom meson

Meson of unspecified mass with antibeauty quark

Excited bottom meson

Vector beauty meson

$B(5270)^{+}$bottom meson

$B(5270)^{-}$bottom meson

Beauty-antistrange meson

Antibeauty-strange meson

$B(5270)^{0}$ bottom meson

"Buddha" meson

"Buddha" meson

"Buddha" meson

Barium nucleus

Barjum-128 nucleus

Barium-131 nucleus

Barium-134 nucleus

Barium-136 nuclet::

Barium-138 nucleus

Barium-140 nucleus

Unspecified baryon

Unspecified antibaryon

Unspecified nucleon-antinucleon particle

Strange mesons that couple predominately to baryon-antibaryon

Strange mesons that couple predominately to baryon-antibaryon $\vec{B}(\mathbf{5 2 7 0 )}$ antibottom meson

Meson of unspecified mass with beauty quark

$\bar{B}(5270)^{\circ}$ antibottorn meson

Beryllium nucleus

Beryllium-10 nucleus

Beryllium-10 hypernucleus with strangeness $=-2$

Beryllium-11 nucleus

Beryllium-12 nucleus

Beryllium-14 nucleus

Beryllium-7 nucleus

Beryllium-8 nucleus

Excited beryllium-8 nucleus

Beryllium-9 nucleus

Beryllium-9 hypernucleus with strangeness $=-1$

Bismuth nucleus

Bismuth-202 radioactive isotope

Bismuth-203 radioactive isotope

Bismuth-204 radioactive isotope

Bismuth-206 radioactive isotope

Bismuth-209 nucleus

Heavily jonizing track in emulsion

Boron nucleus - note name is not same as chemical symbol

Boron-10 nucleus - note name is not same as chemical symbol

Excited horon-10 nucleus - note name is not same is chemical symbol

Boron-11 nucleus - note name is not same as chemical symbol 


\section{PaRTIClename}

${ }^{11}$ Bor"

12 Bor

${ }^{13}$ Bor

${ }_{13}^{14}$ Bor

3 Bor

${ }^{\circ}$ Bor

bottom

bottom

$\frac{b}{b}$

$b^{\prime}$

$\bar{b}^{\prime}$

Br

${ }^{76} \mathrm{Br}$

${ }^{77} \mathrm{Br}$

${ }^{81} \mathrm{Br}$

$\mathrm{C}$

$C(1480)$

$C(1480)^{+}$

$C(1480)^{-}$

$C(1480)^{0}$

$\mathrm{C}^{-}$

${ }^{10} \mathrm{C}$

${ }^{11} \mathrm{C}$

${ }^{12} \mathrm{C}$.

${ }^{13} \mathrm{C}$

${ }^{14} \mathrm{C}$

$\mathrm{Ca}$.

${ }^{40} \mathrm{Ca}$

${ }^{42} \mathrm{Ca}$

${ }^{44} \mathrm{Ca}$

${ }_{{ }^{48} \mathrm{Ca}} \mathrm{Ca}$

Cd

${ }^{100} \mathrm{Cd}$

${ }^{112} \mathrm{Cd}$

${ }^{113} \mathrm{Cd}$

${ }^{114} \mathrm{Cd}$

${ }^{110} \mathrm{Cd}$

$\mathrm{Ce}$

$\mathrm{Ce}^{-}$

${ }^{132} \mathrm{Ce}$

${ }_{139}^{133} \mathrm{Ce}$

${ }^{140} \mathrm{Ce}$

centauro

cbarged

charged ${ }^{+}$

charged ${ }^{-}$

charged-hadron

charged-lepton

charged-meson

chargino

chargino +

chargino-

charm

charm

charmed-meson

charmed-meson

charmed-nucleus

$x$ (unspec)

$x_{b}$ (unspec)

$x_{60}(1 P)$

$x_{60}(2 P)$

$x_{61}(1 P)$

$x_{b 1}(2 P)$

$\chi_{62}(1 P)$

$x_{62}(2 P)$

$\chi_{c}(3455) \dagger$

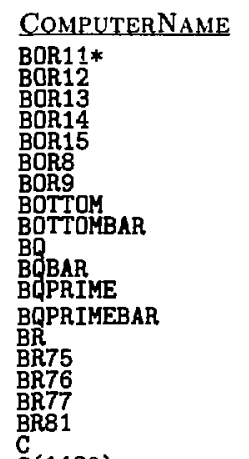

C(1480)

$C(1480)+$

$\mathrm{C}(1480)-$

$C(1480) 0$

C*

C/S

C12

C12*

cis.

C13*

C14

CA

CA*

CA40

CH42

CA 44

CA47

CA48

CD

CD100

CD112

CD113

CD114

CD116

CE

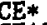

CE132

CE133

CE139

CE140

CENTAURO

CHARGED

CHARGED +

CHARGED-

CHARGED-HADRON

CHARGED-LEPTON

CHARGED-MESON

CHARGINO

CHARGINO +

CHARGINO-

CHARM

CHARMBAR

CHARMED-MESON

CHARMED-MESONBAR

CHARMED-NUCLEUS

CHI (UNSPEC)

CHI/B(UNSPEC)

$\mathrm{CHI} / \mathrm{BO}$ (1P)

$\mathrm{CHI} / \mathrm{BO}(2 \mathrm{P})$

CHI/B1 (1P)

$\mathrm{CHI} / \mathrm{B1}$ (2P)

$\mathrm{CHI} / \mathrm{B} 2$ (1P)

$\mathrm{CHI} / \mathrm{B} 2$ (2P)

CHI/C(3455)

\section{EXPLANATION}

Excited boron-11 nucleus - note name is not same as chemical symbol

Boron 12 nucleus - note name is not stme as chemical symbol

Boron-13 nucleus - note name is not same as chemical symbol

Boron-14 nucleus - note name is not same as chemical symbol

Boron-15 nucleus - note name is not same as chemical symbol

Boron-8 nucleus - note name is not same as chemical symbol

Boron-9 nucleus - note name is not same as chemical symbol

Unspecified particle with naked bottom

Unspecified particle with naked antibottom

Bottom quark

Antibottom quark

Bottom quark of fourth generation

Antibottom quark of fourth generation

Bromine nucleus

Bromine-75 radioactive isotope

Bromine-76 radioactive isotope

Bromine-77 radioactive isotope

Bromine-81 radioactive isotope

Carbon nucleus

Meson decaying into $\phi \pi$

Meson Jecaying into $\phi \pi^{+}$

Meson decaying into $\phi \pi^{-}$

Meson decaying into $\phi \pi^{\circ}$

Excited carbon nucleus

Carbon hypernucleus with strangeness $=-1$

Carbon-10 nucleus

Carbon-11 nucleus

Carbon-12 nucleus

Excited carbon-12 nucleus

Carbon-13 nucleus

Excited carbon-13 nucleus

Carbon-14 nucleus

Calcium nucleus

Excited calcium nucleus

Calcium- 40 nucleus

Calcium-42 nucleus

Calcium-44 nucleus

Calcium- 47 nucleus

Calcium- 48 nucleus

Cadmium nucleus

Cadmium-100 nucleus

Cadmium-112 nucleus

Cadmium-113 nucleus

Cadmium-114 nucleus

Cadmium-116 nucleus

Cerium nucleus

Excited cerium nucleus

Cerium-132 radioactive isotope

Cerium-133 radioactive isotope

Cerium-139 nucleus

Cerium- 140 nucleus

Final state with 50 or more charged particles and no $\pi^{0}, \mathrm{~s}$

Unspecified charged particle

Positive particle of unspecified type

Negative particle of unspecified type

Unspecified charged hadron

Unspecified charged lepton

Unspecified charged meson

Mixture of wino and charged higgsino

Mixture of wino and cliarged higgsino

Mixture of wino and charged higgsino

HPWF's $Y$-particle, mass 2-4 GeV, probably hadron

Unspecified anticharmed particle

Unspecified charmed meson

Unspecified anticharmed meson

Unspecified charmed nucleus

Unspecified radiative decay product of $\psi(2 S)$

Bottomonium meson

Bottomonium meson

Bottomonium meson

Bottomonium meson

Botiomoniurn meson

Bottomonium meson

Bottomonium meson

Radiative decay product of $\psi(2 S)$ 


\section{PaRticlename}

$x_{c}$ (unspec)

$\chi_{\mathrm{eo}}(1 P)$

$x_{c 1}(1 P)$

$x \times 2(1 P)$

Cl

${ }^{34} \mathrm{Cl}$

${ }^{35} \mathrm{Cl}$

${ }^{96} \mathrm{Cl}$

${ }^{37} \mathrm{Cl}$

$\mathrm{Cm}$

Co

${ }^{35} \mathrm{Co}$

${ }^{56} \mathrm{Co}$

${ }^{37} \mathrm{Co}$

${ }^{38} \mathrm{Co}$

${ }^{59} \mathrm{Co}$

${ }^{60} \mathrm{Co}$

${ }^{61} \mathrm{Co}$

${ }^{62} \mathrm{Co}$

c

$\mathrm{Cr}$

${ }^{48} \mathrm{Cr}$

${ }^{51} \mathrm{Cr}$

${ }^{133} \mathrm{Cs}$

$\mathrm{Cu}$

$\mathrm{Cu}$

$\mathrm{CL}$

${ }^{81} \mathrm{Cu}$

${ }^{63} \mathrm{Cu}$

${ }^{64} \mathrm{Cu}$

${ }_{65}^{65} \mathrm{Cu}$

${ }^{67} \mathrm{Cu}$

$D$

$D$ (unspec)

$D^{*}(2010)$

$D^{*}(2010)^{+}$

$D^{-}(2010)^{ \pm}$

$D^{*}(2010)^{-}$

$D^{*}(2010)^{0}$

$D^{*}(2150)^{\circ} \dagger$

$D^{-(2300)^{0} \dagger}$

$\bar{D}^{*}(2010)^{0}$

$\bar{D} *(2150)^{0} t$

$D^{+}$

$D^{ \pm}$

$D^{-}$

$D_{S}$ (unspec) $^{+}$

$D_{s}^{*}$

$D_{s}^{*}(2547)^{+} \dagger$

$D_{S}^{*}(2547)^{-\dagger}$

$D_{S}^{*}(2790)^{+}$

$D_{s}^{*}$

$D_{s}^{-}$

$D_{s}^{+}$

$D_{s}^{ \pm}$

$D_{s}^{-}$

$D^{0}$

$D_{1}(2420)^{+}$

$D_{1}(2420)^{-}$

$D_{1}(2420)^{0}$

$\bar{D}_{1}(2420)^{\circ}$

$D_{2}^{*}(2460)^{+}$

$D_{2}^{*}(2460)^{-}$
Computername

CHI/C (UNSPEC)

$\mathrm{CHI} / \mathrm{CO}$ (1P)

CHI/C1 (1P)

$\mathrm{CHI} / \mathrm{C} 2$ (1P)

CL

CL34

CL34*

CL35

CL36

CL37

C.

CO

C054

$\mathrm{CO55}$

C056

CO57

C058

C059

C060

C061

$\mathrm{CO} 62$

Co

CABAR

CR

CR48

CR5O

CR51

CS133

CU

CU:

cu60

CU61

CU63

CU64

CU65

Cu65*

CU67

D

D(UNSPEC)

D* (2010)

$D *(2010)+$

$D *(2010)+-$

$D *(2010)-$

$D *(2010) 0$

$D *(215 \%) 0$

D* (2300)0

$D * B A R(2010) 0$

D*BAR (2150) 0

D+

D+-

D-

D/S (UNSPEC) +

$D / S *$

$\mathrm{D} / \mathrm{S} *(2547)+$

$\mathrm{D} / \mathrm{S} *(2547)-$

$D / S *(2790)+$

$D / S *+$

$D / S *-$

D/S+

D/S+-

D/S-

Do

D1 2420$)+$.

D1 (2420)- $\therefore$

D1 $(2420) 0$

D1BAR (2420)0

$\mathrm{D} 2 *(2460)+$

D2*(2460)-

\section{EXPLANATION}

Unspecified radiative decay product of any $\psi$ meson

Particle observed in $e^{+} e^{-} \rightarrow \mu^{+} \mu^{-} 2 \gamma$

Observed in $e^{+} e^{-2} \gamma$ final state

Charmonium meson

Chlorine nucleus

Chlorine-34 nucleus

Excited chlorine-34 radioaclive isotope

Chlorine-35 nucleus

Chlorine-36 nucleus

Chlorine-37 nucleus

Curium nucleus

Cobalt nucleus

Cobalt-54 nucleus

Cobalt-55 nucleus

Cobalt-56 nucleus

Cobalt-57 nucleus

Cobalt-58 nucleus

Cobalt-59 nucleus

Cobalt-60 nucleus

Cobalt-61 nucleus

Cobalt-62 nucleus

Charmed quark

Anticharmed quark

Chromium nucleus

Chromium-48 nucleus

Chramium-50 nucleus

Chromium-51 nucleus

Cesium-133 nucleus

Copper nucleus

Excited copper nucleus

Copper-60 nucleus

Copper-61 nucleus

Copper-63 nucleus

Copper-64 nucleus

Copper-65 nucleus

Excited copper-65 nucleus

Copper-67 nucleus

$D^{+}$or $D^{0}$ charmed meson

Unspecified charmed nonstrange meson

Excited charmed nonstrange meson

Excited charmed nonstrange meson

Excited charmed nonstrange meson

Excitud charmed nonstrange meson

Excited charmed nonstrange meson

Excited charmed nonstrange meson

Excited charmed nonstrange meson

Excited anticharmed nonstrange meson

Excited anticharmed nonstrange meson

$D(1869)^{+}$charmed nonstrange meson

$D(1869)^{+}$or $D(1869)^{-}$charmed nonstrange meson

$D(1869)^{-}$charmed nonstrange meson

Unspecified charmed positive strange meson

Was $F^{*}(2140)$. Excited charmed strange meson

Was $F^{*}(2140)$. Excited charmed strange meson

Was $F^{*}(2140)$. Excited charmed strange meson

Was $F$. $D_{S}(1971)^{+}$charmed strange meson

Was F. $D_{S}(1971)^{+}$or $D_{S}(1971)^{-}$charmed strange meson

Was $F$. $D_{S}(1971)^{-}$charmed strange moson

$\eta(1865)^{0}$ charmed nonstrange meson

Entries in order of the equivalent English spelling of the particle same.

$\dagger$ Particle not listed in 1990 Review of Particle Properties. Name may not conform to naming conventions. 


\section{Particlename}

$D_{2}^{*}(2460)^{\circ}$
$\bar{D}_{2}^{*}(2460)^{0}$
$\bar{D}$
$\frac{D}{D}$ (unspec)
$\bar{D}^{\circ}$
$D$ (unspec)
DD

$\Delta\left(1232 P_{33}\right)$

$\Delta\left(1232 P_{33}\right)^{+}$

$\Delta\left(1232 P_{33}\right)^{++}$

$\triangle\left(1232 P_{33}\right)^{-}$

$\Delta\left(1232 P_{33}\right)^{0}$

$\Delta\left(1620 S_{31}\right)^{-}$

$\Delta\left(1700 D_{33}\right)^{-}$

$\Delta(1950 B)^{++}+$

$\bar{\Xi}\left(1232 P_{33}\right)^{--}$

$\bar{\Delta}\left(1232 P_{33}\right)^{0}$

$\bar{\Delta}(1950 B)^{-\top} \dagger$

demon

deuteron

deuteron

dibaryon

dibaryon $(S=-1)$

dibaryon $(S=-2)$

Dy

${ }^{15} \mathrm{D}$ Dy

${ }^{163} \mathrm{Dy}$.

$e^{* t}$

$e^{- \pm}$

$e^{*-}$

$e^{t}$

$e^{-}$

e-color ${ }^{ \pm}$

$\eta$

$\eta(1295)$

$\eta(1440)$

$\eta_{b}$

$\eta_{c}(1 S)$

$\eta^{\prime}$

Eu

even-charged

exotic

exotic-meson

exotjc-nucleon

$f_{0}(1240)$

$f_{0}(1400)$

$f_{0}(1525)$

$f_{0}(1590)$

$f_{0}(1750)$

$f_{0}(700) \dagger$

$f_{0}(975)$

$f_{1}(1285)$

$f_{1}(1420)$

$f_{1}(1510)$

$f_{2}(1270)$

$f_{2}(1720)$

$f_{2}(1810)$

$f_{2}(2010)$

$f_{2}(2300)$

$f_{2}(2340)$

$f_{2}^{\prime}(1525)$

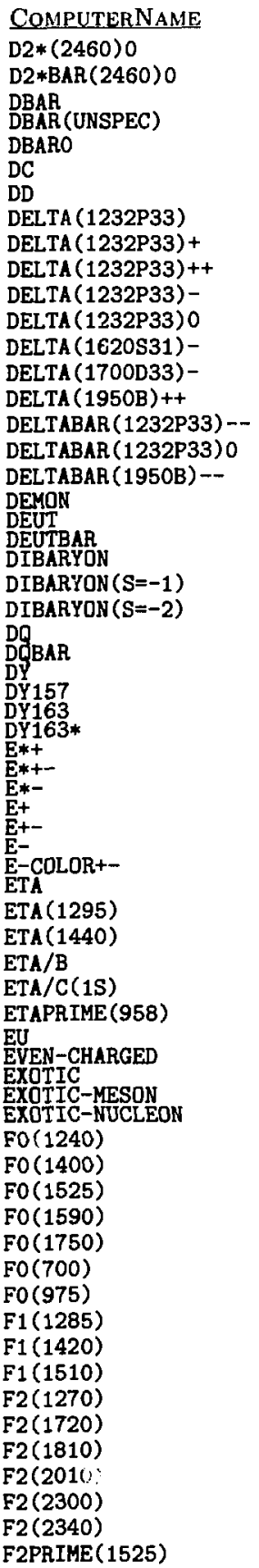

\section{EXPLANATION}

$D^{-}$or $\bar{D}^{\mathbf{0}}$ cliarmed meson

Unspecified anticharmed nonst range meson

$\bar{D}(1865)^{\circ}$ anticharmed nonstrange meson

$D$ or $\bar{D}$ charmed meson

Unspecified diffraction dissociation final state

Bump in production experiment

Bump in production experiment

Exotic 6-quark deuteron-like state

Deuteron

Antideuteron

Dibaryon resonance

Unspecified $S=-1$ dibaryon resonance

Unspecified $S=-2$ dibaryon resonance

Dowil quark

Antidown quark

Dysprosium nucleus

Dysprosium-157 radioactive isotope

Dysprosium-163 radioactive isotope

Excited dysprosium-163 radioact.ve isotope

Excited positron

Excited positron or electron

Excited electron

Positron

Positron or electron

Electron

Colored electron of unspecified charge

$\eta(549)$ meson

Was $\iota(1440)$ - glueball candidate

Lowest mass $J^{P}=0^{-} b \bar{b}$ state

Charnionium meson

$\eta^{\prime}(958)$ meson

Europium nucleus

An even number of charged particles

Unspecified particle which cannot be fit into $q \bar{q}$ or $q q q$ model

Reported manifestiy exotic $\bar{\lambda} \Delta$ meson

Cannot be formed of $q q 9$

Was gs $(1240)$

Was $\epsilon$ (1300). $\pi \pi S$-wave (near $1300 \mathrm{MeV}$ )

Was $S(1730)$

Was $\epsilon(700) . \pi r S$-wave (near $700 \mathrm{MeV}$ )

$I=1 . S$-wave $K \bar{K}$ enhancement

Was $D(1285)$

Was $E(1420)$

Was $D(1530)$

Was $\theta(1690)$ - glueball candidate

Glueball candidate

Was $g_{T}(2320)$ 


\begin{tabular}{|c|c|}
\hline PARTICLENAME & COMPUTERNAMIE \\
\hline$f_{4}(2050)$ & $\mathrm{F} 4(2050)$ \\
\hline$f_{4}(2220)$ & F4(2220) \\
\hline $\begin{array}{l}\text { familon } \\
\mathrm{Fe}\end{array}$ & $\begin{array}{l}\text { FAMILON } \\
\text { FE }\end{array}$ \\
\hline $\begin{array}{l}\mathrm{Fe} \\
\mathrm{Fe}^{2} \mathrm{Fe}^{\circ}\end{array}$ & FE* \\
\hline $\begin{array}{l}33_{\mathrm{Fe}}^{5 e} \\
3^{3+} \mathrm{Fe}\end{array}$ & FE53 \\
\hline 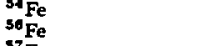 & FE54 \\
\hline $\begin{array}{l}{ }^{57} \mathrm{Fe} \\
{ }^{30} \mathrm{Fe}\end{array}$ & $\begin{array}{l}\text { FE56 } \\
\text { FE57 }\end{array}$ \\
\hline${ }^{61} \mathrm{Fe}$ & $\begin{array}{l}\text { FE59 } \\
\text { FE61 }\end{array}$ \\
\hline $\begin{array}{l}\text { fireball } \\
\mathrm{FI}\end{array}$ & FIREBALL \\
\hline $\begin{array}{l}{ }^{18} \mathrm{FJ} \\
{ }^{10} \mathrm{FJ}\end{array}$ & FL18 \\
\hline $\begin{array}{l}{ }^{10} \mathrm{FJ} \\
\text { frag }\end{array}$ & FL19 \\
\hline $\begin{array}{l}\text { Irag } \\
\text { fragb }\end{array}$ & $\begin{array}{l}\text { FRAG } \\
\text { FRAGB }\end{array}$ \\
\hline $\begin{array}{l}\text { fragt } \\
\text { Ga }\end{array}$ & FRAGT \\
\hline${ }_{\gamma}^{+1 / a} \mathrm{Ga}$ & GA71 \\
\hline $\begin{array}{l}\gamma \\
\text { gaugino }\end{array}$ & $\begin{array}{l}\text { GAMMA } \\
\text { GAUGINO }\end{array}$ \\
\hline${ }_{146}^{\mathrm{Gd}} \mathrm{Gd}$ & $\mathrm{GD}$ \\
\hline $\mathrm{Ge}_{70}^{\mathrm{Gd}}$ & GD146 \\
\hline $\begin{array}{l}{ }^{70} \mathrm{Ge} \\
{ }^{71} \mathrm{Ge}\end{array}$ & GE70 \\
\hline${ }^{72} \mathrm{Ge}$ & GE72 \\
\hline $\begin{array}{l}{ }^{76} \mathrm{Ge} \\
\text { glueball }\end{array}$ & $\overline{G E 7} \overline{6}$ \\
\hline $\begin{array}{l}\text { glueball } \\
\text { gluinium } \\
\text { gluino }\end{array}$ & $\begin{array}{l}\text { GLUEBALL } \\
\text { GLUINIUIN } \\
\text { GLUIN }\end{array}$ \\
\hline gluon & GLUDN \\
\hline $\begin{array}{l}\text { goldst tino } \\
\text { goldstone }\end{array}$ & $\begin{array}{l}\text { GOLDSTINO } \\
\text { GOLDSTONE }\end{array}$ \\
\hline gravitino & GRAVITINO \\
\hline grey & GREY \\
\hline
\end{tabular}

$h_{1}(1170)$

$h_{1}(1380)$

${ }^{3} \mathrm{H}_{S}$

${ }^{4} \mathrm{H}$

${ }^{4} \mathrm{H}_{\mathrm{S}}$

${ }_{\text {hadron }}^{\mathrm{H}}$

nadron(s)

hadron ${ }^{+}$

hadron-

$\mathrm{He}$

$\mathrm{He}^{-}$

${ }^{3} \mathrm{He}$

${ }^{3} \mathrm{He}$

"He

${ }^{3} \mathrm{He}$

${ }^{5} \mathrm{He}_{S}$

$\mathrm{He}$

Hess

${ }^{6} \mathrm{He}$

heavy-e

heavy-lepton

beavy-iepton ${ }^{+}$

heavy-leptont

heavy-lepton-

heavy-iepton ${ }^{0}$

heavy-lepton

heavy- $\nu$

beavy $\nu_{e}$

beavy- $\nu_{\mu}$

Hf

${ }_{173} \mathrm{Hf}$

${ }_{18} \mathrm{H}_{\mathrm{H}}$

higgs

higgst

higgs \pm

\section{EXPLANATION}

Wes $h(2030) . I=0, J^{P}=4^{+}$meson resonance

Was $\xi(2220)$. Mlessn seen in $J / \psi(1 S)$ decays

Massless axion-like Nambu-Goldstone boson Iron nucleus

Excited iron nucleus

1ron-52 nucleus

Jron-53 nucleus

Iron-54 nucleus

Iron-56 nucleus

Iron. 57 nucleus

Iron-59 nucleus

Iron-61 nucleus

Fluorine nucleus - note name is not same as chemical symbol

Fluorine-18 nucleus - note name is not same as chemical symbol

Fluorine-19 nucleus - note name is not same as chemical symbol Nuclear fragment

Fragment of beam

Fragment of target

Gallium nucleus

Gallium-71 nucleus

Photon

Spin-1/2 supersymmetric partner of any gauge boson

Gadolinium nucleus

Gadolinium-146 nucleus

Germanium nucleus

Germanium-70 nucleus

Germanium-71 nucleus

Germanium-72 nucleus

Germanium-76 nucleus

Unspecified glueball

Bound state of gluinos

Spin-1/2 supersymmetric partner of the gluon

Supersymmetric partner of the Goldstone boson

Goldstone boson

Spin-3/2 supersymmetric partner of graviton

Emulsion track reported as grey

(mostly protons in the range $30-400 \mathrm{MeV} / \mathrm{c}$ )

Hypernucleus with $\Lambda$ instead of neutron

Hydrogen-4 nucleus

Hypernucleus with $\Lambda$ instead of neutron

Hydrogen-5 nucleus

Unspecified hadron

One or more unspecified hadrons

Unspecified positive hadron

Unspecified negative hadron

Helium nucleus

Excited helium nucleus

Helium-3 nucleus

Antihelium-3 nucleus

Helium-4 nucleus

Helium-5 nucleus

Helium -5 hypernucleus with strangeness $=-1$

Helium-6 nucleus

Helium-6 hypernucleus with strangeness $=-2$

Helium-8 nucleus

Unspecified heavy electron

Unspecified heavy lepton

Unspecified positive heavy lepton

Unspecifed charged heavy lepton

Unspecifird negative heavy lepton

Unspccified neutral heavy lepton

Unspecified heavy lepton

Unspecified heavy neutrino

Unspecified heavy electron theutrino

Unspecified heavy muon neutrino

Hafnium nucleus

Hafnium-173 radjoactiva isotope

Mercury nucleus

Mercury-196 nucleus

Higgs boson

Positive Higgs boson

Charged Higgs of unspec,.led clarge

Entries in order of the equivalent English spejling of the particle name.

$t$ Particle not listed in 1990 Review of Parlicle Properties. Namic may not conform to naning conventions. 


\begin{tabular}{|c|c|c|}
\hline PARTIClENaAE & COMPUTERNAME & EXPLANATION \\
\hline $\begin{array}{l}\text { higgs- } \\
\text { higgsino }\end{array}$ & $\begin{array}{l}\text { HIGGS- } \\
\text { HIGGSINO }\end{array}$ & $\begin{array}{l}\text { Negative Higgs boson } \\
\text { Spin-1/2 supersymmetric partner of any Higgs boson }\end{array}$ \\
\hline $\begin{array}{l}\text { Ho } \\
{ }_{103} \mathrm{Ho} \\
{ }^{105} \mathrm{Ho}\end{array}$ & $\begin{array}{l}\text { Hू } \\
\text { HOํ. } \\
\text { Ho163 }\end{array}$ & $\begin{array}{l}\text { Holmium nucleus } \\
\text { Holmium- } 163 \text { nucleus } \\
\text { Holmium- } 165 \text { nucleus }\end{array}$ \\
\hline ht rack & HTRACK & Heavy tracks (black or grey) in emulsion \\
\hline $\begin{array}{l}\text { hypernucleus } \\
\text { hyperon } \\
\text { hyperon }\end{array}$ & $\begin{array}{l}\text { HYPERNUCLEUS } \\
\text { HYPERRON } \\
\text { HYPERONBAR }\end{array}$ & $\begin{array}{l}\text { Unspecified hypernucleus, generally containing more than two baryons } \\
\text { Unspecified hyperon } \\
\text { Unspecified antihyperon }\end{array}$ \\
\hline $\begin{array}{l}\text { hyperon } \\
\text { hiso } \\
\text { 120 }\end{array}$ & $\begin{array}{l}\text { HYPERONBAR } \\
\text { I119 } \\
\text { I120 }\end{array}$ & $\begin{array}{l}\text { lodine-119 radioactive isotope } \\
\text { lodine-120 radioa'tive isotope }\end{array}$ \\
\hline & I1 & Iodine-121 radioacuive isotope \\
\hline 123 & $\begin{array}{l}\mathrm{I} 125 \\
\mathrm{I} 127\end{array}$ & $\begin{array}{l}\text { Iodine- } 125 \text { radioactive isotope } \\
\text { lodine-127 radioactive isotope }\end{array}$ \\
\hline $\ln _{115}$ & In & Indium nucleus \\
\hline 年 & IN115 & \\
\hline inelastic & INELASTIC & $\begin{array}{l}\text { Excited indiurn-116 radiaactive isotope } \\
\text { Same as } \mathrm{X} \text {, (ANYTHING), except elastic excluded }\end{array}$ \\
\hline${ }^{186} \mathrm{Ir}$ & IR186 & Iridium- 186 radioactive isotope \\
\hline$J / \psi(1 S)$ & J/PSI (IS) & \\
\hline jet & JET & $\begin{array}{l}\text { One or more jets } \\
K \text { meson }\end{array}$ \\
\hline$K^{*}(1370)^{+}$ & $\mathrm{K} *(1370)+$ & \\
\hline$K^{*}(1370)^{-}$ & $K *(1370)-$ & \\
\hline$K^{*}(1680)^{+}$ & $K *(1680)+$ & \\
\hline$K^{*}(1630)^{-}$ & $k *(1680)-$ & \\
\hline$K^{-1}(1680)^{0}$ & $K *(1680) 0$ & \\
\hline $\begin{array}{l}K^{*}(892) \\
K^{*}(892)^{+}\end{array}$ & $\begin{array}{l}K *(892) \\
K *(892)+\end{array}$ & \\
\hline $\begin{array}{l}K^{*}(892)^{+} \\
K^{*}(892)^{ \pm}\end{array}$ & $\begin{array}{l}\mathrm{K} *(892)+ \\
\mathrm{K} *(892)+-\end{array}$ & \\
\hline $\begin{array}{l}K^{*}(\mathbf{8 9 2})^{ \pm} \\
K^{*}(\mathbf{8 9 2})^{-}\end{array}$ & $\begin{array}{l}\mathrm{K} *(892)+- \\
\mathrm{K} *(892)-\end{array}$ & \\
\hline $\begin{array}{l}K^{*}(892)^{-} \\
K^{*}(892)^{0}\end{array}$ & $\begin{array}{l}K *(892)- \\
K *(892) 0\end{array}$ & \\
\hline$K^{*}$ (unspec) & K* (UNSPEC) & Unspecified $K^{*}$ \\
\hline$K^{*}$ (unspec) ${ }^{+}$ & $K *($ UNSPEC $)+$ & Unspecified $K^{*+}$ \\
\hline$K^{*}$ (unspec) $^{-}$ & $\mathrm{K} *$ (UNSPEC) - & Unspecified $K^{*-}$ \\
\hline$K^{\bullet}$ (unspec) $^{0}$ & $\mathrm{~K} *(\mathrm{UHSPEC}) \mathrm{O}$ & Unspecified $K^{* 0}$ \\
\hline $\bar{K}^{\prime \prime}(1370)^{0}$ & $K * B A R(1370) 0$ & \\
\hline $\bar{K}^{*}(1680)^{0}$ & $K * B A R(1680) 0$ & \\
\hline$K^{*}(892)$ & $\mathrm{K}$ *BAR(892) & \\
\hline $\bar{K}^{*}(\mathbf{8 9 2})^{\circ}$ & $K * B A R(892) 0$ & \\
\hline $\bar{K}^{*}$ (unspec) $^{0}$ & K*BAR(UNSPEC) 0 & Unspecified $\bar{K}^{* 0}$ \\
\hline $\begin{array}{l}K^{+} \\
K^{ \pm}\end{array}$ & $\begin{array}{l}\mathrm{K}+ \\
\mathrm{K}+-\end{array}$ & $\begin{array}{l}\text { Ordinary } K^{+} \text {meson } \\
\text { Ordinary } K^{+} \text {or } K^{-} \text {mesor }\end{array}$ \\
\hline$K^{-}$ & $\mathrm{k}-$ & $\begin{array}{l}\text { Ordinary } K^{+} \text {or } K^{-} \text {meson } \\
\text { Ordinary } K^{-} \text {meson }\end{array}$ \\
\hline $\boldsymbol{K}^{0}$ & KO & Ordinary $K^{0}$ meson \\
\hline$\kappa_{0}^{*}(1950)^{-}$ & $\mathrm{K} 0 *(1950)-$ & \\
\hline $\bar{K}_{0}^{*}(1430)^{0}$ & $K 0 * B A R(1430) 0$ & Was $\kappa(1350)$. Claimed different than $K^{*}(1430)$ \\
\hline $\bar{K}_{0}^{*}(1950)^{0}$ & $K O * B A R(1950) 0$ & \\
\hline$K_{1}(1270)^{+}$ & $K 1(1270)+$ & Was $Q(1280)^{+}$ \\
\hline$\kappa_{1}(1270)^{-}$ & K1(1270)- & Was $Q(1280)^{-}$ \\
\hline$\kappa_{1}(1270)^{0}$ & $K 1(1270) 0$ & Was $Q(1280)^{0}$ \\
\hline$K_{1}(1400)^{+}$ & K1 $(1400)+$ & Was $Q(1400)^{+}$ \\
\hline$K_{1}(1400)^{-}$ & K1 (1400)- & Was $Q(1400)^{-}$ \\
\hline$K_{1}(1400)^{0}$ & $K 1(1400) 0$ & Was $Q(1400)^{0}$ \\
\hline $\bar{K}_{1}(1270)^{0}$ & $\operatorname{K1BAR}(1270) 0$ & Was $\bar{Q}_{1}(1280)^{0}$ \\
\hline $\bar{K}_{1}(1400)^{D}$ & $\operatorname{K1BAR}(1400) 0$ & $W_{a x} \bar{Q}_{1}(1100)^{0}$ \\
\hline$K_{2}^{*}(1430)^{+}$ & $K 2 *(1430)+$ & \\
\hline$K_{2}^{*}(1430)^{-}$ & $K 2 *(1430)-$ & \\
\hline$K_{2}^{*}(1430)^{0}$ & $K 2 *(1430) r$ & \\
\hline $\boldsymbol{K}_{2}^{*}(\mathbf{1 4 3 0})^{0}$ & $K 2 * B A R(: i i) 0$ & \\
\hline$K_{2}^{*}(1980)^{0}$ & K2*BAR ( , ,80j0 & \\
\hline $\mathrm{K}_{\mathbf{3}}(\mathbf{1 7 8 0})^{+}$ & $\mathrm{K} 3 *(1780)+$ & \\
\hline$K_{\mathbf{3}}^{\mathbf{*}(1780)^{-}}$ & $K 3 *(1780)-$ & \\
\hline$K_{3}^{*}(1780)^{\circ}$ & $K 3 *(1780) 0$ & \\
\hline $\boldsymbol{K}_{3}^{*}(1780)^{0}$ & $K 3 * B A R(1780) 0$ & \\
\hline
\end{tabular}




\section{Particlename}

$K_{4}^{*}(2045)^{+}$

$K_{4}^{*}(2045)^{-}$

$K_{4}^{*}(2045)^{0}$

$\bar{K}_{4}^{*}(2045)^{\circ}$

$\bar{K}_{5}^{*}(2380)^{0}$

kaon

$\bar{K}$

kink +

kink-

KK

$K K(L=0)$

${ }^{38} \mathrm{KK}$

${ }^{40} \mathrm{KK}$

${ }^{42} \mathrm{KK}$

${ }^{43} \mathrm{KK}$

${ }^{47} \mathrm{KK}$

$K_{L}$

${ }^{\mathrm{K}} \mathbf{r}$

${ }^{77} \mathrm{Kr}$

${ }^{82} \mathrm{Kr}$

${ }^{84} \mathrm{Kr}$

${ }^{85} \mathrm{Kr}^{*}$

${ }^{80} \mathrm{Kr}$

Ks

${ }_{132} \mathrm{La}$

$132 \mathrm{La}$

${ }^{139} \mathrm{~L}$

$A$

$\Lambda\left(1405 S_{01}\right)$

$\Lambda\left(1520 D_{03}\right)$

$\Lambda N\left(2130^{3} S_{t}\right)^{+} t$

$\Lambda_{c}^{+}$

$\bar{\Lambda}_{c}^{-}$

lepton-quark

e

$e^{+}$

$e^{ \pm}$

tepton-colored

$e^{0}$

$\bar{\ell}^{0}$

$\mathrm{Li}$

${ }^{{ }_{10}^{1 *}} \mathbf{L i}$

${ }^{11} \mathrm{Li}$

${ }^{6} \mathrm{Li}$

${ }^{0} \mathrm{Li}$

${ }^{7} \mathrm{Lis}_{\mathrm{s}}$

${ }^{0} \mathrm{Li}$

${ }^{9} \mathrm{Li}$

longlived

Lu

majoron

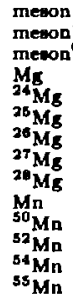

COMPUTERNAME

$\mathrm{K} 4 *(2045)+$

K4* (2045) -

$K 4 *(2045) 0$

K4*BAR (2045) 0

K5*BAR (2380) 0

KAON

KBAR

KBARO

KINK+

KINK-

KK

$\mathrm{KK}(\mathrm{L}=0)$

KK38

KK40

KK42

KK43

Kरㄱ․

KL

KR

KR77

KR81

KR82

$\mathrm{KR} 84$

KR85*

KR88

KS

I

LA131

LA 132

LA139

LAPBDA

LAMBDA (1405S01)

LAMBDA (1520D03)

LAMBDA -N (2130/3S1) +

LAHBDA/C+

LAHBDA/CBAR-

LAMBDABAR

LEPTO-QUARK

LEPTON

LEPTON+

LEPTON+-

LEPTON-

LEPTON-COLOR

LEPTONO

LEPTONBARO

LI.

LI*

LI10

LI6

LI6*

I7

LI7/S

LI8

LI9

LI9/S

LOHGLIVED

LU

MA JORON

MESON

MESON-

MESONO

MG

MG24

G25

726

MC2 7

MG28

T.

H50

M52

754

M55

\section{EXPLANATION}

Kaon or antikson of unspecified charge

$\boldsymbol{K}^{-}$or $\overline{\boldsymbol{K}}^{0}$ ineson

Ordinary $\bar{K}^{0}$ meson

Positive kinking track observed in track detector

Negative kinking track observed in track detector

Potassium nucleus - note name is not same as chemical symbol

Potassium-38 nucleus - note name is not same as chemical symbol Potassium-40 nucleus - note name is not same as chemical symbol Potassium-42 nucleus - note name is not same as chemical symbol Potassium-43 nucleus - note name is not same as chemical symbol Potassium-47 nucleus - note name is not same as chemical symbol $K_{\text {long, }}$ neutral $\boldsymbol{K}$ meson

Krypton nucleus

Krypton-77 radioactive isotope

Krypton-81 nucleus

Krypton-82 nucleus

Krypton-84 nucleus

Excited krypton-85 radioactive irotope

Krypton-88 nucleus

$K_{\text {short, }}$ neutral $K$ meson

Lanthanum nucleus

Lanthanum-131 nucleus

Lantanium- 132 radioactjve isotope

Lanthanum-139 nucleus

Ordinary $\Lambda$ hyperon

$S=-1$ dibaryon resonance

$\Lambda_{c}(2281)^{+} I=0$ charmed baryon

$\bar{\Lambda}_{e}(2281) l=0$ charmed antibaryon

Ordinary $\bar{\Lambda}$ antihyperon

Unspecified lepton

Unspecified positive lepton

Unspecified charged lepton

Unspecified negative lepton

Unspecified lepton carrying color

Unspecified neutral lepton

Unspecified neutral antilepton

Lithium nucleus

Excited lithium nucleus

Lithium-10 nucleus

Lithium-11 nucleus

Lithium-6 nucleus

Excited Jithium-6 nucleus

Lithium-7 nucleus

Hypernucleus with $\Lambda$ instead of neutron

Lithium-8 nucleus

Lithium-9 nucleus

Hypernucleus with $\Lambda$ instead of neutron

Unspecified particle stable under strong and electromagnetic decay

Lutetium nucleus

Hypothetical neutral, spinless, light or massless, penetrating particle. Predicted in some models in which lepton charge conservation is spontaneously broken

Unspecified meson

Unspecified negative meson

Unspecified neutral meson

Magnesium nucleus

Magnesium-24 nucleus

Magnesium-25 nucleus

Magnesium-26 nucleus

Magnesium-27 nucleus

Magnesium-28 nucleus

Manganese nucleus

Manganese-50 nucleus

Manganese-52 nucleus

Manganese-54 nucleus

Manganese-55 nucleus

Entries in o,,$f$ the equivalent English spelling of the particle name.

$\nmid$ Partic. + . in 1990 Review of Particle Properties. Name may not coniorm to naming conventions. 


\section{ParTiclename}

${ }^{\mathrm{se}} \mathrm{Mn}$

Mo

${ }_{90}^{300} \mathrm{Mo}$

${ }^{90} \mathrm{Mo}$

${ }^{20} \mathrm{Mo}^{\circ}$

${ }^{93} \mathrm{Mo}^{*}$

${ }^{24} \mathrm{Mo}$

$98 \mathrm{Mo}$

Ds Mo

monopole

$\mu * t$

$\mu * \pm$

$\mu+$

$\mu^{ \pm}$

$\mu$

muonjum

muonium

mult [black]

mult [charged]

mult [charged $^{+}$]

mult [charged ${ }^{-}$]

mult [charged-hadron]

mult[charged-meson]

mult[deuteron]

mult $\left[\mathrm{e}^{+}\right\}$

mult $\left[e^{-}\right]$

mult $[\eta]$

muit|frag]

inult[fragb]

mult[fragt]

mult $|\gamma|$

mult/grey]

mult|hadron]

mult|hadron ${ }^{+}$]

mult:hadron-\}

mult[hadron ${ }^{0}$ ]

mult[He]

mult[htrack]

mult[jet]

mult $\left.\mid \boldsymbol{K}^{+}\right]$

mult $\left[\boldsymbol{K}^{ \pm}\right]$

mult $\left[\boldsymbol{K}^{-}\right]$

mult $\left[K^{0}\right]$

mult[kaon]

mult[Ks]

mult $[\Lambda]$

mult $[\bar{\Lambda}]$

mult[iepton]

mult/meson]

mult $[\mu]$

mult [n]

mult\{neutral\}

mult:[p]

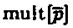

mult $\left[\pi^{+}\right]$

mult $\left\{\pi^{ \pm}\right]$

mult $\left[\pi^{-}\right]$

mult $\left[\pi^{0}\right]$

mult $[\pi]$

mult[shawer]

mult[strange]

$N(1440 B)^{+} \dagger$

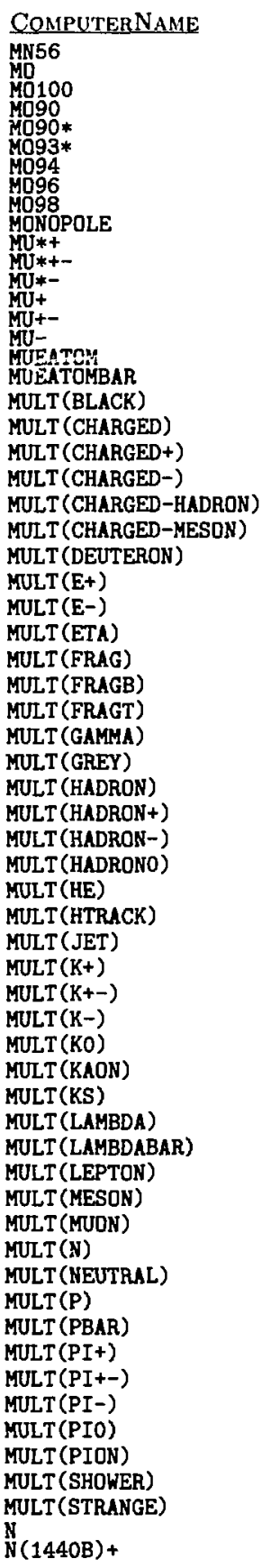

\section{EXPLANATION}

Manganese-56 nucleus

Molybdenum nucleus

Molybdenum-100 nucleus

Molybdenum-90 radionctive isotope

Excited molybdenum-90 radioactive isotope

Excited molybdenum-93 radioactive isotope

Molybdenunl-94 nucleus

Molybdenum-96 nucleus

Molybdenum-98 nucleus

Magnetic monopole

Excited $\mu^{+}$

Excited charged muon

Excited $\mu^{-}$

Ordinary $\mu^{+}$lepton

Ordiuary charged inuon

Ordinary $\mu^{-}$lepton

$\mu^{+} e^{-}$atom

$\mu^{-} e^{+}$atom

Multiplicity distribution for black track

Multiplicity distribution for unspecified charged particle Multiplicity distribution for unspecified positive particle Multiplicity distribution for unspecified negative particle Multiplicity distribution for unspecified charged hadron Multiplicity distribution for unspecified charged meson Multiplicity distribution for deuteron Multiplicity distribution for positron Multiplicity distribution for electron Multiplicity distribution for $\eta(549)$

Multiplicity distribution for nuclear fragment

Multiplicity distribution for beam fragment

Multiplicity distribution for target fragment

Multiplicity listribution for $\gamma$

Multiplicity distribution for grey track

Multiplicity distribution for unspecified hadron

Multiplicity distribution for unspecified positive hadron Multiplicity distribution fot unspecified negative hadron Multiplicity distribution for unspecified neutral hadron Multiplicity distribution for helium nucleus

Multiplicity distribution for heavy tracks (black or grey) in emulsion Multiplicity distribution for jet

Multiplicity distribution for $K^{+}$

Multiplicity distribution for $K^{+}$or $K^{-}$

Multiplicity distribution for $\boldsymbol{K}^{-}$

Multiplicity distribution for $K^{0}$

Multiplicity distribution for kaon of unspecified charge

Multiplicity distribution for $K_{S}$

Multiplicity distribution for $\Lambda$

Multiplicity distribution for $\overline{\boldsymbol{\Lambda}}$

Multiplicity distribution for unspecified lepton

Multiplicity distribution for unspecified meson

Multiplicity distribution for muon of unspecified charge

Multiplicity distribution for neutron

Multiplicity distribution for unspecified neutral particle

Multiplicity distribution for proton

Multiplicity distribution for antiproton

Alultiplicity distribution for $\pi^{+}$

Multiplicity distribution for $\pi^{+}$or $\pi^{-}$

Multiplicity distribution for $\pi^{-}$

Multiplicity distribution for $\pi^{0}$

Multiplicity distribution for pion of unspecified charge

Multiplicity distribution for shower track

Multiplicity distribution for unspecified strange particle

Neutron

Bump in production experiment 
PARTICLENAME

$N(1440 B)^{0} \dagger$

$N\left(1440 P_{11}\right)$

$N\left(1440 P_{11}\right)^{+}$

$N\left(1440 P_{11}\right)^{0}$

$N(1520 B)^{+} \dagger$

$N\left(1680 F_{15}\right)^{+}$

$N\left(1680 F_{15}\right)^{0}$

$N(1700 B)^{+} \dagger$

$N(2100 B)^{+} \dagger$

n(spect)

$N^{*}$ (unspec) ${ }^{+}$

$N_{5 / 2}^{*}(1380)^{+++} \dagger$

$\mathrm{N}_{\mathrm{s} / 2}^{*}(1390)^{+++}$

$N_{s / 2}^{*}(1480)^{+++} \dagger$

$N_{5 / 2}^{*}(1650)^{+++} \dagger$

$N_{\xi / 2}^{*}(1760)^{+++} \dagger$

$N_{5 / 2}^{*}(2070)^{+++}+$

$\mathrm{N}_{\mathrm{s} / 2}$ (unspec) $^{+++} \dagger$

$\mathrm{Na}$

24 $\mathrm{Na}$

$\mathrm{Nb}$

${ }_{90} \mathrm{Nb}$

${ }_{93}^{92} \mathrm{Nb}$

$\overline{\mathbf{n}}$

$\frac{\bar{n}}{N} N(I=0)$

$\bar{N} N(I=1)$

Nd

$\mathrm{Ne}$

${ }^{18} \mathrm{Ne}$

${ }^{20} \mathrm{Ne}$

neutral

neut ralino

$\mathrm{Ni}$

${ }^{56} \mathrm{Ni}$

${ }^{57} \mathrm{Ni}$

${ }^{58} \mathrm{Ni}$

${ }^{60} \mathrm{Ni}$

${ }_{62} \mathrm{Ni}$

${ }_{64}^{62} \mathrm{Ni}$

${ }^{65} \mathrm{Ni}$

Nit

Nit"

${ }^{13} \mathrm{Nic}$

${ }^{14} \mathrm{Nit}$.

${ }^{15} \mathrm{Nit}$

${ }^{16} \mathrm{Nit}$

$N N(2900)^{1} \mathrm{H}_{6}{ }^{++} \dagger$

$N N(2900)^{1} H_{6}^{0} \dagger$

nonres

${ }^{237} \mathrm{~Np}$.

$N \phi(1950) \dagger$

$\nu$

$\nu^{*}$

$\bar{\nu}$

nuclearite

nucleon

nucleus

$\nu_{e}$

$\bar{\nu}_{e}$

$\nu_{\mu}$

$\overline{\boldsymbol{\nu}}_{\mu}$

\section{COMPUTER NAME \\ N $(1440 B) 0$ \\ EXPLANATION \\ Bump in production experiment}

N (1440P11)

N (1440P11)+

$N(1440 \mathrm{P} 11) 0$

$N(1520 \mathrm{~B})+$

$N(1680 F 15)+$

$N(1680 F 15) 0$

$N(1700 \mathrm{~B})+$

$N(2100 B)+$

$N(S P E C T)$

$\mathrm{N} *$ (UNSPEC) +

$N * 5 / 2(1380)+++$

$N * 5 / 2(1390)+++$

$\mathrm{N} * 5 / 2(1480)+++$

$N * 5 / 2(1650)+++$

$N * 5 / 2(1760)+++$

$\mathrm{N} * 5 / 2(2070)+++$

$\mathrm{N} * 5 / 2$ (UNSPEC) +++

NA

$\mathrm{NA} 23$

NA24

$\mathrm{NB}$

NB90

NB92*

NB93

NBAR

NBARN $(I=0)$

$\operatorname{NBARN}(I=1)$

ND

ND 150

NE 18

NE20

NE22

NEUTRAL

NEUTRALINO

NI

NI56

NI57

NI58

NI60

NI60

NI62

Ni64

NI64

NI66

NIT

NIT13

NIT 14

NIT14*

NIT15

NIT16

$\mathrm{NH}(2900 / 1 \mathrm{H} 6)++$

NN $(2900 / 1 \mathrm{H} 6) 0$

NONRES

NP237

NP237*

NPHI (1950)0

NU

NU*

NU*BAR

NUBAR

NUCLEARITE

NUCLEON

NUCLEUS

NUE

NUEBAR

NUMU

NUMUBAR

Bump in production experinent

Bump in production experiment

Spectator neutron

Exotic baryon

$I=5 / 2, S=0$ baryon (exotic)

Exotic baryon

Exotic baryon

Sodium nucleus

Sodium-23 nucleus

Sodium-24 nucleus

Niobium nucleus

Niobium-90 nucleus

Niobium-93 nucleus

Antineutron

Neodymium nucleus

Neodymium- 150 nucleus

Neon nucleus

Neon- 18 nucleus

Neon-20 nucleus

Neon-22 nucleus

Unspecified neutral particle

Nickel nucleus

Nickel-56 nucleus

Nickel-57 nucleus

Nickel-58 nucleus

Nicke!-60 nucleus

Nickel-61 nucleus

Nickel-62 nucleus

Nickel-64 nucleus

Nickel-65 nucleus

Nickel-66 nucleus

Dibaryon resonance

Dibaryon resonance

Unspecified nonresonant state

Neptunium-237 nucleus

Excited neptunium-237 nucleus

Unspecified antineutrino

Unspecified nucleon

Unspecified nucleus

Electron neut rino

Antielectron neutrino

Muon neutrino

Ani,imuon neutrino
$I=$ unspecified, $S=0$ baryon of unspecified mass

$I=5 / 2$ nonstrange baryon (exotic)

$I=5 / 2$ nonstrange baryon (exotic)

Unspecified $I=5 / 2, S=0$ baryon

Excited niobium-92 radioactive isotope

$\bar{N} N I=0$ initial state (and elastic final state)

$\bar{N} N I=1$ initial state (and elastic final state)

Any supersymmetric partner of an ordinary neutral particle

Nitrogen nucleus - note name is not same as chemical symbol

Excited nitrogen nucleus - note name is not same as chemical symbol

Nitrogen-13 nucleus - note name is not same as chemical symbol

Nitrogen-14 nucleus - note name is not same as chemical symbol

Excited nitrogen-14 nucleus - note name is not same as chemical symbol

Nitrogen-15 nucleus - note name is not same as chemical symbol

Nitrogen-16 nucleus - note name is not same as chemical symbol

Reported baryon with $s \bar{s}$ and 3 other quarks

Unspecified neutrino or antineutrino

Excited generic neutrino. Diferent with heavy-lepton ${ }^{\circ}$

Excited generic anti-neutrino. Diferent with heavy-lepton ${ }^{0}$

Proposed new form of strange hadronic matter. Quark nuggets.

Entries in order of the equivalent English spelling of the particle nane.

$\dagger$ Particle not listed in 1990 Review of Particle Properties. Nane may not conform to naming conventions. 


\section{Particlename}

$U_{T}^{U_{T}}$

${ }^{\mathrm{O}} \mathrm{S}$

140

${ }^{16} \mathrm{O}$

${ }^{16} \mathrm{O}^{\circ}$

${ }^{17} \mathrm{O}$

180

odd-charged

$\Omega(2250)^{-}$

$\Omega(2470)^{-}$

$\omega$

$\Omega^{*}$ (unspec)

$\Omega^{-}$

$\Omega_{c}$

$\omega_{3}(1670)$

$\bar{\Omega}+$

$p$

p(spect)

$p^{ \pm}$

Pb

${ }^{\mathrm{Pb}}{ }^{\circ} \mathrm{Pb}$

$190 \mathrm{~Pb}$

${ }^{200} \mathrm{~Pb}$

$201 \mathrm{~Pb}$

$202 \mathrm{~Pb}$

${ }^{202} \mathrm{~Pb}$

${ }^{203} \mathrm{~Pb}$

${ }^{208} \mathrm{~Pb}$

$\bar{p}$

$\bar{p}$ (spect)

${ }^{\mathrm{Pd}} \mathrm{Pd}$

$\mathrm{Ph}$

${ }^{30} \mathrm{Ph}$

$\phi$

$\phi(1680)$

$\phi_{3}(1850)$

photino

$\pi+$

$\pi+$

$\pi^{ \pm}$

$\pi^{\circ}$

$\pi_{2}(1670)^{-}$

$\pi_{2}(1670)^{0}$

atom $(\pi \mu)$

$\pi \pi(L=0)$

pomeron

positronium

posit ronium

${ }_{130} \mathrm{Pr}_{\mathrm{r}}$

$\psi(2,5)$

$\psi(3770)$

$\psi(4040)$

$\psi(4160)$

$\psi(4415)$

Pt

${ }^{106} \mathrm{Pt}$

9

$q^{*}$

\begin{tabular}{|c|}
\hline 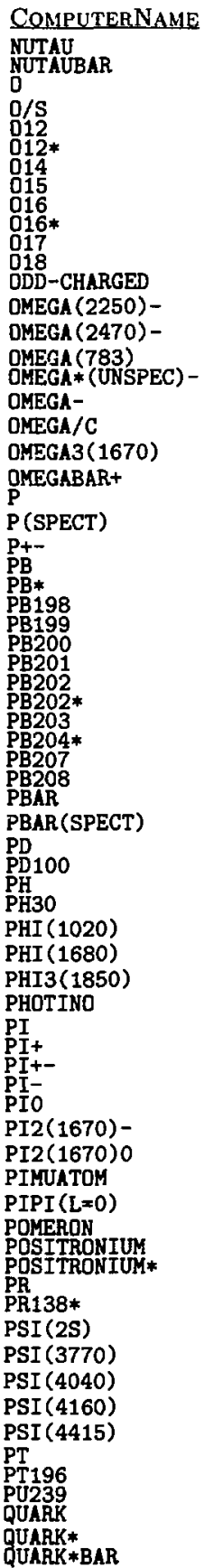 \\
\hline
\end{tabular}

\author{
EXPLANATION \\ $\tau$ neutrino \\ Anti-t neutrino \\ Oxygen nucleus \\ Oxygen hypernucleus \\ Oxygen-12 nucleus \\ Excited oxygen-12 nucleus \\ Oxygen-14 nucleus \\ Oxygen-15 nucleus \\ Oxygen-16 nucleus \\ Excited oxygen-16 nucleus \\ Oxygen- 17 nucleus \\ Oxygen-18 nucleus \\ An odd number of charged particles
}

$I=$ unspecified, $S=-3$ baryon of unspecified mass

Ordinary $\Omega^{-}$hyperon

$\Omega_{c}(2740)^{0} I=0$ charmed doubly strange baryon

Ordinary $\bar{\Omega}^{+}$antihyperon

Proton

Spectator proton

Proton or antiproton

Lead nucleus

Excited lead nucleus

Lead-198 radioactive isotope

Lead-199 radioactive isotope

Lead-200 radioactive isotope

Lead-201 radioactive isotope

Lead-202 radioactive isotope

Excited lead-202 radioactive isotope

Lead-203 radioactive isotope

Excited lead-204 radioactive jsotope

Lead-207 nucleus

Lead-208 nucleus

Antiproton

Spectator antiproton

Palladium nucleus

Palladium-100 radioactive isotope

Phosphorus nucleus - note name is not same as chemical symbol

Phosphorus-30 nucleus - note name is not same as chemical symbol

Bump in $K^{+} K^{-}$mass

Spin-1/2 supersymmetric partner of the photon

Pjon of unspecified charge

Ordinary $\pi^{+}$meson

Ordinary $\pi^{+}$or $\pi^{-}$meson

Ordinary $\pi^{-}$meson

Ordinary $\pi^{0}$ meson

Was $A(1680)^{-}$

Was $A(1680)^{\circ}$

$\pi-\mu$ coulomb bound state

$\pi \pi S$-wave state

Excited positronium

Praseodymium nucleus

Excited praseodymium-138 radioactive isotope
Platinum nucleus

Platinum-196 nucleus

Plutonium-238 nucleus

Quark of charge 2/3

Excited quark

Excited antiquark 


\section{Particlename}

$\overline{\mathbf{9}}$

$\mathrm{Ri}_{\mathrm{Rb}}$

${ }^{81} \mathrm{Rb}$.

Re

Rb

$\rho(1450)^{0}$

$\rho(1700)$

$\rho(1700)^{\circ}$

$\rho$

$\rho^{+}$

$\rho^{-}$

$\rho_{3}(1690)^{0}$

$\mathbf{R u}_{100}$

$100 \mathrm{Bu}$ *

${ }^{103} \mathrm{Ru}$

$\mathrm{S}_{28 \mathrm{~S}}$

${ }^{28} \mathrm{~S}$

${ }^{32} \mathrm{~S}$

${ }^{35} \mathrm{~S}$

${ }^{36} \mathrm{~S}$

${ }_{110}^{\mathrm{Sb}}$

$118 \mathrm{Sb}$

${ }^{120} \mathrm{Sb}$

${ }^{122} \mathrm{Sb}$

${ }^{124} \mathrm{Sb}$

${ }^{\mathrm{Sc}} \mathrm{Sc}$

${ }^{43 \mathrm{Sc}}$

4 Sc

${ }^{45} \mathrm{Sc}$

${ }^{47} \mathrm{Sc}$

Se

${ }^{73} \mathrm{Se}$

${ }^{70} \mathrm{Se}$

${ }^{80} \mathrm{Se}$

$\overline{\boldsymbol{e}}$

$\frac{e^{+}}{e^{-}}$

shower

shower ${ }^{+}$

ghower-

Si

${ }^{24} \mathrm{Si}$.

${ }^{28} \mathrm{Si}$

${ }^{30} \mathrm{Si}$

$\Sigma$

$\Sigma\left(1385 P_{13}\right)^{+}$

$\Sigma\left(1385 P_{13}\right)^{-}$

$\Sigma\left(1385 P_{13}\right)^{0}$

$\Sigma\left(1660 P_{11}\right)^{+}$

$\Sigma(3170 B)^{+}$

$\Sigma^{+}$

$\Sigma_{c}(2455)^{+}$

$\Sigma_{c}(2455)^{++}$

$\Sigma_{c}(2455)^{0}$

$\Sigma_{c}(2510)^{++} \dagger$

$\bar{\Sigma}_{c}(2455)^{--}$

$\Sigma_{c}(2455)^{0}$

$\Sigma^{0}$

$\Sigma\left(1385 P_{13}\right)^{+}$
ComputerName

QUARKBAR

$\mathrm{RB}$

$\mathrm{RB} 81$

RB81*

$\mathrm{RE}$

내

RHO (1450) 0

RHO (1700)

$\mathrm{RHO}(1700) 0$

RHO (770)

$\mathrm{AHO}(770)+$

RHO (770) -

RHO (770) 0

RHD3 (1690)O

RU

RU100

RU100*

RU103

$S$

S28

S28*

S32

S35

S36

SB

SB116

SB118

SB120

SB122

SB124

C

$\mathrm{SC} 42$

$\mathrm{SC} 43$

SC44

SC45

SC46

SC48

SCA

SE*

SE73

SE76

SE82

SE88

SELECTRO

SELECTRON+

SEIECTRON-

SHOWER

SHOWER+

SHOWER-

SI

$\mathrm{SI} 24$

SI24*

SI28

SI30

SIGKA

SIGKA (1385P13)+

SIGKA (1385P13)-

SIGKA (1385P13)0

SIGKA (1660P11)+

SIGKA (3170B)+

SIGMA+

SIGKA-

SIGKA/C (2455)+

SIGKA/C(2455)++

SIGKA/C (2455) 0

SIGMA/C $(2510)++$

SIGKA/CBAR (2455)--

SIGKA/CBAR (2455) 0

SIGKAO

SIGMABAR(1385P13)+

\section{EXPLANATION}

Antiquark of charge 2/3

Rubidium nucleus

Rubidium-81 radioactive isotope

Excited rubidium-81 radioactive isotope

Rhenium nucleus

Rhodium nucleus

Was $g(1690)$

Ruthenium nucleus

Ruthenium-100 nucleus

Excited ruthenium-100 nucleus

Ruthenium-103 radioactive isotope

Sulfur nucleus

Sulfur-28 nucleus

Excited sulfur-28 nucleus

Sulfur-32 nucleus

Sulfur-34 nucleus

Sulfur-35 nucleus

Sulfur-36 nucleus

Antimony nucleus

Antimony-116 nucleus

Antimony-118 nucleus

Antimony-120 nucleus

Antimony-122 nucleus

Antimony-124 nucleus

Scandium nucleus

Scandium-42 nucleus

Scandium-43 nucleus

Scandium-44 nucleus

Scandium-45 nucleus

Scandium- 46 nucleus

Scandium-47 nucleus

Scandium- 48 nucleus

Selenium nucleus

Excited selenium nucleus

Selenium-73 radioactive isotope

Selenium-76 nucleus

Selenium-82 nucleus

Selenium-88 nucleus

Spin-0 superaymmertic partner of the positron or electron

Spin-0 supersymmetric partner of the positron

Spin-0 supersymmetric partner of the electron

Shower track

Positive shower track

Negative shower track

Silicon nucleus

Silicon-24 nucleus

Excited silicon-24 nucleus

Silicon-28 nucleus

Silicon-30 nucleus

Ordinary $\Sigma$ hyperon

Bump in production experiment

Ordinary $\boldsymbol{\Sigma}^{+}$hyperon

Ordinary $\boldsymbol{\Sigma}^{-}$hyperon

$I=1$ charmed baryon

$I=1$ charmed baryon

$I=1$ charmed baryon

Charmed baryon

$I=1$ charmed antibaryon

$I=1$ charmed antibaryon

Ordinary $\Sigma^{0}$ hyperon

Entries in order of the equivalent Engligh spelling of the particle name.

† Particle not listed in 1990 Review of Particle Properties. Nane may not conform to naming conventiony. 


\section{Particlename}

$\bar{\Sigma}\left(1385 P_{13}\right)^{-}$

$\mathbb{E}\left(1385 P_{13}\right)^{0}$

$\Sigma+$

$\bar{\Sigma}-$

ह

$\tilde{\boldsymbol{\varepsilon}}$

$\bar{\ell}^{+}$

$\bar{\varepsilon}^{-}$

${ }_{130}$

${ }_{152}^{152} \mathrm{Sm}$

$\tilde{\mu}^{+}$

Sn

Sn"

${ }_{112} \mathrm{Sn}$

$115 \mathrm{Sn}$

${ }^{116} \mathrm{Sn}$

${ }^{116} \mathrm{Sn}$

$120 \mathrm{sn}$

${ }^{122} \mathrm{Sn}$

${ }^{124} \mathrm{Sn}$

$\bar{v}$

$\bar{\nu}_{e}$

$\vec{\nu}_{e}$

$\tilde{\nu}_{\mu}$

政

sparticle

s

$\overline{\underline{q}}$

star

$\tilde{\tau}^{+}$

strange

supernucleus

Tsi

tachyon ${ }^{+}$

tachyon ${ }^{-}$

$\tau$

T.t

$+$

$\pm$

$r^{-}$

Tb

${ }^{149} \mathrm{~Tb}$

${ }^{259} \mathrm{~Tb}$

Te

${ }^{93} \mathrm{Tc}$

${ }^{94} \mathrm{Tc}$

${ }^{94} \mathrm{Tc}^{*}$

${ }^{95} \mathrm{Tc}$

${ }^{95} \mathrm{TC}$

$\mathrm{Te}$

${ }^{117} \mathrm{Te}$

${ }^{125} \mathrm{Te}$

${ }^{128} \mathrm{Te}$

technipion +

echnipion -

Th

$\mathrm{Ti}$

${ }^{40} \mathrm{Ti}$

${ }^{40} \mathrm{Tj}^{*}$

${ }^{40} \mathrm{Ti}$

${ }^{48} \mathrm{Ti}$

${ }^{198} \mathrm{Tl}$
COMPUTERNAME
SIGMARAR(1385P13)-
SIGMABAR (1385P13)0

SIGMABAR+

SIGMABAR-

SIGMABARO

SLEPTON

SLEPTON+

SLEPTON-

Sy

SM150

SH152

SH152*

SMUDN+

SMUON-

SN

SN*

SN 112

SN115

SN116

SN116*

SN118

SN120

SH 122

SN124

SNU

SNUBAR

SNUE

SNUEBAR

SNUNU

SNUMUBAR

SPARTICLE

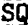

SCBAP

SUUARK

SDUARKBAR

STAR

STAU+

STAU-

STRANGE

SUPERNUCLEUS

$\mathrm{T}$

TA181

TACHYON+

TACYON-

TAU*

TAU*+

TAU*-

TAU+

TAU+-

TAU-

TB

TB149

TB159

TC

TC93

TC93*

TC94

TC94*

TC95

TC98

TE

TE117

TE124

TE125

TE128

TE130

TECHNIPION+

TECHNIPION-

$\mathrm{TH}$

TH232

TI

TI40

TI40*

TI46

TI48

TL198

TL198*

\section{EXPLANATION}

Ordinary $\bar{\Sigma}^{+}$antihyperon

Ordinary $\bar{\Sigma}^{-}$antihyperon

Ordinary $\overline{\mathbf{\Sigma}}^{0}$ antiliyperon

Spin-0 supersymmetric lepton partner

Positive spin-0 supersymmet ric lepton partner

Negative spin- 0 supersymmetric lepton partner

Samarium nucleus

Samarium-150 nucleus

Samarium- 152 nucleus

Excited samarium-152 nucleus

Spin-0 supersymmetric partner of $\mu^{+}$

Spin-0 supersymmetric partner of $\mu^{-}$

Tin nucleus

Excited tin nucleus

Tin-112 nucleus

Tin-115 nucleus

Tin-116 nucleus

Excited tin-116 nucleus

Tin-118 nucleus

Tin-120 nucleus

Tin-122 nucleus

Tin-124 nucleus

Spir-0 supersymmetric part ner of the neutrino

Spin-0 supersymmetric partner of the antineutrino

Spin-0 superyymmetric partner of the $\nu_{c}$

Spin-0 supersymmetric part ner of the $\bar{\nu}_{c}$

Spin-0 supersymmetric partner of $\nu_{\mu}$

Spin-0 supersymmetric partner of the $\bar{\nu}_{\mu}$

Supersymmetric partner of any ordinary particle

Strange quark

Antistrange quark

Spin-0 supersymmetric quark partner

Spin-0 supersymmetric antiquark partner

High charged multiplicity final state

Spin-0 supersymmetric partner of $\tau^{+}$lepton

Spin-0 supersymmetric partner of $\tau^{-}$leptor

Unspecified strange particle

Super heavy nucleus

Tantalum nucleus

Tantalum-181 nucleus

Excited $\tau$ of unspecified charge

Excited $\tau^{+}$

Excited $\tau^{-}$

Ordinary $\tau^{+}$lepton

Ordinary $r$ lepton of unspecified charge

Ordinary $\tau^{-}$lepton

Terbium nucleus

Terbium- 149 nucleus

Terbium-159 nucleus

Technetium nucleus

Technetium-93 radioactive isotope

Excited technetium-93 radioactive isotope

Technetiunl-94 radioactive isotope

Excited technetium-94 radioactive isotope

Technetium- 95 radioactive isotope

Technetium-98 nucleus

Tellurium nucleus

Tellurium-117 radioactive isotope

Tellurium-124 nucleus

Tellurium-125 nucleus

Tellurium-128 nucleus

Tellurium-130 nucleus

Positive technicolor pion

Negative technicolor pion

Thorium nucleus

Thorium-232 nucleus

Titanium nucleus

Titanium-40 nucleus

Excited titanium-40 nucleus

Titanium-46 nucleus

Titanium-48 nucleus

Thallium-198 radioactive isotope

Excited thallium- 198 radioactive isotope 


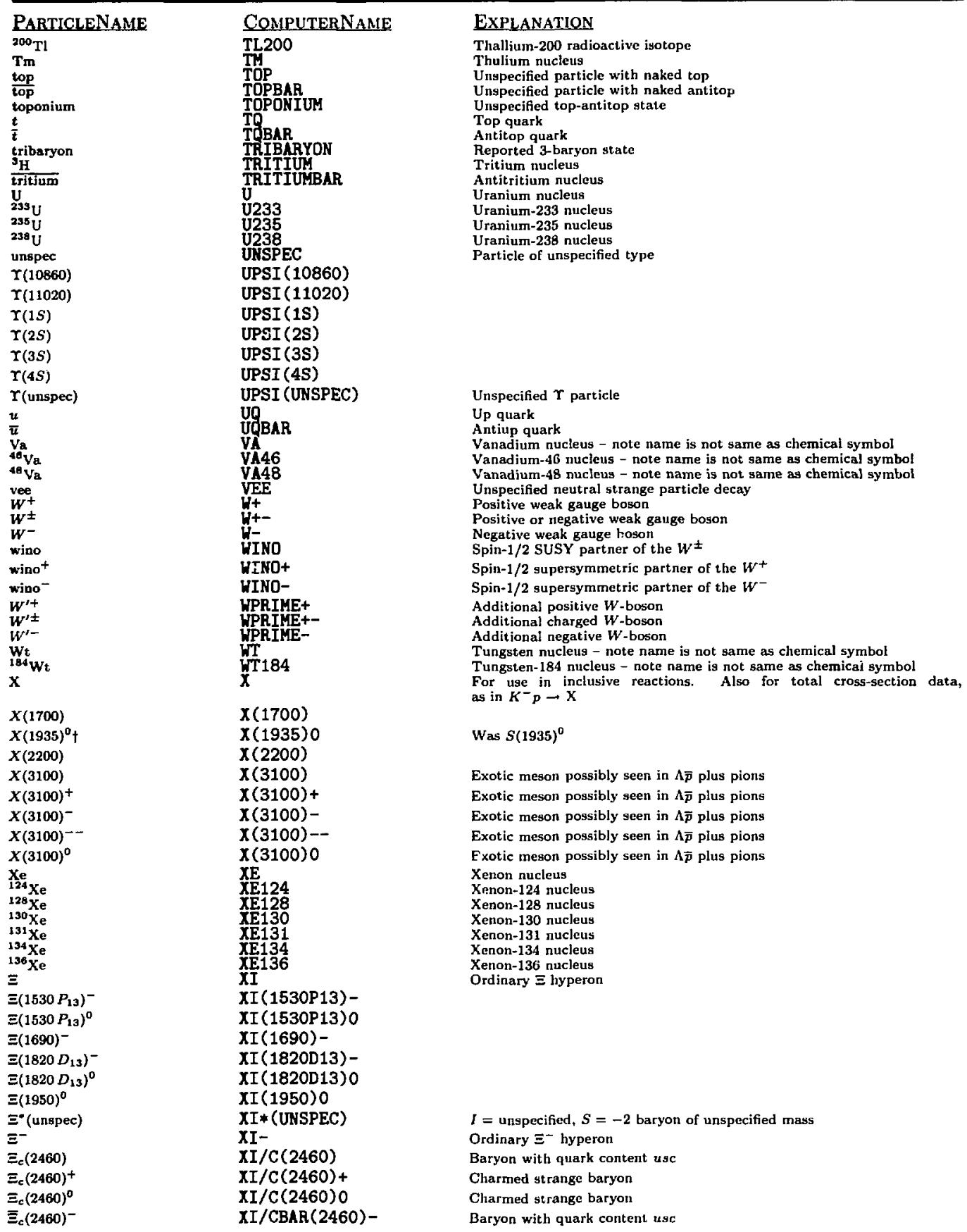

Entries in order of the equivalent English apelling of the particle name.

t Particle not listed in 1990 Review of Particle Properties. Name may not conform to naming conventions. 


\section{Particlename}

$\overline{\mathrm{B}}_{\mathrm{c}}(\mathbf{2 4 6 0})^{0}$

$\Xi^{0}$

$\bar{\Xi}\left(1530 P_{13}\right)^{+}$

$\Xi\left(1530 P_{13}\right)^{0}$

$\bar{\Xi}^{+}$

$\Xi$

$Y^{*}$ (unspec)

$Y^{*}$ (unspec) $^{0}$

$\mathrm{Yb}$

${ }^{Y_{t}} \mathrm{Yt}_{\mathrm{t}}$

${ }^{86} Y_{t}$

${ }^{87} \mathrm{Yt}^{8}$

${ }^{80} \mathrm{Yt}^{0}$

$z^{0}$

zino

$\mathrm{Zn}_{62}$

${ }^{63} \mathrm{Zn}$

${ }^{65} \mathrm{Zn}$

722

$Z^{\prime}$

$\mathrm{Zr}_{\mathbf{2}}$

${ }^{\circ} \mathrm{Zr}$

${ }^{00} \mathrm{Zr}$

${ }^{02} \mathrm{Zr}$

$9 Z_{r}$

${ }^{2} Z_{r}$
COMPUTERNAME

XI/CBAR (2460) O

XIO

XIBAR(1530P13)+

XIBAR (1530P 13) O

XIBAR+

XIBARO

$Y *$ (UNSPEC)

$Y *$ (UNSPEC) 0

YB

YT

YT84

rT

YI87*

YT89

7

ZINO

ZH

2N62

ZN63

2N65

2N72

ZPRIME

$\mathrm{ZR}$

ZR86

2R89

ZR90

ZR92

ZR94

2R96

\section{EXPLANATION}

Charmed strange antibaryon

Ordinary $\Xi^{0}$ hyperon

Ordinary $\bar{\Xi}^{+}$antihyperon

Ordinary $\Xi^{0}$ antihyperon

$I=$ unspecified, $S=-1$ baryon of unspecified mass

$I=$ unspecified, $S=-1$ baryon of unspecified mass

$Y$ tterbium nucleus

Yttrium nucleus - note name is not same as chemical symbol

Ytrium-84 radioactive isotope - note name is not same as chemical symbol Yttrium-86 radioactive isotope - note name is not same as chemical symbol Yttrium-87 radioactive isotope - rote name is not same as chemical symbol Excited yttrium-87 radioactive isotope - note name is not same as chemical symbol

Yttrium-89 nucleus - note name is not same as chemicnl symbol Neutral weak gauge boson

Spin-1/2 supersymmetric partner of the $Z^{0}$

Zinc nucleus

Zinc-62 nucleus

Zinc-63 nucleus

Zinc-65 nucleus

Zinc-72 nucleus

Additional $Z$-boson

Zirconium nucleus

Zirconiunl- 86 radioactive isotope

Zirconium-89 radioactive isotope

Zirconium-90 nucleus

Zirconium-92 nucleus

Zirconiurn-94 nucleus

Zirconium-96 nucleus 
Our names for accelerators are collected below. In most cases, the name is simply an abbreviation of the name of the institution at which the accelerator is located. Where there is more than one accelerator at the same institution, an appropriate modifier is appended, as in CERN-SPS.

For a cosmic ray experiment, we use COSM as the accelerator name, sometimes combined with a specification of the source of the rays.

We use NONE for certain cases, such as proton decay experiments, in which no particle beam is used. We use MANY for certain rapporteur talks or other papers surveying a number of experiments at several (often unspecified) accelerators.

Energies listed are the approximate maximum energies of the circulating beams. 


ACCELERATOR
AERE-HELIOS-NS
ANIK-MEA
ANL
BNL
BONN
BONN-5OO
CERN-ISR
CERN-LEAR
CERN-LEP
CERN-PBAR/P
CERN-PS
CERN-SC
CERN-SPS
CESR
CIT-PELLETRON
COSM
COSM-CYGNUS-X-3
COSM-HERCULES-X-1
COSM-LMC-X-4
COSM-SN1987A
COSM-SLN
DARE-NIIA
DARM-LINAC
DESY
DESY-DORIS-1]
DESY-PETRA
DGSI
FNAL
FNAL-COLLIDER
FNAL-TEV
FRAS-ADONE
GANIL
IND-CYC
INRU-240
ITEP
JINR
JINR-600
KEK-PF-LINAC
KEK-PS
KEK-TRISTAN
KHAR
LAMPF
LASER
LBL-BEVALAC

LBL-CYC-184N

LEBD-650

LEBD-PAHRA

LEN]

LVLN-CYC

MANY

MANZ-LINAC

MIT-BLA

MSU-CYC

MUNT

NBS-IINAC

NONE

NOVO-VEPP-2N

NOVO-VEPP-4

ORSA

ORSA-CYC

ORSA-DCI

PSI

REACTOR

SAAC-CYC

SACL

SACL-LINAC

SACL-SATURNE-II

SERP

SIEG-BEVALAC

SLAC

SLAC-NPI

SLAC-PEP

SLAC-SLC

SLAC-SPEAR

TMSK

TOKY

TRIUMF

VAN-DE-GRAAFF

YERE-ARUS
EXPLANATION

HELIOS ueutron source at Harwell

MEA $e^{-}$linac at NJKHEF

Argonne (ZGS) proton synchrotron $\left(12.7 \mathrm{GeV} / c p_{\mathrm{lnb}}\right)$

Brookhaven (AGS) proton synchrotron $\left(33 \mathrm{GeV} / c p_{\text {(ab }}\right)$

Bonn electron synch rotron $(2.5 \mathrm{GcV} / c$ Piab)

Bonn electron synch rot ron $\left(500 \mathrm{MleV} / \mathrm{c} p_{\text {lab }}\right.$ )

CERN proton-proton collider ( $62 \mathrm{GeV} E_{\mathrm{cm}}$ )

CERN low-energy antiproton rits

CERN large electron-positron collider $\left(120 \mathrm{GeV} E_{\mathrm{cm}}\right)$

CERN $\vec{p} p$ collider $\left(540 \mathrm{GeV} E_{c \mathrm{~m}}\right)$

CERN proton synchrotron ( $28 \mathrm{GeV} / c p_{\text {lat }}$ )

CERN synchrocyclotron ( $600 \mathrm{MeV} T_{\text {lab }}$ )

CERN super proton synchrotron $(450 \mathrm{GeV} / \mathrm{c} \mathrm{par}$ )

Cornell electron-positron storage ring ( $\left.12 \mathrm{GcV} E_{c \mathrm{mn}}\right)$

California Institute of Technology 3-MeV Pelletron accelerator

Cosmic rays

Local source of cosmic ray particles from direction of Cygnus X-3

Local source of cosmic ray particles from direction of Hercules $X-1$

Local source of cosmic ray particles from direction of LMC-X-4

Local source of cosmic ray particles from direction of SN $1987 \mathrm{~A}$

Local source of cosmic ray particles from direction of the Sun

Daresbury electron synch rotron $\left(5.2 \mathrm{GeV} / \mathrm{C} \mathrm{p}_{\mathrm{lab}}\right)$

Darmstadt $65 \mathrm{MeV} / c$ plab linac

Hamburg Deutches electron synchrotron $\left(7.5 \mathrm{GeV} / c p_{l \mathrm{ab}}\right)$

DESY DORIS upgraded in $1977\left(11.2 \mathrm{GeV} E_{\mathrm{cm}}\right)$

DESY electron-positron collider ( $\left.40 \mathrm{GeV} E_{\mathrm{cm}}\right)$

Darmstadt heavy ion facility

FNAL proton synchrotron $\left(500 \mathrm{GeV} / c p_{\mathrm{lab}}\right)$

FNAL $\bar{p} p$ collider ( $2000 \mathrm{GeV} E_{\mathrm{cm}}$ )

FNAL fixed target machine $(1000 \mathrm{GeV})$

Frascatì electron-positron ring $\left(3 \mathrm{GeV} E_{\mathrm{cm}}\right)$

Two coupled isochronous cyclotrons for heavy ions

Indiana University cyclotron facility

Cyclotron of Institute for Nuciear Research, Academy of Sc:-rre Ukr. SSR

ITEP Moscow proton synchrotron ( $7 \mathrm{GeV} / \mathrm{c} p_{\mathrm{lab}}$ )

JINR Dubna proton synchirotron ( $\left.10 \mathrm{GeV} / \mathrm{c} p_{\text {lab }}\right)$

JINR Dubsa synchrocyclotron $\left(600 \mathrm{MeV} T_{\operatorname{lnb}}\right)$

KEK electron linac $(2.5 \mathrm{GeV})$ for photon factory and TRISTAN

KEK proton synchrotron (12 GeV plab)

KEK electron-positron collider $\left(64 \mathrm{GeV} E_{\mathrm{cm}}\right)$

Electron linear accelerator ( $2 \mathrm{GeV}$ plnb) at Kharkov Physico-Technical Insl., Ukr. Acad. Sci.

Los Alamos meson/proton factory

Laser as a source of $\gamma$ 's

Tanciem com'snation of LBL-HLLAC atd Bevatron. Accelerates ions up to Fe $(2.1 \mathrm{GeV} /$ nucleon for charge/masy $=$ $0.5,4.9$ ?or protons)

LBL 184-inch cyclotron (934 MeV for * He). Shut down in 1987

Lebedev Physics Inst. synchrotron $\left(650 \mathrm{MeV} / \mathrm{c} p_{\mathrm{ksb}}\right)$

Lebedev Physics Inst. 1.2 GeV electron synchrotron

Leningrad Inst. of Nucl. Phys. synchrocyclotron ( $1 \mathrm{GeV} T_{\mathrm{lab}}$ )

Isochronous cyclotron at University of Louvain

Used (rarely) for reviews and compilations

Electron LiNAC at Mainz $\left(300 \mathrm{MeV} / c p_{\text {lab }}\right)$

MIT electron LINAC ( $780 \mathrm{MeV} E_{\text {lab }}$ )

Michigan State Univ. superconducting cyclot ron to $40 \mathrm{MeV}$

Accelerator of the Munich Technical University (Munich. FRG)

Linear $100 \mathrm{MeV}$ accelerator

No accelerator used

Electron-positron storage ring at Novosibirsk (1.4 GeV $\left.E_{\mathrm{cm}}\right)$

Electron-positron ring at Novosibirsk, also a syr:chrotron radiation source $\left(7-10.4 \mathrm{GeV} E_{\mathrm{cm}}\right)$

Orsay electron linear accelesator $(2 \mathrm{GeV} / c$ p plo $)$

Orsay synchrocyclotron $(150 \mathrm{MeV} / \mathrm{c}$ prab $)$

Orsay electron-positron storage ring $\left(3.4 \mathrm{GeV} E_{\mathrm{cm}}\right)$

Schweizerische Inst. für Nuklearforschung ( $590 \mathrm{MeV} T_{\text {lab }}$ )

General nuclear reactor

National Accelerator Center Cyclotron, South Africa

Saclay (Saturne) proton synchrotron (3 GeV/c plab)

Saclay electron LINAC

Saclay proton, deuteron, alpha accelerator $\left(2.55 \mathrm{GeV} T_{\mathrm{lab}}\right)$

IHEP Serpukhov proton syuchiotron ( $76 \mathrm{GeV} / \mathrm{c}$ piab)

Accelerator at Siegen Univ. (FRG)

Stanford electron linear accelerator $\left(40 \mathrm{GeV} / \mathrm{c} \mathrm{plab}_{\mathrm{ab}}\right)$

Stanford nuclear physics injector

Stanford electron-positron ring $\left(30 \mathrm{GeV} E_{\mathrm{cm}}\right)$

Stanford linear electron-positron collider ( $\left.100 \mathrm{GeV} E_{\mathrm{cm}}\right)$

Stanford electron-positron ring $\left(8.4 \mathrm{GeV} E_{\mathrm{ctm}}\right)$

Tomsk electron synclirotron $(1.5 \mathrm{GeV} / c$ plab $)$

INS 'Tokyo electron synclurotron $\left(1.3 \mathrm{GeV} / c p_{\operatorname{lnb}}\right)$

Canadian TRlangle University Mleson Facility (520 MoV $T_{\mathrm{lab}}$ )

General Van-de-Graaff accelerator

Yerevan (ARUS) electron synchrotron $\left(6.1 \mathrm{GeV} / \mathrm{c} \mathrm{m}_{\mathrm{ab}}\right.$ ) 
Here we list detectors and the laboratories at which they are used. The Particle Data Group publication, G. Gidal et al., "Major Detectors in Elementary Particle Physics," LBL-91 Supplement (1985), contains a description and a diagram of about 50 of the largest detectors.

Bubble chamber detector names indicate the fill [we distinguish hydrogen (HBC), deuterium (DBC), helium (HEBC), and heavy liquids (HLBC)], then the chamber name (which is usually simply its size), and finally any qualifiers. The qualifiers are HYB for a predominantly hybrid mode of operation, RAP for a rapid-cycling chamber, and TST for a chamber containing a track-sensitive target. When more than one qualifier is appropriate, we use the one most important to the data at hand. 


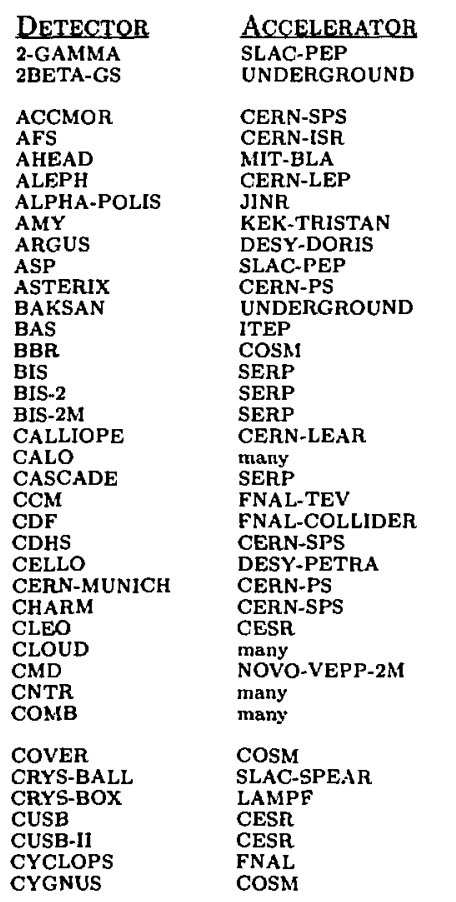

\begin{tabular}{ll} 
DAS & many \\
DBC-12FT & ANL \\
DBC-15FT & FNAL \\
DBC-2M & ITEP,CERN-PS \\
DBC-30IN & ANL,BNL,FNAL,LBL \\
DBC-3OIN-HYB & FNAL \\
DBC-35CM & SACL \\
DBC-40IN-HYB & SLAC \\
DBC-7FT & BNL \\
DBC-801N & BNL \\
DBC-BEBC & CERN-PS \\
DEIS & COSM \\
DELCO & SLAC-PEP \\
DELOS & PSI \\
DELPHI & CERN-LEP \\
DEUTRON-2 & YERE \\
DIOGENE & SACL-SATURNE-II \\
DISC & JINR \\
DISC-3 & JINR \\
DLS & LBL-BEVALAC \\
DM1 & ORSA-DCI \\
DM2 & ORSA-DCI \\
EAS-TOP & COSM \\
& \\
EHS & CERN-SPS \\
ELSSY & MIT-BLA \\
EMC & CERN-SPS \\
EMS & ANL \\
EMUL & ManY \\
EPICS & LAMPF \\
EPOS & DARM-LINAC \\
FANCY & KEK-PS \\
FAS-1 & ITEP \\
FLYSEYE & COSM \\
FMPS & FNAL \\
FODS & SERP \\
FREJUS & UNDERGROUND \\
& \\
& \\
\hline &
\end{tabular}

\section{EXPLANATION}

System of forward detectory for studying mainly the 2- $\gamma$ process

Underground experiment on double $\beta$ decay at the Gran Sasso National Laboratory, LAquila, Italy

Large aperture forward magnetic spectrometer

Axial field spectrometer

Alberta high efficiency analyzer for deutcrons

LEP detector

High resolution lepton detector

Anomalous single photon detector

Antiproton stop experiment $w$ ith trigger on initial $x$ rays

Spectrometer without magnetic field

Cosmic-ray facility at the Black Birch Range in New Zealand

JINR spectrometer, now at JHEP, Serpukhov

Upgrade of BIS

Modification of BIS-2

Magnetic spectrometer at LEAR

Calorimeter

Single crystal target, goni,meter, magnetic spectrometer

Chicago cyclotron magnet spectronieter

Collider detector at Fermilab

CERN-Dortmund-Heidelberg-Saclay-Bologna neutrino detector at SPS (135 tons)

CERN-Max Planck I (Munich) spectrometer

CERN-Hauburg-Amsterdam-Rome-Moscow neut rino detector

Solenoidal magnetic spectrometer

Cloud chamber

Counters (no chambers)

Combinations of different types of detectors. Can include a hybrid system involving a bubble charnber, if the bubble chamber is a minor part oi the uystem

Shower detector at Baksan

Crystal ball

Crystal array detector

High resolution calorimeter

Upgraded CUSB detector

An air-shower array located around the end station of the LAMPF accelerator in Los Alamos. Uses the LAMPF-225 neutrino detector as a inuon detector, with an effective area for muon detection of about $44 \mathrm{~m}^{2}$.

Double arm spectrometer

Deuterium bubble chamber

Deuterium bubble chamber

Deuterium bubble chamber

Deuterium bubble chamber

Deuterium bubble chamber

Deuterium bubble chamber

Deuteriun bubble chamber

Deuterium bubble chamber

Deuterium bubble chamber

Deuterium bubble chamber

Cosmic ray muon spectrometer

LEP detector

Modification of DEUTRON detector

Pictorial drift chamber

Double-arm magnetic spectrometer

Double arm spectrometer

Magnetic detector at Orsay

Detecteur magnetique no. 2

Extended air shower detector on top of the Gran Sasso Mt., at the Gran Sasso National Laboratory, L'Aquila, Italy

European hybrid spectrometer

Electron spect rometer system

European muon collaboration

Effective mass spectrometer

Emulsion. Also used for detectors like PLASTIC where tracks are "frozen" in a solid medium

Energetic pion spectrometer and detection system

Electron-positron solenoid spect rometer

Forward and cylindrical detector system, large acceptance spectrometer covering both projectile and target regions

FLY'S EYE cosmic ray detector

Multiparticle spectrometer at Fermilab

Double-arm spectrometer

Proton decay experiment, tracking calorimeter in the Alps 


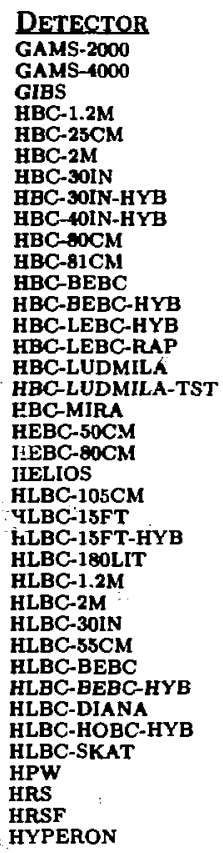

\section{IKAR}

IMB

ISTRA

ISTRA-3

JADE

JANUS

JETSET

KAMIOKANDE-I

KAMIOKANDE-II

KASPI

KGF

L3

LAB-E

LAFRS

LAS

LASS

LEPTON-F

LSD

MAC

MACRO

MANY

MARK-II

MARK-III

MARK-J

MASPIC

MD-1

MEGA

MIS

MMS

MPS

MPS-II

MRS

MTS

ND

NICE

NUSEX

OLYA

OMEGA

OMEGAPRIME

OMICRON

OPAL

\author{
ACCELERATOR \\ SERP \\ CERN-SPS \\ JINR \\ CERN-PS,KEK,JINR \\ JINR.ITEP,JINR \\ ITEP,CERN:-PS \\ ANL, BNL, FNAL,LBL \\ FNAL \\ RHEL,SLAC \\ ITEP,DESY, CERN-PS Hydrogen bubble chamber \\ SACL \\ CFRN-PS \\ CERN-PS \\ CERN-SPS \\ CERN-SPS \\ SERP \\ SERP \\ SERP \\ ANL,ANL

\section{EXPLANATION} \\ Hodoscope gamma-spect rometer \\ Hodoscope gamma-spect rometer \\ Combination of scintillator counters and streamer chamber \\ Hydrogen bubble chamber \\ Hydrogen bubble chamber \\ Hydrogen bubble chamber \\ Hydrogen bubble chamber \\ Hydrogen bubble chamber \\ Hydrogen bubble chamber \\ Little European bubble chamber with hybrid system \\ Little European Bubble Chamber made of lexan \\ Also known as DUBNA chamber, but at Serpukhov \\ Also known as DUBNA chamber, but at Serpukhov \\ $4.5 \mathrm{~m} \times 1.6 \mathrm{~m} \times 1.1 \mathrm{~m}$ cold chamber \\ Northwestern 50-cm helium bubble chamber at ANL
}

ITEP

CERN-SPS

ITEP

FNAL

FNAL

ITEP

CERN-PS,JINR

SERP,JINR

FNAL

JINR

CERN-PS

CERN-PS

ITEP

CERN-SPS

SERP

BNL

SLAC-PEP

LAMPF

SERP

SACL-SATURNE-II

UNDERGROUND

SERP

ITEP

DESY-PETRA

LAMPF

CERN-LEAR

UNDERGROUND

UNDERGROUND

JINR

UNDERGROUND

CERN-LEP

FNAL

LAMPF

LAMPF

SLAC

SERP

UNDERGROUND

SLAC-PEP

UNDERGROUND

many

SLAC.SPEAR

SLAC-SPEAR

DESY-PETRA

JINR

NOVO-VEPP-4

LAMPF

SERP

FNAL

BNL

BNL

TRIUMF

ITEP

VEPP-2M

SERP

UNDERGROUND

NOVO-VEPP-4

CERN-SPS

CERN-SPS

CERN-SC

CERN-LEP

Heavy-liquid bubble chamber

Heavy-liquid bubble chamber

Heavy-liquid bubble chamber

Heavy-liquid bubble chamber

Heavy-liquid bubble chamber

180-liter propane chamber

Heavy-liquid bubble chamber

Propane or xenon chamber

Heavy-liquid bubble chamber

Heavy-liquid bubble chamber

Heavy-liquid bubble chamber

Heavy-liquid bubble chamber

$4.5 \mathrm{~m} \times 1.6 \mathrm{~m}$ warm chambers

Harvard-Penn-Wisconsin neutrino detector at BNL

High resolution spectrometer

High resolution spectrumeter facility doscope counter

Many electrodes ionization chamber

Proton polarimete

compact general purpose detector

Kamioka nucleon decay detector

Kamioka nucleon decay detector, stage-2

LEP detecter

High resolution proton spectrometer

Large aperture solenoid spectrometer

Magnetic calorimeter

Large area detector

Many different detectors

Array of electron and photon spectronieters

Multiparticle spectrometer

Multimuon spectrorjeter with hadron calorimeter

Multiparticle spectrometer

Updated BNL MPS

Medium resolution spectrometer

3-m magnetic spectrometer with spark chambers

Neutral detector at VEPP-2M

Nonmagnetic precision spectrometer at Serpukhov

NUSEX nucleon decay detector, Mont Blanc tunnel
Single arm magnetic spectrometer with big spark and proportional chambers and gas ho-

Irvine-Michigan-Brookhaven nucleon decay detector, Ohio

Channel and $\pi$-meson spectrometer with final particle energy up to $1 \mathrm{GeV}$

1100-ton target-calorimeter muon-spectrometer detector for neutrino physics

SLAC-SPEAR spectrometer system (not related to MARK-II)

LEP detector 


\section{DETECTOR}

ORANGE

OSPK

OTHER

PHOTON

PIOSPEC

PION

PLASTIC

PLASTIC-BALL

PLUTO

PROZA

PROZA-M

RISK

RMS

RONS

SAC-600

SAC-900

SAS

SEMI

SFM

SHIP

SIGMA

SINDRUM!

SINDRUAI-I

SKM-200

SLAC-8GEV

SMM-GRS

SOKOL

SOUDAN-I

SOUDAN-II

SPASE

SPEC

SPEC-6M

SPES-I

SPES-II

SPES-1II

SPES-IV

SPIN

SPRF

STRC

SUPERBENKEI

SUS!

SYSTEMA-1

SYSTEMA-IJ

TAGX

TASSO

TELAS

TISS-3

TOPAZ

TPC

TPS

TRAD

TREAD

TRIUMF-TPC

UAI

UA2

UA4

UA5

UCI-TPC

VENUS

WAS

WIRE
ACCeleratur

DARM-LINAC

many

many

many

LAMPF

COSM

many

LB̈L-BEVALAC

DESY-DORIS

SERP

SERP

SERP

RHEL

REACTOR

SACL-LINAC

SACLAY-LINAC

many

many

CERN-PS

KEK-TRISTAN

SERP

PSI

PSI

JINR

SLAC

COSM

COSM

UNDERGROUND

UNDERGROUND

COSM

many

SERP

SACL-SATURNE-II

CERN-LEAR

SLAC-SATURNE-II

SACL-SATURNEII

ITEP

many

inany

KEK

PSI

COSM

Cosi

TOKY

DESY-PETRA

KEK

ITEP

KEK-TRISTAN

SLAC-PEP

FNAL

many

FNAL

TRIUAF

CERN-PBAR/P

CERN-PBAR/P

CERN-PBAR/P

CERN-PBAR/P

NONE

KEK-TRISTAN

many

many

\section{EXPLANATION}

Beta spect rometer

Optical spark chamber

Rare nonelectronic dctectors (a.g. moon, ocean floor)

Photon spect rometer

Loy Alamos $\pi^{0}$ spectrometer

Hadronic calorimeter with pion-proton identification

Lexan or other such material in wbich tracks are frozen (except enulsion)

Plactic ball detector

Superconducting solenoid spect rometer

Polarized proton target with frozen polarization, gamma spectrometer, neutron detector

Modified PROZA

$4.7 \times 0.9 \times 0.8 \mathrm{mi}^{3}$ streanter charnoer in magnetic field

Magnetic spectrotncter facility

Rovno neutrino spectrometer at Rovmo AES

High resolution electroll scat tering detector

High resolution electron scattering detector

Single arm spect romet er

Semiconductor detector

Split ficld magnet

Detector for search for highly ionizing particles

CERN-[HEP magnetic spectroneter at Serpuklov.

Large-sulid-angle magnetic detector

2-m neon filled streamer chamber

8-GeV spectrometer

Gamma ray spectrometer of the solar maximum mission satellite facility

South Pole air shower experiment

General spectrometer sytitem not filling one of the others or where specific type not given 6-ai spect rometer

High resolution spectrometer

High resolution spectrometer

Saclay Sat urne spect ronieter

High resolution spectroneter

Spark chamber of unspecified type (use WIRE ur OSPK. if possible)

Streanier chamber

Superconducting spectrometer system

Pion spectrometer

Scintillator-ionization spectrometer of two electrons with nultidimensional analysis

Latge-aperture spectroneter system

KEK target-embodied large-aperture spectrotneter

Solenoidal spectrometer with time projection chamber

Time projection chamber

Tagged photon spectrometer

A general transition radiation detector

Fecoil energy and angle detector with mini-time projection chamber

Time projection chamber

Versatile economical and novel universal spectrumeter

Wide angle spectrometer

Wire chambers (proportional wire chambers, drift chambers). Includes all nonoptical spark chambers 
The symbols used to indicate what quantities are measured in an experiment are listed here. They are used in the Reaction/Momentum/Data Descriptor Index. 
DATADESCRIPTOR

\section{A-DEP}

AMP

ANG

ANGP

ASYM

COL

CONST

COR

CS

DME

ET

FLUX

MANY

MASS

MULT

$\mathbf{P}$

POL

PT

PWA

QNC
EXPLANATION

Atomic number dependence

Anplitudes not decomposed into states of definite angular momentum: Re/Im ratio, helicity amplitude, etc. Angular distributions between particles in the finsl state. Includes also angular distributions involving decay producty of particles listed in the reaction, even though those decay products are not themselves explicitly listed. Includes angles used to study the decay of a system in the final state, even though the coordinate system axes may be defined with respect to the incident particley (c.g., Jackson angles, etc.). Also the equivalent, expressed as moments, elc.

Production angular distributions of one or more of the outgoing particles relative to one of the incident particles. Also the equivalent, expressed as moments or polynomial expansion coefficients. Also invariant cross sections a a function of production angle or $t$. By convention, does not include rapidity or its approximation (see P)

Asymmetry in scattering off a polarized target and/or with a polarized beam (with exception of special case noted under POL)

Collective variables (sphericity, thrust, etc.)

Physical constant (Fermi constant, Weinberg angle, ctc.). Used to express that model parameters are extracted from data

General correlator (on momentum, rapidity, etc.)

Cross section, cross section ratio, or cross section upper limit. Can also be listed for very rare reactions whose existence is being established, even though the number of events has not been converted to a cross section. Does not include parametrizations of the cross section, e.g. as a furction of energy

Density matrix elements, including joint density matrix elements

Transverse energy

Cosmic-ray particle Hux

For rare cases when there are many types of data measured

Mass spectrum, or invariant cross section as a funetion of mass

Multiplicity distribution, its average, ratio or moments

Any function of outgoing monentum or energy not included in any others. Includes rapidity and Feynman scaling variables

Final state spin polarization measurement, including Wolfenstein spin rotation parameters, and measurements of the asymmetry off a polarized target when it is equal to the final state polarization

Transverse monientum spectrum, or inviriant cross section as a function of above. Does not include a momentum transfer spectrum (see $A$ NGP). In iudes transverse mas, unlesy the particle mass is also variable

Partial wave amplitudes, including formation partial waves and production partial waves. Any attempt to measure amplitudes of definite angular momentum. Includes scattering length and effective range

Test of quantum-number conservation 